SIATH OF NH W Y OEX.

LIST OF LANDS

IN THE

FOREST PRESERVE.

1814 


$$
\text { ช }
$$





\section{STATE OF NEW YORK \\ CONSERVATION COMMISSION}

\section{List of Lands in the Forest}

Preserve

JANUARY 1, 1914

APPENDIX TO THE THIRD ANNUAL REPORT

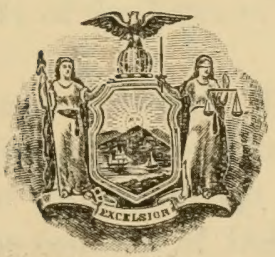




$$
\begin{aligned}
& \frac{712}{1778} \\
& 1914
\end{aligned}
$$

T. AP DE

MIAR 231914 


\section{LIST OF LANDS IN THE FOREST PRESERVE}

\section{REPORT OF THE SUPERINTENDENT OF FORESTS}

Albany, N. Y., January 1, 1914.

Conservation Commission, Albany, N. Y.:

Gentlemen.- I submit herewith a list of lands constituting the Forest Preserve. This information forms the basis for the the entire landed business of this Bureau. The data contained herein is in many ways most important, and the publication from an administrative standpoint more valuable than any other issued by this Division. In this list will be found full information as to what lands the State owns in the Forest Preserve, the acreage of each lot and its location. The source of title is also indicated whether acquired at tax sales, by purchase, or otherwise. The years of the tax sales at which the State acquired a parcel of land are stated. This publication is essential for every one of our forest rangers, and is required for general use in this Division, and there is a demand for this information from a large number of people generally.

Section 50, chapter 444 of the Laws of 1912, defines the Forest Preserve as follows:

"Section 50. Forest Preserve. The Forest preserve shall include the lands owned or hereafter acquired by the state within the county of Clinton, except the towns of Altona and Dannemora, and the counties of Delaware, Essex, Franklin, Fulton, Hamilton, Herkimer, Lewis, Oneida, Saratoga, St. Lawrence, Warren, Washington, Greene, Vlster, and Sullivan, except

1. Lands within the limits of any village or city, and

2. Lands not wild lands acquired by the state on foreclosure of mortgages made to loan commissioners." 
This section provides that all the lands owned by the State within the above named counties, except within the limits of incorporated villages and cities, and certain mortgage title lands, constitute a part of the Forest Preserve. Lands acquired by the foreclosure of United States Deposit Fund mortgages, if not wild, are not part of the Forest Preserve. The Attorney-General has advised that the classification as to whether or not lands are wild should apply as of the time of the foreclosure of the mortgage. Since the last publication of this kind was issued in 1909 an examination of these mortgage lands has been made and those which do not come within the definition have been separated and are included in an appendix to this report. The Attorney-General has also rendered opinions to the effect that lands acquired by the State within these counties for specific purposes and so used did not become a part of the Forest Preserve and, therefore, they are listed separately. Lands within ten miles of Clinton Prison, although within a Forest Preserve county, are not included as a portion of the Forest Preserve.

A list of the various parcels in the St. Lawrence Reservation, or International Park, and other lands owned by the State in the Forest Preserve counties, not classified as Forest Preserve land, are appended for convenient reference but not included in the schedule of the Preserve.

The area of the Preserve by counties, together with information in regard to the number of parcels, sources of titles, ete., are included herewith. 
Conservation Commission.

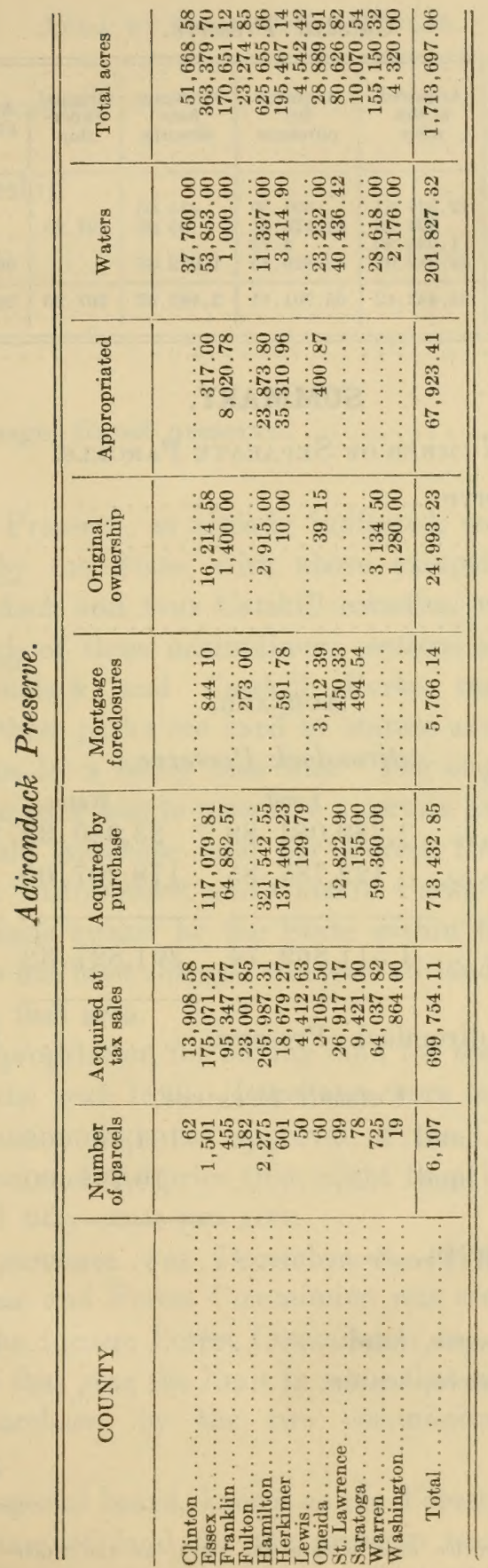


Catskill Preserve.

\begin{tabular}{|c|c|c|c|c|c|c|c|}
\hline COUNTY & $\begin{array}{l}\text { Num- } \\
\text { ber of } \\
\text { parcels }\end{array}$ & $\begin{array}{c}\text { Acquired } \\
\text { at tax } \\
\text { sales }\end{array}$ & $\begin{array}{l}\text { Aequired } \\
\text { by } \\
\text { purchase }\end{array}$ & $\begin{array}{l}\text { Mortgage } \\
\text { fore- } \\
\text { closures }\end{array}$ & $\begin{array}{c}\text { Original } \\
\text { owner- } \\
\text { ship }\end{array}$ & $\begin{array}{l}\text { Appro- } \\
\text { priated }\end{array}$ & $\begin{array}{l}\text { Total } \\
\text { acres }\end{array}$ \\
\hline $\begin{array}{l}\text { Delaware. } \\
\text { Greene... } \\
\text { Sullivan... } \\
\text { Vlster... }\end{array}$ & $\begin{array}{r}105 \\
84 \\
18 \\
536\end{array}$ & $\begin{array}{r}12,527.61 \\
924.00 \\
1,038.80 \\
38,942.01\end{array}$ & $\begin{array}{r}894.50 \\
11,979.01 \\
4.75 \\
42,825.85\end{array}$ & $\begin{array}{r}415.60 \\
95.00 \\
1,972.87\end{array}$ & $\begin{array}{l}297.25 \\
\ldots \ldots \ldots \\
\ldots \ldots\end{array}$ & 2690.0 & $\begin{array}{r}13,837.11 \\
13,295.26 \\
1,043.55 \\
84,009.73\end{array}$ \\
\hline Total. & 743 & $53,432,42$ & $55,701.11$ & $2,482.87$ & 297.25 & 269.00 & $112,185.65$ \\
\hline
\end{tabular}

\section{SUMMARY.}

Nimber of Separate Parcels.

Adirondack Preserve ............... 6,107

Catskill Preserve .................. 743

Total . . . .

Acreage.

Adirondack Preserve.

Land.

*Within the park... 1,389,081.89

Without the park... 122,787.85

Total . . . . 1,511,869.74
Water.

Total.

$23,620.32 \quad 1,412,702.21$ $178,207.00 \quad 300,994.85$

Total Adirondack Preserve....... 1,713,697.06 Catskill Preserve.

*Within the park, land....... 101,436.65

Without the park, land ....... $10,749.00$

Total Catskill Preserve............ 112,185.65

Total Forest Preserve, land . . . . . . . . 1,624,055.39

Total Forest Preserve, water........... 201,827.32

$1,825,882.71$

* Does not include entire area within the park, as the state has acquired at present only a part of the territory. 
Area mi Sourees of Tithes.

Purchased $769,136.96$

Mortgage foreclosures

$8,249.01$

Tax sales $753,186.53$

()riginal ownership

$25,290.48$

Appropriated.

$68,192.41$

Areit, land

$1,62 \pm, 055.39$

Waters.

$201,827.32$

Iotal acreage, forest preserve

$1,825,882.71$

The Forest Preserve, as already indicated, comsists of all the lauds owned by the State, with above exceptions. within the twelve Adirondack and four Catskill counties, while the central fortions of each of these mountainous sections are distinguished as the Adirondack and Catskill Parks respectively. The kunndaries of these parks are fixed by statute and indicated upon our forest maps by a heary blue line. The original purpose of estathlishing the park was to prescribe a certain area within which the state would purchase land for Forest Preserve purposes. The definition "Adirondack and ('atskill Parks" until 1912 applied to the lands owned hy the State within the blue line but since that time has been changed to mean all lands both State and private within that area.

The first appropriation fur huring land for the Forest Preserve Wils made in the rear 1890 . Purchases were to be made br the Furest Commission with the approral of the Commissioners of the Land Office, and the price that might be paid was limited to one dollar and fifty cents per acre.

The first purchase was December 8, 1891. In 1895 the Fisheries, Game and Forest ('ommission was ereated taking over the work of the former Forest Commission and under an appropriation made that year the limit in price that might be paid was removed. Purchases be the two (ommissions amounted to 140,305 acres.

In 1897 a special board, known as the Forest Preserve Board, for the purchase of land for the preserve was created. They 
were authorized to make purehases without the approval of the Commissioners of the Land Office.

Appropriations for the extension of the Forest Ireserve have been made as follows:

1890 .

$\$ 25,000$

1895

600,000

1897

$1,000,000$

1898

500,000

1899

350,000

1900 .

250,000

1904

250,000

1906.

400,000

1907

500,000

1909 .

200,000

Total .

$\$ 4,075,000$

\section{ADironidack Park.}

The area of the Adirondack Park, of which the State owns only a portion, is $3,313,56 t$ acres. Within this area the State owns $1,389,081.89$ acres of land and $23,620.32$ acres of water. The remaining area in the Adirondack forest is outside of the Park. The figures as to the area of these land- are based upon old surveys and, in many cases, are less than the actual acreage.

\section{Catshill Park.}

The Catskill Park, as defined by law, contains 576,120 acres and includes a portion of the following named counties: Delaware, Greene, Ulster and Sullivan. Within the park area the State owns 101,436.65 acres. There are without this section in the Catskill Forest Preserve counties 10,749.00 acres.

This list is compiled on the hasis of the best available information at hand and is published as a matter of information and convenience. The omission of any lands does not necessarily mean that the State does not rlaim title to such premises. In ease detailed information, in regard to the title to any specific premises, is required the original reerds shonld be consulted.

C. R. Petris, Superintendent of State Forests. 


\title{
LIST OF LANDS
}

BELONGING TO

\section{THE FOREST IPRESEIRVE}

\author{
JANUARY 1,1914
}

\section{CLINTON COUNTY}

(Total number of acres, 51,668.58.)

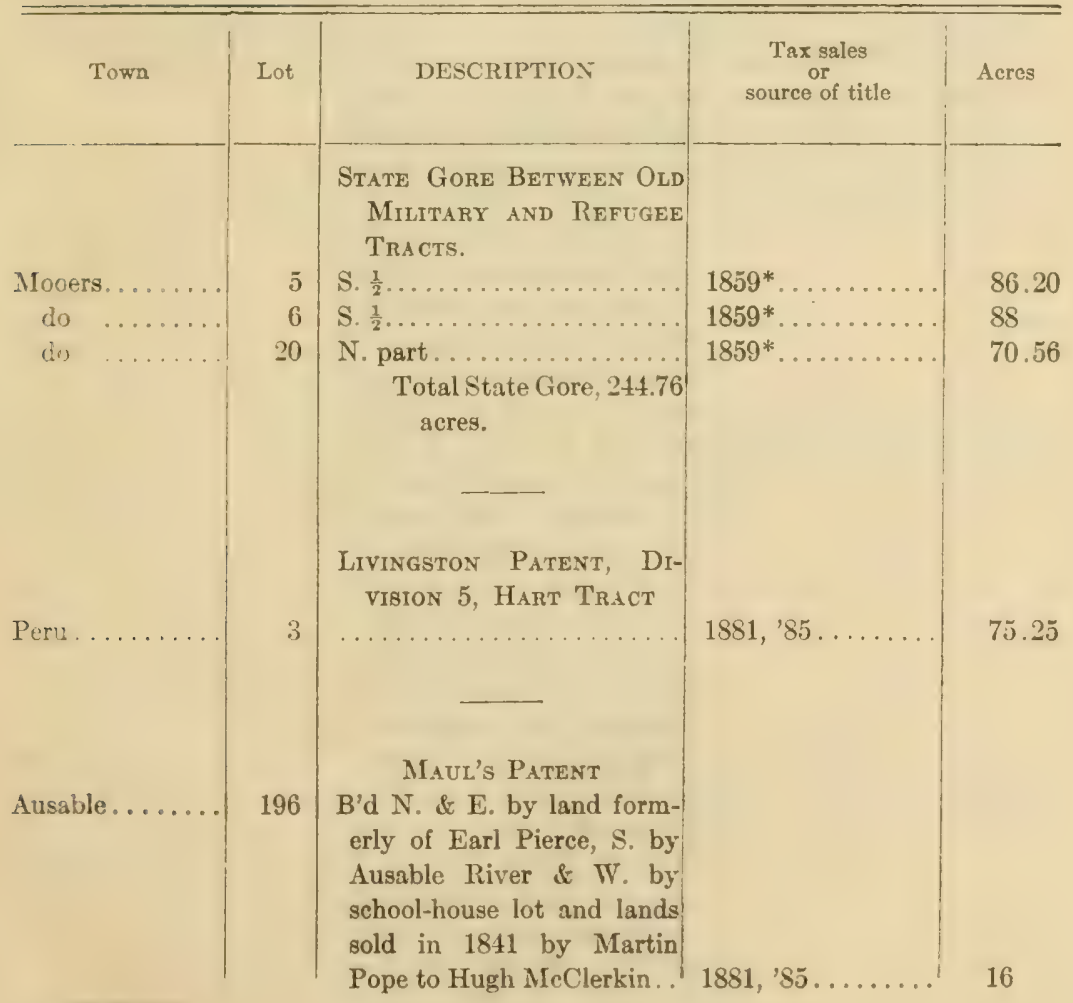


Cuinton County

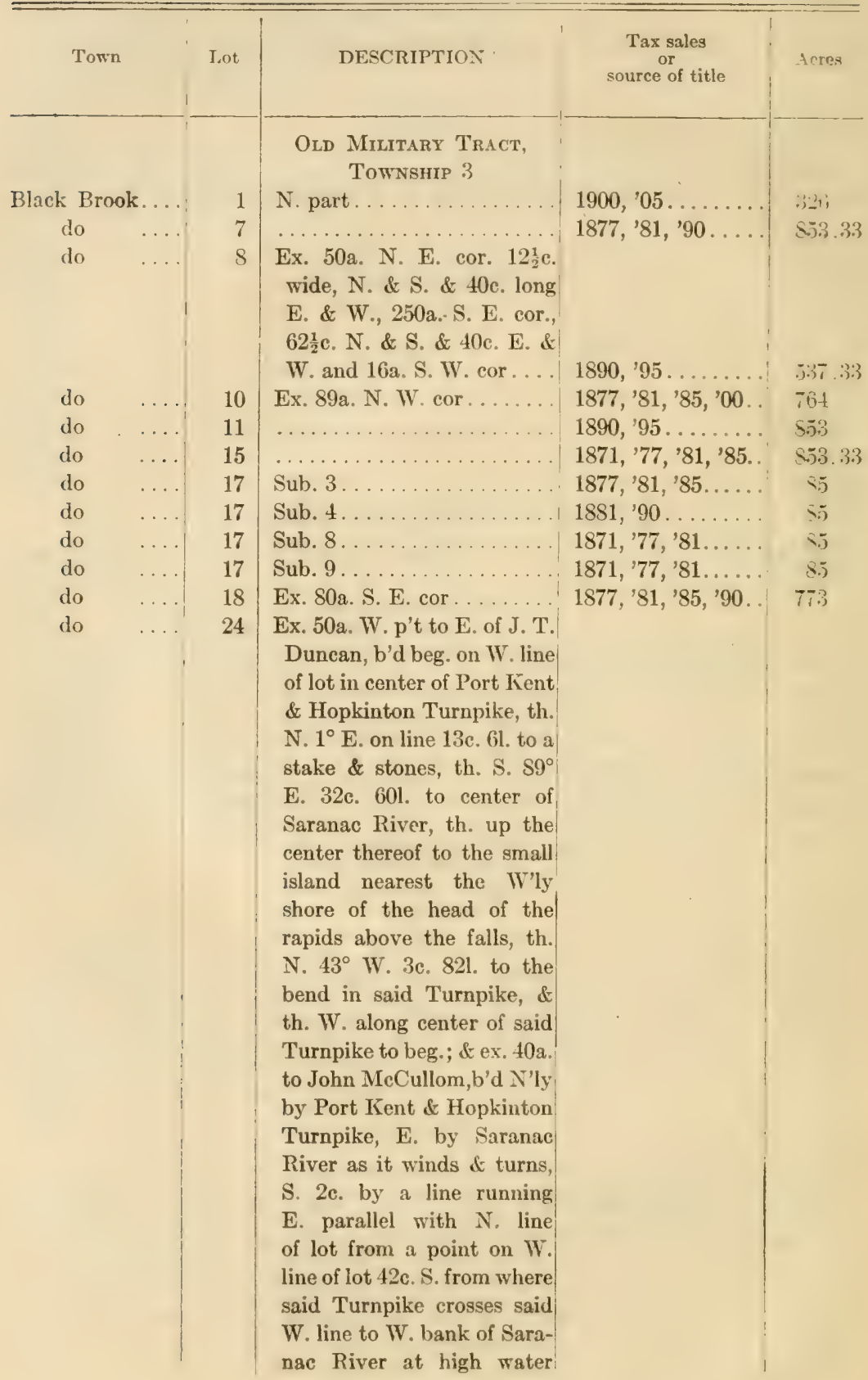


Chinton County

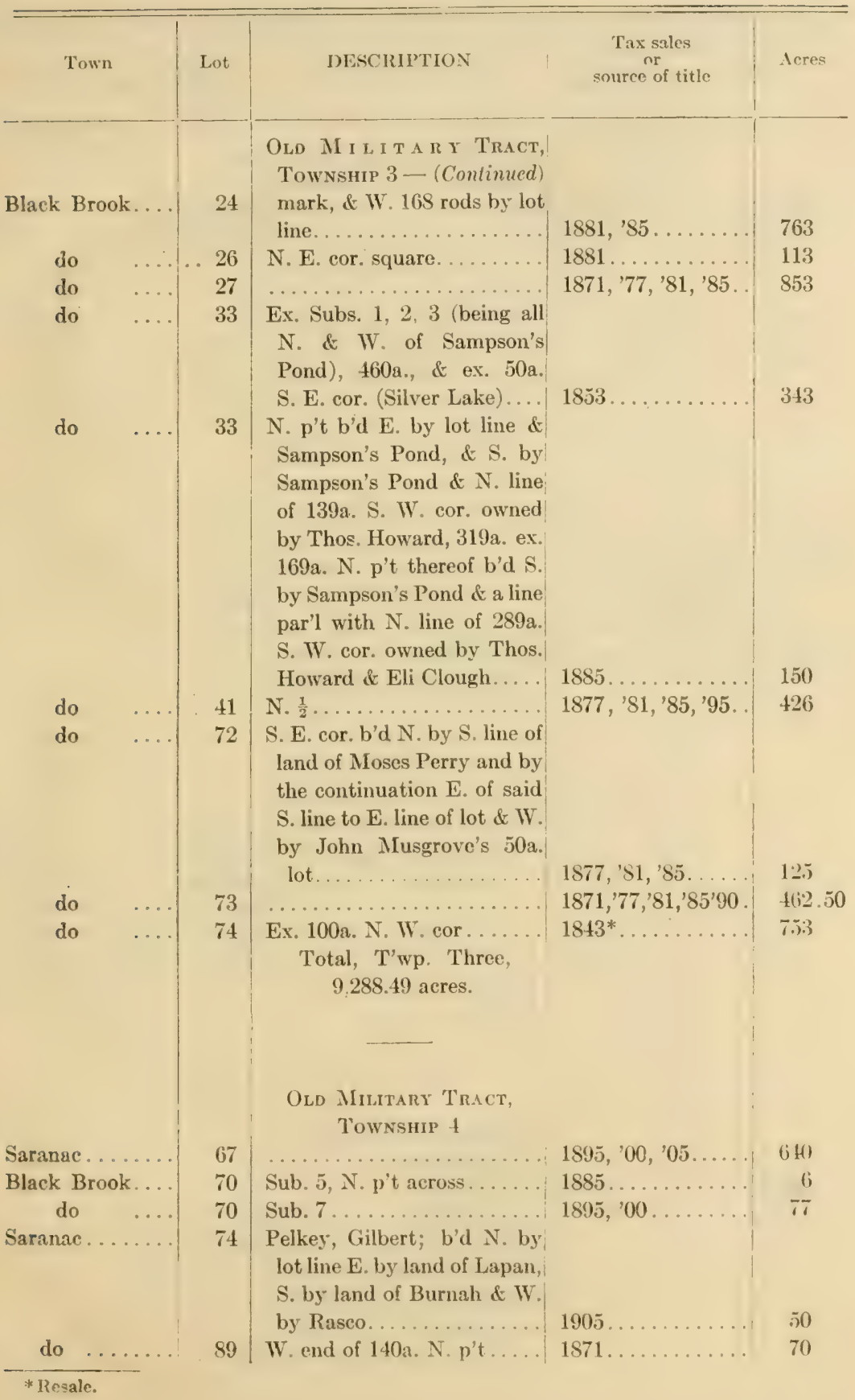


Cunton County

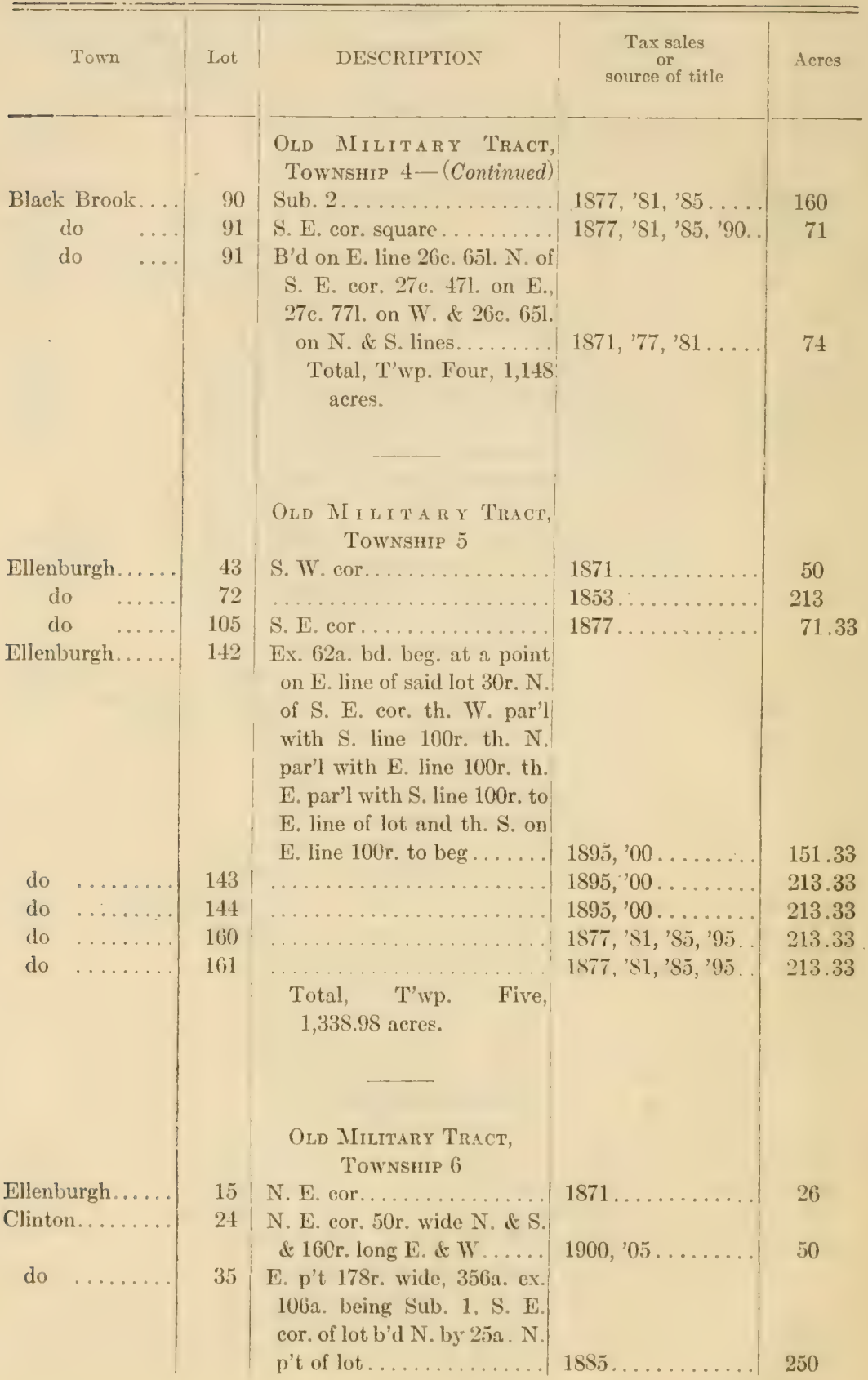


Cuinton County

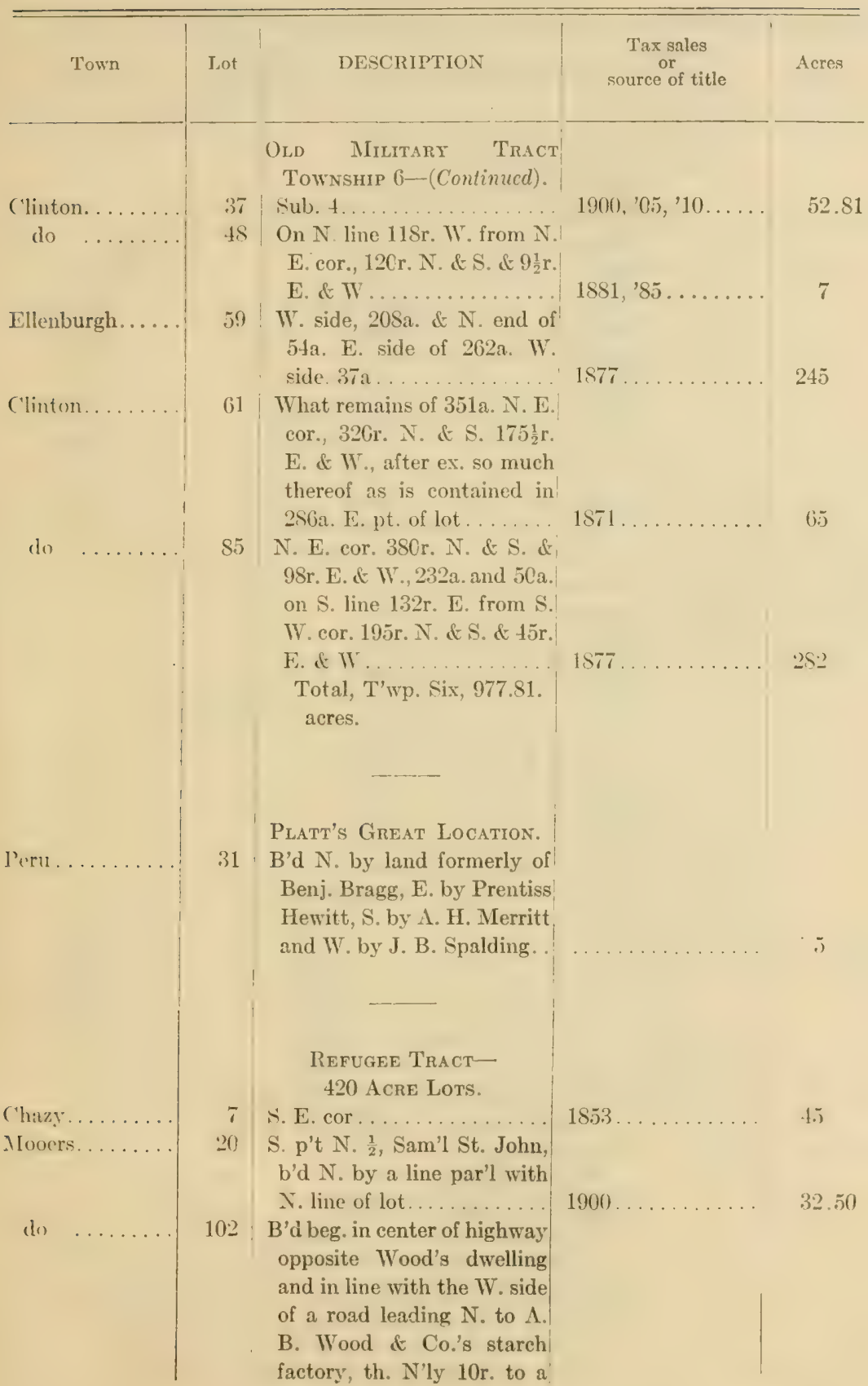




\section{Chinton County}

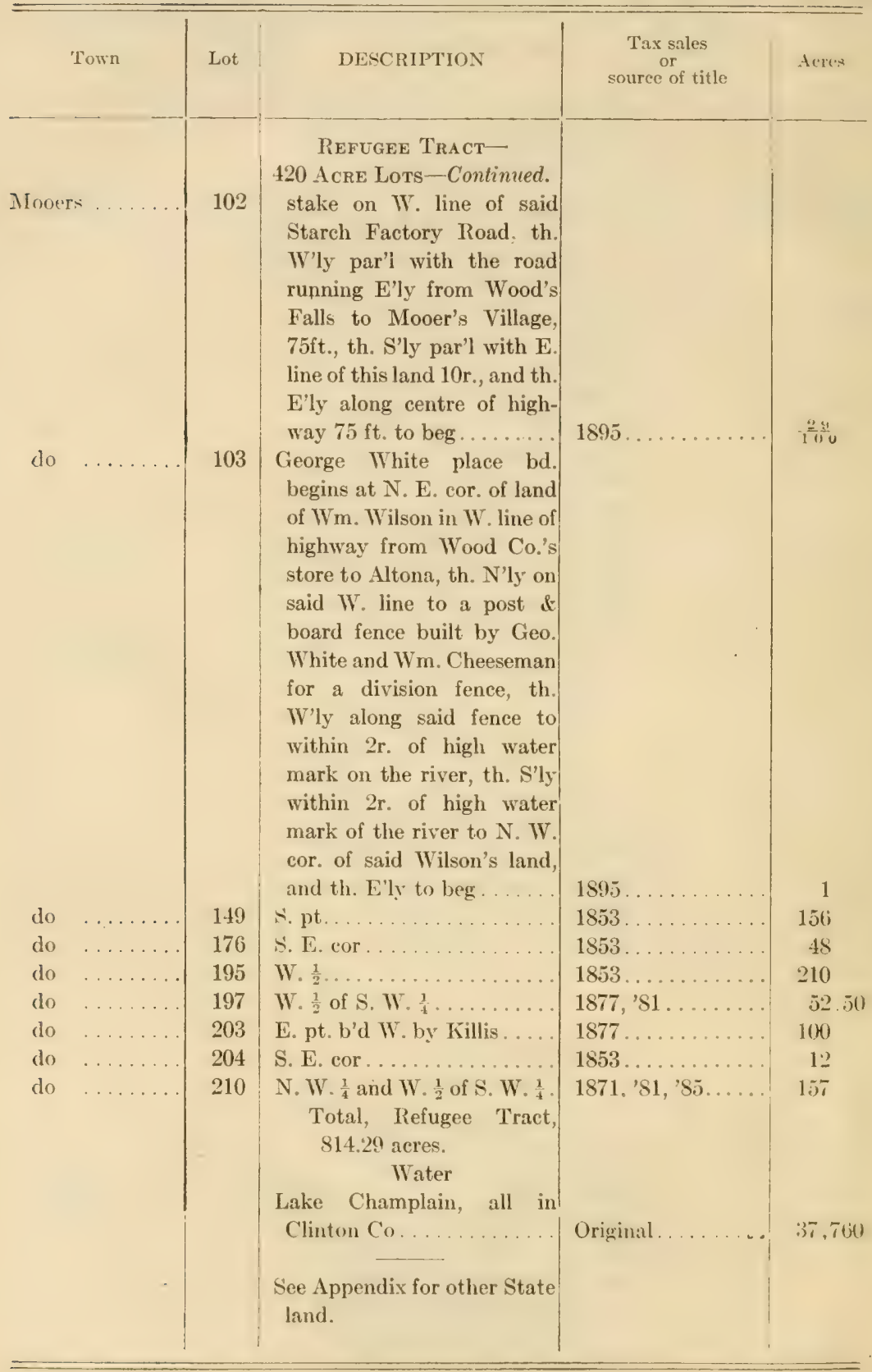


ESSEX COUNTY

(Total number of acres, 363,379.70.)

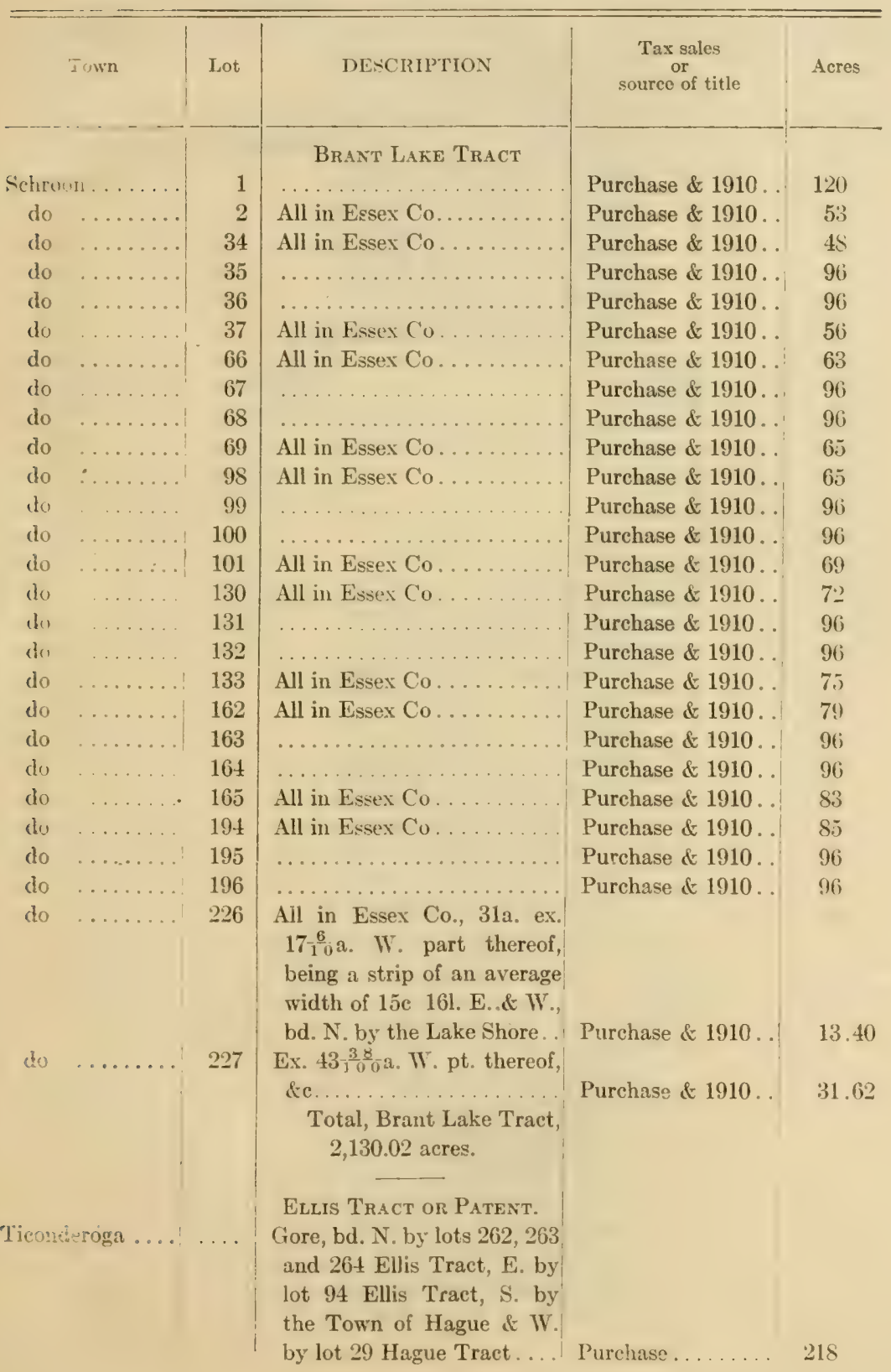




\section{Essex County}

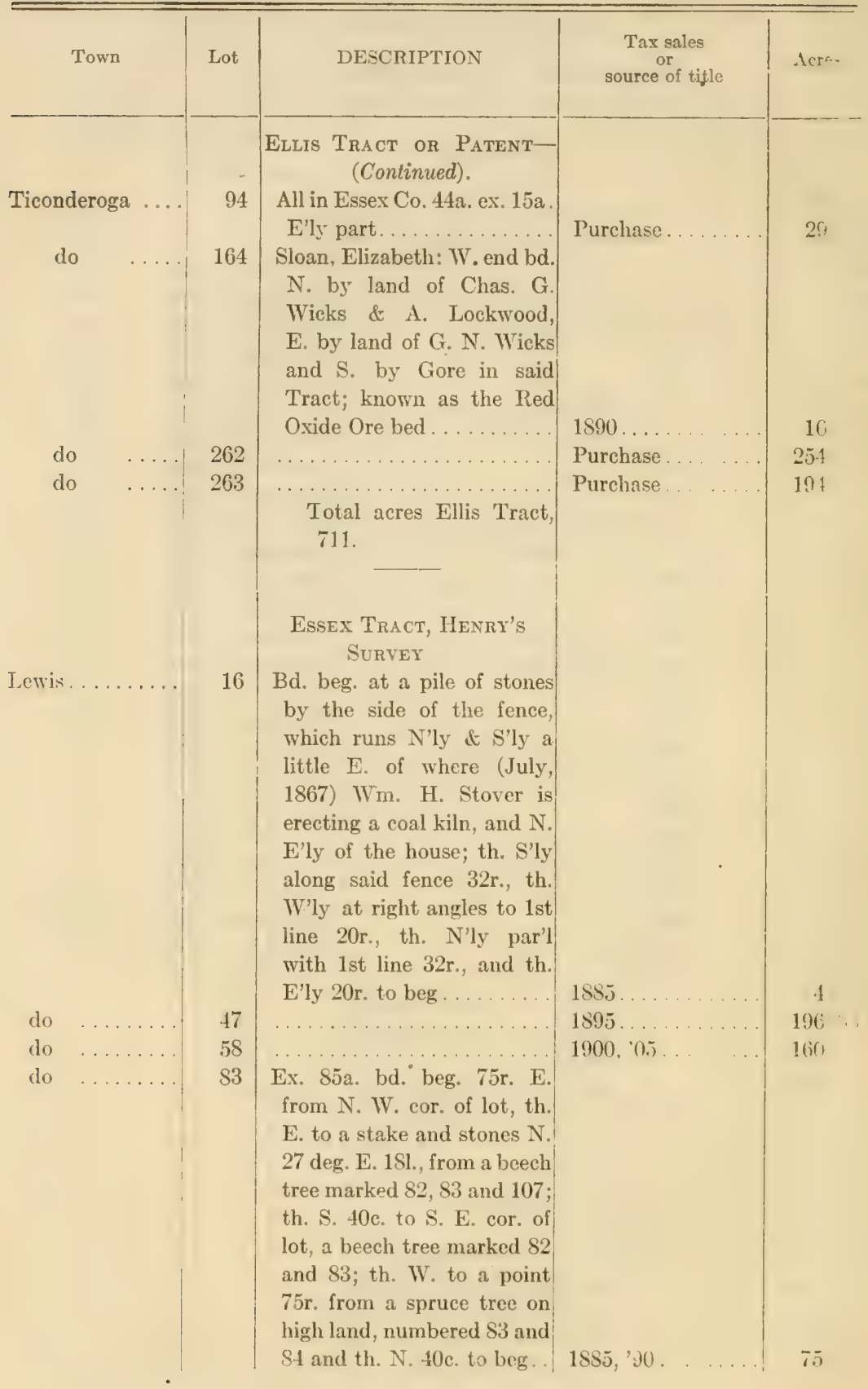




\section{Essex County}

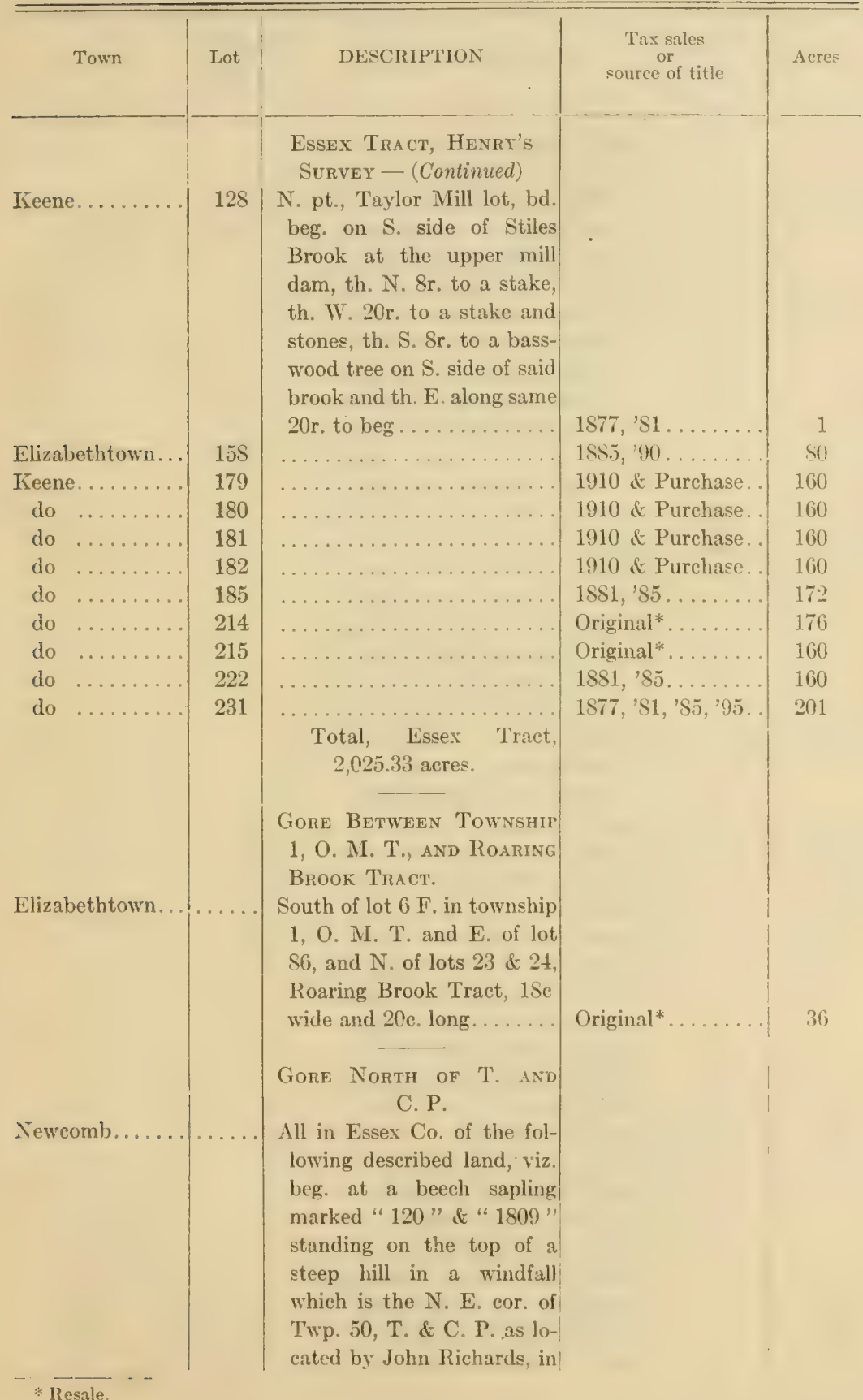




\section{Essex County}

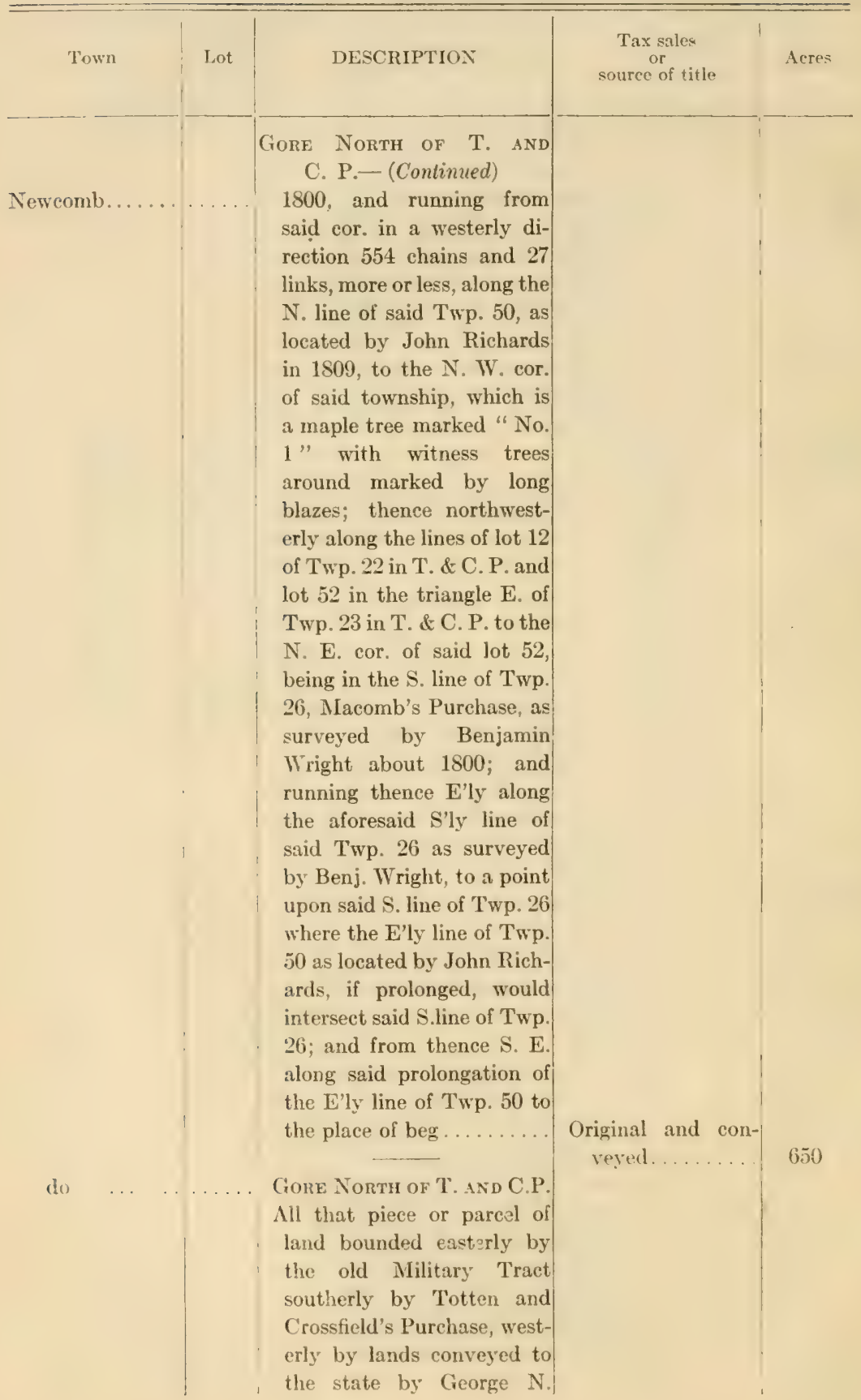


Essex County

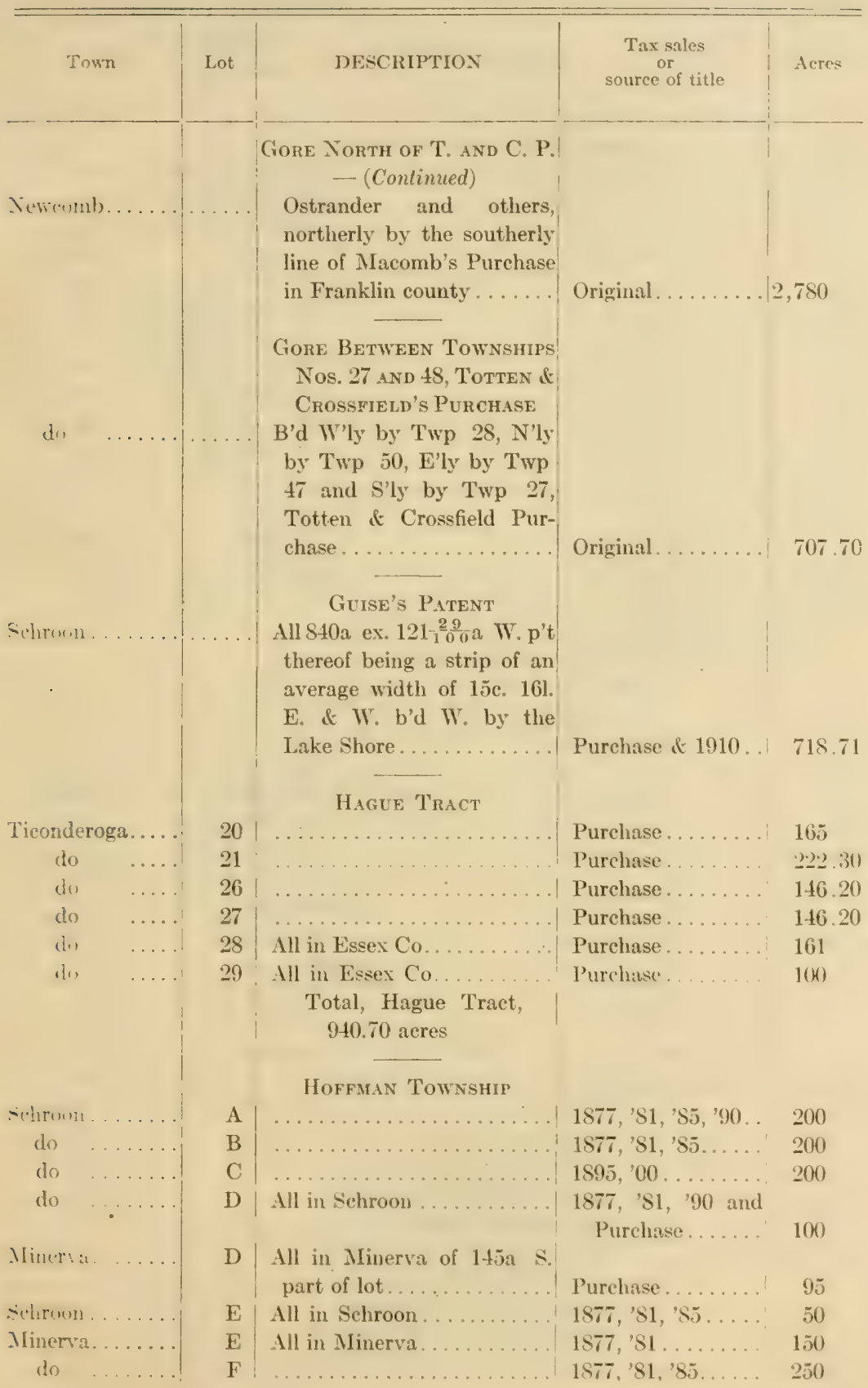




\section{Essex County}

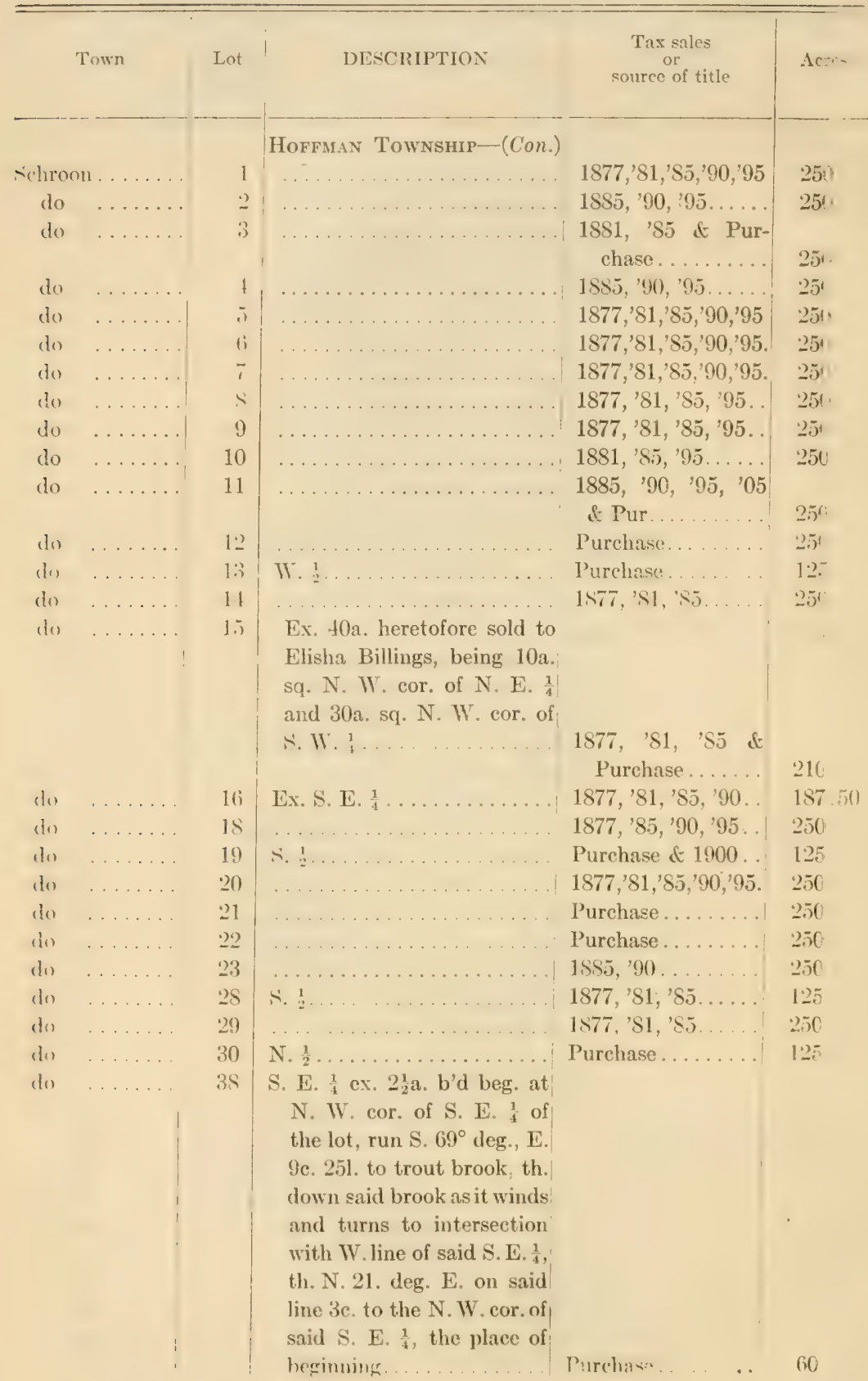


Essex County

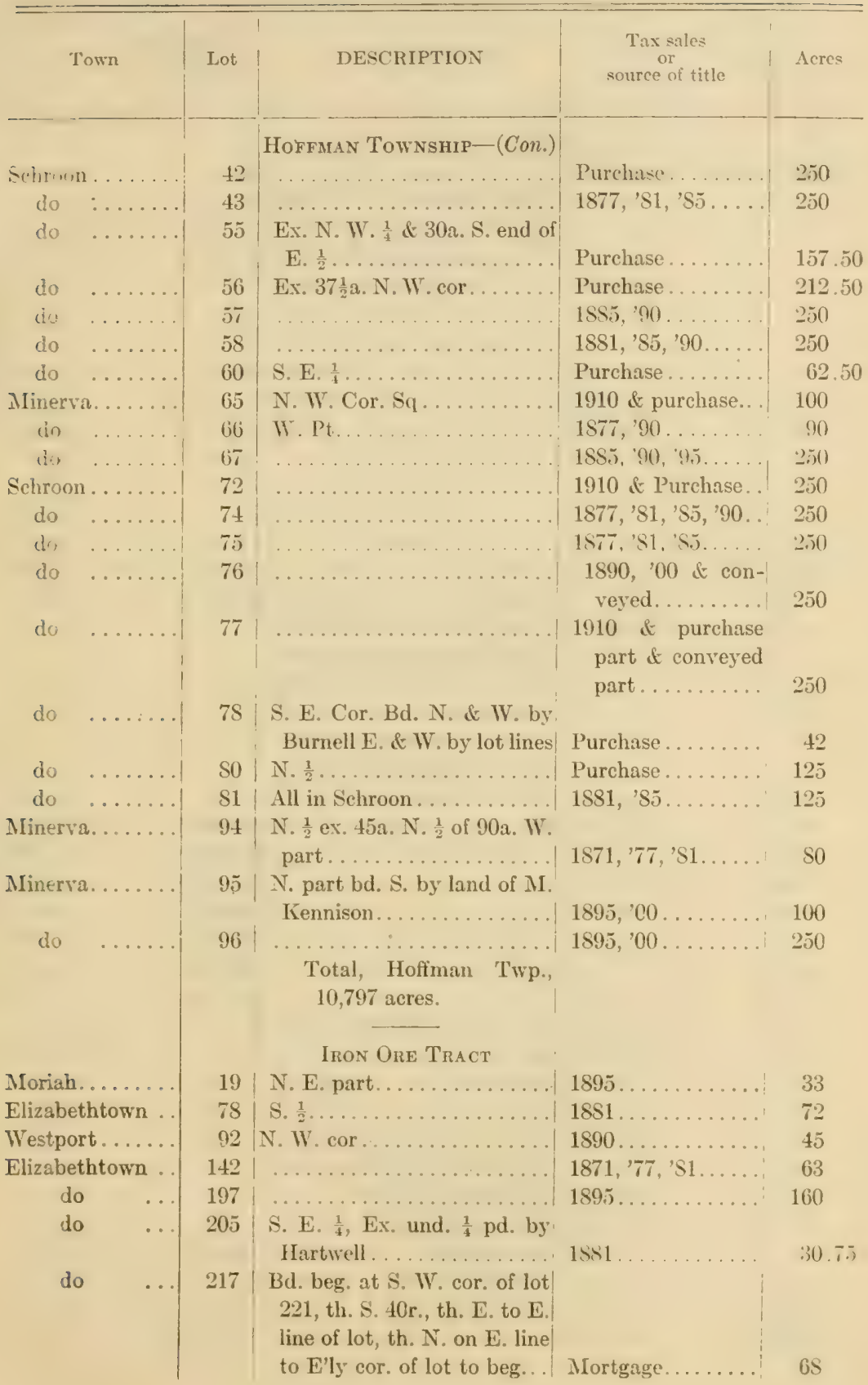


Essex COUNTY

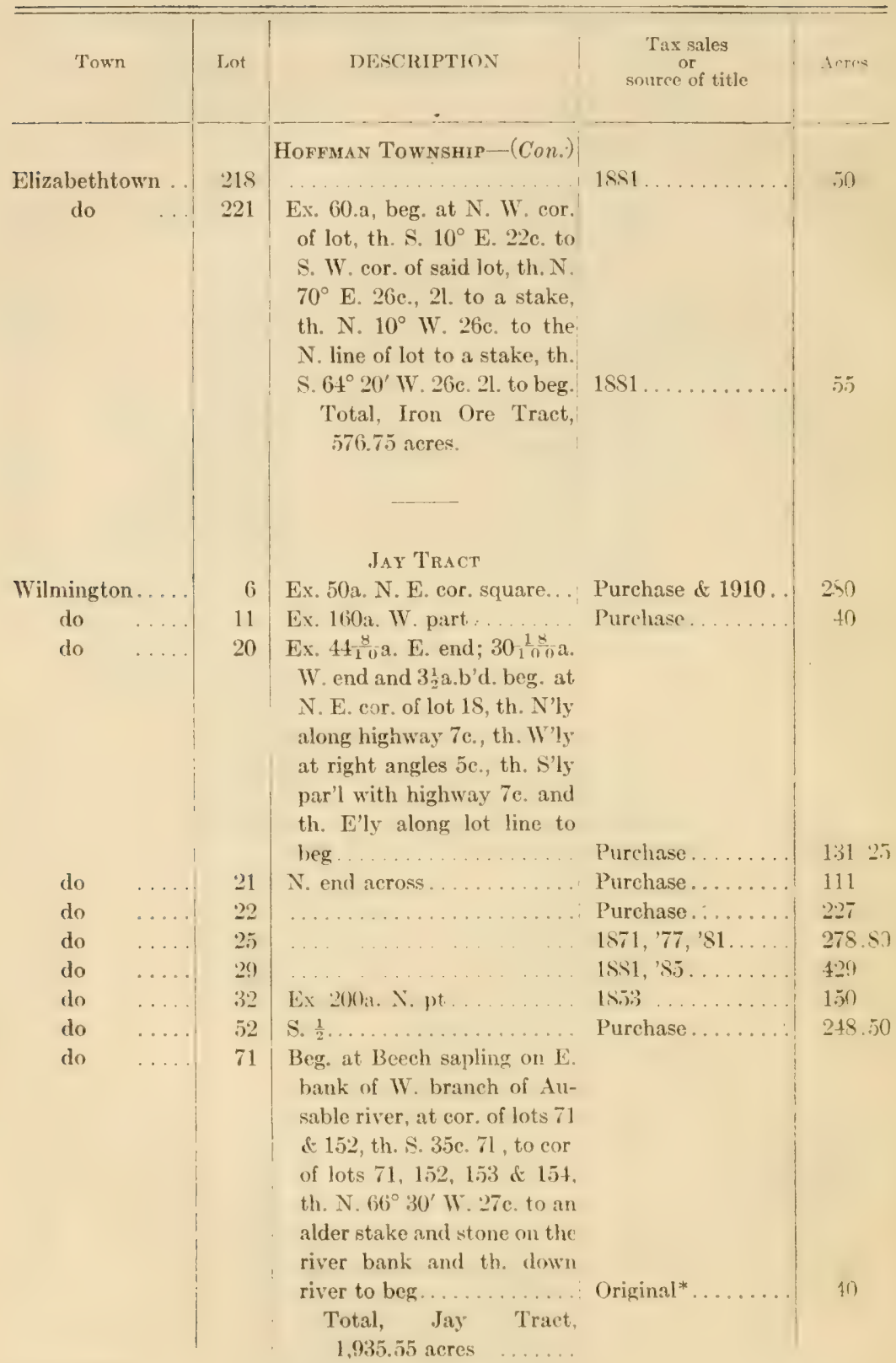


Essex Countr

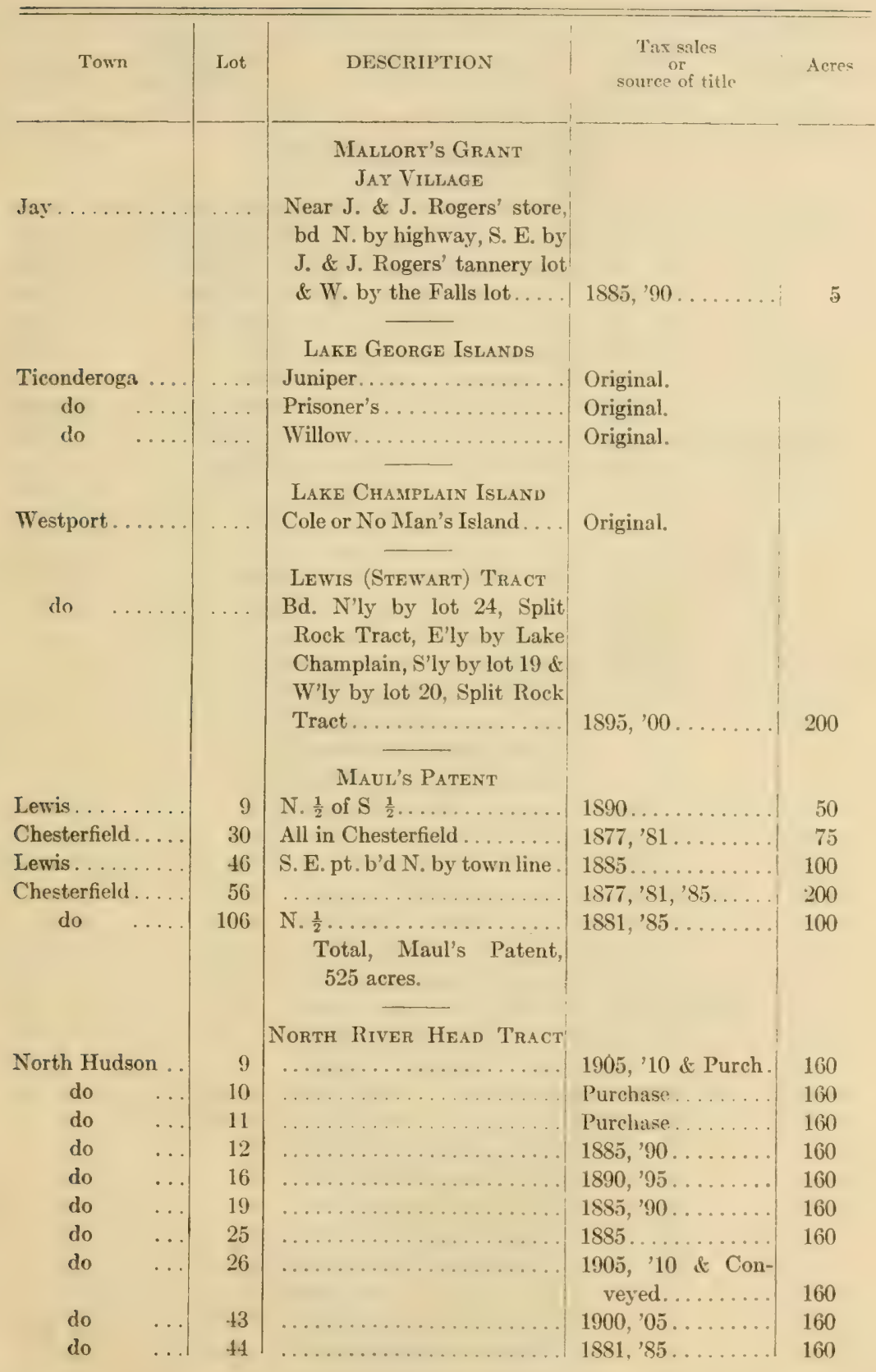


Essex County

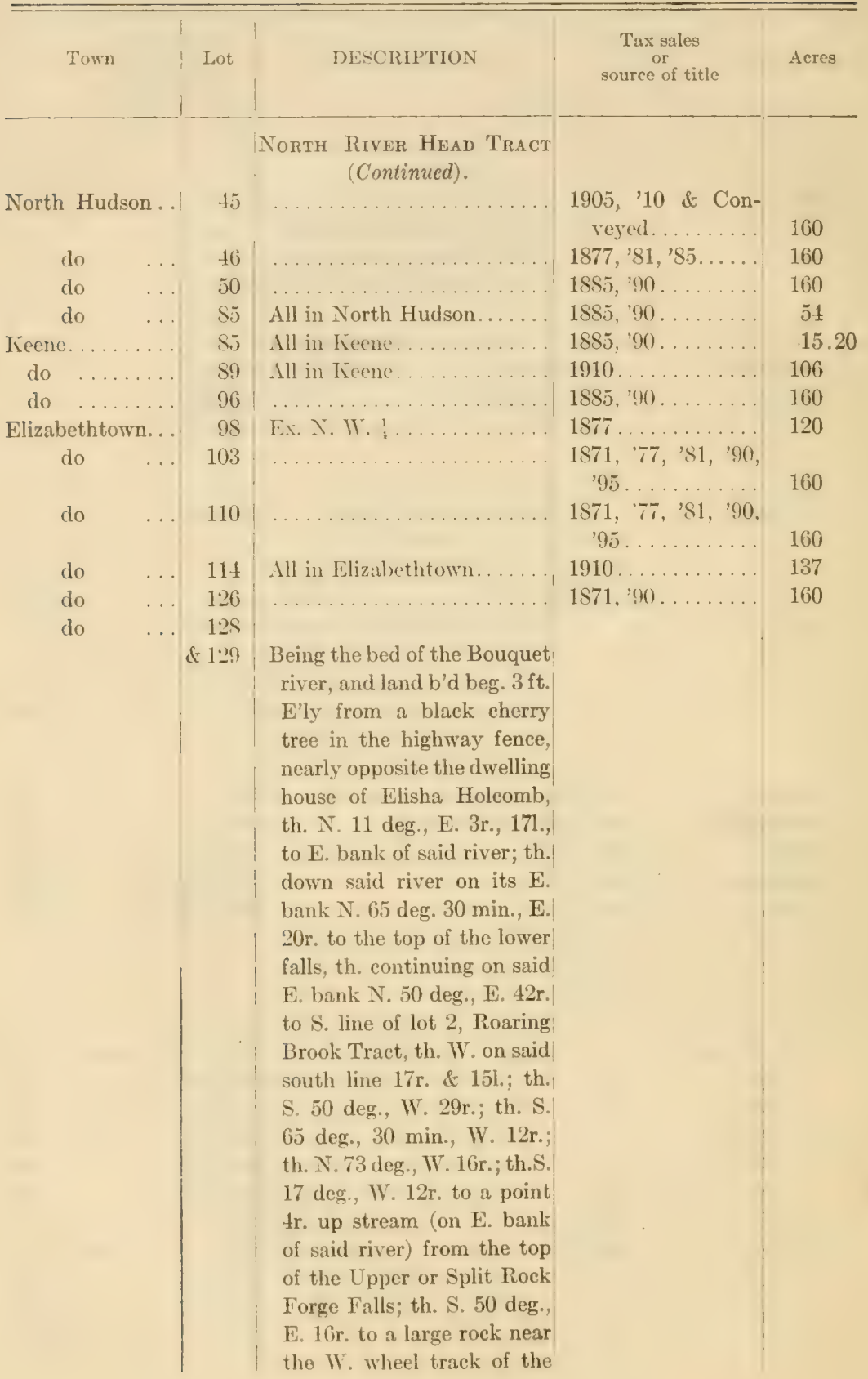


Essex Country

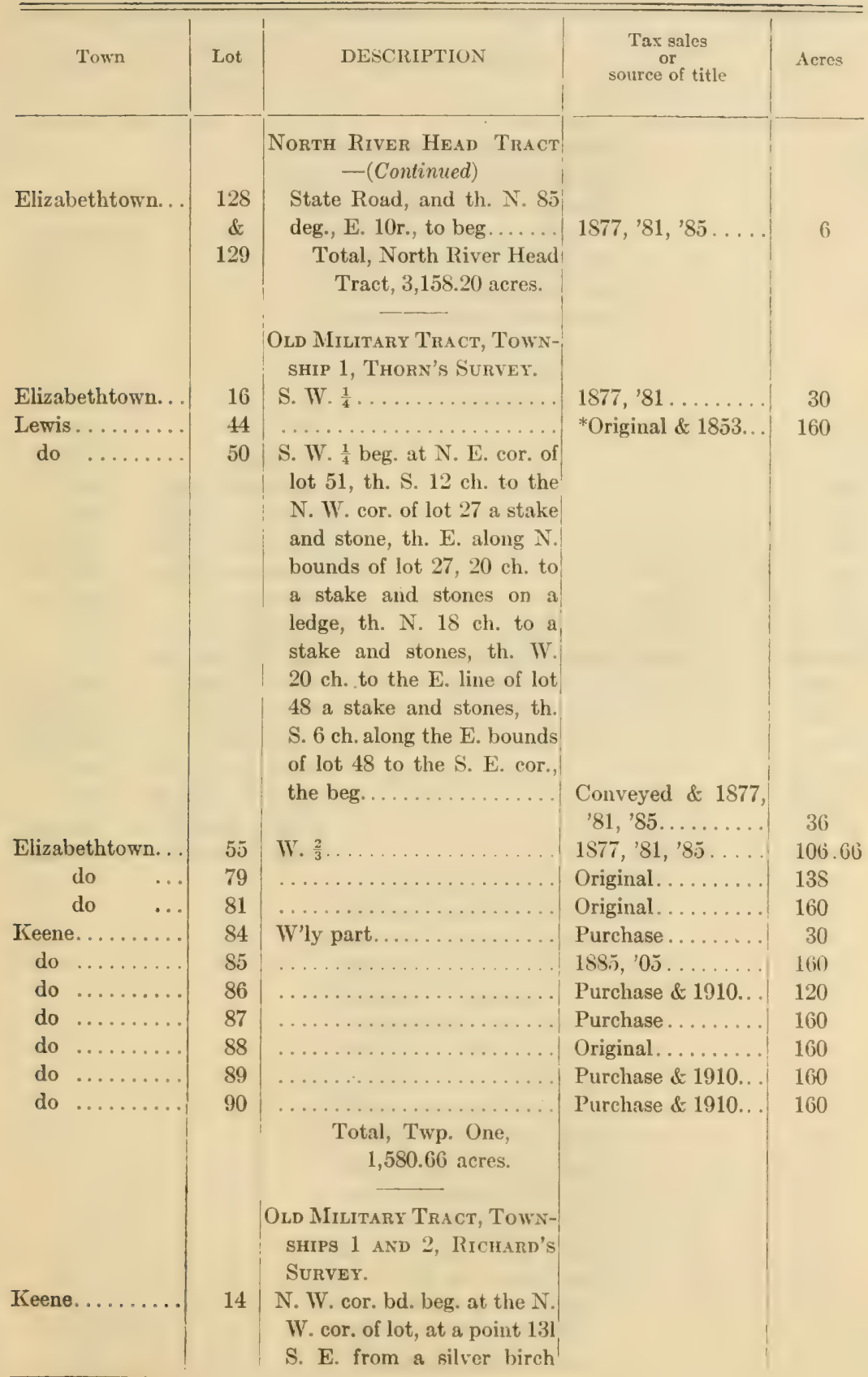




\section{Essex County}

\begin{tabular}{|c|c|c|c|c|}
\hline Town & Lot & DESCRIPTION & $\begin{array}{c}\text { Tax sales } \\
\text { or } \\
\text { source of title }\end{array}$ & Acres \\
\hline Keene.... & & $\begin{array}{l}\text { OLD MILITARY TRACT, ToWN- } \\
\text { SHIPS } 1 \text { AND } 2 \text {, RICHARD's } \\
\text { SURVEY-(Continued) } \\
\text { tree; th. S. 34c. to Walton } \\
\text { brook; th. down said brook } \\
\text { as it winds and turns to a } \\
\text { spruce tree blazed on the } \\
\text { N. and S. sides; th. N. 17c. } \\
\text { to S. line of Lot } 15, \text { and th. } \\
\text { W. } 26 \mathrm{c} \text {. to beg............... }\end{array}$ & $1877, ' 81 \ldots$ & 57 \\
\hline do & 15 & $\ldots \ldots \ldots \ldots \ldots \ldots \ldots$ & $1877, ' 81 \ldots$ & 350 \\
\hline do .. & 42 & & Purchase........ & 299.25 \\
\hline do ... & 45 & & Purchase \& 1905.. & 256.75 \\
\hline do ... & 46 & & Purchase \& 1905.. & 328 \\
\hline do ... & 47 & $\ldots \ldots \ldots \ldots$ & Purchase \& $1905 .$. & 568 \\
\hline do $\ldots$ & 48 & & Purchase \& 1905.. & 518.30 \\
\hline do $\ldots$. & 49 & 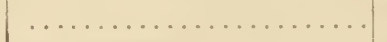 & Purchase........ & 299.30 \\
\hline do ... & 50 & All in Keene. . . . . . . . . & $1885,{ }^{\prime} 90, ' 95 \ldots$ & 375 \\
\hline Wilmington. & 50 & All in Wilmington........ & $1871,777,91, ' 85 \ldots$ & 187 \\
\hline do & 51 & N. pt. in Wilmington..... & $1871,777, ' 81 \ldots$ & 205 \\
\hline do & 52 & All in Wilmington........ & $1877, ' 81, ' 85 \ldots \ldots$ & 247.50 \\
\hline Keene. ... . . & 52 & All in Keene............. & $1881,85 \ldots \ldots \ldots$ & 514.75 \\
\hline Wilmington. & 54 & $\ldots \ldots \ldots \ldots \ldots \ldots \ldots \ldots \ldots \ldots$ & $1877, ' 81, ' 85$, , '95. . & 1,002 \\
\hline do & 56 & $\ldots \ldots \ldots \ldots \ldots \ldots \ldots \ldots \ldots$ & $1877, ' 81, ' 85, ' 90$. & 382 \\
\hline do & 57 & $\ldots \ldots \ldots \ldots \ldots \ldots \ldots \ldots \ldots$ & 1905 \& purch'se .. . & 436.18 \\
\hline do & 58 & $\ldots \ldots \ldots \ldots \ldots \ldots \ldots \ldots$ & $1905 \&$ purch'se ... & 487.28 \\
\hline do & 60 & $\begin{array}{l}\text { Ex. } 100 \text { acres b'd. beg. at a } \\
\text { point in W. line of said lot } \\
60 \text { in center of Cascade } \\
\text { Lake, th. N. } 5 \text { deg. } 50 \text { min. } \\
\text { E. } 700 \mathrm{ft} \text {. to a stake \& } \\
\text { stones, th. N. } 65 \text { deg. } 10 \\
\text { min. E. } 3,300 \mathrm{ft} \text {. to a stake } \\
\text { \& stones, th. S. } 5 \mathrm{deg} .50 \\
\text { min. W. } 1,400 \mathrm{ft} . \mathrm{th} . \mathrm{S} .65 \\
\text { deg. } 10 \mathrm{~min} \text {. W. } 3,300 \mathrm{ft} \text {. } \\
\text { and th. N. } 5 \text { deg. } 50 \mathrm{~min} . \\
\text { E. } 700 \mathrm{ft} \text {. to center of Cas- } \\
\text { cade Late, and ex. } 27.77 \\
\text { acres b'd, beg. at N. E. } \\
\text { cor. of lot } 60 \text {, th. N. } 84 \\
\text { deg. } 10 \mathrm{~min} \text {. W. along lot } \\
\text { line } 1,900 \mathrm{ft} \text {. to a stake, } \\
\text { th. S. } 5 \text { deg. } 50 \text { min. W. }\end{array}$ & & \\
\hline
\end{tabular}


Essex Countr

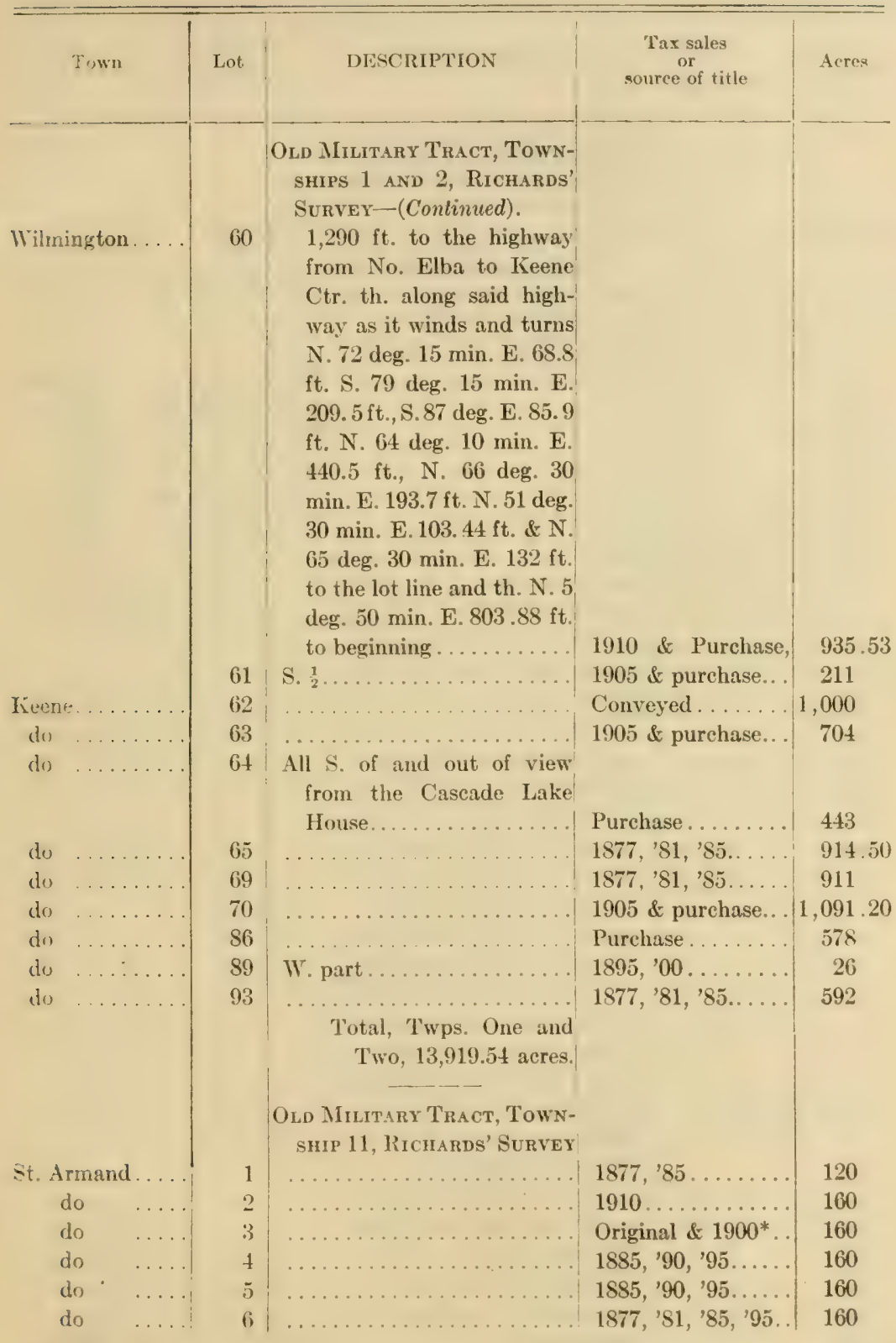




\section{Essex County}

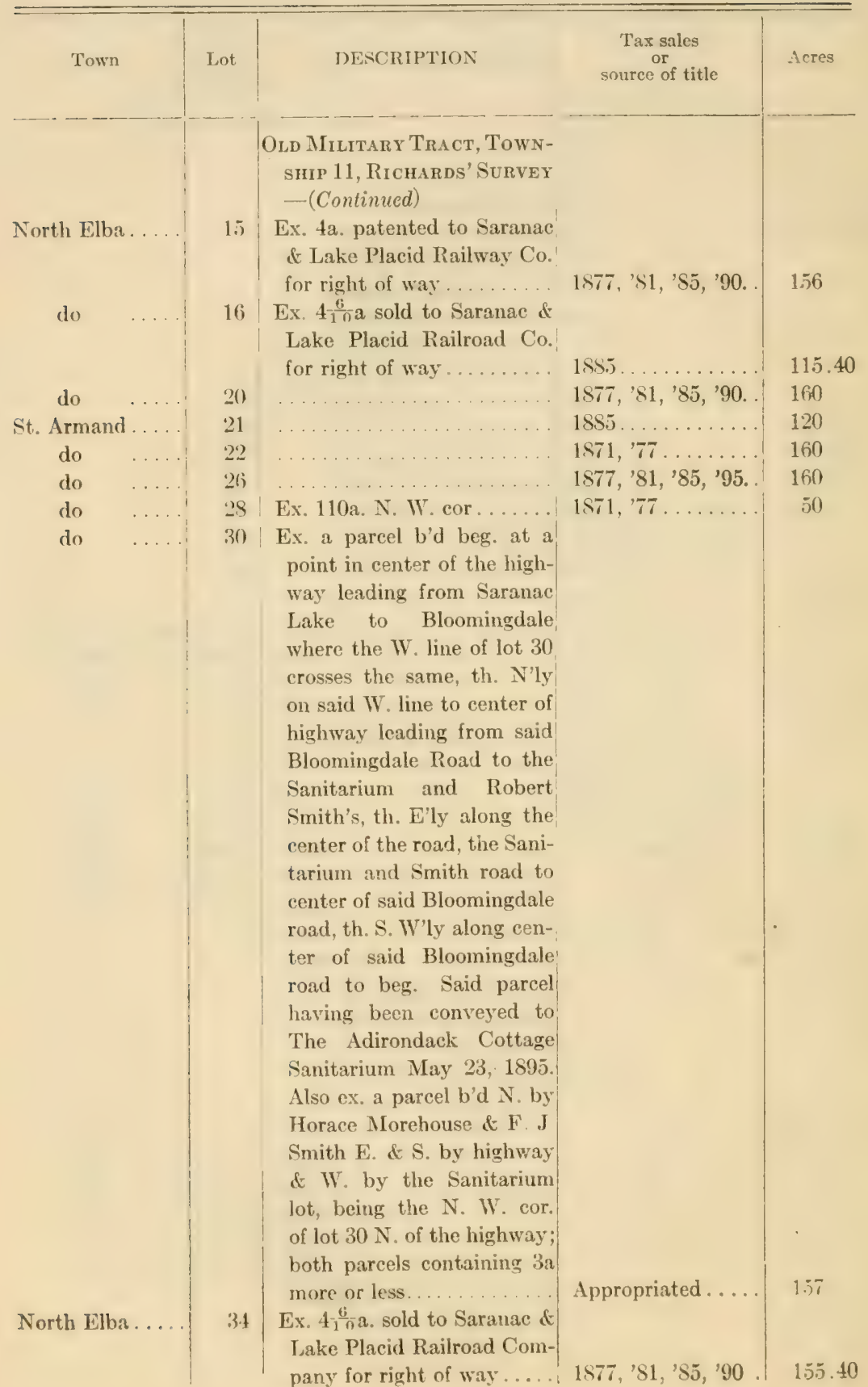


Essex Country

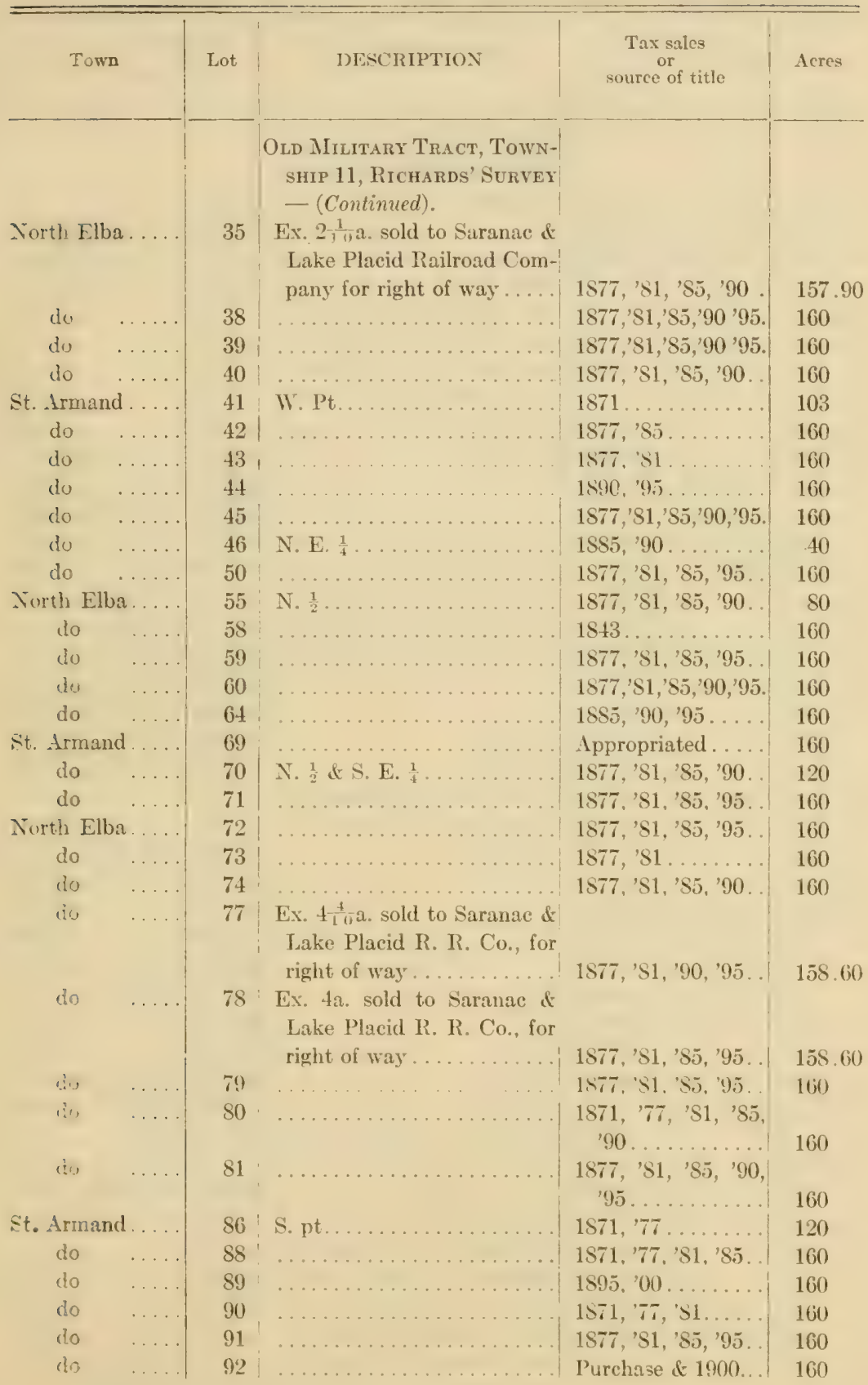


Essex County

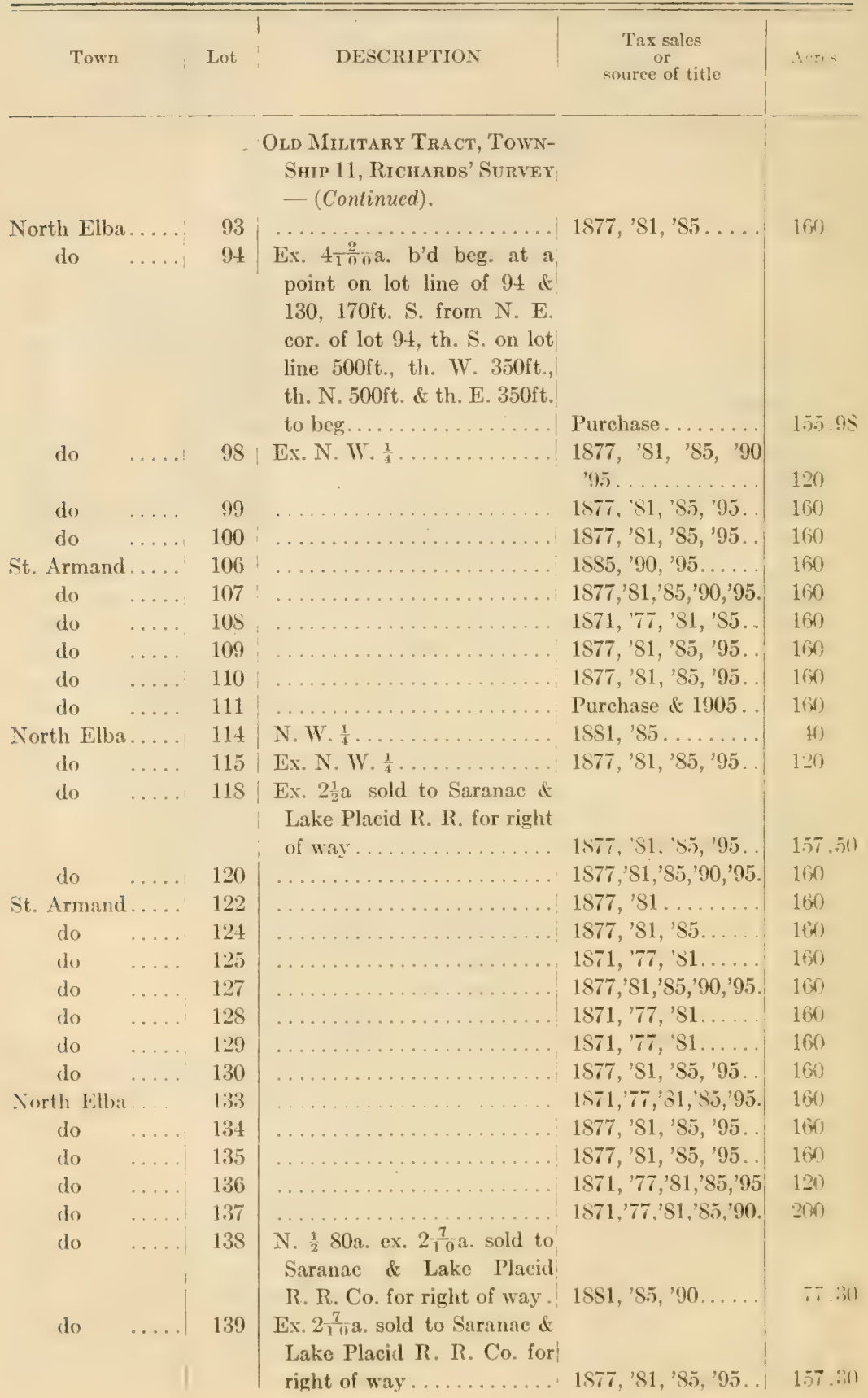


Essex County

\begin{tabular}{|c|c|c|c|c|}
\hline Town & \multirow[t]{2}{*}{ Lot } & DESCRIPTION & \multirow{2}{*}{$\begin{array}{c}\text { Tax sales } \\
\text { or } \\
\text { source of title }\end{array}$} & \multirow[t]{2}{*}{ Acres } \\
\hline & & $\begin{array}{l}\text { Old Military Tract, Town- } \\
\text { ship } 11 \text {, Richards' Survey } \\
\text {-(Continued }) \text {. }\end{array}$ & & \\
\hline North Elba & 140 & $\ldots \ldots \ldots \ldots \ldots \ldots \ldots \ldots$ & $1877, ' 81, ' 85,{ }^{\prime} 95$. & 160 \\
\hline St. Armand & 144 & .. & $1877, ' 81, ' 85, ' 90 \ldots$ & 160 \\
\hline do & 145 & $\ldots \ldots \ldots \ldots \ldots \ldots \ldots \ldots$ & $1877, ' 81, ' 85, ' 95 \ldots$ & 160 \\
\hline do & 146 & & $1877, ' 81, ' 85,{ }^{\prime} 95$. & 160 \\
\hline do & 147 & & $1877, ' 81, ' 85,90, ' 95$. & 160 \\
\hline do & 148 & & $1871, ' 77, ' 81,{ }^{\prime} 90 \ldots$ & 160 \\
\hline do & 149 & & 1877, '81, '85, '95. . & 160 \\
\hline do & 150 & $\ldots \ldots \ldots$ & $1877, ' 81, ' 85,900,95$. & 160 \\
\hline North Elba & 154 & & 1877, ' 81, ' 85, '95. . & 160. \\
\hline do & 155 & $\ldots \ldots \ldots \ldots$ & $1877, ' 81, ' 85,95$. & 160 \\
\hline do & 156 & N. W. cor ............. & $1877, ' 81, ' 85, ' 95$. & 70 \\
\hline do & 157 & $\ldots \ldots \ldots \ldots$ & $1877, ' 81, ' 85,90,995$. & 200 \\
\hline do & 159 & $\begin{array}{l}\text { N. } \frac{1}{2} \& \text { S. W. } \frac{1}{4}, 120 \text { a. ex. } \\
5 \frac{6}{10} \text { a. sold to Saranac } \& \\
\text { Lake Placid R. R. Co. for } \\
\text { right of way. . . . . . . }\end{array}$ & $187 \%, 95,90,95$. & 114.40 \\
\hline do & 160 & $\ldots \ldots \ldots$ & $1877, ' 81, ' \$ 55,90,995$. & 160 \\
\hline St. Armand & 163 & $\ldots \ldots \ldots \ldots \ldots \ldots \ldots$ & $1885,90,95 \ldots \ldots$ & 200 \\
\hline do & 164 & $\ldots \ldots \ldots \ldots \ldots \ldots \ldots \ldots$ & $1877, ' 81 \ldots \ldots \ldots$ & 200 \\
\hline do & 165 & & $1877, ' 81$, ' $85,95$. & 200 \\
\hline do & 166 & & $1877, ' 81, ' 85,95$. & 200 \\
\hline do & 167 & & $1877, ' 81, ' 85, ' 95$. & 200 \\
\hline do & 168 & .. & $1877, ' 81, ' 85 \ldots \ldots$ & 200 \\
\hline do & 169 & . & $1877, ' 81,95,90,995$. & 200 \\
\hline do & 170 & & $1885,{ }^{\prime} 90,95 \ldots$. & 200 \\
\hline North Elba & 174 & Ex. 70a. N. W. cor. & $1877, ' 81, ' 85, ' 90$. & 90 \\
\hline do & 175 & $\ldots \ldots \ldots \ldots \ldots$ & $1877, ' 81, ' 85,900,95$. & 160 \\
\hline do & 176 & $\ldots \ldots \ldots \ldots$ & $\begin{array}{r}\text { Conveyed \& } 1877, \\
181, ' 85,95 \ldots .\end{array}$ & 120 \\
\hline do & 177 & WI. $\frac{1}{2}$. & $1881, ' 85$. & 50 \\
\hline do & 178 & $\ldots \ldots \ldots$ & $1877, ' 81, ' 85 \ldots$. & 200 \\
\hline do & 179 & $\begin{array}{l}\text { Ex. } 3 \frac{1}{2} \text { a. sold to Saranac \& } \\
\text { Lake Placid R. R. Co. for } \\
\text { right of way . . . . . . }\end{array}$ & $1871,77,85$. & 126.50 \\
\hline do & 180 & & $1877, ' 81,95,90,95$. & 160 \\
\hline St. Armand & 181 & W. side, $35 \mathrm{c}$. wide. . & $1877, ' 81, ' 85 \ldots$ & 105 \\
\hline do & 182 & & $1877, ' s 1,90 \ldots$. & 300 \\
\hline do & 183 & & $1885,990,995 \ldots$ & 300 \\
\hline do & 184 & & 1877, ' 81, ' 85, , '95. . & 300 \\
\hline do & 185 & & 1877, ' 81, ' 85, '95. . & 300 \\
\hline do & 186 & & $1877, ' 81, ' 85 \ldots$ & 300 \\
\hline
\end{tabular}


Essex County

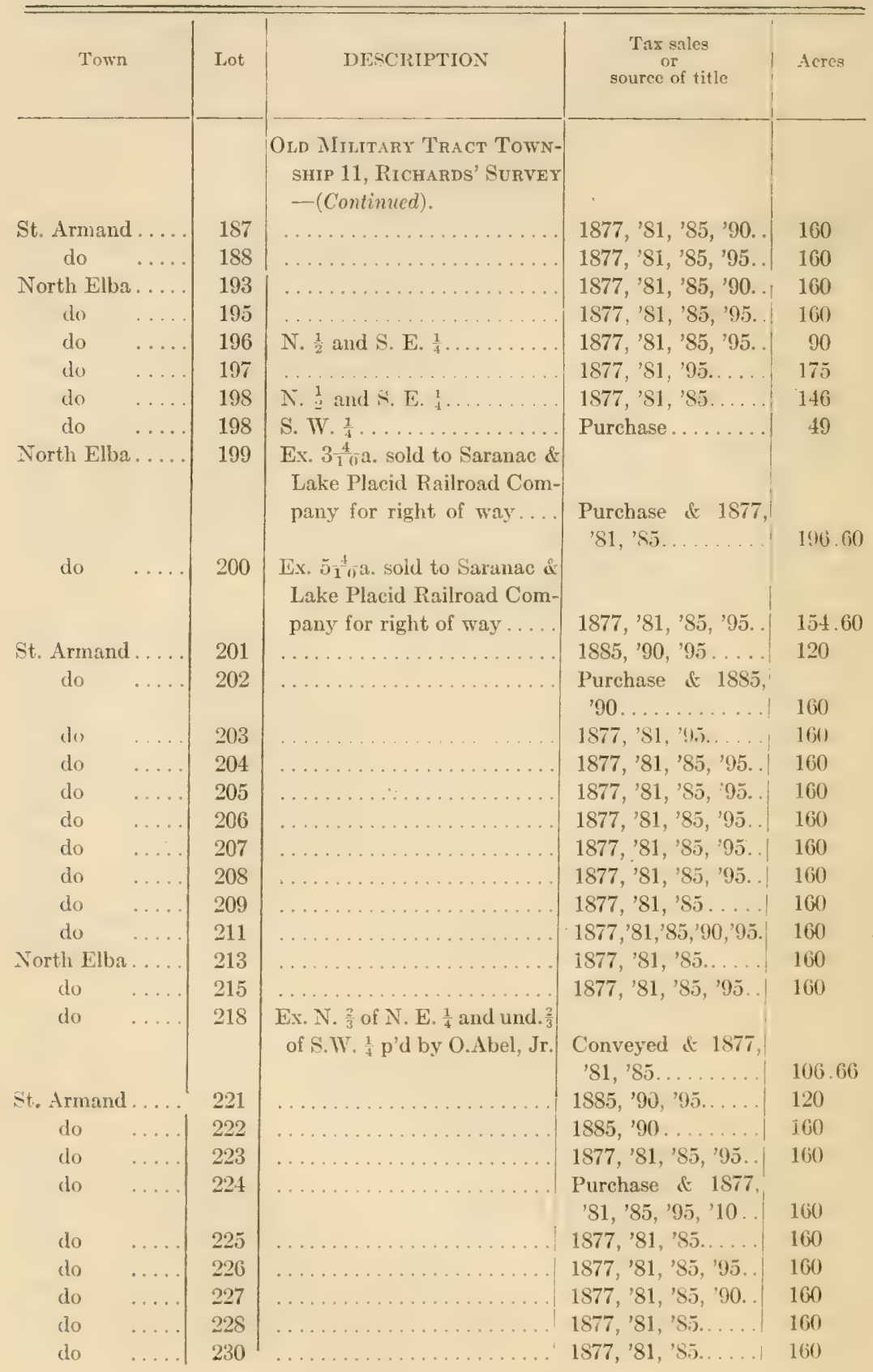


Comservation Commssion.

Essex County

\begin{tabular}{|c|c|c|c|c|}
\hline Town & \multirow[t]{2}{*}{ Lot } & DESCRIPTION & $\begin{array}{c}\text { Tax sales } \\
\text { or } \\
\text { source of title }\end{array}$ & Acres \\
\hline & & \multicolumn{2}{|l|}{$\begin{array}{l}\text { Old Mrlitary Tract, Town-, } \\
\text { ship 11, Richards' Survey } \\
\text {-(Contimued). }\end{array}$} & \\
\hline St. Armand. & 231 & $\ldots . .$. & $1877, ' 81, ' 8.5 \ldots$ & 160 \\
\hline North Elba. & 232 & $\ldots \ldots \ldots \ldots \ldots \ldots$ & $1877, ' 81, ' 85,95$. & 160 \\
\hline do . & 233 & $\ldots \ldots \ldots \ldots \ldots$ & $1877, ' 81, ' 85, ' 95$. & 160 \\
\hline do & 234 & Ex. S. E. $\frac{1}{4} \ldots$. . & $1877, ' 81,{ }^{\prime} 85$. & 120 \\
\hline St. Armand. & 241 & $\ldots$. & 1877, ' 81, ' $\$ 5 . \ldots \ldots$ & 120 \\
\hline do & 242 & $\ldots \ldots \ldots$ & $1877, ' 81, ' 85, ' 90,95$. & 160 \\
\hline do & 243 & & $1877, ' 81, ' 85 . \ldots$. & 160 \\
\hline do & 244 & $\ldots \ldots \ldots \ldots \ldots \ldots \ldots$ & $1877, ' 81, ' 85 \ldots \ldots$ & 160 \\
\hline do & 245 & $\ldots$. & $1877, ' 81, ' 85, ' 90$. & 160 \\
\hline do & 246 & & $1877, ' 81, ' 85 \ldots \ldots$ & 160 \\
\hline do & 247 & $\ldots$ & $1877,981,95 \ldots \ldots$ & 160 \\
\hline do & 248 & $\ldots \ldots$ & 1877, ' $81, ' 85, ' 95$. & 160 \\
\hline do & 249 & $\cdots$ & 1877, '81, '85, '95. . & 160 \\
\hline do & 250 & & $1877, ' 81, ' 85,90,995$ & 160 \\
\hline do & 251 & $\ldots \ldots \ldots$ & $1871,77, ' 81,95$. & 160 \\
\hline North Elba. & 252 & & 1877, '81, '85, '95. . & 160 \\
\hline do & 253 & N. E. ${ }_{4}^{1} \ldots \ldots \ldots \ldots$ & 1871,77, ' $81,95$. & 40 \\
\hline St. Armand. & 262 & $\ldots \ldots \ldots \ldots \ldots$ & $1877, ' 81,{ }^{\prime} 85,{ }^{\prime} 95$. & 160 \\
\hline do & 263 & & $1877,81, ' 85 \ldots \ldots$ & 160 \\
\hline do & 264 & . & $1877, ' 81, ' 85, ' 90$. & 160 \\
\hline do & 265 & $\cdots \cdots$ & 1877,91, ' $85,95$. & 160 \\
\hline do & 266 & & $1877, ' 81, ' 85,{ }^{\prime} 95$. & 160 \\
\hline do & 267 & & $1877, ' 81, ' 85, ' 90$. & 160 \\
\hline do & 269 & Ex. 100a. X. W. cor. square. & $1877, ' 81, ' 85,95$. & 60 \\
\hline do & 270 & $\ldots \ldots \ldots \ldots \ldots \ldots \ldots$ & $1885,90,95 \ldots$ & 160 \\
\hline do & 271 & $\ldots \ldots$ & 1877, ' 81, ' 85 , '95. . & 160 \\
\hline North Elba. & 275 & $\ldots \ldots \ldots \ldots$ & $1877, ' 81 \ldots \ldots \ldots$ & 160 \\
\hline St. Armand. & 282 & $\ldots \ldots \ldots \ldots \ldots \ldots$ & $1877, ' 81, ' 85 \ldots$. & 160 \\
\hline do & 283 & $\ldots \ldots \ldots \ldots \ldots$ & $1877, ' 81, ' 85,{ }^{\prime} 95$. & 160 \\
\hline do & 284 & $\ldots \ldots \ldots \ldots$ & $1877, ' 81, ' 85, ' 95$. & 160 \\
\hline do & 285 & $\ldots \ldots \ldots \ldots$ & $1877, ' 81, ' 85,90,995$. & 160 \\
\hline do & 286 & $\ldots \ldots \ldots \ldots \ldots \ldots$ & 1877, '81, '85, '95. & 160 \\
\hline do & 287 & $\ldots \ldots \ldots \ldots \ldots \ldots \ldots$ & $1877, ' 81, ' 85,95$. & 160 \\
\hline do & 288 & $\ldots \ldots \ldots \ldots \ldots \ldots \ldots \ldots$ & $1877, ' 81, ' 85,95$. & 160 \\
\hline do & 289 & $\ldots \ldots \ldots \ldots \ldots \ldots \ldots$ & 1877 , '\$1, '85, '95. .' & 160 \\
\hline do & 290 & & $1877, ' 81, ' 85, ' 95$. & 160 \\
\hline do & 302 & & 1910 \& purchase.. & 120 \\
\hline do & 303 & & 1877, '81, '85, '95. & 120 \\
\hline do & 304 & 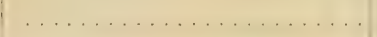 & $1877, ' 81$, '85, '95. . & 120 \\
\hline do & 305 & $\ldots \ldots \ldots \ldots \ldots$ & $1877, ' 81, ' 85, ' 90$. & 120 \\
\hline do & 306 & $\ldots \ldots \ldots \ldots \ldots$ & $1877, ' 81, ' 85, ' 90,955$. & 120 \\
\hline
\end{tabular}


Essex County

\begin{tabular}{|c|c|c|c|c|}
\hline Town & \multirow[t]{2}{*}{ Lot } & DESCRIPTION & \multirow[t]{2}{*}{$\begin{array}{l}\text { Tax sales } \\
\text { or } \\
\text { source of title }\end{array}$} & \multirow[t]{2}{*}{ Acreg } \\
\hline & & $\begin{array}{l}\text { Old Military Tract, Town- } \\
\text { ship } 11, \text { Richards' Survey } \\
- \text { (Continued). }\end{array}$ & & \\
\hline St. Armand. & 307 & $\ldots \ldots \ldots \ldots \ldots \ldots \ldots \ldots \ldots$ & $1877, ' 81, ' 85, ' 95$. & 120 \\
\hline do & 308 & $\ldots \ldots \ldots \ldots \ldots \ldots \ldots$ & $1877, ' 81, ' 85, ' 90, ' 95$. & 120 \\
\hline do & 309 & $\ldots$ & $1877, ' 81, ' 85, ' 95$. & 120 \\
\hline do & 310 & $\ldots$ & $1877, ' 81, ' 85 \ldots \ldots$ & 120 \\
\hline North Elba. & 318 & … $\ldots \ldots \ldots \ldots$ & $1877, ' 81, ' 85, ' 95$. & 160 \\
\hline St. Armand. & 321 & $\ldots \ldots \ldots \ldots \ldots \ldots \ldots \ldots$ & $1877, ' 81, ' 85 \ldots \ldots$ & 120 \\
\hline do & 322 & $\ldots \ldots \ldots \ldots \ldots \ldots \ldots \ldots$ & $1877, ' 81, ' 85, ' 90 .$. & 160 \\
\hline do & 323 & .. & 1877, ' 81, ' 85, , 95. & 160 \\
\hline do & 324 & & $1881,{ }^{\prime} 85,{ }^{\prime} 95 \ldots$. & 160 \\
\hline do & 325 & Ex. N. E. $\frac{2}{1} \ldots \ldots \ldots \ldots$ & $1877, ' 81,{ }^{\prime} 85,95$. & 120 \\
\hline do & 326 & $\ldots \ldots \ldots \ldots \ldots \ldots \ldots \ldots \ldots$ & $1877, ' 81,{ }^{\prime} 85,{ }^{\prime} 95$. & 160 \\
\hline do & 327 & $\ldots \ldots \ldots \ldots \ldots \ldots \ldots \ldots$ & $1877, ' 81,{ }^{\prime} 85, ' 95 \ldots$ & 160 \\
\hline do & 328 & & $1877, ' 81,{ }^{\prime} 85, ' 95 \ldots$ & 160 \\
\hline do & 329 & $\ldots \ldots$ & $1877, ' 81, ' 85, ' 95 \ldots$ & 160 \\
\hline do & 330 & ... & $1877, ' 81, ' 85, ' 95 \ldots$ & 160 \\
\hline North Elba. & 337 & Ex. N. E. $\frac{1}{1}, \ldots \ldots \ldots \ldots$ & $1877,81,{ }^{\prime} 85, ' 90,95$. & 150 \\
\hline do & 338 & & $1877, ' 81,{ }^{\prime} 85 \ldots \ldots$ & 160 \\
\hline St. Armand. & 341 & S. part 17c. N. \& S...... & Purchase ......... & 68 \\
\hline do & 347 & $\ldots \ldots \ldots \ldots \ldots \ldots$ & $1877, ' 81,{ }^{\prime} 85, ' 95$. & 160 \\
\hline do & 350 & .. & $1877,{ }^{\prime} 81, ' 85 \ldots$ & 160 \\
\hline North Elba. & 354 & & 1877, '81, '85, '95. & 160 \\
\hline do & 355 & ... & $1877, ' 81, ' 85, ' 95 \ldots$ & 160 \\
\hline do & 357 & $\ldots \ldots \ldots$ & $1877,{ }^{\prime} 81, ' 85 \ldots \ldots$ & 200 \\
\hline do & 358 & $\ldots \ldots \ldots \ldots \ldots \ldots \ldots \ldots \ldots \ldots$ & $1877, ' 81, ' 85,95$. & 160 \\
\hline do & 359 & S. E. $\frac{1}{3} \ldots \ldots \ldots \ldots \ldots$ & $1877, ' 81 \ldots \ldots$ & 40 \\
\hline do & 360 & N. $\frac{1}{2} \ldots \ldots \ldots \ldots \ldots \ldots$ & $1877, ' 81,95 \ldots$ & 80 \\
\hline do & 361 & $\begin{array}{l}\text { S. W. cor. b'd E. by Mill } \\
\text { Brook \& N. by line parallel } \\
\text { to and } 17 \mathrm{c} \text {. from S. line of }\end{array}$ & & \\
\hline & & 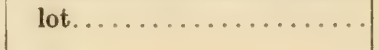 & Purchase ......... & 37 \\
\hline do & 369 & $\ldots \ldots \ldots \ldots \ldots \ldots \ldots \ldots$, & $1905 \&$ purchase. . & 160 \\
\hline St. Armand. & 375 & $\begin{array}{l}\text { Total, Township } 11,32,- \\
608.74 \text { acres. }\end{array}$ & Mortgage \& 1900 & 160 \\
\hline & & $\begin{array}{l}\text { Old Military Tract, Town- } \\
\text { ship 12, Richarda' Survey. }\end{array}$ & & \\
\hline North Elba. & $\begin{array}{l}1 \\
2\end{array}$ & Sub. $3 \ldots \ldots \ldots \ldots \ldots$ & Original....... & 155.80 \\
\hline do & 2 & Sub. 1, ex. N. W. $\frac{x}{4} \ldots$ & $1881,, 85, ’ 90$ & 123 \\
\hline
\end{tabular}


Essex County

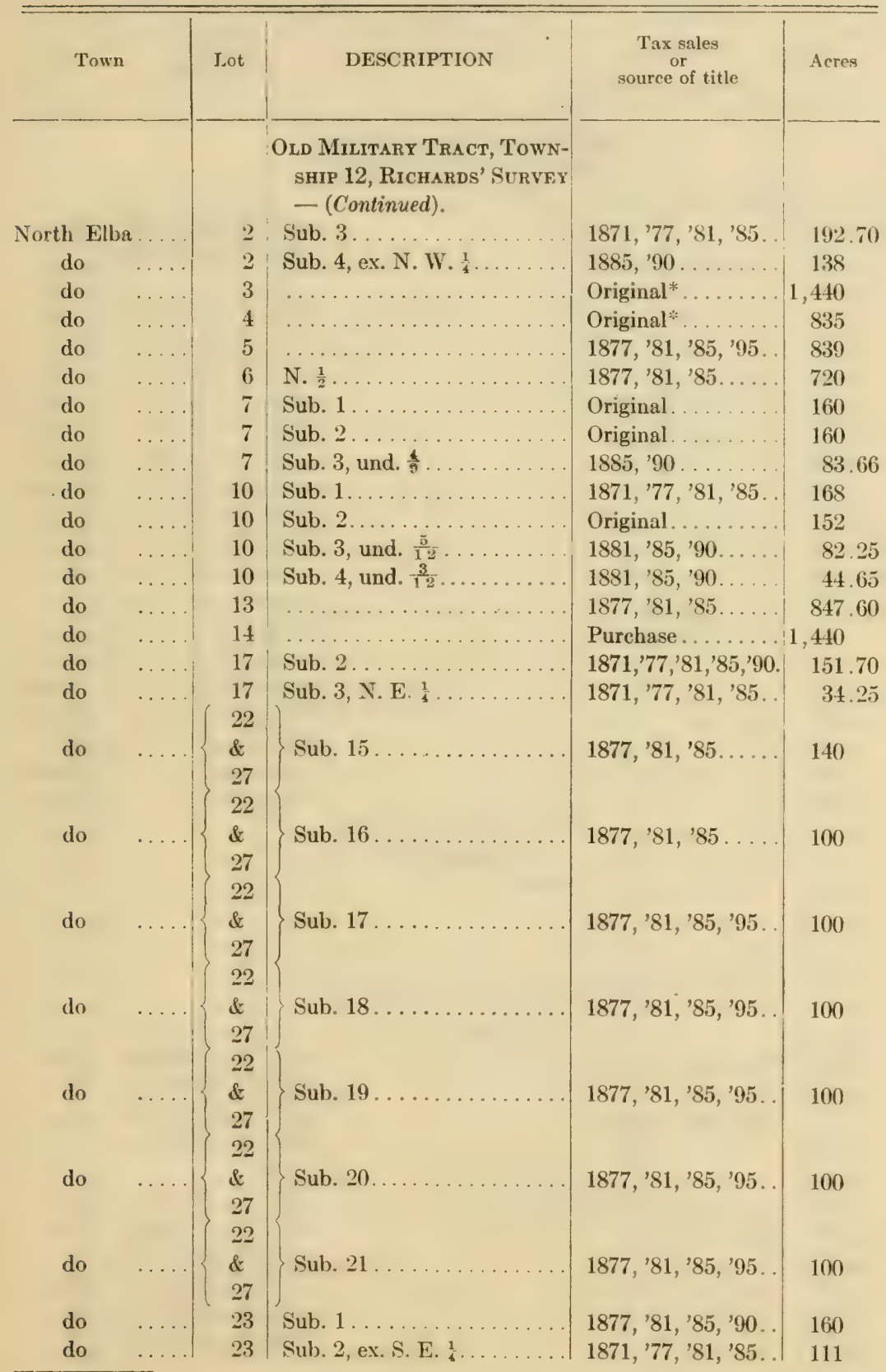

* Resale. 
Essex County

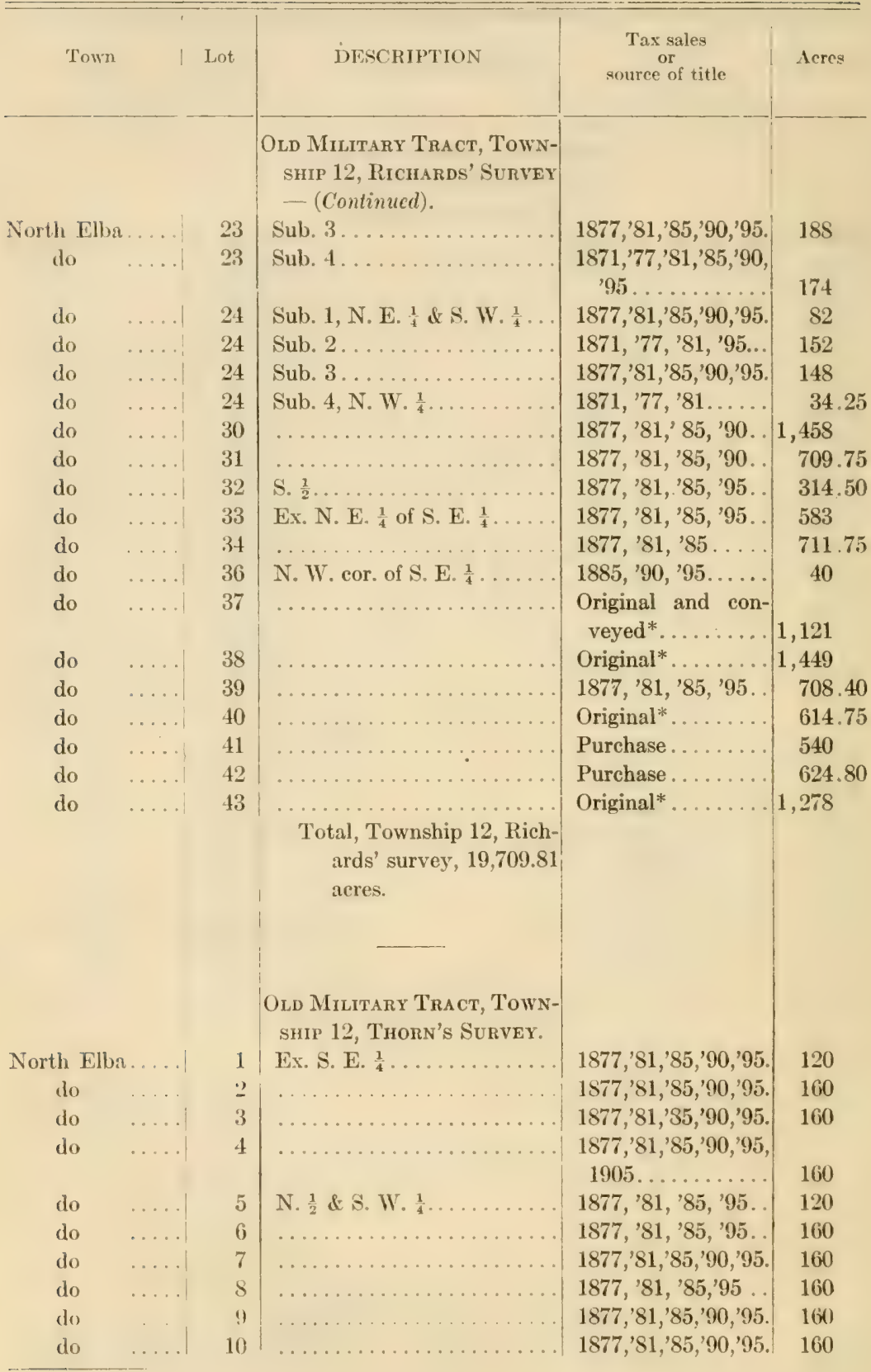

* Resale. 
Essex County

\begin{tabular}{|c|c|c|c|c|}
\hline Town & Lot & DESCRIPTION & $\begin{array}{c}\text { Tax sales } \\
\text { or } \\
\text { source of title }\end{array}$ & Acres \\
\hline & & $\begin{array}{l}\text { Old Military Tract, Town- } \\
\text { Ship } 12 \text {, Thorn's Survey- } \\
\text { (Continued). }\end{array}$ & & \\
\hline North Elba. & 11 & $\cdots$ & $1877, ' 81, ' 85,{ }^{\prime} 90, ' 95$. & 160 \\
\hline do & 12 & & $1877, ' 81, ' 85, ' 90, ' 95$ & 160 \\
\hline do & 13 & $\cdots \cdots$ & 1905 \& purchase. . & 160 \\
\hline do & 14 & $\ldots \ldots$ & $1877, ' 81, ' 85, ' 95 \ldots$ & 160 \\
\hline do & 15 & S. $\frac{1}{2}$ and N. E. $\frac{1}{4}$. & $1877, ' 81, ' 85, ' 95 \ldots$ & 120 \\
\hline do & 16 & $\ldots \ldots \ldots \ldots \ldots \ldots$ & $1877, ' 81, ' 85, ' 95 \ldots$ & 160 \\
\hline do & 17 & & $1877, ' 81, ' 35,90,95$. & 160 \\
\hline do & 18 & & $1877,91,85,90,95$. & 160 \\
\hline do & 19 & . & $1877, ' 81,95,{ }^{\prime} 90,95$. & 160 \\
\hline do & 20 & & 1877 , '81, 'S5, '95 . . & 160 \\
\hline do & 21 & S. $\frac{1}{2}$ and N. W. $\frac{1}{4}$. & $1877, ' 81, ' 85,995$. & 120 \\
\hline do & 22 & $\ldots \ldots \ldots \ldots \ldots$ & $1877, ' 81, ' 85,{ }^{\prime} 95,{ }^{\prime} 05$. & 160 \\
\hline do & 23 & N. W. $\frac{1}{4}$ and S. $\frac{1}{2} \ldots \ldots$ & $\begin{array}{r}1877, ' 81,95,90,{ }^{\prime} 95 \\
1905 \ldots \ldots \ldots \ldots \ldots\end{array}$ & 120 \\
\hline do & 23 & N. E. $\frac{1}{4}$, S. E. cor. sq . . . . & Purchase........ & 15 \\
\hline do & 24 & 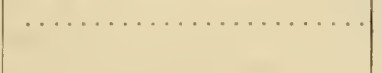 & $\begin{array}{r}1877, ' 81, ' 85, ' 90,95, \\
1905 \ldots \ldots \ldots \ldots \ldots\end{array}$ & 160 \\
\hline do & 25 & & $1877, ' 81,85,900,95$ & 160 \\
\hline do & 26 & & $1877, ' 81, ' 85, ' 90,95$ & 160 \\
\hline do & 27 & 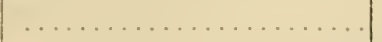 & $1877, ' 81,95,90,95$. & 160 \\
\hline do & 28 & & $1877, ' 81,95,995 \ldots$ & 160 \\
\hline do & 29 & . & $1877, ' 81,95, ' 95 \ldots$ & 160 \\
\hline do & 30 & & 1877 , '81, '85, '95. . & 160 \\
\hline do & 31 & S. $\frac{1}{2} \ldots$. & $1877, ' 81,95, ' 90, ' 95$. & So \\
\hline do & 32 & $\ldots \ldots \ldots$ & $1877, ' 81, ' 85 \ldots \ldots$ & 160 \\
\hline do & 33 & $\ldots \ldots \ldots$ & $1877, ' 81, ' 85 \ldots \ldots$ & 160 \\
\hline do & 34 & $\ldots \ldots \ldots \ldots$ & $1877, ' 81, ' 85,{ }^{\prime} 90, ' 95$. & 160 \\
\hline do & 35 & Ex. N. W. $\frac{1}{4} \ldots \ldots \ldots$ & $1877, ' 81, ' 85, ' 95 \ldots$ & 120 \\
\hline do & 36 & $\ldots$ & $1877, ' 81, ' 85, ' 95 \ldots$ & 160 \\
\hline do & 37 & $\ldots$. & $1877, ' 81, ' 85 \ldots \ldots$ & 160 \\
\hline do & 40 & & $1877, ' 81, ' 85 \ldots \ldots$ & 160 \\
\hline do & 41 & & $1877, ' 81,{ }^{\prime} 85, ' 90, ' 95$. & 160 \\
\hline do & 42 & & $\begin{array}{r}1877, ' 81,85, ' 90,95, \\
1900 \ldots \ldots \ldots \ldots\end{array}$ & 160 \\
\hline do & 43 & & $1877, ' 81,95 \ldots$. & 160 \\
\hline do & 47 & & $1877, ' 81, ' 85, ' 95 \ldots$ & 160 \\
\hline do & 48 & & $1877, ' 81, ' 85, ' 95$. & 160 \\
\hline do & 49 & Ex. S. W. $\frac{1}{1}$ & $1877, ' 81, ' 85, ' 90$. & 120 \\
\hline do & 50 & & $1871, ' 77, ' 91, ' 95$. & 160 \\
\hline do & 53 & & $1877, ' 81, ' 85, ' 95 \ldots$ & 160 \\
\hline do & 54 & N. $\frac{1}{2}$ and $S . M . \frac{1}{4}$. & $1877, ' 81, ' 85,95 . . \mid$ & 120 \\
\hline
\end{tabular}


Essex County

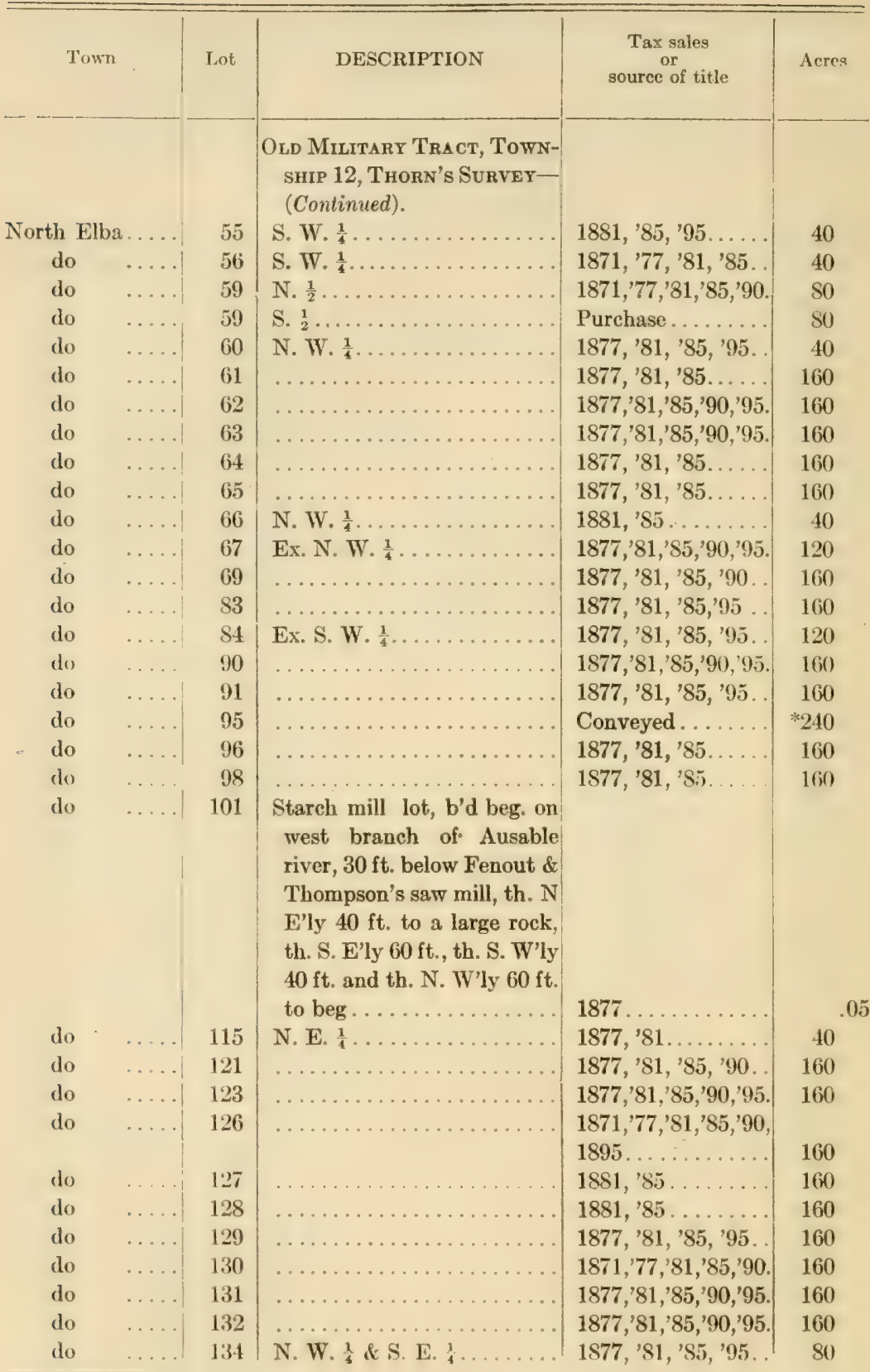

* John Brown farm. 
Essex County

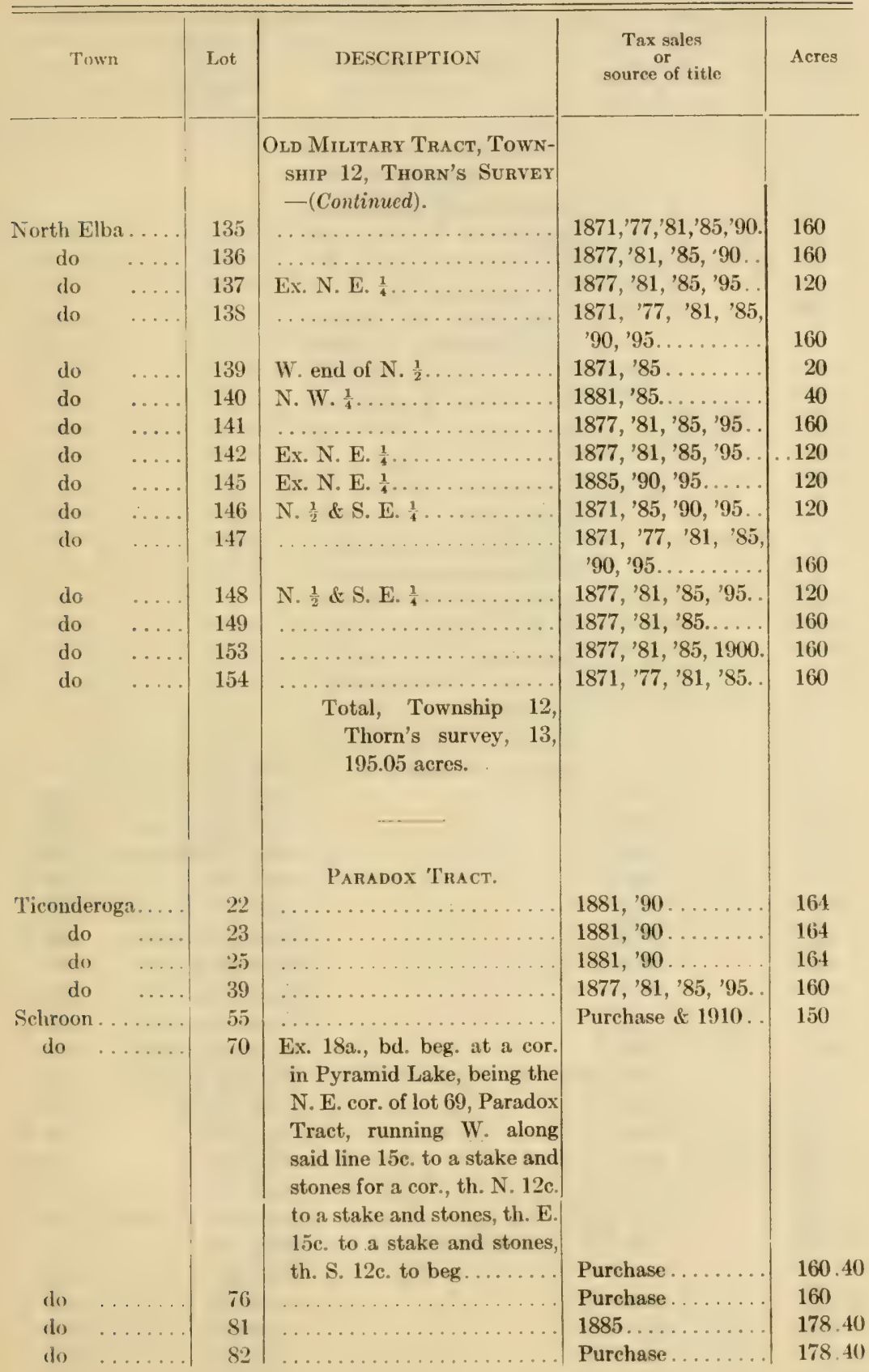


Essex County

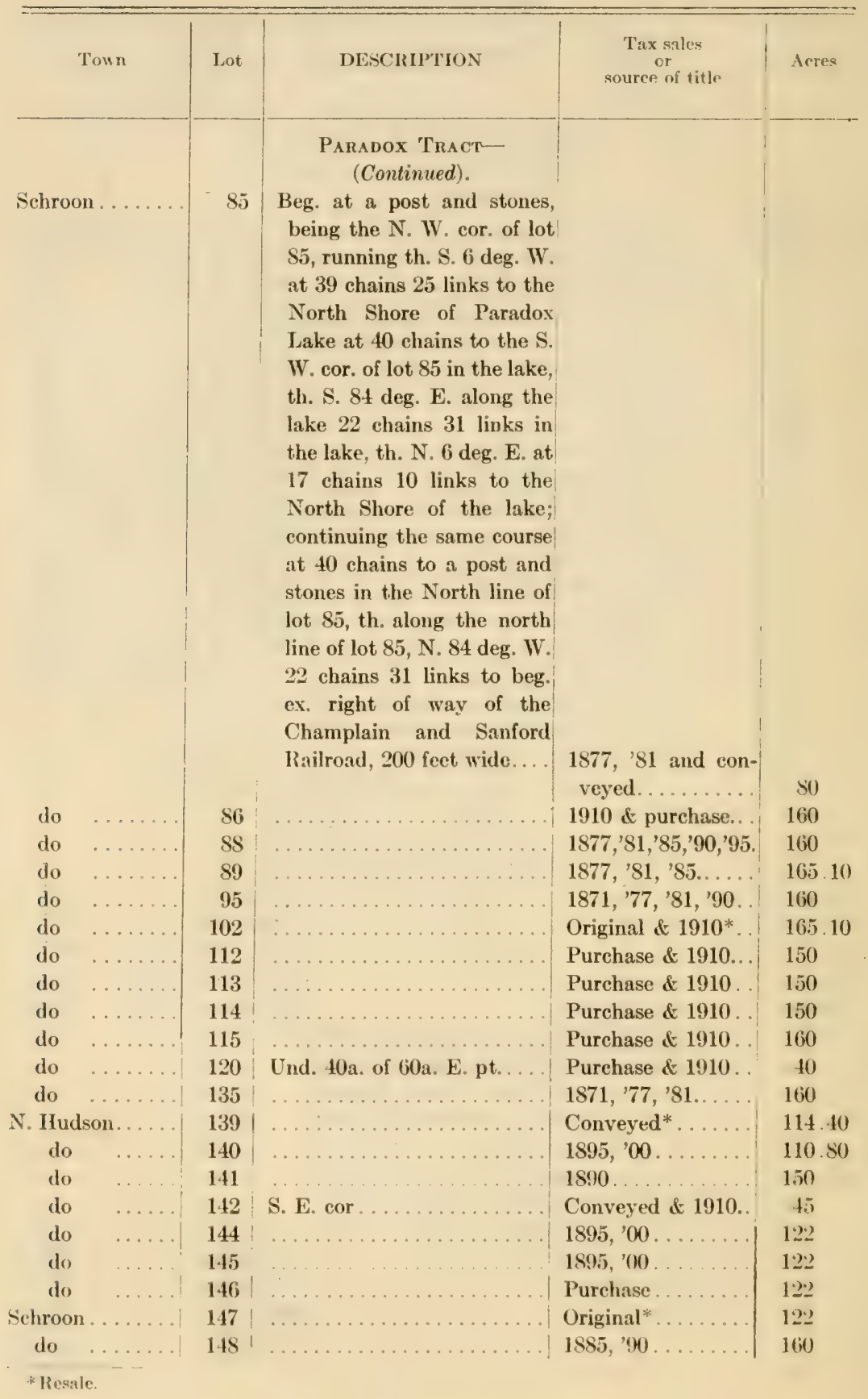


Essex County

\begin{tabular}{|c|c|c|c|c|}
\hline Town & rot & DESCRIPTION & $\begin{array}{l}\text { Tax sales } \\
\text { or } \\
\text { souree of title. }\end{array}$ & Arres \\
\hline & & $\begin{array}{c}\text { Paradox Tract- } \\
\text { (Continued) }\end{array}$ & & \\
\hline Sehroon & 149 & $\ldots \ldots \ldots \ldots \ldots \ldots$ & $1885,90$. & 160 \\
\hline do & 165 & & $1885,90 \ldots \ldots$ & 160 \\
\hline do & 166 & . & $1885,{ }^{\prime} 90,{ }^{\prime} 95 \ldots$. & 160 \\
\hline N. Hudson. & 171 & $\ldots \ldots \ldots \ldots \ldots \ldots \ldots$ & $1890,95 \ldots \ldots \ldots$. & 160 \\
\hline do & 172 & $\begin{array}{c}\text { Ex. und. } \frac{1}{2} \text { Crown Point } \\
\text { Iron Co } \ldots \ldots \ldots \ldots \ldots\end{array}$ & $1877,81,955 \ldots$ & 84.30 \\
\hline do & $17+$ & $\ldots \ldots \ldots$ & $1877, ' 81,85 \ldots \ldots$ & 150 \\
\hline do & 184 & $\ldots \ldots \ldots \ldots \ldots \ldots$ & $1877, ' 81, ' 85, ' 95 \ldots$ & 160 \\
\hline do & 185 & $\ldots \ldots \ldots \ldots \ldots \ldots$ & $1877, ' 81, ' 85,{ }^{\prime} 95 \ldots$ & 149.30 \\
\hline do & 188 & Und. $\frac{1}{2} \ldots \ldots \ldots \ldots$ & Conveyed....... & 80 \\
\hline do & 195 & $\ldots \ldots \ldots \ldots \ldots \ldots \ldots \ldots$ & Purchase....... & 165.20 \\
\hline do & 196 & $\ldots \ldots \ldots \ldots \ldots \ldots \ldots$ & $1895,{ }^{\prime} 00 \ldots \ldots \ldots$ & 160 \\
\hline do & 197 & $\ldots \ldots \ldots \ldots \ldots \ldots \ldots$ & $1890, ' 95, ' 00 \ldots \ldots$ & 160 \\
\hline do & 199 & $\ldots \ldots \ldots$ & Conveyed....... & 152 \\
\hline do & 201 & $\ldots \ldots \ldots \ldots \ldots \ldots \ldots$ & Conveyed....... & 160 \\
\hline do & 203 & $\ldots \ldots \ldots \ldots \ldots \ldots$ & Purchase........ & 152.70 \\
\hline do & 212 & S. $\frac{1}{2} \ldots \ldots \ldots \ldots$ & $1910 \&$ Purchase . . & 80 \\
\hline do & 217 & $\ldots \ldots \ldots \ldots \ldots$ & $\begin{array}{r}1871,777,81,95,90 \\
95 \ldots . . .\end{array}$ & 160 \\
\hline do & 218 & $\ldots \ldots \ldots$ & Conveyed \& 1910. & 160 \\
\hline do & 220 & $\ldots \ldots \ldots \ldots \ldots \ldots \ldots$ & Purchase \& $1910 \ldots$ & 160 \\
\hline do & 221 & & Purchase \& $1910 \ldots$ & 160 \\
\hline do & 222 & & Purchase \& 1910 . I & 160 \\
\hline do & 2.3 & . & Purchase \& $1910 \ldots$ & 160 \\
\hline do & 224 & .. & $1871,77, ' 81, ' 85$. & $165 \cdot 20$ \\
\hline do & 230 & $\ldots \ldots \ldots$ & Conveyed....... & 160 \\
\hline do & 231 & & Conveyed ....... & 160 \\
\hline do & 234 & & Purchase......... & 160 \\
\hline do & 23:3 & & $\begin{array}{c}1910 \text { \& Conveyed } \\
\& \text { Purchase.... }\end{array}$ & 160 \\
\hline do & 236 & & $\begin{array}{c}\text { Conveyed \& Pur- } \\
\text { chase.......... }\end{array}$ & 160 \\
\hline do & 237 & . & Conveyed \& 1910 & 160 \\
\hline do & 238 & $\ldots \ldots \ldots \ldots \ldots \ldots \ldots \ldots \ldots$ & Conveyed \& 1910. & 160 \\
\hline do & 239 & . & $1871, ' 77, ' 81, ' 10 \ldots$ & 160 \\
\hline Crown Point & 240 & $\ldots \ldots \ldots \ldots \ldots \ldots \ldots$ & Purchase........ & 160 \\
\hline do & 247 & Und. $\frac{1}{8}$ for. pd. by Russell. & $1895,{ }^{\prime} 00 \ldots \ldots \ldots$ & 20.75 \\
\hline do & 259 & $\ldots \ldots \ldots \ldots$ & Purchase ........ & 160 \\
\hline N. Hudson. & 260 & . & Purchase ........ & 160 \\
\hline do $\ldots$ & 261 & & Conveyed \& 1910. & 160 \\
\hline do & 262 & & Purchase \& $1910 \ldots$ & 160 \\
\hline do & 263 & & Purchase \& 1910 & 160 \\
\hline
\end{tabular}


Essex County

\begin{tabular}{|c|c|c|c|c|}
\hline Town & Let & DESCRIPTION & $\begin{array}{c}\text { Tax sales } \\
\text { or } \\
\text { source of title }\end{array}$ & Acres \\
\hline & & $\begin{array}{c}\text { Paradox Tract- } \\
\text { (Continued })\end{array}$ & & \\
\hline N. Hudson. & 266 & & Purchase. & 160 \\
\hline Moriah.... & 289 & & $1885,90$. & 160 \\
\hline do & 292 & & $1871,77, ' 81, ' 90$. & 160 \\
\hline do & 293 & Ex. 80a. N. W. cor. & $\begin{array}{r}1871,77,91,85 \\
95 \ldots \ldots \ldots\end{array}$ & So) \\
\hline do & 294 & & $\begin{array}{r}1871, ' 77, \quad 81,95 \\
90,95 \ldots \ldots \ldots\end{array}$ & 160 \\
\hline do & 295 & $\begin{array}{l}\text { Ex, und. } \frac{2}{3} \text { of W. } 110 \mathrm{a} . \text {, pd. } \\
\text { by A. F. Edwards . . . . . }\end{array}$ & $1871, ' 77, ' 85, ' 90$. & 86.66 \\
\hline Crown Point & 297 & $\ldots \ldots \ldots \ldots \ldots \ldots \ldots$ & $1885,90,95 \ldots$. & 160 \\
\hline do & 312 & $\begin{array}{l}\text { All of N. } \frac{1}{2} \text {, in Schroon in } \\
1857 \& 1860 \text {, ex. und. } \frac{2}{3} \\
\text { thereof paid by D. Rider . } \\
\text { Und. } \frac{1}{3} \text { of S. } \frac{1}{2} \text {, rem. } \frac{2}{3} \text { for. pd. } \\
\text { by D. Rider. . . . . . . . . . }\end{array}$ & $\begin{array}{l}1890,{ }^{\prime} 00 \ldots \ldots \\
1895 \ldots \ldots \ldots\end{array}$ & $\begin{array}{r}6.66 \\
26.66 i\end{array}$ \\
\hline Moriah. & 314 & 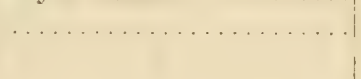 & $\begin{array}{r}1871,77, \quad 81, .85 \\
990,95 \ldots \ldots \ldots\end{array}$ & 160 \\
\hline do & 315 & & $1871, ' 77, ' 81, ' 95$. & 160 \\
\hline N. Hudson. & 349 & & Purchase \& 1910. & 160 \\
\hline do & 350 & 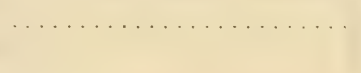 & $\begin{array}{r}1871,77,91,95, \\
90,95 \ldots \ldots \ldots\end{array}$ & 160 \\
\hline do & 351 & & Conveyed . . . . . . . & 16() \\
\hline do & 352 & & Conveyed. & 160 \\
\hline do & 353 & & $1871, ' 77, ' 81, ' 85$. & 167 \\
\hline do & 354 & 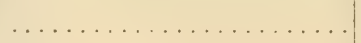 & $1871 \ldots \ldots \ldots$ & 37.50 \\
\hline do & 355 & & Purchase . . . . . . . & 89.50 \\
\hline do & 356 & & Purchase \& 1910. & $16 \overline{7}$ \\
\hline do & 358 & & $1871,{ }^{\prime} 90,95 \ldots$ & 160 \\
\hline do & 377 & & Purchase \& 1910. & 160 \\
\hline do & 378 & & Purchase \& 1910 . & 160 \\
\hline do & 379 & $\ldots \ldots \ldots \ldots \ldots$ & $\begin{array}{c}\text { Conveyed \& } 1905, \\
\text { '00 and } 10 \ldots \ldots\end{array}$ & INt \\
\hline do & 380 & & Purchase ....... & $18 !$ \\
\hline do & 381 & $\begin{array}{c}\text { Ex. mill \& water power } \\
\text { thereon . . . . . . . }\end{array}$ & Mortgage. . & 124.60 \\
\hline do & 382 & 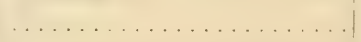 & Mortgage . . . . . . & $4 ; 3$ \\
\hline do & 385 & & $1885,90 \ldots$ & 160 \\
\hline do & 386 & & Purchase \& 1910. & 160 \\
\hline do & 387 & & Purchase \& 1910. & 160 \\
\hline do & 397 & & Purchase \& 1910. & 145.40 \\
\hline do & 398 & & Purchase \& 1910. & 5.3 .40 \\
\hline do & 399 & & 1881, ' 85. & 12 \\
\hline
\end{tabular}


Essex County

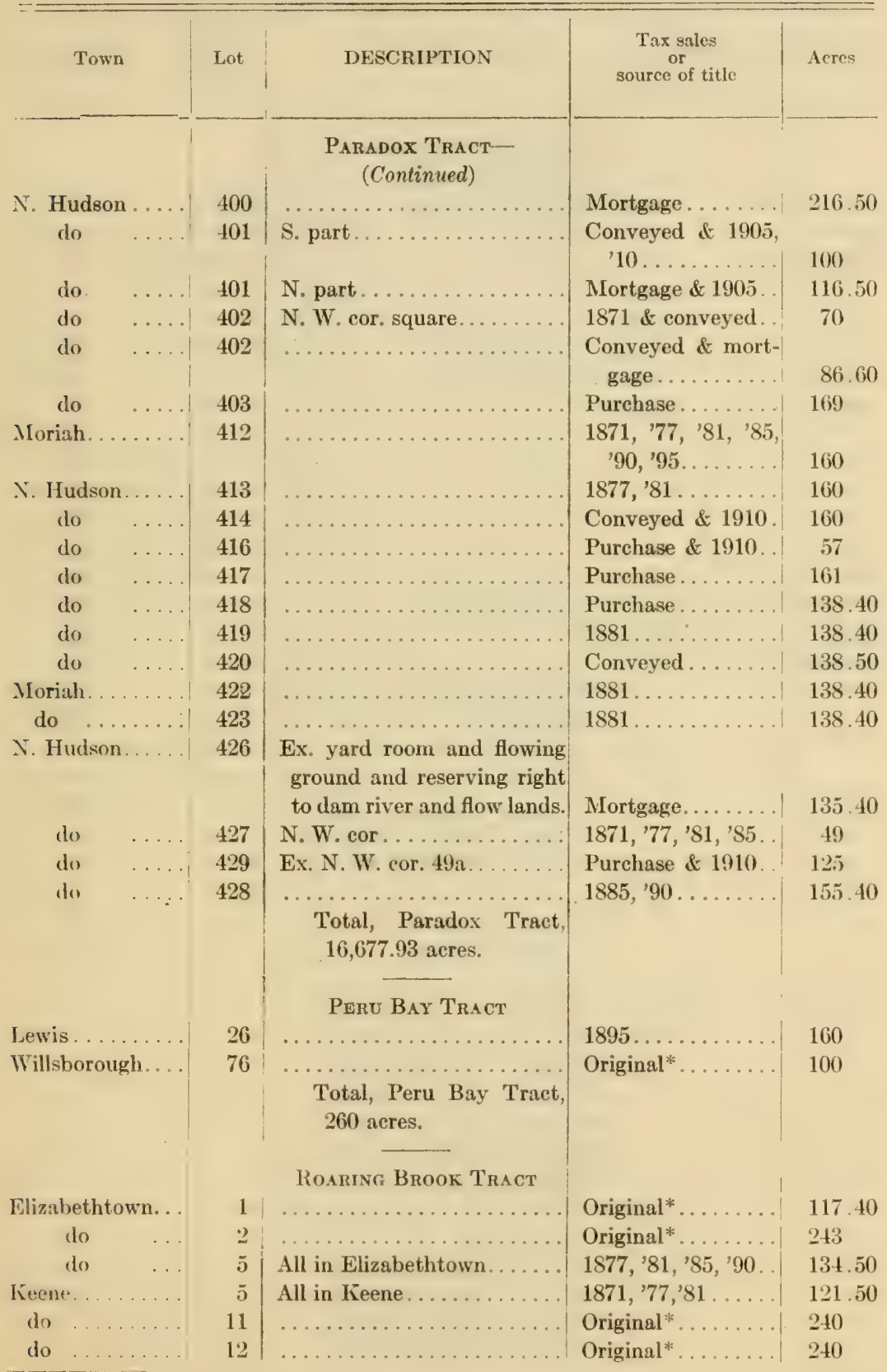

* Resale. 
E'ssex ( County

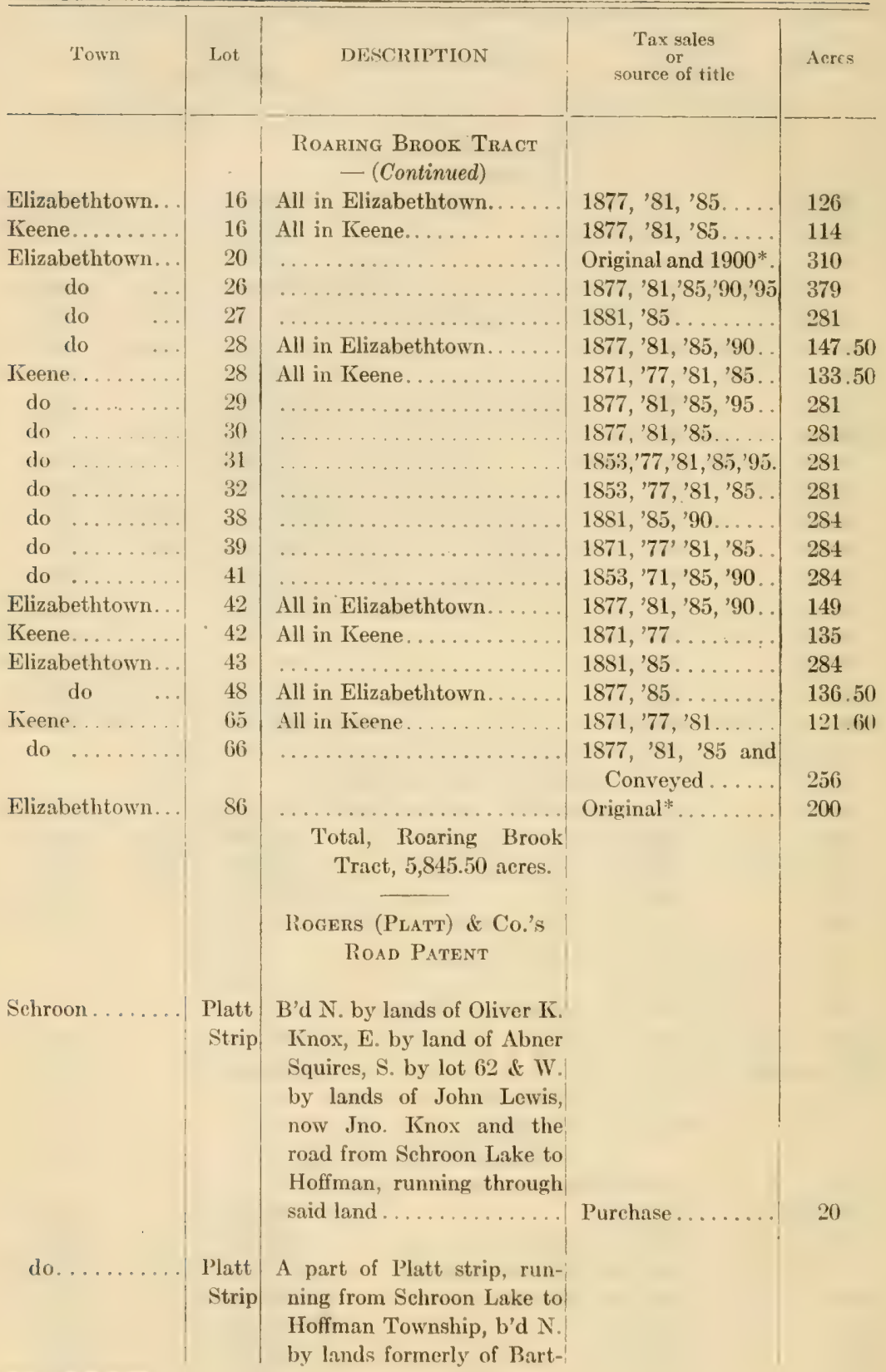

* Resale. 
Essex Countr

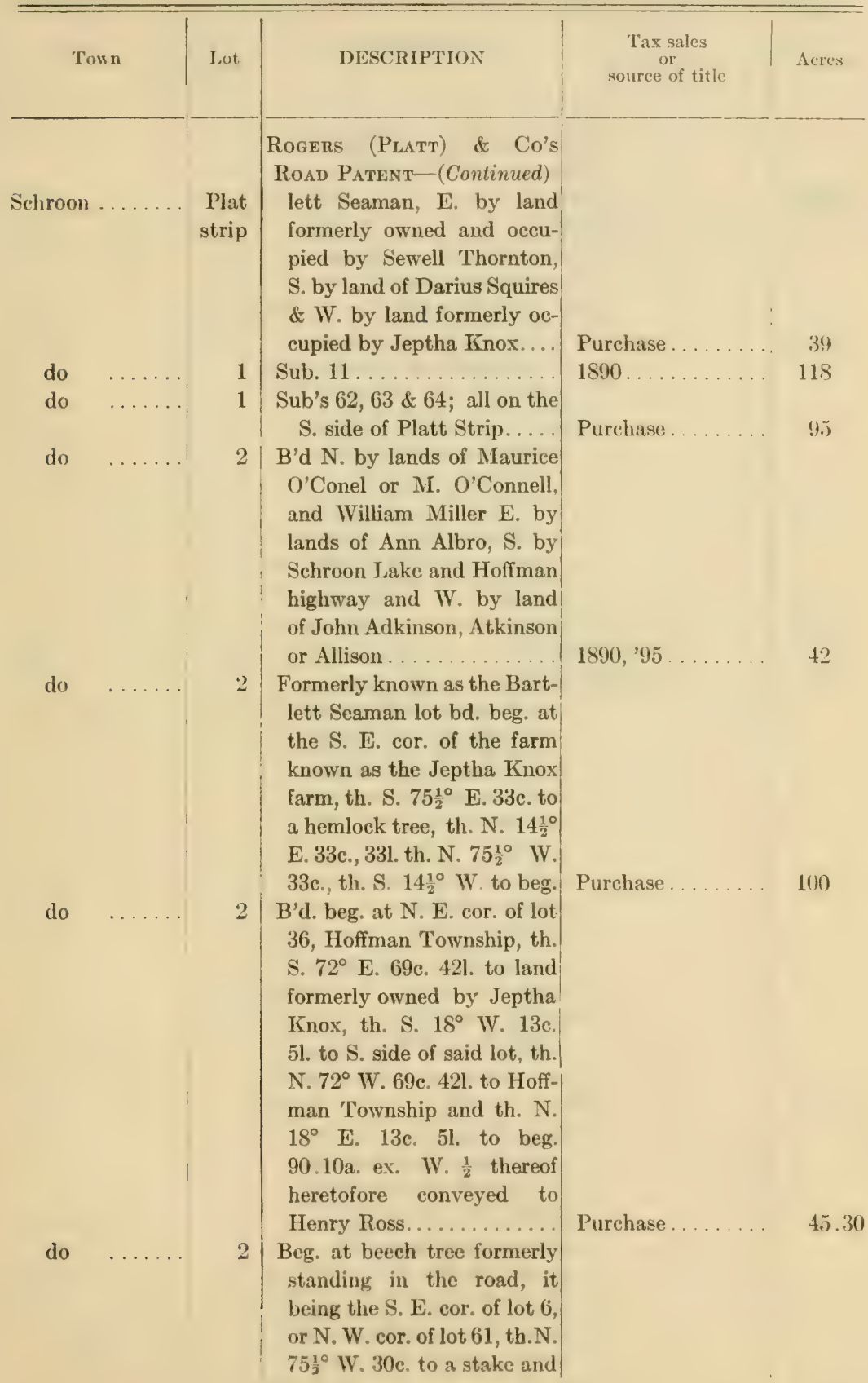


Essex County

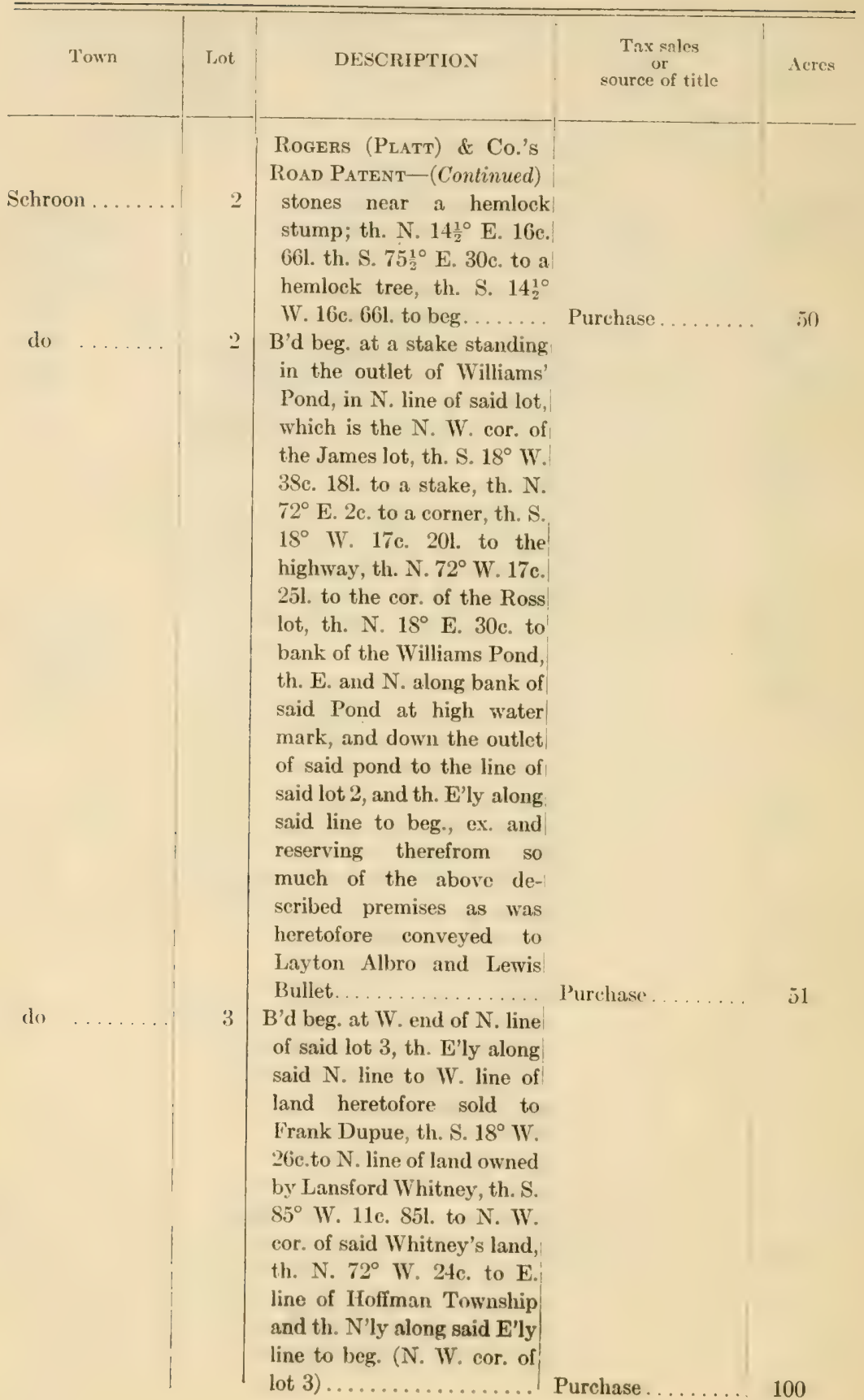


Essex County

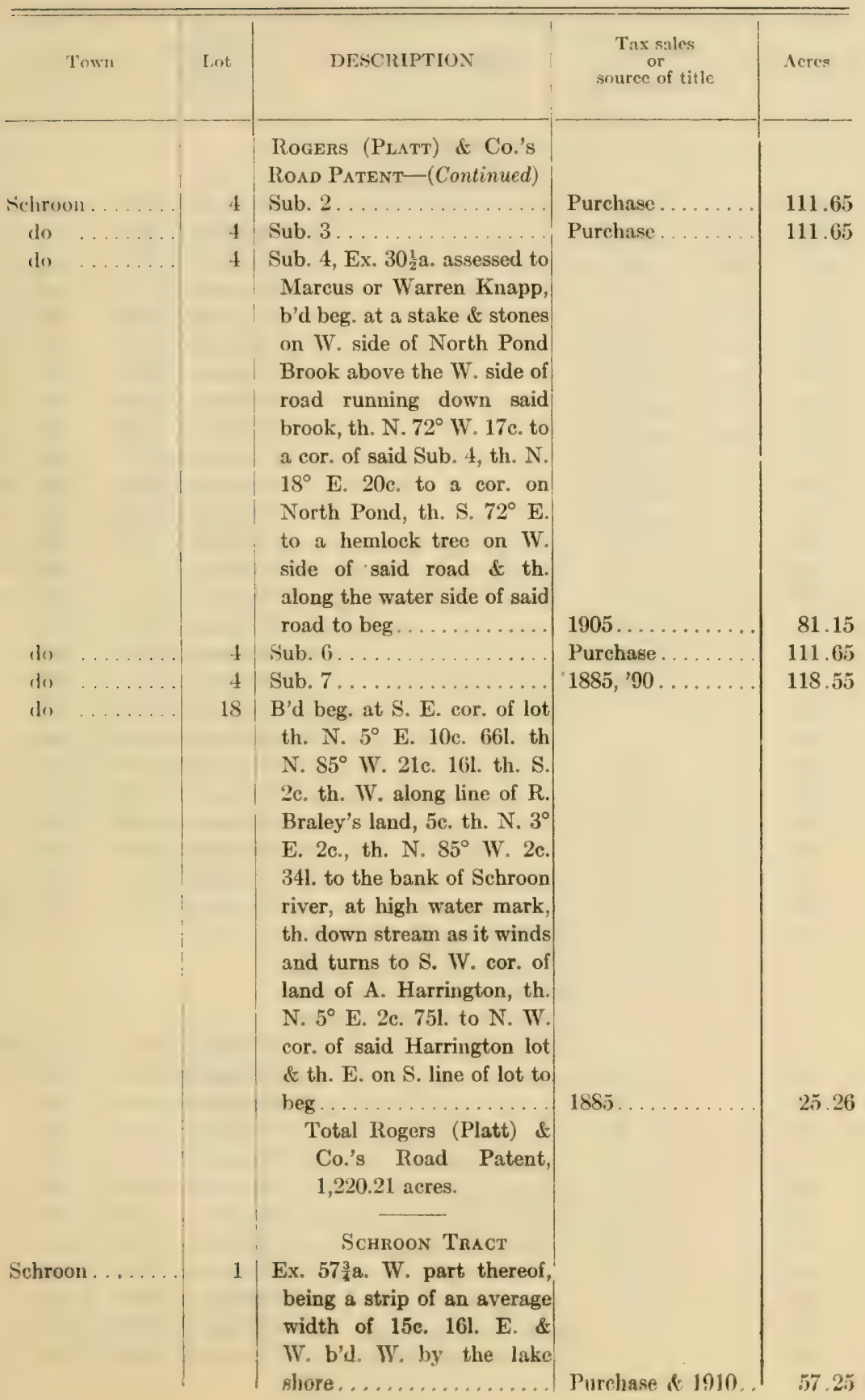


Essex County

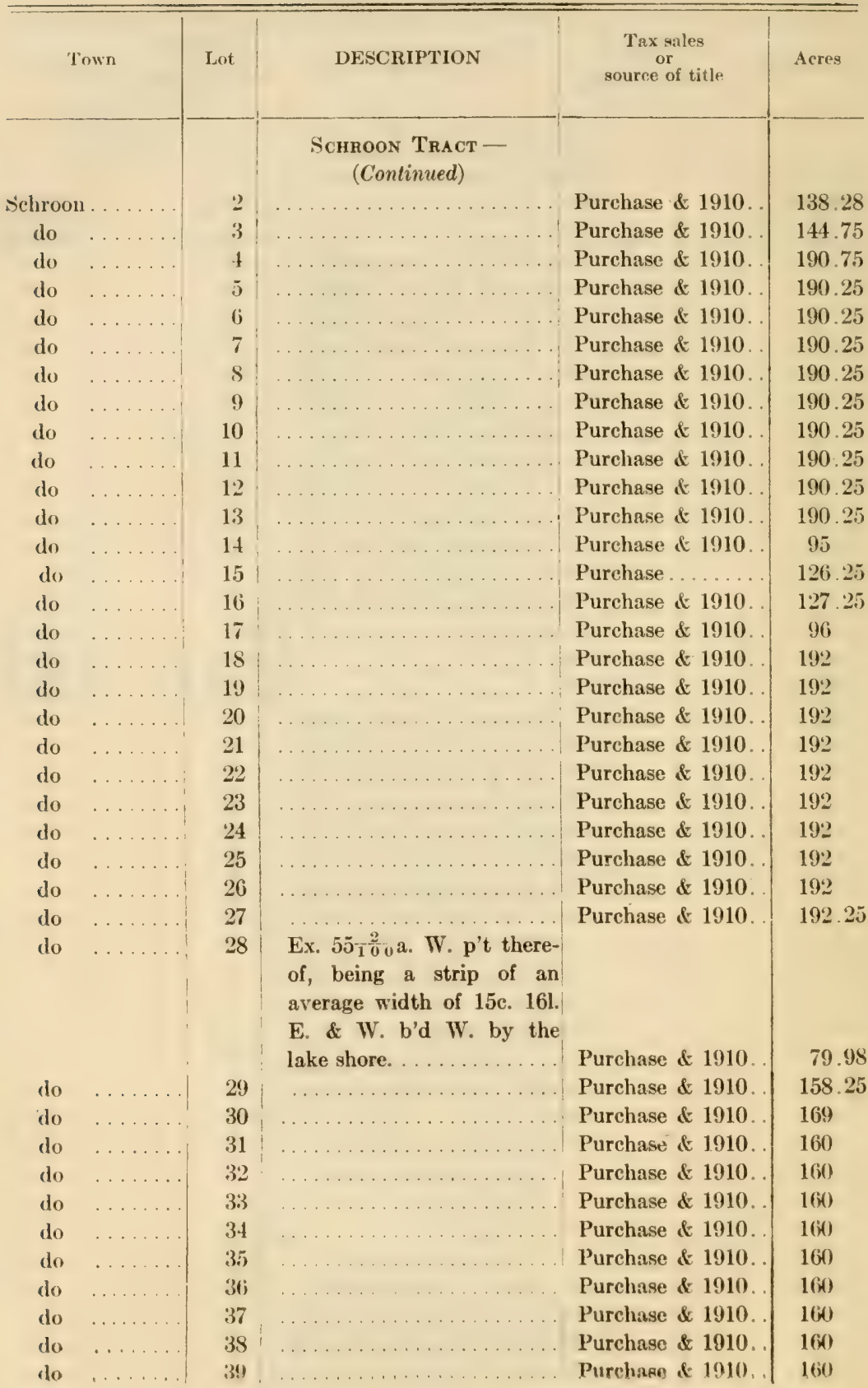


Essex Country

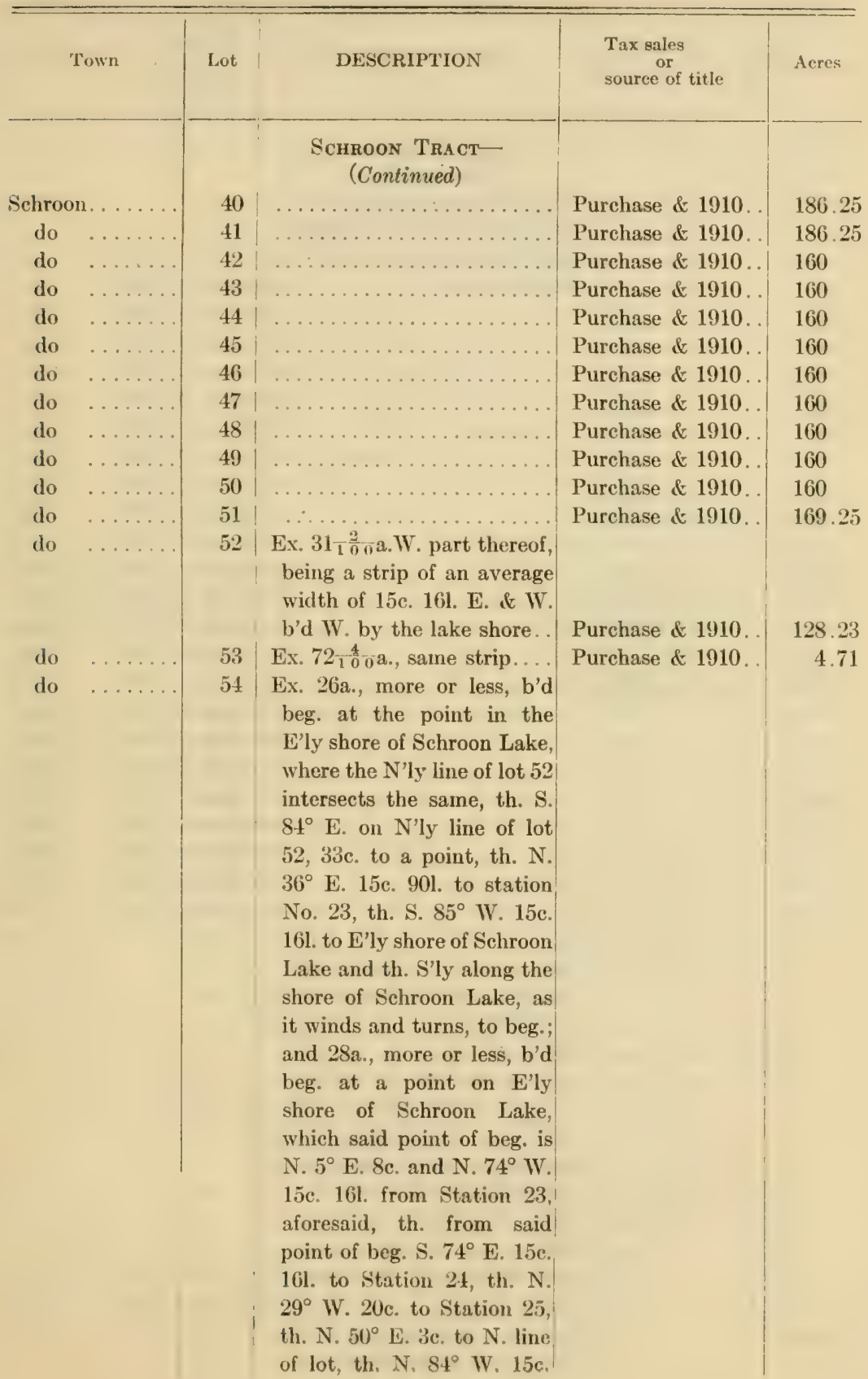


Essex Countr

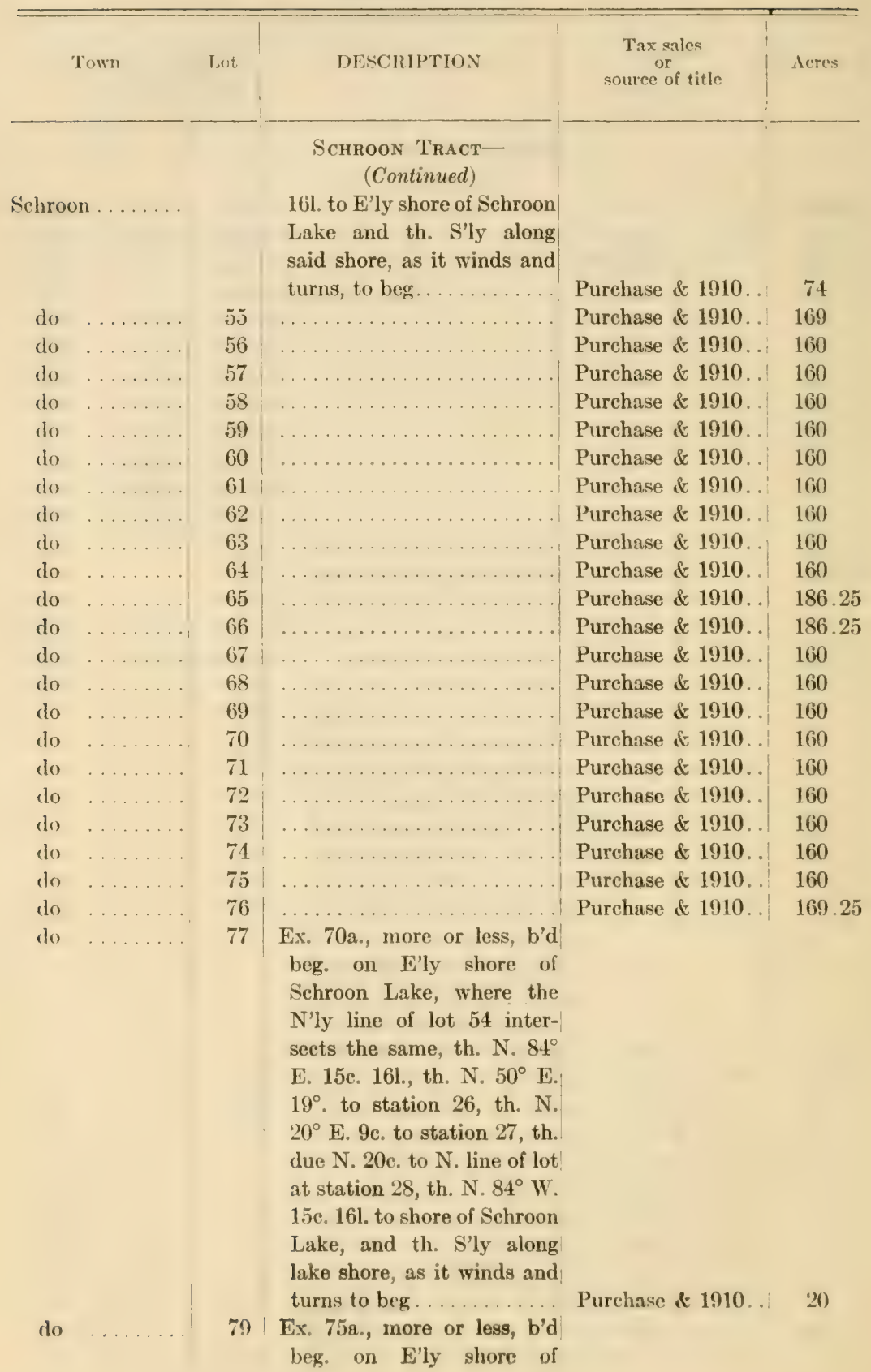


ESSEX COUNTY

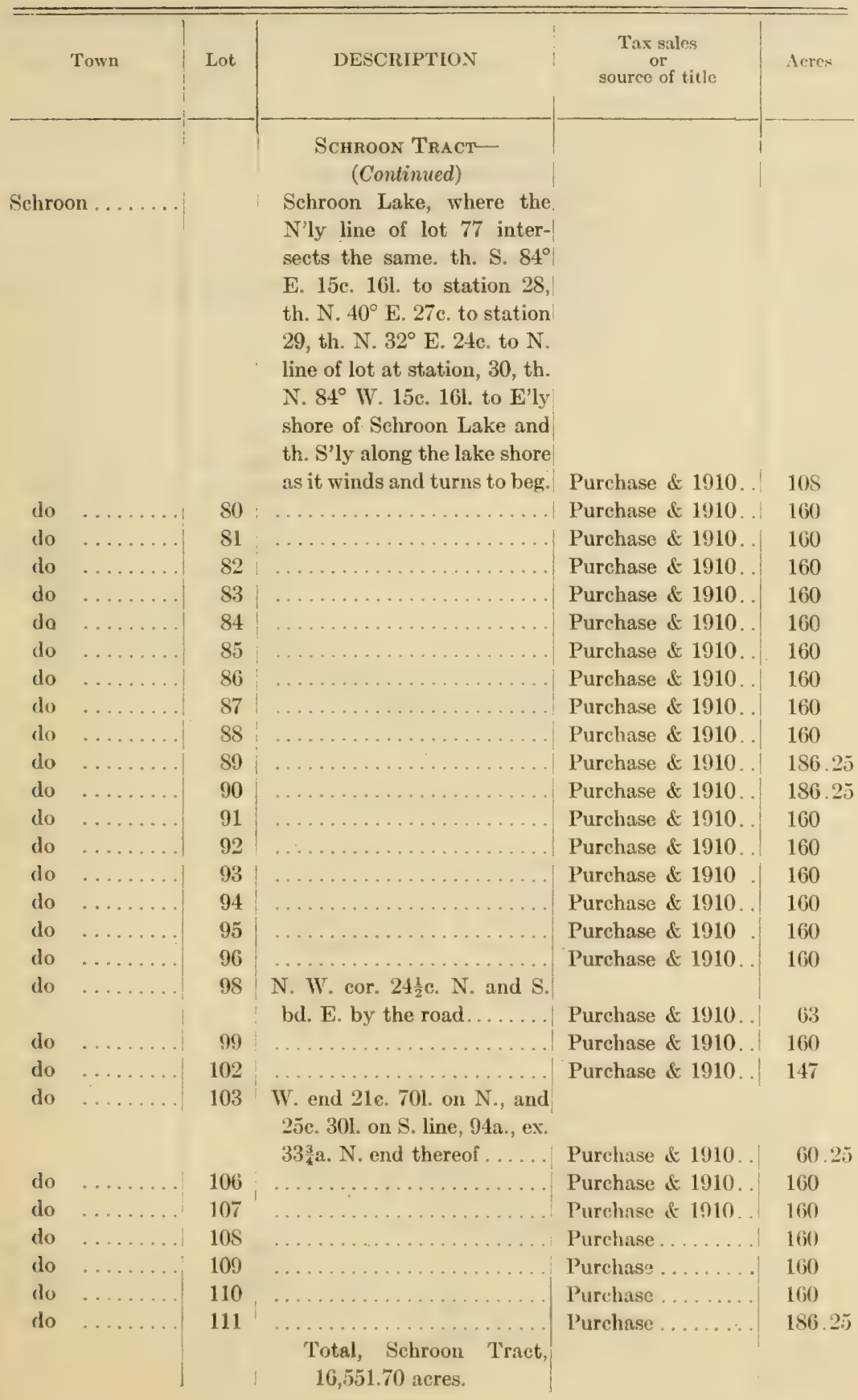




\section{Essex County}

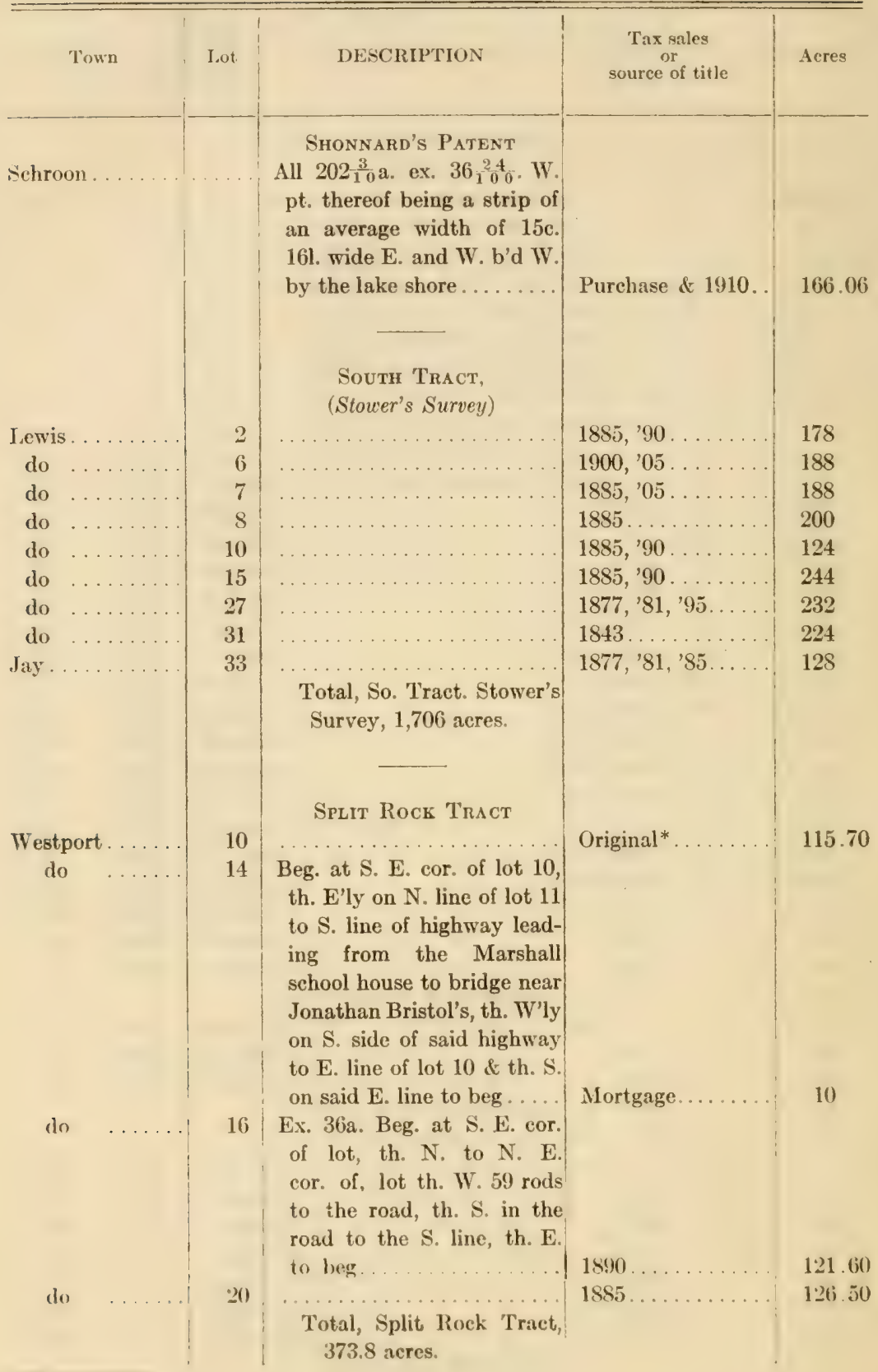


Essex County

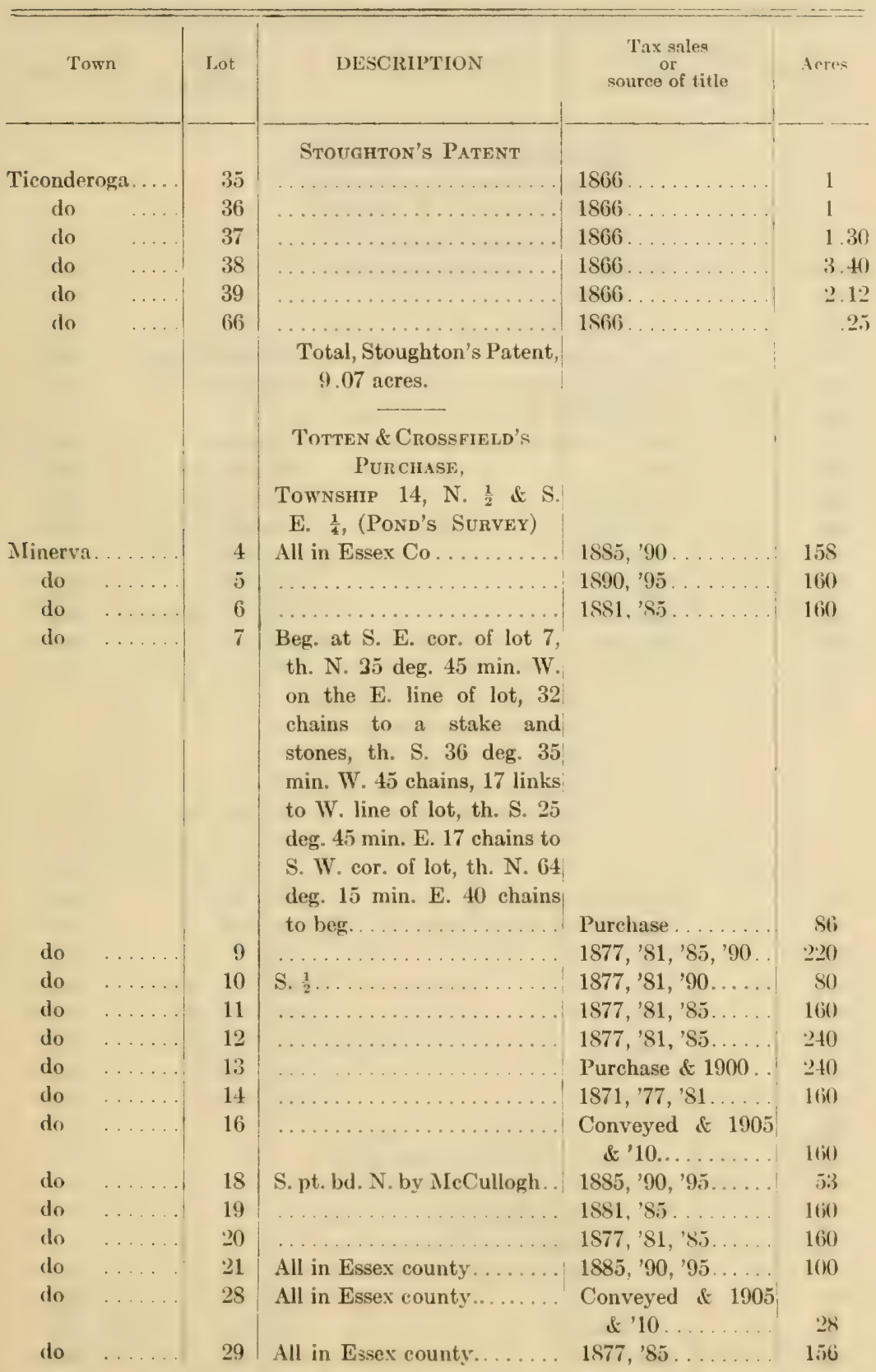


Essex County

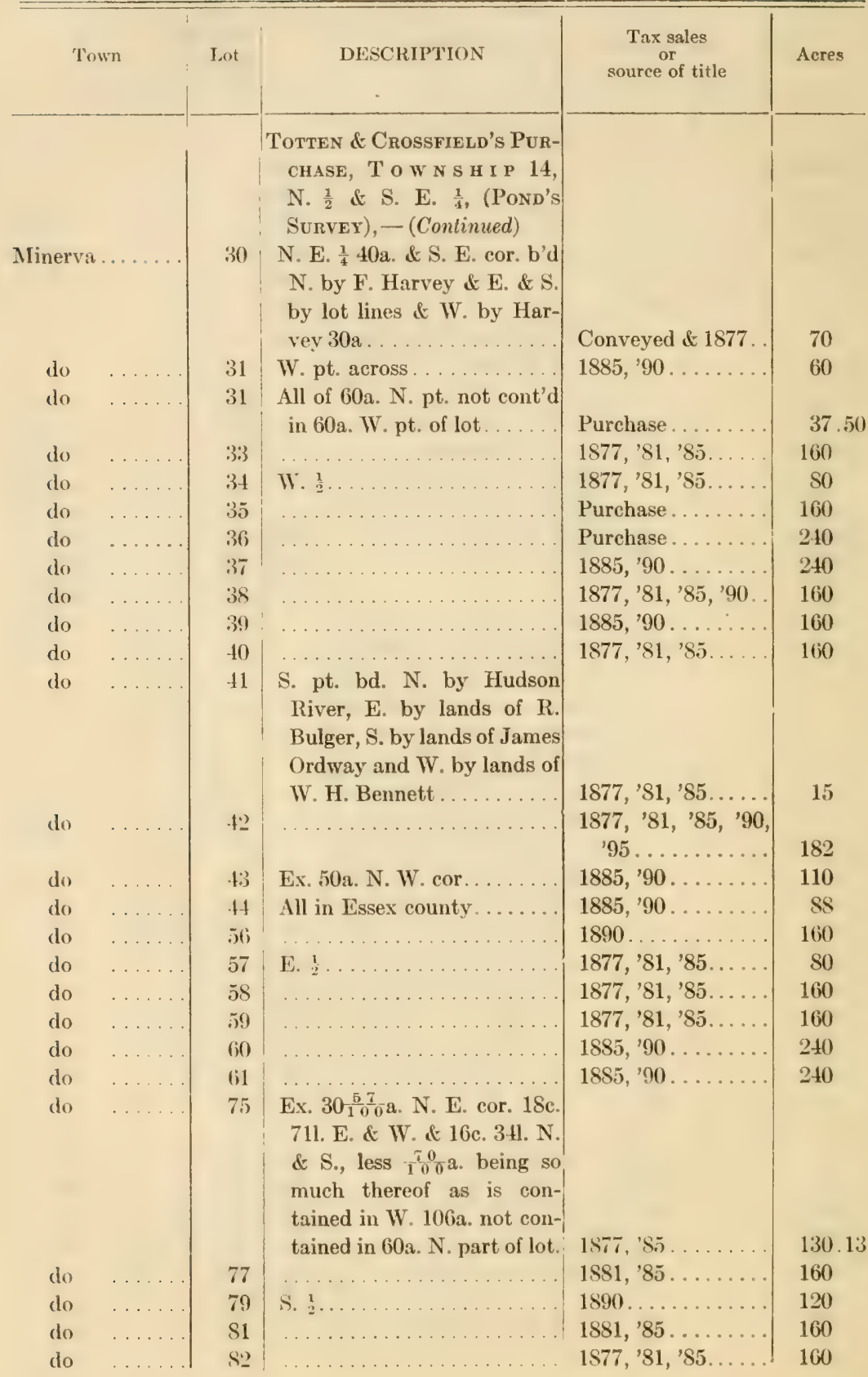




\section{Essex County}

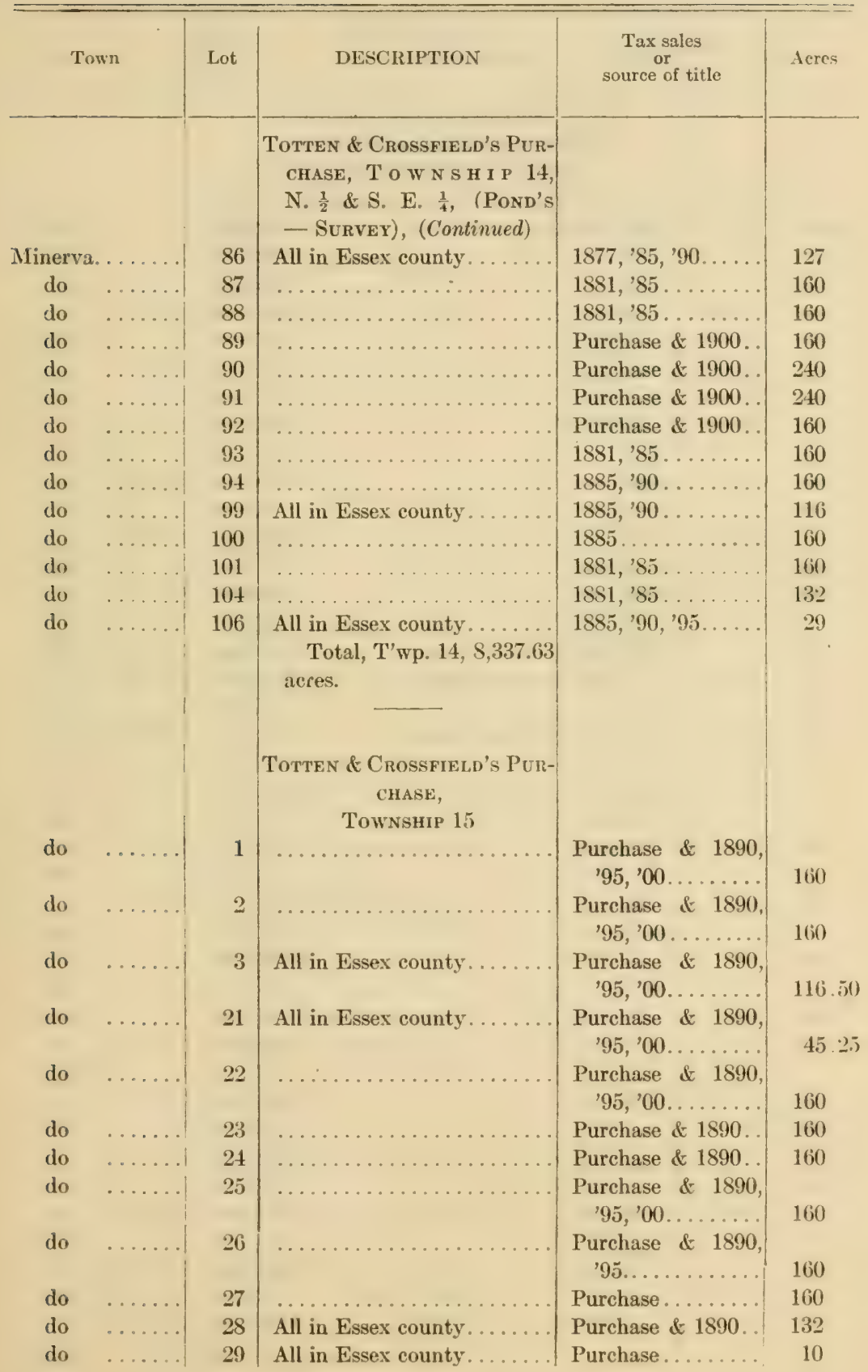




\section{Essex County}

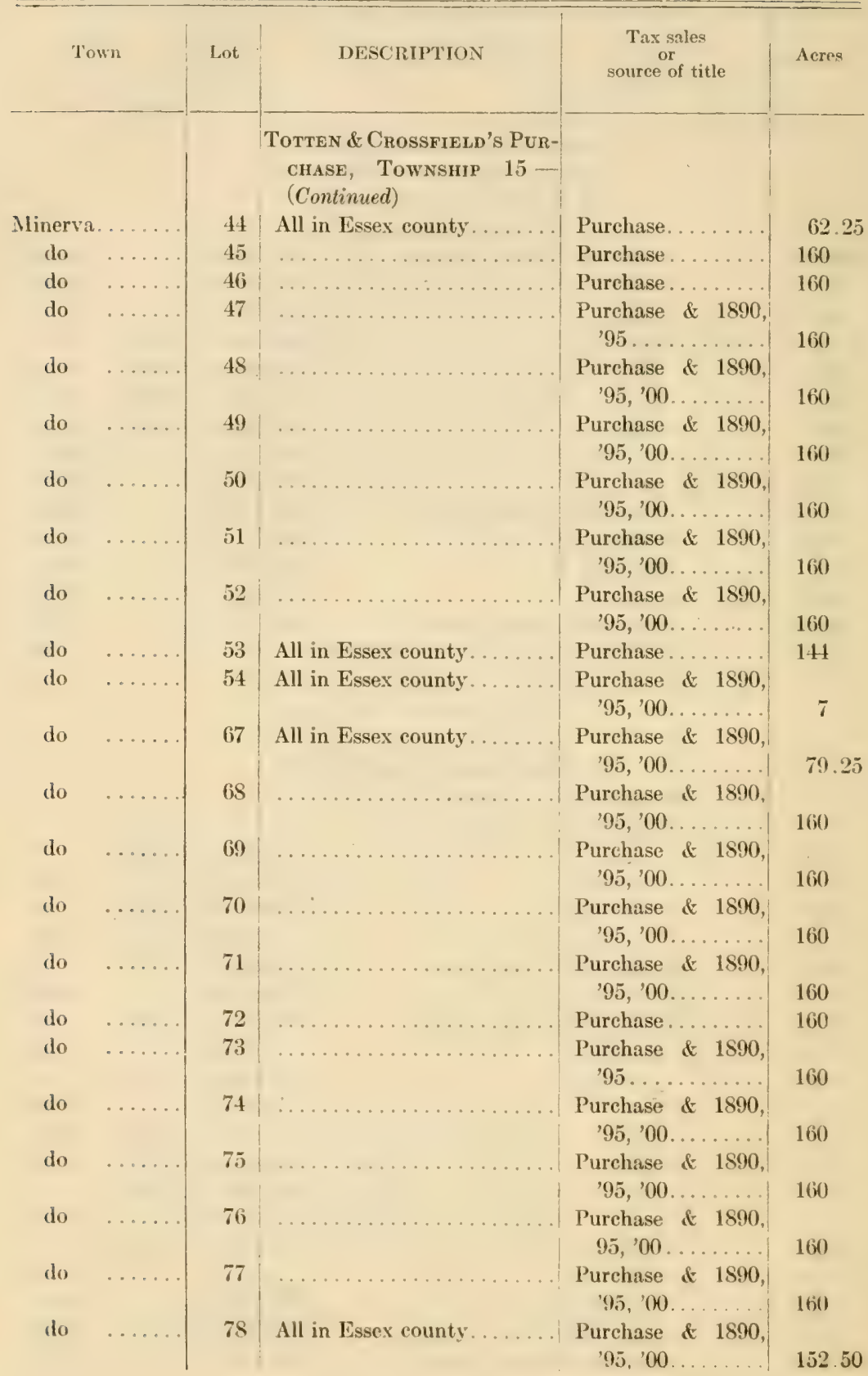


Essex County

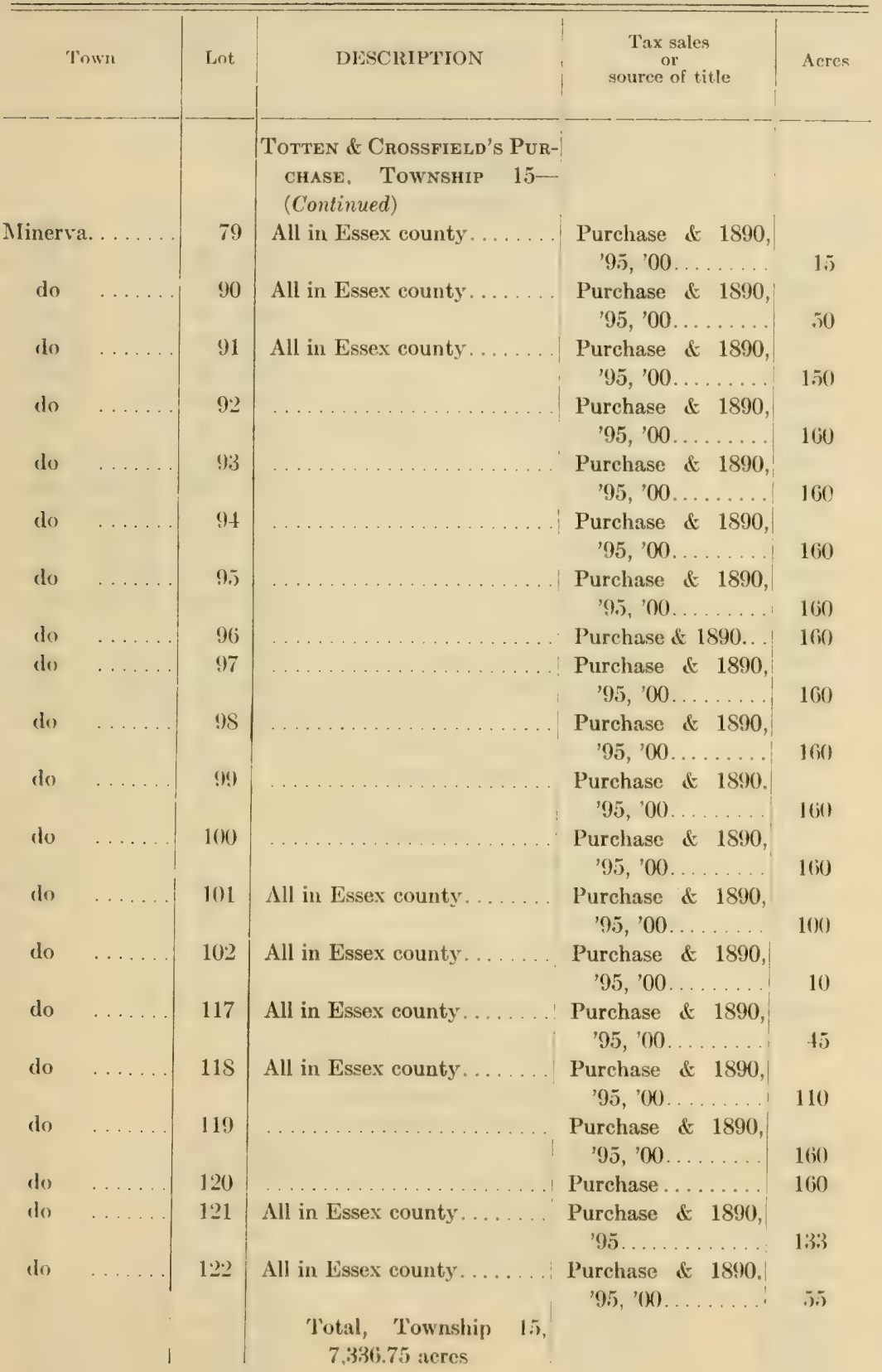


Essex County

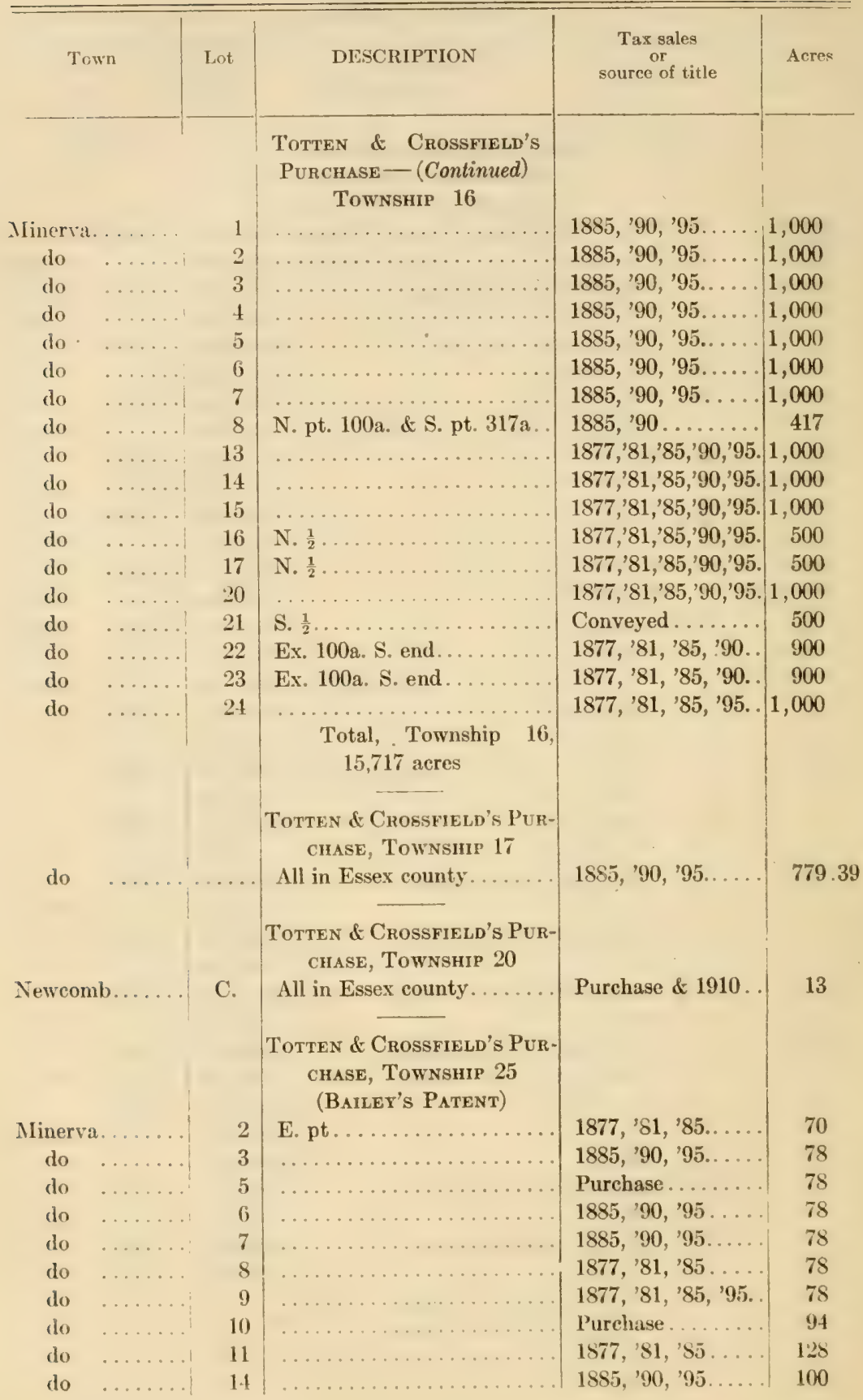


Essex County

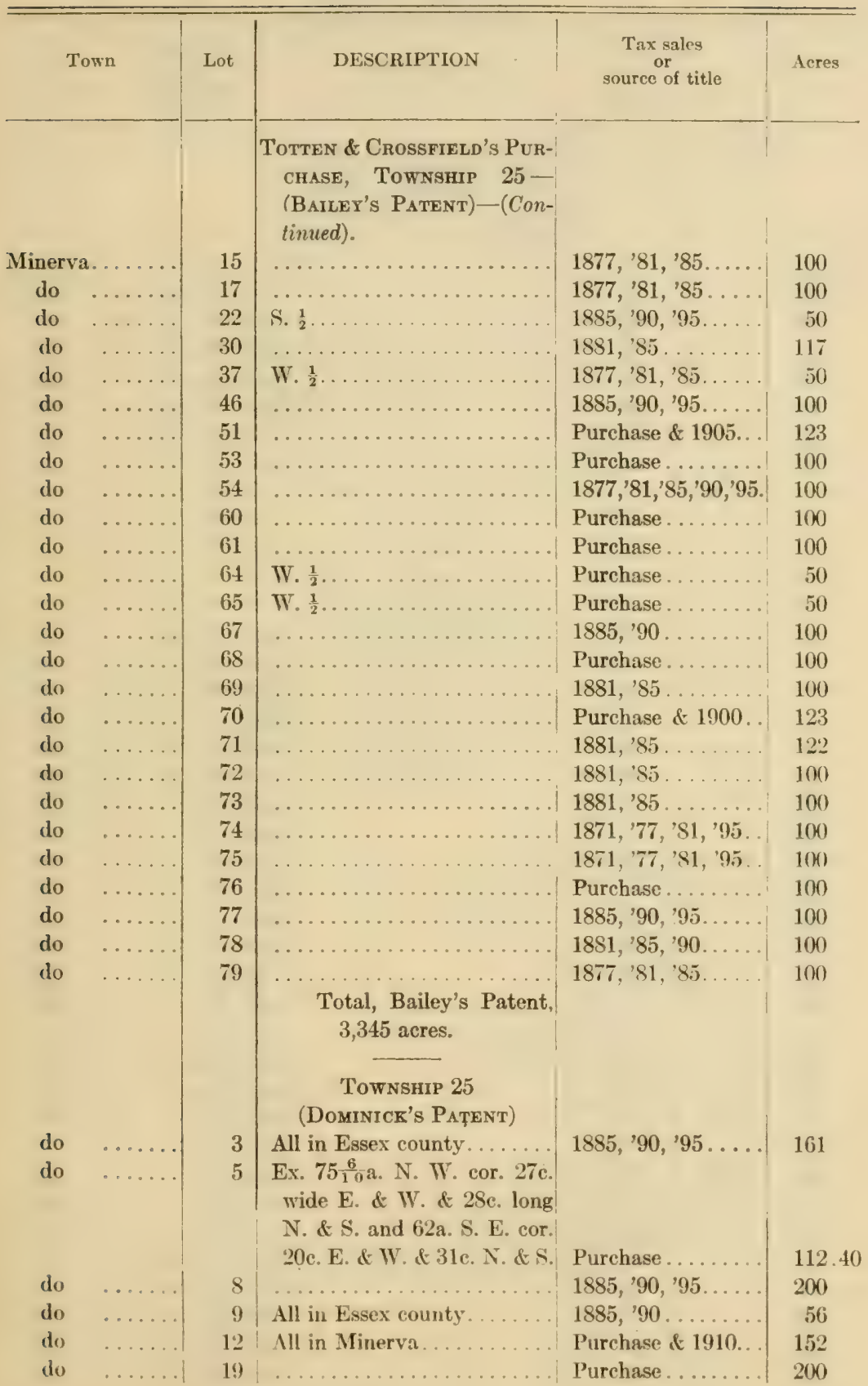


Essex County

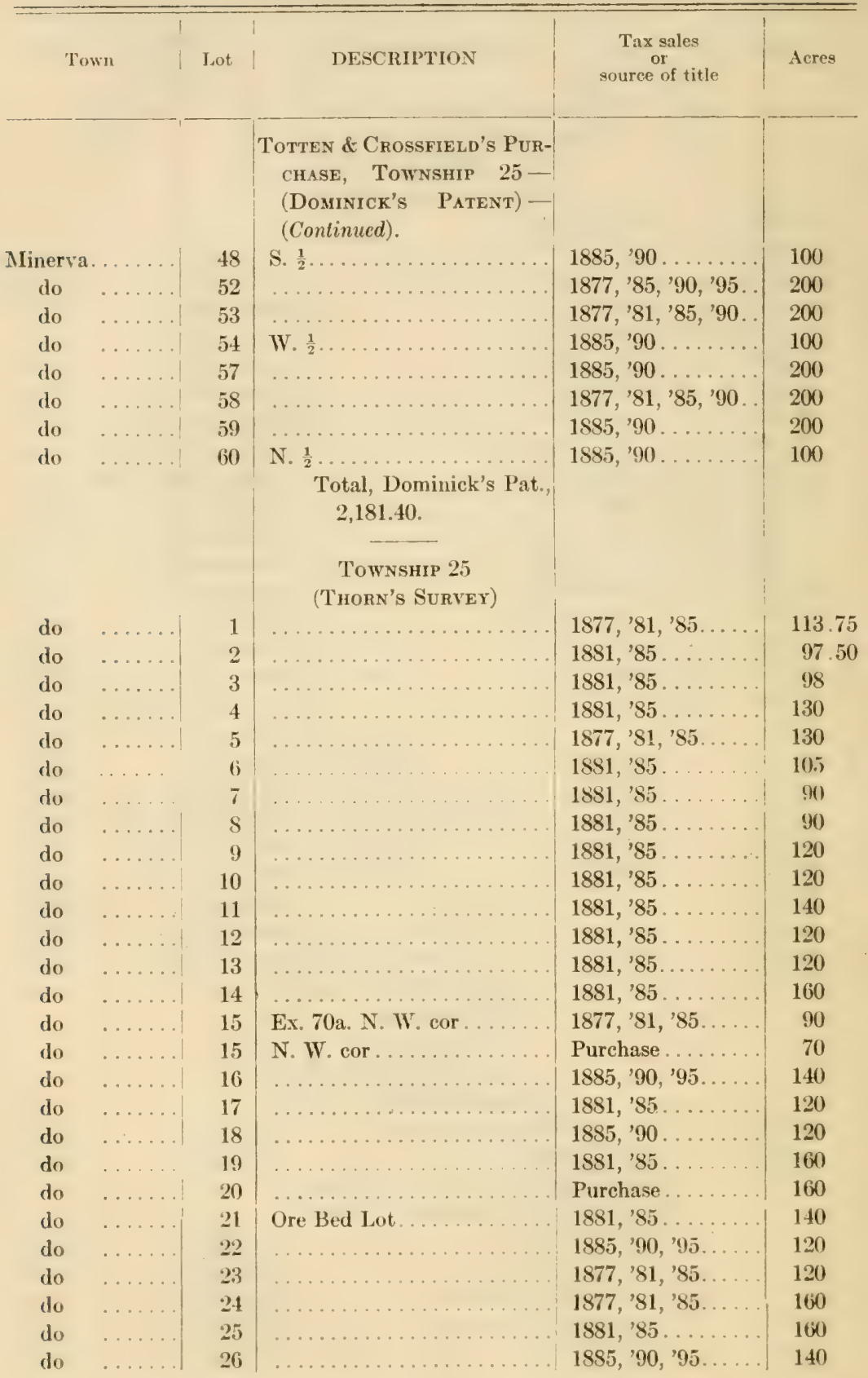


Essex County

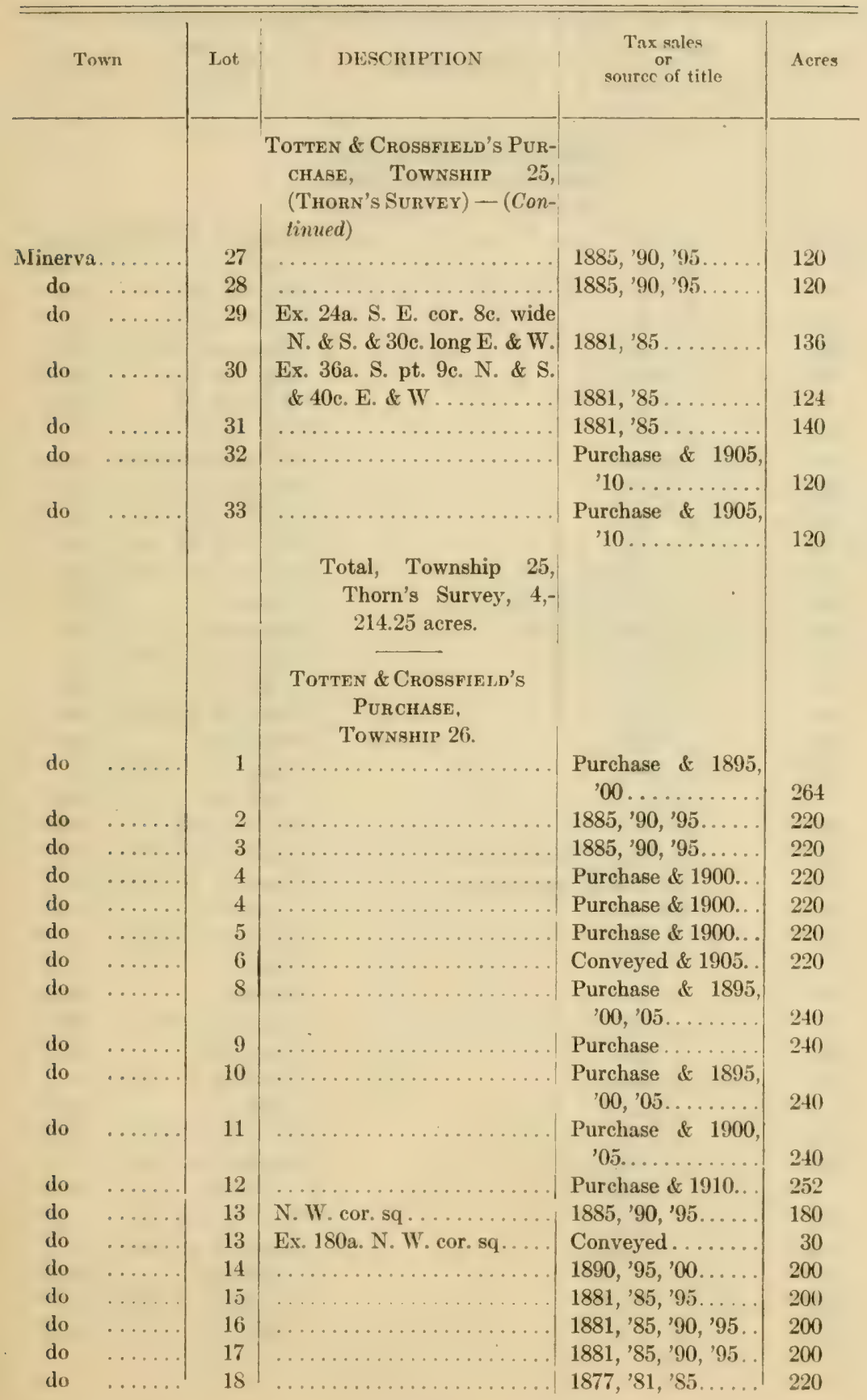


Essex County

\begin{tabular}{|c|c|c|c|c|}
\hline Town & Lot & DESCRIPTION & $\begin{array}{c}\text { Tax sales } \\
\text { or }\end{array}$ & Acres \\
\hline \multirow[b]{2}{*}{ Minerva. } & & $\begin{array}{l}\text { Totten \& Crossfield's PUR- } \\
\text { chase, T o W N S H I P } 26- \\
\text { (Continued) }\end{array}$ & & \\
\hline & 19 & $\ldots \ldots \ldots$ & Purchase \& 1895 & 220 \\
\hline do & 20 & 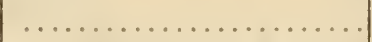 & $1877,{ }^{\prime} 81, ' 85$, '90. & 220 \\
\hline do & 21 & $\ldots \ldots \ldots$ & $1877,{ }^{\prime} 81, ' 85 \ldots$. & 220 \\
\hline do & 22 & & Purchase \& $1900 \ldots$ & 220 \\
\hline do & 23 & & $1885,{ }^{\prime} 90, ' 95 \ldots$. & 220 \\
\hline do & 24 & & $1885,{ }^{\prime} 90, ' 95 \ldots$. & 264 \\
\hline do & 25 & & $1877, ' 81, ' 90$. & 192 \\
\hline do & 26 & & $1877, ' 81, ' 85,90, ' 95$. & 160 \\
\hline do & 27 & & Purchase \& 1900. & 160 \\
\hline do & 28 & & $1877, ' 81, ' 85, ' 95$. & 160 \\
\hline do & 29 & & 1877, ' 81, ' $85, ' 95$. & 160 \\
\hline do & 30 & Ex. 100a. N. part.... & $1877, ' 81, ' 85, ' 95, ' 00$. & 60 \\
\hline do & 30 & N. W. $\operatorname{cor} \ldots \ldots \ldots \ldots$ & Purchase........ & 50 \\
\hline do & 30 & N. E. cor............ & Conveyed . . . . . . & 50 \\
\hline do & 31 & & 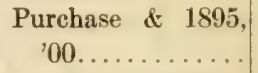 & 160 \\
\hline do & 32 & & Conveyed \& 1905. & 160 \\
\hline do & 33 & & $1881, ' 85 \ldots \ldots$. & 160 \\
\hline do & 34 & & $\begin{array}{r}\text { Purchase \& 1890, } \\
' 95,{ }^{\prime} 00 \ldots \ldots \ldots\end{array}$ & 160 \\
\hline do & 35 & & $1877, ' 81, ' 85 \ldots \ldots$ & 160 \\
\hline do & 36 & E. part across.. & 1881, ' 85, '90, '95. . & 92 \\
\hline do & 36 & W. part across... & $\begin{array}{r}\text { Purchase } \& \text { 1885 } \\
' 90,95 \ldots \ldots \ldots\end{array}$ & 76 \\
\hline do & 37 & ... & $\begin{array}{c}\text { Purchase \& } 1890 \& \\
\quad 00 \ldots \ldots \ldots \ldots\end{array}$ & 210 \\
\hline do & 39 & & Purchase........ & 200 \\
\hline do & 40 & & Purchase \& 1905. & 200 \\
\hline do & 41 & & Purchase...... & 200 \\
\hline do & 42 & & $\begin{array}{c}\text { Purchase \& 1895, } \\
\quad 00 \ldots \ldots \ldots \ldots\end{array}$ & 200 \\
\hline do & 43 & & $1871,777, ' 81, ' 85,95$. & 200 \\
\hline do & 47 & & $\begin{array}{r}\text { Purchase \& } 1890, \\
\text { '95, '00........ }\end{array}$ & 200 \\
\hline do & 48 & & 1877, '81, '85, '95. . & 240 \\
\hline do & 49 & & $\begin{array}{c}\text { Purchase } \& \text { 1881, } \\
\text { '85, '95 } \ldots \ldots \ldots \ldots\end{array}$ & 240 \\
\hline do & 50 & & $\begin{array}{r}\text { Purchase \& } 1890 \\
95,90 \ldots \ldots \ldots\end{array}$ & 200 \\
\hline
\end{tabular}


Essex County

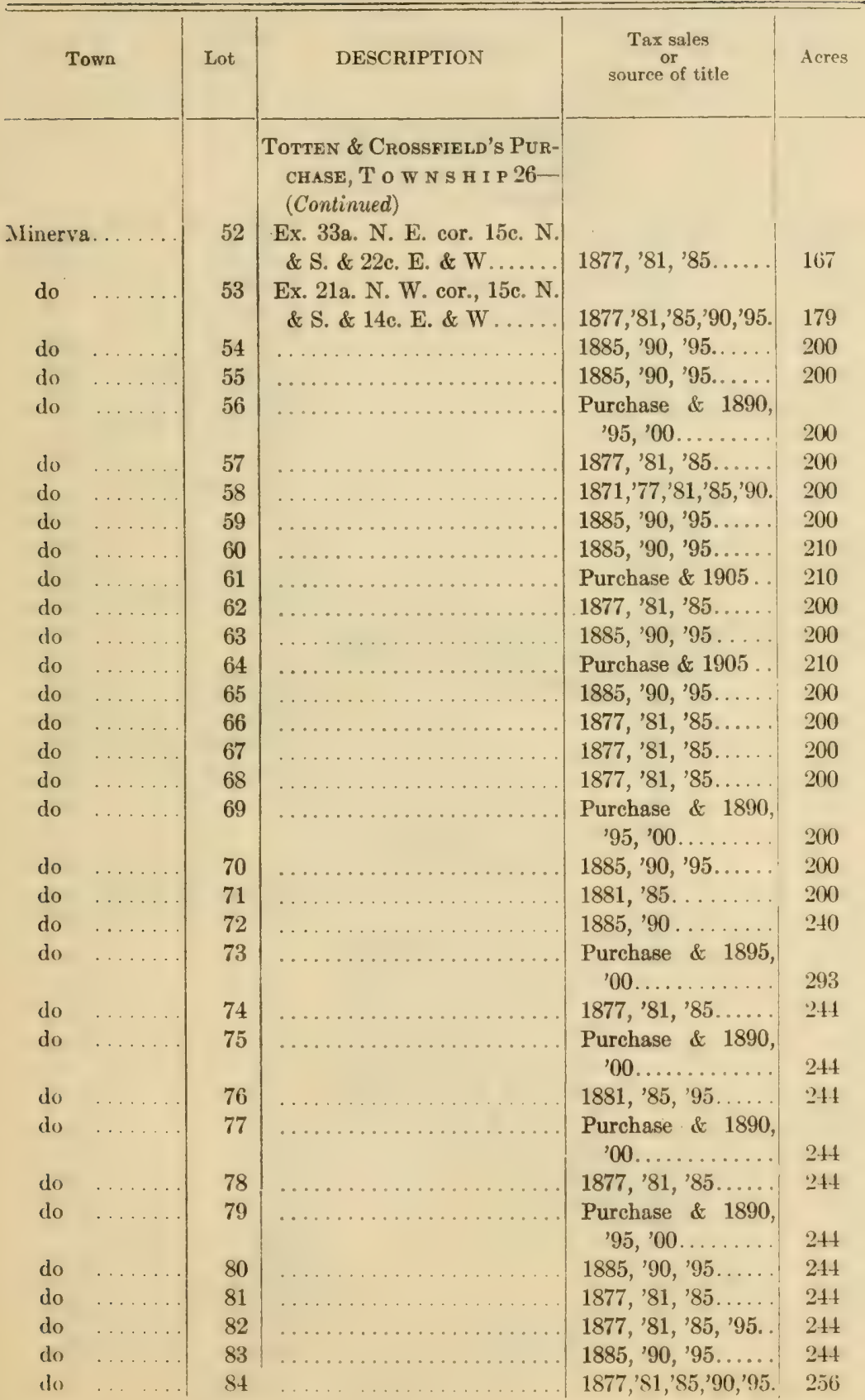




\section{Essex County}

\begin{tabular}{|c|c|c|c|c|}
\hline Towr & Lot & DESCRIPTION & $\begin{array}{l}\text { Tax sales } \\
\text { or } \\
\text { source of title }\end{array}$ & Acres \\
\hline & & $\begin{array}{l}\text { Totten \& Crossfield's PUR- } \\
\text { CHase, Township } 26- \\
\text { (Continued) }\end{array}$ & & \\
\hline Minerva. & 85 & $\ldots \ldots$ & $\begin{array}{r}\text { Purchase it 1890, } \\
905,00 \ldots \ldots\end{array}$ & $247+10$ \\
\hline do & 86 & Ex. 7 ta. S. part . . . . . & Purchase........ & 162 \\
\hline do & 88 & & $\begin{array}{r}\text { Purchase \& } 1890, \\
\text { '95, '00 } \ldots \ldots \ldots\end{array}$ & 236 \\
\hline do & 89 & & $1877, ' 81,{ }^{\prime} 85,95,90$. & 236 \\
\hline do & 90 & & $1885,{ }^{\prime} 90,95$. & 236 \\
\hline do & 91 & 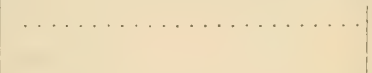 & $\begin{array}{r}\text { Purchase \& 1890, } \\
' 95,90 \ldots \ldots \ldots\end{array}$ & 236 \\
\hline do & 92 & & $\begin{array}{r}1871,77,91,85,90 . \\
95 \ldots \ldots \ldots \ldots \ldots\end{array}$ & 236 \\
\hline do & 93 & $\ldots$ & $1877, ' 81, ' 85 \ldots$. & 236 \\
\hline do & 94 & $\ldots \ldots \ldots \ldots \ldots \ldots$ & $1877, ' 81, ' 85 \ldots \ldots$ & 236 \\
\hline do & 95 & ... & Purchase \& 1900. & 236 \\
\hline do & 96 & $\ldots \ldots \ldots \ldots \ldots \ldots \ldots \ldots \ldots$ & $1881, ' 85 \ldots \ldots \ldots$ & 283 \\
\hline do & 97 & $\begin{array}{l}\text { Ex. 183a. N. pt. pd. by W. } \\
\text { H. Halsted.......... }\end{array}$ & $1885,90,95 \ldots$. & 100 \\
\hline do & 98 & $\ldots \ldots \ldots \ldots \ldots \ldots \ldots \ldots$ & $1877,81 \ldots \ldots$ & 236 \\
\hline do & 99 & $\ldots \ldots \ldots \ldots \ldots \ldots \ldots \ldots$ & $1877,81, ' 85, ' 90,{ }^{\prime} 95$. & 236 \\
\hline do & 100 & $\ldots \ldots$ & Purchase \& $1905 .$. & 236 \\
\hline do & 101 & $\ldots \ldots \ldots \ldots \ldots \ldots \ldots$ & $1877,91,8.5 \ldots$ & 236 \\
\hline do & 102 & $\begin{array}{l}\text { Ex. } 100 \mathrm{a} . \text { N. pt. \& } 50 \mathrm{a} . \mathrm{N} \\
\text { W. cor. of remainder ..... }\end{array}$ & $1877, ' 81,{ }^{\prime} 85,95$. & 86 \\
\hline do & 103 & $\ldots \ldots \ldots \ldots \ldots \ldots \ldots$ & $\begin{array}{r}\text { Purchase \& } 1881, \\
' 85,{ }^{\prime} 95,{ }^{\prime} 00 \ldots \ldots\end{array}$ & 236 \\
\hline d" & 104 & $\begin{array}{l}\text { E. pt. } 122 \text { a., ex. 7a. being so } \\
\text { much thereof as is included } \\
\text { in } 41{ }_{10}^{7} \text { a. S. W. cor. of lot, } \\
21 \text { c. N. \& S. \& } 31 \text { c. on N. \& } \\
\text { Sc. on S. line, bd. E. by lake } \\
\text { and } 2 a . \text { S. E. cor. thereof, } \\
\text { 5c. on E. \& 6c. on S. line, } \\
\text { bd. N. W. by lake. ...... }\end{array}$ & 1877. & 113 \\
\hline do & 105 & N. W. pt............ & $1881, ' 85 \ldots \ldots \ldots$ ! & 50 \\
\hline do & 106 & S. pt.......... & $1881,{ }^{\prime} 85 \ldots \ldots \ldots$ & 76 \\
\hline do & 107 & - & $\begin{array}{l}\text { Purchase \& 1890, } \\
\quad 95,{ }^{\prime} 00 \ldots \ldots \ldots\end{array}$ & 236 \\
\hline do & 109 & & $1877, ' 81, ' 85 . \ldots$. & 247.80 \\
\hline do & 110 & S. pt. . & $1881,{ }^{\prime} 85 \ldots \ldots \ldots$ & 36 \\
\hline do & 110 & Ex. 36r. S. pt. & Purchase ........ & 200 \\
\hline
\end{tabular}




\section{Essex County}

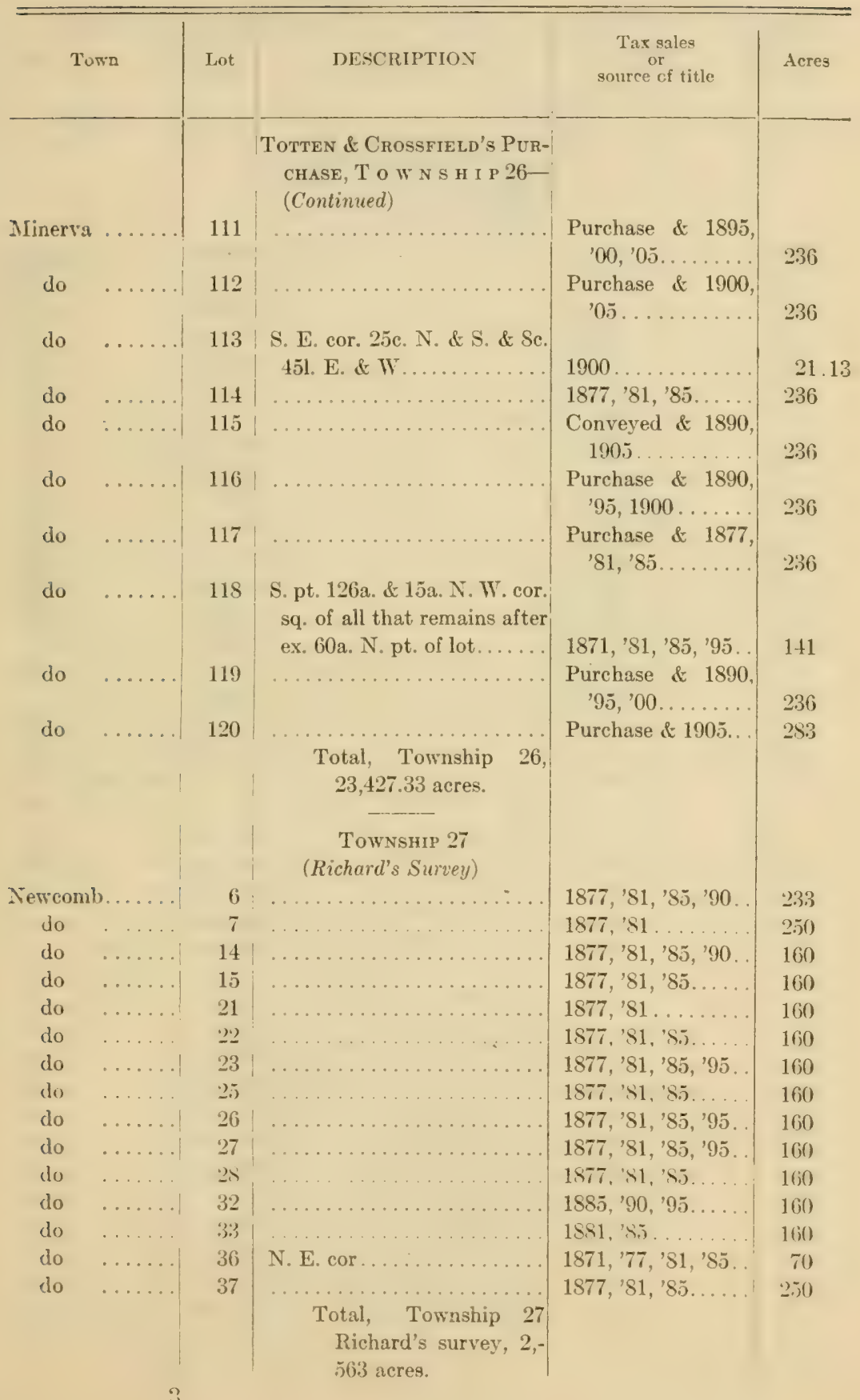


Essex County

\begin{tabular}{|c|c|c|c|c|}
\hline Town & Lot & DESCRIPTION & $\begin{array}{c}\text { Tax sales } \\
\text { or } \\
\text { source of title }\end{array}$ & Acres \\
\hline \multirow[b]{2}{*}{ Newcomb. . } & & $\begin{array}{l}\text { Totten \& Crossfield's PUR- } \\
\text { Chase, Township } 27- \\
\text { (Continued) } \\
\text { (Thorn's Survey) }\end{array}$ & & \\
\hline & 10 & $\ldots \ldots \ldots \ldots \ldots \ldots \ldots$ & Purchase ..... . & 68.12 \\
\hline do & 11 & $\ldots \ldots \ldots \ldots \ldots$ & Purchase..... & 68.12 \\
\hline do & 12 & $\ldots \ldots \ldots \ldots \ldots \ldots$ & Purchase. & 68.12 \\
\hline do & 13 & $\ldots \ldots \ldots \ldots \ldots \ldots \ldots$ & Purchase....... & 65.12 \\
\hline do & 14 & $\ldots \ldots \ldots \ldots \ldots \ldots \ldots$ & Purchase ....... & 68.12 \\
\hline do & 16 & $\ldots \ldots \ldots \ldots \ldots$ & $1871,77, ' 81, ' 90$. & so \\
\hline do & 17 & Ex. 55a. Х. W. cor....... & $1877, ' 81, ' 85 \ldots$. & 25 \\
\hline do & 18 & $\ldots \ldots \ldots \ldots \ldots \ldots \ldots \ldots$ & $1877, ' \$ 1, ' \$ 5 \ldots$ & so \\
\hline do & 19 & $\ldots \ldots \ldots \ldots \ldots \ldots \ldots$ & $\begin{array}{r}1877, \text { ' } 11,95, ' 90, \\
995 \ldots \ldots \ldots\end{array}$ & so \\
\hline do & 23 & $\ldots$ & $1877, ' 81, ' 85 \ldots .$. & 160 \\
\hline do & 24 & $\ldots \ldots \ldots \ldots$ & $1877, ' 81, ' 85 \ldots \ldots$ & 160 \\
\hline do & 26 & $\ldots$ & Purchase ........ & 160 \\
\hline do & 27 & $\ldots \ldots \ldots \ldots \ldots$ & Purchase...... & 160 \\
\hline do & 28 & $\ldots \ldots \ldots \ldots$ & $\begin{array}{c}\text { Purchase \& } 1905, \\
10 \ldots \ldots \ldots\end{array}$ & 160 \\
\hline do & 29 & & $\begin{array}{c}\text { Purchase \& 1905, } \\
\quad 10 \ldots \ldots \ldots \ldots\end{array}$ & 160 \\
\hline do & 32 & & $1885,{ }^{\prime} 90,{ }^{\prime} 95 \ldots \ldots$ & 160 \\
\hline do & 33 & $\ldots$ & $\begin{array}{r}1871,77,981, ' 85 \\
90 \ldots \ldots \ldots \ldots\end{array}$ & 160 \\
\hline do & 34 & & $1885, ' 90 \ldots \ldots$ & 160 \\
\hline do & 35 & $\ldots$ & $1877.81,95 \ldots$. & 160 \\
\hline do & 36 & $\ldots \ldots$ & $1877, ' 81, ' 85 \ldots \ldots$ & 160 \\
\hline do & 37 & $\ldots$ & Purchase......... & 160 \\
\hline do & 38 & & $\begin{array}{c}\text { Purchase \& 1905, } \\
110 \ldots \ldots \ldots\end{array}$ & 160 \\
\hline do & 39 & & $\begin{array}{c}\text { Purchase \& } 190 . \bar{j} \\
10 \ldots \ldots \ldots \ldots\end{array}$ & 160 \\
\hline do & 41 & N. W. cor. & $1885, ' 90$. & 70 \\
\hline do & 42 & $\ldots \ldots \ldots \ldots$ & $1877,81,85 \ldots$ & 160 \\
\hline do & 43 & & $1877, ' \$ 1, ' 85 \ldots$ & 160 \\
\hline do. & 44 & & $1877,81, ' \$ 5 \ldots$ & 160 \\
\hline do & 45 & & $1877,81,85 \ldots$ & 160 \\
\hline do & 40 & & $1877,81, ' 85 \ldots$ & 160 \\
\hline do & 47 & & 1877 '\$1 'S5.... & 160 \\
\hline do & 48 & & 1877 '81 '85.... & 160 \\
\hline do & 49 & $\ldots$ & 1877 '81 '85..... & 160 \\
\hline do & 50 & & Purchase. . & 132 \\
\hline do & 51 & & 1877 '81 '85..... & 160 \\
\hline
\end{tabular}


Conservation Commesion.

Essex County

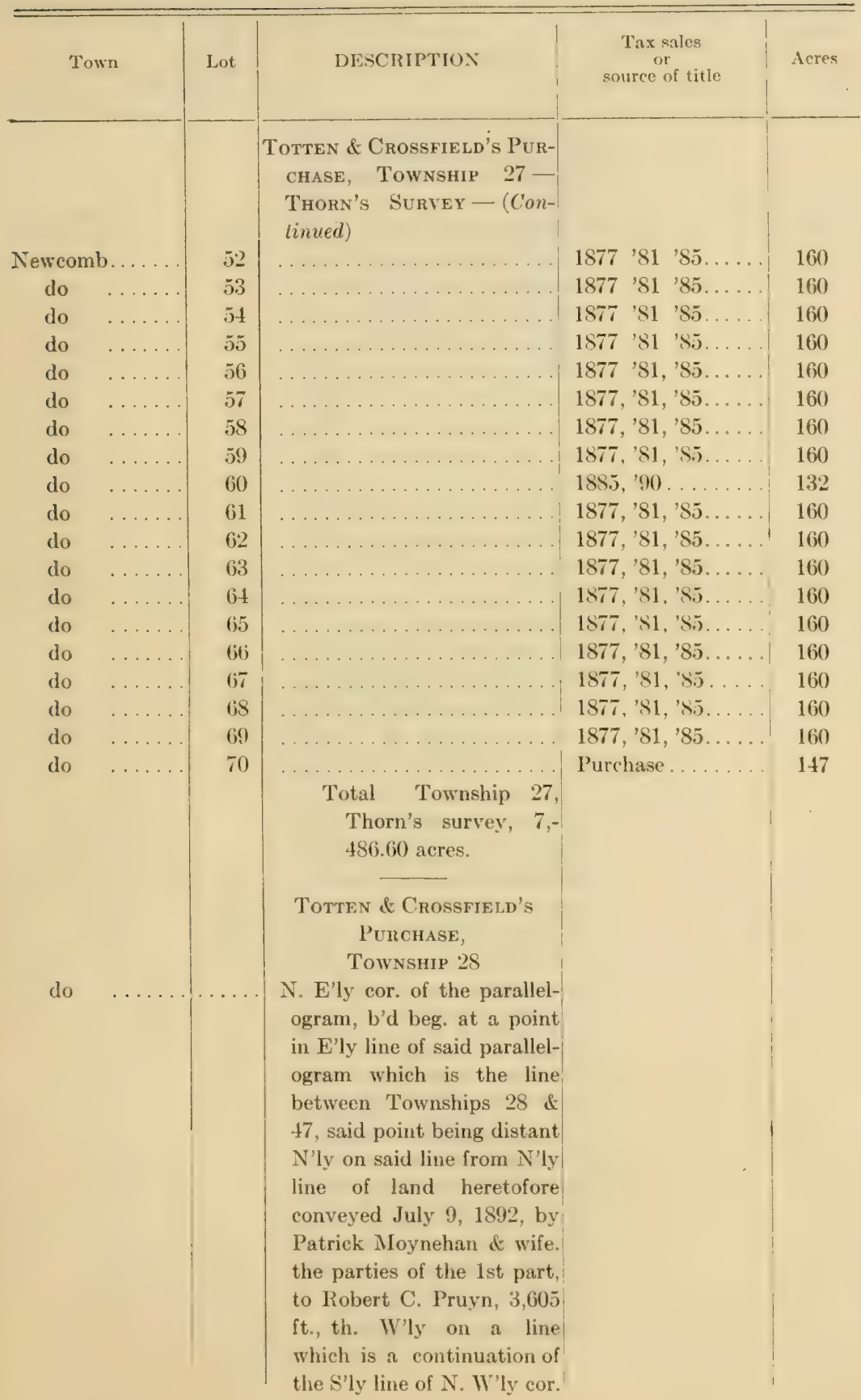


Essex County

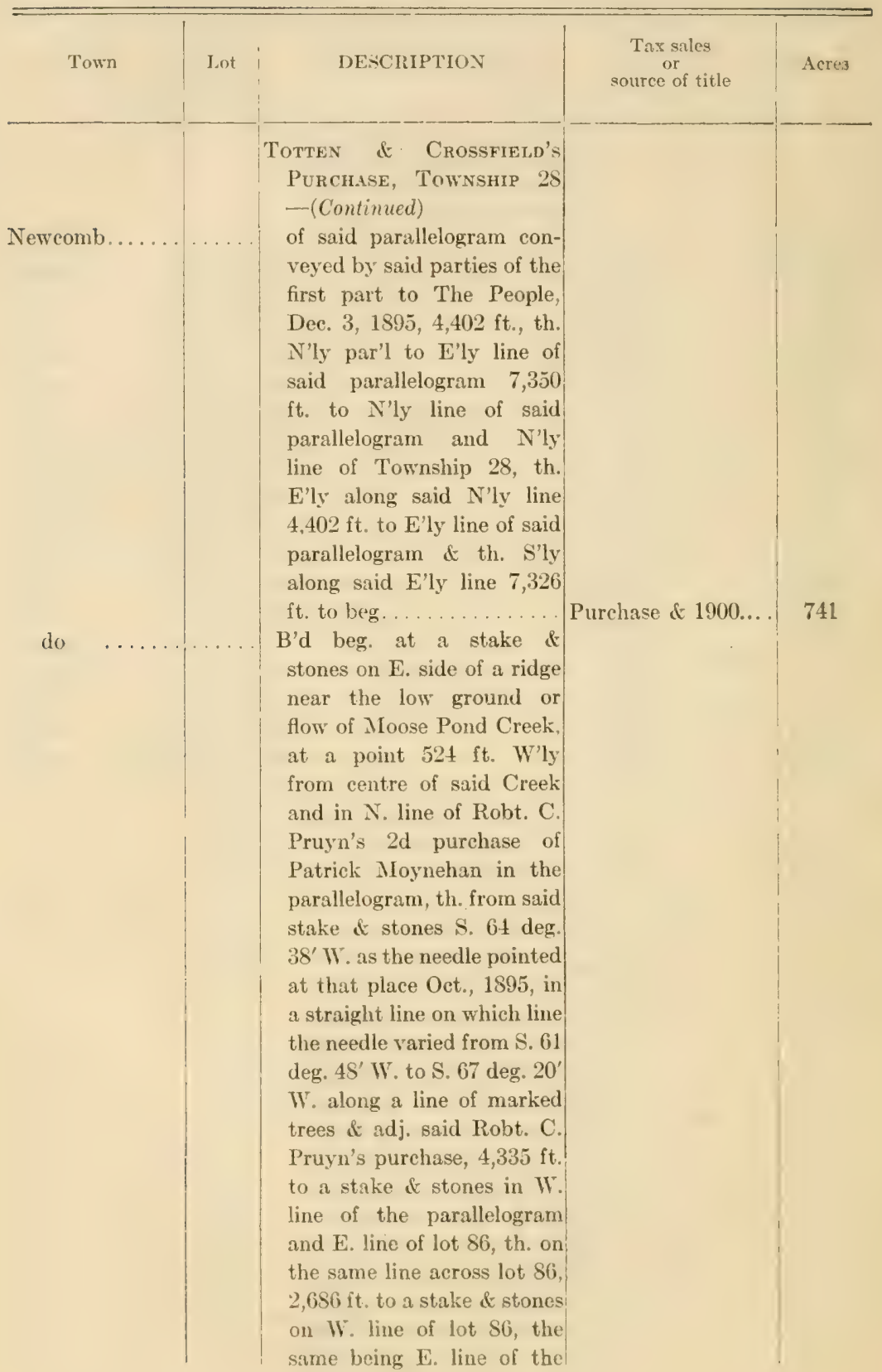


Essex County

\begin{tabular}{|c|c|c|c|c|}
\hline Town & Lot & DESCRIPTION & $\begin{array}{c}\text { Tax sales } \\
\text { or } \\
\text { source of title }\end{array}$ & Acres \\
\hline Newcomb... & & 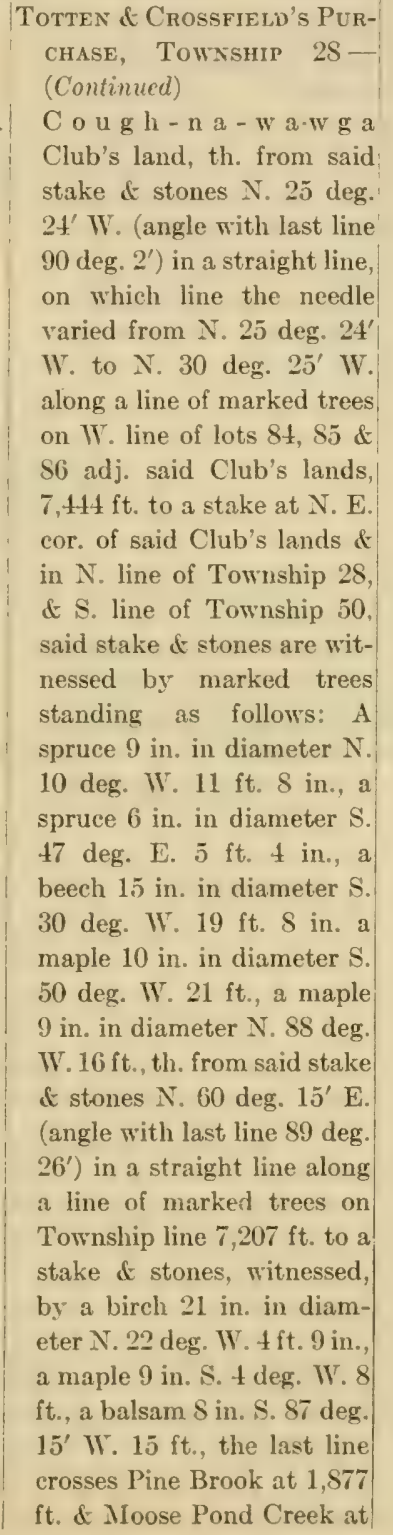 & & \\
\hline
\end{tabular}


Essex County

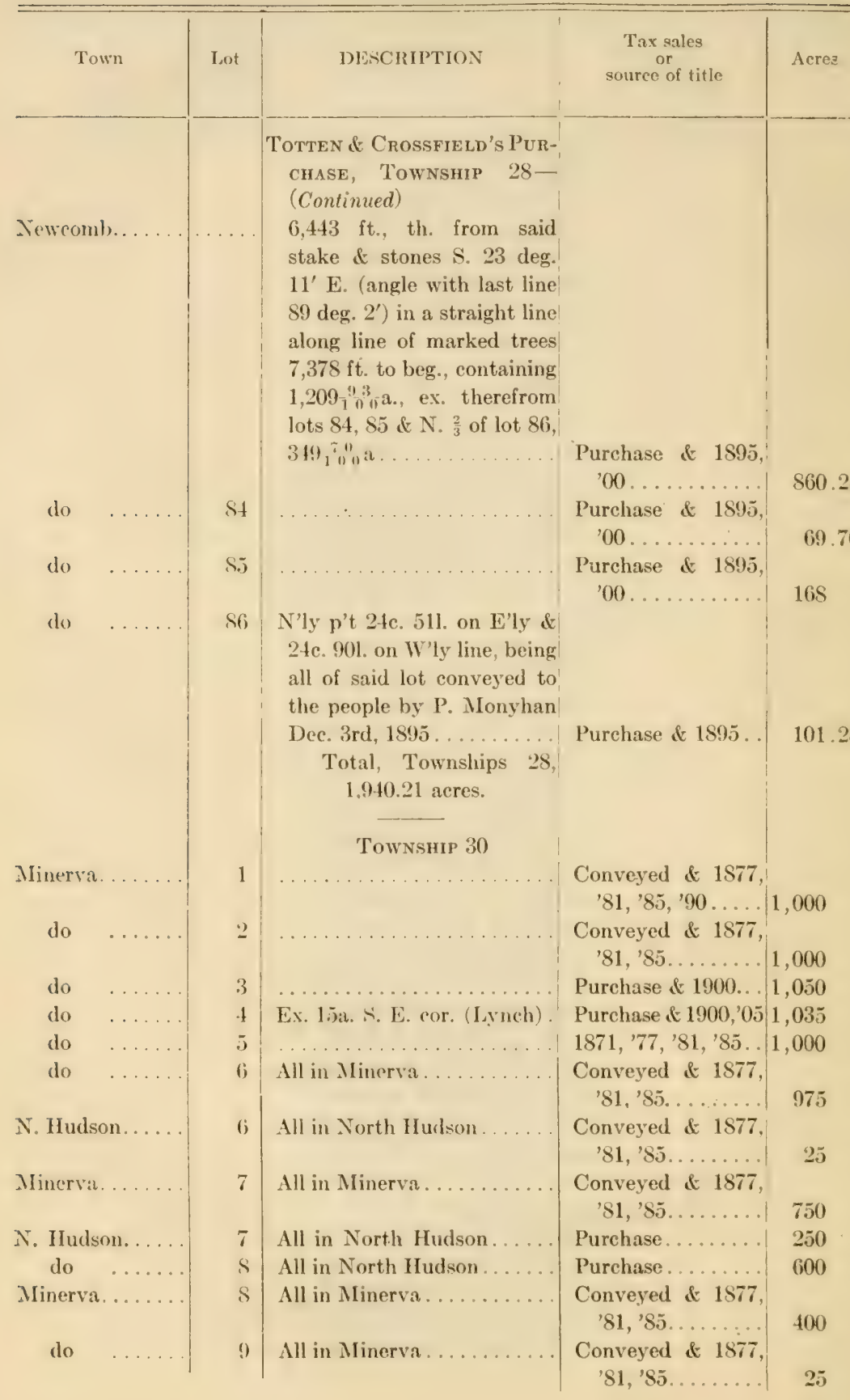


Essex County

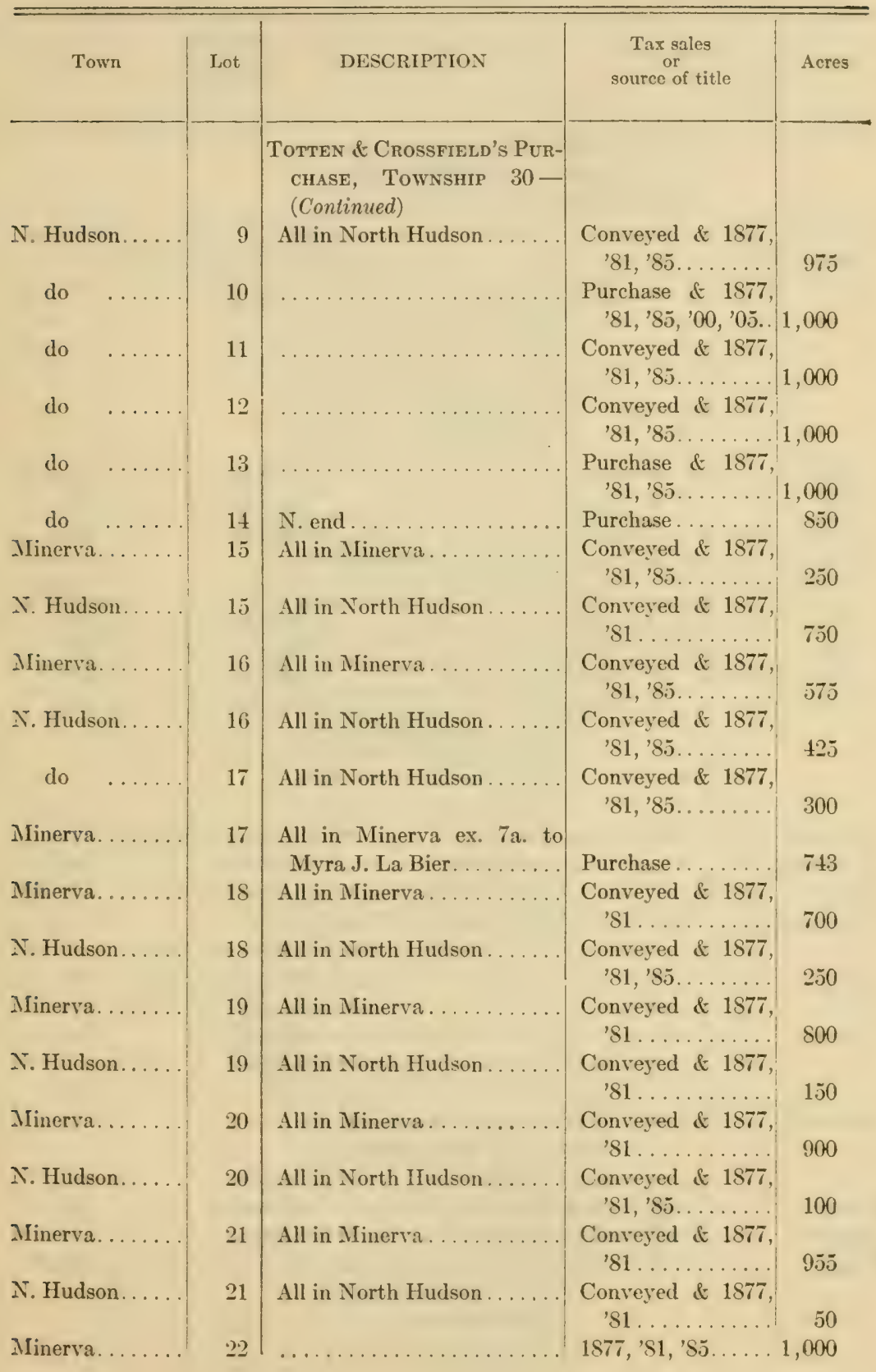


Essex County

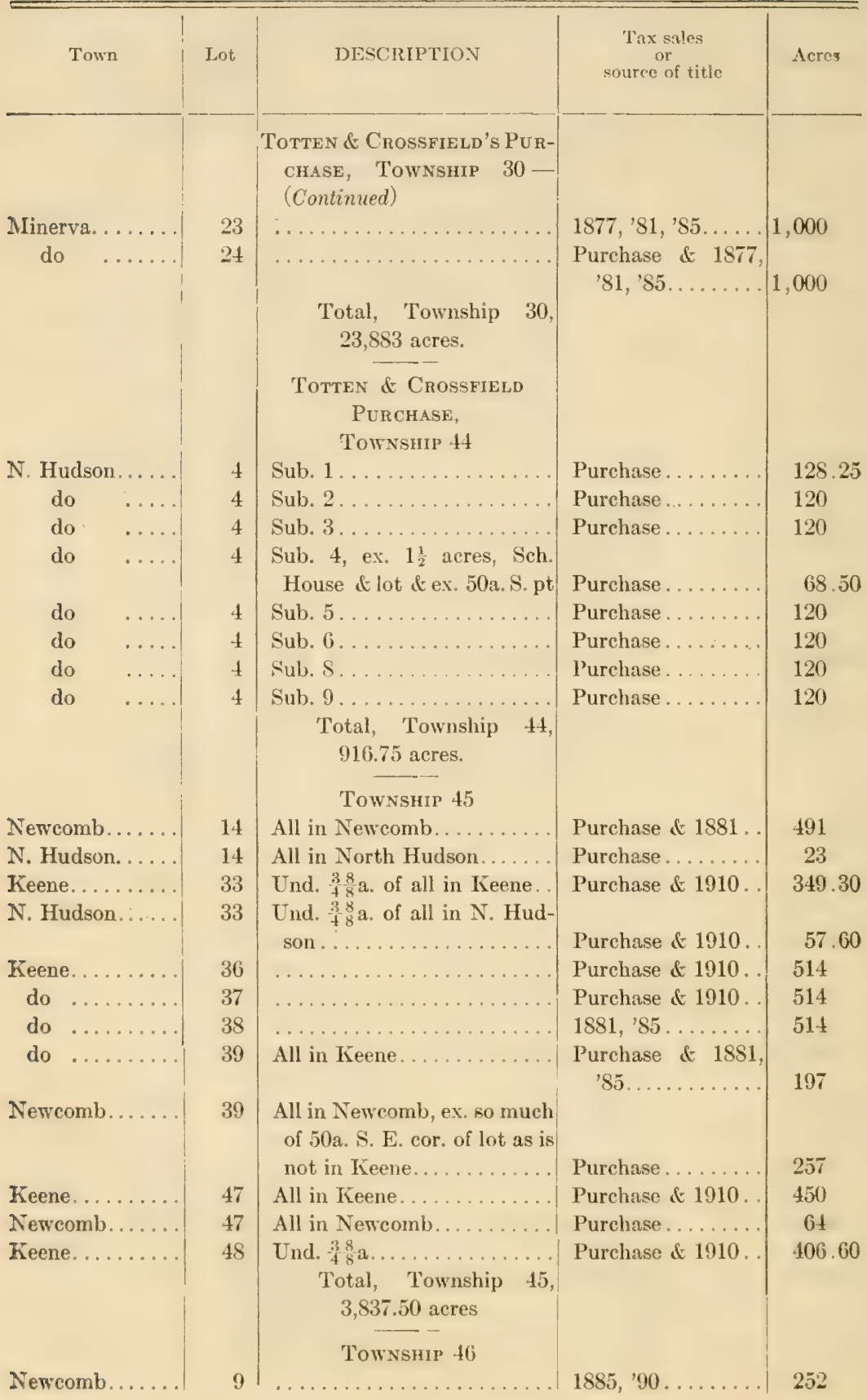


Essex County

\begin{tabular}{|c|c|c|c|c|}
\hline Town & Lot & DESCRIPTION & $\begin{array}{c}\text { Tax sales } \\
\text { or } \\
\text { source of title }\end{array}$ & Acres \\
\hline Newcomb. & & 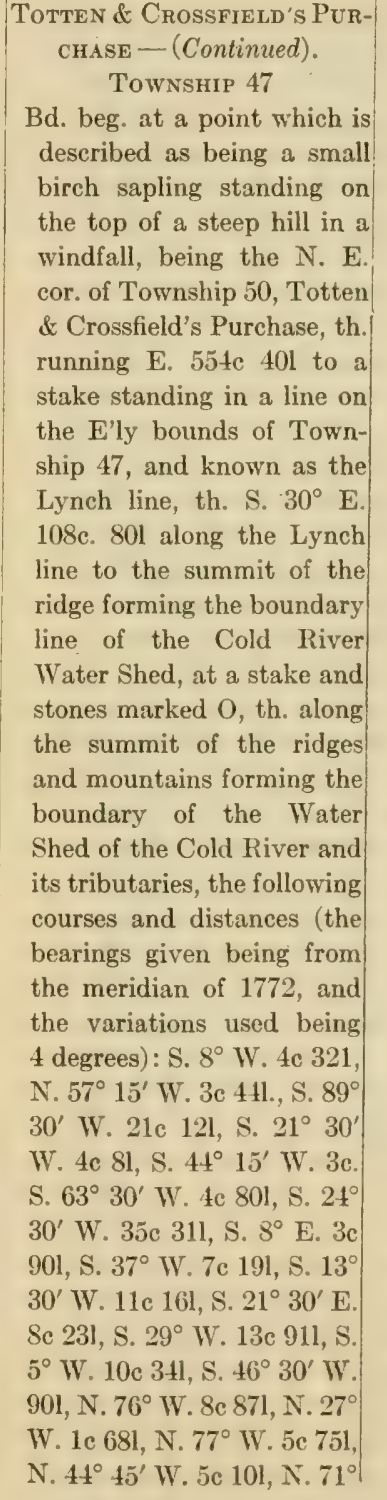 & & \\
\hline
\end{tabular}


Essex County

\begin{tabular}{|c|c|c|c|c|}
\hline Town & Lot & DESCRIPTION & $\begin{array}{c}\text { Tax sales } \\
\text { or } \\
\text { source of title }\end{array}$ & Acres \\
\hline Newcomb.. & & 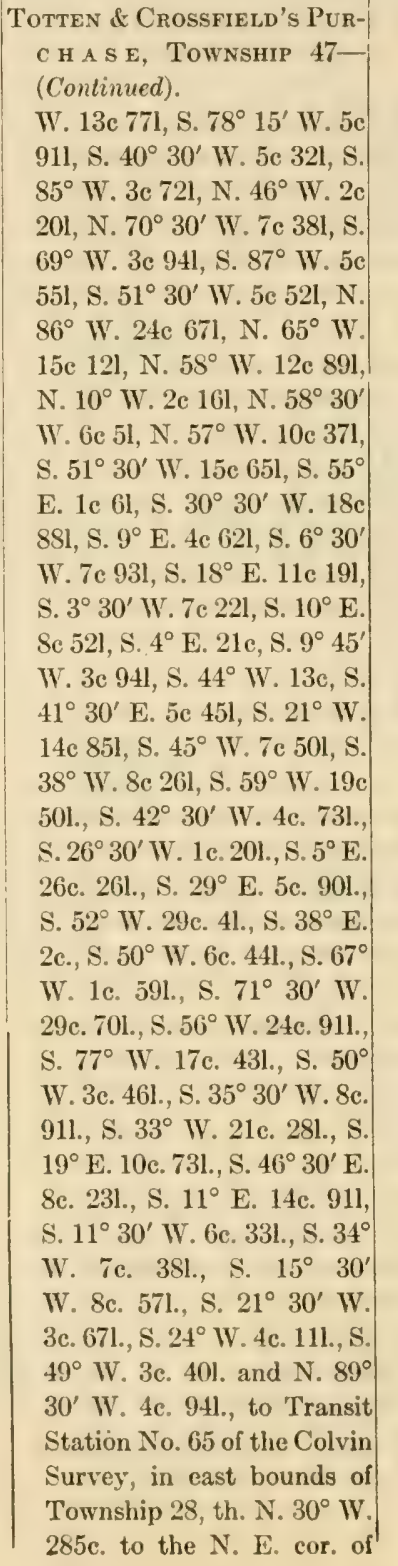 & & \\
\hline
\end{tabular}




\section{Essex County}

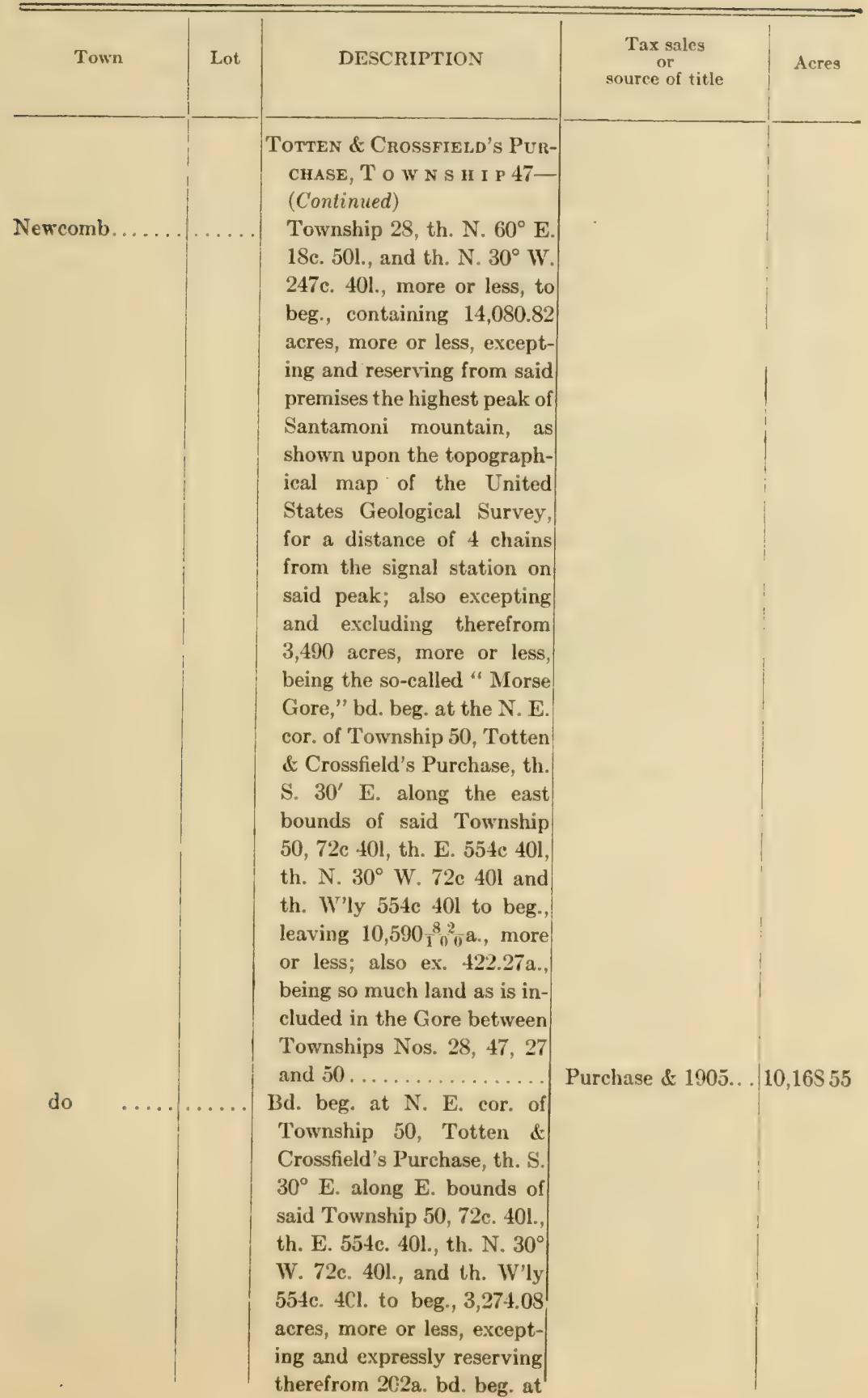


Essex County

\begin{tabular}{l|l|l}
\hline Town & Lot & DESCRIPTION \\
\hline & $-\begin{array}{c}\text { TotTEN \& Crossfield's PUR- } \\
\text { CHASE, Township } \\
\text { (Continued) }\end{array}$ \\
\hline
\end{tabular}
stones standing in $\mathrm{N}$. line of Township 47 and in the S. line of the Gore N. of Township 47 , 6c. 501 . E. of the point in $\mathrm{N}$. line of Township 47 , where the line run East from the N. W. cor. of Township 47 comes to Cold River and S. $60^{\circ} 30^{\prime} \mathrm{W} .7 \mathrm{c}$. from the North wing of a dam across Cold River now owned by John Anderson, Jr, th. S. $5^{\circ}$ W. 9c. 401. to a cedar post and stones Marked "A. O." and standing in a small brook, th. S. $85^{\circ}$ E. $39 \mathrm{c}$. 91. to a cedar post standing on a hill in hardwood timber, th. S. $40^{\circ}$ IV. 2Se. 50l. to a cedar post. and stones, th. S. $30^{\circ}$ E. $11 \mathrm{c}$ 12l. to a cedar post and stones, th. N. $70^{\circ}$ E. 20 c. 65 l. to a cedar post and stones, th. S. $30^{\circ}$ E. $11 \mathrm{c}$. 121 . to a cedar post and stones, th. N. $80^{\circ}$ E. 20 c. 501 . to a cedar post and stones standing on the South end of a small hill, th. N. $55^{\circ}$ E. 31e. 931, to a cedar post and stones, th. $\mathrm{N}$ $35^{\circ}$ W. 3c. 75l. to a cedar post and stones, th. N. $55^{\circ}$ E. 7c. 611. to a cedar post and stones standing in N. line of Township 47 , and th. N. $84^{\circ} 45^{\prime} \mathrm{W}$. along $\mathrm{N}$. line of Township 47, 95c. 50l. to beg................ Total, Township 47, $13,412.63$ acres.

\section{Tax sales source of title}

Acres 
Essex County

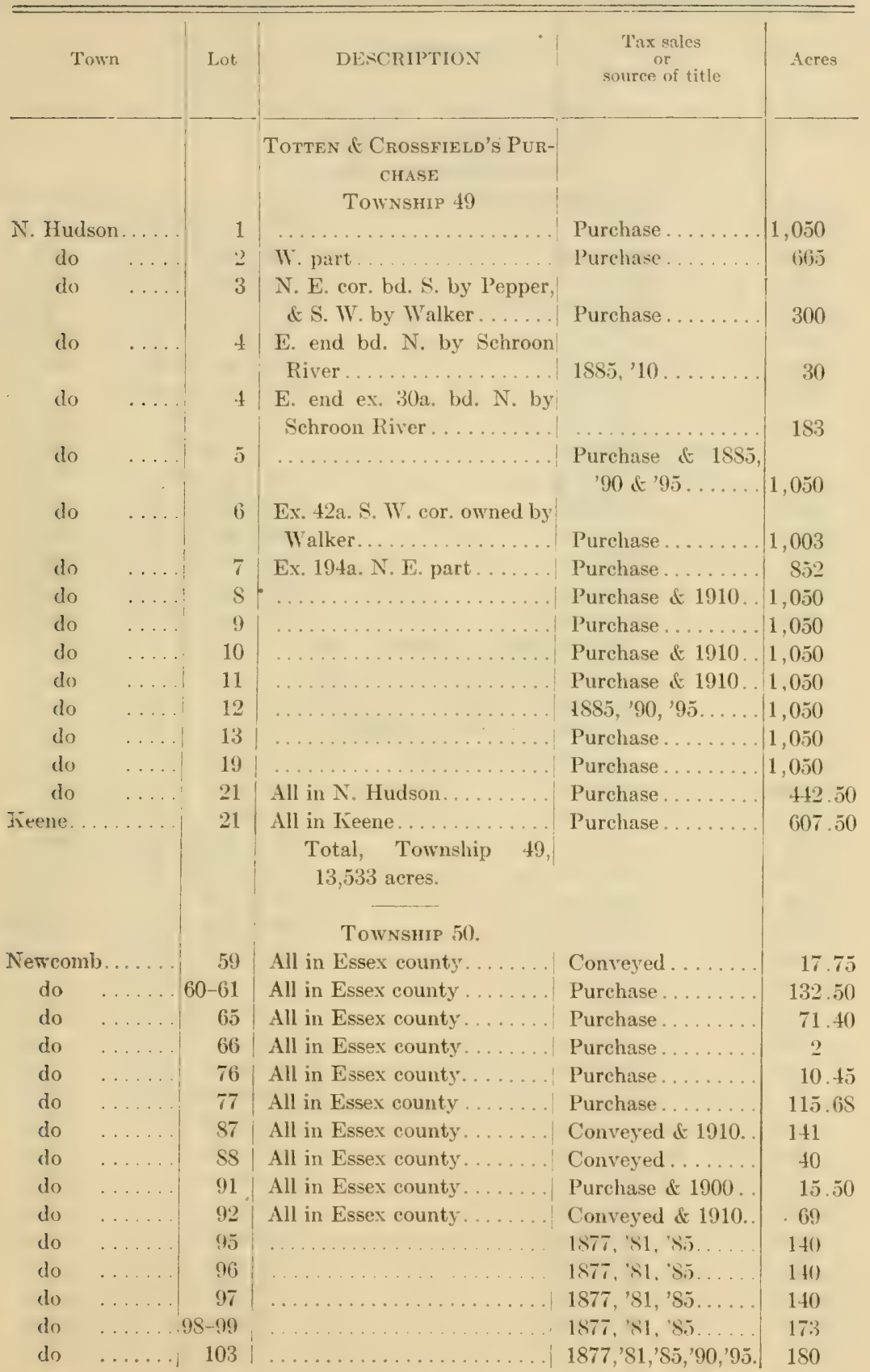


Essex County

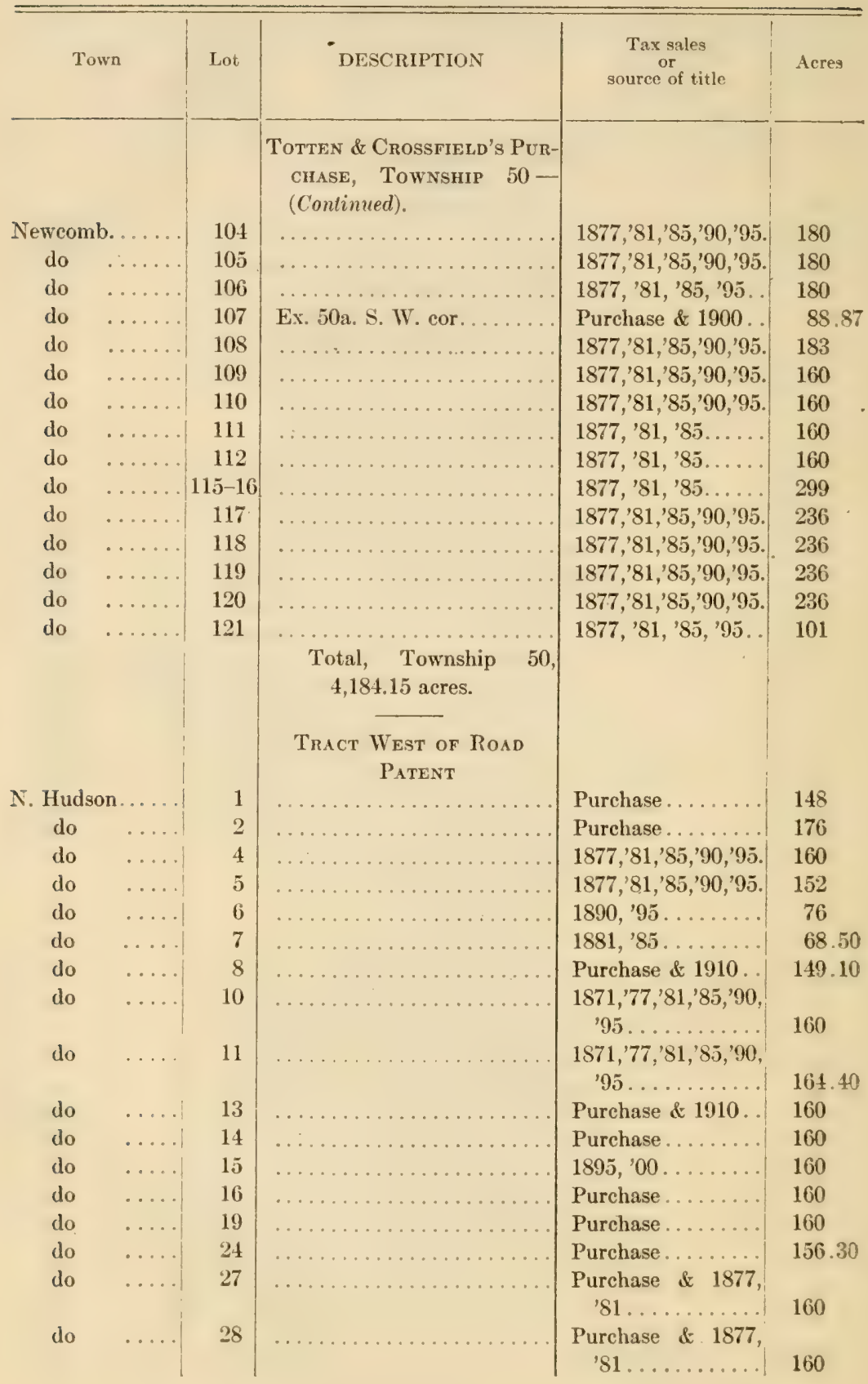


Conservatiox Commssion.

Essex County

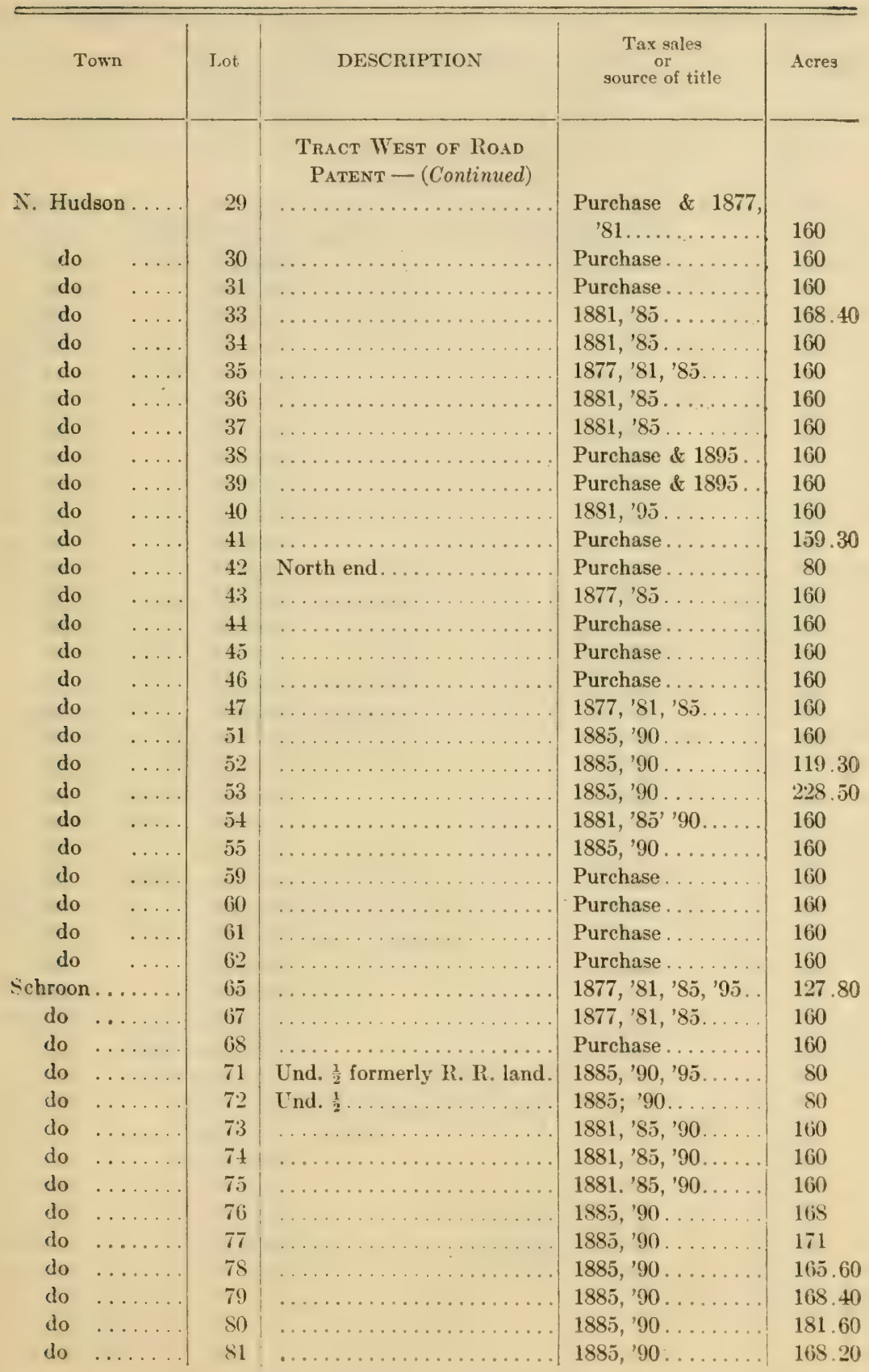


Essex County

\begin{tabular}{|c|c|c|c|c|c|}
\hline \multicolumn{2}{|c|}{ Town } & \multirow[t]{2}{*}{ Lot } & DESCRIPTION & \multirow{2}{*}{$\begin{array}{c}\text { Tax sales } \\
\text { or } \\
\text { source of title }\end{array}$} & \multirow[t]{2}{*}{ Acres } \\
\hline & & & $\begin{array}{l}\text { Tract West of Road } \\
\text { Patent - (Continued })\end{array}$ & & \\
\hline Schroon. & $\ldots \ldots$ & 82 & $\ldots \ldots \ldots \ldots \ldots \ldots \ldots$ & 1881, ' $85, ' 90$. & 160 \\
\hline do . & $\ldots \ldots$ & 85 & Und. $\frac{1}{2}$ formerly R. R. land. & $1885,{ }^{\prime} 90,95$. & 80 \\
\hline do & $\ldots \ldots$ & 86 & Und. $\frac{1}{2} \ldots \ldots \ldots \ldots \ldots$ & $1885,90,95 \ldots$ & 80 \\
\hline do & & 88 & $\ldots \ldots \ldots \ldots \ldots \ldots$ & Purchase... . & 160 \\
\hline do & ... & 89 & $\ldots \ldots \ldots \ldots \ldots$ & Purchase....... & 160 \\
\hline do & $\ldots \ldots$ & 90 & & Purchase... & 160 \\
\hline do & .... & 91 & $\ldots \ldots \ldots \ldots \ldots \ldots \ldots$ & $1881,{ }^{\prime} 85,{ }^{\prime} 90$. & 116.10 \\
\hline do & $\therefore \ldots$ & 93 & $\ldots \ldots \ldots \ldots \ldots \ldots$ & $1881,{ }^{\prime} 85,90 \ldots$ & 120 \\
\hline do & ... & 94 & $\ldots \ldots \ldots \ldots \ldots \ldots$ & $1885,90 \ldots$ & 160 \\
\hline do & .... & 100 & $\ldots \ldots \ldots \ldots \ldots \ldots \ldots$ & $1885,{ }^{\prime} 90 \ldots \ldots$ & 160 \\
\hline do & $\ldots \ldots$ & 101 & $\ldots \ldots \ldots$ & $1881, ' 85,{ }^{\prime} 90 \ldots$ & 160 \\
\hline do & $\ldots \ldots$ & 102 & $\ldots \ldots \ldots \ldots$ & $1885,90 \ldots$. & 168.20 \\
\hline do & $\ldots \ldots$ & 103 & $\ldots \ldots \ldots \ldots \ldots$ & $1885,90 \ldots$ & 181.60 \\
\hline do & ... & 104 & & $1885,90 \ldots$ & 148.50 \\
\hline do & $\ldots$ & 105 & $\ldots$ & 1881 , 'S5, '90. & 115 \\
\hline do & $\ldots \ldots$ & 106 & $\ldots \ldots \ldots \ldots$ & $1885,90 \ldots \ldots$ & 158 \\
\hline do & .. & 110 & & Purchase \& 1905. & 160 \\
\hline do & ... & 111 & . . & Purchase........ & 160 \\
\hline do & $\therefore \ldots$ & 112 & . & Purchase........ & 160 \\
\hline do & $\ldots \ldots$ & 113 & & 1877, '81. '85, '90. & 120 \\
\hline do & $\therefore \ldots$ & 117 & & $1881, ' 85,90 \ldots$. & 160 \\
\hline do & $\ldots$ & 118 & & Purchase \& 1895. & 160 \\
\hline do & $\ldots \ldots$ & 119 & $\ldots$ & Purchase \& 1900. & 155 \\
\hline do & $\ldots \ldots$ & 120 & $\ldots \ldots \ldots$ & Purchase ........ & 143 \\
\hline do & & 121 & & Purchase \& 1900. & 157 \\
\hline do & . & 122 & & Purchase........ & 156.70 \\
\hline do & .. & 123 & & Purchase \& 1910. & 156.70 \\
\hline do & $\therefore$ & 129 & . & $1885,900 \ldots$ & 160 \\
\hline do & $\ldots$ & 130 & . & Purchase. & 160 \\
\hline do & & 131 & & Original \& $1900^{*}$. & 105.80 \\
\hline do & . & 132 & & Purchase \& 1900. & 95 \\
\hline do & .. & 133 & $\ldots$ & Original \& $1900^{*}$. & 137 \\
\hline do & . & 134 & & 1881, ' $85,{ }^{\prime} 90 \ldots$. & 180.80 \\
\hline do & . & 135 & & Purchase \& 1910. & 160 \\
\hline do & . & 138 & & $1881, ' 85, ' 90 \ldots$. & 96 \\
\hline do & $\therefore$ & 139 & & $1881, ' 85, ' 90 \ldots$. & 50.80 \\
\hline do & . & 141 & & $1881,95 \ldots \ldots$ & 83 \\
\hline do & . & 142 & & $1881, ' 85,90 \ldots$ & 74.80 \\
\hline do. & ... & 143 & & $1885, ' 90$. & 28.60 \\
\hline
\end{tabular}


Essex County

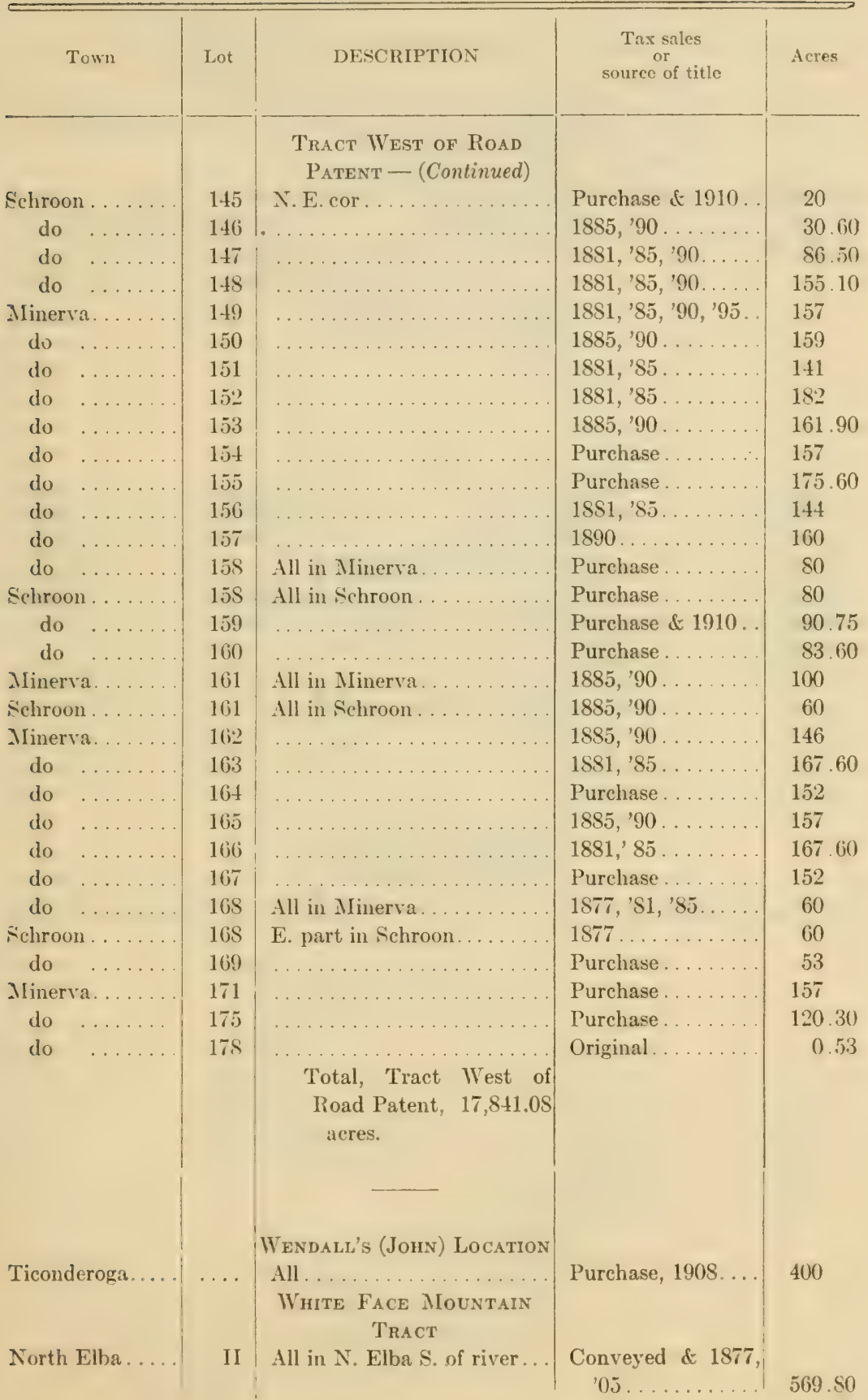


Essex County

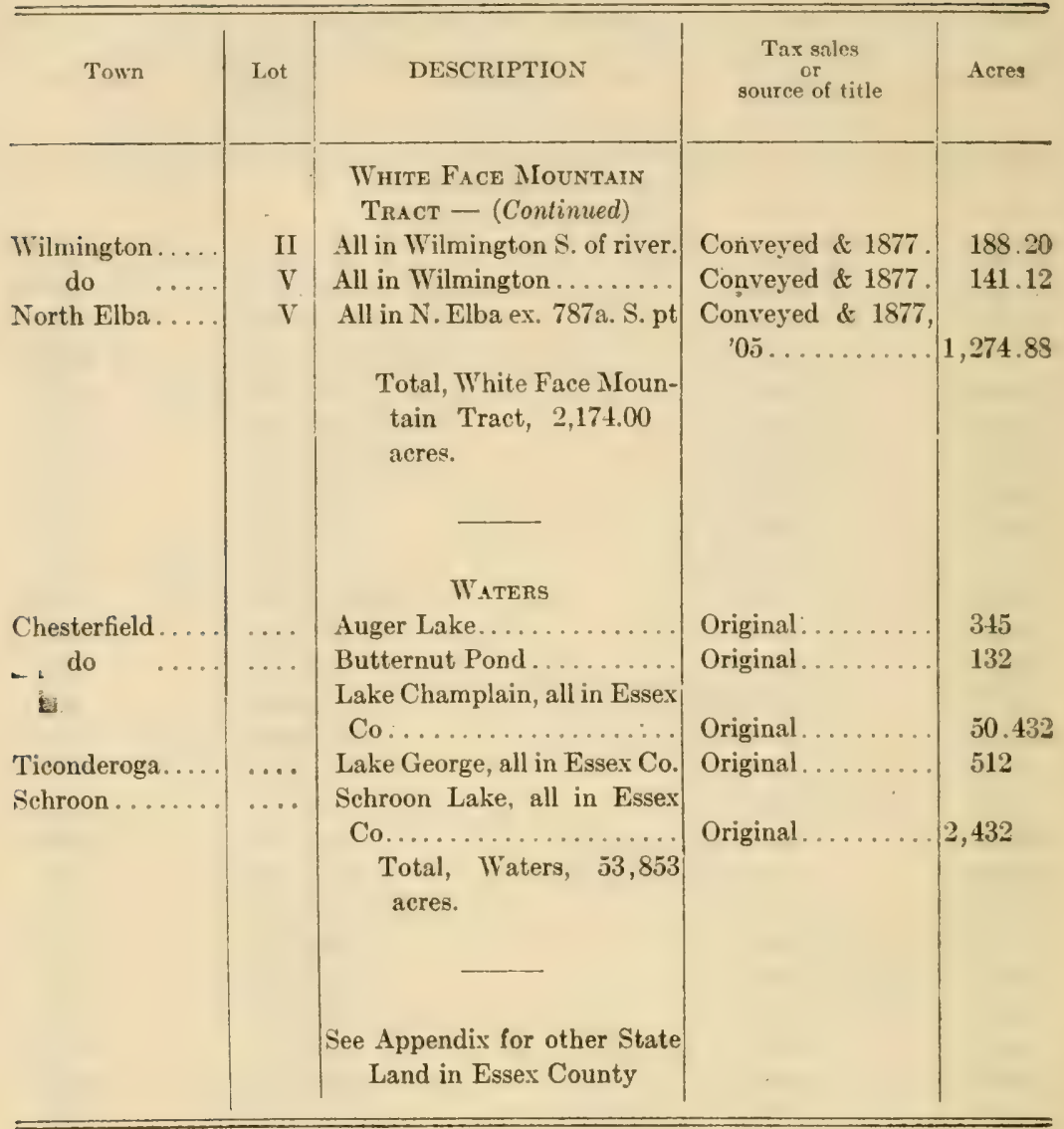




\section{FRANKLIN COUNTY}

(Total number of acres, 170,651.12.)

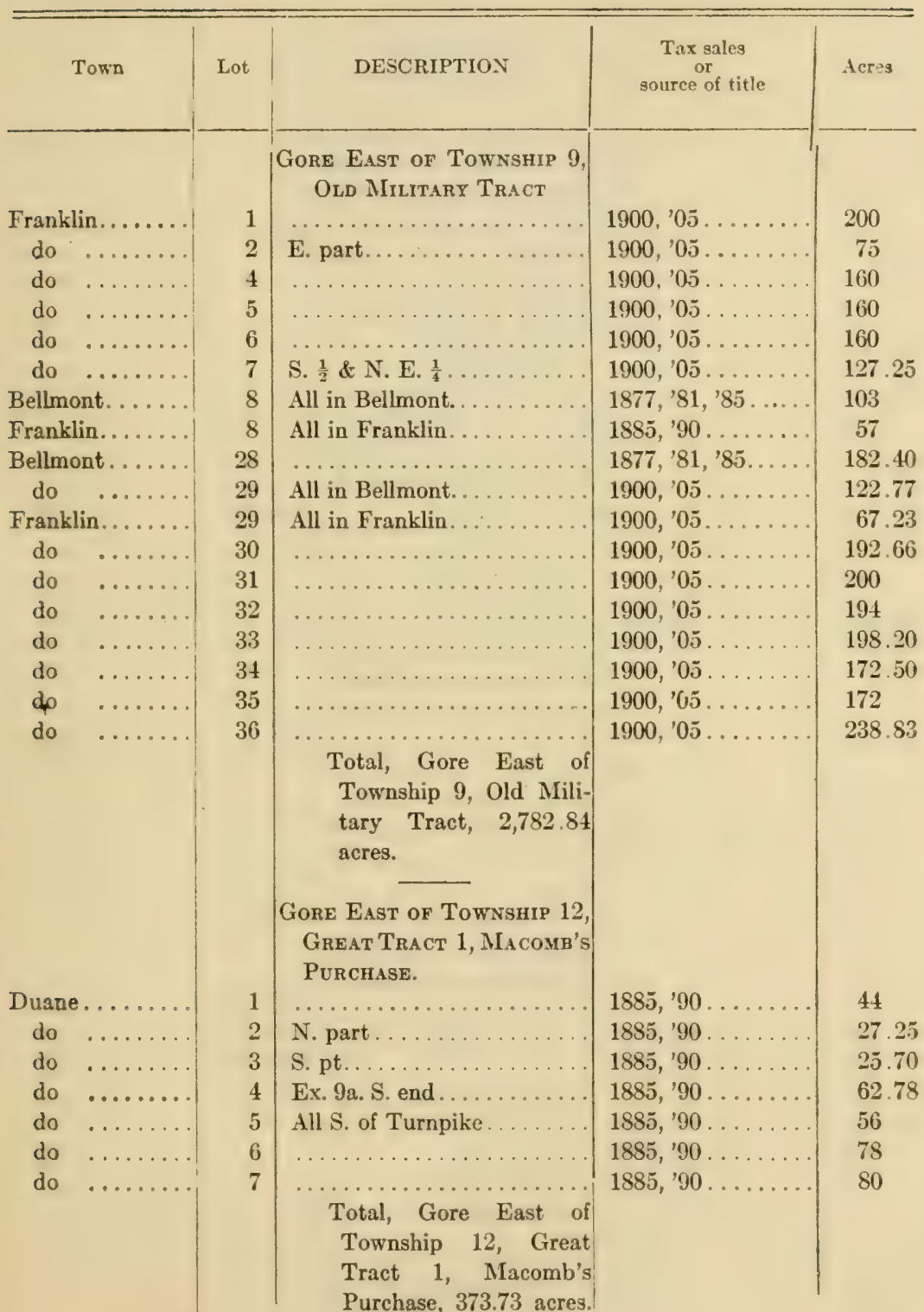


Franklin County

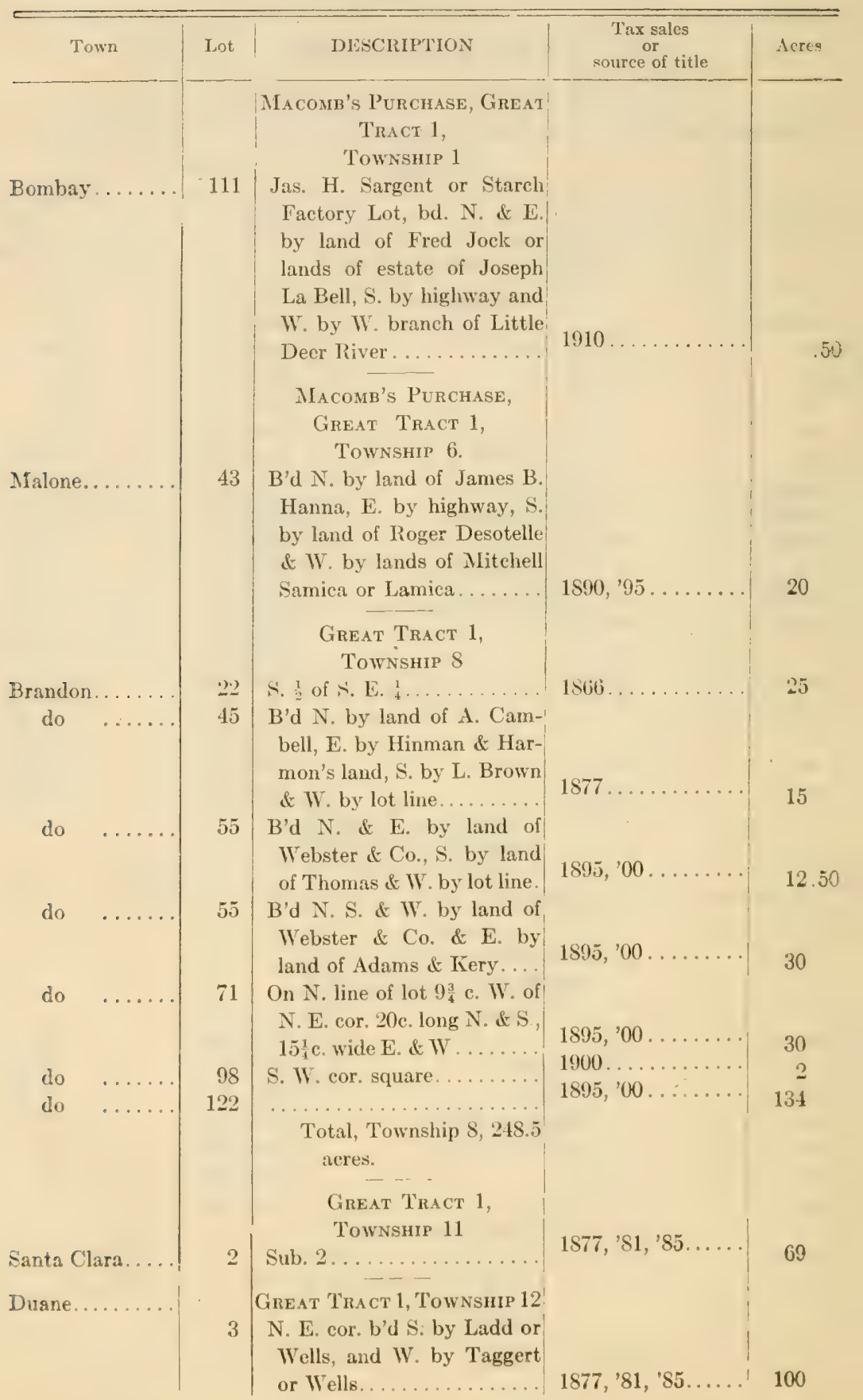




\section{Franklin County}

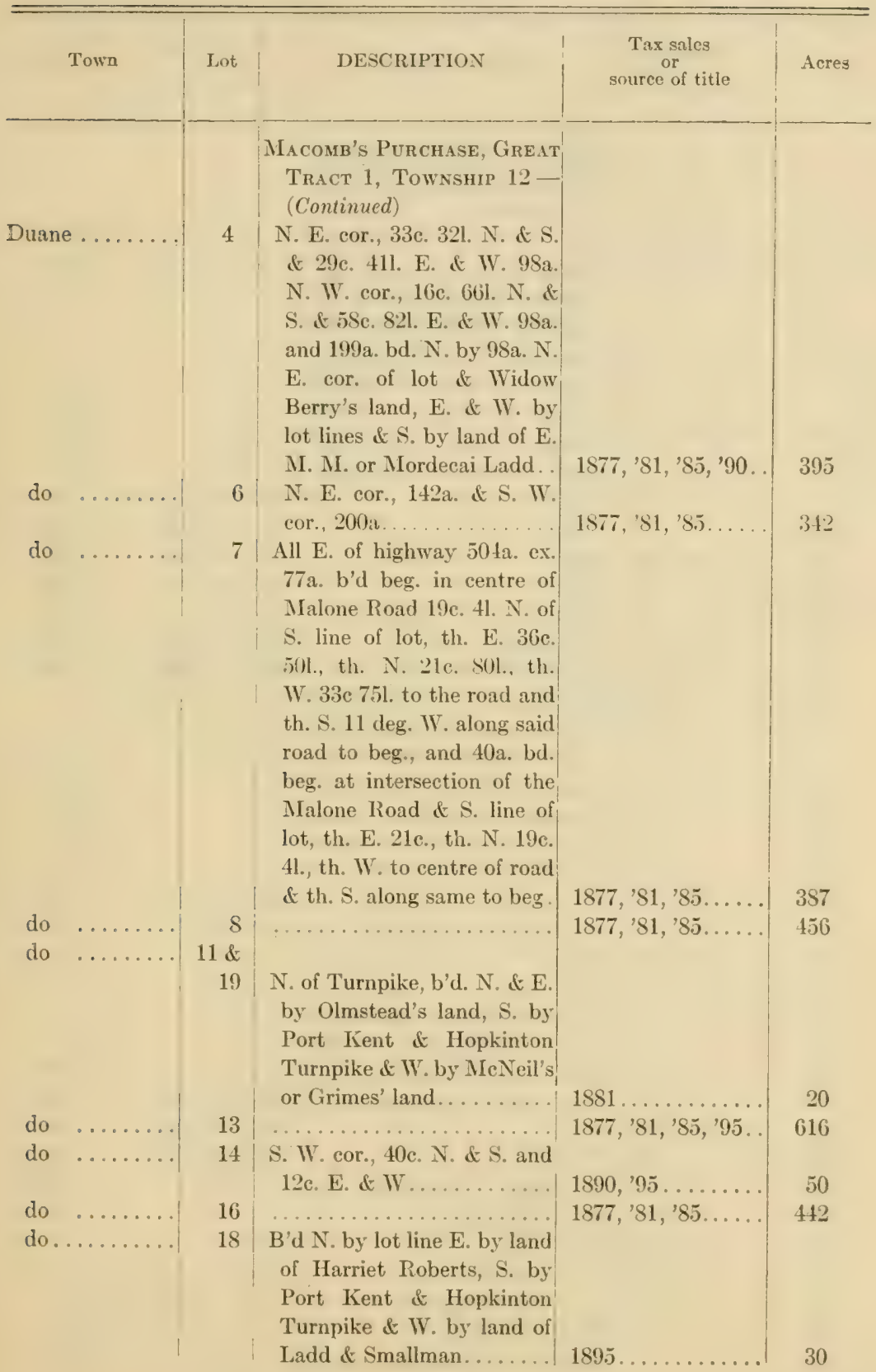


Franklin County

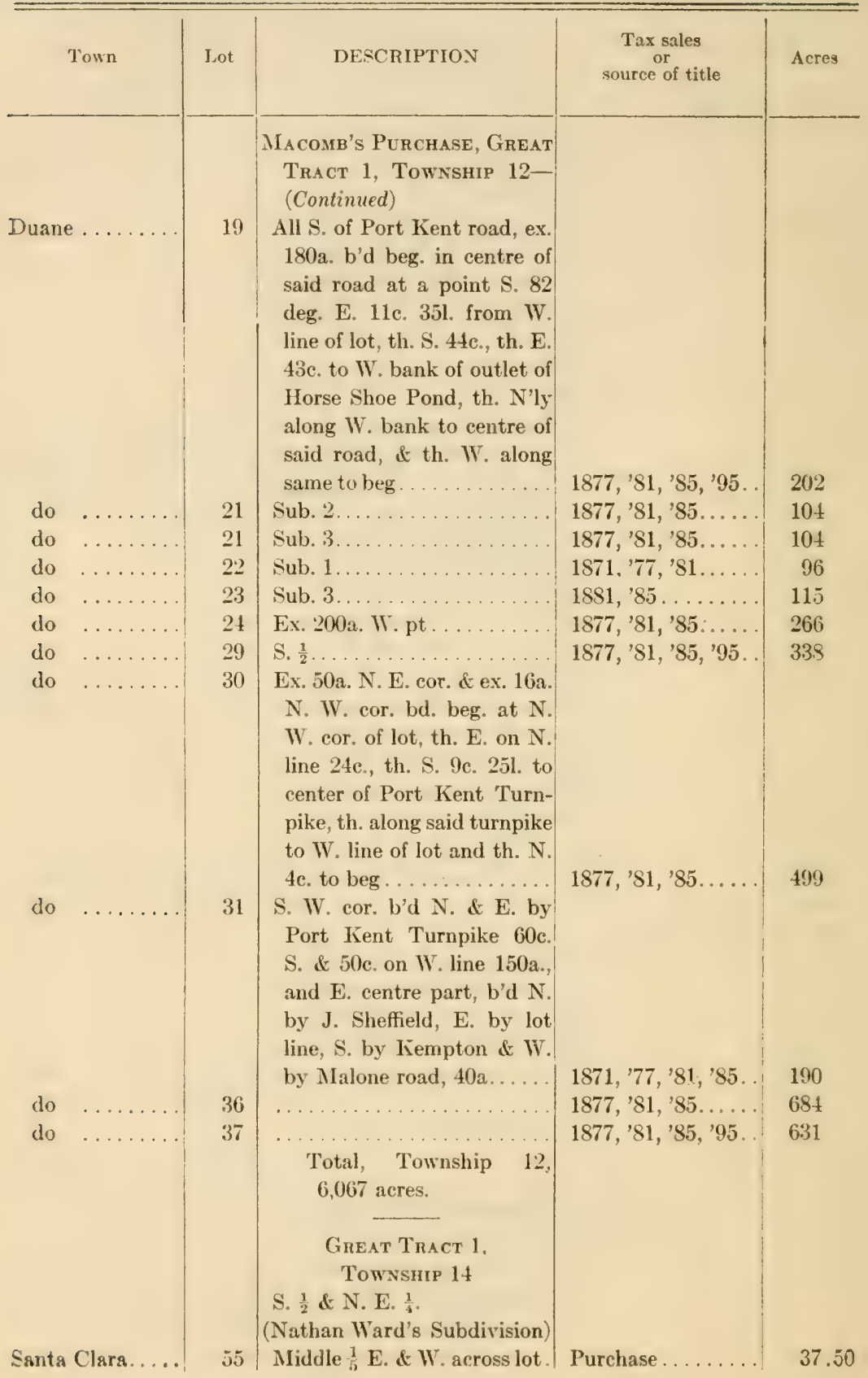


Conservation Commission.

Franklin County

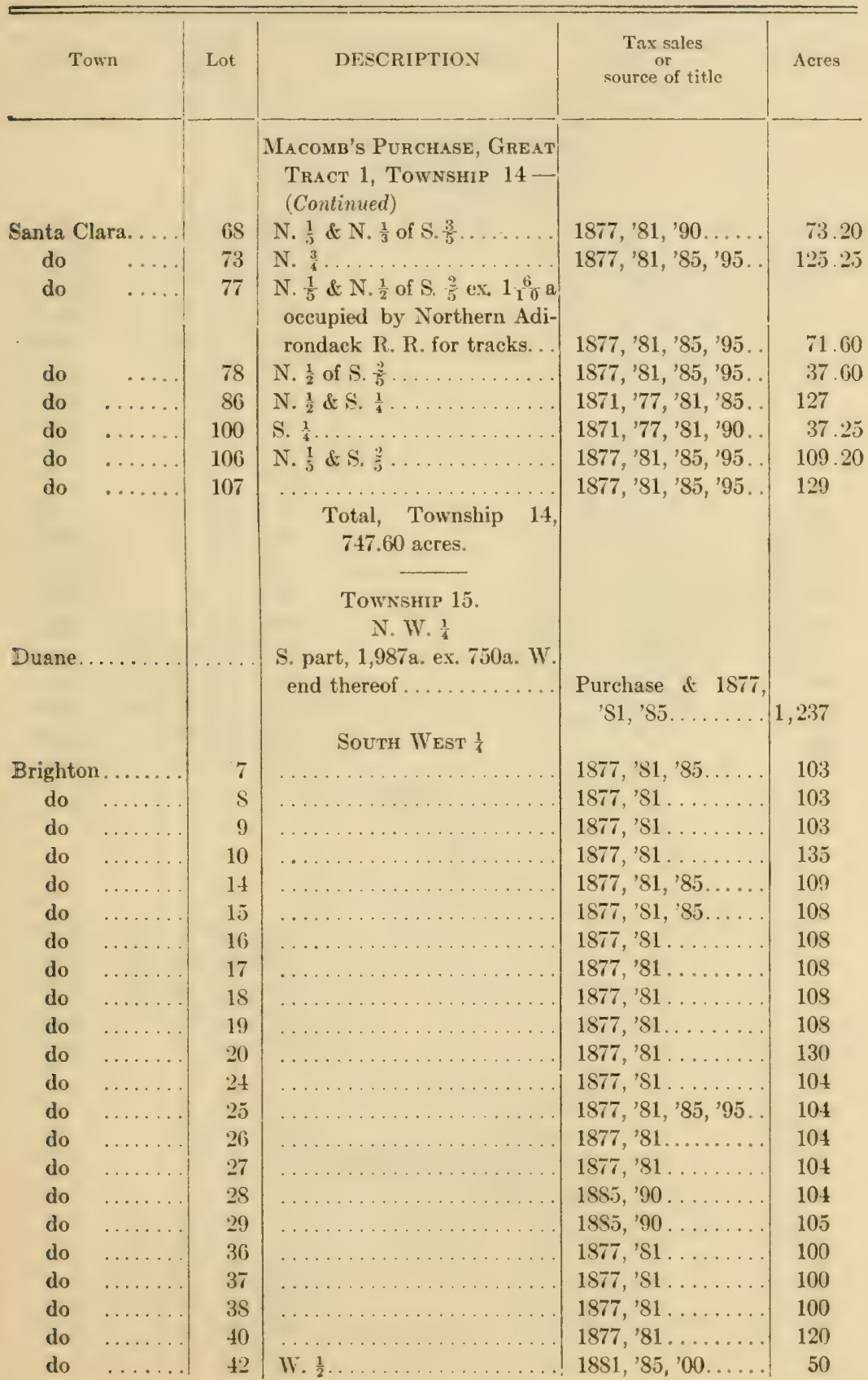


Franklin County

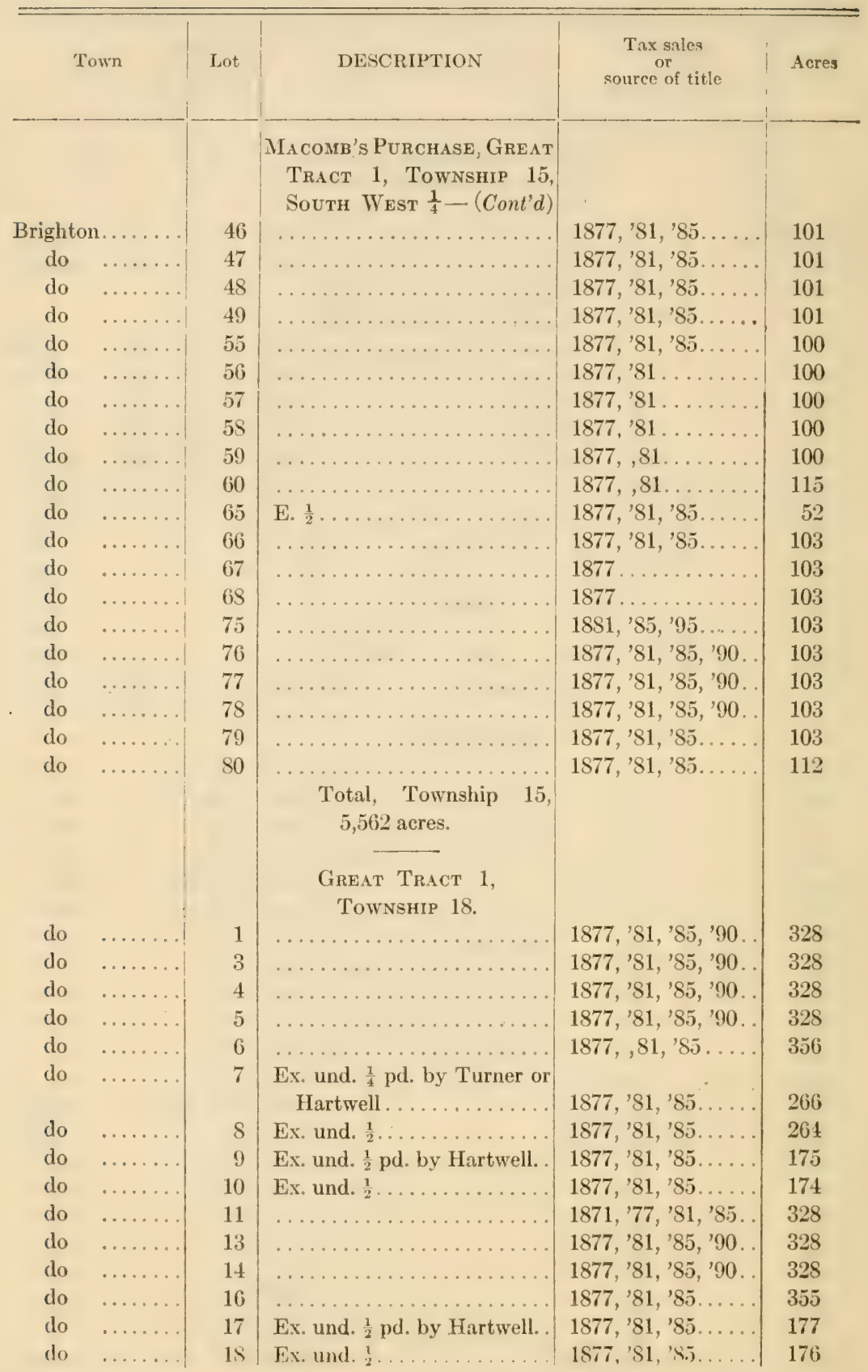


Franklin County

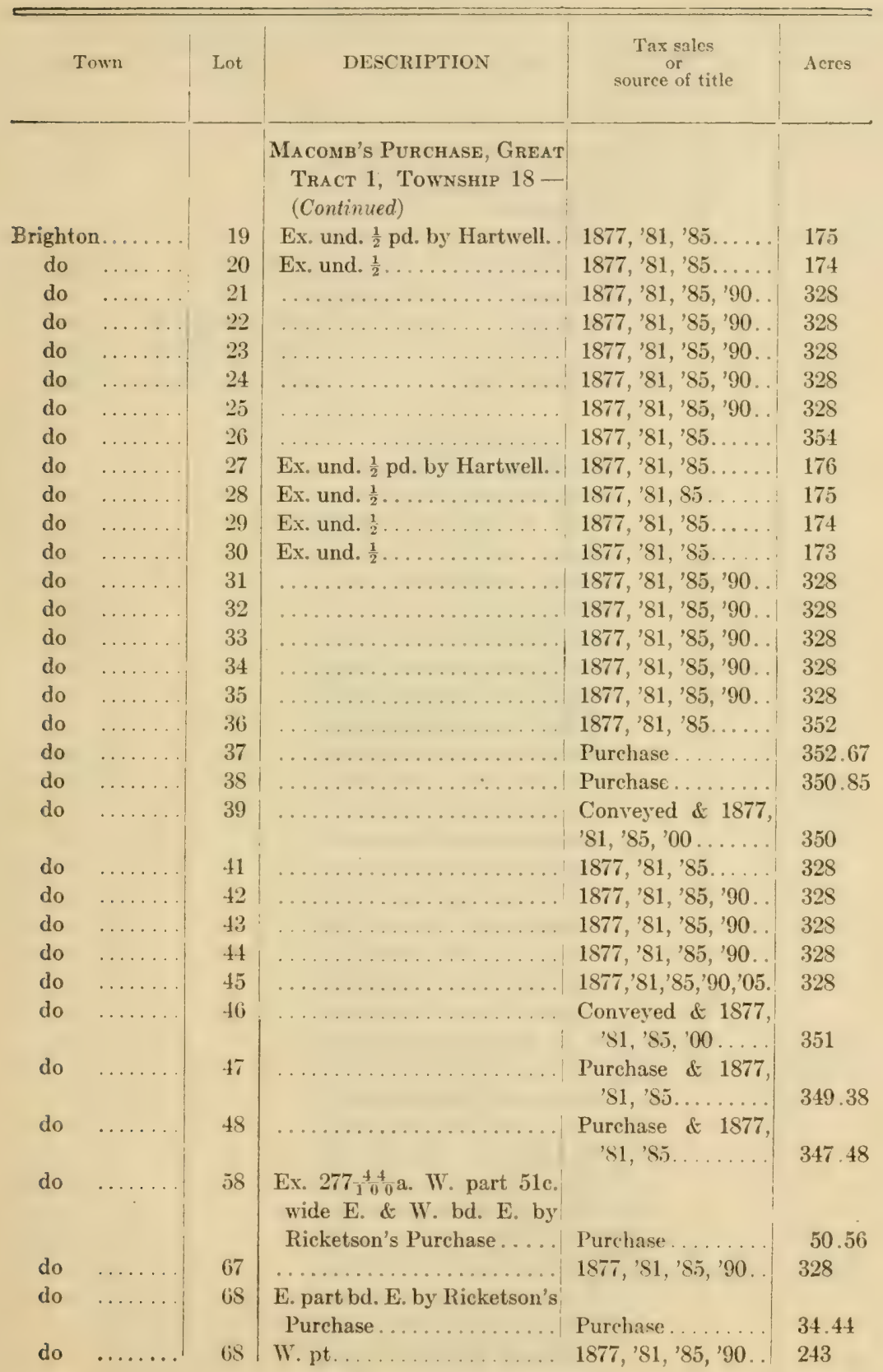




\section{Franklin Countx}

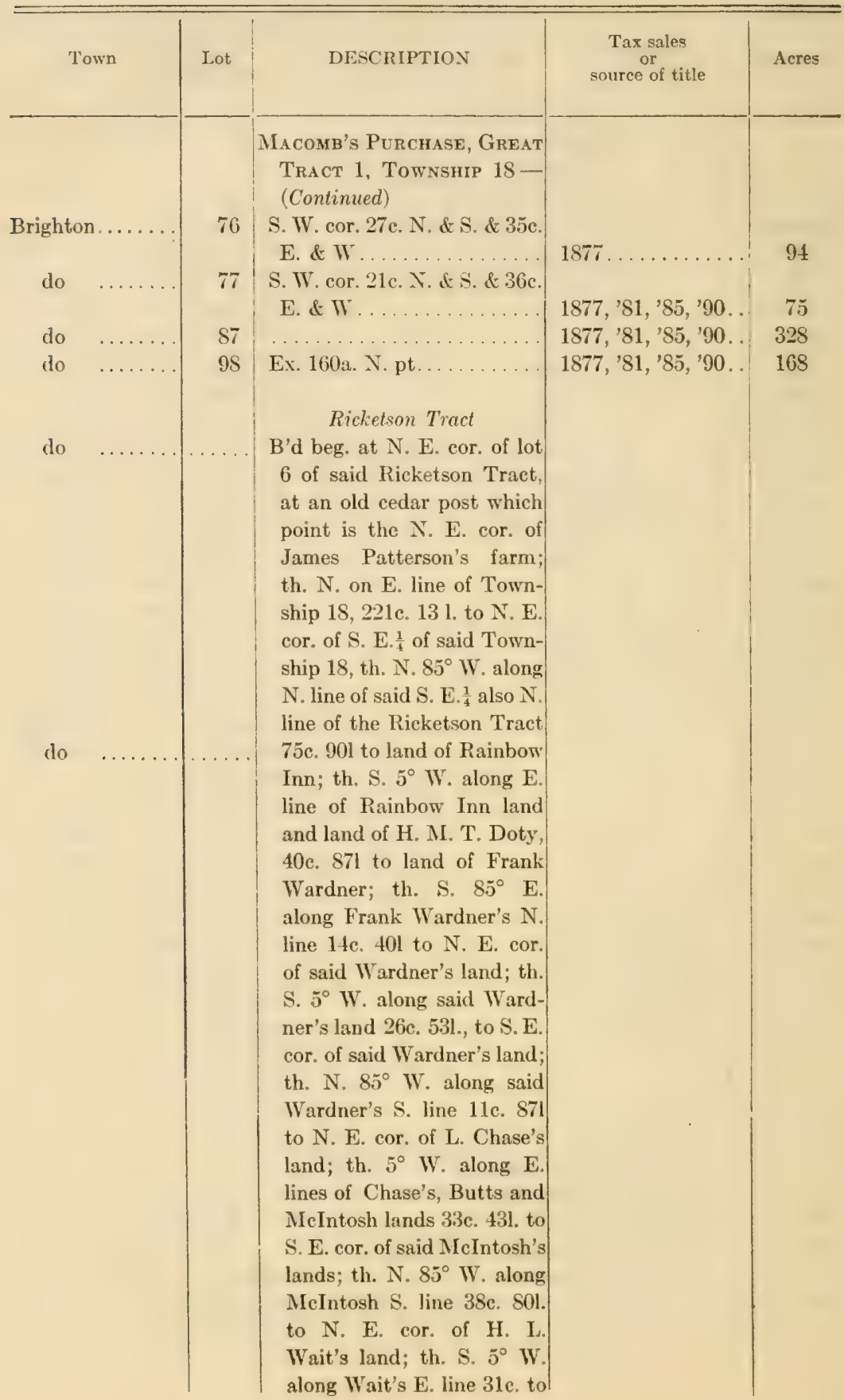


Franklin County

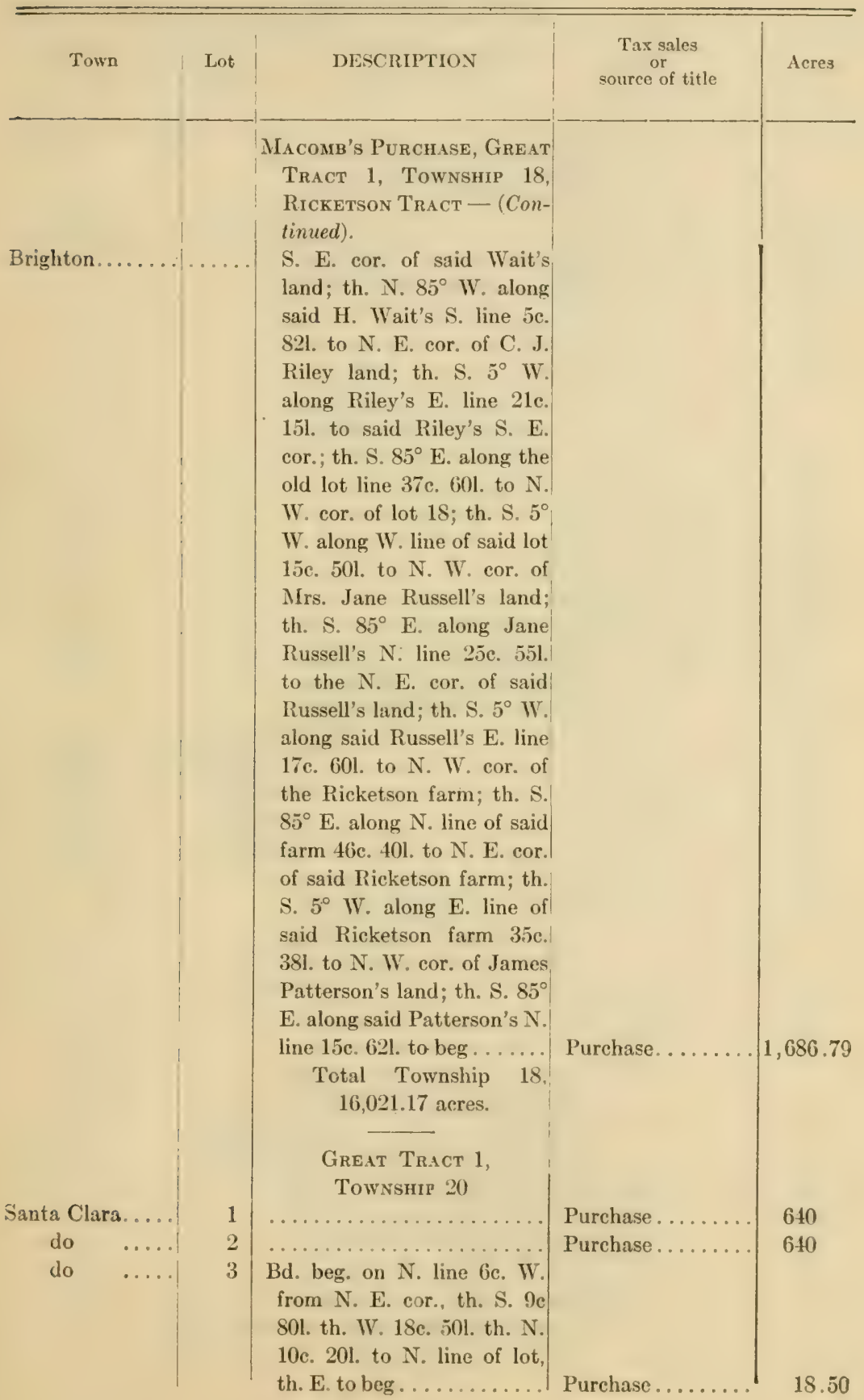


Franklin County

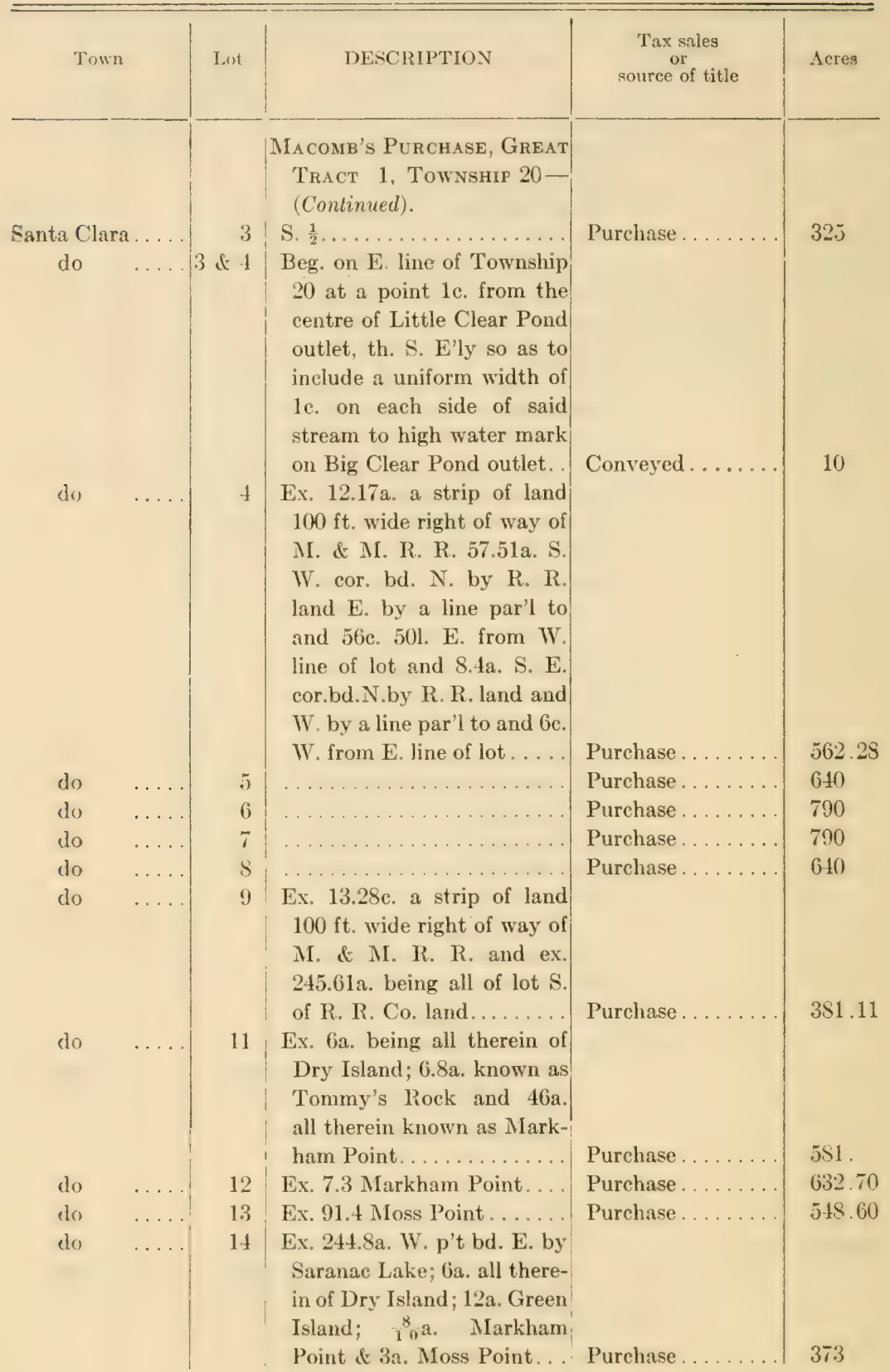


Franklin Countr

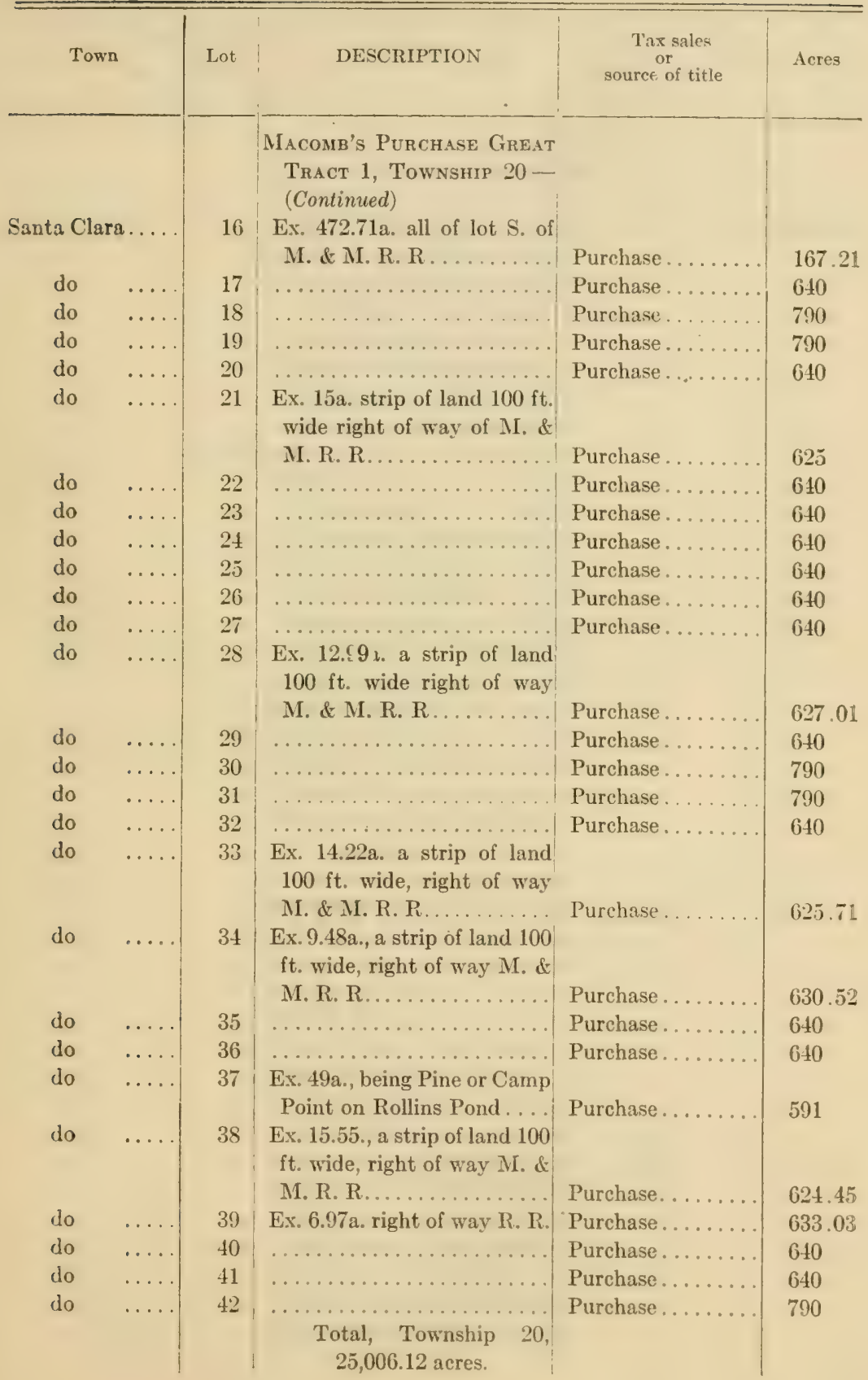


Franklin County

\begin{tabular}{|c|c|c|c|c|}
\hline Town & Lot & DESCRIPTION & $\begin{array}{c}\text { Tax sales } \\
\text { or } \\
\text { source of title }\end{array}$ & Acres \\
\hline Harrietstown. . & & 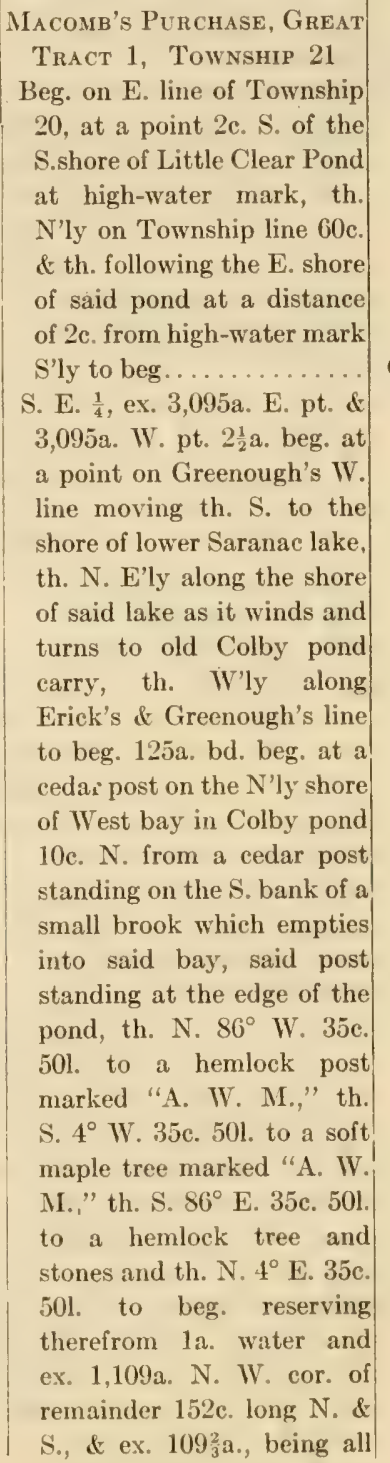 & Conveyed...... & 40 \\
\hline
\end{tabular}


Franklin Countr

\begin{tabular}{|c|c|c|c|c|}
\hline Town & Lot & DESCRIPTION & $\begin{array}{c}\text { Tax sales } \\
\text { or } \\
\text { source of title }\end{array}$ & Acres \\
\hline Harrietstown. . & $\ldots$ & 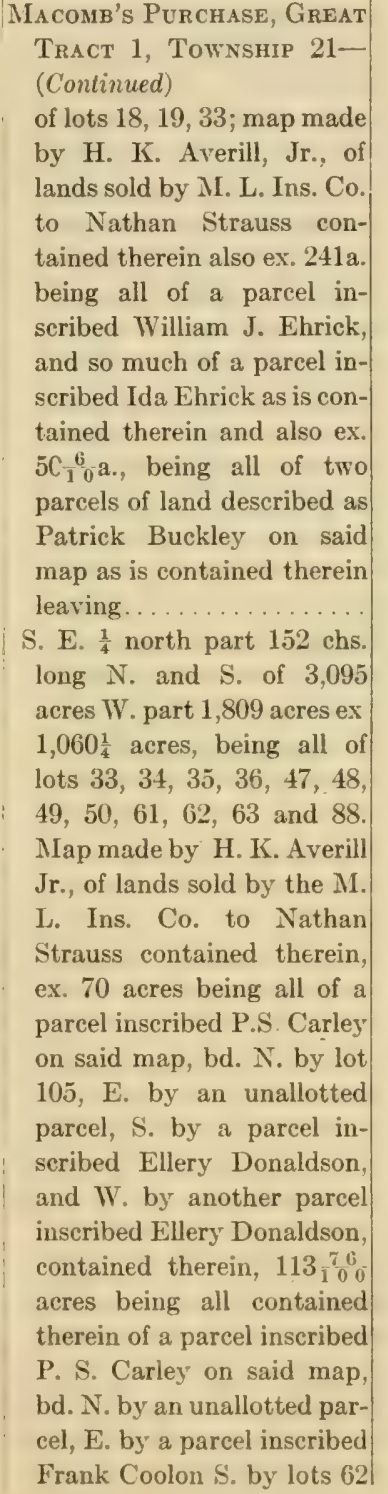 & 1895. & $5 S j$ \\
\hline
\end{tabular}


Franklin County

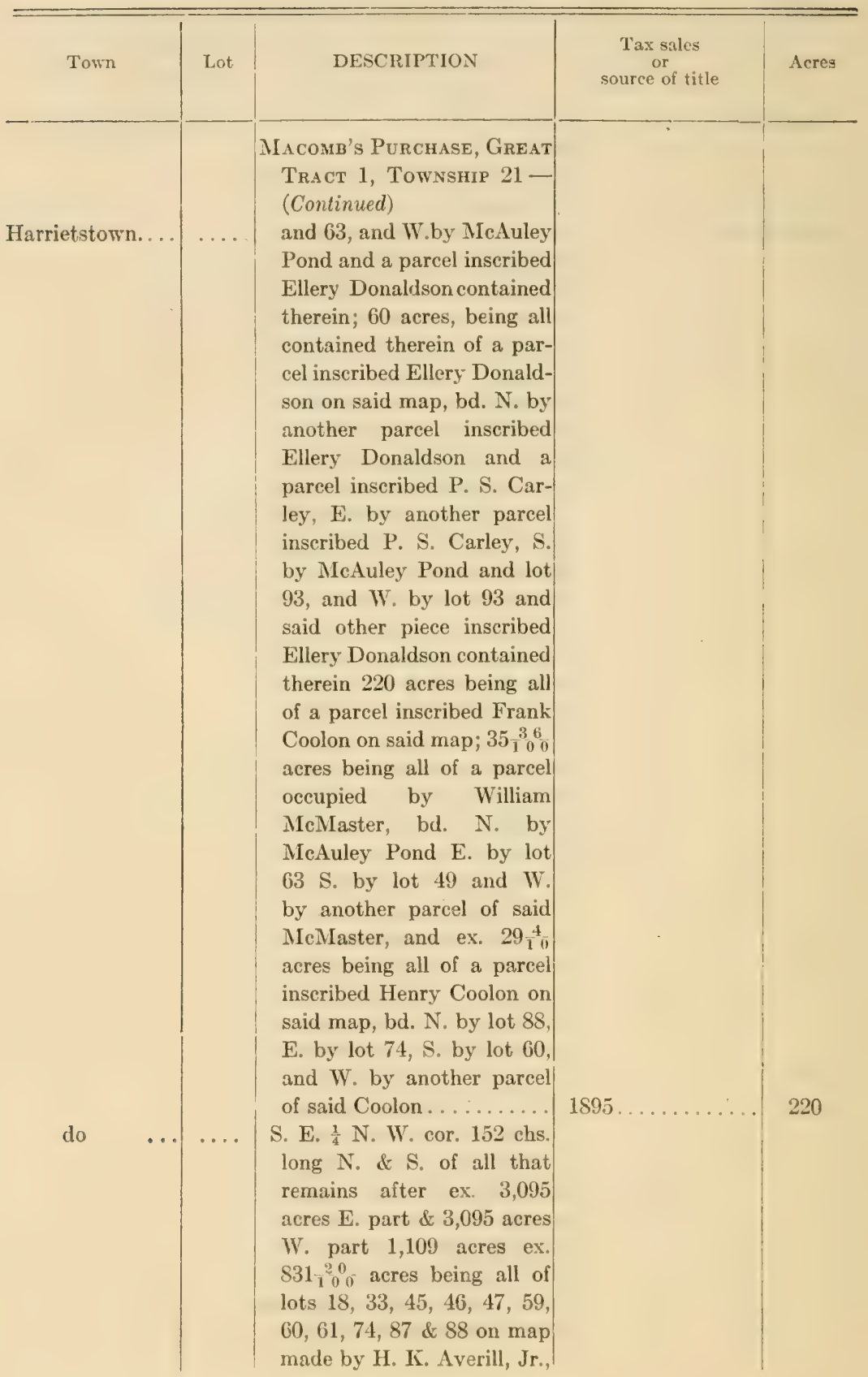


Franklin County

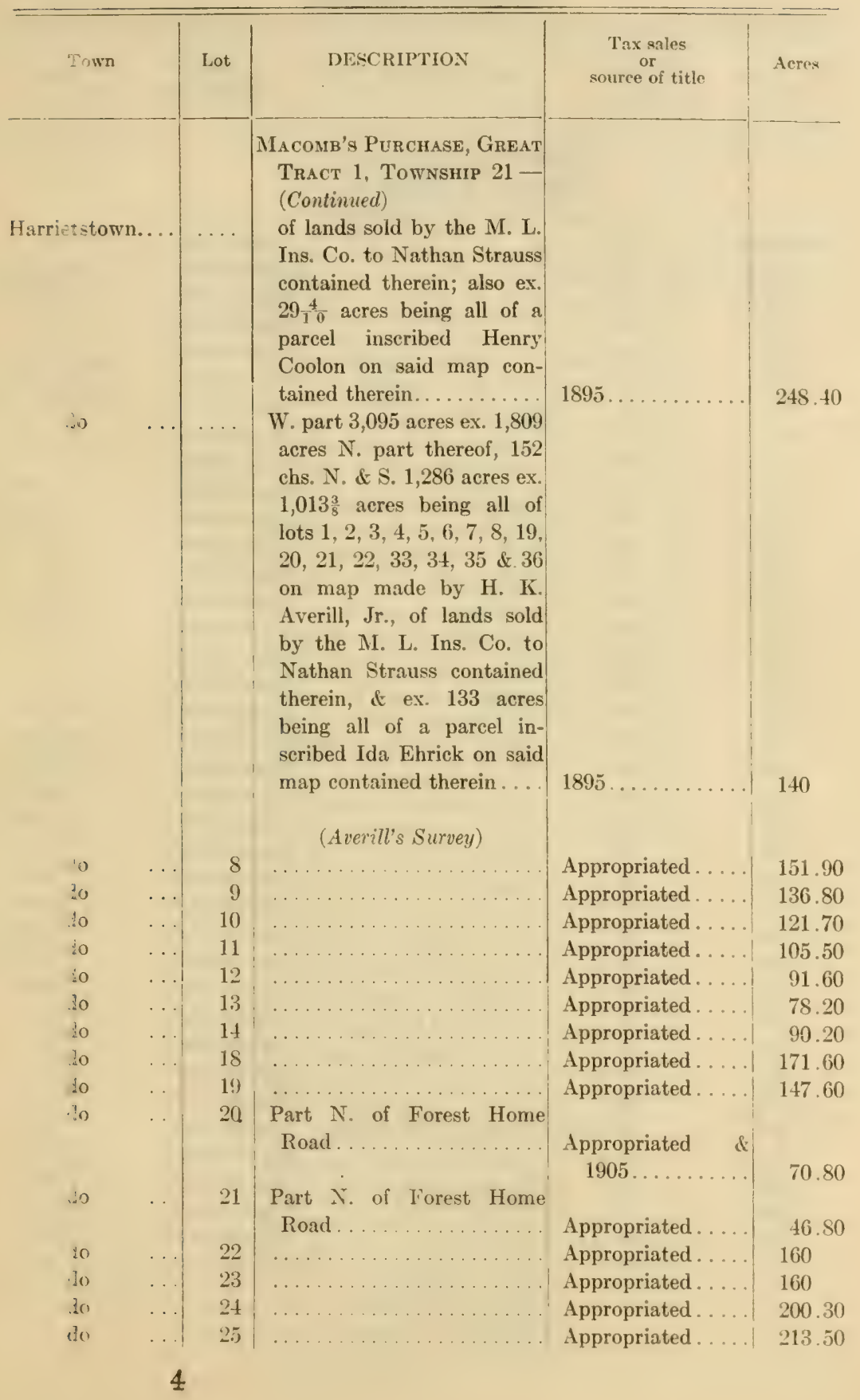


Franklin County

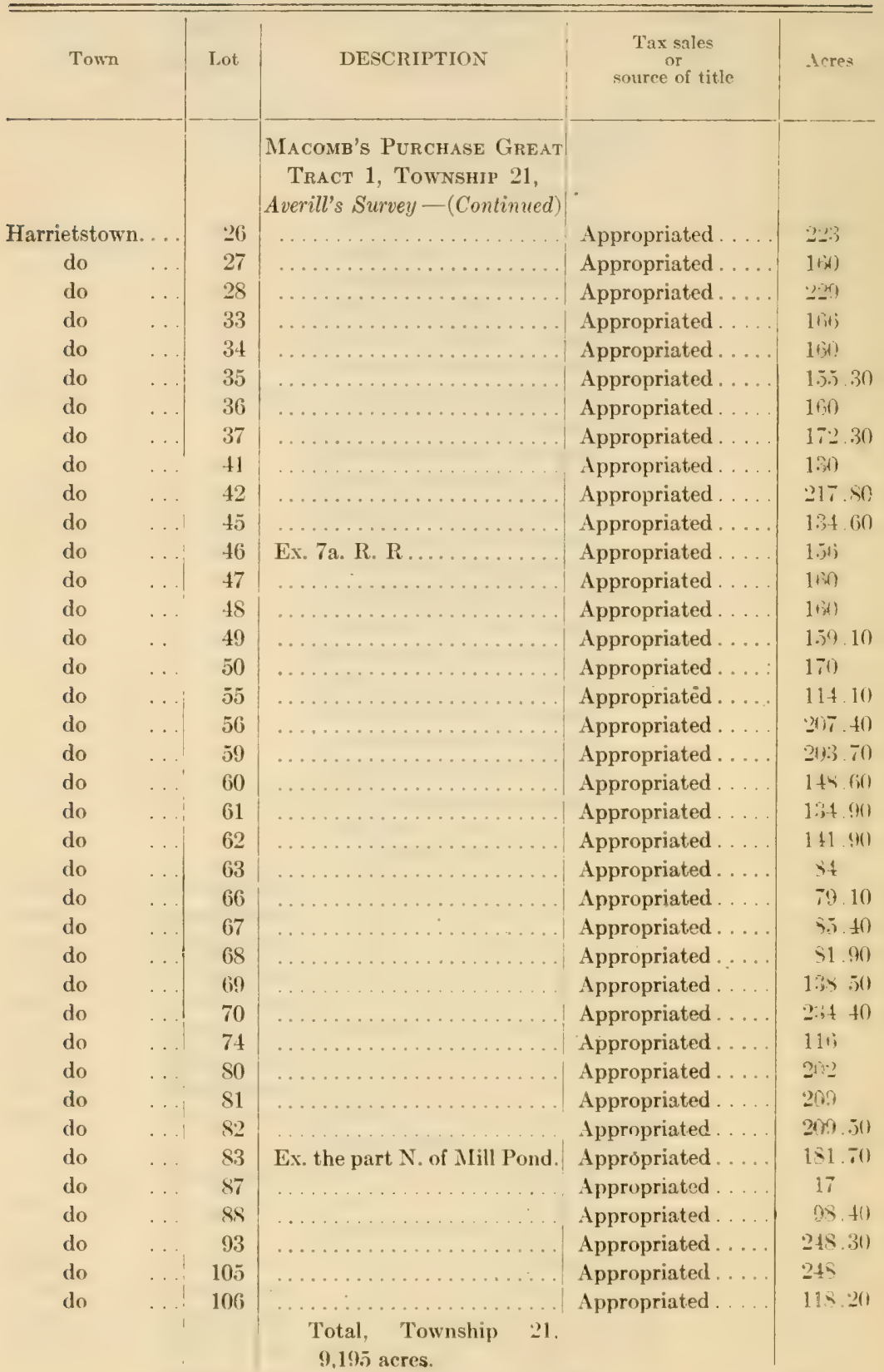


Franklin County

\begin{tabular}{|c|c|c|c|c|}
\hline Town & T.ot & DESCRIPTION & $\begin{array}{c}\text { Tax sales } \\
\text { or } \\
\text { source of title }\end{array}$ & Acres \\
\hline $\begin{array}{l}\text { Santa Clara. } \\
\text { do } \\
\text { Harrietstown } \\
\text { do }\end{array}$ & $\ldots$ & 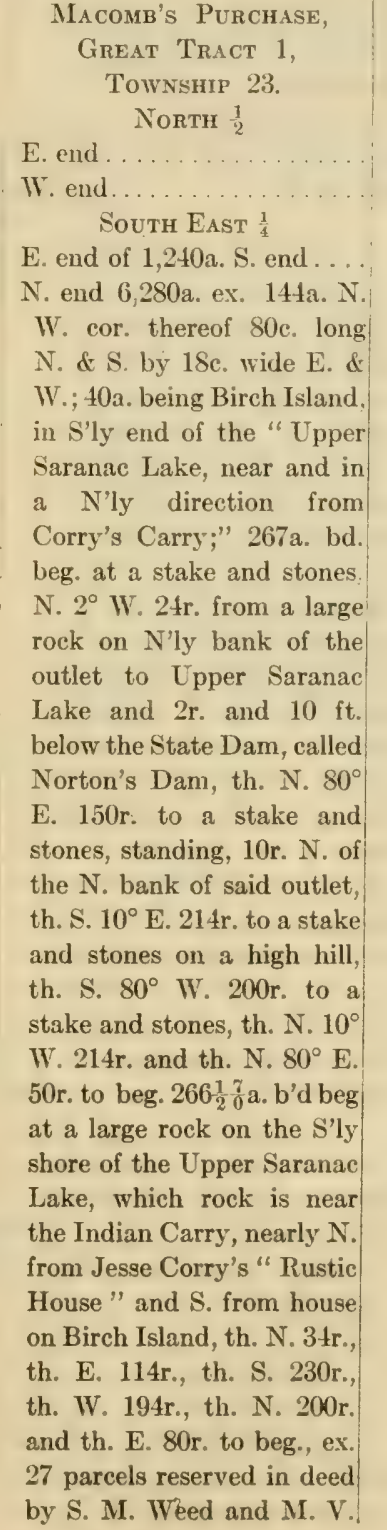 & $\begin{array}{l}1877, ' \$ 1, \text { '85... } \\
1898 \ldots \ldots \\
\text { Purchase........ }\end{array}$ & $\begin{array}{r}4,379 \\
6,661 \\
300\end{array}$ \\
\hline
\end{tabular}


Franklin County

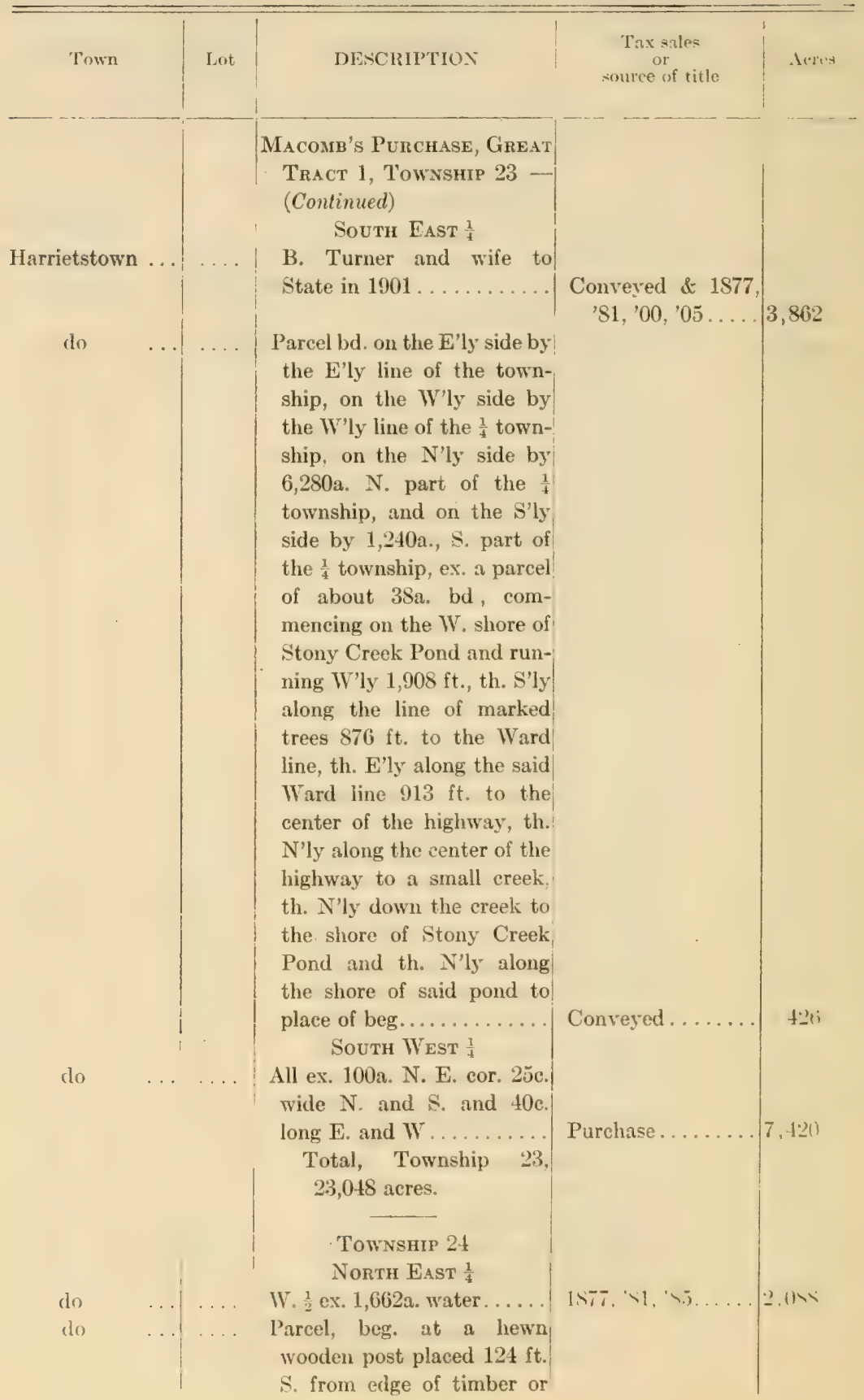




\section{Franklin County}

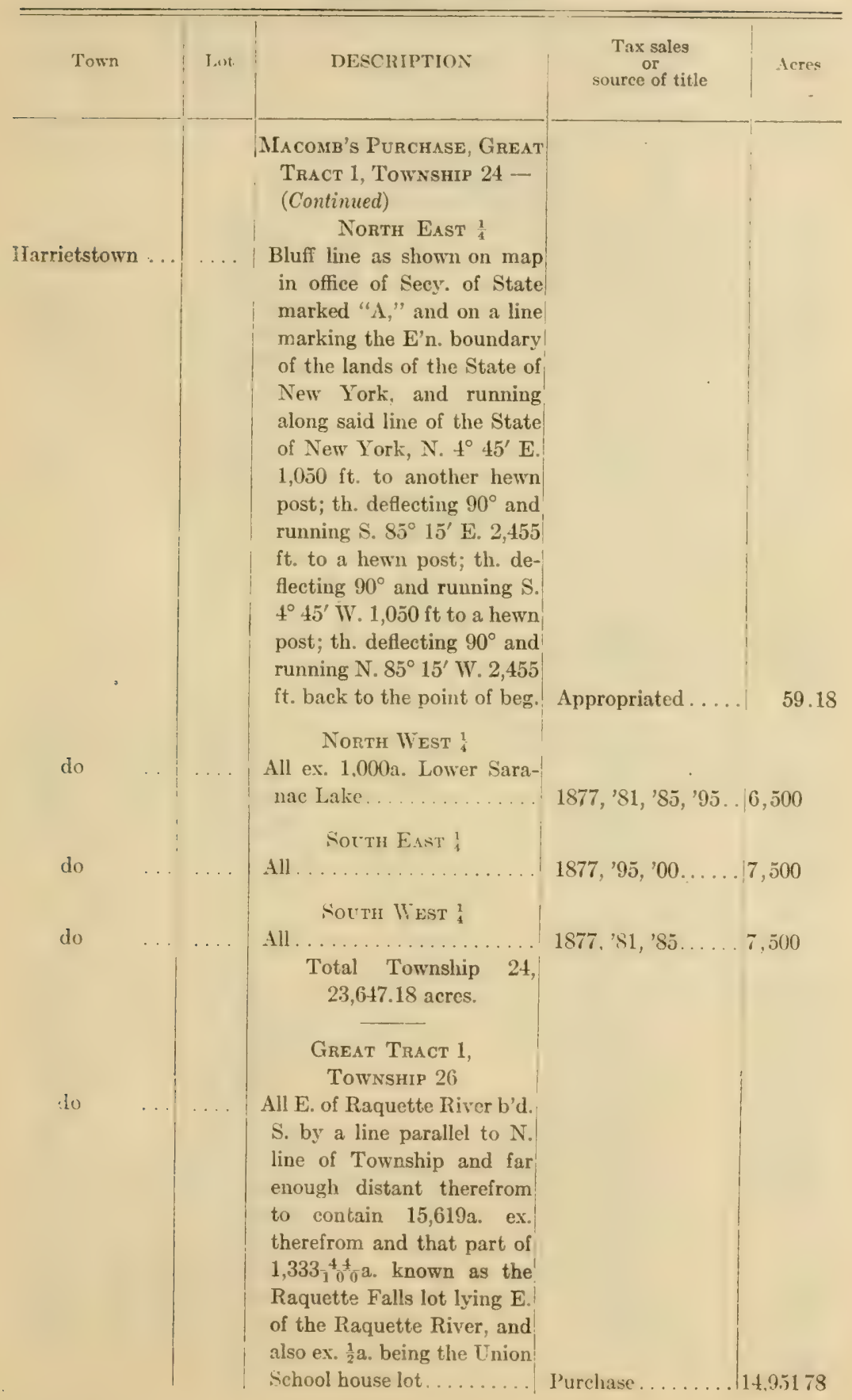


Franklin. County

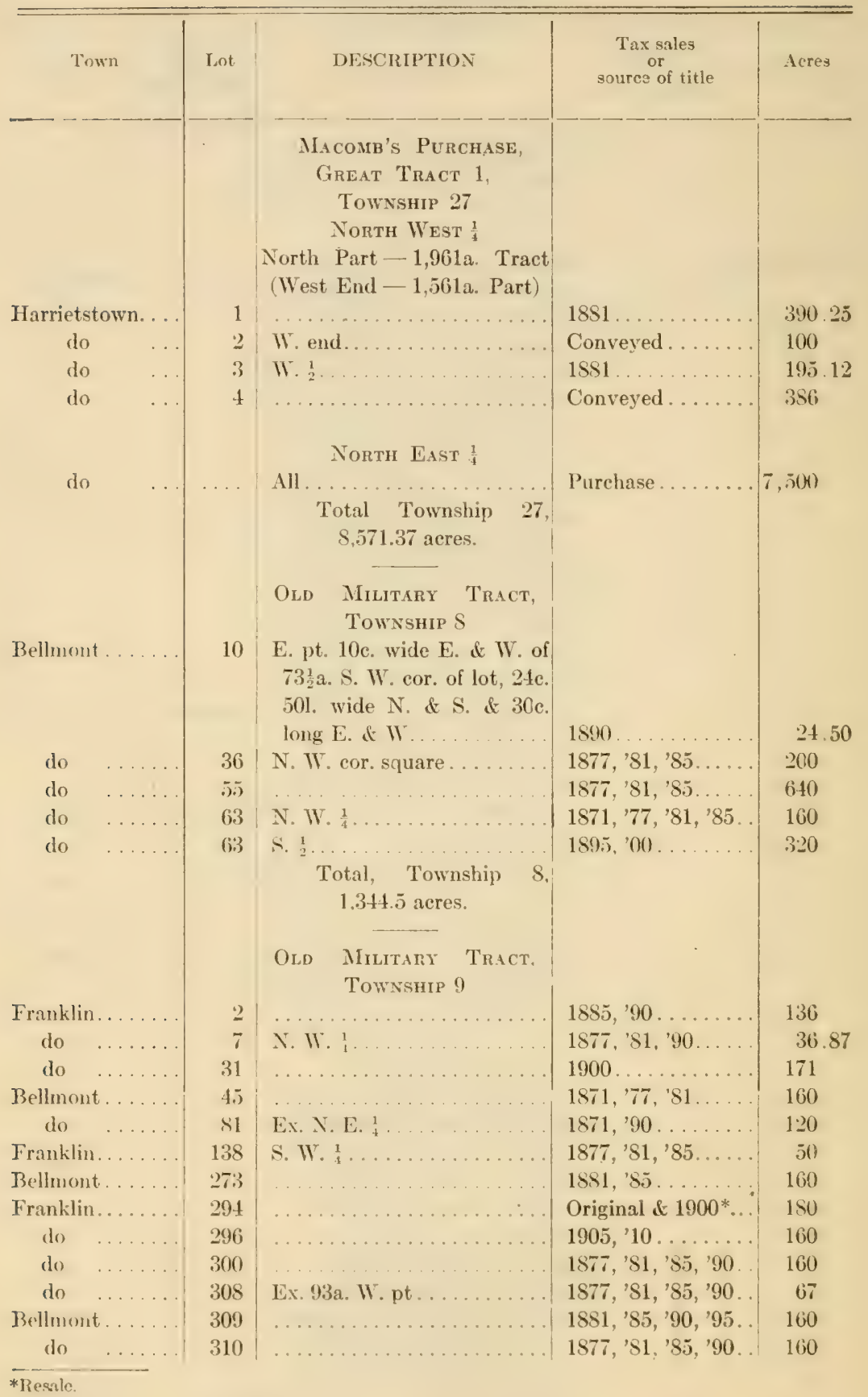


Franklin County

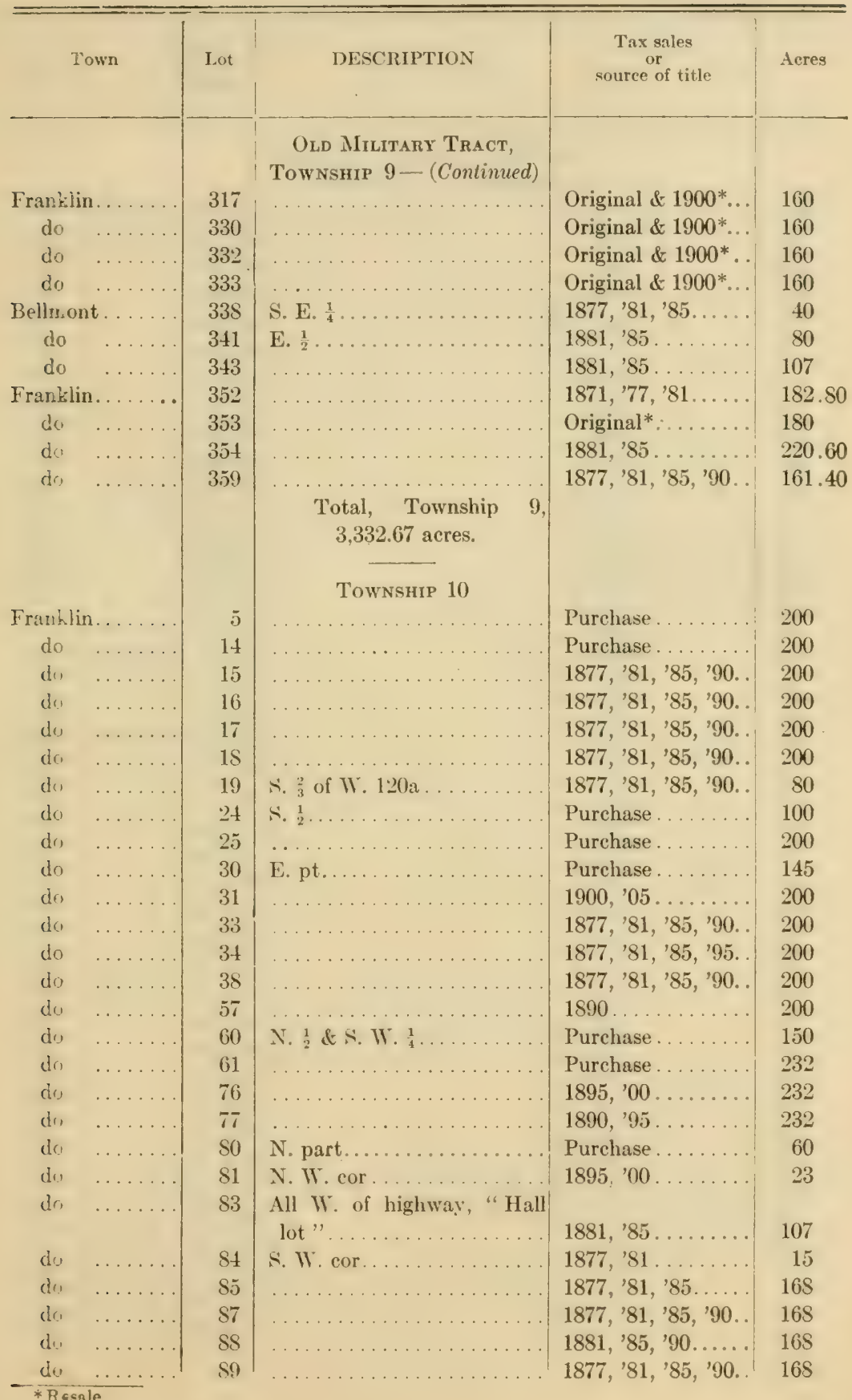


Franklin Countr

\begin{tabular}{|c|c|c|c|c|}
\hline Town & Lot & DESCRIPTION & $\begin{array}{l}\text { Tax sales } \\
\text { or } \\
\text { source of title }\end{array}$ & Acres \\
\hline \multirow[b]{2}{*}{ Franklin. . } & & $\begin{array}{l}\text { Old MiL I ta R T Tract, } \\
\text { Township } 10 \text {-(Continued })\end{array}$ & & \\
\hline & 90 & $\ldots \ldots \ldots \ldots \ldots \ldots$ & $1877, ' 81, ' 85, ' 90$. & $16 s$ \\
\hline do & 96 & $\ldots \ldots \ldots$ & $1877, ' 81, ' 85, ' 90$. & $16 \mathrm{~S}$ \\
\hline do & 97 & & $1895,{ }^{\prime} 00 \ldots \ldots \ldots$ & 169 \\
\hline do & 99 & $\cdots$ & Purchase........ . & 169 \\
\hline do & 100 & Ex. 33a. W. pt...... & Purchase ... . . . . . & 135 \\
\hline do & 101 & S. $\frac{1}{2} \ldots \ldots \ldots$ & Purchase ........ & 100 \\
\hline do & 102 & $\ldots \ldots \ldots \ldots \ldots$ & Purchase........ & 200 \\
\hline do & 103 & & Purchase... & 200 \\
\hline do & 104 & $\ldots \ldots \ldots$ & Purchase........ & 200 \\
\hline do & 105 & $\ldots \ldots \ldots \ldots \ldots$ & $1900,{ }^{\prime} 05 \ldots \ldots \ldots$ & 200 \\
\hline do & 106 & 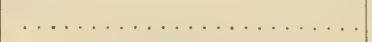 & $1877, ' 81, ' 85$, '95. . & 200 \\
\hline do & 107 & & $1877, ' 81, ' 85, ' 95$. & 200 \\
\hline do & $10 \mathrm{~s}$ & & Purchase ........ & 200 \\
\hline do & 109 & & Purchase ........ & 200 \\
\hline do & 112 & 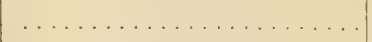 & $1877, ' 81, ' 85, ' 90$. & 200 \\
\hline do & 113 & & $1877, ' 81, ' 85, ' 90$. & 200 \\
\hline do & 114 & 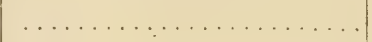 & $1871,77, ' 81, ' 85, ' 90$. & 200 \\
\hline do & 115 & $\ldots$ & $1871,77, ' 81, ' 85$. & 200 \\
\hline do & 116 & & $1881, ' 85 \ldots \ldots \ldots$ & 200 \\
\hline do & 126 & & $1871,77, ' 81, ' 85, ' 90$. & 200 \\
\hline do & 127 & & $1871,77, ' 81 \ldots$. & 200 \\
\hline do & 128 & & $1871,77,91 \ldots$. & 200 \\
\hline do & 130 & . & Purchase \& 1905. & $2(0)$ \\
\hline do & 131 & & Purchase........ & $2(x)$ \\
\hline do & 137 & & $1900,05 \ldots \ldots \ldots$ & 200 \\
\hline do & 138 & & $1877, ' 81, ' 85 \ldots \ldots$ & 200 \\
\hline do & 140 & ... & Purchase ........ & 200 \\
\hline do & 141 & $\ldots$ & 1871, '77, '81, 'S5. . & 200 \\
\hline do & 144 & Ex. N. $\frac{1}{2}$ of $\mathrm{W} .120 \mathrm{a}$. & $1871, ' 77, ' 81, ' 85$. & 160 \\
\hline do & 150 & $\ldots \ldots \ldots \ldots \ldots$ & Original $^{*} \ldots \ldots$ & 200 \\
\hline do & 151 & E. ${ }_{2}^{1} \ldots$ & Purchase....... & 100 \\
\hline do & 152 & & $1877, ' 85 \ldots$ & 200 \\
\hline do & 153 & & $18 s 1, ' 85 \ldots \ldots$. & 210 \\
\hline do & 154 & . . & $1885,90 \ldots \ldots$ & $2(10)$ \\
\hline do & 160 & ... & $1890,95 \ldots$ & 200 \\
\hline do & 166 & Ex. $40 \mathrm{a}$. N. ${ }_{3}^{1}$ of W. $120 \mathrm{a}$. & $1877, ' 81, ' 85 \ldots$. & 160 \\
\hline do & 167 & & $1877, ' 81, ' 85 \ldots$ & 200 \\
\hline do & 168 & & $1877, ' 81, ' 85 \ldots$. & $2(H)$ \\
\hline do & 171 & & $1871,77, ' 81, ' 85, ' 90$. & $2(16)$ \\
\hline do & 172 & & $\begin{array}{c}* \text { Original \& con- } \\
\text { veyed .......... }\end{array}$ & 200 \\
\hline
\end{tabular}

* Resale. 
Franklin County

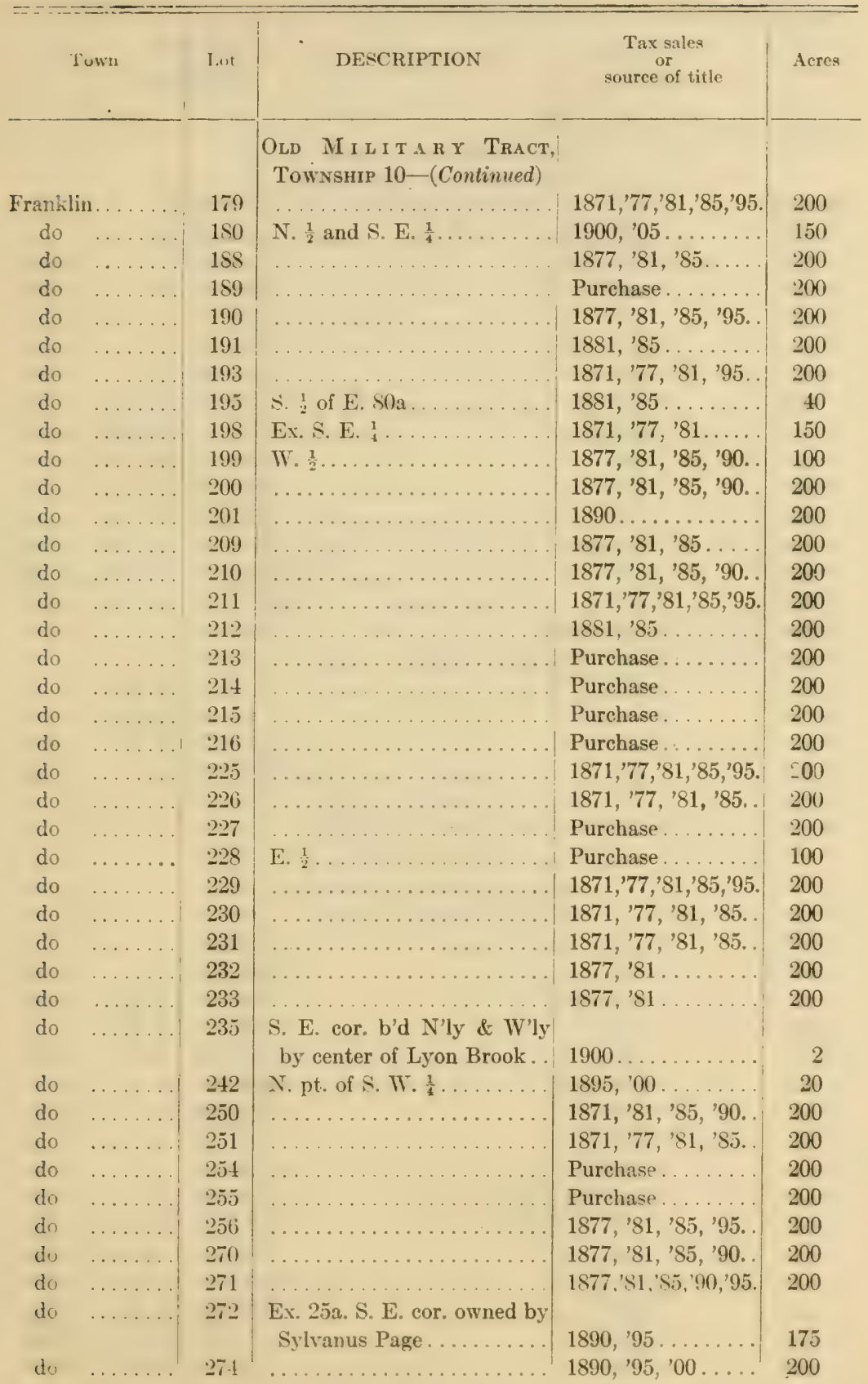


Franklin Country

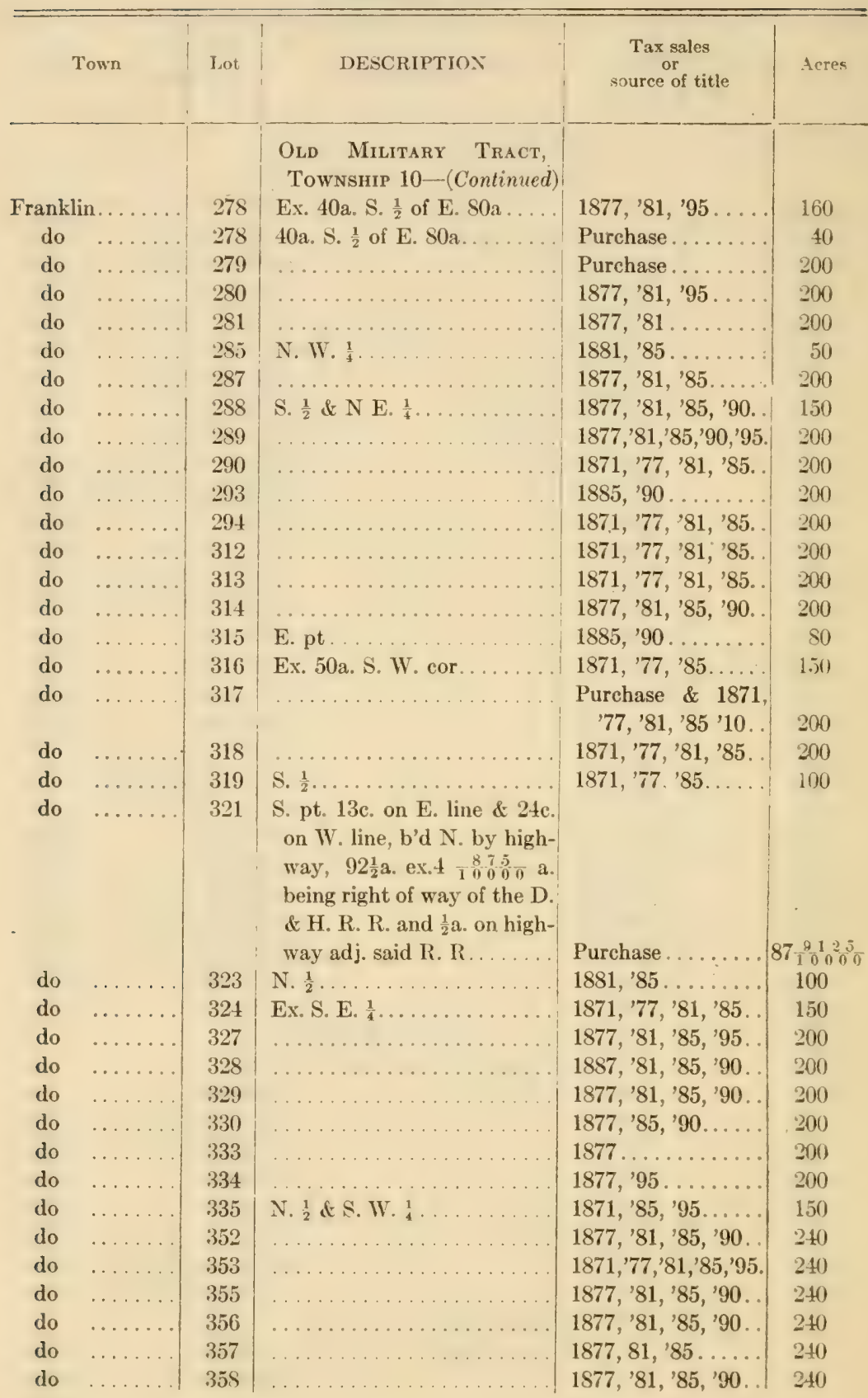




\section{Franklin County}

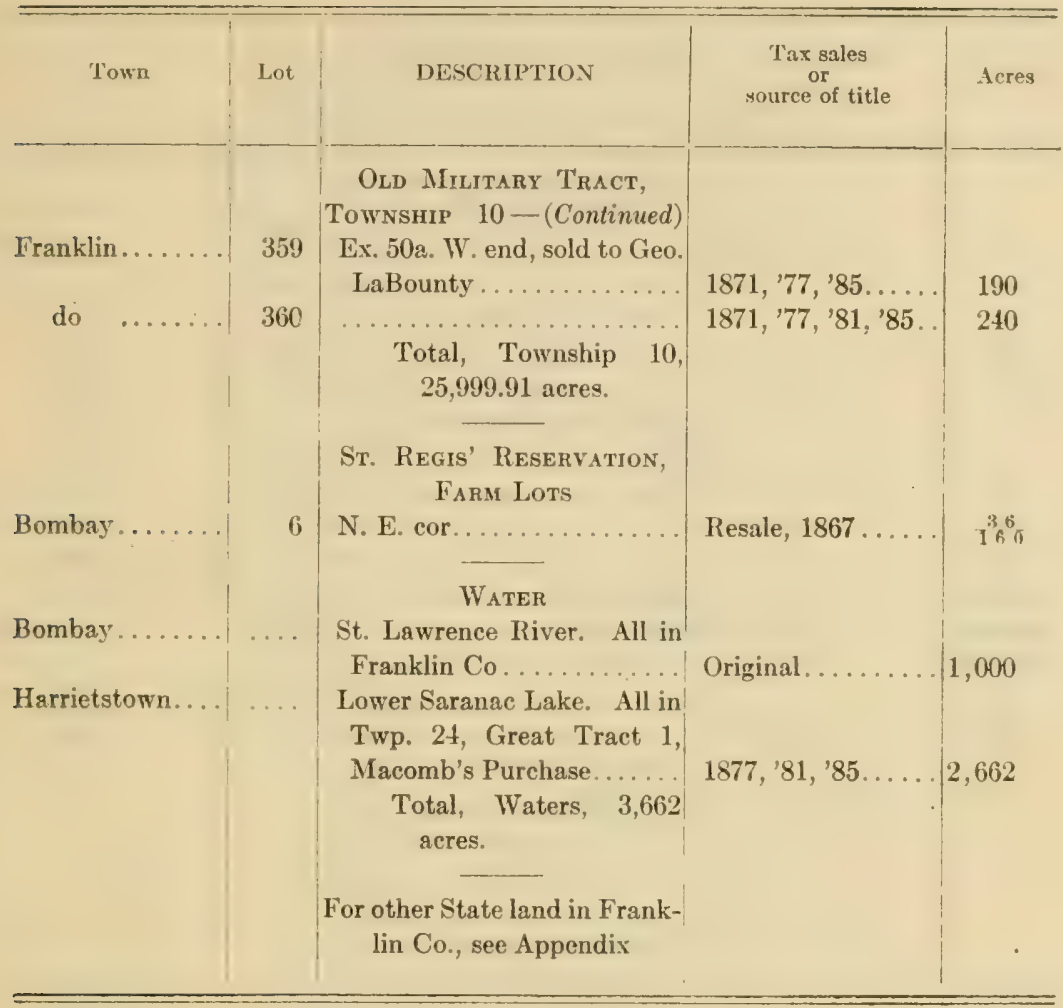


FULTON COUNTY

(Total number of acres, $23,274.85$.)

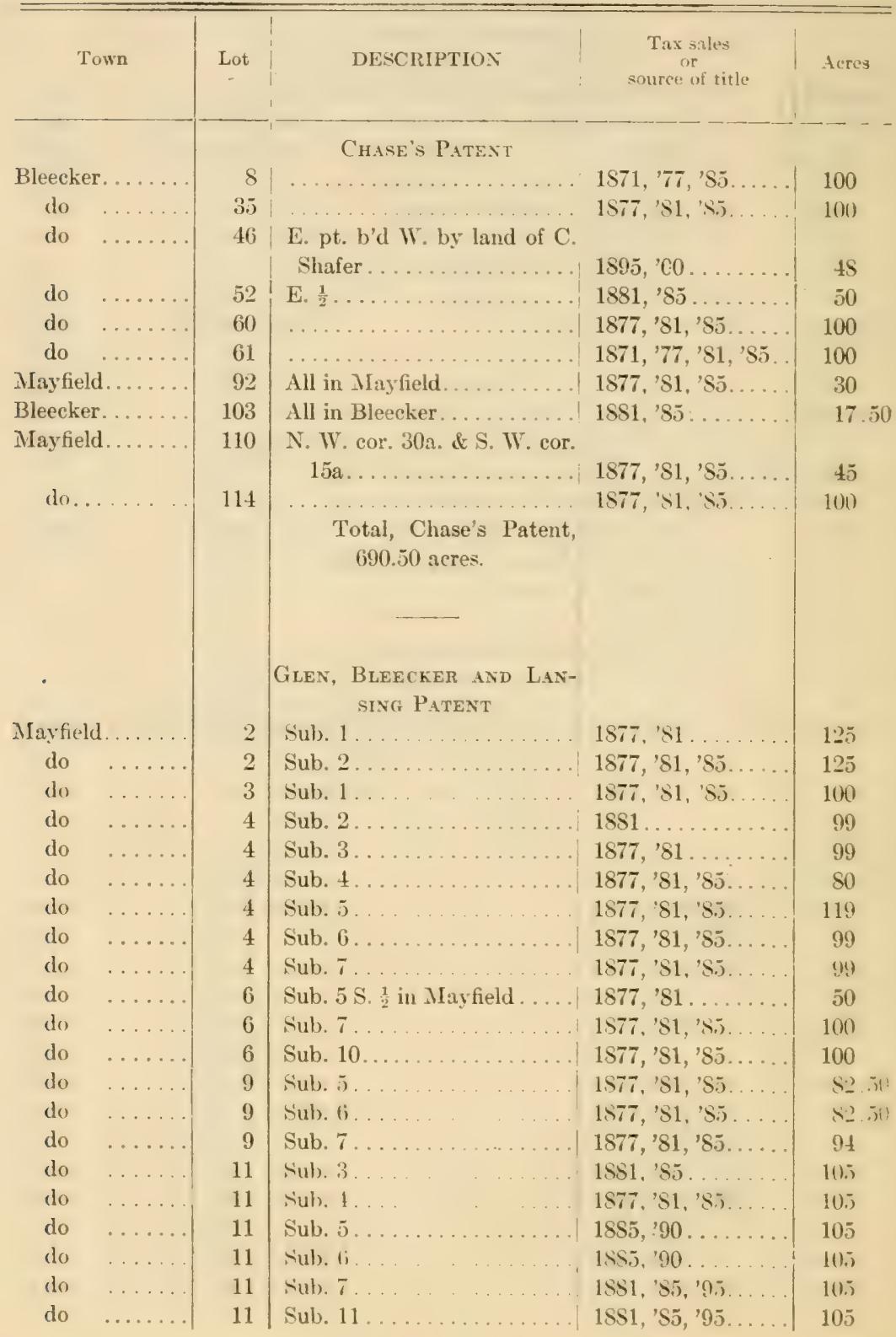




\section{Fulton County}

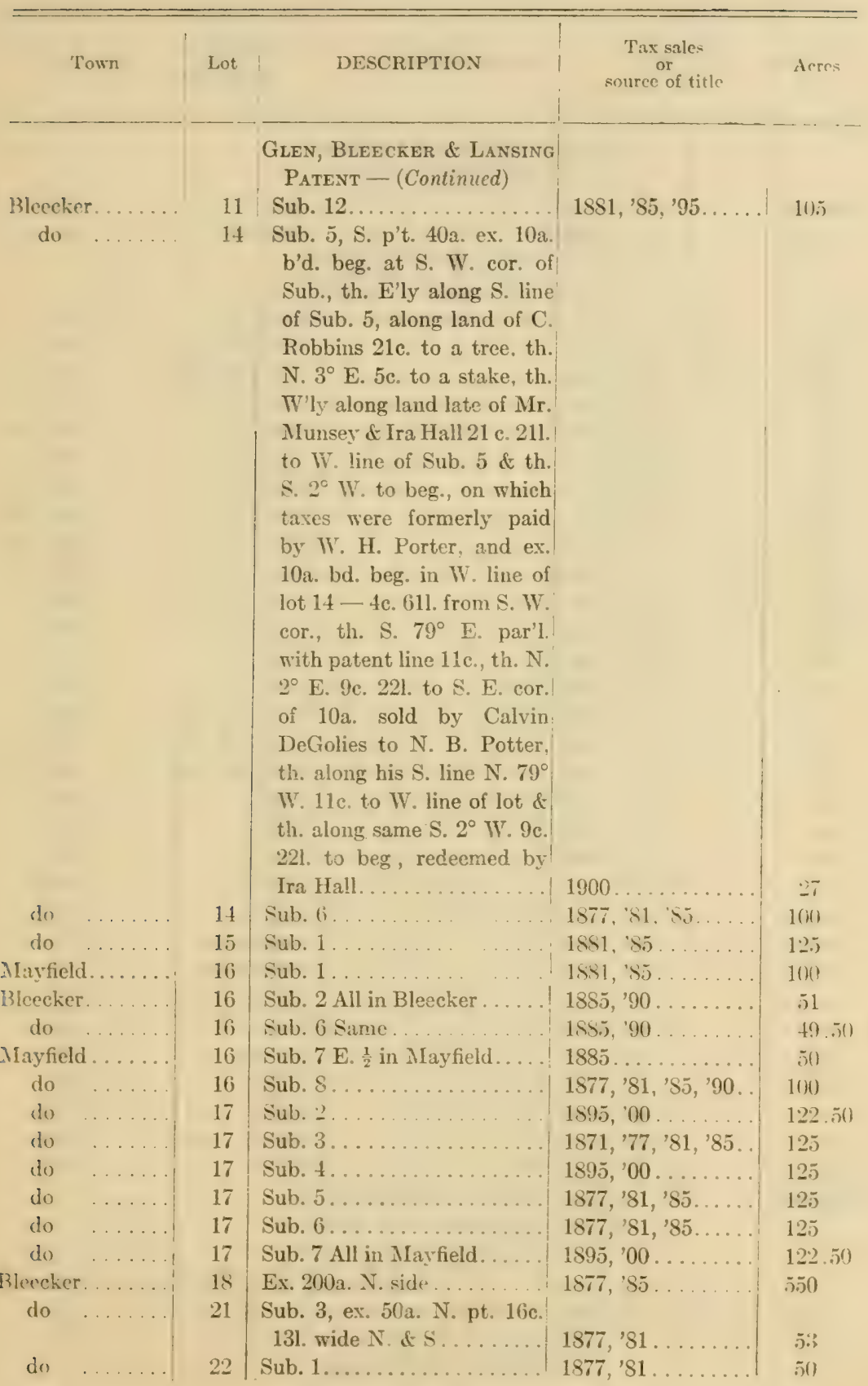




\section{Fulton County}

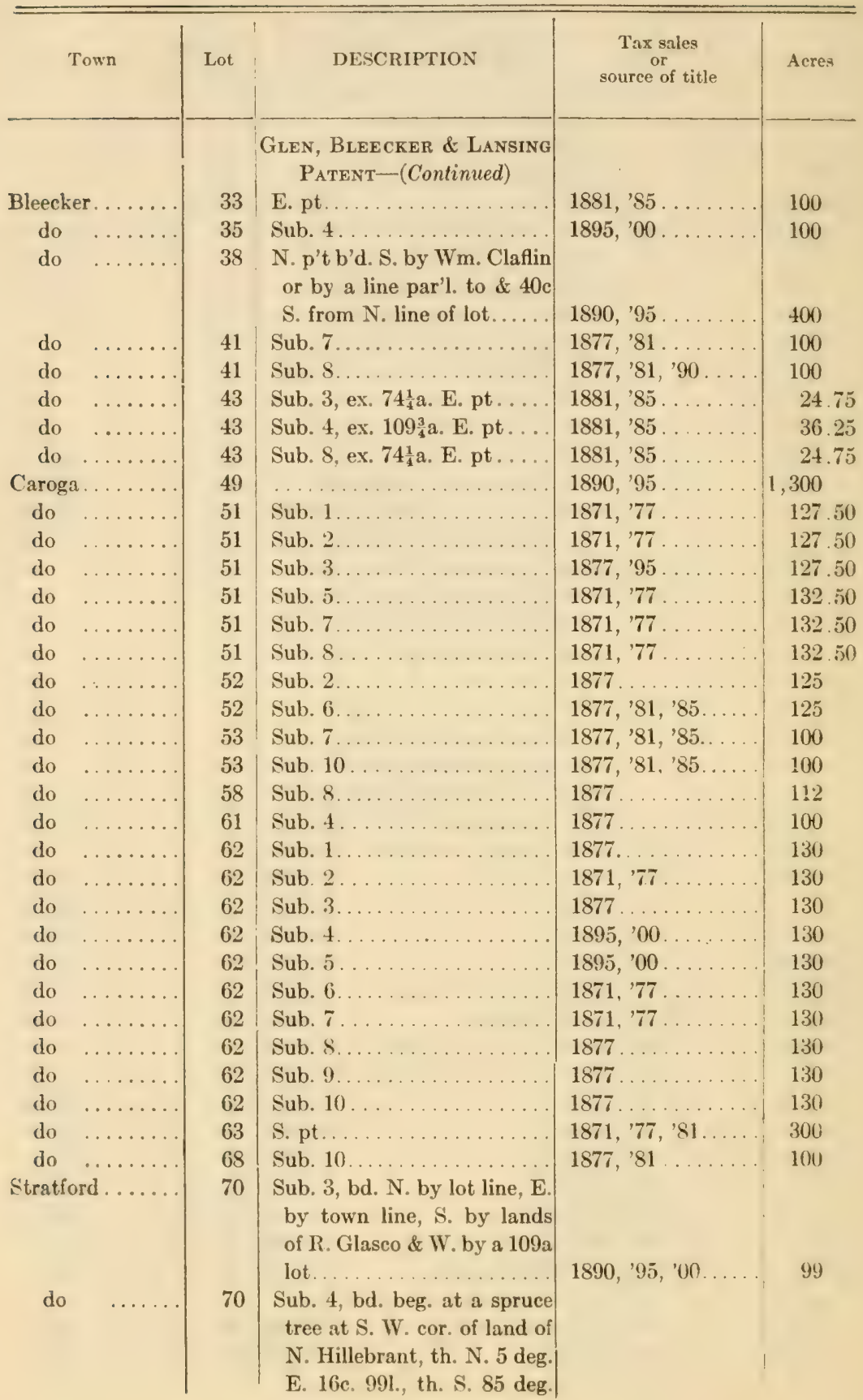


Conservation Commission.

Fulton County

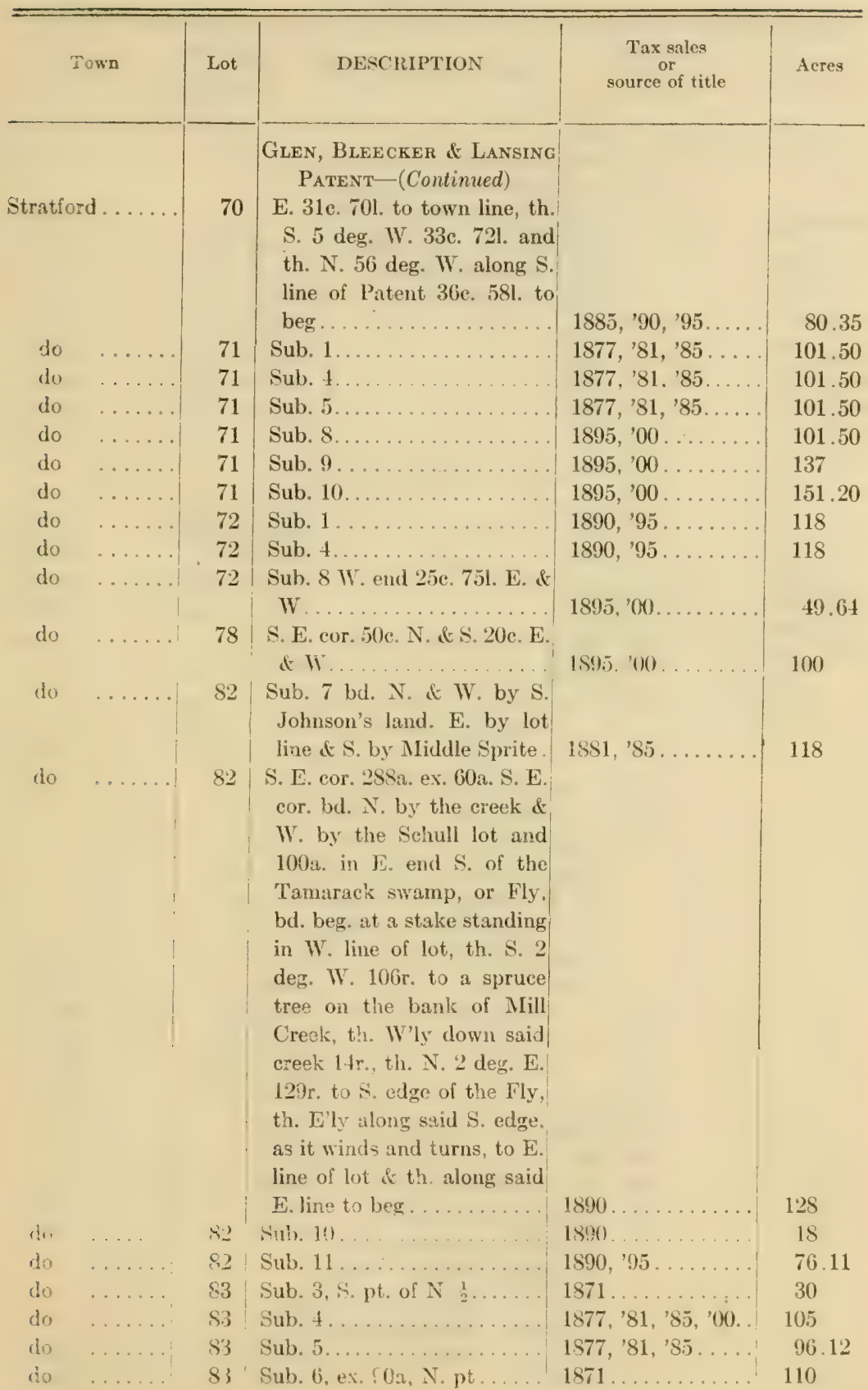


Fulton Countr

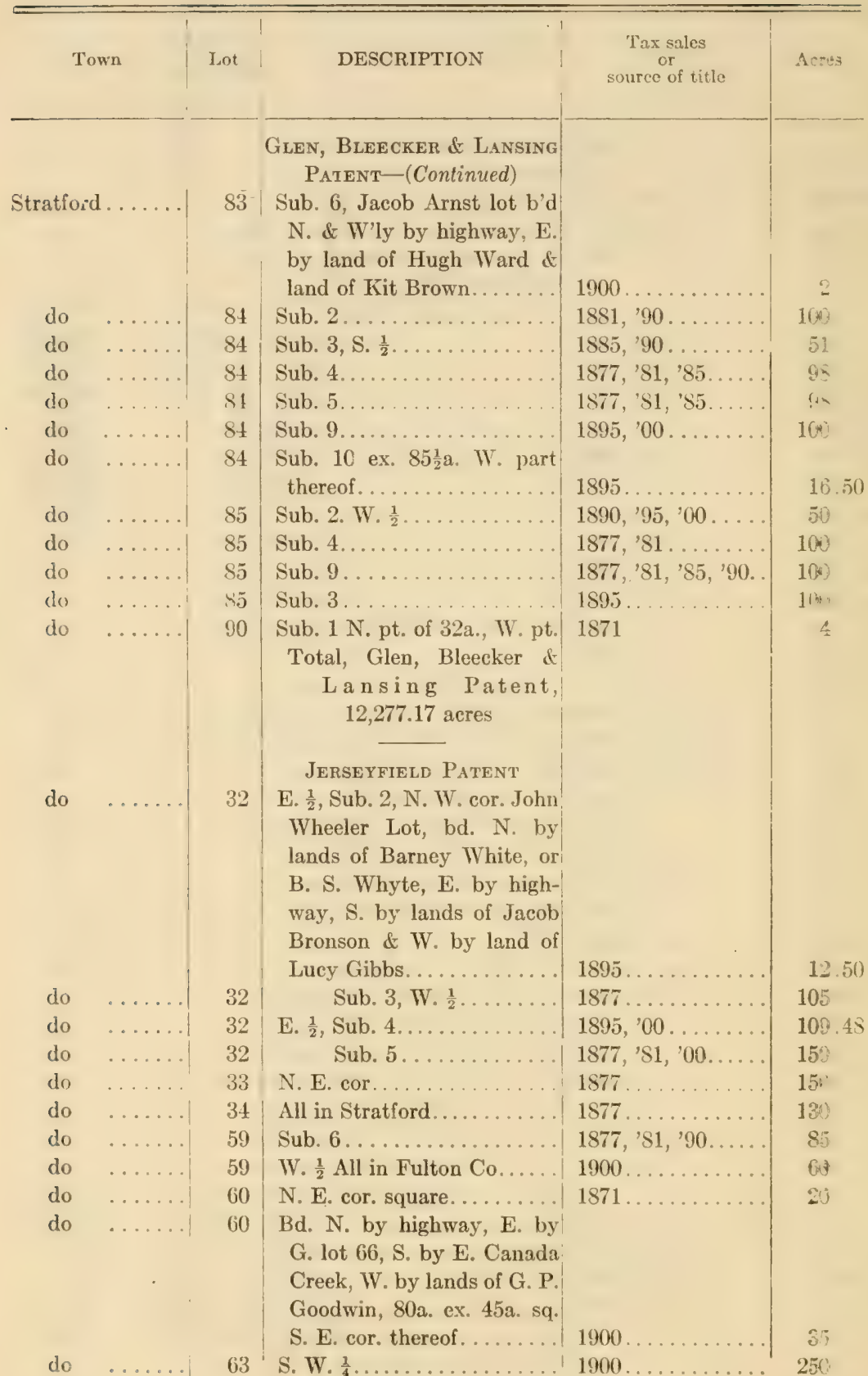




\section{Fulton County}

\begin{tabular}{|c|c|c|c|c|}
\hline Town & Lot & DESCRIPTION & $\begin{array}{c}\text { Tax sales } \\
\text { or } \\
\text { source of title }\end{array}$ & Acres \\
\hline \multirow[b]{2}{*}{ Stratford } & & $\begin{array}{l}\text { Jerseyfield Patent - } \\
\text { (Continued })\end{array}$ & & \\
\hline & 63 & E. $\frac{1}{2} \ldots \ldots \ldots \ldots \ldots \ldots$ & $1900,{ }^{\prime} 05 \ldots$ & 500 \\
\hline do & 64 & Sub. 1, Peck lot.......... & $1900 \ldots \ldots$ & 106.20 \\
\hline do & 64 & Sub. $2 \ldots \ldots \ldots \ldots \ldots$ & $1877, ' 81 \ldots$ & 106.20 \\
\hline do & 64 & Sub. $3 \ldots \ldots \ldots \ldots$ & $1877, ' 81$. & 106.20 \\
\hline do & 64 & Sub. $4 \ldots \ldots$ & 1900. & 106 \\
\hline do & 64 & Sub. $5 \ldots \ldots \ldots \ldots$ & 1900 & 106 \\
\hline do & 66 & $W . \frac{1}{2} \ldots \ldots \ldots \ldots \ldots \ldots$ & $1877, ' 81, ' 85 \ldots$ & 525 \\
\hline do & 66 & S. E. $\frac{1}{1} \ldots \ldots \ldots \ldots \ldots$ & $1895,{ }^{\prime} 00 \ldots \ldots$ & 262.50 \\
\hline do & 67 & $\begin{array}{c}\text { All in Fulton Co., 934a., ex. } \\
40 \text { a. N. E. cor. thereof..... }\end{array}$ & $1877, ' 81, ' 85 \ldots$ & \\
\hline do & 68 & All in Stratford ........... & $1900,05 \ldots$ & 135 \\
\hline do & 88 & $\begin{array}{l}\text { All in Stratford, 365a., ex. } \\
100 a ., \text { N. E. cor. thereof... }\end{array}$ & $1871, ' 77, ' 81$. & 265 \\
\hline do & 90 & $N, W . \frac{1}{4} \ldots \ldots \ldots \ldots \ldots$ & $1871, ' 77, ' 81$. & 262 \\
\hline do & 91 & $\ldots \ldots \ldots \ldots \ldots \ldots \ldots$ & $1971,77,91 \ldots$ & 1,000 \\
\hline do & 92 & $\ldots \ldots \ldots \ldots$ & $1585 . \ldots \ldots$ & 1,000 \\
\hline do & 93 & $\ldots \ldots \ldots \ldots \ldots \ldots$ & $1885,90$. & 1,080 \\
\hline do & 94 & "N." ${ }^{\prime} \ldots \ldots \ldots \ldots \ldots \ldots$ & 1900. '05. . & 821 \\
\hline do & 95 & $\begin{array}{l}\text { "West" Old Porter Place, } \\
\text { bd. N. by lands of Powley } \\
\text { or Livingston, E'ly by } \\
\text { Ayers or Cayers. Creek, } \\
\text { W'ly by lands of Shaad, } \\
\text { being a triangle.......... }\end{array}$ & $1900,{ }^{\prime} 05 \ldots$ & $\frac{1}{2}$ \\
\hline do & 95 & $\begin{array}{l}\text { Bd. N. by W. } \frac{1}{2} \text { of lot } 62, \mathrm{E} . \\
\text { by lands of A. A. Bullock } \\
\text { \& Wm. Doxtater or Dox- } \\
\text { sader, S. by land of John } \\
\text { Keller or Nora or Norah } \\
\text { Bracey or J. Keck, \& W. } \\
\text { by land of Parmeliah Newell } \\
\text { \& Martin Bracey or Nora } \\
\text { Brocey \& Jason Hall. ..... } \\
\text { Total, Jerseyfield Patent, } \\
\text { 8,516.58 acres. }\end{array}$ & $1890,95 \ldots$ & 125 \\
\hline Johnstow & 516 & $\begin{array}{l}\text { Kingsboro Patent, } \\
\text { W'Ly Allotment } \\
\text { N'ly pt. J. Wiley lot.... }\end{array}$ & Mortgage, 1830. & 25 \\
\hline
\end{tabular}




\section{Fulton County}

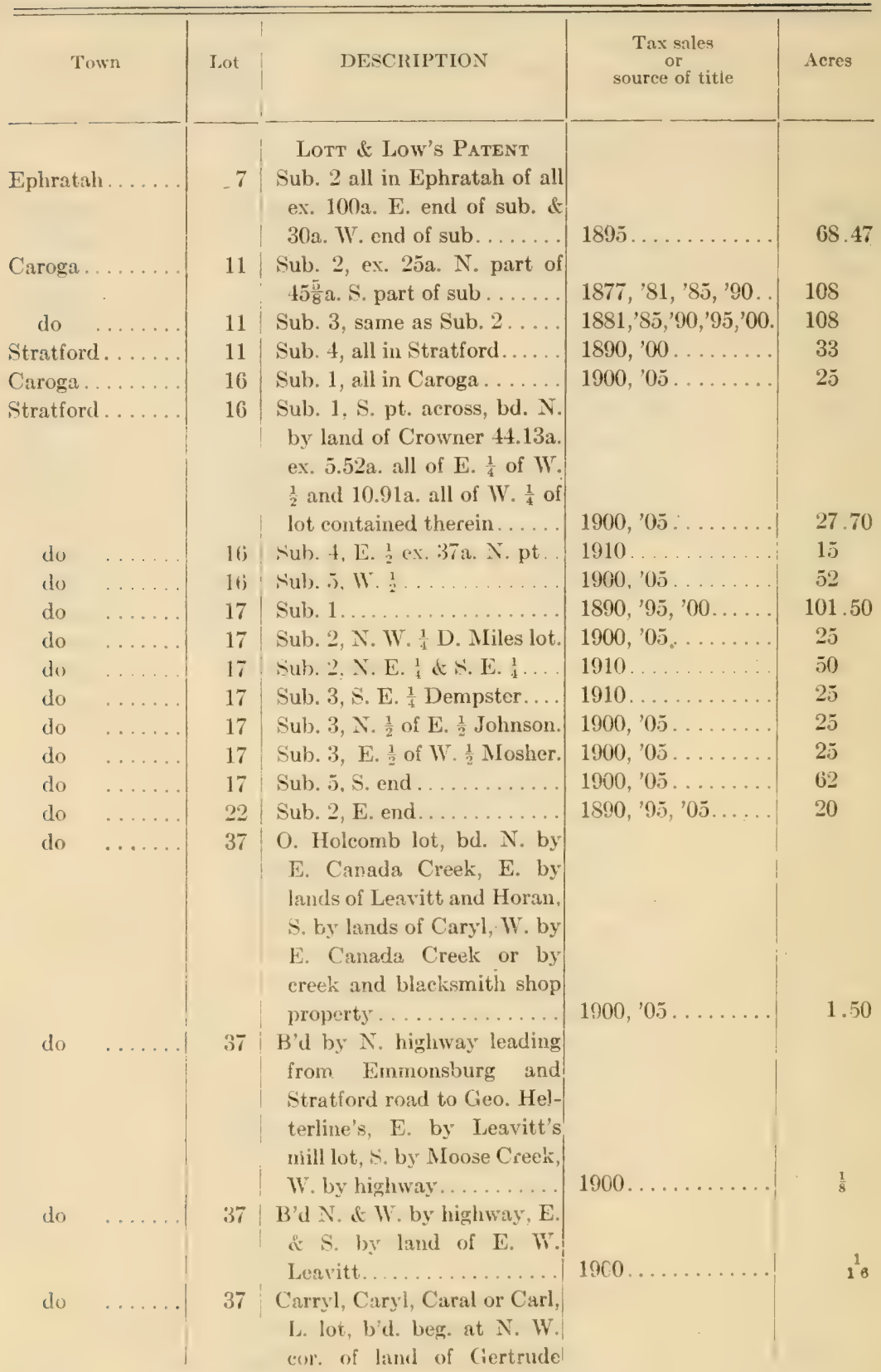




\section{Fulton Countr}

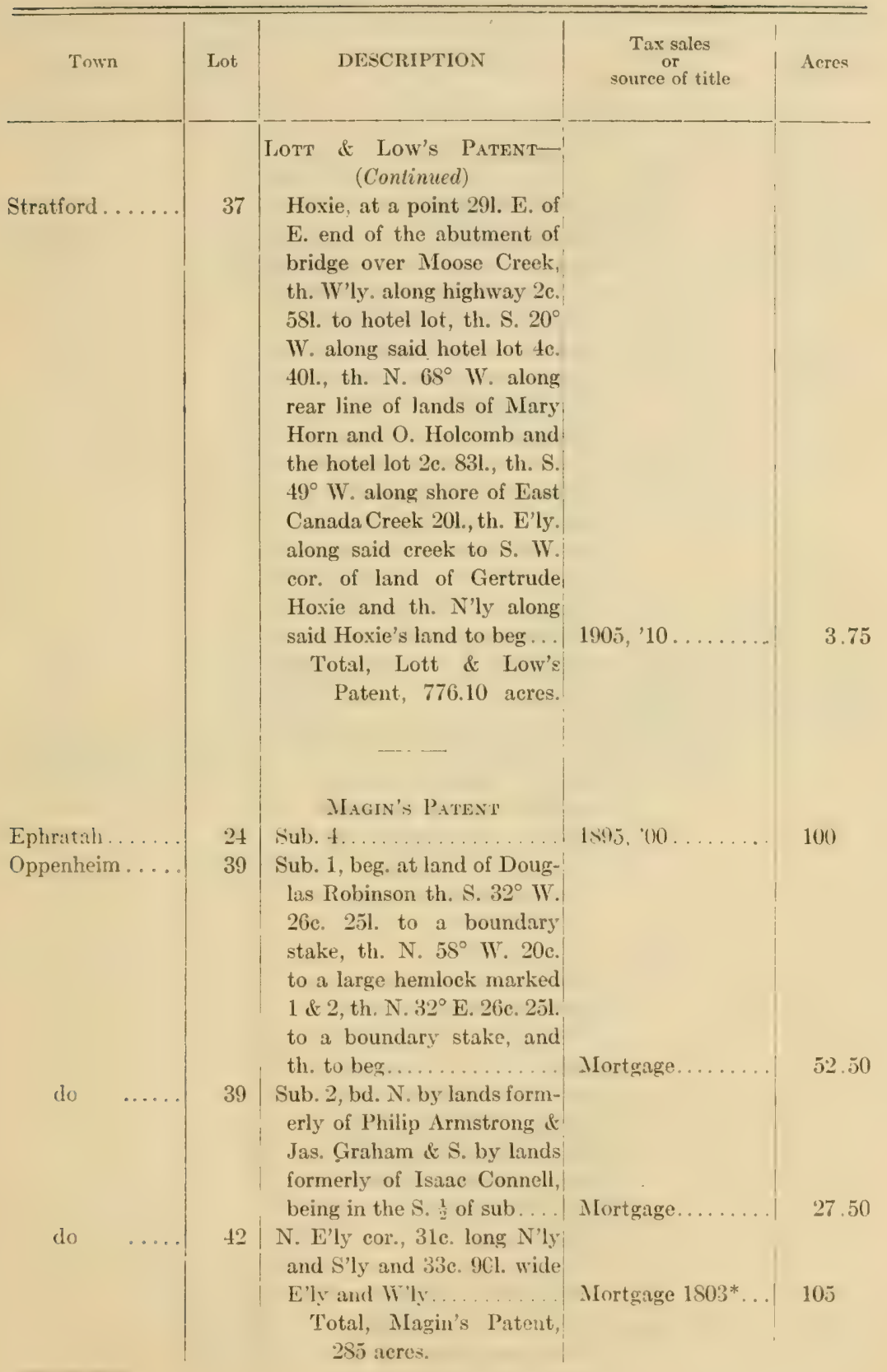


Fulton Country

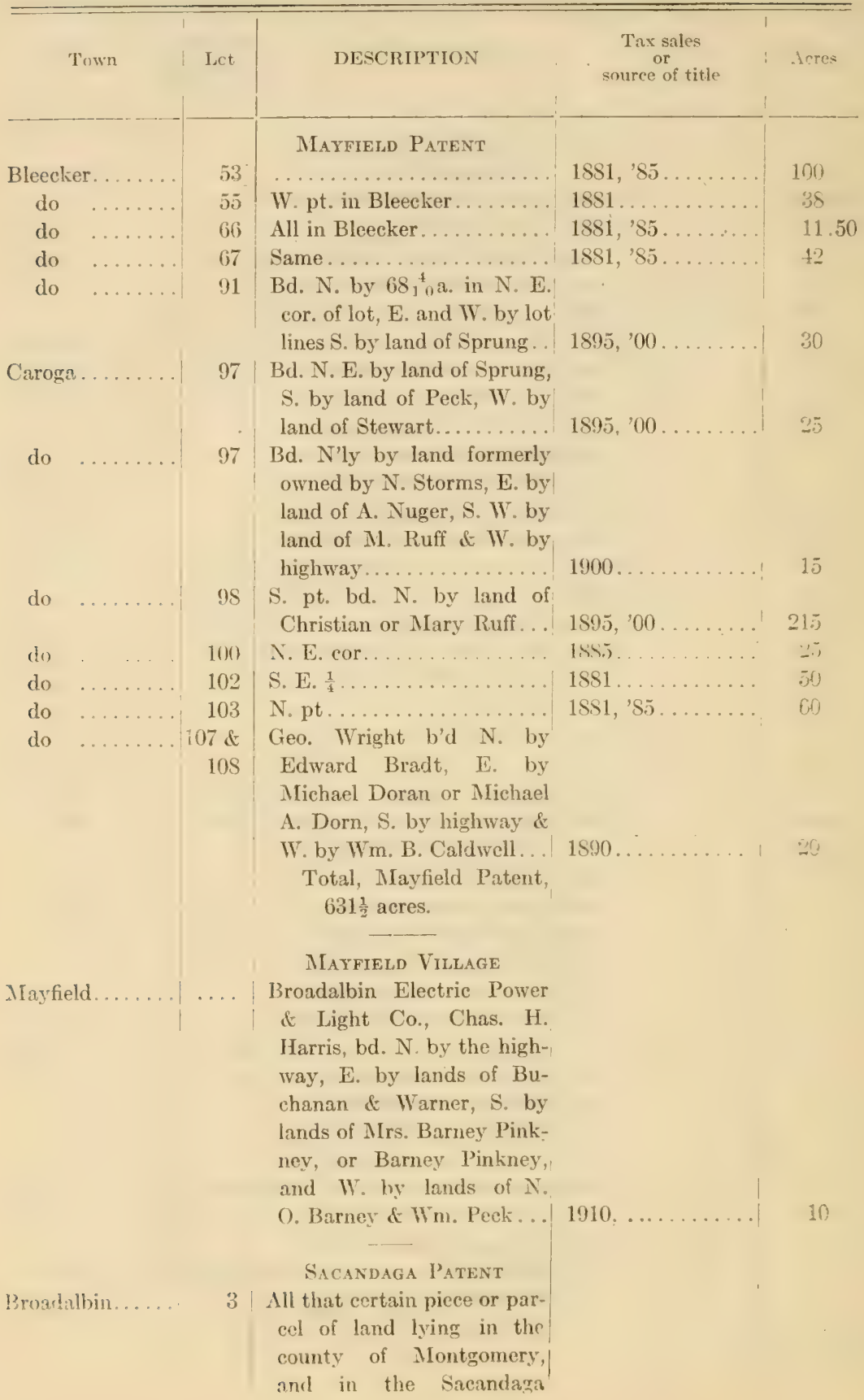


Conservition Commssiox.

Fulton County

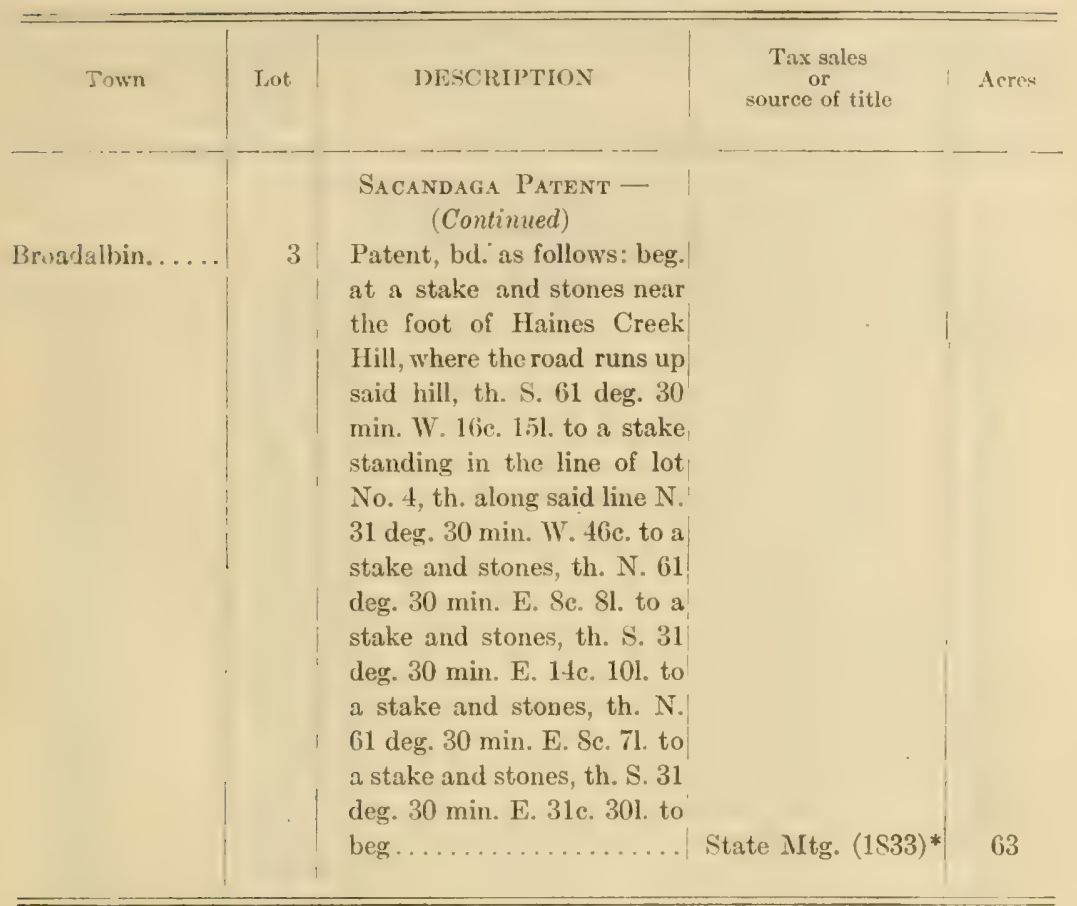

* Resule.

For other State land in Fulton County, Ses Appondix. 


\section{HAMILTON COUNTY}

(Total number of acres, 625,655.66.)

\begin{tabular}{|c|c|c|c|c|}
\hline Town & \multirow[t]{2}{*}{ Lot } & DESCIRIPTION & $\begin{array}{c}\text { Tax sales } \\
\text { or }\end{array}$ & Aeres \\
\hline & & $\begin{array}{c}\text { Arthurboro PAtent, } \\
\text { North }{ }_{2}^{-1}\end{array}$ & & \\
\hline Morehouse. & 1 & & Purchase .... . & 200 \\
\hline do & 2 & $\ldots \ldots$ & Purchase. . . . . . & 200 \\
\hline do & 3 & $\ldots \ldots \ldots \ldots \ldots \ldots \ldots$ & $1895,{ }^{\prime} 00 \ldots$ & 200 \\
\hline do & 4 & $\ldots \ldots \ldots \ldots \ldots \ldots \ldots$ & $1895,{ }^{\prime} 00 \ldots \ldots \ldots$ & 200 \\
\hline do & 5 & $\ldots \ldots \ldots \ldots \ldots \ldots \ldots$ & $1895,00 \ldots \ldots \ldots$ & $2(0)$ \\
\hline do & 7 & $\ldots \ldots \ldots \ldots \ldots \ldots$ & Purchase..... . & $20)$ \\
\hline do & 8 & $\ldots \ldots \ldots \ldots \ldots \ldots$ & Purchase........ & 200 \\
\hline do & 13 & $\ldots \ldots \ldots \ldots \ldots \ldots \ldots$ & Purchase........ & 200 \\
\hline do & 15 & & Purchase . . . . . . & 200 \\
\hline do & 16 & $\ldots \ldots$ & Purchase ........ & 200 \\
\hline do & 17 & $\ldots \ldots$ & Purchase . . . . . & 200 \\
\hline do & 19 & $\ldots \ldots \ldots \ldots \ldots$ & Purchase........ & 200 \\
\hline do & 21 & $\ldots \ldots \ldots \ldots \ldots$ & Purchase ........ & 200 \\
\hline do & 22 & $\ldots \ldots \ldots \ldots \ldots$ & Purchase...... . & $2(x)$ \\
\hline do & 23 & $\ldots \ldots \ldots \ldots \ldots \ldots$ & Purchase......... & $2(\theta)$ \\
\hline do & 24 & & Purchase........ & 200 \\
\hline do & 25 & & Purchase........ & 200 \\
\hline do & 26 & . & Purchase ........ & 200 \\
\hline do & 27 & . & $\begin{array}{r}1877, ' \text { 's1 and Con- } \\
\text { veyed. . . . . . . }\end{array}$ & 200 \\
\hline do & 28 & & $\begin{array}{r}1877,91, ' 85 \text { and } \\
\text { Conveyed } \ldots . .\end{array}$ & 200 \\
\hline do & 29 & & Purchase........ & $2(x)$ \\
\hline do & 30 & & Purchase . . . . . . . & 200 \\
\hline do & 31 & & Purchase ........ & 200 \\
\hline do & 32 & . & Purchase... & 200 \\
\hline do & 33 & & $1877,81, \$ .7$. & $\ddot{3}(0)$ \\
\hline do & 34 & & Purchase........ & 200 \\
\hline do & 35 & & P'urchase ........ & 200 \\
\hline do & 36 & & $1877, ' 81, ' 85 \ldots$ & $20(0)$ \\
\hline do & 37 & & Purchase........ & 200 \\
\hline do & 38 & Ex. 30a. S. W. pt.... & $1881 \ldots \ldots \ldots \ldots$ & 1711 \\
\hline do & 39 & & Purchase........ & $2(k)$ \\
\hline do & 41 & & $\begin{array}{l}\text { 1877, ' } 81, \text { ' } 85 \text { and } \\
\text { Conveyed...... }\end{array}$ & $2(x)$ \\
\hline do & 42 & & Purehase........ & 200 \\
\hline do & 46 & & Purchase....... & 200 \\
\hline do & 47 & & $1.877,81,85 \ldots$ & 200 \\
\hline do & 48 & & $1877, ' 81, ' 85 \ldots$ & 200 \\
\hline do & 49 & & $1 \times 77,81, ' 85$. & 200 \\
\hline do & 50 & & $1877,81.85$ & 200 \\
\hline
\end{tabular}


Conservation Commeston.

Hamilton Countr

\begin{tabular}{|c|c|c|c|c|}
\hline Town & Lot & DESCRIPTION & $\begin{array}{c}\text { Tax sales } \\
\text { or } \\
\text { source of title }\end{array}$ & Arres \\
\hline & & $\begin{array}{c}\text { Arthurboro P A T E N T- } \\
\text { North } \frac{1}{2}-\text { (Continued) }\end{array}$ & & \\
\hline Morehouse. & 51 & $\ldots \ldots \ldots \ldots \ldots$ & $1877, ' 81, ' 85$. & 200 \\
\hline do & 59 & & Purchase. . & $2(16)$ \\
\hline do & 62 & $\ldots \ldots \ldots \ldots$ & $1877,81,85$. & $20(1)$ \\
\hline do & 63 & $\ldots \ldots \ldots \ldots \ldots \ldots \ldots$ & $1877 . ' 81,85 \ldots$ & 2001 \\
\hline do & 64 & & $\begin{array}{c}\text { Purchase \& 1900, } \\
05 \ldots \ldots \ldots \ldots\end{array}$ & 2011 \\
\hline do & 65 & & $1877, ' 81,85,90$. & $20 \times 1$ \\
\hline do & 66 & N. $\frac{1}{2} \ldots$ & $1877,81, \$ 5 . \ldots$ & $10)$ \\
\hline do & 66 & S. $\frac{1}{2} \ldots \ldots \ldots$ & Purchase . .... & $10(1$ \\
\hline do & 75 & $\ldots \ldots \ldots \ldots \ldots \ldots \ldots$ & Purchase. . & 200 \\
\hline do & 76 & $\ldots \ldots \ldots$ & $1577,81,85 \ldots$ & 2010 \\
\hline do & 77 & & $\begin{array}{c}\text { Purchase \& 1900, } \\
05 \ldots \ldots \ldots \ldots\end{array}$ & $201)$ \\
\hline do & 78 & & $\begin{array}{c}\text { Purchase \& 1900, } \\
\text { '05..... }\end{array}$ & 200 \\
\hline do & 79 & ...... & $1877, ' 81$, ' $85 \ldots \ldots$ & 200 \\
\hline do & 80 & $\ldots \ldots$ & Purchase. ...... & 200 \\
\hline do & 81 & $\cdots$ & Purchase.... . & 200 \\
\hline do & $\$ 3$ & $\ldots \ldots \ldots \ldots \ldots \ldots$ & $1877,81,85 . \ldots$ & 200 \\
\hline do & 85 & $\ldots$ & $\begin{array}{c}\text { Purchase \& 1900, } \\
05 \ldots \ldots \ldots\end{array}$ & 200 \\
\hline do & 86 & & $\begin{array}{c}\text { Purchase \& 1900, } \\
\text { (0;) . . . . . . }\end{array}$ & 200 \\
\hline do & 89 & & $\begin{array}{l}1877, \quad 81,85 \text { and } \\
\text { conveyed....... }\end{array}$ & 200 \\
\hline do & 90 & & $\begin{array}{l}1877, \text { ' } 81, \text { ' } 85 \text { and' } \\
\text { conveyed....... }\end{array}$ & 200 \\
\hline do & 91 & & $\begin{array}{c}\text { Purchase \& 1900 } \\
\text { (1.) . . . . . . . }\end{array}$ & 200 \\
\hline do & 92 & .. & $\begin{array}{r}1877, \quad 81, \quad 85 \\
\text { conveyed....... }\end{array}$ & 200 \\
\hline do & 95 & $\ldots \ldots \ldots \ldots$ & $\begin{array}{l}1877, \text { ' } 81, \text { '85 \& } \\
\text { conveyed. ...... }\end{array}$ & $2(x)$ \\
\hline do & 96 & & $\begin{array}{c}\text { Purchase \& 1900, } \\
05 \ldots . . . \ldots \ldots\end{array}$ & 200 \\
\hline do & 97 & $\ldots \ldots \ldots \ldots$ & $1843, ' 85,{ }^{\prime} 90^{*} \ldots$. & 200 \\
\hline do & 99 & & Conveyed. & 200 \\
\hline do & 100 & & Conveyed.... & 231 \\
\hline do & 101 & $\begin{array}{c}\text { Total, } \\
\text { acres. }\end{array}$ & Conveyed. & 231 \\
\hline
\end{tabular}

* Resale. 
Hamiton County

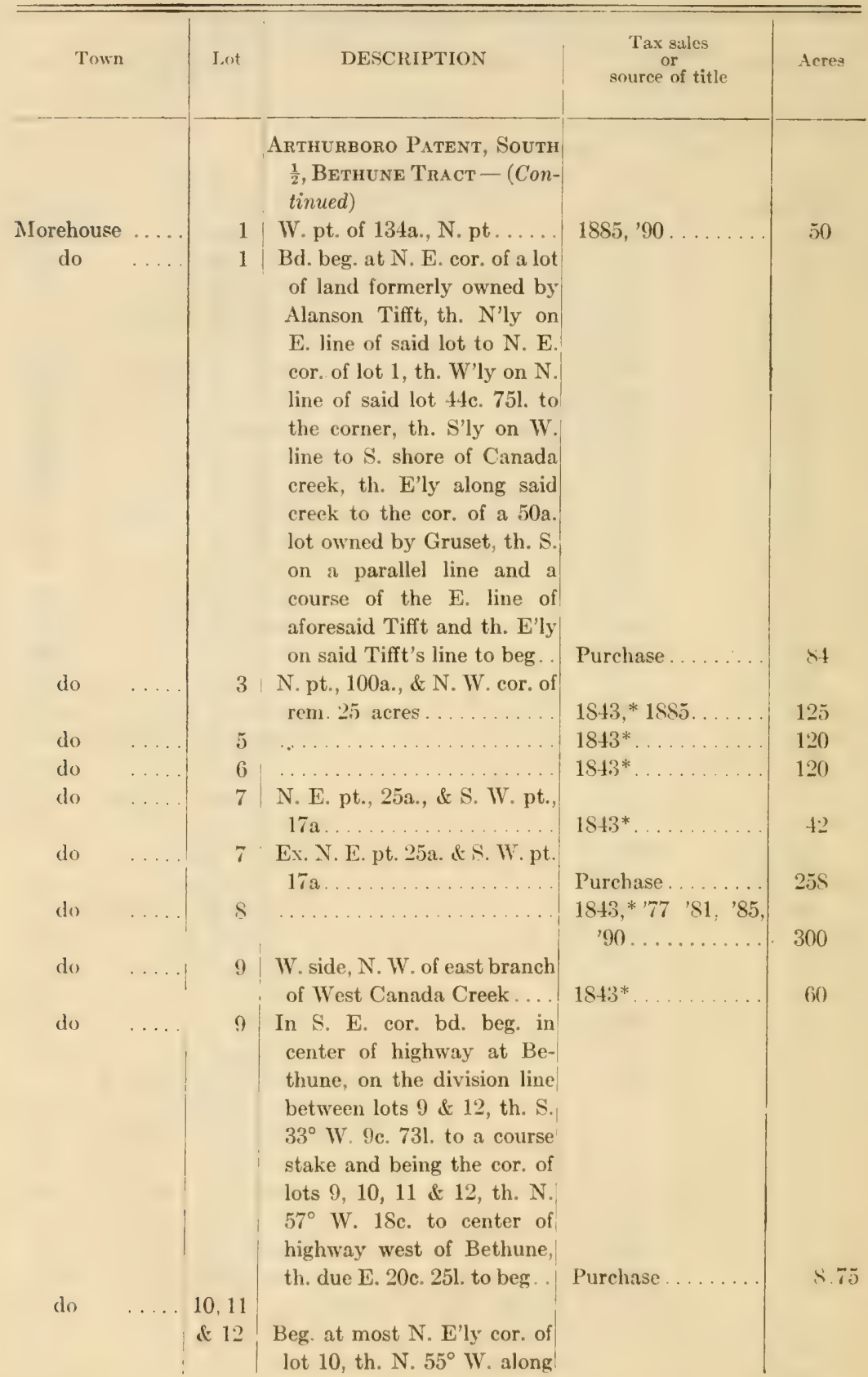


Conservation Commission.

Hamilon County

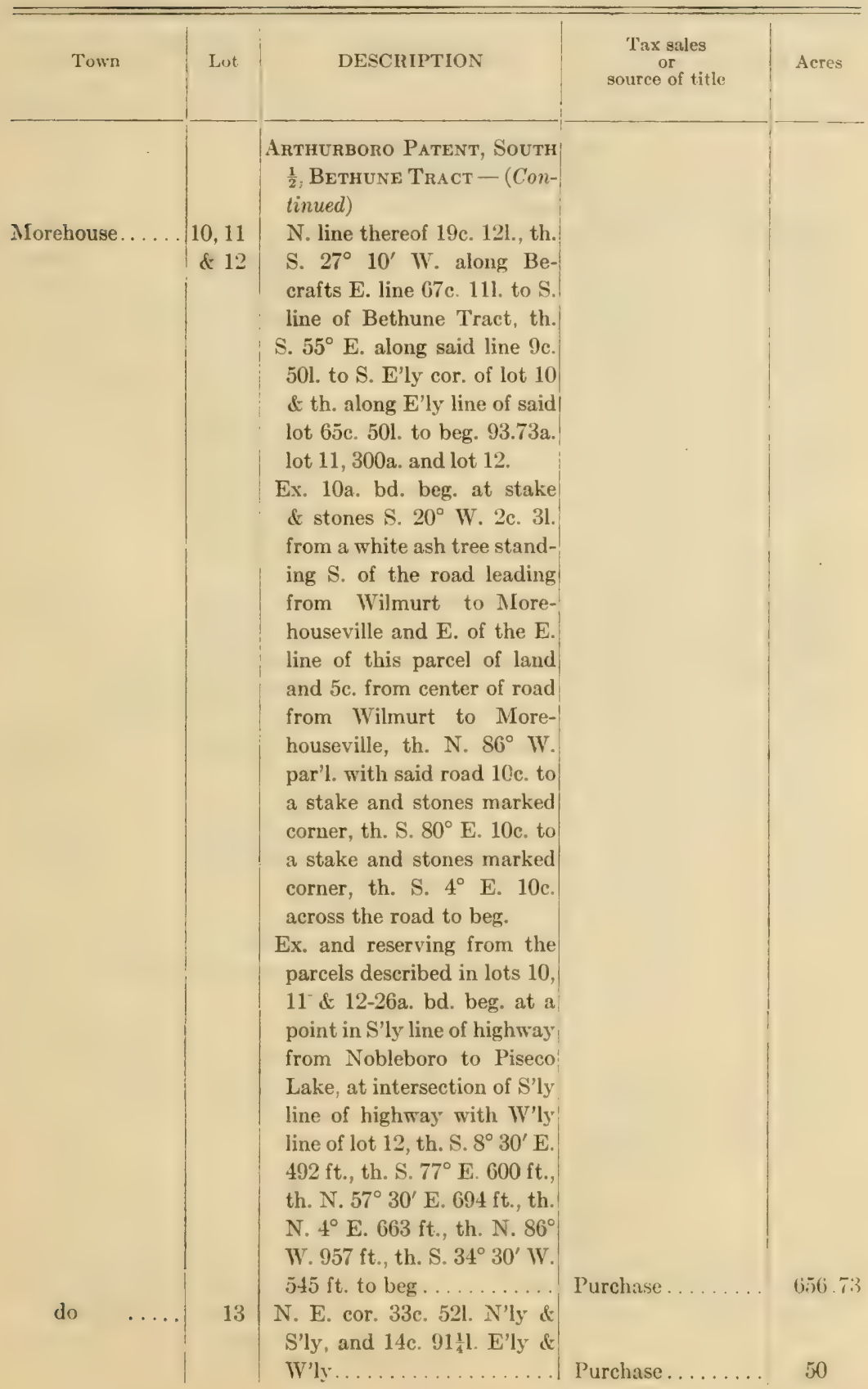




\section{Hamiton County}

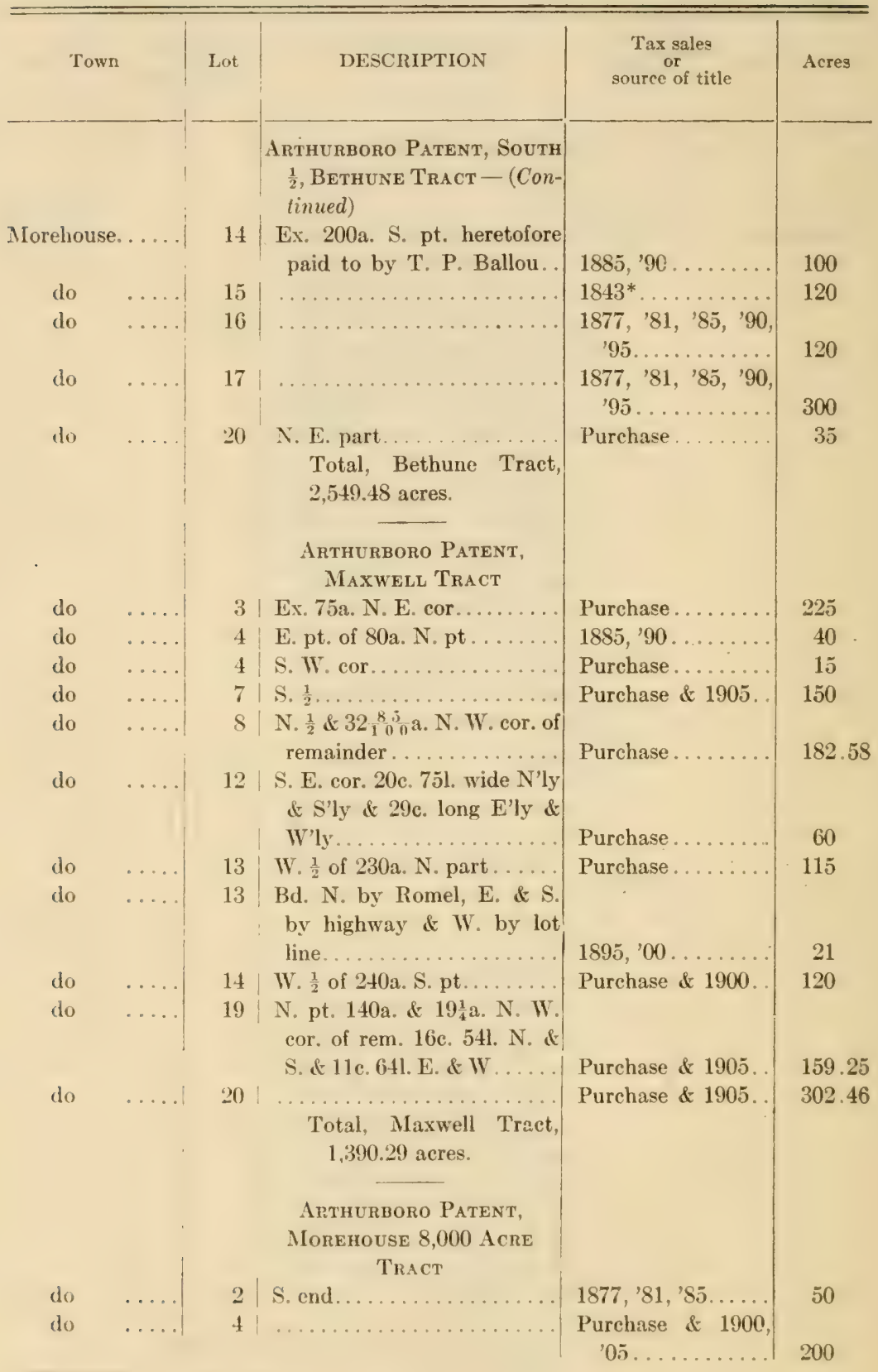




\section{Hamiton County}

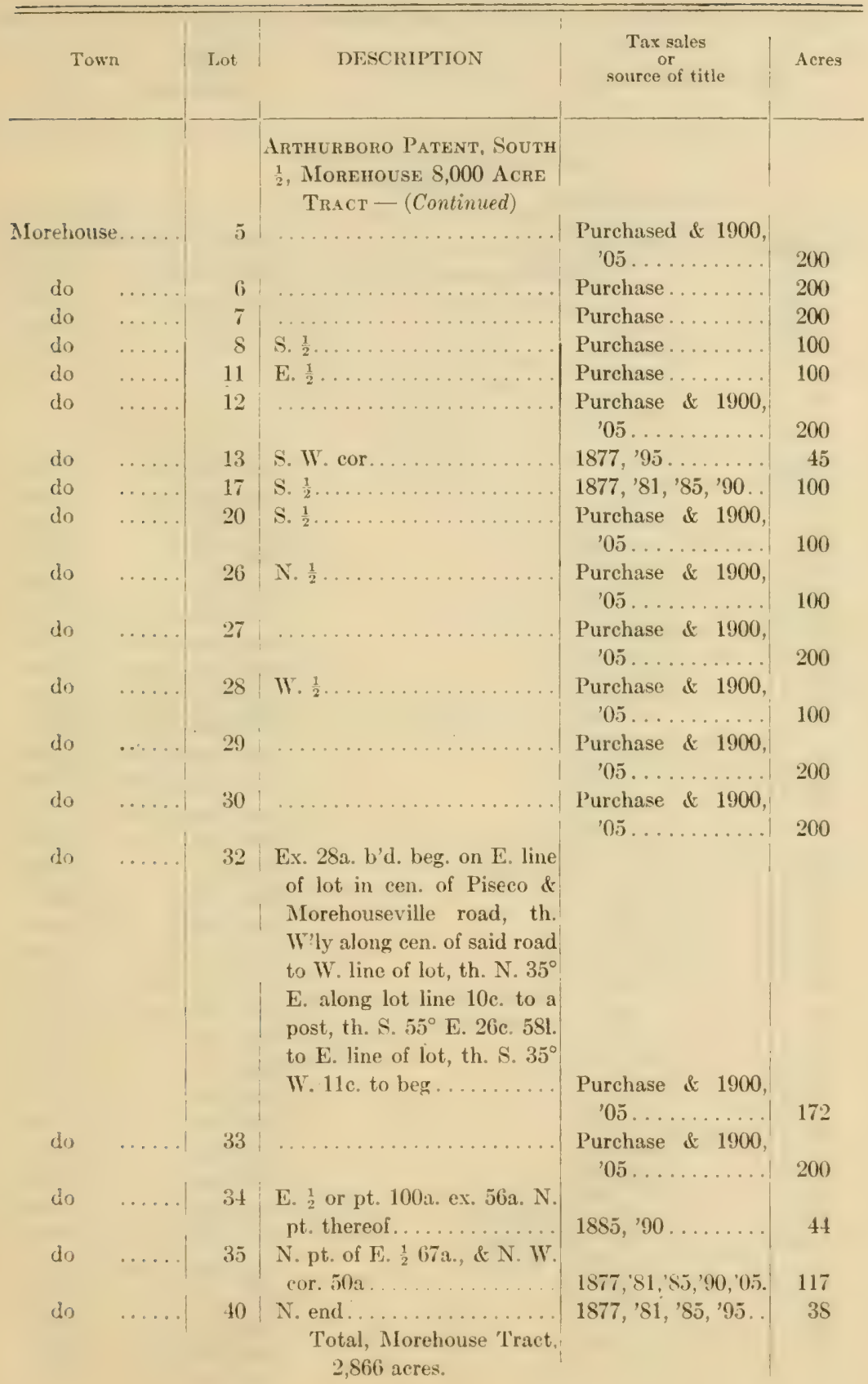


Hamilton County

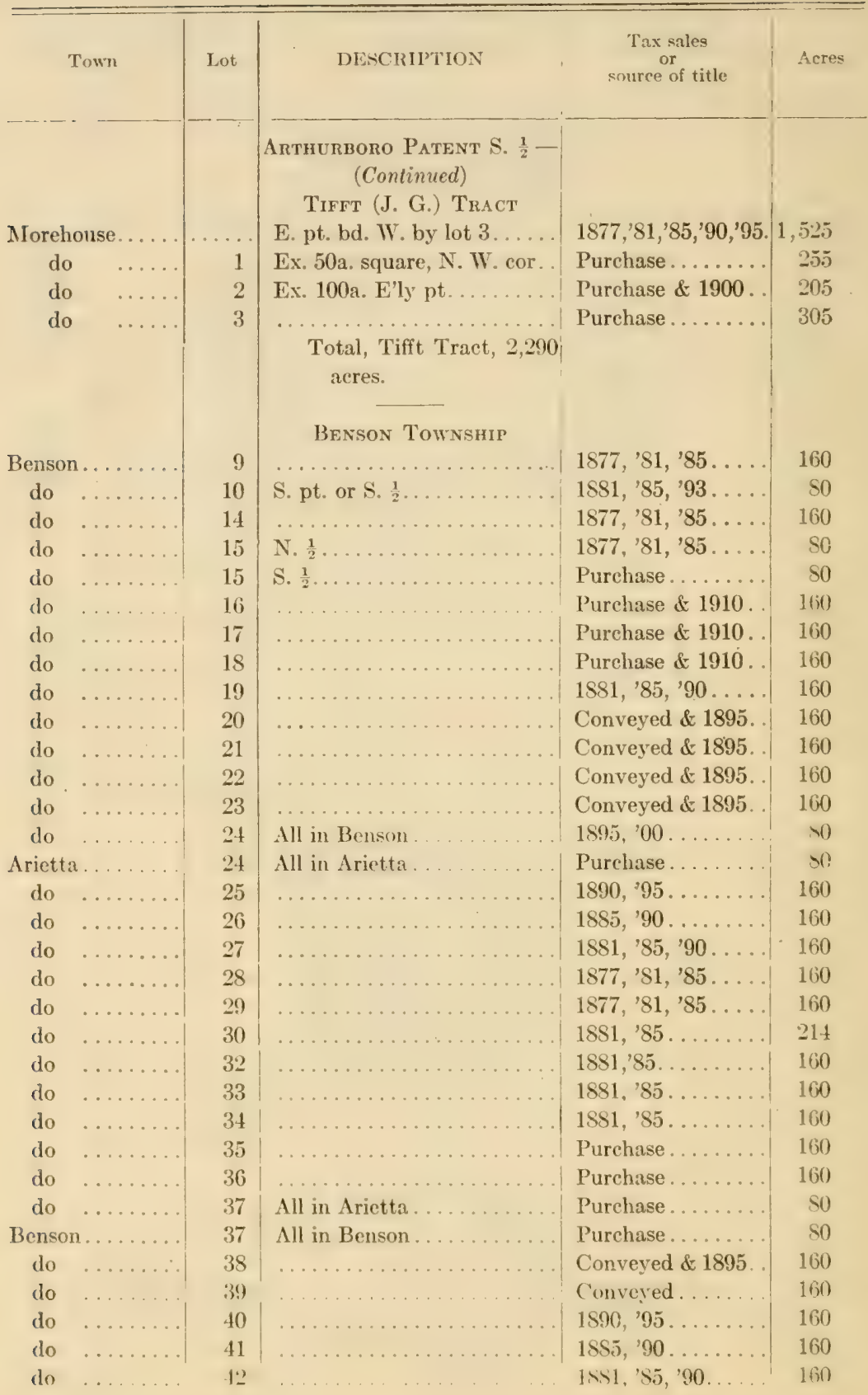


Hamilton Country

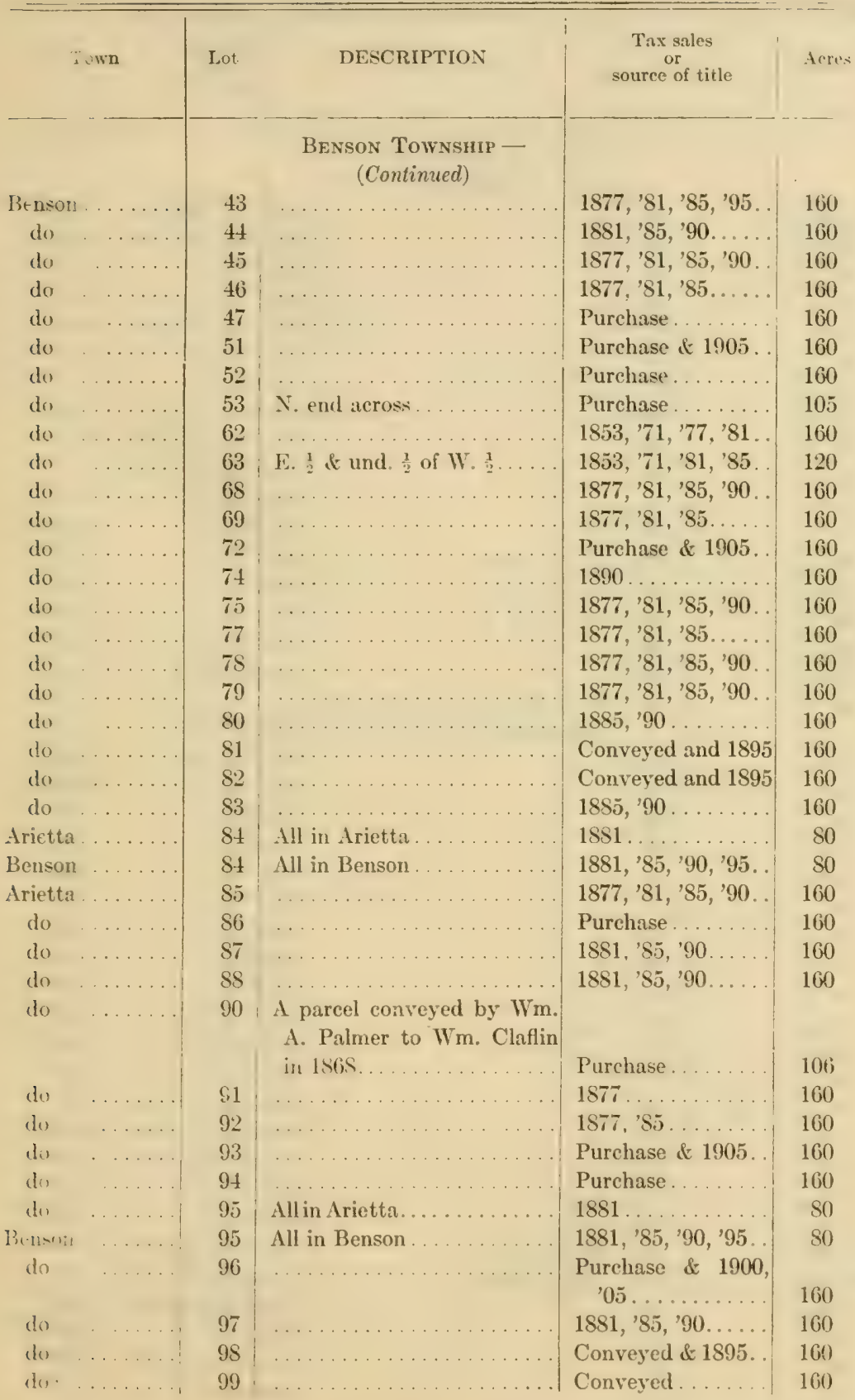


Hamiton County

\begin{tabular}{|c|c|c|c|c|}
\hline To & Lot & DESCRIITTION & $\begin{array}{c}\text { Tax sales } \\
\text { or } \\
\text { source of title }\end{array}$ & Acres \\
\hline & $=$ & $\begin{array}{c}\text { Benson Township - } \\
\text { (Continued) }\end{array}$ & & \\
\hline Benson. & 100 & $\ldots \ldots \ldots \ldots \ldots \ldots \ldots$ & 1877 , '81, '85, '90. & 160 \\
\hline do & 101 & $\ldots \ldots \ldots \ldots \ldots \ldots \ldots$ & 1877, ' $81, ' 85, ' 90$. & 169) \\
\hline do & 102 & & $1877, ' 81,85 \ldots$ & 160 \\
\hline do & 103 & & $1885,90 \ldots \ldots$ & 160 \\
\hline do & 104 & & $1877, ' 81, ' 85, ' 90$. & $16 n$ \\
\hline do. & 106 & $\ldots \ldots \ldots$ & $1885,90,{ }^{\prime} 95 \ldots .$. & 16() \\
\hline do & 107 & & Purchase....... & 160 \\
\hline do. & 109 & & Purchase.... & 160 \\
\hline do . & 110 & . & $1877, ' 81, ' 85, ' 90$. & 160 \\
\hline do & 111 & & $\begin{array}{c}\text { Purchase \& 1885, } \\
' 90,10 \ldots \ldots \ldots\end{array}$ & 160 \\
\hline do & 112 & & Purchase. & 160 \\
\hline do. & 113 & W. pt. across... & $1890,{ }^{\prime} 95 \ldots$ & 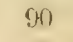 \\
\hline do & 116 & & Purchase ... & 160 \\
\hline do & 117 & Ex. 70a.s. pt...... & $1885,{ }^{\prime} 90$. & $11 \frac{1}{2}$ \\
\hline do & 118 & & $1853, ' 81,85$. & 24 \\
\hline do & 119 & $\ldots \ldots \ldots \ldots \ldots \ldots \ldots$ & Conveyed...... & 160 \\
\hline do & 120 & $\begin{array}{l}\text { N. pt. 40a. ex. und. } \frac{1}{4} \text { thereof } \\
\text { Adirondack Co........... }\end{array}$ & 1877, '81, '85... & 3 \\
\hline do & 121 & & $1871,77,81 \ldots$ & 1601 \\
\hline do & 123 & & $1571,85 \ldots \ldots$ & 160 \\
\hline do & 124 & $\ldots \ldots$ & Purchase...... & 161 \\
\hline do & 125 & & Purchase.... & 164 \\
\hline do & 126 & & $1890,{ }^{\prime} 95$ & 160 \\
\hline do & 127 & 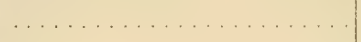 & $1885, ' 90 \ldots$ & 160 \\
\hline do & 128 & & $1885,{ }^{\prime} 90 \ldots$ & 160 \\
\hline do & 129 & . & Purchase....... & 1601 \\
\hline do & 130 & $\therefore$ & Purchase ....... & $16 n$ \\
\hline do & 131 & $\cdots$ & $1877, ' 81, ' 85,90$. & 160 \\
\hline do & 132 & $\ldots \ldots \ldots$ & $1885,{ }^{\prime} 90 \ldots \ldots$ & $16+1$ \\
\hline do & 133 & & Purchase \& 1905. & $160)$ \\
\hline do & 134 & $\cdots$ & Purchase \& 1905. & $16 n$ \\
\hline do & 135 & $\ldots \ldots \ldots$ & Purchase \& 1905. & $16 \sqrt{11}$ \\
\hline do & 136 & & Conveyed \& 1895. & 160 \\
\hline do & 137 & & $1881, ' 85,{ }^{\prime} 90 \ldots$. & 160 \\
\hline do & 138 & & Purchase... & $16 n$ \\
\hline Arietta. & 139 & All in Arietta... & Conveyed \& 1895. & (n) \\
\hline Benson. & 139 & All in Benson... & Conveyed \& 1895. & 81 \\
\hline Arietta. & 140 & $\ldots \ldots \ldots \ldots \ldots \ldots \ldots \ldots$ & Conveyed \& 1895. & 16 \\
\hline do & 141 & & Purchase....... & $16 n$ \\
\hline do & 142 & & Purchase \& 1900. & $16 n$ \\
\hline do & 14.3 & & Purchase. & $17=$ \\
\hline
\end{tabular}




\section{Hamilton County}

\begin{tabular}{|c|c|c|c|c|}
\hline & Lot & DESCRIPTION & $\begin{array}{c}\text { Tax sales } \\
\text { or } \\
\text { source of title }\end{array}$ & Acres \\
\hline & & $\begin{array}{l}\text { Benson Township - } \\
\text { (Continued })\end{array}$ & & \\
\hline Arietta . & 144 & $\ldots \ldots \ldots \ldots \ldots \ldots$ & Purchase. & 92 \\
\hline do & 145 & $\ldots \ldots \ldots$ & $1871,77, ' 81$. & 160 \\
\hline do & 146 & & Purchase ........ & 160 \\
\hline do & 147 & $\therefore$ & $1871,77,81 \ldots$. & 160 \\
\hline do & 148 & All in Arietta. . . . . . . . . & Conveyed \& 1895. & 80 \\
\hline Benson. & 148 & All in Benson . . . . . . . & Conveyed \& 1895. & 80 \\
\hline do & 149 & $\ldots \ldots \ldots \ldots \ldots \ldots$ & $1871,77,91 \ldots$. & 160 \\
\hline do & 150 & $\ldots \ldots \ldots \ldots \ldots \ldots$ & $1877, ' 81, ' 85 \ldots \ldots$ & 160 \\
\hline do & 151 & & Conveyed . . . . . . . & 160 \\
\hline do & 152 & . & 1881 , '\$5, '90.... & 160 \\
\hline do & 153 & & $1881, ' 85, ' 90 \ldots$. & 160 \\
\hline do & 154 & & $1881, ' 85,90 \ldots$. & 160 \\
\hline do & 155 & & $1877, ' s 1, ' 85, ' 90$. & 160 \\
\hline do & 156 & & 1877, ' $81, ' 85, ' 90$. & 160 \\
\hline do & 157 & & $1887, ' 81, ' 85 . ' 90$. & 160 \\
\hline do & 158 & & $1877,85 \ldots \ldots$ & 160 \\
\hline do & 159 & & $1877,90,95$. & 160 \\
\hline do & 162 & & $1885,90,95 \ldots$ & 160 \\
\hline do & 163 & $\begin{array}{l}\text { Ex. und. } \frac{1}{2} \text { pd. by Finch, } \\
\text { Pruyn \& } \mathrm{Co} \ldots \ldots \ldots \ldots\end{array}$ & 1877, '8:, '85, '90. & 80 \\
\hline do & 168 & $\ldots \ldots \ldots \ldots \ldots \ldots$ & $1877, ' 81,{ }^{\prime} 85,{ }^{\prime} 90 \ldots$ & 160 \\
\hline do & 169 & & $1881, ' 85,{ }^{\prime} 90 \ldots \ldots$ & 154 \\
\hline do & 170 & & Purchase ........ . & 220 \\
\hline do & 171 & & $\begin{array}{r}1877, ' 81, ' 85 \& \\
\text { conveyed...... }\end{array}$ & 160 \\
\hline do & 177 & . & $\begin{array}{r}1877,981,85,90, \\
95 \ldots \ldots \ldots\end{array}$ & 160 \\
\hline do & 179 & & $1877, ' 81, ' 85, ' 90$. & 160 \\
\hline do & 180 & & $1877, ' 81, ' 85 \ldots$ & 160 \\
\hline do & 181 & & $1877, ' \$ 1, ' 85, ' 90$. & 160 \\
\hline do & $18: 2$ & & $1881, ' 85, ' 90 \ldots \ldots$ & 160 \\
\hline do & 183 & & $1881, ' 85,90 \ldots$. & 160 \\
\hline do & 184 & & $\begin{array}{r}1877,91,85,90, \\
95 \ldots \ldots \ldots\end{array}$ & 160 \\
\hline do & 185 & & $1890,95 \ldots \ldots$ & 160 \\
\hline do & 186 & & $1890,95 \ldots \ldots \ldots$ & 160 \\
\hline do & 187 & & $1813,{ }^{\prime} 00^{*}$. & 160 \\
\hline do & 188 & & $1877, ' 81, ' 85 \ldots \ldots$ & 160 \\
\hline do & 189 & & $1877, ' 81, ' 85, ' 90 \ldots$ & 160 \\
\hline Arietta . & 190 & All in Arietta. & $1877,81 \ldots \ldots$ & (x) \\
\hline
\end{tabular}

* Resale. 
Hamilton County

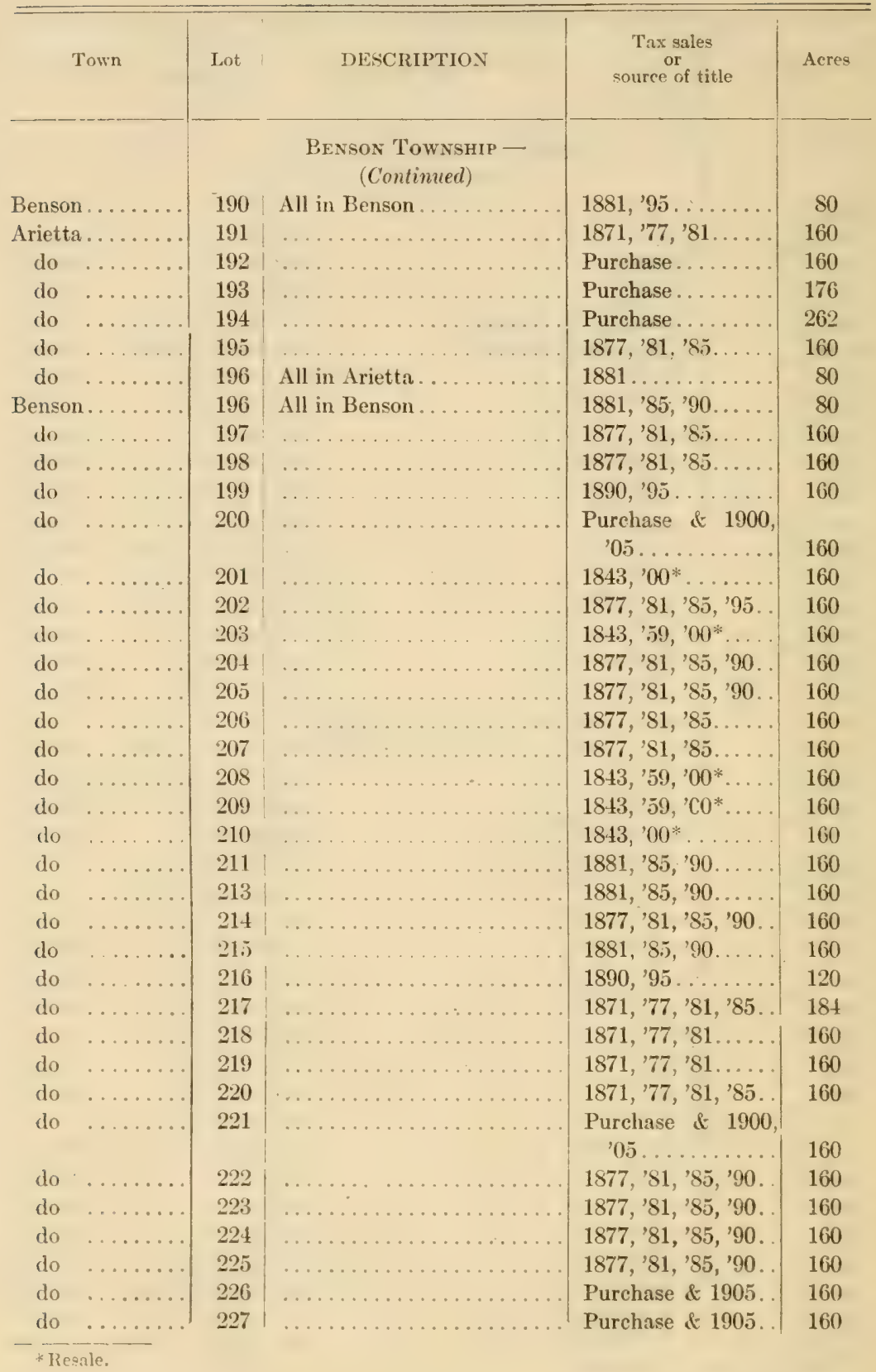


Hamlton County

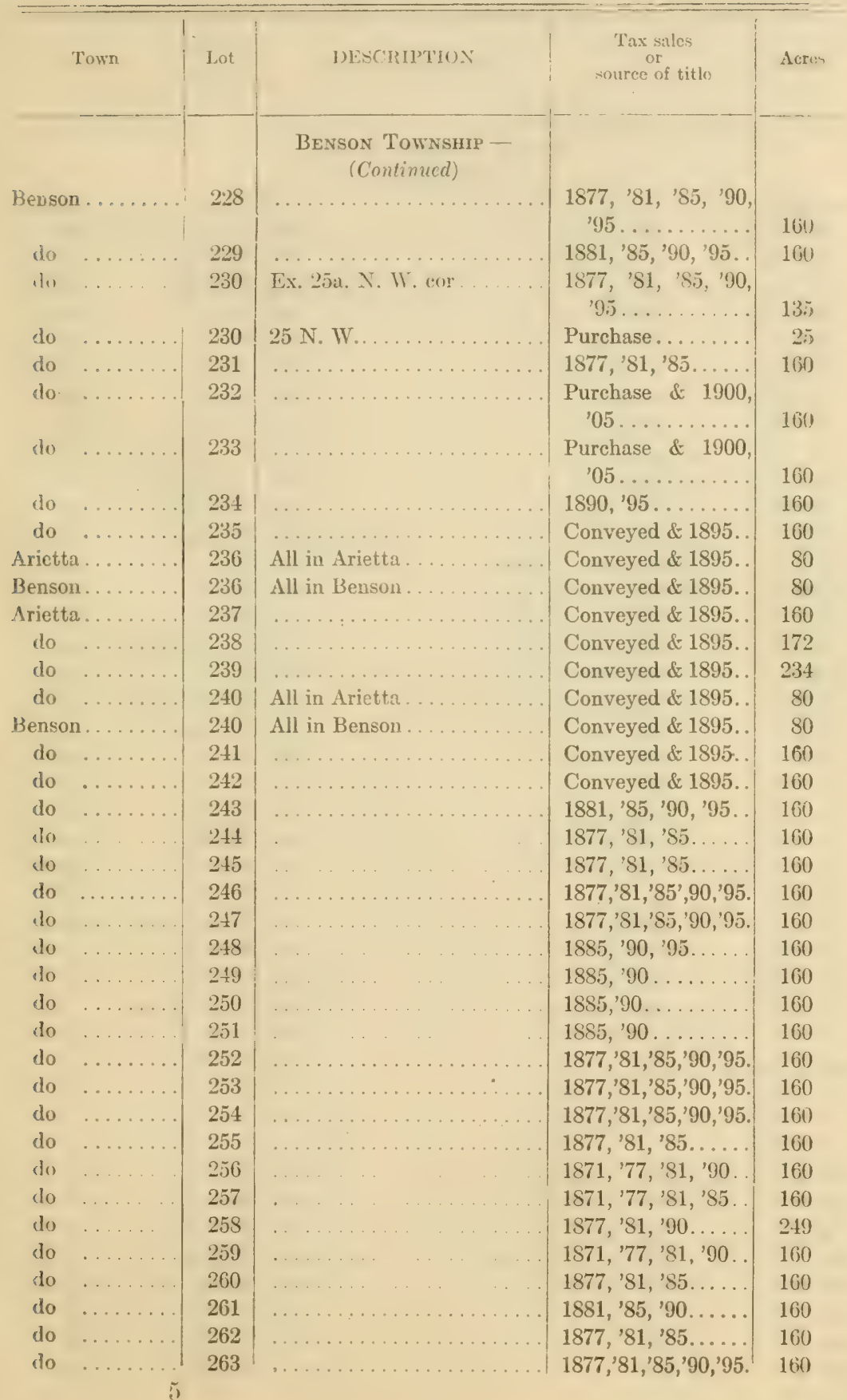


Hamilton County

\begin{tabular}{|c|c|c|c|c|c|}
\hline & Fown & Lot & DESCRIPTION & $\begin{array}{c}\text { Tax sales } \\
\text { or } \\
\text { source of title }\end{array}$ & Acre: \\
\hline & & & $\begin{array}{c}\text { Benson Township - } \\
\text { (Continued })\end{array}$ & & \\
\hline Benson. & & 264 & $\ldots \ldots \ldots \ldots \ldots$ & $1890,95 \ldots$ & 160 \\
\hline do & & 265 & $\ldots \ldots \ldots \ldots \ldots$ & $1877, ' 81, ' 85,{ }^{\prime} 90,{ }^{\prime} 95$. & 160 \\
\hline do & . & 266 & & $1885,90,95$. & 160 \\
\hline do & & 267 & & 1881, 'S5, '90. & 160 \\
\hline do & $\ldots$ & 268 & $\ldots \ldots \ldots \ldots \ldots$ & $1885,90 \ldots$ & 160 \\
\hline lio & $\ldots$ & 269 & & $1877,81$. & 160 \\
\hline do & $\ldots$ & 270 & $\ldots$ & $1885,90 \ldots$ & 160 \\
\hline do & $\ldots$ & 271 & $\ldots \ldots \ldots \ldots \ldots \ldots$ & $1877, ' 81, ' 85 \ldots$. & 160 \\
\hline do & $\ldots$ & 272 & $\ldots \ldots \ldots$ & $1881, ' 85 . ' 90 \ldots$ & 160 \\
\hline do. & $\ldots \ldots$ & 273 & $\ldots \ldots \ldots \ldots \ldots$ & $1877, ' 81, ' 85, ' 90$. & 160 \\
\hline do . & $\ldots \ldots$ & 274 & $\ldots \ldots \ldots \ldots \ldots \ldots$ & $1881, ' 85,90, ' 95 \ldots$ & 160 \\
\hline do. & $\ldots$. & 275 & $\ldots \ldots \ldots$ & $1881,{ }^{\prime} 85,90 \ldots$. & 160 \\
\hline do. & ... & 276 & $\ldots \ldots \ldots$ & $1881, ' 85,90,95$. & 160 \\
\hline do. & ... & 277 & All in Benson ........ & $1890,95 \ldots \ldots$ & 80 \\
\hline Arietta. & . & 277 & All in Arietta..... & Purchase... . & 80 \\
\hline do. & . . & 278 & $\ldots \ldots \ldots \ldots \ldots$ & $1890,{ }^{\prime} 95 \ldots \ldots$ & 146 \\
\hline do . & $\ldots$ & 279 & All in Arietta.......... & Conveyed \& 1895. & 138 \\
\hline Renson. & a... & 279 & All in Benson ........... & Purchase . . . . . . & 80 \\
\hline do & .. & 280 & $\ldots \ldots \ldots \ldots \ldots \ldots \ldots$ & Conveyed \& 1895. & 160 \\
\hline do & $\cdots$ & 281 & $\ldots \ldots \ldots \ldots \ldots \ldots \ldots$ & 1881, '85, '90, '95. & 160 \\
\hline do . & $\ldots$. & 282 & $\ldots \ldots \ldots \ldots \ldots \ldots$ & $1885,90,95 \ldots$. & 160 \\
\hline do. & $\ldots \ldots$ & 283 & $\ldots \ldots$ & $1885,90 \ldots \ldots \ldots$ & 160 \\
\hline do . & $\ldots \ldots$ & 284 & $\ldots \ldots \ldots \ldots$ & $1885,90 \ldots$ & 160 \\
\hline do & $\ldots$. & 285 & $\ldots \ldots$ & $1881, ' 85,{ }^{\prime} 90 \ldots \ldots$ & 160 \\
\hline do & ... & 286 & $\ldots \ldots$ & $1881, ' 85,90 \ldots$. & 160 \\
\hline do & $\ldots$ & 287 & $\ldots \ldots \ldots$ & $1877, ' 81, ' 85,{ }^{\prime} 90$. & 160 \\
\hline do & $\ldots$ & 288 & $\ldots \ldots \ldots$ & $1881,{ }^{\prime} 85,90, ' 95$ & 160. \\
\hline do & . & 289 & & $1885,90,95 \ldots$ & 160 \\
\hline do & ... & 290 & & $1885,{ }^{\prime} 90 \ldots \ldots$ & 160 \\
\hline do & .. & 291 & $\ldots$ & $\begin{array}{r}1877, \quad ' 81, ' 85,90, \\
995 \ldots \ldots \ldots \ldots\end{array}$ & 160 \\
\hline do & & 292 & . & $\begin{array}{r}1877,91,95,90 \\
95 \ldots \ldots \ldots \ldots\end{array}$ & 160 \\
\hline do & .. & 293 & . & $\begin{array}{r}1877, \quad 81, \quad 85,90, \\
95 \ldots \ldots \ldots \ldots\end{array}$ & 160 \\
\hline do & . & 294 & & $1877, ' 81, ' 85 \ldots$. & 160 \\
\hline do & . . & 295 & $\ldots \ldots$ & $1881, ' 85,{ }^{\prime} 90,{ }^{\prime} 95$. & 160 \\
\hline do & . . & 296 & $\ldots \ldots \ldots \ldots \ldots$ & $1877, ' s 1, ' 85 \ldots \ldots$ & 210 \\
\hline do & . & 297 & $\ldots \ldots \ldots \ldots \ldots$ & $1877, ' 81, ' 85 \ldots \ldots$ & 92 \\
\hline do & & 298 & $\ldots \ldots$ & $1877, ' 81, ' 85, ' 90$. & 160 \\
\hline do & . & 299 & $\ldots \ldots \ldots$ & $1877, ' 81, ' 85, ' 90$. & 160 \\
\hline do & . & 300 & & $1877, ' 81, ' 85 \ldots$. & 160 \\
\hline do & $\ldots \ldots$ & 301 & & $1877, ' 81, ' 85 \ldots \ldots$ & 160 \\
\hline
\end{tabular}




\section{Hamilton County}

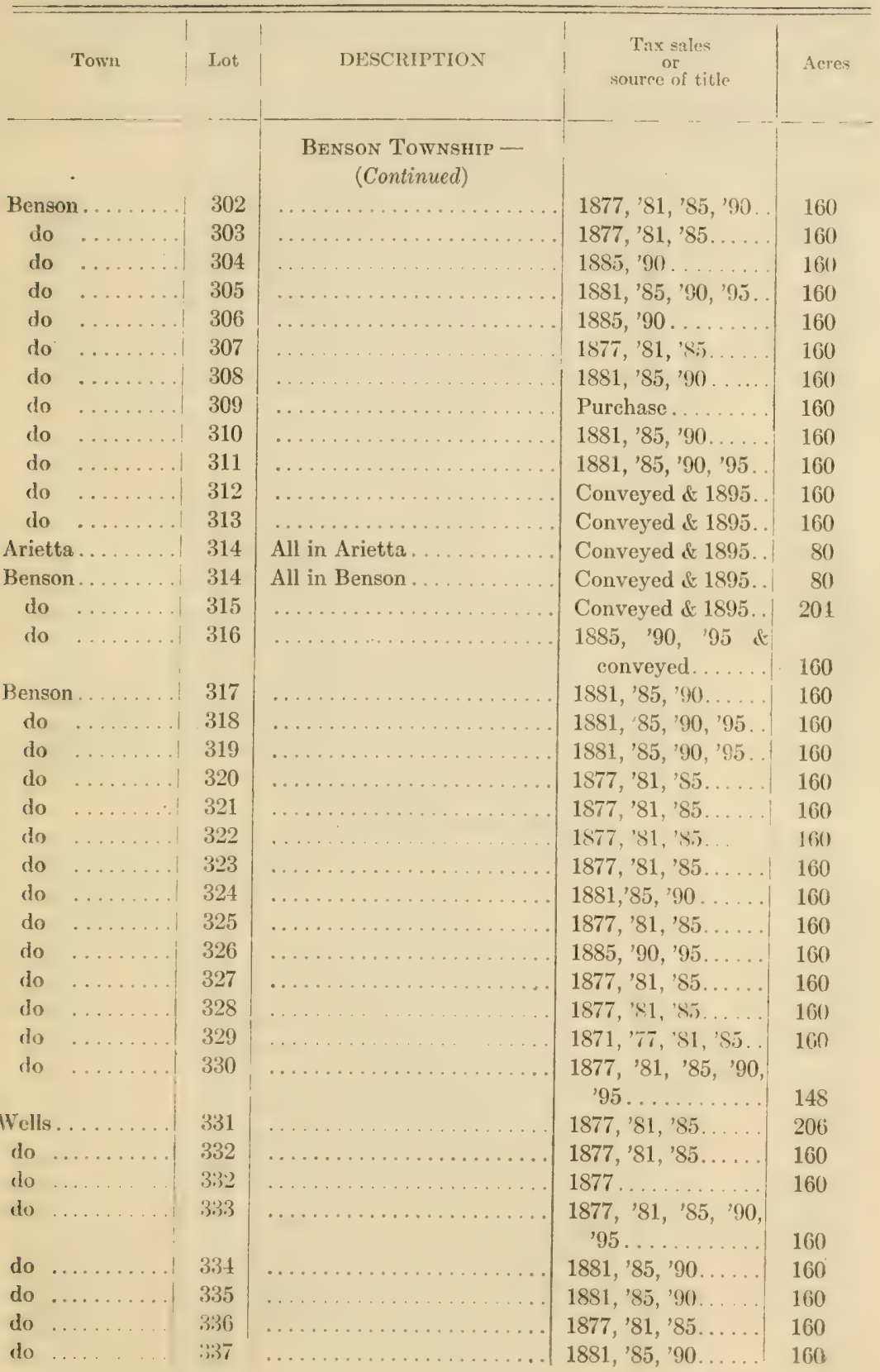


Hamiton County

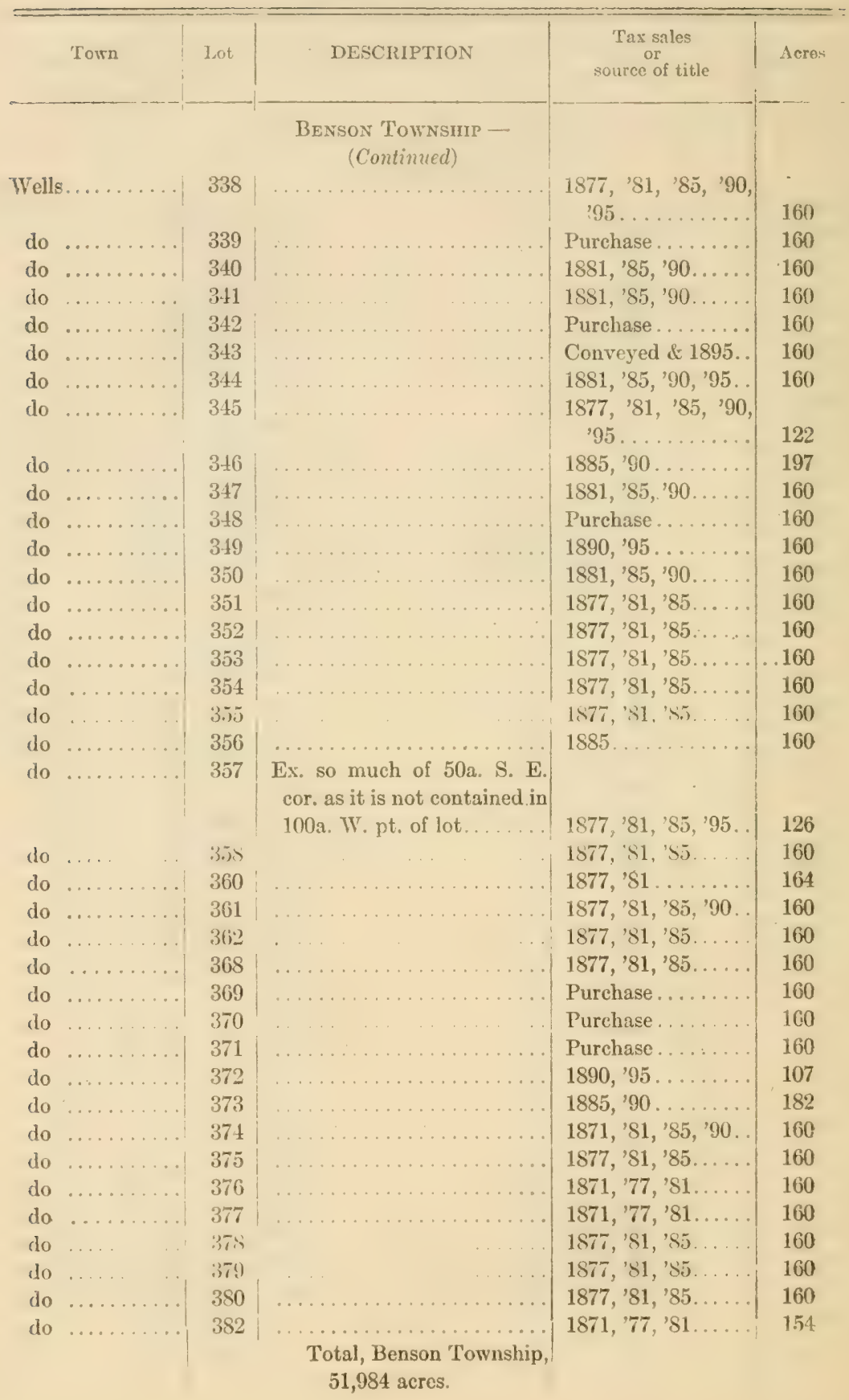


Hamilton County

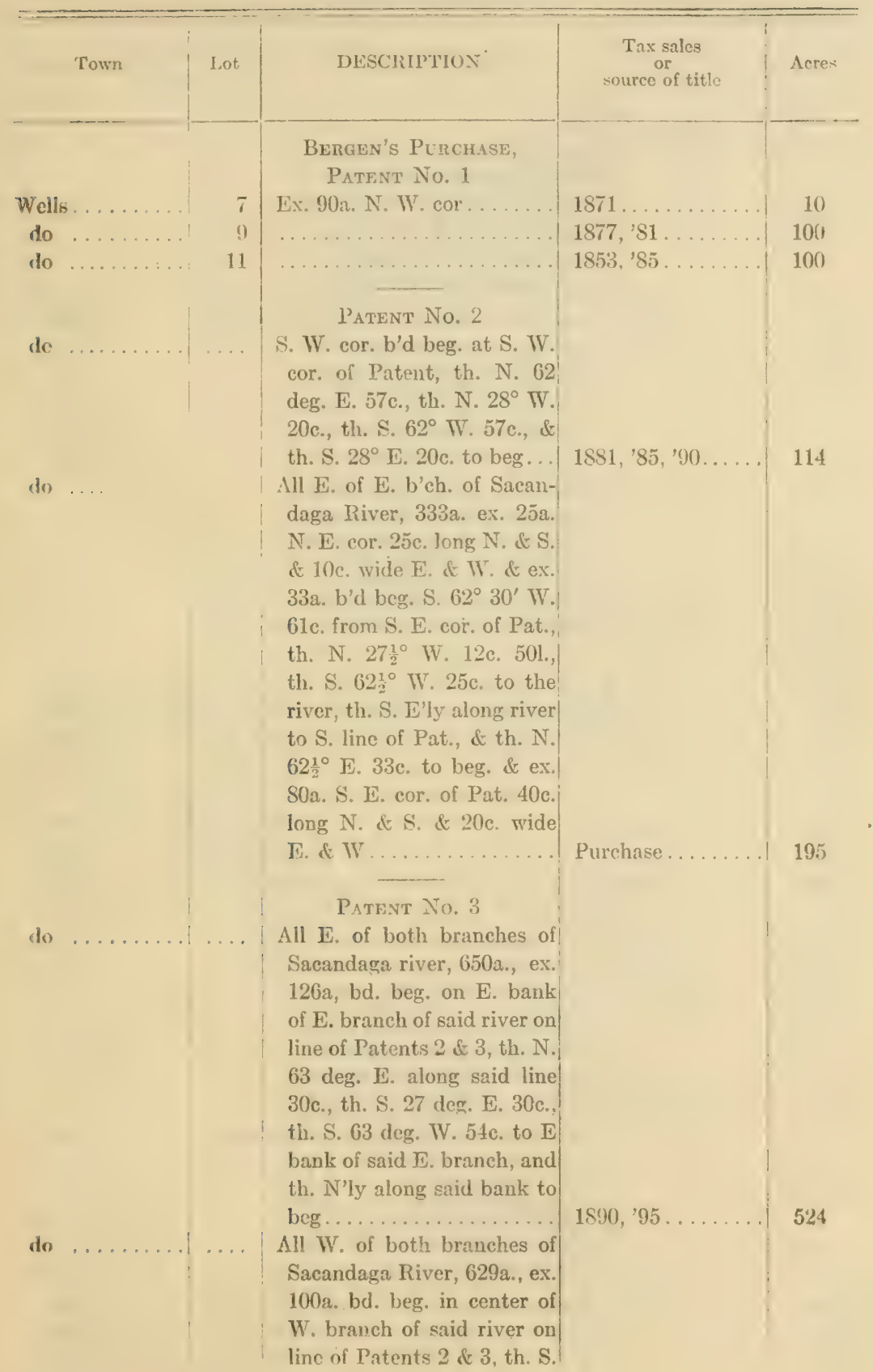


Hamilton County

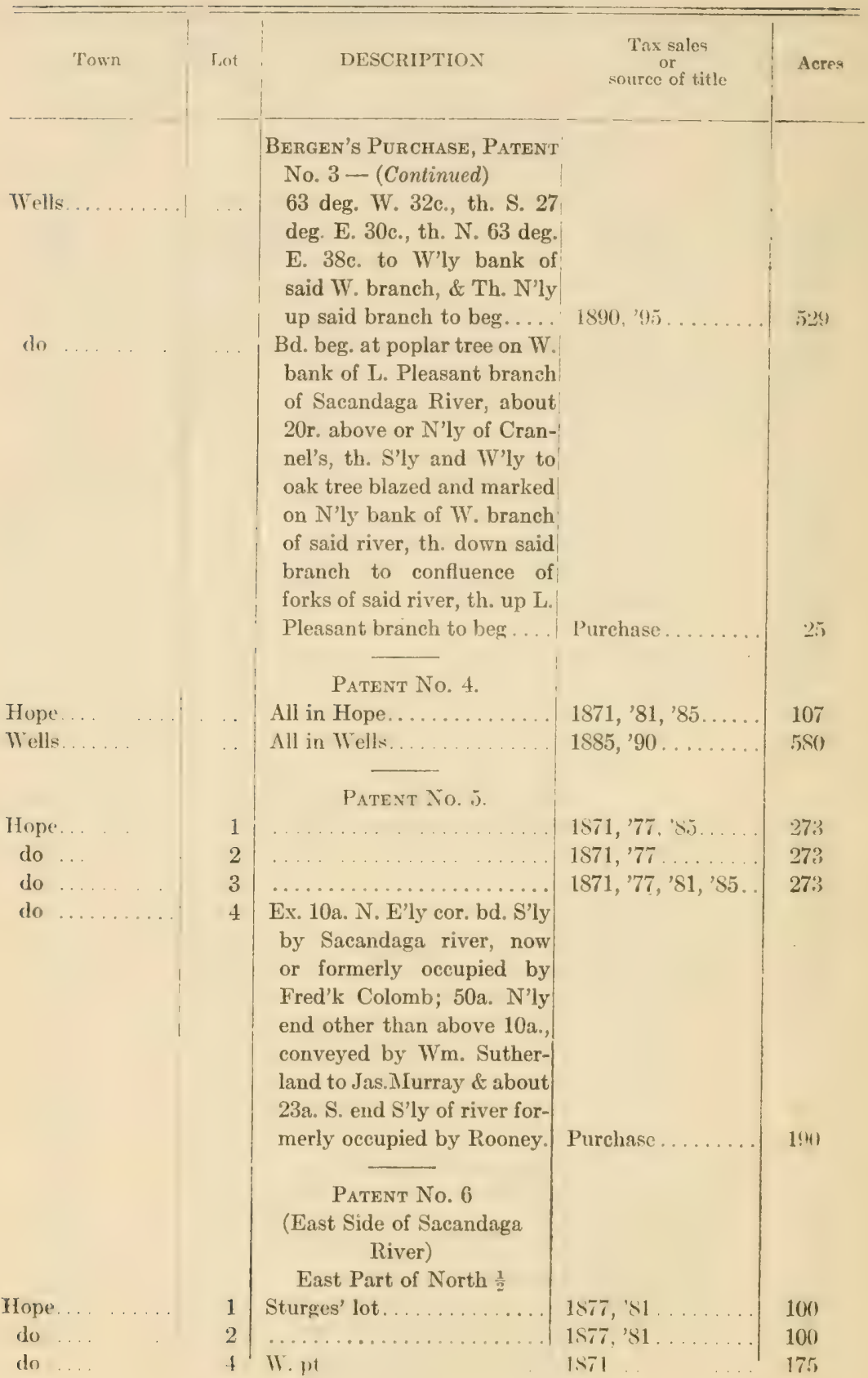


Hamilton County

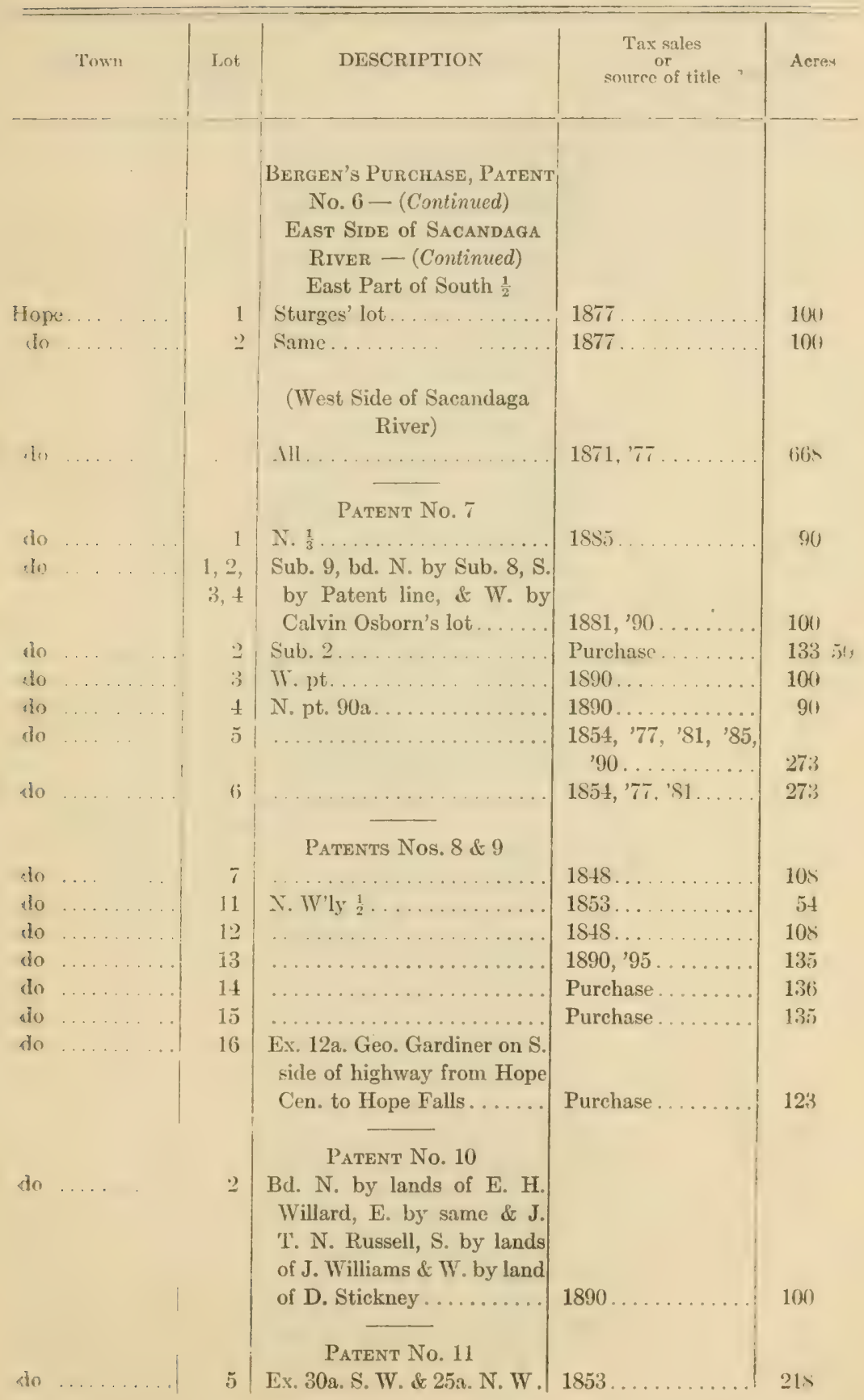


Hamilton County

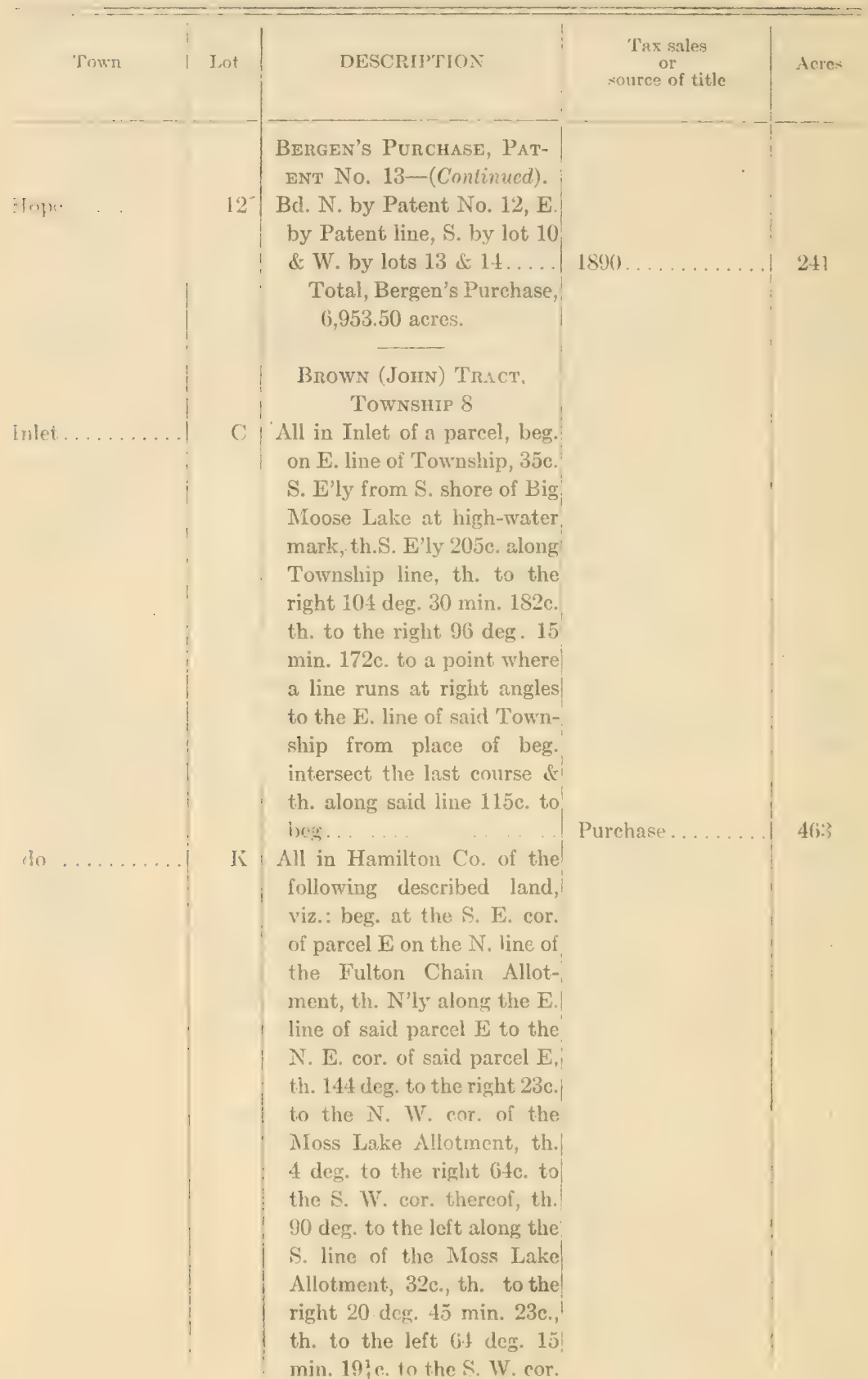




\section{Hamilton County}

\begin{tabular}{|c|c|c|c|c|}
\hline Town & L,ot. & DESCRIPTION & $\begin{array}{l}\text { Tnx sales } \\
\text { or } \\
\text { souree of title }\end{array}$ & Acres \\
\hline In\}ent. & & 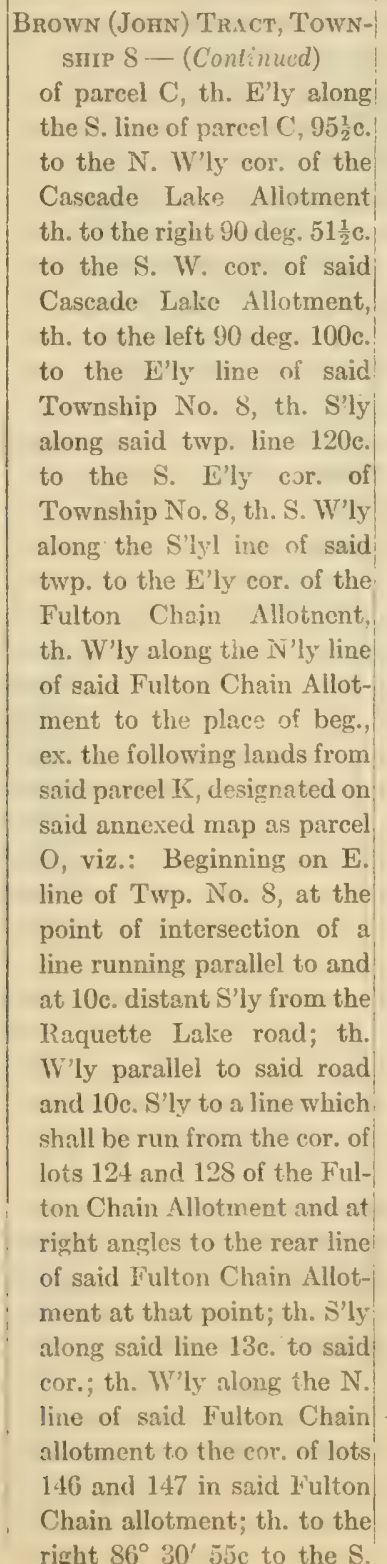 & & \\
\hline
\end{tabular}


Hamilton County

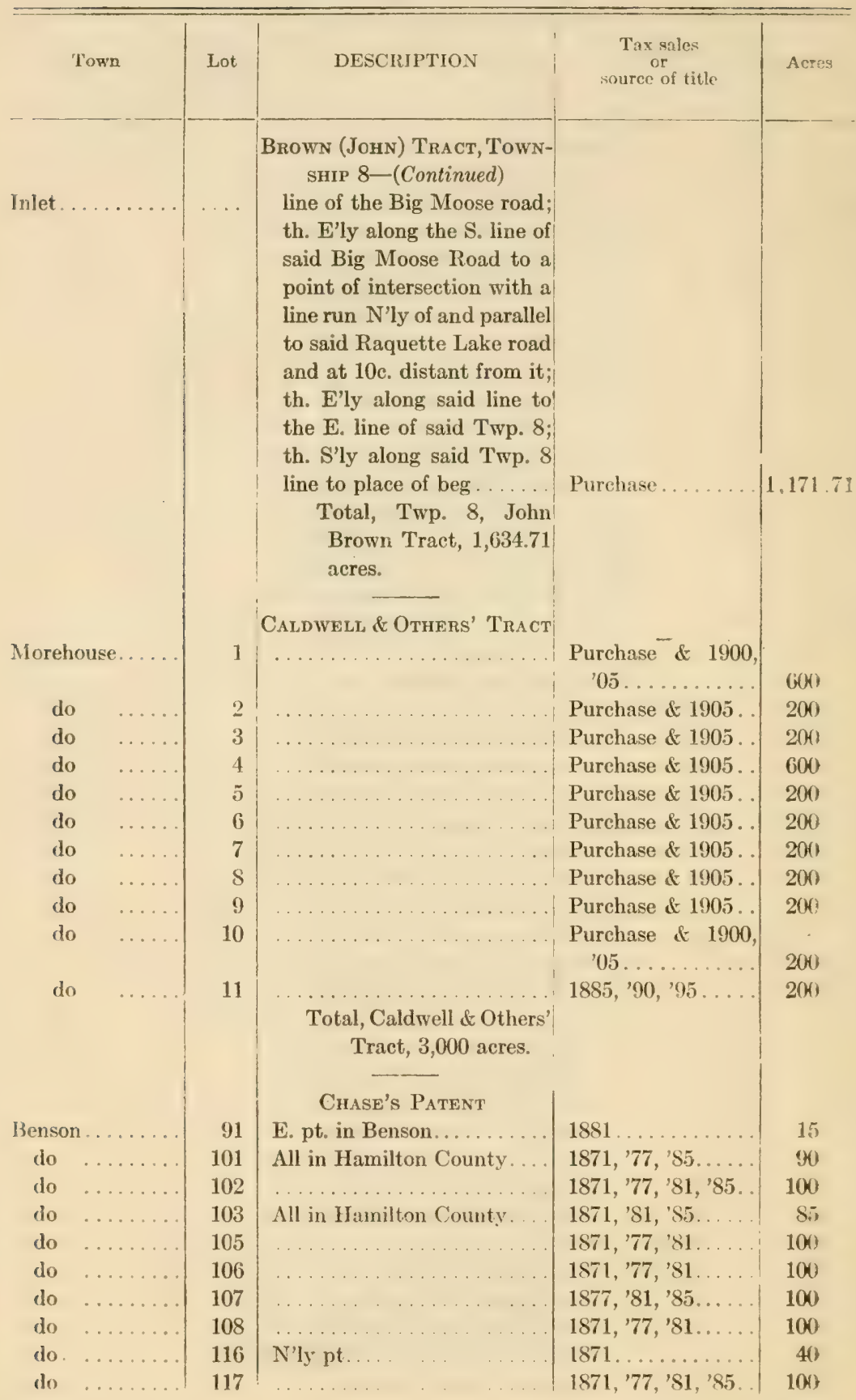




\section{Hamilton County}

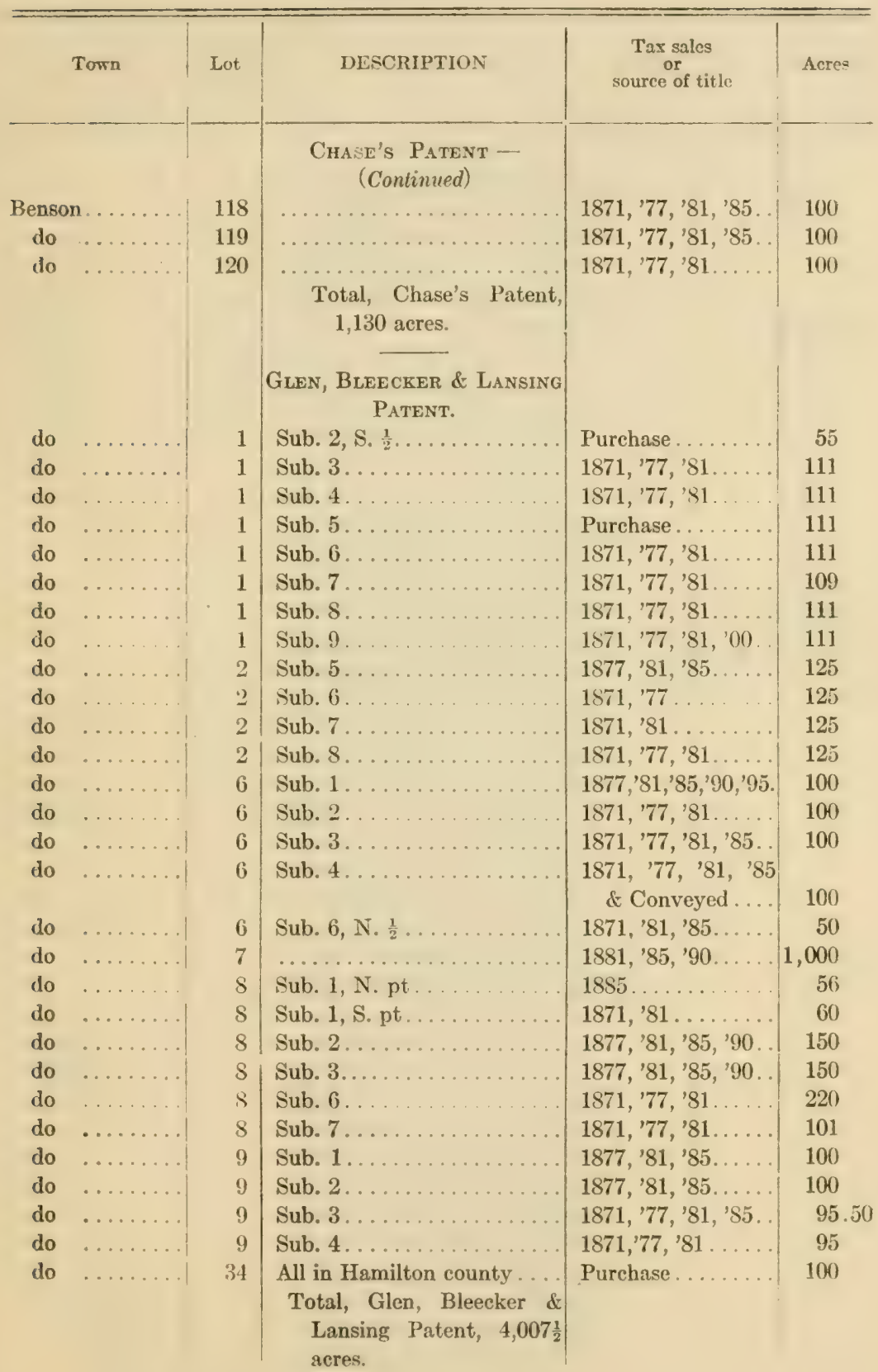


Hamiluon County

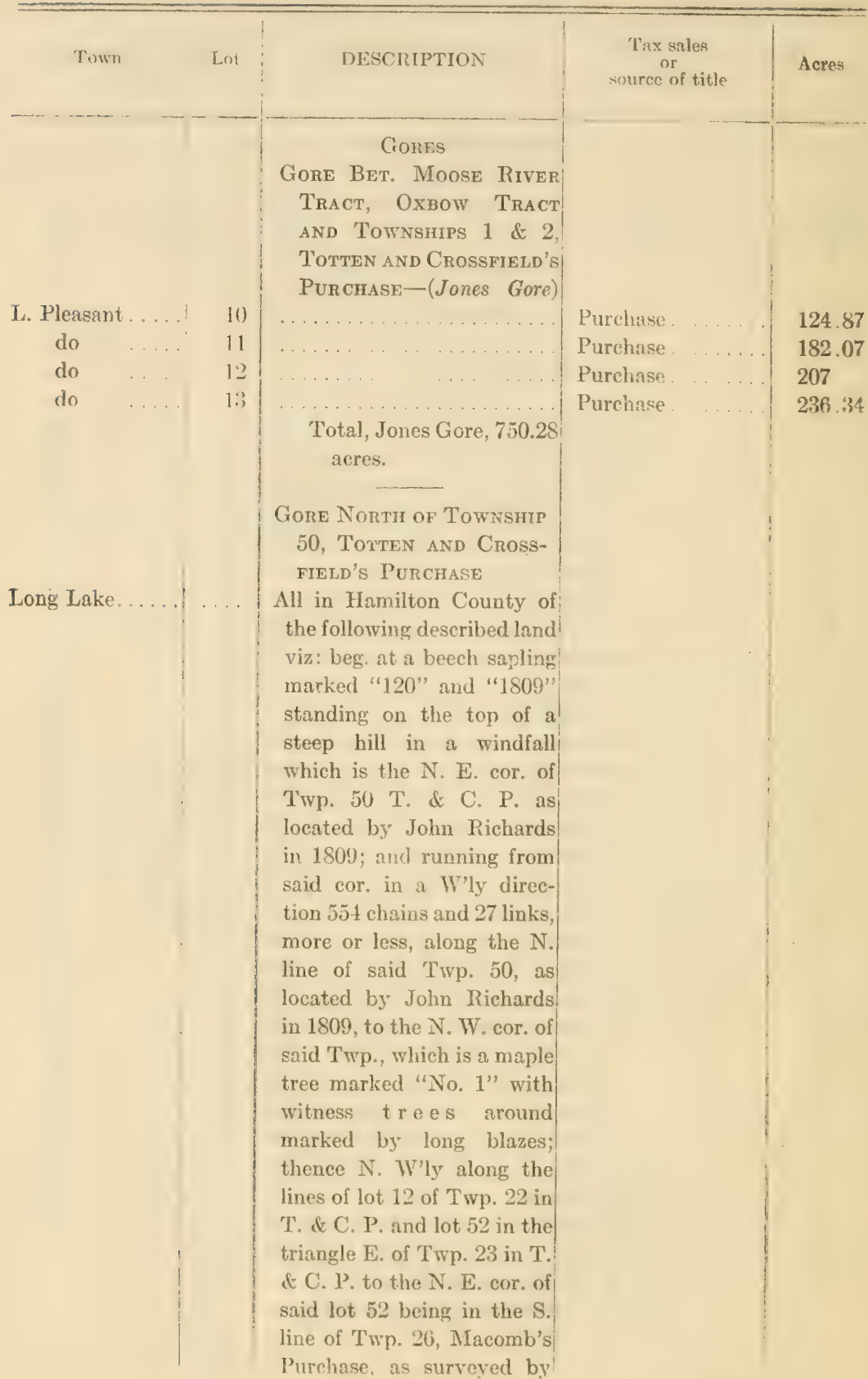


Hamlton County

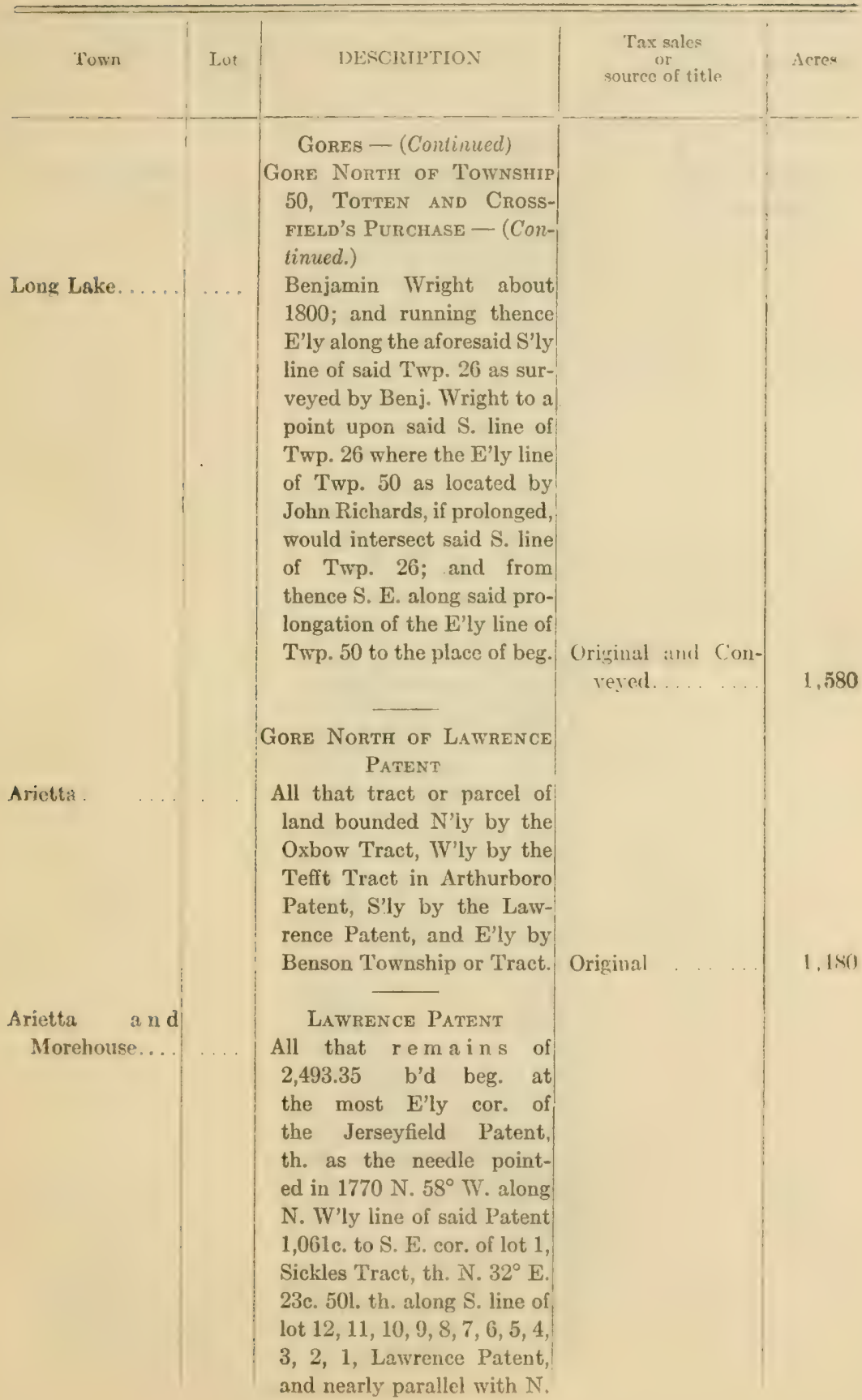


Hamilton County

\begin{tabular}{|c|c|c|c|c|}
\hline Town & Lot. & DESCRIPTION & $\begin{array}{c}\text { Tax sales } \\
\text { or } \\
\text { source of title }\end{array}$ & Iere: \\
\hline $\begin{array}{l}\text { Arietta and } \\
\text { Morehouse... }\end{array}$ & $\ldots$ & 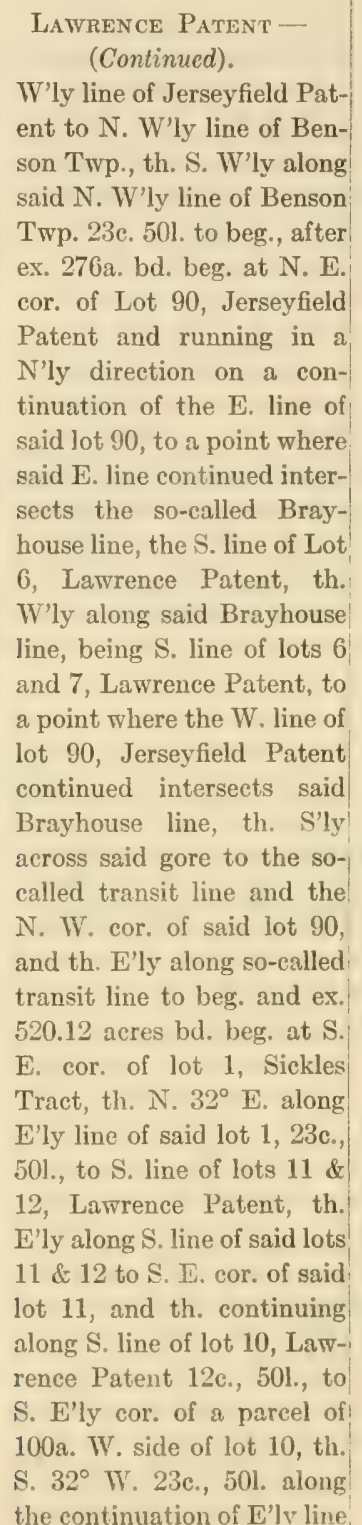 & & \\
\hline
\end{tabular}


Conservation Commisston.

Hamilton County

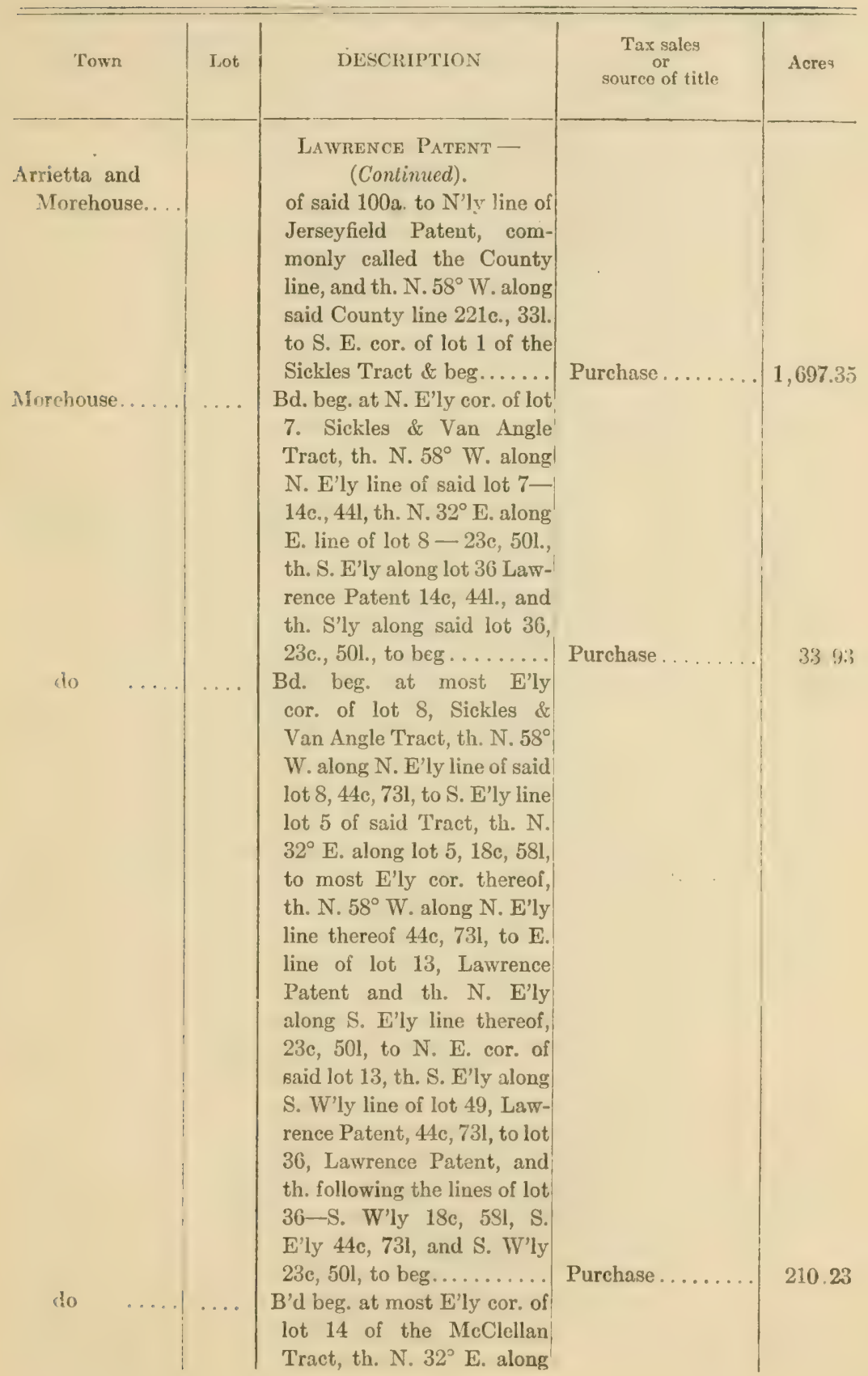


Hamilton County

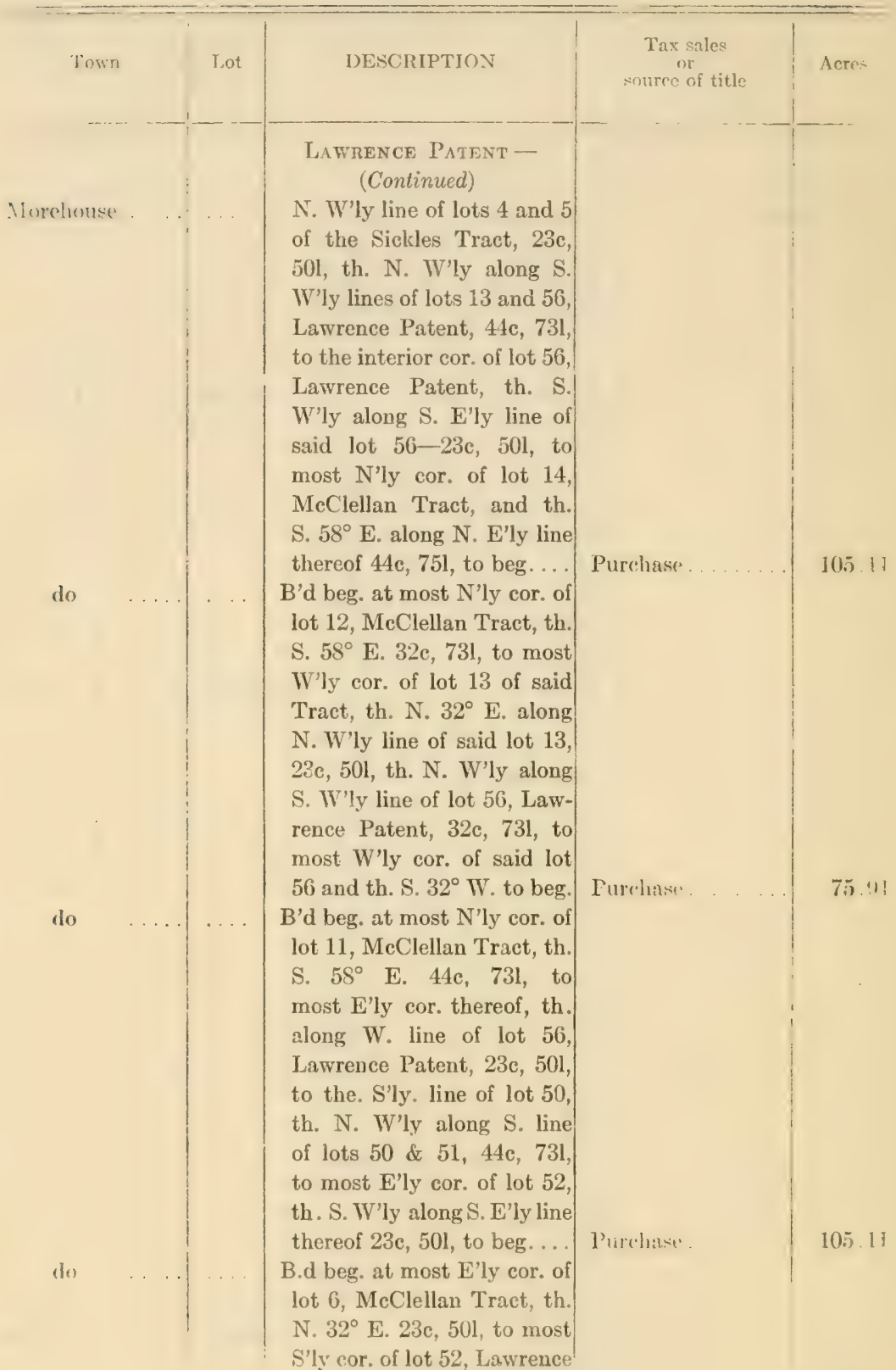


Hamilton County

\section{IAWRENCE PATENT -}

(Continued)

Patent, th. N. W'ly along S. W'ly line of lots $52 \& 55$, Lawrence Patent, 44c, 731, to an interior cor. of said lot! 55 , th. S. W'ly along S. E'ly line lot $55-23 \mathrm{c}, 501$, to most N'ly cor. of lot 6 , MeClellan Tract, \& th. S. $58^{\circ}$ E. $44 \mathrm{c}, 731$, to beg..... B'd beg. at most W'ly cor. of lot 5, McClellan Tract, th. N. $32^{\circ}$ E. along N. W'ly line of said lot $5-23 \mathrm{c}, 501$, th. N. W'ly along S. W'ly line of lot 55, Lawrence Patent, $32 \mathrm{c}, 731$, to $\mathrm{E}$. line of lot 2 , Caldwell's Tract, th. S. $32^{\circ}$ W. along S. E'ly line of said lot 2, to most E'ly cor. of lot 1 of said Tract \&, th. S. $58^{\circ}$ E. along N. E'ly line of lot 4, McClellan Tract, 32c,73l, to beg . . . . . . . . . . . . B'd beg. at most E'ly cor. of lot 3, Caldwell's Tract, th. N. E'ly along N. W'ly line of lot 55, Lawrence Patent, $23 \mathrm{c}, 50 \mathrm{l}$, to an interior cor of said lot 55 , th. N. W'l along $\mathrm{S}$. W'ly lines of lots 5 : \& 54, Lawrence Patent, 44c 73l, th. S. W'ly along S. E'l line of said lot $54,23 \mathrm{c}, 501$. to most N'ly cor. of lot 3 . Caldwell's Tract, th. S. $58^{\circ}$ E. $44 \mathrm{c}, 731$, to beg........ B'd beg. at most N'ly cor. of Lot 1 Caldwell Tract, th. S. $58^{\circ}$ E. $32 \mathrm{c}, 731$, th. N. $32^{\circ}$ E. along N. W'ly line of lot 2 of said Tract, $23 \mathrm{c}, 50 \mathrm{l}$, th. N. W. along S. W. line of
Purchase........

76.91
Acres

105.11

Purchase .

105.11 
Hamilton County

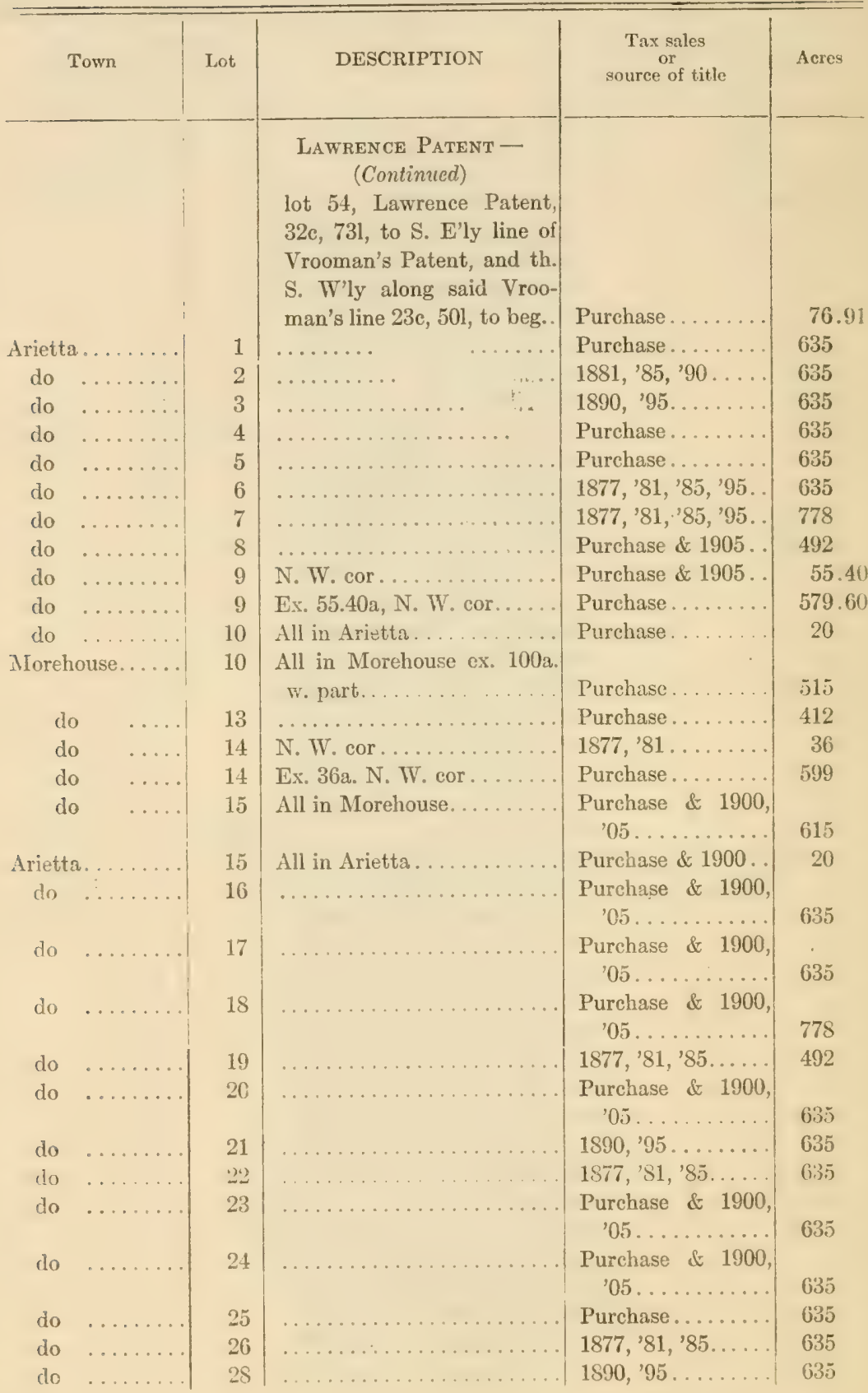




\section{Hamilton County}

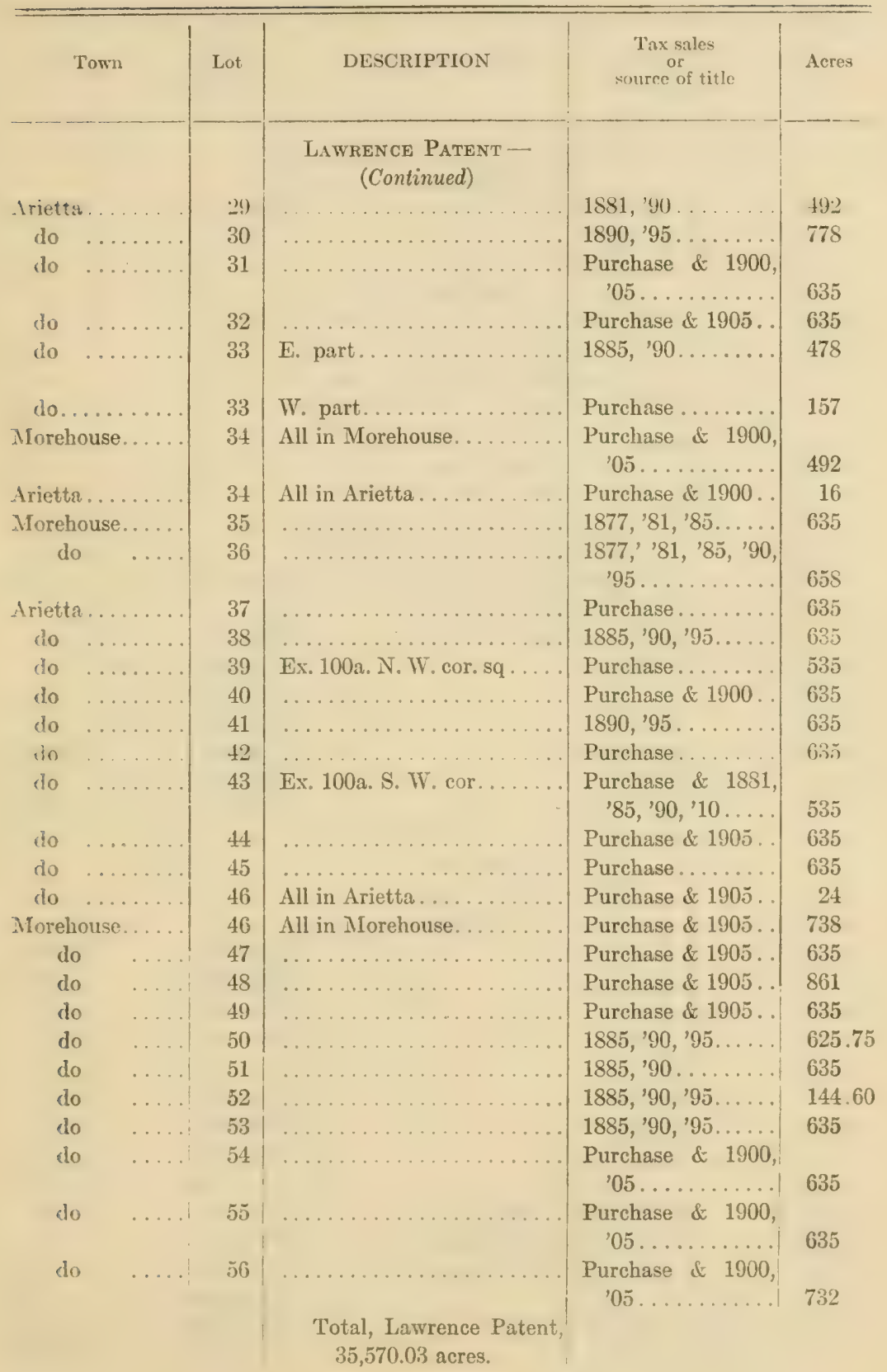


Hamilton County

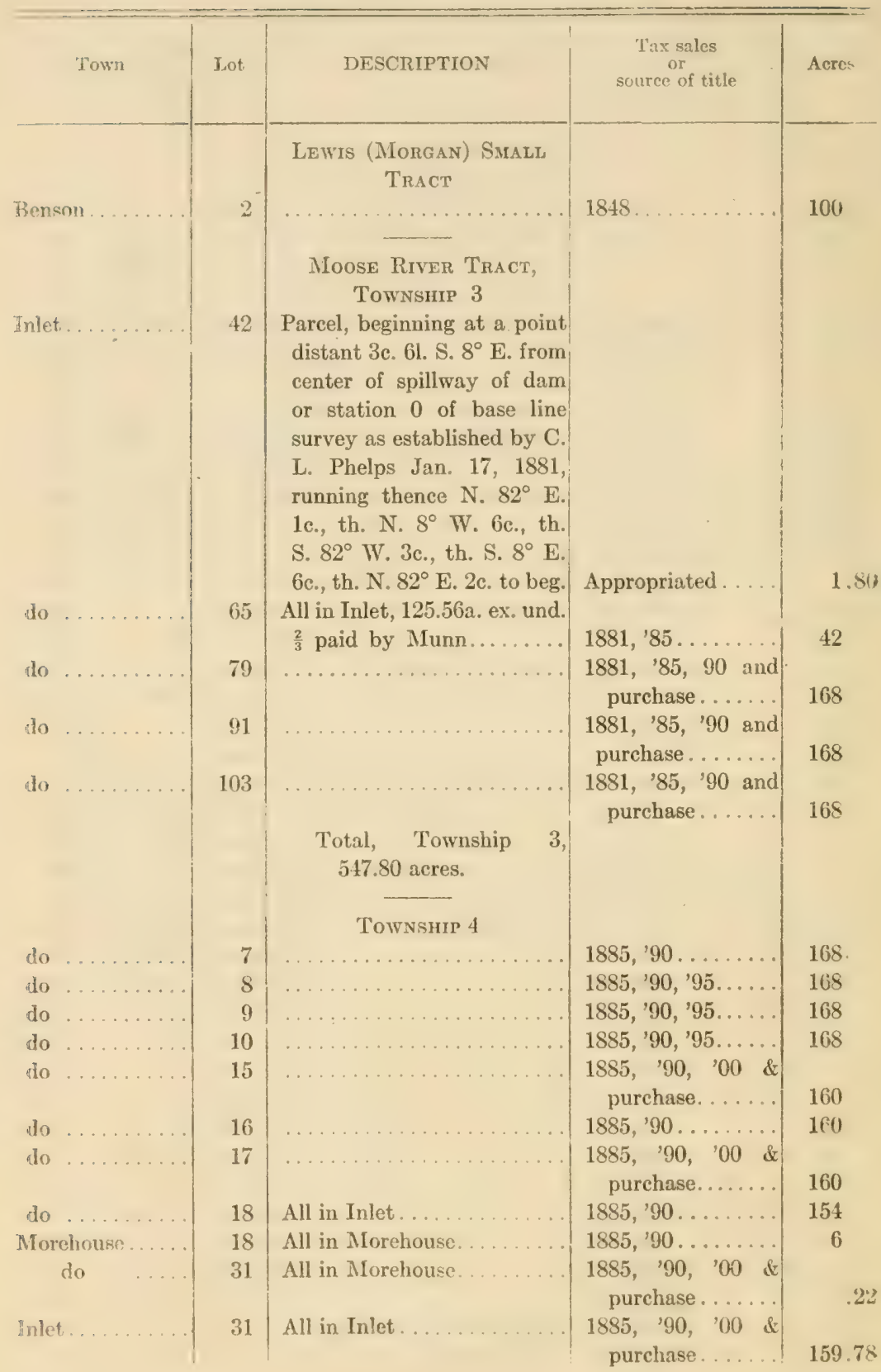




\section{Hanilton County}

\begin{tabular}{|c|c|c|c|c|}
\hline Town & Lot & DESCRIITION & $\begin{array}{l}\text { Titx sales } \\
\text { or } \\
\text { source of title }\end{array}$ & Acret \\
\hline & & $\begin{array}{l}\text { Moose River Tract, Town- } \\
\text { Smip } 4-\text { (Continued })\end{array}$ & & \\
\hline Inlet & $: 32$ & $\cdots+1$ & $\begin{array}{l}\text { 1881, '\$ร̃, '90, '00 } \\
\text { \& purchase..... }\end{array}$ & 160 \\
\hline $4: 0$ & $: 3 ;$ & & $\begin{array}{r}1881, \quad 85, ' 90, ' 00 \\
\text { \& purchase..... }\end{array}$ & 160 \\
\hline (i) & $\therefore 1$ & & 1881 , 'S5, '30, '00. . & 160 \\
\hline do & 39 & & $\begin{array}{r}\text { 1881, ' } 85, \text { '90, '00 } \\
\text { \& purchase..... }\end{array}$ & \\
\hline (8) & 40 & & $\begin{array}{l}\text { 1881, '\$5, '90, '00' } \\
\text { \& purchase..... }\end{array}$ & 160 \\
\hline do & 41 & & $\begin{array}{l}\text { 1881, '85, '90, '00! } \\
\text { \& purchase..... }\end{array}$ & $161 \%$ \\
\hline do & 42 & All in Inlet. & $\begin{array}{r}1885, \text { '90, } 90 \quad \& \\
\text { purchase. } \ldots . . .\end{array}$ & \\
\hline Morehouse. & 42 & All in Morehouse. & 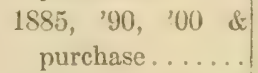 & 17 \\
\hline do & $5 \check{5}$ & All in Morehouse. . & $\begin{array}{c}1885, \quad 90, \quad 00 \quad \& \\
\text { purchase } \ldots \ldots \ldots\end{array}$ & 180 \\
\hline lnlet & 5 & All in Inlet & 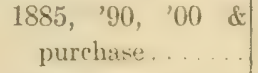 & 81 \\
\hline do & $\tilde{5} 6$ & All in Inlet... & $\begin{array}{r}1885, \quad 90, \quad 00 \quad \& \\
\text { purchase, } 1901 \ldots\end{array}$ & 164.7 \\
\hline Morehouse. & 56 & All in Morehouse..... . & $\begin{array}{r}1885, \quad 90, \quad 00 \\
\text { purchase, } 1901 \ldots\end{array}$ & $472:$ \\
\hline Inlet. & 57 & & $\begin{array}{c}\text { 1885, '90 \& }{ }^{9} \text { pur- } \\
\text { rhasr } . . .\end{array}$ & 21: \\
\hline do & 64 & All in Inlet. & $\begin{array}{l}\text { 1881, ' } 85,90, ' 00 \\
\text { \& purchase..... }\end{array}$ & $163 \quad 13$ \\
\hline Morehouse. & 64 & All in Morehouse... & $\begin{array}{r}1881, \quad 85, ' 90, ' 00 \\
\& \text { purchase ..... }\end{array}$ & 49.87 \\
\hline do & 65 & All in Morehouse. & $\begin{array}{l}\text { 1881, '85, '90, '00 } \\
\text { \& purchase'.... }\end{array}$ & 207 \\
\hline lulet. & (i.j) & . 111 in Inlet & 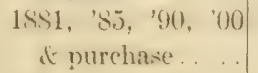 & bi \\
\hline Morehouse. & 66 & & 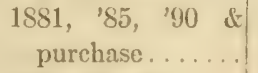 & 213 \\
\hline do & 79 & & 1SS1, '১5. "U!. & 213 \\
\hline do & 81 & Und. $\frac{1}{3}$ of all in Morehouse.. & Purchase........ & $\tilde{5} 5.5$ \\
\hline Inlet. . & 81 & Und. $\frac{1}{3}$ of all in Inlet...... & Purchase........ & 15. . . in! \\
\hline do & $S:$ & . 11 in Inlet... & Purchase \& 1900. & 208 \\
\hline M rehouse. & 82 & All in Morehouse. . . . & Purchase \& 1900 . & 5 \\
\hline Inlet.... & 83 & & $\begin{array}{c}1881, ' 85, ' 90, \text { \& } \\
\text { purrhas. }\end{array}$ & $21: 3$ \\
\hline
\end{tabular}


Hamilton County

\begin{tabular}{|c|c|c|c|c|}
\hline Town & Lot & DESCRIPTION & $\begin{array}{c}\text { Tax sales } \\
\text { or } \\
\text { source of title }\end{array}$ & Acres \\
\hline & $\begin{array}{c}\text { Moose River Tract, Town- } \\
\text { Ship } 4-\text { - Continued })\end{array}$ & & \\
\hline Inlet. & 84 & $\cdots \cdots \cdots \cdots \cdots \cdots$ & $\begin{array}{r}1881, \quad ' 85, \quad 90 \quad \& \\
\text { purchase. . . . . }\end{array}$ & 213 \\
\hline do & 85 & & Purchase ........ & 213 \\
\hline do & 86 & . & Purchase......... & $21: 3$ \\
\hline do & 87 & All in Inlet.... & $\begin{array}{l}\text { 1881, '85, '90 } \& \\
\text { Purchase....... }\end{array}$ & $6 \%$ \\
\hline Morchouse. . & 87 & All in Morehouse... & $\begin{array}{c}1881, \text { '85, '90 \& } \\
\text { purchase ....... }\end{array}$ & 150 \\
\hline do & 88 & & 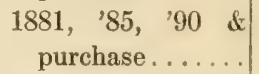 & $21 ;$ \\
\hline do & 89 & & $\begin{array}{r}1885, \quad 90, \quad 95 \quad \& \\
\text { purchase }\end{array}$ & 213 \\
\hline do & 90 & & Puchase......... & 21: \\
\hline do & 97 & $\ldots$. & $\begin{array}{r}1881, .,{ }^{85}, \quad 90 \quad \& \\
\text { purchase. ...... }\end{array}$ & 213 \\
\hline do & 98 & & $\begin{array}{c}1881, \text { ' } 85, \quad ' 90 \quad \& \\
\text { purchase. . . . . . }\end{array}$ & 213 \\
\hline do & 99 & & $\begin{array}{c}1881, \quad \text { '85, '90 } \quad \& \\
\text { purchase. . . . . . }\end{array}$ & 213 \\
\hline do & 100 & & Purchase........ & 213 \\
\hline do & 101 & All in Morehouse..... & Purchase........ & 106.50 \\
\hline Inlet . . & 101 & All in Inlet . . . . . . . . & Purchase........ & 106.50 \\
\hline do. & 102 & $\ldots \ldots \ldots \ldots \ldots \ldots$ & Purchase \& 1900. & 213 \\
\hline \multirow{2}{*}{$\begin{array}{l}\text { do } \ldots . . . \\
\text { Morehouse. }\end{array}$} & 103 & All in Inlet....... & Purchase........ & 134 \\
\hline & 103 & All in Morehouse.... & Purchase........ & 30 \\
\hline $\begin{array}{l}\text { Morehouse. } \\
\text { do }\end{array}$ & 104 & All in Morehouse..... & Purchase ........ & 180.70 \\
\hline Inlet....... & 104 & All in Inlet.......... & Purchase......... & 32.30 \\
\hline Morehouse...... & 105 & $\ldots \ldots \ldots \ldots \ldots \ldots \ldots$ & $\begin{array}{r}1881, '{ }^{\prime} 85, ' 90 \quad \& \\
\text { purchase. . . . . }\end{array}$ & 213 \\
\hline do & 106 & & $\begin{array}{r}\text { 1881, '85, } 90 \quad \& \\
\text { purchase. } . . .6 .\end{array}$ & 213 \\
\hline do & 107 & & $\begin{array}{c}1881,{ }^{\prime} 85,90 \quad \text { ' } 90 \\
\text { purchase....... }\end{array}$ & 213 \\
\hline \multirow[t]{2}{*}{ do } & 108 & $\begin{array}{l}\text { Total, Township } \\
8,574.95 \text { acres. }\end{array}$ & Purchase....... & $21: 3$ \\
\hline & & $\begin{array}{c}\text { Moose River Tract, } \\
\text { Township } 7\end{array}$ & & \\
\hline do & 1 & $\ldots \ldots \ldots \ldots \ldots \ldots$ & Purchase... & 198 \\
\hline do & 2 & 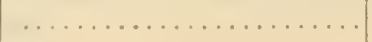 & Purchase........ & 160 \\
\hline do & 23 & $\cdots$ & Purchase ........ & 160 \\
\hline
\end{tabular}




\section{Hamilton County}

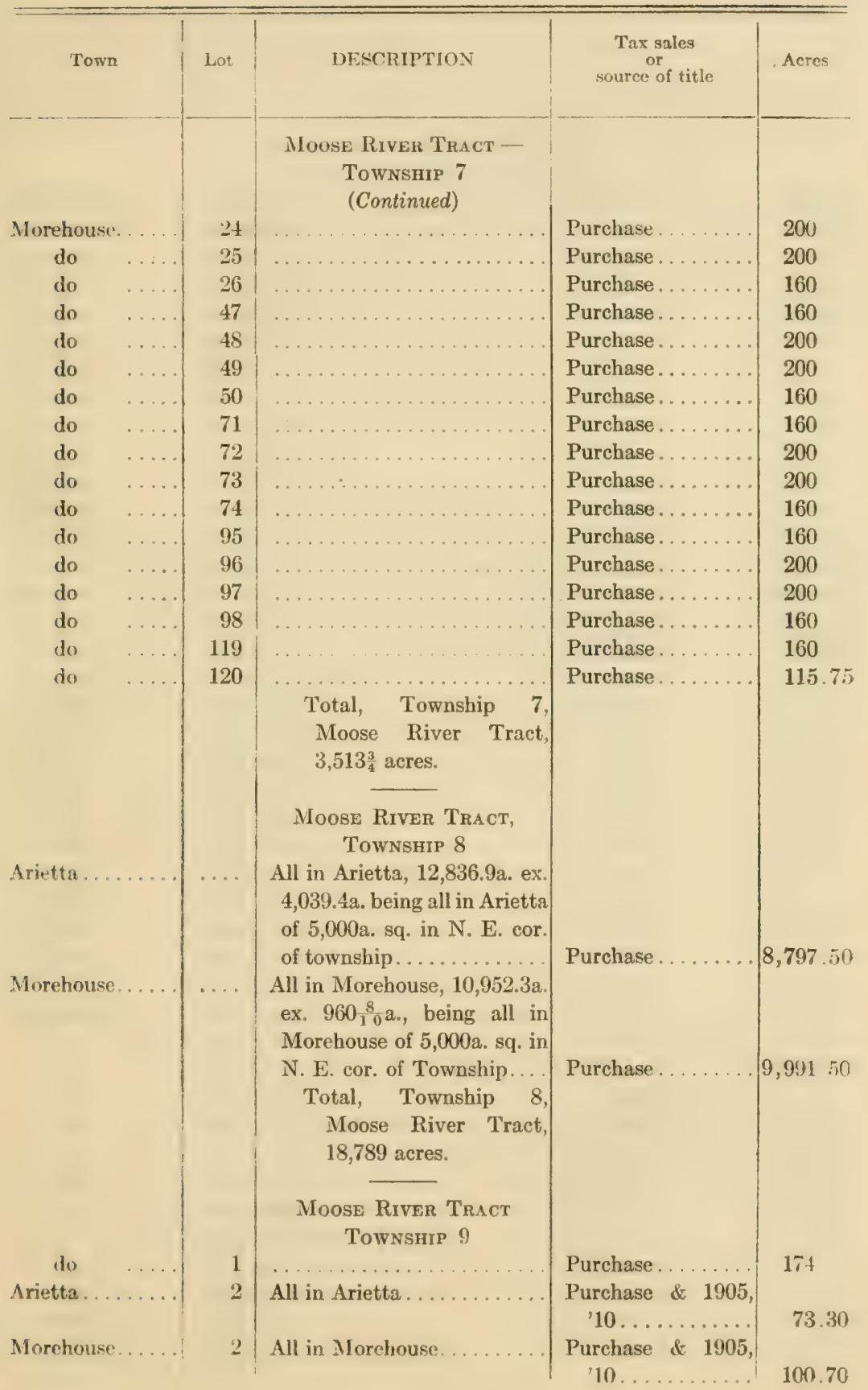


Hamilton County

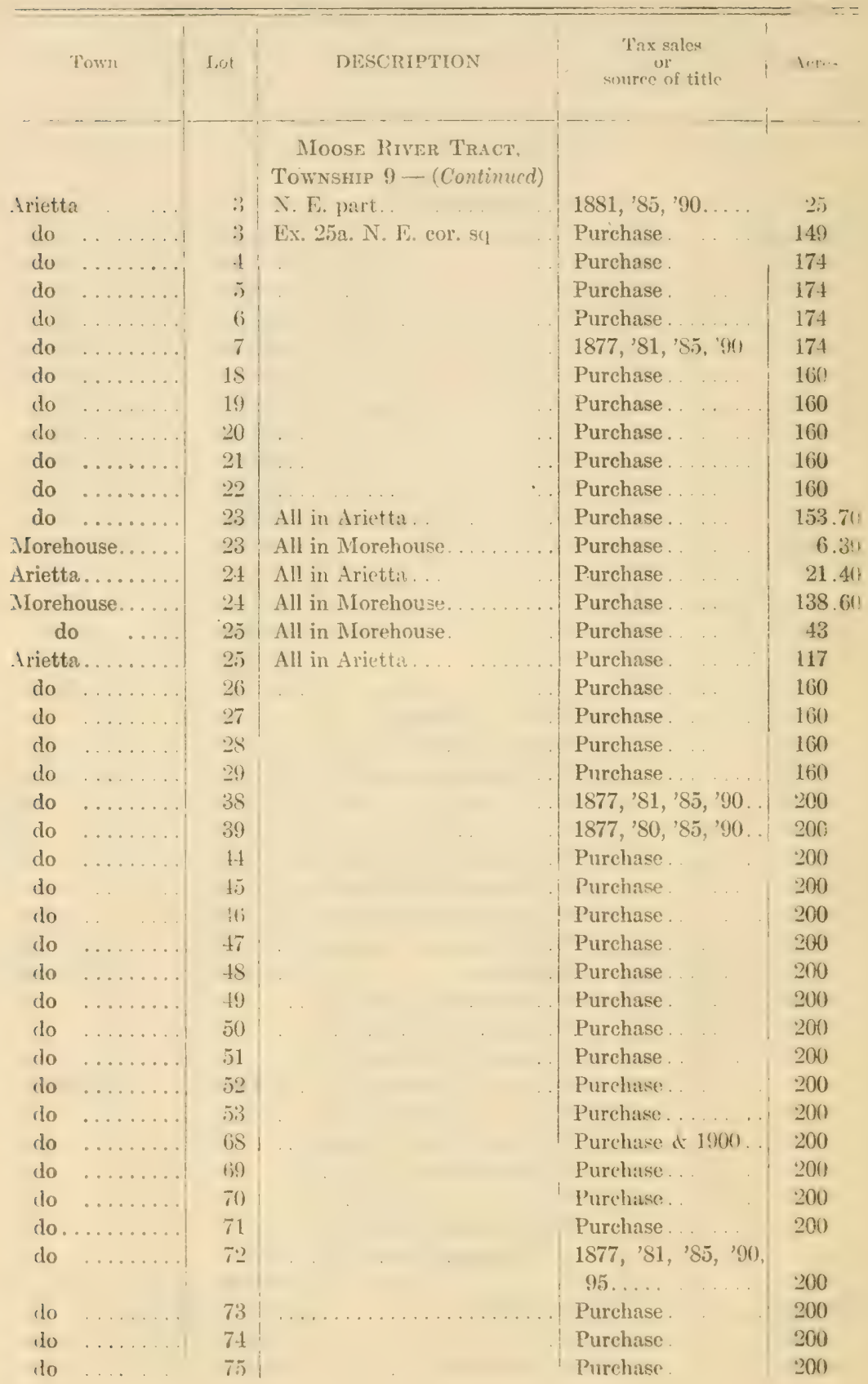


Hamiton County

\begin{tabular}{|c|c|c|c|c|}
\hline Town & Lom & DFACRTPTIOA & $\begin{array}{l}\text { Tax sales } \\
\text { or } \\
\text { source of title }\end{array}$ & Acres \\
\hline & & $\begin{array}{l}\text { Moose River Tract, Town- } \\
\quad \text { ship } 9-\text { (Continued) }\end{array}$ & & \\
\hline Arietta........ & 76 & & $\begin{array}{l}\text { Conveyed \&. Pur- } \\
\text { chased ........ }\end{array}$ & 200 \\
\hline do & 77 & & $\begin{array}{l}\text { Conveyed \& Pur- } \\
\text { chase.......... }\end{array}$ & \\
\hline do & 78 & & Conveyed ........ & 200 \\
\hline do & 79 & & Conveyed ..... & 200 \\
\hline .. & 80 & & Purchase..... & 200 \\
\hline do $\ldots$ & 81 & & Purchase...... & 200 \\
\hline do $\ldots$ & 82 & & Purchase...... & 200 \\
\hline do ... & 83 & & Purchase..... . & 200 \\
\hline d:s & sit & & Purchose. & 210 \\
\hline do & 85 & & Purchase..... . & 210 \\
\hline do $\ldots$ & S6 & & Purchase . . . . . . & 200 \\
\hline (o) $\ldots$. & 87 & & Purchase ...... . & 200 \\
\hline do $\ldots$ & 88 & & Purchase ...... & 200 \\
\hline do $\ldots$ & 89 & & Purchase....... & 200 \\
\hline do & 90 & & Conveyed ...... & 200 \\
\hline do & 91 & & Conveyed..... & 200 \\
\hline do $\ldots$. & 92 & & Conveyed . . . . . & 200 \\
\hline do $\ldots \ldots \ldots$. & 93 & & Conveyed...... & 200 \\
\hline do $\ldots \ldots \ldots$. & 94 & & Conveyed...... & 200 \\
\hline do $\ldots . . .$. & 95 & & Conveyed...... & 200 \\
\hline do $\ldots$ & 96 & & Conveyed ..... & 200 \\
\hline do & 97 & & Conveyed ...... & 200 \\
\hline do & 98 & & Conveyed . . . . . . & $\cdot 200$ \\
\hline (i) & 99 & & Conveyed... & 204 \\
\hline do & 100 & & Conveyed...... & 200 \\
\hline do ... & 101 & & Conveyed...... & 209 \\
\hline do & 102 & & Conveyed ....... & 200 \\
\hline do & 103 & & Conveyed...... & 200 \\
\hline do & 104 & & Purchase \& 1900. & 200 \\
\hline$i(u)$ & 105 & & P'urchase....... & 200 \\
\hline do .. & 106 & & Purchase \& 1900. & 200 \\
\hline do & 107 & & Purchase \& 1900. & 200 \\
\hline do $\ldots$ & 108 & All in Arietta...... & Purchase \& 1900. & 133.30 \\
\hline Jake Pleasant. & 108 & All in Take Pleasant. & Purchase....... & 76.70 \\
\hline do & 109 & All in Lake Pleasant. & Purchase... . . & 200.30 \\
\hline Arictla & 109 & All in Ariettat. & Purchase. & 9.311 \\
\hline do $\ldots$ & 110 & All in Arietta.. & Purchase. . & 178 \\
\hline Iuake Pleasant. . & 110 & All in Lake Pleasant. & Purchase........ & 32 \\
\hline Arietta....... & 111 & & Conveyed \& 1895. & 200 \\
\hline do & 112 & & Conveyed \& 1895.. & 200 \\
\hline
\end{tabular}




\section{Hamiton County}

\begin{tabular}{|c|c|c|c|c|}
\hline Town & Lot & DESCRIPTION & $\begin{array}{c}\text { Tax sales } \\
\text { or } \\
\text { source of title }\end{array}$ & Acres \\
\hline & & $\begin{array}{c}\text { Moose River Tract, Town- } \\
\text { ship } 9-(\text { Continued })\end{array}$ & & \\
\hline Irietta .... . & 113 & & $\begin{array}{l}\text { Purchase \& } 1900, \\
' 05 \ldots \ldots \ldots \ldots\end{array}$ & 200 \\
\hline do & 114 & & Conveyed \& 1895. & 200 \\
\hline do & 115 & $\ldots$ & Conveyed....... & 200 \\
\hline do $\ldots$. & 116 & & Purchase... & 200 \\
\hline do & 117 & & Purchase.... & 200 \\
\hline do & 118 & Ех. 7.ја. W. pt.... & $1877, ' 81$, 'S5.... & 125 \\
\hline do & 119 & $\ldots \ldots \ldots$ & Purchase \& 1900. & 200 \\
\hline do $\ldots$. & 120 & & Purchase \& 1900. & 244 \\
\hline do $\ldots$ & 121 & & Purchase....... & 120.78 \\
\hline do $\ldots$ & 122 & & Purchase... & 382.42 \\
\hline do & 123 & & Purchase. . & 200 \\
\hline do & 124 & & Conveyed.... & 200 \\
\hline$\ldots \ldots$ & 125 & & $1885,{ }^{\prime} 90,{ }^{\prime} 00 \ldots$ & 200 \\
\hline$\ldots \ldots$ & 126 & $\ldots$ & Conveyed \& 1895. & 200 \\
\hline$\ldots \ldots \ldots 1$ & 127 & & Conveyed \& 1895. & 200 \\
\hline do & 128 & W. pt......... & Conveyed \& 1900. & 180 \\
\hline do & 128 & Ex. 180 a. W. pt. . & Purchase........ & 20 \\
\hline do & 129 & All in Arietta..... & Conveyed \& 1895. & 201 \\
\hline Lake Pleasant. & 129 & All in Lake Pleasant... & Conveyed \& 1900. & 4 \\
\hline do & 131 & & Purchase. & 210 \\
\hline do & 132 & & 1890, '95. & 210 \\
\hline do & 133 & & $1890, ' 95$ & 200 \\
\hline Arietta. . & 134 & All in Irietia. & $18(90,9) 5,00$. & 93.75 \\
\hline Lake Pleasant. . & 134 & All in Lake Pleasant... . & $1885, .90,{ }^{\prime} 00 \ldots$ & 112.25 \\
\hline Arietta ........ & 135 & $\ldots \ldots \ldots \ldots \ldots \ldots$ & $1885,{ }^{\prime} 90,{ }^{\prime} 00 \ldots$. & 200 \\
\hline do & 136 & & Conveyed. . . . & 200 \\
\hline do & 137 & & $1885, ' 90,{ }^{\prime}(10 \ldots \ldots$ & 200 \\
\hline do & 140 & Triangle N. W. cor . . . . . . & $1890 \ldots \ldots \ldots \ldots$ & 2 \\
\hline do & 144 & $\begin{array}{l}\text { Ex. 200a. N. pt. Adiron- } \\
\text { dack Co.'s land .......... }\end{array}$ & 1881, 'S5, '90, '95. & 70 \\
\hline do & 144 & N. pt.............. & Conveyed........ & 200 \\
\hline do & 145 & $\ldots \ldots \ldots \ldots \ldots \ldots$ & $1885,90,95,{ }^{\prime} 00 \ldots$ & 200 \\
\hline do & 116 & All in Arietta. . . . . & Purchase ....... & 150 \\
\hline Lake Pleasant. . & 146 & All in Lake Pleasant. & Purchase....... & $5 i$ \\
\hline do & 147 & All in Lake Pleasant. . & $1885,{ }^{\prime} 95,{ }^{\prime} 00 \ldots \ldots$ & 204 \\
\hline do & 148 & $\ldots \ldots \ldots \ldots \ldots \ldots$ & $1885,{ }^{\prime} 90, ' 00 \ldots$. & 200 \\
\hline do & 149 & S. E. cor. of E. $\frac{1}{2}$.. & 1881, ' $85,90,95$. & 75 \\
\hline do & 149 & W. $\frac{1}{2} \ldots \ldots \ldots \ldots$ & Conveyed ...... & 105 \\
\hline do & 149 & E. $\frac{1}{2}$ ex. 75 a. S. E. cor . & Purchase ........ & 30 \\
\hline do & 150 & N. E. $\frac{1}{2} \ldots$ & Purchase.... . & 105 \\
\hline do & 150 & N.W. ${ }_{4}^{2}$ & $1881,8.5,90$. & 52.50 \\
\hline
\end{tabular}


Hamilton Country

\begin{tabular}{|c|c|c|c|c|}
\hline 'Town & Lot & DESCRIPTION & $\begin{array}{l}\text { Tax sales } \\
\text { or } \\
\text { source of title }\end{array}$ & Acres \\
\hline & & $\begin{array}{l}\text { Moose RIVER Tract, } \\
\text { Township } 9-\text { (Continued) }\end{array}$ & & \\
\hline \multirow{7}{*}{$\begin{array}{l}\text { Lake Pleasant } \\
\text { do } \\
\text { do } \\
\text { do } \\
\text { do } \\
\text { do } \\
\text { do }\end{array}$} & 150 & S. W. part.......... & Purchase. & 20 \\
\hline & 151 & $\ldots \ldots \ldots \ldots \ldots \ldots$ & Conveyed \& $1895 .$. & 200 \\
\hline & 152 & N. pt. & Conveyed \& $1895 .$. & 128 \\
\hline & 152 & Ex. 128a. N. pt....... & Purchase....... & 92 \\
\hline & 153 & Ex. 150a. E. pt. of S. ${ }_{2}^{1} \ldots$ & Purchase........ & 128 \\
\hline & 153 & E. $150 \mathrm{a}$. of S. $250 \mathrm{a} . .$. & Conveyed \& $1595 .$. & $150.7 \pi$ \\
\hline & 154 & $\begin{array}{l}\text { All in Lake Pleasant of 100a. } \\
\text { E. pt . . . . . . . . . . }\end{array}$ & Purchase.... . & 22 \\
\hline \multirow{4}{*}{$\begin{array}{l}\text { Arietta ........ } \\
\text { Lake Pletwant. } \\
\text { do }\end{array}$} & 154 & All in Arietta of $100 \mathrm{a}$. E. pt. & Purchase... . & 78 \\
\hline & 156 & & Conveyed \& $1895 .$. & 200 \\
\hline & 160 & $\begin{array}{l}\text { Total, Township 9, } \\
\text { Moose River Tract, } \\
22,095.34 \text { acres. }\end{array}$ & Purchase..... & $137: 24$ \\
\hline & & $\begin{array}{c}\text { Moose River Tract, } \\
\text { Township } 10\end{array}$ & & \\
\hline Irictlit. & 1 & $\ldots \ldots \ldots \ldots \ldots$ & Purchase.... . & 212 \\
\hline do & 2 & & Purchase.... . & 200 \\
\hline do & 3 & & Purchase..... & 200 \\
\hline do & + & & Purchase.... & 210 \\
\hline do $\ldots$ & ¿ & All in Arietta..... & Purchase.... & 113.5 \\
\hline Morehouse..... & 5 & All in Morehouse.... & Purchase... . & 92.70 \\
\hline Arietta....... & 6 & $\ldots \ldots \ldots \ldots \ldots$ & Purchase...... & 160 \\
\hline do. & 7 & $\ldots$ & Purchase... . & 160 \\
\hline do. & s & & Purchase... . & 160 \\
\hline do & 9 & & Purchase.... & 160 \\
\hline do .. & 10 & & Purchase... & 160 \\
\hline Morehouse... & 11 & All in Morehouse. . & Purchase ... . . & 9.30 \\
\hline Arietta. & 11 & All in Arietta. . & Purchase... . & 150.70 \\
\hline do & 12 & All in Arietta . . . . . & Purchase.... & 17.70 \\
\hline Morehouse... & 12 & All in Morehouse... . : & Purchase.... & 142.30 \\
\hline do & 13 & $\ldots \ldots \ldots \ldots \ldots$ & Purchase.... & 128 \\
\hline do & 14 & $\ldots \ldots \ldots$ & Purchase.... & 72 \\
\hline do & 15 & $\ldots \ldots \ldots$ & Purchase.... & 200 \\
\hline do & 16 & & Purchase. . & 200 \\
\hline do & 17 & All in Morehouse...... & Purchase... & 135.25 \\
\hline Arietta & 17 & All in Arietta...... & Purchase. & 64.75 \\
\hline do & 18 & $\ldots \ldots \ldots \ldots \ldots \ldots \ldots$ & Purchase. & 200 \\
\hline do & 19 & $\ldots \ldots \ldots \ldots \ldots \ldots \ldots$ & Purchase. & 200 \\
\hline do & 20 & & Purchase. . & 200 \\
\hline do & 21 & All in Arietta. . & Purchase ....... & 104 \\
\hline
\end{tabular}


Hamilton Countr

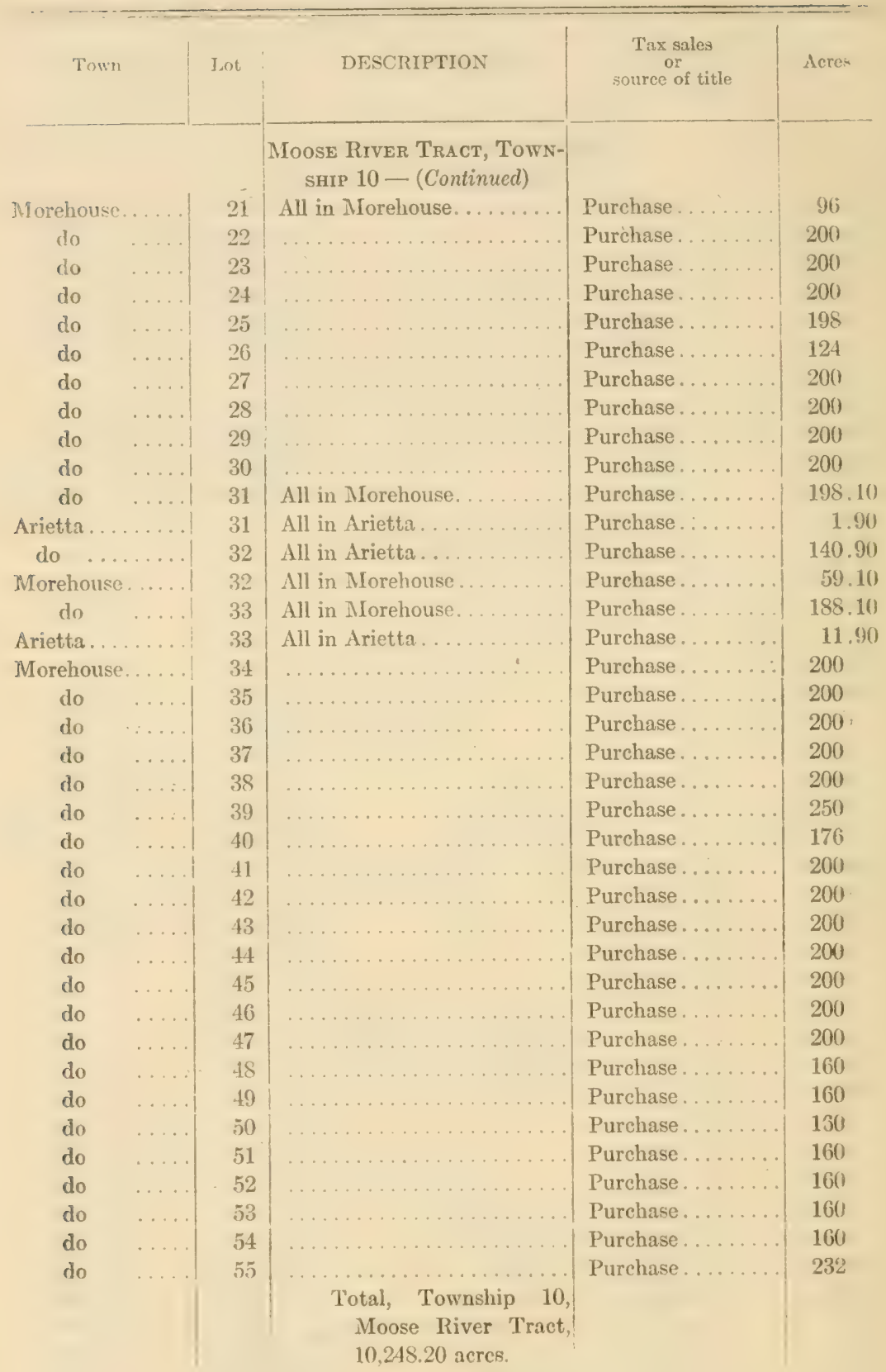




\section{Hamiton County}

\begin{tabular}{|c|c|c|c|c|}
\hline Town & Lot & DESCRIPTION & $\begin{array}{c}\text { Tax sales } \\
\text { or } \\
\text { source of tithe }\end{array}$ & Acres \\
\hline & & Oxbow Tract. & & \\
\hline Wells. & * & & Purchase.... & 3.50 \\
\hline do & 1 & & $\begin{array}{r}\text { Purchase \& } 1890 \\
95 \ldots \ldots \ldots\end{array}$ & (1:3) \\
\hline Lake Pleasant. & $\ddot{2}$ & & Purchase. & 315 \\
\hline do & 3 & & $1890,95$. & 219 \\
\hline do & 4 & . & $189): 95$ & $14 !$ \\
\hline do & 5 & & $1890,{ }^{\prime} 95 \ldots$ & 247 \\
\hline do & 6) & & $1855.90 \ldots$ & 345 \\
\hline do & 7 & & 18 si, , 90. & $4 \pm 1$ \\
\hline do & 8 & & $1590,95$. & 320 \\
\hline do & 9 & & Purchase ....... & 320 \\
\hline Wils... & 10 & & $1577, ' s 1,85 .$. & 646 \\
\hline Iake Pleasant. & 12 & & Purchase......... & 320 \\
\hline do & 13 & & $1 \times \$ 1,85,900$ & 320 \\
\hline do & 14 & 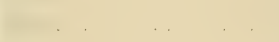 & 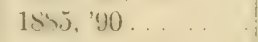 & 320 \\
\hline do & 15 & $\ldots$ & $1885,90 \ldots \ldots$ & 320 \\
\hline do & 16 & & $1855,90,95 \ldots$ & 320 \\
\hline do & 17 & & $1890,95 \ldots \ldots$ & 320 \\
\hline do & 18 & $\ldots$ & $\begin{array}{r}1877, ' 81, ' 85,90, \\
95, ' 00 \ldots \ldots\end{array}$ & 373 \\
\hline do & 19 & & $1871,77, ' 81, ' 85$. & $27 \frac{1}{2}$ \\
\hline do & 23 & E. $\frac{1}{2}$ & $1890,{ }^{\prime} 95$. & 160 \\
\hline do & 24 & $\ldots$ & $1890, ' 95,{ }^{\prime} 00 \ldots$. & 320 \\
\hline do & 25 & $\ldots$ & $1890,{ }^{\prime} 95,{ }^{\prime} 00 \ldots$ & 320 \\
\hline do & 26 & & $1885, ' 90, ' 95,{ }^{\prime} 00$. & 320 \\
\hline do & 27 & & $1890,95 \ldots \ldots$ & 320 \\
\hline do & 28 & & $1843,00 \dagger \ldots \ldots$ & 320 \\
\hline do & 29 & & $1890,95 \ldots$ & 320 \\
\hline do & 30 & & Purchase...... & 320 \\
\hline Wells... & 31 & All in Wells.. & Purchase....... & 195 \\
\hline do & 32 & All in Wells. & $1881,{ }^{\prime} 85,{ }^{\prime} ' 30 \ldots$. & 17 \\
\hline dn & 33 & $\ldots \ldots \ldots \ldots$ & Purchase \& 1905. & 166 \\
\hline do & $3 t$ & & $1877, ' 81,{ }^{\prime} 85$. & 271 \\
\hline rlo ... & 35 & & $1813, ' 00 \nmid$ & 310 \\
\hline do $\ldots$ & 36 & All in Well: & $1885,90 \ldots$ & 307 \\
\hline do ... & 37 & All in Wells... & $1843,{ }^{\prime} 00 \dagger \ldots$ & 248 \\
\hline Irietta & 37 & All in Ariettat. & 1813, '00广் . & 62 \\
\hline do & 38 & All in Ariettit. & Purchase. . & 140 \\
\hline Wells.. & $3 S$ & All in Wells. . & Purchase.... & 170 \\
\hline do $\ldots$ & 39 & All in Wells... & Purchase.... & 33.65 \\
\hline Benson. . & 39 & All in Benson. & Purchase. & $41.8 \%$ \\
\hline
\end{tabular}

* Albany corporation lot. $\dagger$ Resale. 
Hamilton County

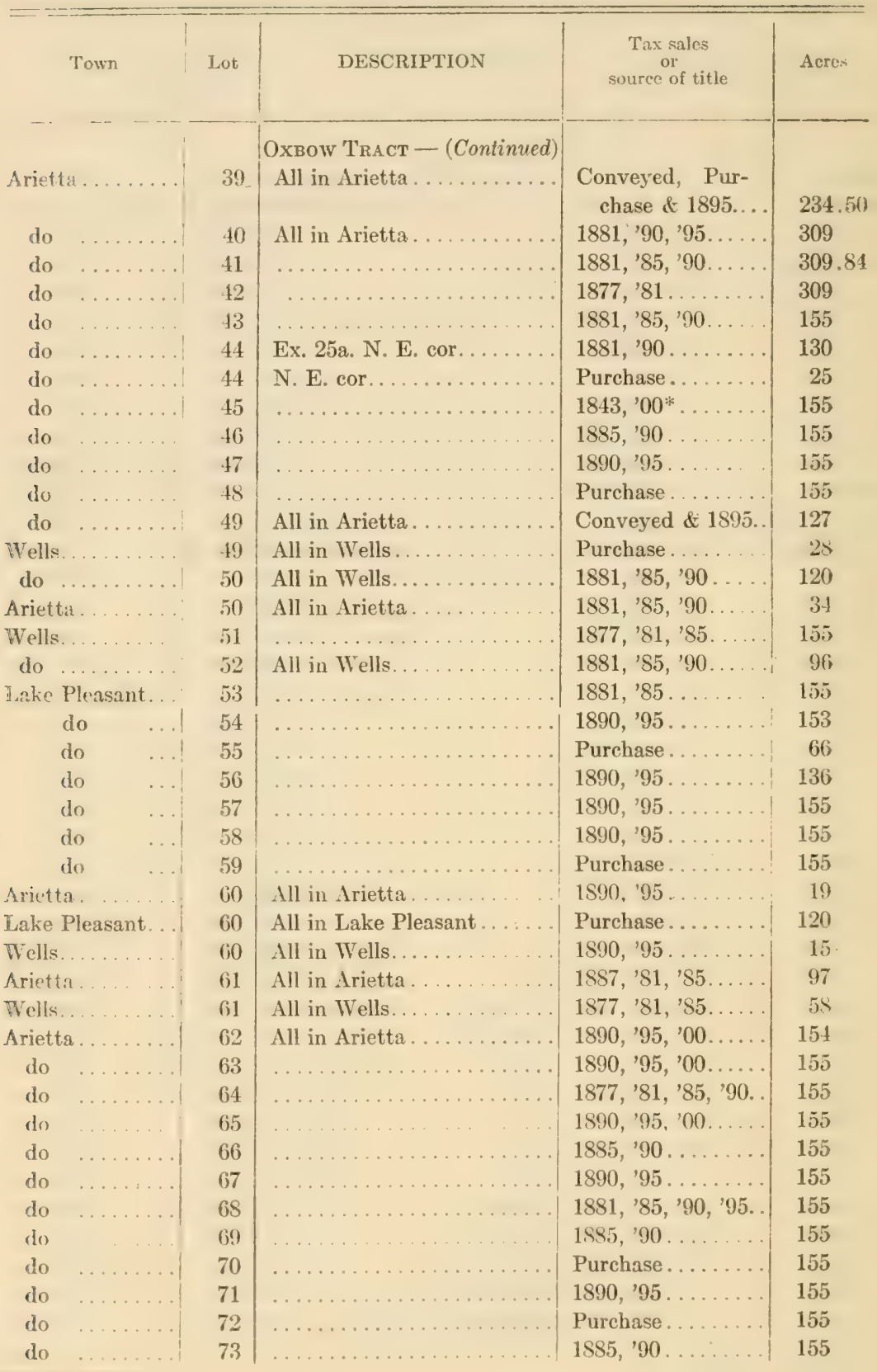

kesale. 
Hamilton County

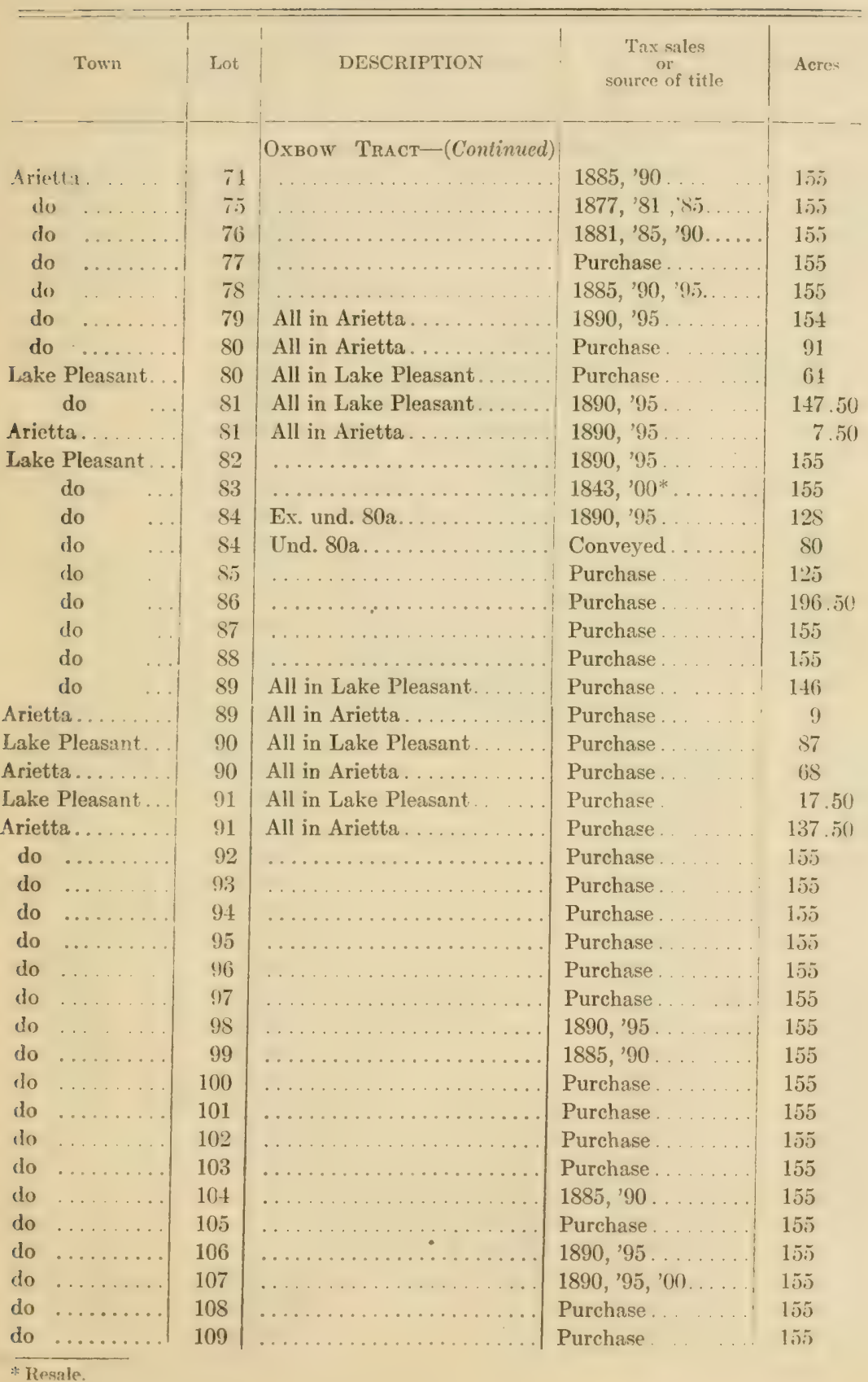


Hamlton County

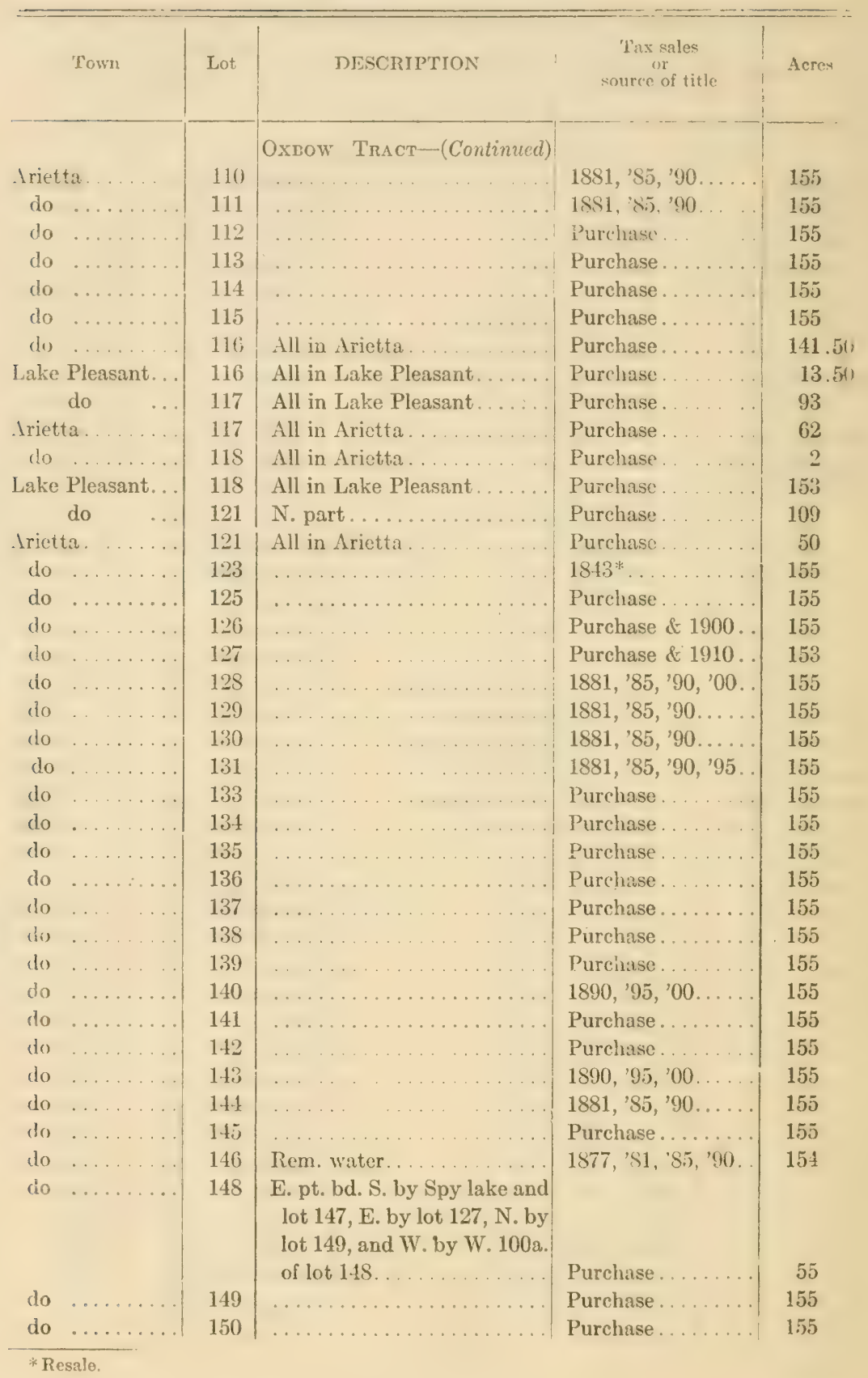


Hammon County

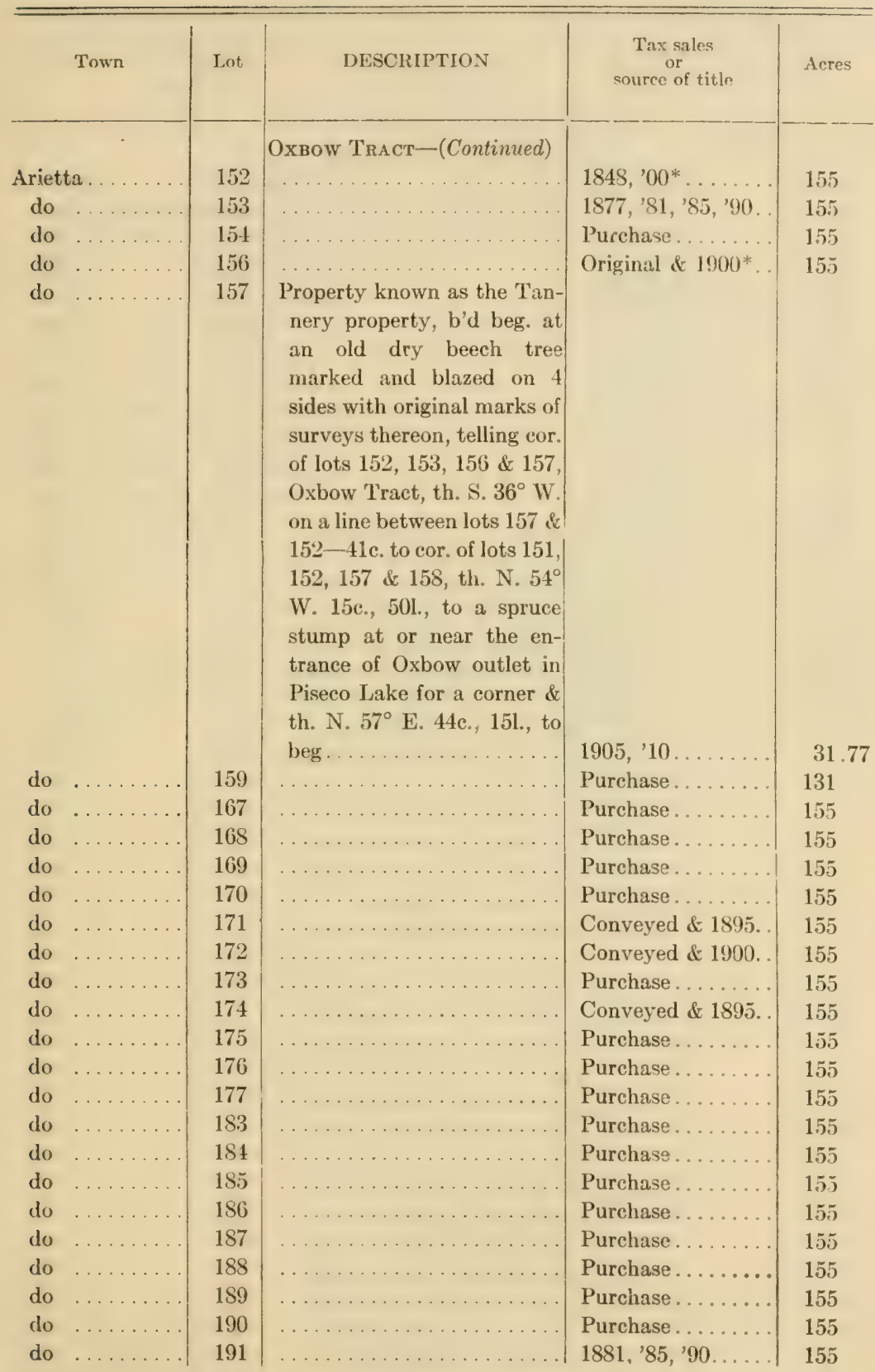

* Resale. 
Hamilton County

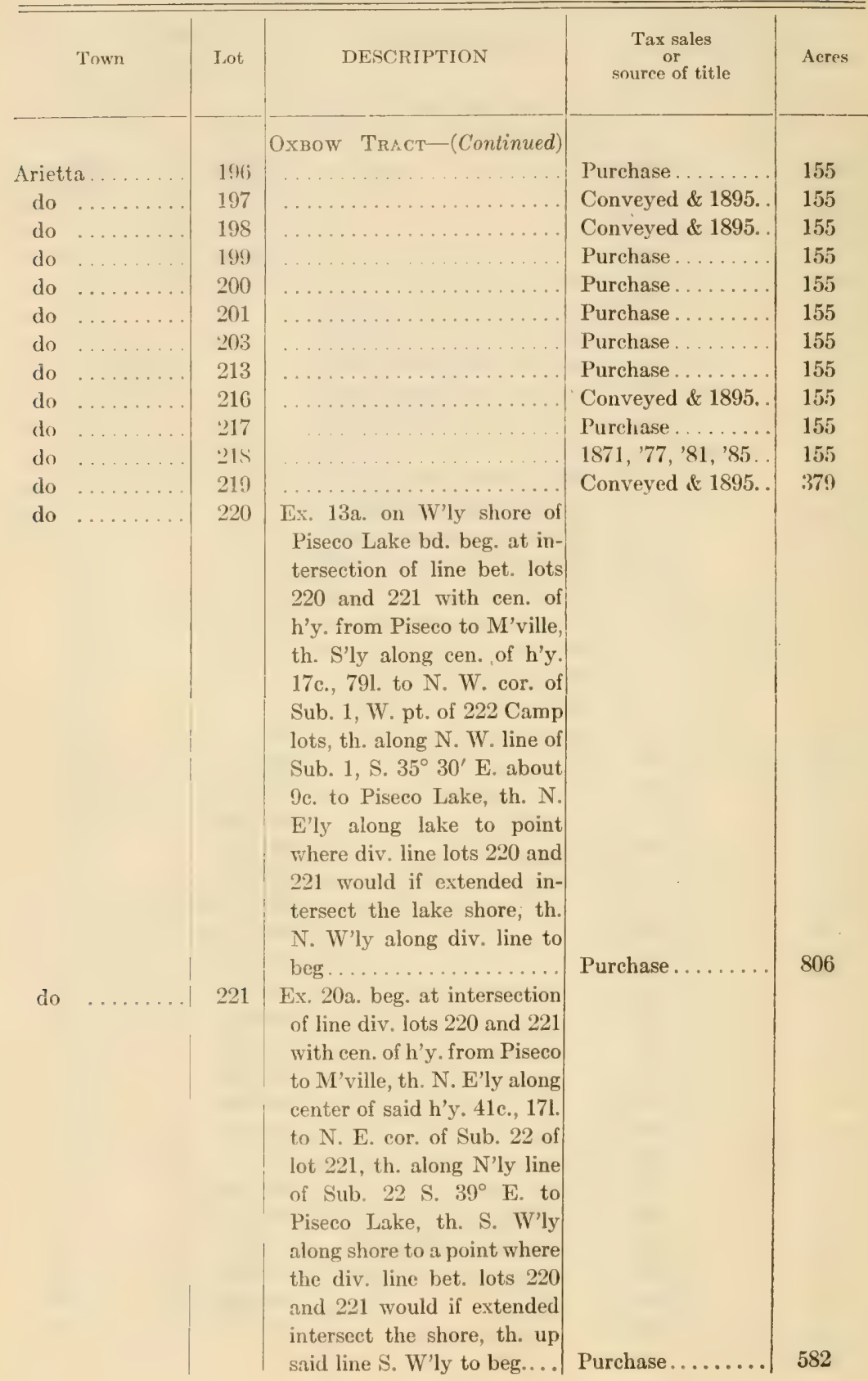


Hamilton County

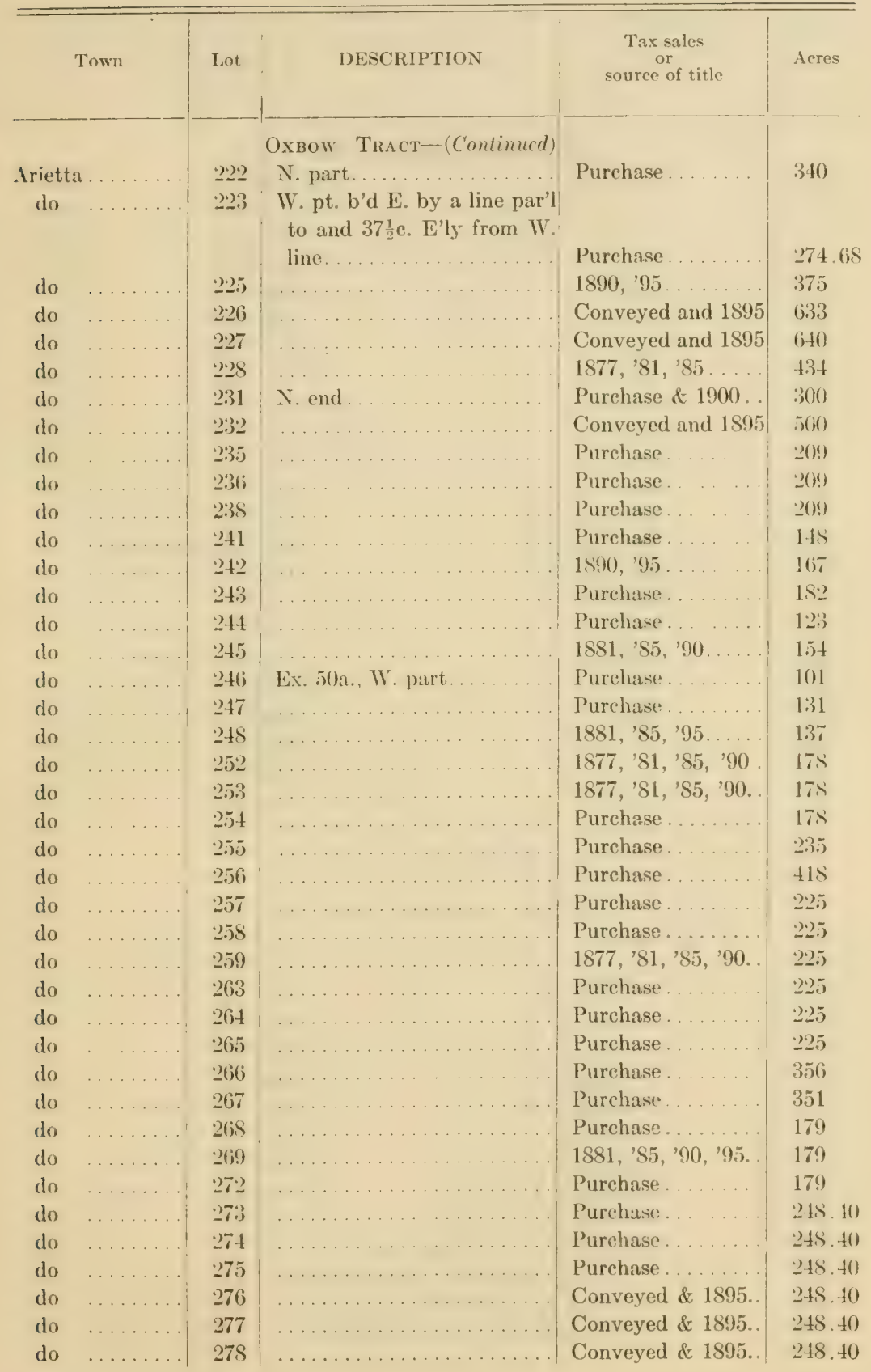


Hamiton County

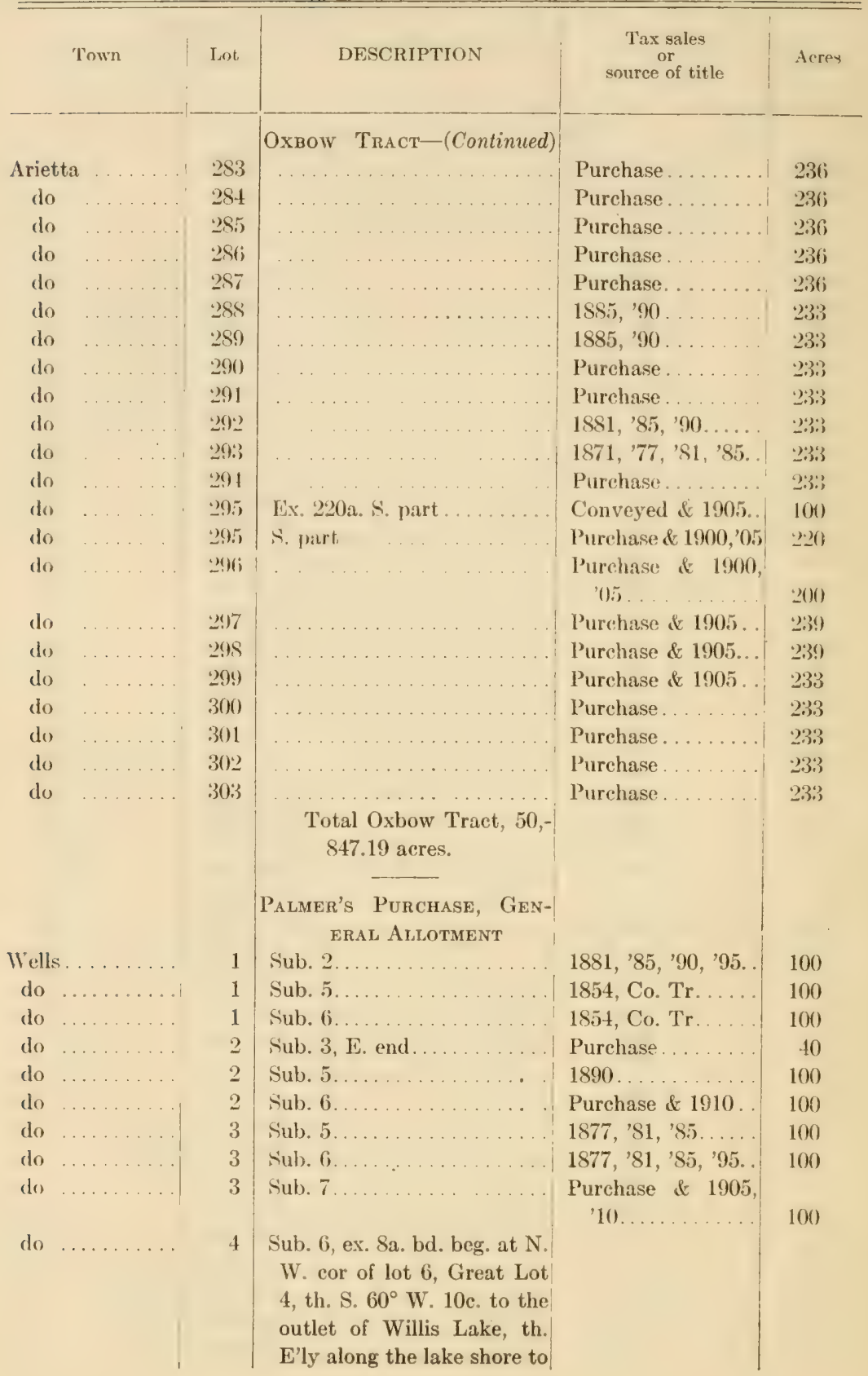


Hamilton County

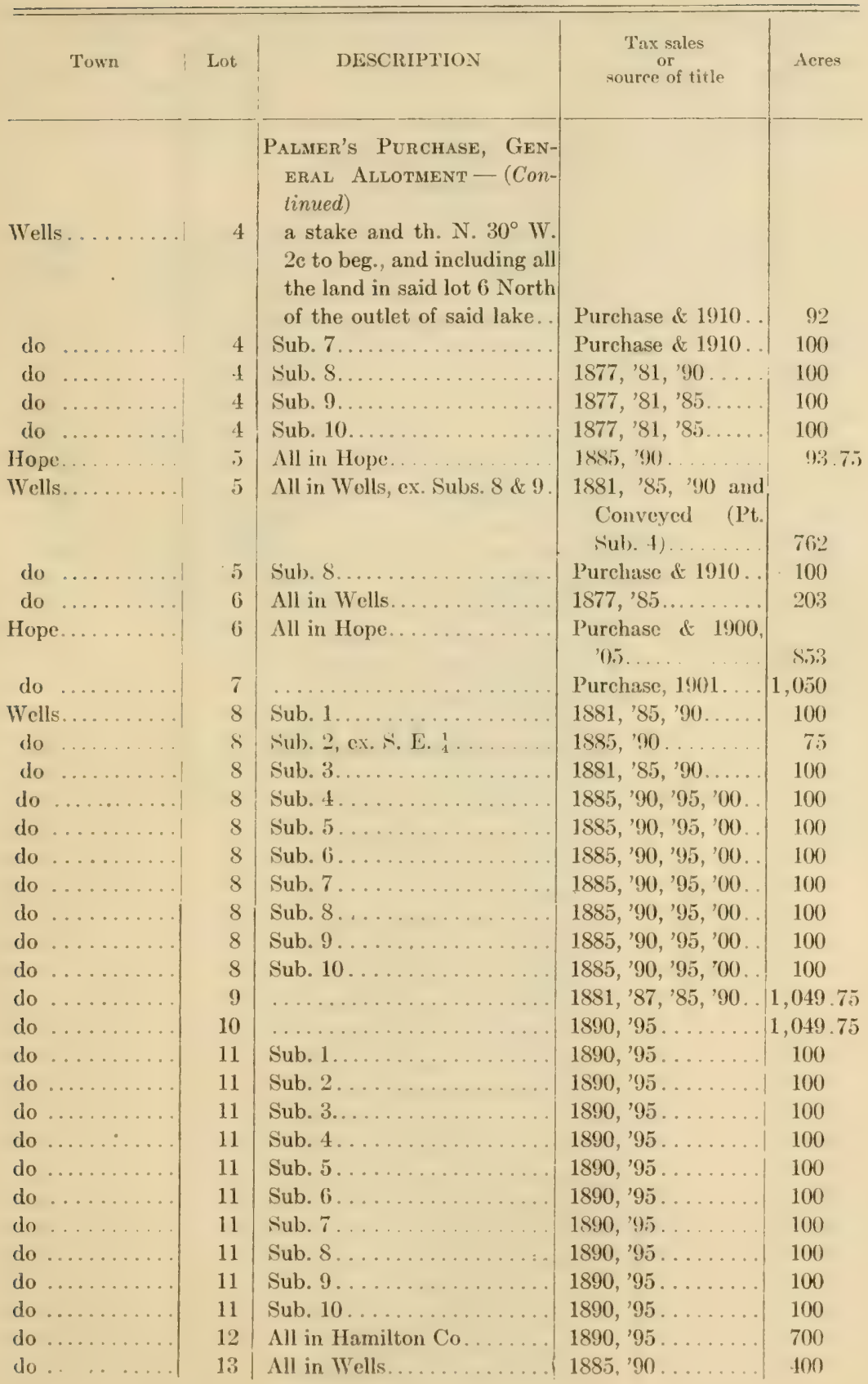




\section{Hamiton County}

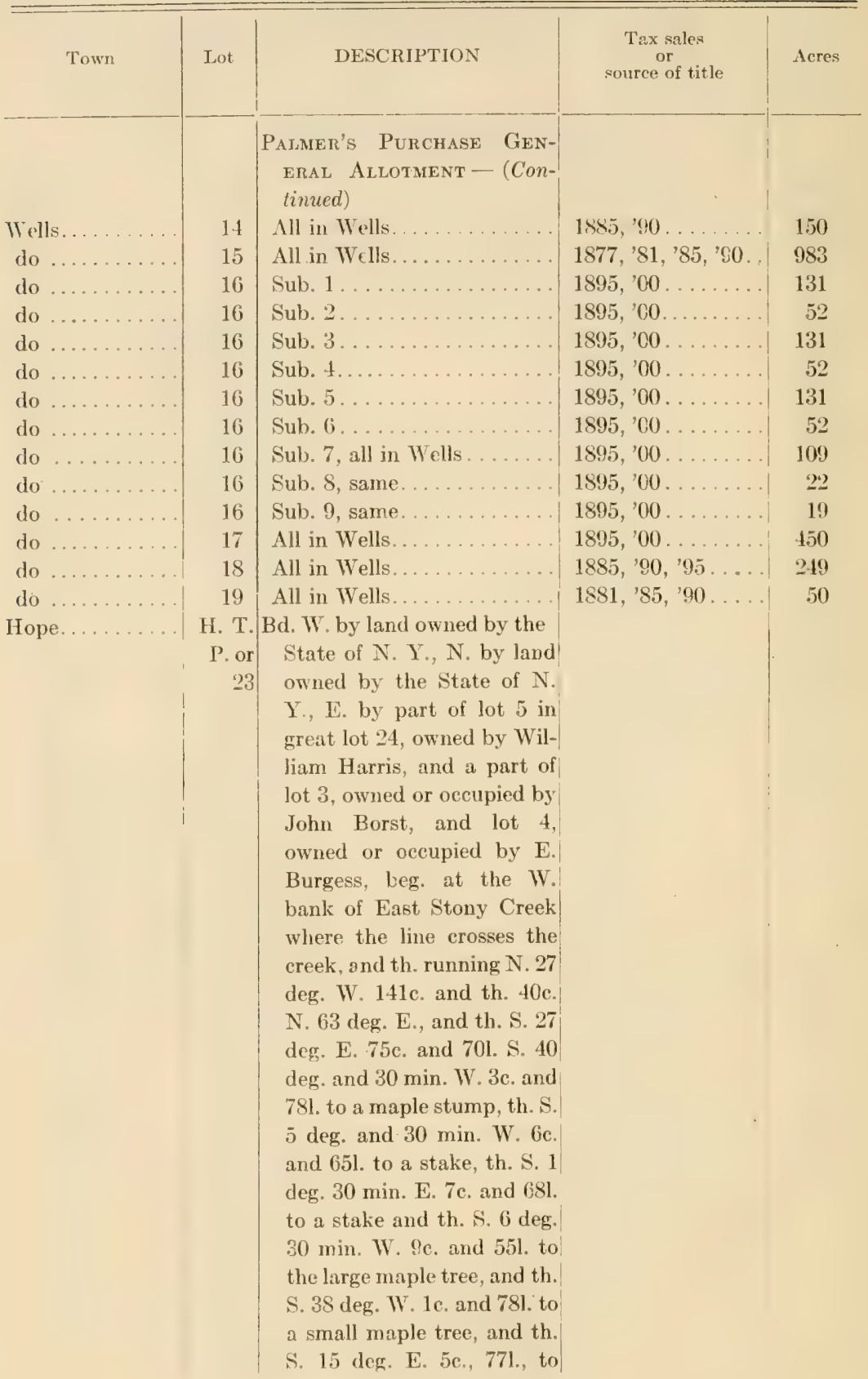


Hamilton County

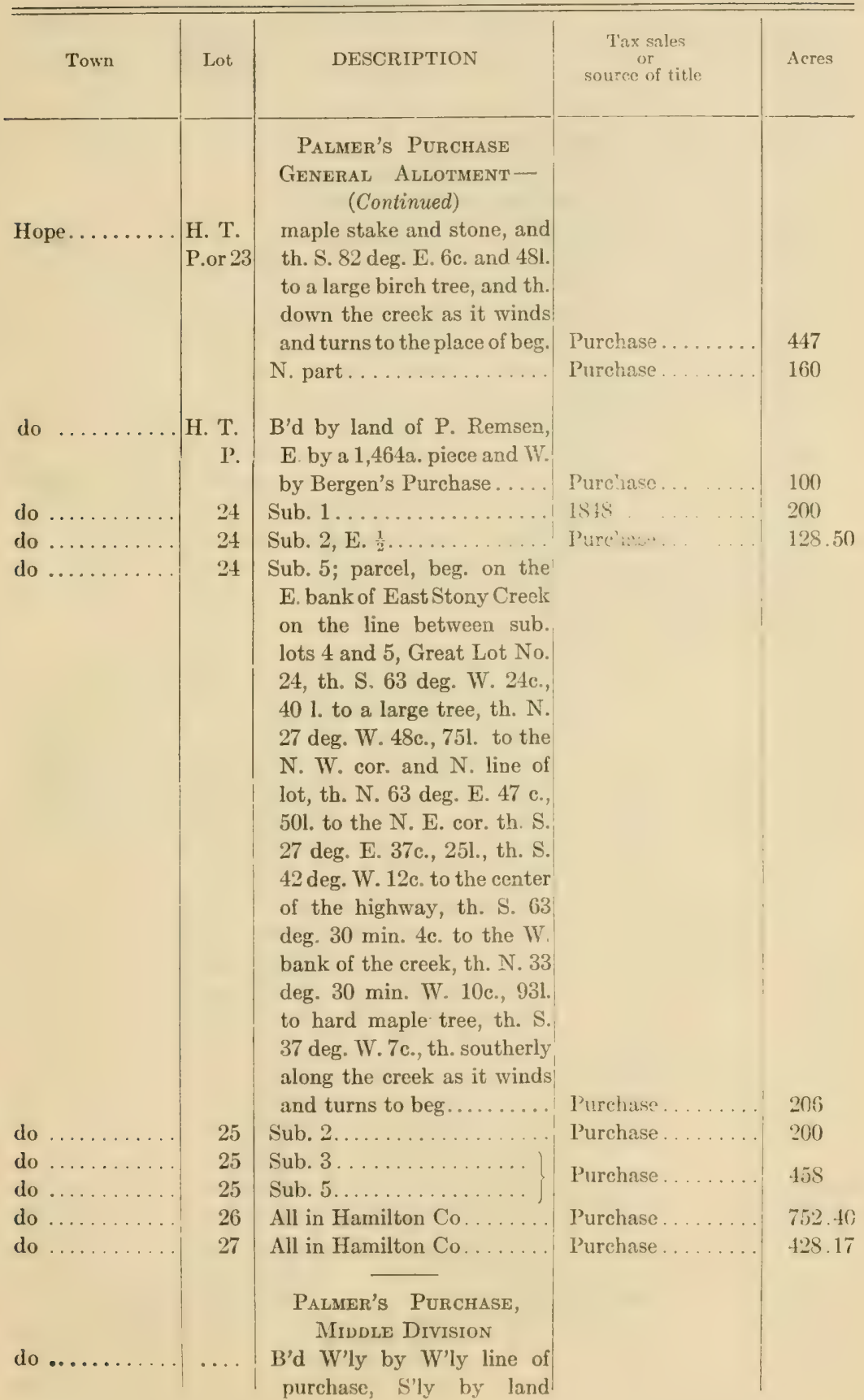


Hamliton County

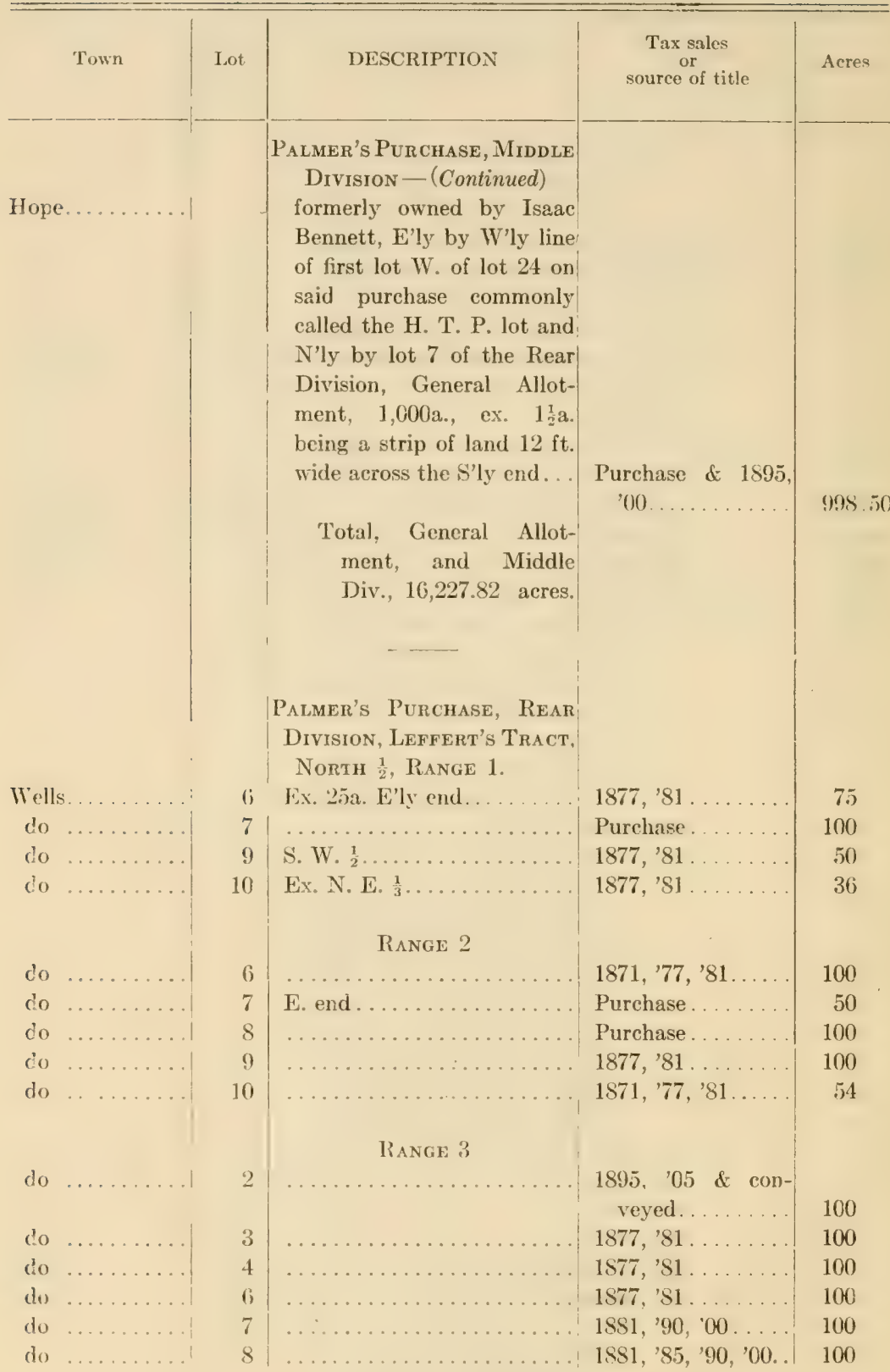




\section{Hamliton Countx}

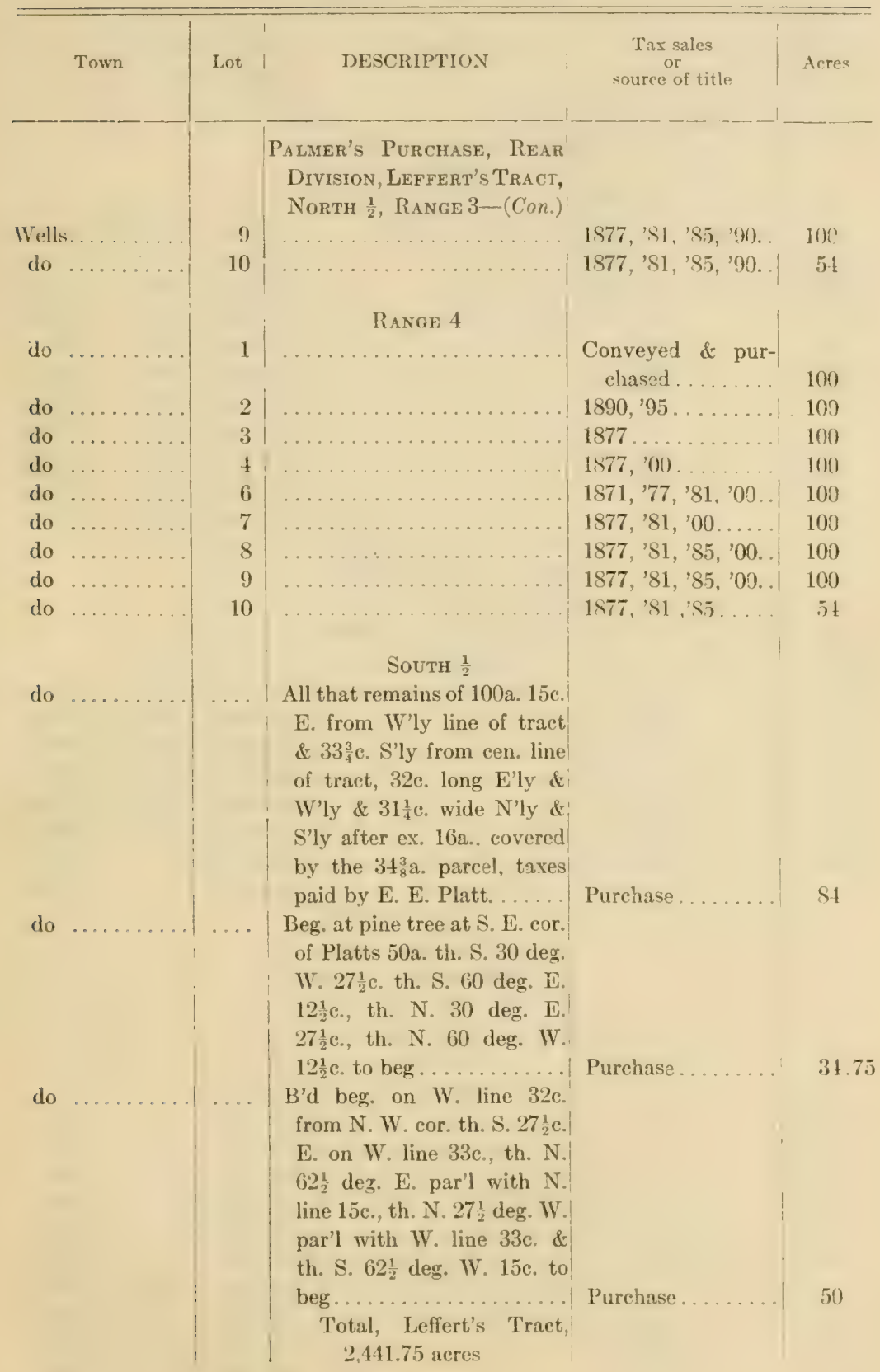


HAMILTON CoUNTY

\begin{tabular}{|c|c|c|c|c|}
\hline Town & Lot & DESCRIPTION & $\begin{array}{c}\text { Tax sales } \\
\text { or } \\
\text { source of title }\end{array}$ & Acres \\
\hline 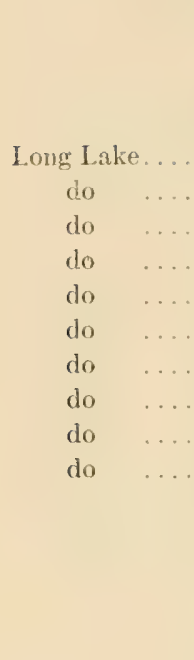 & $\begin{array}{l}27 \\
34 \\
35 \\
36 \\
37 \\
38 \\
39 \\
42 \\
43 \\
44\end{array}$ & 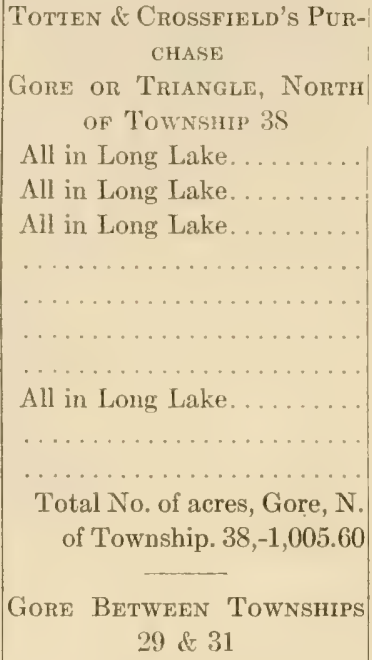 & $\begin{array}{l}\text { Purchase \& } 1900 \ldots \\
\text { Purchase \& } 1900 \ldots \\
\text { Purchase \& } 1900 \ldots \\
\text { Purchase \& } 1900 \ldots \\
\text { Purchase \& } 1900 \ldots \\
\text { Purchase \& } 1900 \ldots \\
\text { Purchase \& } 1900 \ldots \\
\text { Purchase \& } 1900 \ldots \\
\text { Purchase \& } 1900 \ldots \\
\text { Purchase \& } 1900 \ldots\end{array}$ & $\begin{array}{r}62.86 \\
15.20 \\
149.50 \\
160 \\
160 \\
145.76 \\
48.60 \\
.68 \\
180.12 \\
82.88\end{array}$ \\
\hline 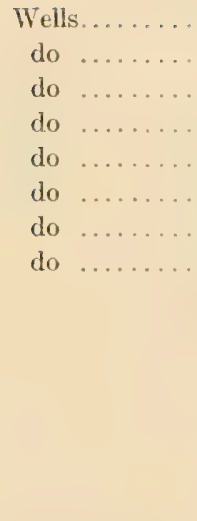 & $\begin{array}{l}1 \\
2 \\
3 \\
4 \\
5 \\
6 \\
7 \\
8\end{array}$ & 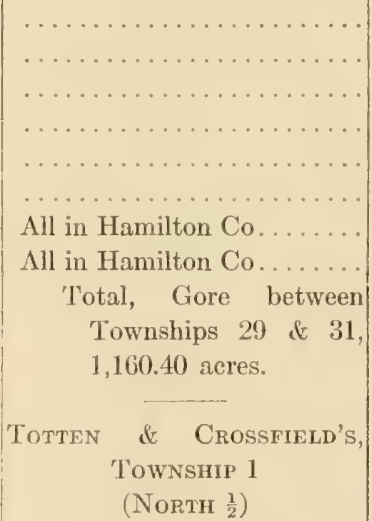 & $\begin{array}{l}\text { Conveyed \& 1905.. } \\
\text { Conveyed \& 1905.. } \\
\text { Conveyed \& 1905.. } \\
\text { Conveyed \& 1905.. } \\
\text { Conveyed \& 1905.. } \\
\text { Conveyed \& 1905.. } \\
\text { Conveyed........ } \\
\text { Conveyed. . . . . . }\end{array}$ & $\begin{array}{c}169.60 \\
169.60 \\
169.60 \\
169.60 \\
169.60 \\
169.60 \\
131 \\
11.80\end{array}$ \\
\hline $\begin{array}{l}\text { Lake Pleasant. } \\
\text { do } \\
\text { do } \\
\text { do } \\
\text { do } \\
\text { do } \\
\text { do } \\
\text { do } \\
\text { do } \\
\text { do }\end{array}$ & $\begin{array}{r}1 \\
2 \\
3 \\
4 \\
5 \\
6 \\
7 \\
12 \\
13 \\
14\end{array}$ & 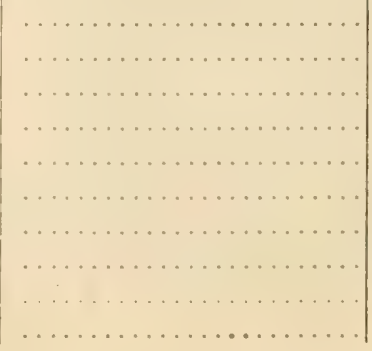 & 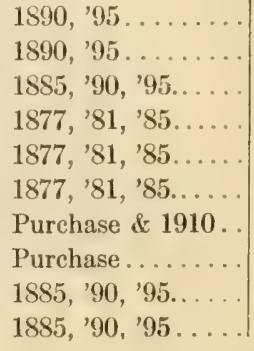 & $\begin{array}{l}170 \\
170 \\
250 \\
250 \\
250 \\
250 \\
250 \\
250 \\
250 \\
250\end{array}$ \\
\hline
\end{tabular}


Hamiton County

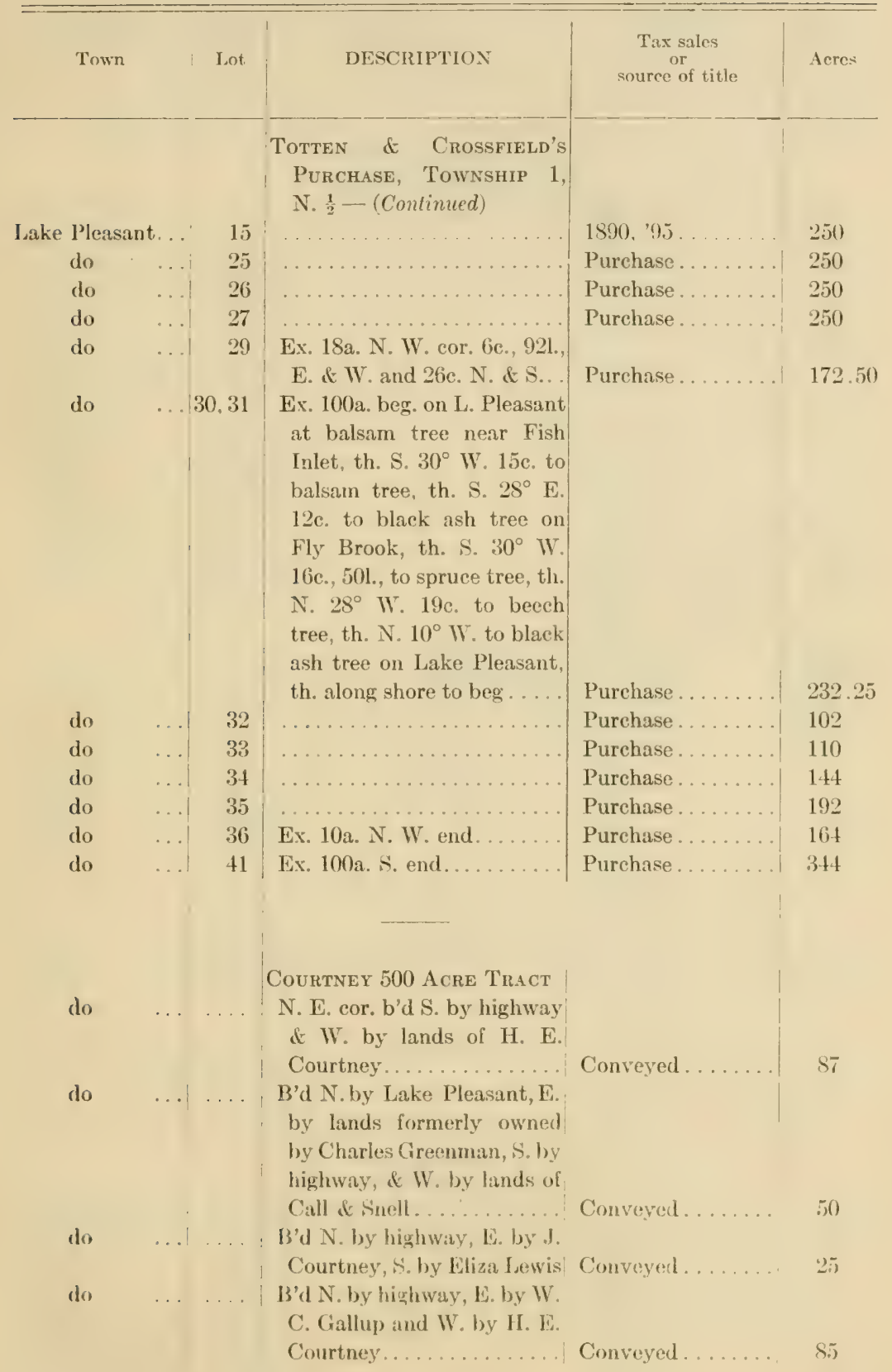


Hamlton County

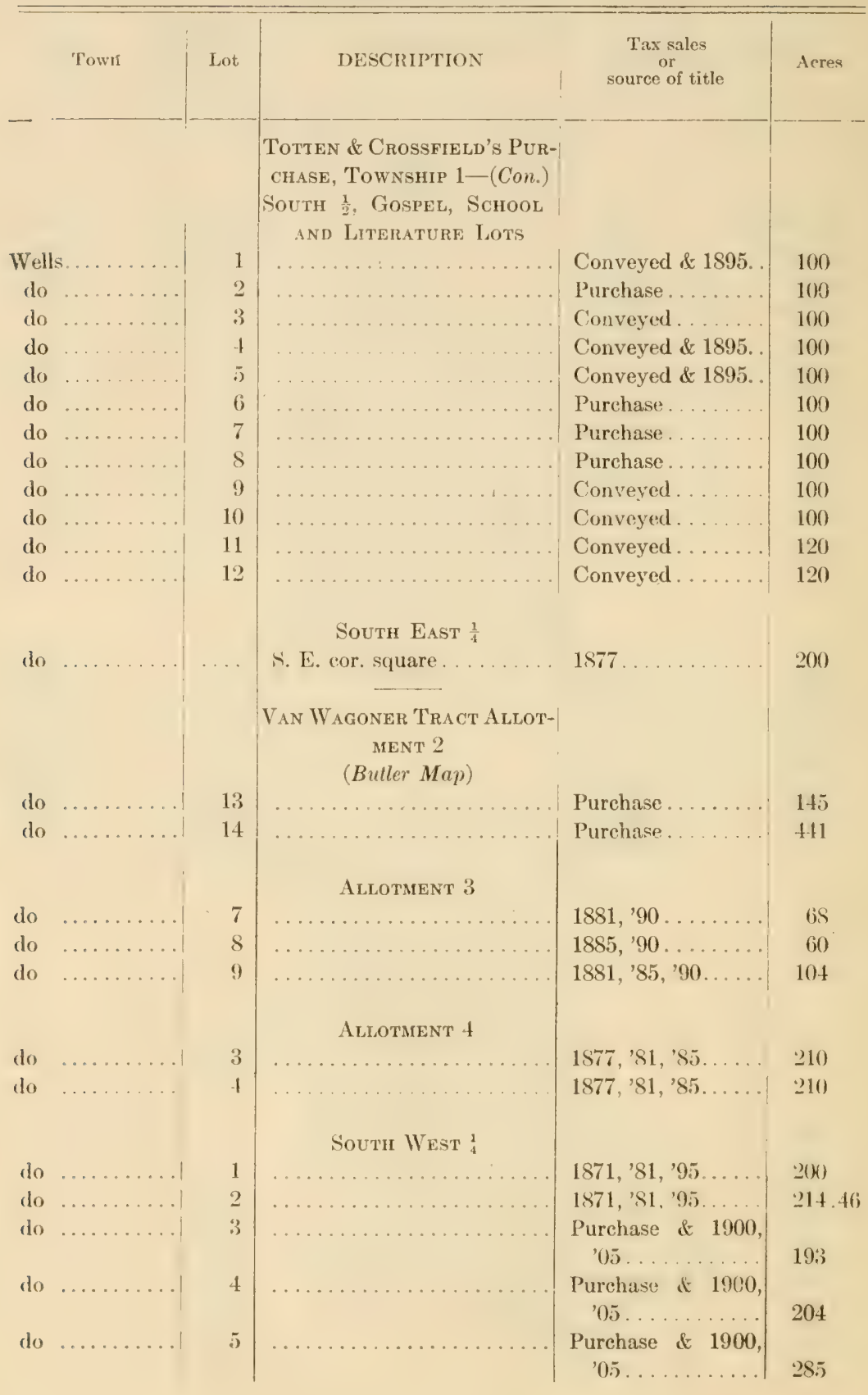


Conservation Commission.

Hamilton County

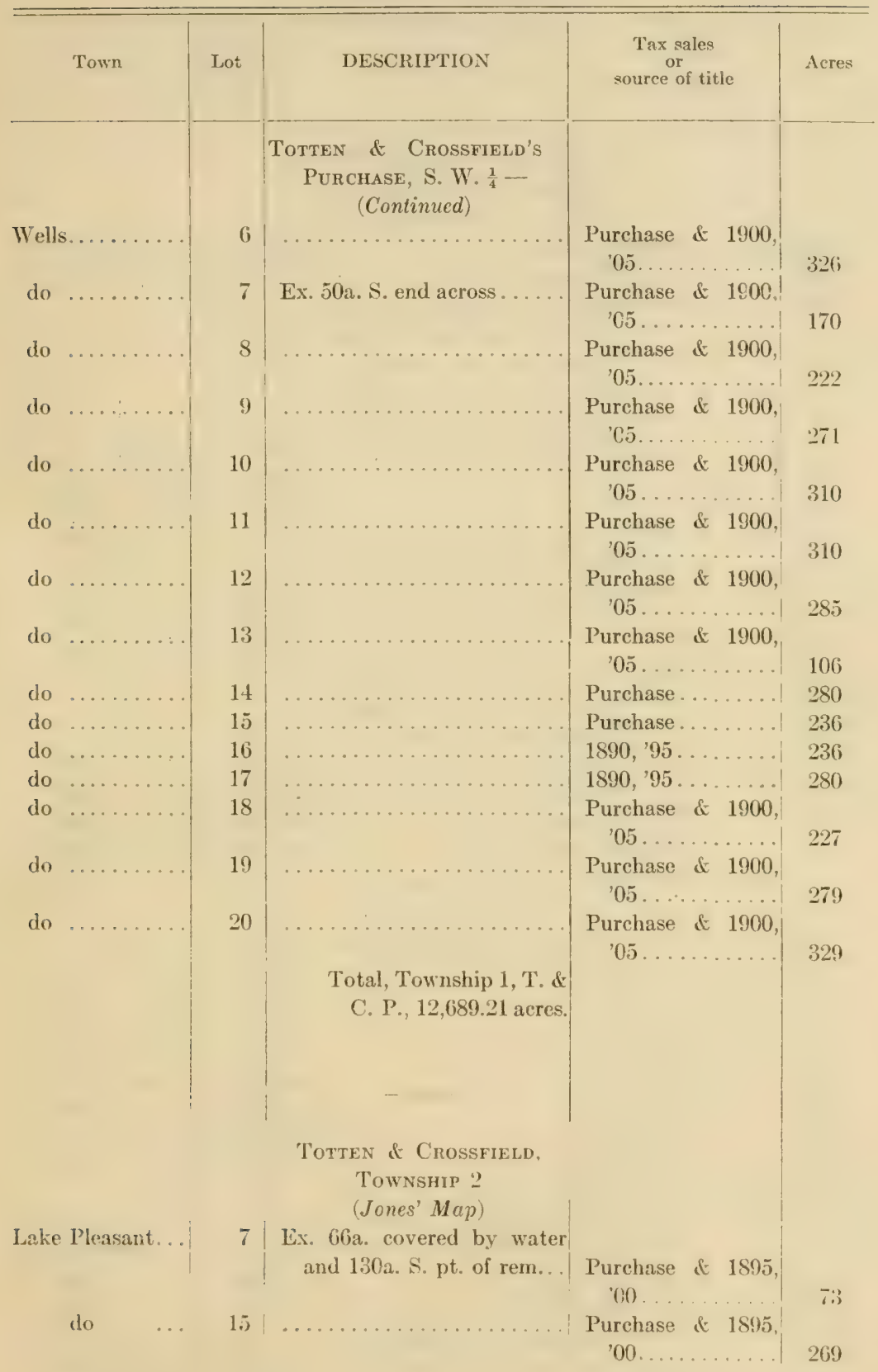


HAMILTON County

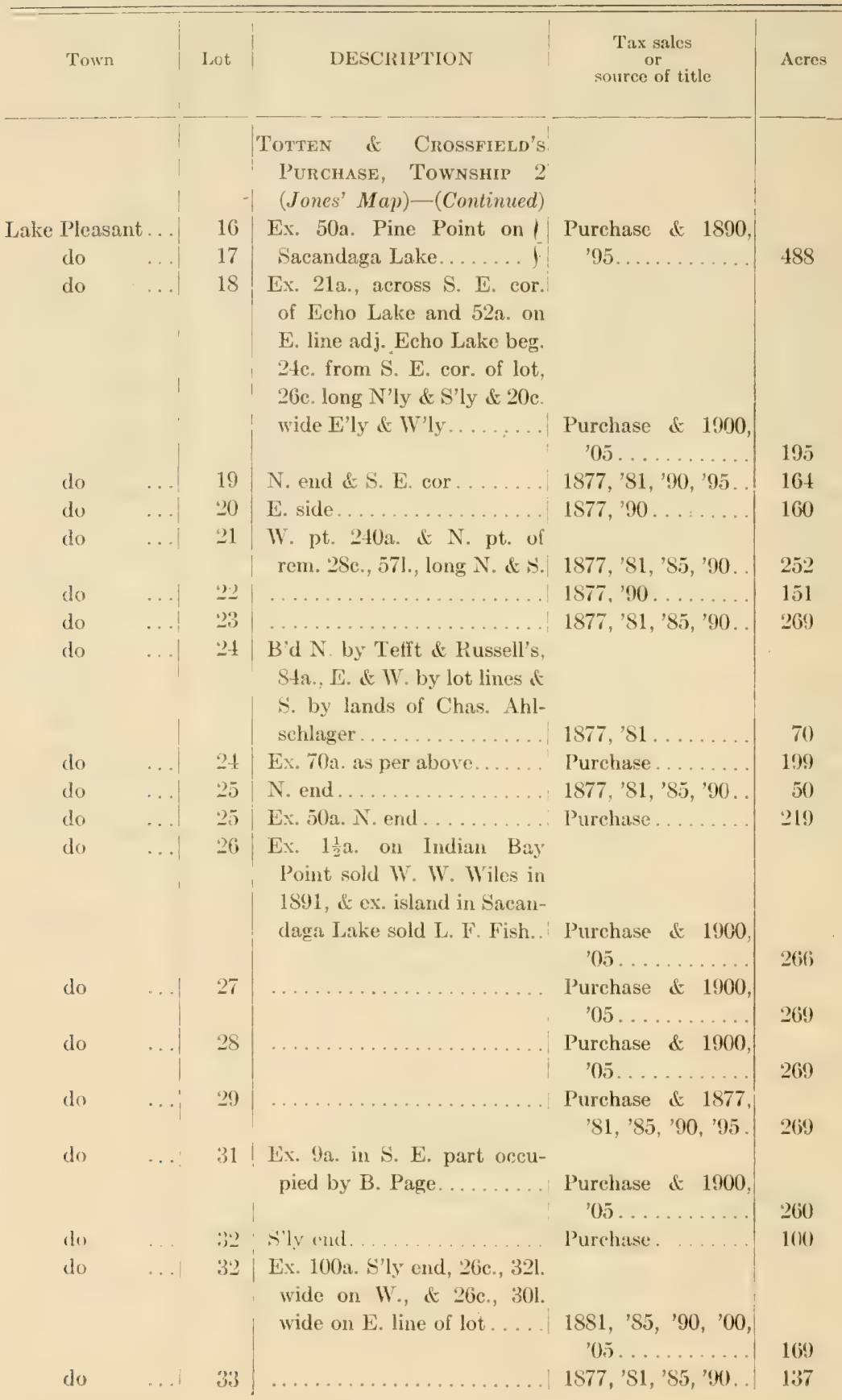


Conservation Commisston.

Hamilon (olinte

\begin{tabular}{|c|c|c|c|c|c|}
\hline \multicolumn{2}{|r|}{ Town } & \multirow[t]{2}{*}{ Lot } & DESCRIPTION & \multirow[t]{2}{*}{$\begin{array}{c}\text { Tax sales } \\
\text { or } \\
\text { souree of title }\end{array}$} & \multirow[t]{2}{*}{ Acres } \\
\hline & & & $\begin{array}{l}\text { Totren \& Crossfield's Pur- } \\
\text { Chase, Township } 2 \text { (Jones' } \\
\text { Map) - (Continued) }\end{array}$ & & \\
\hline Lake & Pleasant & 34 & $\ldots \ldots \ldots \ldots \ldots \ldots \ldots$ & Purchase & 269 \\
\hline & do & 35 & All not under water... & $1885, ' 90, ' 95, ' 00 \ldots$ & 217 \\
\hline & do & 36 & $\ldots \ldots \ldots \ldots \ldots \ldots$ & Purchase \& 1910. & 269 \\
\hline & do & 37 & .. & $\begin{array}{c}\text { Purchase \& } 1900 . \\
\text { (0.5. . . }\end{array}$ & 269 \\
\hline & do & 38 & . & $\begin{array}{l}\text { Purchase \& } 1900 \text {, } \\
\text { (vis . . . . . . }\end{array}$ & 269 \\
\hline & do & 39 & & $\begin{array}{r}\text { Purchase \& } 1900 \text {, } \\
05 \ldots \ldots \ldots \ldots\end{array}$ & \\
\hline & do & 40 & . & $1877, ' 81, ' 85, ' 90 \ldots$ & 269 \\
\hline & do & 41 & & $\begin{array}{l}\text { Purchase \& 1900, } \\
\text { (05. } \ldots \ldots \ldots\end{array}$ & 269 \\
\hline & do & 42 & & $\begin{array}{c}\text { Purchase is } 1900, \\
(0.5 \ldots\end{array}$ & 269 \\
\hline & do & 43 & $\begin{array}{l}\text { Ex. 20a. } N \text {. W. cor d tas } \\
\text { E. cor }\end{array}$ & $1890,95 \ldots$ & 245 \\
\hline & do & 44 & $\ldots \ldots \ldots \ldots \ldots$ & $1877, ' 81, ' 85, ' 90 \ldots$ & 124.40 \\
\hline & do & 15 & & $1885, ' 90, ' 95, ' 00 \ldots$ & 269 \\
\hline & do & 46 & & 1877, '\$1, '85, '90. & 269 \\
\hline & do & 47 & Und. $\frac{1}{2}$ & $189 \pi \ldots \ldots$ & $13+.50$ \\
\hline & do & 47 & Lnd. $\frac{1}{2}$. & Purchave \& 1000. & 134.50 \\
\hline & do & 48 & $\ldots \ldots$ & $1890,05 \ldots$. & 269 \\
\hline & do & 51 & 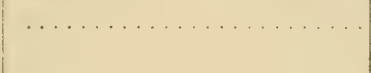 & $\begin{array}{r}1877,91,85,90 \text {, } \\
4, \ldots \ldots \ldots\end{array}$ & 269 \\
\hline & do & 53 & . . & $\begin{array}{r}1877, ' 81,95,90, \\
95 \ldots \ldots \ldots \ldots\end{array}$ & $2(i)$ \\
\hline & do & 55 & & $1877, ' 81, '>5, ' 90 \ldots$ & $10 S$ \\
\hline & do & 56 & - & Purchase ..... . & 269 \\
\hline & do & 57 & & Purchase. & $26 !$ \\
\hline & do & 58 & & $1885,{ }^{\prime} 90, ' 95 \ldots$. & 269 \\
\hline & do & 62 & & $1885,90 \ldots \ldots$ & 269 \\
\hline & do & 63 & & $1885,90 \ldots \ldots$ & $20 ?$ \\
\hline & do & 64 & . & $1890,{ }^{\prime} 95 \ldots \ldots$ & $26 ! 9$ \\
\hline & do & 66 & & 1877, ' 81, ' 85, , '90. & 93.60 \\
\hline Ariet & ta...... & 67 & All in Arietta . ...... & Purchase ..... . . & 19.5() \\
\hline Lake & Pleasant & 67 & All in Lake Pleasant . . & Purchase. & 249.50 \\
\hline & do & 68 & . & Purchase & 269 \\
\hline & do & 69 & & 1890, , 95 & $2(i !)$ \\
\hline & do & 70 & & $1890,95 \ldots \ldots$ & $2(i)$ \\
\hline & do & 71 & & Purchase. & 2 (i) \\
\hline & do & 72 & $\ldots$ & $1890,99$. & $\because(j !)$ \\
\hline
\end{tabular}




\section{Hamilion County}

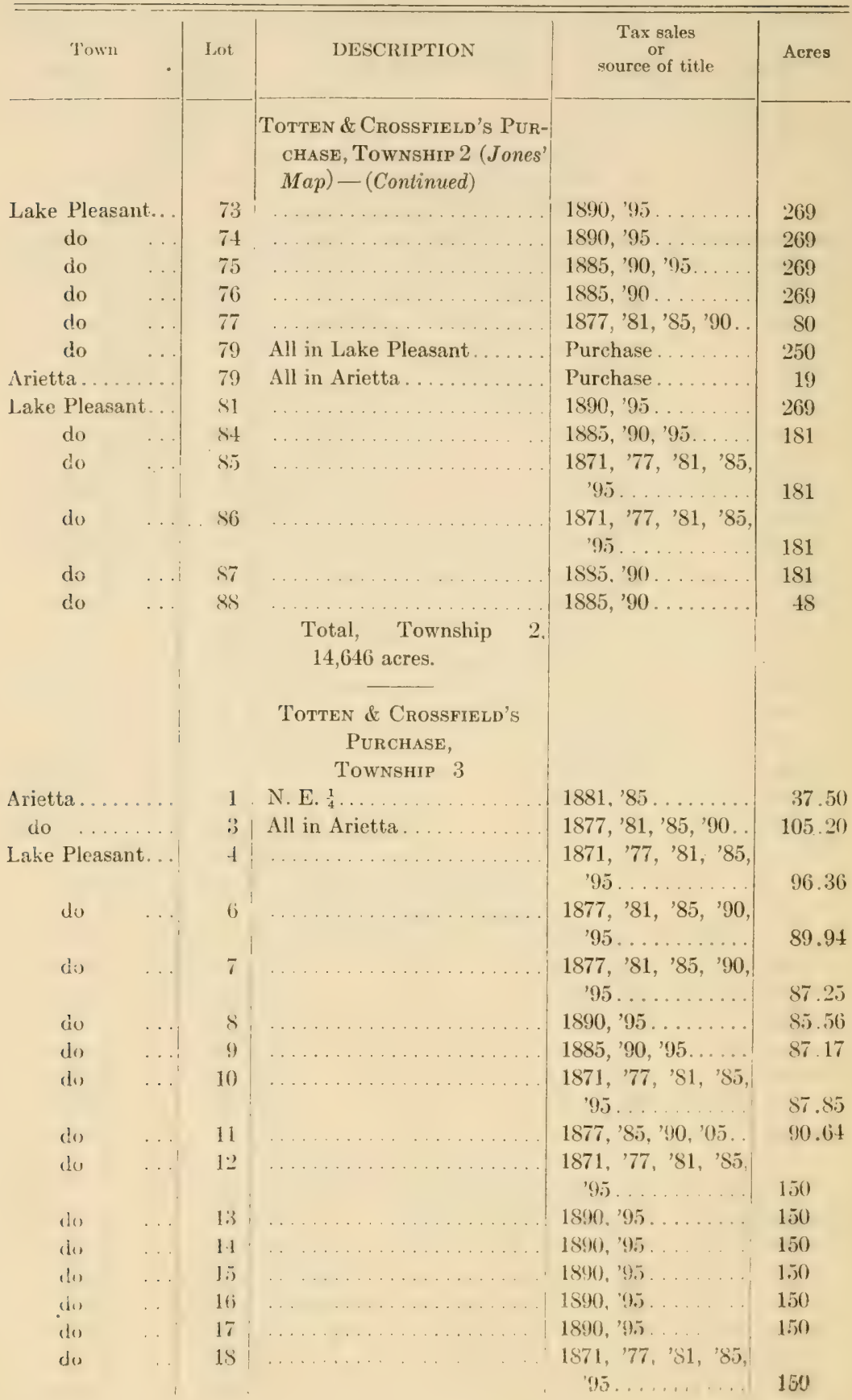




\section{HaMILTON COUNTY}

\begin{tabular}{|c|c|c|c|c|}
\hline Town & Lot & DESCRIPTION & $\begin{array}{c}\text { Tax sales } \\
\text { or } \\
\text { source of title }\end{array}$ & Acres \\
\hline & & $\begin{array}{l}\text { Totten \& Crossfield's Pur- } \\
\text { chase, Township } \\
\text { (Continued) }\end{array}$ & & \\
\hline Arictta. & 20 & $\ldots \ldots \ldots \ldots \ldots \ldots \ldots \ldots$ & 1877, ' 81 , ' 85 , '90. . & 150 \\
\hline do & 21 & $\begin{array}{r}\text { Ex. } 37 \frac{1}{2} a . N . W . \frac{1}{4}, \& 25 a . E \text {. } \\
\text { end of } S . \frac{1}{2} \text { across } \ldots \ldots \ldots\end{array}$ & $1877, ' 81$, ' $85 \ldots$ & 87.50 \\
\hline do & 22 & $\ldots \ldots \ldots \ldots \ldots \ldots \ldots \ldots$ & $1877, ' 85, ' 90 \ldots \ldots$ & 150 \\
\hline do & 24 & $\ldots \ldots \ldots \ldots \ldots \ldots$ & $\begin{array}{r}1877, ' 81, ' 85,90, \\
95 \ldots \ldots \ldots \ldots\end{array}$ & 150 \\
\hline do & 25 & & $1877, ' 85, ' 90 \ldots \ldots$ & 150 \\
\hline Lake Pleasan & 27 & All in Lake Pleasant. . & $\begin{array}{r}1881, \quad 85, \quad 90 \quad \& \\
\text { conveyed. . . . } \ldots\end{array}$ & 144 \\
\hline do & 28 & & $\begin{array}{r}1877,77,81,85, \\
95 \ldots \ldots \ldots\end{array}$ & 150 \\
\hline do & 29 & . & $\begin{array}{r}1871, \quad 77, \quad 81,95, \\
995 \ldots \ldots \ldots\end{array}$ & 150 \\
\hline do & 30 & $\ldots \ldots \ldots$ & $\begin{array}{r}1871, ' 77, ' 81,95 \\
95 . \ldots \ldots\end{array}$ & 150 \\
\hline do & 31 & $\ldots \ldots \ldots$ & $\begin{array}{r}1871,77,91,95, \\
95 \ldots \ldots \ldots \ldots\end{array}$ & 150 \\
\hline do & 32 & $\ldots \ldots \ldots$ & $\begin{array}{r}1871,{ }^{\prime} 77, \quad 91, ' 85, \\
95 \ldots \ldots \ldots\end{array}$ & 150 \\
\hline do & 33 & $\ldots: \ldots \ldots$ & $\begin{array}{r}1871,76,91,95 \\
95, \ldots \ldots \ldots\end{array}$ & 150 \\
\hline do & $3 \pm$ & $\ldots \ldots \ldots \ldots \ldots$ & $\begin{array}{r}1871, ' 77, \quad 81,95, \\
95 \ldots \ldots \ldots\end{array}$ & 150 \\
\hline do & 35 & . & $\begin{array}{r}1871,77,91,85, \\
95 \ldots \ldots \ldots \ldots\end{array}$ & 150 \\
\hline do & 36 & . & $\begin{array}{r}1877,91,90,95 \\
900 \ldots \ldots \ldots\end{array}$ & 150 \\
\hline do & 37 & & $\begin{array}{r}1877, \quad ' 81, \quad 90,95, \\
900 \ldots \ldots \ldots\end{array}$ & 150 \\
\hline do & 38 & $\ldots \ldots \ldots$ & $\begin{array}{r}1877, \quad, 81, \quad 90,95 \\
900 \ldots \ldots\end{array}$ & 150 \\
\hline do & 39 & 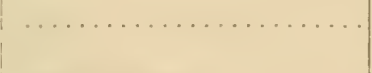 & $\begin{array}{r}1877,91,90,95 . \\
900 \ldots \ldots \ldots \ldots\end{array}$ & 150 \\
\hline Arietta... & 40 & All in Arietta. . & $1881,{ }^{\prime} 85, ' 95, ' 00$. & 70 \\
\hline Lake Pleasan & 40 & All in Lake Pleasant... & $1881, ' 85,{ }^{\prime} 90,{ }^{\prime} 00 \ldots$ & 80 \\
\hline Arietta..... . & 41 & $\ldots \ldots \ldots \ldots \ldots \ldots$ & $\begin{array}{r}1877,91,85,95, \\
905 \ldots \ldots \ldots \ldots\end{array}$ & 150 \\
\hline do & 43 & Ex. 30a. N. W. cor. square. : & $1881,{ }^{\prime} 85, ' 90 \ldots$. & 120 \\
\hline do & 46 & S. W. $\frac{1}{1} \ldots \ldots \ldots \ldots \ldots \ldots$ & $1881,85 \ldots \ldots \ldots$ & 37.50 \\
\hline .... & 47 & $\ldots \ldots \ldots \ldots \ldots \ldots \ldots \ldots$ & $1877, ' 81, ' 85, ' 90 \ldots$ & 1.50 \\
\hline
\end{tabular}


Hamilton County

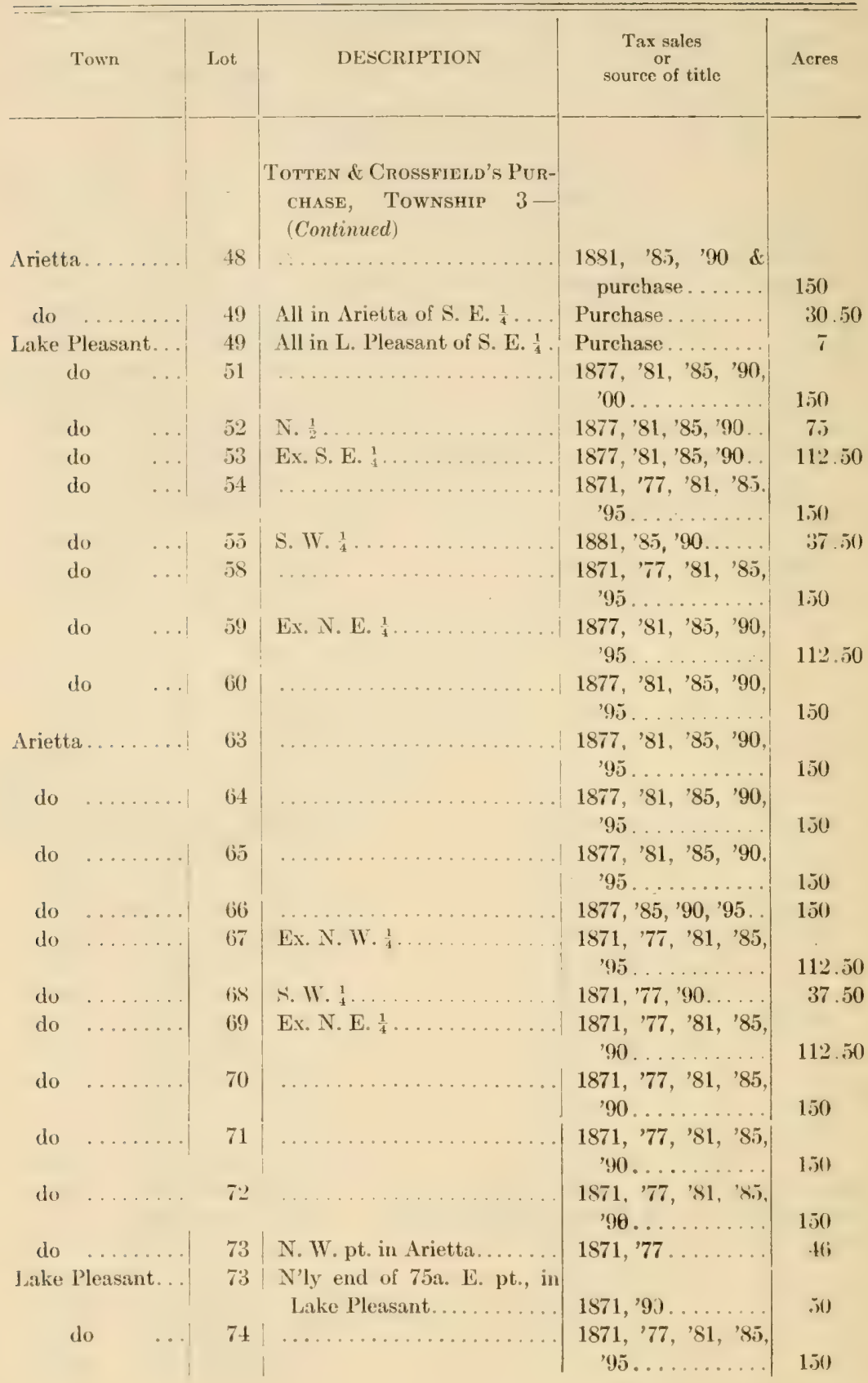


Conservation Commission.

Hamiton County

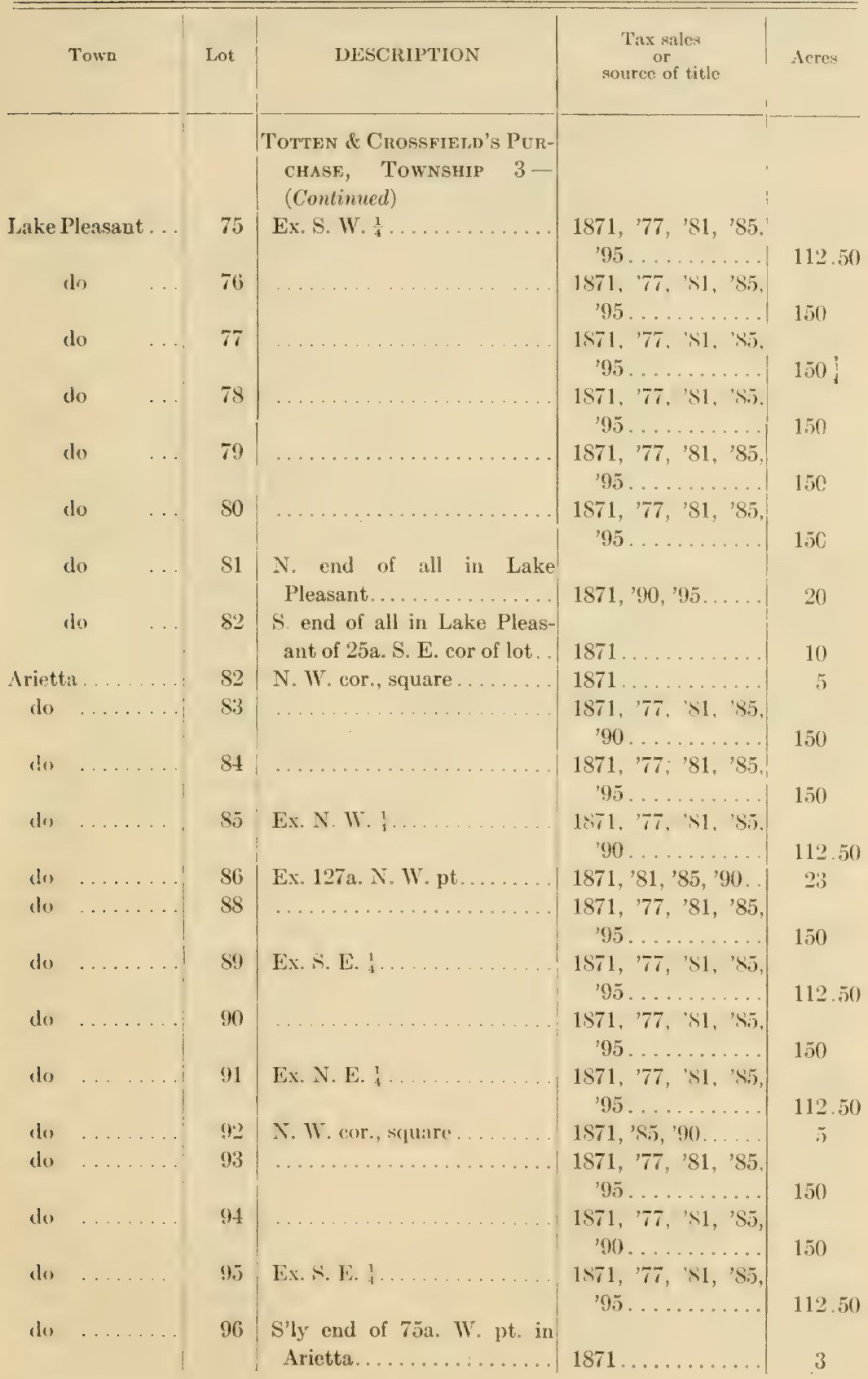




\section{Hamliton Countey}

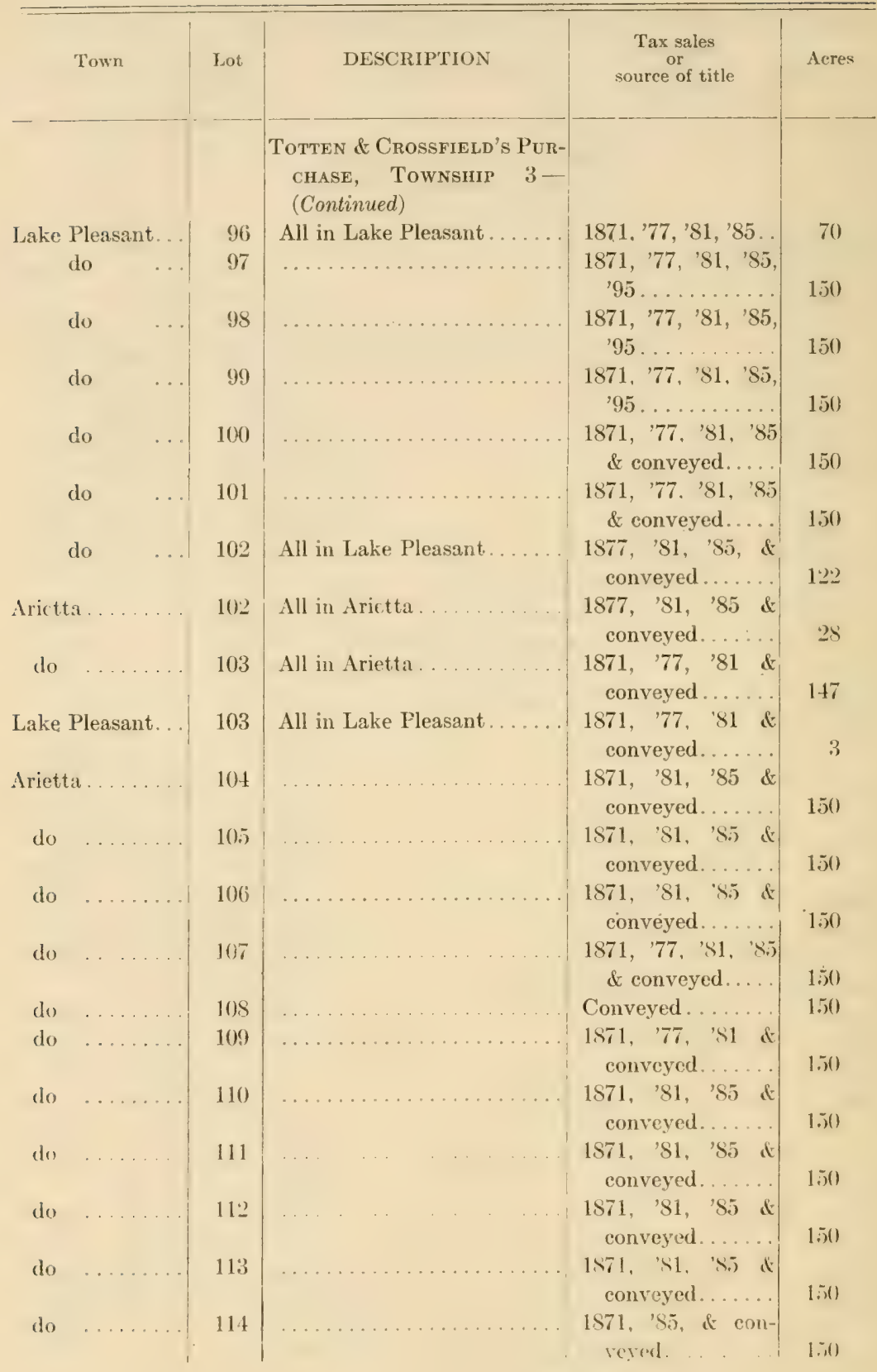


Hamilton Countr

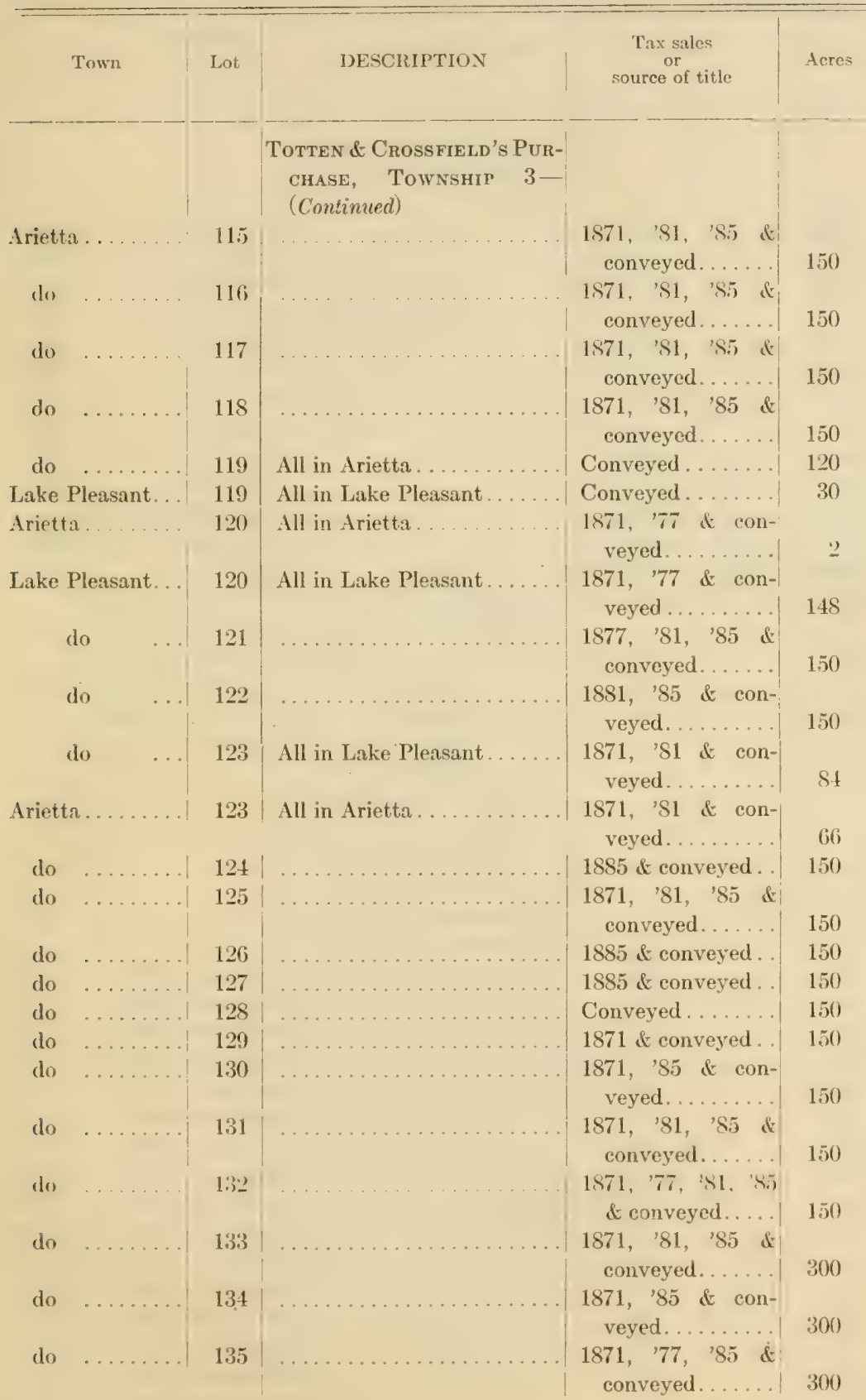


Hamilton County

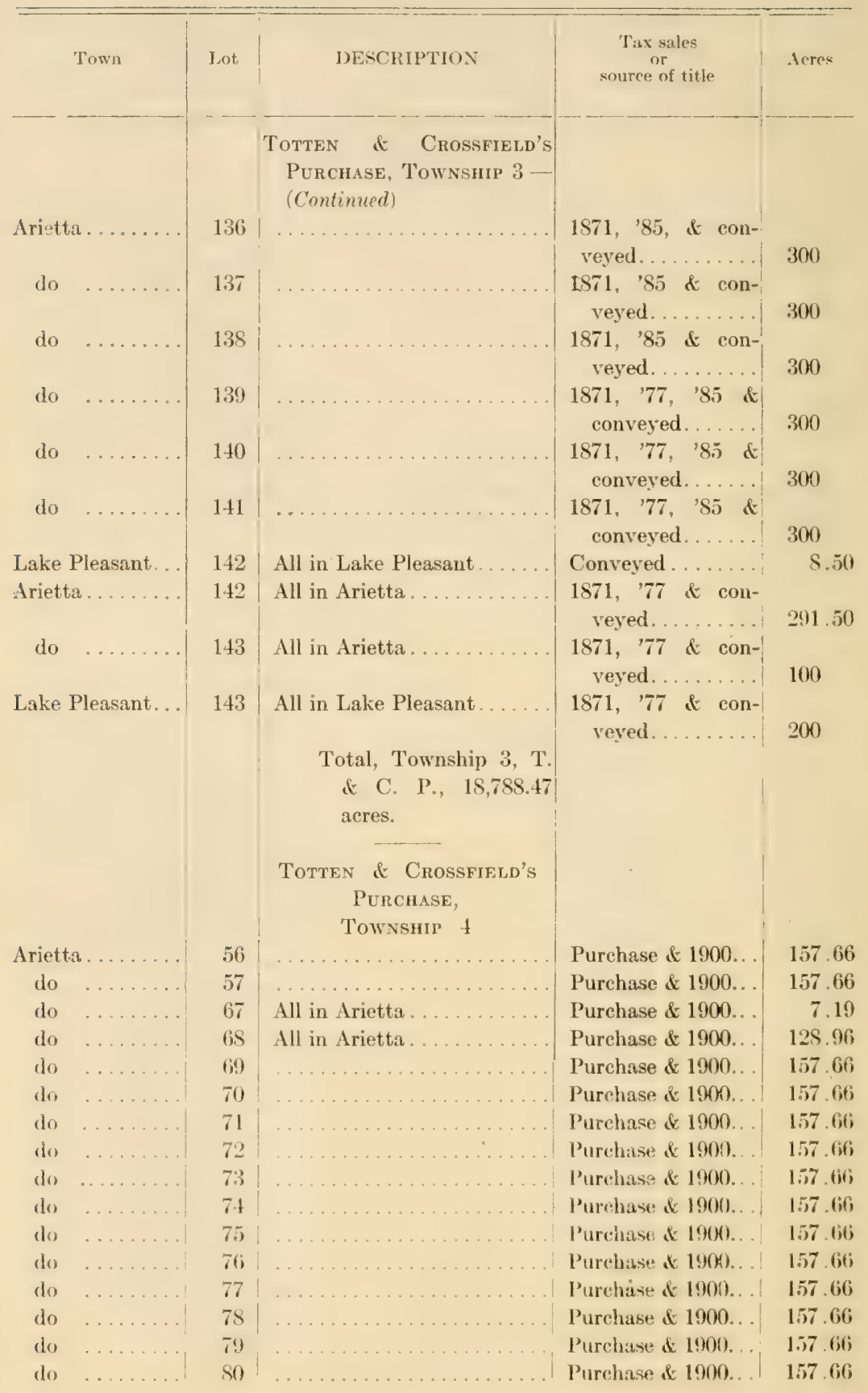


Hamiton County

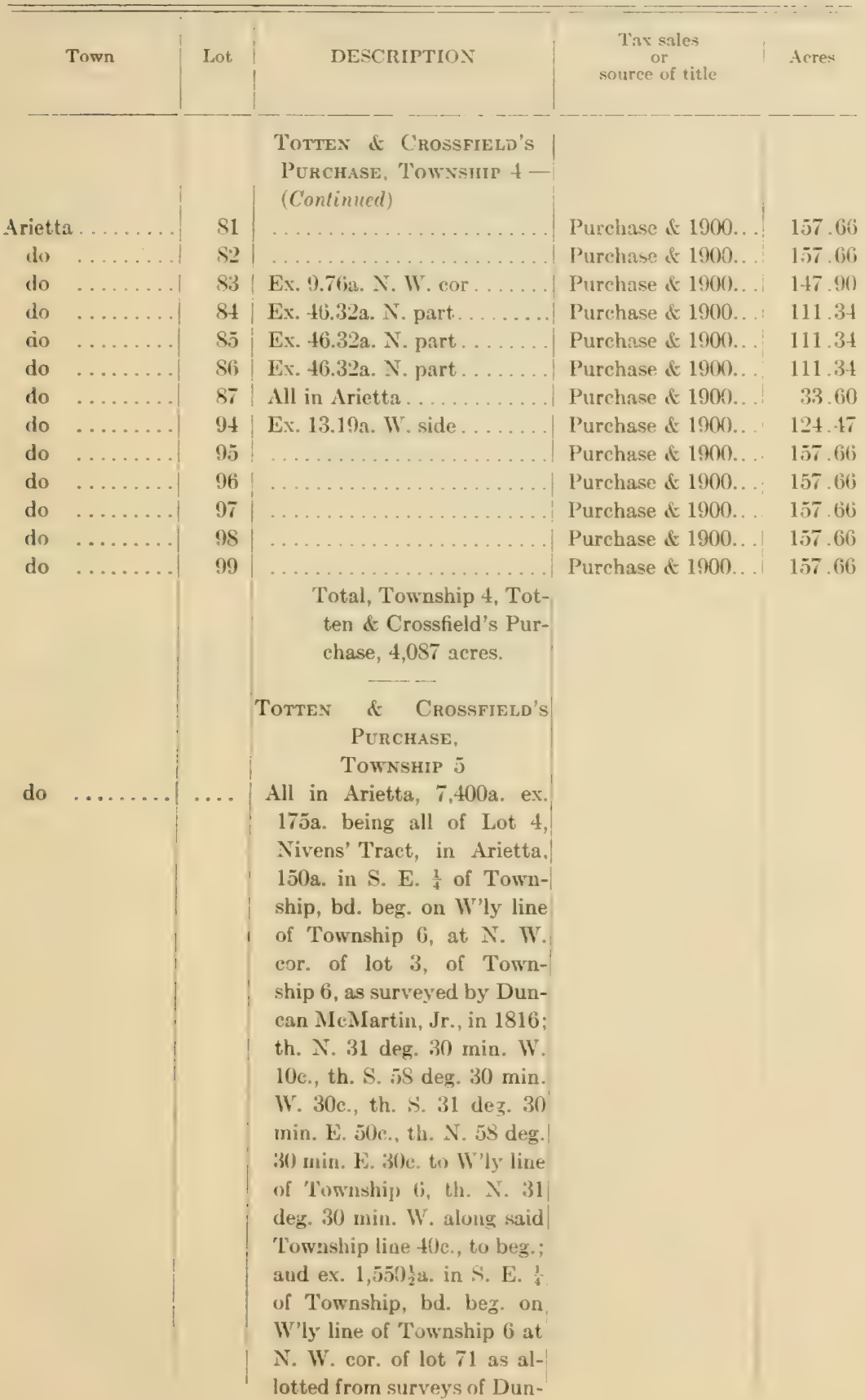


Hamitron County

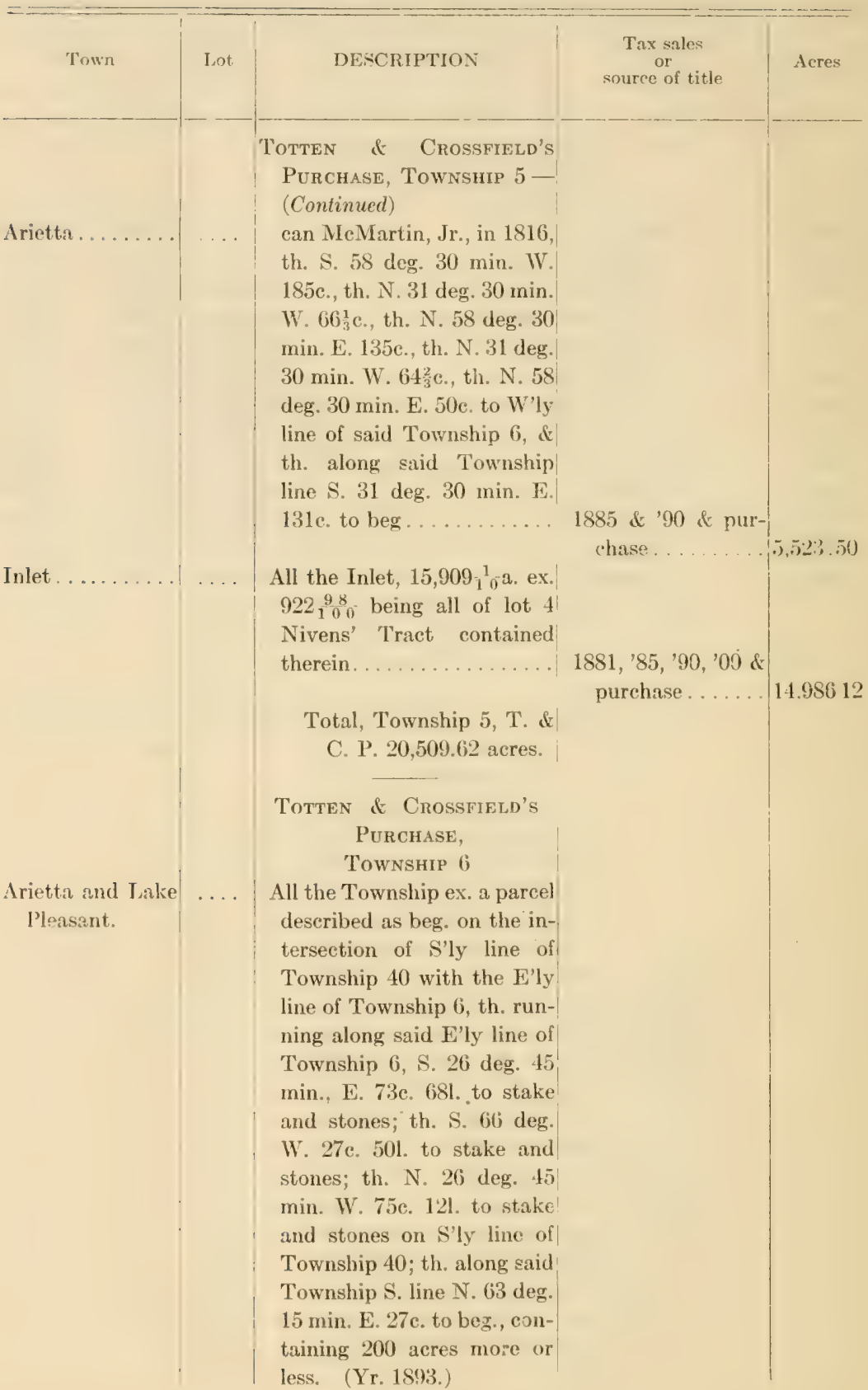


Hamiton County

\begin{tabular}{|c|c|c|c|c|}
\hline Town & Lot & DESCRIPTION & $\begin{array}{c}\text { Tax sales } \\
\text { or } \\
\text { source of title }\end{array}$ & Acres \\
\hline $\begin{array}{l}\text { Arietta and Lake } \\
\text { Pleasant. . . . . }\end{array}$ & . & 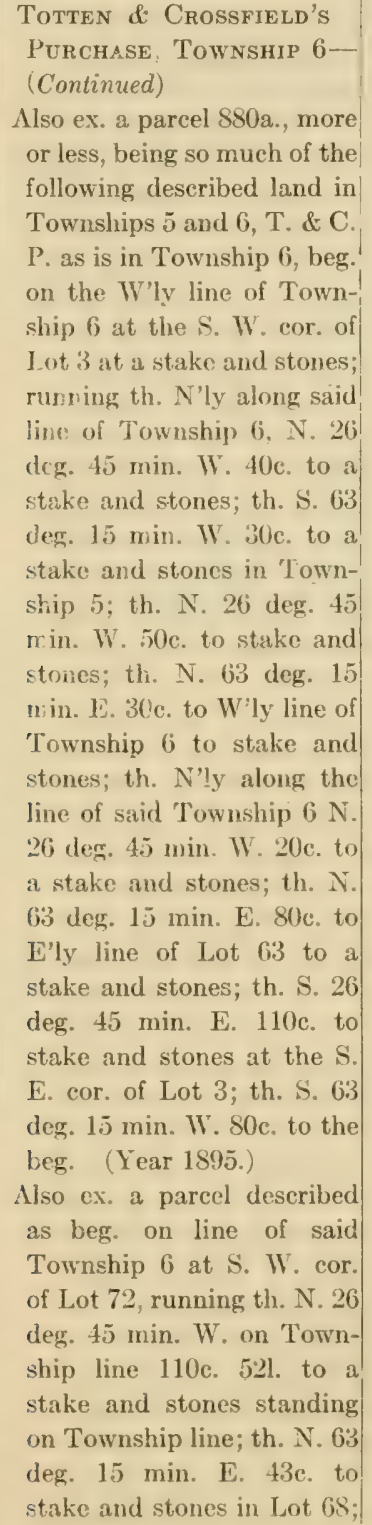 & & \\
\hline
\end{tabular}


Hamilton County

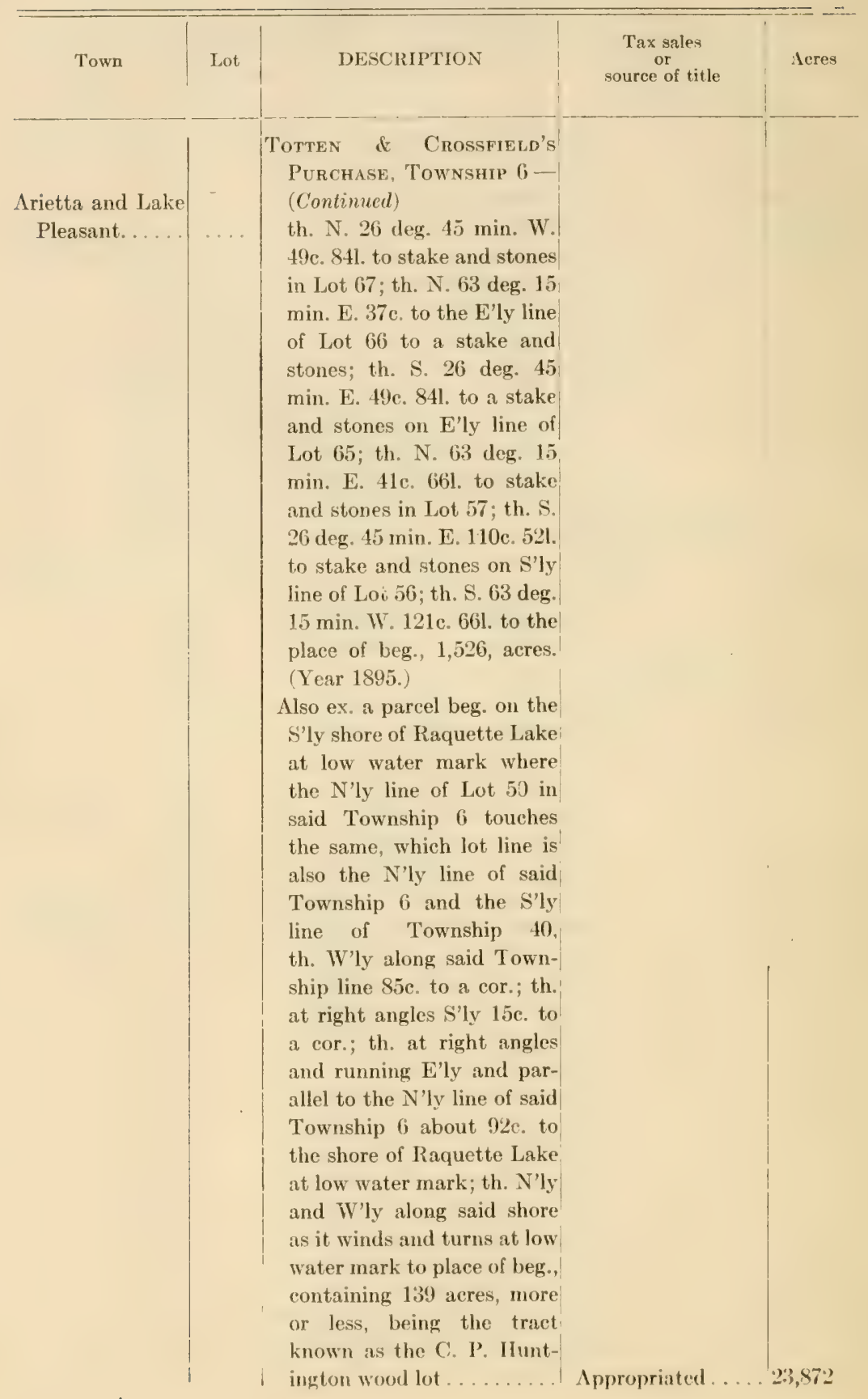


Conservation Commission.

Hamilton County

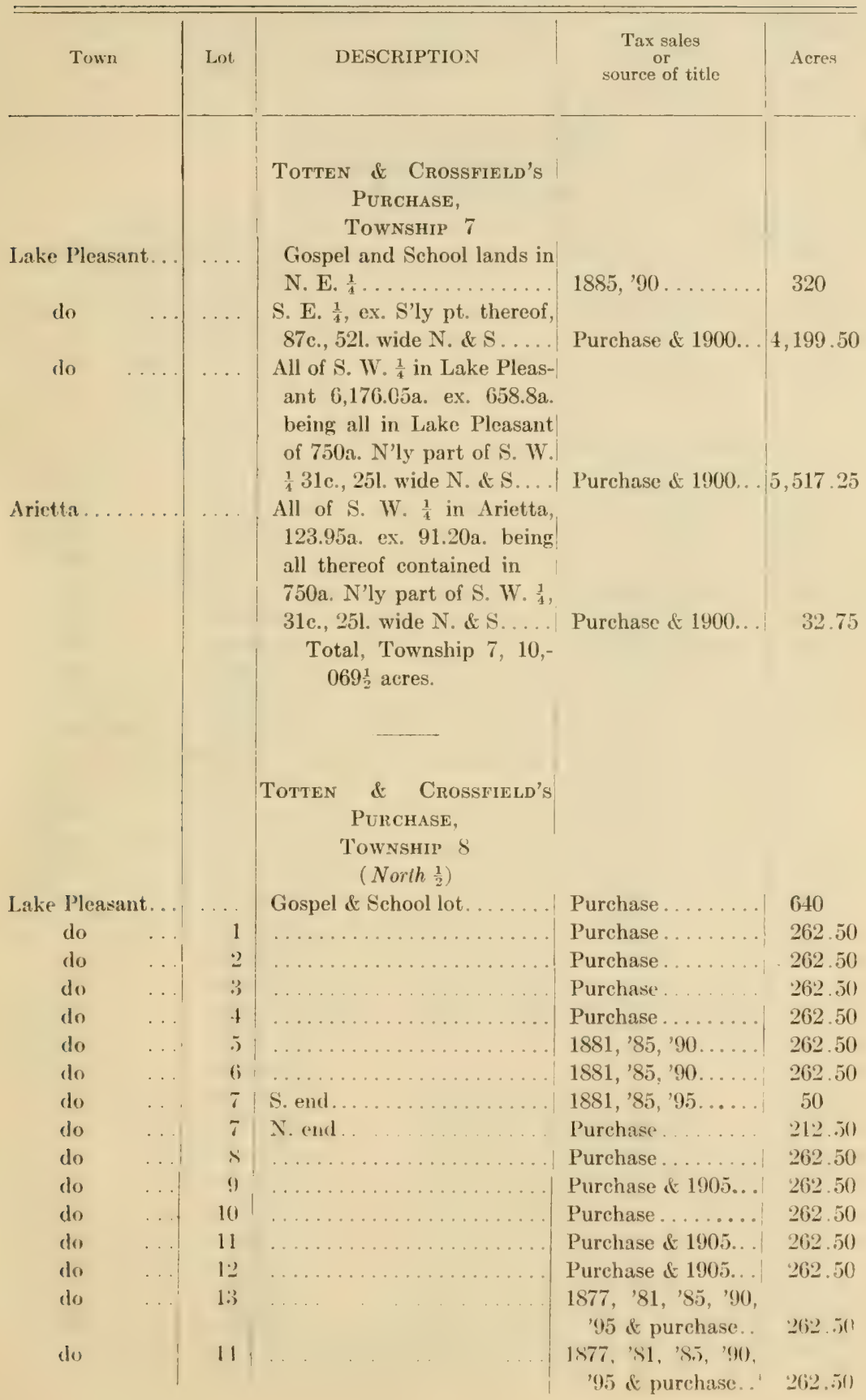


Hamilton County

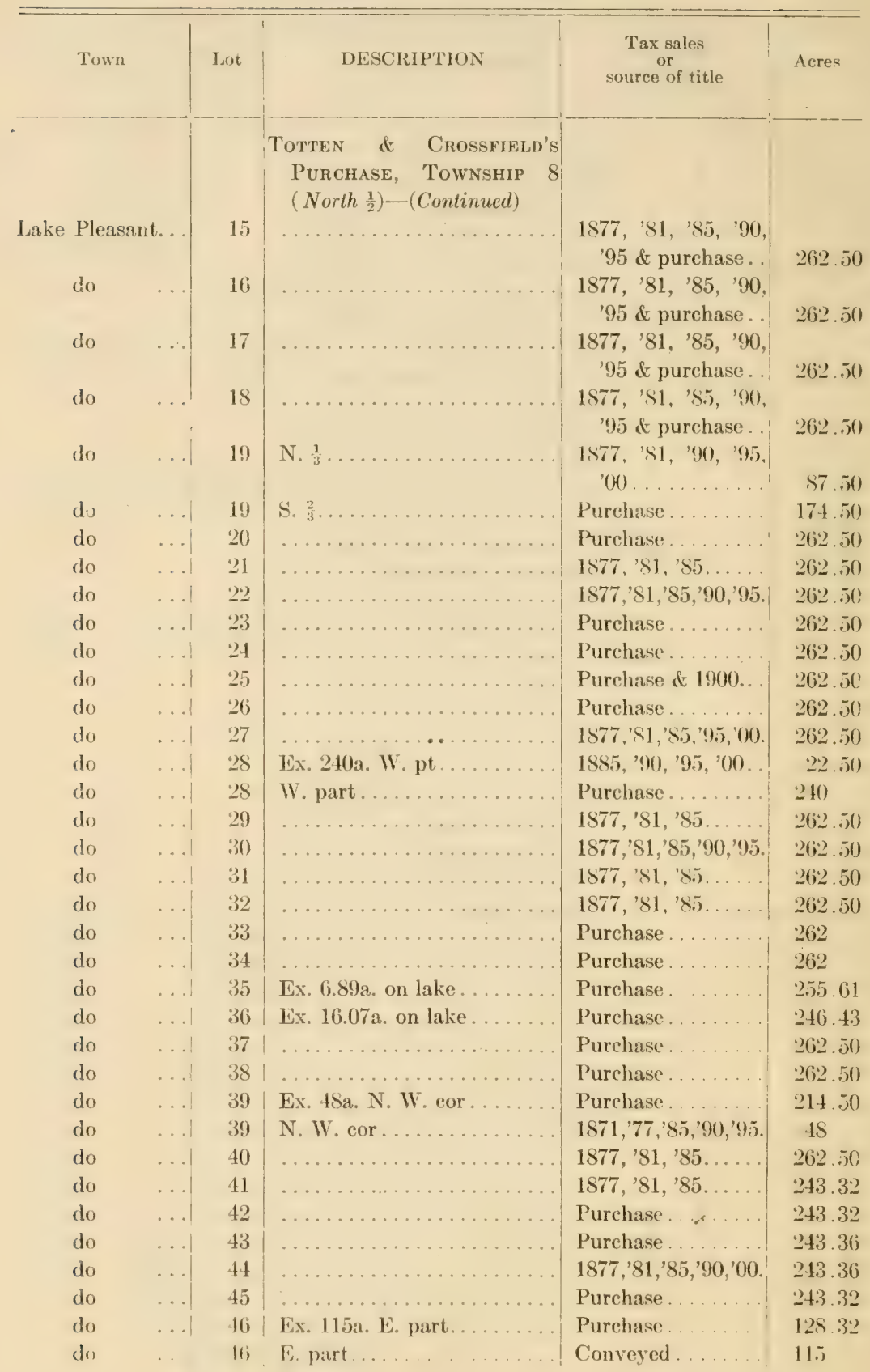


Conservation Commesion.

Hamlton County

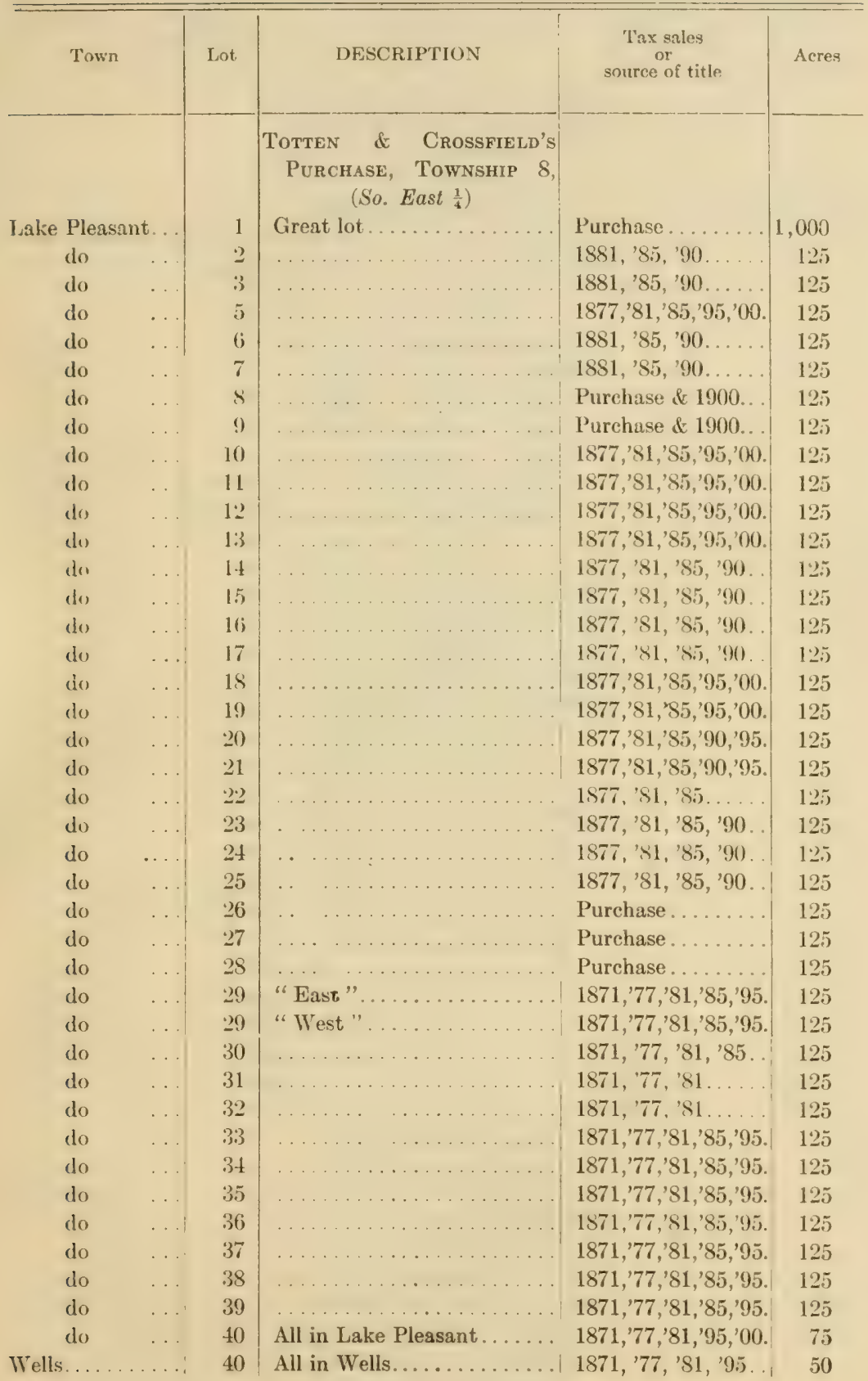


Hamilton County

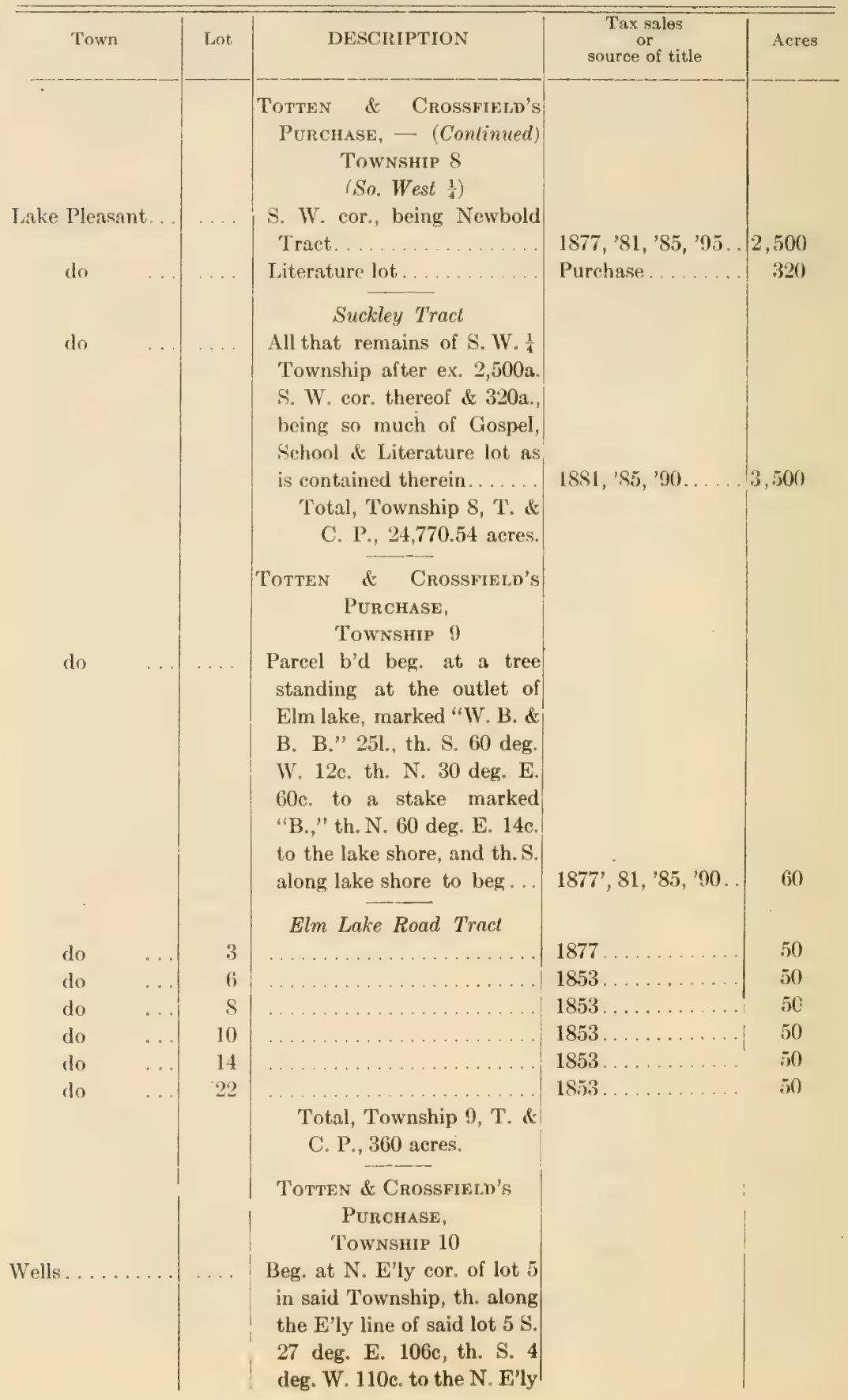


Hamilton County

\begin{tabular}{|c|c|c|c|c|}
\hline Town & I Jot & DESCRIPTION & $\begin{array}{c}\text { Tax sales } \\
\text { or } \\
\text { source of title }\end{array}$ & Acres \\
\hline Wells.......... & $1 \ldots$ & 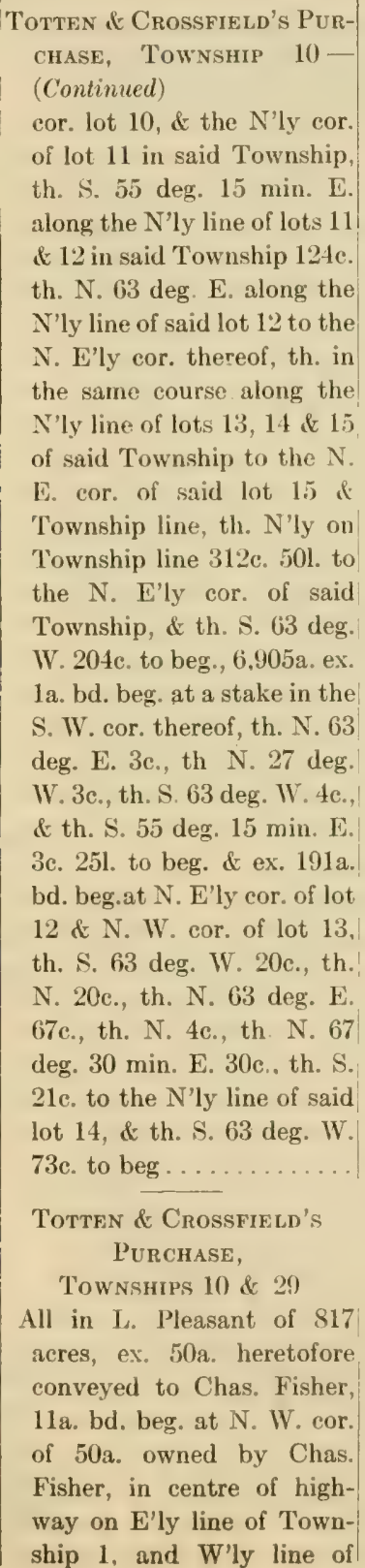 & Purchase. & 6,713 \\
\hline
\end{tabular}


Hamiton County

\begin{tabular}{|c|c|c|c|c|}
\hline Town & Lot & DESCRIPTION & $\begin{array}{c}\text { Tax sales } \\
\text { or } \\
\text { source of title }\end{array}$ & Acres \\
\hline Lake Pleasant. . & 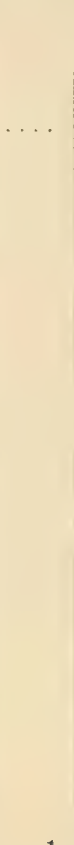 & 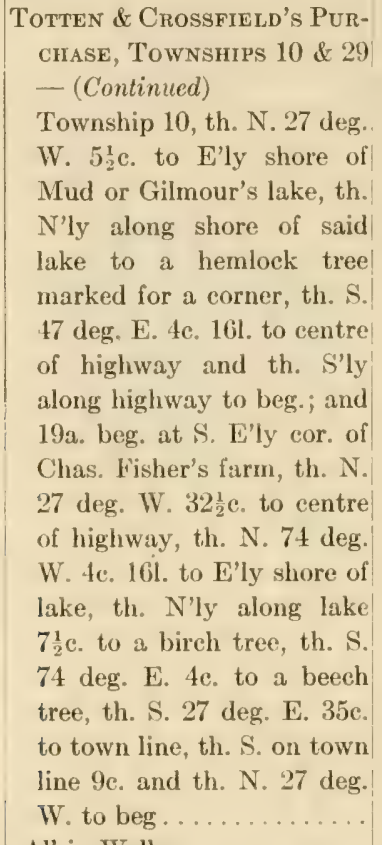 & Purchase... & 737 \\
\hline Wells......... & 1 & All in Wells............. & Purchase... & 250 \\
\hline Lake Pleasant... & 2 & $\begin{array}{c}\text { All of } 361 \mathrm{a} . \text { N. pt. in Lake } \\
\text { Pleasant................... }\end{array}$ & Purchase ........ & 323 \\
\hline $\begin{array}{l}\text { Wells...... } \\
\text { do ....... }\end{array}$ & $\begin{array}{l}2 \\
2\end{array}$ & $\begin{array}{l}\text { All of } 361 \mathrm{a} . \text { N. pt. in Wells. } \\
\text { Parcel beg. at S. W. cor. of } \\
\text { lot, th. N. } 27 \text { deg. W. on lot } \\
\text { line } 75 \mathrm{c} .501 . \text { to town line, } \\
\text { th. N. } \frac{1}{4} \text { deg. W. on town } \\
\text { line } 28 \mathrm{c} . \text { th. S. } 59 \text { deg. E. } \\
21 \mathrm{c}, \text { th. N. } 31 \mathrm{deg} \text {. E. } 16 \mathrm{c} . \\
\text { th. N. } 59 \text { deg. W. } 15 \mathrm{c} \text {, th. } \\
\text { N. } 31 \text { deg. E. } 31 \mathrm{c} \text { to E. line } \\
\text { of lot, th. S. } 27 \text { deg. E. on } \\
\text { E. line of lot } 131 \mathrm{c} \text {. to S. E. } \\
\text { cor., th. S. } 63 \text { deg. W. on } \\
\text { S. line } 53 \mathrm{c} \text { to beg....... }\end{array}$ & $1885,90,95 \ldots$. & 38 \\
\hline do & 3 & Sub. $1 \ldots \ldots \ldots \ldots \ldots$ & $1895,{ }^{\prime} 00 \ldots \ldots \ldots$ & 108.80 \\
\hline do $\ldots . . .$. & 3 & Sub. $2 \ldots \ldots \ldots \ldots \ldots$ & Conveyed \& 1895. & 108.80 \\
\hline do $\ldots . . .$. & 3 & Sub. $3 \ldots \ldots \ldots \ldots \ldots$ & Conveyed \& 1895. & 108.80 \\
\hline do $\ldots . .$. . & 3 & Sub. $4 \ldots$. & Conveyed \& 1895.. & 108.80 \\
\hline & 3 & Sub. $5 \ldots \ldots \ldots \ldots \ldots$ & Conveyed \& 1895. & 108.80 \\
\hline
\end{tabular}




\section{Hamilton County}

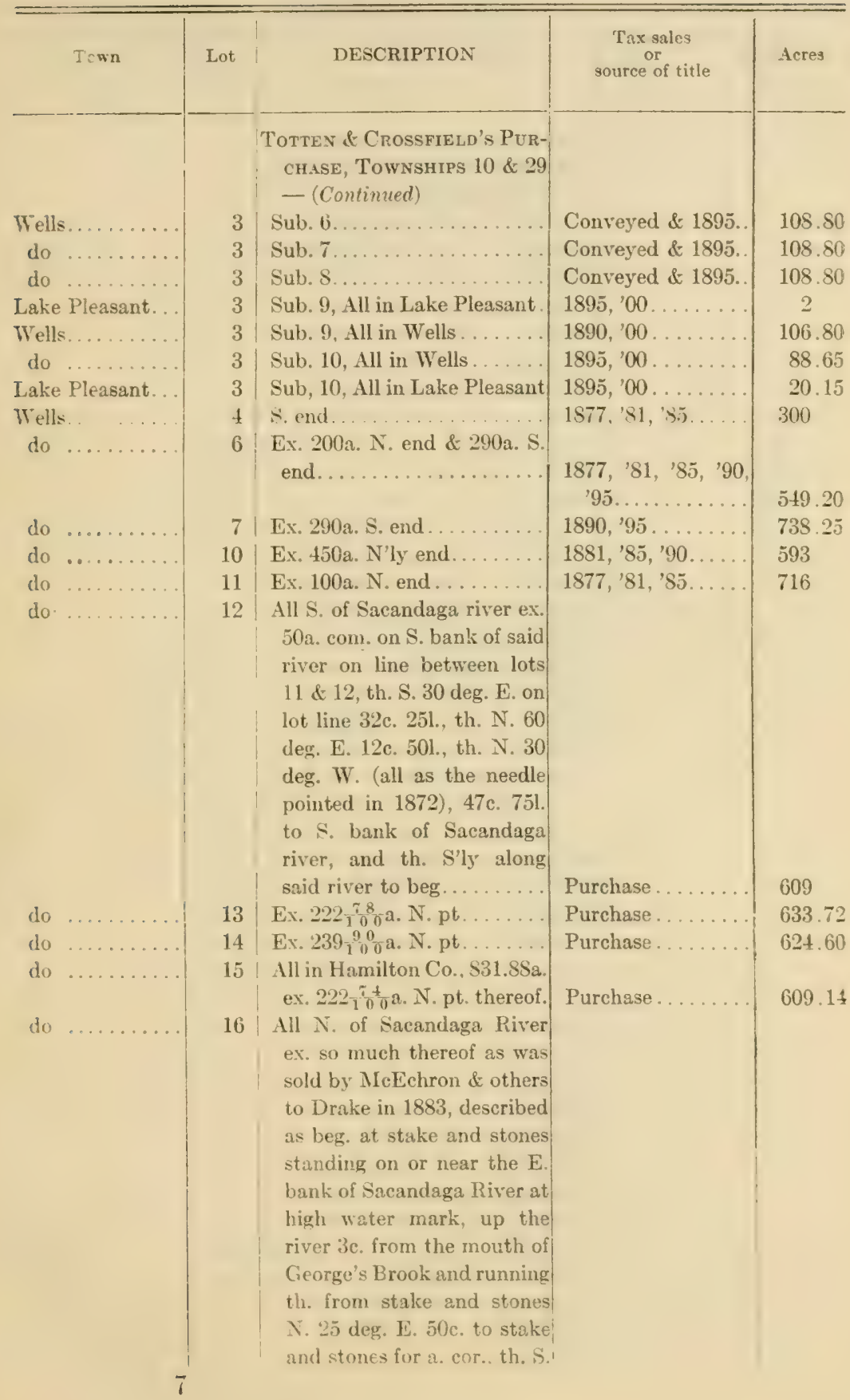


Hamilton County

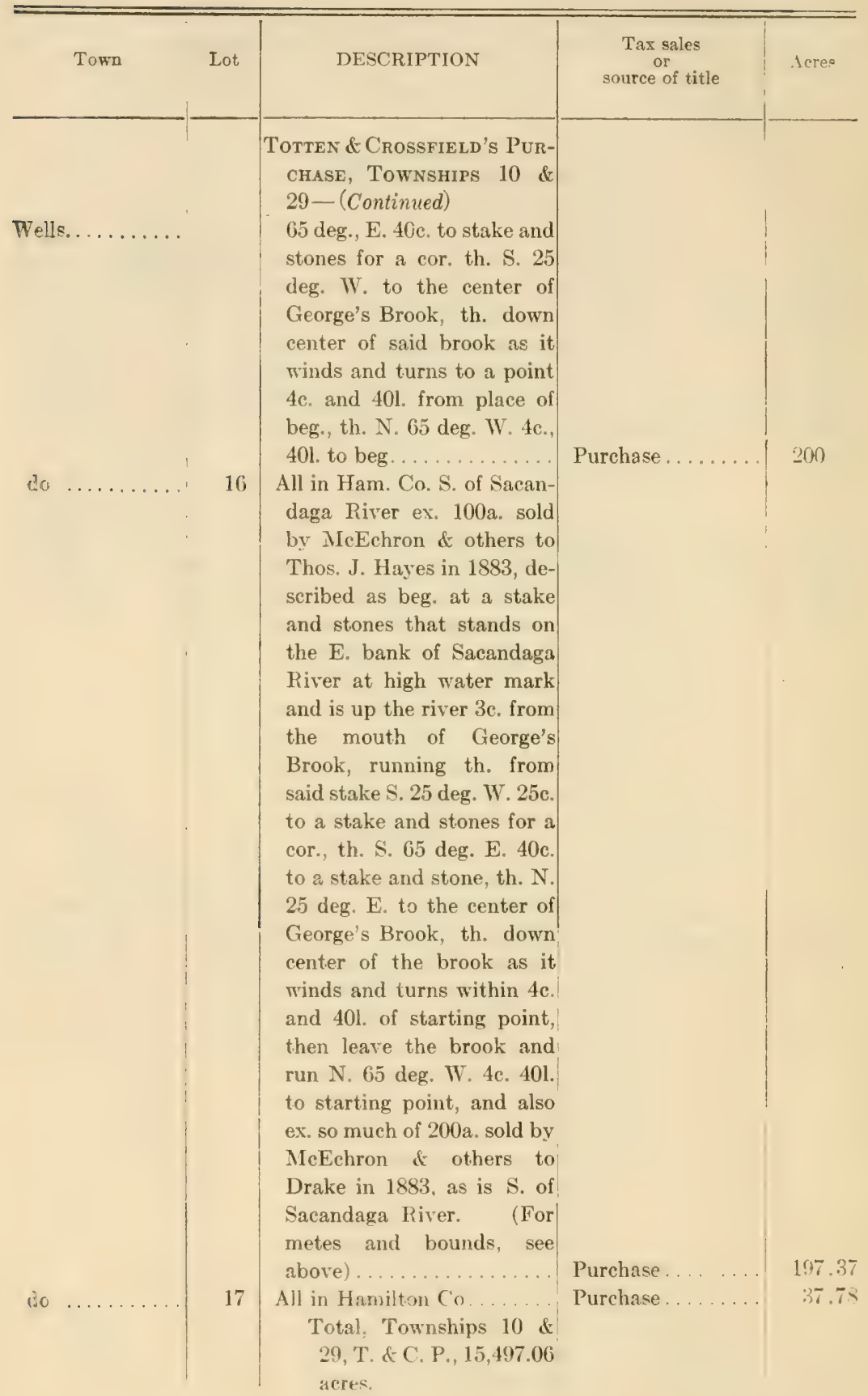




\section{Hainlton County}

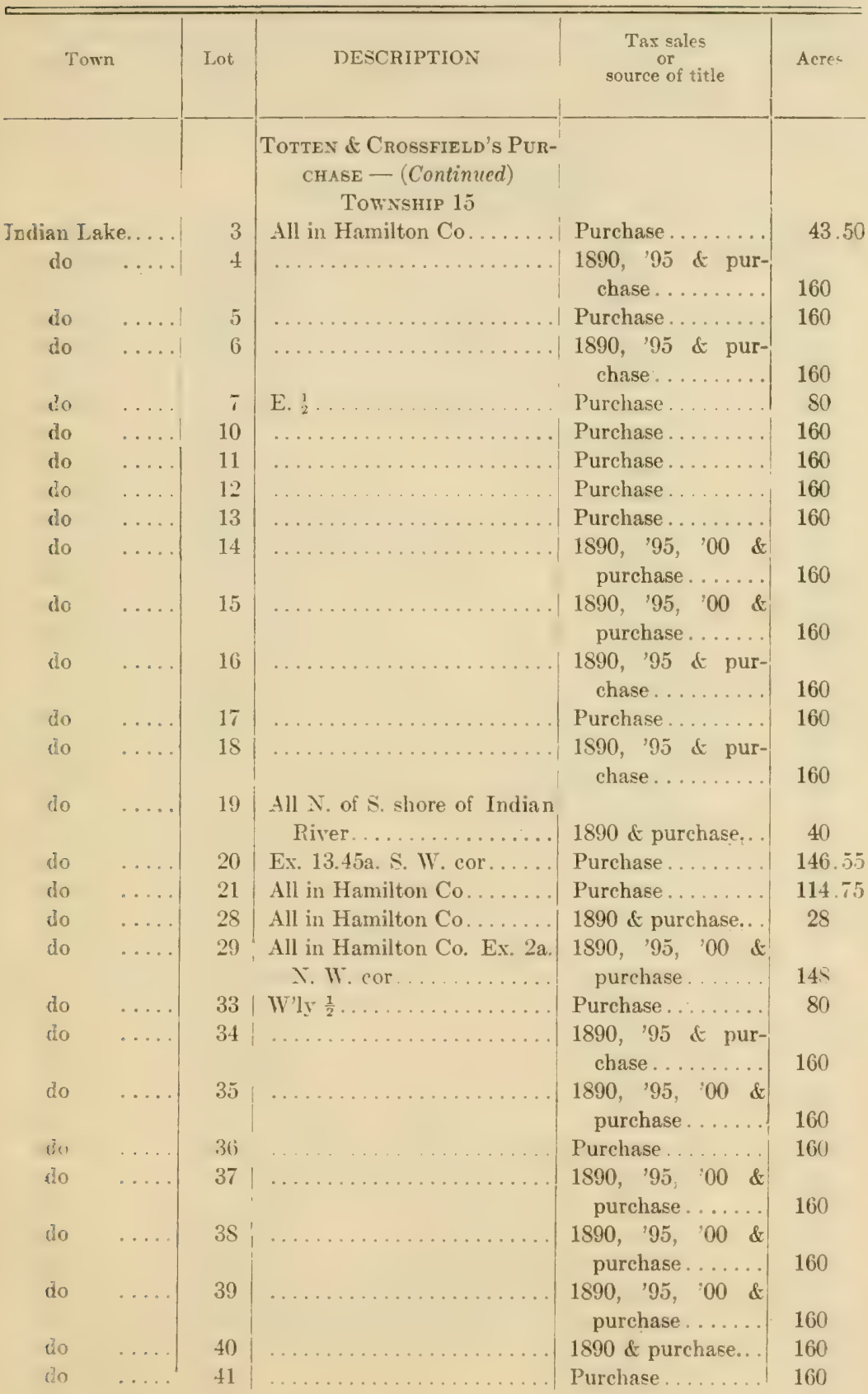




\section{Hamltoy County}

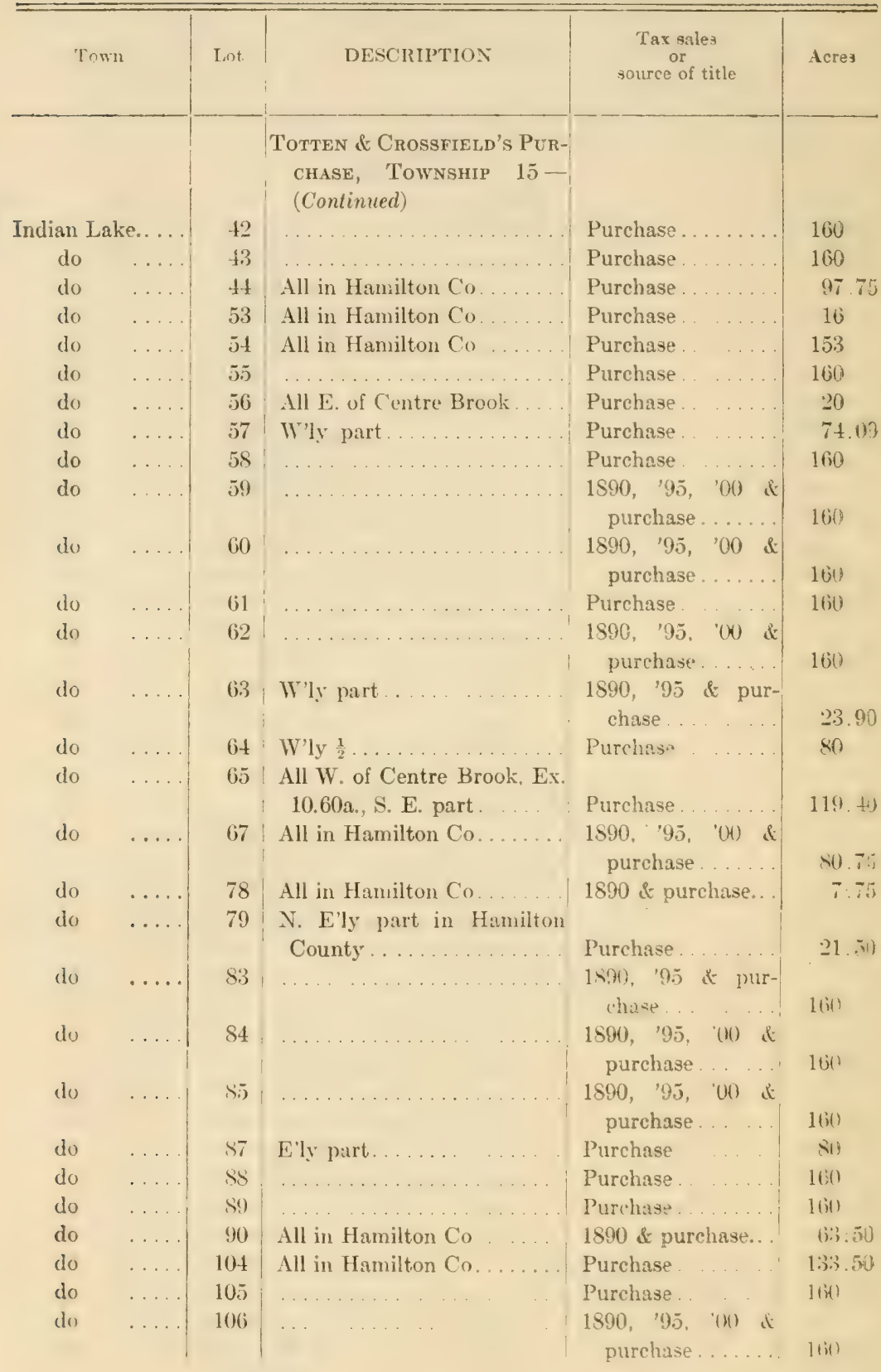


Hamimton County

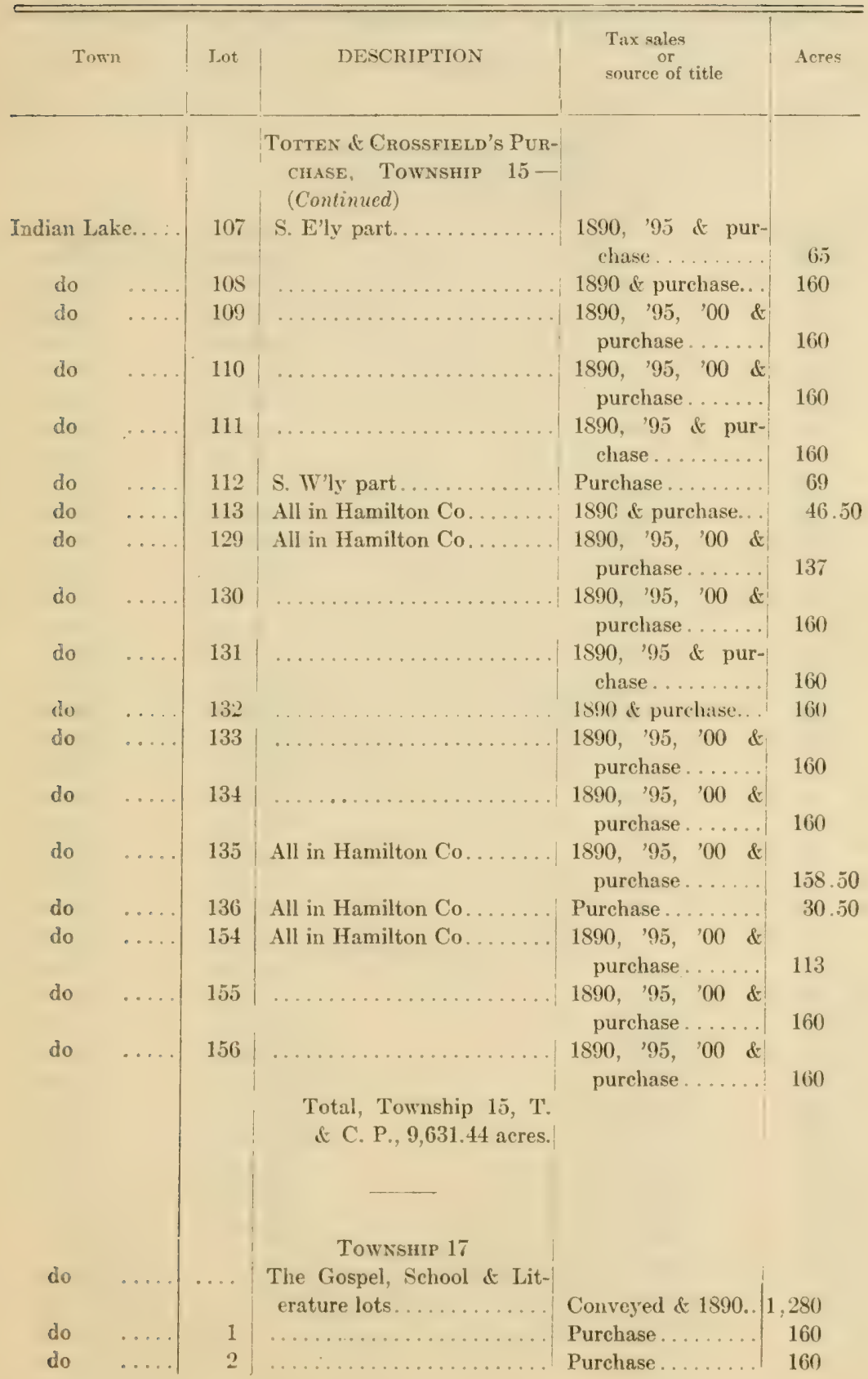




\section{Hanilton County}

\begin{tabular}{|c|c|c|c|c|}
\hline Town & \multirow[t]{2}{*}{ Lot } & DESCRIPTION & \multirow{2}{*}{$\begin{array}{c}\text { Tax sales } \\
\text { or } \\
\text { source of title }\end{array}$} & \multirow[t]{2}{*}{ Acres } \\
\hline & & $\begin{array}{l}\text { Tomten \& Crossfield's } \\
\text { Purchase, Township 17- } \\
\text { (Contimued) }\end{array}$ & & \\
\hline Indian Lake. & 3 & $\ldots \ldots \ldots \ldots$ & Purchase..... . & 160 \\
\hline do & 4 & $\cdots \cdots \cdots \cdots \cdots \cdots \cdots \cdots \cdots$ & Purchase...... & 160 \\
\hline do & 5 & $\ldots \ldots \ldots \ldots \ldots \ldots$ & Purchase .... . . & 160 \\
\hline do & 6 & & Purchase..... & 160 \\
\hline do & 7 & $\ldots \ldots \ldots \ldots \ldots \ldots \ldots$ & Purchase..... & 160 \\
\hline do & 8 & $\ldots \ldots \ldots$ & Purchase ..... . & 160 \\
\hline do & 9 & & Purchase..... . & 160 \\
\hline do & 10 & $\ldots \ldots \ldots \ldots \ldots \ldots$ & Purchase ..... . & 160 \\
\hline do & 11 & & Purchase..... & 160 \\
\hline do & 16 & & Purchase..... & 160 \\
\hline do & 17 & & Purchase ..... . & 160 \\
\hline do & 18 & & Purchase ..... . & 160 \\
\hline do & 19 & .. & Purchase..... & 160 \\
\hline do & 20 & & Purchase ..... . & 160 \\
\hline do & 21 & & Purchase..... . & 160 \\
\hline do & 22 & $\ldots \ldots \ldots$ & Purchase..... . & 160 \\
\hline do & 23 & & Purchase...... & 160 \\
\hline do & 24 & . & Purchase..... & 160 \\
\hline do & 27 & & Purchase...... & 160 \\
\hline do & 29 & & Purchase..... & 160 \\
\hline do & 32 & & Purchase ...... & 160 \\
\hline do & 33 & & Purchase..... & 160 \\
\hline do & 34 & & Purchase..... . & 160 \\
\hline do & 35 & . . & Purchase ..... . & 160 \\
\hline do & 36 & & Purchase..... & 160 \\
\hline do & 37 & $\ldots$ & Purchase ..... . & 160 \\
\hline do & 38 & $\ldots$ & Purchase ...... & 160 \\
\hline do & 39 & & Purchase..... . & 160 \\
\hline do & 45 & & Purchase...... & 160 \\
\hline do & 46 & & Purchase..... . & 160 \\
\hline do & 47 & & Purchase..... . & 160 \\
\hline do & 48 & .. & Purchase...... & 160 \\
\hline do & 49 & & Purchase...... & 160 \\
\hline do & 50 & & Purchase..... & 160 \\
\hline do & 51 & $\ldots$ & Purchase..... . & 160 \\
\hline do & 52 & $\ldots \ldots \ldots$ & Purchase..... & 160 \\
\hline do & 56 & $\begin{array}{l}\text { Bd. beg. at cor. of lots } 42,43 \\
55 \text { \& } 56 \text {, th. N. } 25^{\circ} 30^{\prime} \mathrm{W} \\
23 \mathrm{c} .191 \text {. to S'ly shore of } \\
\text { Cedar river, th. along the } \\
\text { shore of said river } \mathrm{N} .1^{\circ} 30^{\prime}\end{array}$ & & \\
\hline
\end{tabular}




\section{Hamiton County}

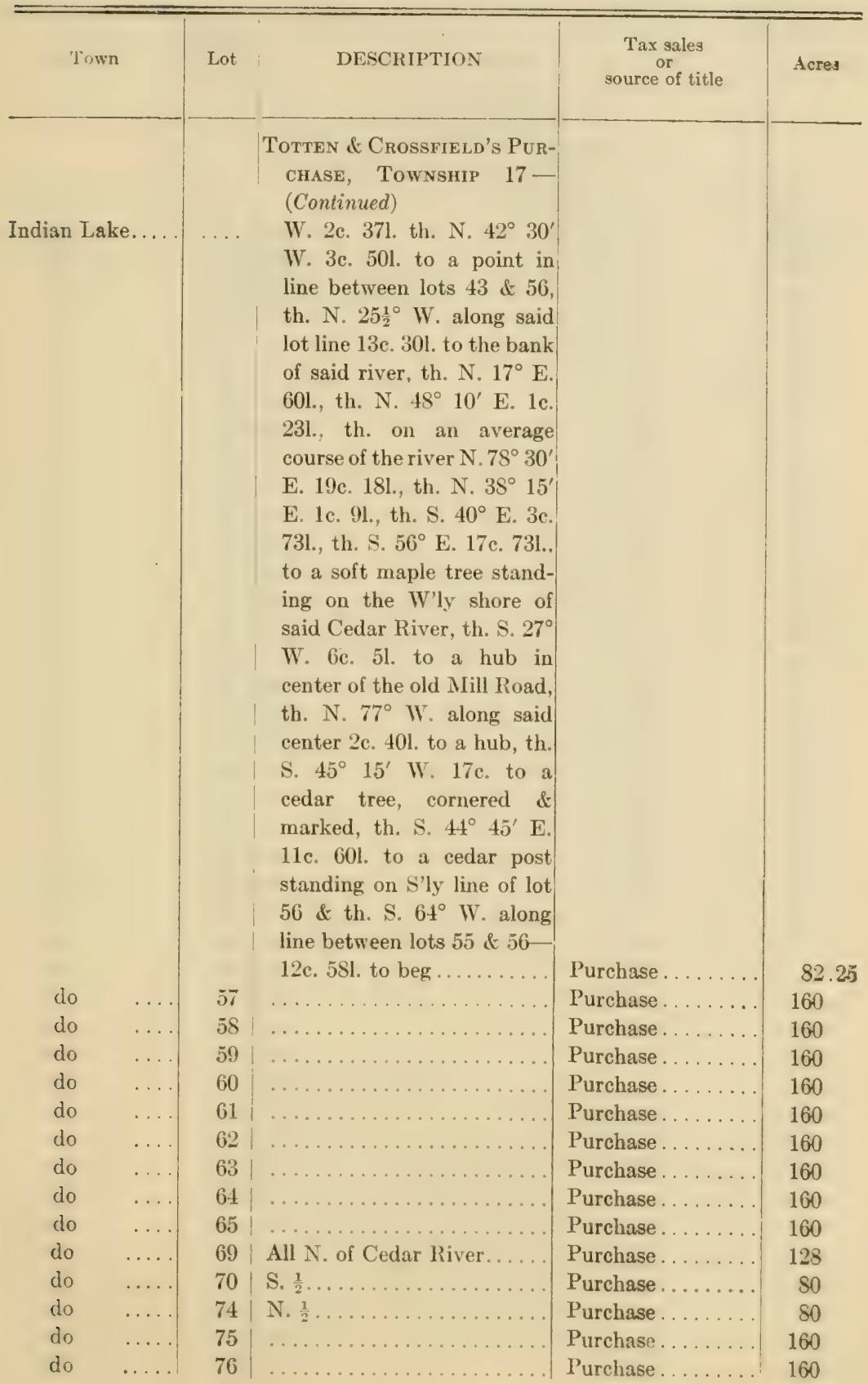


Hamilton County

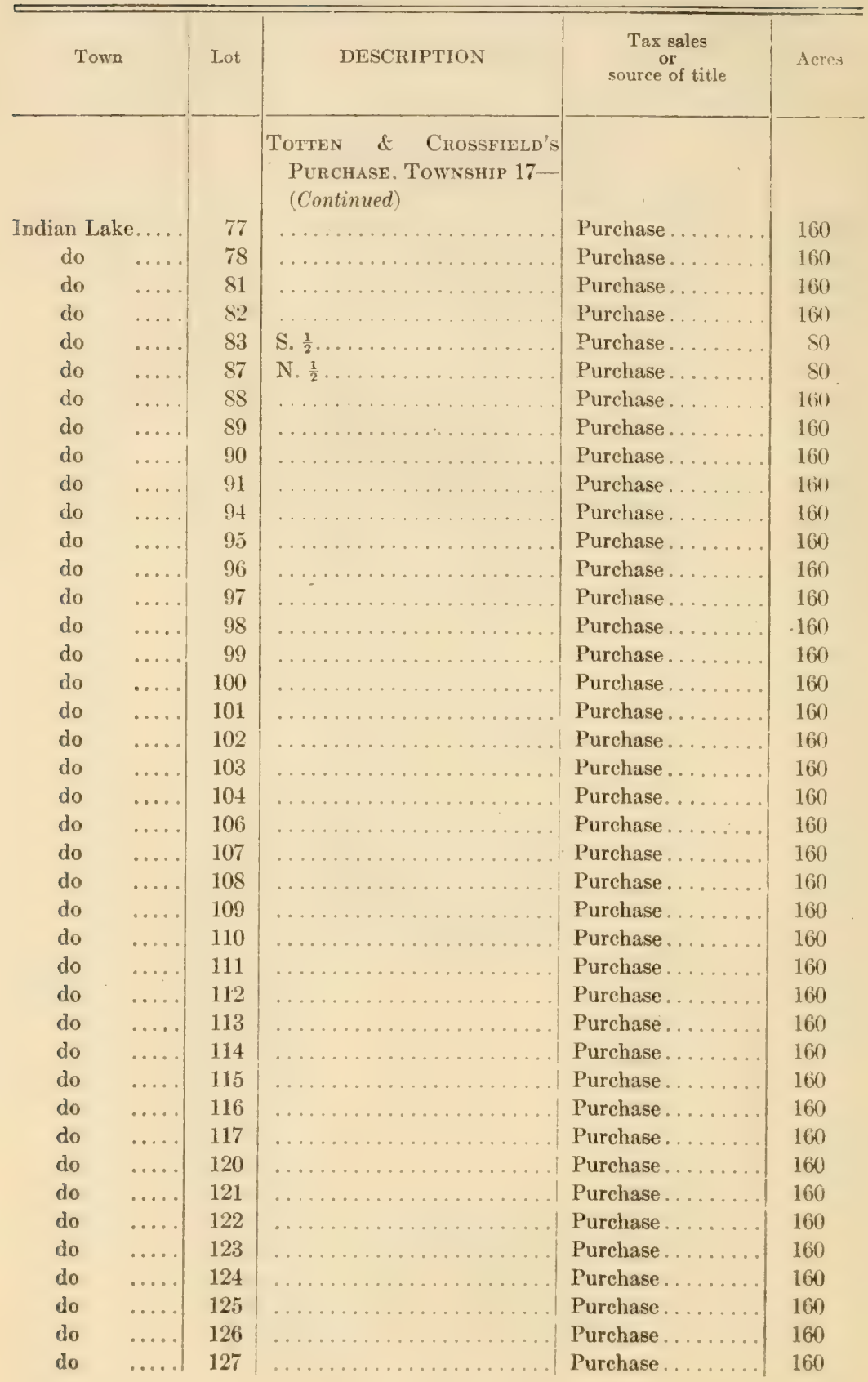


Conservation Commission.

Hamiton County

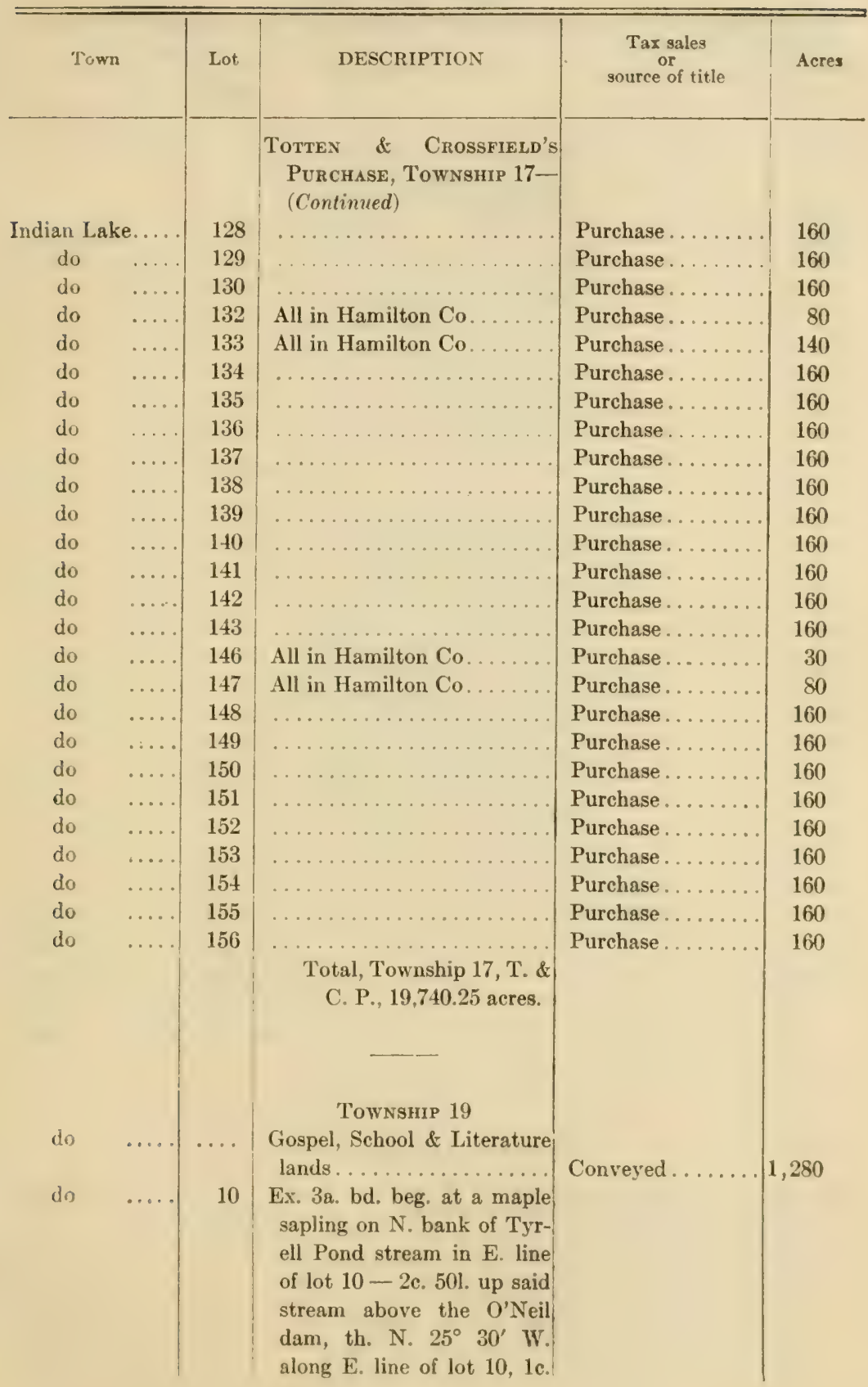




\section{Hamiton County}

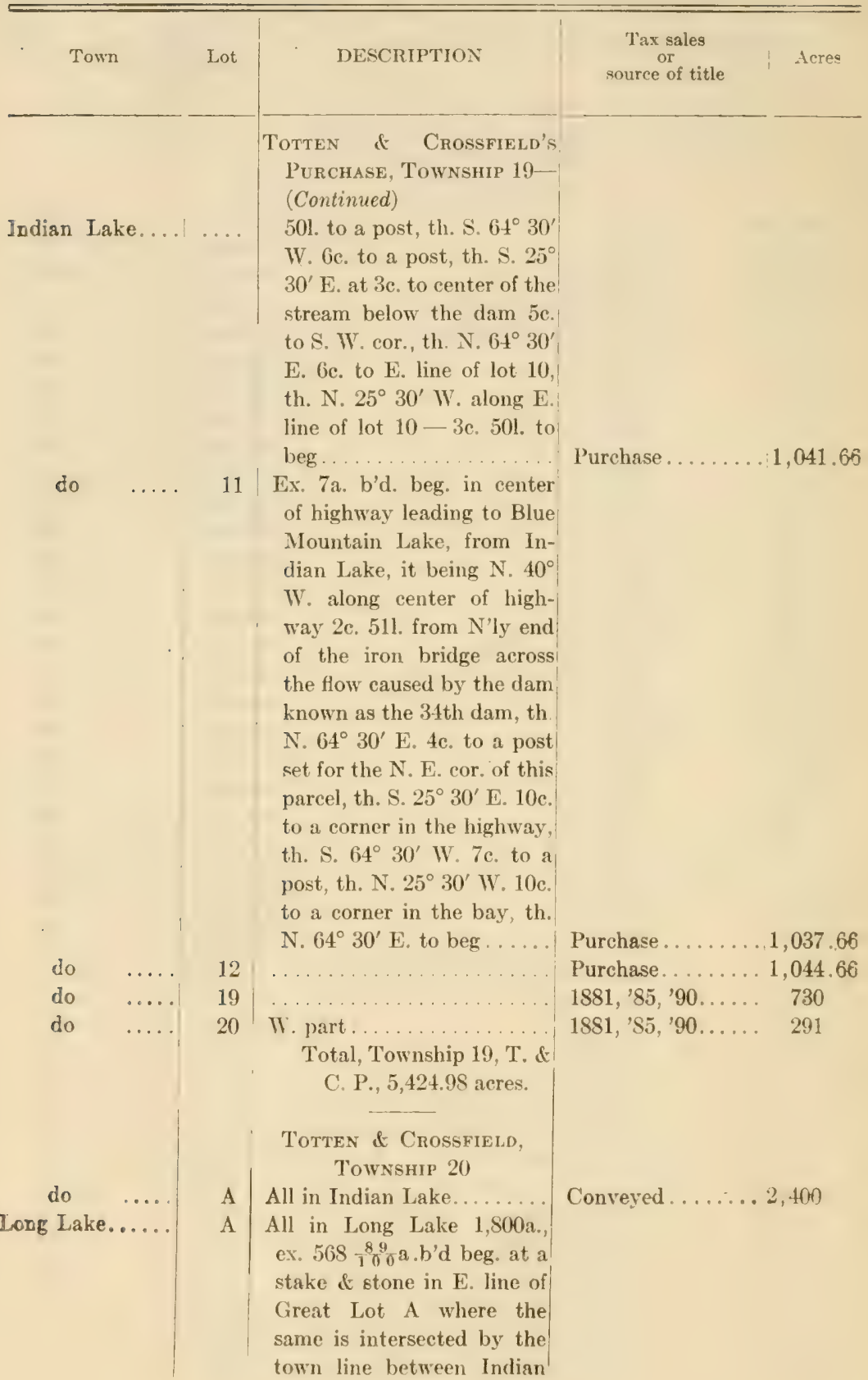




\section{Hamimon Counte}

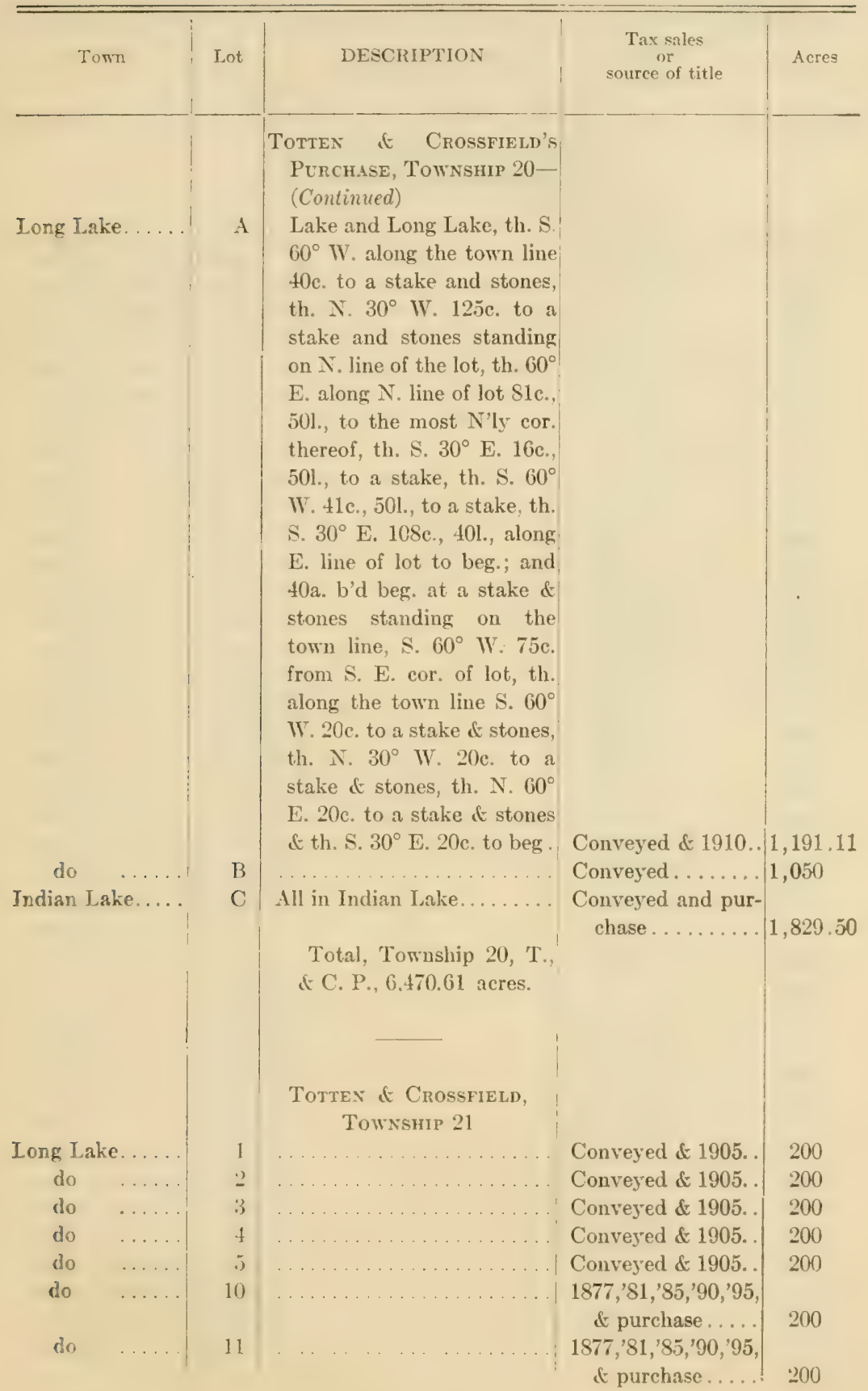




\section{Hamiton County}

\begin{tabular}{|c|c|c|c|c|}
\hline Town & Lot & DESCRIPTION & $\begin{array}{c}\text { Tax salez } \\
\text { or } \\
\text { source of title }\end{array}$ & Acres \\
\hline \multirow[b]{2}{*}{ Long Lake. } & & $\begin{array}{l}\text { Totten \& Crossfield's } \\
\text { Purchase, Township 21- } \\
\text { (Continued) }\end{array}$ & & \\
\hline & 12 & $\ldots \ldots \ldots \ldots \ldots \ldots$ & $\begin{array}{r}\text { 1877, ' } 81, \text { ' } 85, \text { ' } 90 \\
\& \text { purchase..... }\end{array}$ & 200 \\
\hline do & 13 & . & Conveyed \& 1905. & 200 \\
\hline do & 14 & $\ldots \ldots \ldots \ldots$ & Conveyed \& 1905. & 200 \\
\hline do & 15 & $\ldots \ldots$ & Conveyed \& 1905. & 200 \\
\hline do & 16 & & Conveyed \& 1905. & 200 \\
\hline do & 17 & & Conveyed \& 1905 & 200 \\
\hline do & 18 & $\ldots$ & $\begin{array}{r}187 \overline{7}, ' \$ 1, \text { ' } \$ \tilde{5}, 90 \\
\& \text { purchase..... }\end{array}$ & 200 \\
\hline do & 21 & $\ldots \ldots \ldots$ & $1877, ' 81, ' 85 \ldots$. & 200 \\
\hline do & 22 & $\ldots \ldots \ldots$ & 1877, '81, '\$5..... & 200 \\
\hline do & 23 & $\ldots \ldots \ldots$ & $1877, ' \$ 1, ' 85 \ldots$. & 200 \\
\hline do & 21 & & $187 ., 81,85 \ldots$. & 200 \\
\hline do & 25 & & Conveyed \& 1905. & 200 \\
\hline do & 26 & . & Conveyed it 1905. & 200 \\
\hline do & 27 & & Conveyed \& 1905: & 200 \\
\hline do & 28 & & Conveyed \& 1905. & 200 \\
\hline do & 29 & $\ldots \ldots \ldots$ & Conveyed \& 1905. & 200 \\
\hline do & 30 & & $1877, ' 81, ' 85 ., 90 \ldots$ & 200 \\
\hline do & 31 & & $1877, ' 85, ' 90 \ldots \ldots$ & 200 \\
\hline do & 32 & $\ldots \ldots$ & $1877, ' 81, ' 85, ' 90 \ldots$ & 200 \\
\hline do & 33 & ... & $1877, ' 81, ' 85,90 \ldots$ & 200 \\
\hline do & 37 & $\ldots \ldots \ldots$ & Conveyed \& 1905. & 200 \\
\hline do & 38 & & Conveyed \& 1905. & 200 \\
\hline do & 39 & & Convered \& 1905. . & 200 \\
\hline do & 40 & & Conveyed \& 1905. & 200 \\
\hline do & 41 & & Conveyed \& 1905. . & 200 \\
\hline do & 42 & & $\begin{array}{l}1885, \quad 90 \& \text { \& pur- } \\
\text { chase } \ldots \ldots \ldots\end{array}$ & 200 \\
\hline do & 44 & & $1881,{ }^{\prime} 85,{ }^{\prime} 90, ' 95 \ldots$ & 200 \\
\hline do & 45 & & Purchase........ & 200 \\
\hline do & 46 & & $\begin{array}{c}1877, \text { ' } 81, \text { '\$5 \& } \\
\text { purchase. . . . }\end{array}$ & 200 \\
\hline do & 47 & & $1877, ' 81, ' 85, ' 90 \ldots$ & 200 \\
\hline do & 49 & & Conveyed \& 1905. & 200 \\
\hline do & 50 & & Conveyed \& 1905. & 200 \\
\hline do & 51 & & Conveyed \& 1905. & 200 \\
\hline do & 52 & & Conveyed \& $100 . \mathrm{s}$ & 2001 \\
\hline do & 53 & & Conveyed \& 1905. & 200 \\
\hline do & 54 & $\ldots$ & $\begin{array}{r}1877, ' \$ 1, \text { ' } 85,90 \& \\
\text { purchase. . . . . }\end{array}$ & 200 \\
\hline
\end{tabular}


Hamuton County

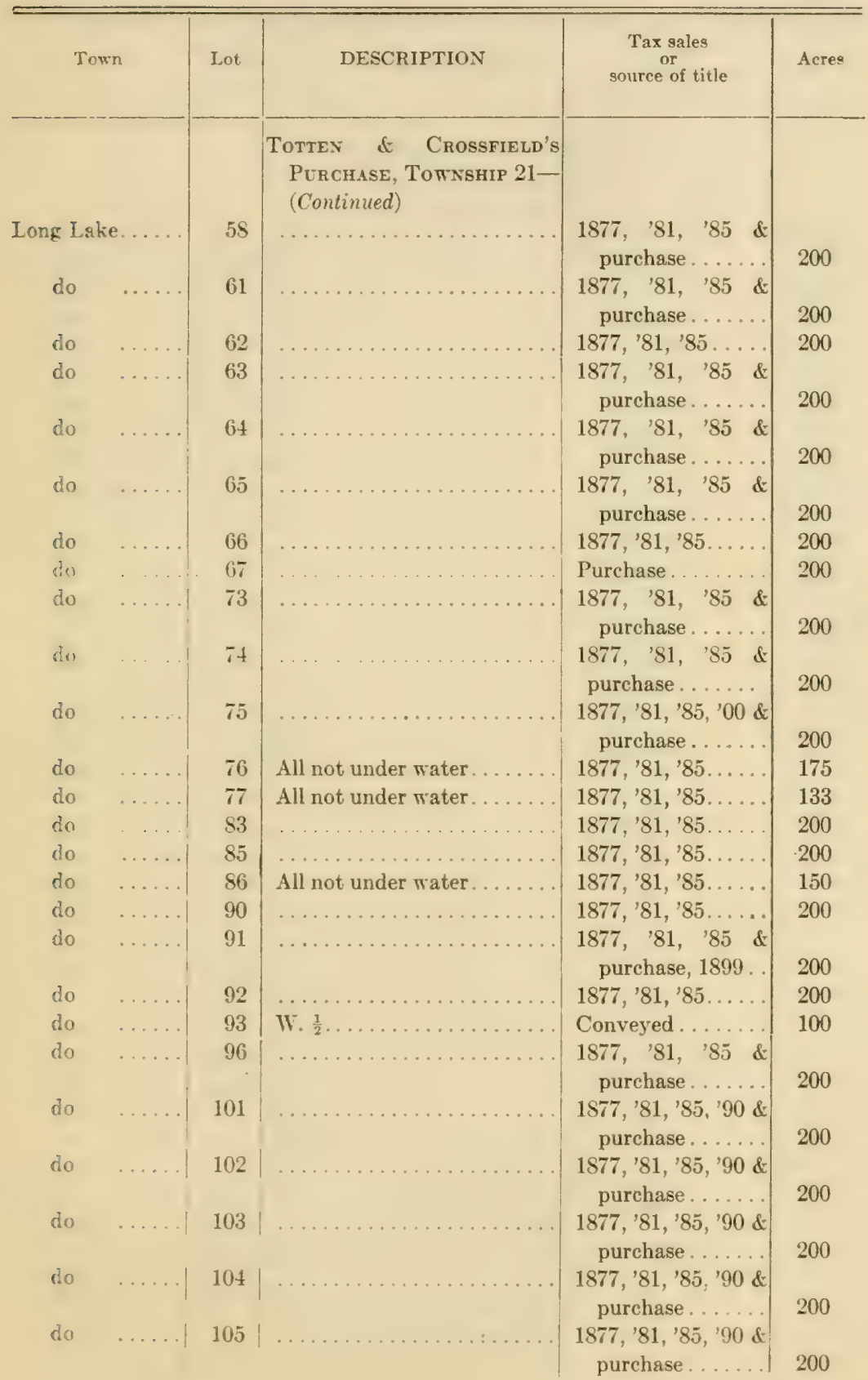


Hamitox Couyty

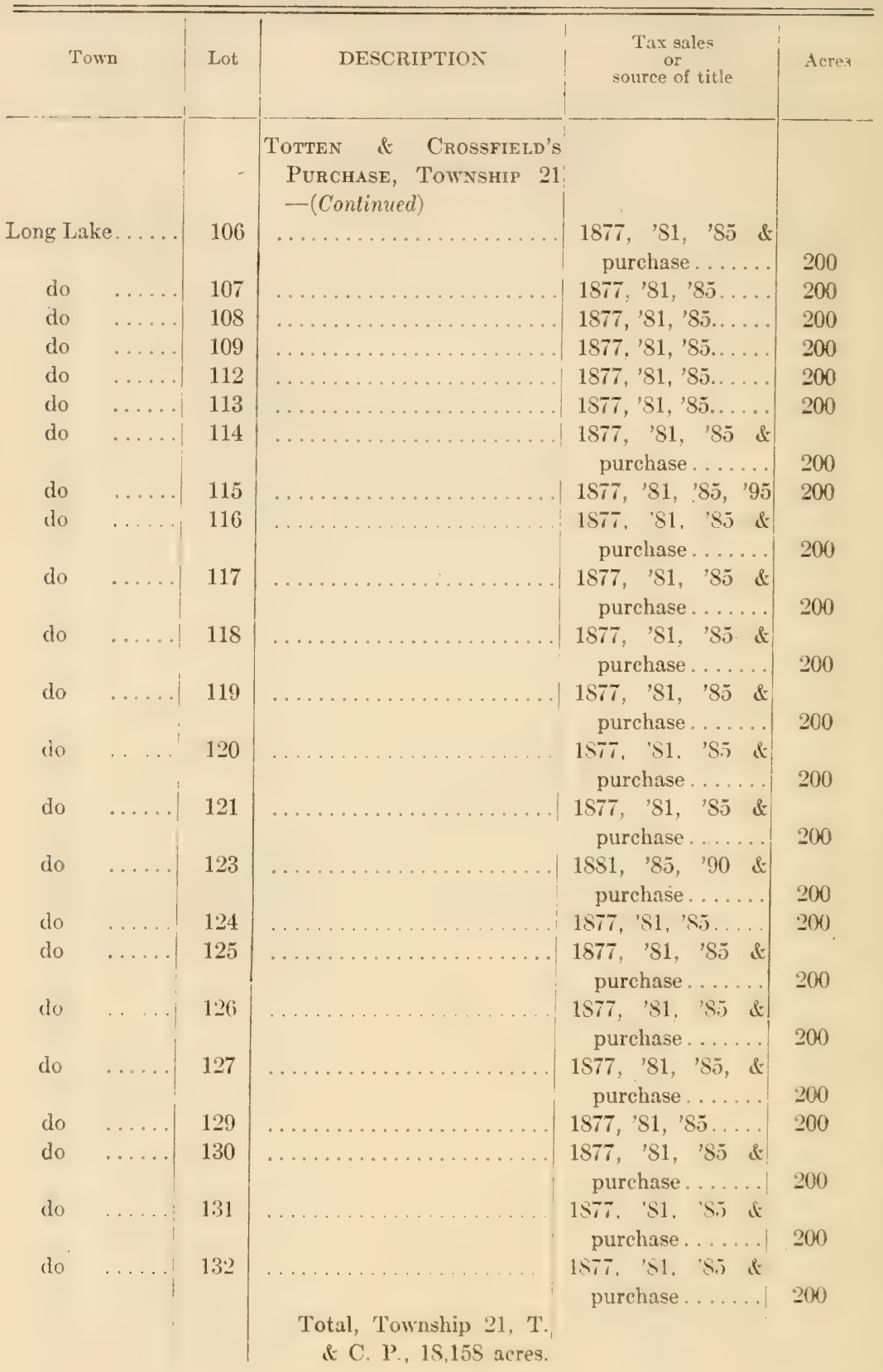


Hamilton County

\begin{tabular}{|c|c|c|c|c|}
\hline Town & Lot & DESCRIPTION & $\begin{array}{c}\text { Tax sales } \\
\text { or } \\
\text { source of title }\end{array}$ & Acres \\
\hline & & $\begin{array}{c}\text { TOTTEN \& CROSSFIELD, } \\
\text { Township } 22 \\
\left(\text { South } \frac{1}{2}\right)\end{array}$ & & \\
\hline Long Lake. & 1 & $\ldots \ldots \ldots \ldots \ldots \ldots \ldots$ & Conveyed....... & 176 \\
\hline do & 2 & & Conveyed....... & 169 \\
\hline do & 3 & 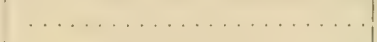 & Conveyed....... & 170 \\
\hline do & 4 & . & Conveyed........ & 162 \\
\hline 10 & 9 & $\cdots \cdots$ & $\begin{array}{c}1881, \quad ' 85, \quad ' 90 \quad \& \\
\text { purchase. . } \ldots . . .\end{array}$ & 158 \\
\hline (io) & 10 & & Conveyed........ & 154 \\
\hline do & 11 & & Conveyed....... & 147.50 \\
\hline do & 12 & 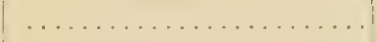 & Conveyed....... & 158 \\
\hline do & 13 & & Conveyed....... & 166.75 \\
\hline do & 14 & & Purchase........ & 151.50 \\
\hline do & 15 & & Conveyed....... & 158 \\
\hline do & 22 & 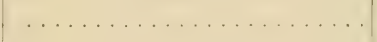 & Conveyed........ & 160 \\
\hline do & 23 & & $1877, ' 81, ' 85 \ldots$. & 155 \\
\hline do & 24 & & Purchase........ & 167.50 \\
\hline do & 25 & & Purchase........ & 172 \\
\hline do & 48 & $\ldots$ & Purchase......... & 163 \\
\hline do & 49 & & Purchase........ & 161.40 \\
\hline do & 55 & Ex. 14:a., s. pt. & Conveyed....... & 52 \\
\hline do & 59 & All not covered by water. & Purchase........ & 124 \\
\hline do & 60 & $\cdots$ & $1877, ' 81, ' 85 . \ldots$ & 160 \\
\hline do & 61 & $\ldots \ldots \ldots \ldots$ & $1877, ' 81, ' 85 \ldots .$. & 160 \\
\hline do & 62 & All not covered by water... & Purchase........ & 106 \\
\hline do & 63 & $\begin{array}{l}\text { N. end, S. pt. water....... } \\
\text { Total, Township } 22, \text { T. \& } \\
\text { C. P., 3,381.65 acres. }\end{array}$ & 1877. & 30 \\
\hline & & \begin{tabular}{|c} 
Totten \& Crossfield's \\
Purchase, Township 23, \\
Including Triangle
\end{tabular} & & \\
\hline$d x$ & 1 & $\ldots \ldots \ldots \ldots \ldots \ldots \ldots \ldots$ & 1871, '81, '85, ’90. & 148 \\
\hline Hells ...... & $\ldots$ & \begin{tabular}{|} 
TotTeN \& CRossfield's \\
Township 29 \\
Beg. at N. W'ly cor. of lot 16 \\
in said township, th. along \\
the N'ly line of lots $16 \& 17$ \\
in said township, N. $63 \mathrm{deg}$. \\
E. $86 \mathrm{c} .211$. to the county \\
line, th. N'ly along the \\
county line to the N'ly line \\
of township 29, th. S. 63
\end{tabular} & & \\
\hline
\end{tabular}




\section{Hamilton County}

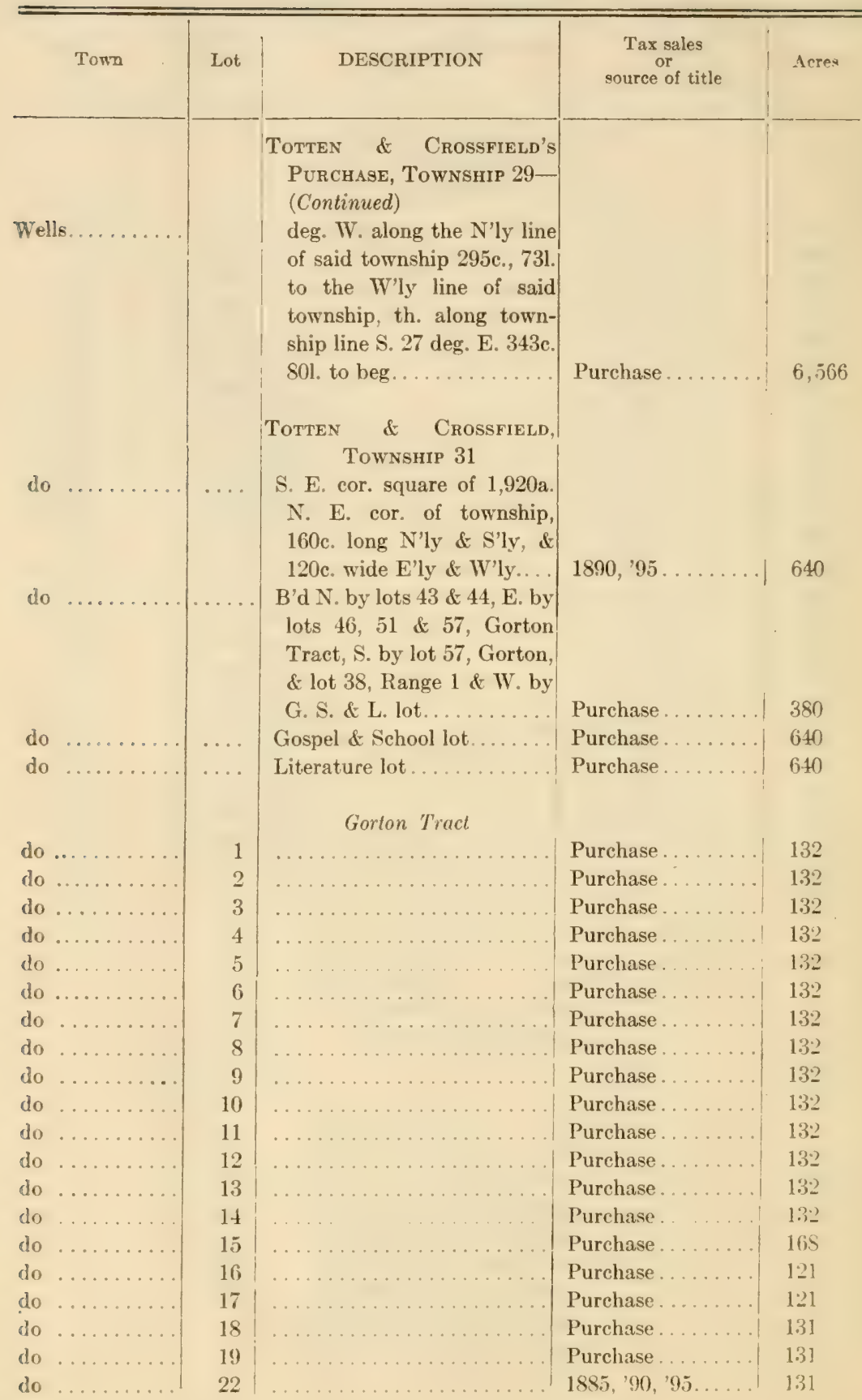


Hamilton County

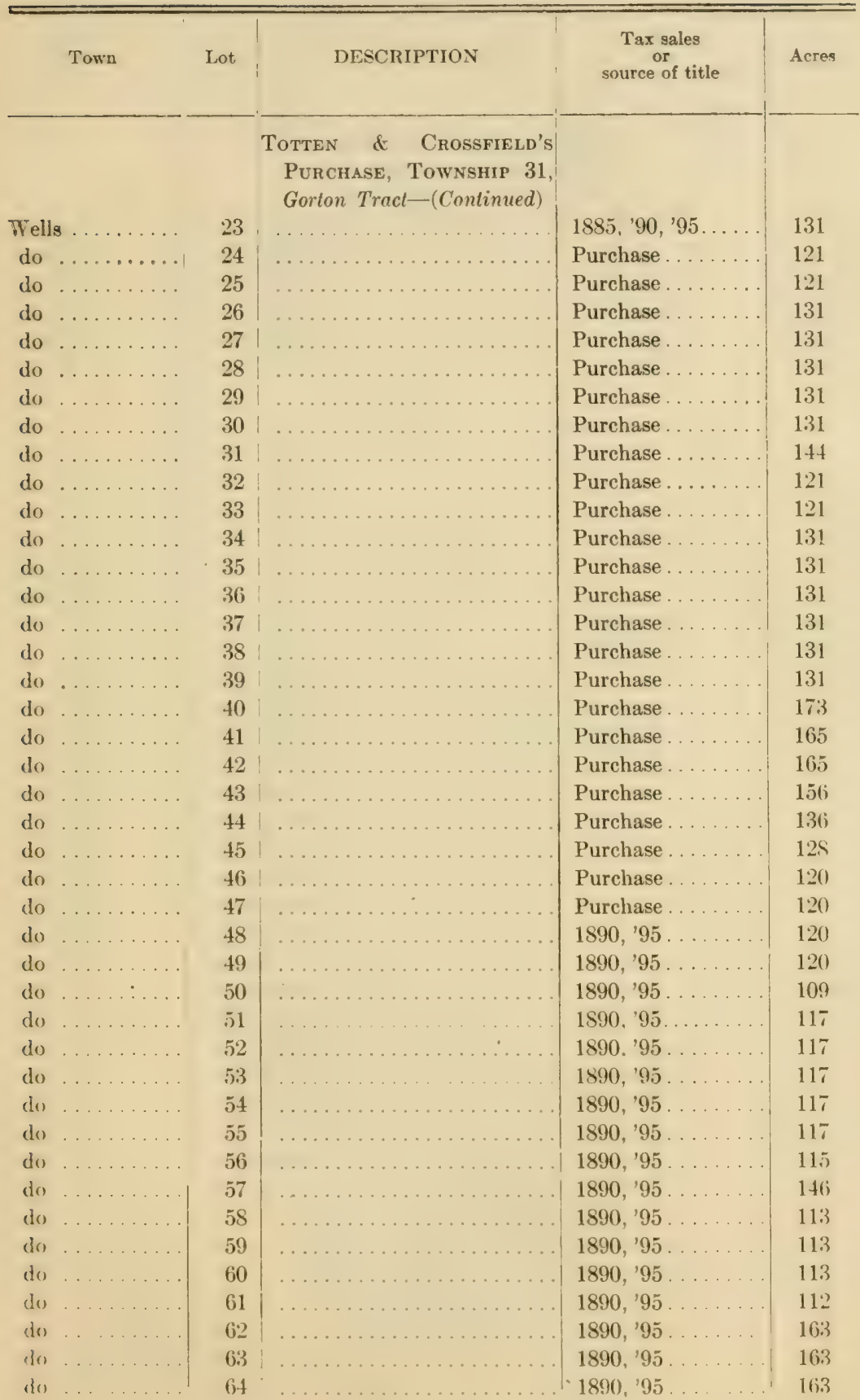


Hamilton County

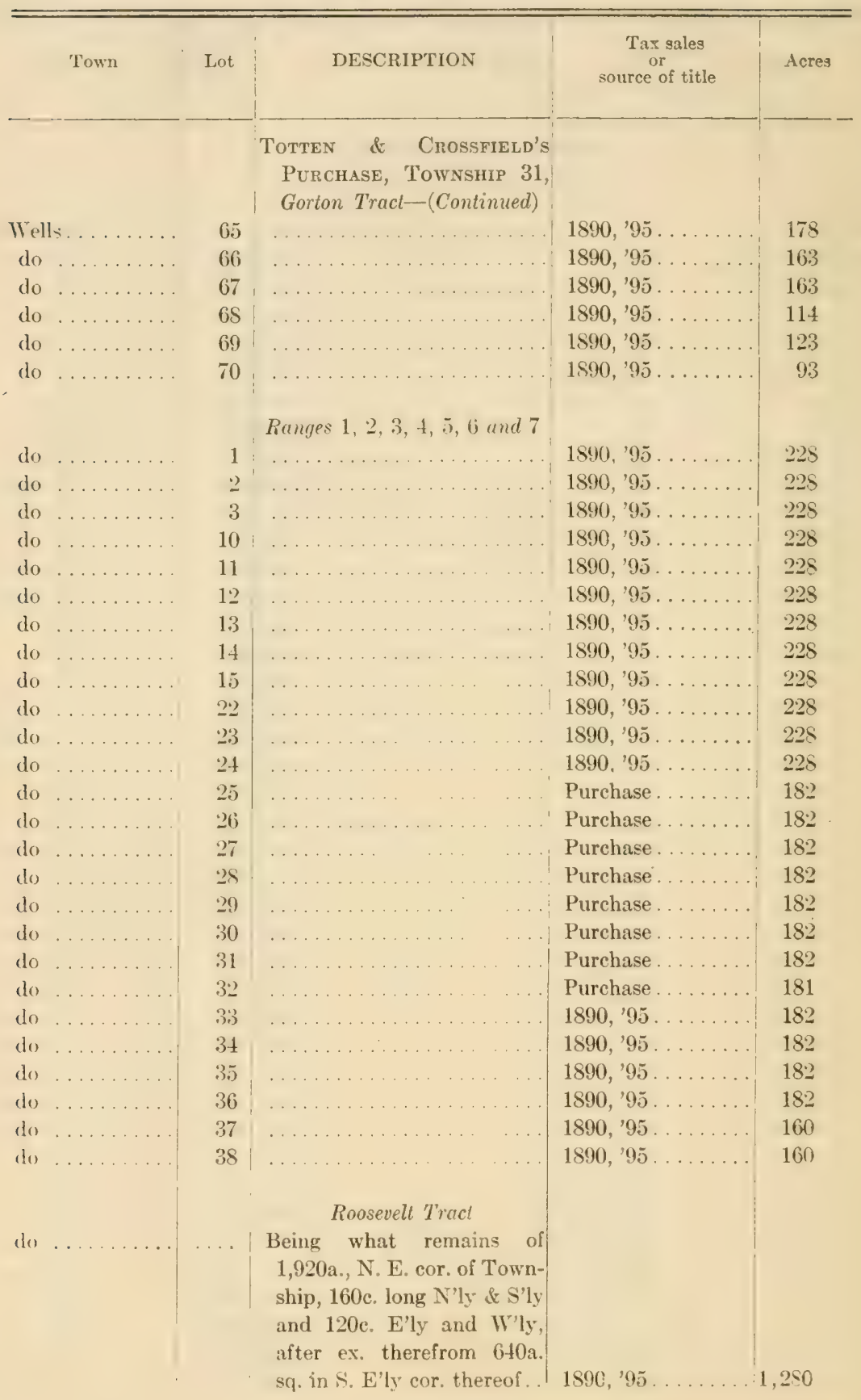


Hamilton County

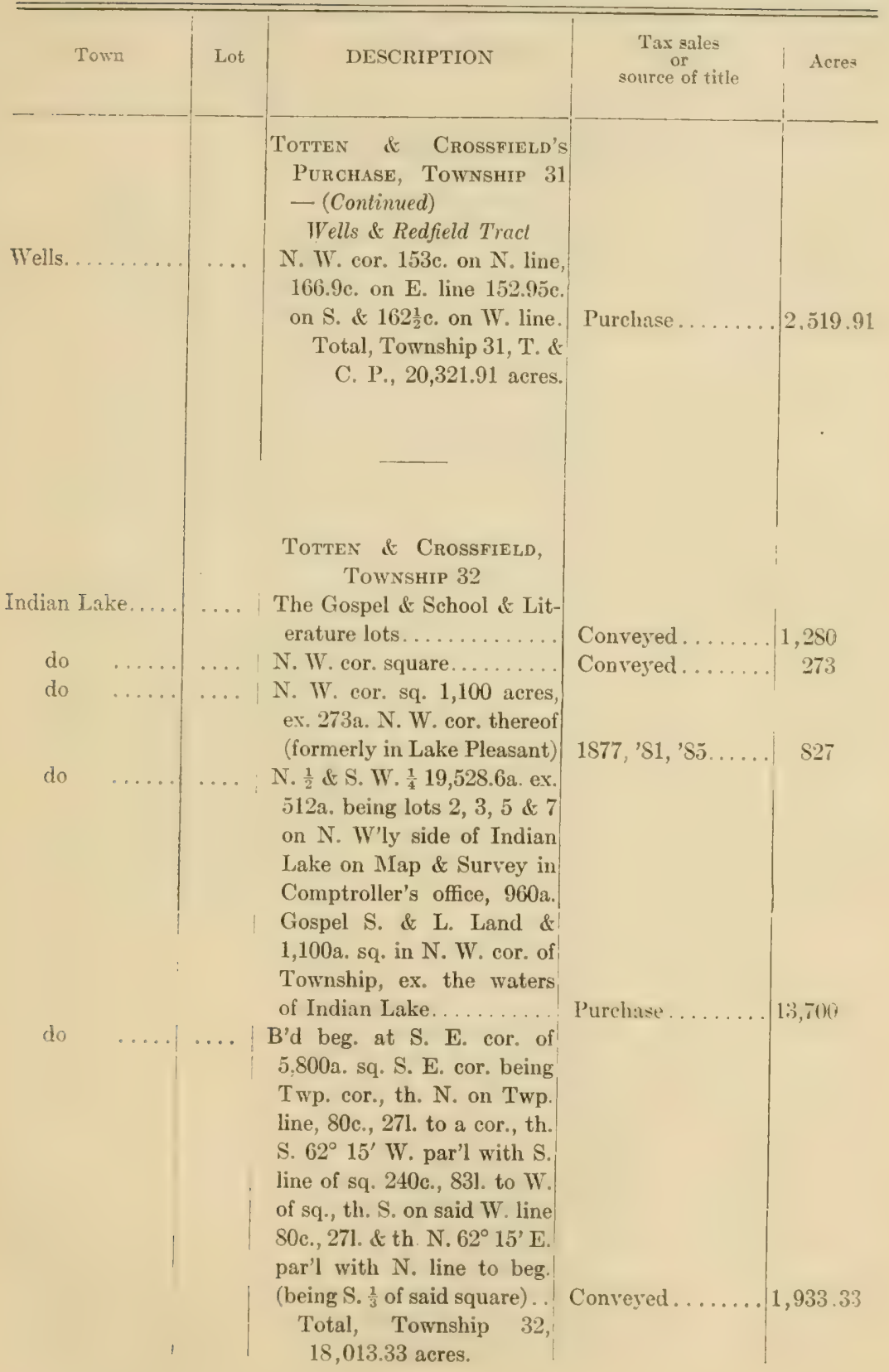




\section{Hamilton Counti}

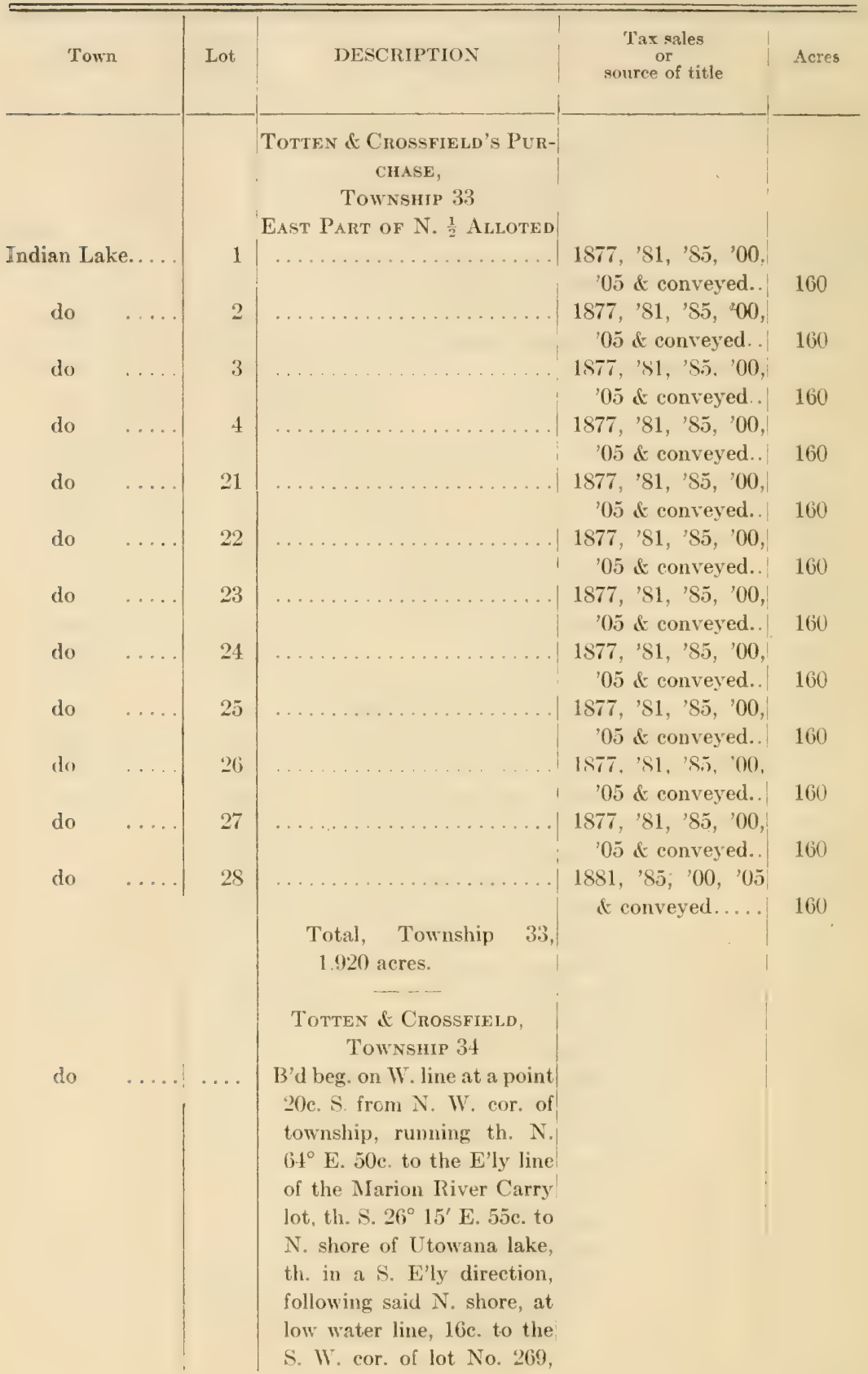




\section{Hamiton County}

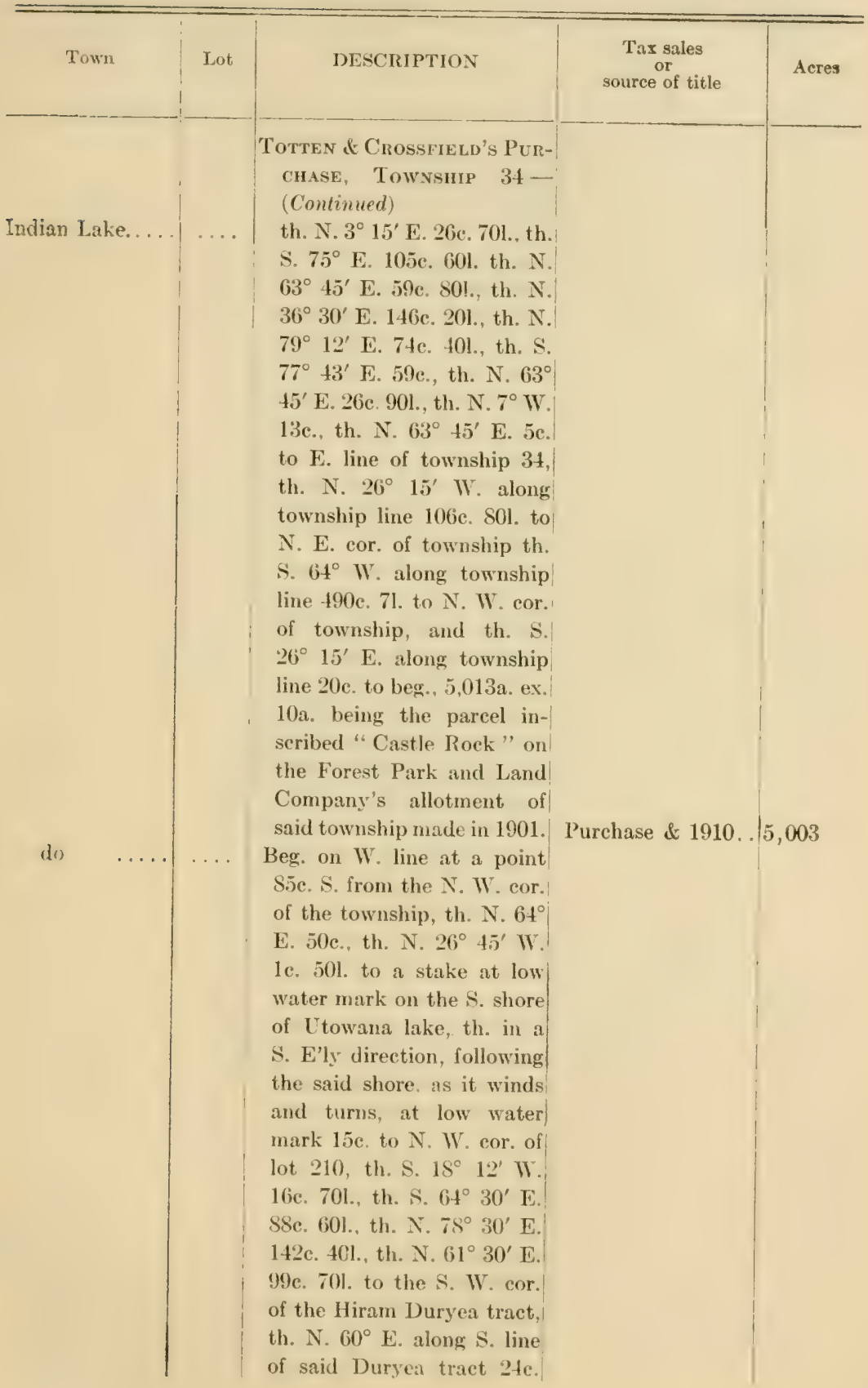


Hanilton County

\begin{tabular}{|c|c|c|c|}
\hline Town & Lot & DESCRIPTION & $\begin{array}{c}\text { Tax sales } \\
\text { or } \\
\text { source of title }\end{array}$ \\
\hline Indian Lake... & $\ldots$ & 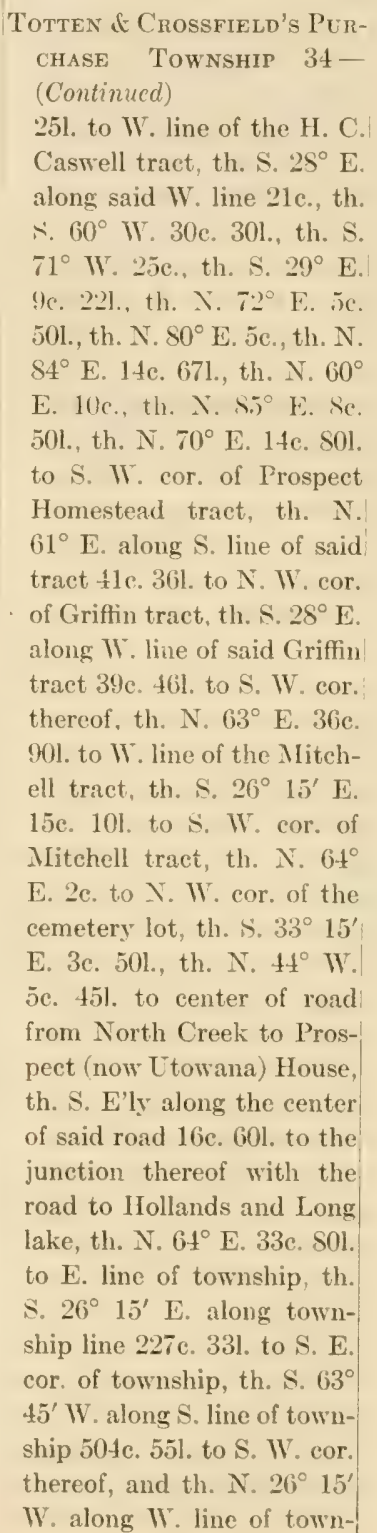 & \\
\hline
\end{tabular}


Hamiton County

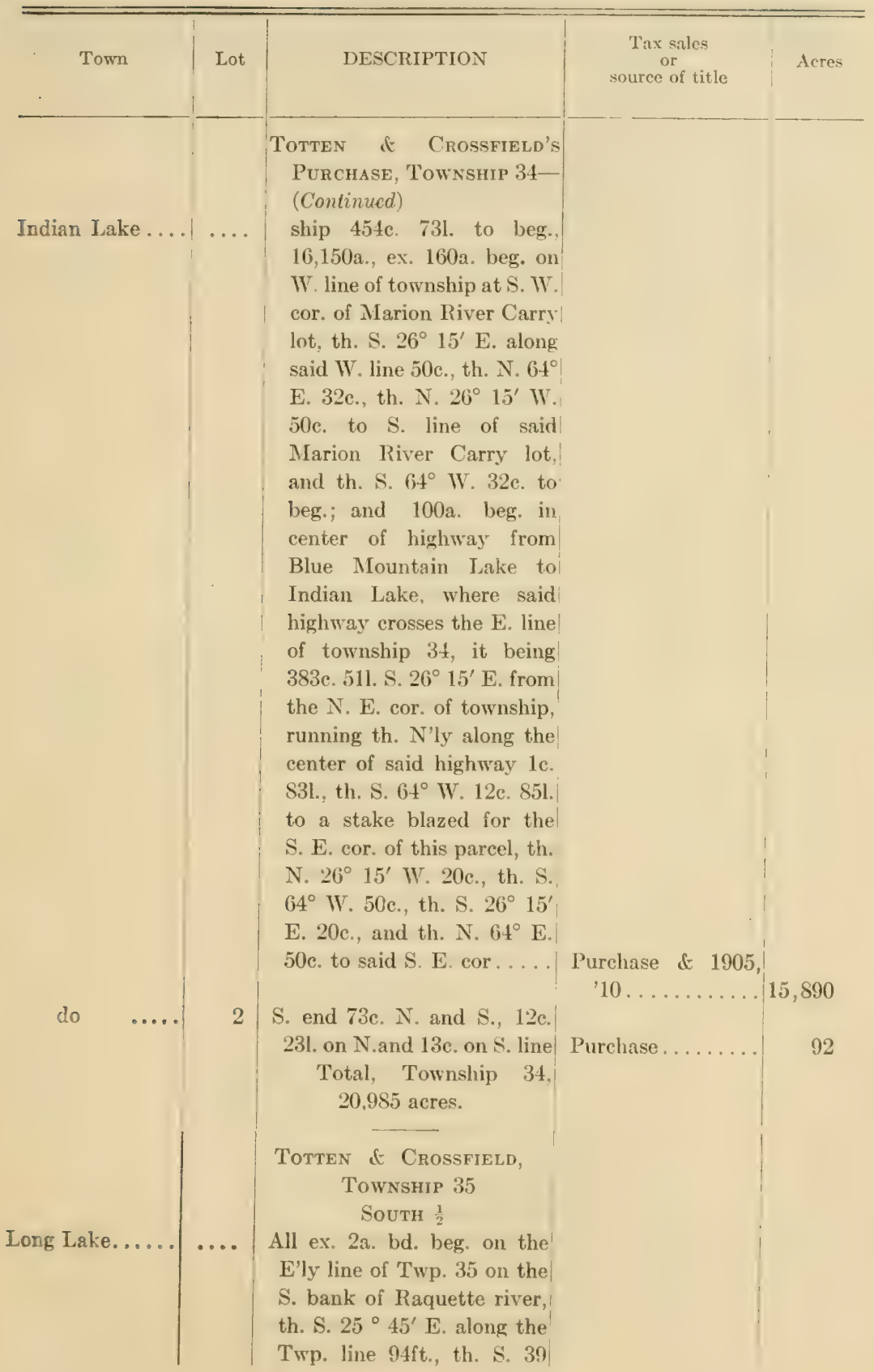




\section{Hamilton County}

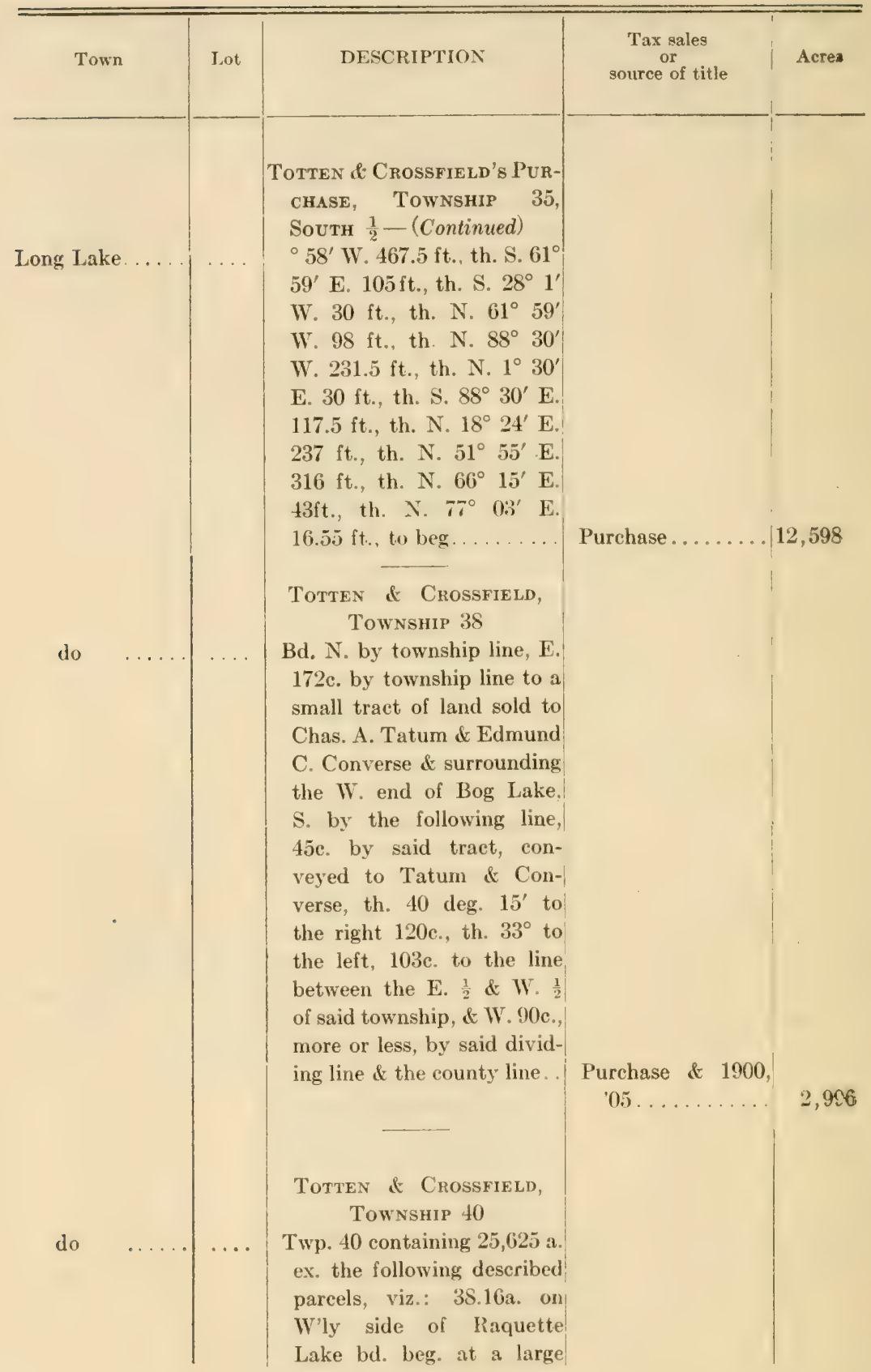




\section{Hamiton County}

\begin{tabular}{|c|c|c|c|}
\hline Town & Lot & DESCRIPTION & $\begin{array}{c}\text { Tax sales } \\
\text { or } \\
\text { source of title }\end{array}$ \\
\hline Long Lake.. . & $\ldots$ & 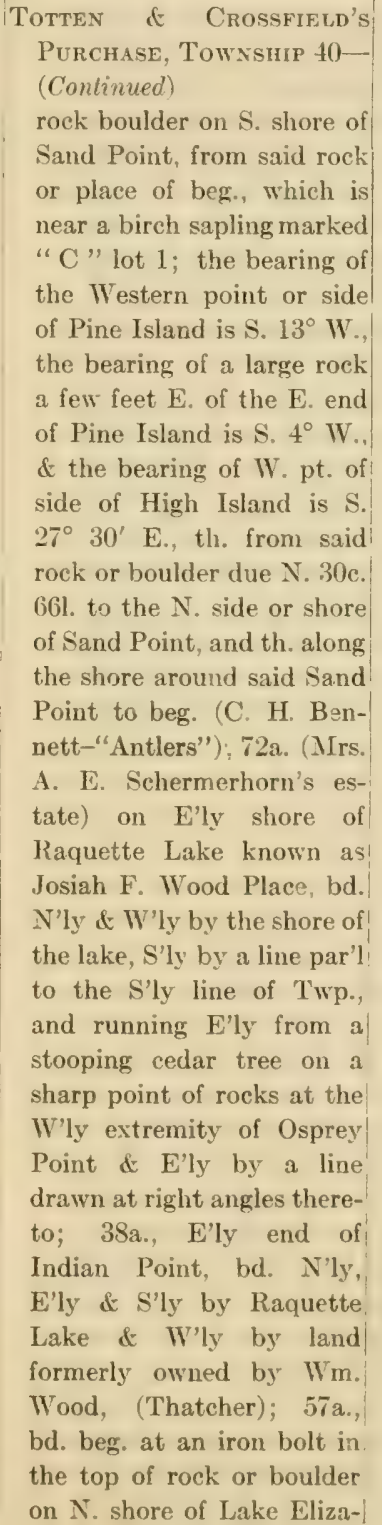 & \\
\hline
\end{tabular}




\section{Hamilton County}

\begin{tabular}{|c|c|c|c|c|}
\hline Town & Lot & DESCRIPTION & $\begin{array}{c}\text { Tax sales } \\
\text { or } \\
\text { source of title }\end{array}$ & Acres \\
\hline Long Lake... & $\ldots$ & 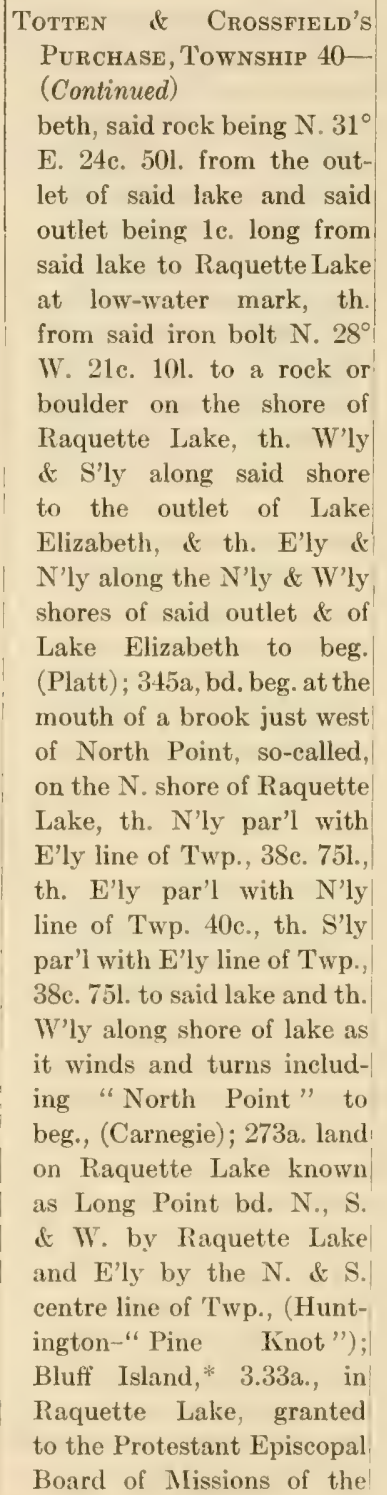 & & 6 \\
\hline
\end{tabular}

* Linown also as Church Island and as St. Hubert's Island. 
Hamilton County

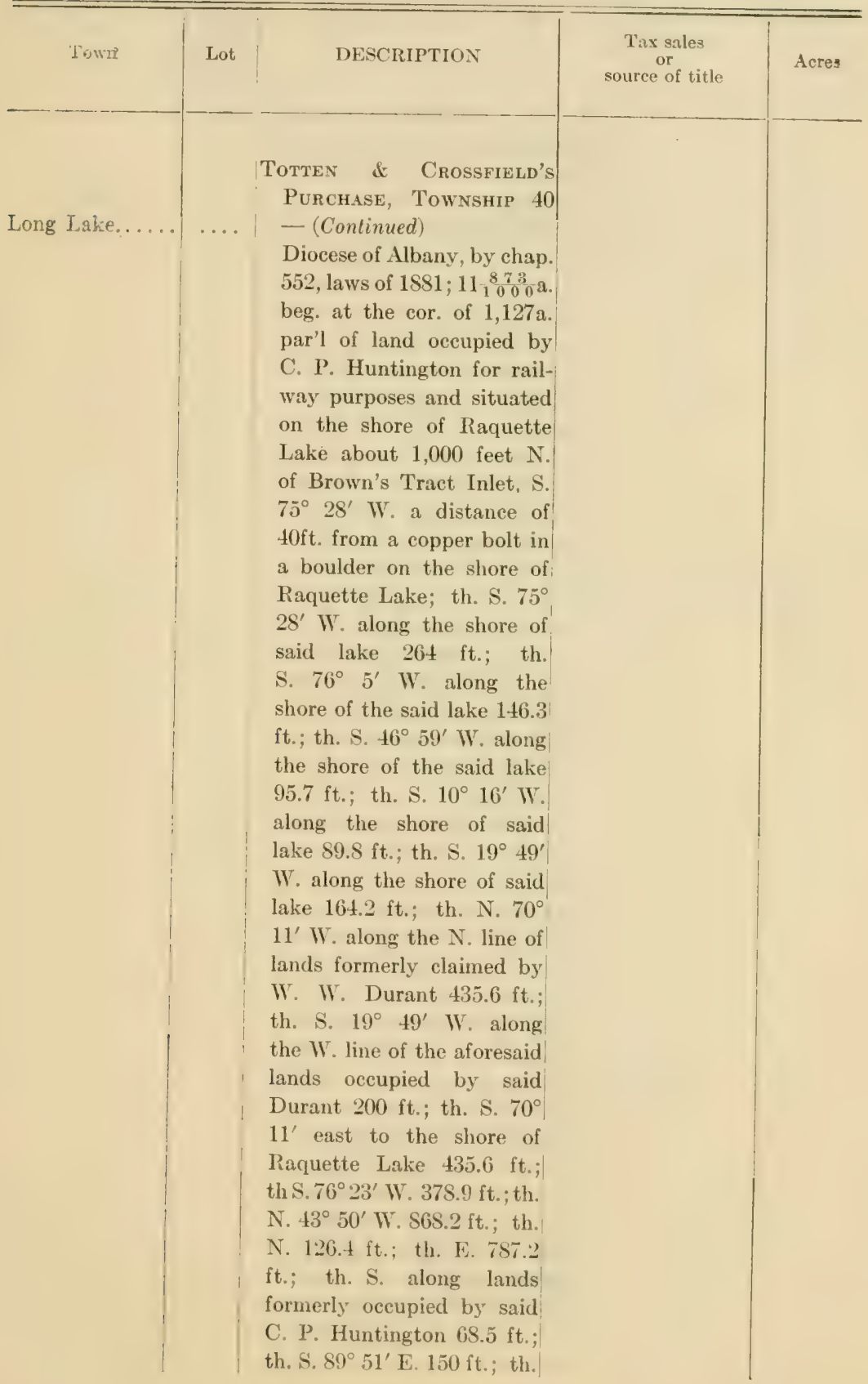




\section{Hamilton Countr}

\begin{tabular}{|c|c|c|c|c|}
\hline Town & Lot & DESCRIPTION & $\begin{array}{c}\text { Tax sales } \\
\text { or } \\
\text { source of title }\end{array}$ & Acres \\
\hline Long Lake.. & $\ldots$ & 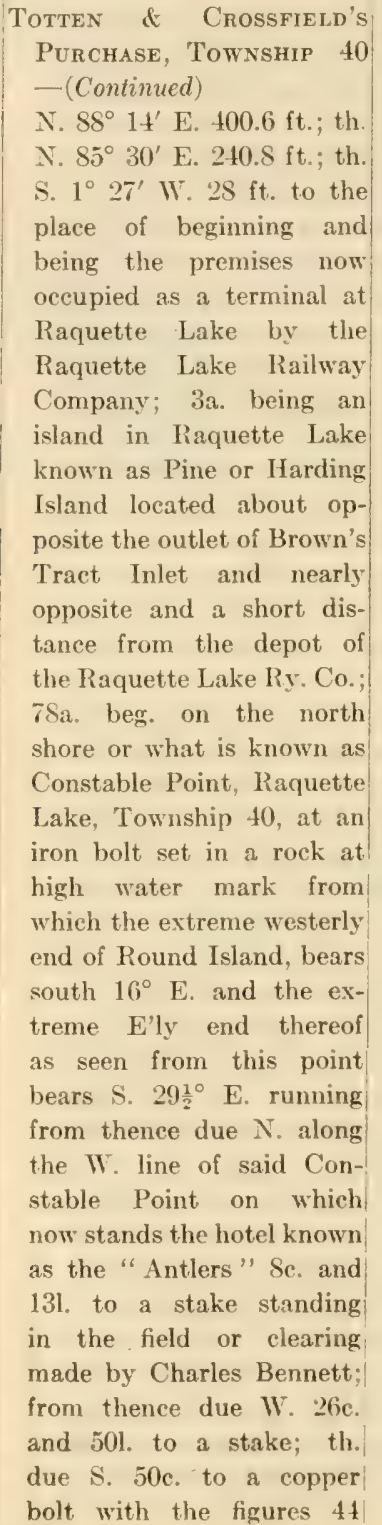 & & \\
\hline
\end{tabular}


Hamition County

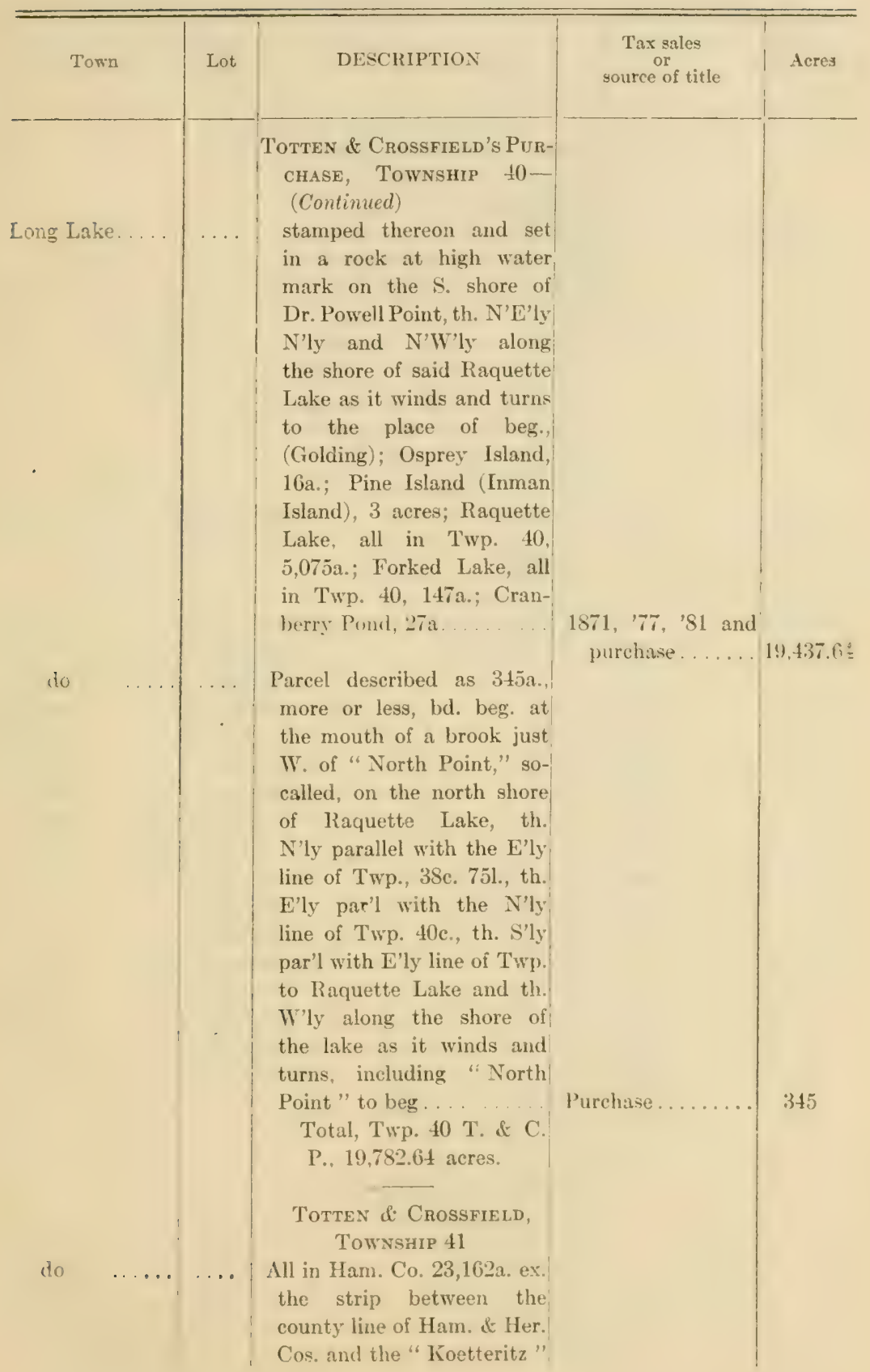




\section{Hamiton County}

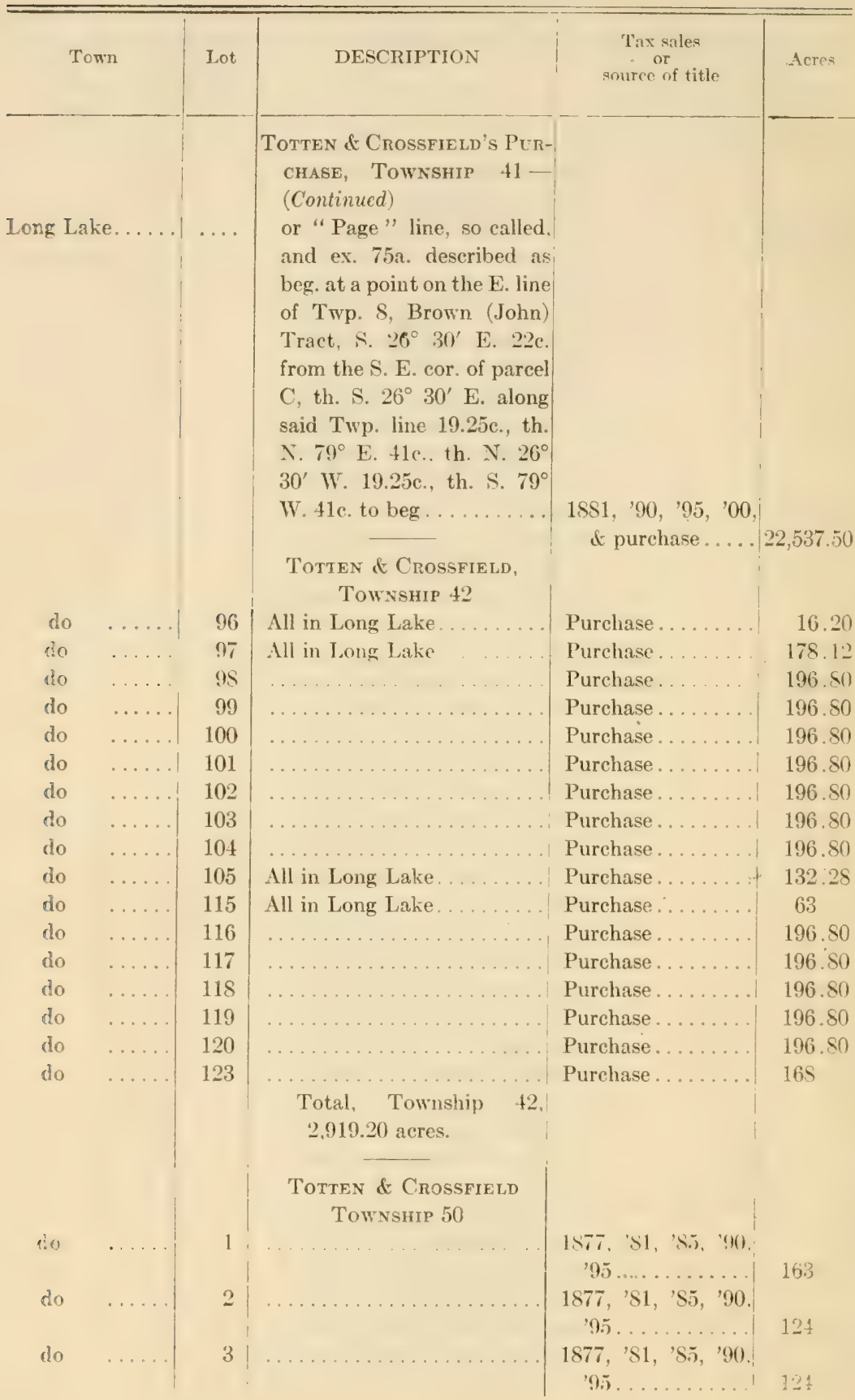




\section{Hamilton County}

\begin{tabular}{|c|c|c|c|c|}
\hline Town & Lot & DESCRIPTION & $\begin{array}{c}\text { Tax sales } \\
\text { or } \\
\text { source of title }\end{array}$ & Acres \\
\hline \multirow[b]{2}{*}{ Long I Lake. } & & $\begin{array}{l}\text { TotTen \& CRossfield's PUR- } \\
\text { Chase, Township } 50- \\
\text { (Continued) }\end{array}$ & & \\
\hline & 4 & $\ldots \ldots$ & Conveyed....... & 124 \\
\hline do & 7 & & Conveyed....... & 124 \\
\hline do & 9 & & Purchase....... & 124 \\
\hline do & 10 & & Purchase... & 124 \\
\hline do & 11 & 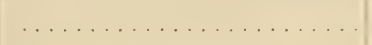 & Purchase \& $1905 \ldots$ & 124 \\
\hline do & 12,13 & & Purchase... & 1.46 \\
\hline do & 14,15 & & Purchase........ & 190 \\
\hline do & 16 & & Purchase . . . . . . . & 160 \\
\hline do & 17 & & Purchase \& 1905. & 160 \\
\hline do & 18 & & Purchase ....... & 160 \\
\hline do & 19 & & Purchase....... & 160 \\
\hline do & 23 & & $1877, ' 81,{ }^{\prime} 85 \ldots$. & 160 \\
\hline do & 24 & & $\begin{array}{r}1871,77, \quad 81, \quad 8 . \\
95, \ldots \ldots \ldots\end{array}$ & 160 \\
\hline do & 26 & & Purchase ........ & 154.87 \\
\hline do & 27 & & $\begin{array}{r}1871, ' 77, \quad 81, \quad 85, \\
95 \ldots \ldots \ldots \ldots\end{array}$ & 120 \\
\hline do & 29 & & $1877, \$ 1,85 \ldots$. & 120 \\
\hline do & 33 & & Conveyed....... & 120 \\
\hline do & 34 & & Purchase ........ & 120 \\
\hline do & 35 & & Purchase ........ & 120 \\
\hline do & 36 & & Purchase ....... & 120 \\
\hline do & 37,38 & & Purchase . . . . . . . & 143 \\
\hline do & 39,40 & & Purehase ........ & 192.50 \\
\hline do & 41 & & Purchase . . . . . . . & 160 \\
\hline do & 42 & & Purchase . . . . . . & 160 \\
\hline do & 43 & & Purchase . . . . . . . & 160 \\
\hline do & 44 & & Purchase........ & 160 \\
\hline do & 45) & & Purchase ........ & 160 \\
\hline do & $4 S$ & & $1877, ' 81, ' 85,{ }^{\prime} 90$. & 160 \\
\hline do & 49 & & $1877, ' 81, ' 85 \ldots$. & 160 \\
\hline do & 50 & & $1877, ' 81, ' \$ 5 \ldots \ldots$ & 160 \\
\hline do & 51 & & $\begin{array}{r}1871,77,81, \quad 85, \\
95 \ldots \ldots \ldots \ldots\end{array}$ & $25 t$ \\
\hline do & 52 & & $\begin{array}{r}1871,77,81,85, \\
95 \ldots \ldots \ldots\end{array}$ & 160 \\
\hline do & 53 & & Purchase........ & 120 \\
\hline do & 54 & & Purchase ........ & 120 \\
\hline do & 56 & & Purchase \& 1900. & 160 \\
\hline do & 57 & & Purchase ...... & 160 \\
\hline do & is & & Purchase. & 160 \\
\hline
\end{tabular}


Hamilton County

\begin{tabular}{|c|c|c|c|c|}
\hline Town & \multirow[t]{2}{*}{ Lot } & DESCRIPTION & \multirow[t]{2}{*}{$\begin{array}{c}\text { Tax sales } \\
\text { or } \\
\text { source of title }\end{array}$} & \multirow[t]{2}{*}{ Acres } \\
\hline & & $\begin{array}{l}\text { Totten \& Crossfield's PUR- } \\
\text { Chase, Township 50- } \\
\text { (Continued) }\end{array}$ & & \\
\hline Long Lake. & 59 & All in Hamilton $\mathrm{Co}$. & Conveyed. & 142.25 \\
\hline do & 60,61 & All in Hamilton Co. & Purchase ... & 60 \\
\hline do & 65 & All in Hamilton $\mathrm{Co}$. & Purchase. . & 88.60 \\
\hline do & 66 & All in Hamilton Co. & Purchase ........ & 158 \\
\hline do & 67 & & Purchase... . & 160 \\
\hline do & $6 s$ & & $1890,{ }^{\prime} 00 \ldots$ & 160 \\
\hline do & 69 & & Purchase ........ & 120 \\
\hline do & 72 & . & Purchase... . & 158.76 \\
\hline do & 73 & & Conveyed... & 120 \\
\hline do & 76 & All in Humilton Co. & Purchase.... & 149.55 \\
\hline do & 77 & All in Hamilton Co. . & Purchise. & 44.32 \\
\hline do & $\$ 7$ & All in Hamilton Co. & Conveyed \& 1910.. & 19 \\
\hline do & SS & All in Hamilton $\mathrm{Co}$. & Conveyed....... & 120 \\
\hline do & 89 & $\ldots \ldots \ldots \ldots$ & Conveyed \& 1910.. & 120 \\
\hline do & 90 & & Purchase........ & 184 \\
\hline do & 91 & All in Hamilton $\mathrm{Co}$. & Conveyed . . . . . . & 68.75 \\
\hline$d$, & 92 & $\begin{array}{l}\text { All in Hamilton } \mathrm{Co} \\
\text { Total, Township , } 0 \text {, } \\
7.860 .60 \text { acres. }\end{array}$ & Conveyed is 1910.. & 36 \\
\hline & & VRooman's PateNt & & \\
\hline Morehouse. & 9 & All in Morehouse... & $1885,90,95$. & 66 \\
\hline do & 10) & . & $1881, ' 85,90$. & 200 \\
\hline do & 11 & & $1881, ' 85, ' 90, \ldots$. & 200 \\
\hline do & 12 & All in Morehouse. & 1881, '85, '90, '95. & 66 \\
\hline do & 17 & All in Morehouse... & $1881, ' 95,90,95$. & 66 \\
\hline do & 18 & $\cdots$ & $1885, ' 00 \ldots$ & 200 \\
\hline do & 19 & & $1885, ' 90 \ldots$ & 200 \\
\hline do & 20 & All in Morehouse... & $15 \$ 1, ' 5.5,90$ & 66 \\
\hline do & 25 & All in Morehouse & $1881, ' 85,{ }^{\prime} 90,95$. & 66 \\
\hline de & 26 & & $1885,{ }^{\prime} 90,95 \ldots \ldots$ & 200 \\
\hline du & 27 & & $\begin{array}{r}\text { Purchase \& 1905, } \\
\text { '10. . . }\end{array}$ & $20 i)$ \\
\hline do & 28 & All in Hamilton $\mathrm{C} n$. & $\begin{array}{c}\text { Purchise \& } 190.5 \\
10 \ldots \ldots\end{array}$ & 66 \\
\hline do & 34 & All in Morehouse. ........ & $1 \S 85,{ }^{\prime} 90,{ }^{\prime} 95 \ldots$. & (i6) \\
\hline do & 3.5 & Ex. 50a. W. pt. of E. $\frac{1}{2} \ldots$ & $1885,90 \ldots \ldots \ldots$ & 150 \\
\hline de & 35) & W. pt. of E. $\frac{1}{2} \ldots$. & Purchase. & 50 \\
\hline do & 12 & & Purchase. . & 200 \\
\hline
\end{tabular}


Hamiton County

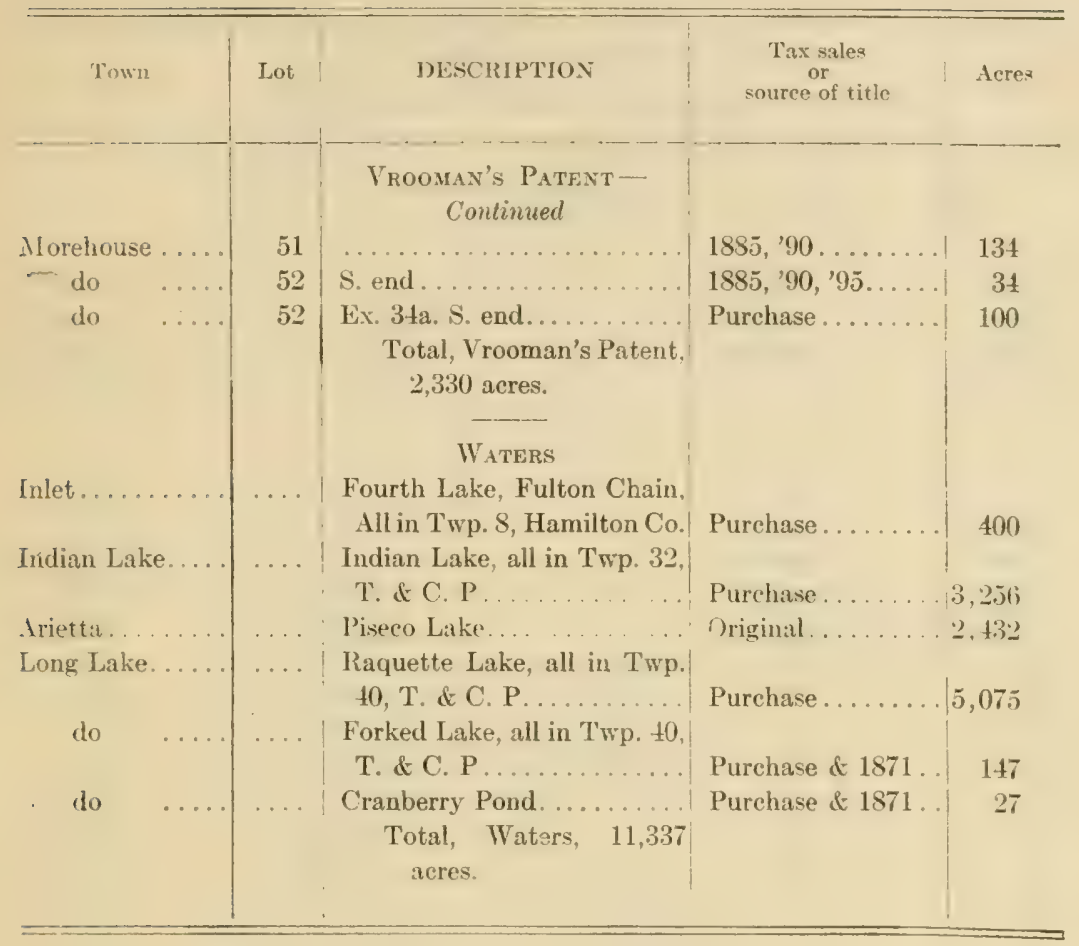


HERKIMER COUNTY

(Total number of acres, 195,467.14)

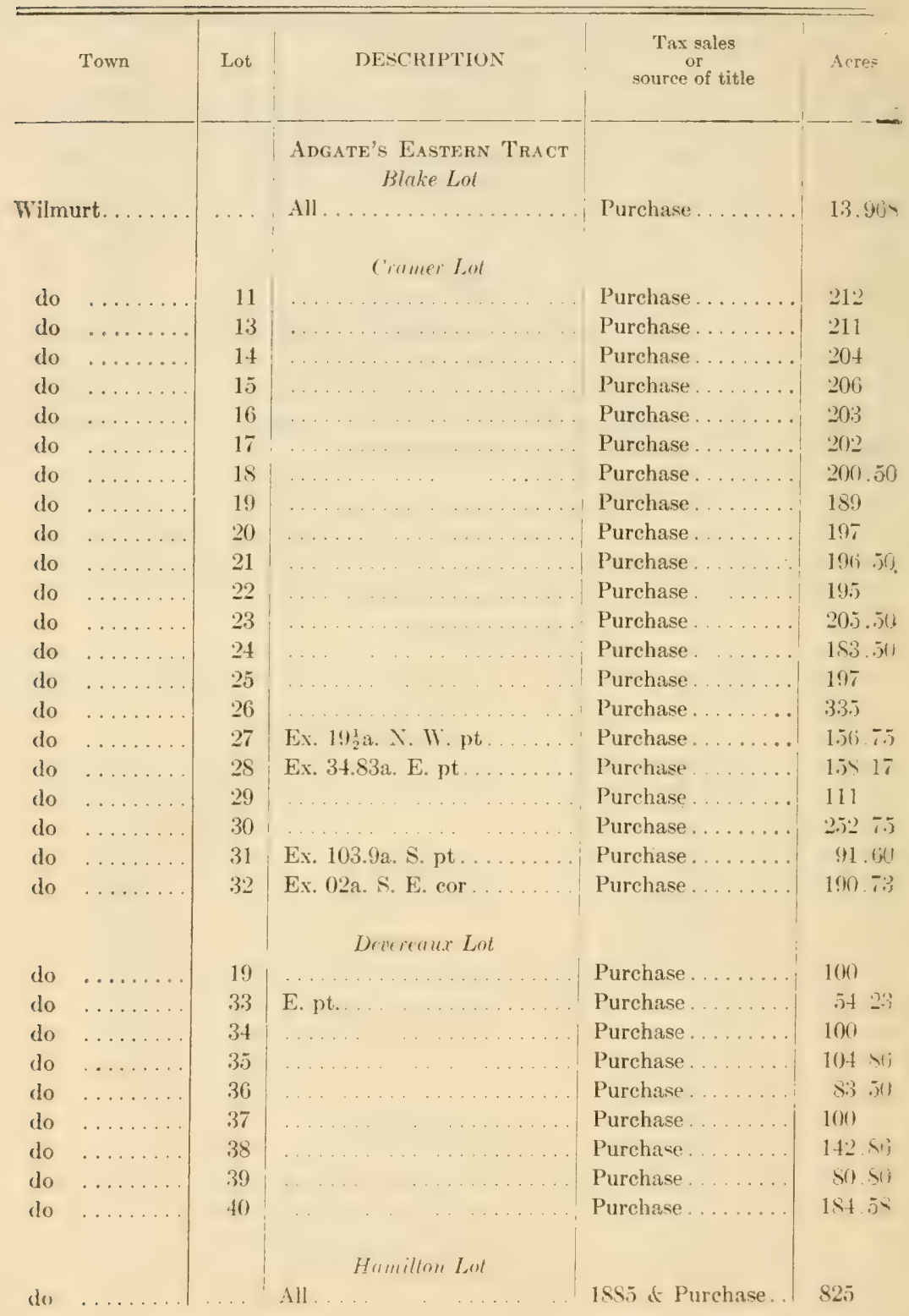




\section{Herkimer County}

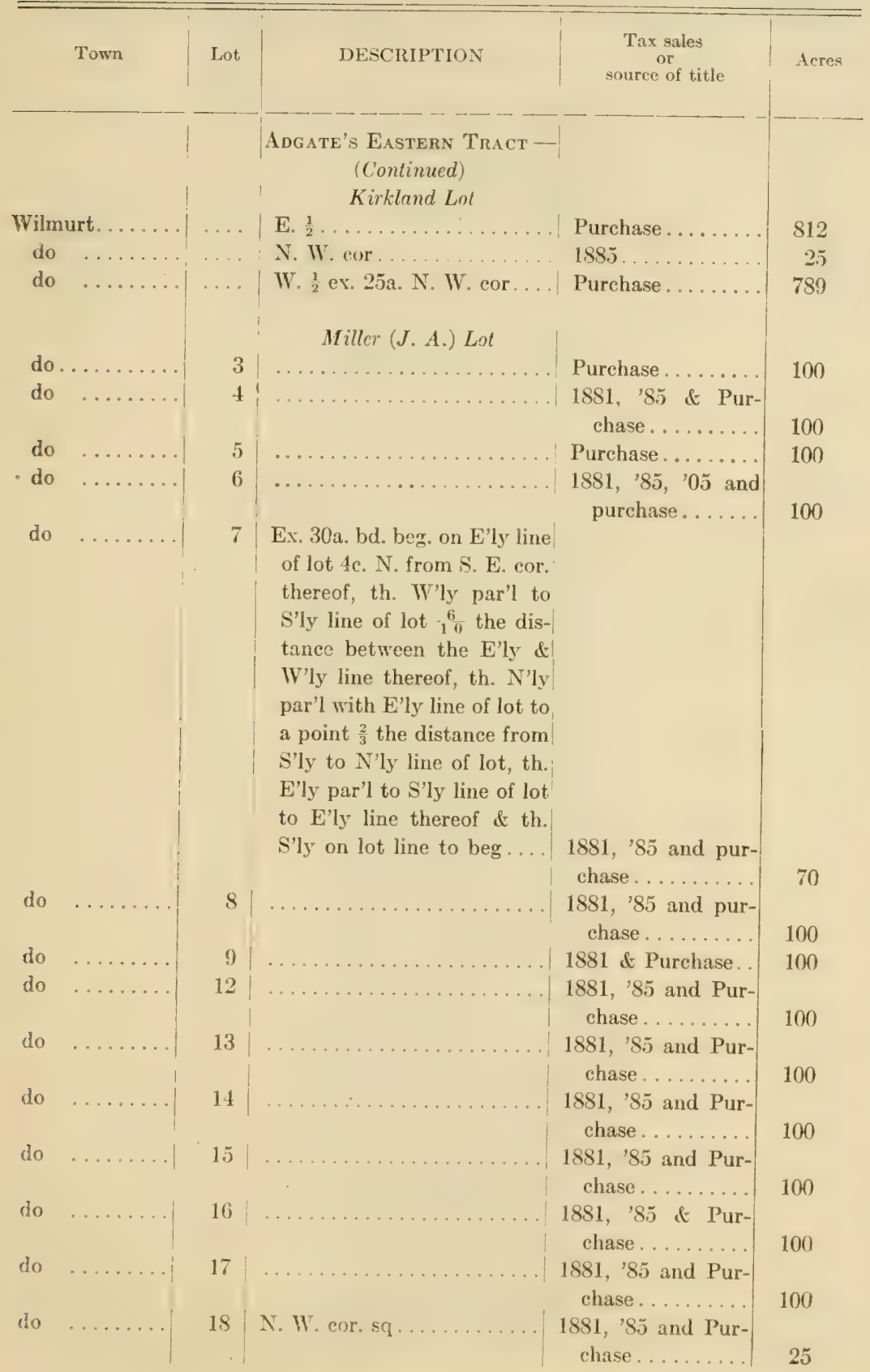


Herkimer Couyty

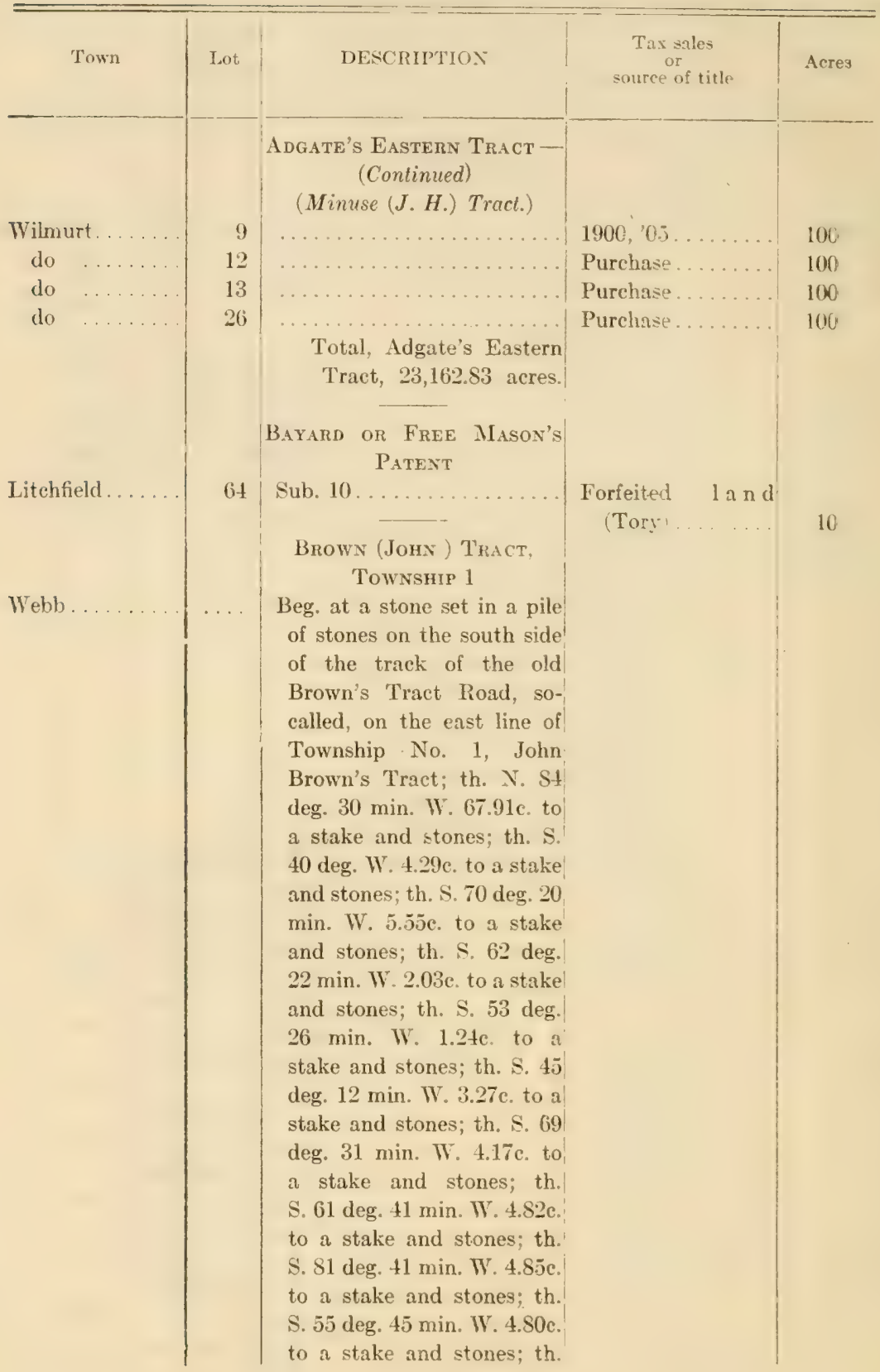




\section{Herkmer County}

\begin{tabular}{|c|c|c|c|c|}
\hline Town & Lot & DESCRIPTION & $\begin{array}{c}\text { Tax sales } \\
\text { or } \\
\text { soure of title }\end{array}$ & Acres \\
\hline Webb.... & $\ldots$ & 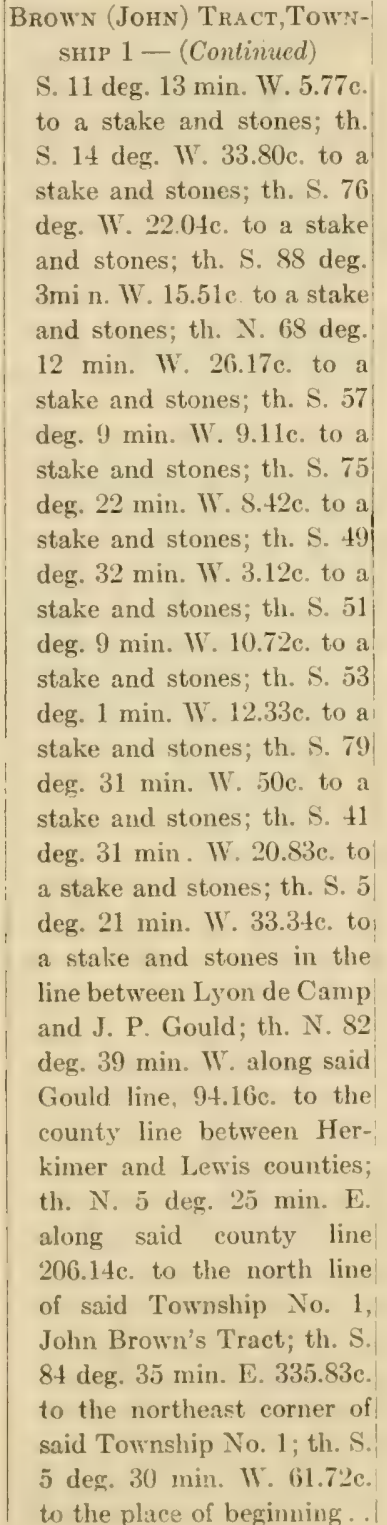 & . & 4.607 .20 \\
\hline
\end{tabular}




\section{Herkmer County}

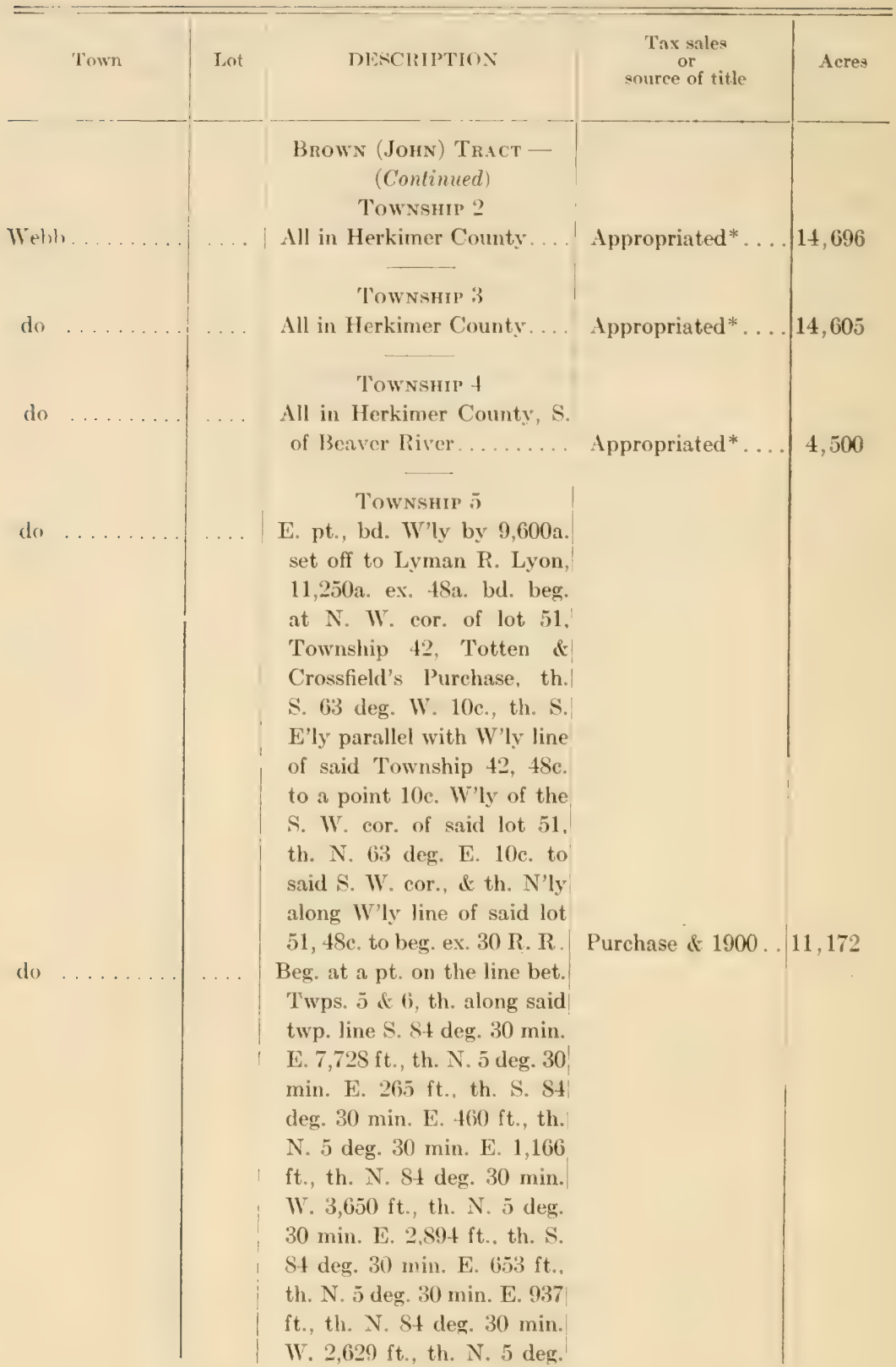

* The validity of this alleged appropriation of land is the subject matter of litigation. 
Herkimer County

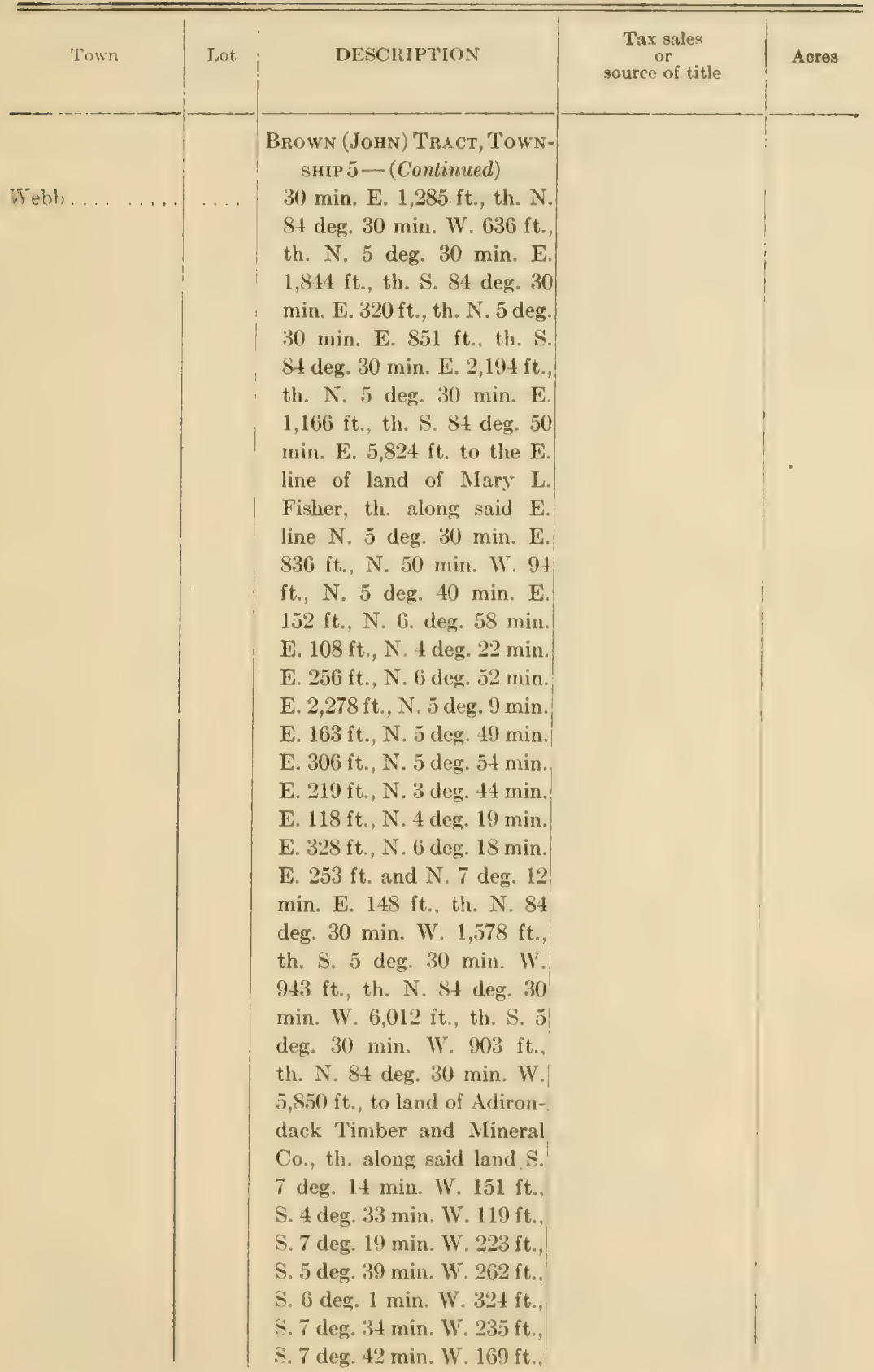




\section{Herkmer County}

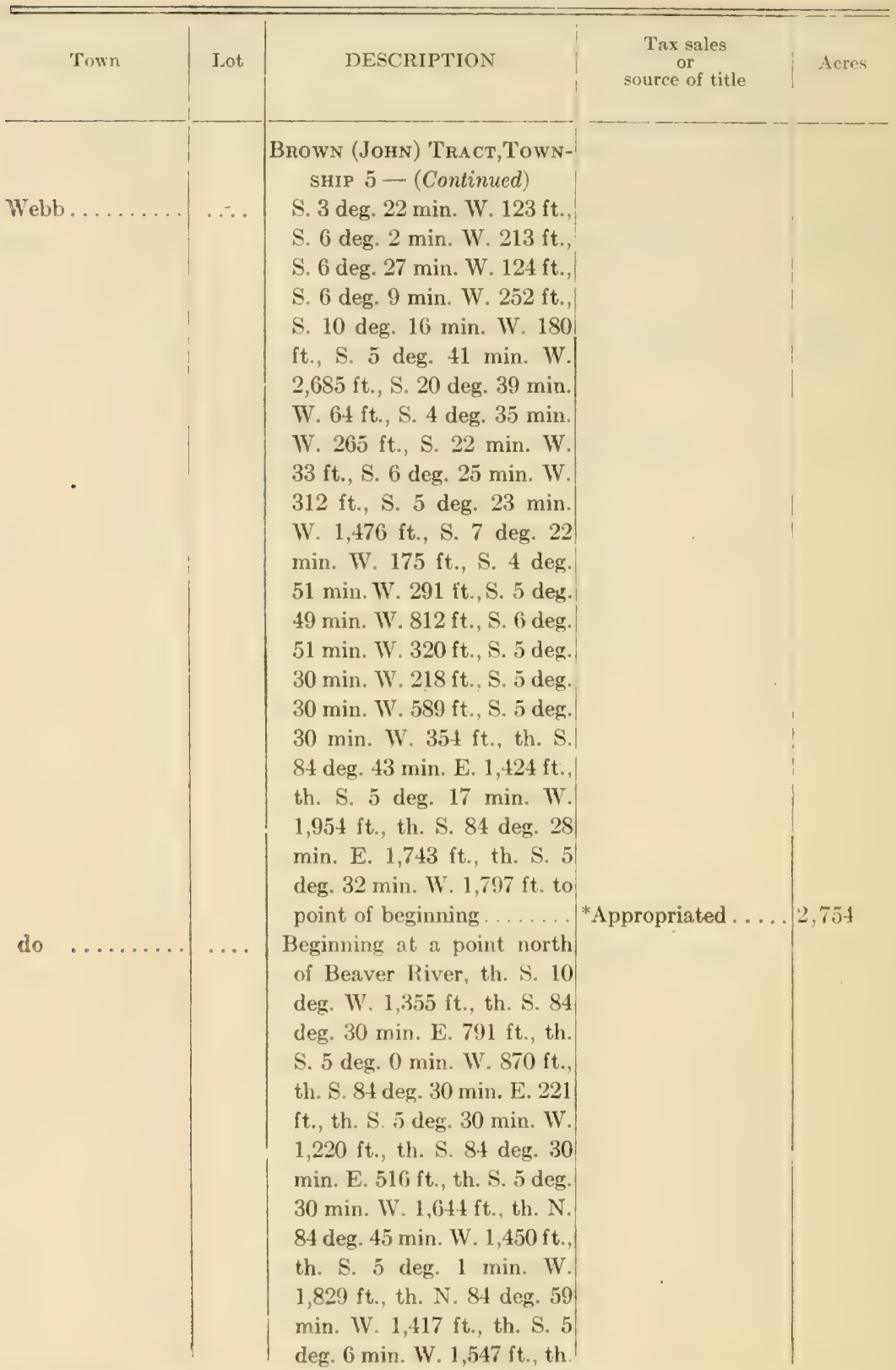

* This land was acquired by condemnation proceedings for reservoir purposes as compensation for water diverted from Black River for uso of Frie canal. The Court of Appeals has decided that the land not flooded is forest preserve land. 


\section{Herkimer County}

\begin{tabular}{|c|c|c|c|c|}
\hline Town & Lot & DESCRIPTION & $\begin{array}{c}\text { Tax sales } \\
\text { or } \\
\text { source of title }\end{array}$ & Acres \\
\hline Webl. & & \begin{tabular}{|} 
Brown (JoHN) T R A C T, \\
Township 5 -(Continued) \\
S. 84 deg. 43 min. E. 3,392 \\
ft., th. N'ly along the W. \\
line of Mrs. Mary L. Fisher \\
$8,437 \mathrm{ft}$. , th. N. 84 deg. 30 \\
min. W. $2,031 \mathrm{ft}$. to the \\
point of beginning....... \\
Total, Township $5,14,-$ \\
$274 \%$ acres, less 2,517 \\
acres, B. R. Reservoir, \\
11.757 .80 acres.
\end{tabular} & *Appropriated . & 345.80 \\
\hline do & & 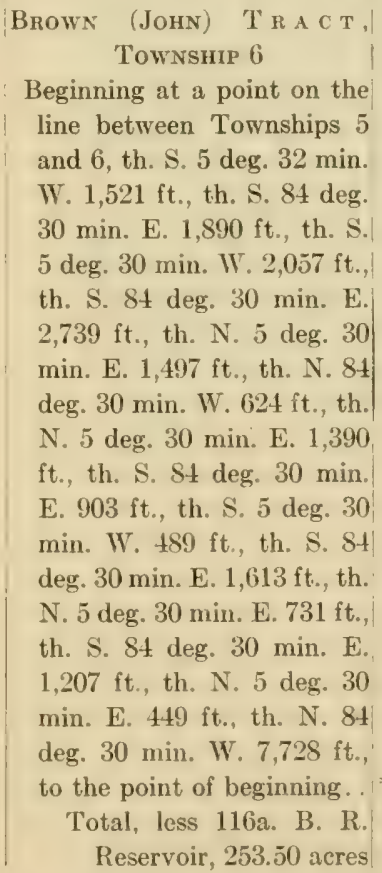 & *Appropriated . & 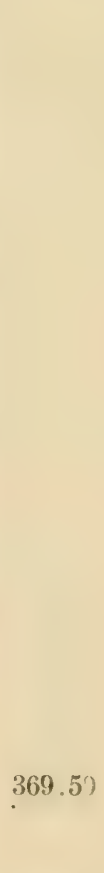 \\
\hline
\end{tabular}

2: This land was aciuired by conclemnation procedings for reservoir purposes as compensation for water diverted from Black River for use of Erie cana!. The land not flooded belongs to the forest preserve. 
Herkimer County

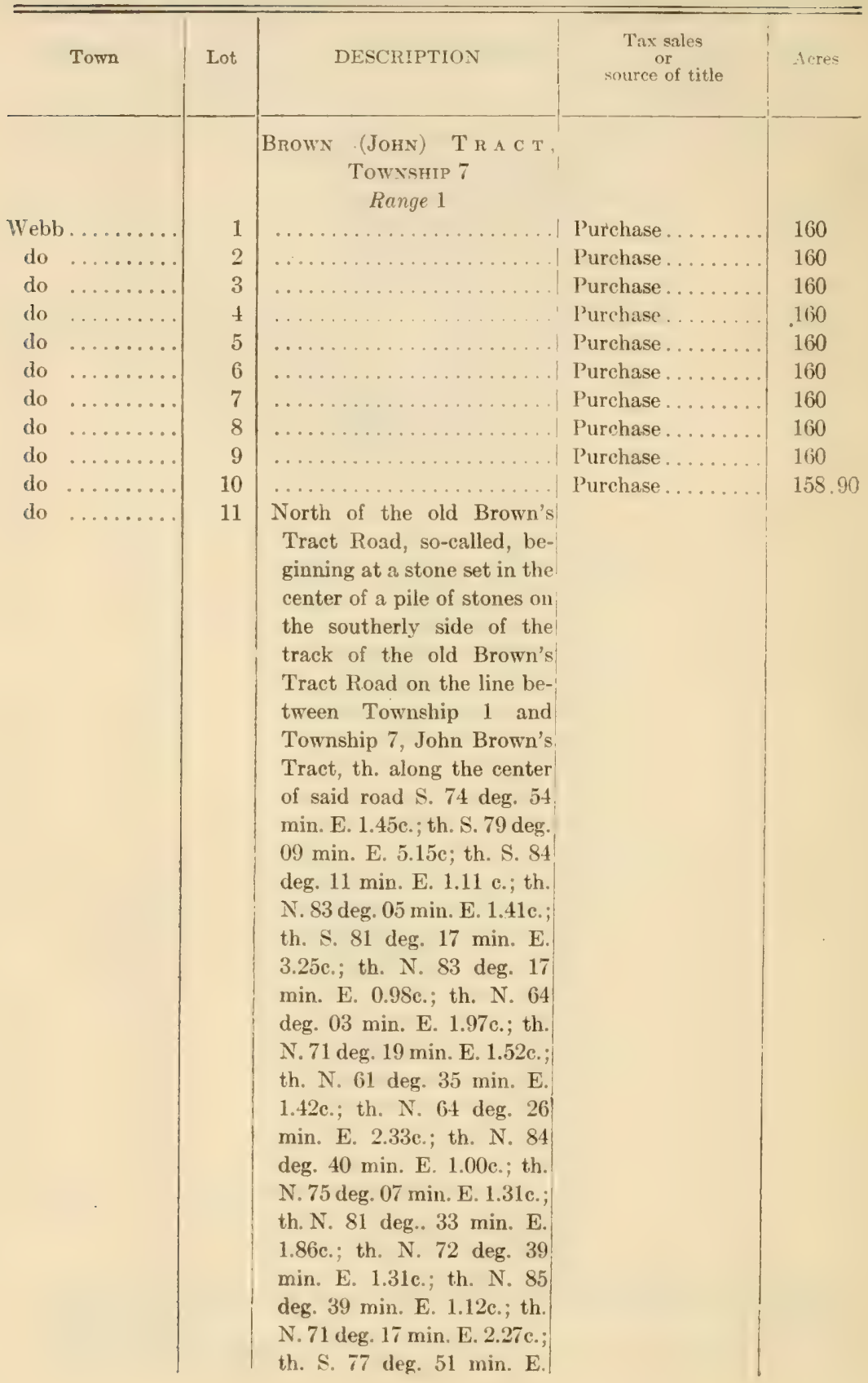


Herkimer County

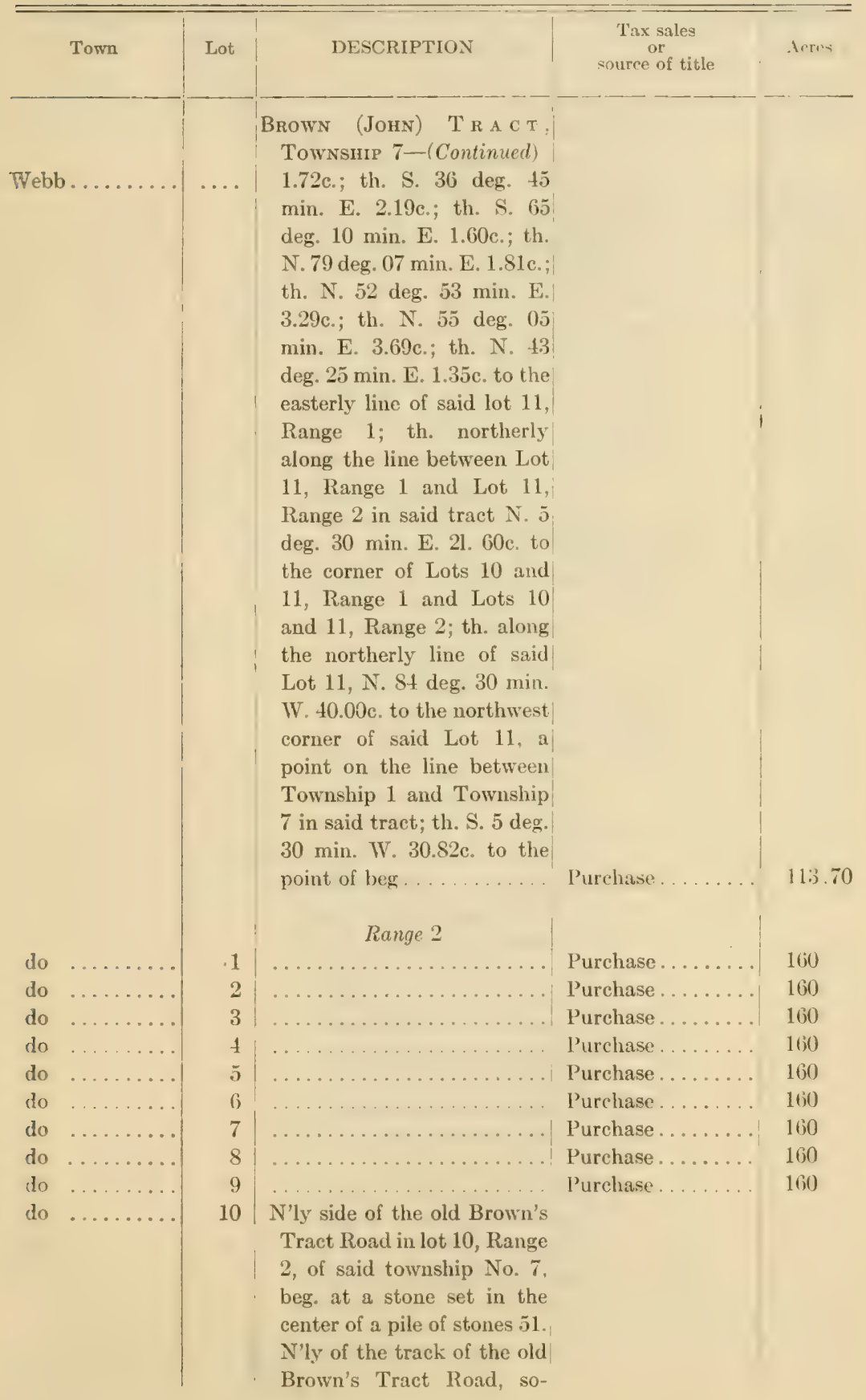


Herkmer County

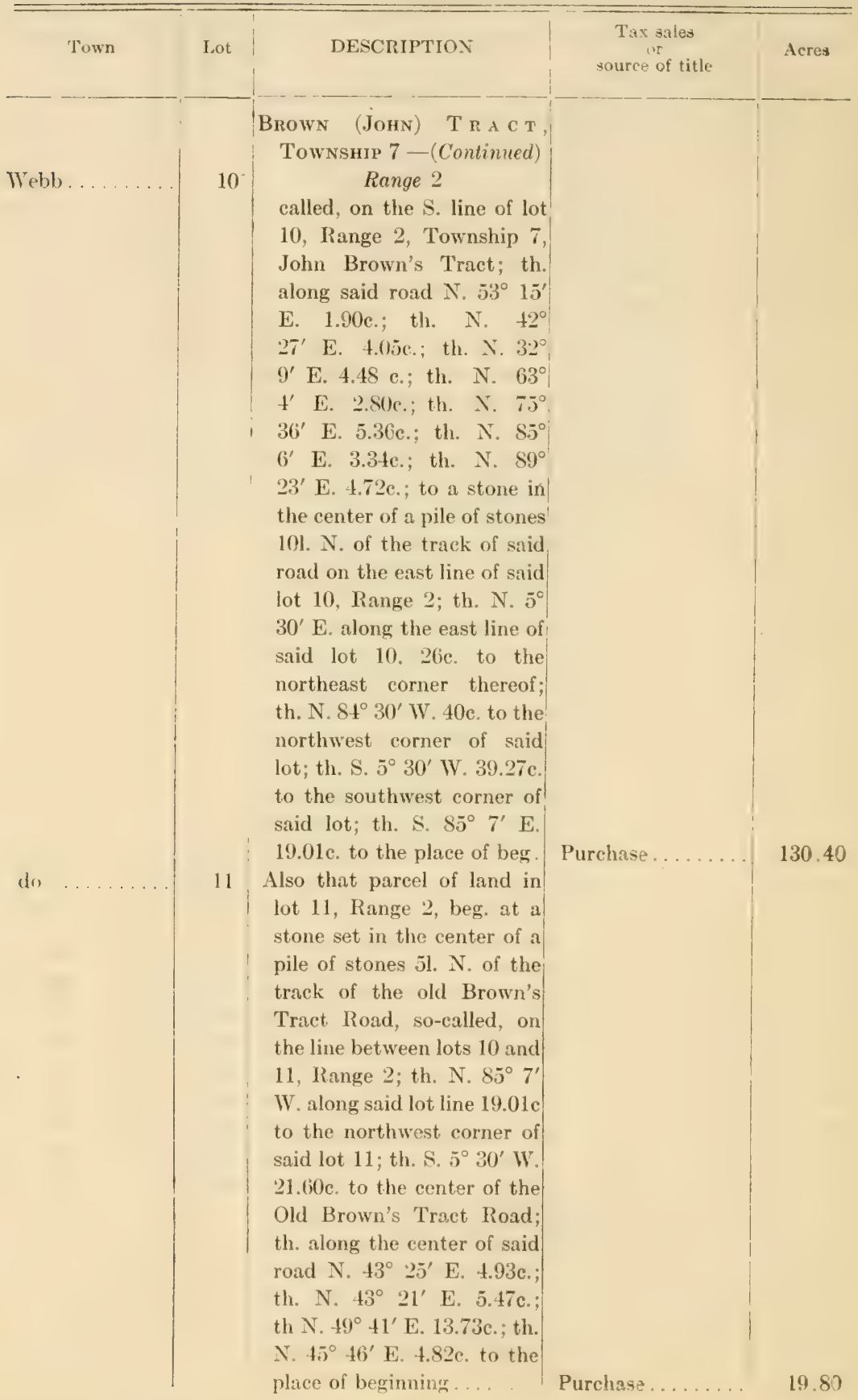




\section{Herkimer County}

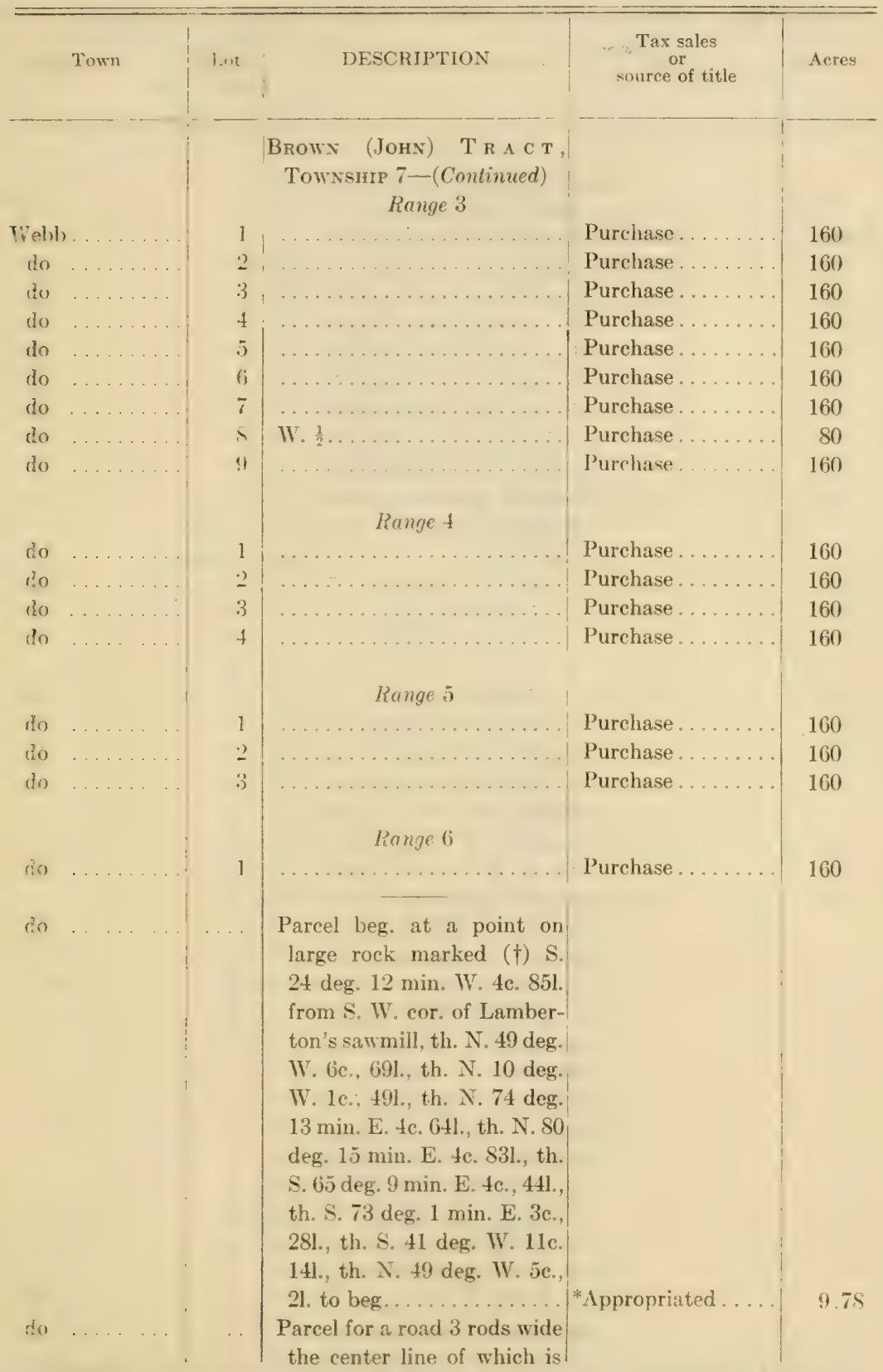

* This land was acquired by condemnation procedings for reservoir purposes as compensation for water diverted from Black River for use of Erie canal. 


\section{Herkimer County}

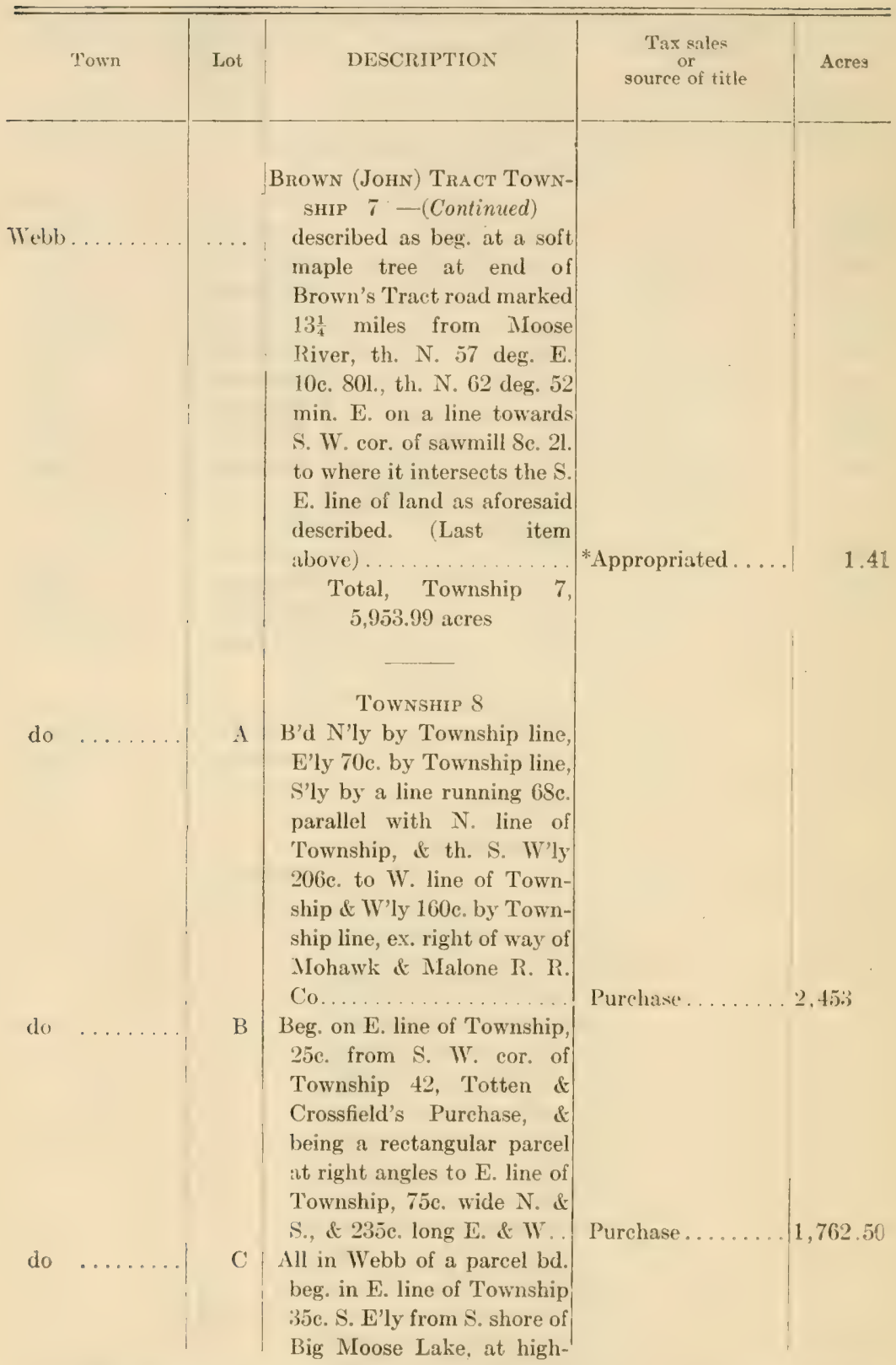

* 'This land was acquired by condemnation proceedings for reservoir purposes as compensation or water diverted from Black River for use of Erie canal. 
Herkmer County

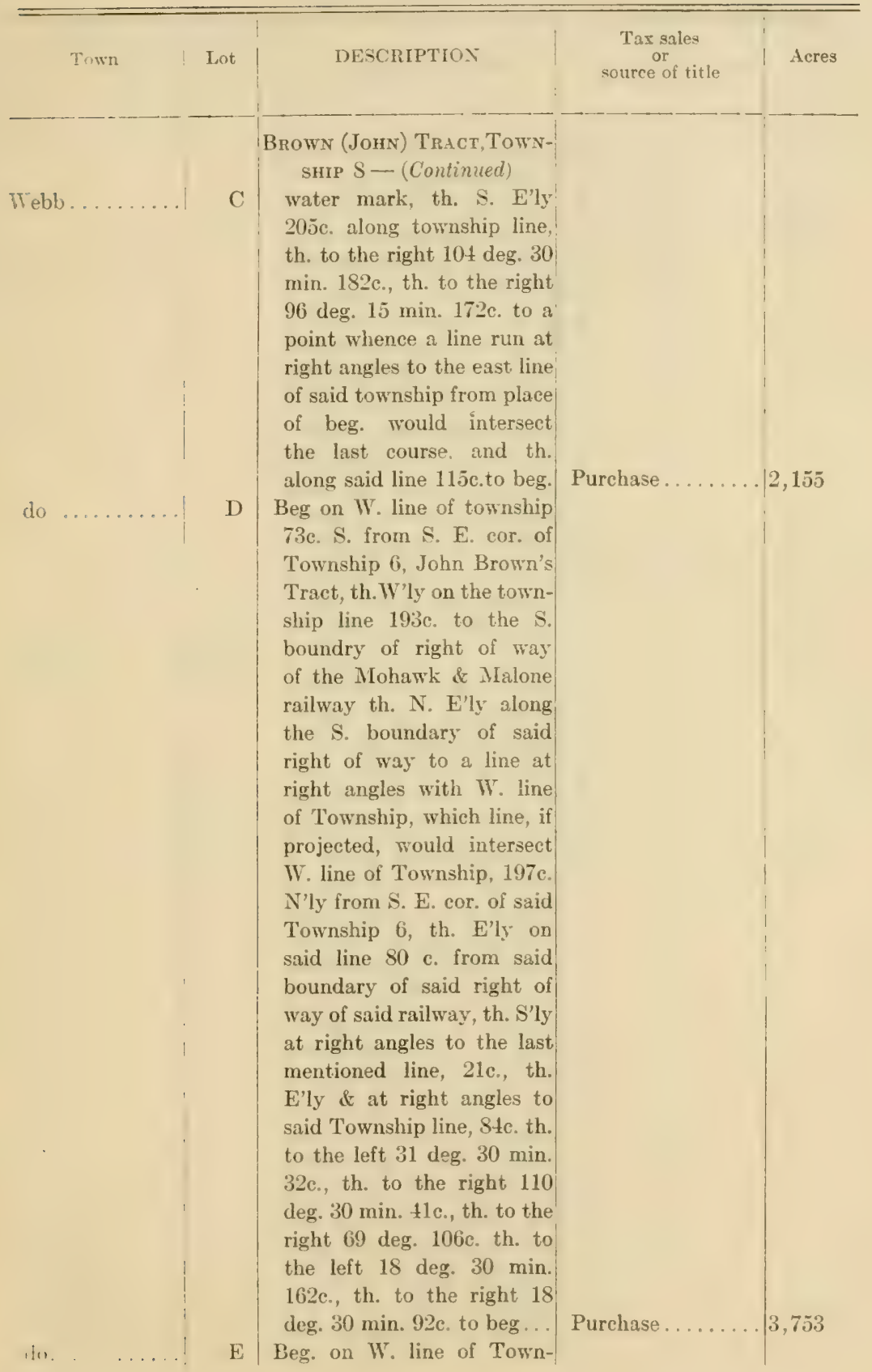


Herkmer County

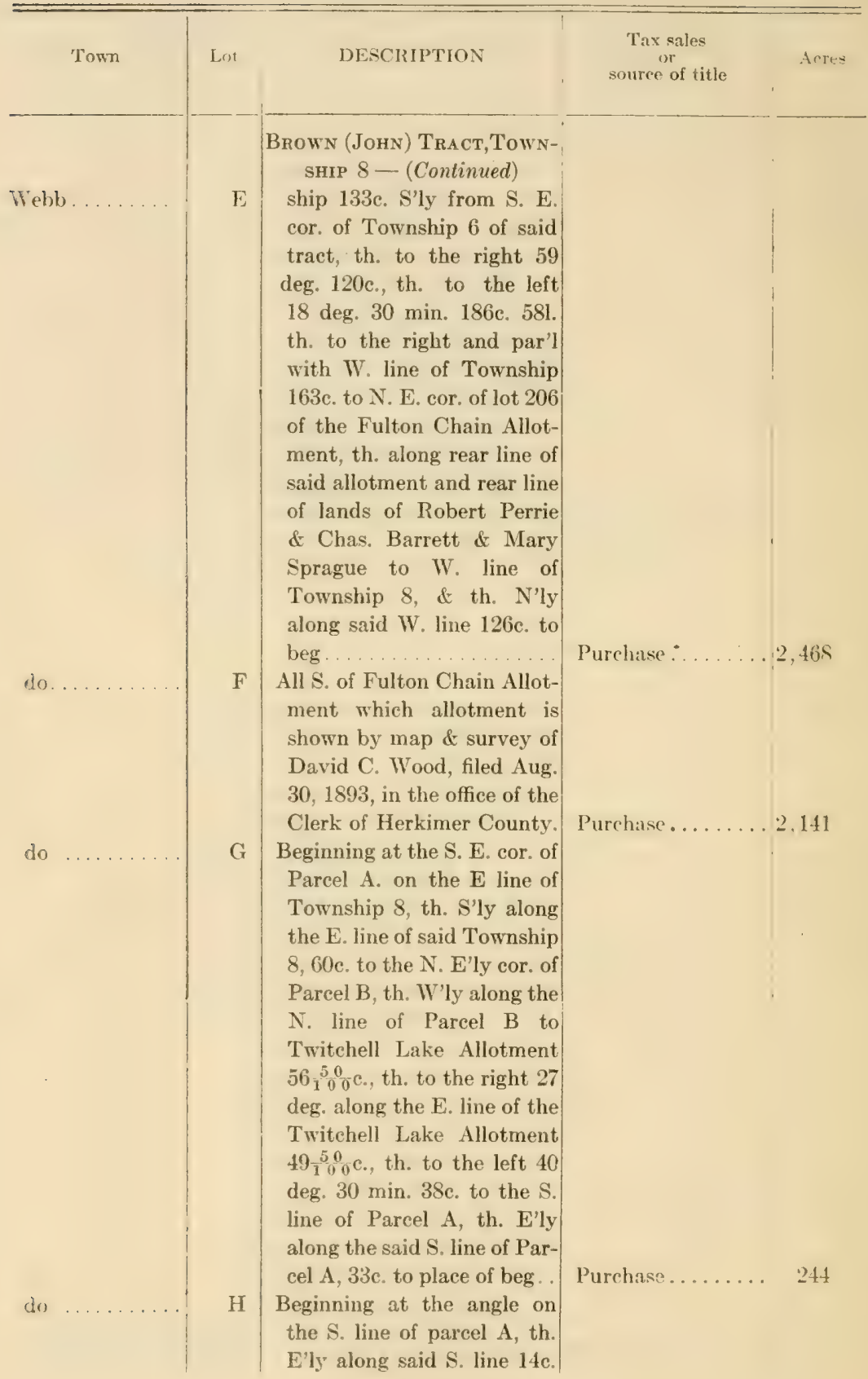


Herkimer County

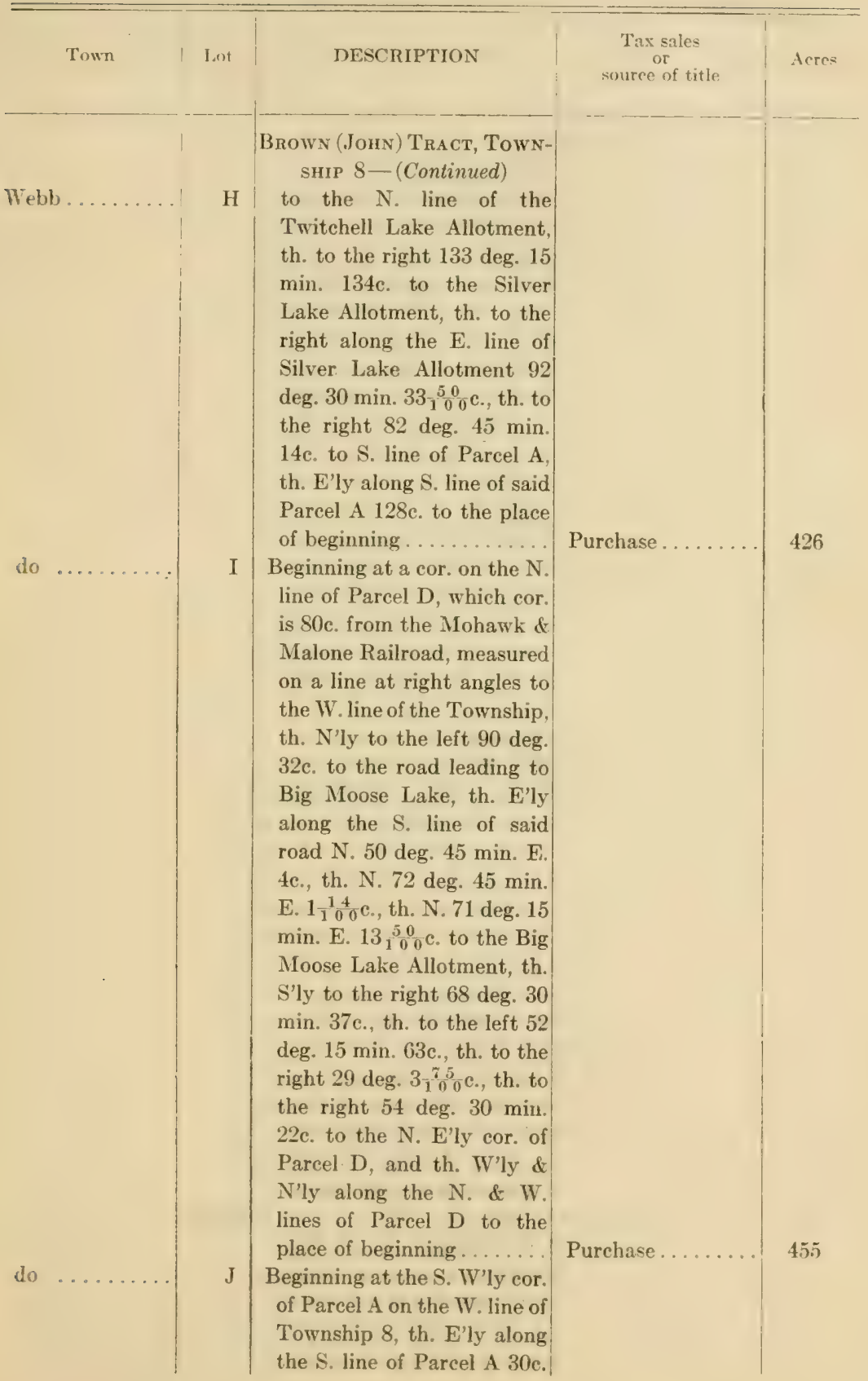


Herkimer County

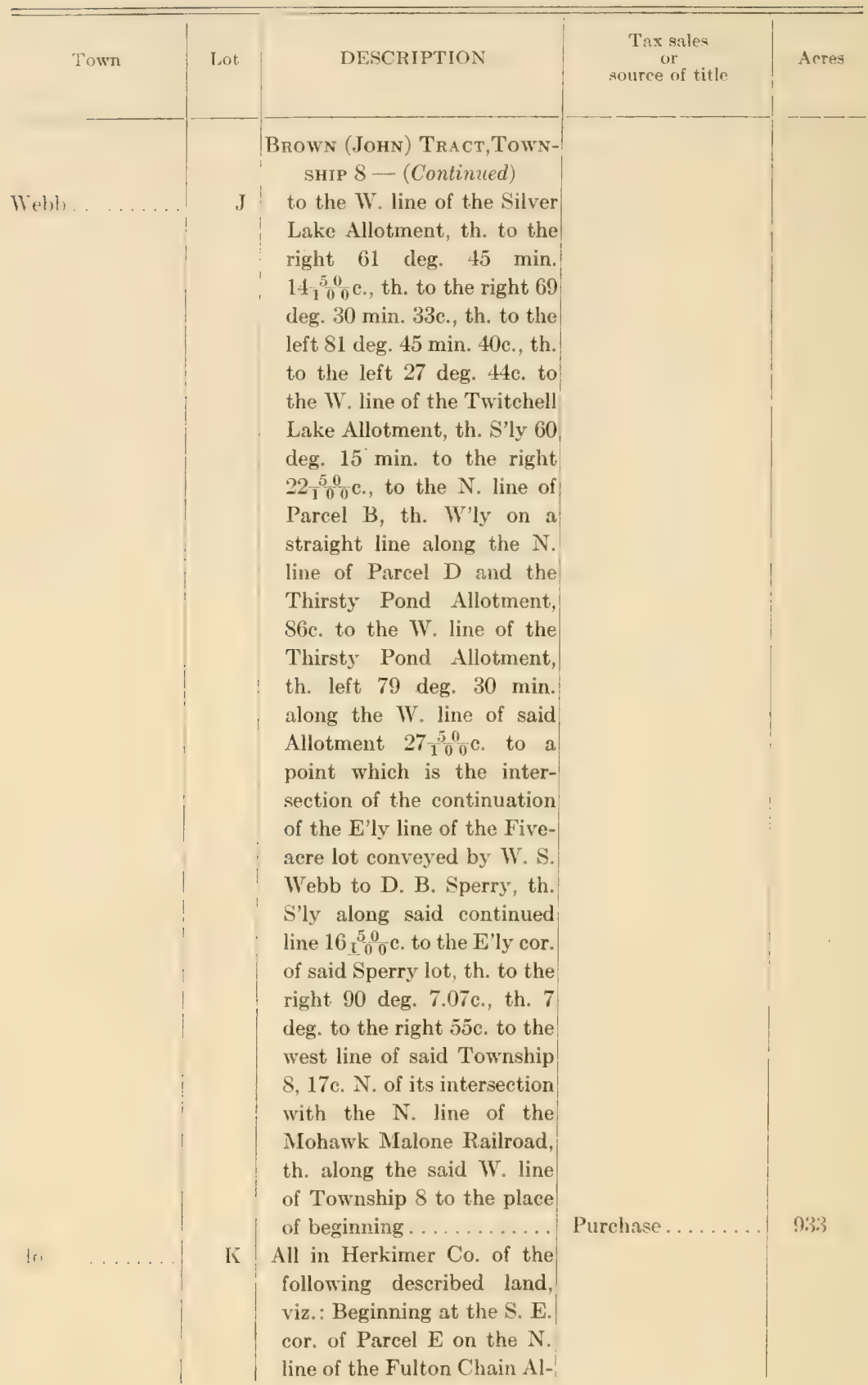


Herkimer County

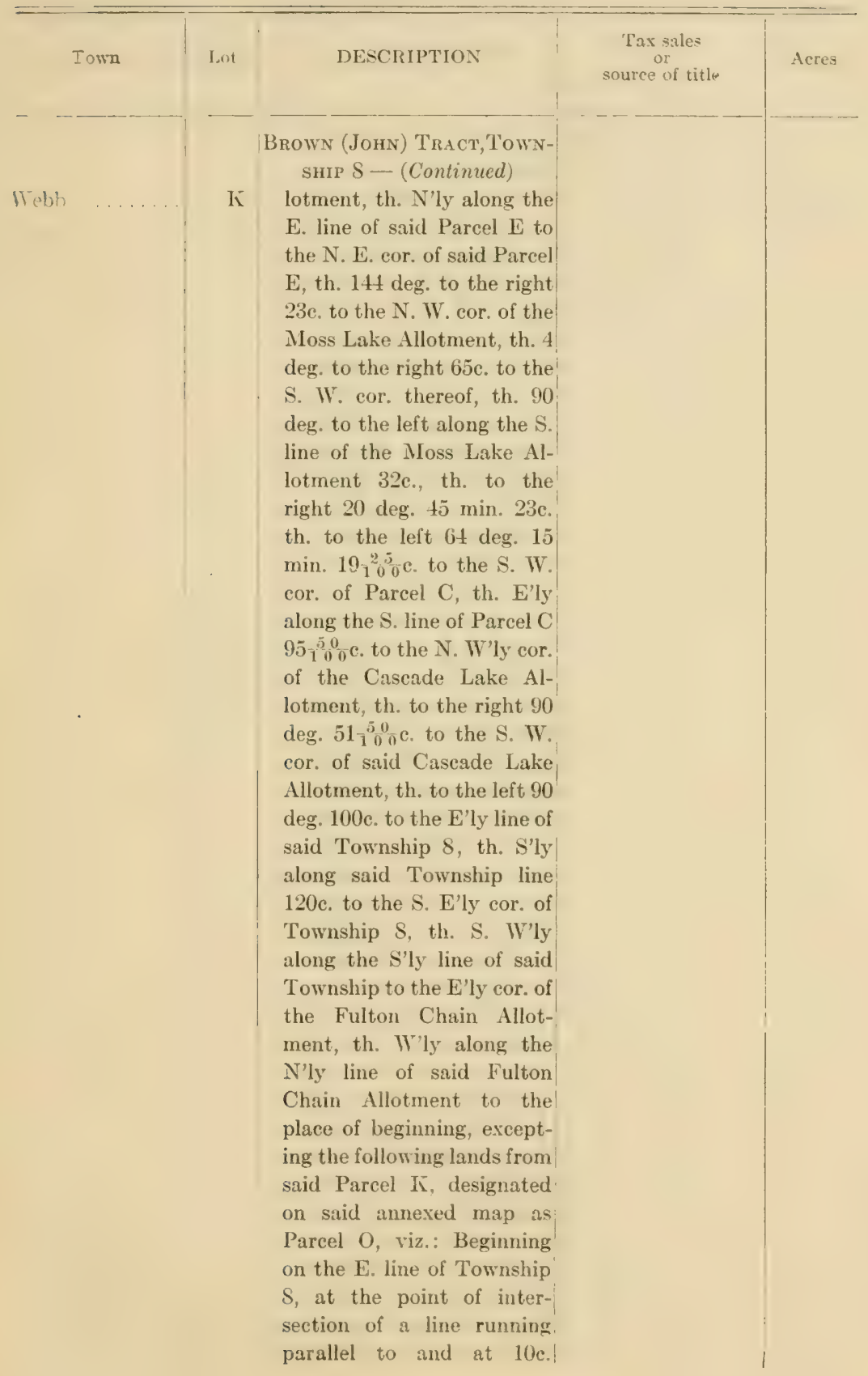


Herkmer Cousty

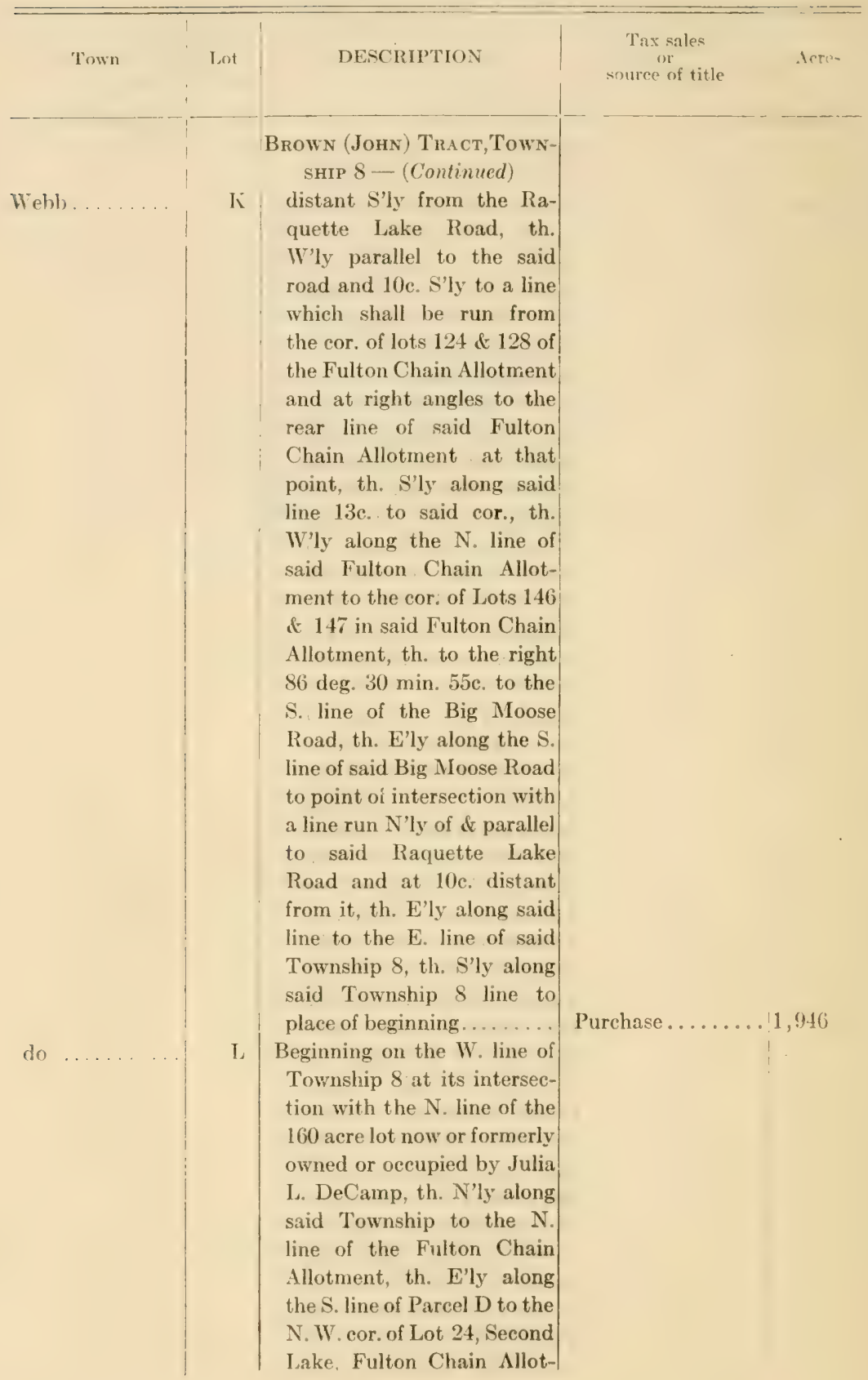


Herkimer County

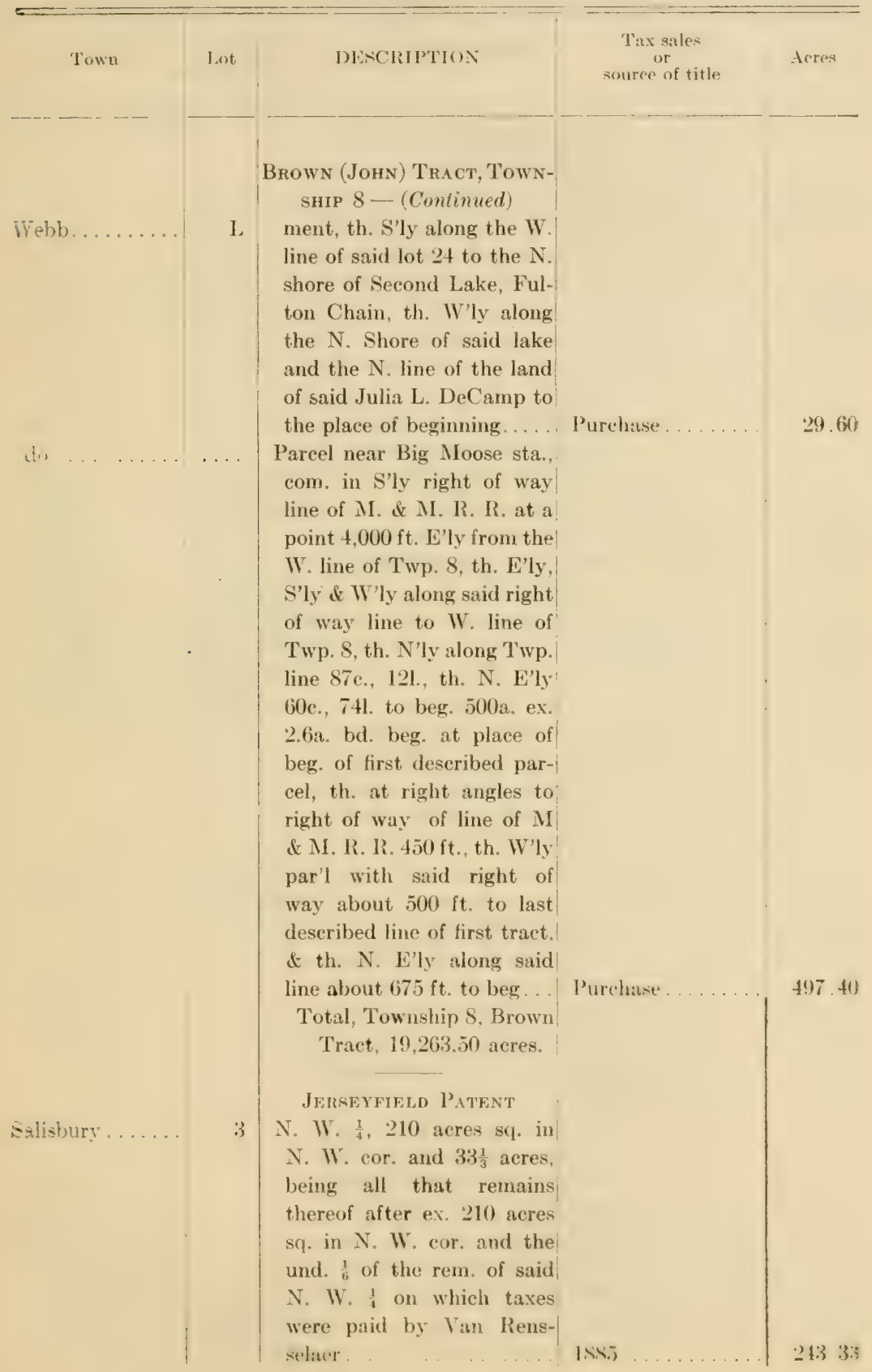




\section{Herkimer County}

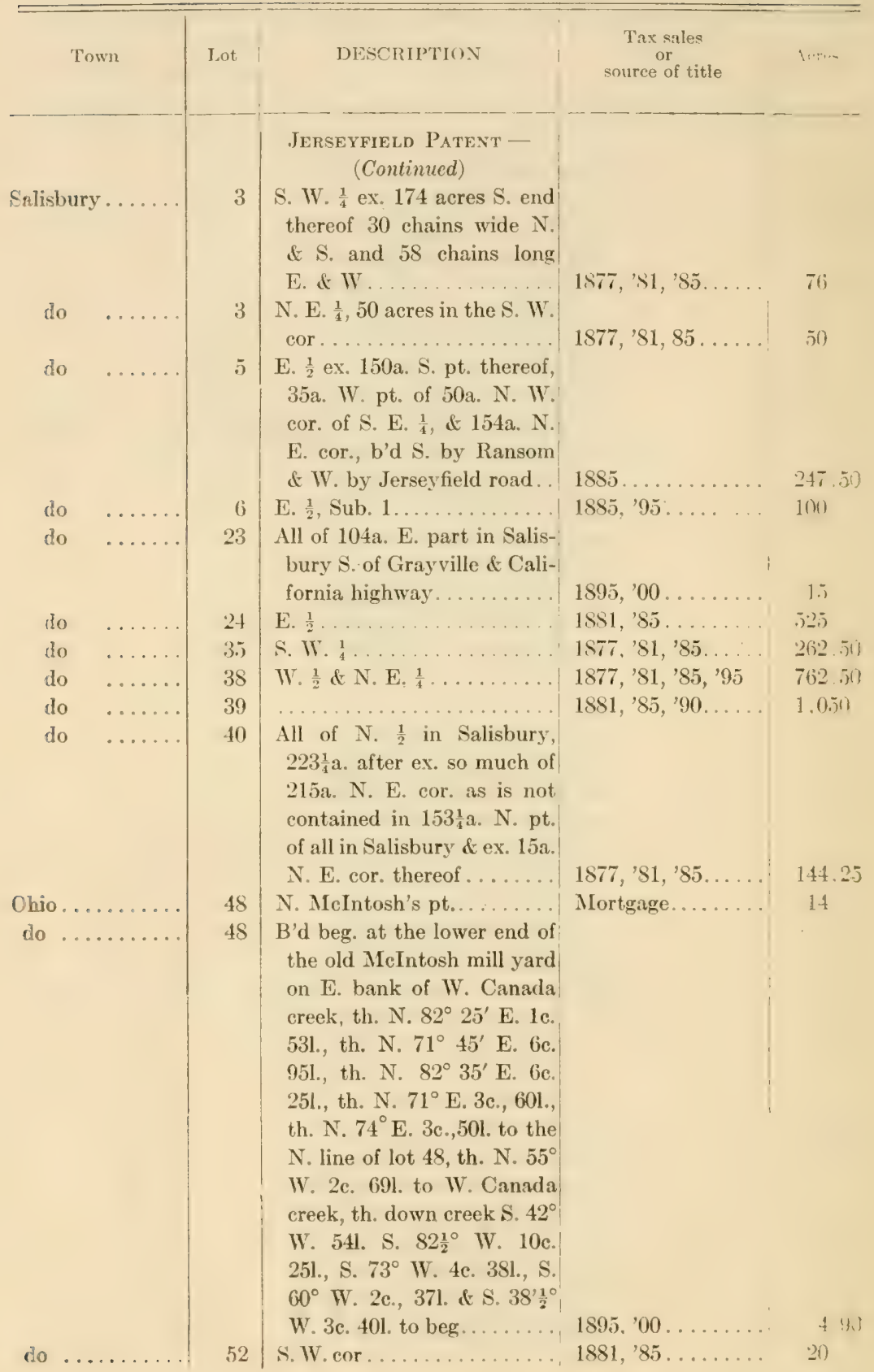




\section{Herhimer County}

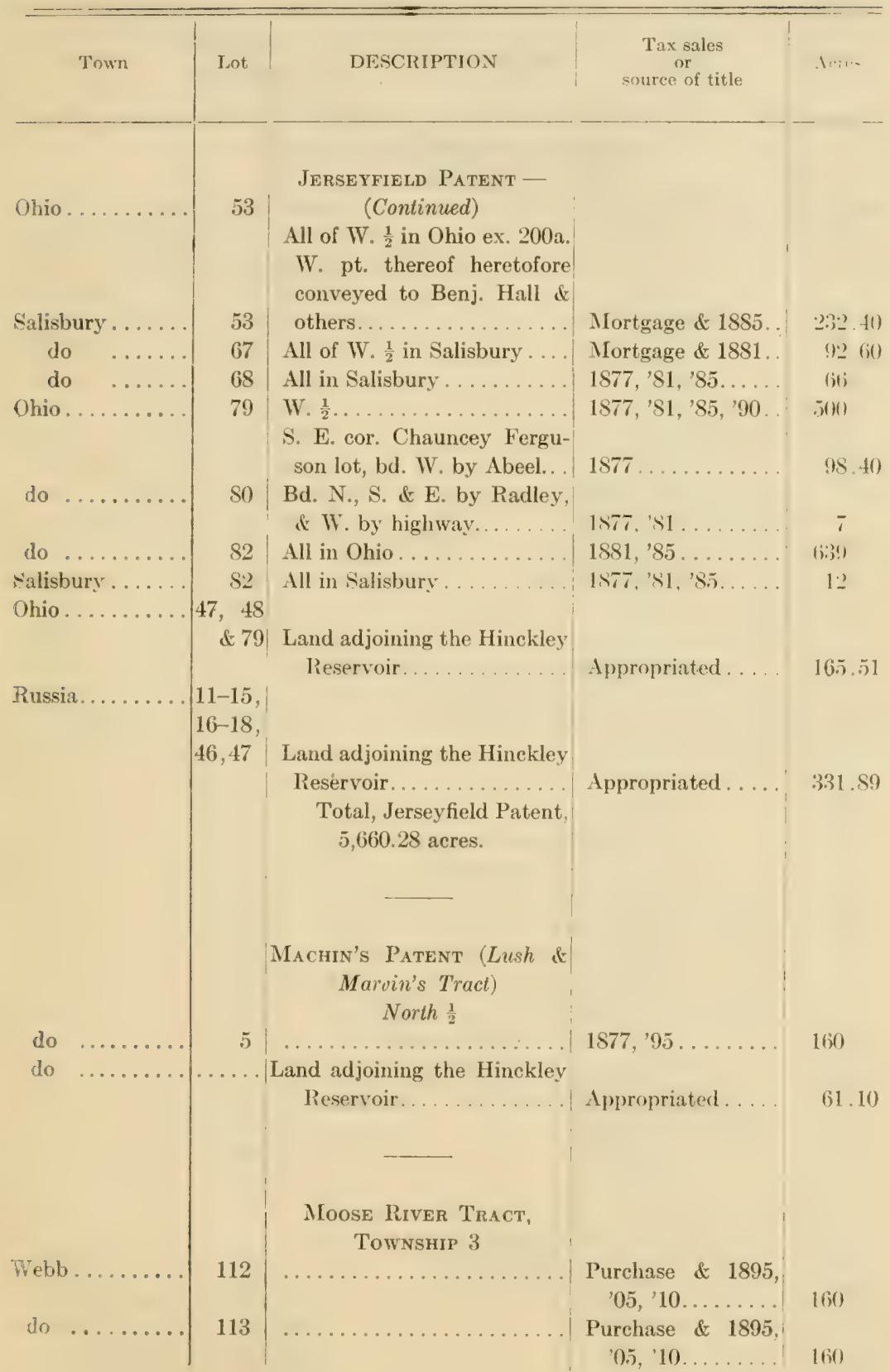


Herkimer County

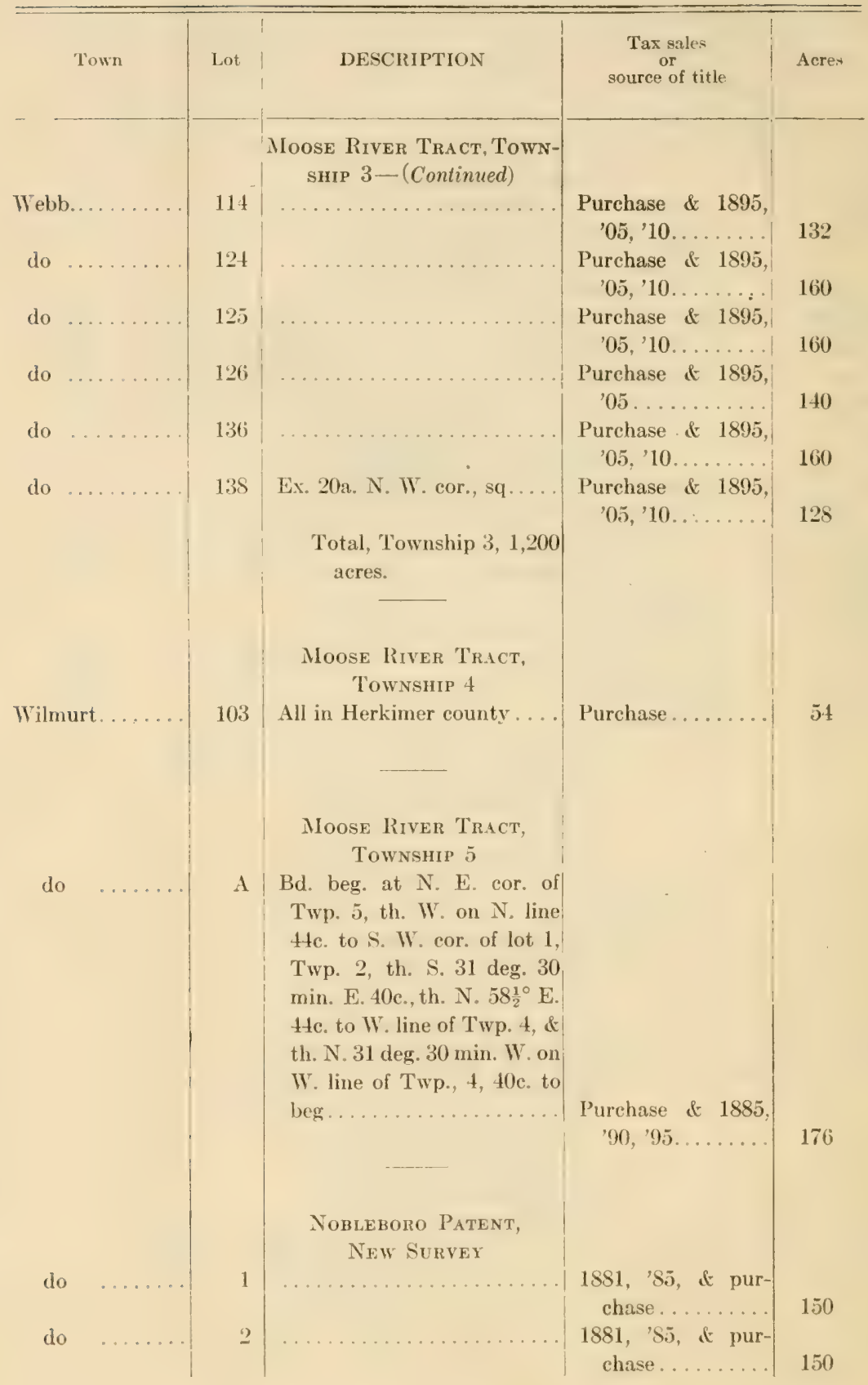


Herkmer County

\begin{tabular}{|c|c|c|c|c|}
\hline lowr & Lot & DESCRIPTION & $\begin{array}{c}\text { Tax sales } \\
\text { or }\end{array}$ & Acres \\
\hline & & $\begin{array}{c}\text { Nobleboro Patent, New } \\
\text { Survey-(Continued) }\end{array}$ & & \\
\hline Wilmurt. & 3 & & 1885 \& purchase. & $1 \tilde{0})$ \\
\hline do & 4 & & 1885 & 150 \\
\hline do & 5 & & $\begin{array}{l}1881,85, \text { and pur- } \\
\text { chase . . . . . . }\end{array}$ & 150 \\
\hline do & 6 & & 1885 \& purchase... & 150 \\
\hline do & 7 & & 1848,00 & 150 \\
\hline do & 8 & & $\begin{array}{l}1881 \text {, ' } 85 \& \text { pur- } \\
\text { chase. . . . }\end{array}$ & 150 \\
\hline do & 9 & & $1885 \&$ purchase. & 150 \\
\hline do & 10 & & 1885 \& purchase... & 150 \\
\hline do & 11 & & $1885 \&$ purchase. & 150 \\
\hline do & 1.2 & & 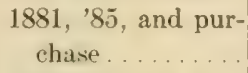 & 150 \\
\hline do & 13 & & $\begin{array}{l}1881, \text { ' } 85, \& \text { pur- } \\
\text { chase } \ldots \ldots \ldots\end{array}$ & 150 \\
\hline do & 14 & & $\begin{array}{l}1881, \text { ' } 85, \text { \& pur- } \\
\text { chase. . . . . . . }\end{array}$ & 150 \\
\hline do & 15 & & $1885 \&$ purchase... & 150 \\
\hline do & 16 & & $\begin{array}{l}1881 \text {, ' } 85 \text {, and pur- } \\
\text { chase. . . . . . . . }\end{array}$ & 150 \\
\hline do & 17 & & $1885 \&$ purchase. & 150 \\
\hline do & 18 & & Purchase. & 150 \\
\hline do & 19 & & Purchase ....... & 150 \\
\hline do & 23 & & 1845, '\$. . . . . . . & 150 \\
\hline do & 24 & & $1881, ' 85$. & 150 \\
\hline do & 25 & & $1885 \&$ purchase. . & 150 \\
\hline do & 26 & & $\begin{array}{l}1881 \text {, ' } 85, \text { \& pur- } \\
\text { chase . . . . . . . }\end{array}$ & 150 \\
\hline do & 27 & & $1885 \&$ purchase. & 150 \\
\hline do & 28 & & $\begin{array}{l}1881 \text {, '85, \& pur- } \\
\text { chase. . . . . . }\end{array}$ & 150 \\
\hline do & 29 & & 1885 \& purchase & 150 \\
\hline do & 30 & & 1SS5 \& purchase. & 150 \\
\hline do & 31 & & $\begin{array}{l}1851 \text {. ' } \$ 5 \text {, and pur- } \\
\text { chase . . . . . . . . }\end{array}$ & 150 \\
\hline do & 32 & & $\begin{array}{l}1881,85, \& \text { pur- } \\
\text { chase . . . . . }\end{array}$ & 150 \\
\hline do & $3: 3$ & & Purchase ... . . . . & 150 \\
\hline do & 34 & & Purchase .... & 150 \\
\hline do & 35 & & 1885 \& purchase. . & 150 \\
\hline do & 36 & & $\begin{array}{c}1881 \text {, ' } 85 \text { \& pur- } \\
\text { chase. . . . }\end{array}$ & 150 \\
\hline
\end{tabular}




\section{Herkimer County}

\begin{tabular}{|c|c|c|c|c|}
\hline Town & \multirow[t]{2}{*}{ Lot } & DESCRIPTION & \multirow{2}{*}{$\begin{array}{c}\text { Tax sales } \\
\text { or } \\
\text { source of title }\end{array}$} & \multirow[t]{2}{*}{ Acres } \\
\hline & & $\begin{array}{l}\text { Nobleboro Patent, New } \\
\text { Survey - (Continued })\end{array}$ & & \\
\hline Wilmurt. & 37 & $\ldots \ldots \ldots \ldots \ldots \ldots$ & $1885 \&$ purchase. & 150 \\
\hline do & 38 & $\ldots \ldots \ldots \ldots \ldots \ldots$ & $1885 \&$ purchase. & 150 \\
\hline do & 39 & $\ldots \ldots \ldots \ldots \ldots \ldots \ldots$ & $1885 \&$ purchase. & 150 \\
\hline do & 40 & $\ldots \ldots \ldots \ldots \ldots \ldots$ & $1885 \&$ purchase. & 150 \\
\hline do & 42 & $\ldots \ldots \ldots \ldots \ldots \ldots \ldots$ & $1885 \&$ purchase.. & 150 \\
\hline do & 43 & $\ldots \ldots \ldots \ldots \ldots \ldots \ldots$ & $1885 \&$ purchase. & 150 \\
\hline do & 44 & $\ldots \ldots \ldots \ldots \ldots \ldots$ & $1885 \&$ purchase.. & 150 \\
\hline do & 46 & $\ldots \ldots \ldots \ldots \ldots \ldots$ & $1881,95 \ldots \ldots$ & 150 \\
\hline do & 47 & & $1877,91,85 \ldots$ & 150 \\
\hline do & 48 & & $1877,81, ' 85 \ldots$ & 150 \\
\hline do & 49 & & $1881,95 \ldots \ldots$ & 150 \\
\hline do & 52 & $\ldots \ldots \ldots \ldots \ldots \ldots$ & $1877, ' 81, ' 85, ' 90$. & 150 \\
\hline do & 53 & $\ldots \ldots \ldots \ldots \ldots \ldots \ldots \ldots$ & $1877, ' 81, ' 85,{ }^{\prime} 90$. & 150 \\
\hline do & 54 & $\ldots \ldots \ldots$ & $1877,81, ' 85 \ldots$ & 150 \\
\hline do & 56 & $\ldots \ldots \ldots \ldots$ & $1881, ' 85 \ldots \ldots$ & 150 \\
\hline do & 57 & 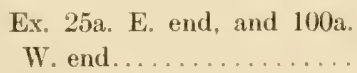 & $1877, ' 81, ' 85, ' 95:$ & 25 \\
\hline do & 58 & $\ldots \ldots \ldots \ldots \ldots \ldots$ & Purchase........ & 150 \\
\hline do & 59 & $\ldots \ldots \ldots \ldots \ldots \ldots \ldots$ & $1877, ' 81, ' 85 \ldots$. & 150 \\
\hline do & 60 & $\ldots \ldots \ldots \ldots \ldots \ldots \ldots \ldots$ & $1877, ' 81, ' 85, ' 95$. & 150 \\
\hline do & 61 & $\ldots \ldots \ldots \ldots \ldots \ldots \ldots \ldots$ & $1877, ' 81, ' 85 \ldots$. & 150 \\
\hline do & 62 & $\ldots \ldots \ldots \ldots \ldots \ldots \ldots$ & 1877, '81, '85, '95. & 150 \\
\hline do & 64 & & Purchase ........ & 150 \\
\hline do & 67 & & $1881,85 \ldots \ldots$ & 150 \\
\hline do & 69 & & Purchase....... & 150 \\
\hline do & 70 & & Purchase....... & 150 \\
\hline do & 71 & & Purchase....... & 150 \\
\hline do & 72 & & Purchase. & 150 \\
\hline do & 74 & & P'urchase ....... & 150 \\
\hline do & 75 & & Purchase........ & 150 \\
\hline do & 76 & & Purchase ........ & 150 \\
\hline do & 77 & & Purchase........ & 150 \\
\hline do & 7s & & Purchase. . . . . . . & 150 \\
\hline do & si & & Purchase . . . . . & 150 \\
\hline do & 87 & & Purchase ....... & 150 \\
\hline do & 90 & & $1871,77, ' 81 \ldots$ & 150 \\
\hline do & 91 & & $1871,77,81,8.7$. & 150 \\
\hline do & 93 & & $\begin{array}{l}1881, \text { 's5 \& pur- } \\
\text { chase......... }\end{array}$ & 150 \\
\hline do & 94 & & $1848 \ldots \ldots \ldots \ldots$ & 150 \\
\hline do & 95 & & $\begin{array}{r}1881 \text {, '\$5 \& pur- } \\
\text { chase.......... }\end{array}$ & 150 \\
\hline do & 97 & & $1871, ' 77, ' s 1 \ldots \ldots$ & 150 \\
\hline
\end{tabular}


Herkimer County

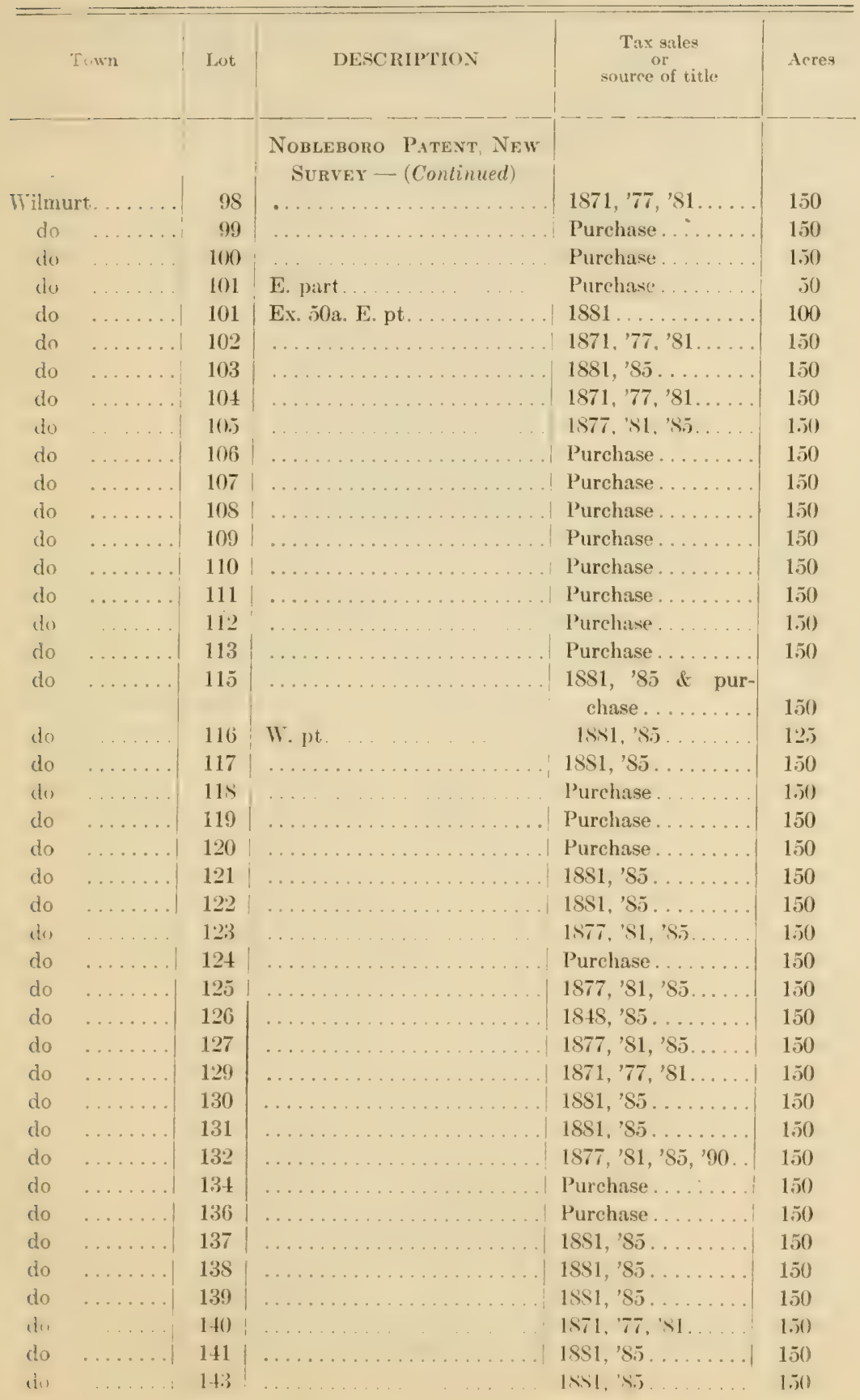


Herkimer County

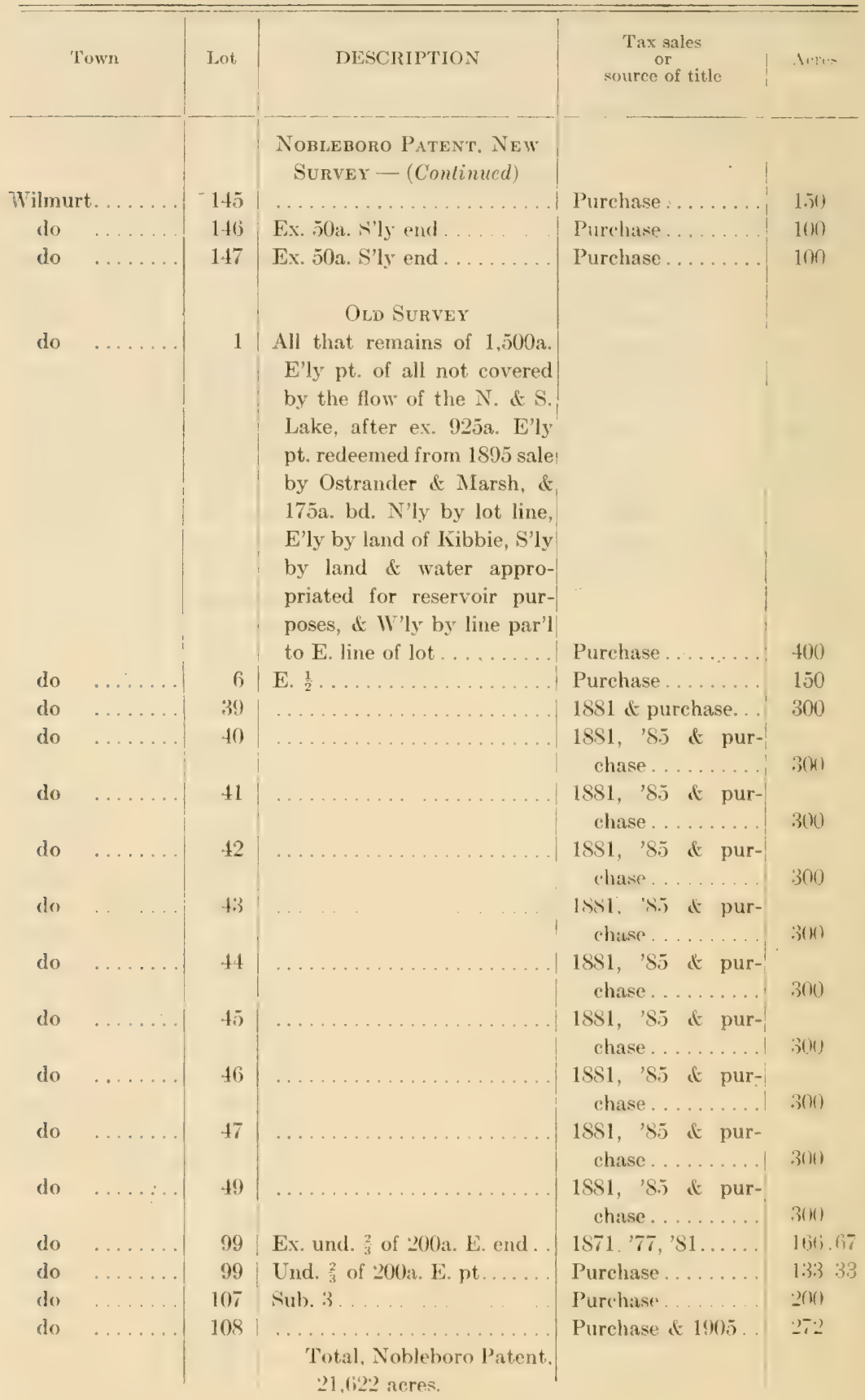




\section{Herkimer County}

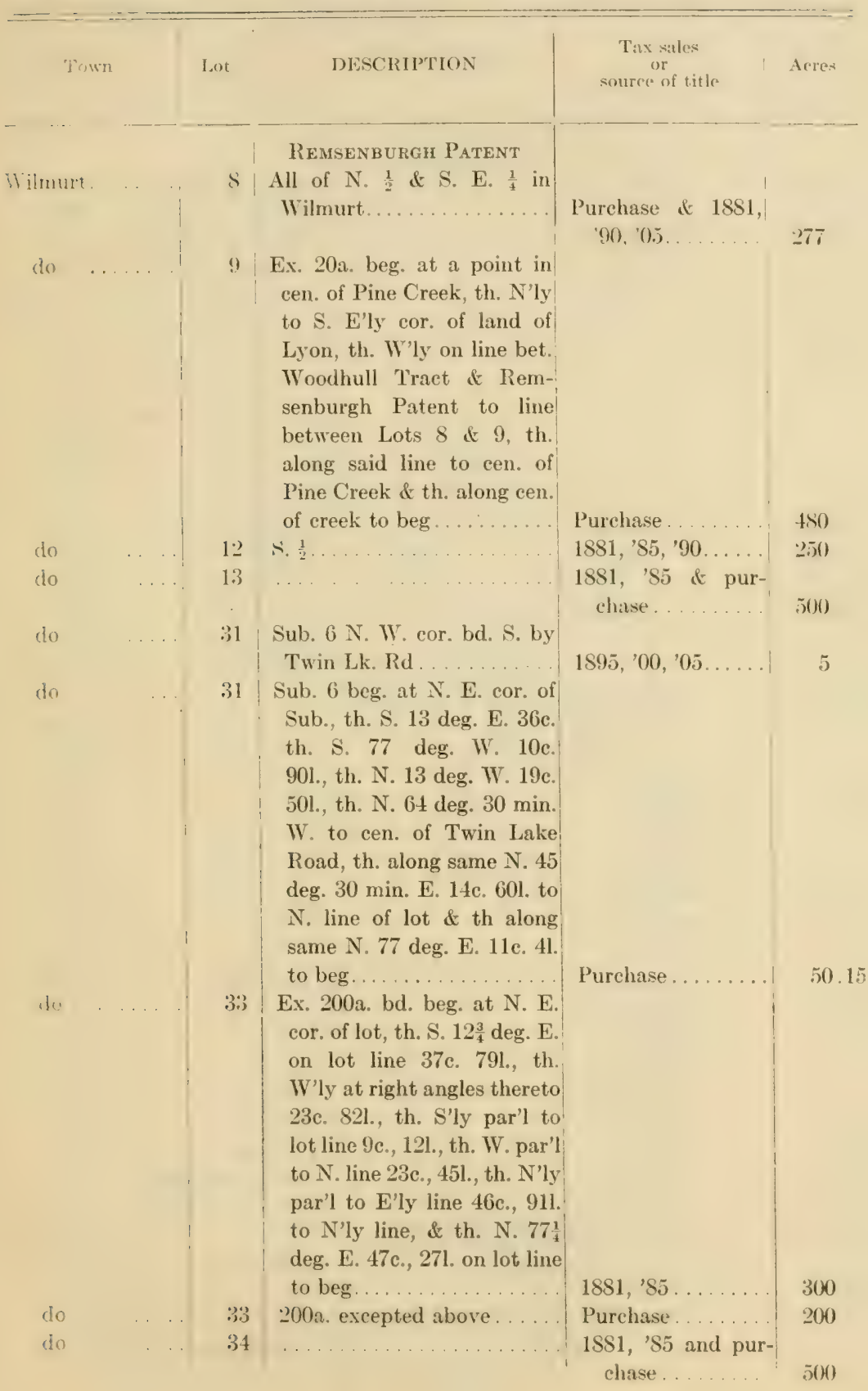




\section{Herkimer County}

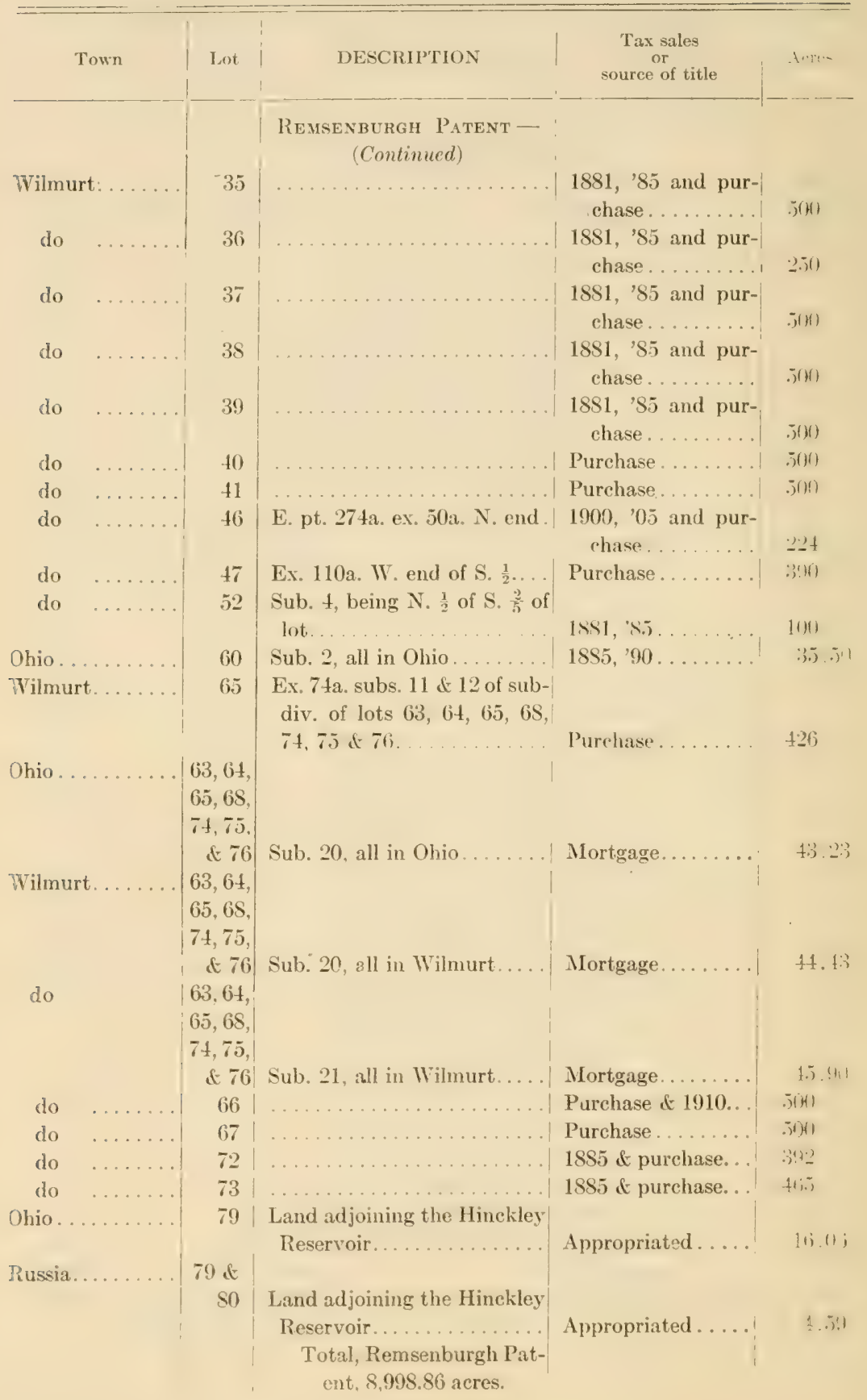


Conservation Commission.

Herkimer County

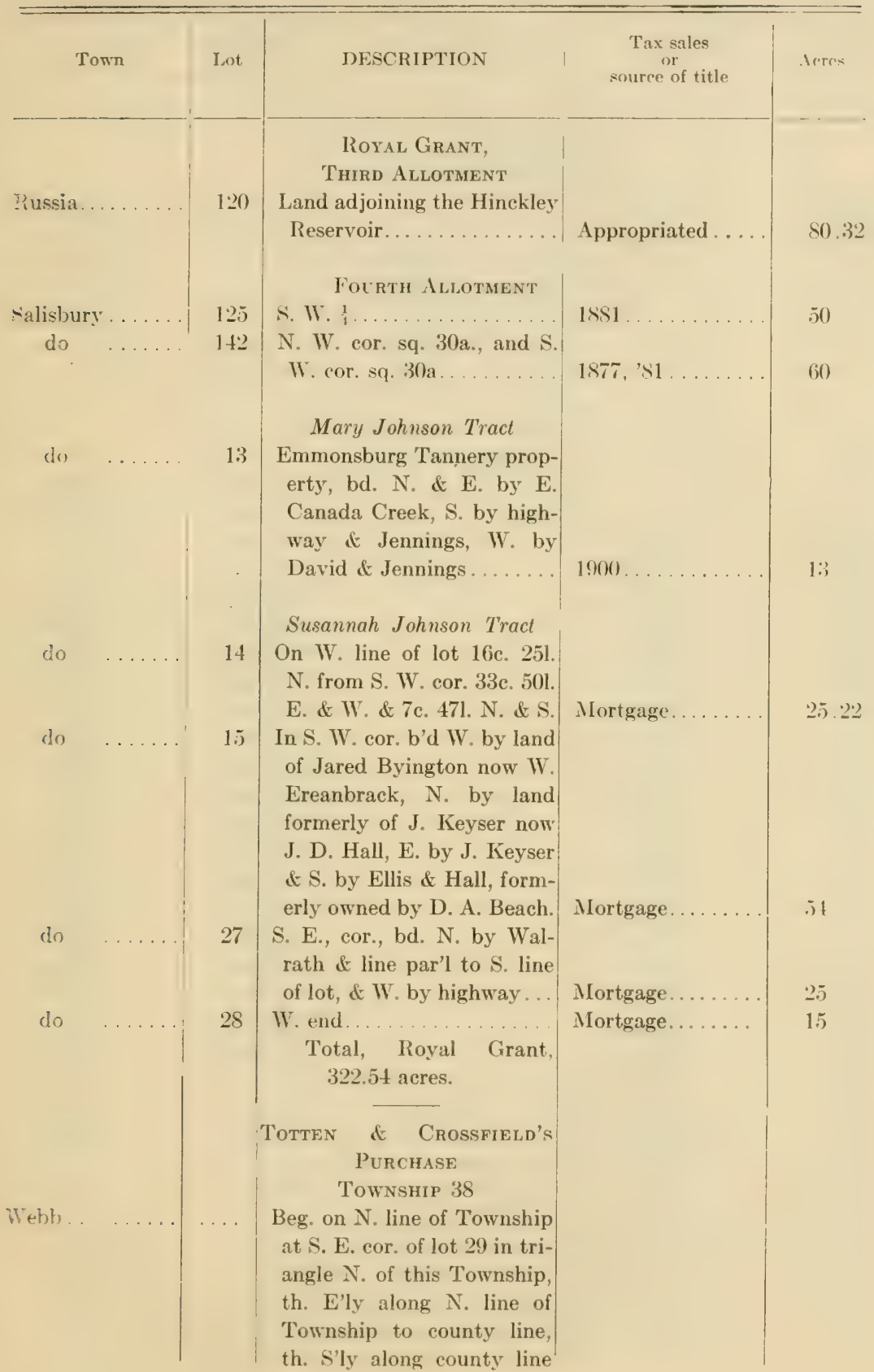




\section{Herkiner County}

\begin{tabular}{|c|c|c|c|c|}
\hline & Lot & DESCRIPTION & $\begin{array}{l}\text { Tax sales } \\
\text { or } \\
\text { source of title }\end{array}$ & Acres \\
\hline Wilbb. & & $\begin{array}{l}\text { TotTEN \& CROSSFiELd's } \\
\text { Purchase, Township } 38 \\
- \text { (Continued) } \\
\text { to the line between the E. } \\
\frac{1}{2} \& \text { W. } \frac{1}{2} \text { of Township, th. } \\
\text { S. E'ly along said line to a } \\
\text { point } 90 \mathrm{c} \text {. along same from } \\
\text { N. line of Township \& th } \\
\text { 199c. to beg.............. } \\
\text { TotTEN \& Crossfield, } \\
\text { Townsip } 42\end{array}$ & Purchase......... & 818 \\
\hline do & 1 & & Purchase..... . & 209 \\
\hline do & 2 & & Purchase ..... & 225.50 \\
\hline (b) & 3 & $\ldots$ & Purchase....... & 225.50 \\
\hline do & 4 & & Purchase..... . & 225.50 \\
\hline do & 5 & & Purchase...... & 225.50 \\
\hline do & 6 & & Purchase....... & 225.50 \\
\hline do & 15 & & Purchase ........ & 225.50 \\
\hline do & 16 & & Purchase ........ & 225.50 \\
\hline do & 17 & & Purchase........ & 225.50 \\
\hline di) & 1s & & Purchase......... & 225.50 \\
\hline do & 19 & & Purchase....... & 225.50 \\
\hline do & 20 & & Purchase ......... & 209 \\
\hline do & 21 & & Purchase.... & 182.40 \\
\hline$d o$ & $2 \cdot 2$ & & Purchase....... & 196.80 \\
\hline do & 23 & & Purchase........ & 196.80 \\
\hline do & 24 & $\cdots$ & Purchase.... & 196.80 \\
\hline do & 37 & & Purchase... & 196.80 \\
\hline do & $3 S$ & & Purchase ........ & 196.80 \\
\hline do & 39 & & Purchase. ....... & 196.80 \\
\hline do & 40 & & Purchase........ & 182.40 \\
\hline do & 41 & & Purchase........ & 182.40 \\
\hline do & 42 & Ex. 6a. Mohawk \& Malone & & \\
\hline & & Railway, right of way..... & Purchase.... & 190.80 \\
\hline do & 43 & Ex. 6a. R. R. right of way. . & Purchase.... & 190.80 \\
\hline & 44 & $\begin{array}{r}\text { Ex. 4a. Mohawk \& Malone } \\
\text { R. R., right of way ........ }\end{array}$ & Purchase......... & 192.80 \\
\hline din & 4) & $\begin{array}{l}\text { Beg. at S. W. cor., th. N. on } \\
\text { W. line } 30 \mathrm{c} \text {, th. S. E. to a } \\
\text { point } 30 \mathrm{c} \text {. E. from W. line } \\
\text { of lot \& } 20 \mathrm{c} \text {. N. from S. } \\
\text { line, th. S. } 20 \mathrm{c} \text {. to lot line, } \\
\& \text { th. to beg............ }\end{array}$ & Purchase... & 75 \\
\hline
\end{tabular}


Herkimer County

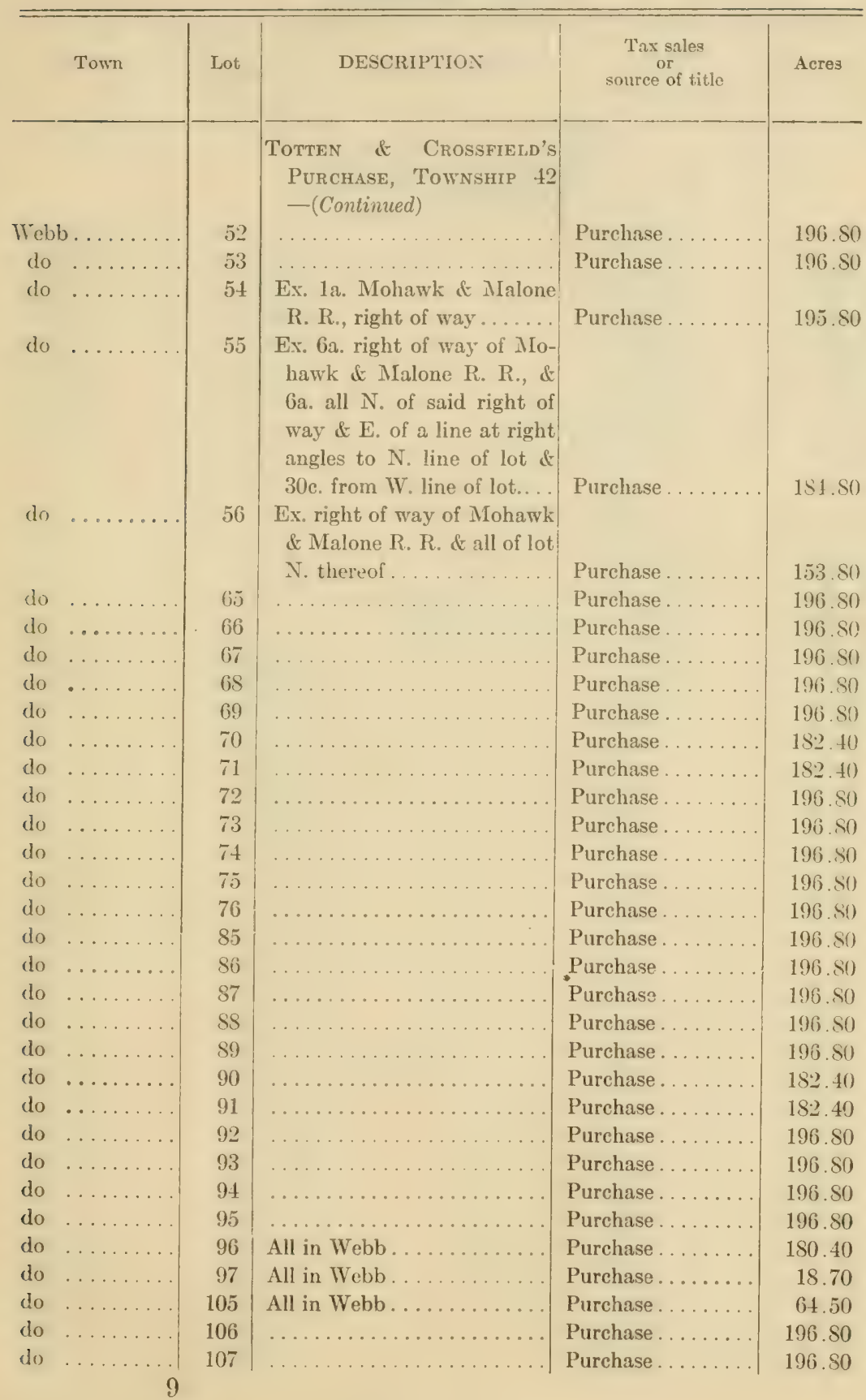


Herktuier County

\begin{tabular}{|c|c|c|c|c|c|}
\hline & Town & Lot & DESCRIPTION & $\begin{array}{c}\text { Tax sales } \\
\text { or } \\
\text { source of title }\end{array}$ & Acres \\
\hline $\begin{array}{l}\text { Webb } \\
\text { do } \\
\text { do } \\
\text { do } \\
\text { do } \\
\text { do } \\
\text { do } \\
\text { do }\end{array}$ & $\begin{array}{l}\ldots \ldots \ldots \\
\ldots \ldots \ldots \\
\ldots \ldots \ldots \\
\ldots \ldots \ldots \\
\ldots \ldots \ldots \\
\ldots \ldots \ldots \\
\ldots \ldots \ldots \\
\ldots \ldots \ldots \\
\ldots \ldots\end{array}$ & $\begin{array}{l}108 \\
109 \\
110 \\
111 \\
112 \\
113 \\
114 \\
115\end{array}$ & 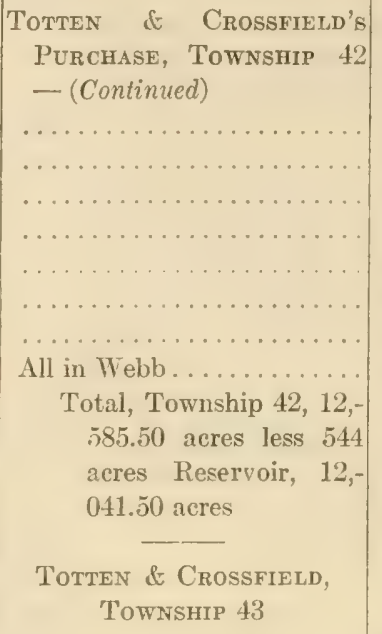 & $\begin{array}{l}\text { Purchase . . . . . } \\
\text { Purchase . . . . } \\
\text { Purchase . . . . } \\
\text { Purchase . . . . } \\
\text { Purchas . . . . } \\
\text { Purchase . . . . } \\
\text { Purchase. . . . . } \\
\text { Purchase. . . . }\end{array}$ & $\begin{array}{l}196.80 \\
196.80 \\
182.40 \\
182.40 \\
196.80 \\
196.80 \\
196.80 \\
132.30\end{array}$ \\
\hline do & $\ldots \ldots \ldots$ & 1 & $\ldots \ldots \ldots \ldots \ldots \ldots \ldots$ & Purchase.... & 166.85 \\
\hline do & $\ldots \ldots \ldots$ & 2 & $\ldots \ldots \ldots \ldots \ldots \ldots \ldots$ & Purchase...... & 166.85 \\
\hline do & $\ldots \ldots \ldots$ & 3 & $\ldots \ldots \ldots \ldots \ldots \ldots \ldots \ldots$ & Purchase...... & 197.40 \\
\hline do & $\ldots \ldots \ldots$ & 4 & $\ldots \ldots \ldots \ldots \ldots \ldots \ldots \ldots$ & Purchase ..... & 197.40 \\
\hline do & $\ldots$. & $\bar{j}$ & $\ldots \ldots \ldots \ldots \ldots \ldots$ & Purchinso..... & 197.40 \\
\hline do & $\ldots \ldots \ldots$ & 6 & $\ldots \ldots \ldots \ldots \ldots \ldots \ldots$ & Purchase....... & 197.40 \\
\hline do & $\cdots \cdots$ & 7 & $\ldots \ldots \ldots \ldots \ldots \ldots \ldots$ & Purchase..... . & 197.40 \\
\hline do & & 8 & $\ldots \ldots \ldots \ldots \ldots \ldots$ & Purchas . . . . & 197.40 \\
\hline do & $\ldots \ldots$ & 9 & $\ldots \ldots \ldots \ldots \ldots \ldots \ldots \ldots$ & Purchase..... & 168 \\
\hline do & $\ldots \ldots \ldots$ & 10 & $\ldots \ldots \ldots \ldots \ldots \ldots \ldots$ & Purchase. .... & 60.80 \\
\hline do & $\ldots \ldots \ldots$ & 11 & & Purchase.... . & 150.40 \\
\hline do & $\ldots \ldots \ldots$ & 12 & $\ldots \ldots \ldots \ldots \ldots \ldots \ldots \ldots$ & Purchase.... . & 197.40 \\
\hline do & $\ldots$. & 13 & $\ldots \ldots \ldots \ldots$ & Purchase..... & 197.40 \\
\hline do . & $\ldots$ & 1. & & Purchase..... & 197.40 \\
\hline do & $\ldots \ldots$ & 15 & & Purcha e.... & 197.40 \\
\hline do & . $\cdots$ & 16 & $\ldots \ldots \ldots \ldots \ldots \ldots \ldots \ldots \ldots \ldots$ & Purchase..... & 197.40 \\
\hline do & $\cdots \cdots$ & 17 & $\cdots$. & Purchase.... & 197.40 \\
\hline do & $\ldots \ldots$ & 18 & $\ldots \ldots \ldots \ldots \ldots \ldots$ & Purchase.... & 197.40 \\
\hline do & ... & 19 & & Purchase..... & 166.85 \\
\hline do . & $\ldots$. & 20 & & Purchase.... . & 166.85 \\
\hline do. & $\ldots \ldots$ & 21 & & Purchase.... . & 166.85 \\
\hline do & & 22 & & Purchase.... & 166.85 \\
\hline do & & 23 & & Purchase.... . & 197.40 \\
\hline do & & 24 & $\ldots \ldots \ldots \ldots$ & Purchase.... . & 197.40 \\
\hline do & & 25 & & Purchase.... . & 197.40 \\
\hline do & & 26 & & Purchase. & 197.17 \\
\hline
\end{tabular}




\section{Herkimer County}

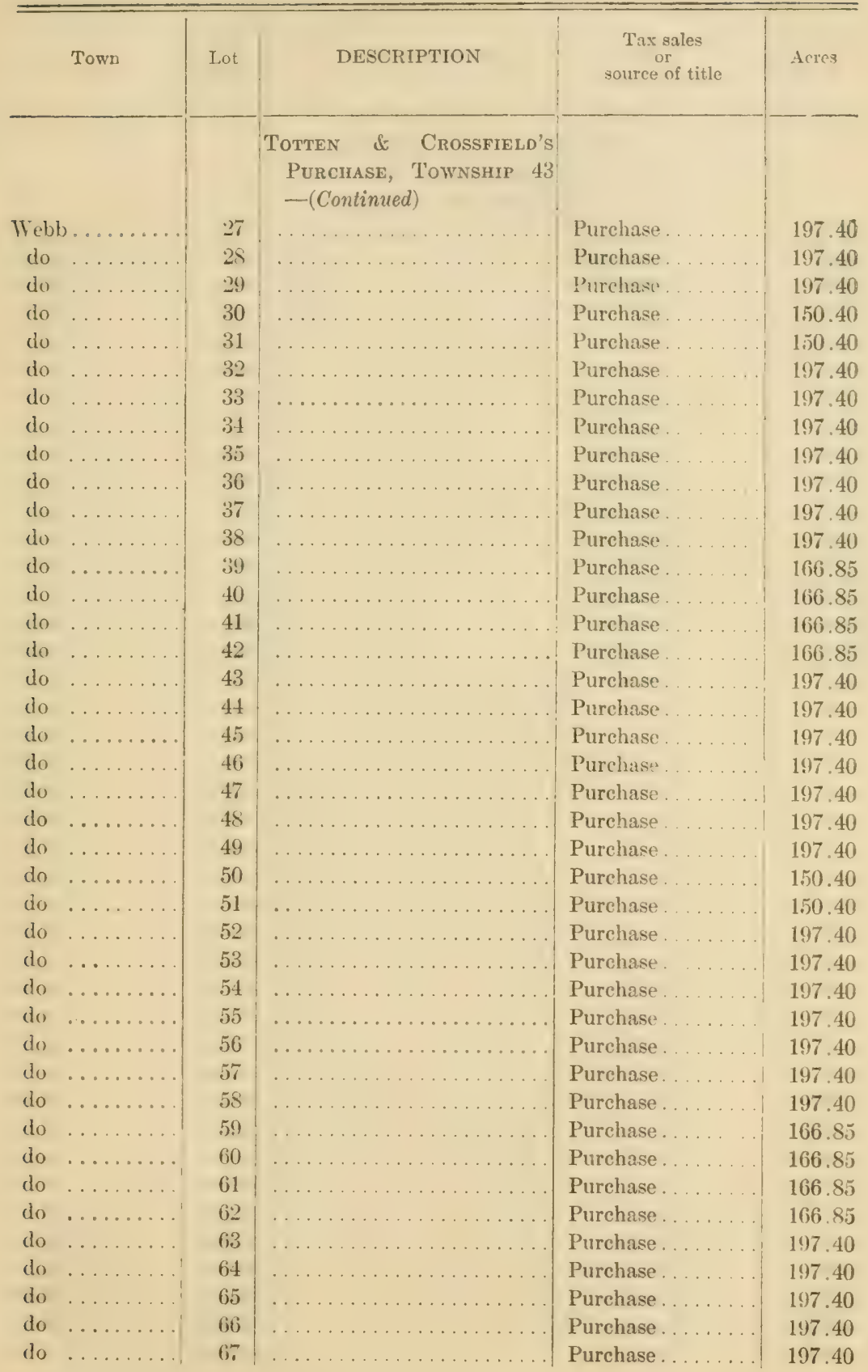


Herkimer County

\begin{tabular}{|c|c|c|c|c|c|}
\hline & Town & Lot & DESCRIPTION & $\begin{array}{c}\text { Tax sales } \\
\text { or } \\
\text { source of title }\end{array}$ & Acres \\
\hline & & & $\mid \begin{array}{c}\text { Totten \& Crossfield's } \\
\text { Purchase, Township } 43 \\
\text { - Continued })\end{array}$ & & \\
\hline Webb. & . & 6.9 & $\cdots$ & P'urchase... & 197.40 \\
\hline do & & 73 & & Purchase.... . & 197.40 \\
\hline do & $\ldots \ldots$ & it & . . & Purchase..... & 197.40 \\
\hline do. & $\ldots \ldots$ & 75 & $\ldots \ldots$ & Purchase...... & 197.10 \\
\hline do. & $\ldots \ldots$ & 76 & 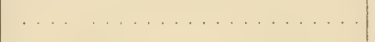 & Purchase..... & 197.40 \\
\hline do. & $\ldots \ldots$ & 77 & $\ldots \ldots \ldots \ldots$ & Purchase.... . & 197.10 \\
\hline do. & $\ldots \ldots \ldots$ & $7 s$ & & P'urchase. & 197.10 \\
\hline do. & $\ldots \ldots \ldots$ & 79 & $\ldots$ & Purchase... . & 166.85 \\
\hline do. & $\ldots$ & 80 & & Purchase..... . & 166.85 \\
\hline do & . & $\$ 1$ & & Purchase..... . & 166.85 \\
\hline do & $\ldots$ & 82 & $\ldots \ldots$ & Purchase...... & 166.85 \\
\hline do & $\ldots$ & 83 & $\ldots$ & Purchase... . . . & 197.40 \\
\hline do & $\ldots$ & 84 & & P'urchase..... & 197.41 \\
\hline do. & $\ldots$ & $8 \tilde{\jmath}$ & $\ldots \ldots \ldots$ & Purchase ..... & $197 \cdot 10$ \\
\hline do. & ........ & S6 & $\ldots \ldots$ & Purchase...... & 197.10 \\
\hline do. & $\ldots \ldots \ldots$ & 87 & 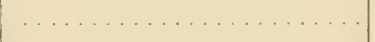 & Purchase. & $197+40$ \\
\hline do. & & is & $\cdots$ & Purchase..... & 197.40 \\
\hline do. & $\ldots \ldots \ldots{ }^{\prime}$ & 93 & & Purchase. . & 181.81 \\
\hline do. & $\ldots \ldots$ & 94 & . & Purchase..... & $18+.80$ \\
\hline do. & $\ldots \ldots$ & 95 & & Purchase ...... & $18+80$ \\
\hline do. & $\ldots \ldots$ & 96 & & Purchase .... . . & 181.8() \\
\hline do. & $\cdots$ & 97 & $\ldots$ & Purchase..... . & $18+.80$ \\
\hline do. & $\ldots \ldots$ & 98 & & Purchase...... & $18+\ldots()$ \\
\hline do. & $\ldots$ & 99 & & Purchase ... & 156.20 \\
\hline do. & $\ldots$ & 100 & & Purchase...... & 156.2() \\
\hline do . & ... & 104 & Ex. $171 \frac{6}{10}{ }^{0}$ a. E. pt... & Purchase..... . & 13.20 \\
\hline do. & $\ldots \ldots \ldots$ & 105 & $\ldots \ldots \ldots \ldots \ldots \ldots$ & Purchase.... . & 181.80 \\
\hline do. & $\cdots \cdots$ & 106 & ․ & Purchase..... . & $18+$ so \\
\hline do . & $\ldots$ & 107 & & Purchase. & $18+. s)$ \\
\hline do. & $\ldots \ldots$ & 108 & & Purchase...... & 181.80 \\
\hline do. & $\ldots \ldots$ & 109 & & Purchase...... & 1.56 .2() \\
\hline do. & $\ldots \ldots$ & 110 & . & Purchase..... & 156.20 \\
\hline do. & $\ldots \ldots$ & 111 & $\ldots \ldots \ldots \ldots \ldots$ & Purchase...... & 182.40 \\
\hline do. & $\ldots \ldots$ & 112 & . & Purchase..... & 196 \\
\hline do. & $\ldots \ldots$ & 113 & $\ldots \ldots \ldots \ldots \ldots$ & Purchase... & 196 \\
\hline do & $\ldots$ & 114 & $\ldots \ldots$ & Purchase..... & 196 \\
\hline do & $\ldots$ & 115 & .. & Purchase.... & 196 \\
\hline do & $\ldots \ldots$ & 116 & $\ldots \ldots \ldots \ldots$ & Purchase... & 196 \\
\hline do & $\ldots$. & 121 & $\ldots$ & Purchase... . & 75.60 \\
\hline do & $\ldots$ & 122 & & Purchase... . & 174.30 \\
\hline do. & $\ldots \ldots \ldots$ & 123 & & Purchase.... & 197.40 \\
\hline
\end{tabular}


Herkimer County

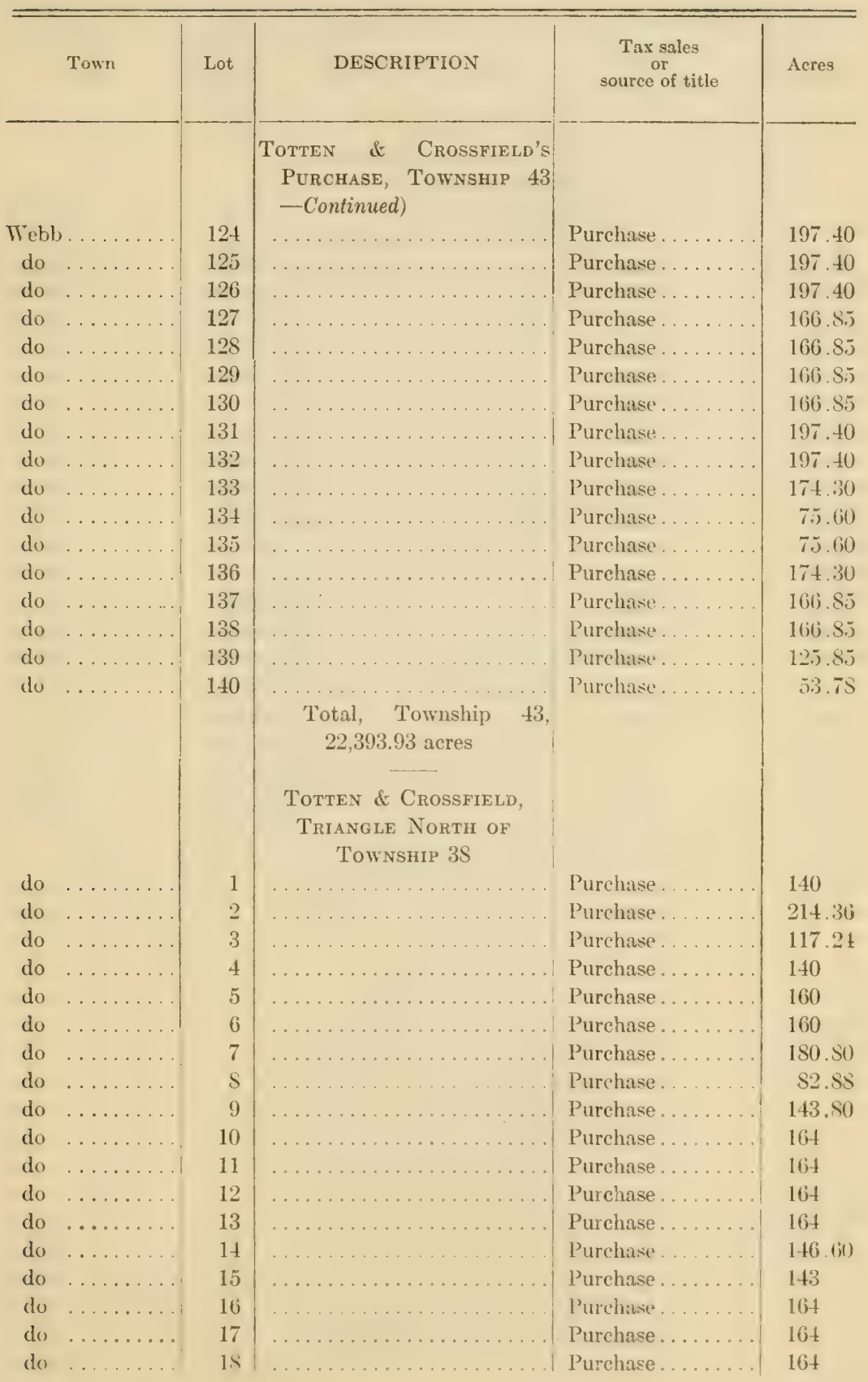


Herkmer County

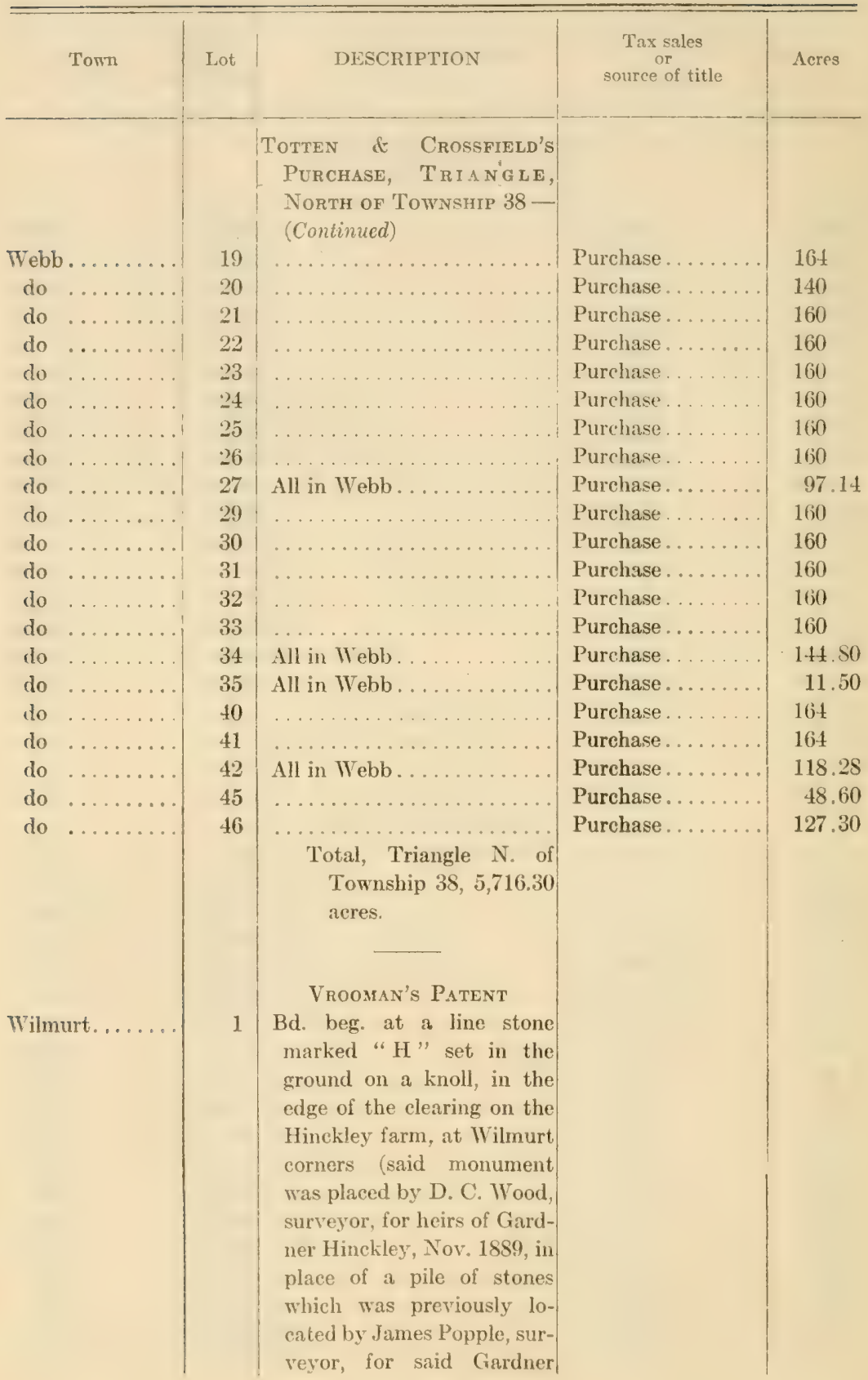




\section{Herkimer County}

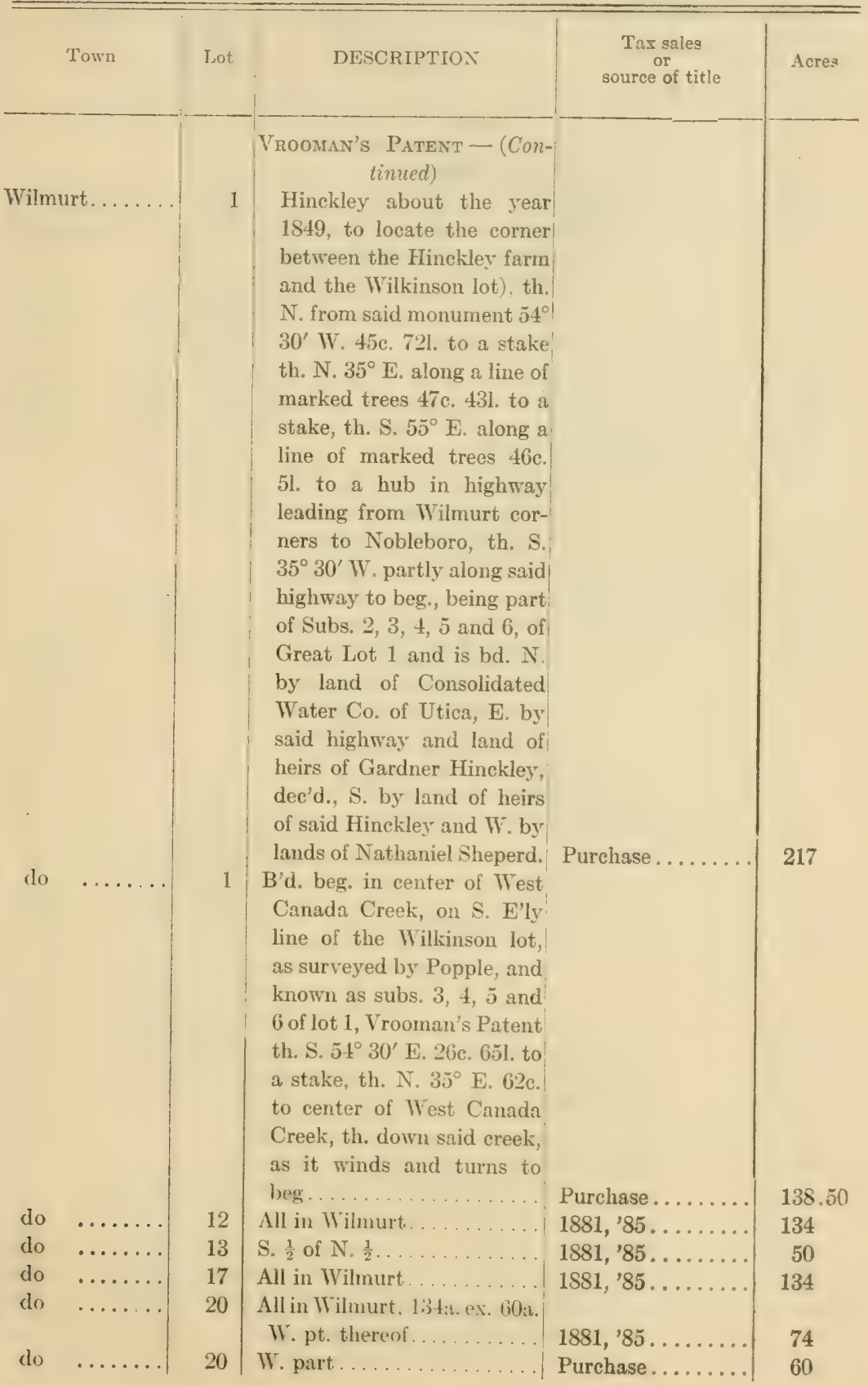




\section{Herkmer County}

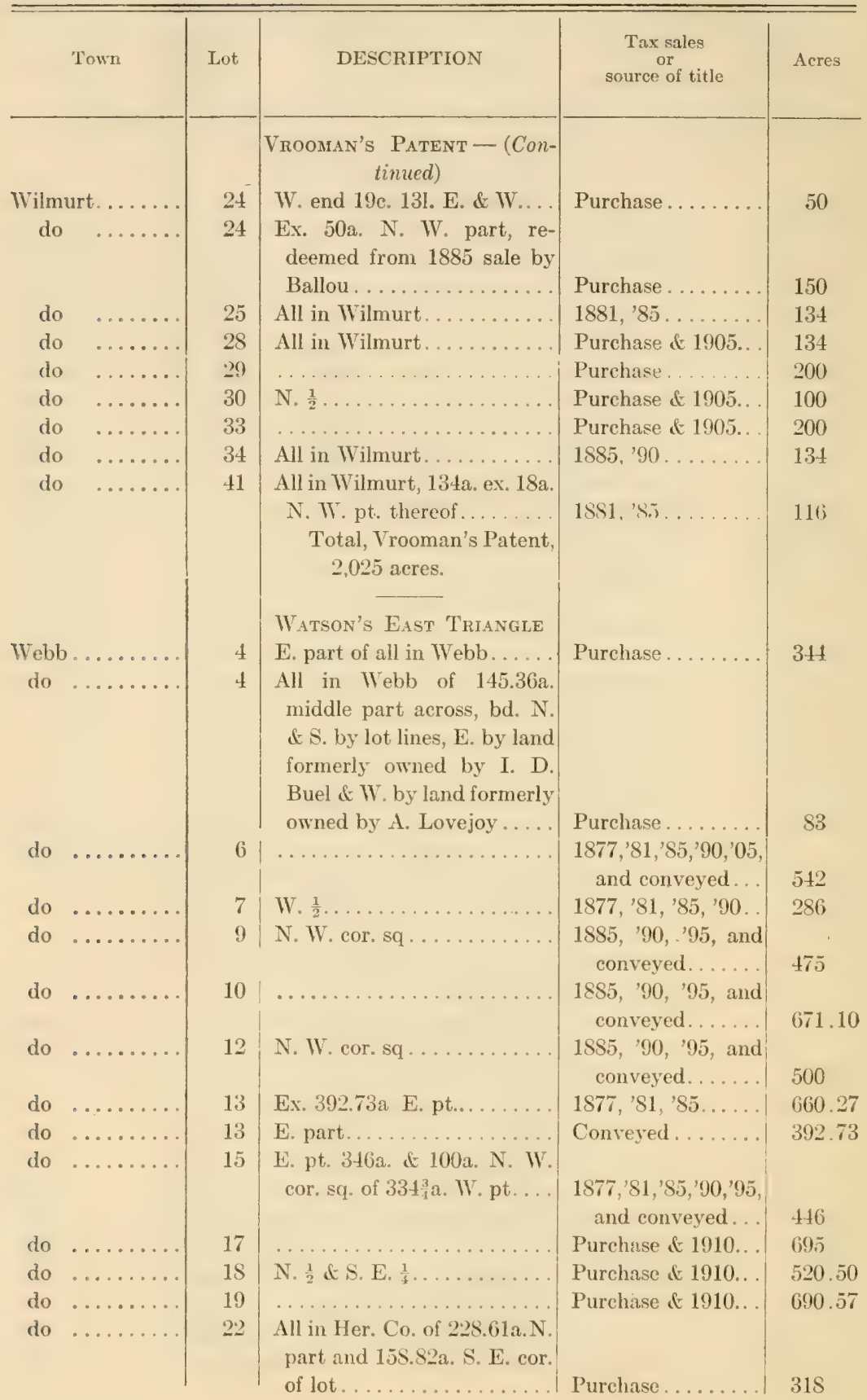


Herkimer County

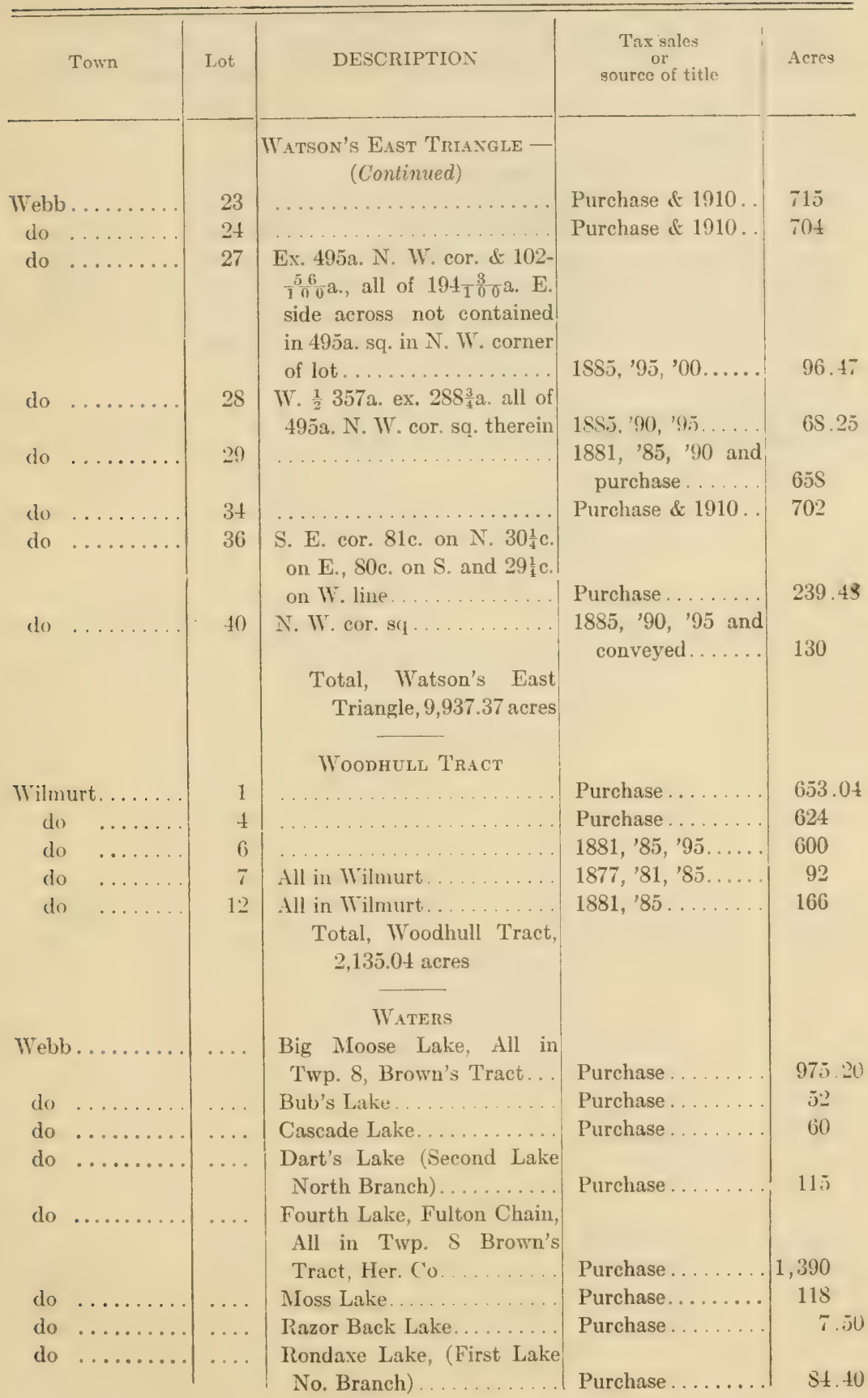




\section{Herkimer County}

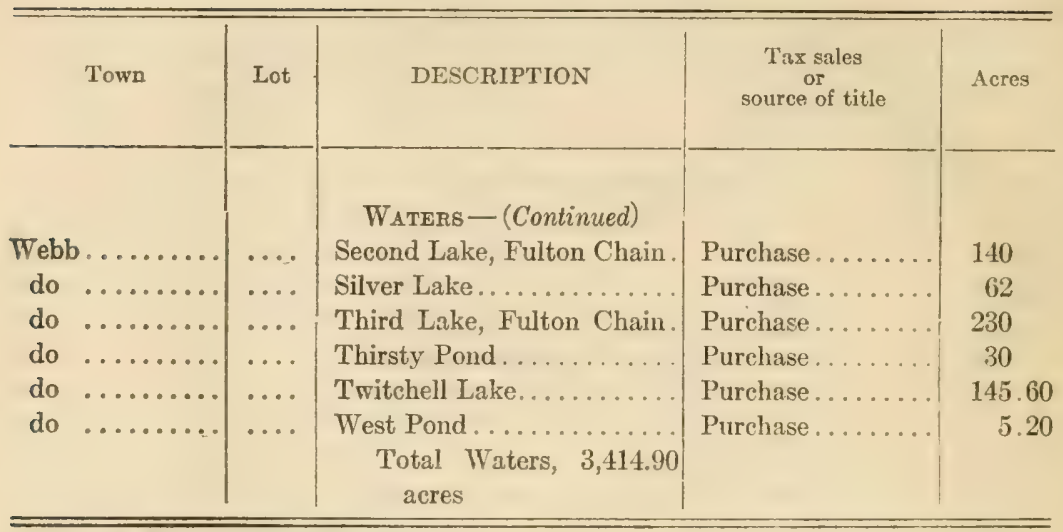

For other State land in Herkimer county, see Appendix. 


\section{LEWIS COUNTY}

(Total number of acres, 4,542.42)

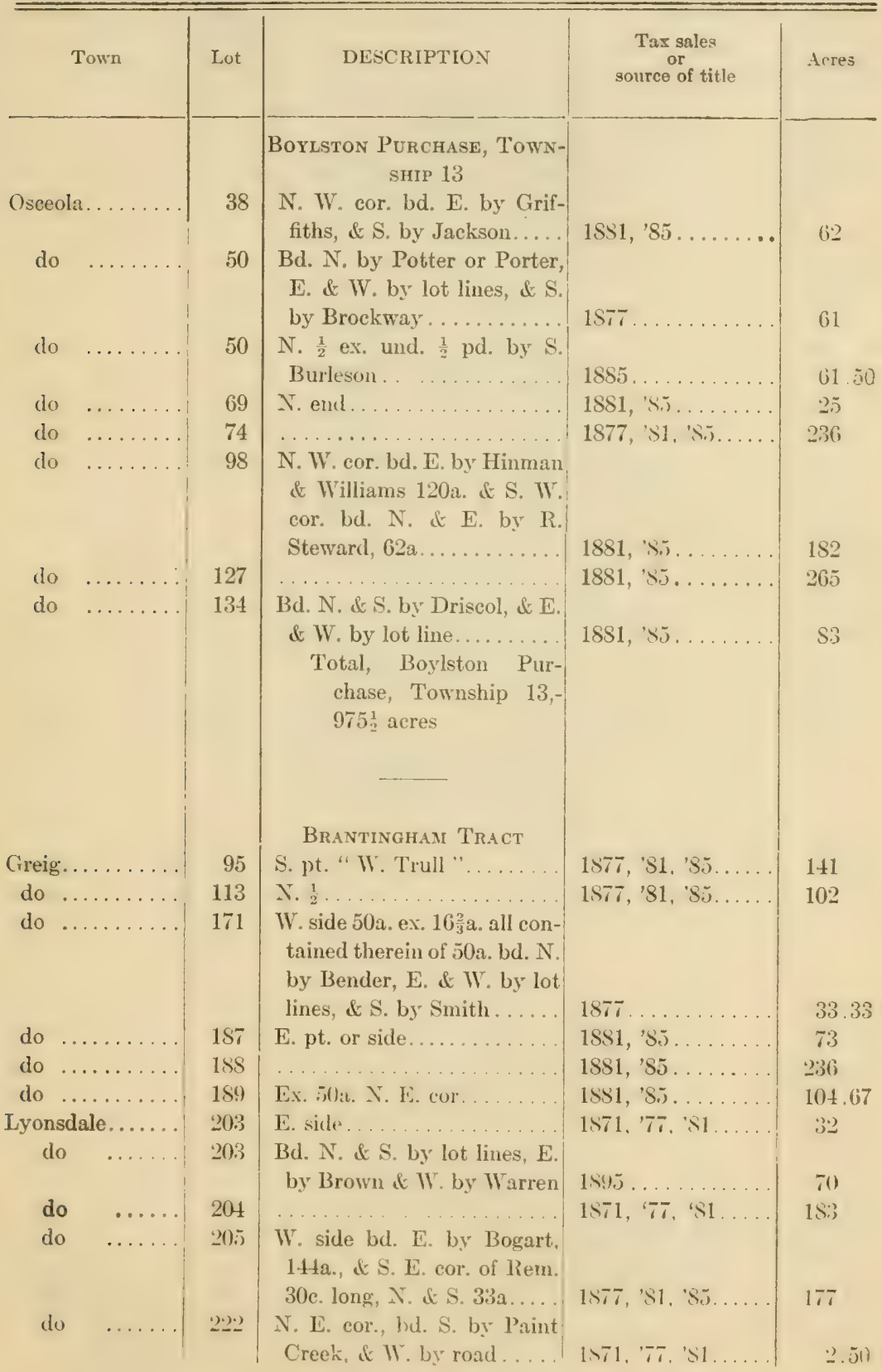


Lewis County

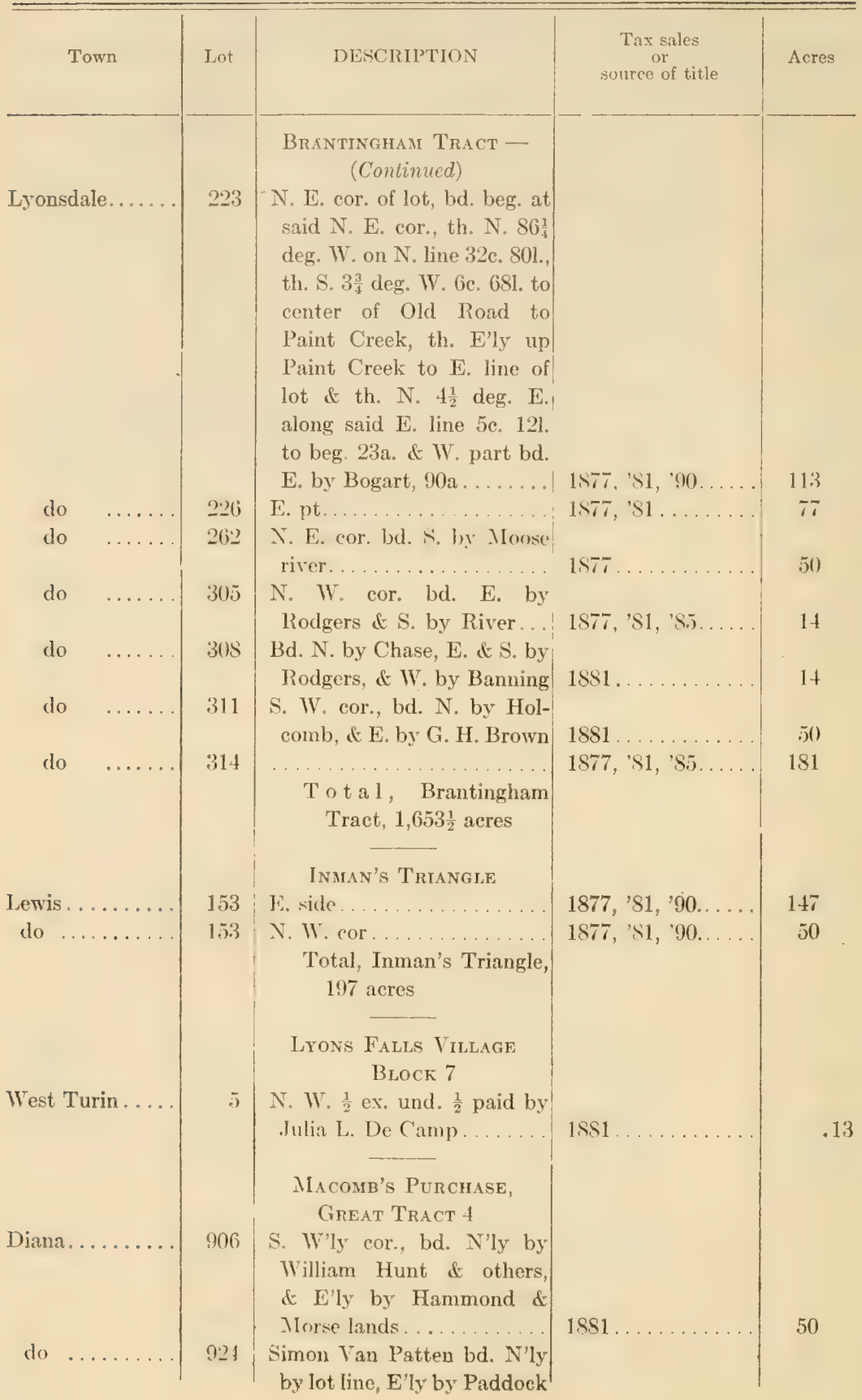


Lewis County

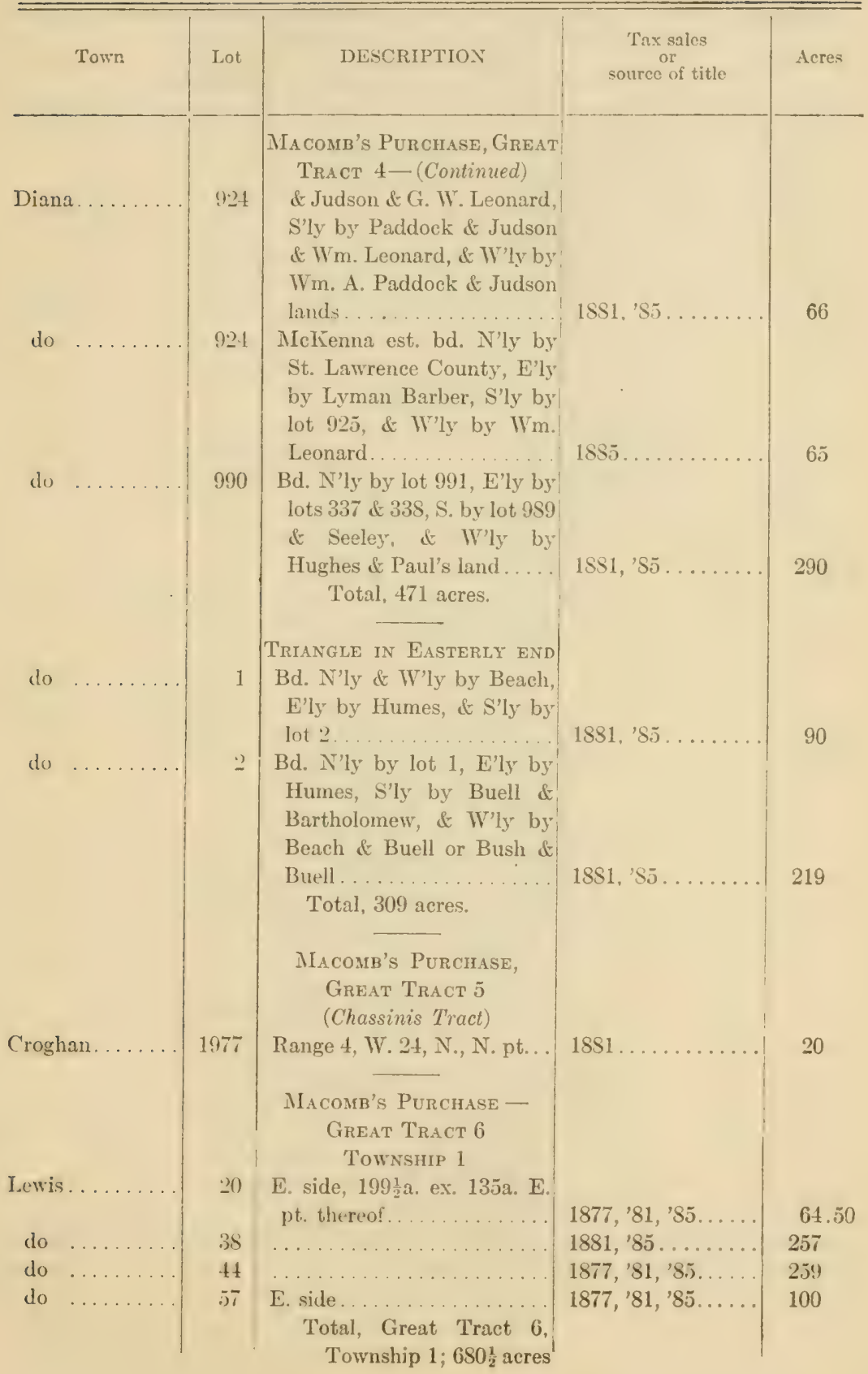




\section{Lewis County}

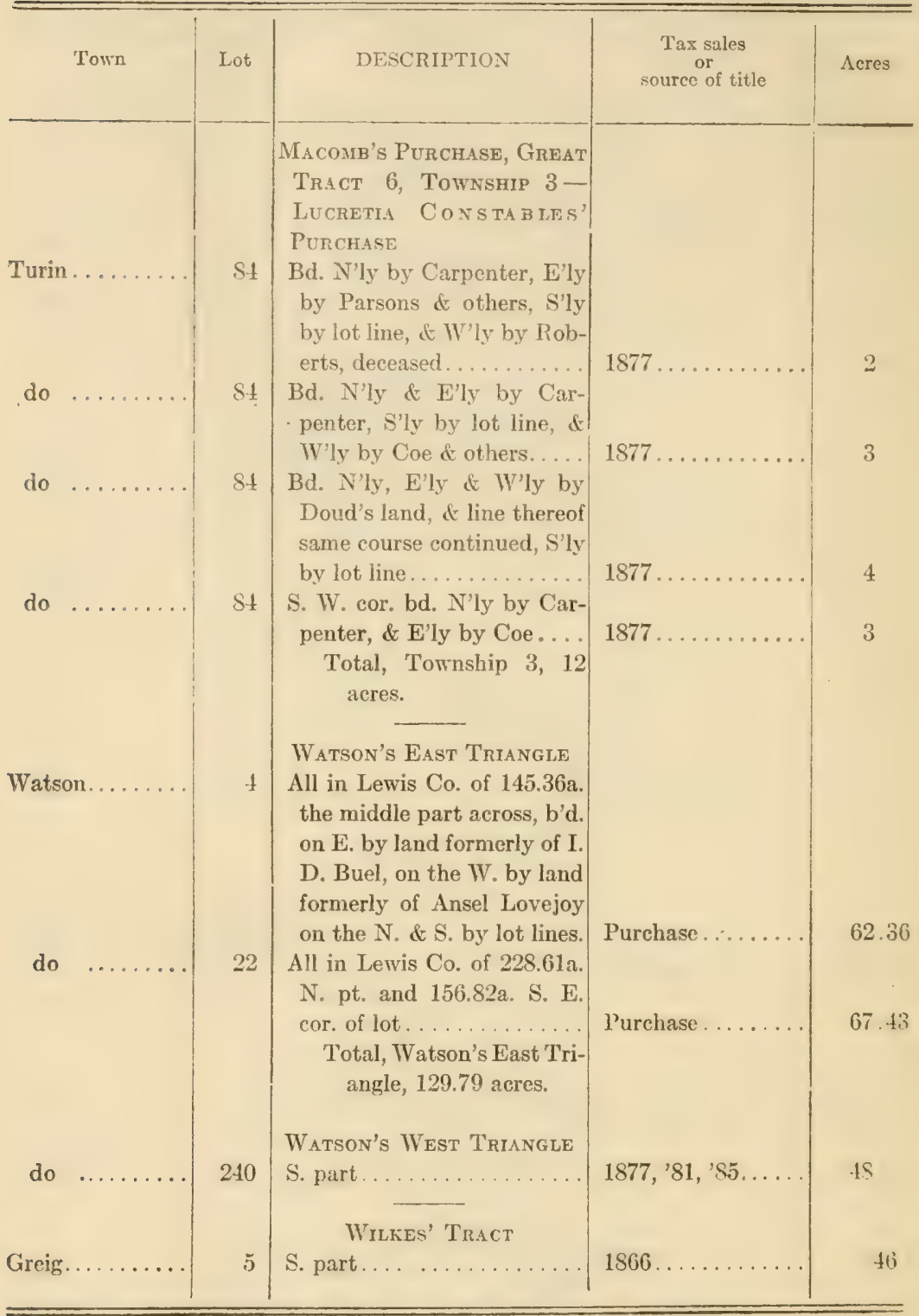

For other State land in Lewis County see Appendix. 


\section{ONEIDA COUNTY}

(Total number of acres, 28,889.91)

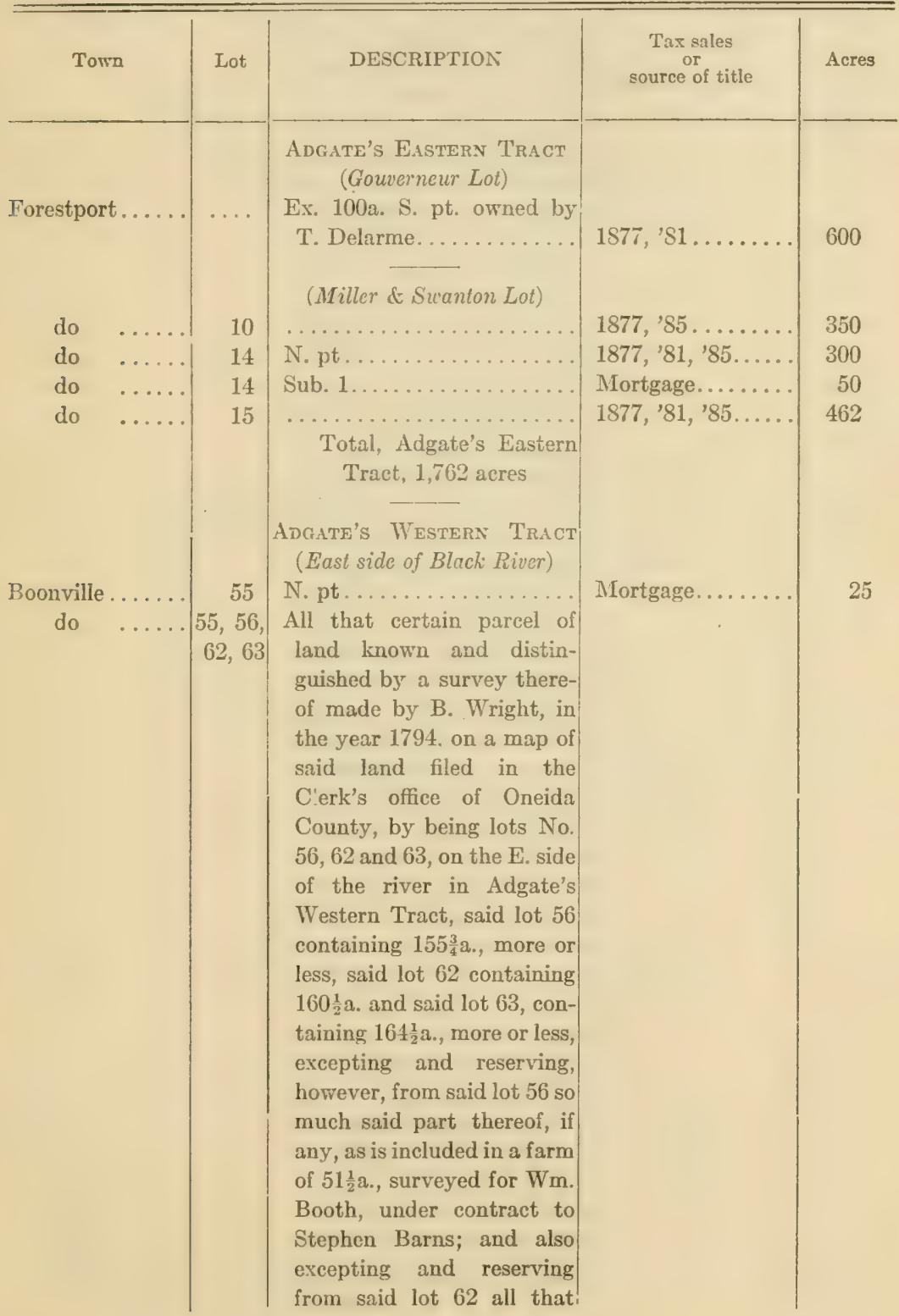


Oneida Countr

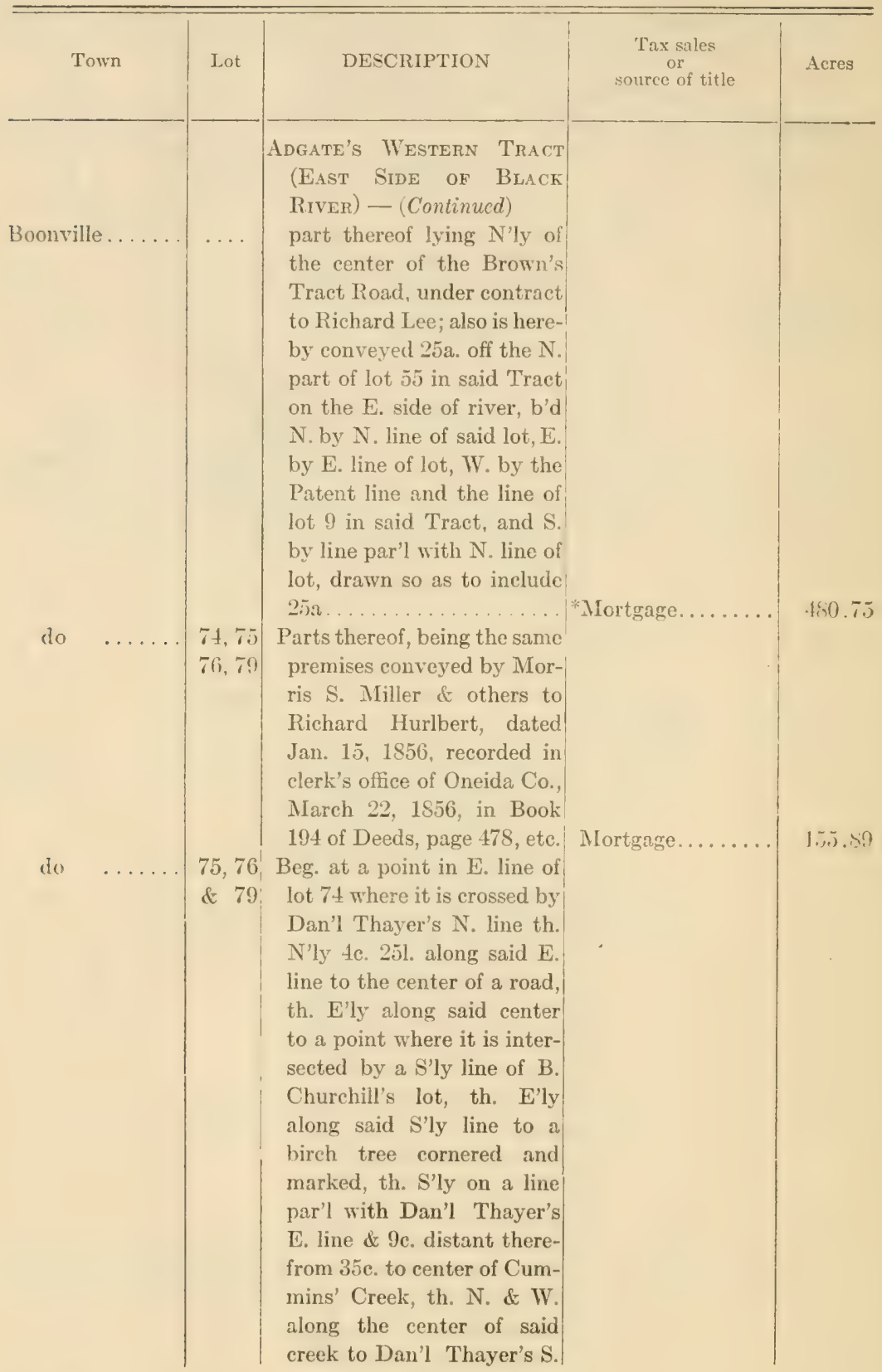

* Resale. 
Oneida Country

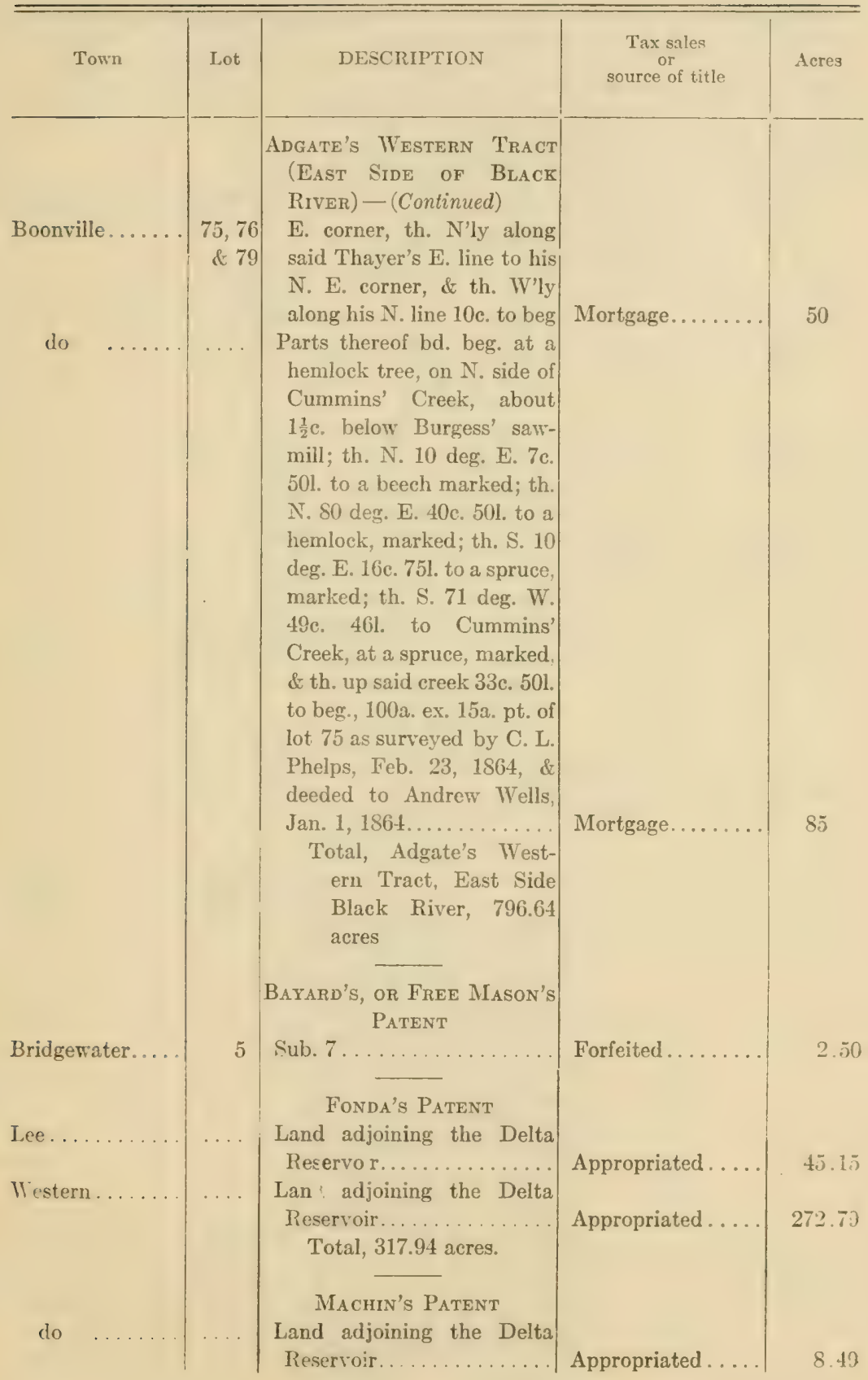


Onerda County

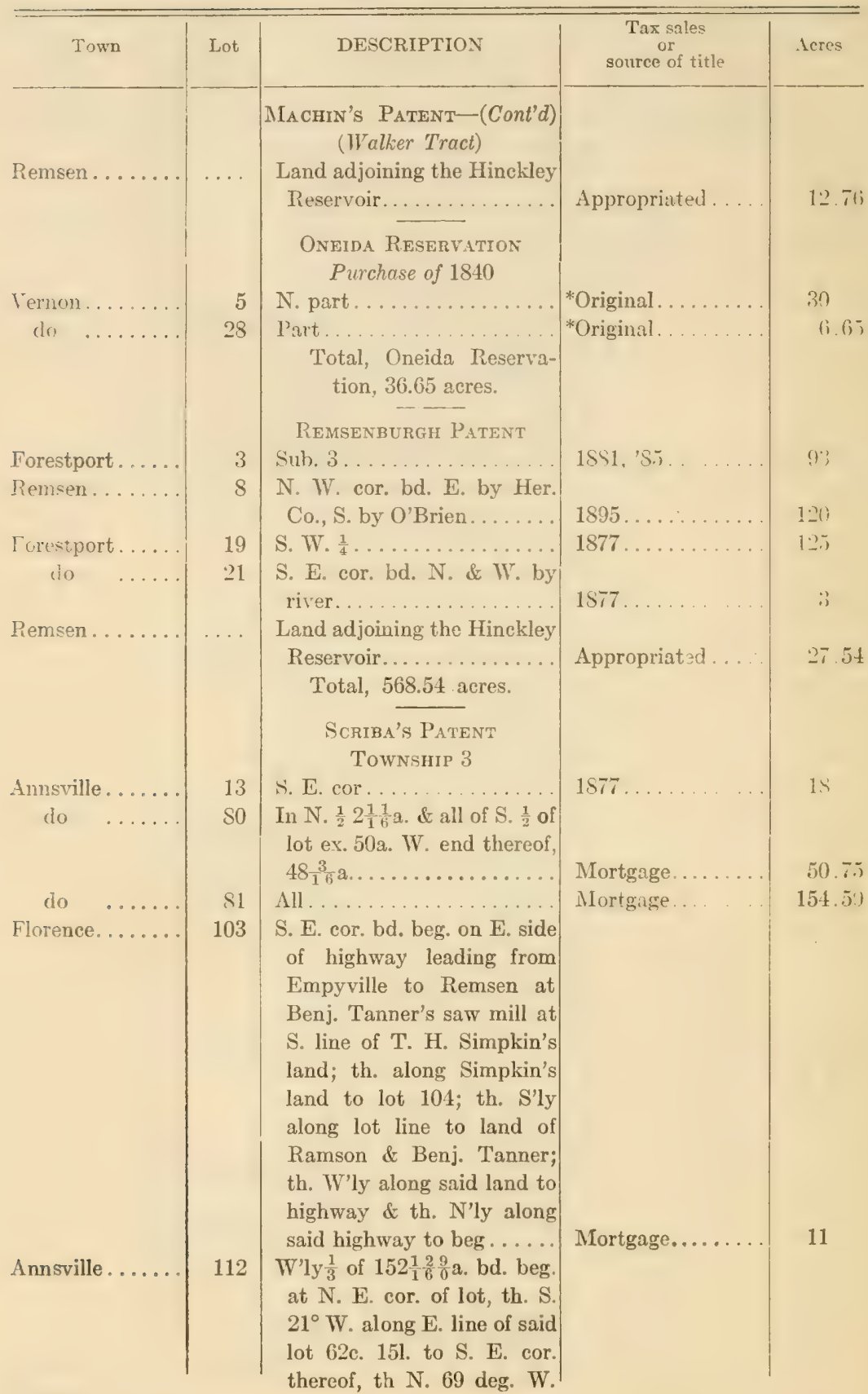


Oneida County

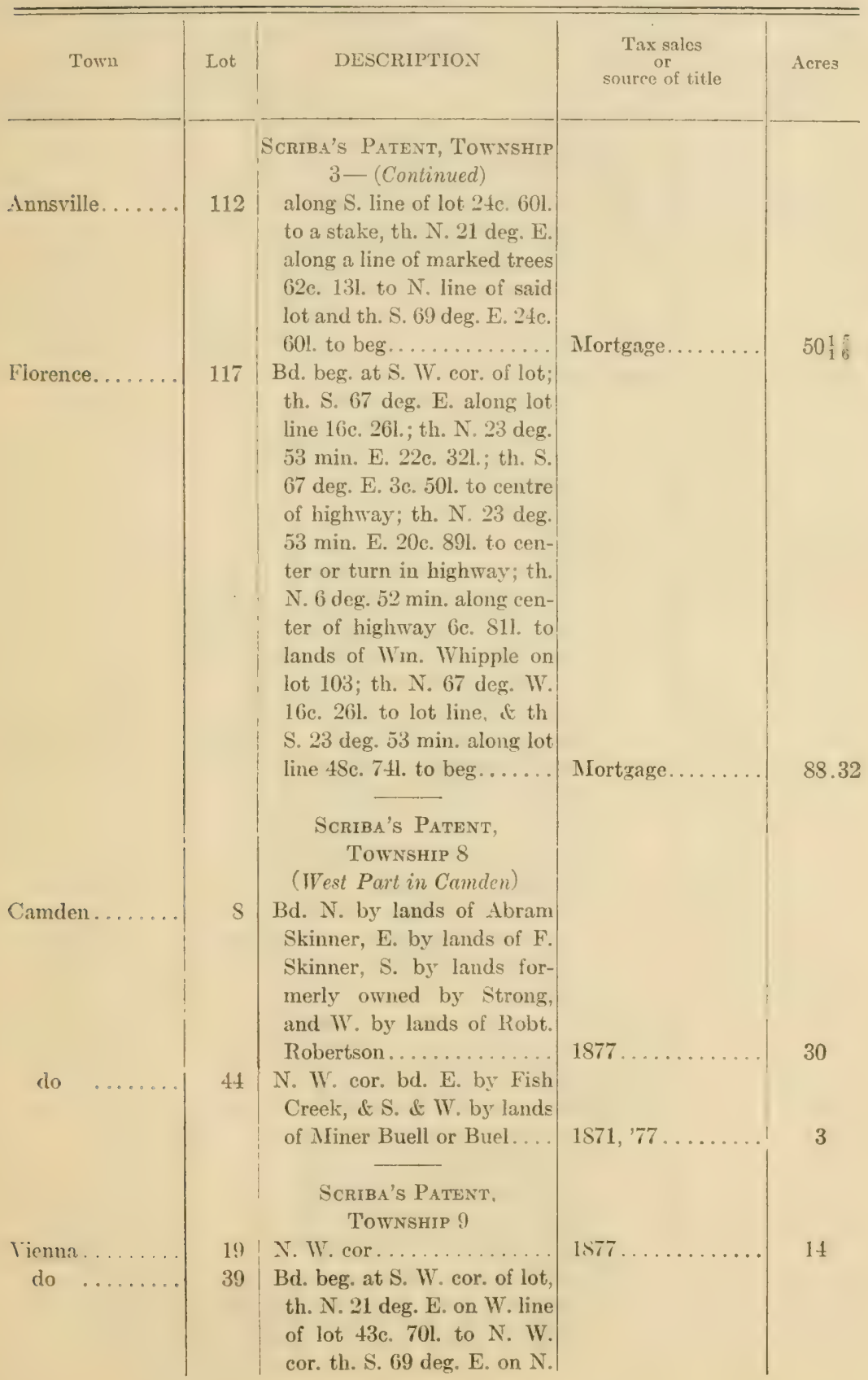


Oneida County

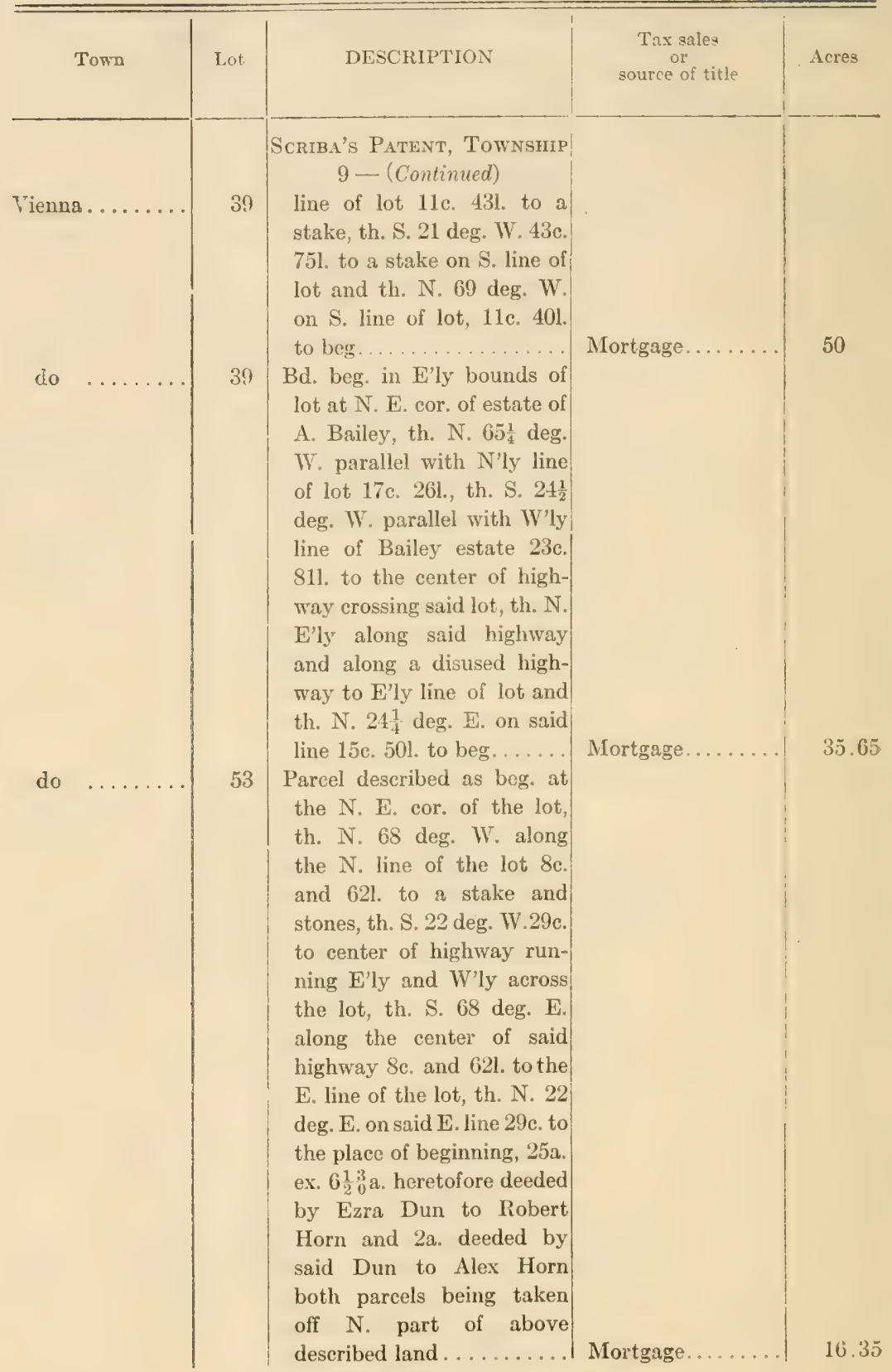




\section{Oneida Countr}

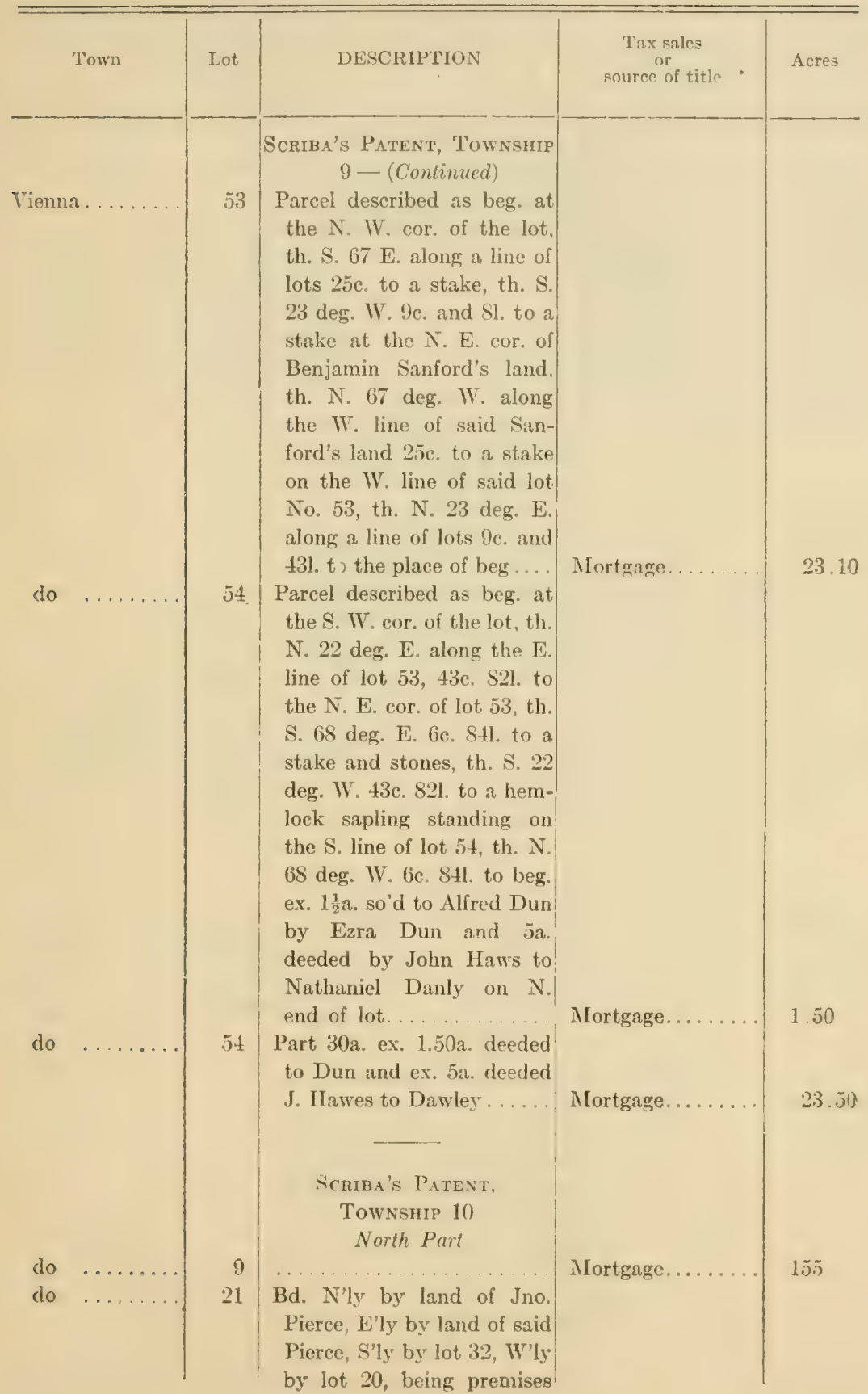


Oneida Countr

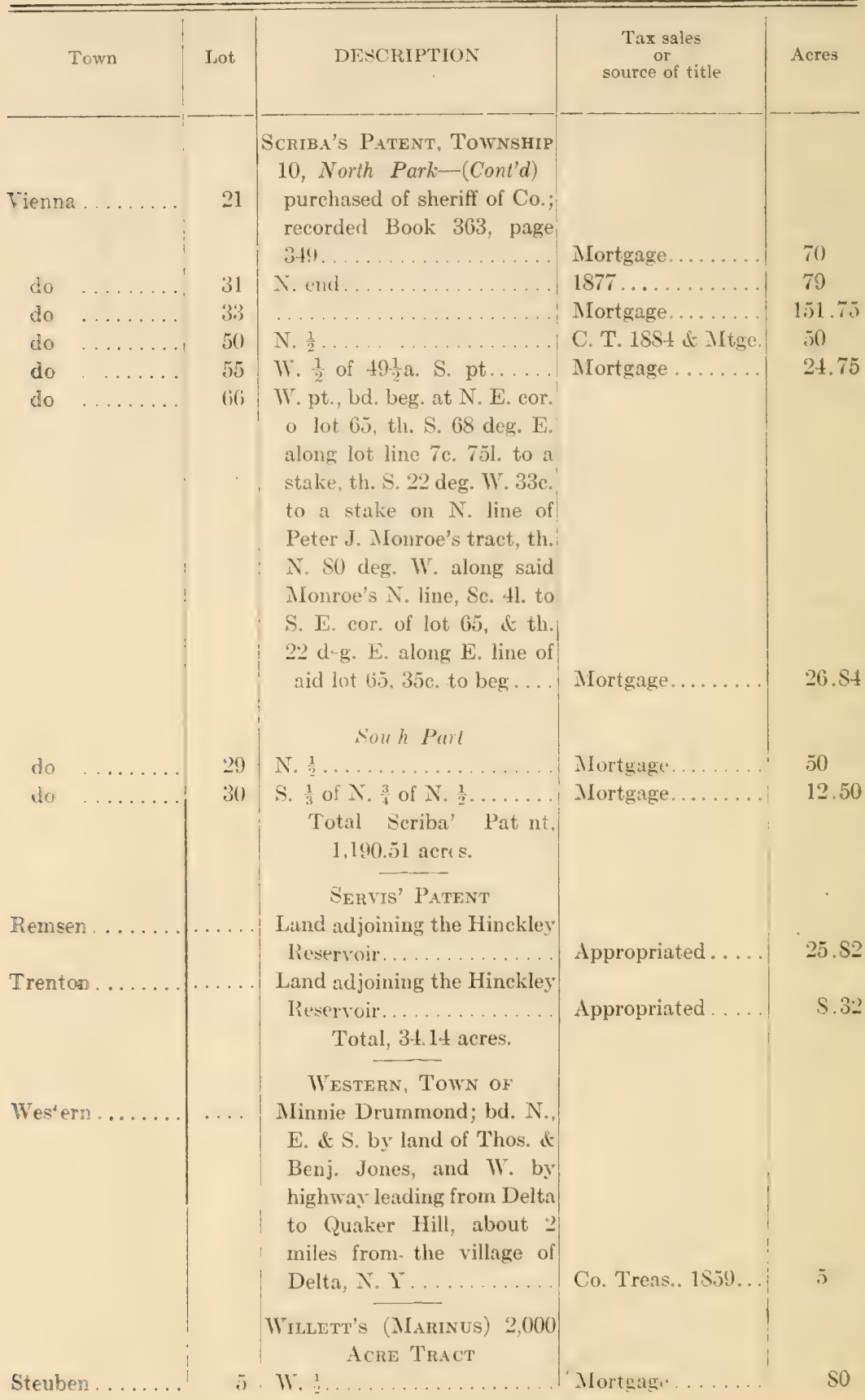


Oneida County

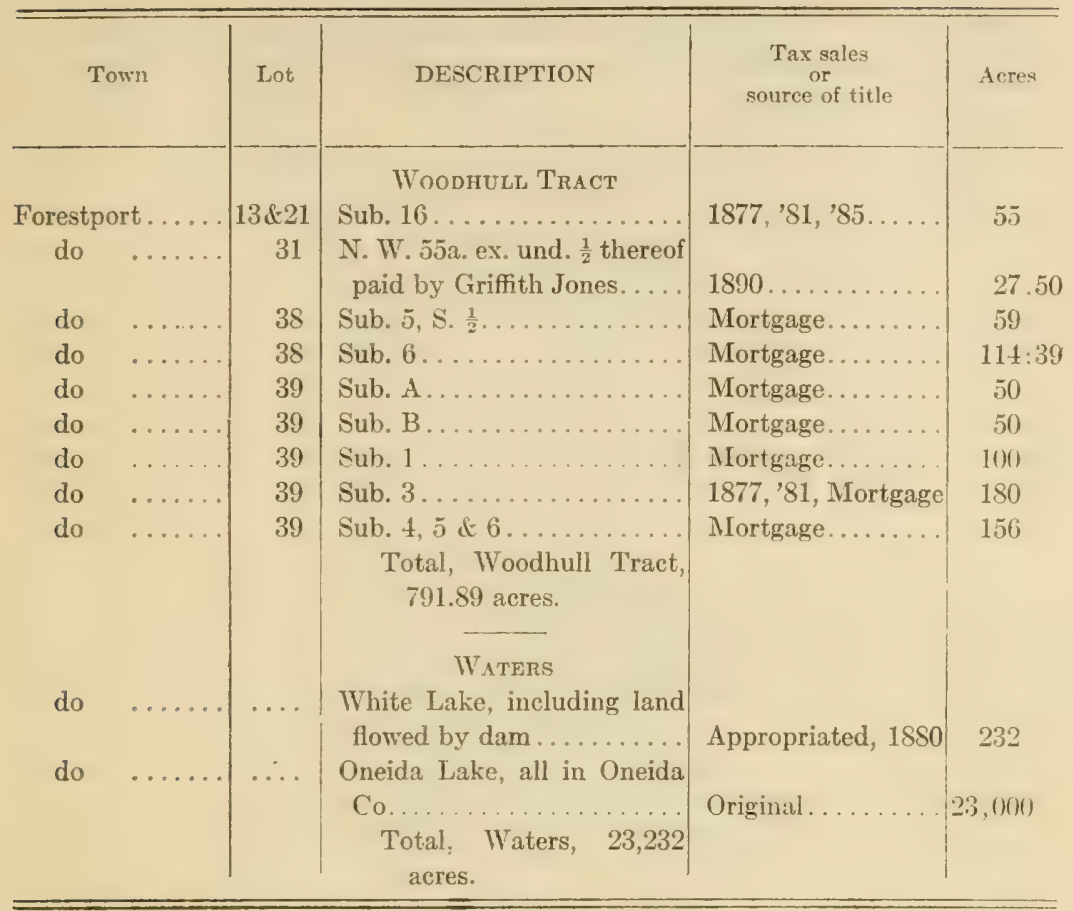

For other State land in Oneida County see Appendix : 
ST. LAWRENCE COUNTY

(Total number of acres, 80,626.82)

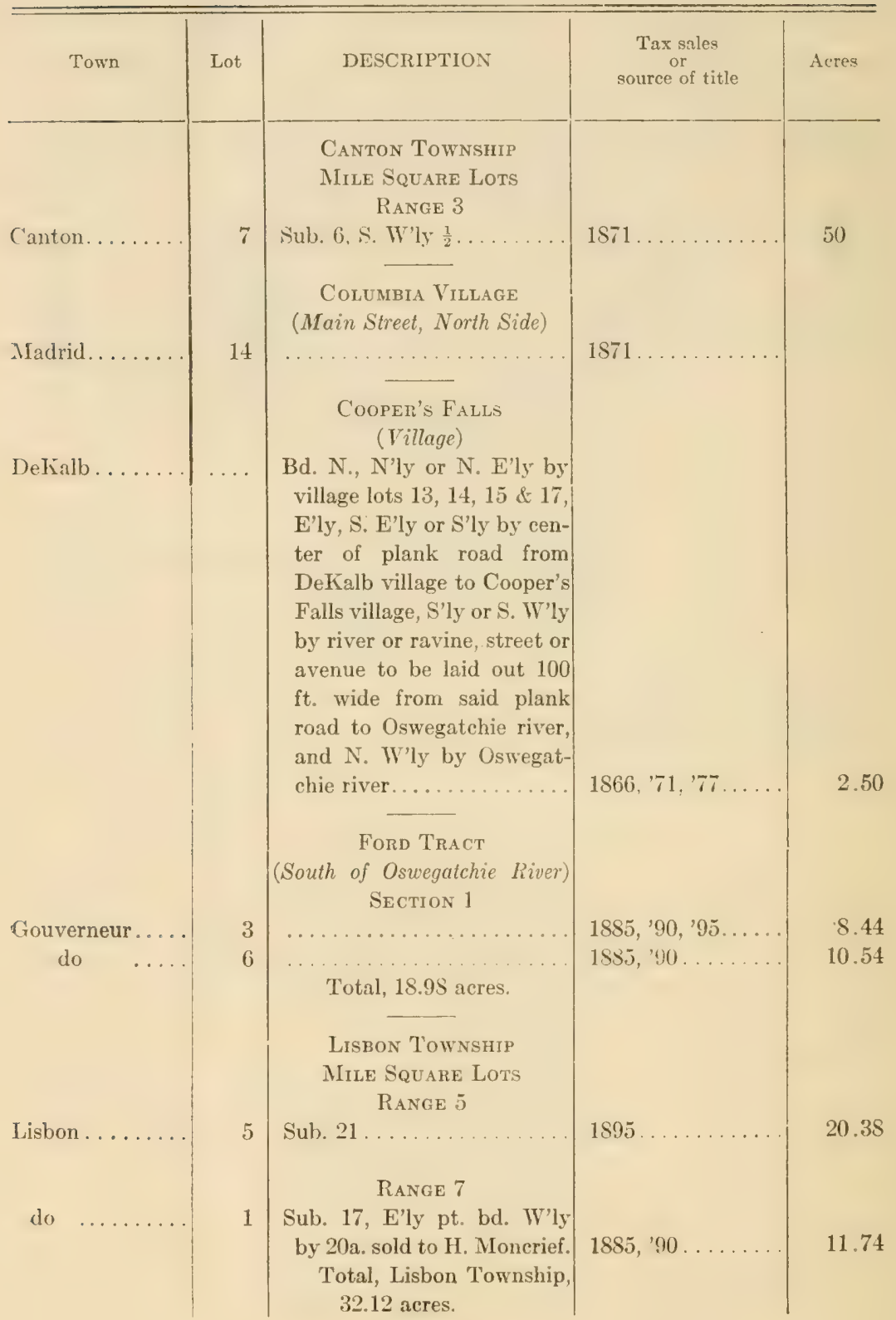


St. Lawrence County

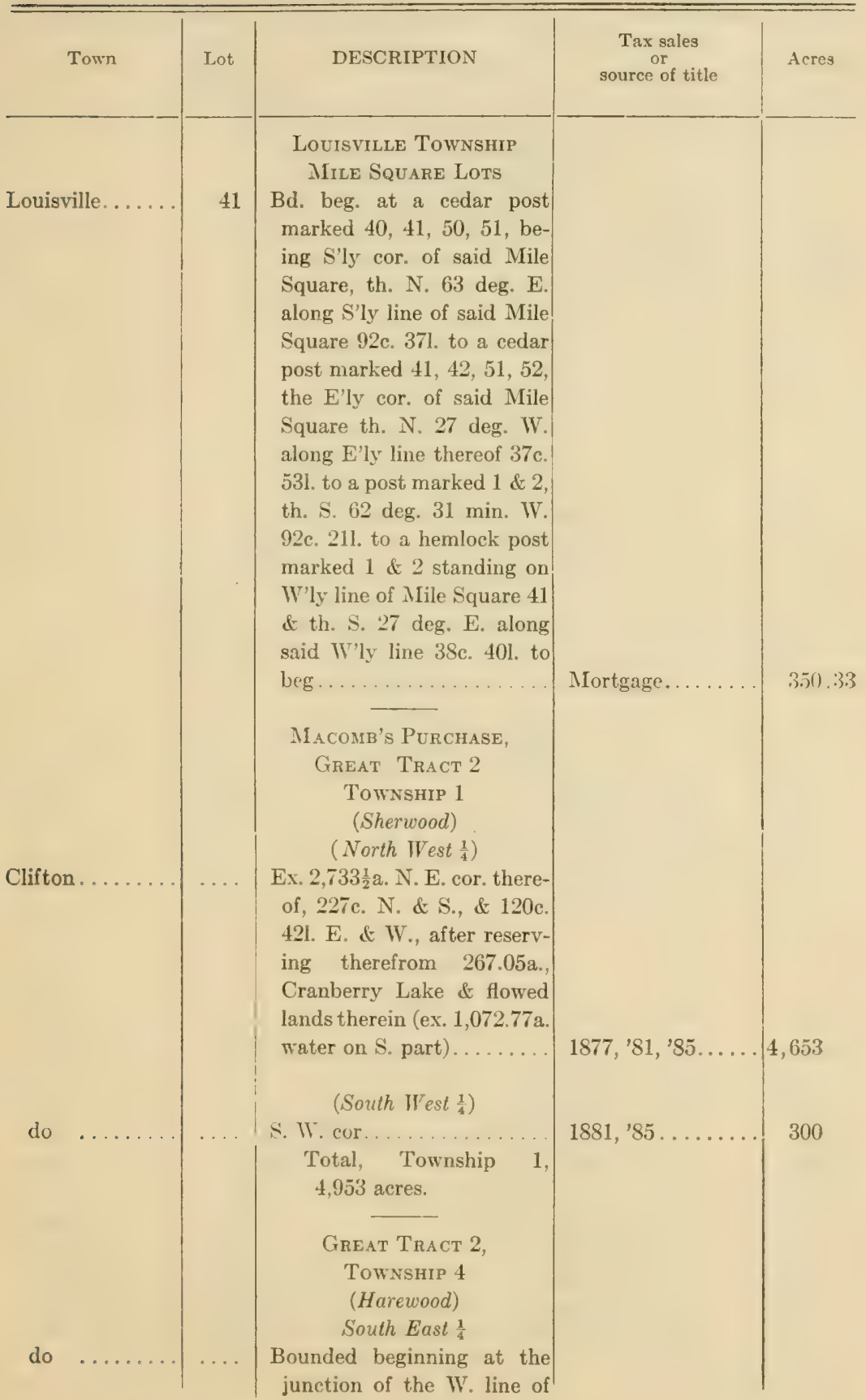


St. Lawrence County

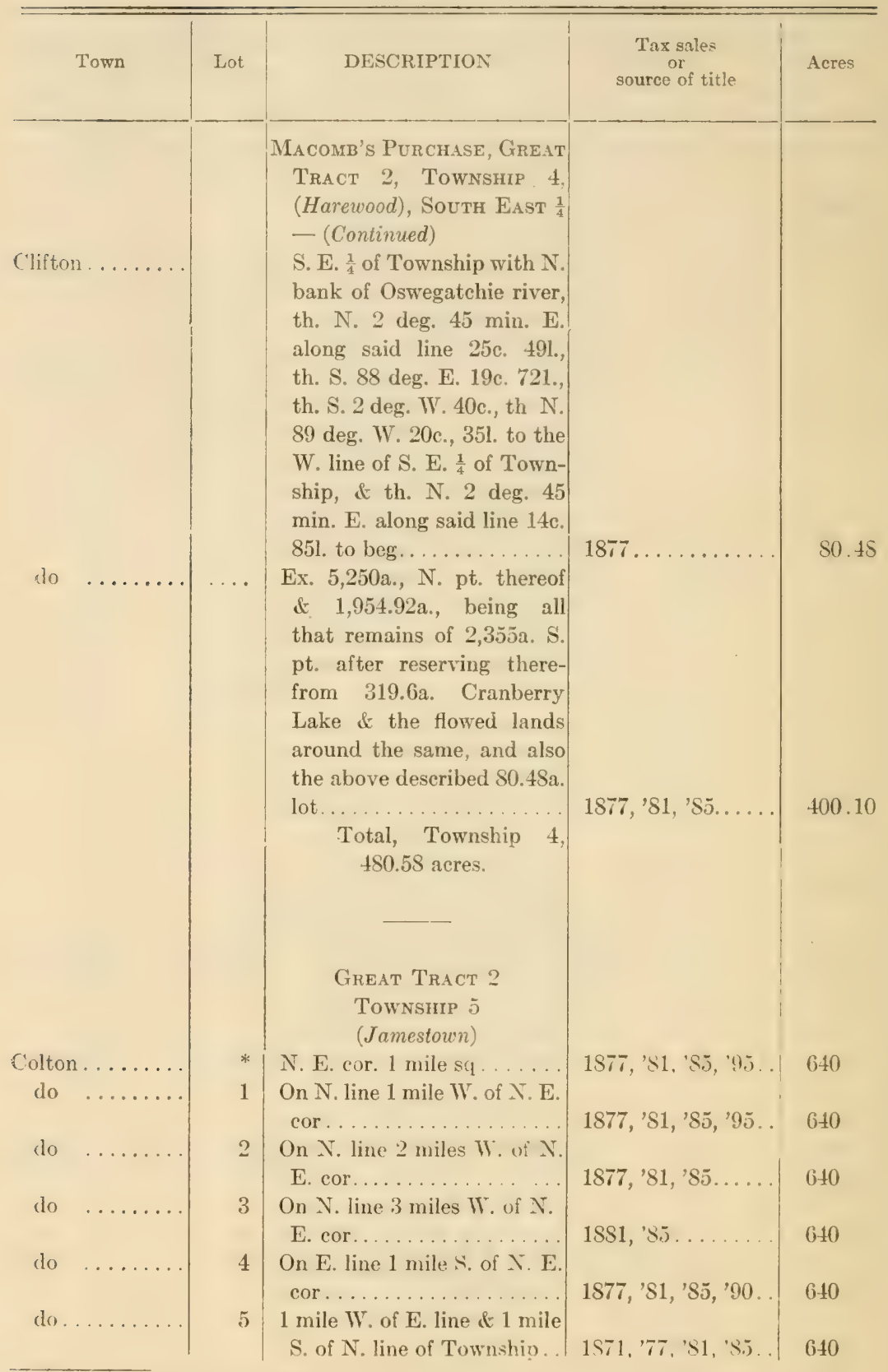

\footnotetext{
* Correct; this lot is not numbered.
} 
St. Lawrence County

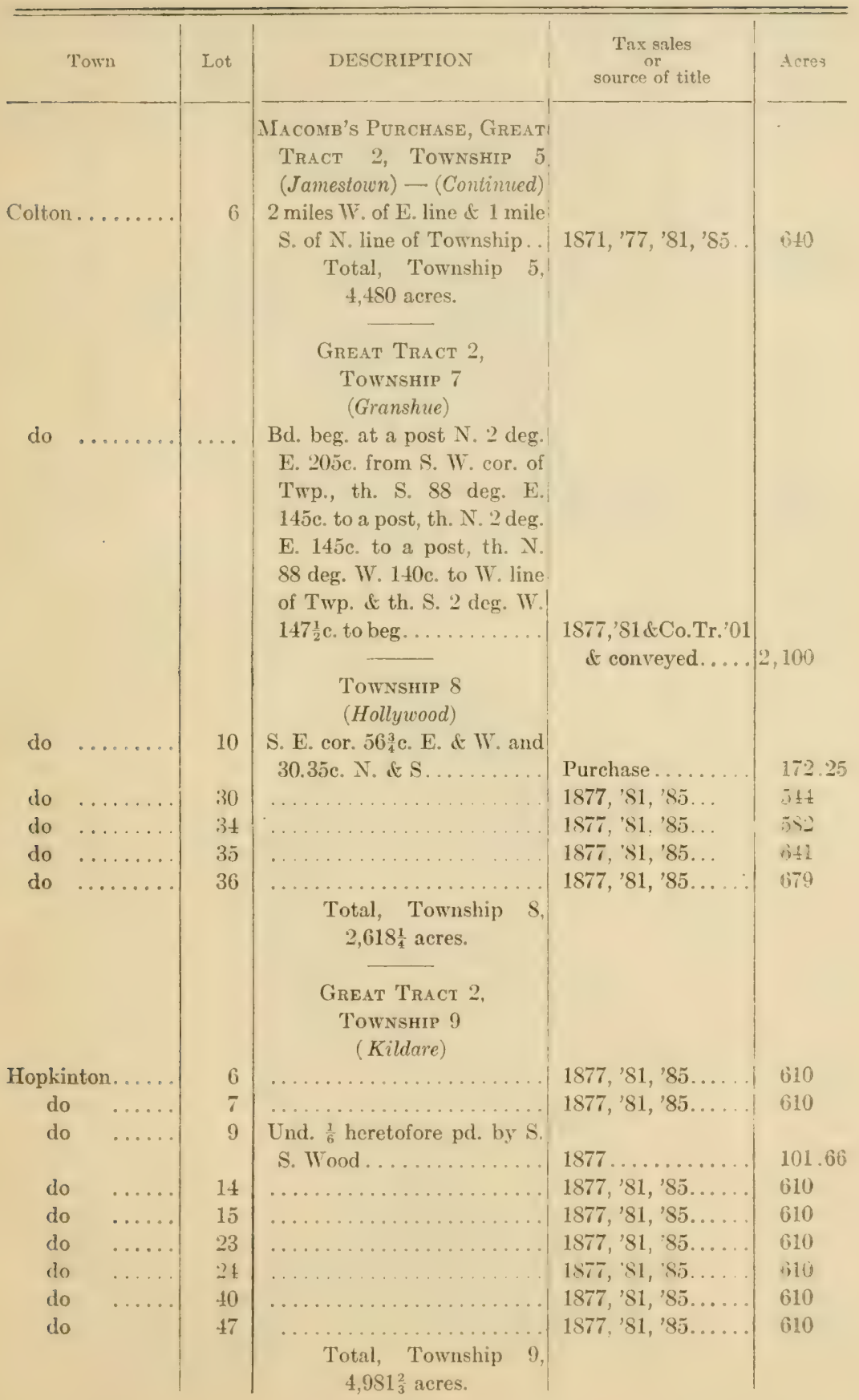


St. Lawrence Countr

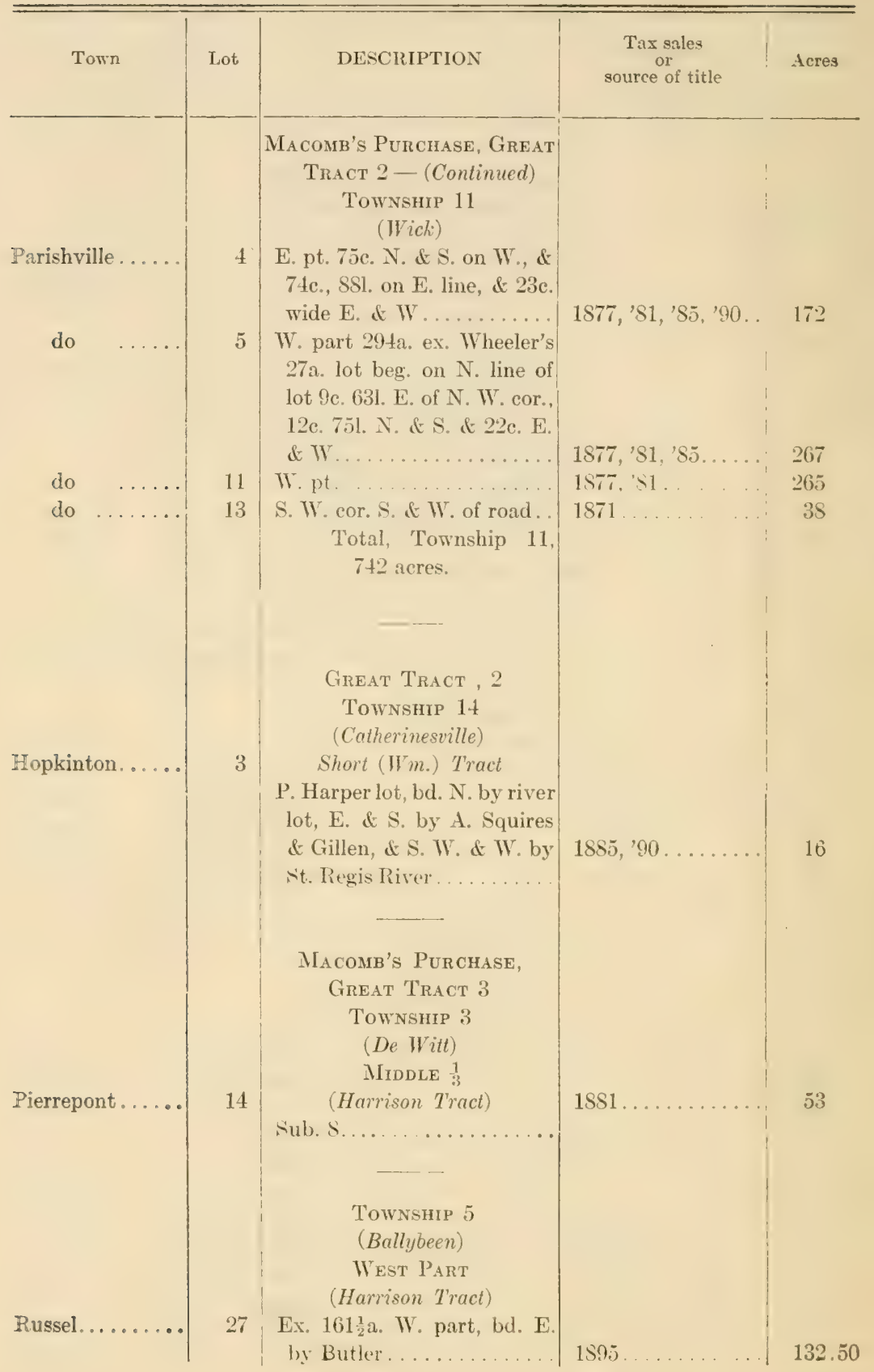


St. Lawrence County

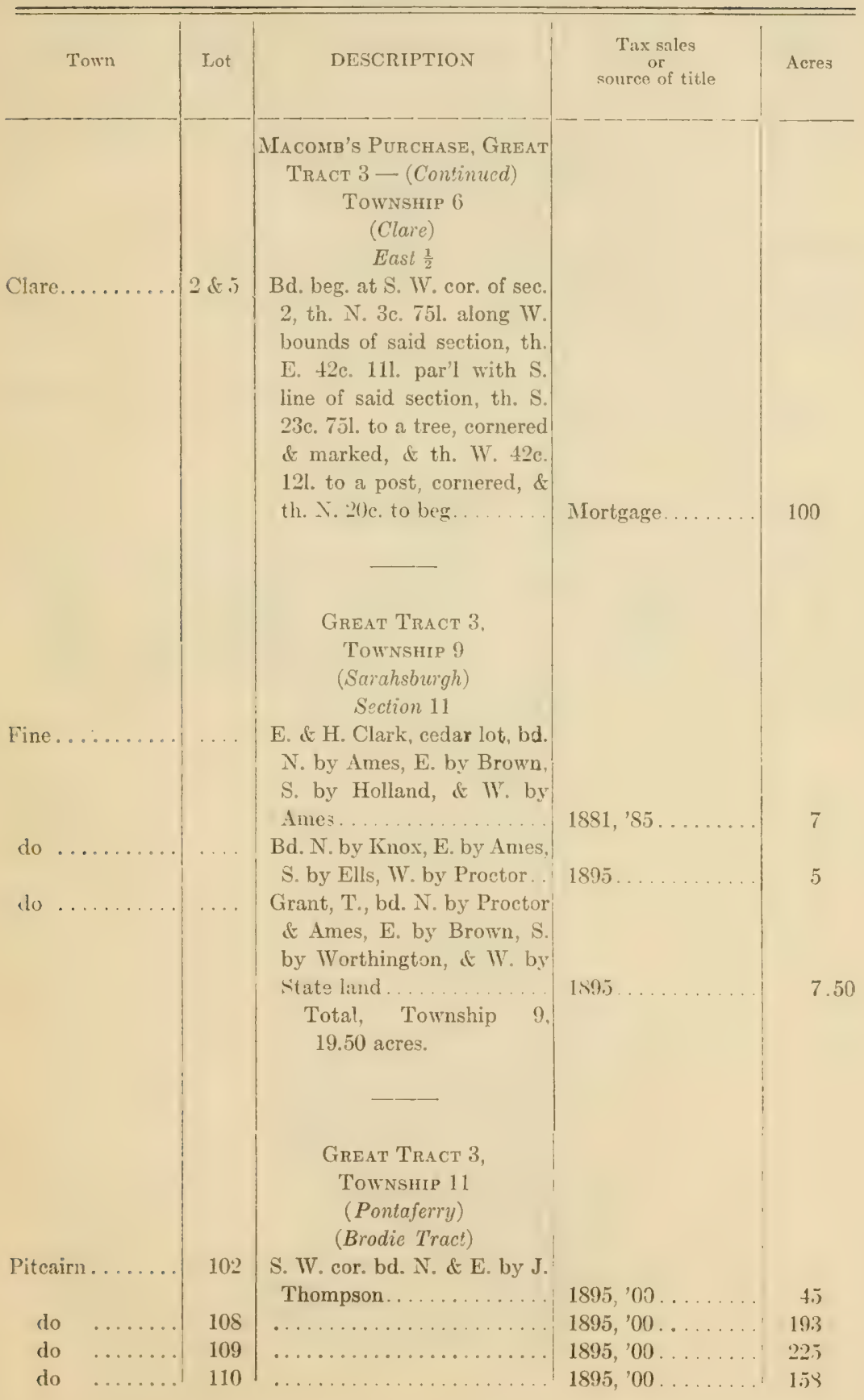


St. Latwence County

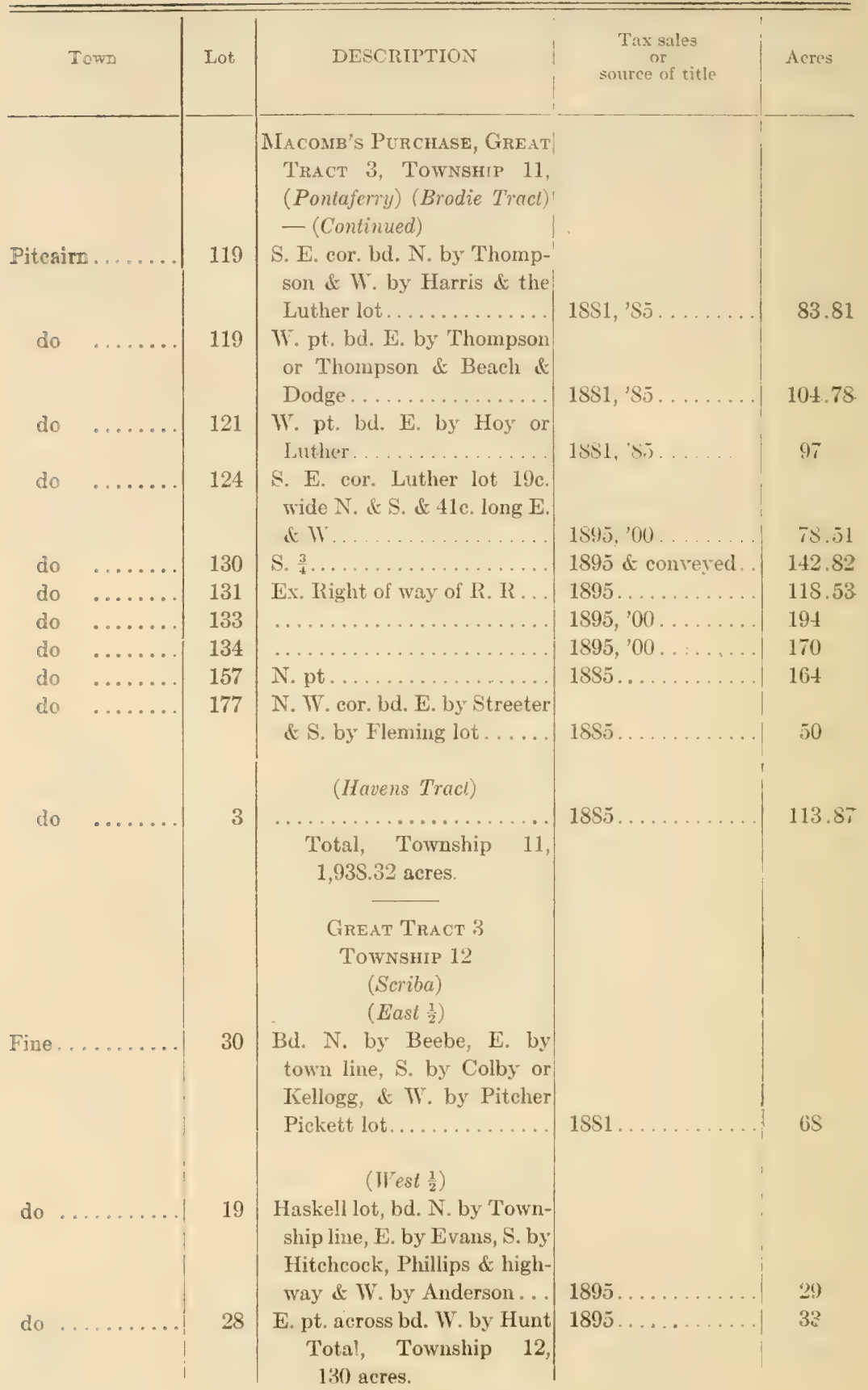


St. Lawrence County

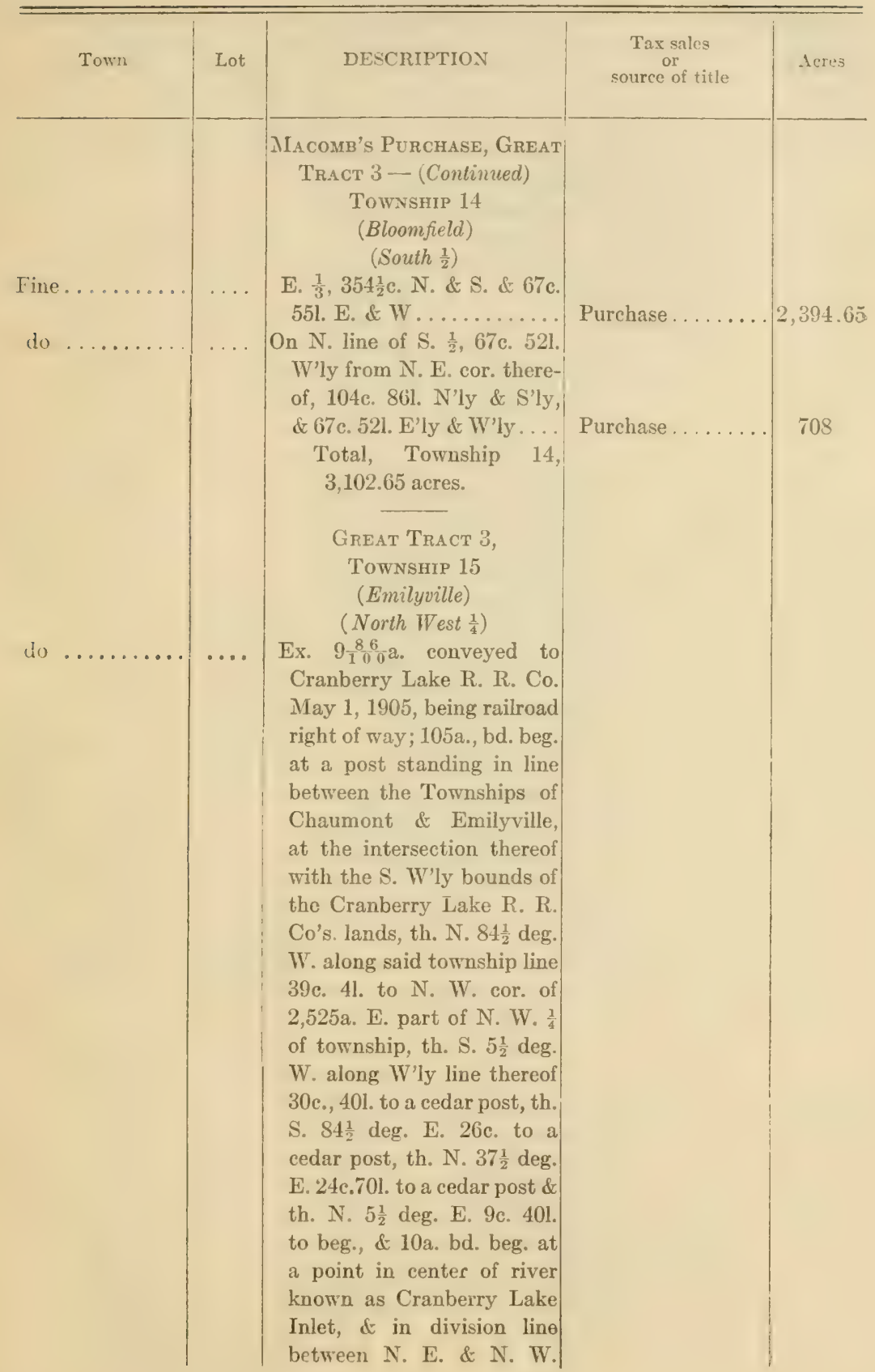


St. Lawrence County

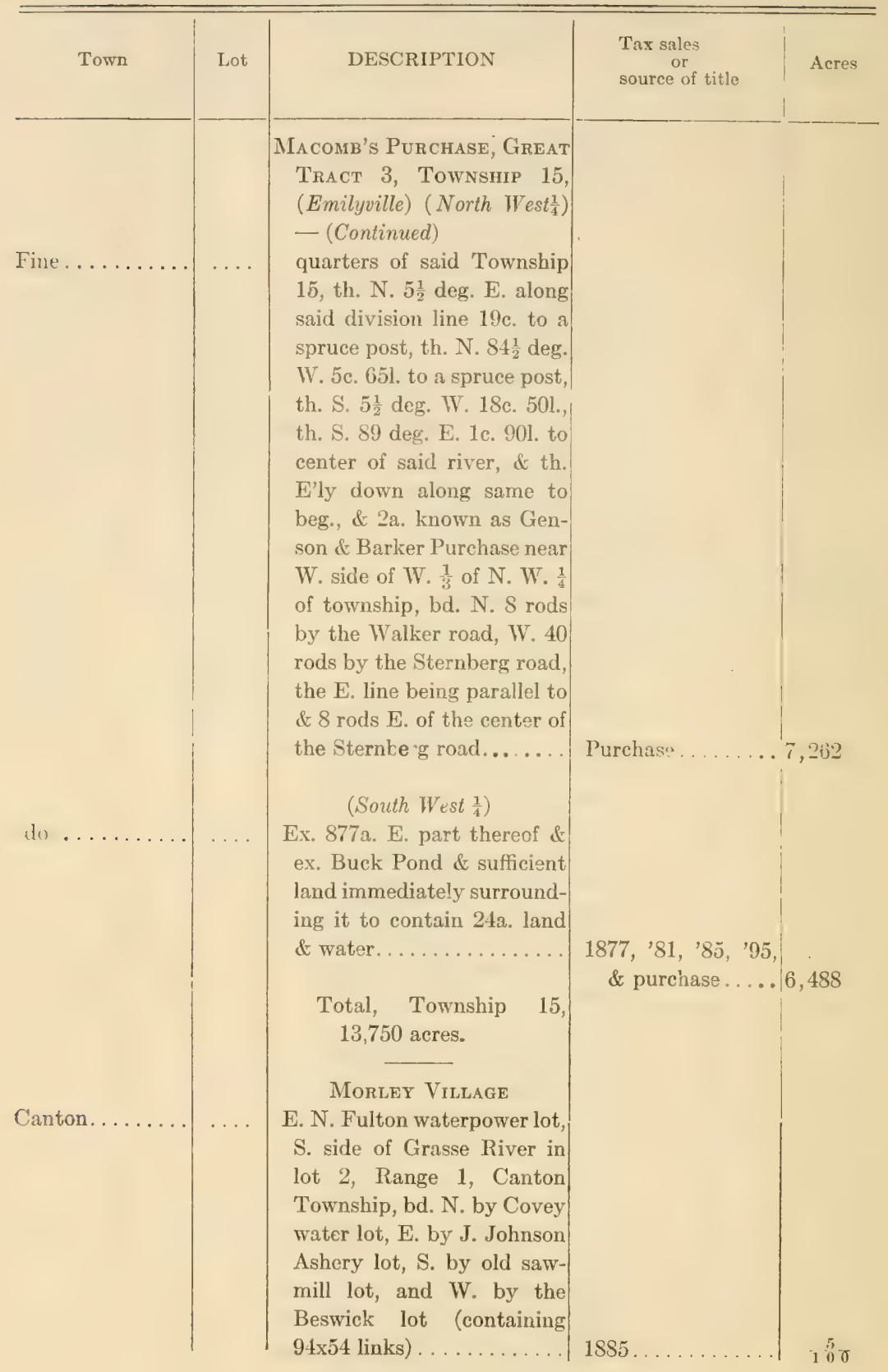


St. Lawrence County

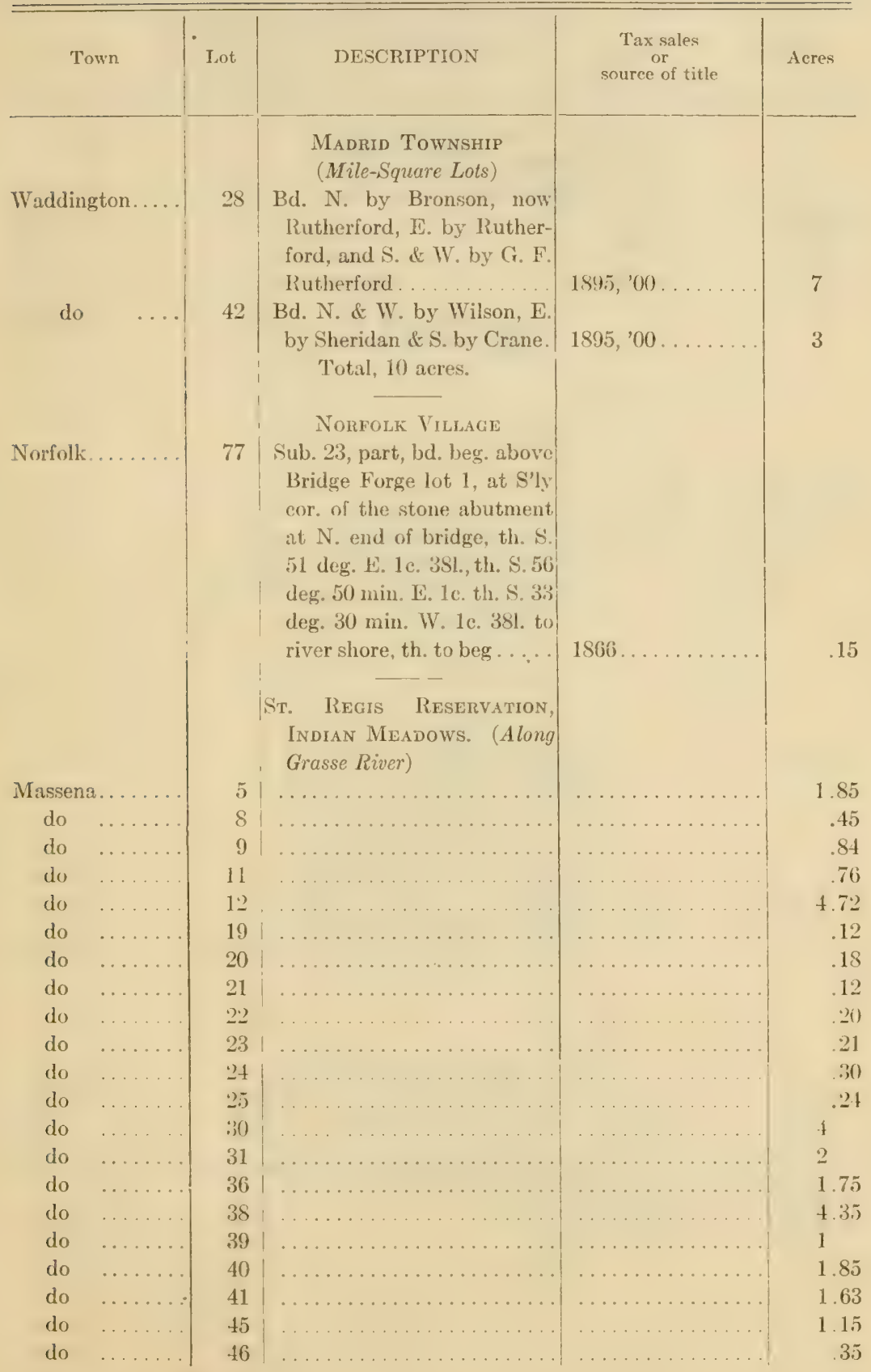


St. Lawkence County

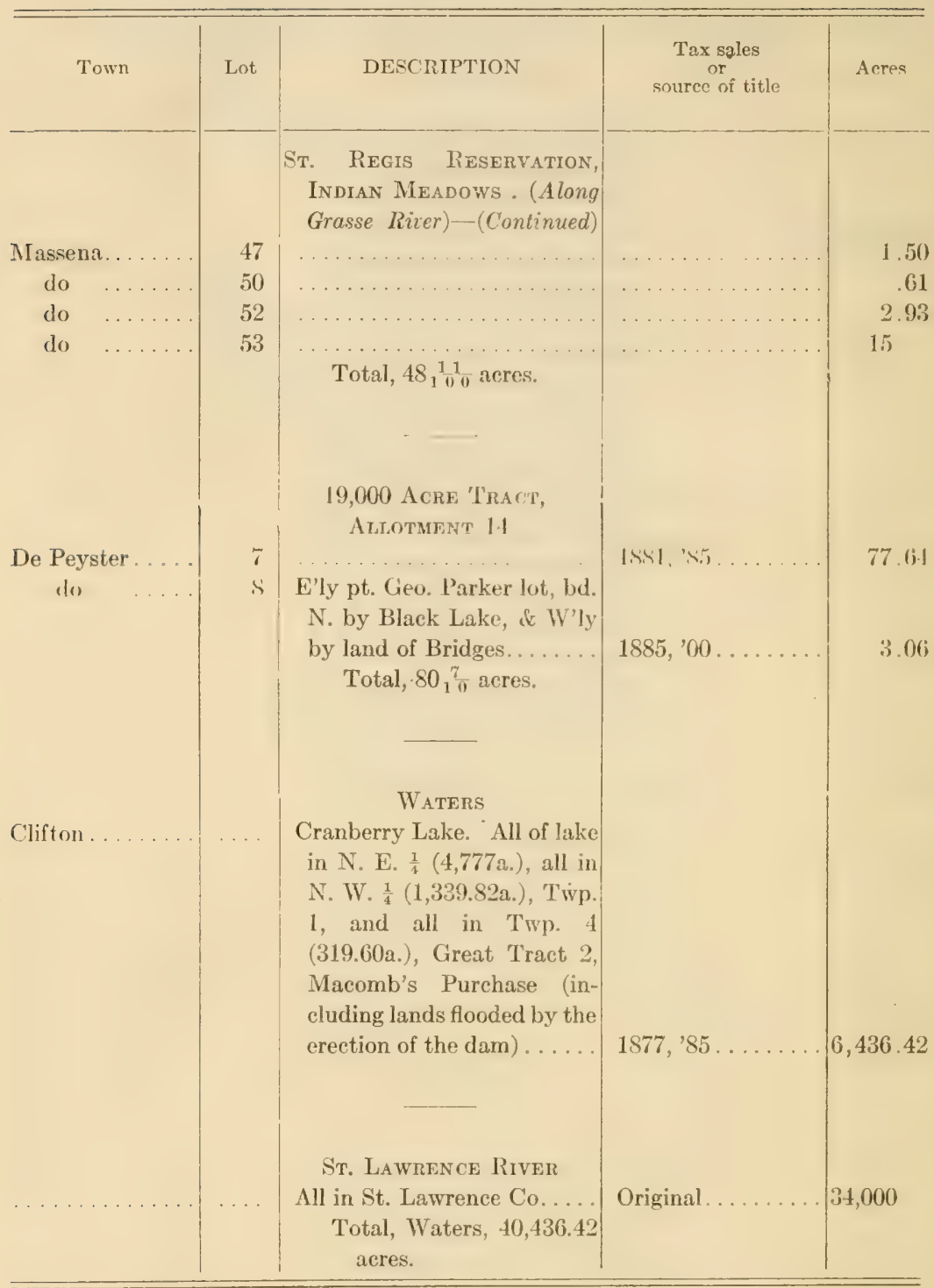

For other State land in St. Lawrence County see Appendix. 


\section{SARATOGA COUNTY}

(Total number of acres, 10,070.54)

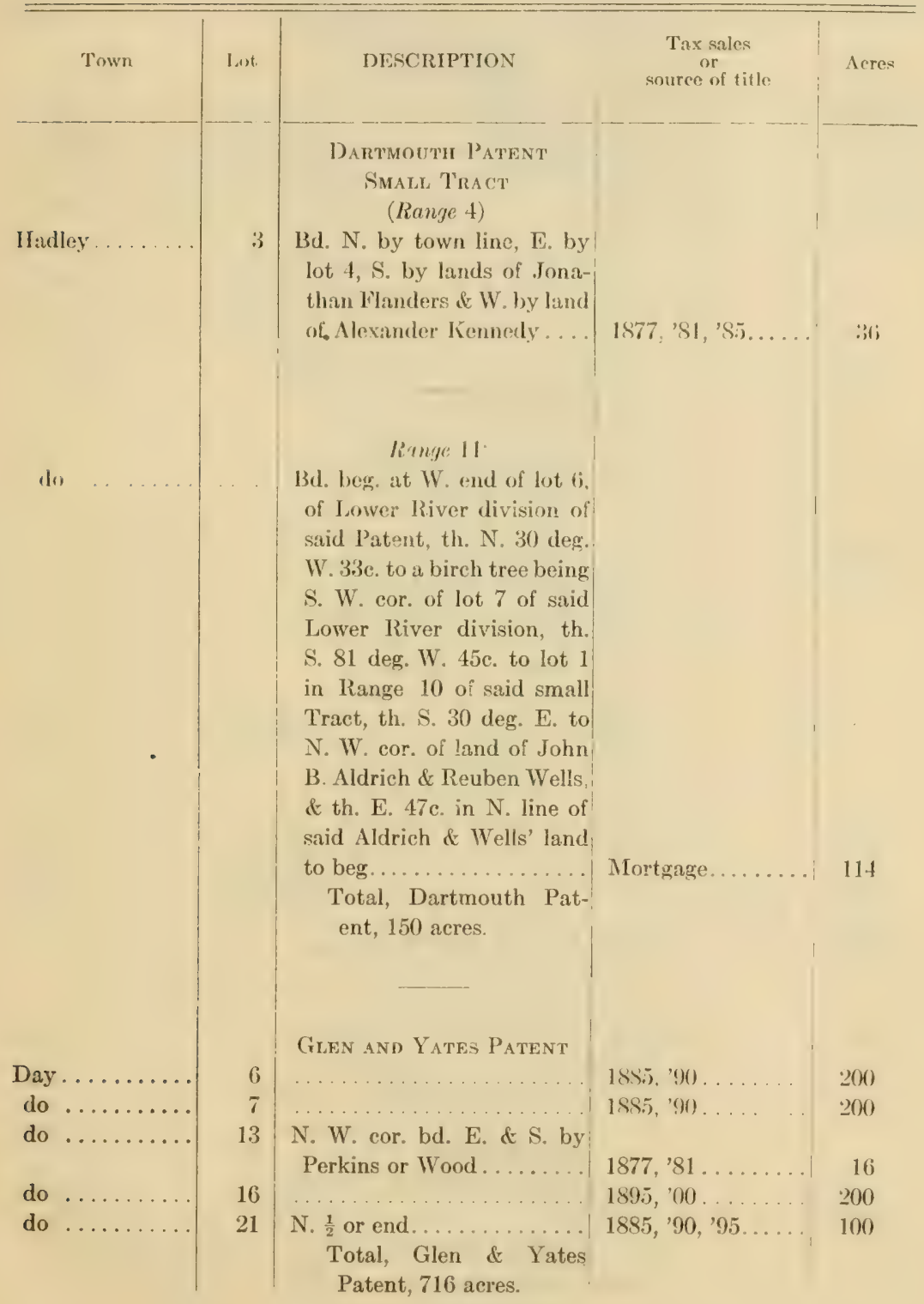


Saratoga Country

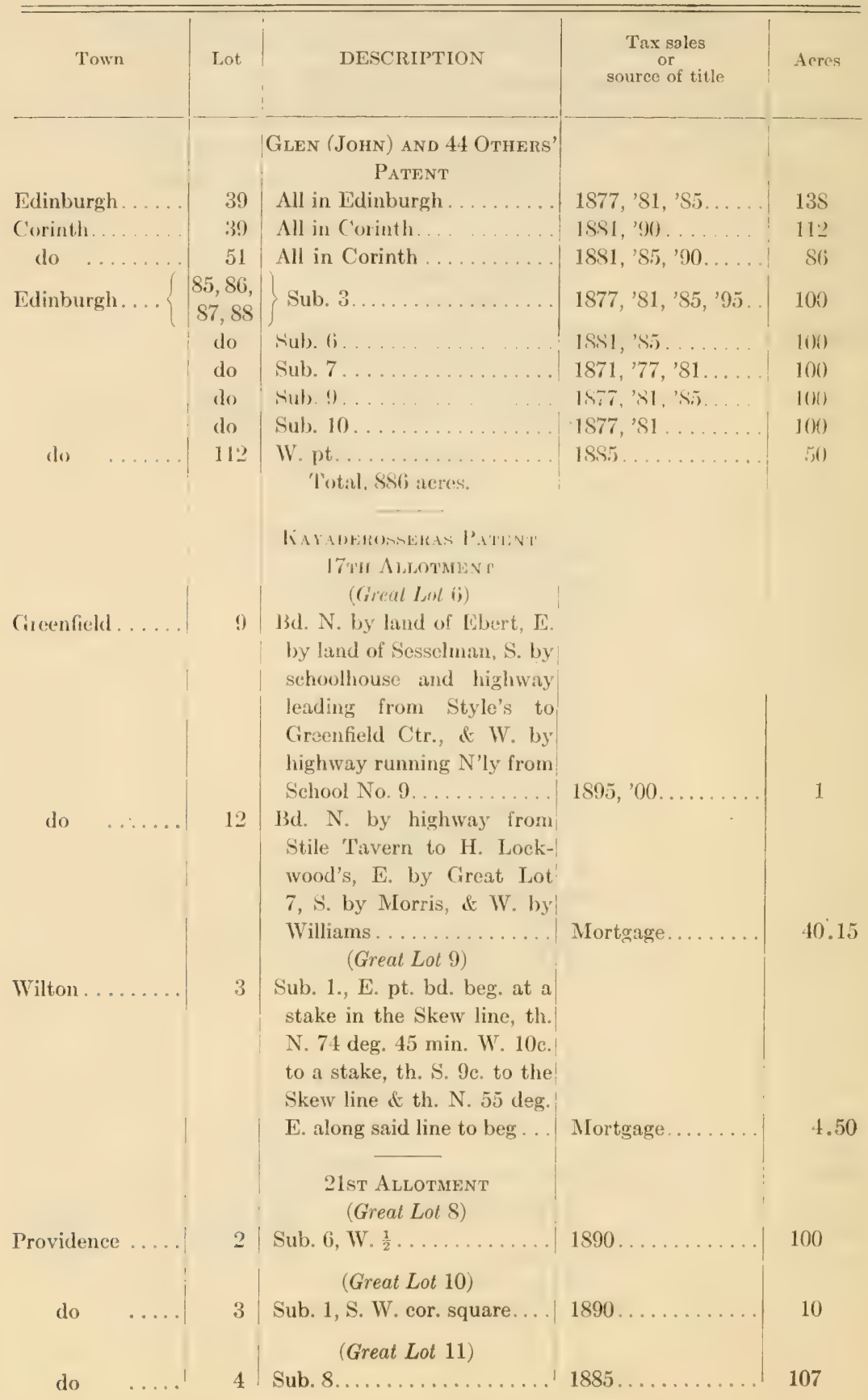




\section{Saratoga County}

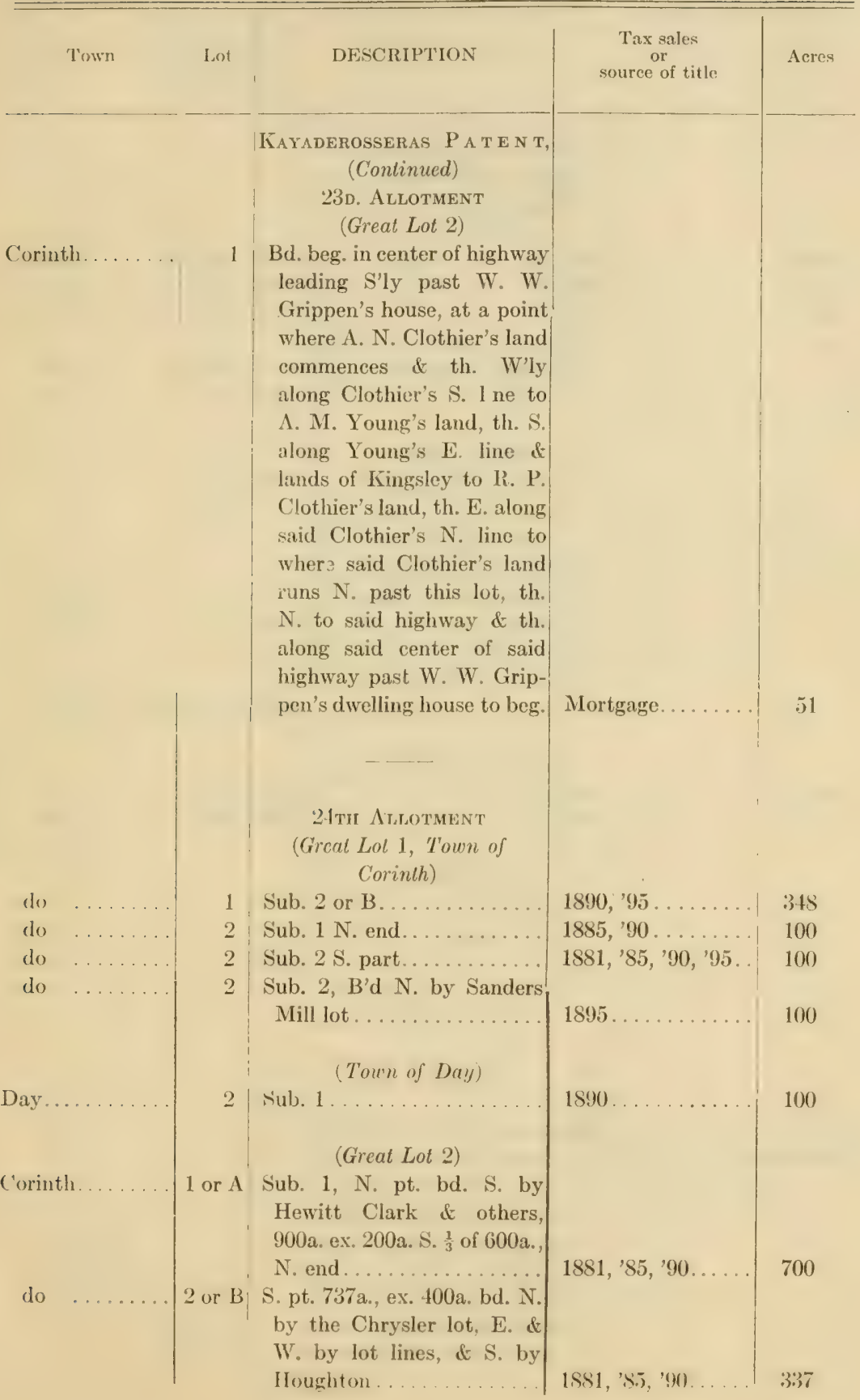


Saratoga County

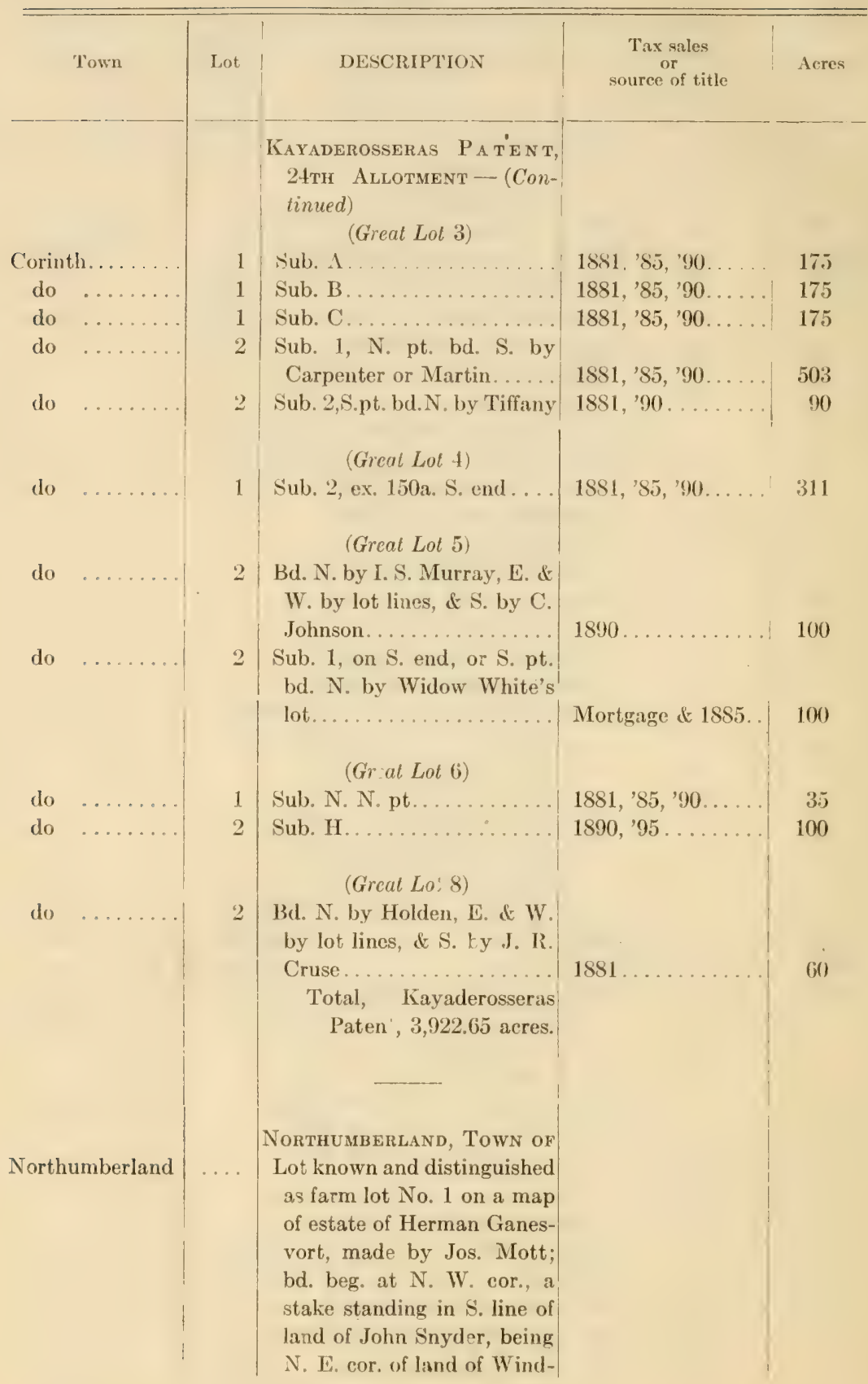




\section{Saratoga County}

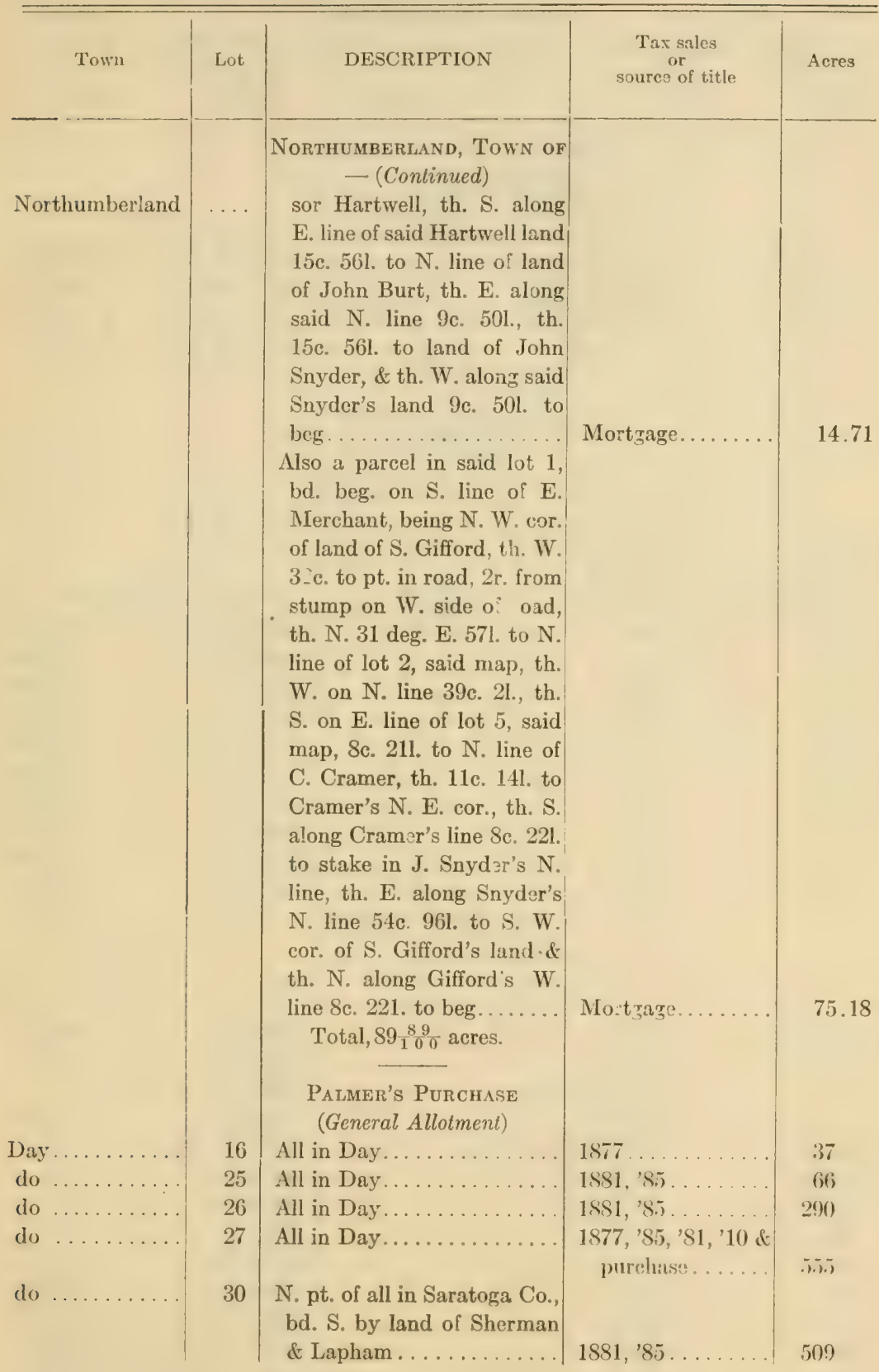


Saratoga County

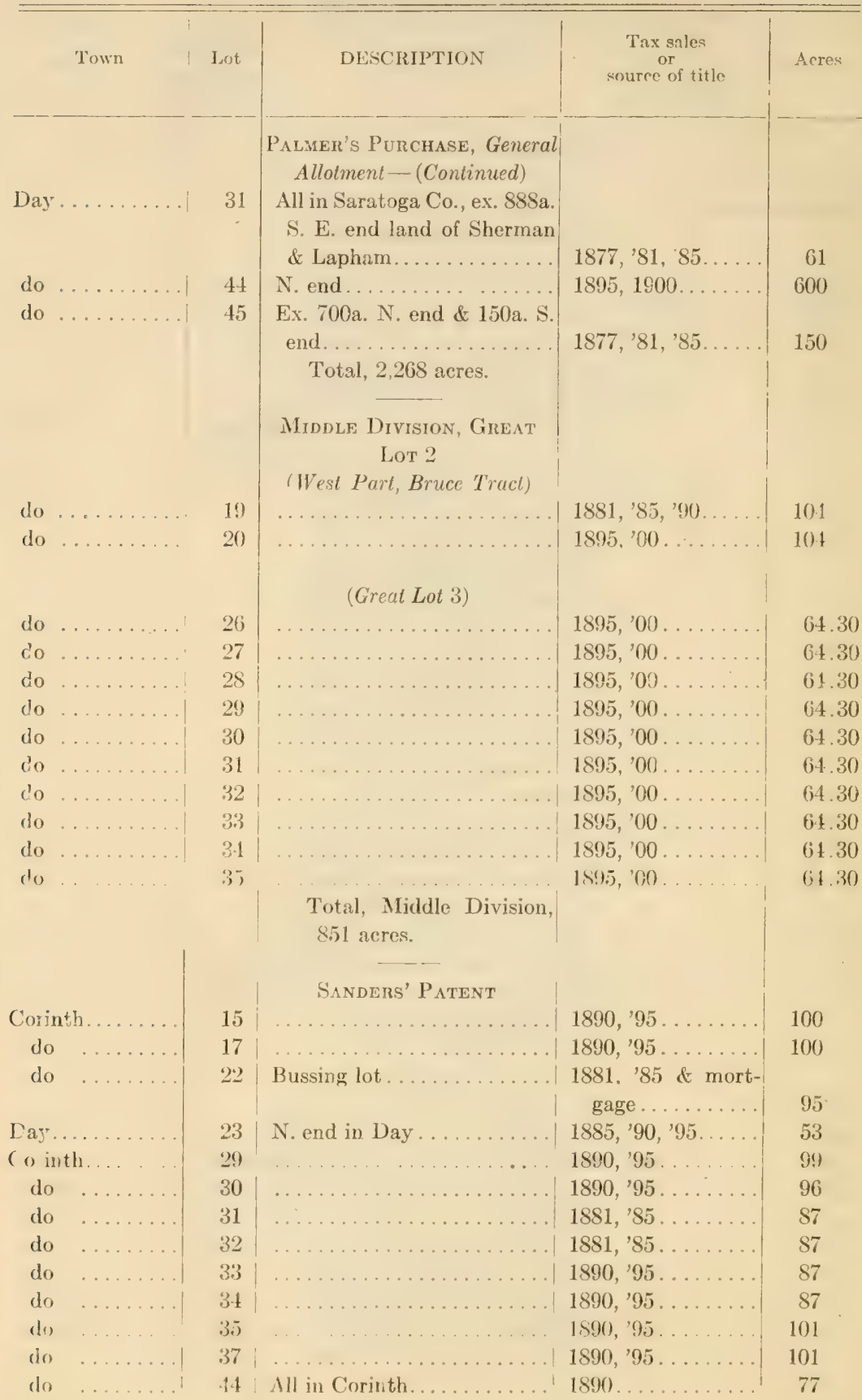


Saratoga County

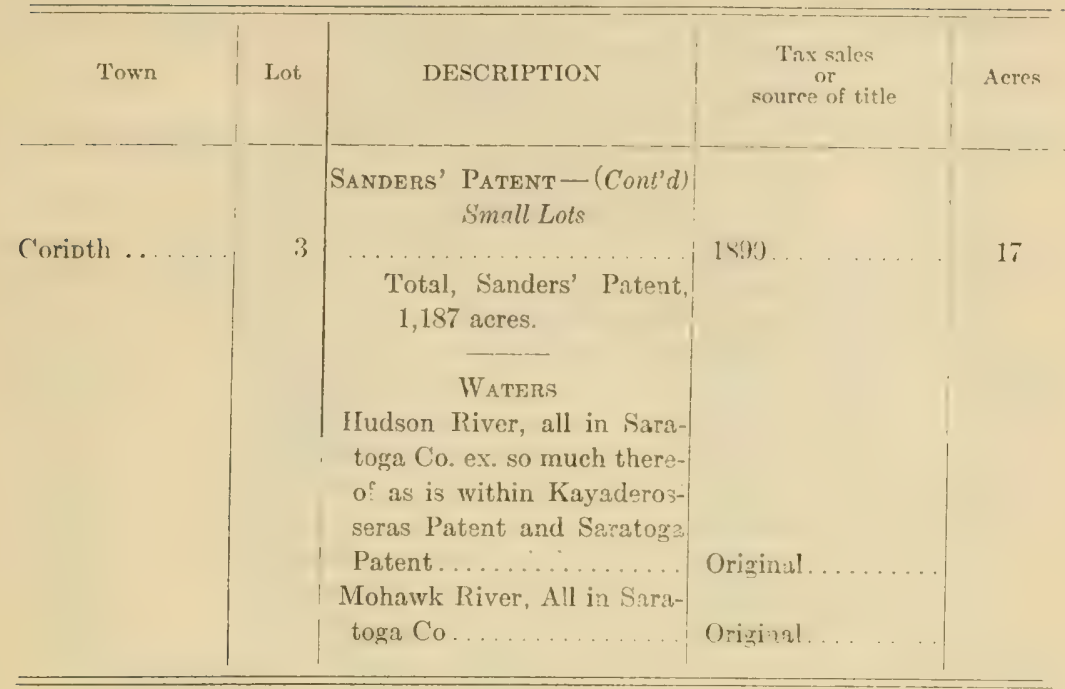

For other State lands in Saratoga County see Appendix. 


\section{WARREN COUNTY}

(Total number of acres, 155,150.32)

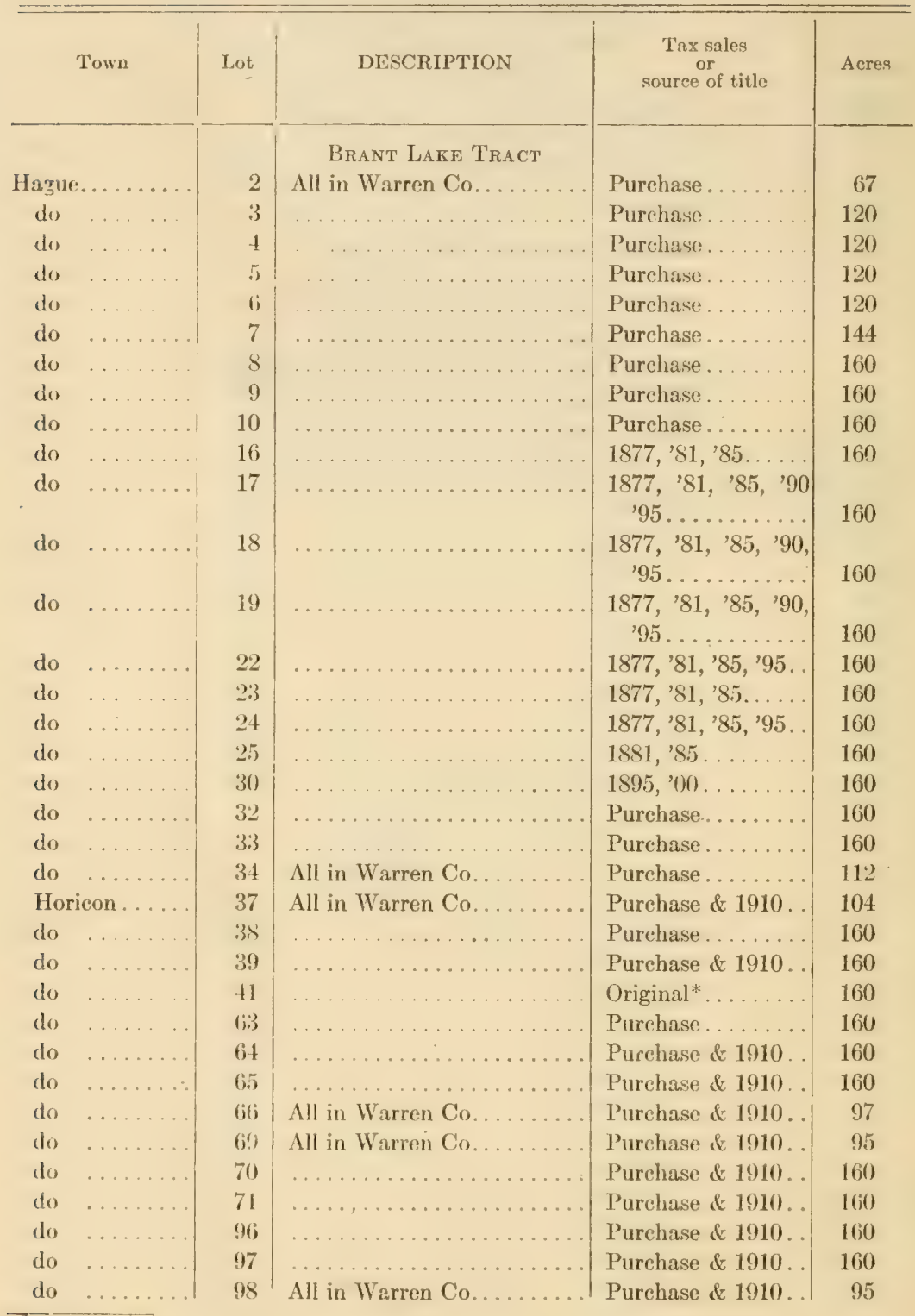


Warren County

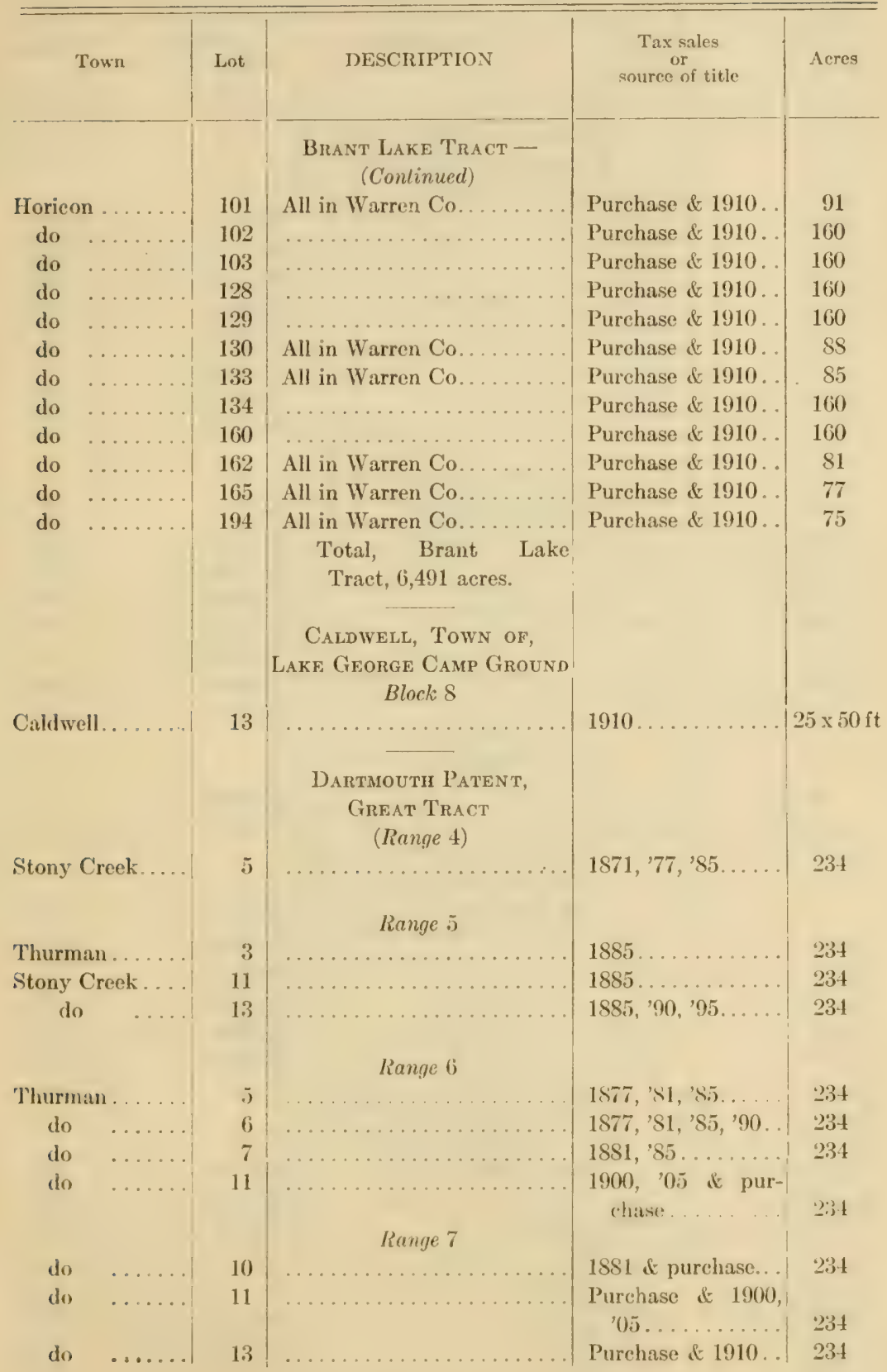


WARREN COUNTY

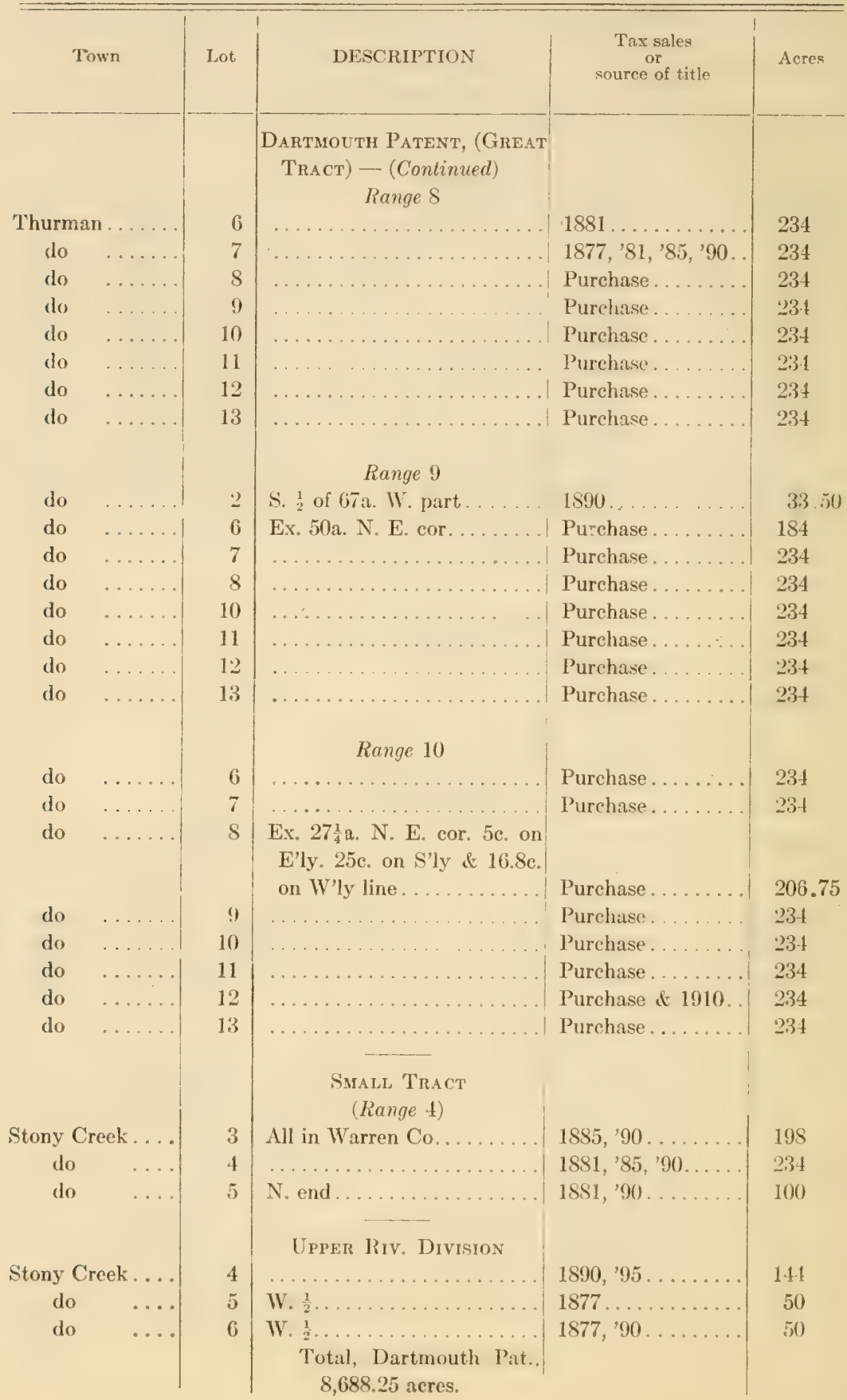




\section{Warren County}

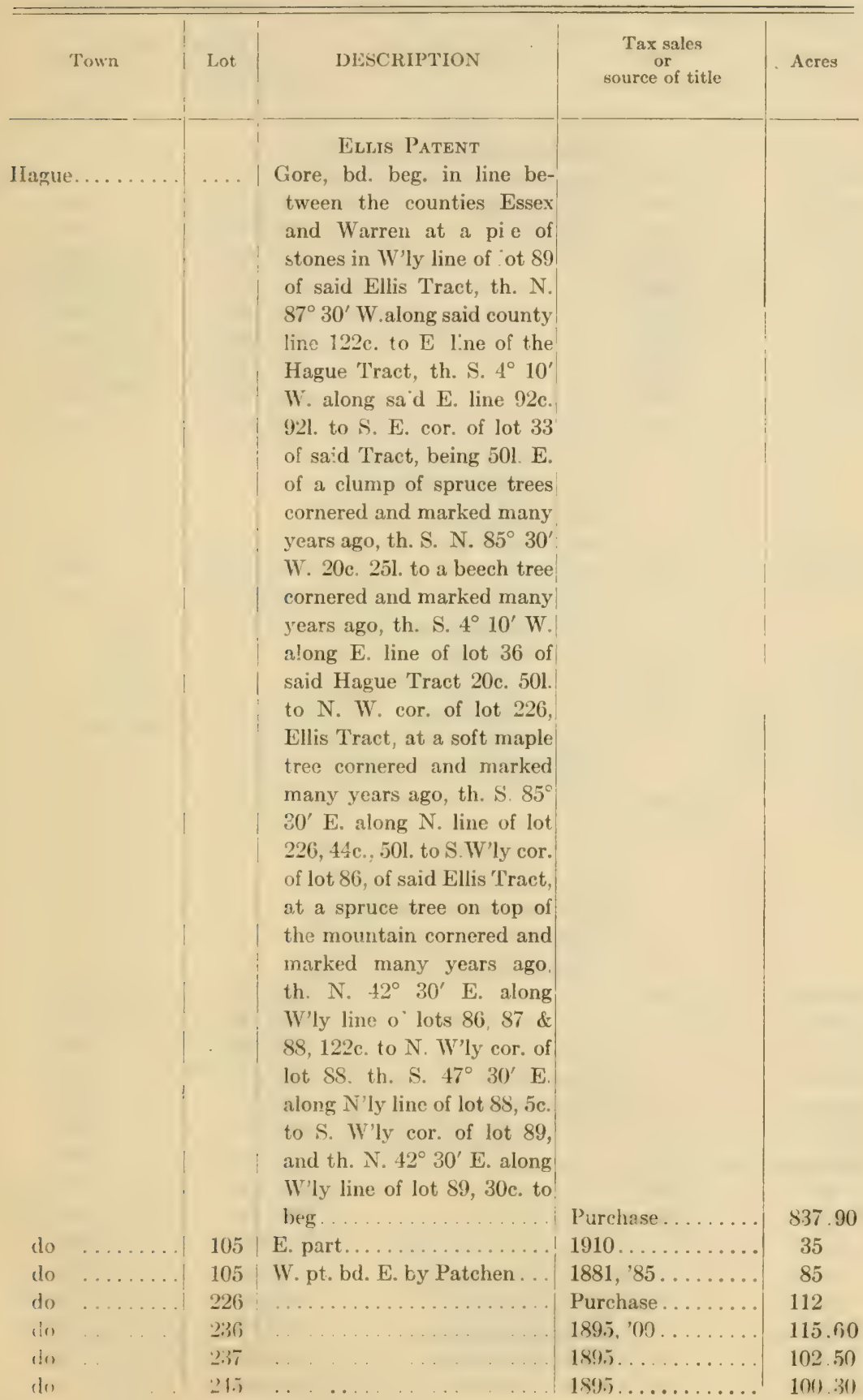


Warren County

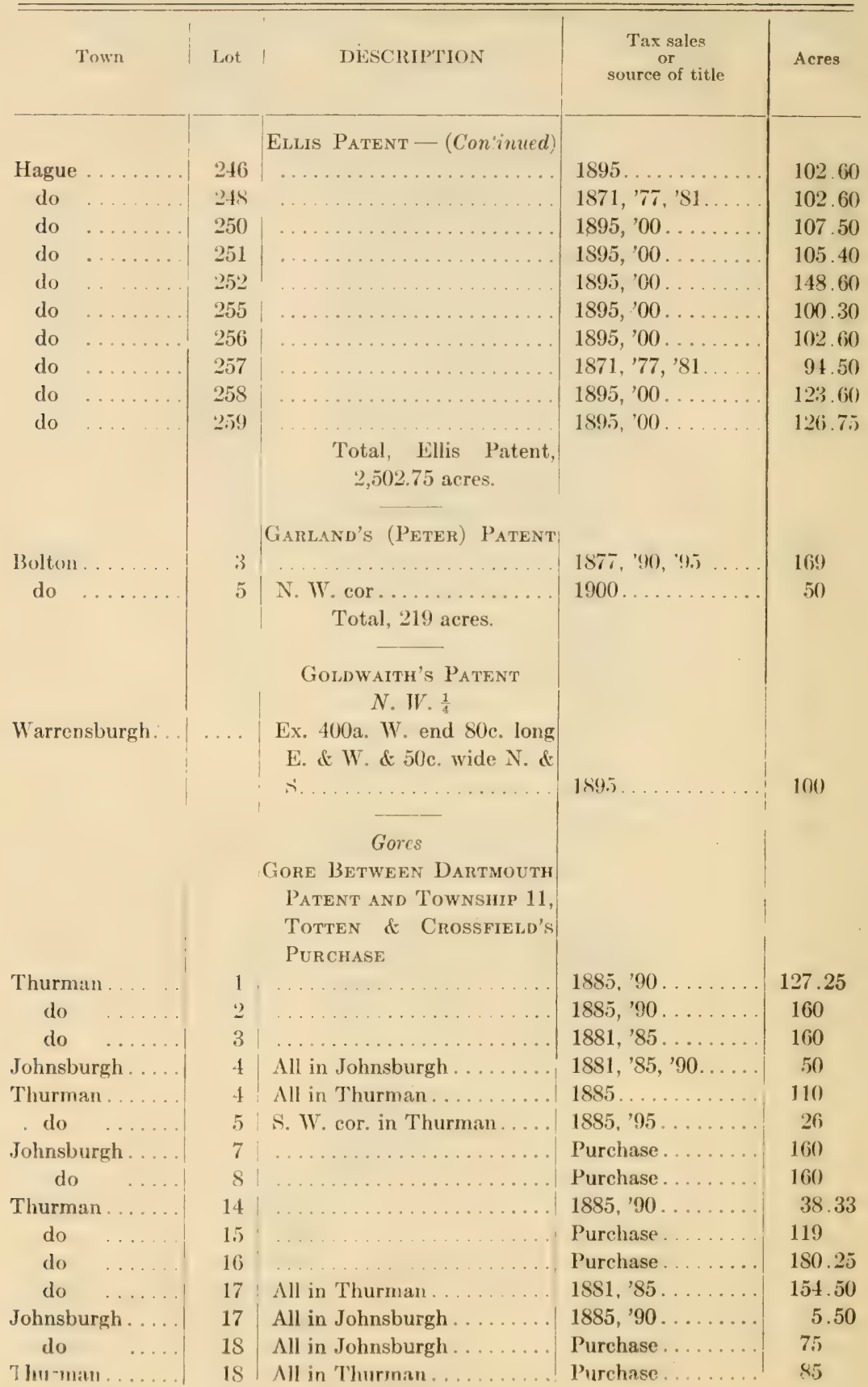


Warren County

\begin{tabular}{|c|c|c|c|c|}
\hline Town & Lot & DESCRIPTION & $\begin{array}{l}\text { Tax sales } \\
\text { or } \\
\text { source of title }\end{array}$ & Acres \\
\hline & & $\begin{array}{c}\text { Gores } \\
\text { Gore Between Dartmouth } \\
\text { Patent and Township 11, } \\
\text { Totten \& Crossfield's } \\
\text { Purchase - Continued })\end{array}$ & & \\
\hline Thurman... & 19 & All in Thurman.......... & $\begin{array}{c}\text { Original \& } 1905 \\
10^{*} \ldots \ldots \ldots \ldots\end{array}$ & 8 \\
\hline Johnsburgh . & 19 & All in Johnsburgh. & Original \& $1905^{*}$. & 152 \\
\hline & 23 & $\ldots \ldots \ldots \ldots \ldots \ldots \ldots \ldots$ & 1895 . & 160 \\
\hline Thurman... & 26 & $\ldots \ldots \ldots \ldots \ldots \ldots \ldots \ldots$ & Purchase....... & 90.32 \\
\hline do & 27 & $\begin{array}{l}\text { Ex. } 56.08 \mathrm{a} . \text { beg. at point on } \\
\text { E'ly line of lot on shore of } \\
\text { Mill Creek Pond at high } \\
\text { water mark, th. S. } 30 \text { deg. } \\
\text { E. to N. line of Dartmouth } \\
\text { Pat., th. W'ly along said N. } \\
\text { line } 27 \frac{3}{4} \text { c. th. N. } 30 \text { deg. W. } \\
9 \frac{2}{10} \text { c. to high water mark on. } \\
\text { shore of Mill Creek Pond \& } \\
\text { th. E'ly along pond to beg. }\end{array}$ & Purchass... & 106.25 \\
\hline do & 28 & All in Thurman....... & $1881 \ldots$ & 142 \\
\hline Johnsburgh . & 28 & All in Johnsburgh. ........ & $1881 \ldots \ldots$ & 18 \\
\hline do & 33 & $\begin{array}{l}\text { All in Johnsburgh } 935 \text { a., cx. } \\
700 \text { a. N. Pt. bd. S. by line } \\
\text { par'l to town line.......... }\end{array}$ & 1890, '95. & 235 \\
\hline Thurman.. & 35 & Total, $2,594.73$ acres. & Purchase... & 72.33 \\
\hline & & $\begin{array}{c}\text { Gore Between Townships } \\
12 \text { and 14, Totten \& } \\
\text { Crossfield's Purchase. }\end{array}$ & & \\
\hline Johnsburgh . & 196 & $\mid \ldots \ldots \ldots \ldots \cdots \cdots \cdots$ & $1885 \ldots$ & 106 \\
\hline do & 7 & $\begin{array}{c}\text { Gore Between Townships } \\
29 \text { and 31, Totten \& } \\
\text { Crossfields' Purchase. } \\
\text { All in Warren Co........... }\end{array}$ & Conveyed. & 38.50 \\
\hline do & 8 & All in Warren Co......... & $1885 \&$ conveyed. & 176 \\
\hline do & 9 & $\ldots \ldots \ldots \ldots \ldots \ldots \ldots \ldots$ & $1885,{ }^{\prime} 05 \ldots \ldots \ldots$ & 187 \\
\hline do & 10 & \begin{tabular}{|} 
Total, Gore between \\
Townships 29 and 31 \\
Totten \& Crossfield's \\
Purchase, 588.50 acres.
\end{tabular} & $1881 \ldots \ldots \ldots$ & 187 \\
\hline
\end{tabular}




\section{Warren County}

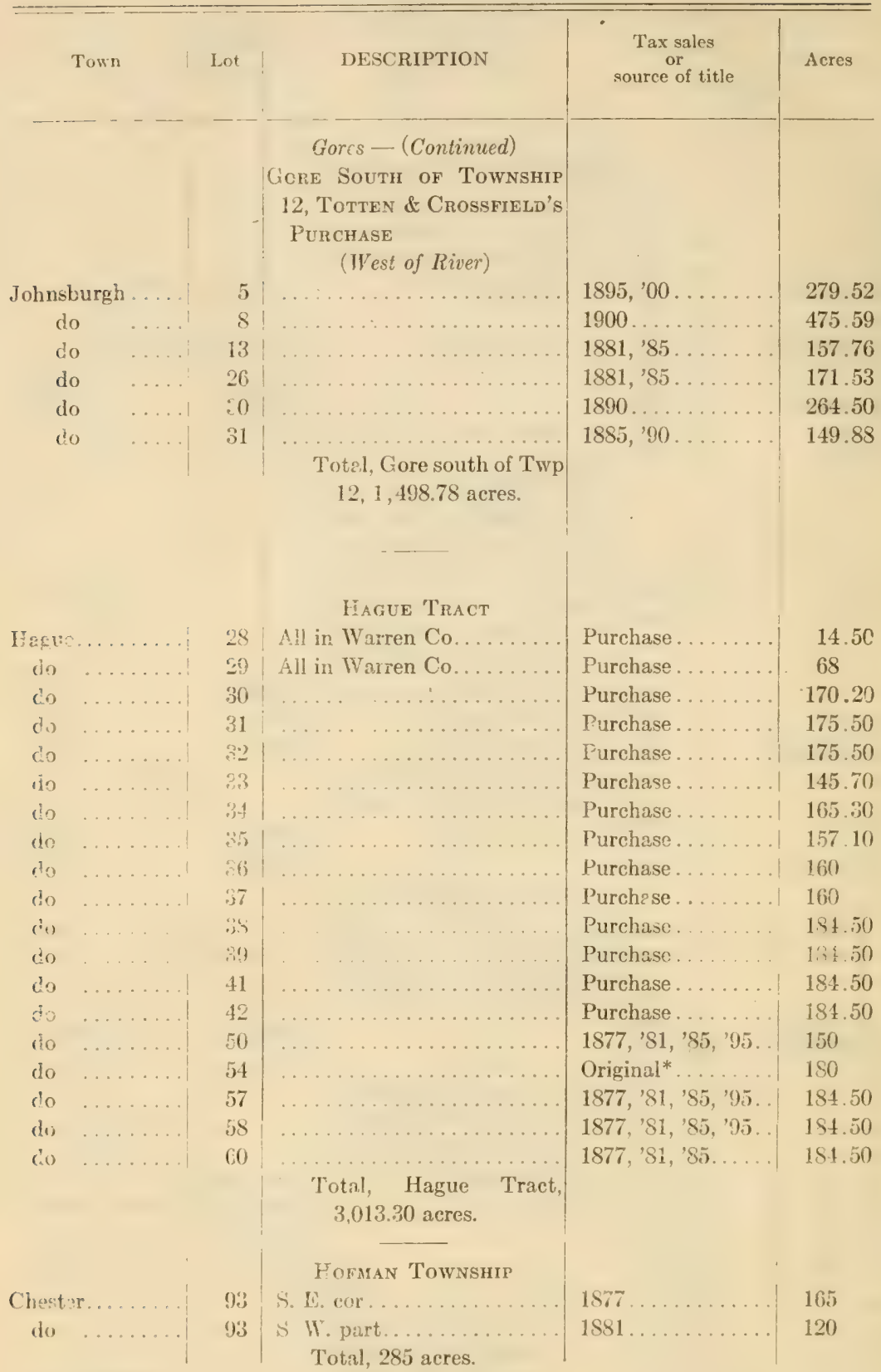

* Hecale. 


\section{W ARREN Countr}

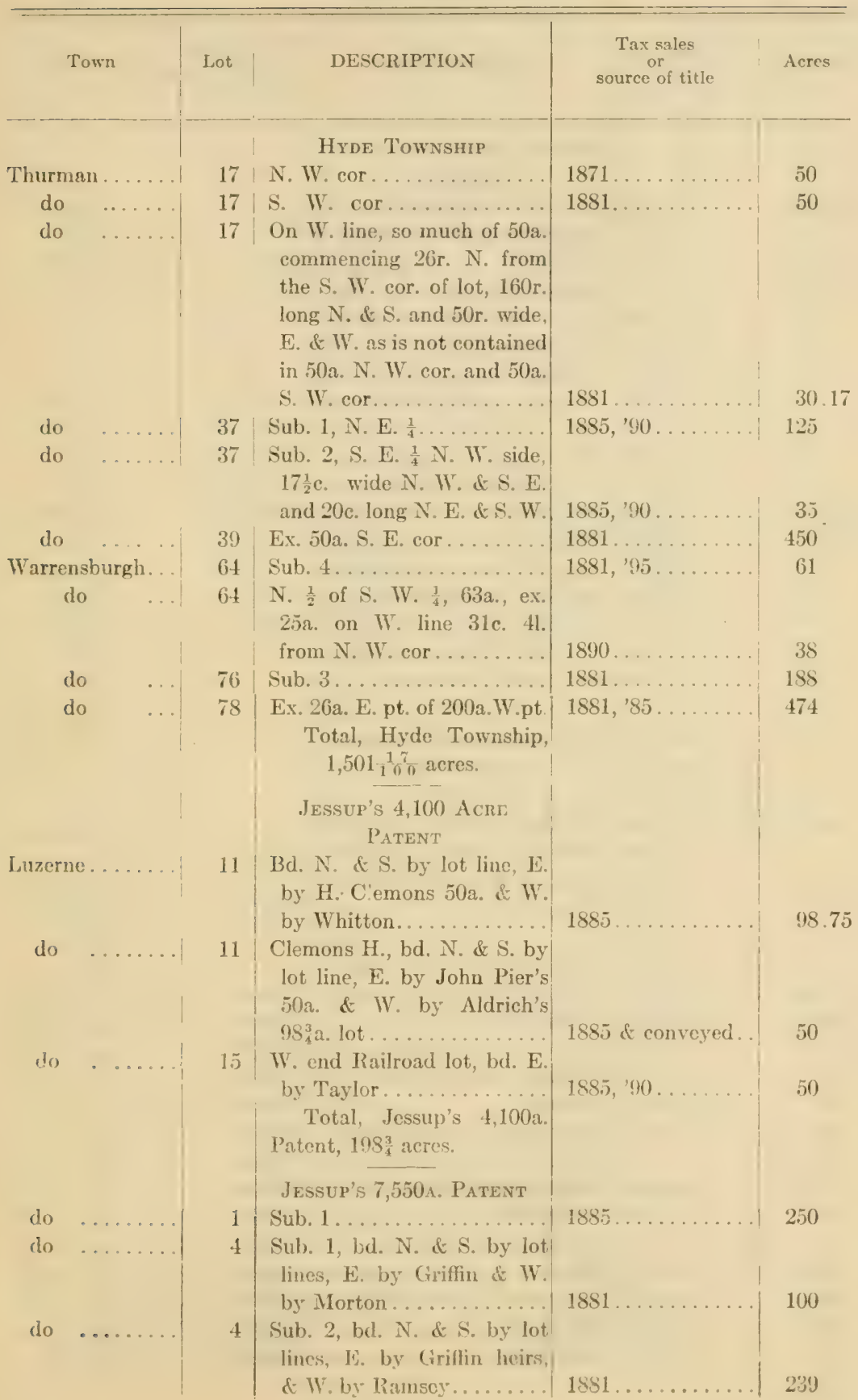




\section{Warren County}

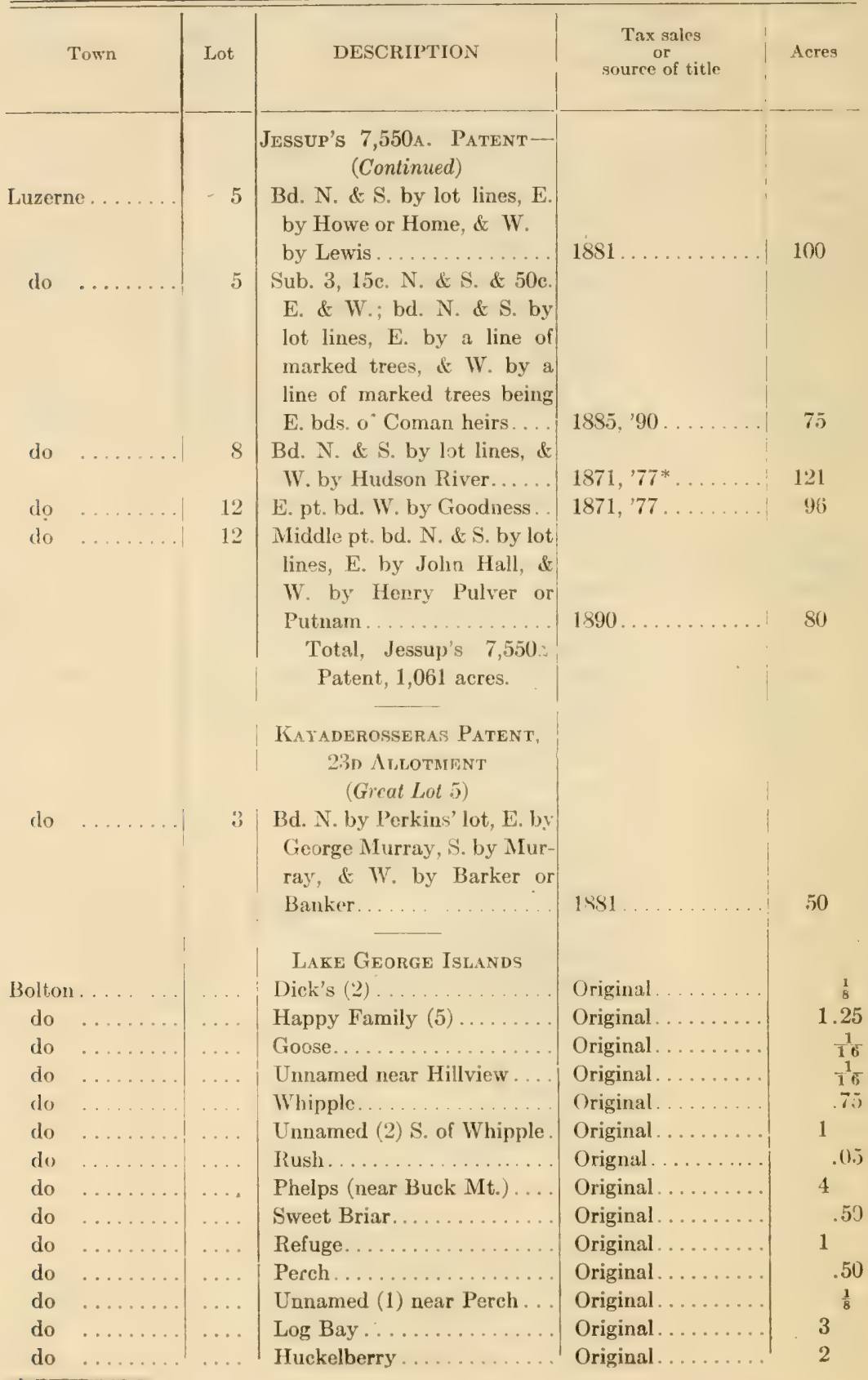

- Regale. 


\section{WARREN CoUnty}

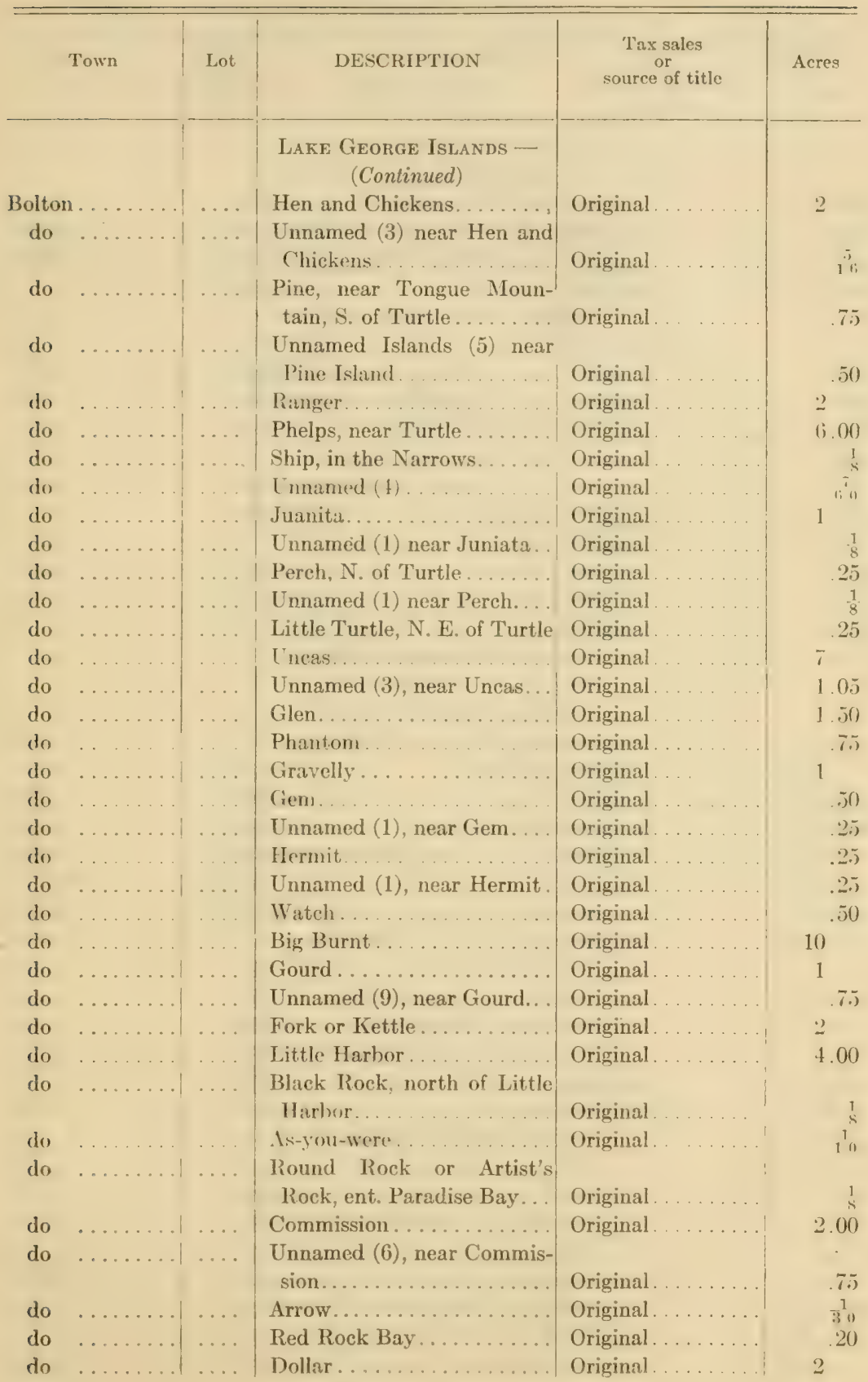




\section{WARREN COUNTY}

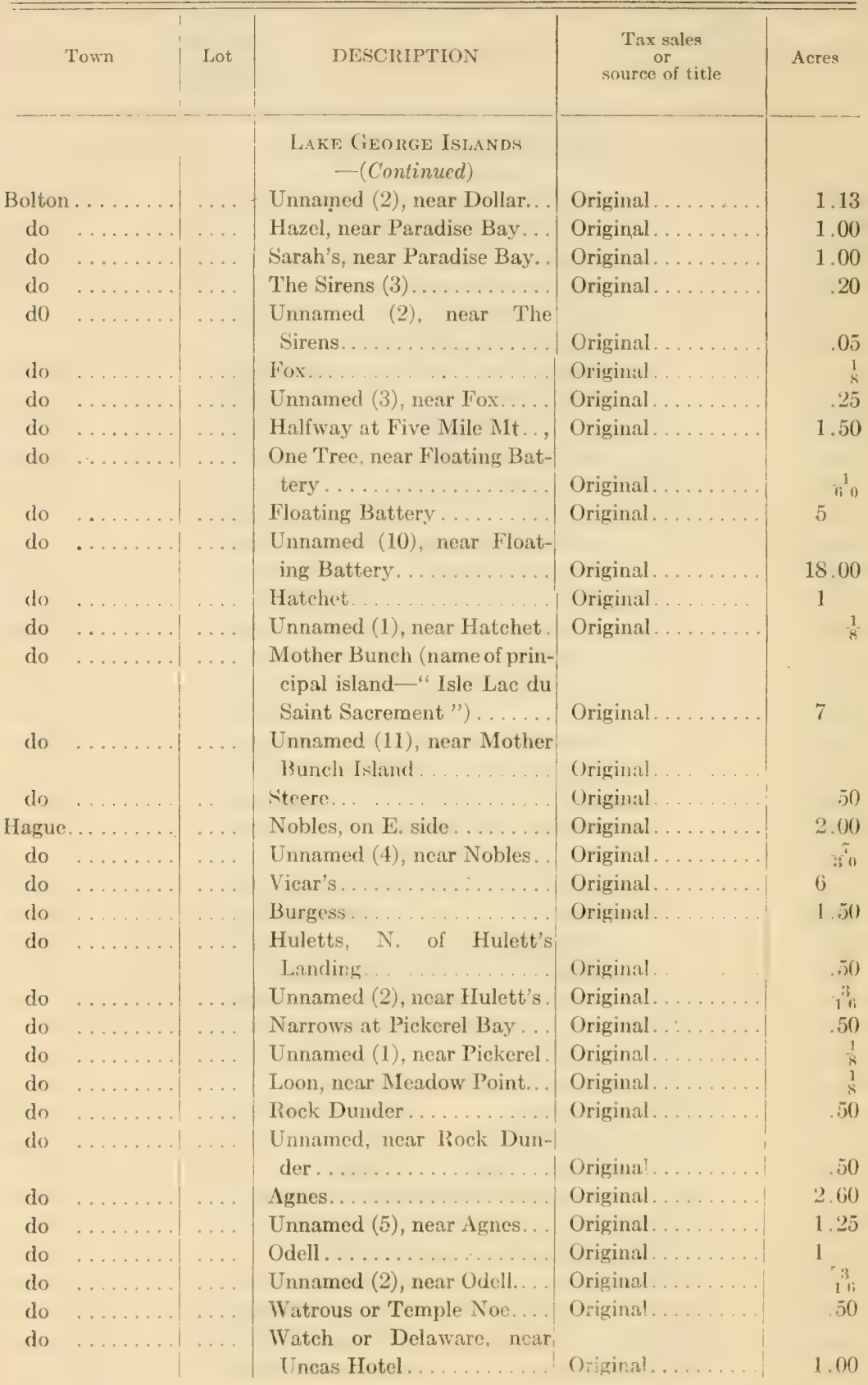


Conservation Commishmon.

Wairren County

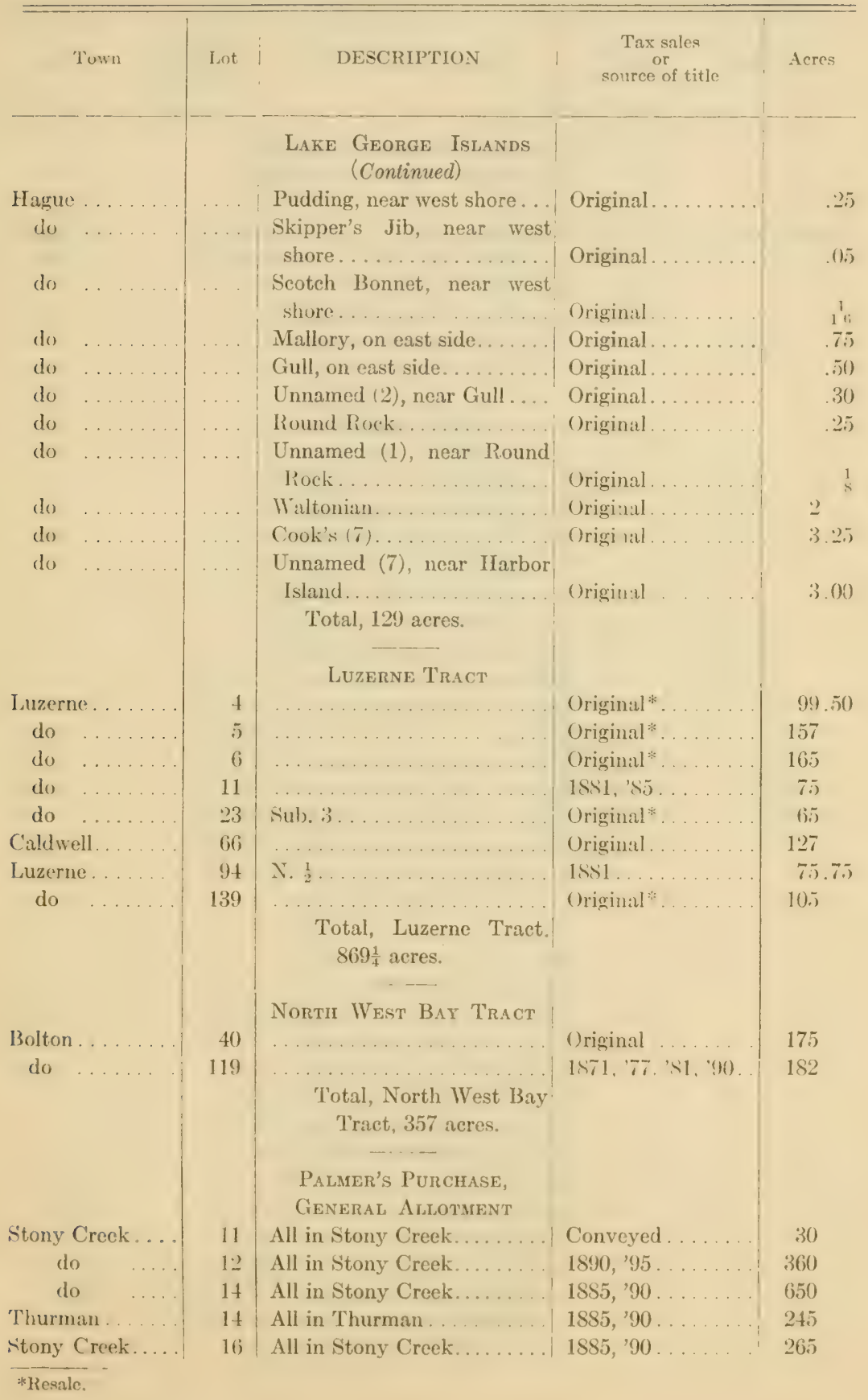


WARREn County

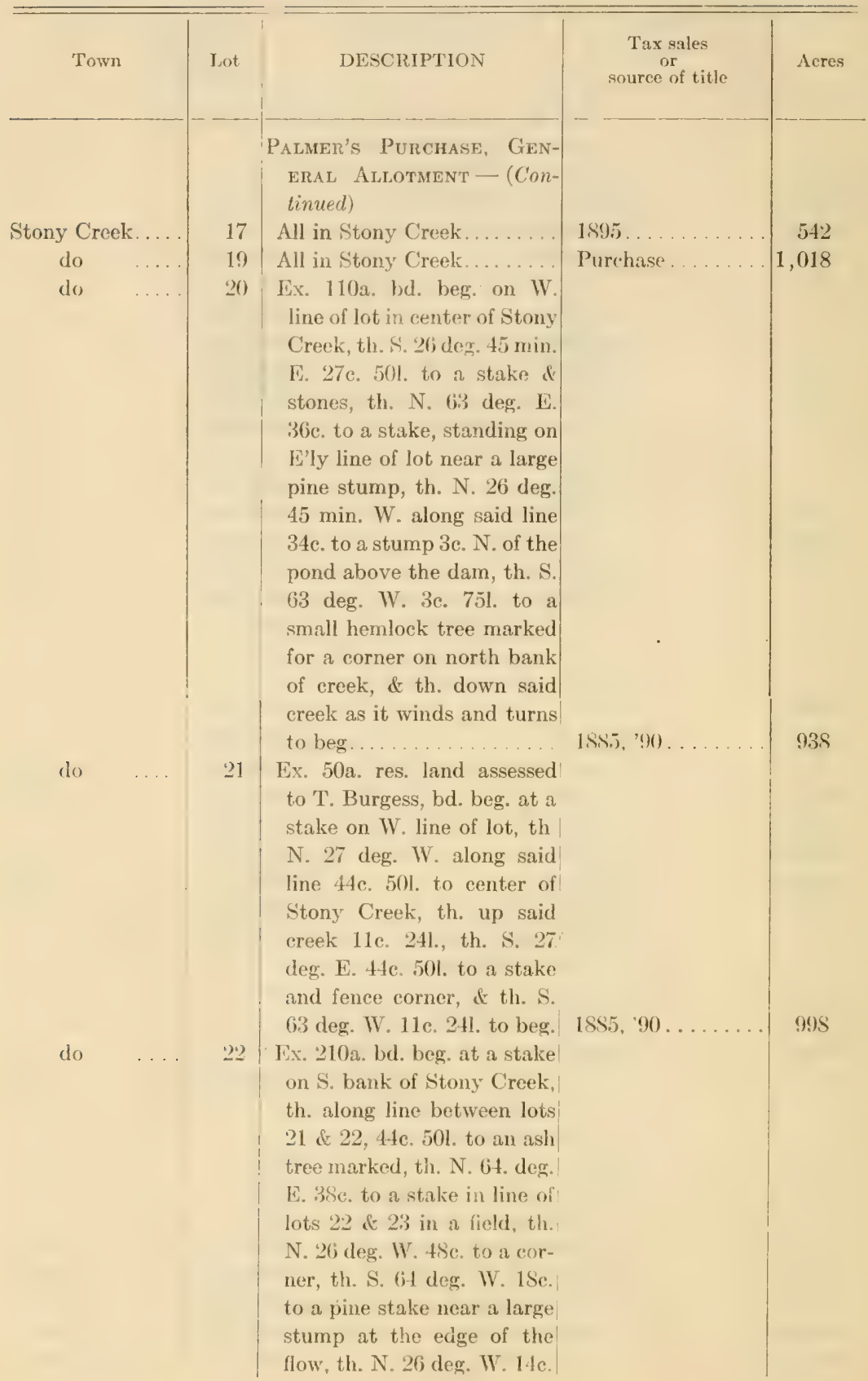




\section{WARren County}

Stony Creek....

do

do

do

do

do

do

do

do

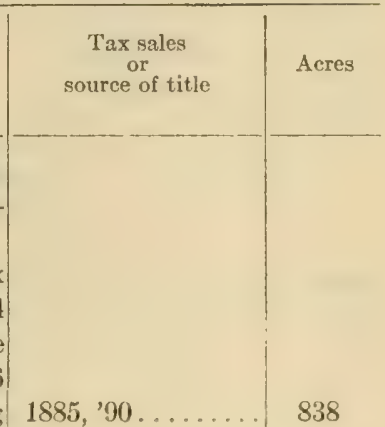

23 Ex, 203a. bd. beg. at a stake in line between lot $23 \&$ Great lot 3, Rear Division, 3e. S. of Bakertown Road, th. S. 64 deg. W. 33c. to lot 22 , th. N. $26 \mathrm{deg}$. W. $61 \frac{1}{2} \mathrm{c}$. to a stake on line of lot 22 , th. N. 64 deg. E. 33c. to Great lot 3 , aforesaid, \& th. S. 26 deg. E. along said Great lot line $61 \frac{1}{2} \mathrm{c}$. to beg. .

All in Stony Creek.

All in Stony Creek........ 1885, '90 . . . . . . 70

All in Stony Creek.........

All in Stony Creek..........

$1895,{ }^{\prime} 00 \ldots \ldots \ldots . . .68$

$1895,{ }^{9} 00 \ldots \ldots \ldots . .156$

All in Stony Creek. ........

All in Stony Creek.........

$1895,{ }^{\prime} 00 \ldots \ldots \ldots+240$

$1895,{ }^{\prime} 00 \ldots \ldots . \quad 300$

All in Stony Creek.

$1895,00 \ldots \ldots \ldots$

360

Total, General Allotment 7,982 acres.

\section{Middle Division,} Great Lot 1

All except the following described parcels, viz.: 100a. in the S. E. cor ..... Sub. 4, 100a. deeded by Hollis Russell to Joseph L. liuller.

Subs. 5 and 6 , 55a., more or less, deeded by Co umbus Gill to Chas. W. Murray, descr bed as beg. at a hemlock tree on the S. line of 
Warren CountT

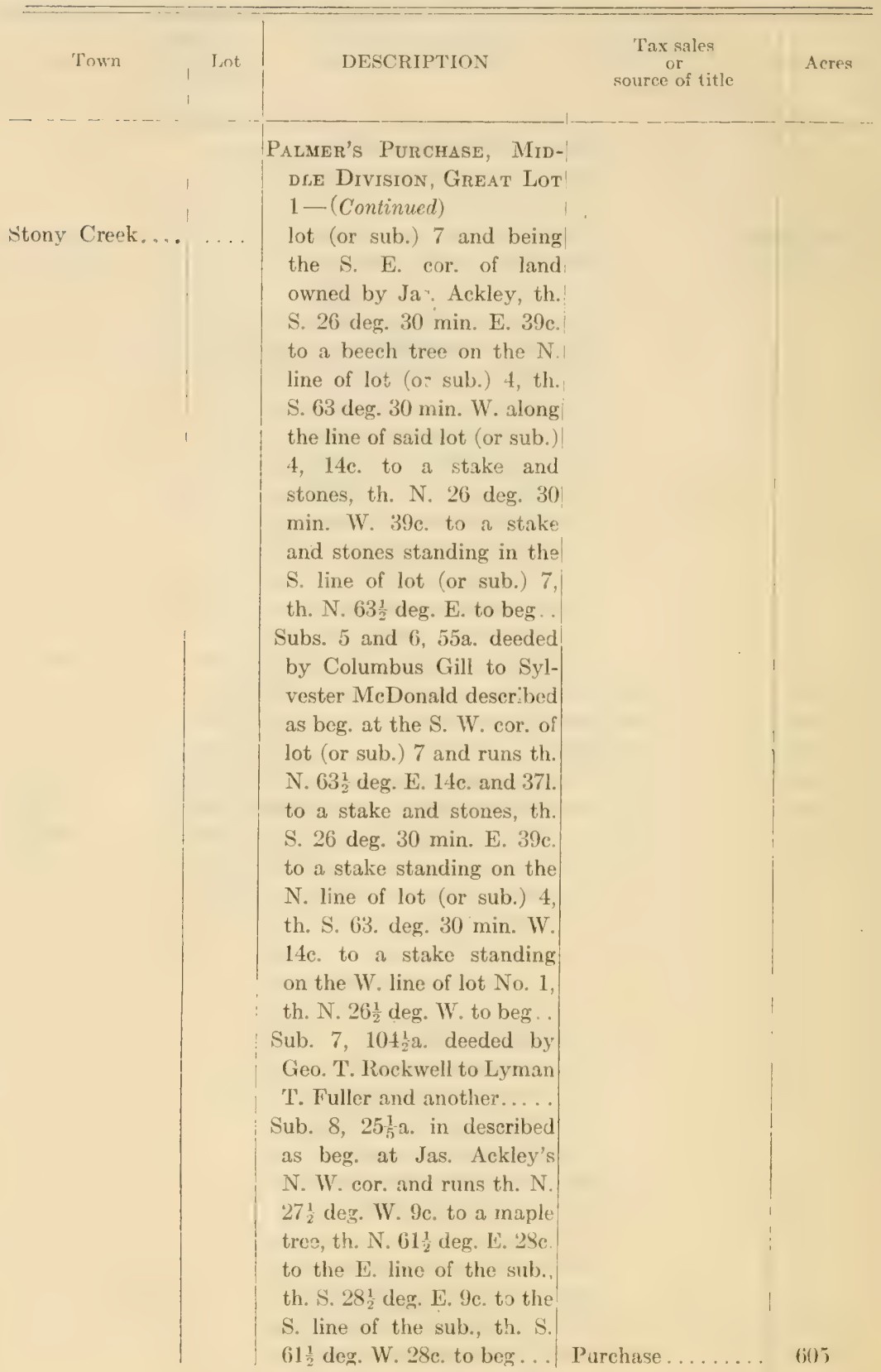




\section{WARREN COUNTY}

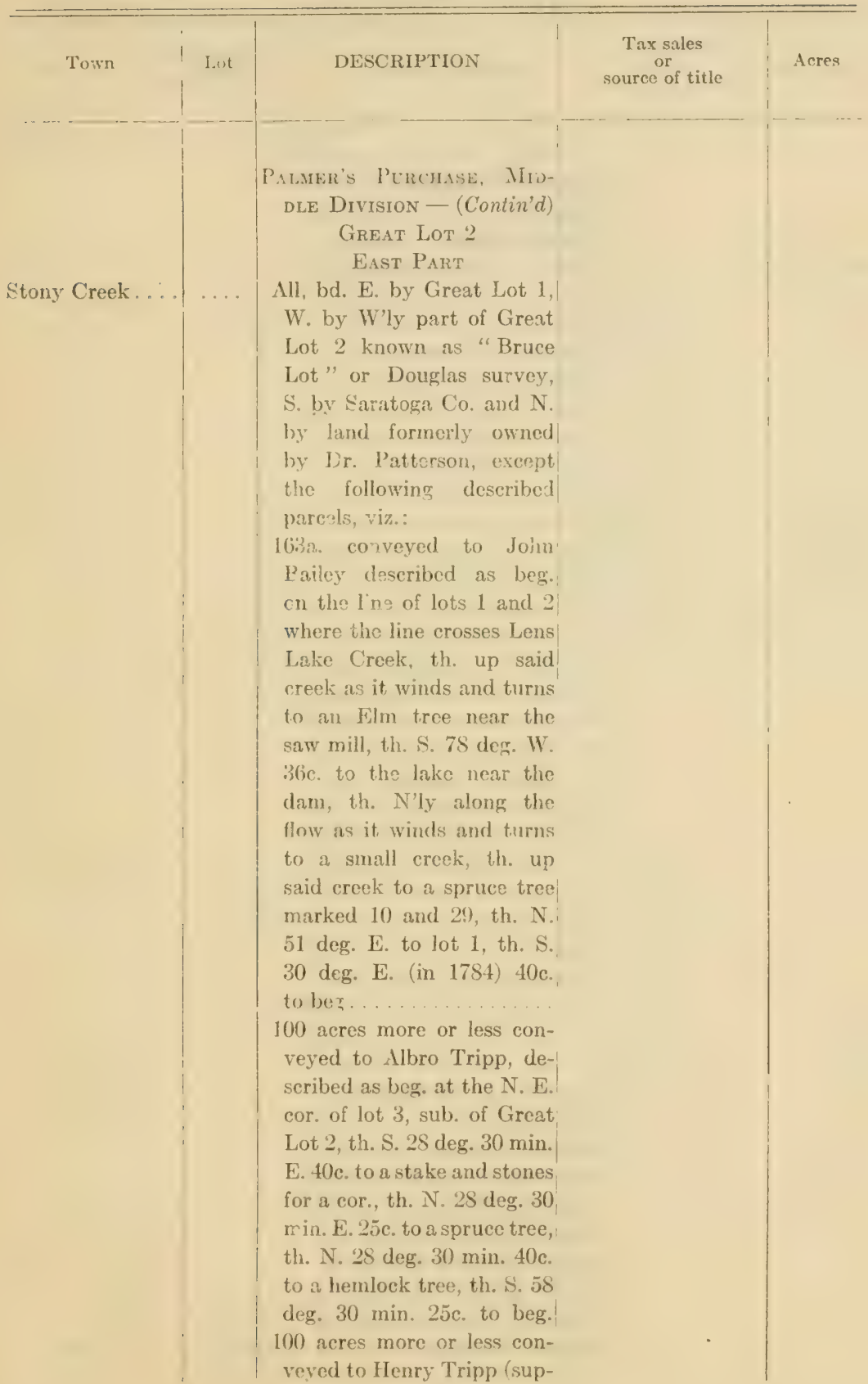


WarRen County

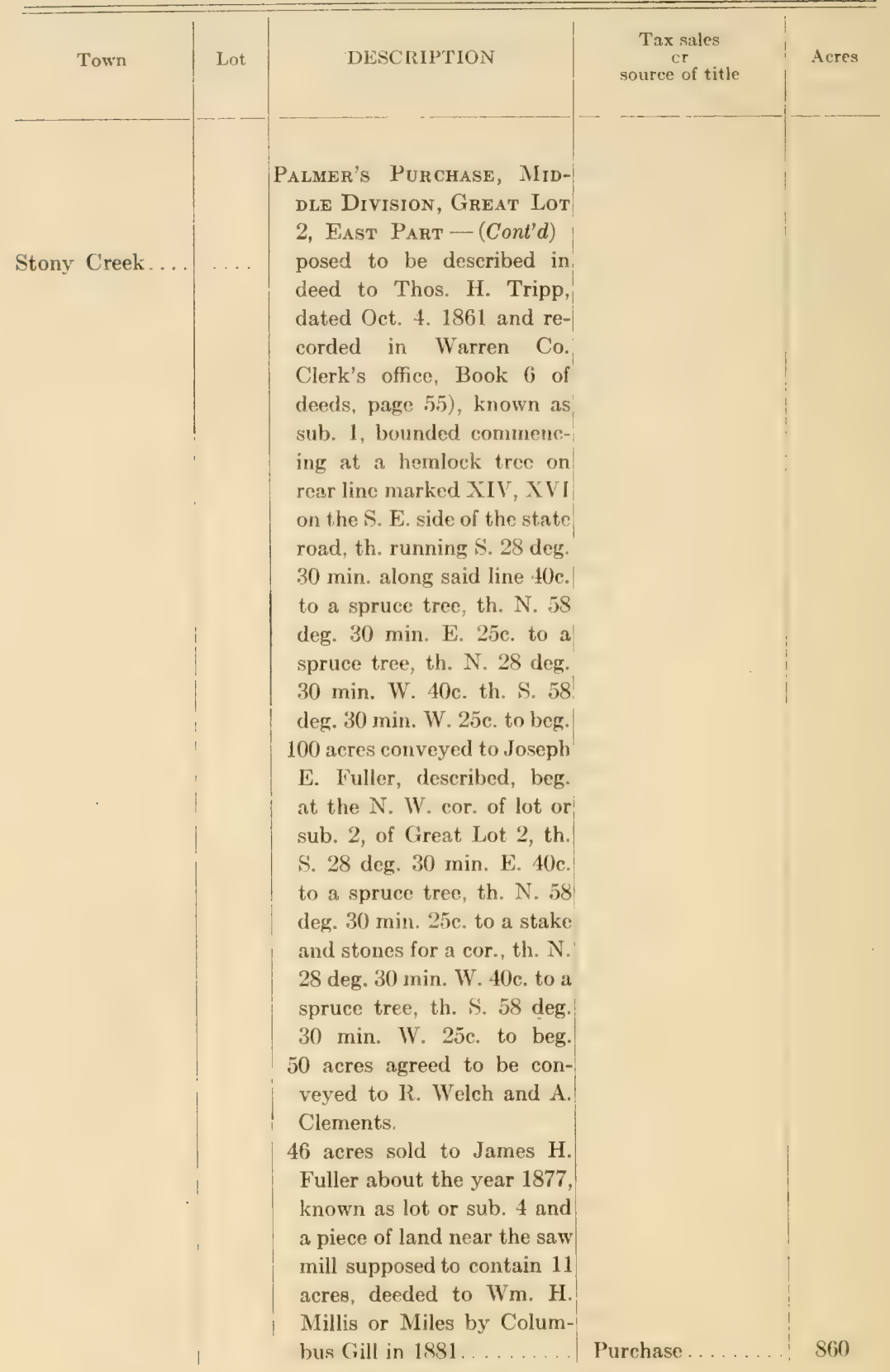


Warren County

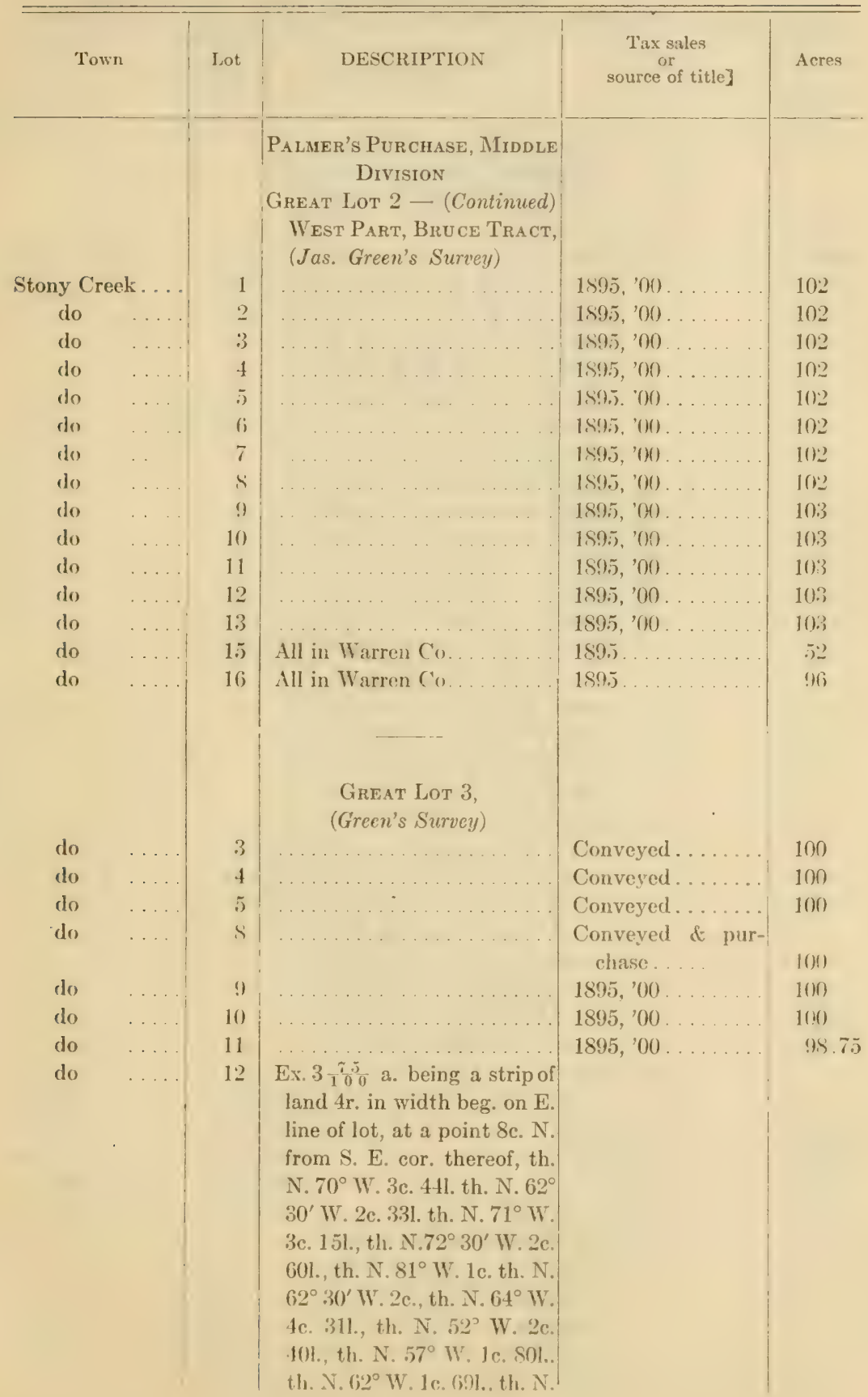


Warren County

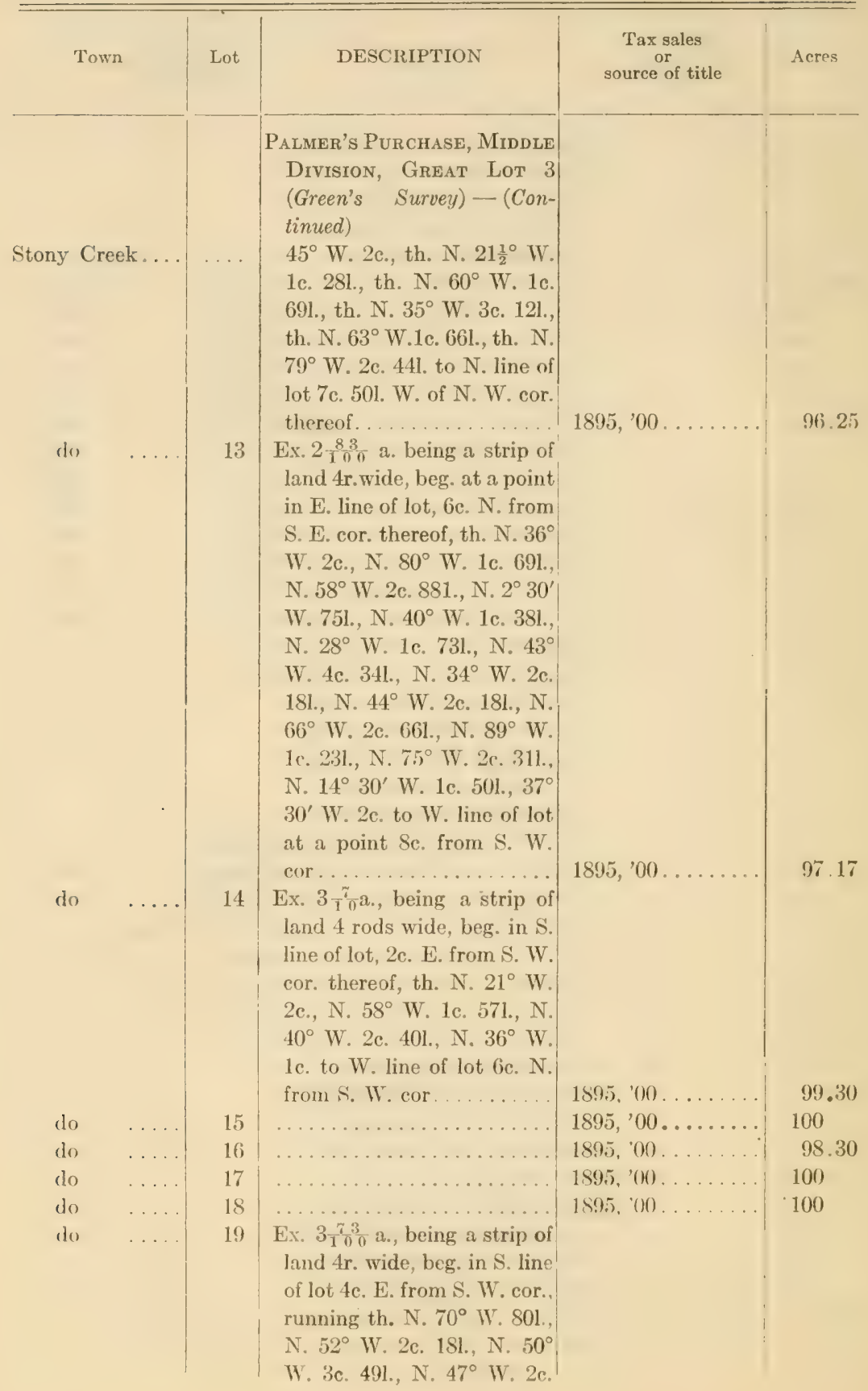




\section{Warren County}

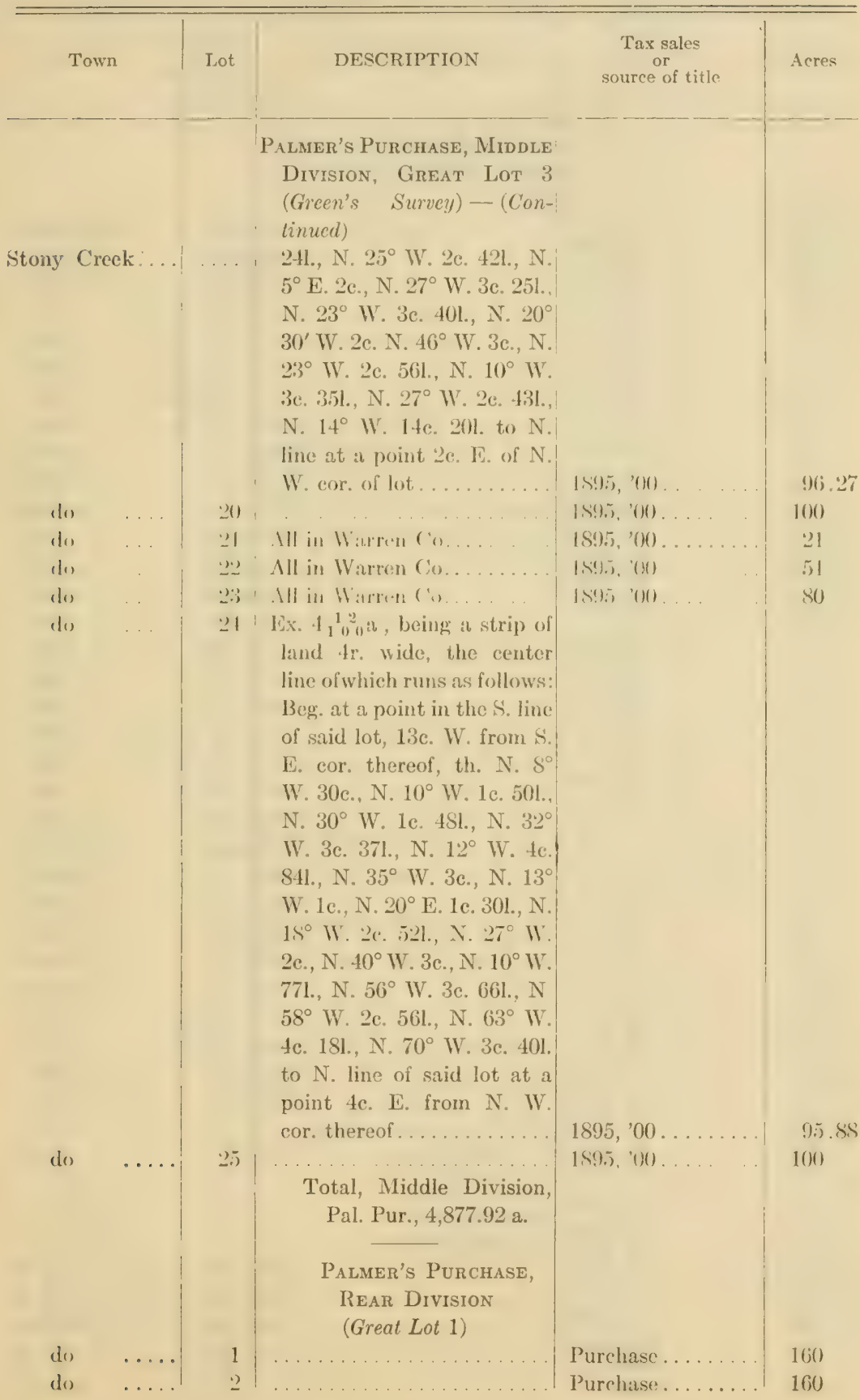




\section{Warren County}

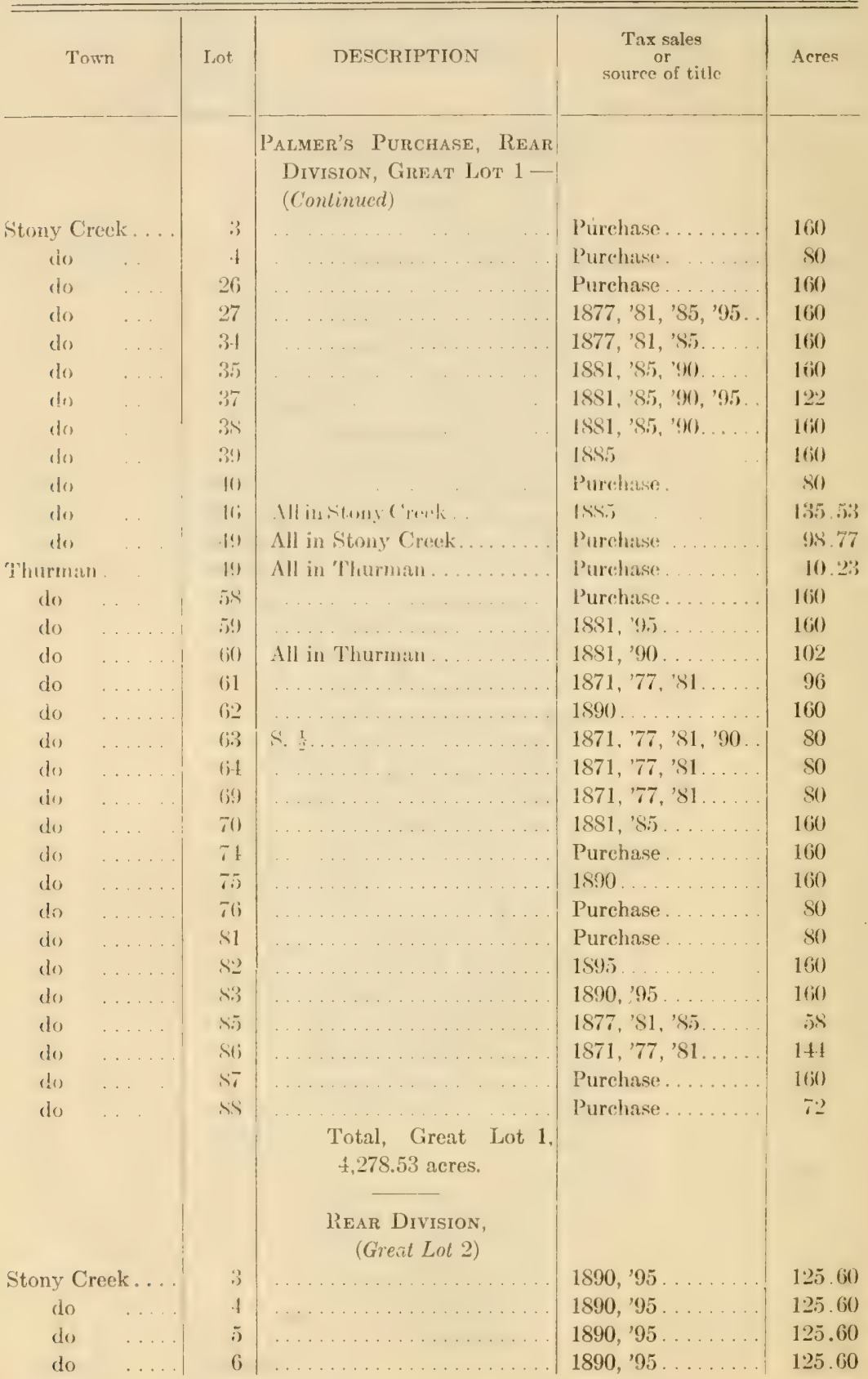


Conservation Commission.

WARREN COUNTY

\begin{tabular}{|c|c|c|c|c|}
\hline Town & Lot & DESCRIPTION & $\begin{array}{c}\text { Tax sales } \\
\text { or } \\
\text { source of title }\end{array}$ & Acres \\
\hline & & $\begin{array}{l}\text { Palmer's Purchase, Rear } \\
\text { Division, Great Lot } 2- \\
\text { (Continued) }\end{array}$ & & \\
\hline Stony Creck. & 7 & $\ldots \ldots \ldots \ldots \ldots \ldots \ldots$ & $1890,95 \ldots \ldots$ & 125.60 \\
\hline do & 8 & & $1890,95 \ldots \ldots$ & 125.60 \\
\hline do & 9 & & $1890,95 \ldots$ & 125.60 \\
\hline do & 10 & & $1890,95 \ldots$ & 125.60 \\
\hline du & 11 & & 1890,95 & 125.60 \\
\hline do & 12 & 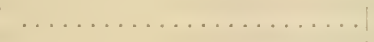 & $1890,95 \ldots$ & $125.0(0)$ \\
\hline do & 13 & & $1890,95 \ldots$ & 125.60 \\
\hline do & 14 & All in Stony Crcek. . & $1890, ’ 95 \ldots$ & 101.60 \\
\hline Thwiman ... & $1 \cdot 4$ & All in Thurman. . . . . . . & $1890,95 . \ldots$. & 21 \\
\hline Stony Creek. & 15 & All in Stony Creek.... & 1890,95 & $9 .(60)$ \\
\hline Thurmsn. & 15 & All in Thurm:th... & $1890,95 \ldots$ & 116 \\
\hline do & 16 & . & $1890,95 \ldots$ & 125.60 \\
\hline do & 17 & & $1890,95 \ldots$ & 125.60 \\
\hline do & 18 & $\ldots$ & $1890,995 \ldots$ & 125.60 \\
\hline do & 19 & & $1890,{ }^{\prime} 95 \ldots$. & 125.60 \\
\hline do & 20 & ….... & $1871, ' 81, ' 85 \ldots \ldots$ & 150.70 \\
\hline do & 21 & & $1871, ' 81, ' 85 \ldots \ldots$ & 150.70 \\
\hline do & 22 & & $1890,{ }^{\prime} 95 \ldots \ldots$ & 125.60 \\
\hline do & 23 & & $1890,95 \ldots$ & 125.60 \\
\hline do & 24 & & $1980,{ }^{\prime} 95 \ldots$ & 125.60 \\
\hline do & 25 & 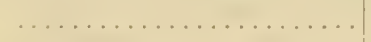 & $1890,{ }^{\prime} 95 \ldots$ & 125.60 \\
\hline do & 26 & & $1890,95 \ldots \ldots \ldots$ & 125.60 \\
\hline Stony Creek. & 27 & All in Stony Creek..... & $1890 \ldots \ldots \ldots \ldots$ & 3 \\
\hline Thurman... & 27 & All in Thurman..... & $\begin{array}{r}1871,91,85,90 . \\
95 \ldots \ldots \ldots \ldots\end{array}$ & 122.60 \\
\hline Stosy Creek. & 28 & All in Stony Creek. & $1890,{ }^{\prime} 95 \ldots \ldots \ldots$ & 93.60 \\
\hline Thurman .... & 28 & All in Thurman.... & $1890,{ }^{\prime} 95 \ldots \ldots$ & 32 \\
\hline Stony Creek. & 29 & $\ldots \ldots \ldots \ldots \ldots$ & $1890,95 \ldots \ldots \ldots$ & 125.60 \\
\hline do & 30 & & $1890,95 \ldots \ldots \ldots$ & 125.60 \\
\hline do & 31 & . & $1890,95 \ldots \ldots$ & 125.60 \\
\hline (d) & 32 & & $1890,95 \ldots \ldots$ & 125.60 \\
\hline do & 33 & & $1890,{ }^{\prime} 95 \ldots \ldots$ & 125.60 \\
\hline do & 34 & & $1890,{ }^{\prime} 95 \ldots \ldots$ & 125.60 \\
\hline do & 35 & & $1890, ' 95 \ldots \ldots \ldots$ & 125.60 \\
\hline do & $3 ̈ 6$ & & $1890,{ }^{\prime} 95 \ldots \ldots \ldots$ & 125.60 \\
\hline do & 37 & & $1890,{ }^{\prime} 95 \ldots \ldots \ldots$ & 125.60 \\
\hline do & 38 & & $1890, ' 95 \ldots \ldots \ldots$ & 125.60 \\
\hline do & 43 & & $1890,95 \ldots \ldots \ldots$ & 125.60 \\
\hline do & 44 & & $1890,{ }^{\prime} 95 \ldots \ldots \ldots$ & 125.60 \\
\hline do & 45 & & 1890 , ’95. & 125.60 \\
\hline do & 46 & & $1890, ' 95 \ldots \ldots \ldots$ & 125.60 \\
\hline do & 47 & & $1890, ' 95, \ldots \ldots$ & 125.60 \\
\hline
\end{tabular}


WARREN County

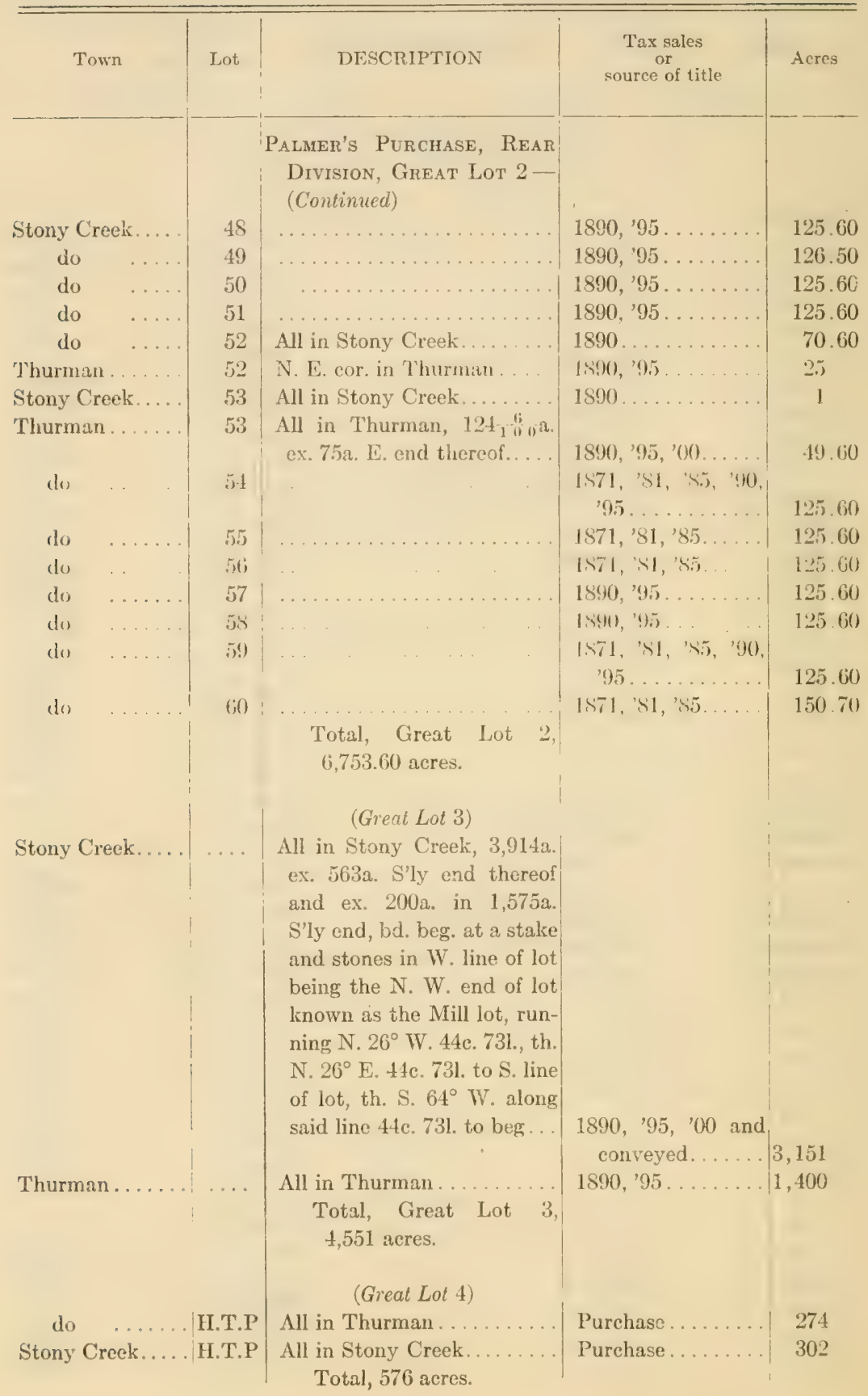




\section{WARREn County}

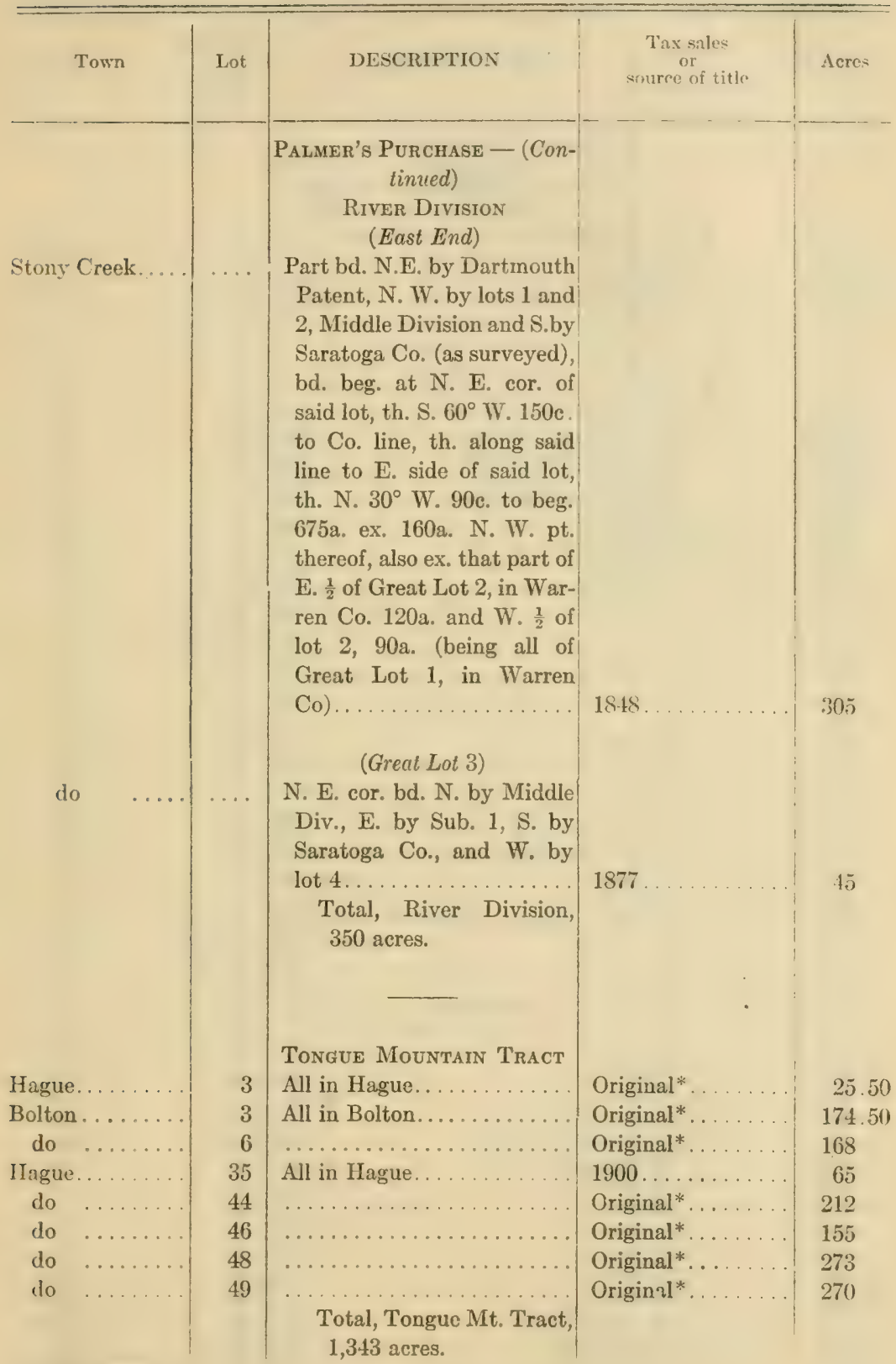

* Resale. 
Warren County

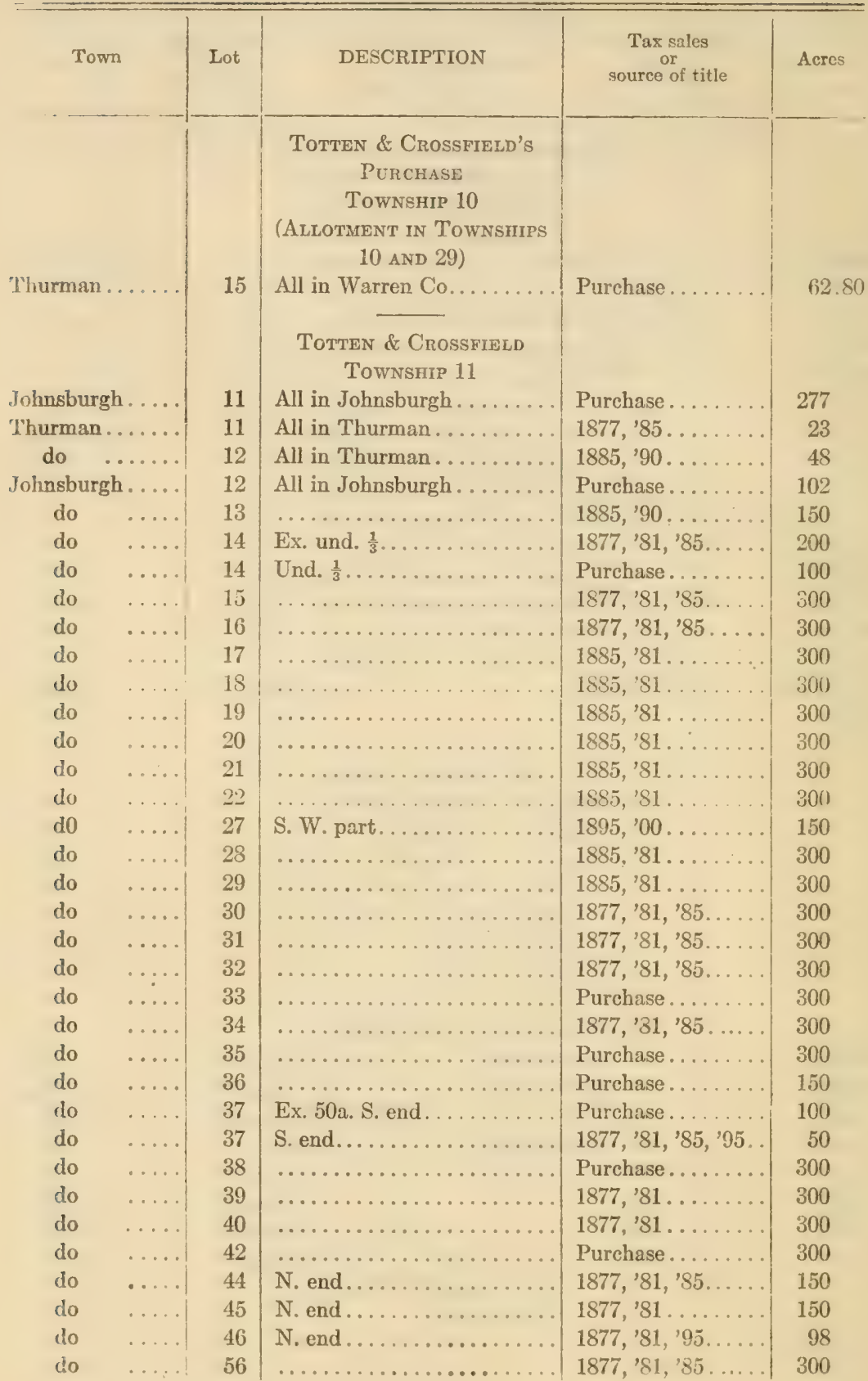


Warren County

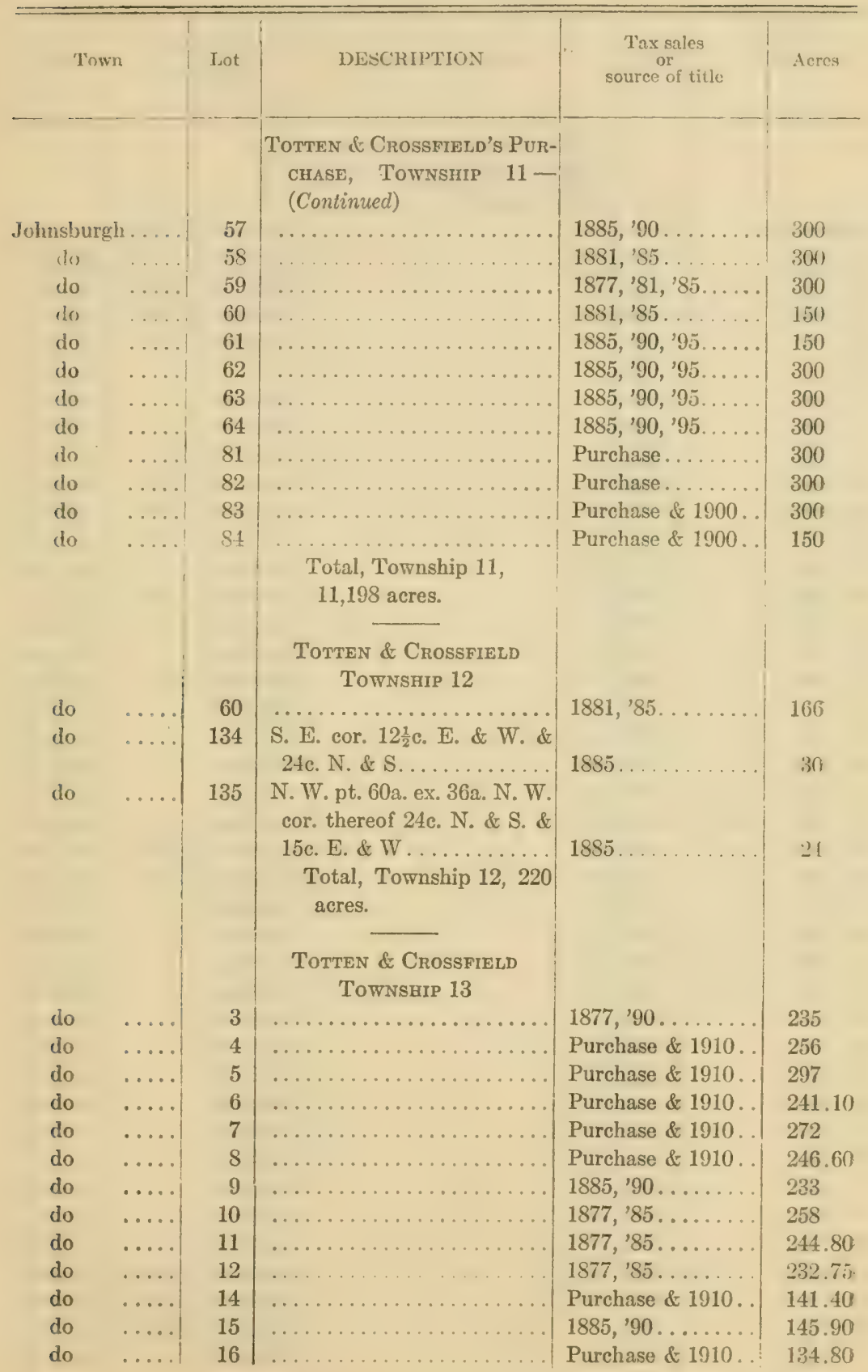


WARREN COUNTY

\begin{tabular}{|c|c|c|c|c|}
\hline Town & Lot & DESCRIPTION & $\begin{array}{c}\text { Tax sales } \\
\text { or }\end{array}$ & Acres \\
\hline \multirow[b]{2}{*}{ Johnsburgh } & & $\begin{array}{l}\text { Totten \& Crossfield's PUR- } \\
\text { CHase, Township } 13- \\
\text { (Continued) }\end{array}$ & & \\
\hline & 17 & $\ldots \ldots \ldots \ldots \ldots$ & Purchase \& 1910. & 137.60 \\
\hline do & 18 & & Purchase \& $1910 \ldots$ & 154 \\
\hline do & 19 & 列 & Purchase \& 1910. & 135 \\
\hline do & 20 & $\ldots \ldots$ & Purchase \& $1910 \ldots$ & 165.20 \\
\hline do & 21 & . & Purchase \& $1910 \ldots$ & 143.40 \\
\hline do & 22 & . & 1877, '85. . . . . . & 131.90 \\
\hline do & 23 & & $1885, ' 90,{ }^{\prime} 95 \ldots$. & 127.75 \\
\hline do & 26 & & $1885,90 \ldots \ldots$ & 145.20 \\
\hline do & 27 & & $1885,90, ' 95 \ldots$. & 151.10 \\
\hline do & 28 & $\cdots$ & Purchase \& $1910 \ldots$ & 164.50 \\
\hline do & 29 & $\ldots \ldots \ldots \ldots \ldots \ldots$ & Purchase \& 1910. & 187.10 \\
\hline do & 30 & 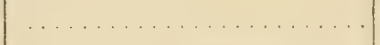 & Purchase \& 1910. . & 152.50 \\
\hline do & 31 & $\ldots \ldots \ldots$ & Purchase........ & 174.30 \\
\hline do & 32 & $\ldots \ldots \ldots$ & Purchase \& $1910 \ldots$ & 155 \\
\hline do & 33 & $\cdots$ & Purchase....... & 152.40 \\
\hline do & 34 & $\ldots \ldots \ldots \ldots \ldots$ & Purchase....... & 163.60 \\
\hline do & 35 & $\ldots$ & Purchuse \& 1910. & 157.50 \\
\hline do & 36 & $\cdots \cdots$ & $1885,{ }^{\prime} 90 \ldots \ldots$ & 152.40 \\
\hline do & 37 & $\ldots \ldots$ & $1885, ' 90 \ldots \ldots$ & 148.30 \\
\hline do & 38 & & $1885,{ }^{\prime} 90 \ldots \ldots$ & 151.70 \\
\hline do & 39 & & Purchase....... & 157.20 \\
\hline do & 40 & & Purchase........ & 146.50 \\
\hline do & 41 & & Purchase \& $1910 \ldots$ & 147.40 \\
\hline do & 42 & $\ldots$ & Purchase \& 1910 & 165.25 \\
\hline do & 43 & & Purchase........ & 143.25 \\
\hline do & 44 & $\cdots$ & Purchase....... & 174.75 \\
\hline do & 45 & . & Purchase........ & 156.10 \\
\hline do & 46 & . & Purchase........ & 140.30 \\
\hline do & 47 & . & Purchase....... & 134.20 \\
\hline do & 49 & $\ldots \ldots$ & Purchase....... & 122.60 \\
\hline do & 49 & & $1877, ' 85 \ldots$ & 122.60 \\
\hline do & 50 & . & $1885,{ }^{\prime} 90 \ldots \ldots$ & 146.90 \\
\hline do & 51 & & Purchase......... & 154.30 \\
\hline do & 52 & & Purchase........ & 169 \\
\hline do & 53 & & Purchase ........ & 187.80 \\
\hline do & 54 & & Purchase........ & 153 \\
\hline do & 55 & . & Purchase \& 1910 . & 175.30 \\
\hline do & 56 & & Purchase \& 1910. & 155.50 \\
\hline do & 57 & & Purchase \& 1910. & 154.60 \\
\hline do & 58 & & Purchase \& 1910. & 163.80 \\
\hline do & 59 & . & $1885,{ }^{\prime} 90 \ldots$. & 155.90 \\
\hline
\end{tabular}




\section{Warren County}

\begin{tabular}{|c|c|c|c|c|}
\hline Town & Lot & DESCRIPTION & $\begin{array}{l}\text { Tax sales } \\
\text { or } \\
\text { source of title }\end{array}$ & Acres: \\
\hline & & $\begin{array}{l}\text { Totten \& Crossfield's PUR- } \\
\text { Chase, Township } 13- \\
\text { (Continued) }\end{array}$ & & \\
\hline Johnsburgh . & 60 & $\ldots \ldots \ldots \ldots \ldots \ldots$ & $1885,{ }^{\prime} 90, ' 95 \ldots$ & 152.50 \\
\hline do & 61 & & $1885,90 \ldots$ & 118.20 \\
\hline do & 62 & & Purchase \& 1910. & 119.80 \\
\hline do & 63 & $\ldots \ldots \ldots \ldots \ldots \ldots$ & Purchase \& 1910. & 125.50 \\
\hline do & 64 & ............. & Purchase....... & 118.40 \\
\hline do & 65 & $\ldots \ldots \ldots$ & Purchase...... & 117.80 \\
\hline do & 66 & $\cdots \ldots \ldots$ & Purchase..... & 132.80 \\
\hline do & 67 & $\ldots \ldots$ & Purchase.... & 114.40 \\
\hline do & 68 & $\ldots \ldots \ldots \ldots$ & $1885,{ }^{\prime} 90 \ldots .$. & 140.70 \\
\hline do & 69 & $\ldots \ldots$ & $1885,{ }^{\prime} 90 \ldots$ & 126.30 \\
\hline do & 70 & & $1885, ' 90 \ldots$ & 115.80 \\
\hline do & 71 & ... & $1885,90 \ldots \ldots$ & 107.60 \\
\hline do & 72 & $\cdots$ & $1885,{ }^{\prime} 90 \ldots$ & 118.30 \\
\hline do & 73 & & $1885, ' 90 \ldots \ldots$ & 136.80 \\
\hline do & 74 & & $1885,90 \ldots \ldots$ & 122.75 \\
\hline do & 75 & & $1885,90 \ldots$ & 131.80 \\
\hline do & 76 & & Purchase ...... & 146.30 \\
\hline do & 77 & & Purchase.... & 161.30 \\
\hline do & 78 & $\ldots$. & Purchase ....... & 132.40 \\
\hline do & 80 & & $1885, ' 90 \ldots \ldots$ & 137.70 \\
\hline do & 81 & & Purchase \& 1910. & 139.75 \\
\hline do & 82 & $\ldots \ldots \ldots \ldots \ldots \ldots$ & Purchase \& 1910. & 147.90 \\
\hline do & 83 & $\cdots$ & Purchase \& 1910. & 140.30 \\
\hline do & 81 & & $1885,{ }^{\prime} 90 \ldots \ldots$ & 140.60 \\
\hline do & 85 & . & $1885, ' 90 \ldots \ldots \ldots$ & 152.60 \\
\hline do & 86 & & Purchase \& 1910. & 153.40 \\
\hline do & 87 & $\ldots$. & Purchase \& 1910. & 161.90 \\
\hline do & 88 & & Purchase \& 1910. & 154.50 \\
\hline do & \$9 & & $1885,90 \ldots$ & 151.90 \\
\hline do & 90 & .. & $1885, ' 90 \ldots \ldots$ & 171.80 \\
\hline do & 91 & & Purchase...... & 147.50 \\
\hline do & 92 & & Purchase.... & 179.80 \\
\hline do & 93 & ... & Purchase....... & 165.70 \\
\hline do & 94 & & Purchase....... & 149.50 \\
\hline do & 9.5 & & $1885,{ }^{\prime} 90 \ldots$ & 138.70 \\
\hline do & 96 & & $1885,{ }^{\prime} 90 \ldots$ & 157.60 \\
\hline do & 97 & & Purchase... . & 178.20 \\
\hline do & 98 & & Purchase........ & 153.70 \\
\hline do & 99 & & Purchase....... & 152.20 \\
\hline do & 100 & & Purchase... & 186.10 \\
\hline do & 101 & & $1877, ' 85 \ldots$ & 200 \\
\hline
\end{tabular}


Warren County

\begin{tabular}{|c|c|c|c|c|}
\hline Town & \multirow[t]{2}{*}{ Lot } & DESCRIPTION & \multirow[t]{2}{*}{$\begin{array}{c}\text { Tax sales } \\
\text { or } \\
\text { source of title }\end{array}$} & \multirow[t]{2}{*}{ Acres } \\
\hline & & $\begin{array}{l}\text { Totten \& Crossfield's Pur- } \\
\text { Chase, Township } 13- \\
\text { (Continued) }\end{array}$ & & \\
\hline Johnsburgh . & 102 & $\ldots \ldots \ldots \ldots \ldots \ldots$ & Purchase. & 163.80 \\
\hline do & 103 & $\ldots \ldots \ldots \ldots \ldots \ldots$ & Purchase.... . & 192.30 \\
\hline do & 104 & & Purchase .... . & 169.80 \\
\hline do & 105 & $\ldots \ldots \ldots \ldots \ldots$ & Purchase...... & 174.20 \\
\hline do & 106 & $\ldots \ldots$ & $1885,{ }^{\prime} 90 \ldots$ & 181.50 \\
\hline do & 107 & $\ldots \ldots \ldots \ldots \ldots \ldots \ldots$ & $1885,90 \ldots$ & 171 \\
\hline do & 108 & $\ldots \ldots \ldots \ldots \ldots \ldots$ & $1885, ' 90 \ldots$ & 171.7 .5 \\
\hline do & 109 & $\ldots \ldots \ldots \ldots \ldots$ & $1885,{ }^{\prime} 90$. & 149.60 \\
\hline do & 110 & $\ldots \ldots \ldots \ldots \ldots$ & $1885,{ }^{\prime} 90$. & 147.40 \\
\hline do & 111 & $\ldots \ldots \ldots \ldots \ldots \ldots \ldots$ & $1885, ' 90 \ldots$ & 156 \\
\hline do & 112 & $\ldots \ldots \ldots \ldots \ldots \ldots \ldots$ & Purchase.... . & 149.6() \\
\hline do & 113 & $\ldots \ldots \ldots \ldots \ldots \ldots \ldots$ & Purchase...... & 143.80 \\
\hline do & 114 & $\ldots \ldots \ldots \ldots \ldots \ldots \ldots$ & Purchase...... & 162.10 \\
\hline do & 115 & $\ldots \ldots \ldots \ldots \ldots \ldots$ & Purchase . . . . . . & 136.75 \\
\hline do & 116 & $\ldots \ldots \ldots \ldots \ldots$ & $1877, ' 85 \ldots \ldots$ & 165.80 \\
\hline do & 117 & $\ldots$ & Purchase...... . & 158.50 \\
\hline do & 118 & $\ldots \ldots \ldots \ldots \ldots \ldots$ & $1885, ' 90 \ldots \ldots$ & 137 \\
\hline do & 119 & .. & Purchase...... & 123.75 \\
\hline do & 120 & $\ldots \ldots \ldots$ & Purchase...... . & 146.75 \\
\hline do & 121 & $\ldots \ldots \ldots \ldots \ldots \ldots$ & $1877, ' 85 \ldots \ldots$ & 167.30 \\
\hline do & 122 & $\ldots \ldots \ldots \ldots \ldots \ldots$ & $1877, ' 85 \ldots \ldots \ldots$ & 138.50 \\
\hline do & 123 & $\ldots \ldots \ldots \ldots \ldots$ & $1877, ' 85 \ldots \ldots \ldots$ & 154 \\
\hline do & 124 & $\ldots \ldots$ & $1877, ' 85 \ldots \ldots \ldots$ & 173.60 \\
\hline do & 125 & $\ldots$ & $1877, ' 85 \ldots \ldots \ldots$ & 184.10 \\
\hline do & 126 & $\ldots \ldots \ldots$ & Purchase........ & 152 \\
\hline do & 127 & & Purchase........ & 181.10 \\
\hline do & 128 & .. & Purchase........ & 159.25 \\
\hline do & 129 & $\ldots \ldots \ldots \ldots$ & $1885, ' 90 \ldots \ldots \ldots$ & 168.75 \\
\hline do & 130 & & $1885,90 \ldots \ldots$ & 174.60 \\
\hline do & 131 & & $1885, ' 90 \ldots \ldots$ & 164.25 \\
\hline do & 132 & $\ldots$ & $1885,90 \ldots$ & 167.50 \\
\hline do & 133 & & $1885,90 \ldots$ & 111.30 \\
\hline do & 134 & . . & $1885,{ }^{\prime} 90 \ldots \ldots$ & 110.10 \\
\hline do & 135 & & $1885,{ }^{\prime} 90 \ldots \ldots \ldots$ & 118.90 \\
\hline do & 136 & & $1885,90 \ldots \ldots \ldots$ & 116 \\
\hline do & 137 & $\ldots \ldots \ldots$ & Purchase ....... & 112.20 \\
\hline do & 138 & & Purchase........ & 128.60 \\
\hline do & 139 & & Purchase . . . . . . . . & 108.90 \\
\hline do & 140 & $\ldots$ & $1877, ' 85 \ldots \ldots$ & 134 \\
\hline do & 141 & $\ldots \ldots$ & $1877,85 . \ldots \ldots$ & 130 \\
\hline
\end{tabular}


WARREN Countr

\begin{tabular}{|c|c|c|c|c|}
\hline Town & Lot & DESCRIPTION & $\begin{array}{c}\text { Tax sales } \\
\text { of } \\
\text { source of title }\end{array}$ & Acrese \\
\hline $\begin{array}{l}\text { Johnsburgh } \\
\text { do } \\
\text { do } \\
\text { do }\end{array}$ & $\begin{array}{r}142 \\
143 \\
144 \\
\text { Gore }\end{array}$ & 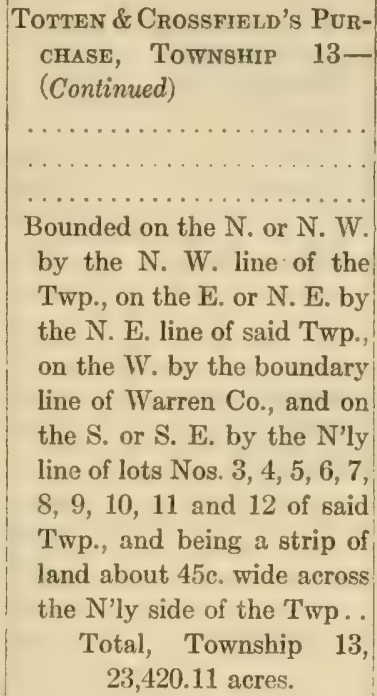 & $\begin{array}{l}1877, ' 85 \ldots \ldots \\
1877, ' 85 \ldots \ldots \\
1877, ' 85 \ldots \ldots\end{array}$ & $\begin{array}{l}115.71 \\
104.7 \% \\
125.80\end{array}$ \\
\hline & & $\begin{array}{c}\text { TOtTEN \& CROSSFIELD's } \\
\text { Township } 14 \\
\text { North } \frac{1}{2} \text { and South East } \frac{1}{4} \\
\text { (Pond's Survey) }\end{array}$ & & \\
\hline do & 50 & $\ldots \ldots \ldots \ldots \ldots \ldots \ldots$ & $1877, ' 81, ' 85 \ldots$ & 160 \\
\hline do & 51 & & $1877, ' 81, ' 85 \ldots$ & 160 \\
\hline do & 99 & S. W. cor.... & $1877, ' 81, ' 85 \ldots$ & 33 \\
\hline do & 106 & $\begin{array}{l}\text { All in Warren Co.......... } \\
\text { South West } \frac{1}{4} \text {. Conklin \& } \\
\text { Others'. Tract (Legget's Sur- } \\
\text { vey) }\end{array}$ & $1881,85 \ldots \ldots$ & 132 \\
\hline do & 6 & Ex. 20a. X. W. cor. & $1895 \ldots \ldots \ldots \ldots$ & 130 \\
\hline do & 13 & $\ldots \ldots \ldots$ & Purchase \& 1910. & 150 \\
\hline do & 14 & & $1881, ' 85 \ldots \ldots \ldots$ & 150 \\
\hline do & 22 & & $1877, ' 81, ' 85 \ldots$. & 112.50 \\
\hline do & 24 & & $1877,81 \ldots \ldots$ & 178 \\
\hline do & 25 & $\begin{array}{c}\text { Total, Township } 14, \\
1,383.50 \text { acres. }\end{array}$ & $1877, ' 81, ' 85 \ldots$. & 178 \\
\hline
\end{tabular}




\section{Warren Cotunty}

\begin{tabular}{|c|c|c|c|c|}
\hline Town & Lot & DESCRIPTION & $\begin{array}{l}\text { Tax sales } \\
\text { or } \\
\text { source of title }\end{array}$ & Aeres \\
\hline & & $\begin{array}{c}\text { Totmen \& Crossfield's Pur- } \\
\text { chase - (Continued }) \\
\text { Township } 15\end{array}$ & & \\
\hline Johnsburgh & 90 & All in Warren Co....... & $1895 \&$ purchase... & 46.50 \\
\hline do & 91 & All in Warren Co.. & Purchase......... & 10 \\
\hline do & 101 & All in Warren Co.. & $\begin{array}{r}1895,{ }^{\prime} 00 \quad \& \text { pur- } \\
\text { chase. . . . . . . . }\end{array}$ & 60 \\
\hline do & 102 & All in Warren Co...... & $\begin{array}{r}1895, \text { '00 \& pur- } \\
\text { chase. . . . . . . . }\end{array}$ & 150 \\
\hline$d p$ & 103 & & $\begin{array}{r}1895,{ }^{\prime} 00 \quad \& \text { pur- } \\
\text { chase } \ldots \ldots\end{array}$ & 160 \\
\hline do & 104 & All in Warren Co...... & $1895 \&$ purchase. . . & $26 . \tilde{0} 0$ \\
\hline do & 112 & All in Warren Co. . & Purchase......... & 3 \\
\hline do & 113 & All in Warren Co....... & Purchase ......... & 113.50 \\
\hline do & 114 & $\ldots \ldots \ldots \ldots \ldots \ldots \ldots$ & Purchase........ & 160 \\
\hline do & 115 & $\cdots \cdots$ & $1895 \&$ purchase. . . & 160 \\
\hline do & 116 & $\ldots \ldots \ldots \ldots \ldots \ldots \ldots \ldots \ldots \ldots$ & $\begin{array}{r}1895, \quad 00 \& \text { \& } \\
\text { chase } \ldots \ldots \ldots\end{array}$ & 160 \\
\hline do & 117 & All in Warren Co.. & $\begin{array}{r}1895, \text { '00 } \quad \& \text { pur- } \\
\text { chase. . . . . . . }\end{array}$ & 115 \\
\hline do & 118 & All in Warren Co. & Purchase........ & 50 \\
\hline do & 121 & All in Warren Co... & Purchase......... & 27 \\
\hline do & 122 & All in Warren Co... & Purchase........ & 105 \\
\hline do & 123 & . & $\begin{array}{r}1895, \text { '00 \& pur- } \\
\text { chase. . . . . . . }\end{array}$ & 160 \\
\hline do & $12 t$ & & $\begin{array}{r}1895, \text { '00 \& pur- } \\
\text { chase. . . . . . . }\end{array}$ & 160 \\
\hline do & $12 j$ & & $\begin{array}{r}1895, \text { ' } 00 \text { \& } \text { pur- } \\
\text { chase } \ldots \ldots \ldots \ldots\end{array}$ & 160 \\
\hline do & 126 & & $\begin{array}{r}1895,{ }^{\prime} 00 \quad \& \quad \text { pur- } \\
\text { chase } \ldots \ldots \ldots \ldots\end{array}$ & 160 \\
\hline do & 127 & & $\begin{array}{r}1895 \text {, 'v0 \& } \quad \text { pur- } \\
\text { chase } \ldots \ldots \ldots \ldots\end{array}$ & 160 \\
\hline do & 128 & & Purchase......... & 160 \\
\hline do & 129 & All in Warren Co. & Purchase........ & 23 \\
\hline do & 135 & All in Warren Co.. & Purchase ......... & 1.50 \\
\hline do & 136 & All in Warren Co. & Purchase ........ . & 129.50 \\
\hline do & 137 & $\ldots \ldots \ldots \ldots$ & $\begin{array}{r}1895,00, \& \text { pur- } \\
\text { chase . . . . . . . }\end{array}$ & 160 \\
\hline do & 138 & & 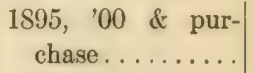 & 160 \\
\hline do & 139 & & $\begin{array}{c}1895 \text {, '00 \& pur- } \\
\text { chase. } \ldots \ldots \ldots\end{array}$ & 160 \\
\hline do & 140 & & $\begin{array}{r}180 \pi, \text { of is pur- } \\
\text { chane. }\end{array}$ & 160 \\
\hline
\end{tabular}




\section{Warren County}

\begin{tabular}{|c|c|c|c|c|}
\hline Town & I,ot & DESCRIPTION & $\begin{array}{c}\text { Tax sales } \\
\text { or } \\
\text { source of title }\end{array}$ & Acrey \\
\hline & & $\begin{array}{l}\text { Totten \& Crossfield's PUR- } \\
\text { Chase, Township } 15- \\
\text { (Continued) }\end{array}$ & & \\
\hline Johnsburgh & 141 & $\ldots \ldots \ldots \ldots \ldots \ldots$ & 1895, '00 \& pur- & 160 \\
\hline do & 142 & & $\begin{array}{l}1895, \text { '00 \& pur- } \\
\text { chase. . . . . . . }\end{array}$ & 160 \\
\hline do & 143 & & $\begin{array}{c}1895, \text { '00 \& pur- } \\
\text { chase......... }\end{array}$ & 160 \\
\hline do & 144 & & $\begin{array}{l}1895, \text { '00 \& pur- } \\
\text { chase.......... }\end{array}$ & 160 \\
\hline do & 145 & & $\begin{array}{r}1895, \text { '00 \& pur- } \\
\text { chase. . . . . . . . }\end{array}$ & 160 \\
\hline do & 146 & 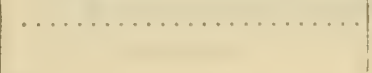 & $\begin{array}{r}1895, \text { '00 \& pur- } \\
\text { chase.......... }\end{array}$ & 160 \\
\hline do & 147 & 年 & $\begin{array}{l}1895, \text { '00 \& pur- } \\
\text { chase.......... }\end{array}$ & 160 \\
\hline do & 148 & & $\begin{array}{c}1895, \text { '00 \& pur- } \\
\text { chase. . . . . . . . }\end{array}$ & 160 \\
\hline do & 149 & . & $\begin{array}{r}1895, \text { '00 \& pur- } \\
\text { chase.......... }\end{array}$ & 160 \\
\hline do & 150 & & $\begin{array}{l}1895, \text { '00 \& pur- } \\
\text { chase......... }\end{array}$ & 160 \\
\hline do & 151 & 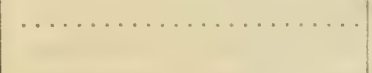 & $\begin{array}{l}1895, \text { '00 \& pur- } \\
\text { chase .......... }\end{array}$ & 160 \\
\hline do & 152 & . & $\begin{array}{r}1895 \text {, '00 \& pur- } \\
\text { chase. . . . . . . . }\end{array}$ & 160 \\
\hline do & 153 & . & $\begin{array}{l}1895,{ }^{\prime} 00 \& \text { pur- } \\
\text { chase.......... }\end{array}$ & 160 \\
\hline - do & 154 & $\begin{array}{c}\text { All in Warren Co......... } \\
\text { Total, Township 15, } \\
5,227 \frac{1}{2} \text { acres. }\end{array}$ & Purchase........ & 47 \\
\hline & & $\begin{array}{c}\text { TotTen \& Crossfield, } \\
\text { Township } 24\end{array}$ & & \\
\hline Chester. & 18 & N.pt........... & $1877, ' 81, ' 85 . \ldots$. & 300 \\
\hline Johnsburgh & 24 & $\ldots \ldots \ldots \ldots \ldots \ldots \ldots \ldots$ & $1881, ' 85, ' 90 \ldots$. & 420 \\
\hline do & 25 & $\ldots \ldots \ldots \ldots \ldots \ldots$ & $1877, ' 85,{ }^{\prime} 95 \ldots$. & 420 \\
\hline Chester... & 29 & $\begin{array}{l}\text { Sub. } 12, \text { N. E. pt......... } \\
\text { Total, Township 24, } \\
1,156 \text { acres. }\end{array}$ & $1877 \ldots \ldots \ldots \ldots$ & 16 \\
\hline
\end{tabular}




\section{Warren County}

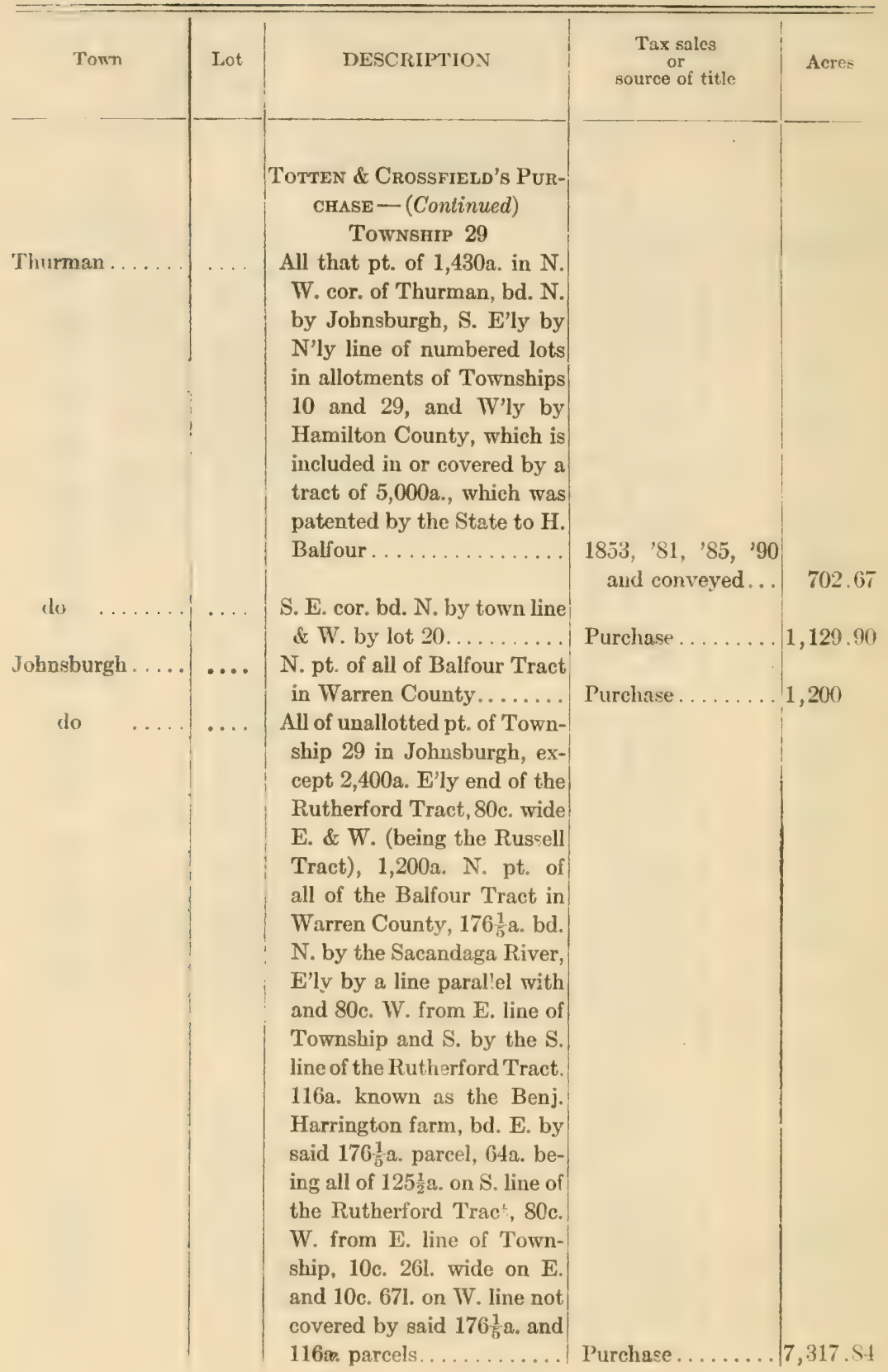


WARREN CoUnty

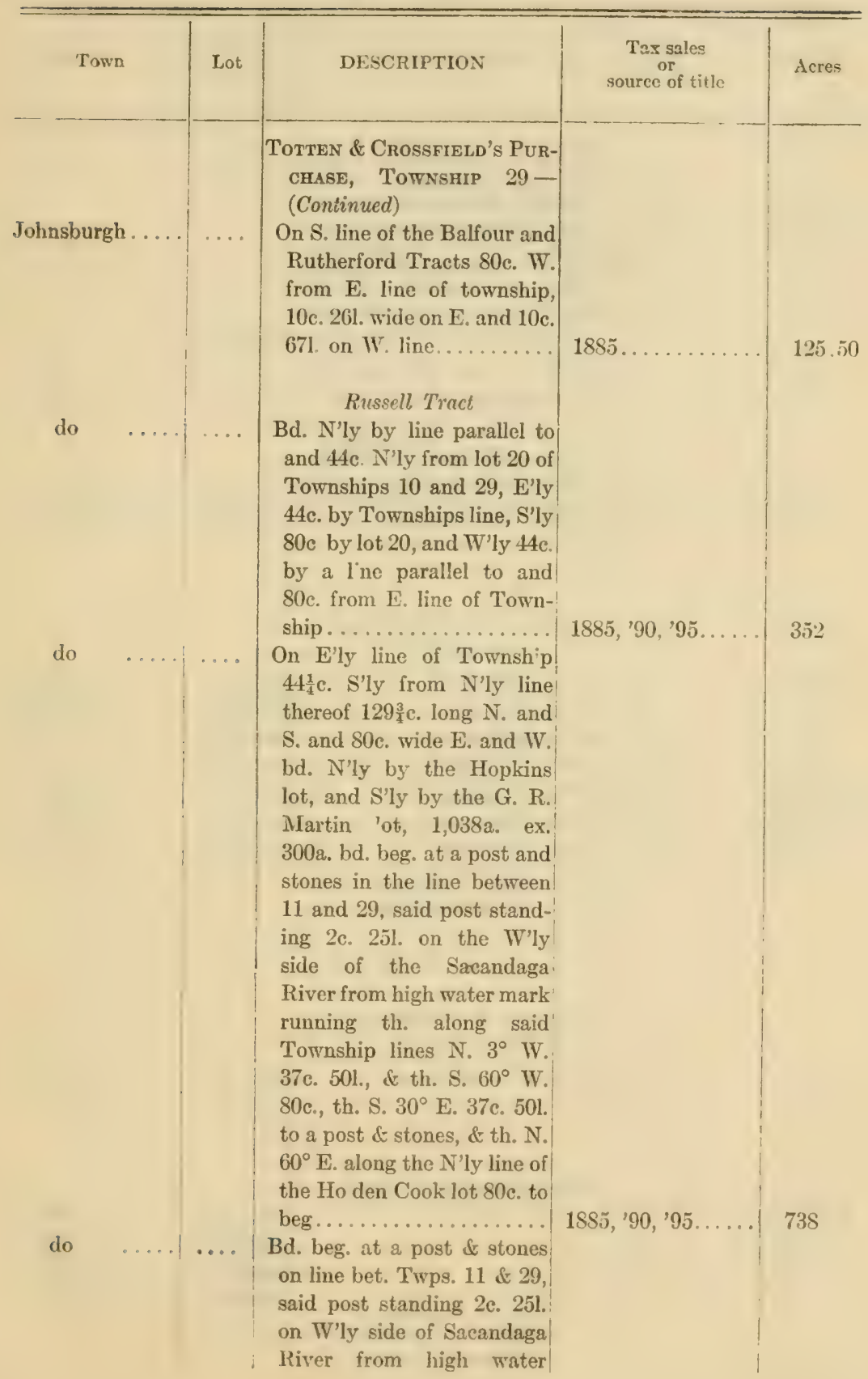




\section{WARREN COUNTY}

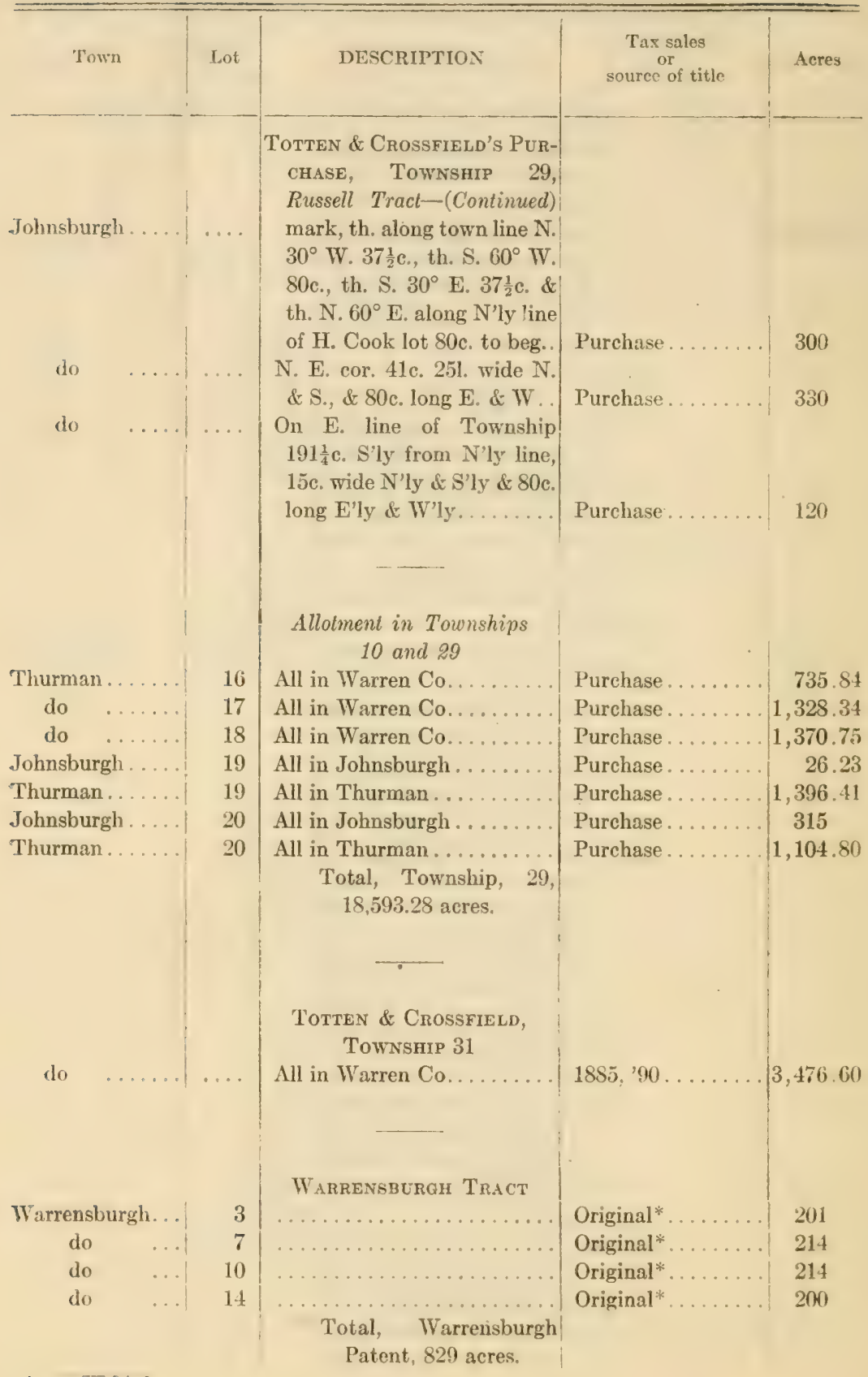


WARREN CoUntY

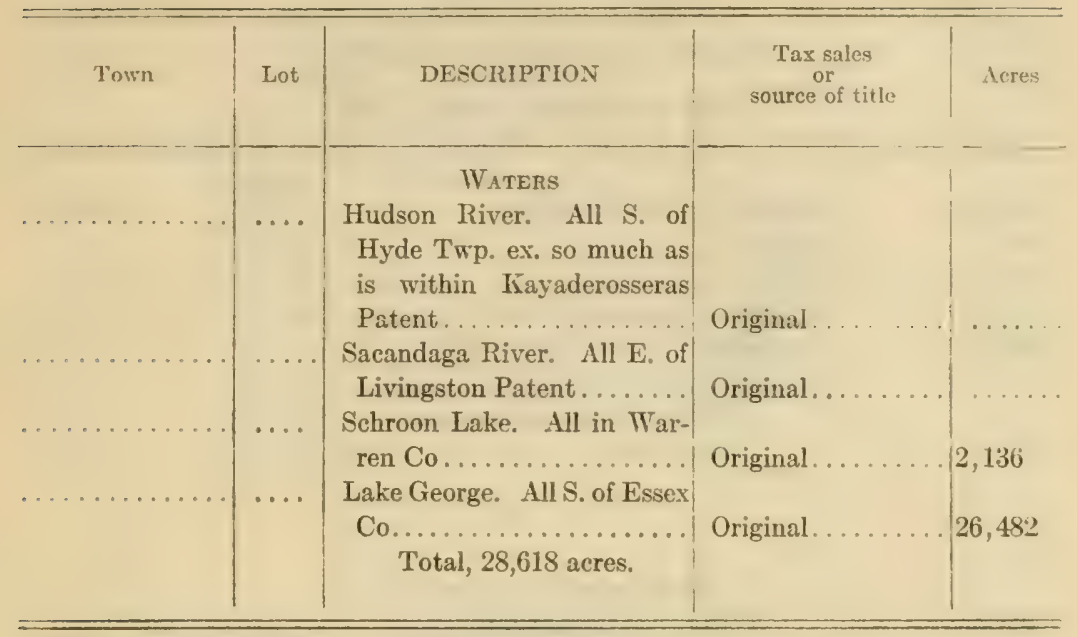

For other State land in Warren County see Appendix. 
WASHINGTON COUNTY

(Total number of acres, 4,320)

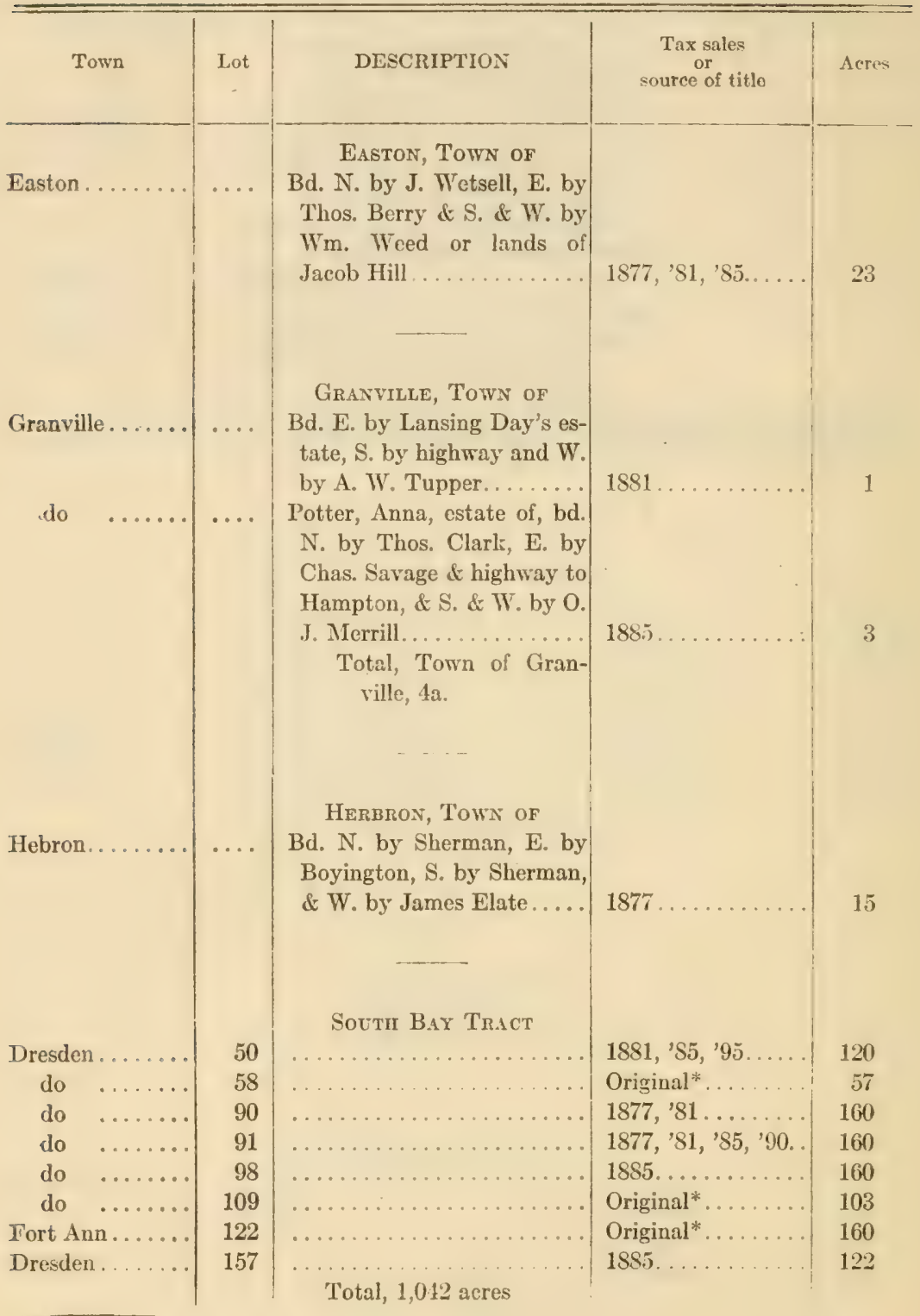


Conservation Commission.

WASHINGTON COUNTY

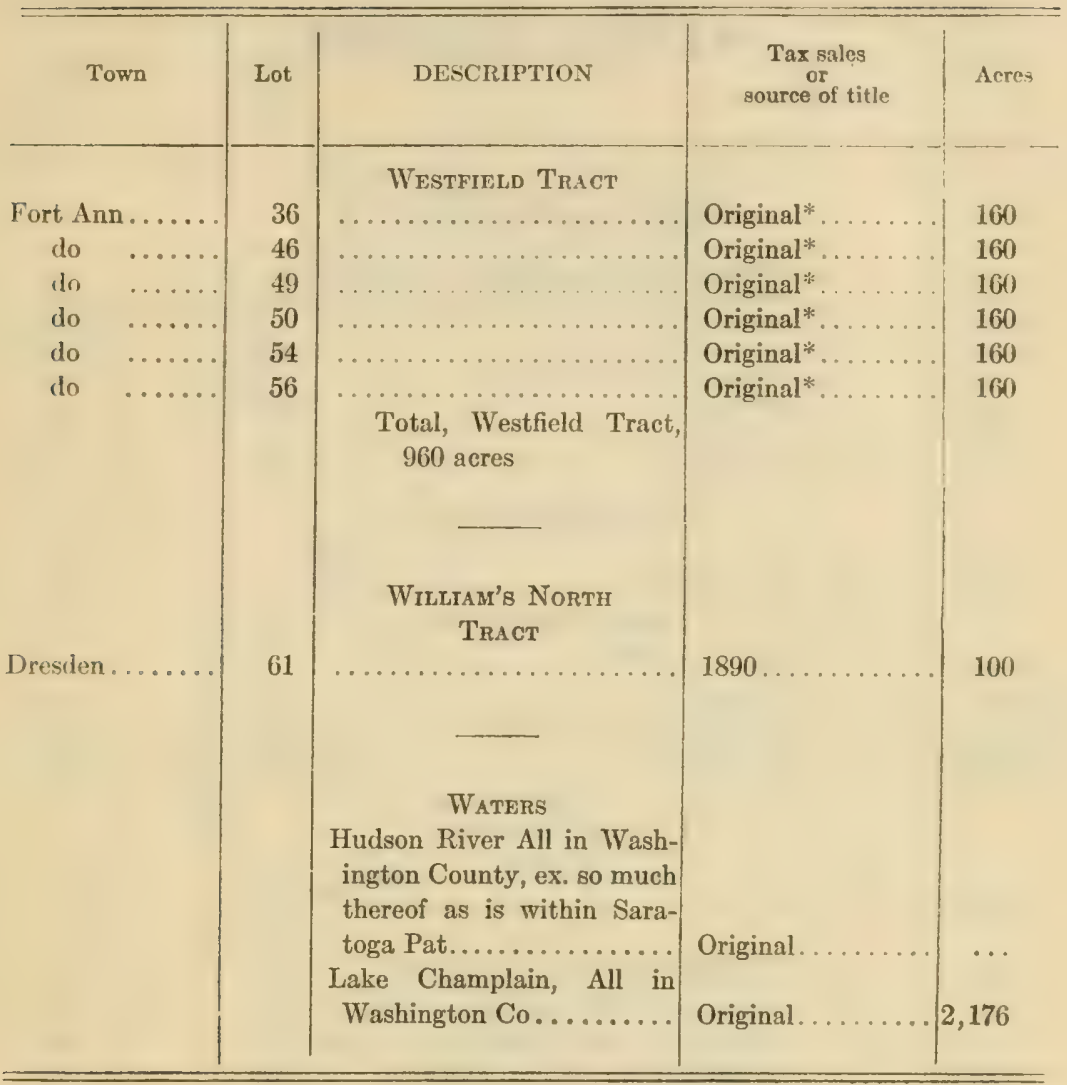

* Resale.

For other State land in Washington County see Appondix. 


\section{CATSKILL PRESERVE}

\section{DELAWARE COUNTY}

(Total number of acres, 13,837.11)

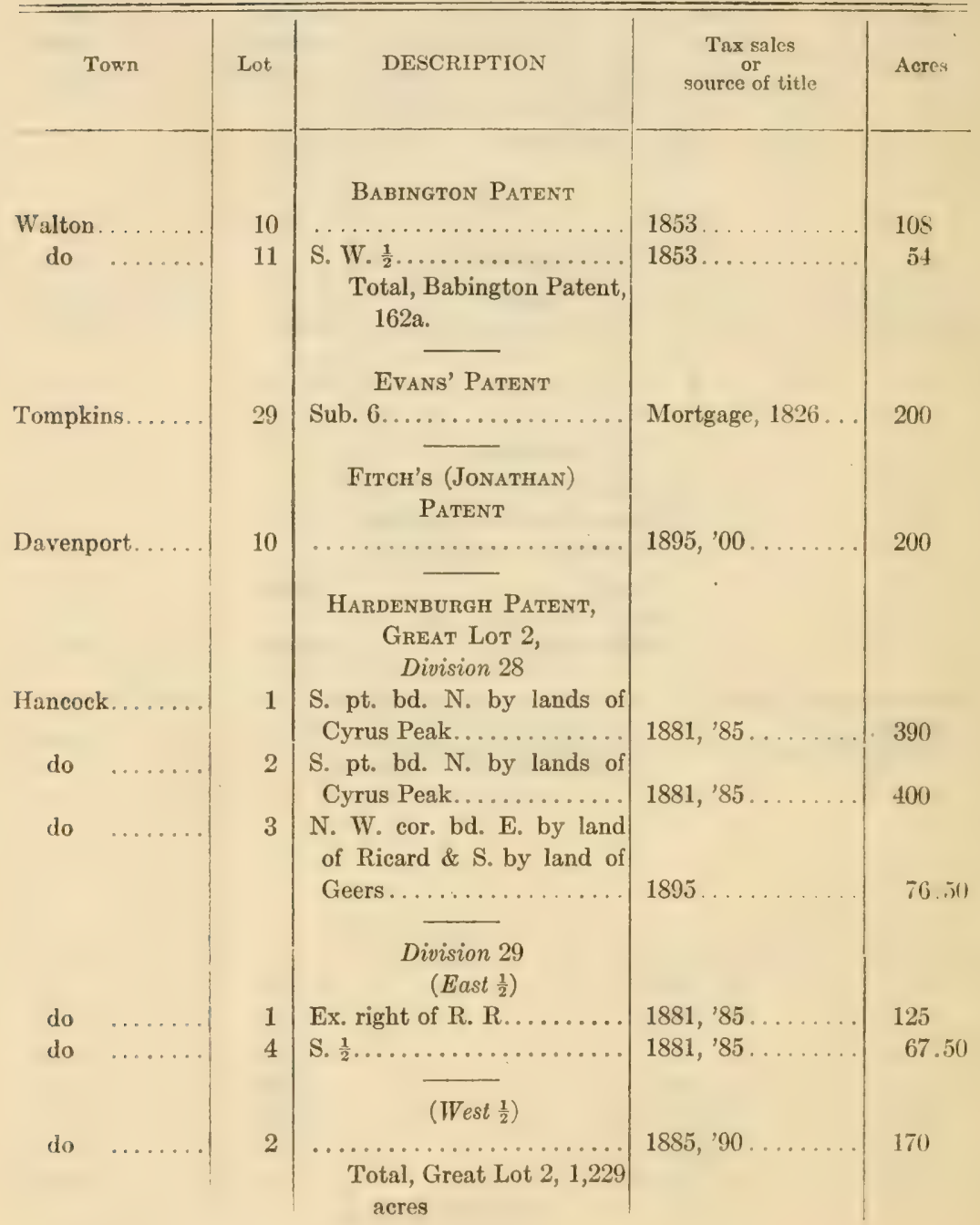




\section{Delaware County}

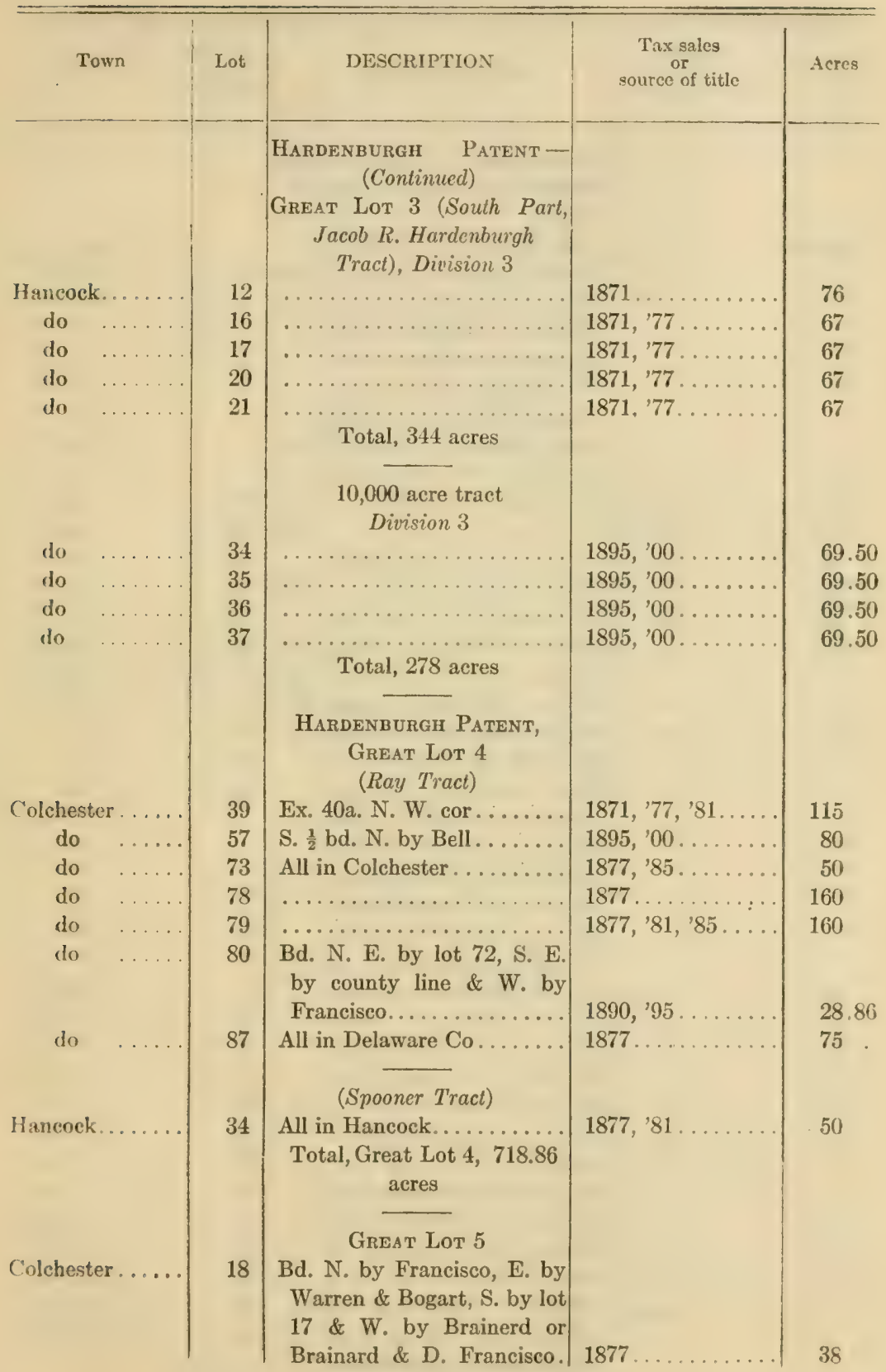


Delaware County

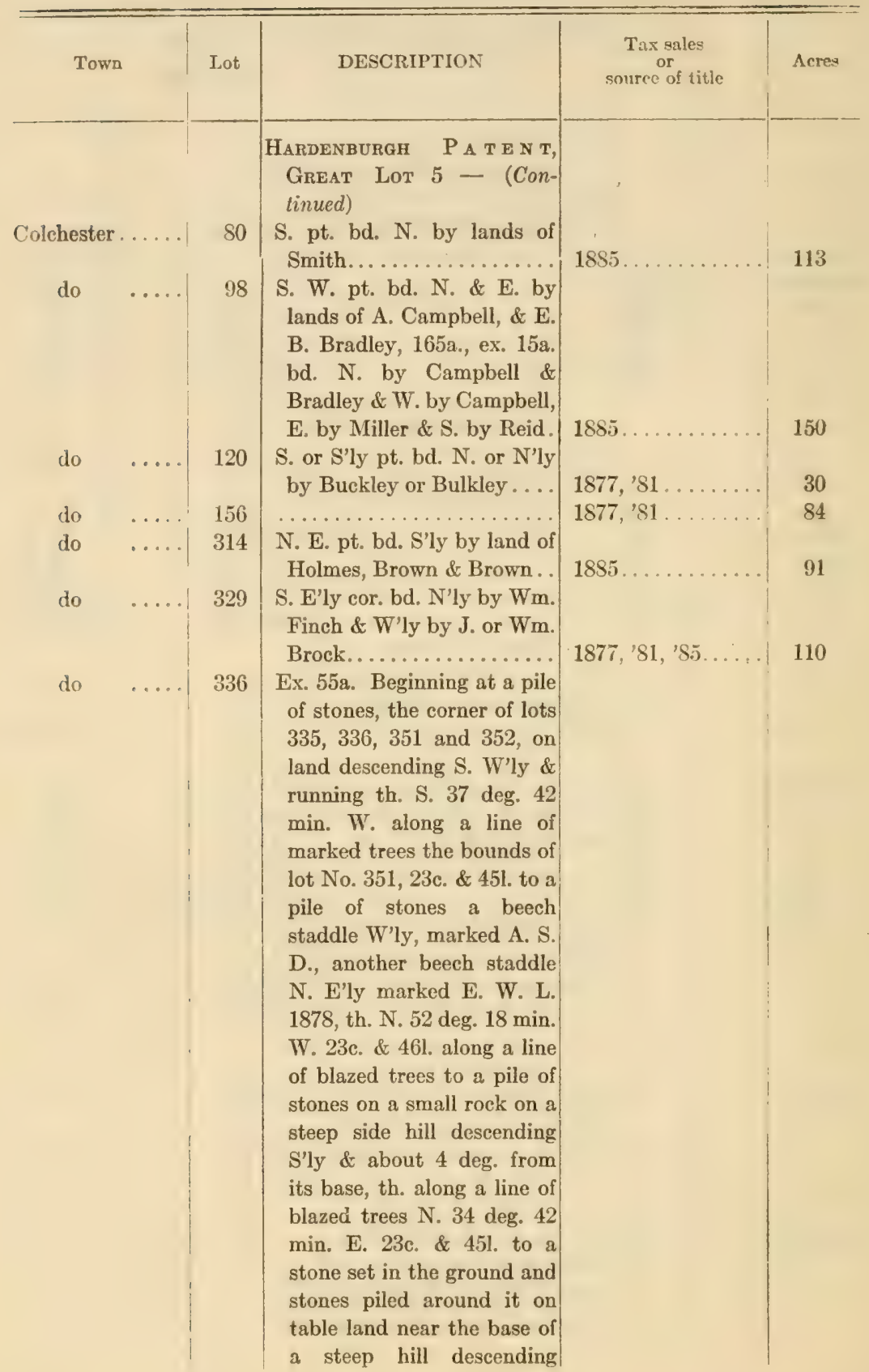


Conservation Commission.

Delaware County

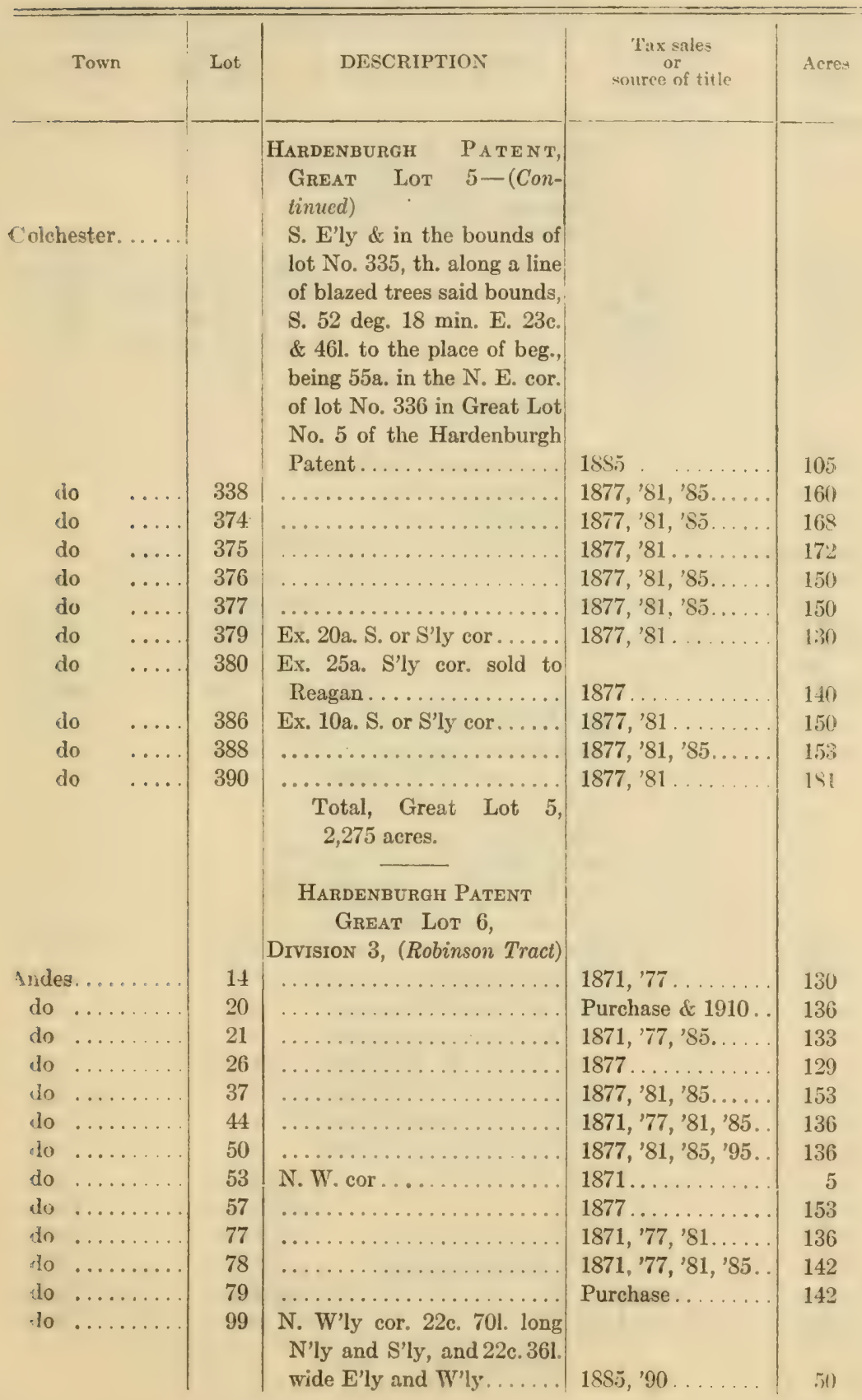


Delaware County

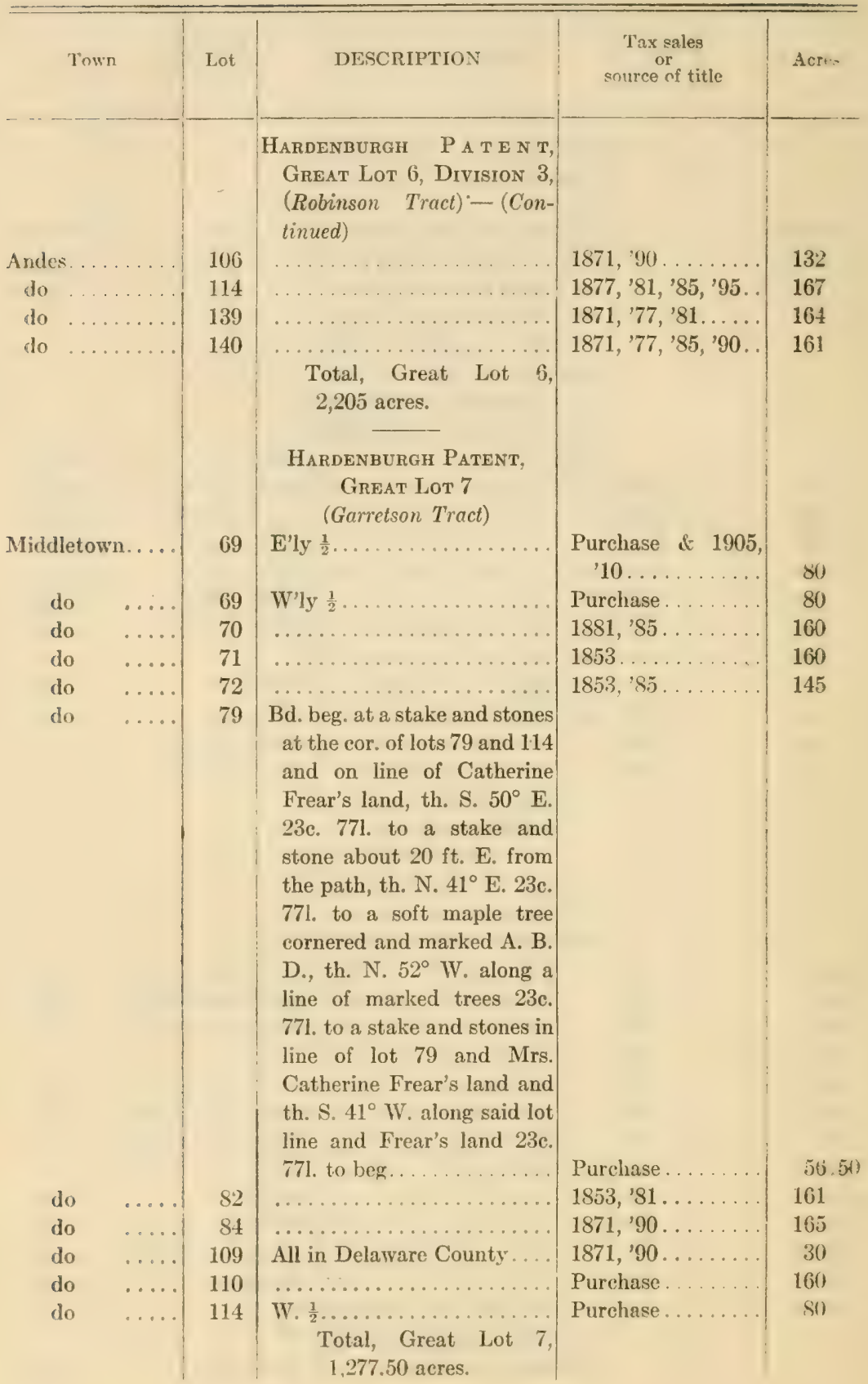


Detaware County

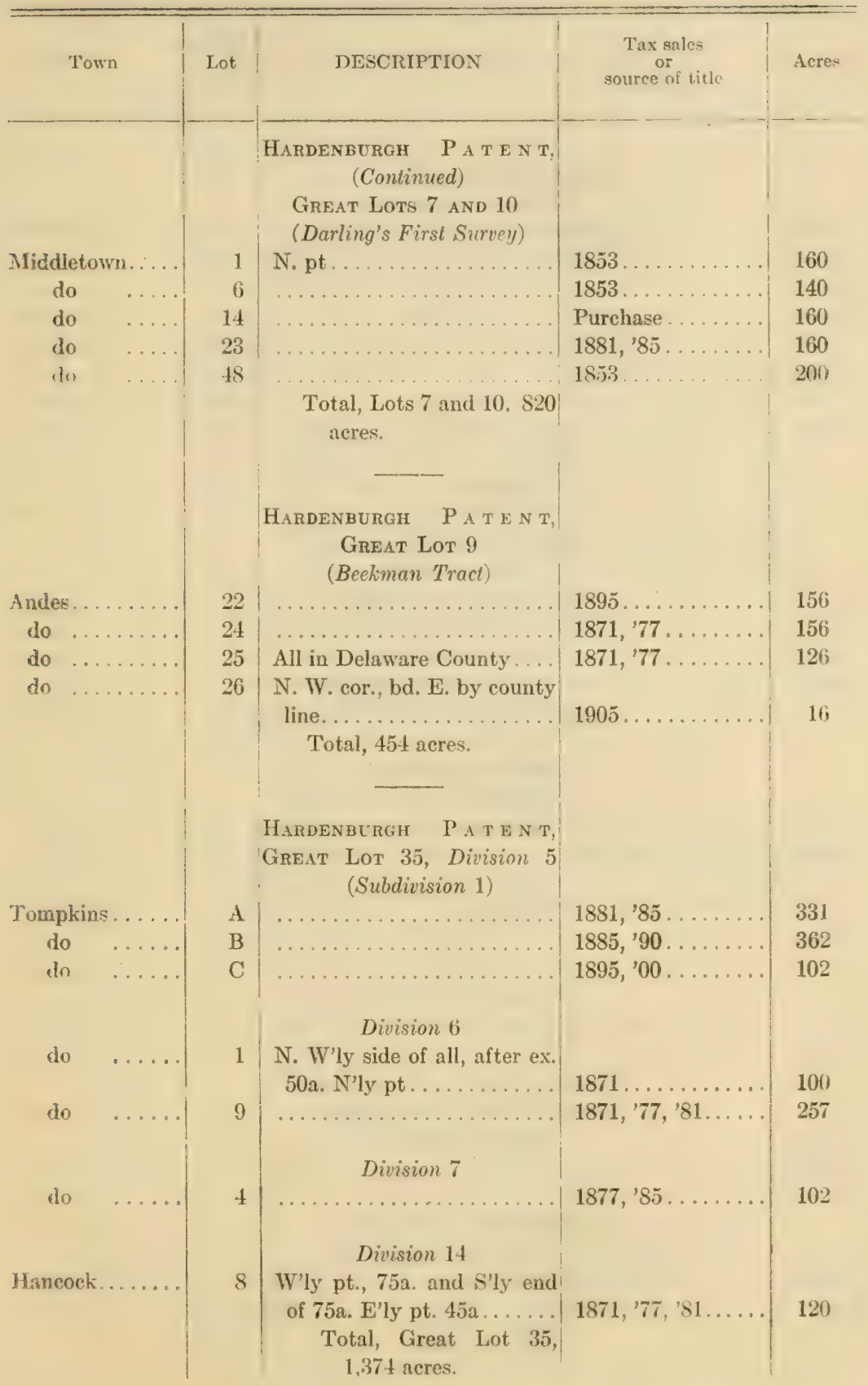


Delaware County

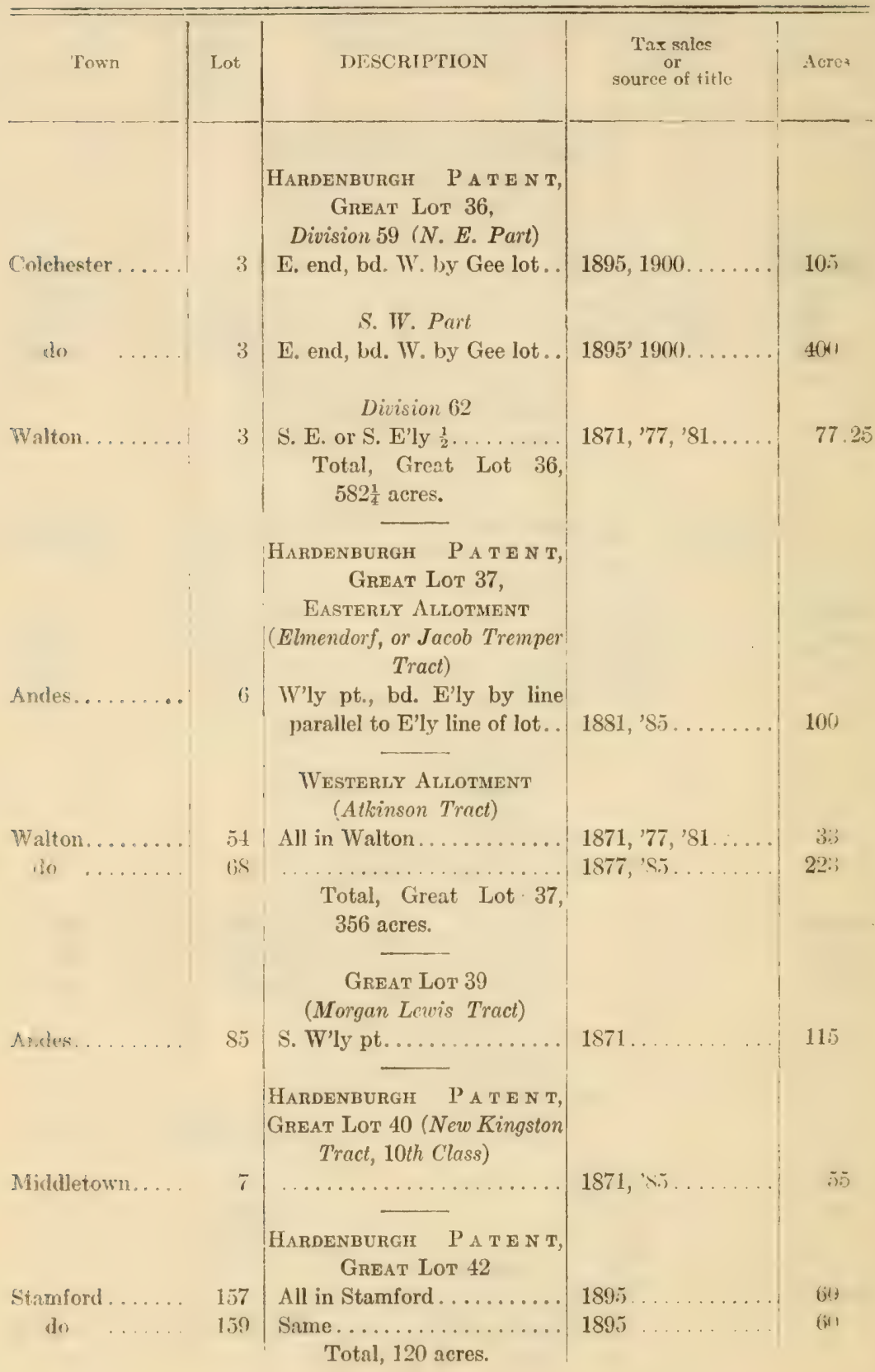


Delaware County

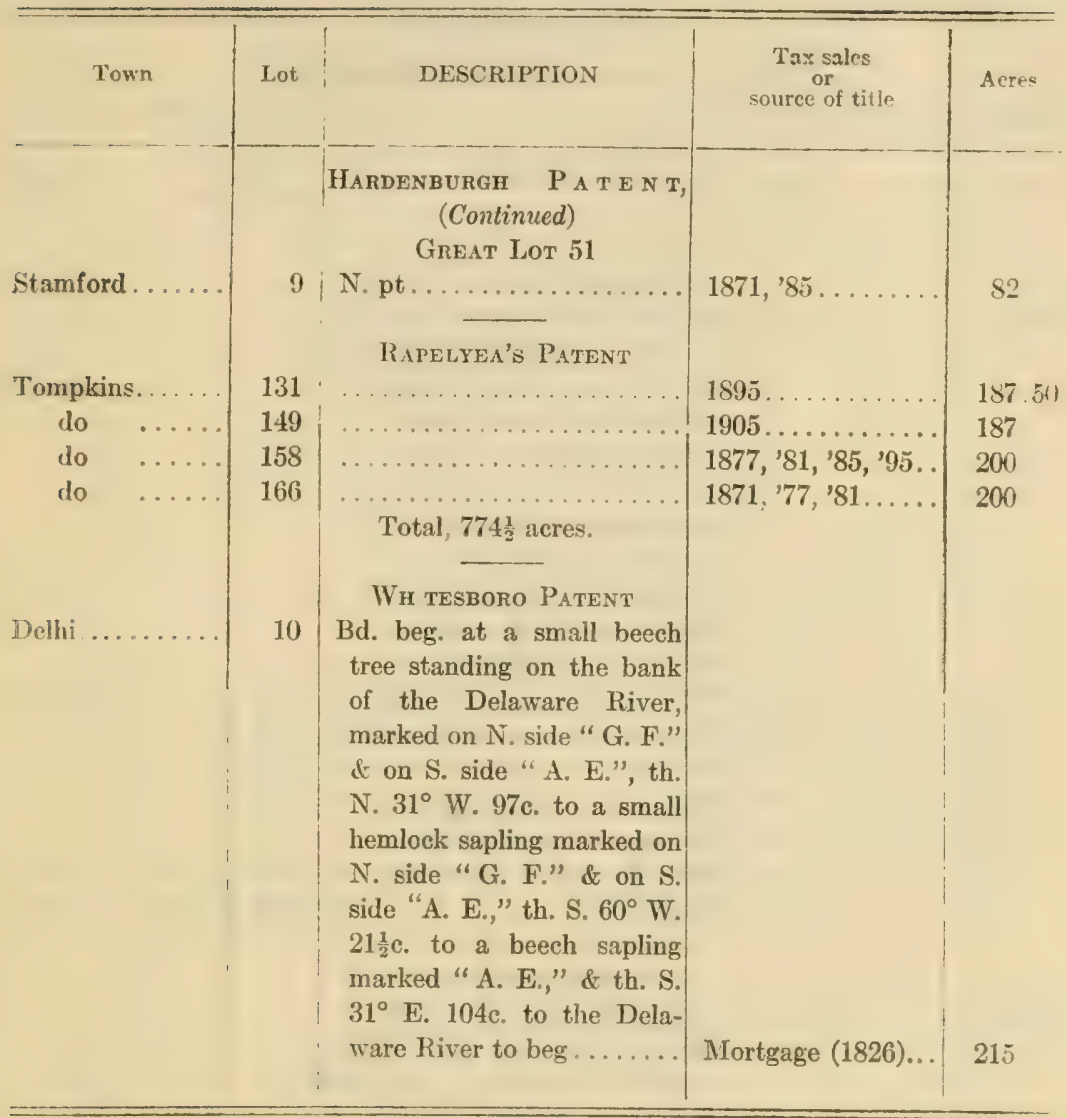

For other State land in Delaware County see Appendix. 
GREENE COUNTY

(Total number of acres, 13,295.26)

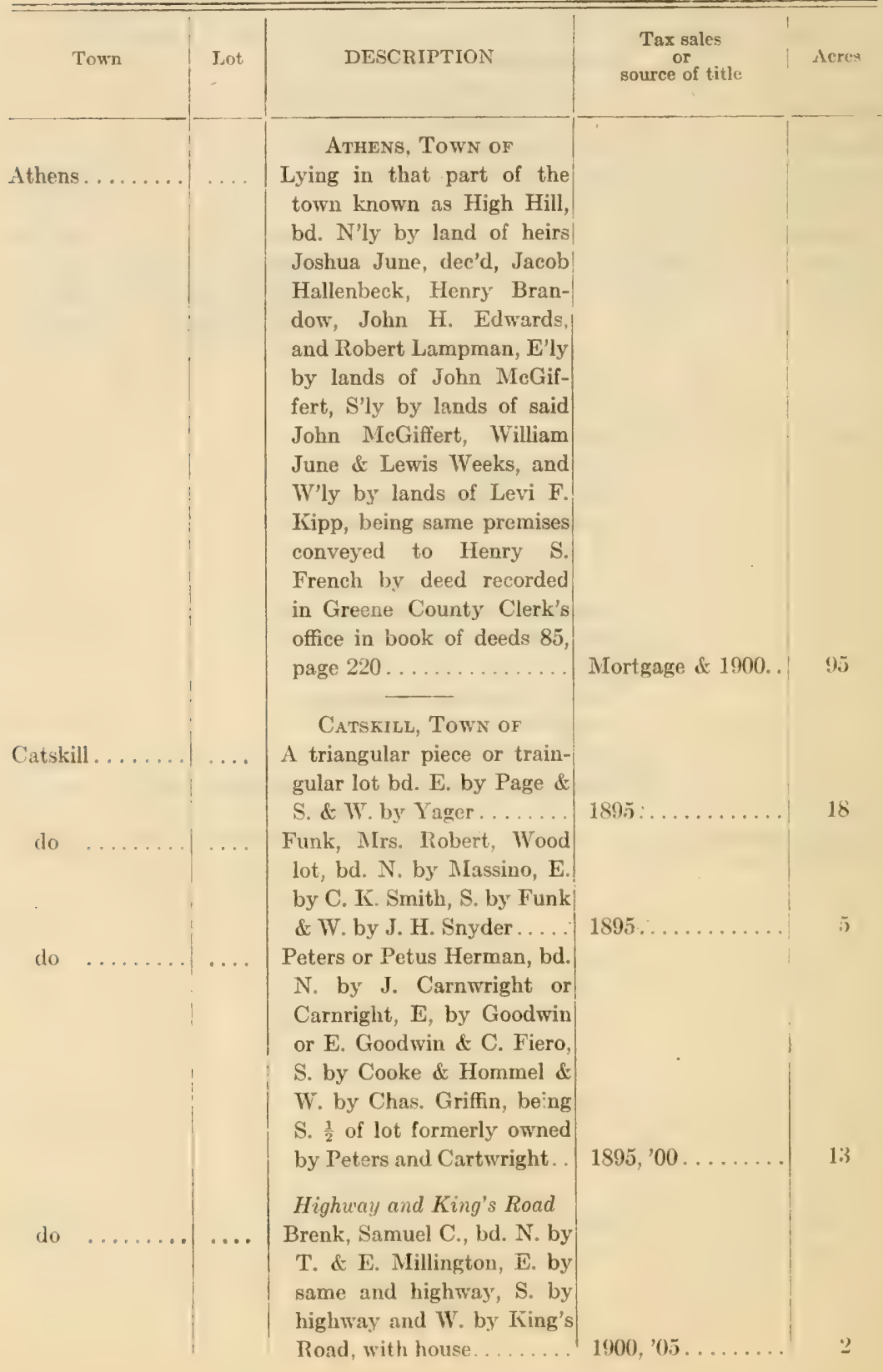


Greene County

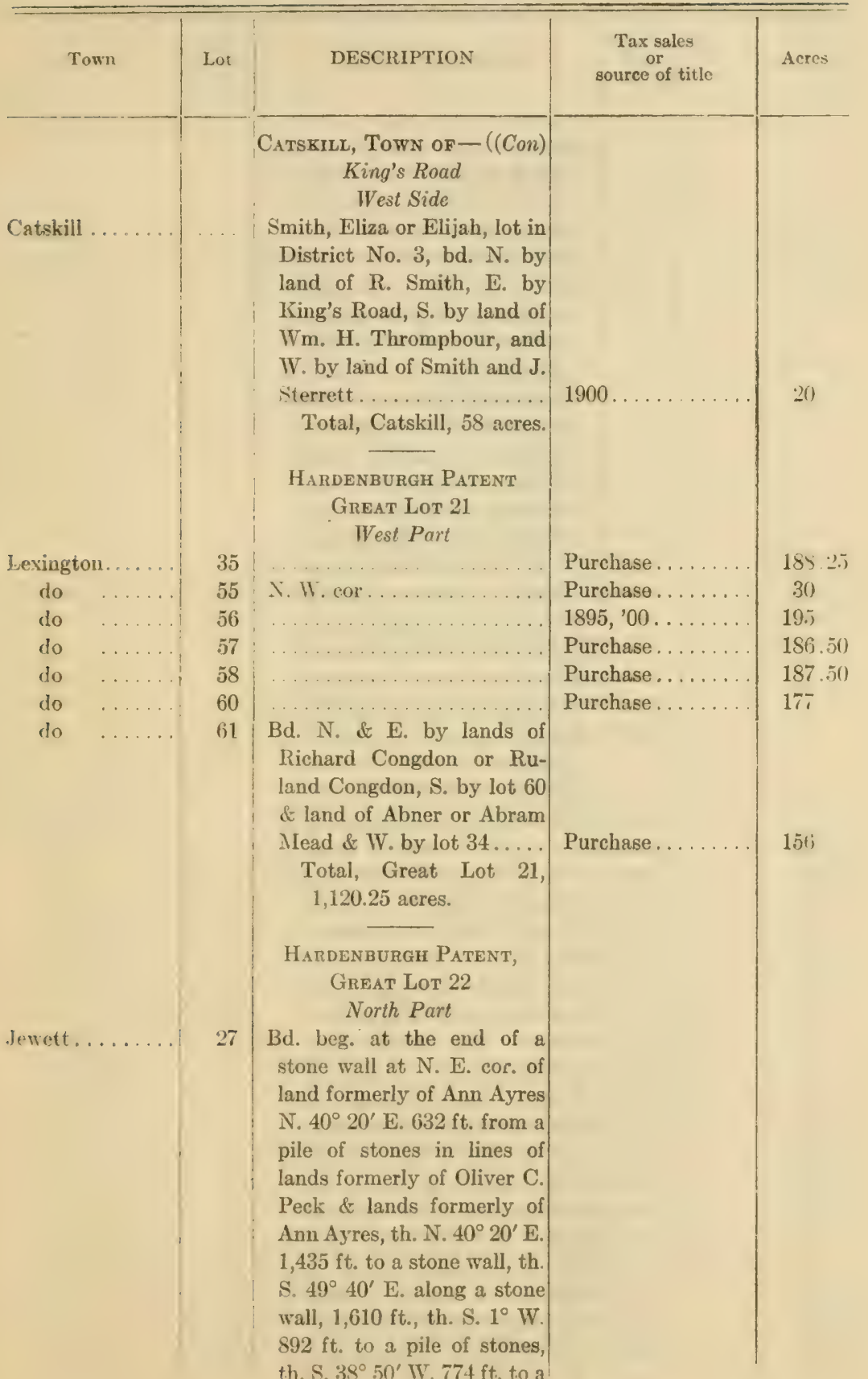




\section{Greene County}

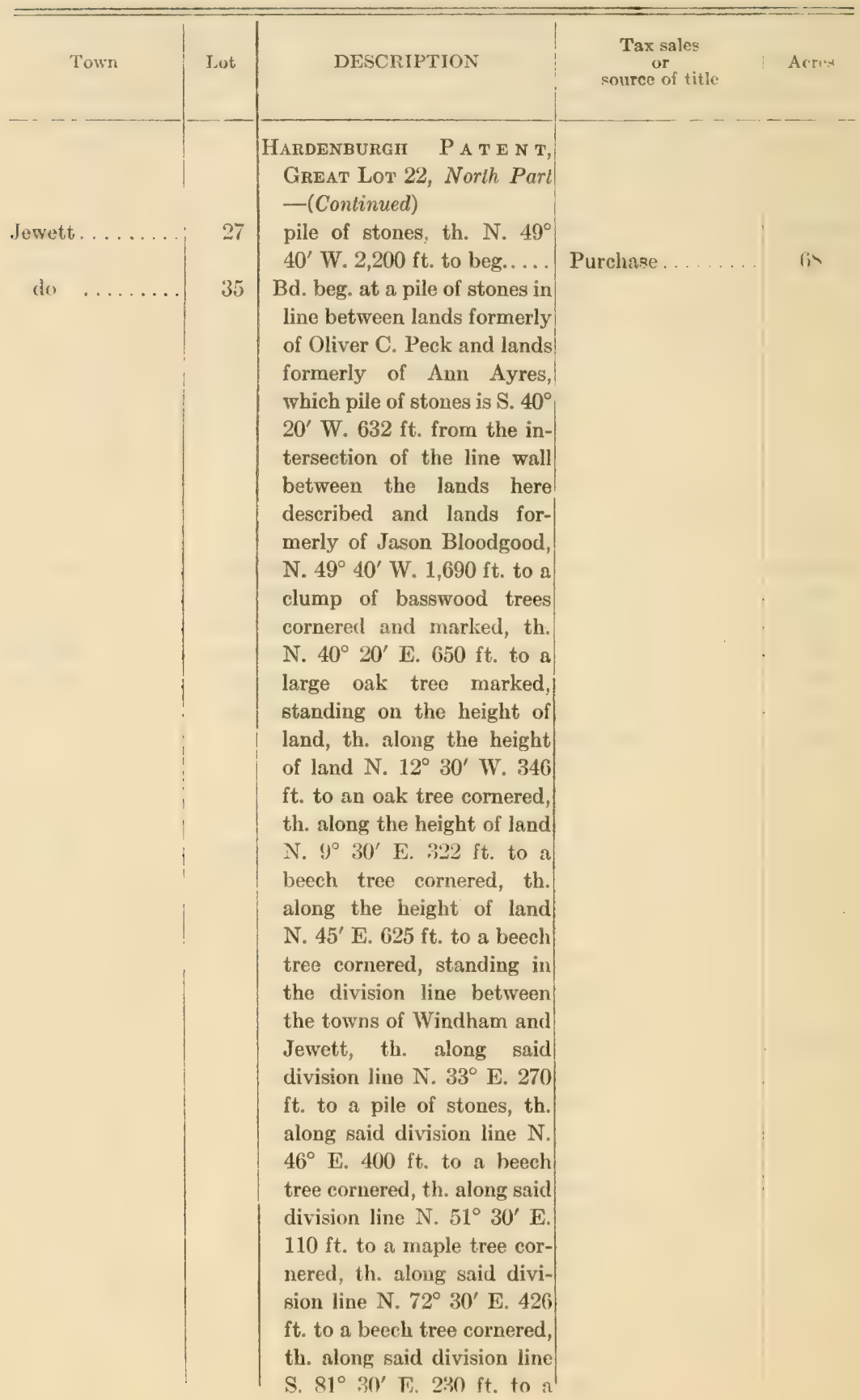


Greene Country

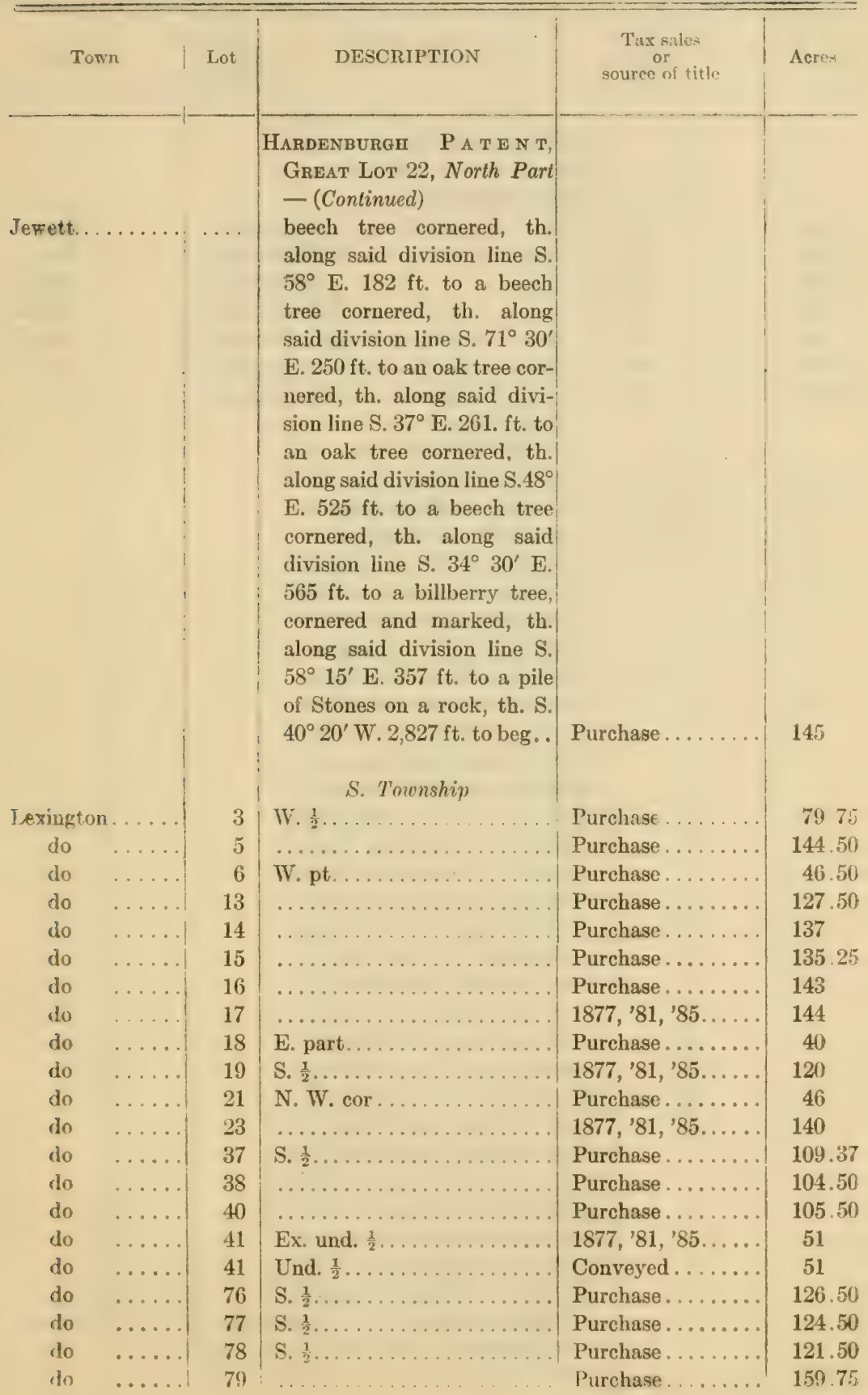


Greene County

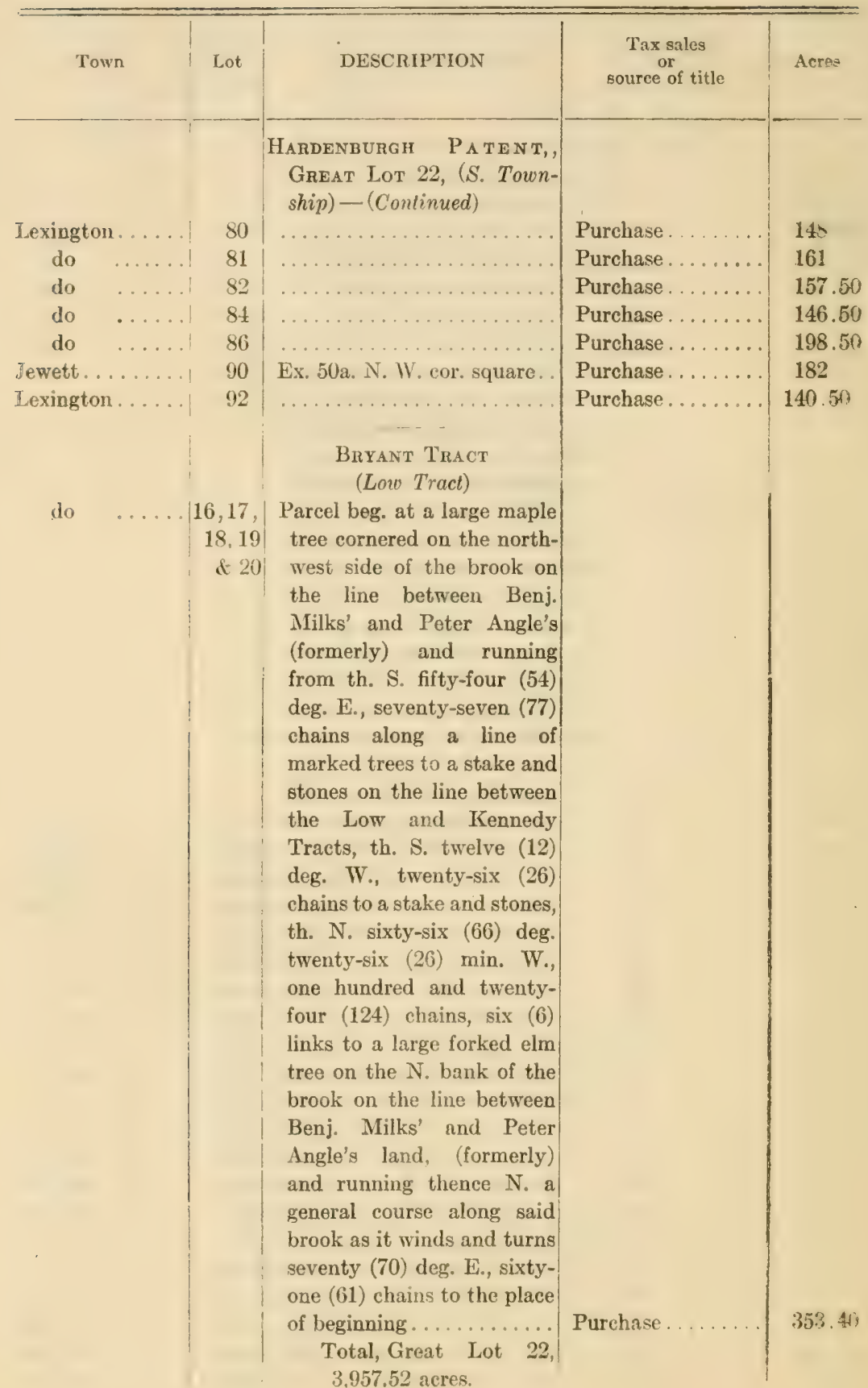


Greenk County

I. wertt

iks

in,
47

N. E. cor. \& water privilege bd. N. \& E. by land of Mills, S. W. by land of Butts .

Webster lot .

$\mathrm{Bd}$. beg. at a pile of stones at S. W. cor. of the Benj. Taylor Tract, th. along W. side of said Tract as the needle now points $\mathrm{N} .38^{\circ} 50^{\prime} \mathrm{E}$. $3,567 \mathrm{ft}$. to a pile of stones at formerly the S. W. cor. of the James Jones farm, and now in S. line of the Milton Jones farm, th. along said Milton Jones farm S. $51^{\circ} 10^{\prime}$ E. $1,222 \mathrm{ft}$. to center line of the old road leading from South Jewett to Spruceton, th. a'ong said road in a S'ly direction to $N$. line of the Benj. Taylor Tract (the courses and distances along said road are as follows, viz.) S. $54^{\circ} 50^{\prime}$ W. $347 \mathrm{ft} .9$ in., S. $40^{\circ} 46^{\prime}$ W. 291 ft. 4 in., S. $23^{\circ} 10^{\prime}$ E. 190 it. 7 in., S. $31^{\circ} 43^{\prime}$ E. $208 \mathrm{ft}$. S. $14^{\circ} 18^{\prime}$ E. 146 ft. 5 in., S. $6^{\circ}$ $1^{\prime}$ W. 147 ft. 5 in., S. $18^{\circ}$ $51^{\prime}$ W. 214 ft. 1 in., S. $21^{\circ}$ $32^{\prime}$ W. 610 ft. 8 in., S. $18^{\circ}$ $25^{\prime}$ W. 556 ft. 2 in., S. $2^{\circ} 4^{\prime}$ E. $128 \mathrm{ft} .8 \mathrm{in}$., S. $8^{\circ} 3^{\prime} \mathrm{E}$. 245 ft. 2 in., S. $10^{\circ} 47^{\prime} \mathrm{E}$. 359 ft. 4 in., S. $2^{\circ} 26^{\prime}$ E. 233 ft. 8 in., S. $16^{\circ} 1^{\prime}$ W. $171 \mathrm{ft}$. 5 in., S. $43^{\circ} 39^{\prime}$ W. 172 ft. 5 . in., S. $54^{\circ} 40^{\prime}$ W. $236 \mathrm{ft}$. 9 in., th. along said $N$. line N. $51^{\circ} 10^{\prime}$ W. $2,838 \mathrm{ft}$. to beg
Acrem

1895

Purchase. . 
Greene County

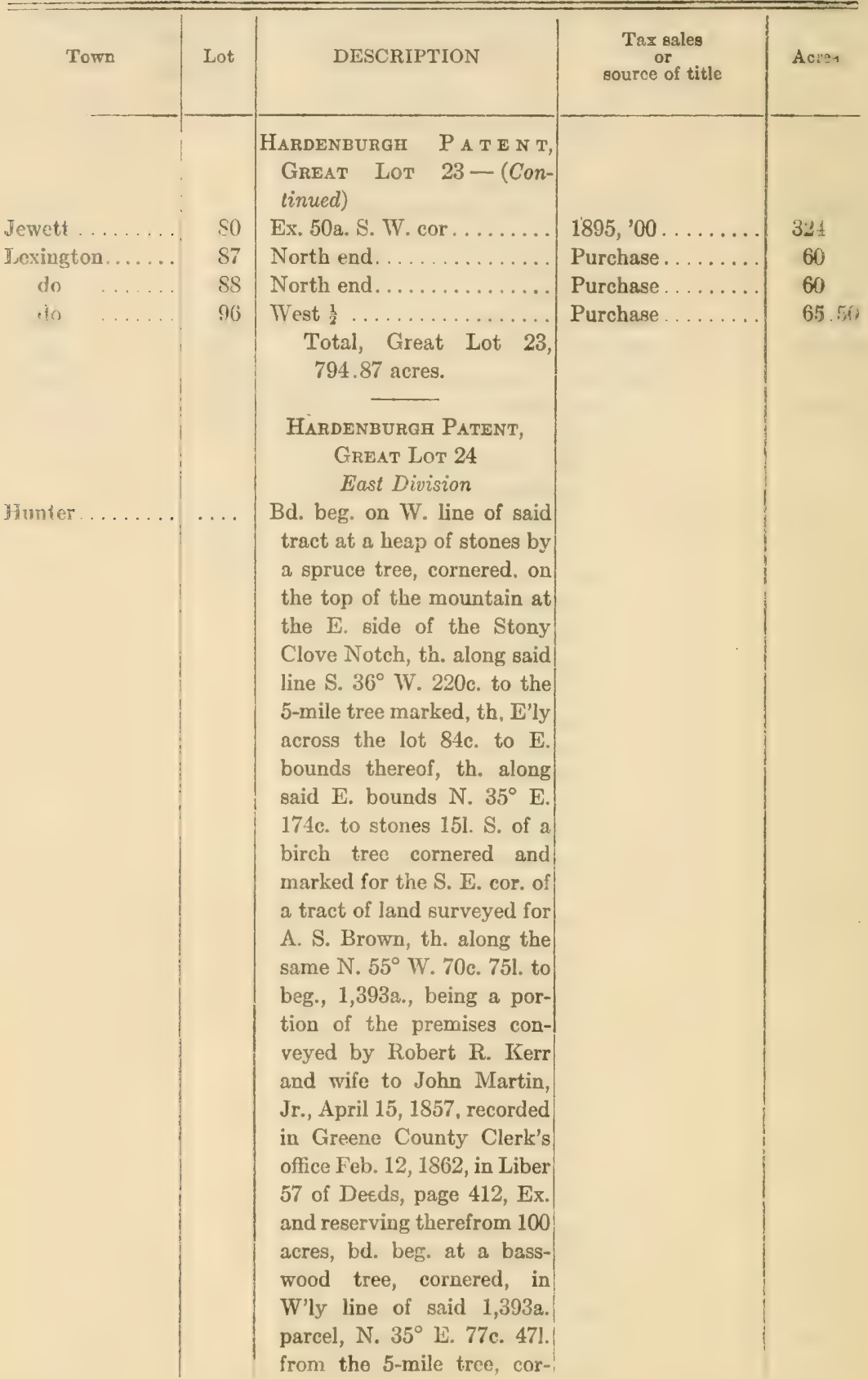


Greene County

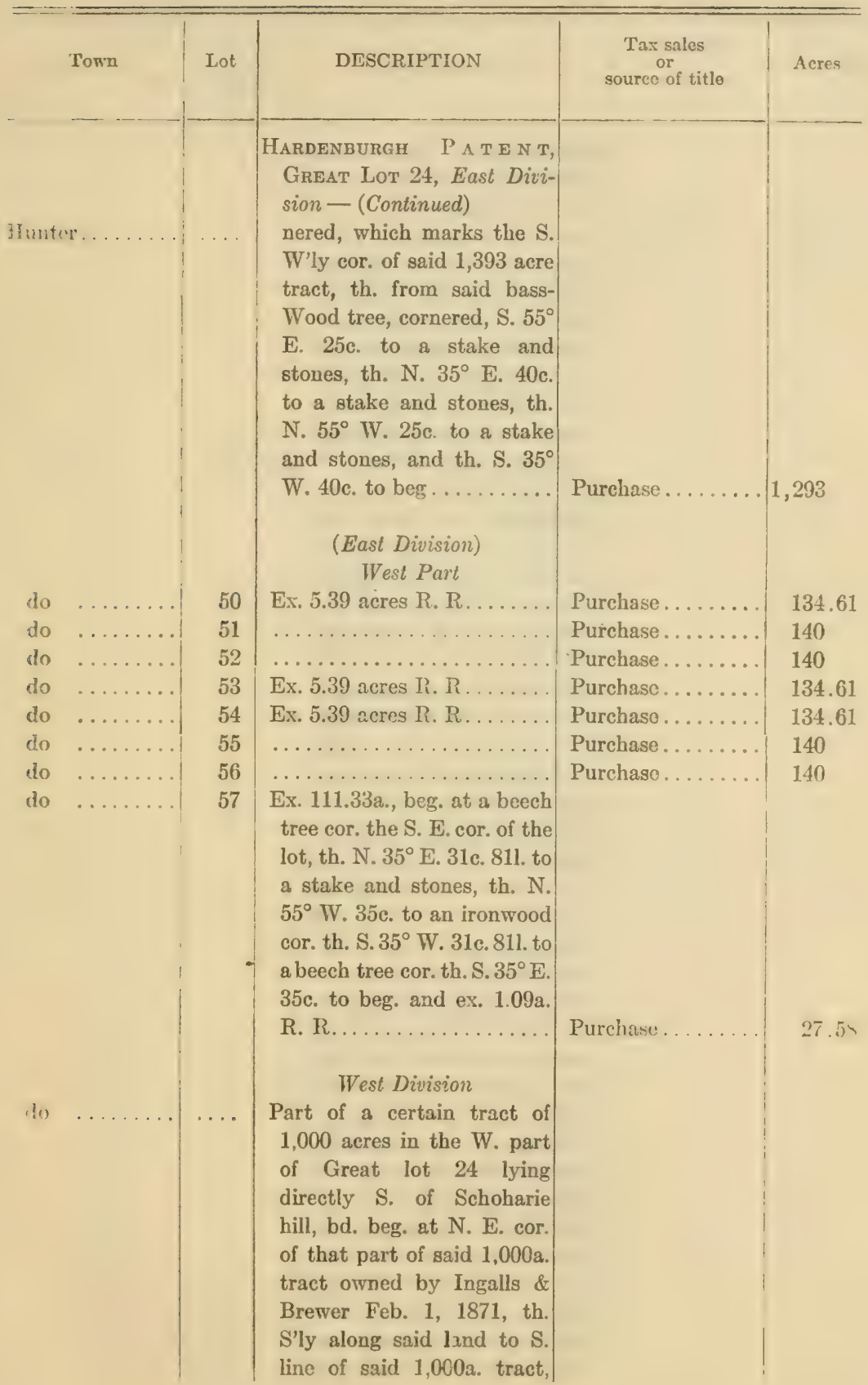


Greene County

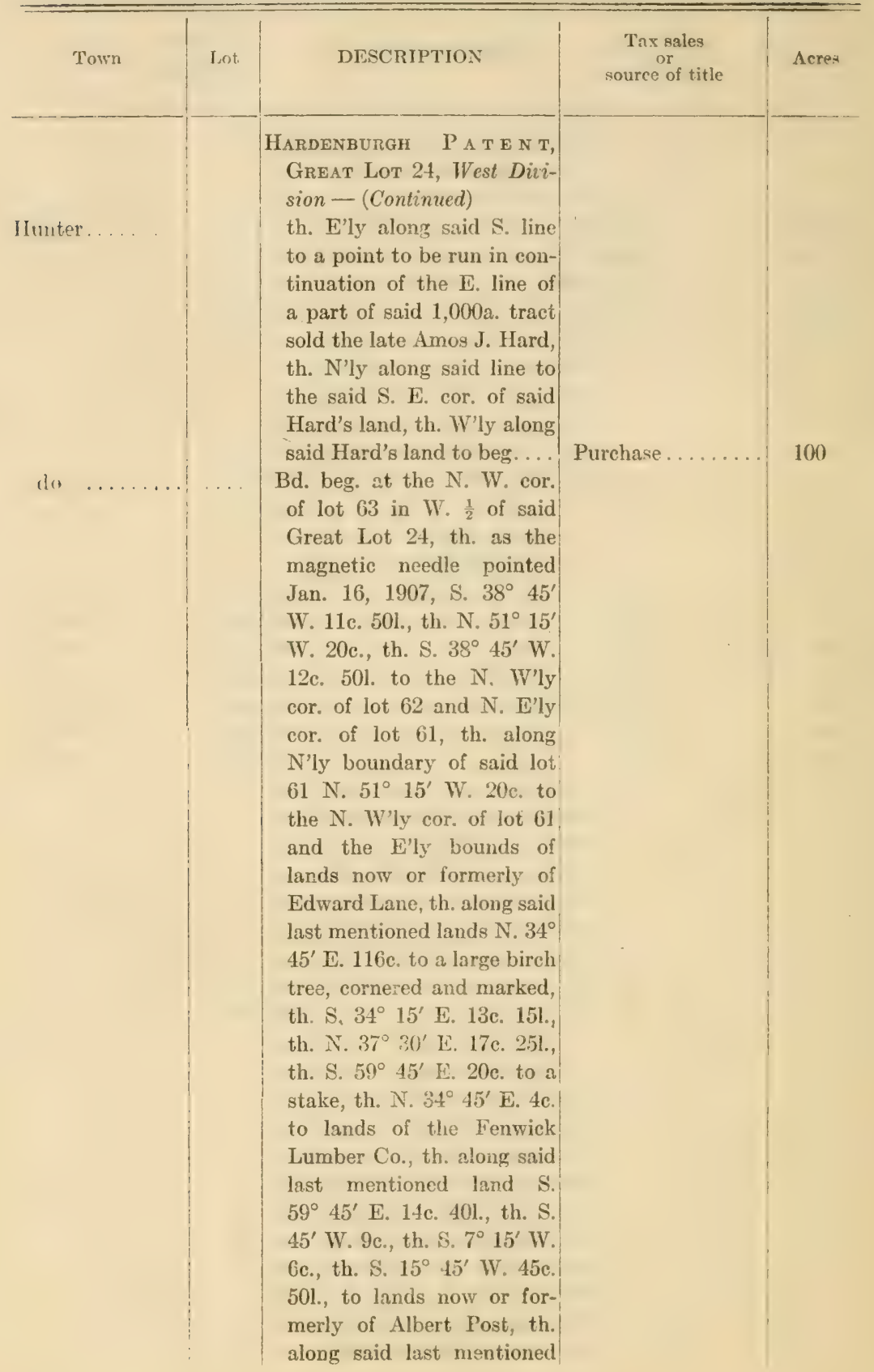


Greene County

\begin{tabular}{|c|c|c|c|c|}
\hline Town & Lot & DESCRIPTION & $\begin{array}{c}\text { Tax sales } \\
\text { or } \\
\text { source of title }\end{array}$ & Acres \\
\hline Hunter. . & $\ldots$ & 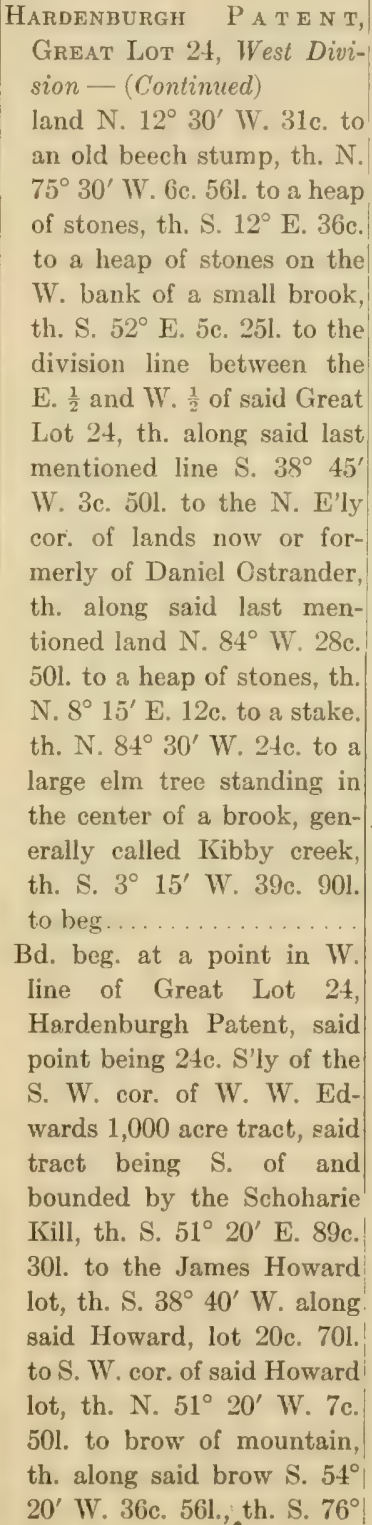 & Purchase. & 585.50 \\
\hline
\end{tabular}


Greene County

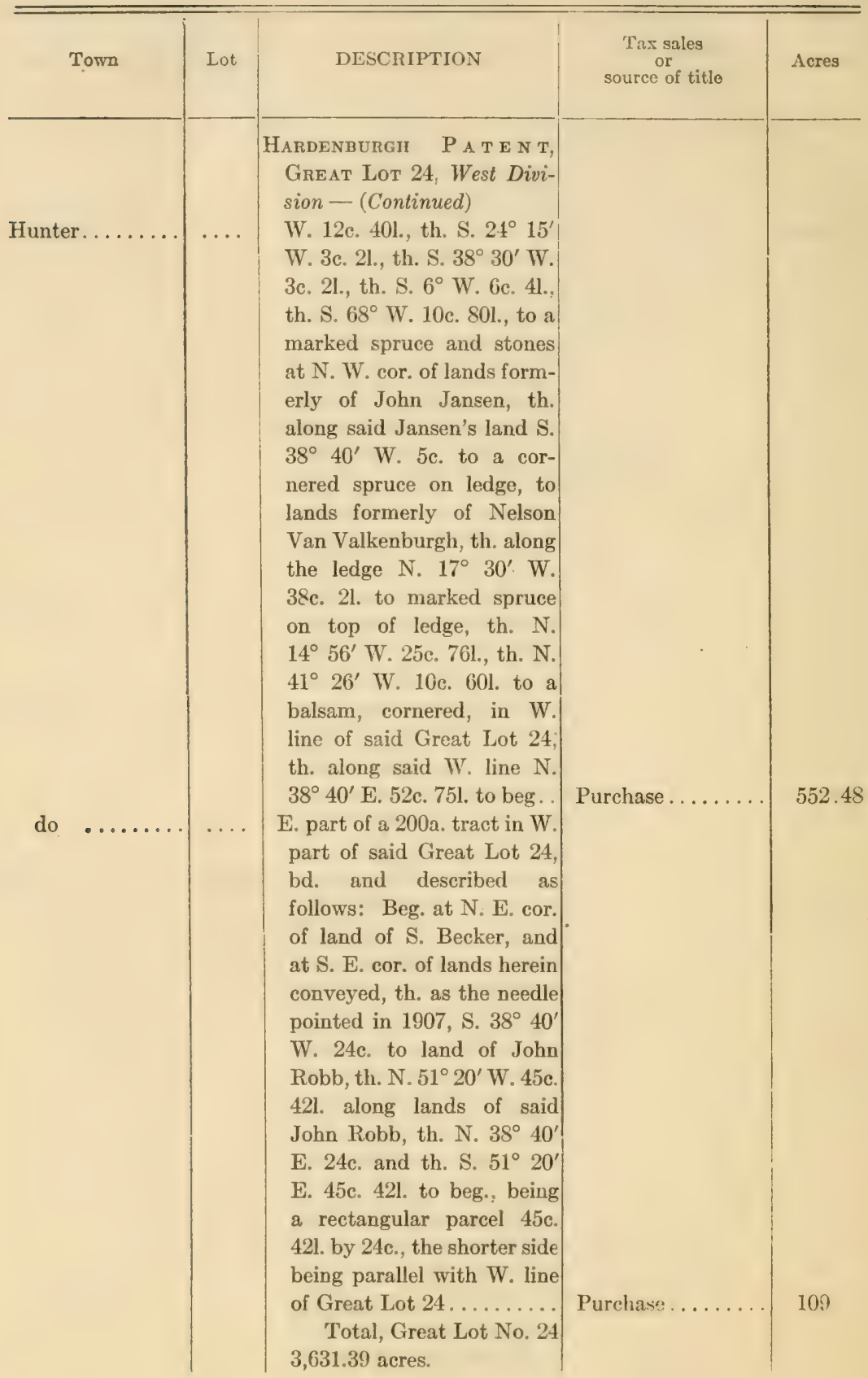


Greene County

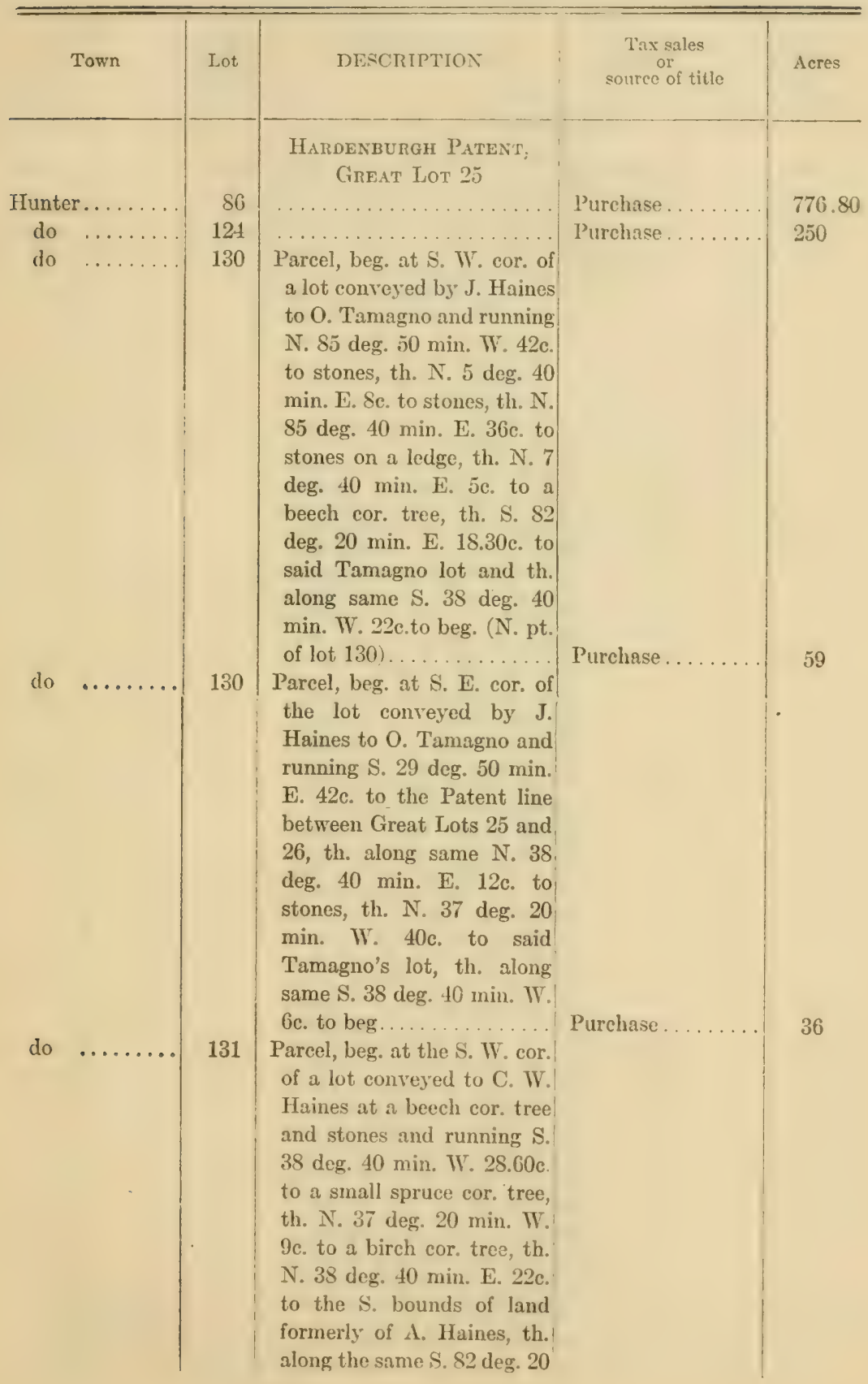


Greene County

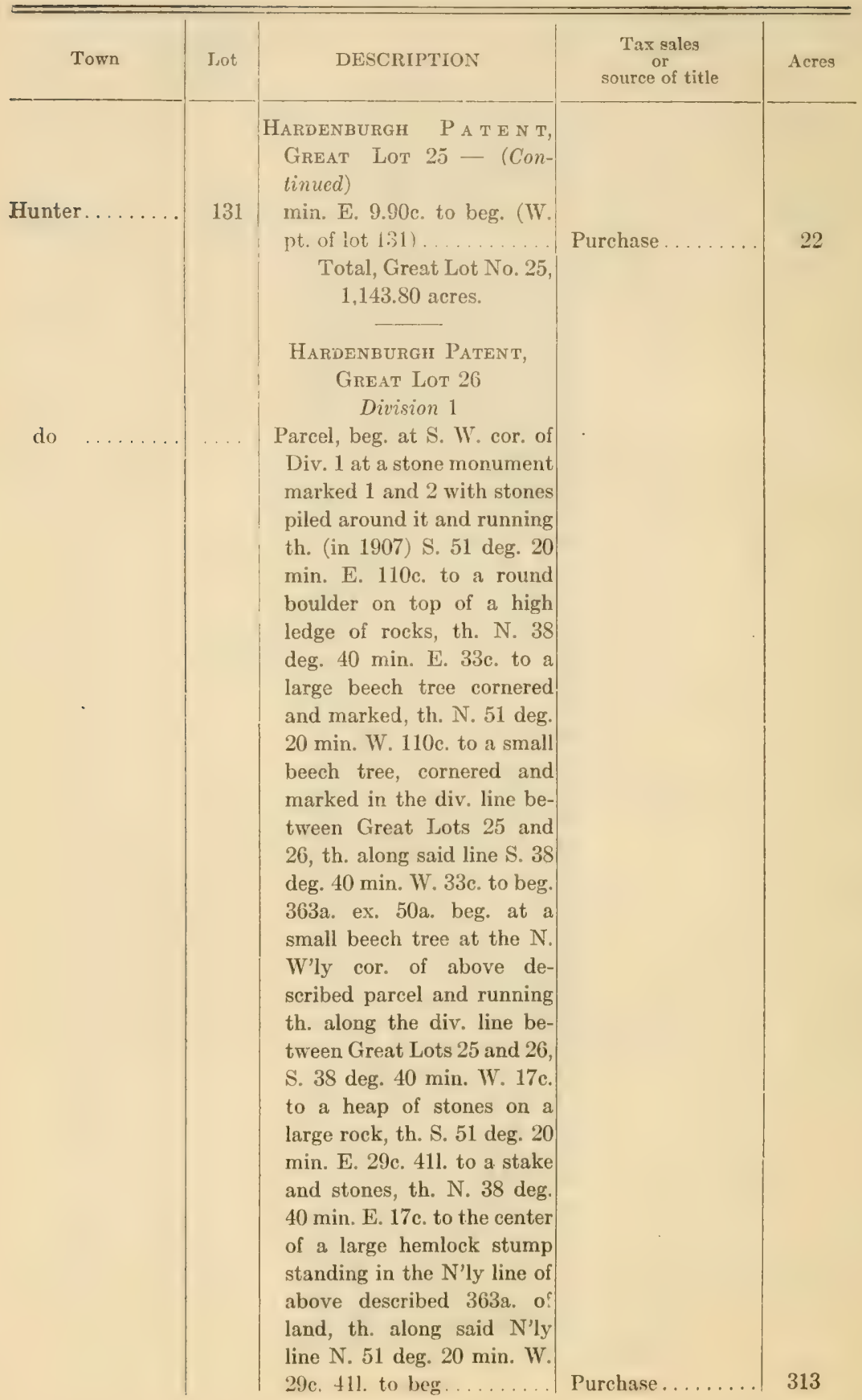


Greene County

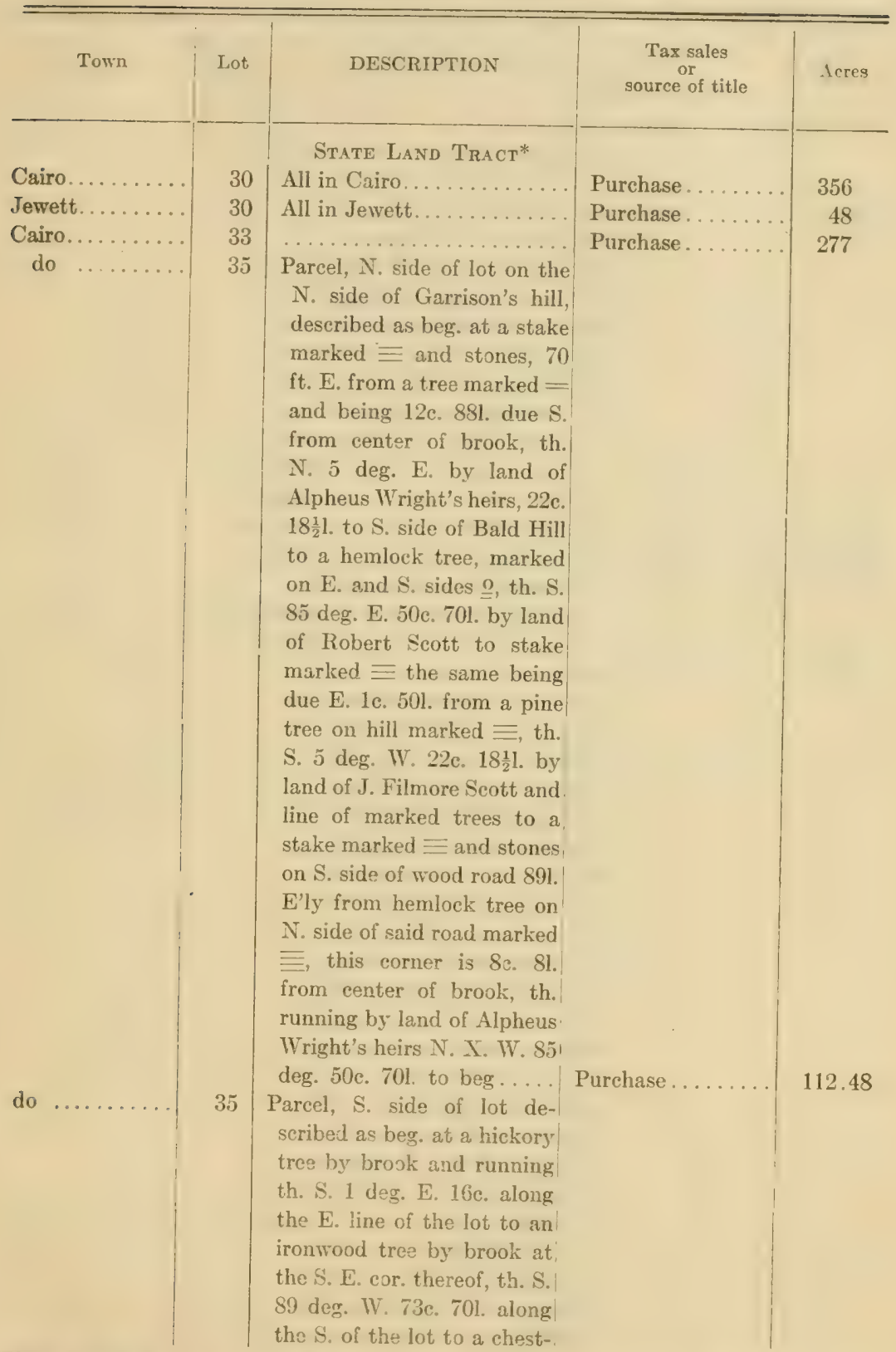

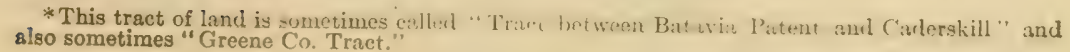


Greene Country

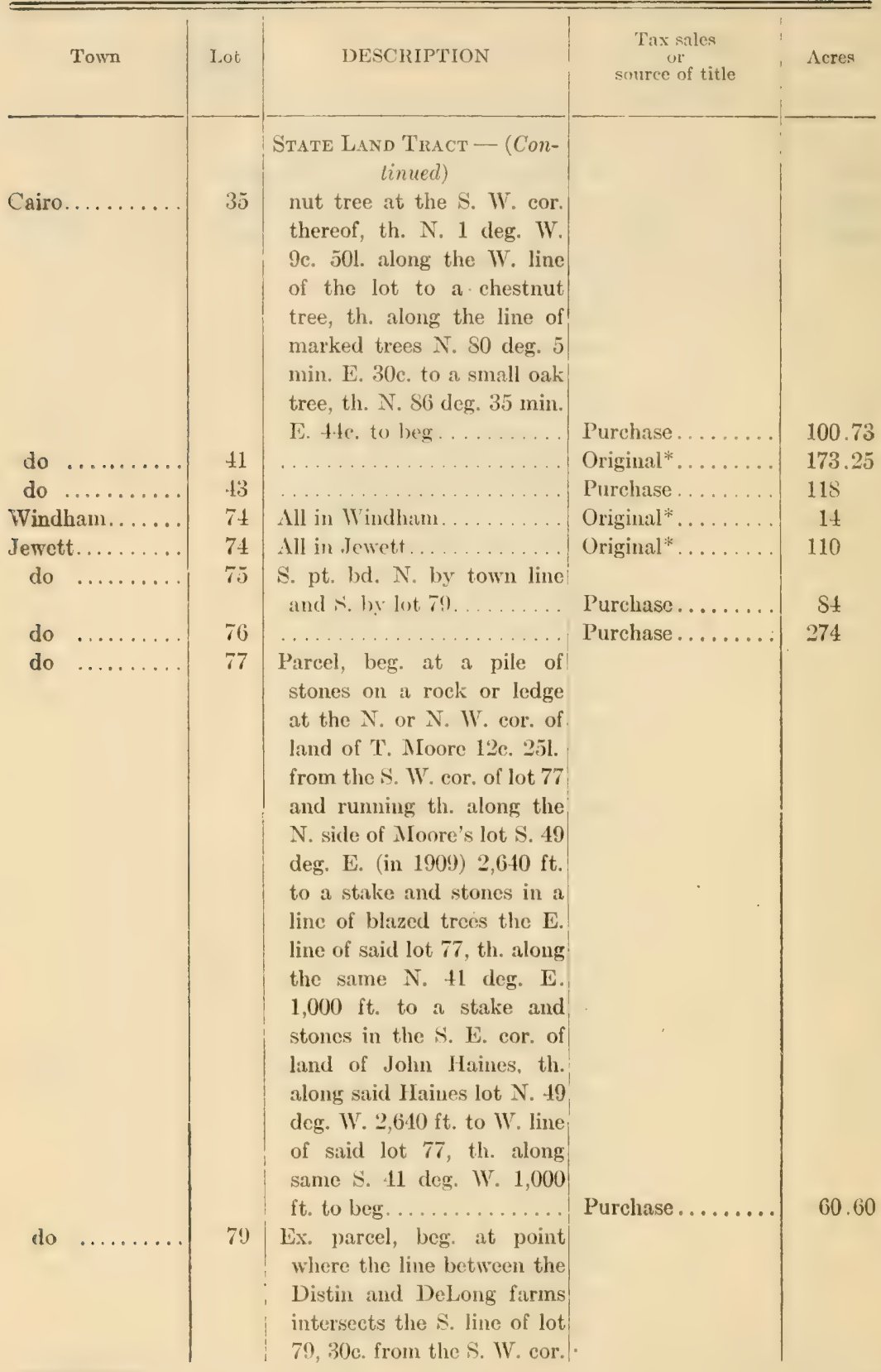


Greene County

\begin{tabular}{|c|c|c|c|c|}
\hline Town & Lot & DESCRIPTION & $\begin{array}{c}\text { Tnx sales } \\
\text { or } \\
\text { source of title }\end{array}$ & Acres \\
\hline Jewett & 79 & 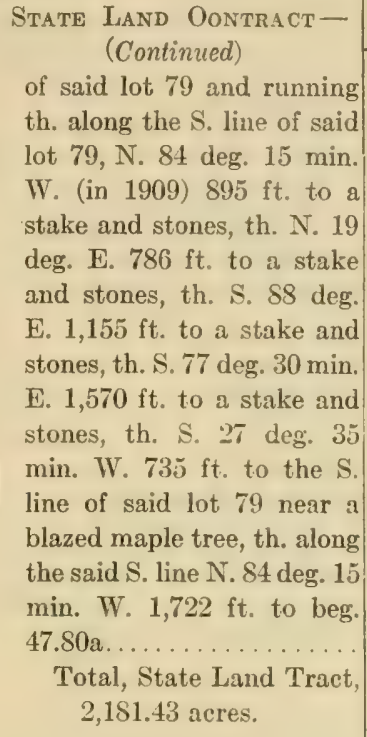 & Purchase.... & 453.37 \\
\hline
\end{tabular}

For other State land in Greene County see Appendix. 
SULLIVAN COUNTY

(Total number of acres, 1,043.55)

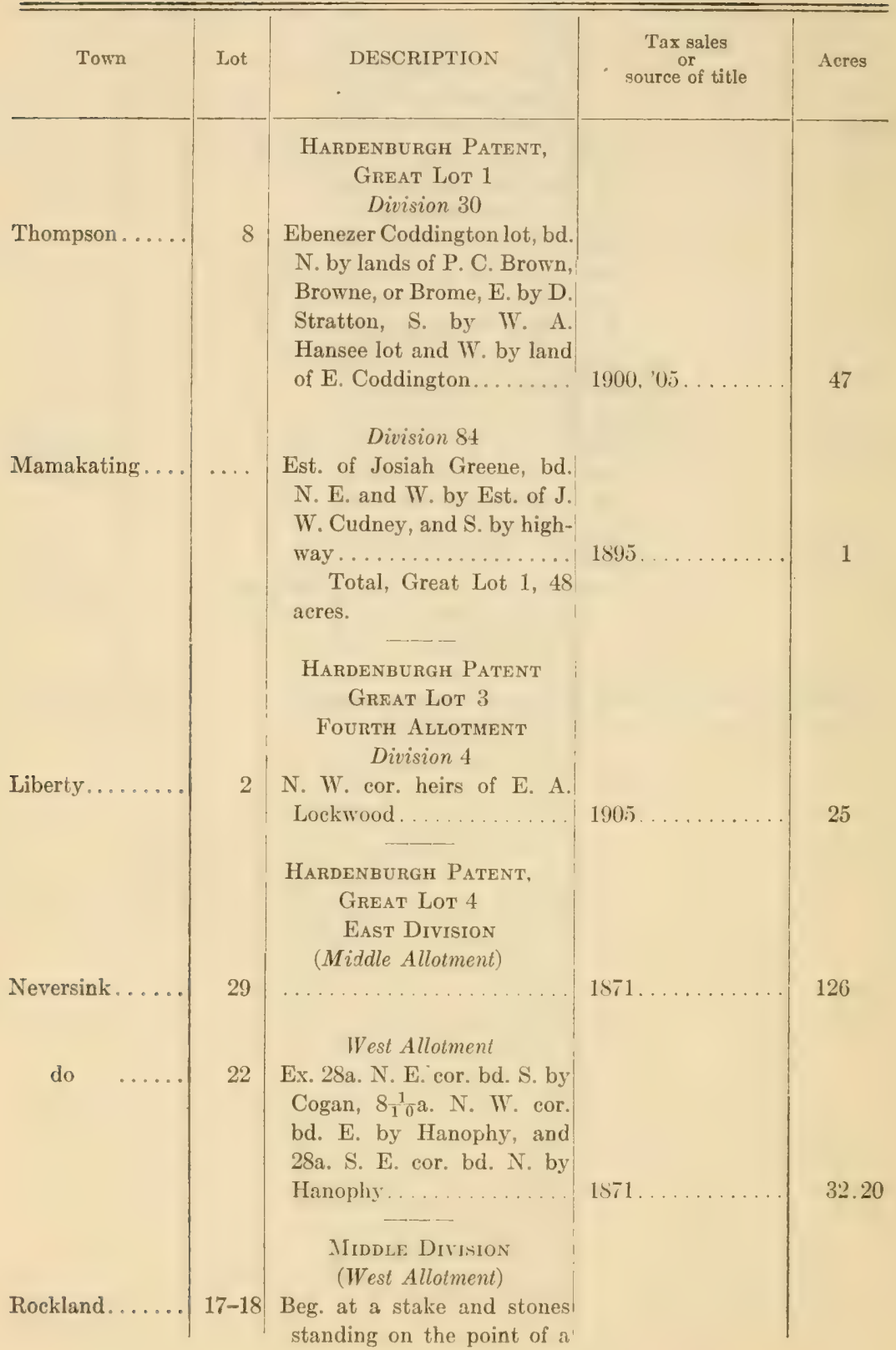


Sullivan County

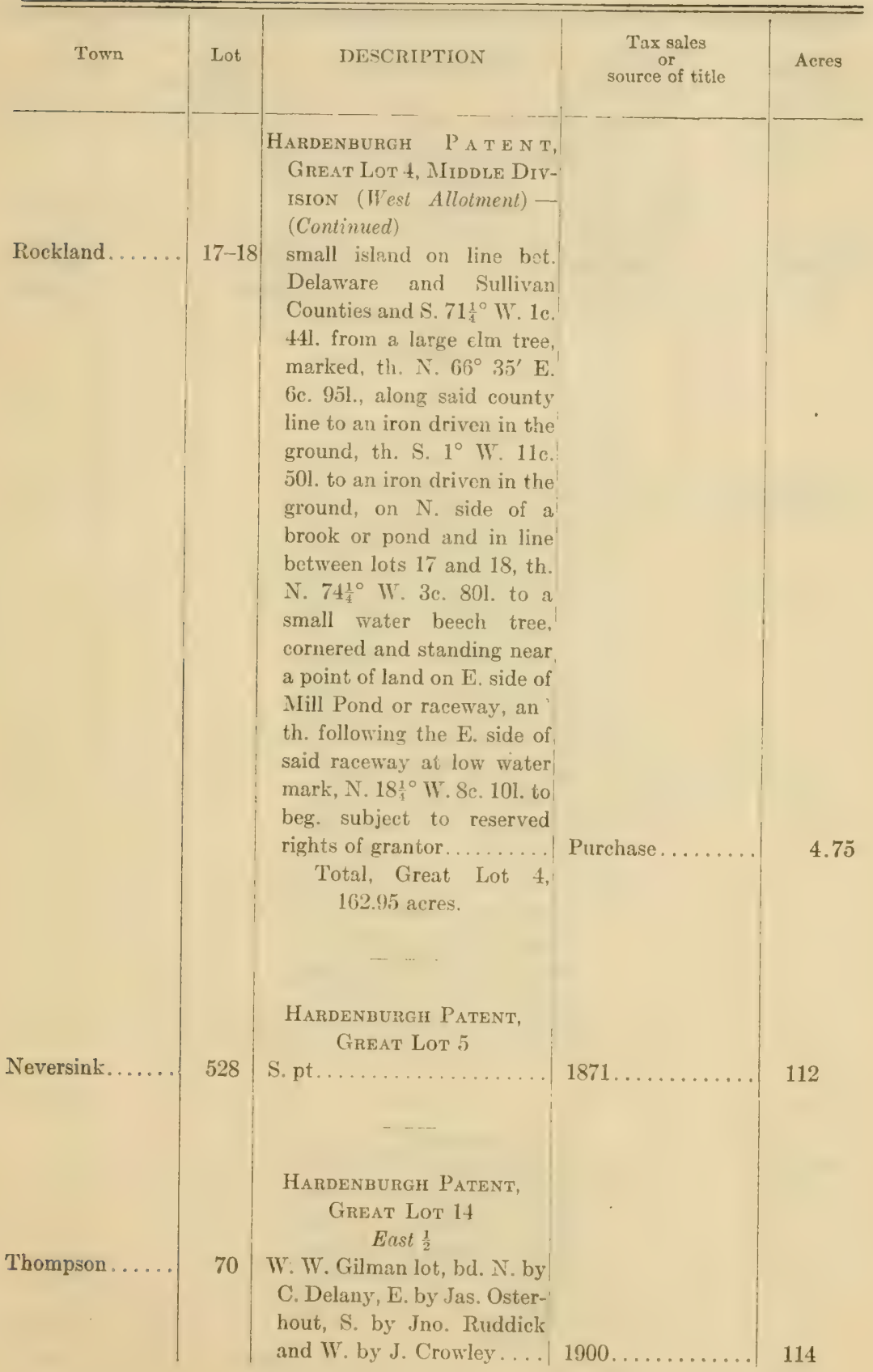


Sullivan County

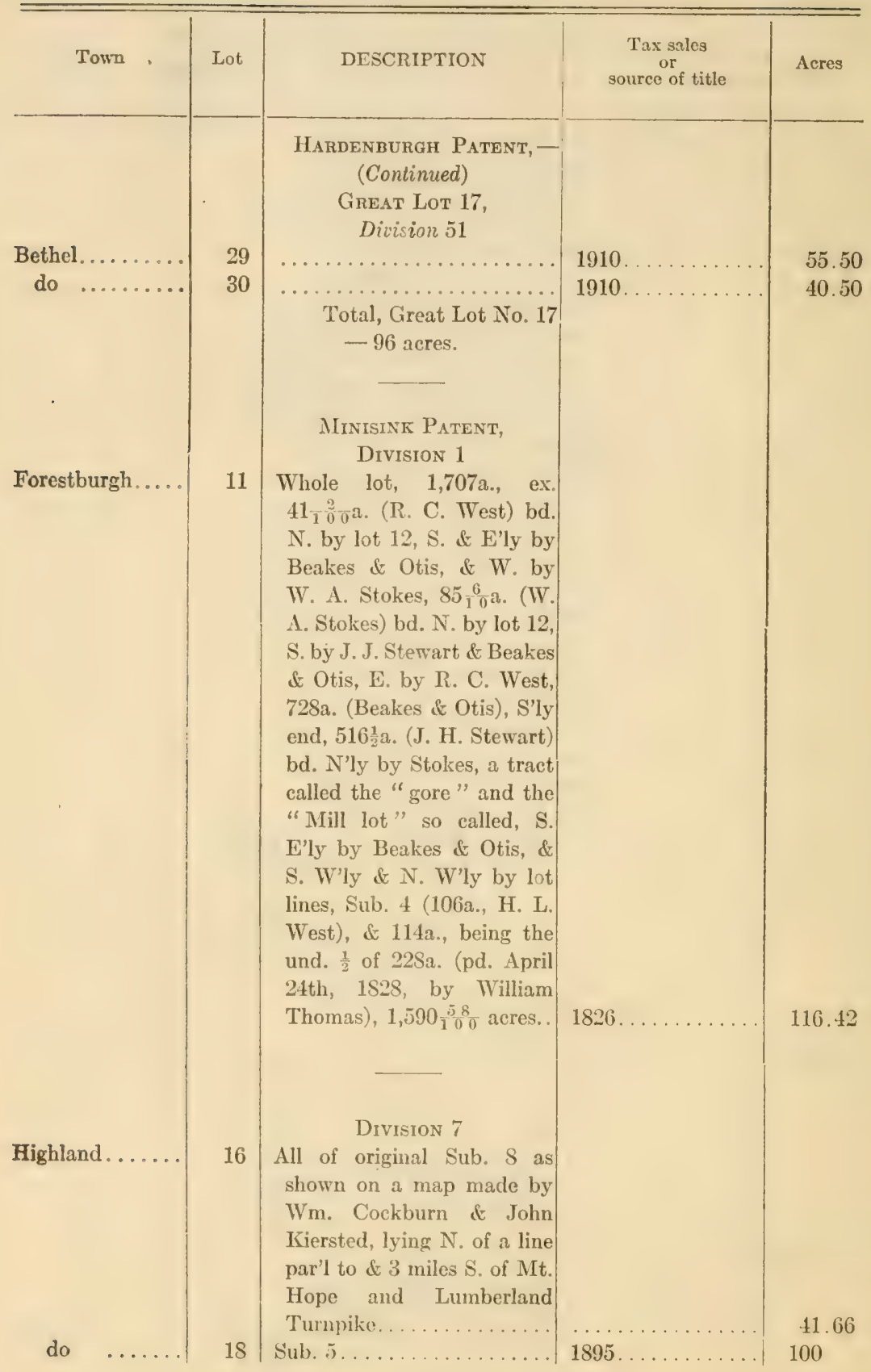


Conserration Commissiox.

Suluivan County

\begin{tabular}{|c|c|c|c|c|}
\hline Town & I.ot & DESCRIPTION & $\begin{array}{c}\text { Tax sales } \\
\text { or } \\
\text { source of title }\end{array}$ & Acres \\
\hline & 18 & $\begin{array}{l}\text { Minisink Patent, Devision } \\
7-\text { (Continued) } \\
\text { Sub. } 1 \mathrm{~s} \ldots \ldots \ldots \ldots \ldots \ldots\end{array}$ & 151701 & \\
\hline Lumberland... & 31 & Sub. $25, \ldots \ldots \ldots \ldots \ldots$ & $1895,(30, \ldots$ & 16.40 \\
\hline do & .31 & Sub. $26 \ldots \ldots \ldots \ldots$ & $1895,00 \ldots$ & 64 \\
\hline do & 37 & $\begin{array}{l}\text { Sub. } 4 \ldots \ldots \ldots \ldots \ldots \\
\text { Total, Minisink Patent, } \\
\text { 485. } 1 \text { acres. }\end{array}$ & 189.), '00. . . & 67 \\
\hline Mamakating. . & & $\begin{array}{l}\text { Prillipsport VildAge } \\
\text { Hanyen or Hanyene, William| } \\
\text { H., heirs; bd. N. by lands of } \\
\text { Mrs. James Allen, E. by the } \\
\text { Kingston Road, or Kingston } \\
\text { Public Road, S. by lands of } \\
\text { Mrs. Berdell S., or Bedel S. } \\
\text { Johnson, and W. by lands } \\
\text { of estate of J. B. Daved or } \\
\text { Deved................... }\end{array}$ & 190.5. & $\frac{1}{8}$ \\
\hline
\end{tabular}


ULSTER COUNTY

(Total number of acres, 84,009.73)

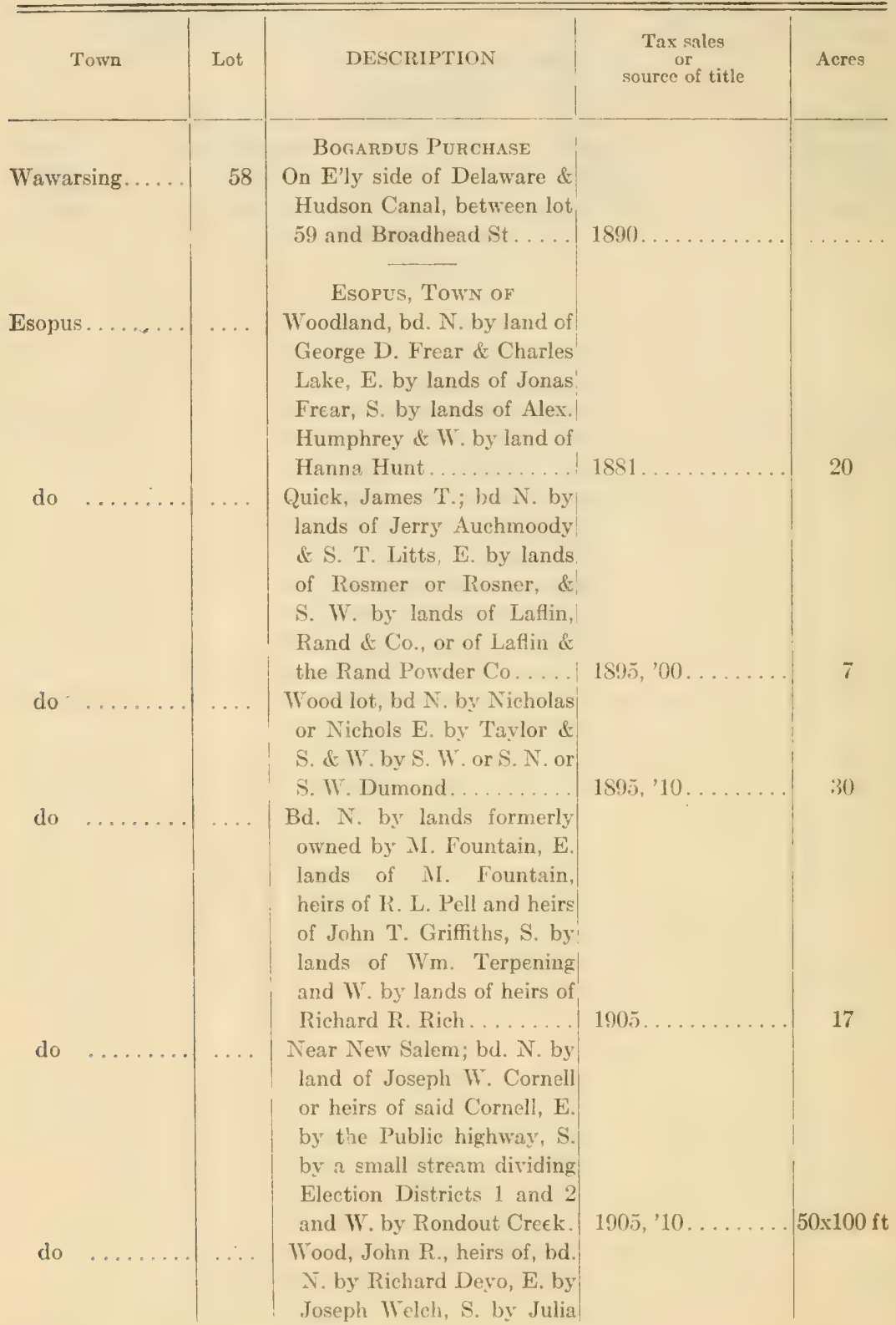




\section{Ulster County}

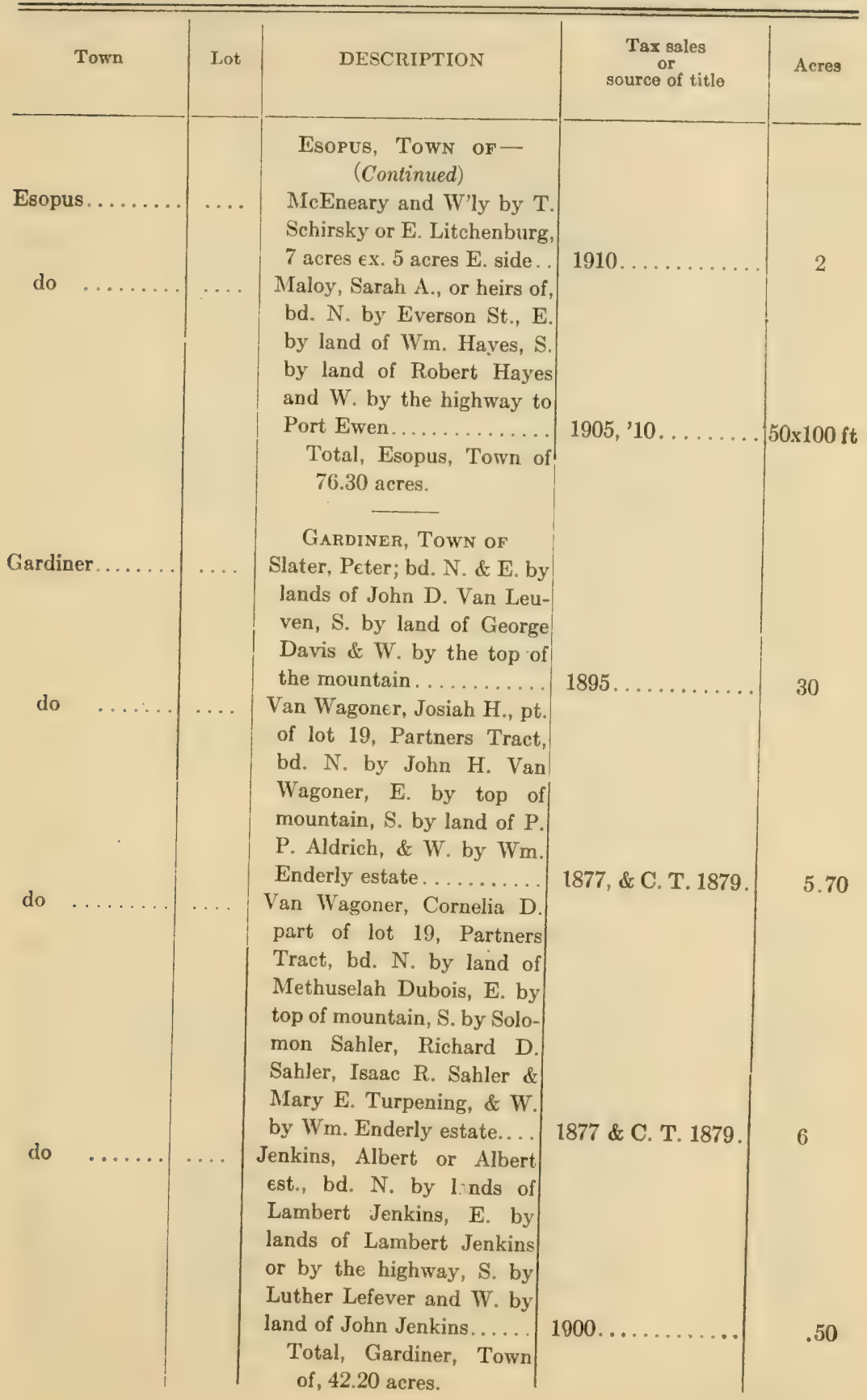


ULster County

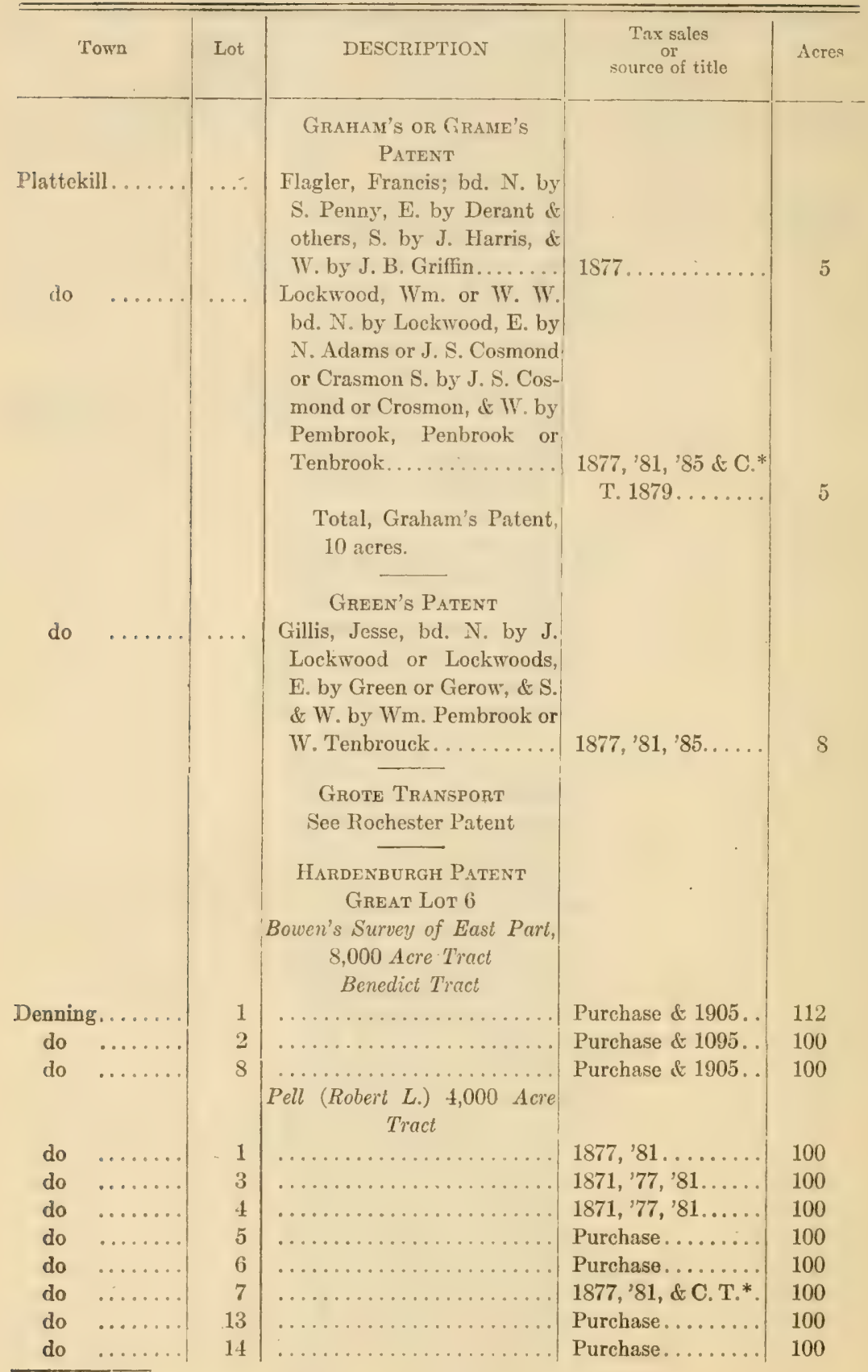

*County 'Treasurer's sales, 1879. 
Ulster County

\begin{tabular}{|c|c|c|c|c|}
\hline Town & \multirow[t]{2}{*}{ Lot } & DESCRIPTION & $\begin{array}{c}\text { Tax sales } \\
\text { or } \\
\text { source of title }\end{array}$ & Acres \\
\hline & & $\begin{array}{c}\text { Hardenburgh Patent } \\
\text { Great Lot 6, EAST Part, } \\
\text { Pell (Robert L.) 4,000 Acre } \\
\text { Tract-(Continued) }\end{array}$ & & \\
\hline Denning. & 15 & $\ldots \ldots \ldots \ldots \ldots \ldots \ldots$ & Purchase......... & 100 \\
\hline do & 16 & W.pt.............. & $\begin{array}{r}1877, ' 81, ' 95 \text { \& C. } \\
\text { T.*......... }\end{array}$ & 50 \\
\hline do & 17 & ......... & $\begin{array}{r}1877,{ }^{\prime} 81,95 \& \text { C. } \\
\text { T.* . . . . . . }\end{array}$ & 100 \\
\hline do & 18 & . & $\begin{array}{r}1877,{ }^{\prime} 81, ' 95 \& \mathrm{C} \\
\text { T.* } \ldots \ldots \ldots \ldots\end{array}$ & 100 \\
\hline do & 19 & • & $\begin{array}{r}1877,{ }^{\prime} 81, ' 95 \& \text { C. } \\
\text { T. }{ }^{*} \ldots \ldots \ldots \ldots\end{array}$ & 100 \\
\hline do & 20 & . & $\begin{array}{r}1877, ' 81, ' 95 \& \mathrm{C} . \\
\mathrm{T}{ }^{*} \ldots \ldots \ldots \ldots\end{array}$ & 100 \\
\hline do & 21 & $\ldots \ldots \ldots \ldots$ & $\begin{array}{r}1877,{ }^{\prime} 81,95 \& \text { C. } \\
\text { T. }{ }^{*} \ldots \ldots \ldots \ldots\end{array}$ & 100 \\
\hline do & 22 & - . & $\begin{array}{r}1877, ' 81, ' 95 \& \text { C. } \\
\text { T.* } \ldots \ldots \ldots \ldots\end{array}$ & 100 \\
\hline do & 23 & . & $\begin{array}{r}1877,{ }^{\prime} 81,{ }^{\prime} 95 \& \mathrm{C} \\
\text { T. } \ldots \ldots \ldots \ldots\end{array}$ & 100 \\
\hline do & 24 & $\ldots \ldots \ldots \ldots \ldots$ & $\begin{array}{r}1877,{ }^{\prime} 81,95 \& \text { C. } \\
\mathrm{T} * \ldots \ldots \ldots \ldots\end{array}$ & 100 \\
\hline do & 25 & $\ldots \ldots \ldots \ldots \ldots$ & $\begin{array}{r}1877,{ }^{\prime} 81, ' 95 \& \text { C. } \\
\text { T. }{ }^{*} \ldots \ldots \ldots \ldots\end{array}$ & 100 \\
\hline do & 26 & $\ldots \ldots \ldots \ldots \ldots \ldots$ & $\begin{array}{r}1877,{ }^{\prime} 81,{ }^{\prime} 95 \& \text { C. } \\
\text { T. } . \ldots \ldots \ldots\end{array}$ & 100 \\
\hline do & 27 & $\cdots$ & $\begin{array}{r}1877,{ }^{\prime} 81,{ }^{\prime} 95 \& \text { C. } \\
\text { T. }{ }^{*} \ldots \ldots \ldots \ldots\end{array}$ & 100 \\
\hline do & 28 & $\ldots \ldots \ldots \ldots \ldots \ldots \ldots \ldots \ldots \ldots \ldots$ & Purchase........ & 100 \\
\hline do & 29 & $\ldots \ldots \ldots \ldots \ldots \ldots \ldots \ldots \ldots$, & Purchase........ & 100 \\
\hline do & 30 & $\ldots \ldots \ldots \ldots \ldots \ldots \ldots \ldots \ldots$ & Purchase........ & 100 \\
\hline do & 31 & $\ldots \ldots \ldots \ldots \ldots \ldots \ldots \ldots \ldots$ & Purchase........ & 100 \\
\hline do & 32 & $\ldots \ldots \ldots \ldots \ldots \ldots \ldots \ldots$ & Purchase........ & 100 \\
\hline do & 33 & $\ldots \ldots \ldots \ldots \ldots \ldots \ldots \ldots \ldots \ldots \ldots$ & Purchase........ & 100 \\
\hline do & 34 & $\ldots \ldots \ldots \ldots \ldots \ldots \ldots$ & Purchase........ & 100 \\
\hline do & 35 & ….... & Purchase........ & 100 \\
\hline do & 36 & $\ldots \ldots \ldots \ldots \ldots \ldots \ldots \ldots$ & Purchase........ & 100 \\
\hline do & 37 & $\ldots \ldots \ldots \ldots \ldots \ldots \ldots \ldots \ldots$ & Purchase........ & 100 \\
\hline do & 38 & $\ldots \ldots \ldots \ldots \ldots \ldots \ldots \ldots$ & Purchase........ & 100 \\
\hline do & 39 & 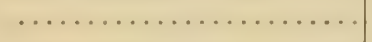 & Purchase........ & 100 \\
\hline do & 40 & $\begin{array}{c}\text { Total, Pell and Benedict } \\
\text { Trncts, } 3,662 \text { acres. }\end{array}$ & Purchase........ & 100 \\
\hline
\end{tabular}

* County Treasurer's sales, 1879. 
Ulster County

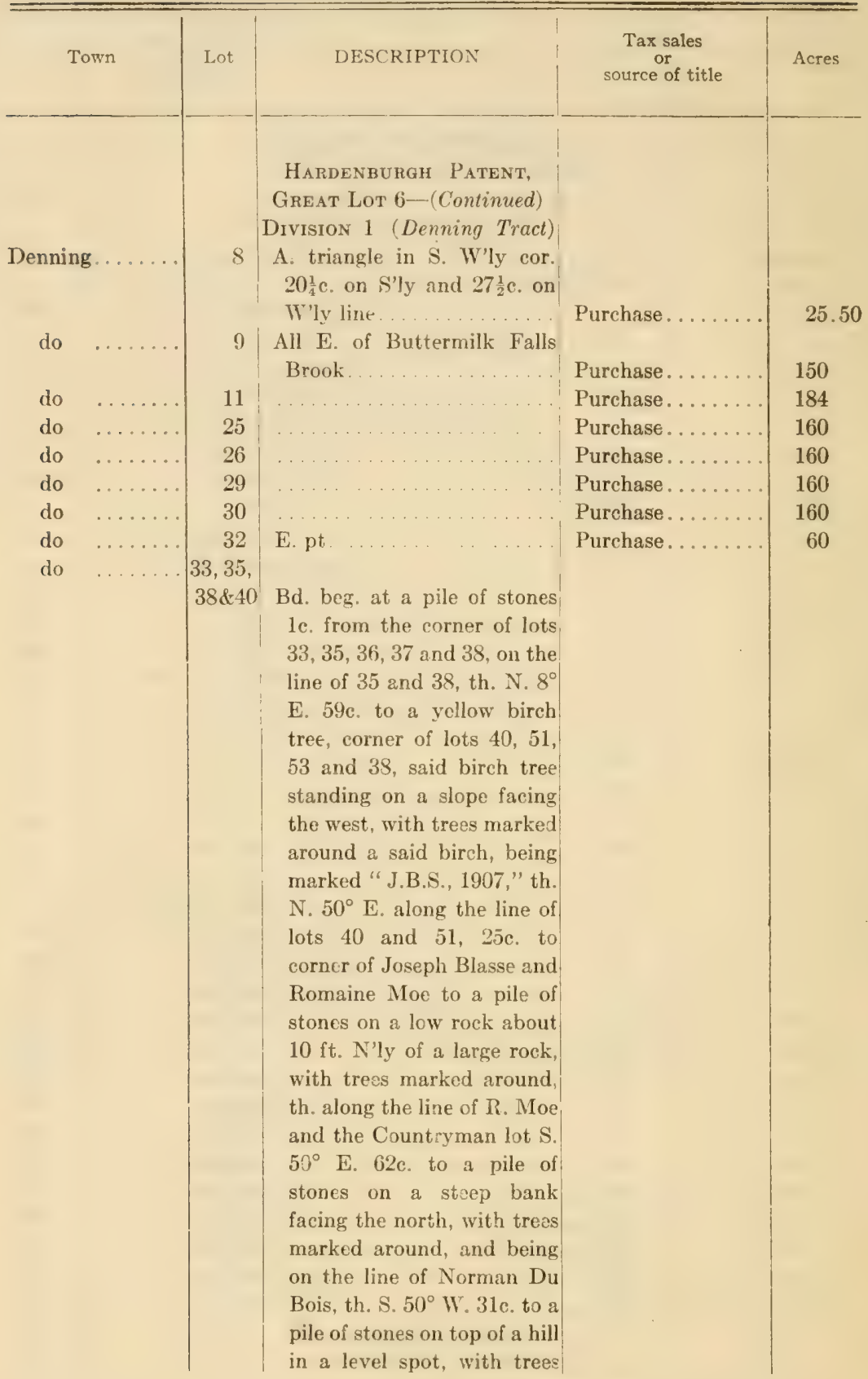




\section{Ulster County}

\begin{tabular}{|c|c|c|c|c|}
\hline Town & Lot & DESCRIPTION & $\begin{array}{c}\text { Tax sales } \\
\text { or } \\
\text { source of title }\end{array}$ & Acres \\
\hline Denning... & $\mid$\begin{tabular}{|}
33,35 \\
$38 \& 40$
\end{tabular} & $\begin{array}{l}\text { HARDENBURGH P A TEN T, } \\
\text { Great Lot 6, Division 1, } \\
\text { (Denning Tract) - (Con- } \\
\text { tinued) } \\
\text { marked around, th. N. } 50^{\circ} \\
\text { W. 22c. to a pile of stones } \\
\text { on the south of an old road } \\
\text { in a swampy place, th. S. } \\
50^{\circ} \mathrm{W} .45 \mathrm{c} \text { to beg......... }\end{array}$ & Purchase... & 266 \\
\hline do & $\begin{array}{l}36,37 \\
\& 38\end{array}$ & 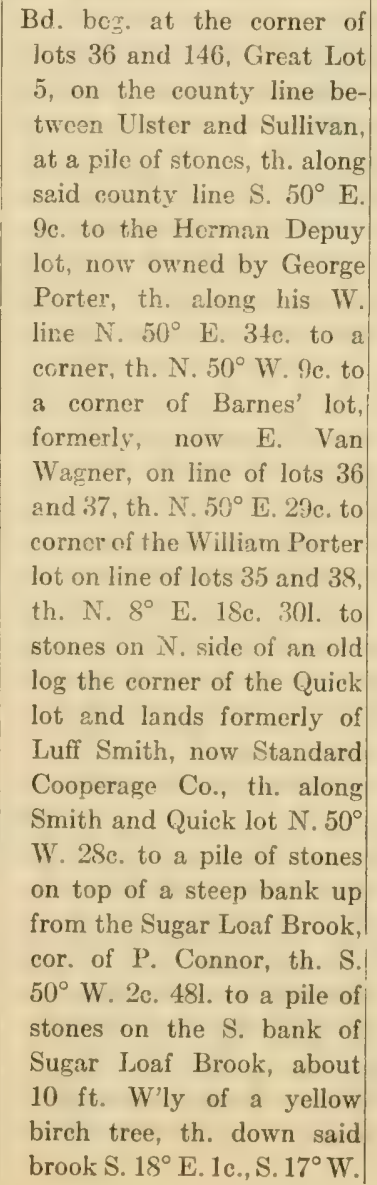 & & \\
\hline
\end{tabular}




\section{Ulster County}

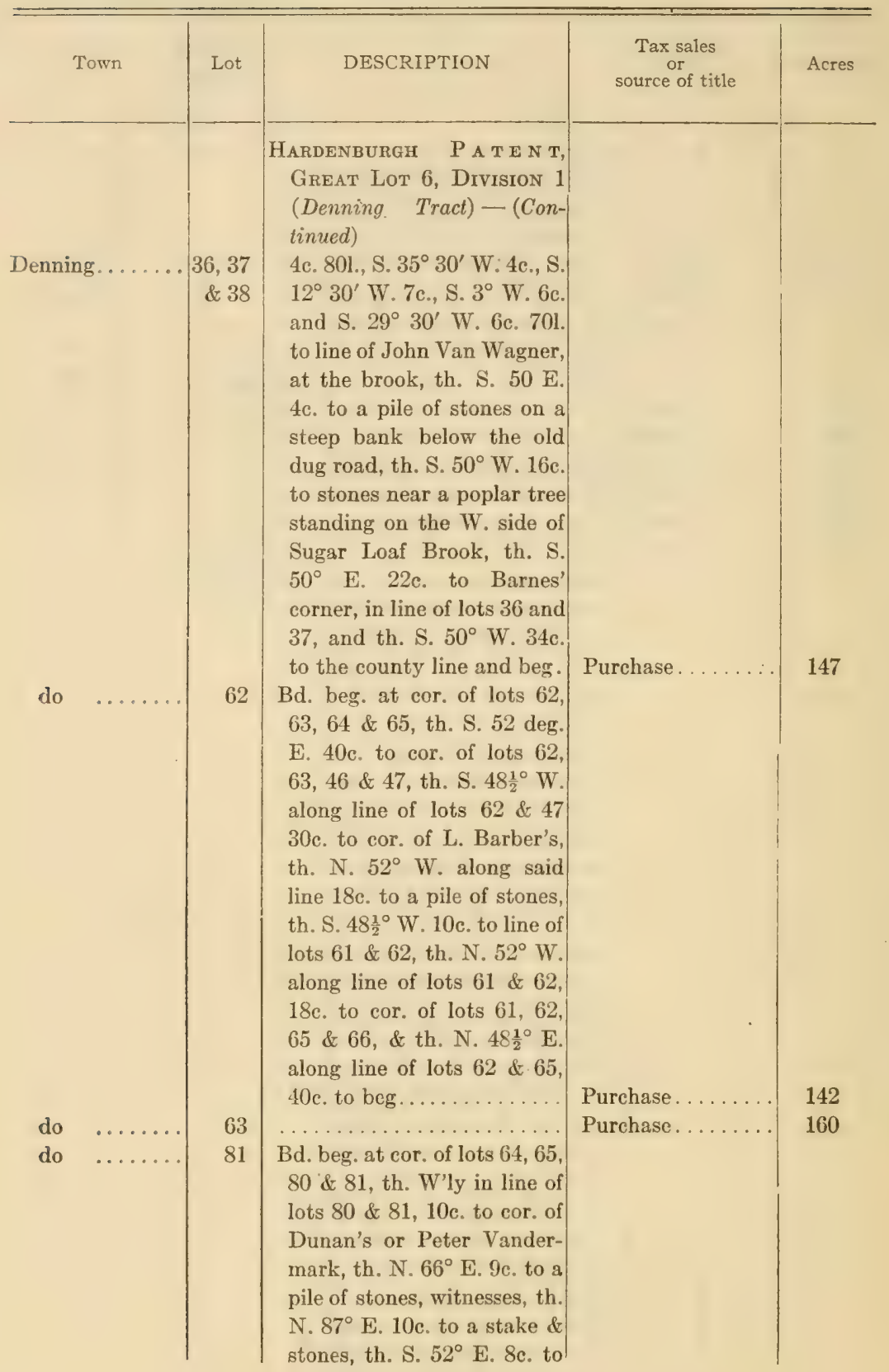


Uluster County

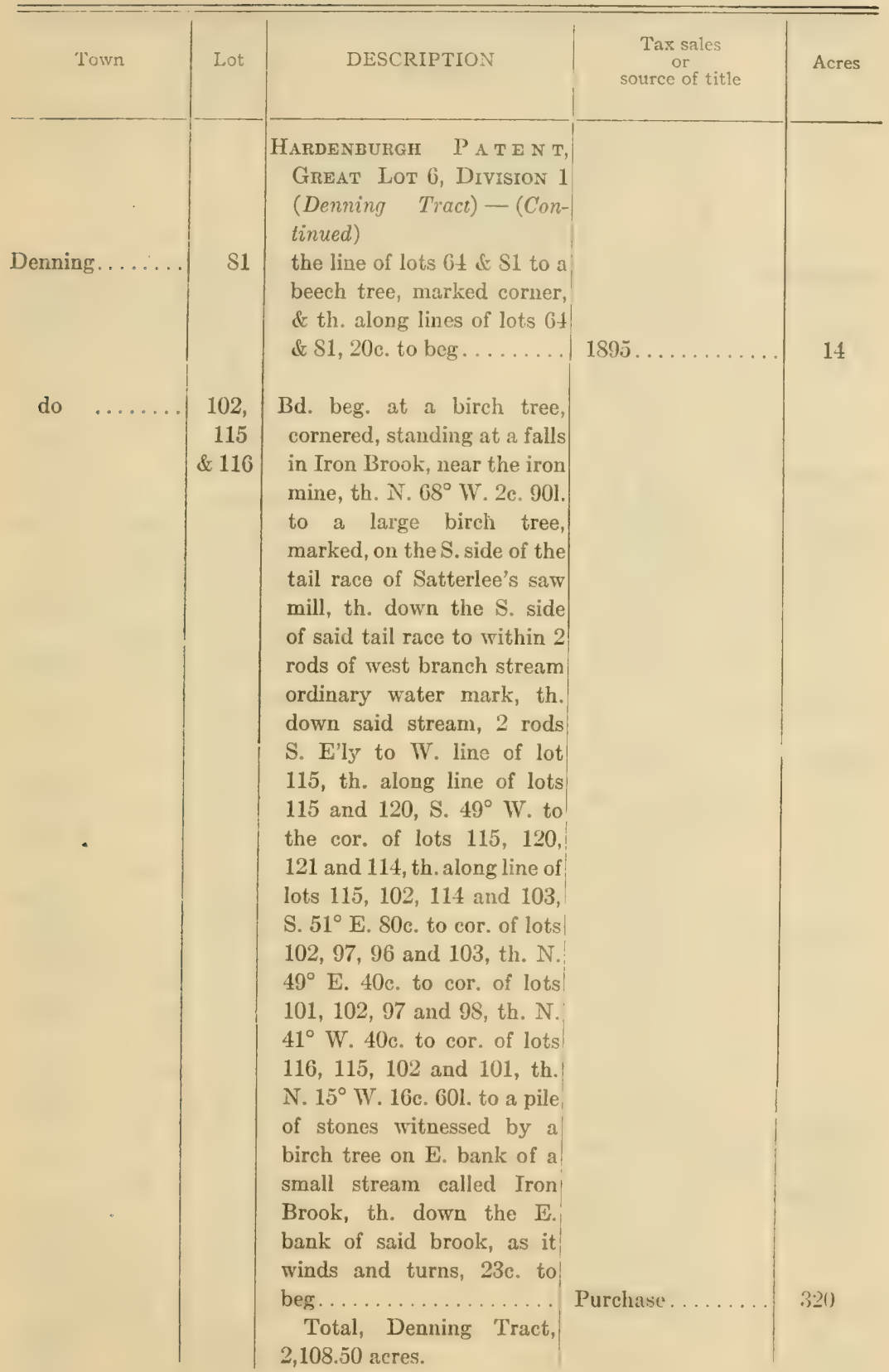


Ulster County

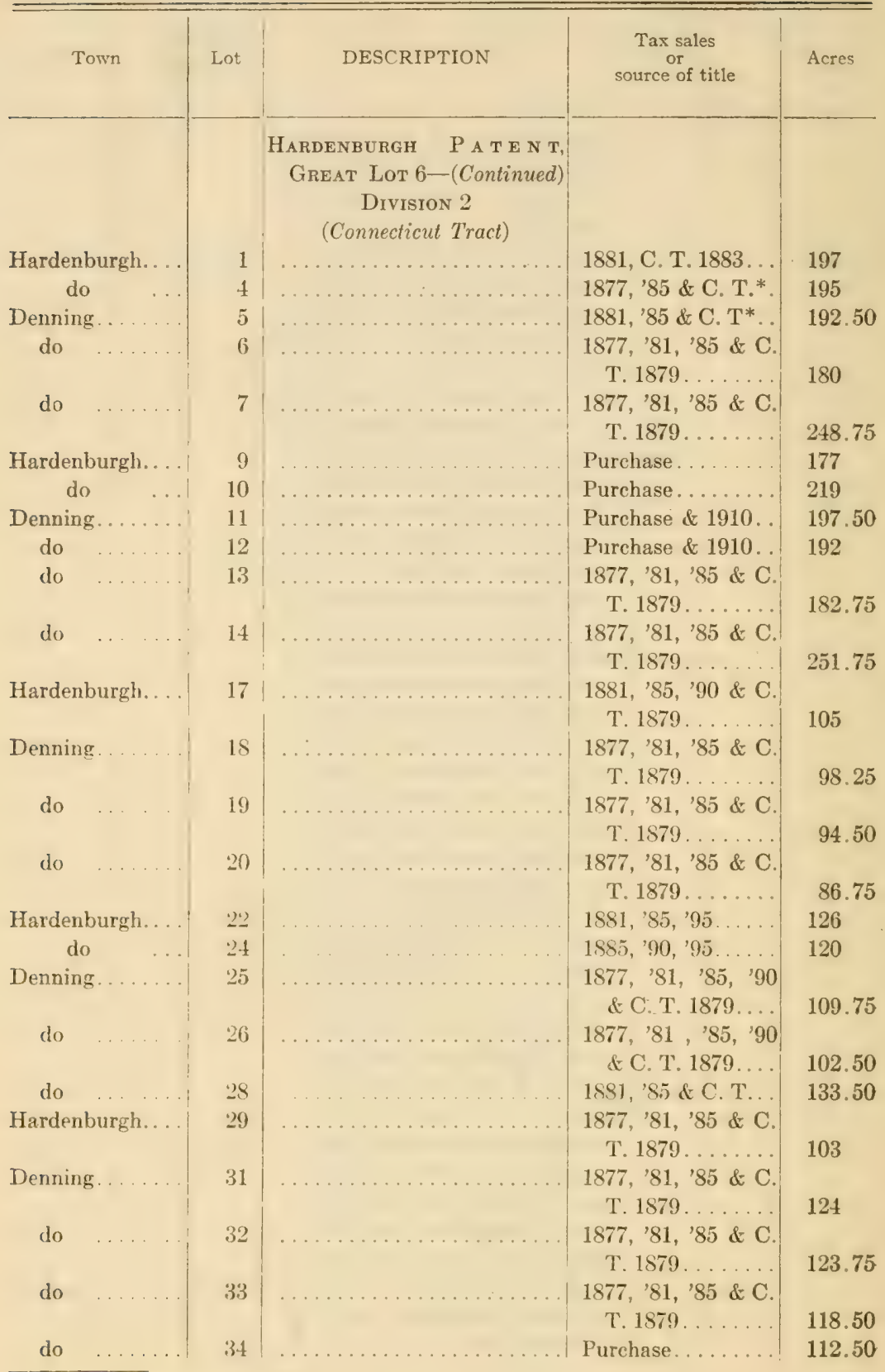

*County Treasurer's sales, 1879 


\section{ULSTER COUNTY}

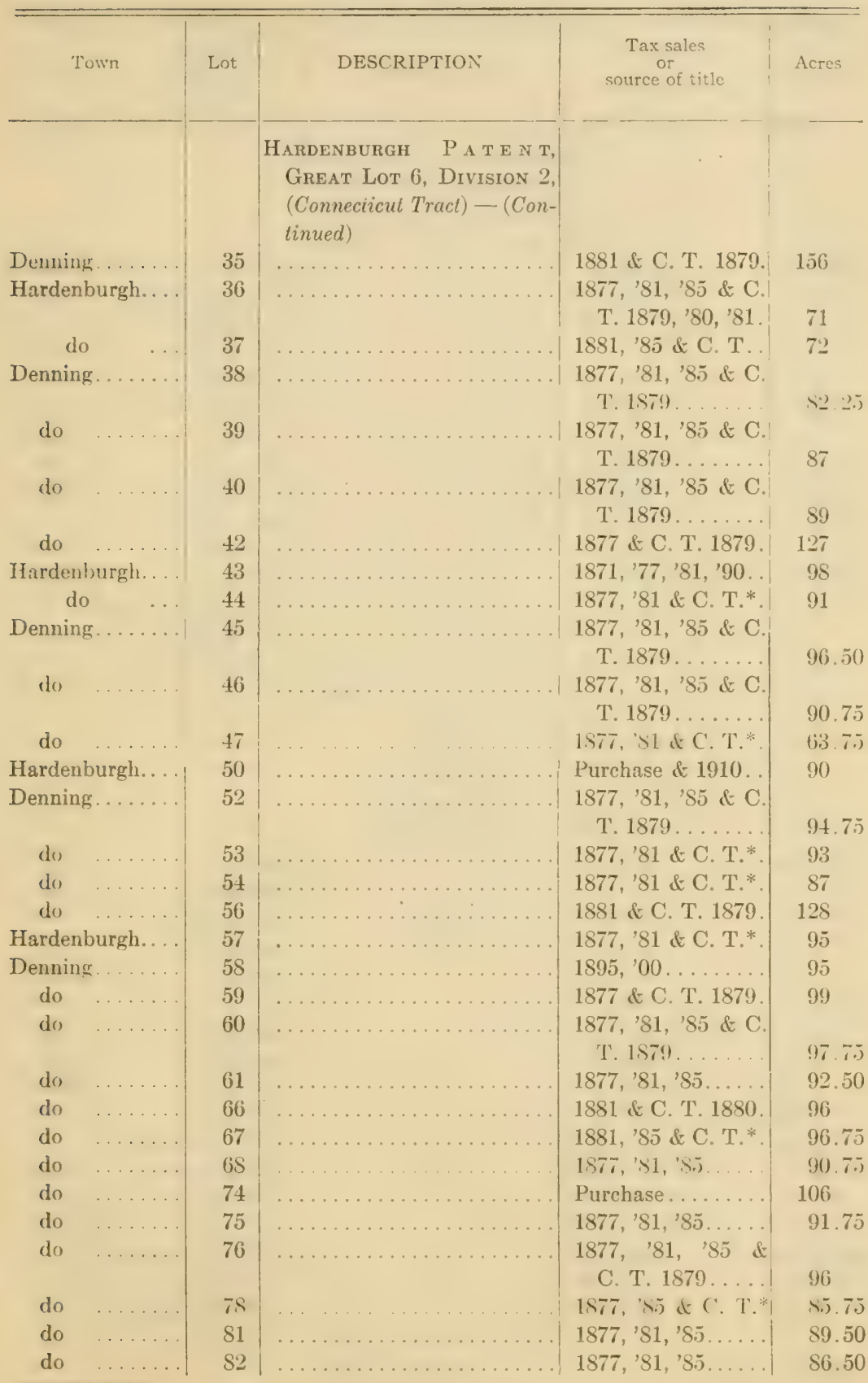


Ulster County

\begin{tabular}{|c|c|c|c|c|}
\hline Town & Lot & DESCRIPTION & $\begin{array}{c}\text { Tax sales } \\
\text { or } \\
\text { source of title }\end{array}$ & Acres \\
\hline & & $\begin{array}{l}\text { Hardenburgh PATEN T, } \\
\text { Great Lot 6, Division } 2- \\
\text { (Connecticut Tract)-(Con- } \\
\text { tinued) }\end{array}$ & & \\
\hline Denning.... & 83 & $\ldots \ldots \ldots \ldots \ldots \ldots \ldots$ & $1877, ' 81,95 \ldots .$. & 85.25 \\
\hline$\ldots \ldots$ & 85 & & 1877,81 , '\$5, '95. . & 100 \\
\hline do & 89 & & $\begin{array}{l}1877, \quad 81, \text { 'S5 } \\
\text { C. T. } 1879 \ldots \ldots\end{array}$ & 109.75 \\
\hline do & 90 & & Purchase......... & 111 \\
\hline do & 93 & . & $\begin{array}{c}1877, ' 81,95 \& \text { C. } \\
\text { T. } * \ldots \ldots \ldots \ldots\end{array}$ & $\begin{array}{l}112.50 \\
129.50\end{array}$ \\
\hline do & 106 & Fx. S2a. X. W. cor. . & $1871,77 \ldots \ldots \ldots$ & 52 \\
\hline do & 106 & X. $\mathrm{H}^{\circ}$. cor . . . . . & Purchase \& $1905 . .$. & 82 \\
\hline do & 108 & $\ldots \ldots \ldots \ldots \ldots \ldots$ & $\begin{array}{r}\text { 1881, 'S5 \& C. T. } \\
\text { 1879, '80, '81.... }\end{array}$ & 153 \\
\hline do & 112 & & Purchase......... & 127 \\
\hline do & 113 & & $\begin{array}{r}1877, \quad 81, \quad 85 \quad \\
\text { C. T. } 1879 \ldots .\end{array}$ & 142.50 \\
\hline do & 118 & $\begin{array}{l}\text { II. part . . . . . . . . . . } \\
\text { Total, Connecticut Tract, } \\
7,878.50 \text { acres. } \\
\text { GREAT Lot } 6, \\
\text { Division } 3 \\
\text { (Robinson Tract) }\end{array}$ & Purchase \& $1905 .$. & 60 \\
\hline Hardenburgh. & 154 & & Purchase........ & 135 \\
\hline do & 156 & & Purchase........ & 135 \\
\hline do & 177 & Ex. 1 ta.N. E. cor. & Appropria ${ }^{\perp}$ ed..... & 122 \\
\hline do & 175 & & Appropriated..... & 147 \\
\hline do & 194 & & Purchase........ & 143 \\
\hline do & 196 & & $1877 \ldots \ldots \ldots \ldots$ & 148 \\
\hline do & 207 & & Purchase........ & 120 \\
\hline do & 215 & & Purchase......... & 143 \\
\hline do & 216 & & Purchase........ & 130 \\
\hline do & 217 & & Purchase........ & 133 \\
\hline do & 218 & & $\begin{array}{r}1877, ' 81, ' 85 \& \mathrm{C} . \\
\text { T. }{ }^{*} 1879 \ldots \ldots\end{array}$ & 127 \\
\hline do & 219 & & $\begin{array}{r}1877, ' 81, ' 85 \& \mathrm{C} . \\
\mathrm{T}, * 1879 \ldots \ldots .\end{array}$ & 129 \\
\hline do & 220 & & $\begin{array}{r}1877, ' 81, ' 85 \& \mathrm{C} . \\
\mathrm{T} .{ }^{*} 1879 \ldots \ldots\end{array}$ & 142 \\
\hline do & 221 & & $\begin{array}{l}1877, ' 81, ' 85 \text { \& C. } \\
\text { T.*1879....... }\end{array}$ & 141 \\
\hline
\end{tabular}

*County Treasurer's sales. 
Ulster County

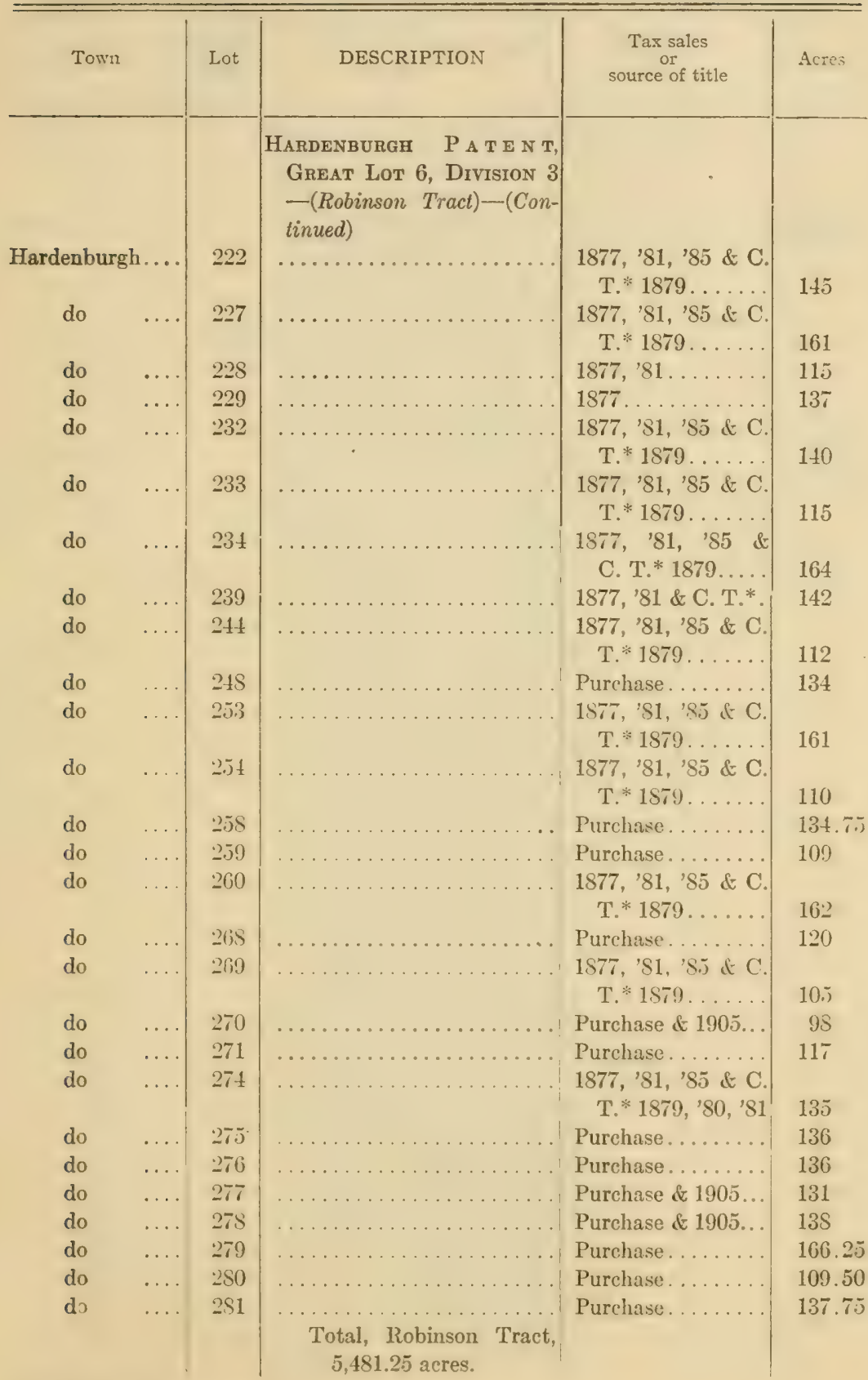




\section{Ulster County}

\begin{tabular}{|c|c|c|c|c|}
\hline Town & Lot & DESCRIPTION & $\begin{array}{c}\text { Tax sales } \\
\text { or } \\
\text { source of title }\end{array}$ & Acres \\
\hline Denning.... & . & $\begin{array}{l}\text { Hardenburgh Patent - } \\
\text { (Continued) } \\
\text { Great Lot } 7 \\
\text { Bd. N'ly by town of Shan- } \\
\text { daken, E. by Vernoy's sur- } \\
\text { vey of } 0 \text { lo s, S. by an } \\
\text { 86ta. tract, \& } 11 \text {. by town of } \\
\text { Harde burgh. (N. } 4 \text { p } 461 \text {.) }\end{array}$ & Purchase. & 1,25 \\
\hline Olive..... & $\cdots$ & 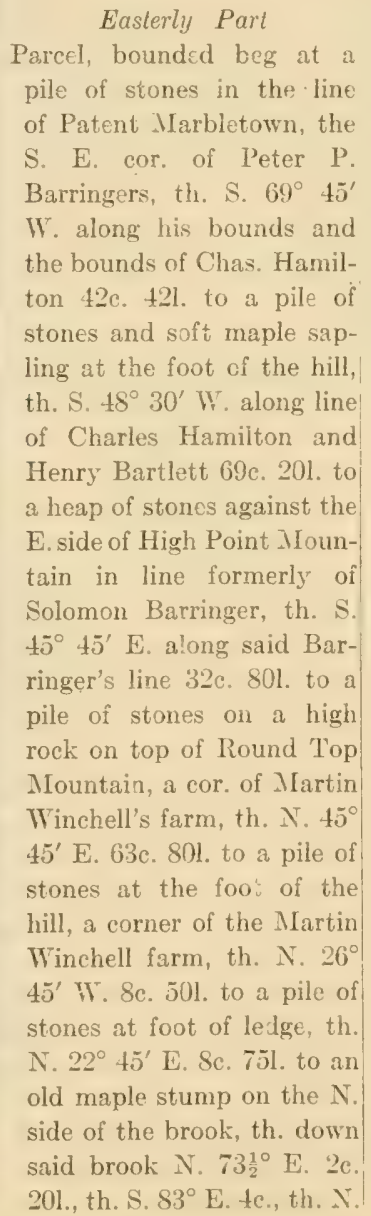 & 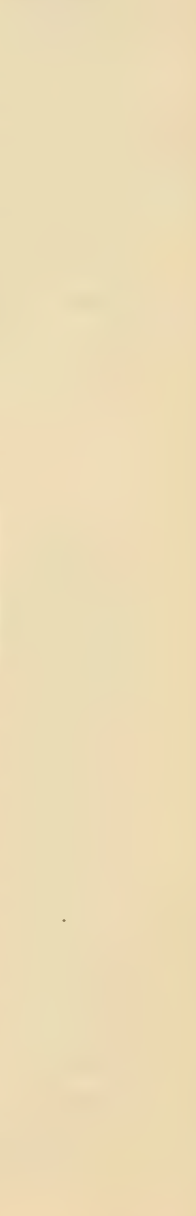 & \\
\hline
\end{tabular}


Ulster County

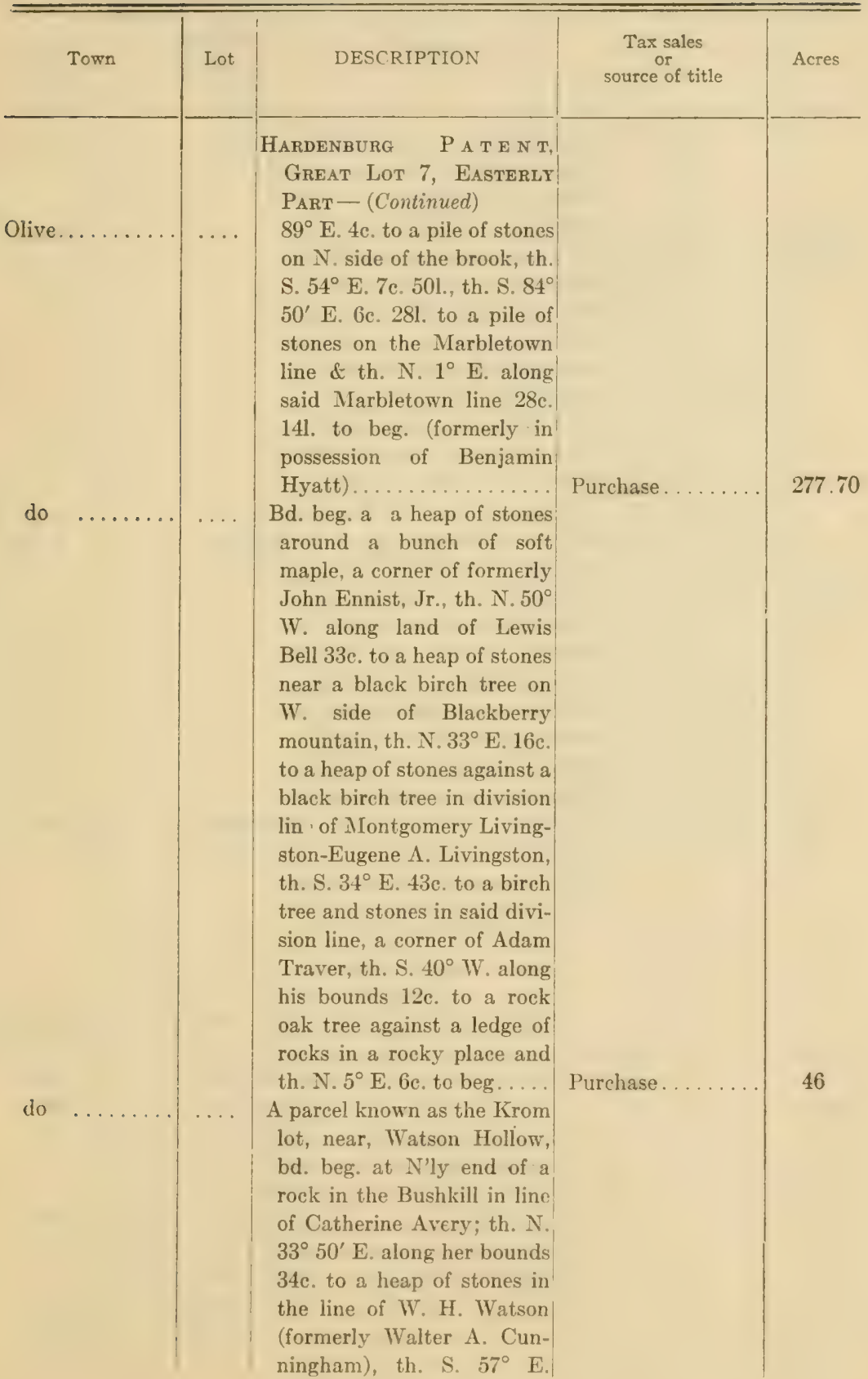


Ulster County

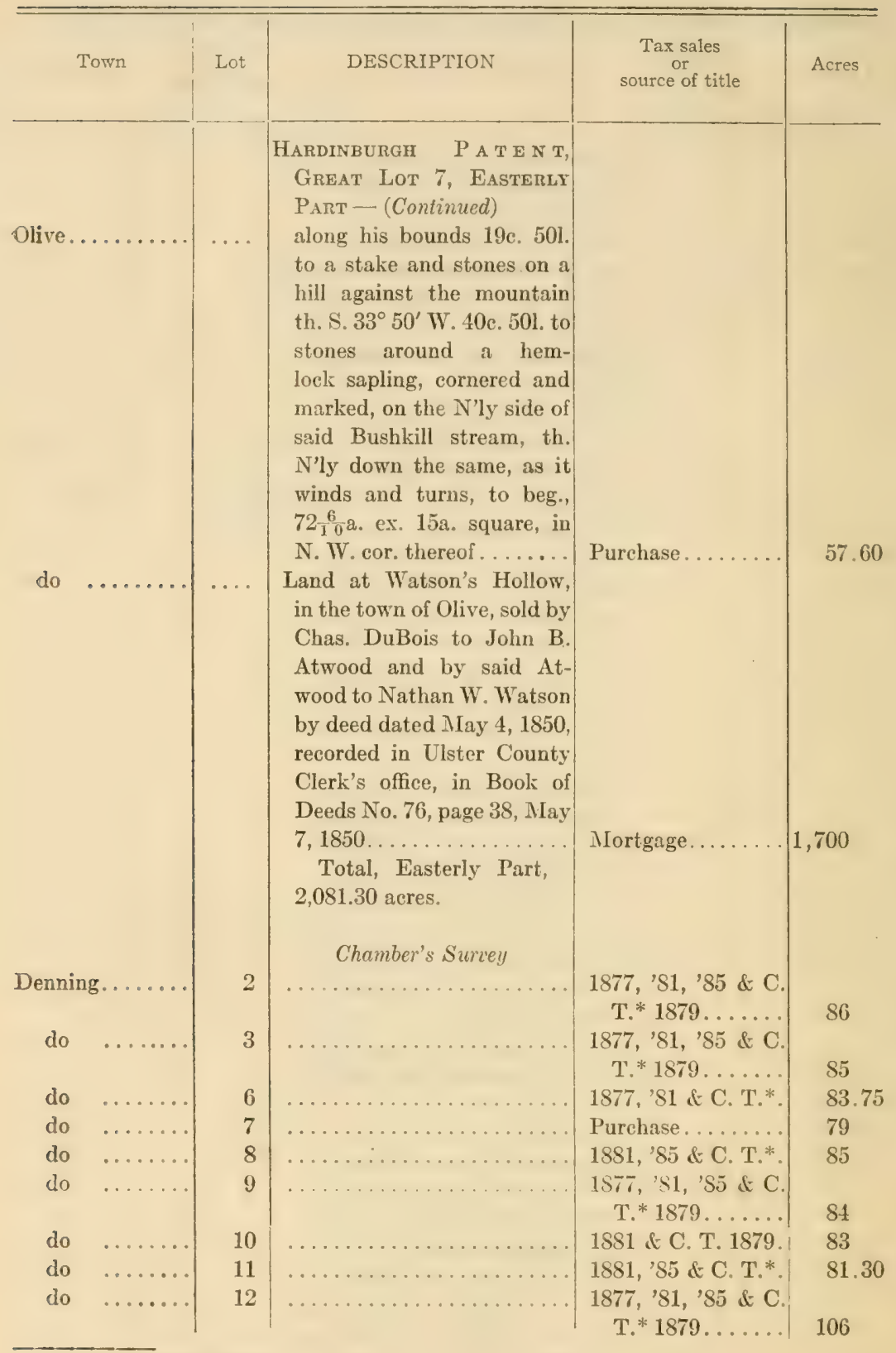

* County Treasurer's sales. 


\section{Ulster Coenty}

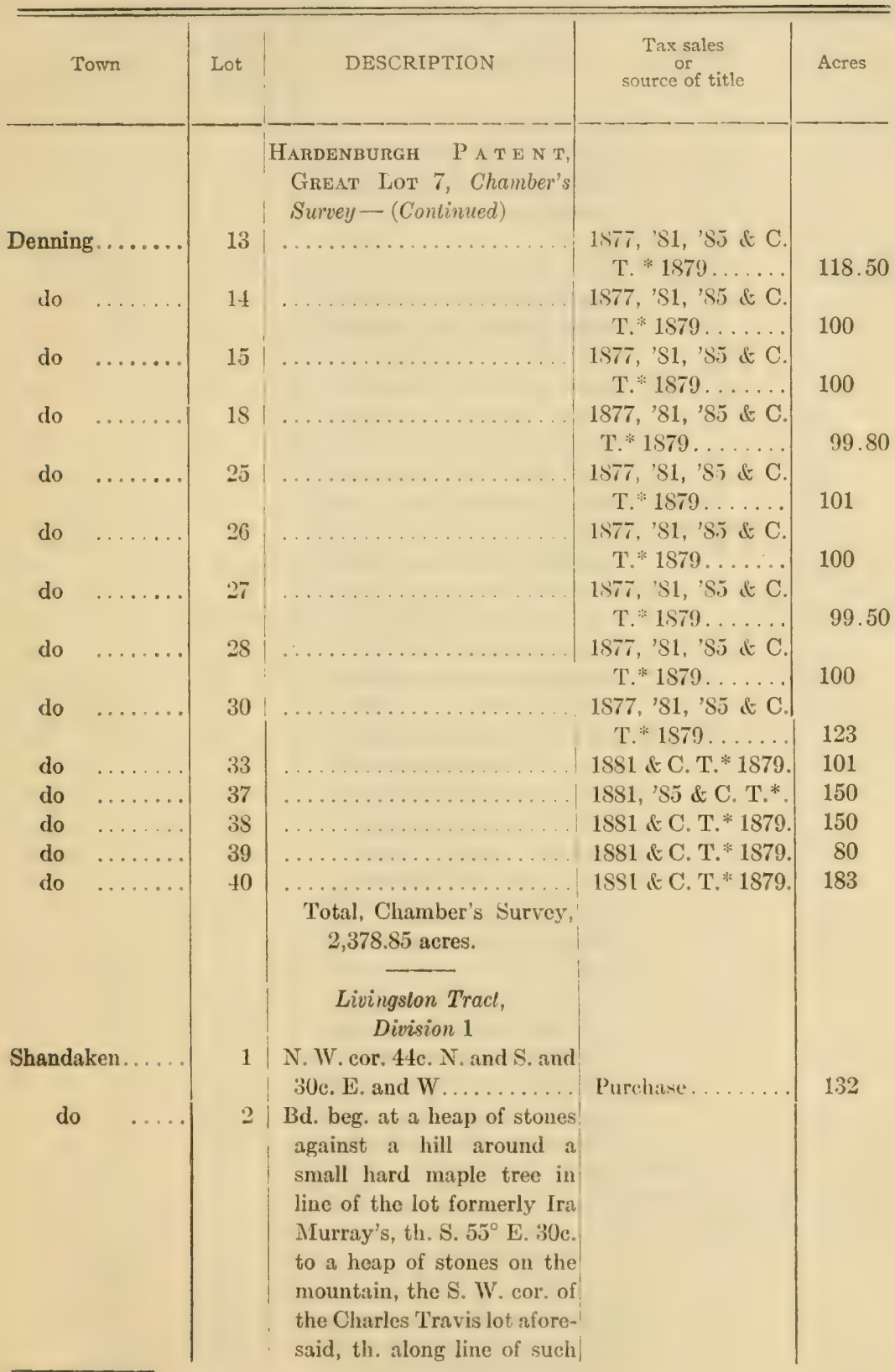

* County 'Treasurer's sales. 
Ulster County

\begin{tabular}{|c|c|c|c|c|}
\hline Town & Lot & DESCRIPTION & $\begin{array}{c}\text { Tax sales } \\
\text { or } \\
\text { source of title }\end{array}$ & Acres \\
\hline Shandaken. & 2 & 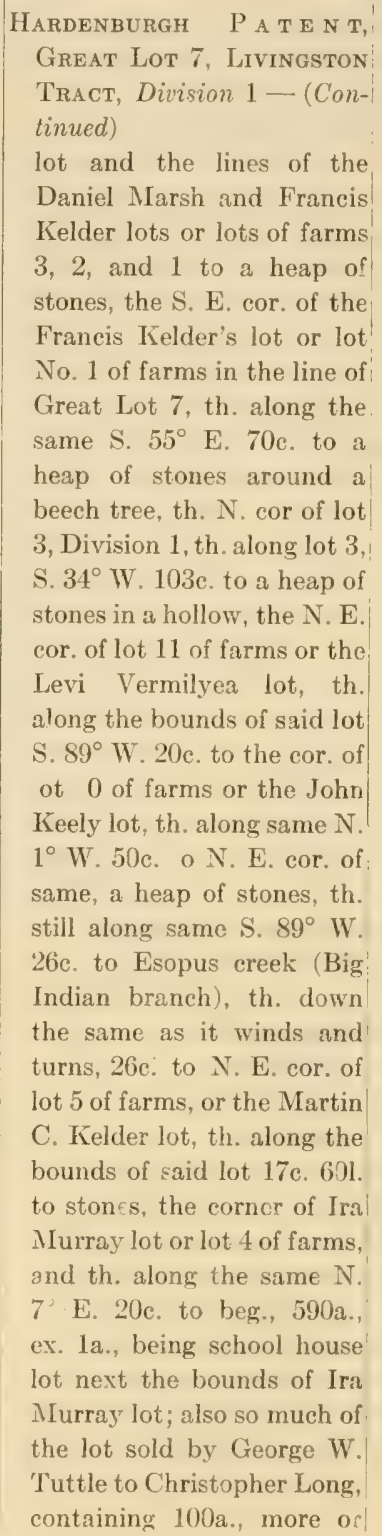 & & \\
\hline
\end{tabular}


Ulster County

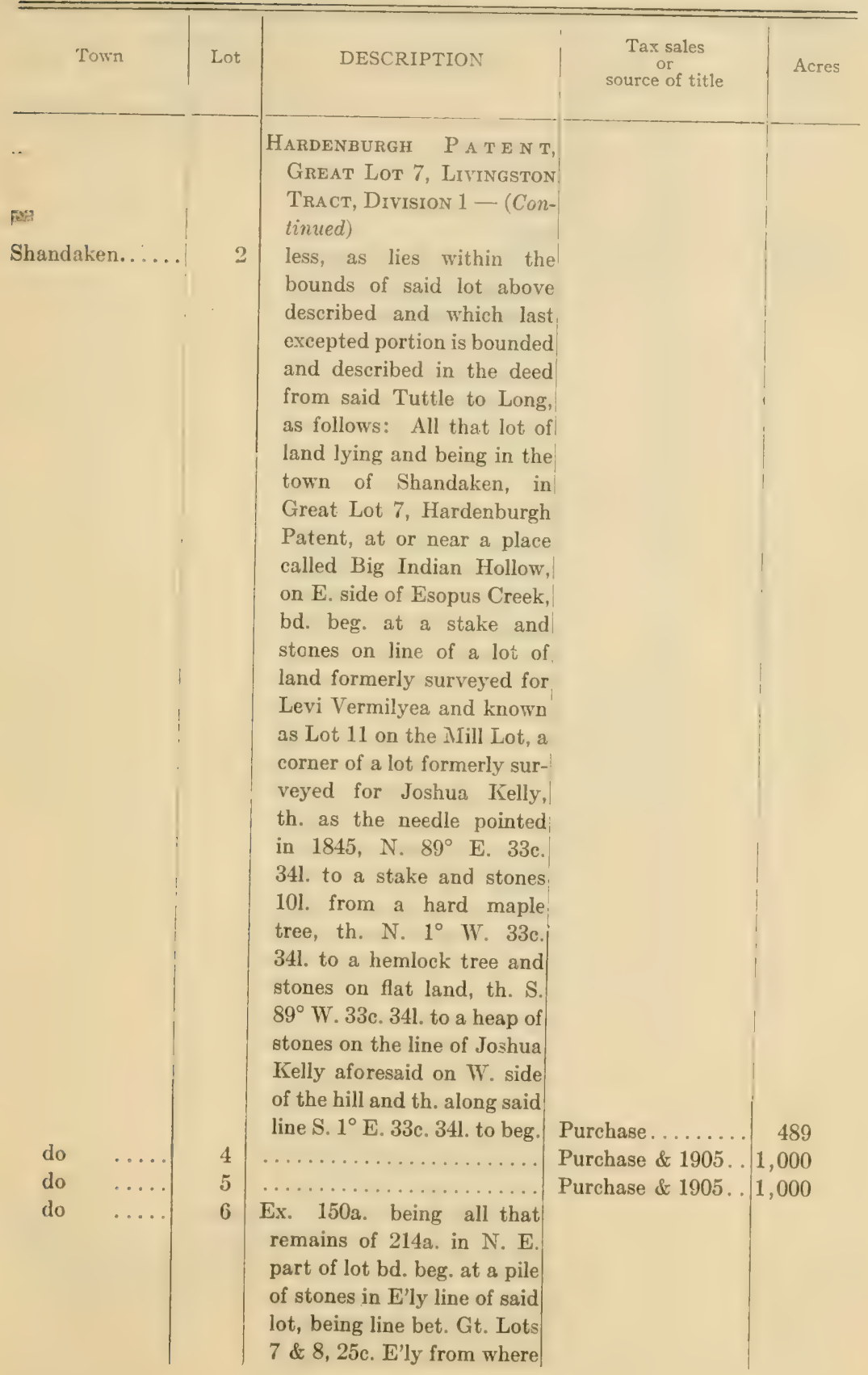




\section{Ulster County}

\begin{tabular}{|c|c|c|c|c|}
\hline Town & Lnt & DESCRIPTION & $\begin{array}{c}\text { Tax sales } \\
\text { or } \\
\text { source of title }\end{array}$ & Acres \\
\hline Shandaken... & $\begin{array}{l}7 \\
8 \\
9\end{array}$ & 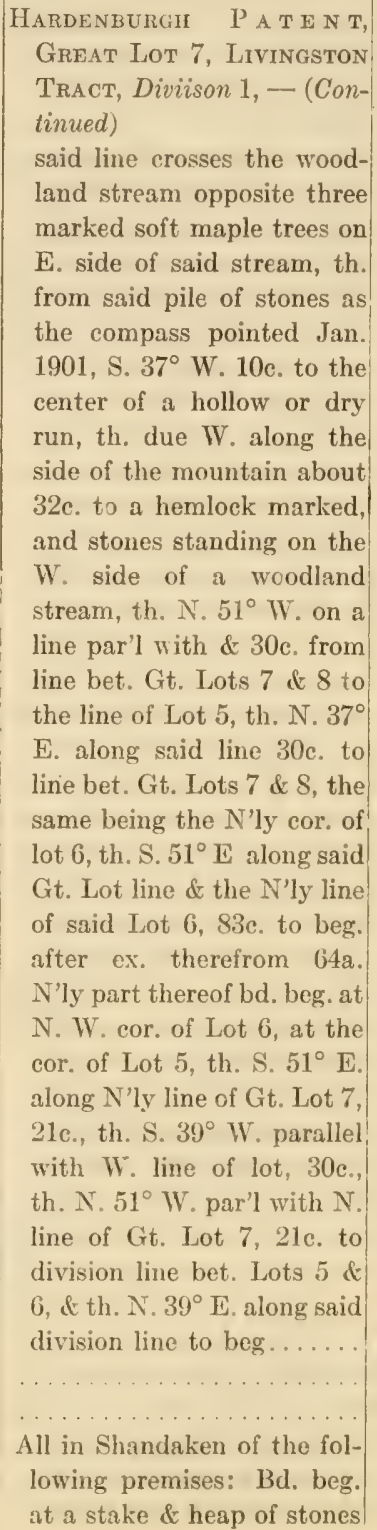 & $\begin{array}{l} \\
\text { Purchase........ } \\
\text { Purchase........ } \\
\text { Purchase \& } 1905 .\end{array}$ & $\begin{array}{r}915 \\
1,065 \\
1,115\end{array}$ \\
\hline
\end{tabular}




\section{Ulster County}

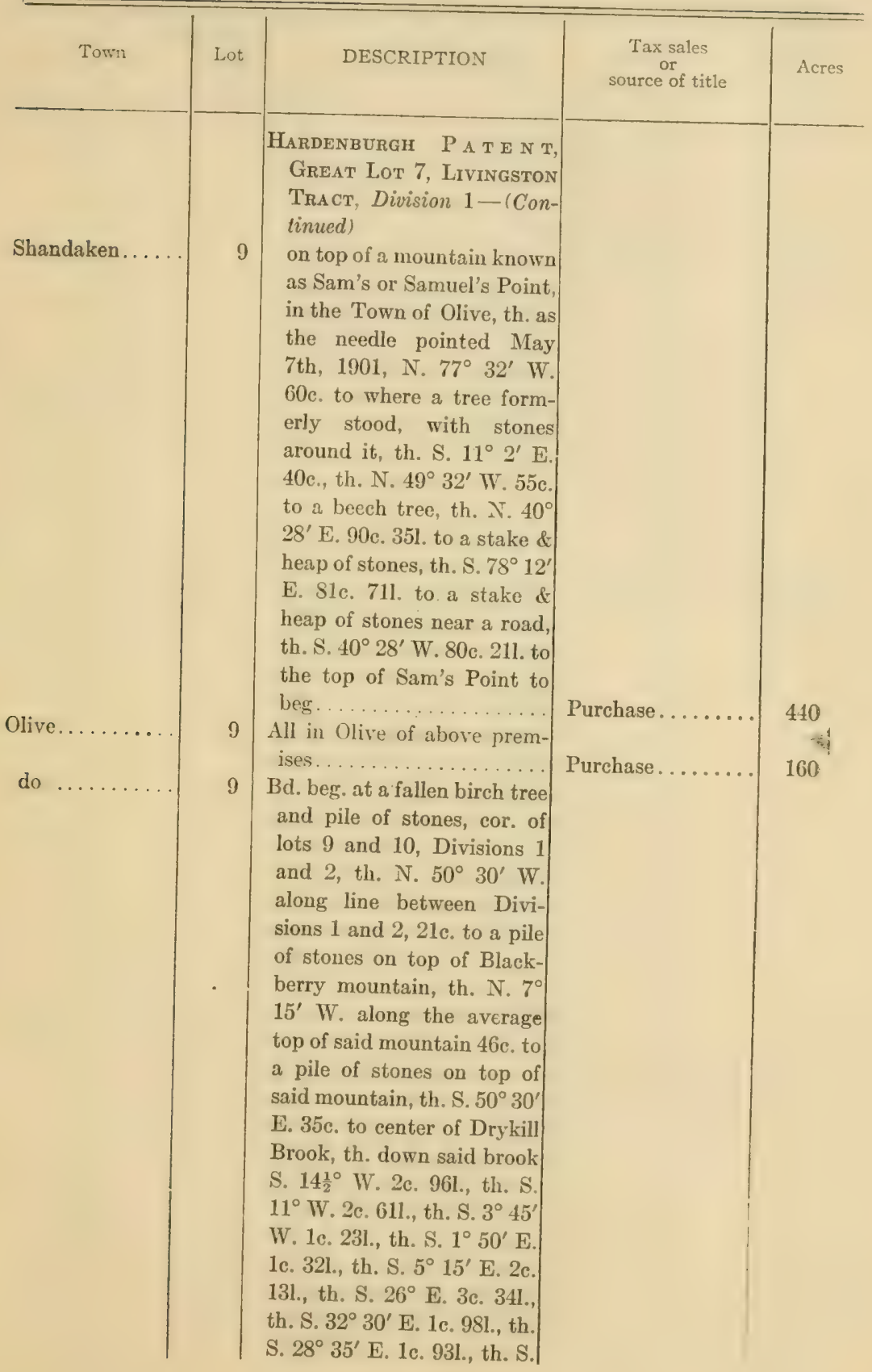


Ulster County

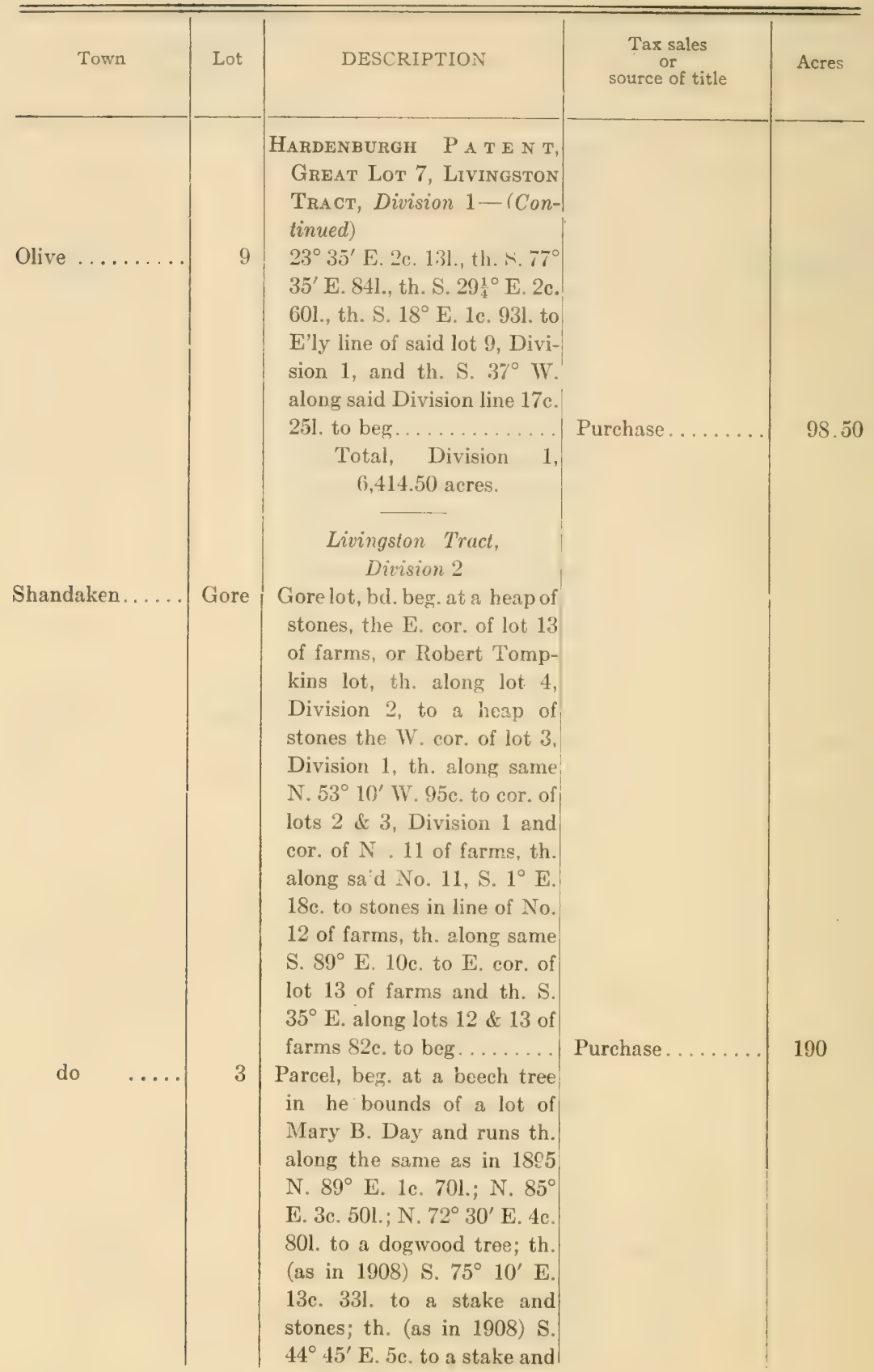




\section{Ulster County}

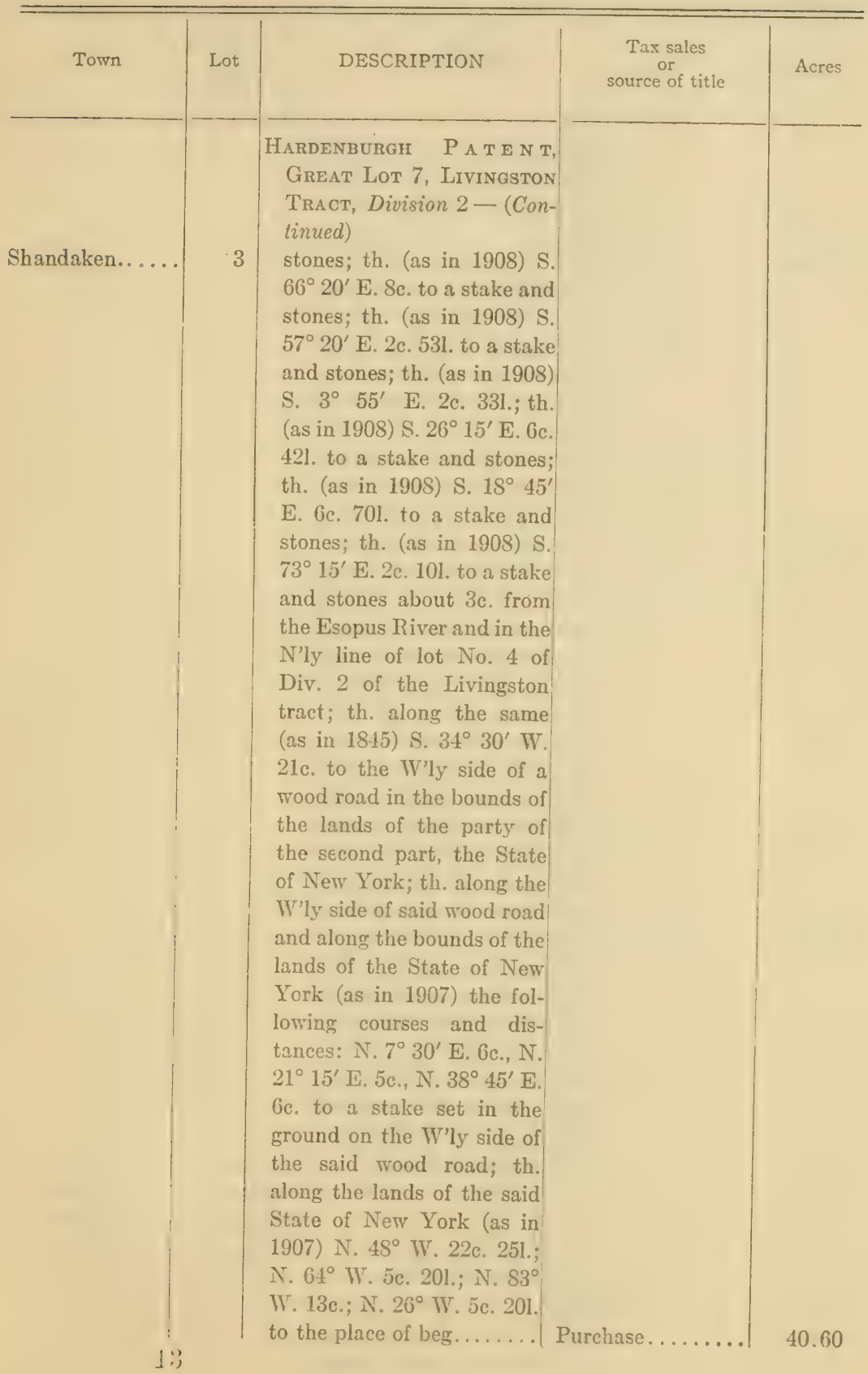


Ulster County

\begin{tabular}{|c|c|c|c|c|}
\hline Town & Lot & DESCRIPTION & $\begin{array}{c}\text { Tax sales } \\
\text { or } \\
\text { source of title }\end{array}$ & Acres \\
\hline $\begin{array}{r}\text { do } \\
\text { do } \\
\text { do } \\
\text { Olive. . }\end{array}$ & $\begin{array}{l}6 \\
7 \\
i \\
9\end{array}$ & 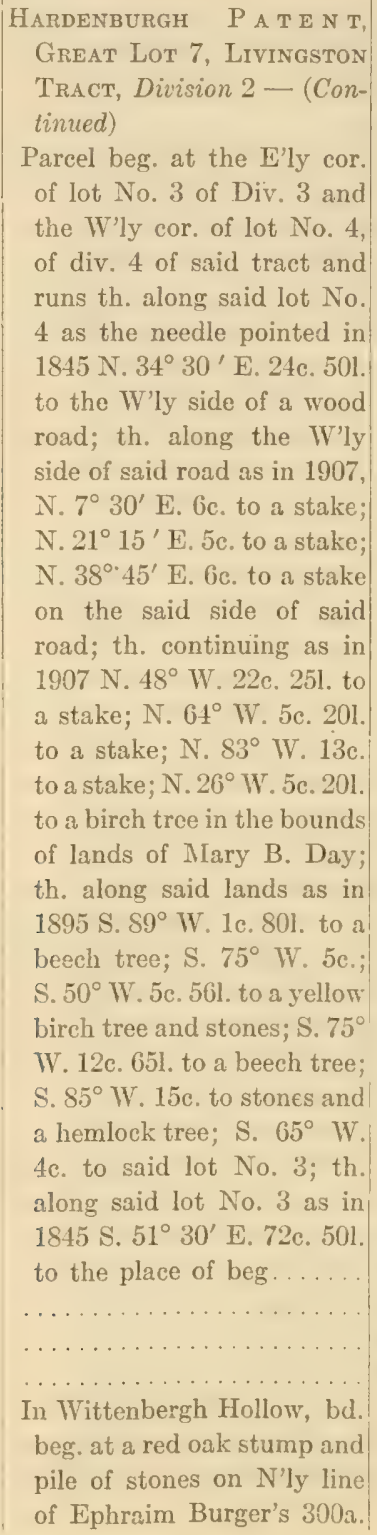 & $\begin{array}{l}\text { Purchase.... . . } \\
\text { Purchase.... . . } \\
\text { Purchase... . . } \\
\text { Purchase. . . . . }\end{array}$ & $\begin{array}{l}213.86 \\
1,000 \\
1,000 \\
1,000\end{array}$ \\
\hline
\end{tabular}




\section{Ulster County}

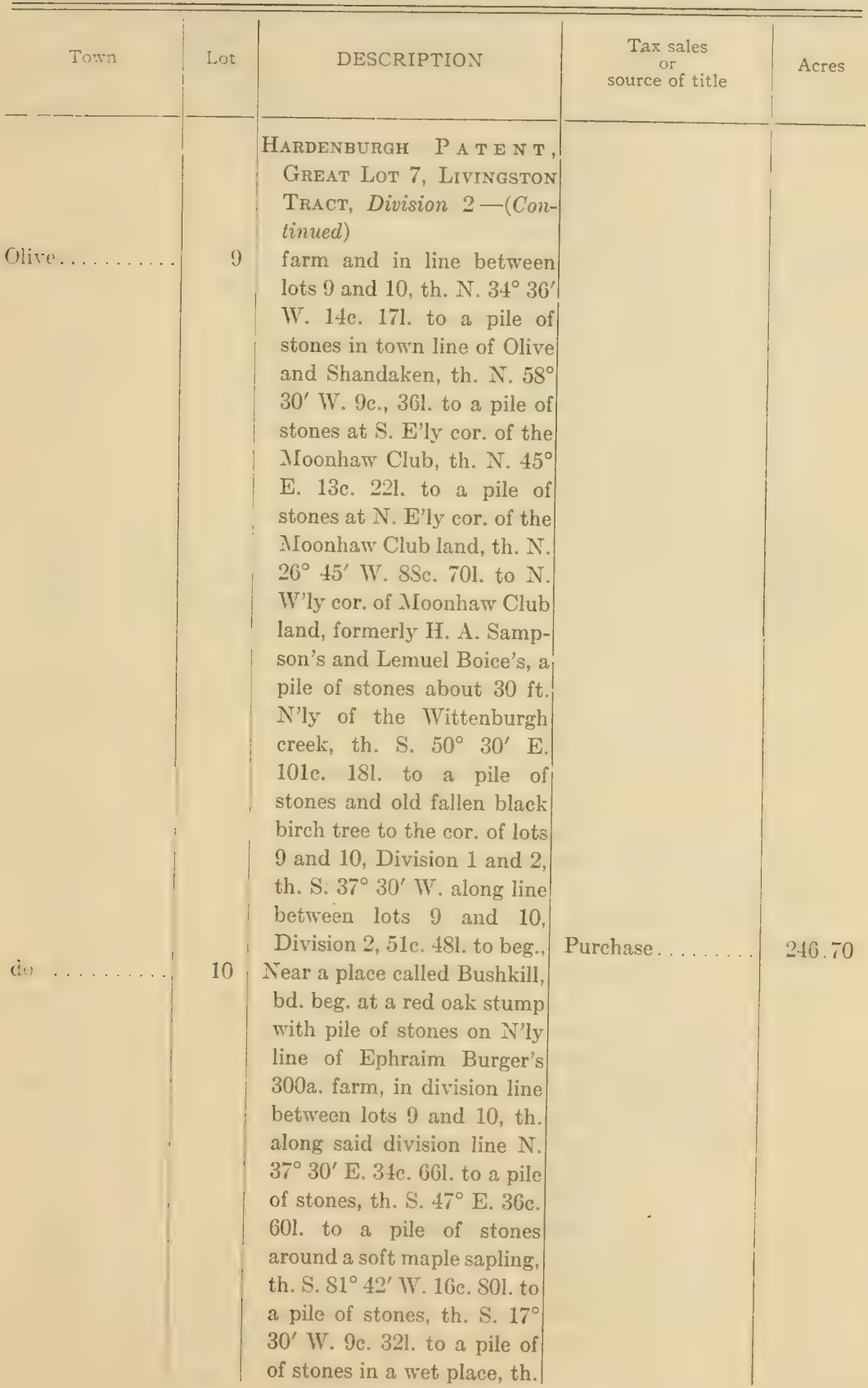


Ulster County

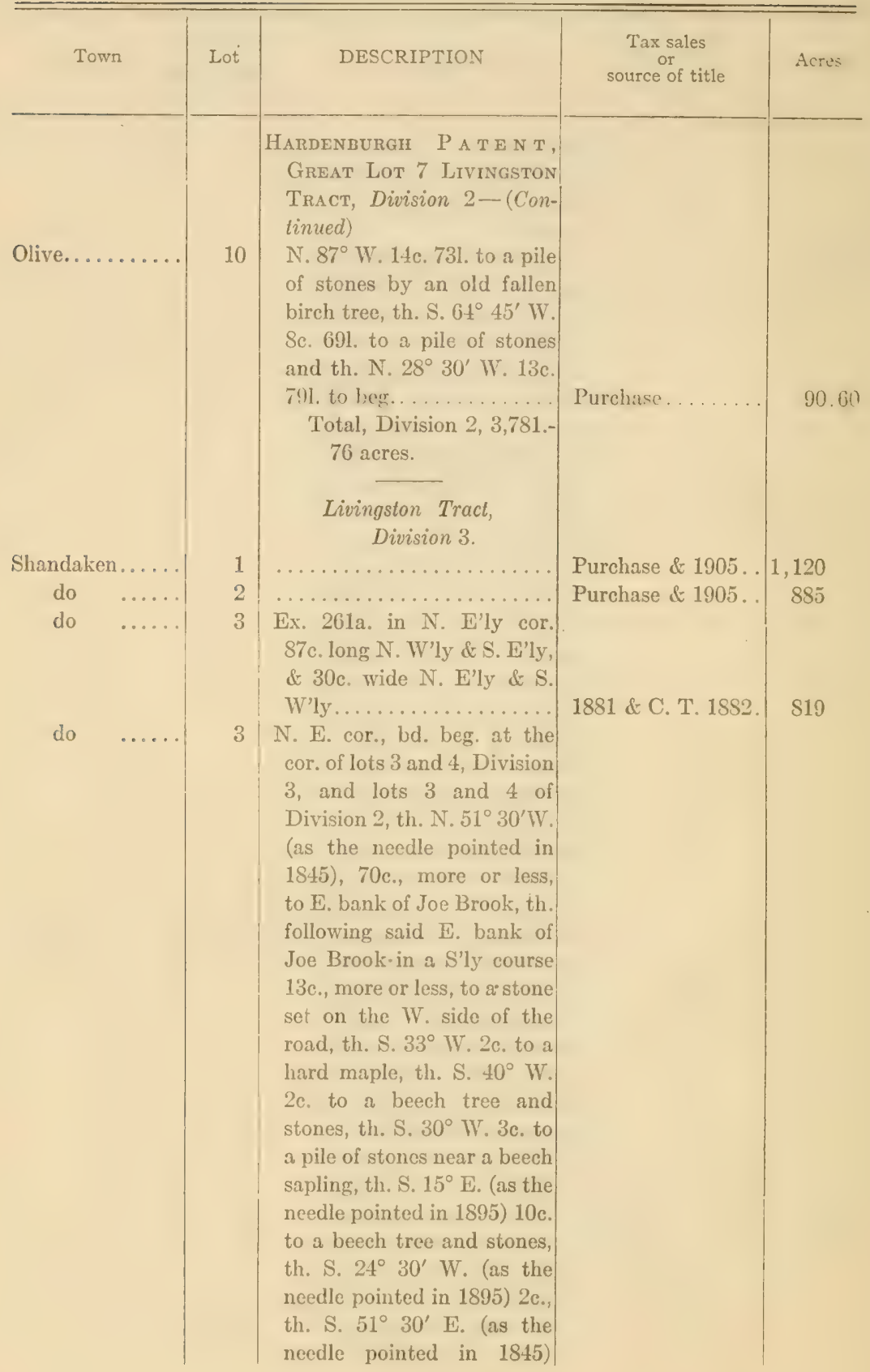




\section{Ulster County}

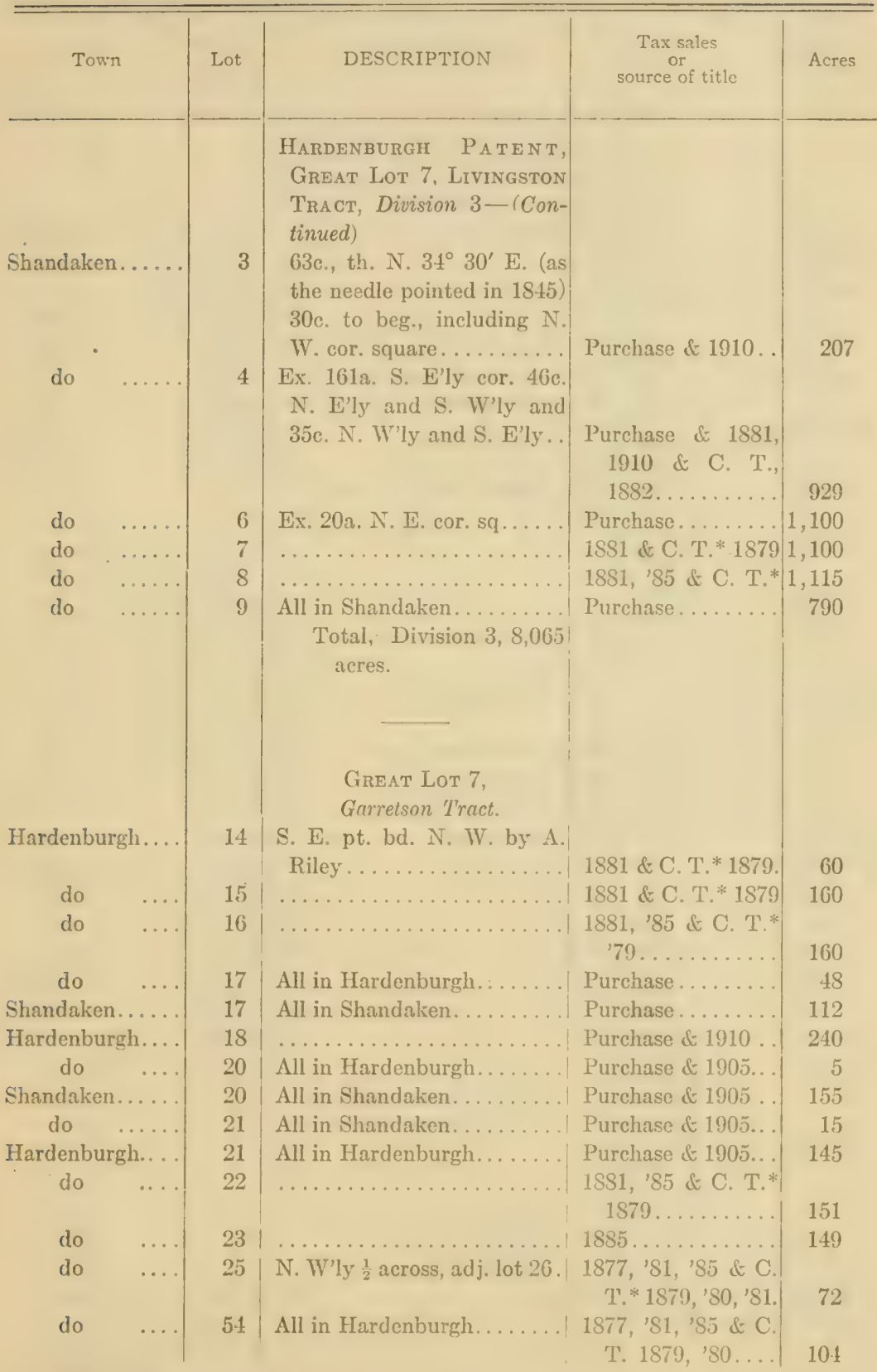

County Treasurer's sale. 


\section{Ulster County}

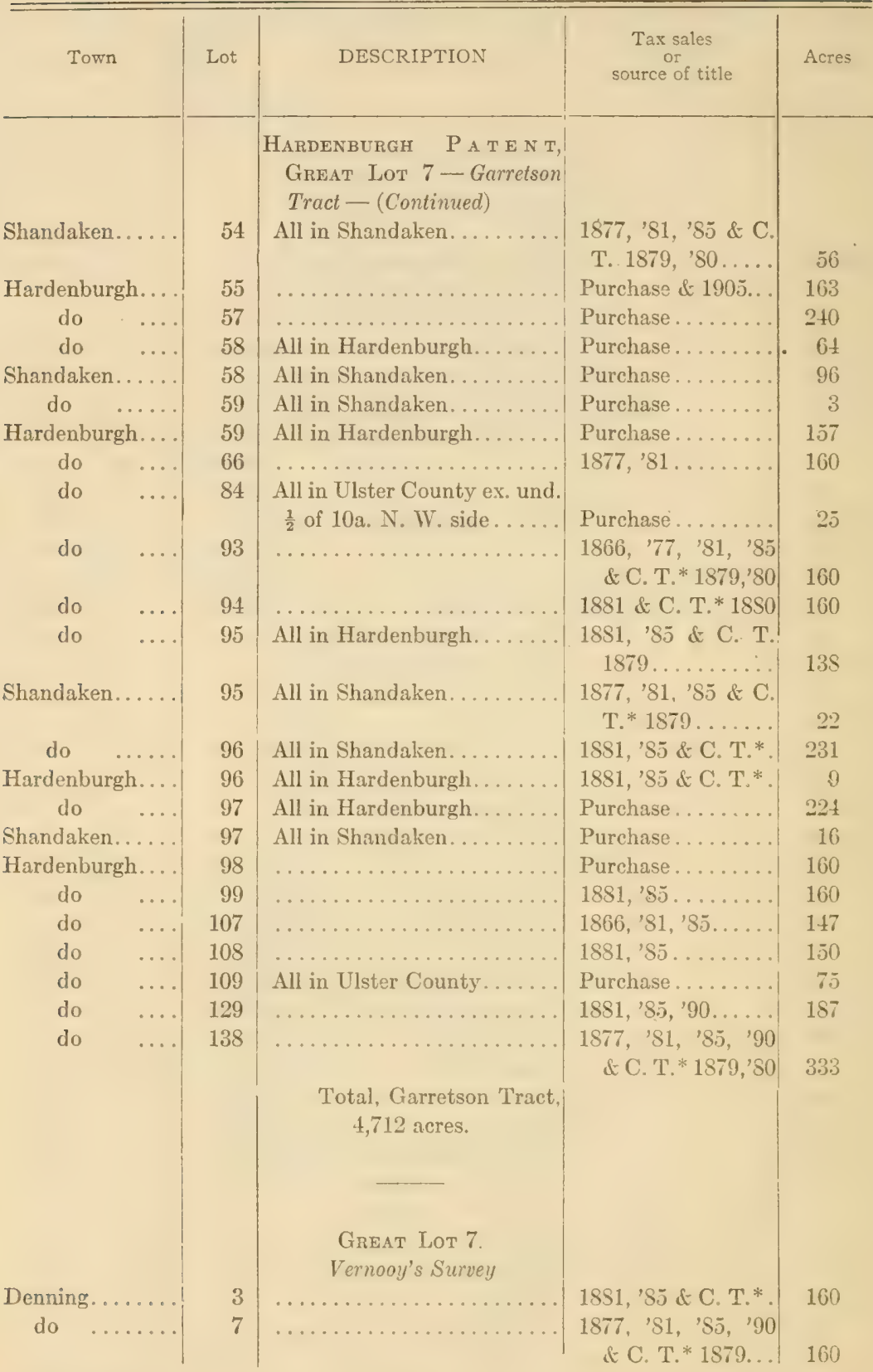

* County Treasuror's sale. 
Conservation Comanissiox.

\section{Ulster County}

\begin{tabular}{|c|c|c|c|c|}
\hline Town & Lot & DESCRIPTION & $\begin{array}{c}\text { Tax sales } \\
\text { or } \\
\text { source of title }\end{array}$ & Acres \\
\hline & & $\begin{array}{l}\text { HaRdenburgh PateN T, } \\
\text { Great Lot 7, Vernooy's } \\
\text { Survey-(Continued) }\end{array}$ & & \\
\hline Denning. & 8 & 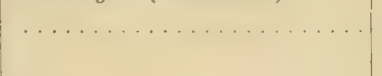 & $\begin{array}{l}\text { 1877, '81, '85, ' } 90 \\
\text { \& C. T.* 1882,' } 84\end{array}$ & 160) \\
\hline do & 9 & & Purchase......... & $161)$ \\
\hline do & 10 & 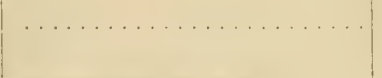 & $\begin{array}{l}\text { 1SS1, 'S.j \& C. T. } \\
18,9 \ldots \ldots \ldots \ldots\end{array}$ & 160 \\
\hline do & 12 & N. side $15 \mathrm{c} . \mathrm{N}$. \& $\mathrm{S}$. & Purchase....... I & 60 \\
\hline do & 13 & $\ldots \ldots$ & $\begin{array}{c}18 \pi, 81 \text { \& C. T.* } \\
1879 \ldots \ldots \ldots\end{array}$ & $160)$ \\
\hline do & 16 & & $\begin{array}{l}\text { 1S7т, '\$1, 'Sј \& C. } \\
\text { T.* 1S79..... }\end{array}$ & 160 \\
\hline do & 17 & & $\begin{array}{c}18 \pi 7, ' 81,9.5 \& \mathrm{C} . \\
\text { T." } 1879 \ldots \ldots\end{array}$ & 160 \\
\hline do & 18 & & 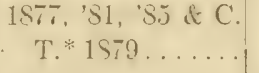 & \\
\hline do & 10 & & 1877 , '81, 'S5, '90.. & 160 \\
\hline do & 22 & & $\begin{array}{r}1881 \text {, ' } 85, \text { ' } 90 \& \mathrm{C} . \\
\text { T. * } 1581, \text { ' } 83 . . .\end{array}$ & \\
\hline do & 23 & & Purchase........ & $1+j)$ \\
\hline do & 24 & & 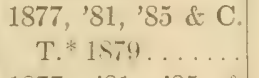 & 170 \\
\hline do & 25 & & $\begin{array}{l}187 ., \text { '\$1, '\$5 } \\
\text { C. T. }{ }^{*} 1879 \ldots \ldots\end{array}$ & \\
\hline do & 26 & $\ldots$ & 1881, \$.5. . . . . & $1 i j)$ \\
\hline do & 27 & & $1 \mathrm{~S} 00,9.5 \ldots \ldots$ & $1(i)$ \\
\hline do & 29 & & Purchase........ & $1(j ! 1$ \\
\hline do & 30 & & 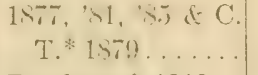 & 160 \\
\hline do ${ }^{\circ}$ & 31 & & Purchase \& 1910... & 160 \\
\hline do & 32 & & $\begin{array}{r}1877, ' 81, ' 85 \& \mathrm{C} . \\
\text { T. } 1.79 \ldots \ldots\end{array}$ & $16 i 0$ \\
\hline do & 33 & & $1877, ' 81$, ' $85 . \ldots$. & 16i) \\
\hline do & 36 & & $\begin{array}{c}1877, \text { '81, 'S5̃ \& C. } \\
\text { T.* } 1879 \ldots \ldots\end{array}$ & 150 \\
\hline do & 37 & & 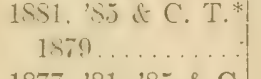 & $2(1)$ \\
\hline do & $3 S$ & & $\begin{array}{r}1877, \text { ' } 81, \text { ' } 85 \text { \& C. } \\
\text { T.* 1879, '80, '81 }\end{array}$ & $20 i$ \\
\hline do & 39 & & $\begin{array}{r}1877, \text { '\$1, '85 \& C. } \\
\text { T.* 1879, '80, '81 }\end{array}$ & $20 !)$ \\
\hline do & 43 & & $\begin{array}{c}\text { 1881. '85 \& C. T.* } \\
1579 \ldots \ldots \ldots \ldots\end{array}$ & $2,1) 0$ \\
\hline
\end{tabular}

* County Trensurer's sale. 


\section{Ulster County}

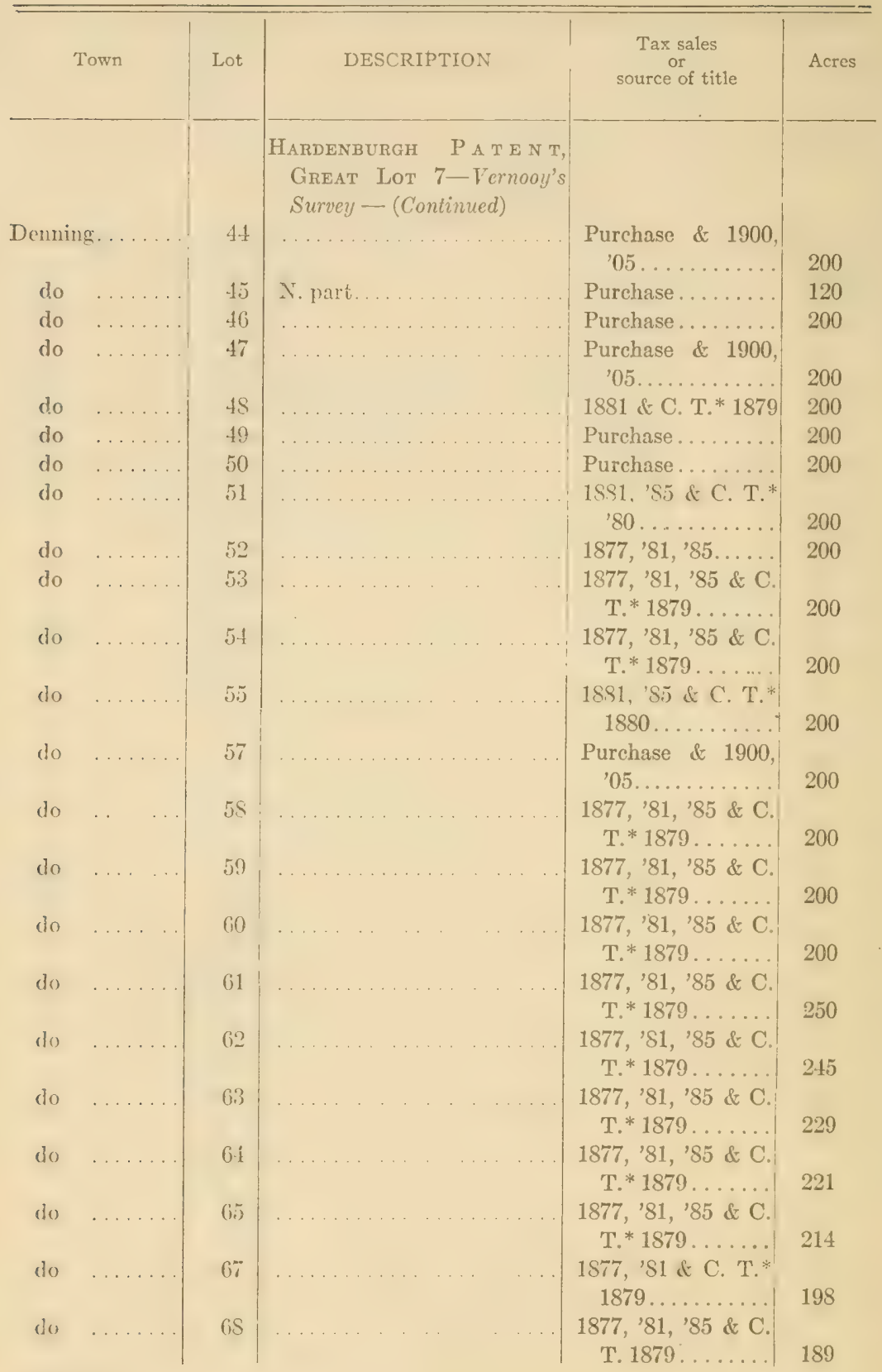

\footnotetext{
* County 'Treasurer's sale.
} 


\section{ULSTER COUnty}

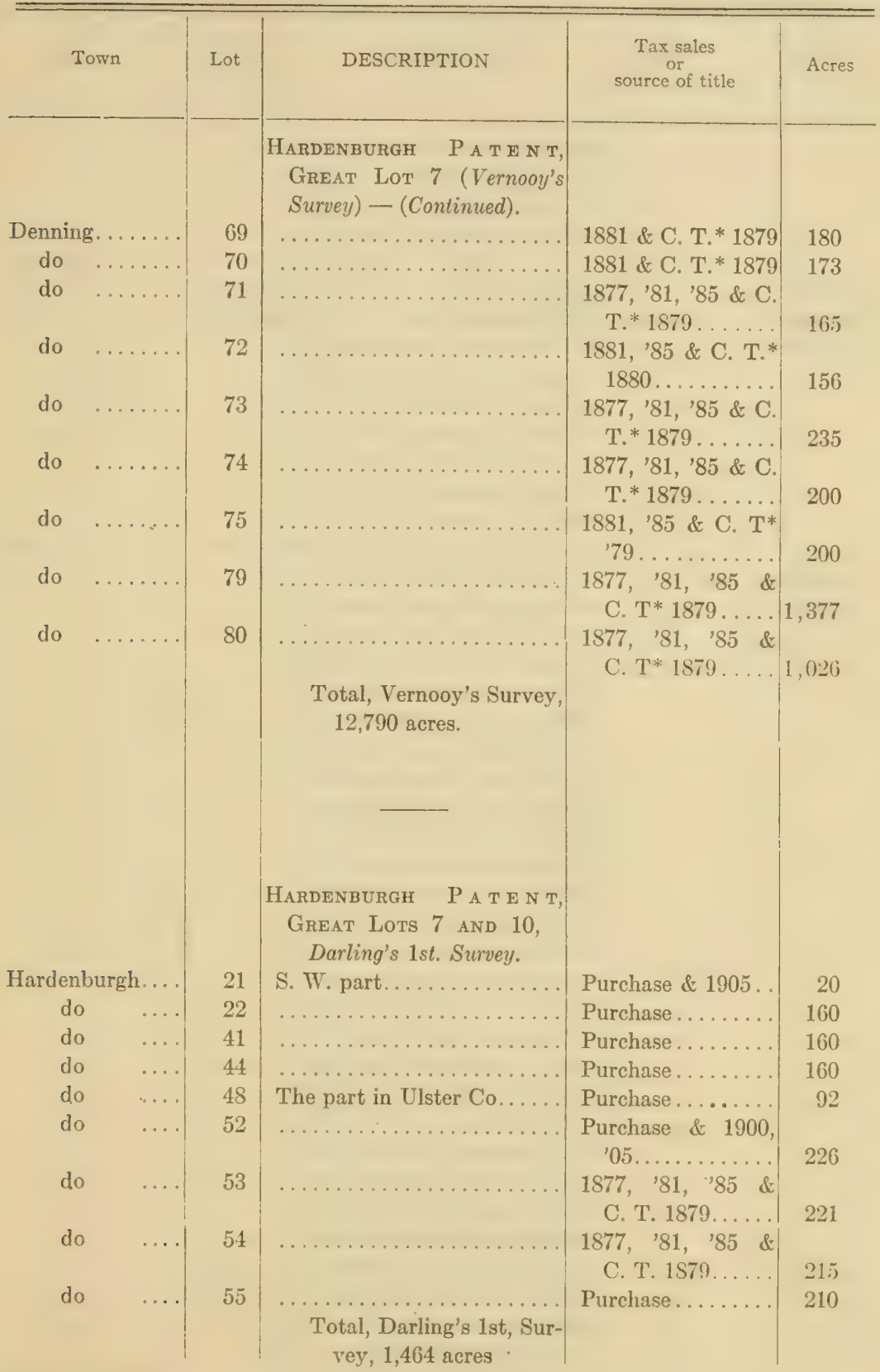

* County Treasurer's sale. 


\section{Uluster County}

\begin{tabular}{|c|c|c|c|c|}
\hline Town & Lot & DESCRIPTION & $\begin{array}{c}\text { Tax sales } \\
\text { or } \\
\text { source of title }\end{array}$ & Acres \\
\hline $\begin{array}{l}\text { Shandaken..... } \\
\text { TWoodstock..... } \\
\text { Shandaken..... } \\
\text { Shandaken and } \\
\text { Woodstock }\end{array}$ & $\begin{array}{l}3 \\
3 \\
4\end{array}$ & 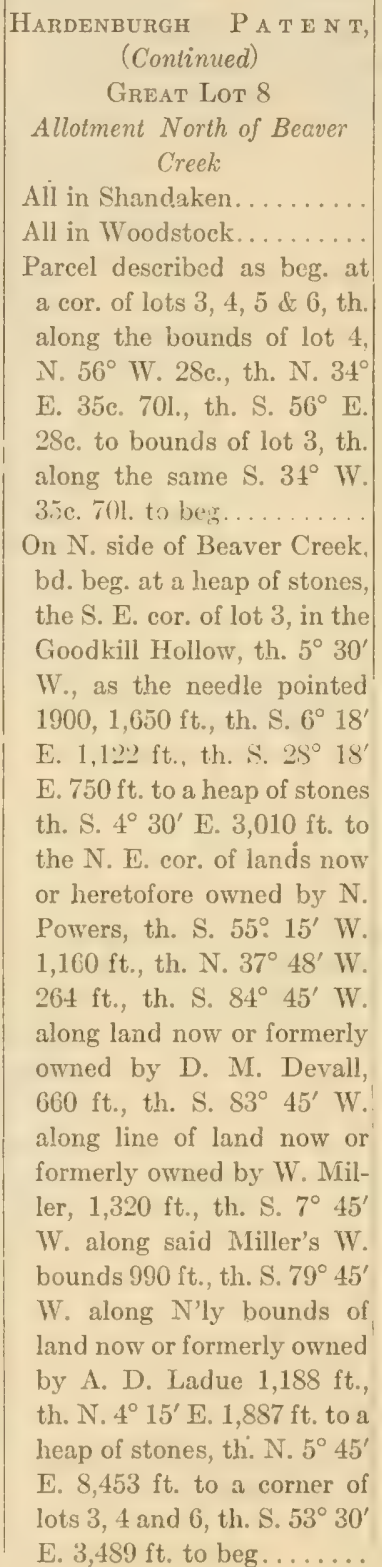 & $\begin{array}{l}\text { Purchase. } \\
\text { Purchase. } \\
\text { Purchase. }\end{array}$ & $\begin{array}{l}254 \\
250\end{array}$ \\
\hline
\end{tabular}




\section{Ulster County}

\begin{tabular}{|c|c|c|c|c|}
\hline Town & Lot & DESCRIPTION & $\begin{array}{c}\text { Tax sales } \\
\text { or } \\
\text { source of title }\end{array}$ & Acres \\
\hline Shandaken... & 6 & 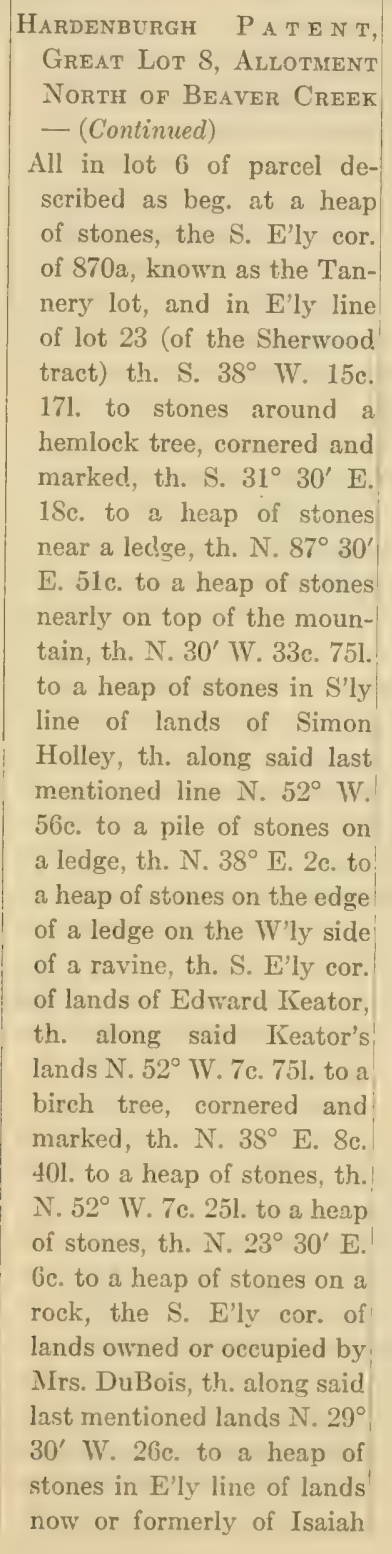 & & \\
\hline
\end{tabular}




\section{Ulster County}

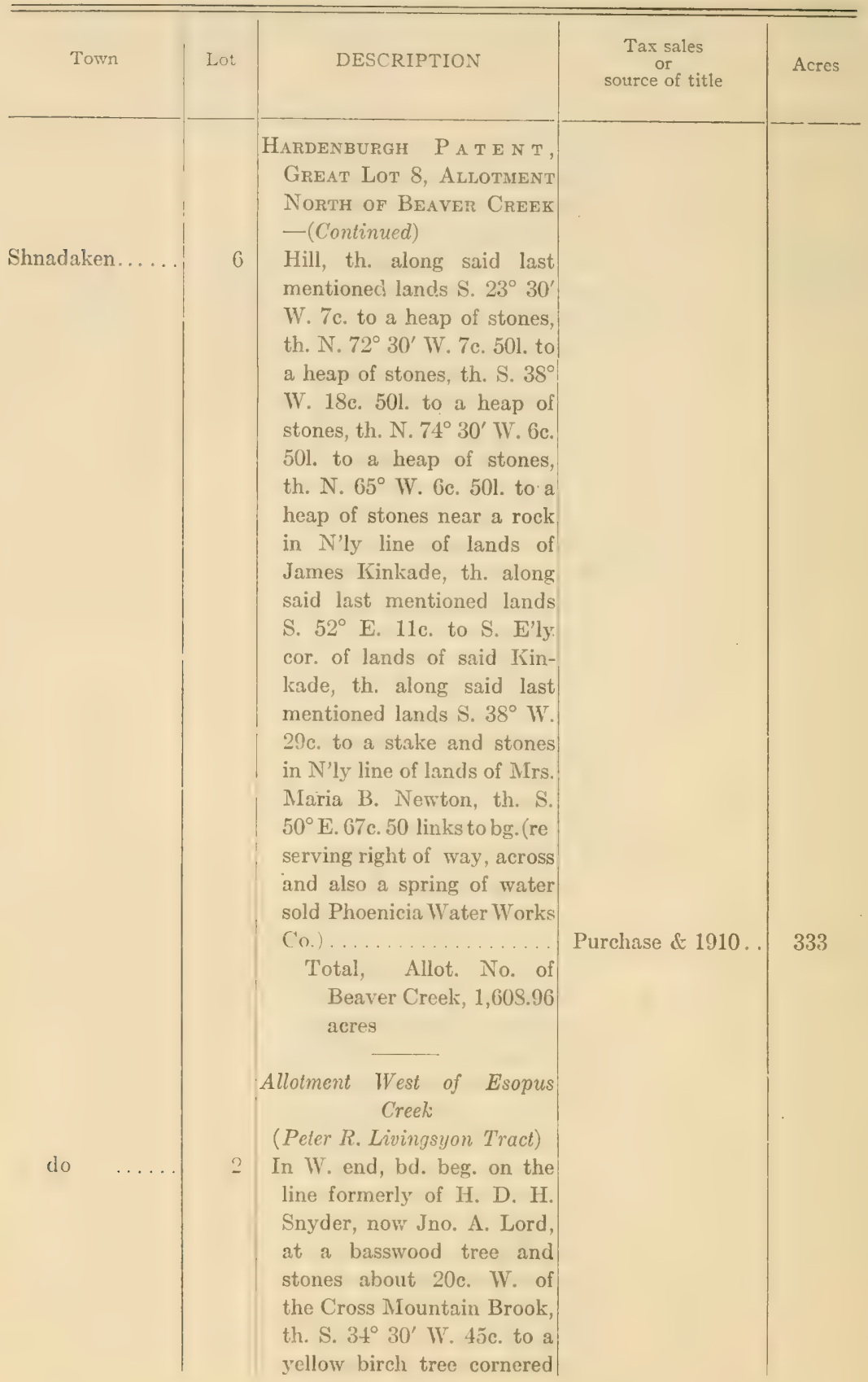




\section{Ulster County}

\begin{tabular}{|c|c|c|c|c|}
\hline Town & Lot & DESCRIPTION & $\begin{array}{c}\text { Tax sales } \\
\text { or } \\
\text { source of title }\end{array}$ & Acres \\
\hline Shandaken. & $2 \& 3$ & 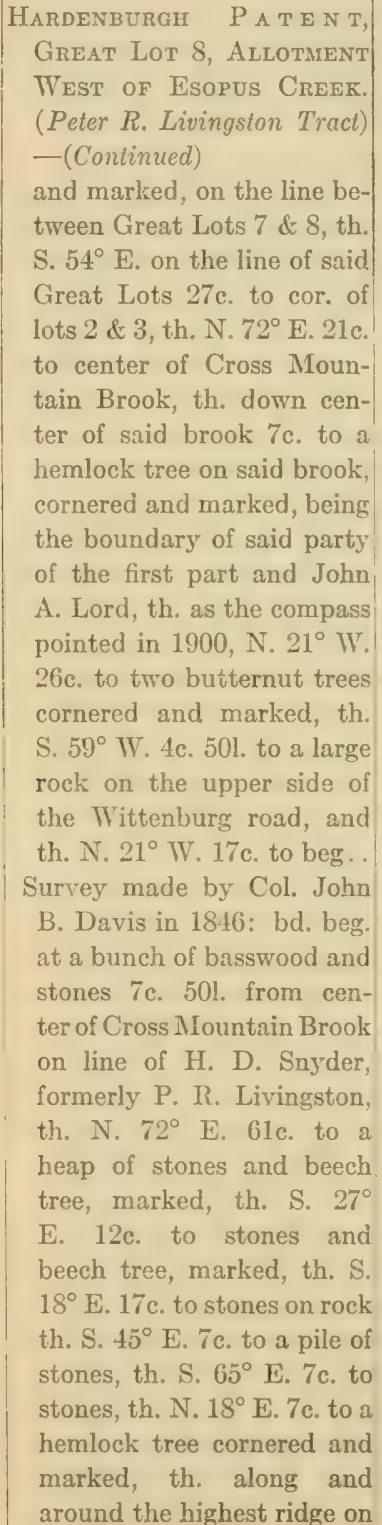 & Purchase.. & 124 \\
\hline
\end{tabular}




\section{Úlster County}

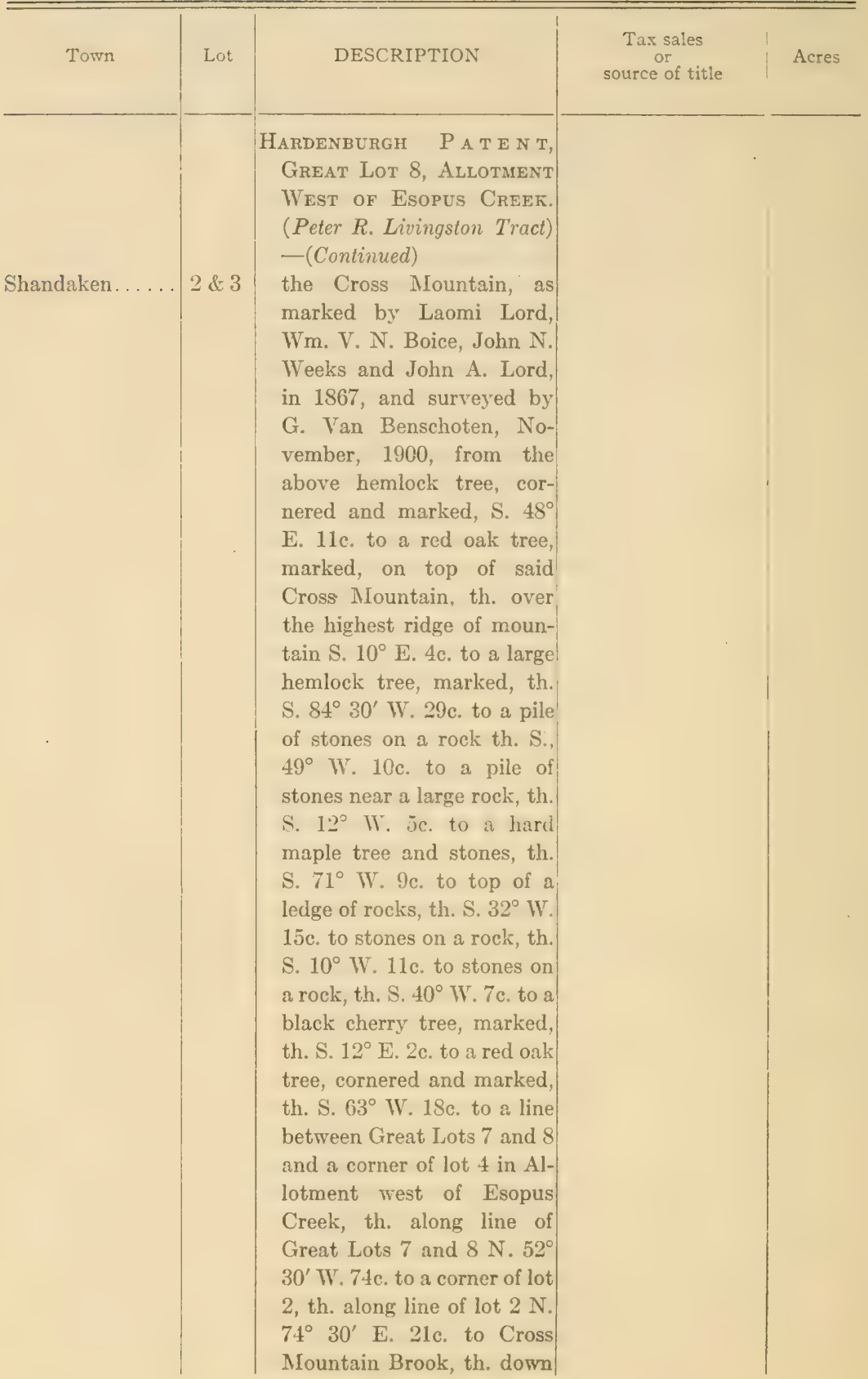




\section{Ulster County}

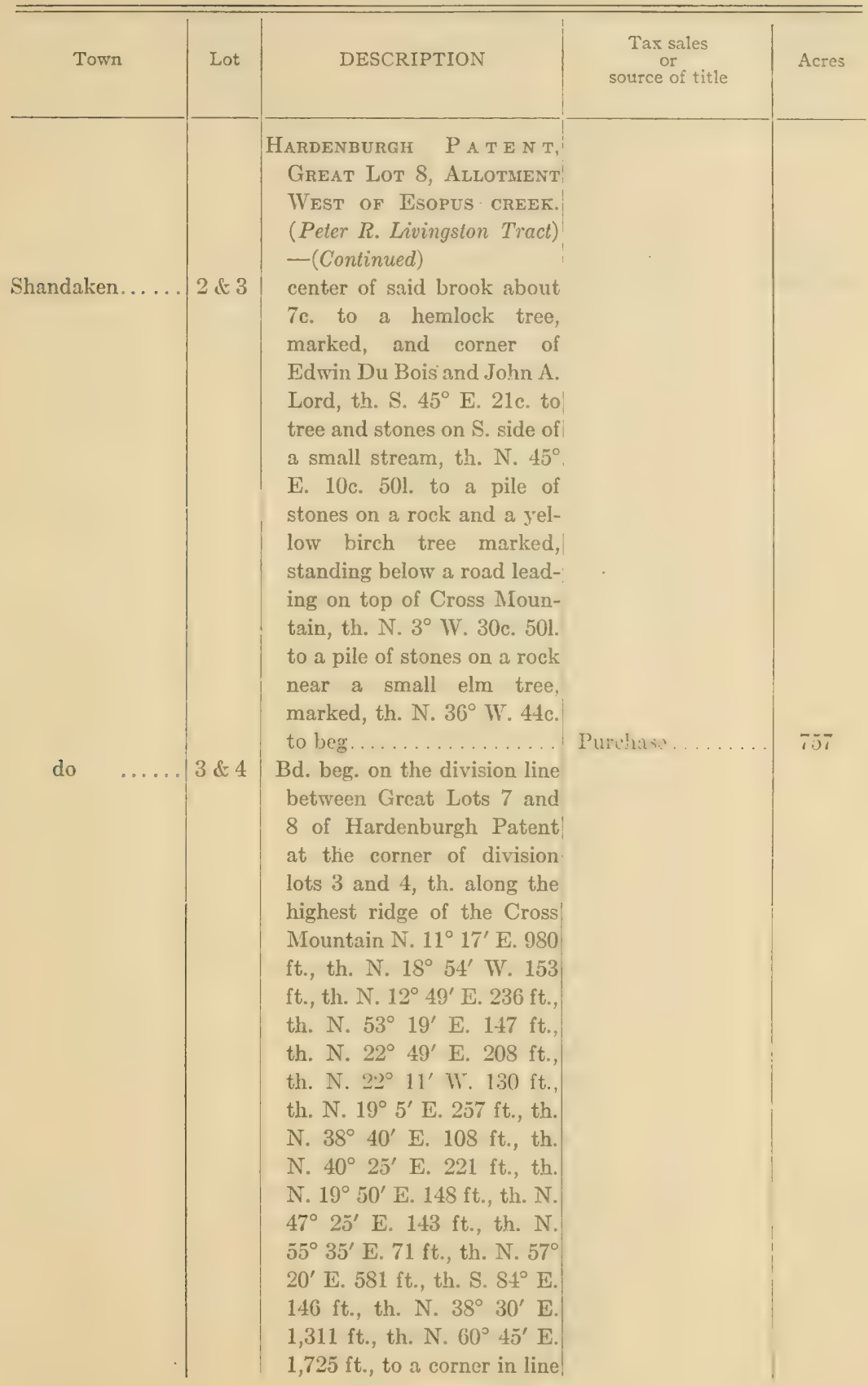


Ulster County

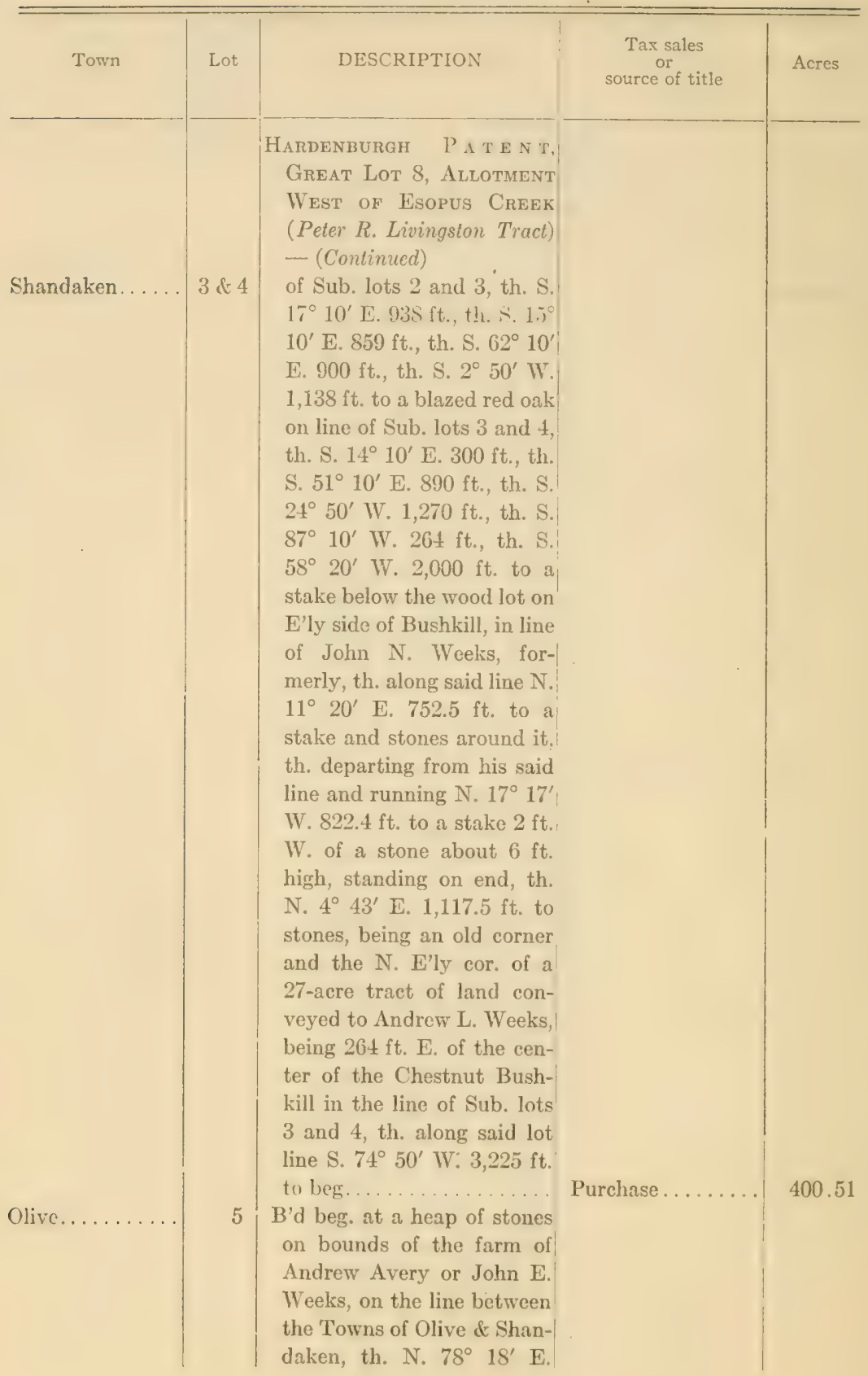


Ulster County

\begin{tabular}{|c|c|c|c|c|}
\hline Torrn & Lot & DESCRIPTION & $\begin{array}{c}\text { Tax sales } \\
\text { or } \\
\text { source of title }\end{array}$ & Acres \\
\hline Olive ...... & 5 & 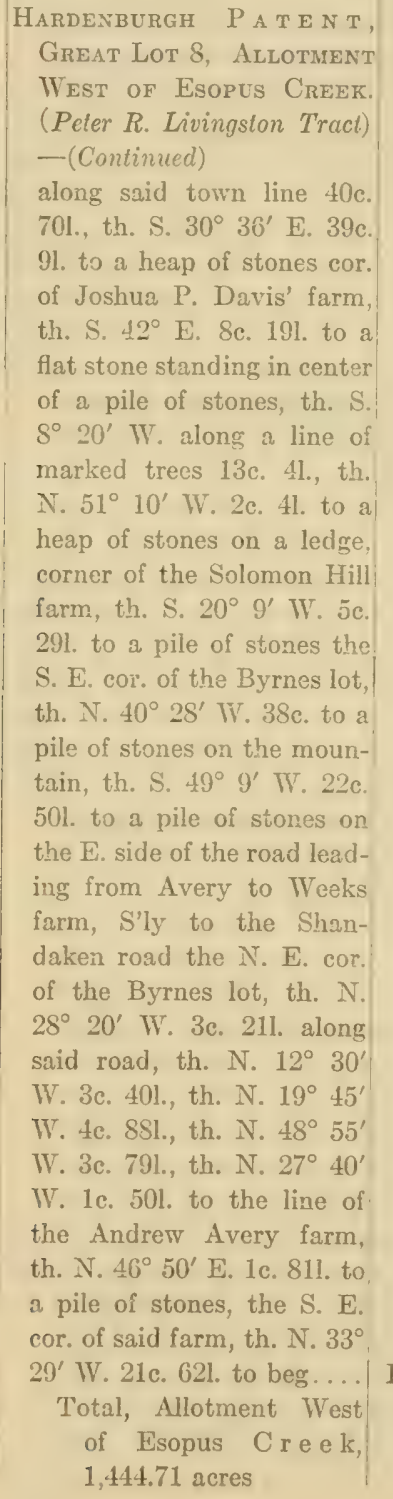 & Purchase. . & 163.20 \\
\hline
\end{tabular}


Ulster County

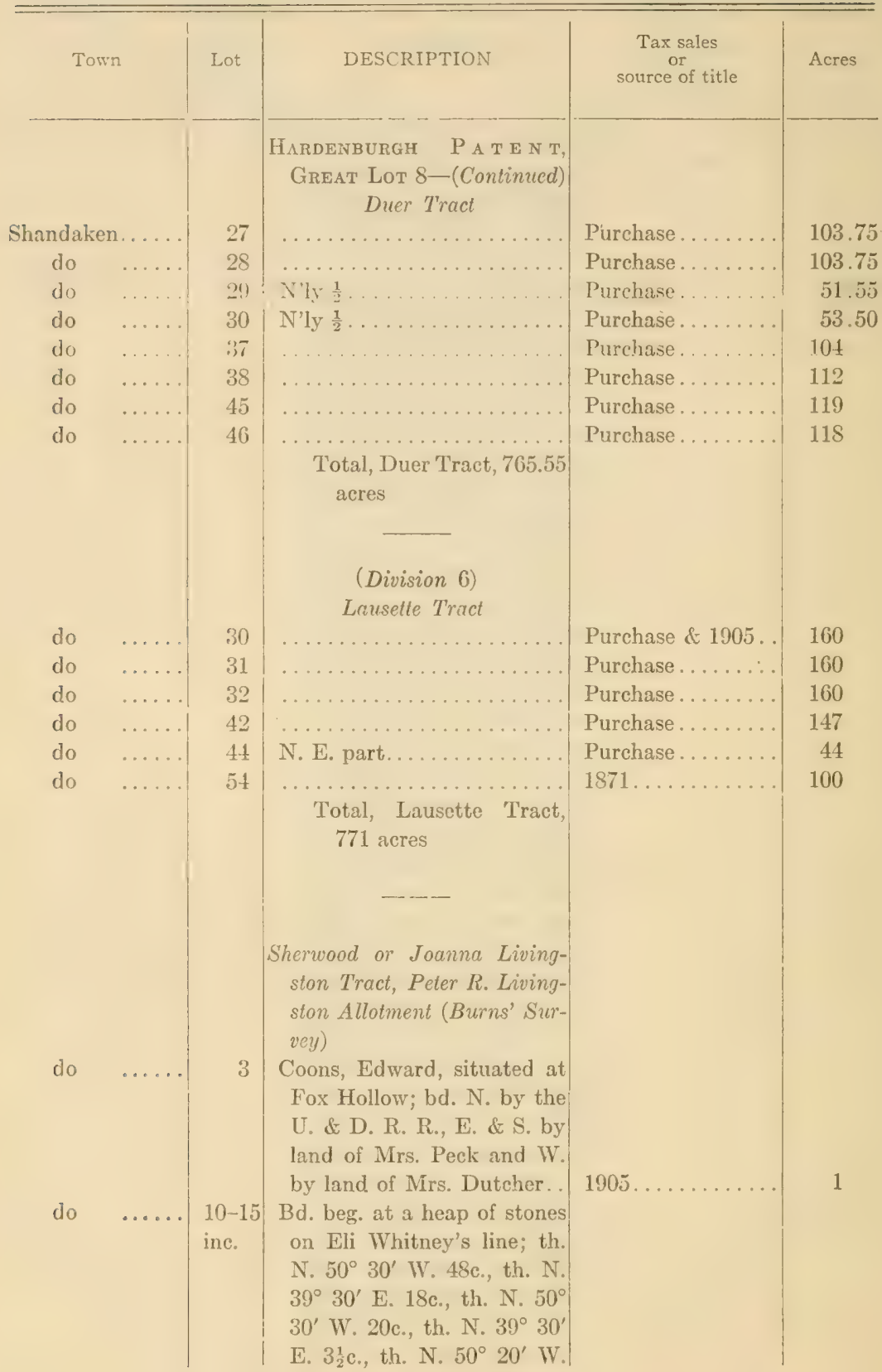


Ulster County

\begin{tabular}{|c|c|c|c|c|}
\hline Town & Lot & DESCRIPTION & $\begin{array}{c}\text { Tax sales } \\
\text { or } \\
\text { source of title }\end{array}$ & Acres \\
\hline Shandaken. . & $\begin{array}{c}10-15 \\
\text { inc. } \\
11-23\end{array}$ & 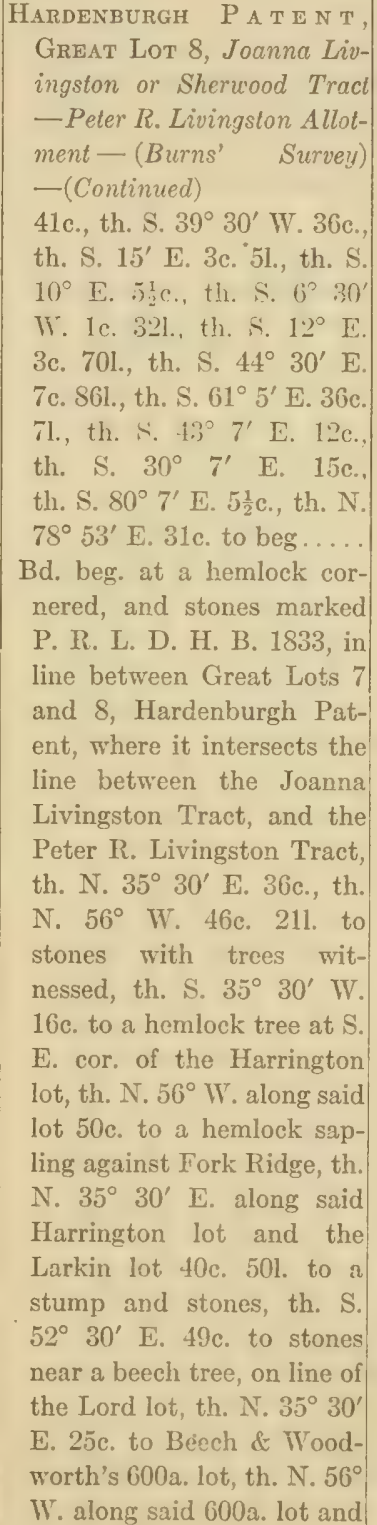 & Purchase... & 359 \\
\hline
\end{tabular}


Ulster County

\begin{tabular}{|c|c|c|c|c|}
\hline Town & L Lot & DESCRIPTION & $\begin{array}{c}\text { Tax sales } \\
\text { or } \\
\text { source of title }\end{array}$ & Acres \\
\hline Shandaken. & $11-23$ & 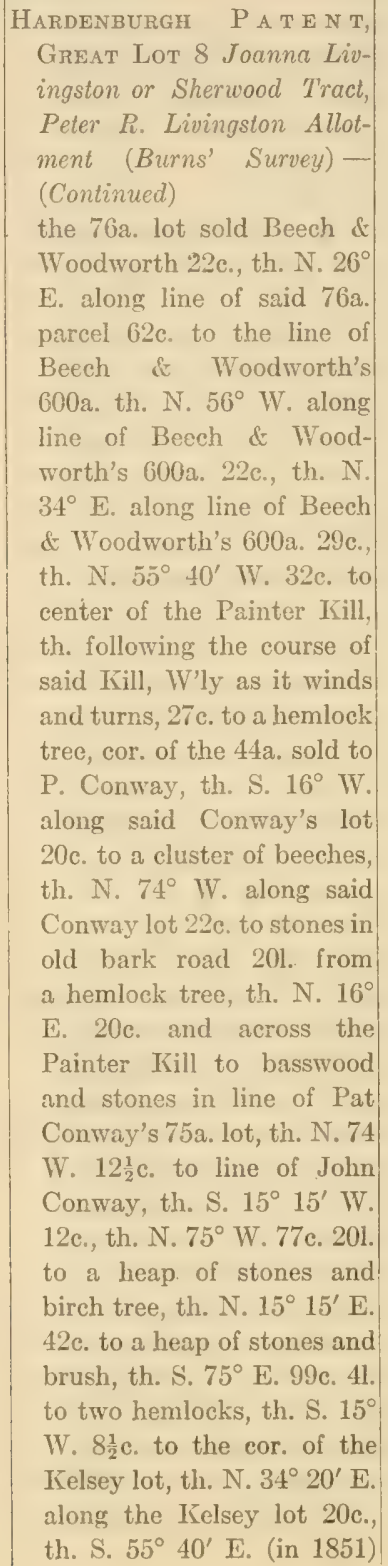 & & \\
\hline
\end{tabular}




\section{Ulster County}

\begin{tabular}{|c|c|c|c|c|}
\hline Torwn & Lot & DESCRIPTION & $\begin{array}{c}\text { Tax sales } \\
\text { or } \\
\text { source of title }\end{array}$ & Acres \\
\hline Shandaken. & $11-23$ & 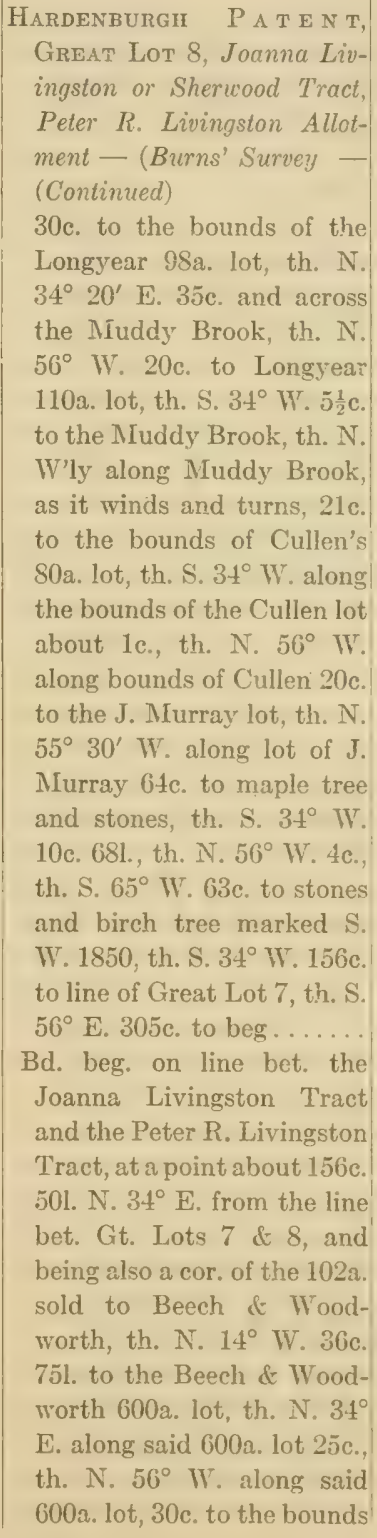 & Purchase. & $4,259.65$ \\
\hline
\end{tabular}




\section{Ulster County}

\begin{tabular}{|c|c|c|c|c|}
\hline Town & Lot & DESCRIPTION & $\begin{array}{c}\text { Tax sales } \\
\text { or } \\
\text { source of title }\end{array}$ & Acres \\
\hline Shandaken. & \begin{tabular}{|r} 
\\
\\
20,21 \\
$22 \&$ \\
23
\end{tabular} & 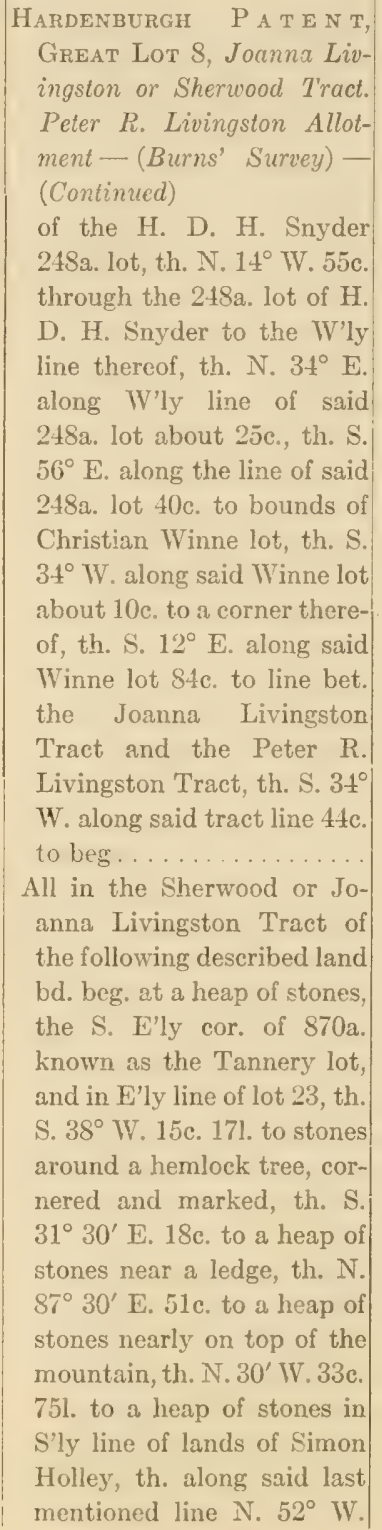 & Purchase. & 419 \\
\hline
\end{tabular}


Ulster County

\begin{tabular}{|c|c|c|c|c|}
\hline Town & Lot & DESCRIPTION & $\begin{array}{c}\text { Tax sales } \\
\text { or } \\
\text { source of title }\end{array}$ & Acres \\
\hline Shandaken.. & $\begin{array}{l}20,21, \\
22 \& 23\end{array}$ & 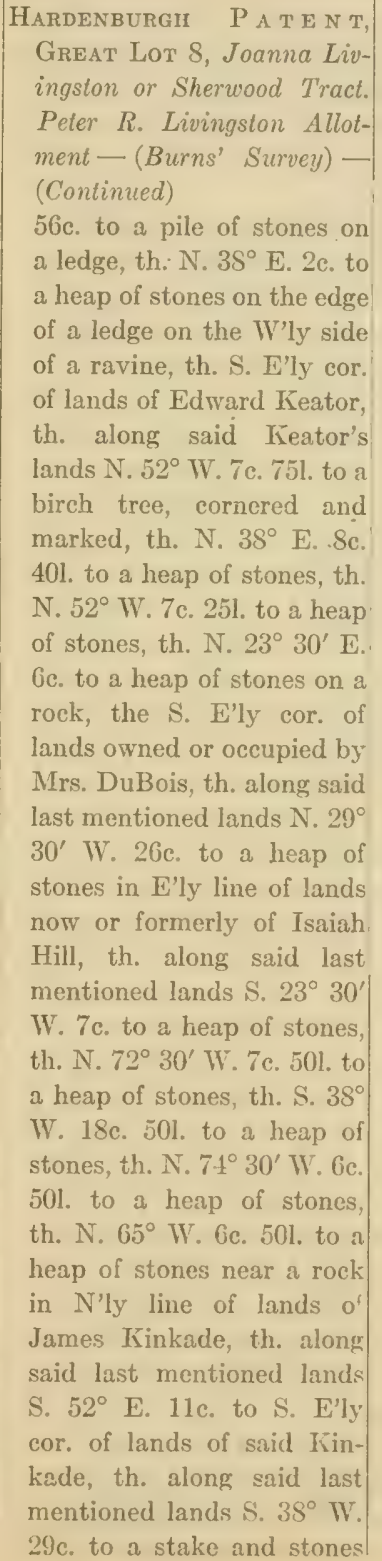 & & \\
\hline
\end{tabular}




\section{Ulster County}

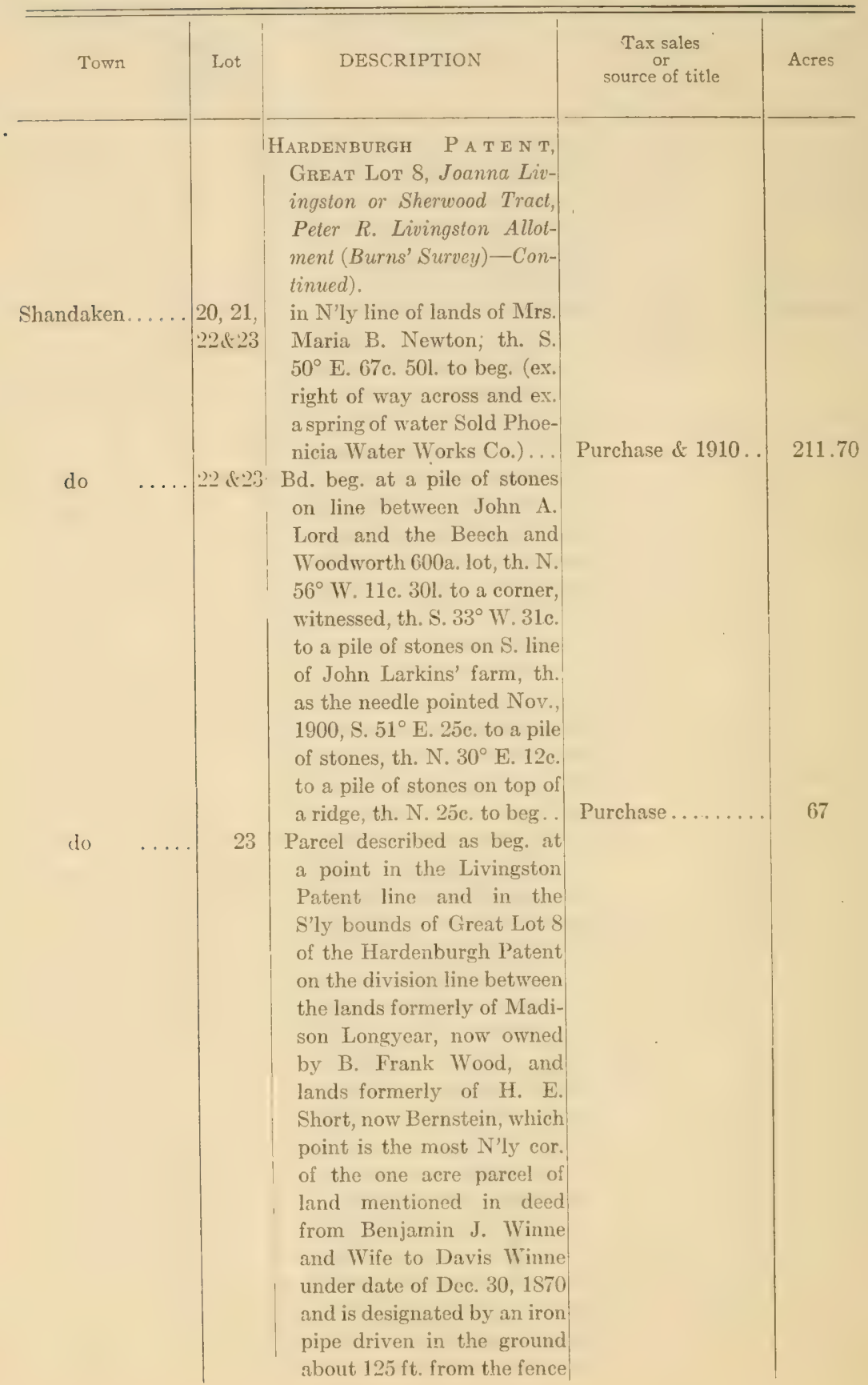


Ulster CoUnty

\begin{tabular}{|c|c|c|c|c|}
\hline Town & Lot & DESCRIPTION & $\begin{array}{c}\text { Tax sales } \\
\text { or } \\
\text { source of title }\end{array}$ & Acres \\
\hline Shandaken. . & 23 & 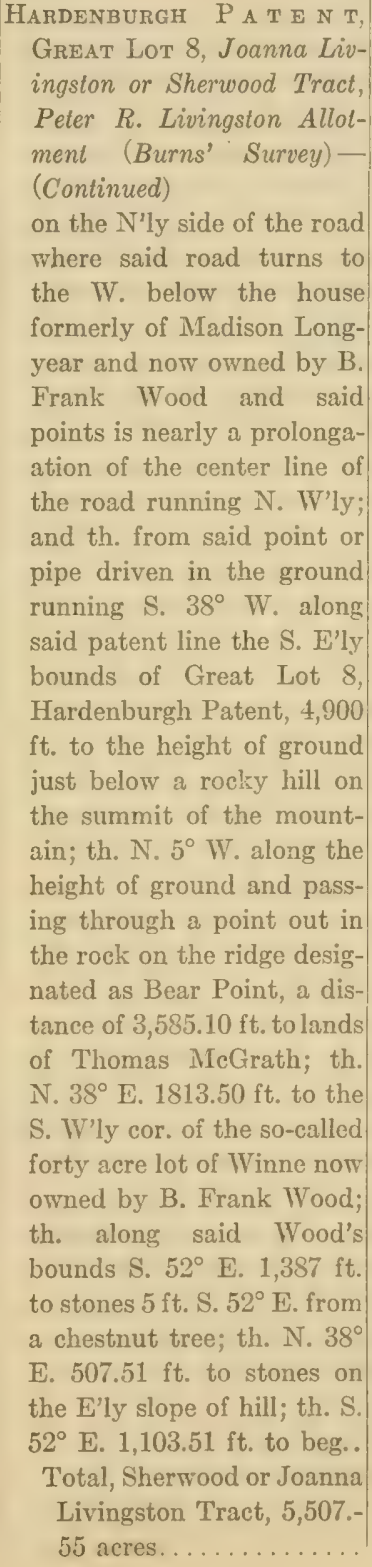 & Purchase \& 1910. & 190.20 \\
\hline
\end{tabular}




\section{ULster County}

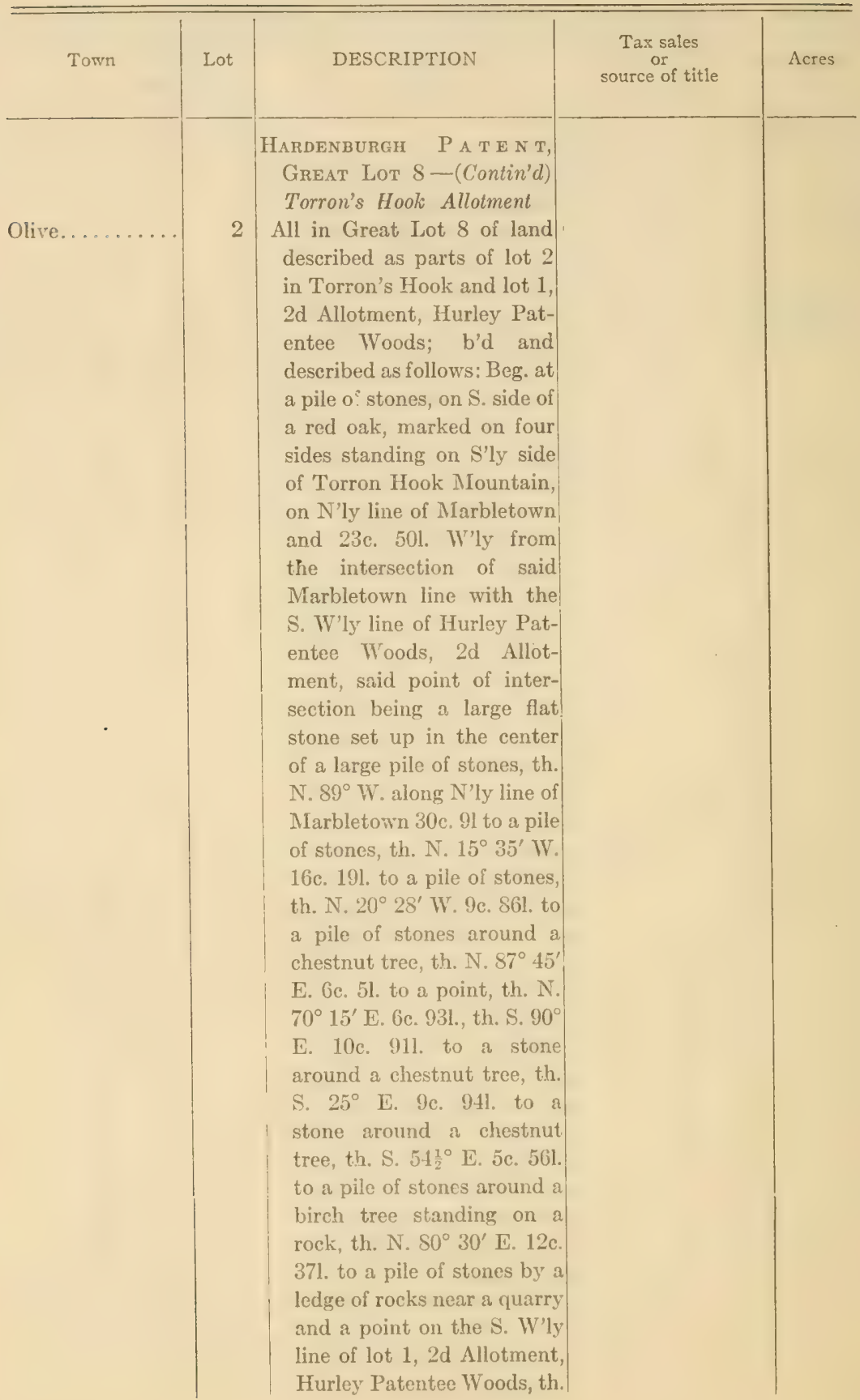




\section{Ulster COUnty}

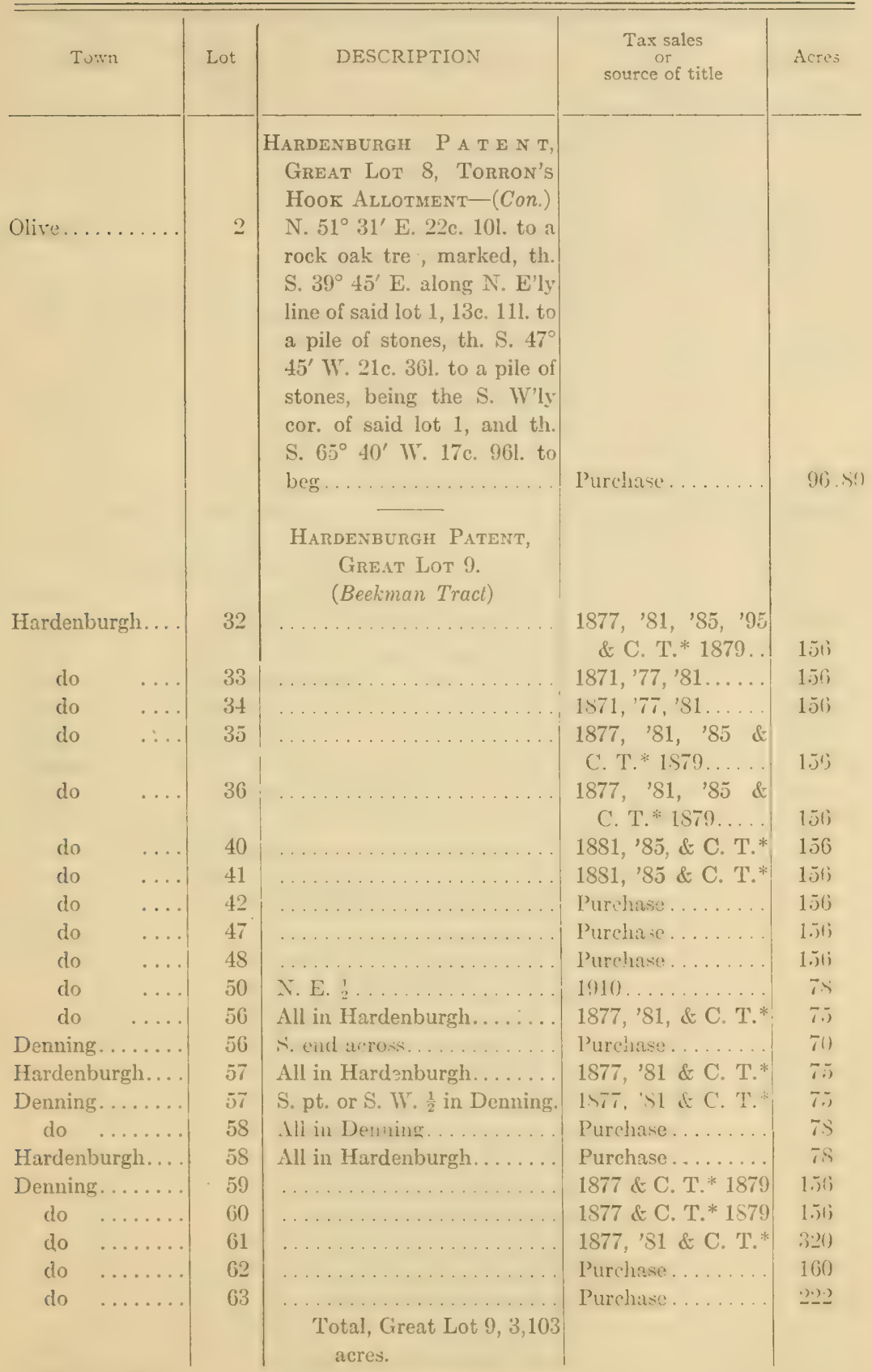

* County Treasurer's sales. 
Ulster County

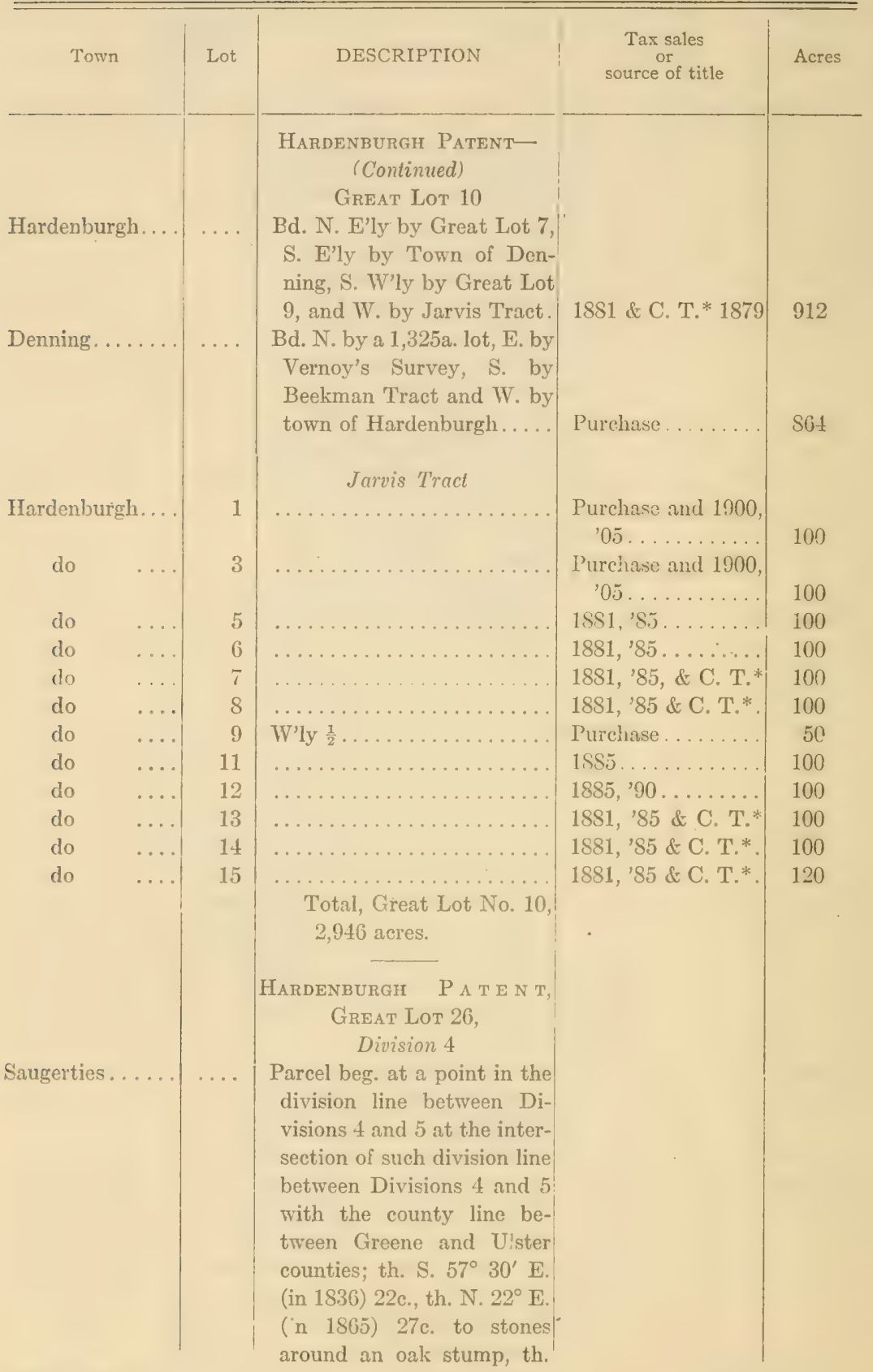

\footnotetext{
* County Treasurer's sales.
} 


\section{Ulster County}

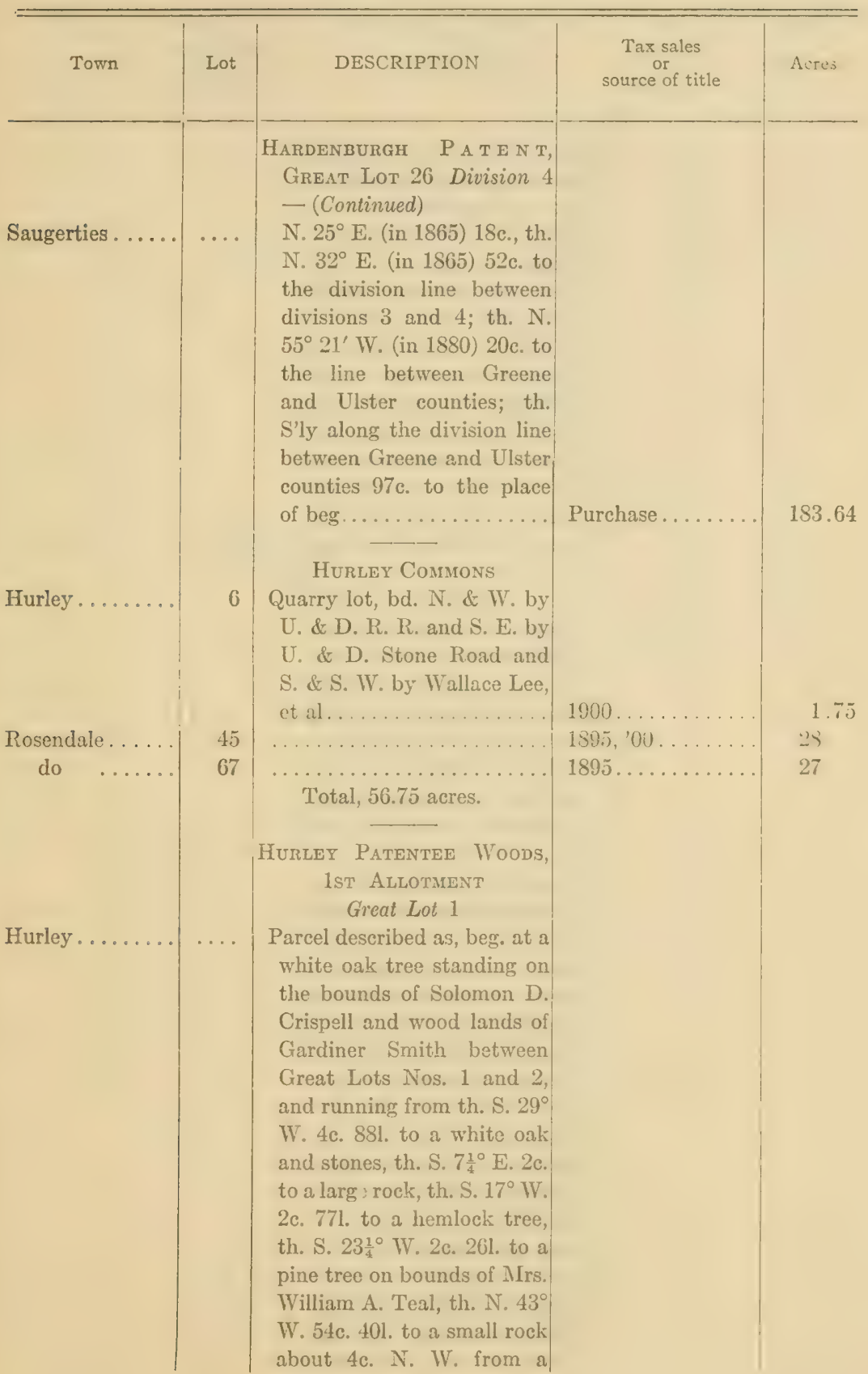


Ulster County

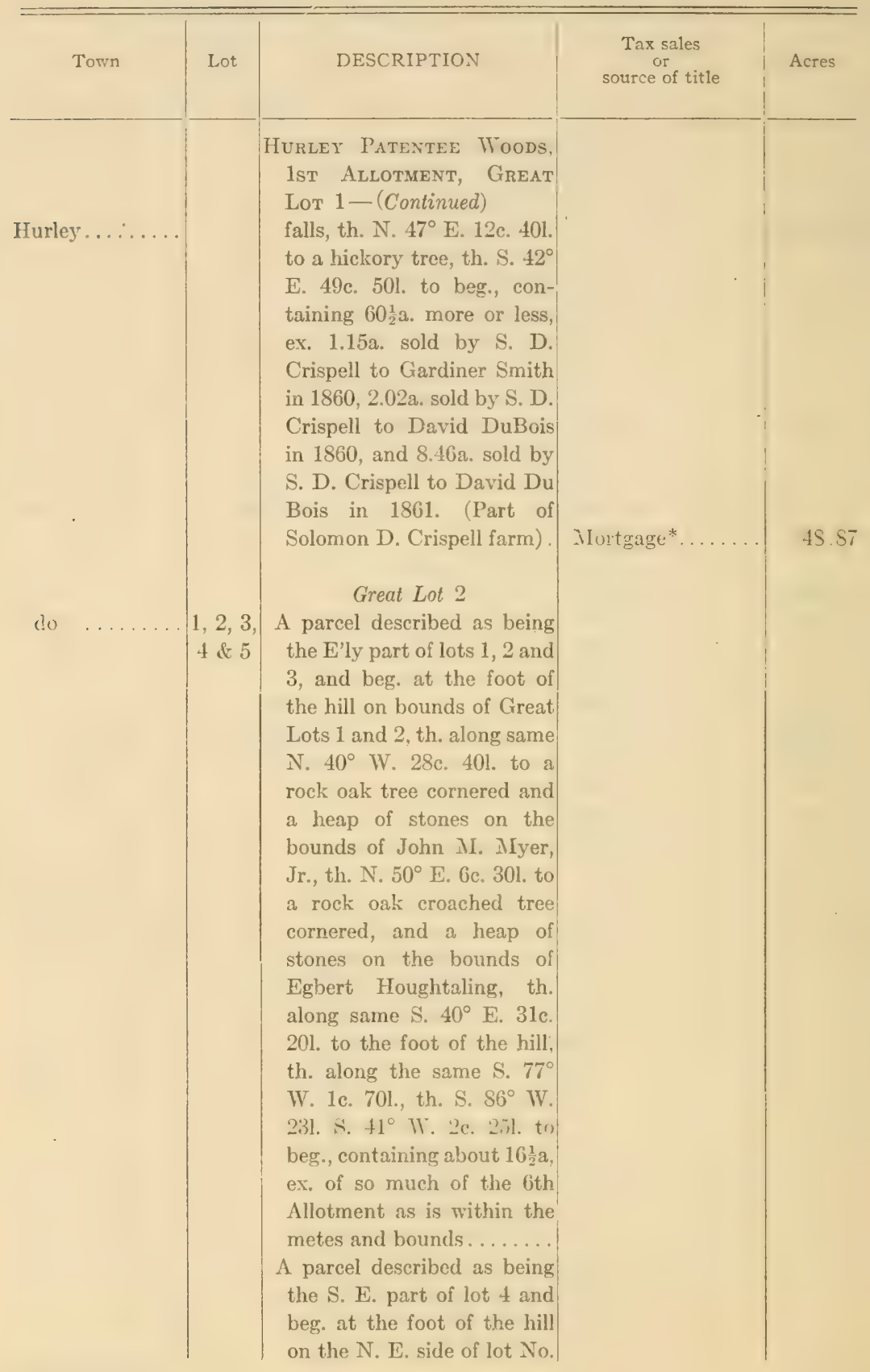




\section{Ulster County}

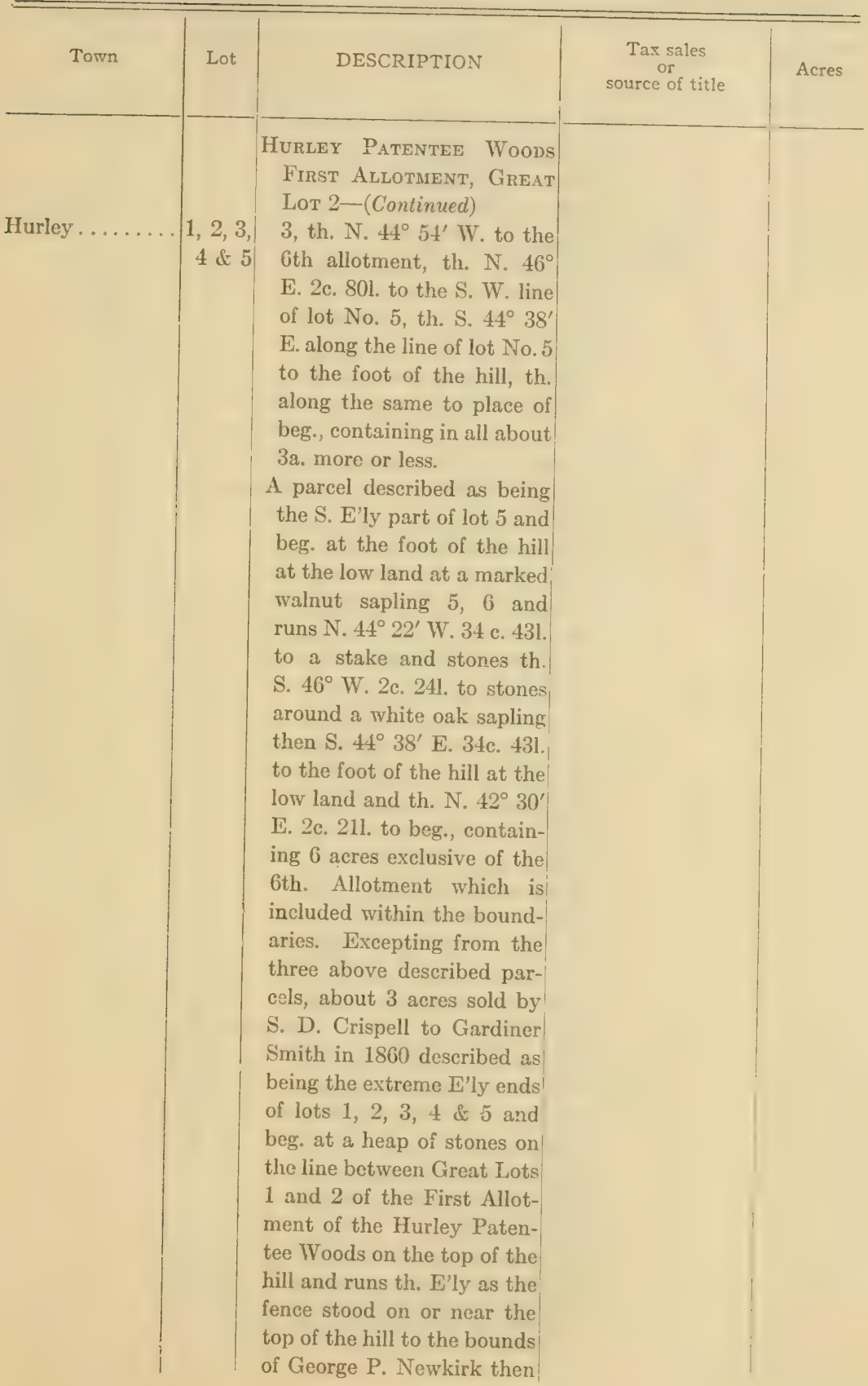




\section{Ulster County}

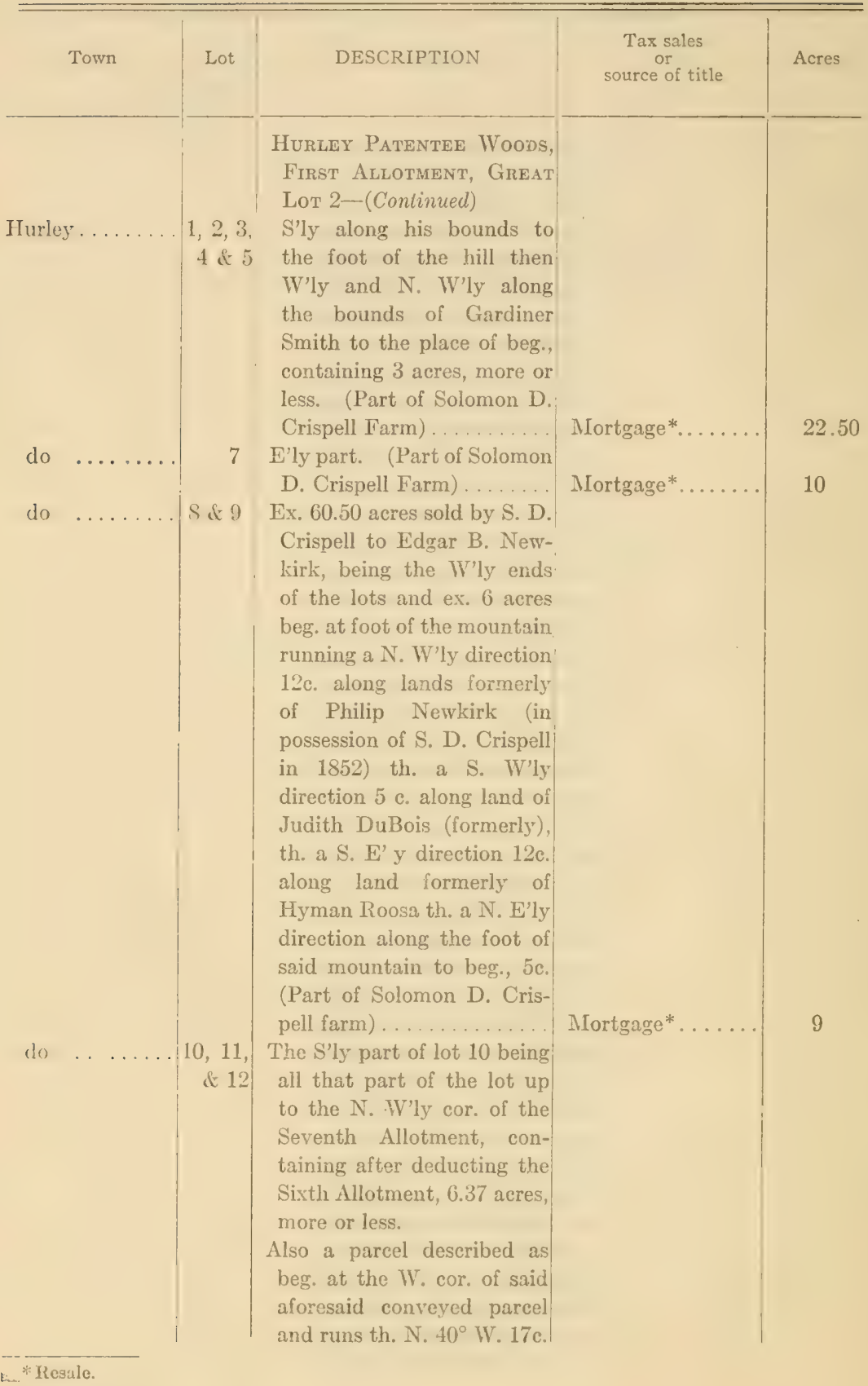


Ulster County

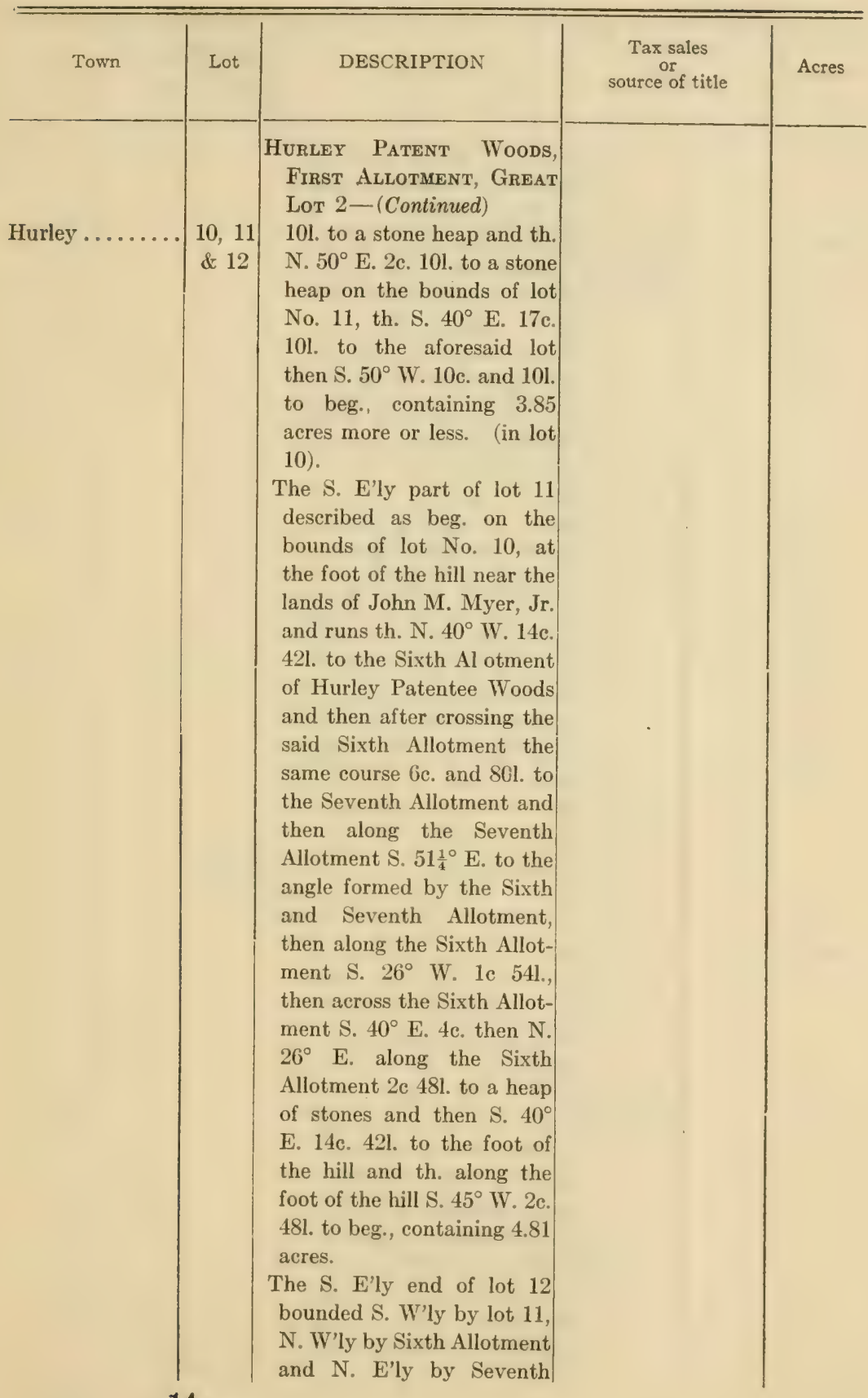


Ulster County

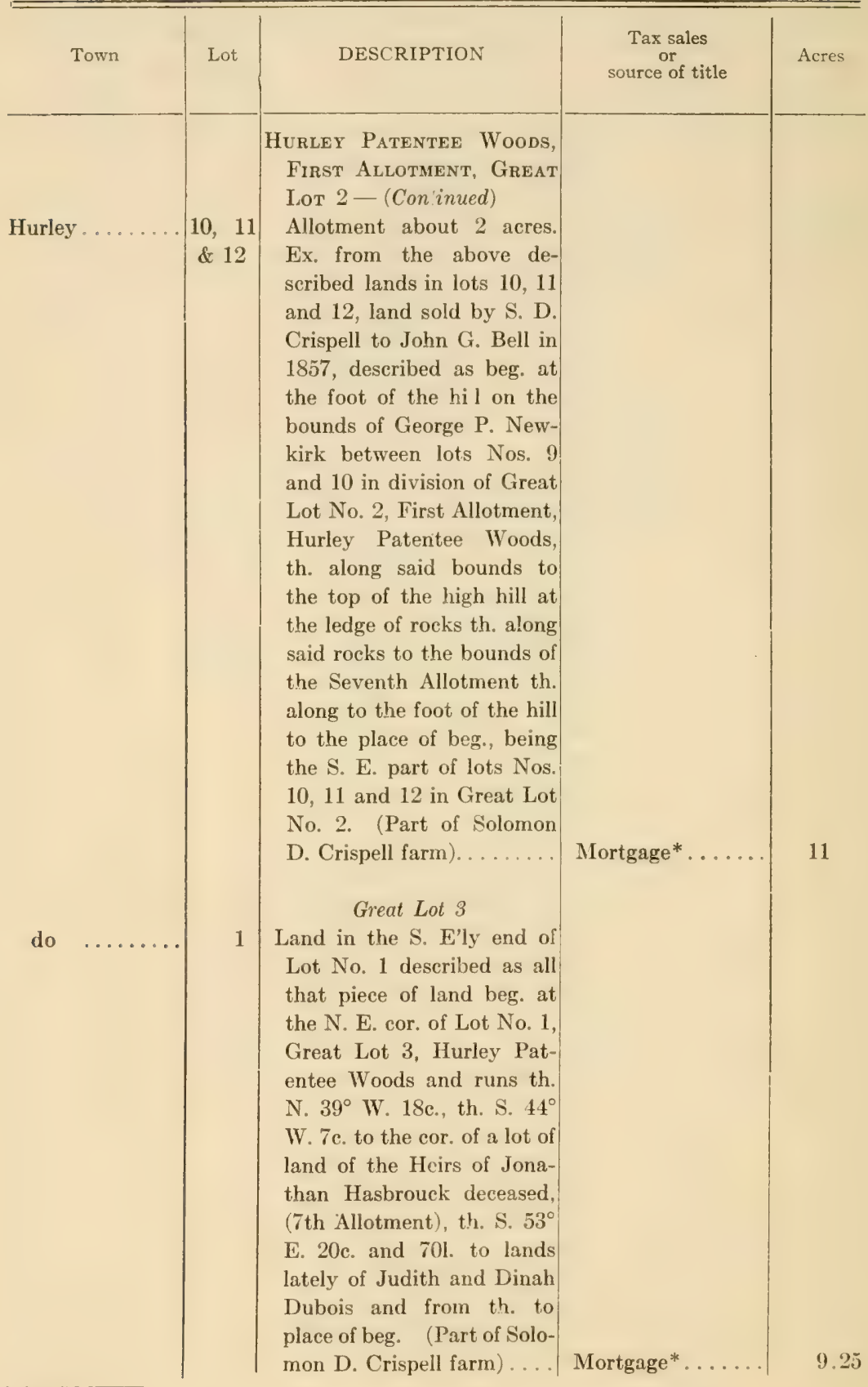




\section{Ulster County}

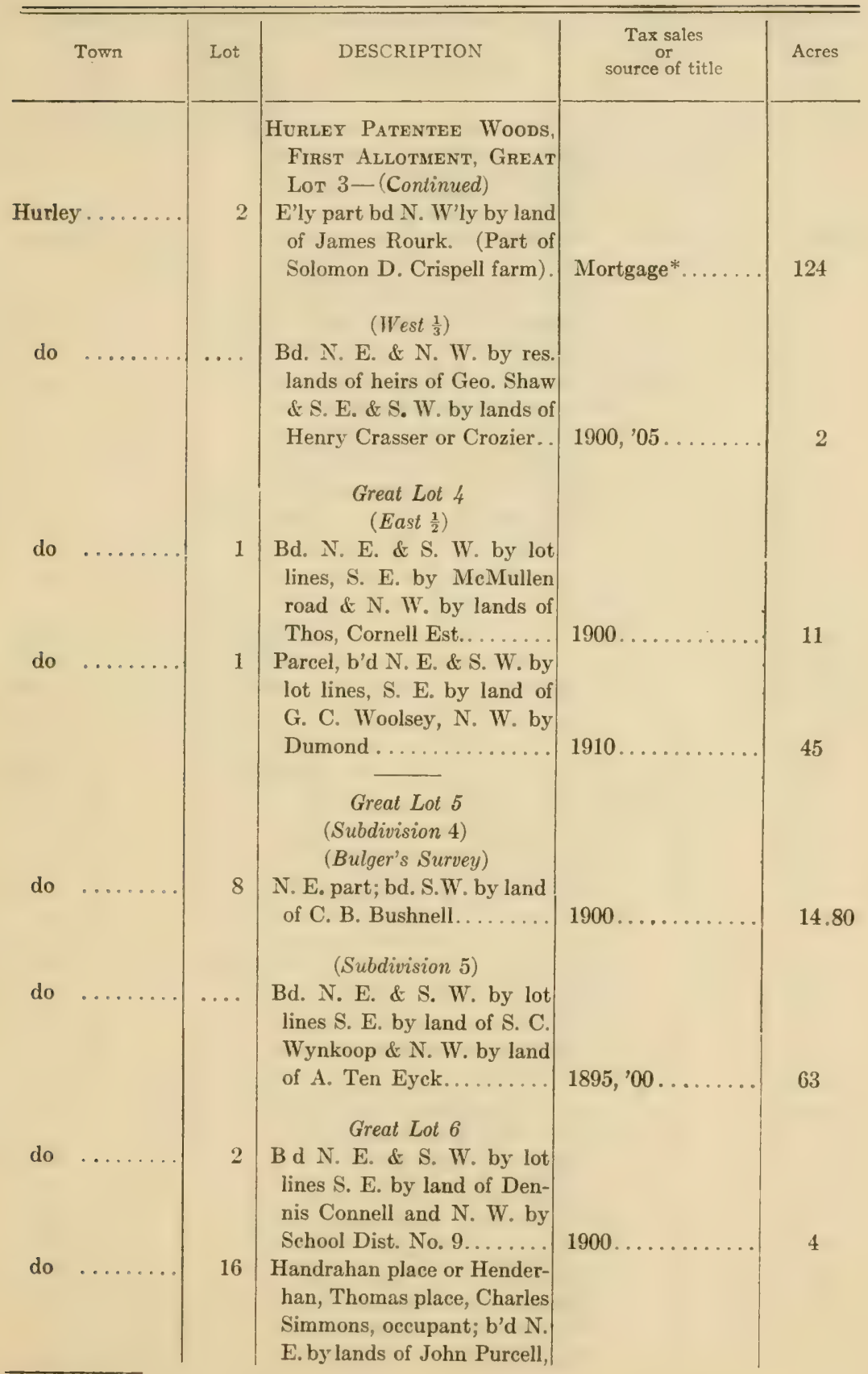




\section{Ulster County}

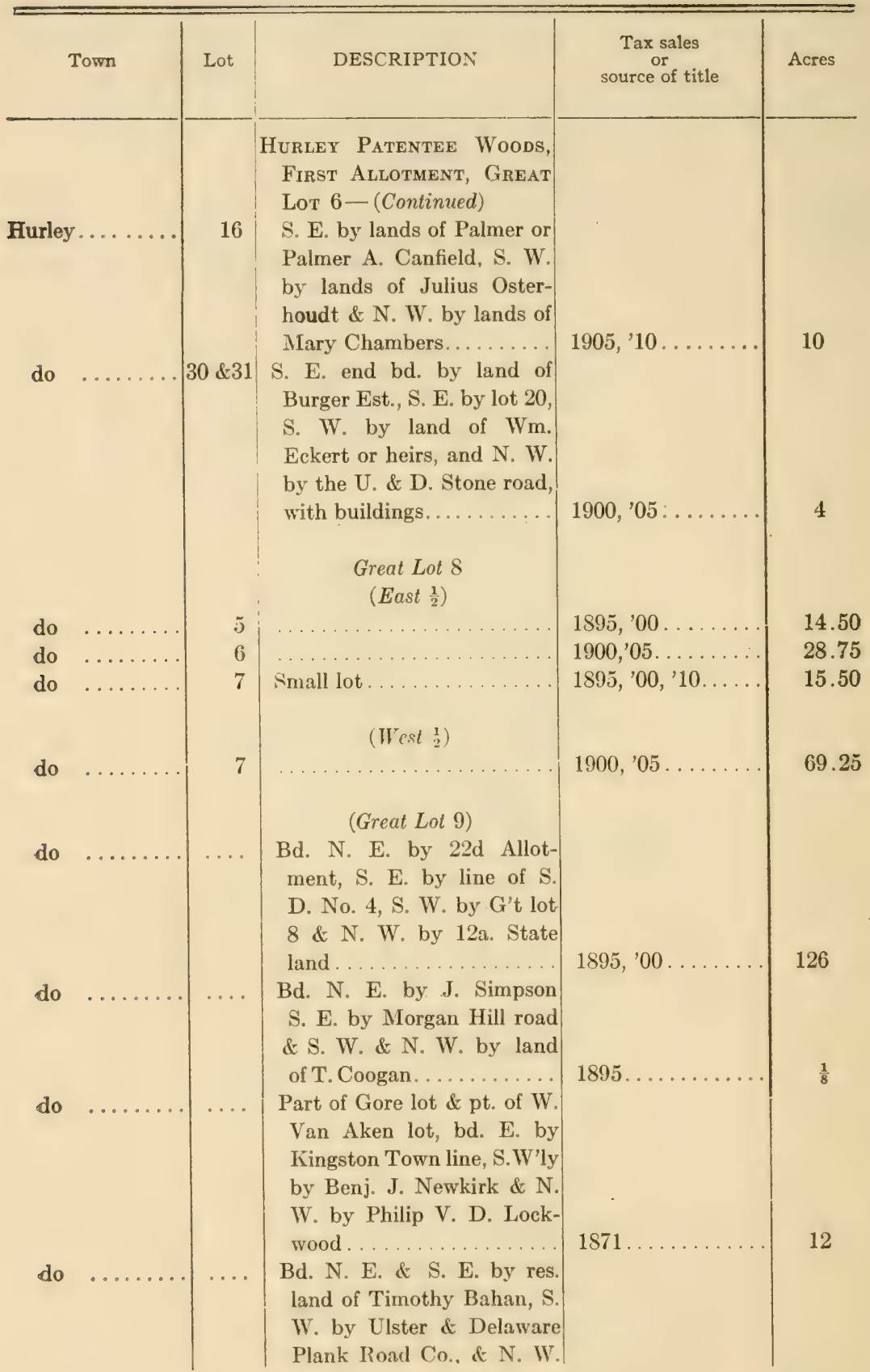


Ulster County

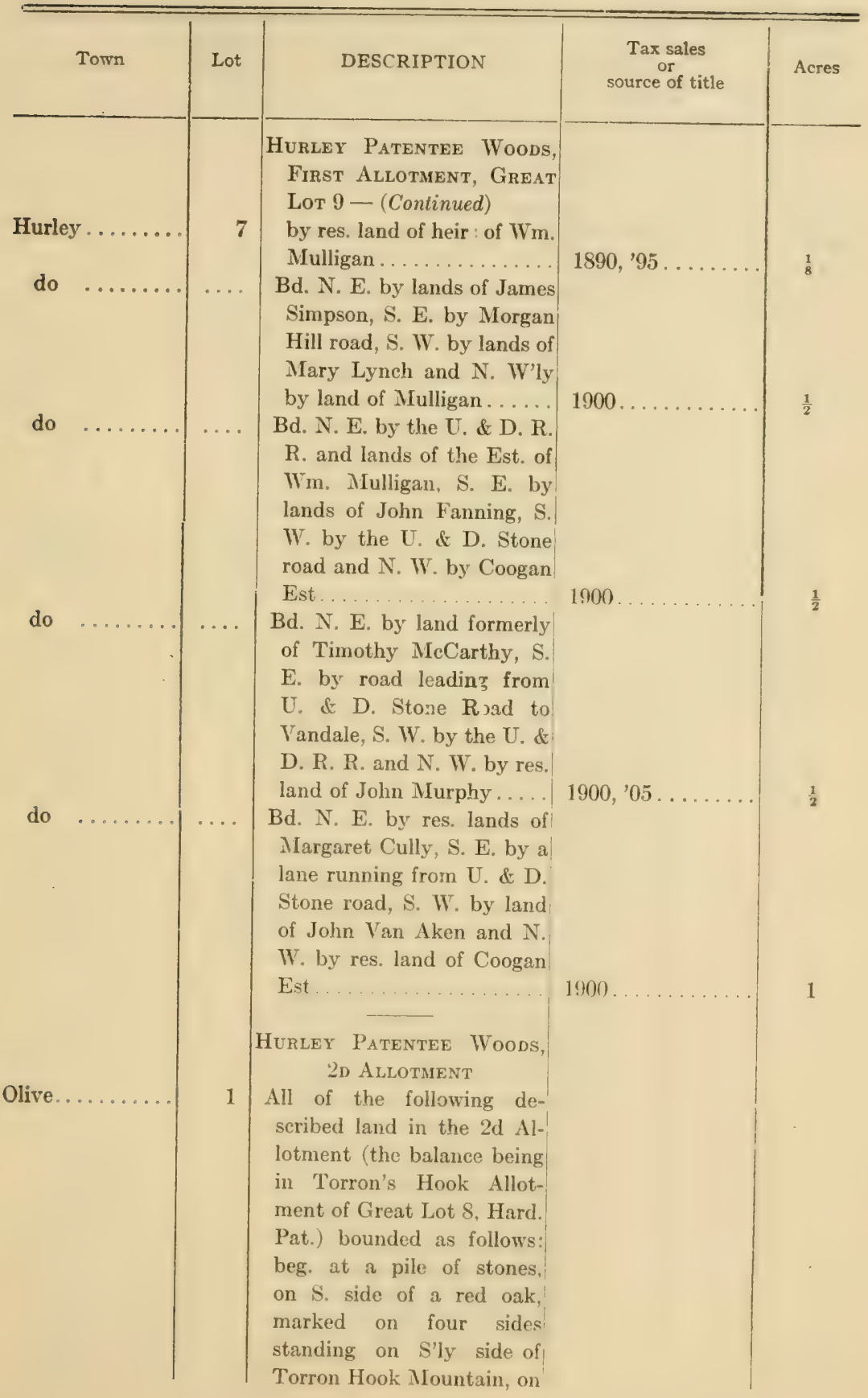


Ulster County

\begin{tabular}{|c|c|c|c|c|}
\hline Town & Lot & DESCRIPTION & $\begin{array}{c}\text { Tax sales } \\
\text { or } \\
\text { source of title }\end{array}$ & Acres \\
\hline Olive..... & 1 & 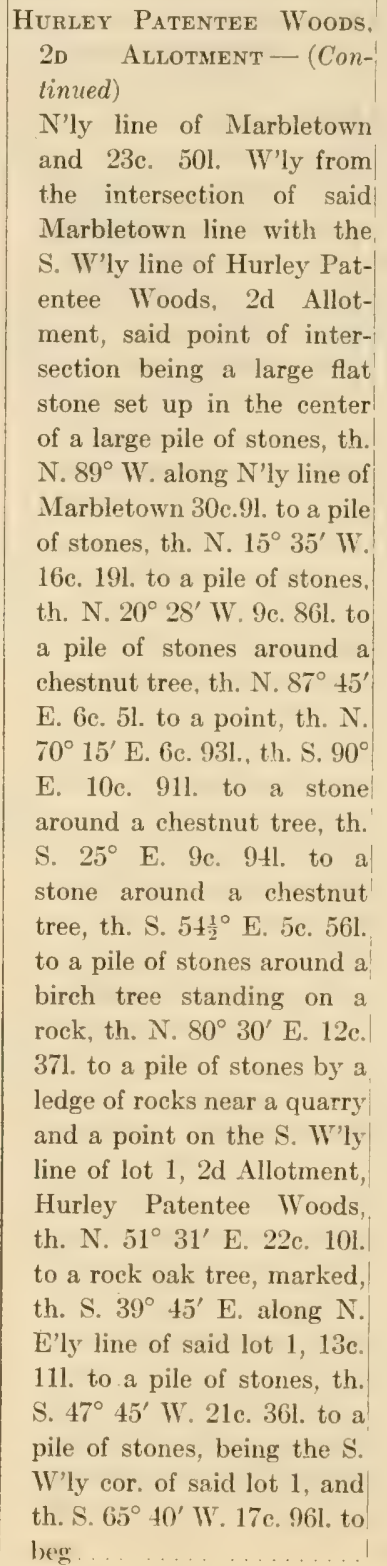 & rchase. . & 29.70 \\
\hline
\end{tabular}


Ulster County

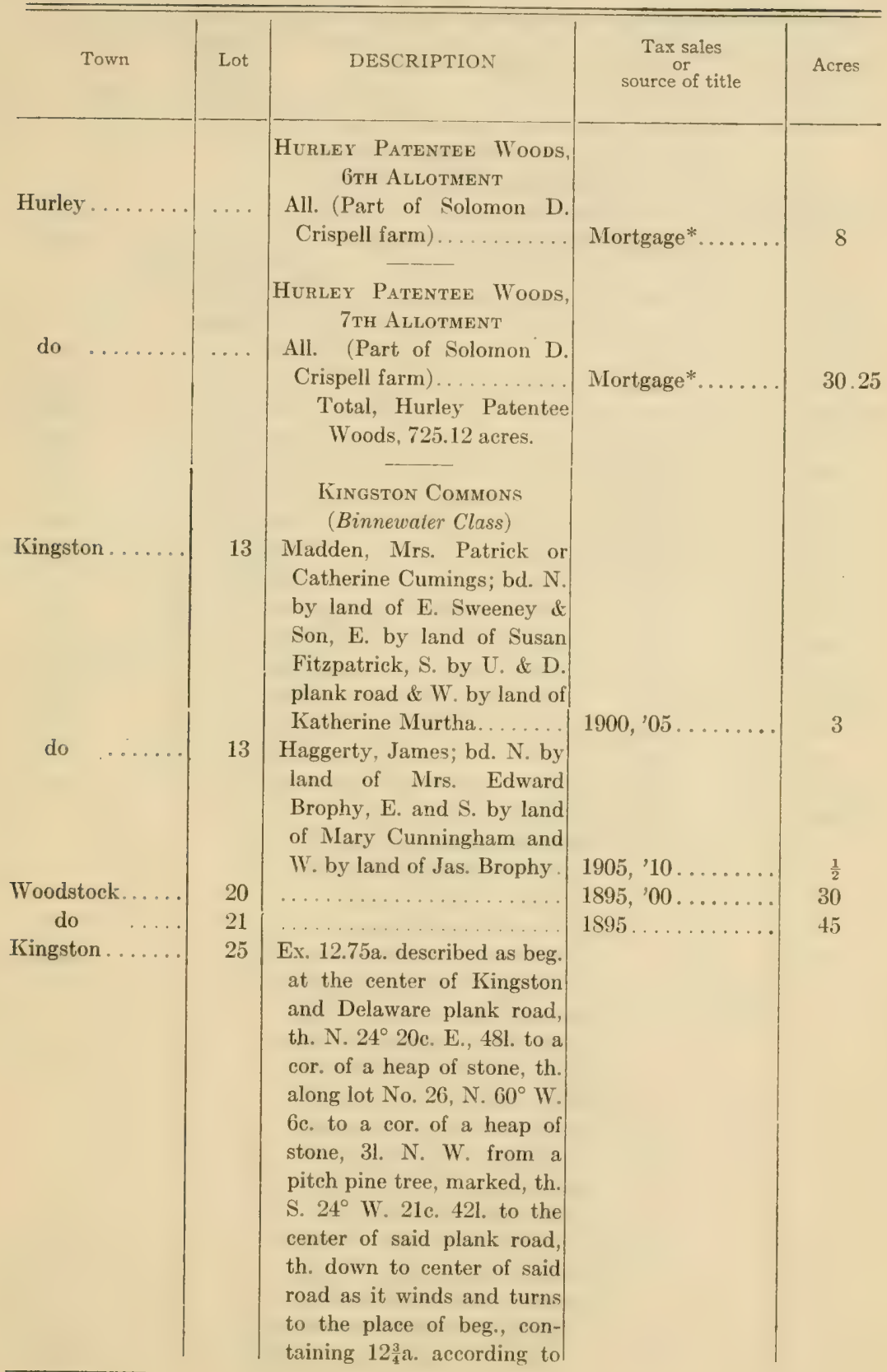




\section{Ulster County}

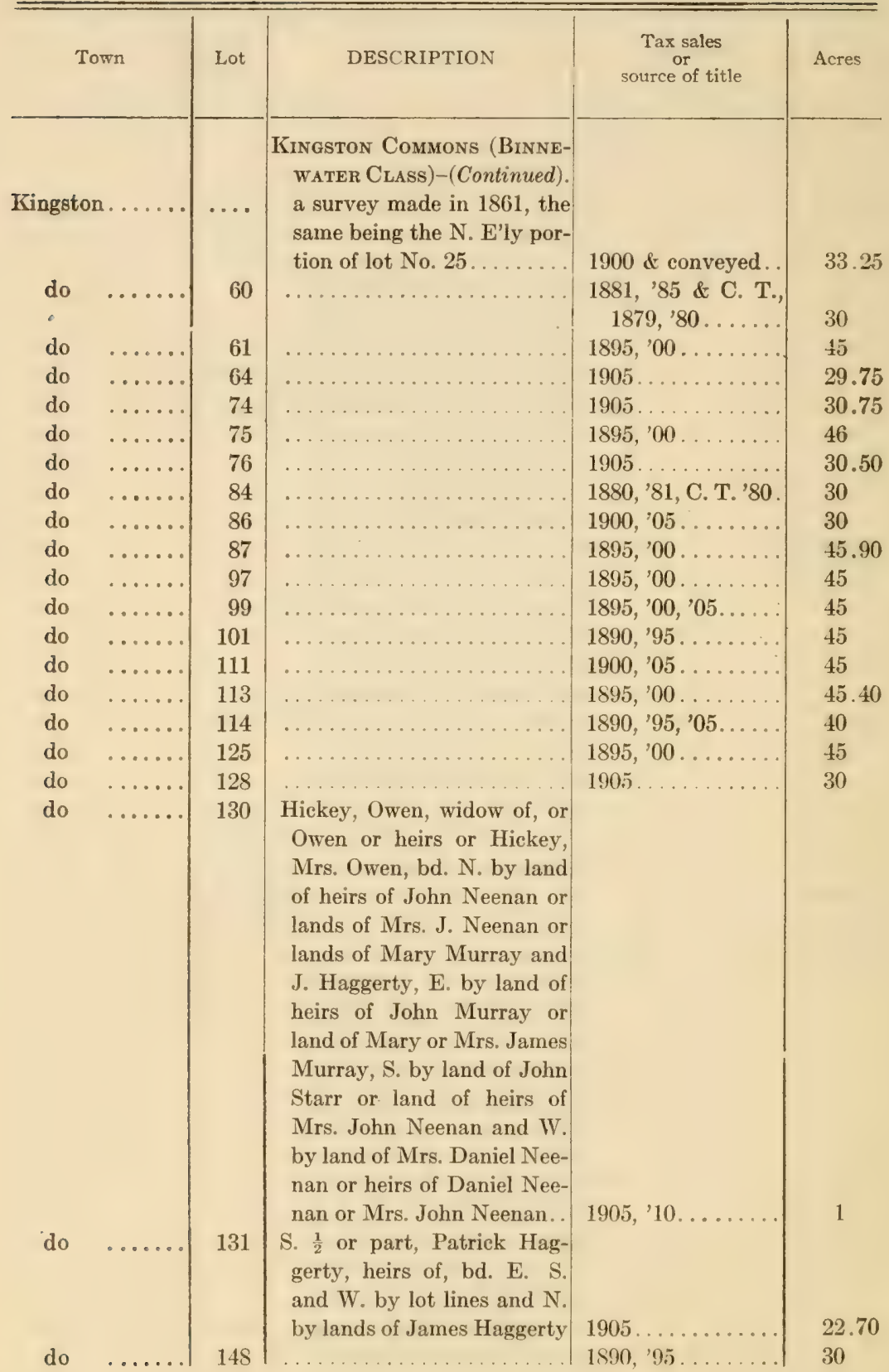




\section{Ulster County}

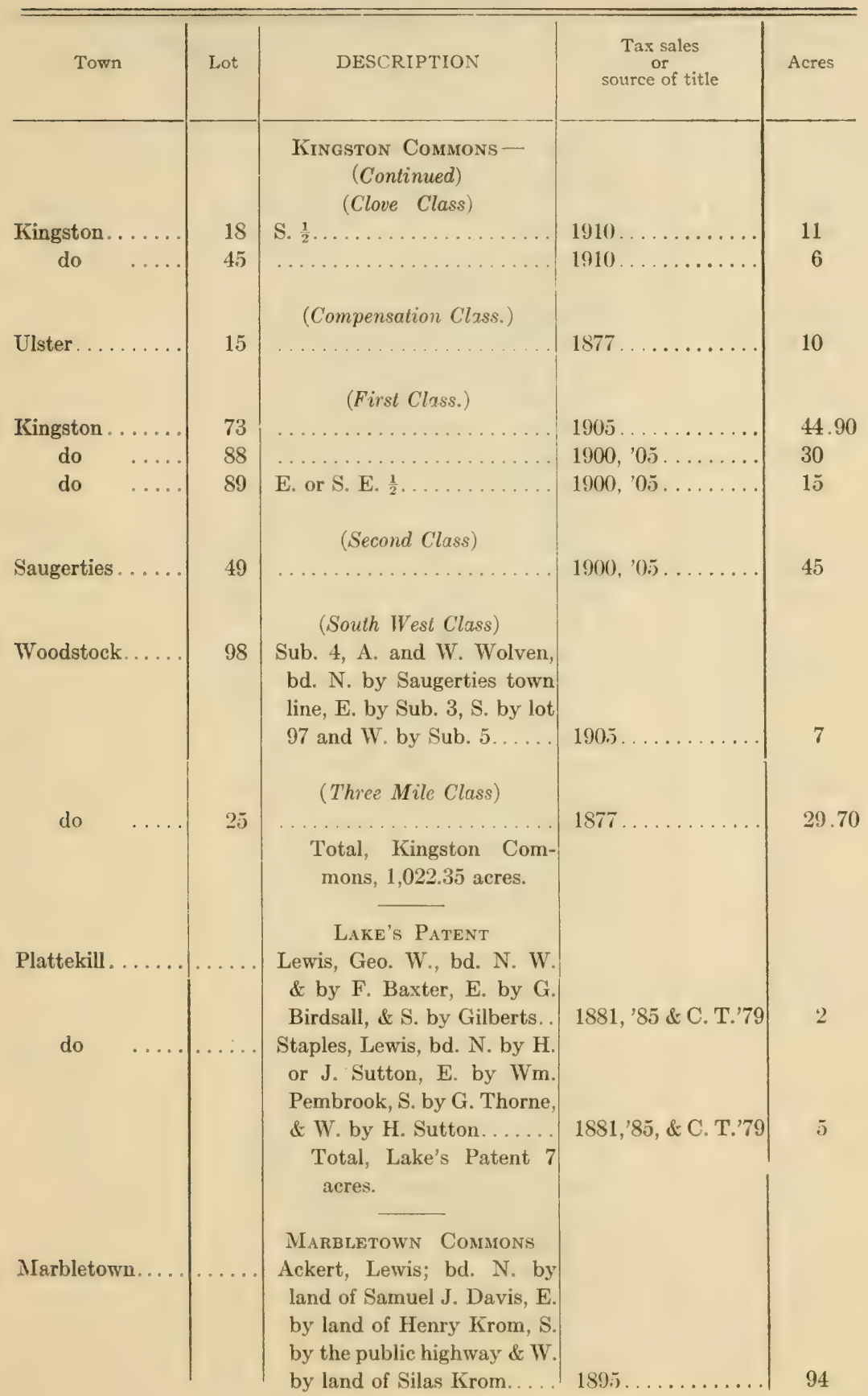


Ulster County

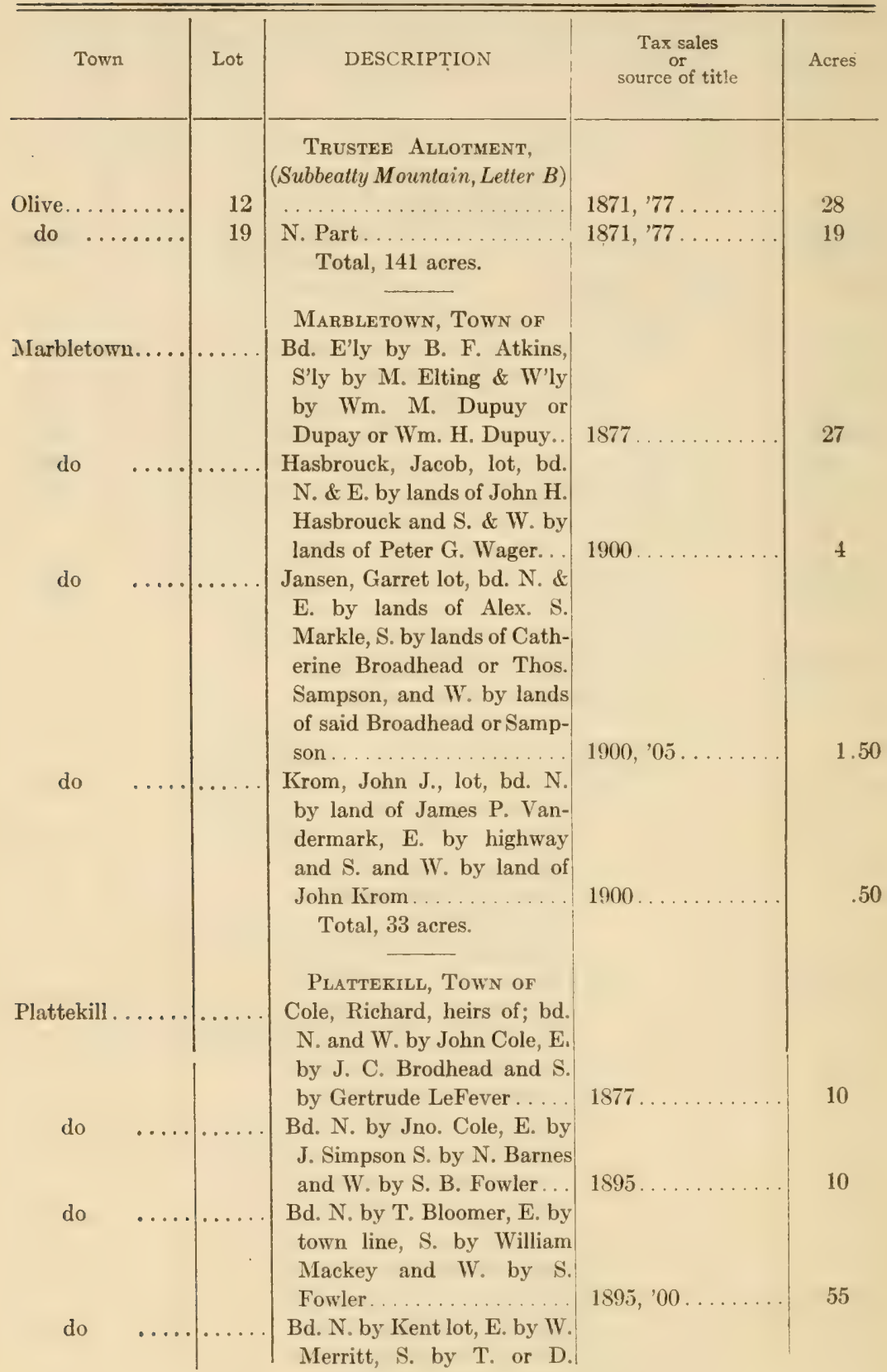


Ulster County

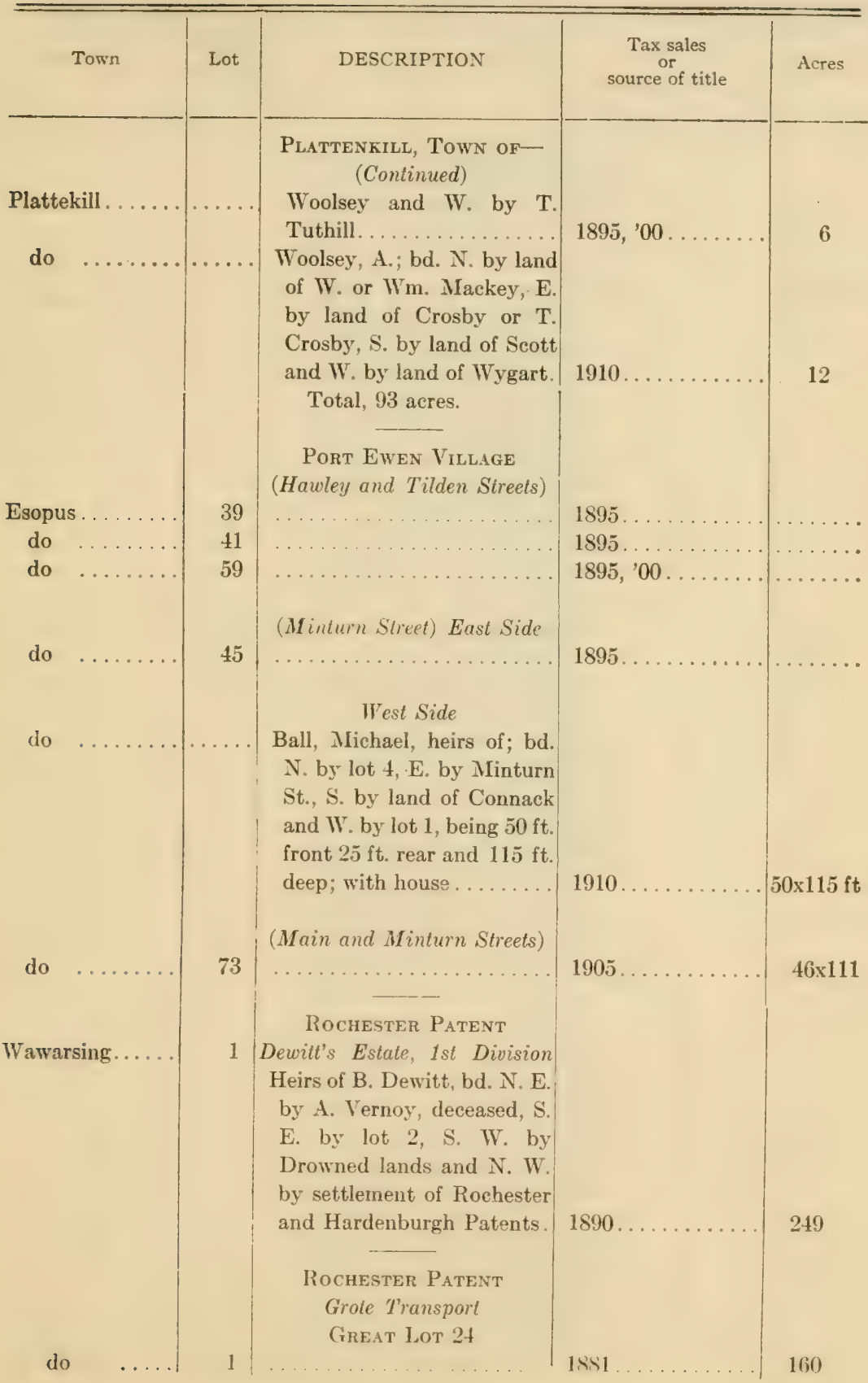




\section{ULSTER COUNTY}

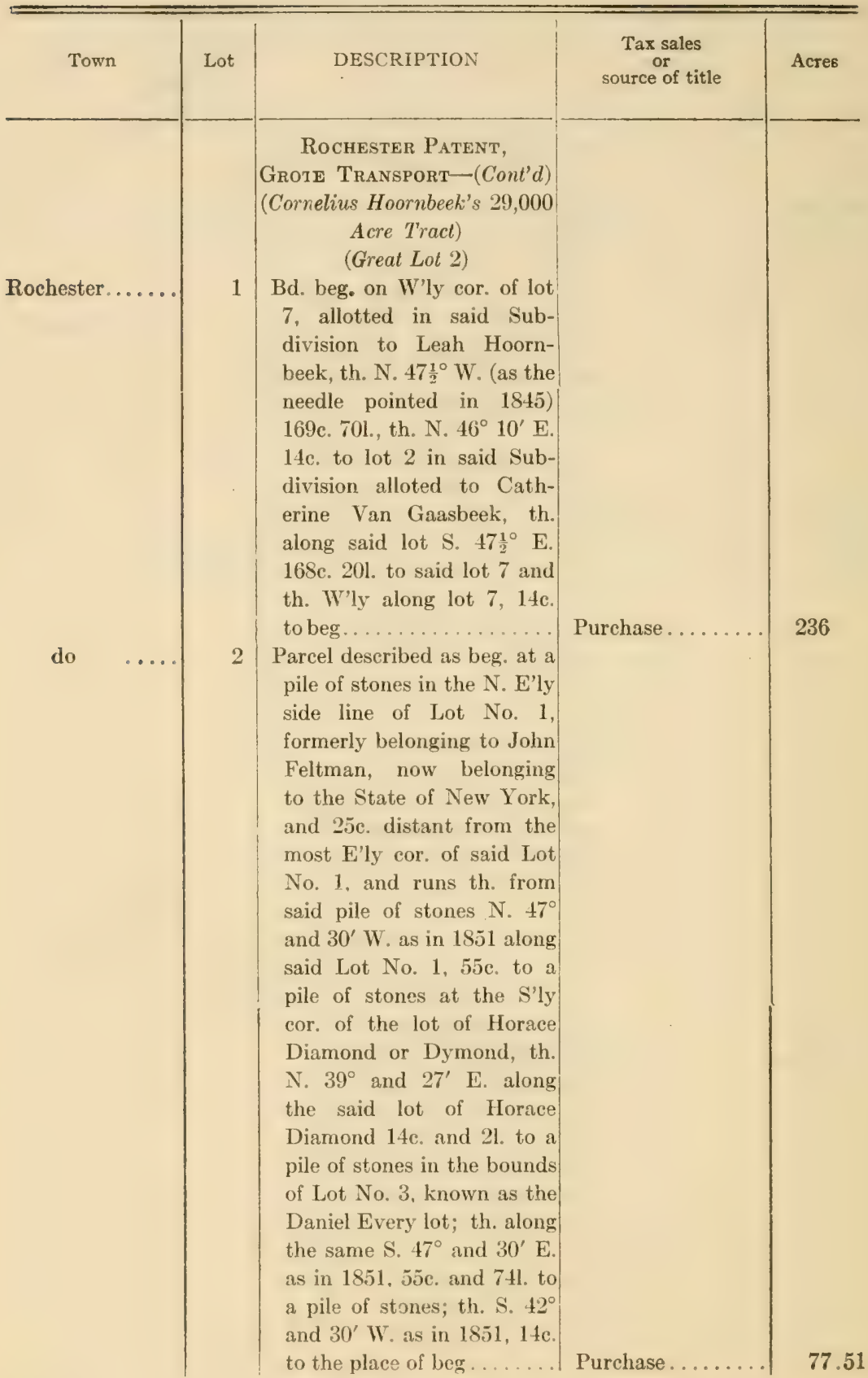




\section{Ulster County}

\begin{tabular}{|c|c|c|c|c|}
\hline Torrn & Lot & DESCRIPTION & $\begin{array}{c}\text { Tax sales } \\
\text { or } \\
\text { source of title }\end{array}$ & Acres \\
\hline Rochester... & 3 & 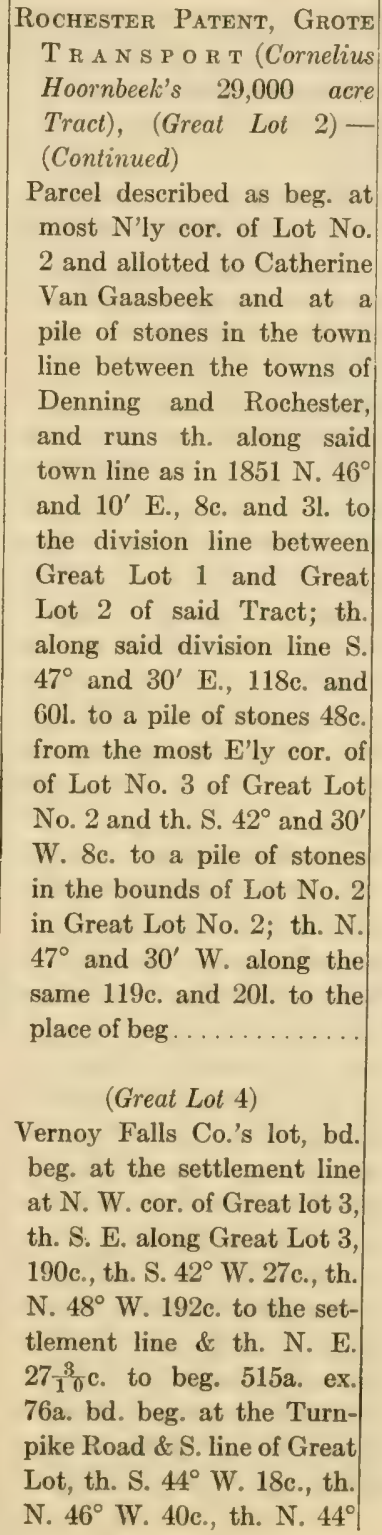 & 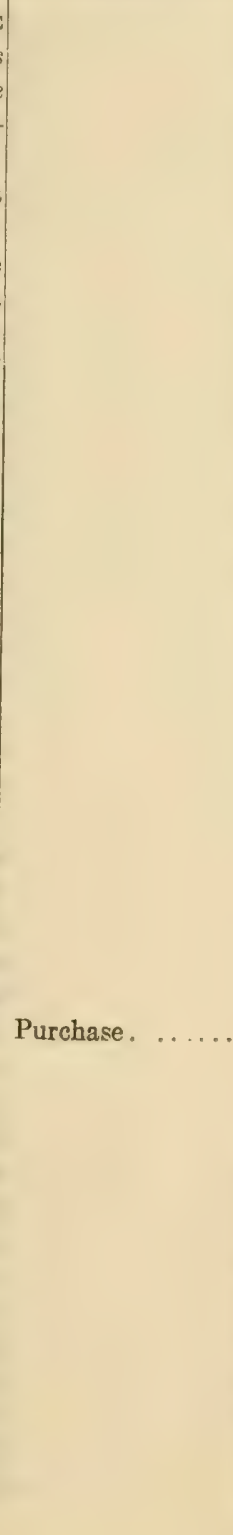 & 95.12 \\
\hline
\end{tabular}




\section{Ulster County}

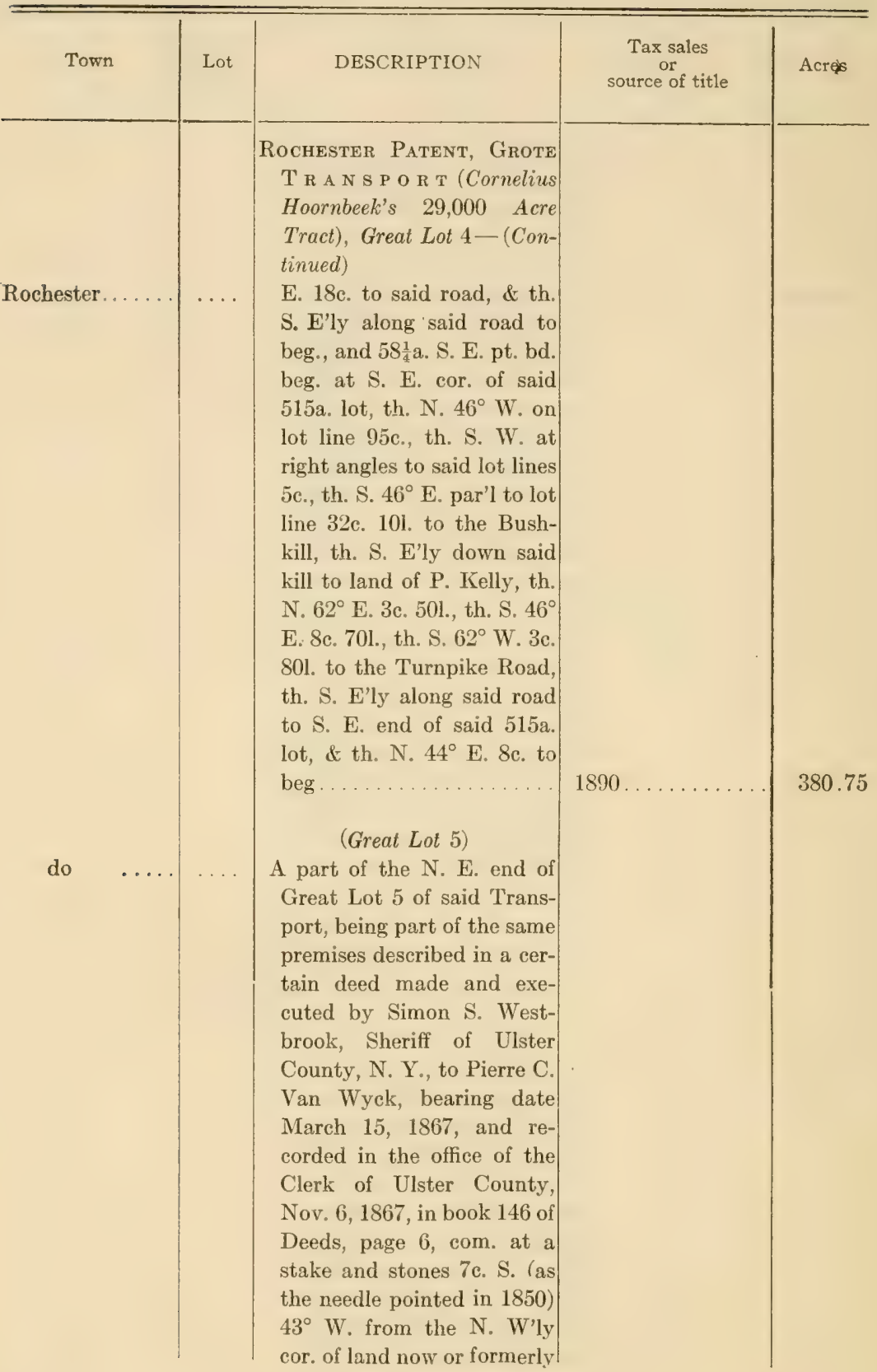




\section{Ulster County}

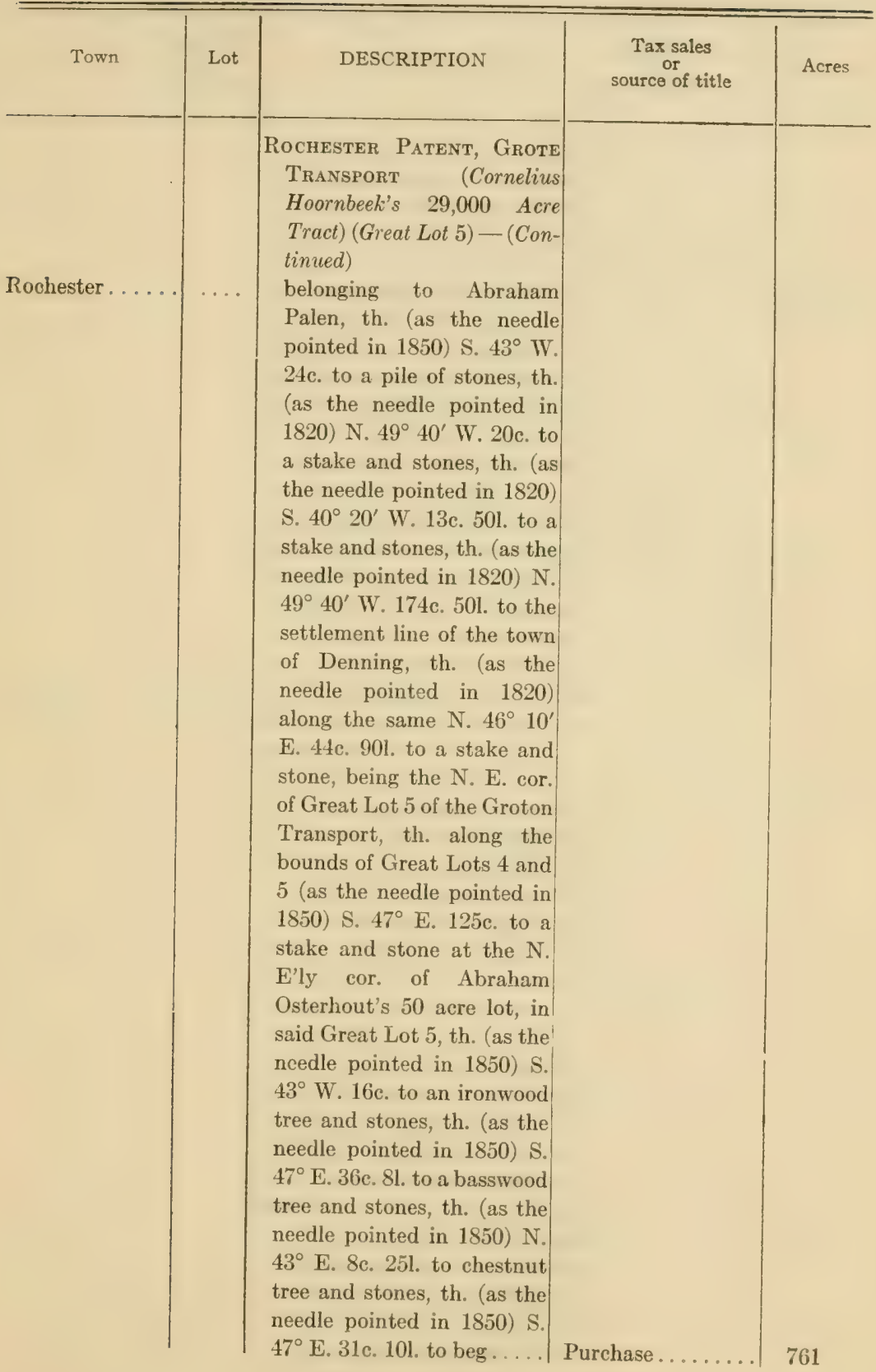




\section{Ulster County}

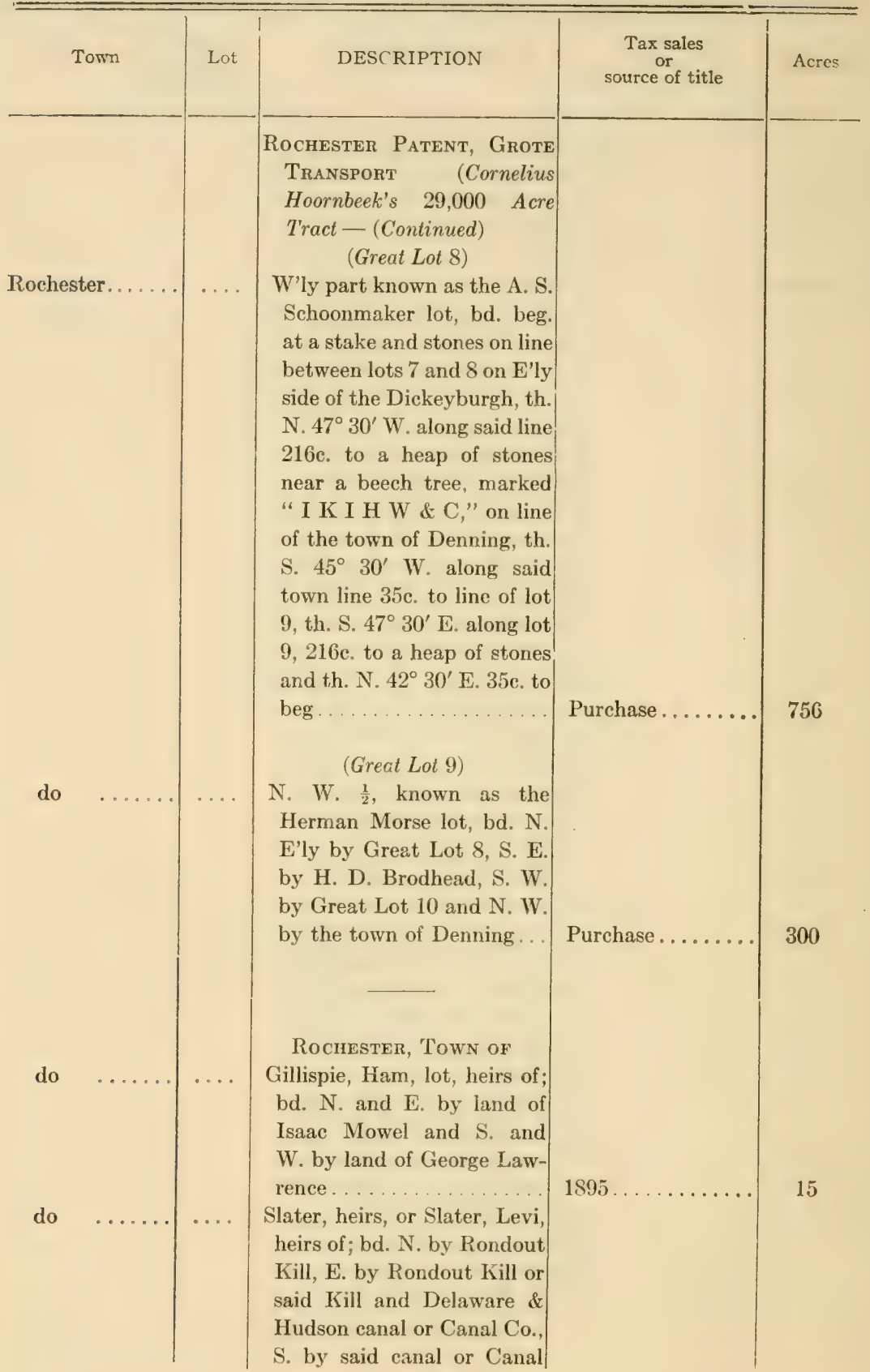


Ulster Countr

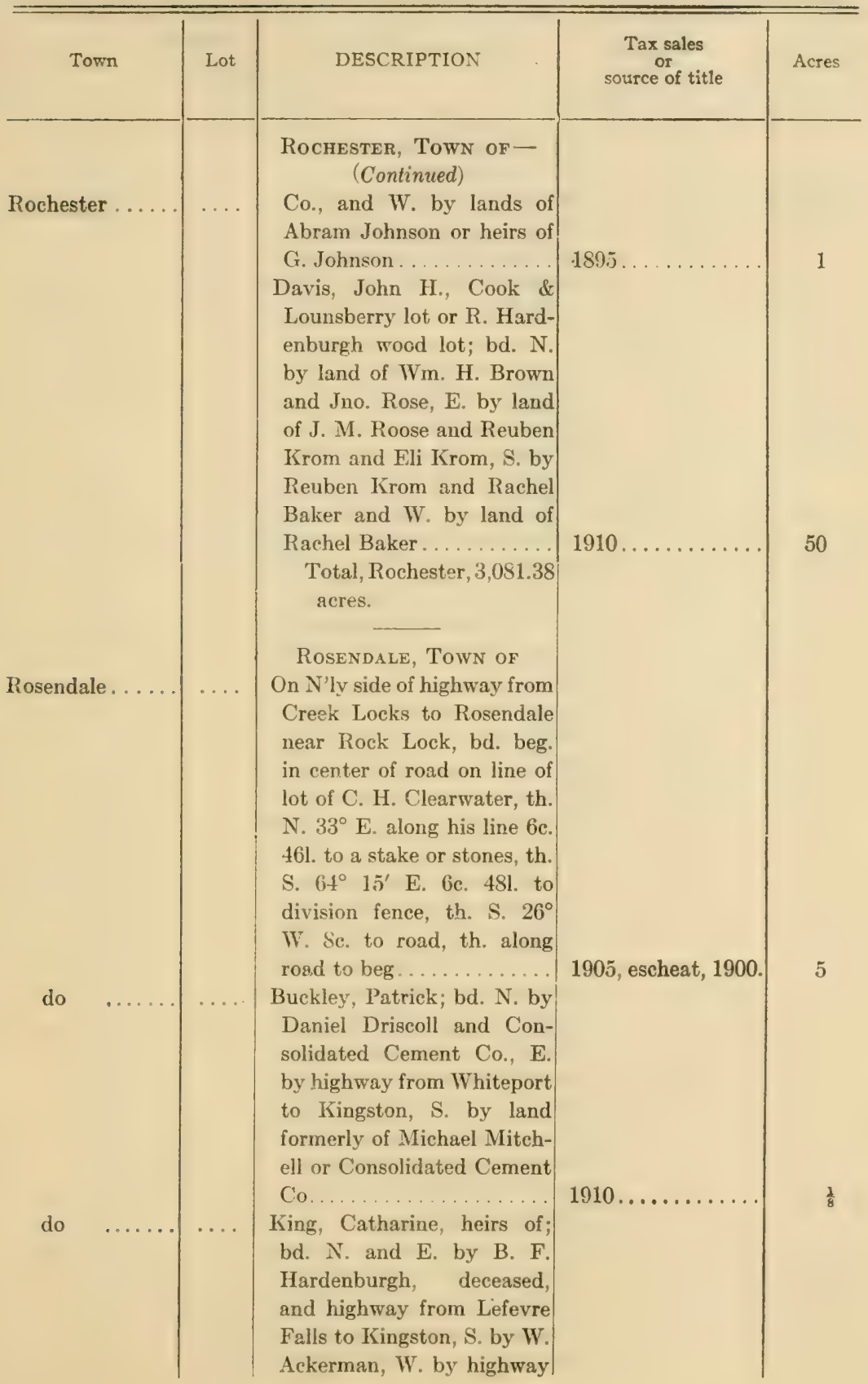


Ulster County

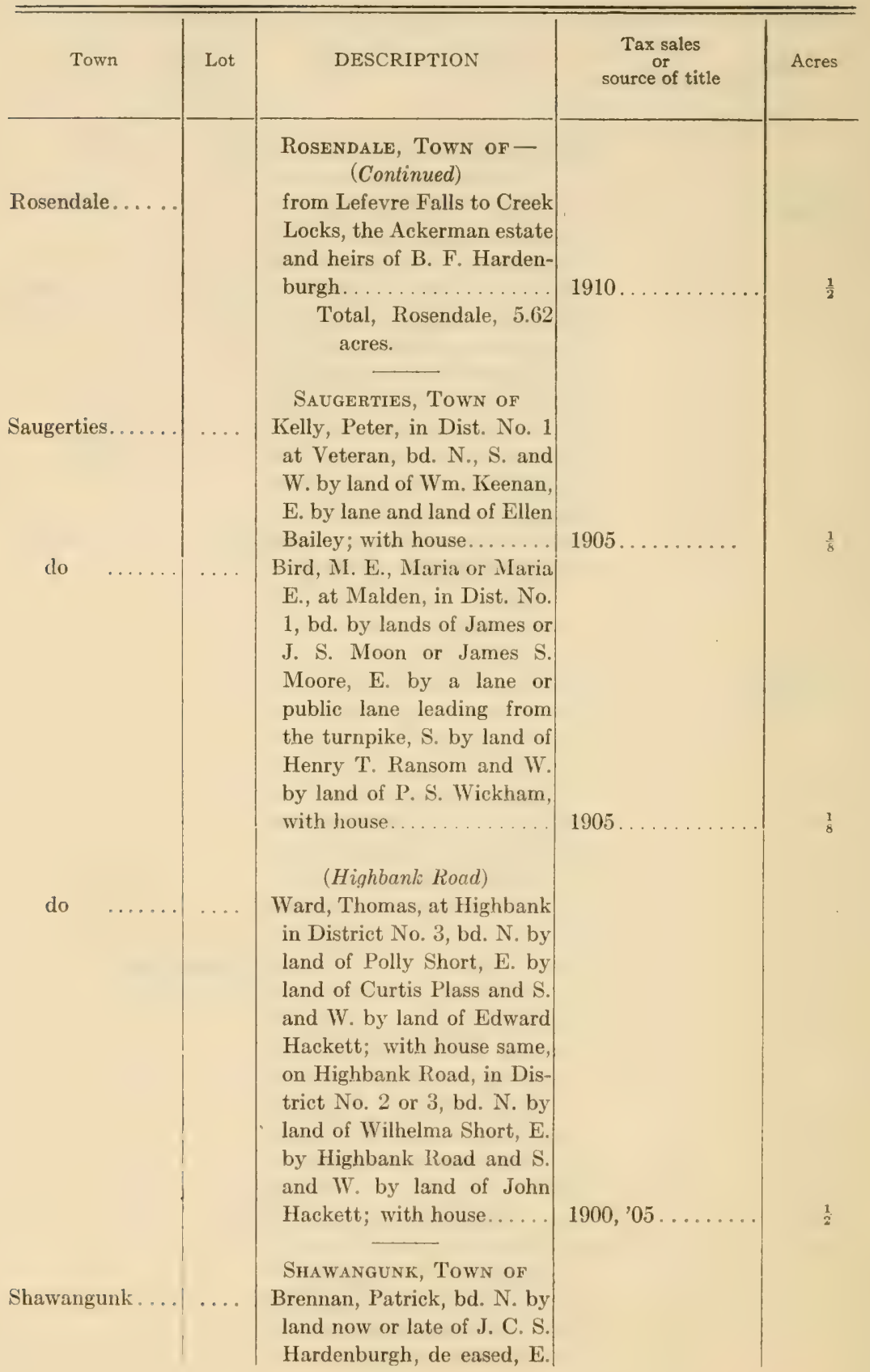




\section{Ulster County}

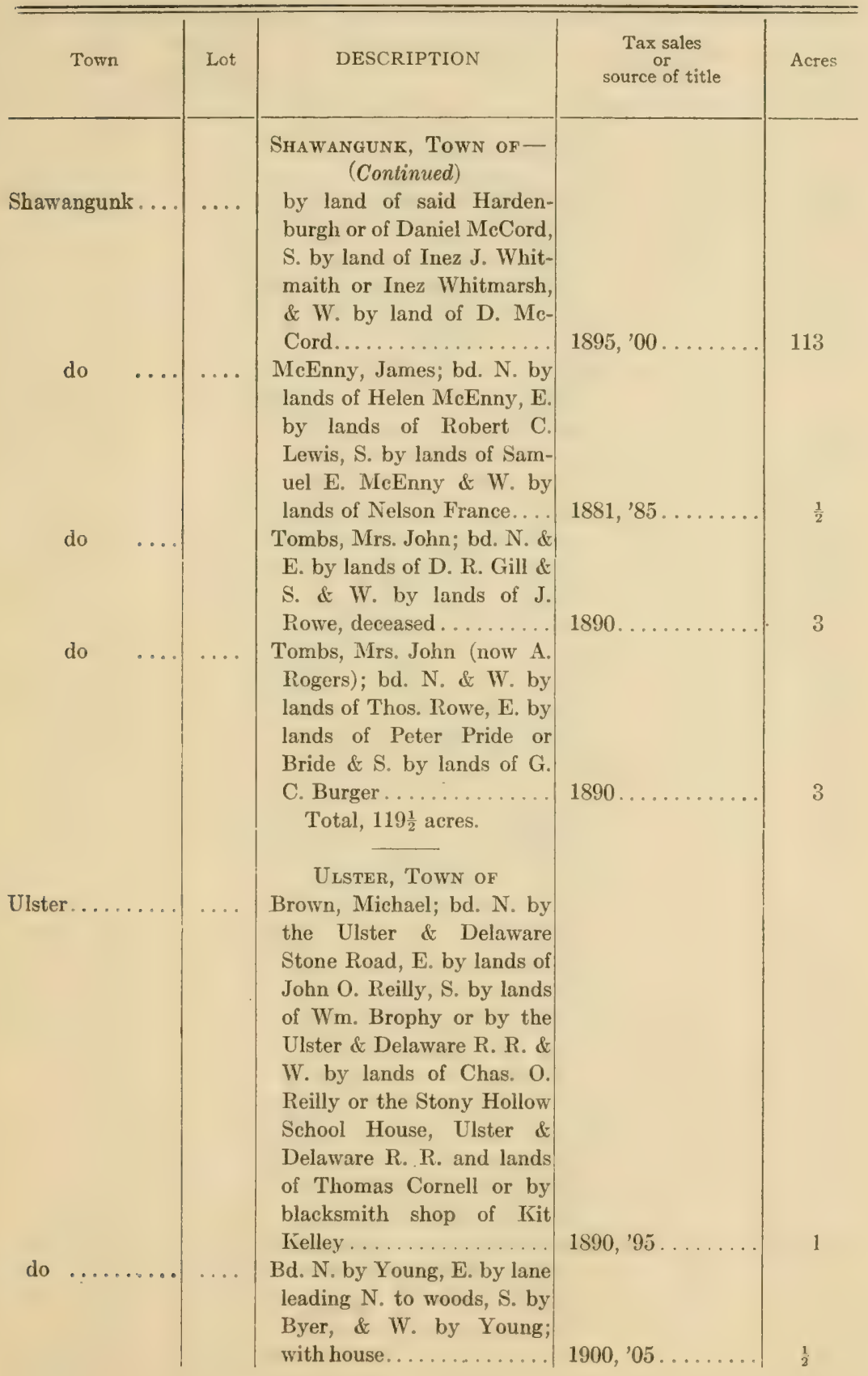




\section{Ulster County}

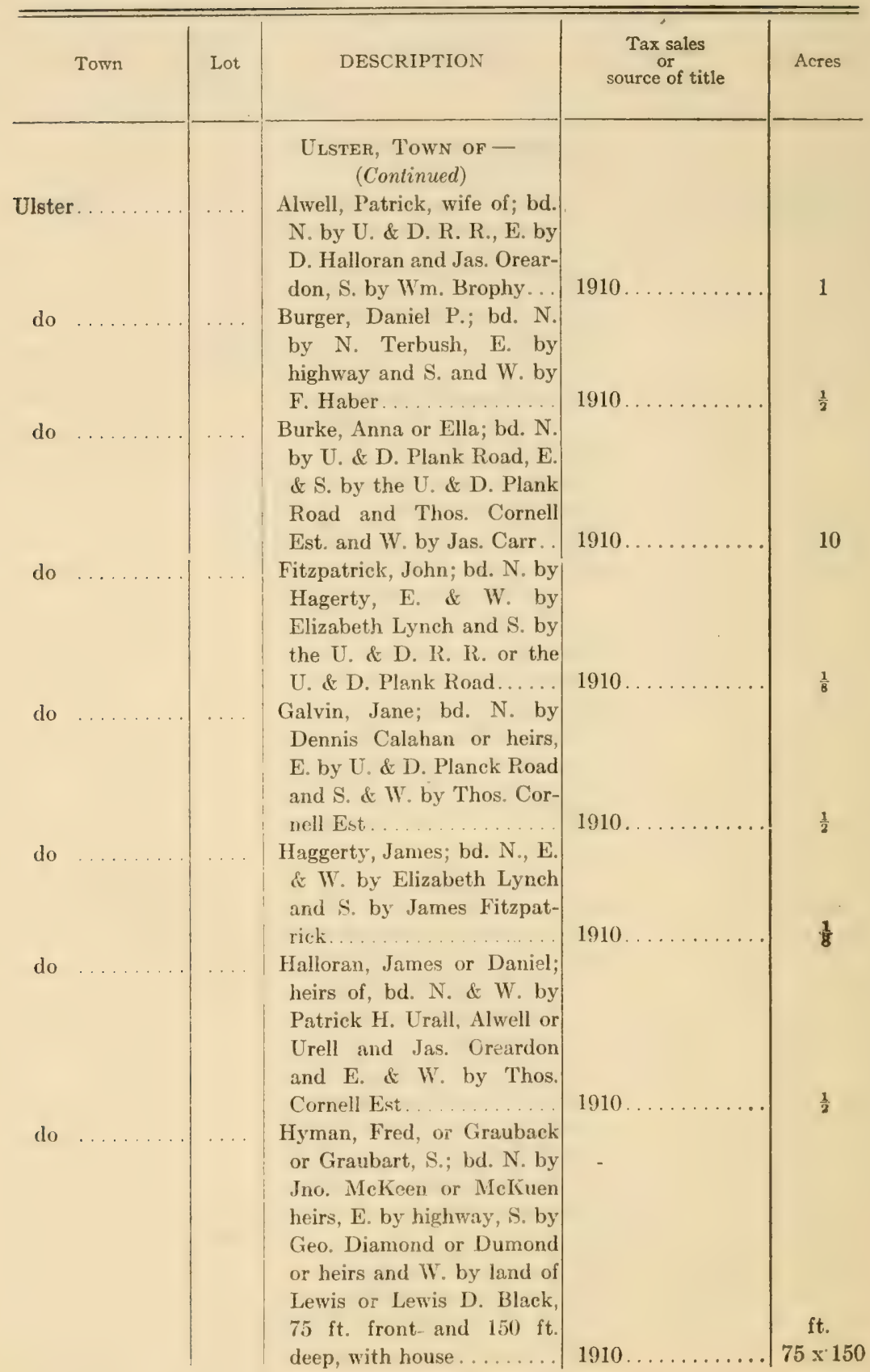




\section{Ulster County}

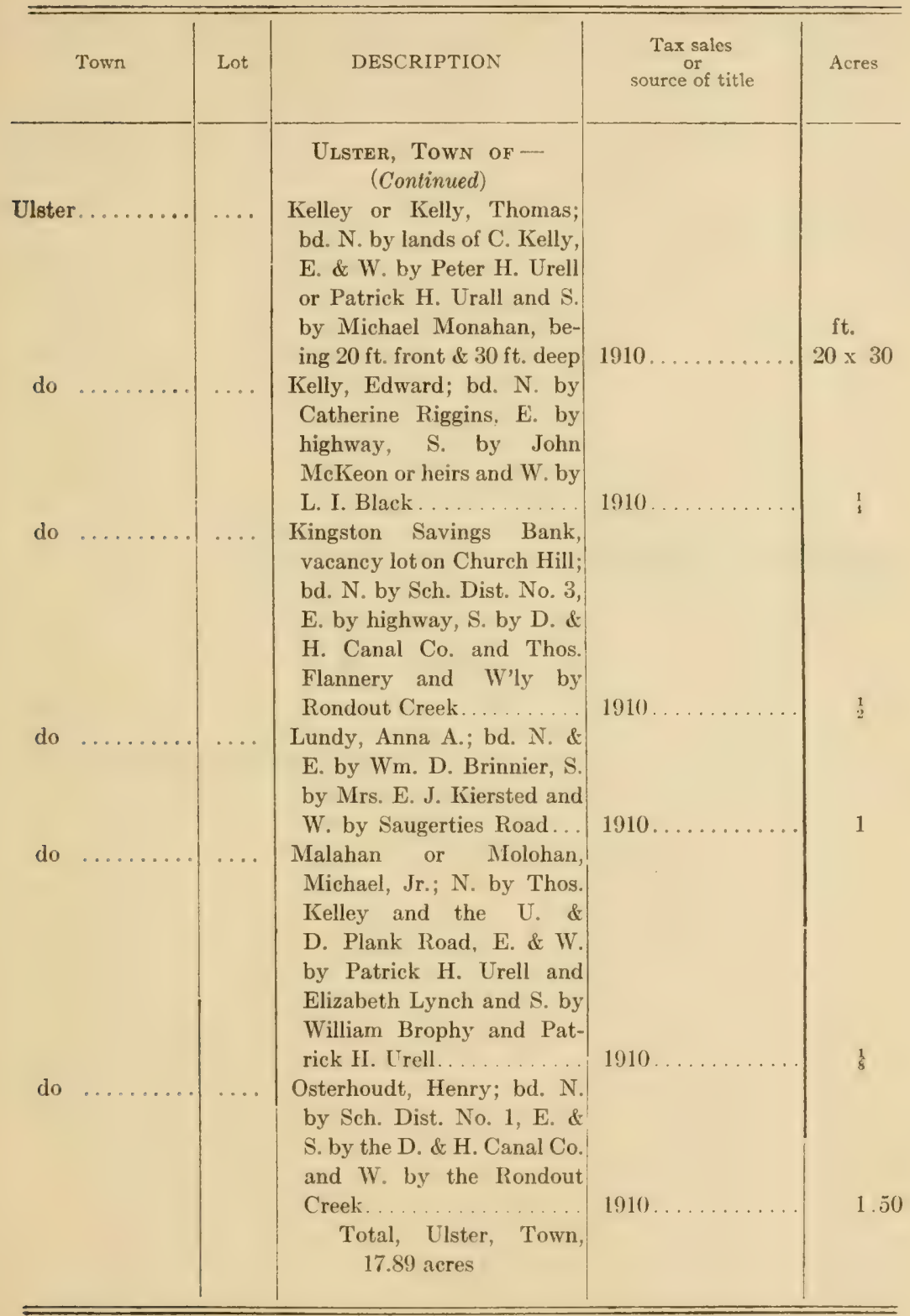

For other State land in Ulster County see Appendix. 

APPENDIX

[439] 



\section{CLINTON COUNTY}

List of State Prison Lands*

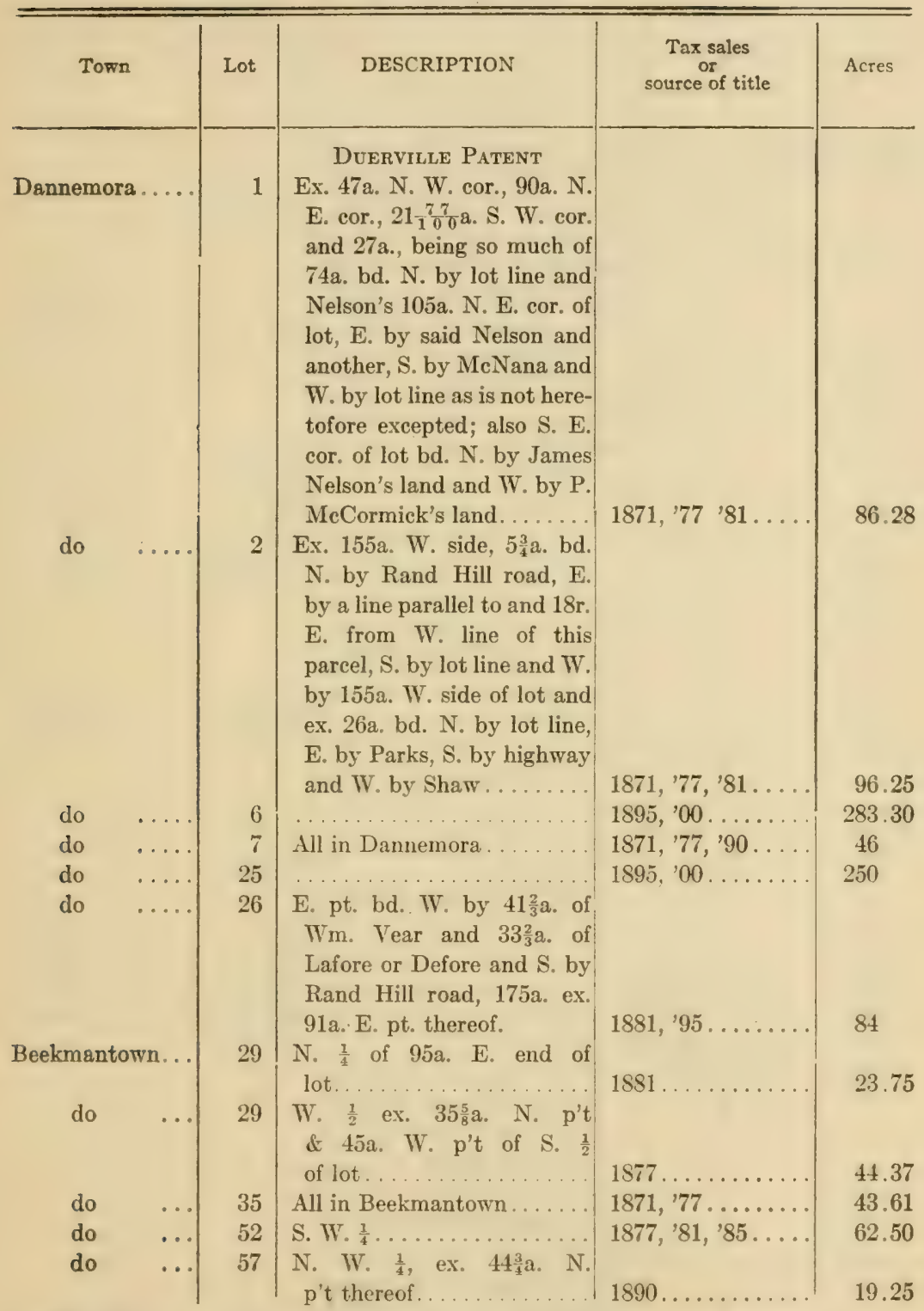

*The prison law provides that all State land within ten miles of Clinton Prison (Dannemora) shall be devoted to the use of the prison. 


\section{Clinton County}

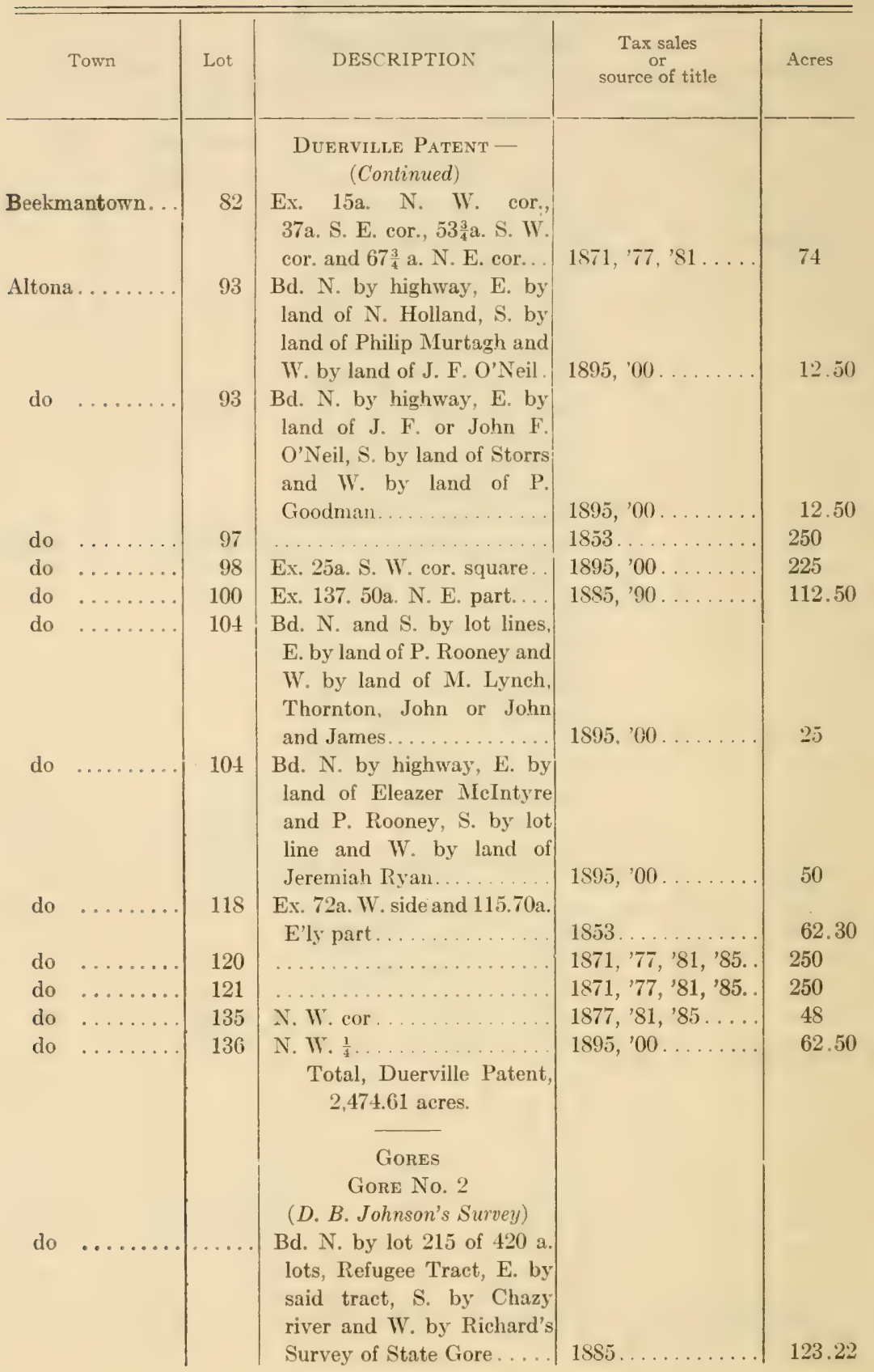




\section{Chinton County}

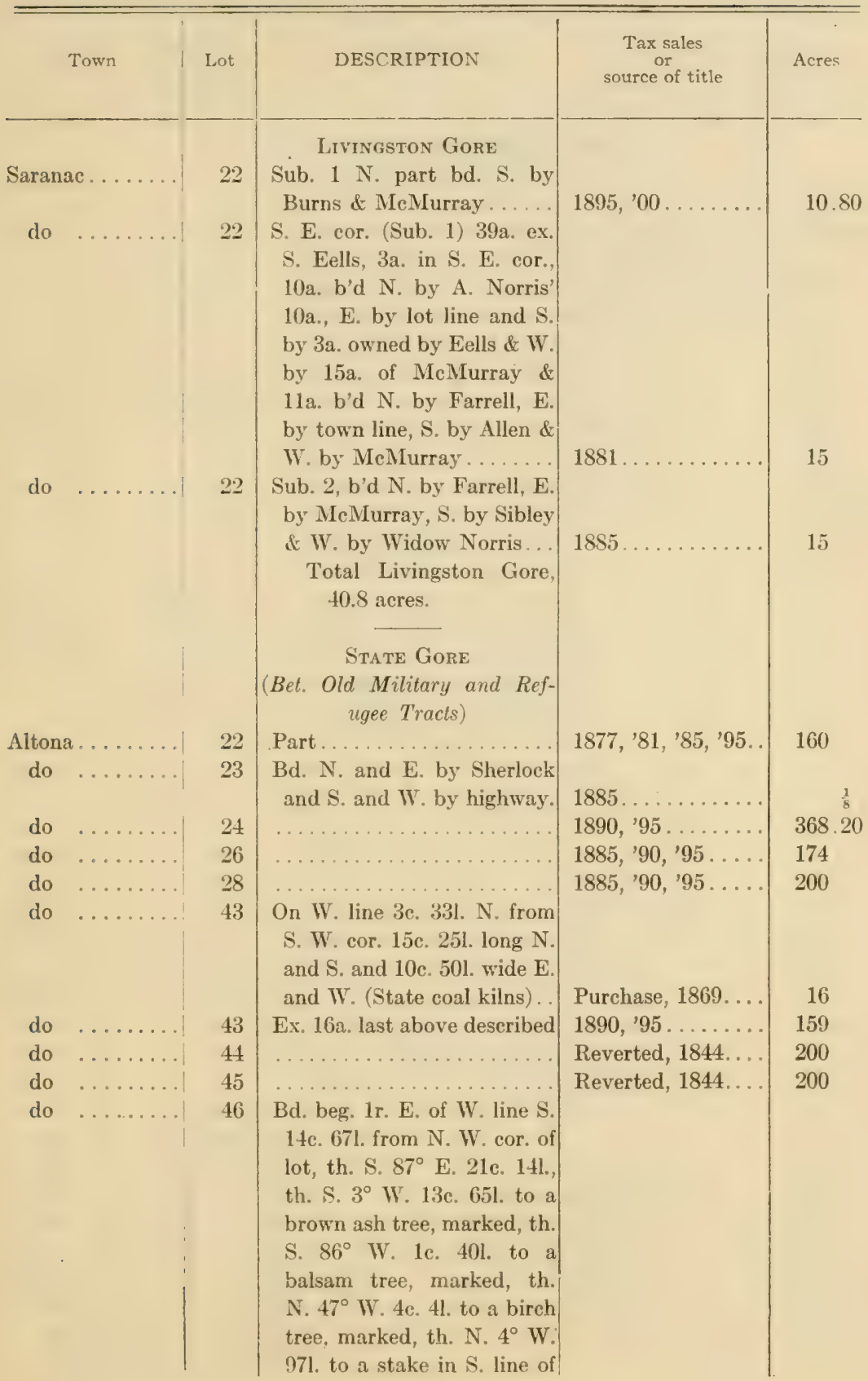


Cuinton County

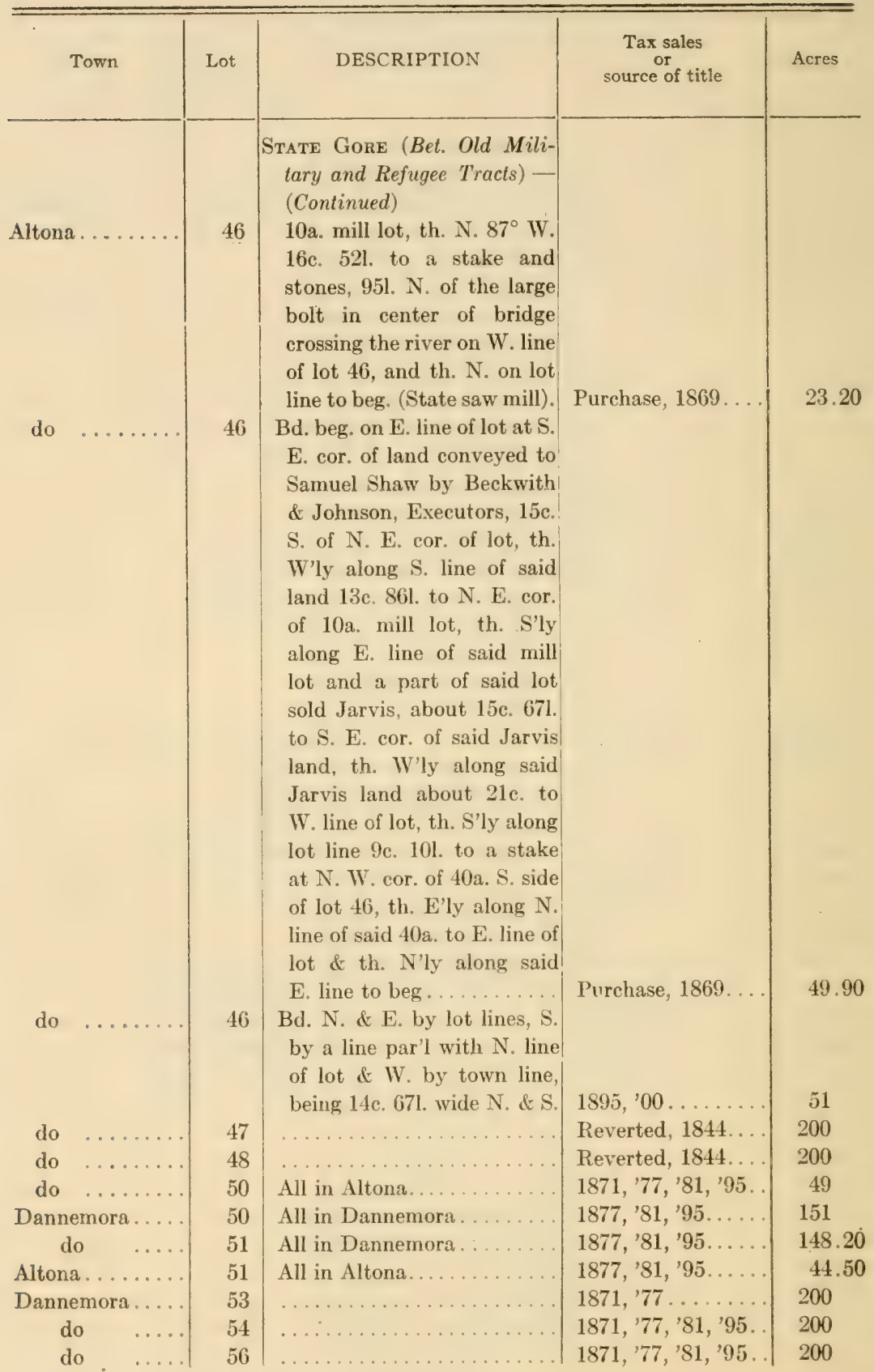




\section{Chinton County}

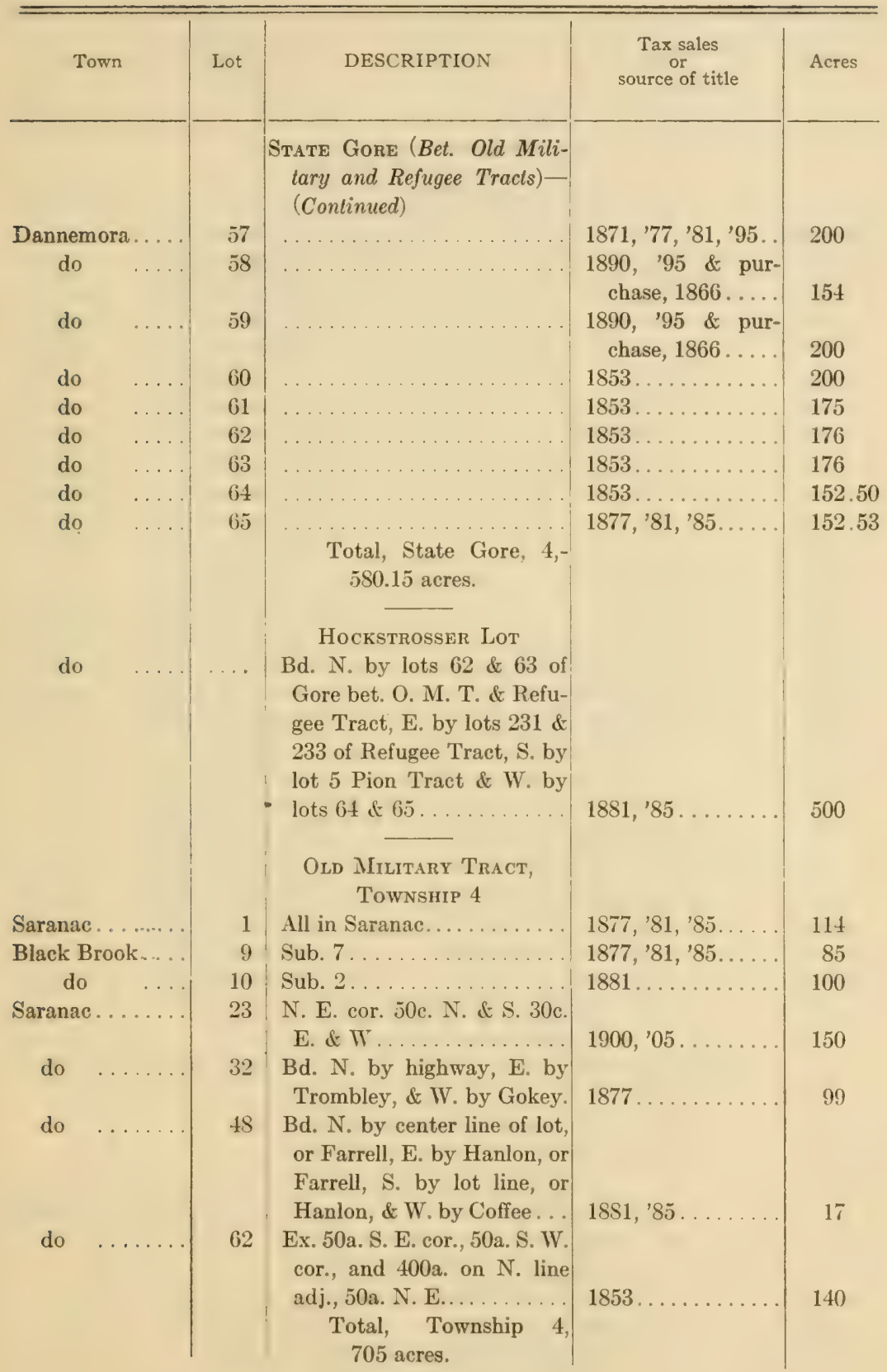


Cuinton County

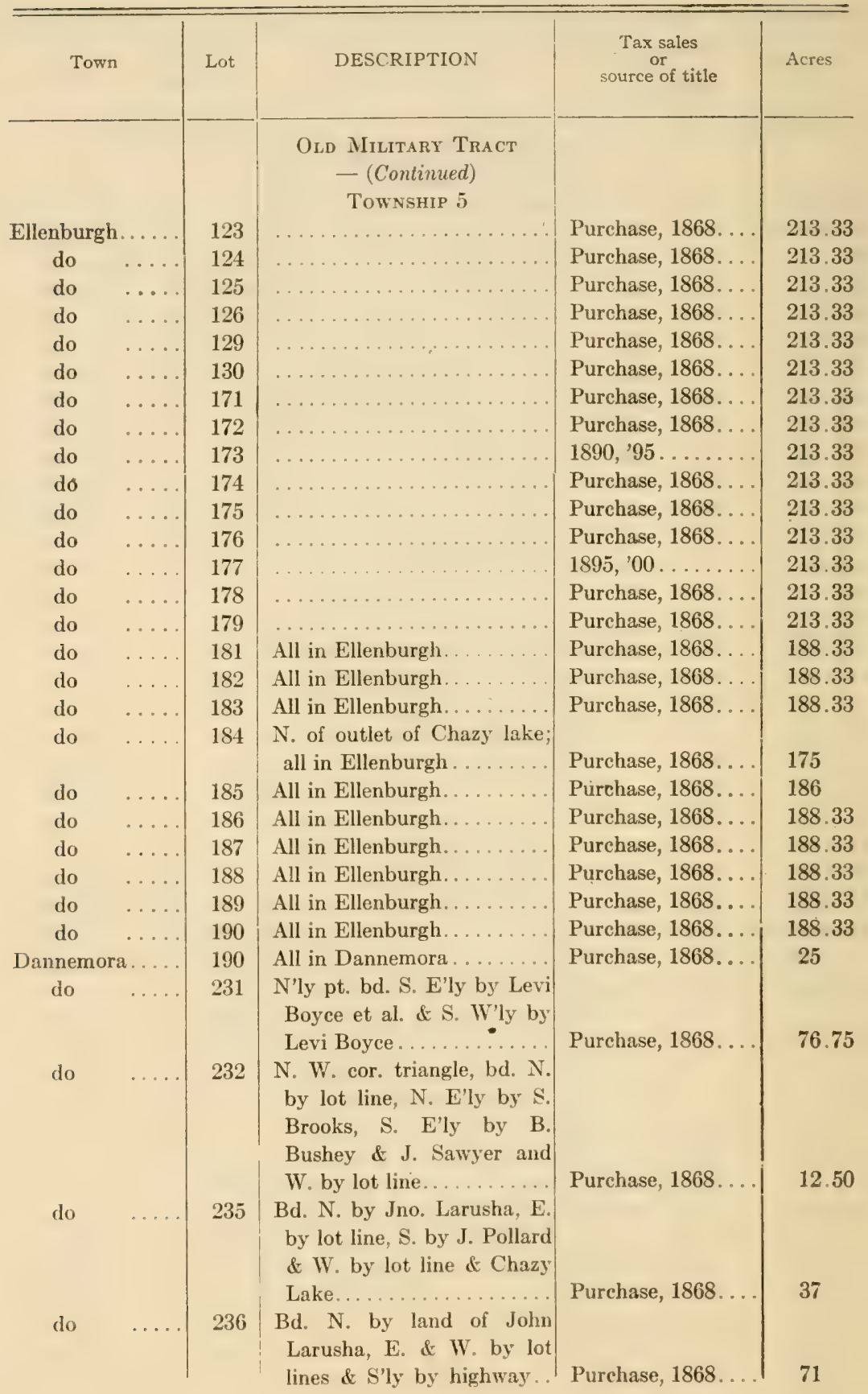


Cuinton County

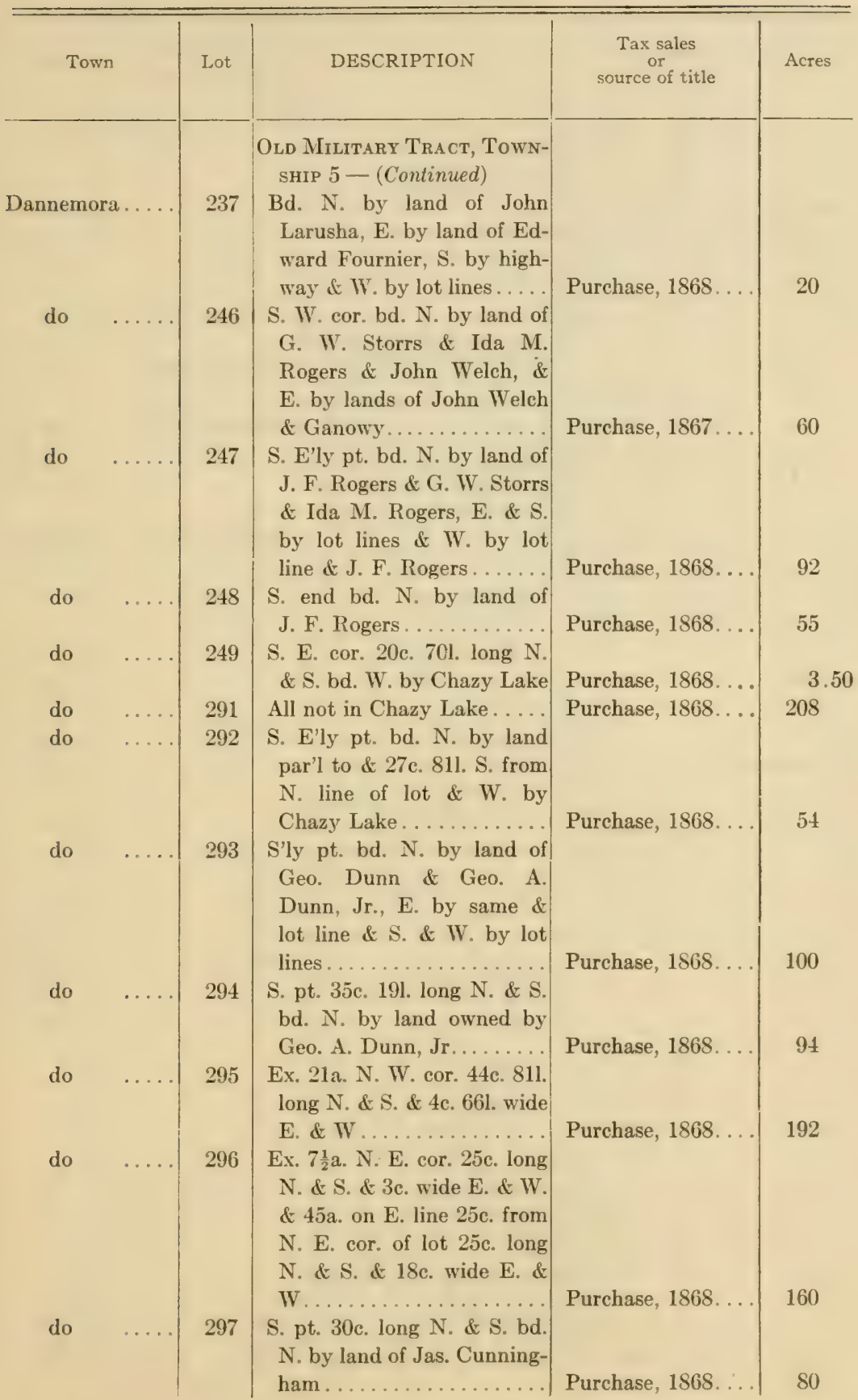


Cuinton County

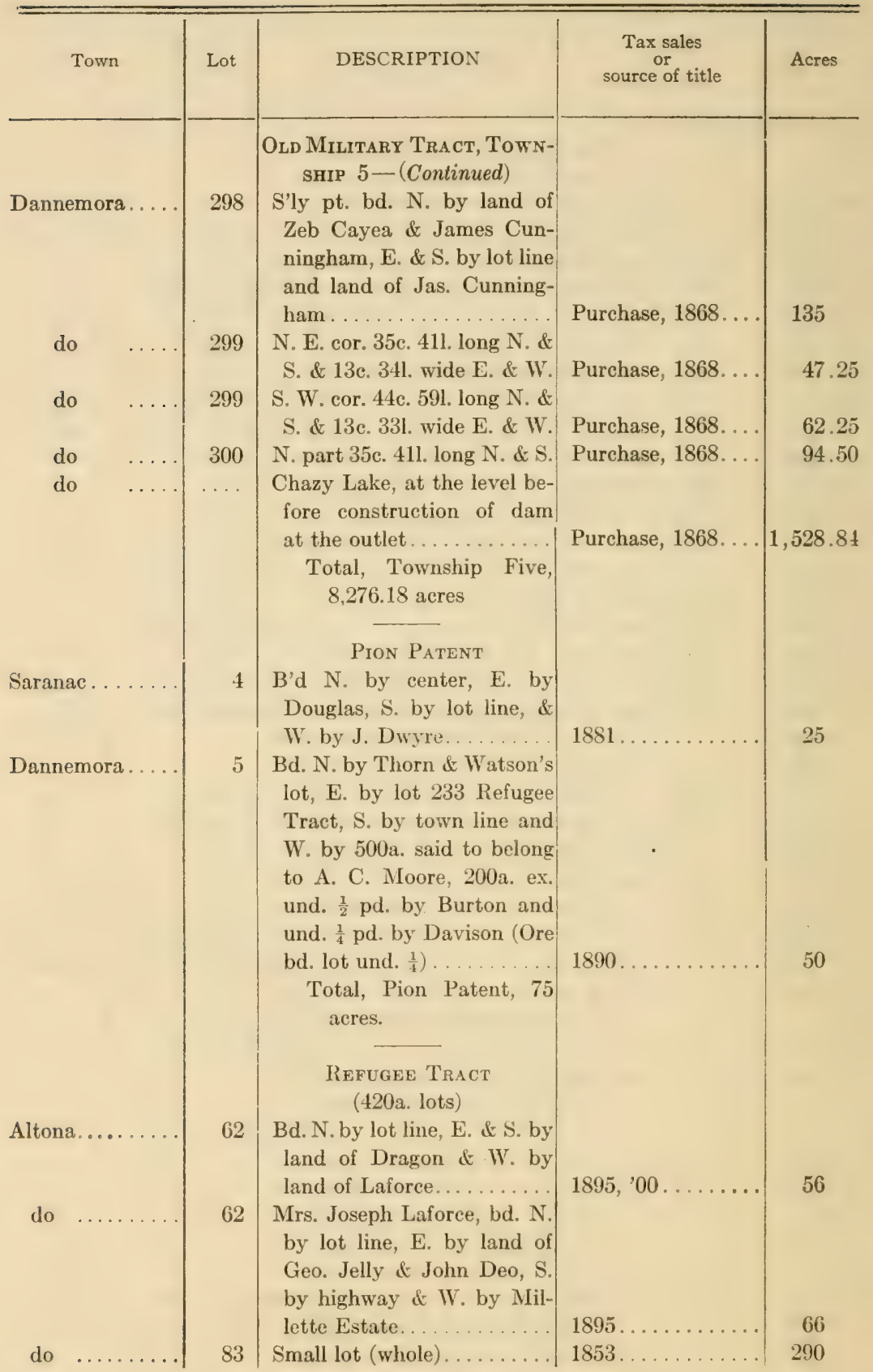


Cunnton County

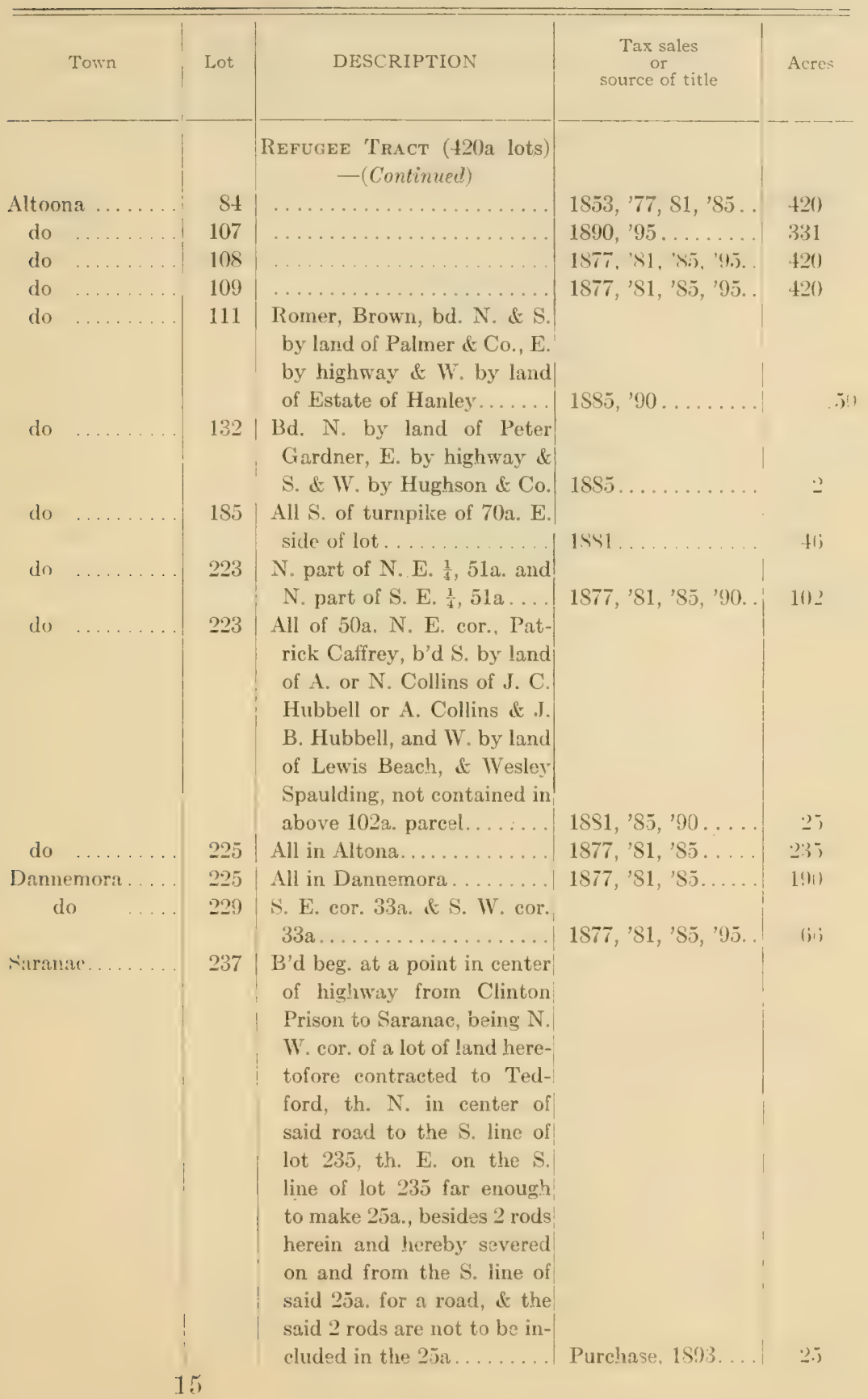


Timrd Axyeat Repolpt of the

\section{Cuinton County}

\begin{tabular}{|c|c|c|c|c|}
\hline Town & Lot & DESCRIPTION & $\begin{array}{c}\text { Tax sales } \\
\text { or } \\
\text { source of title }\end{array}$ & Acres \\
\hline $\begin{array}{c}\text { Plattsburgh. } \\
\text { do } \\
\text { do }\end{array}$ & $\begin{array}{l}241 \\
250 \\
252\end{array}$ & 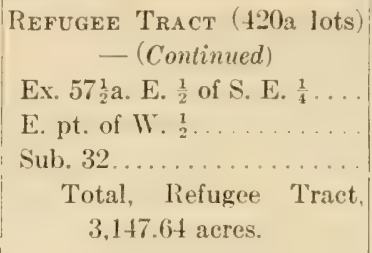 & 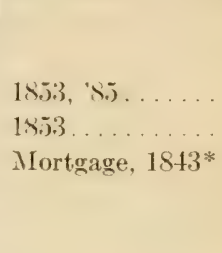 & $\begin{array}{l}367.50 \\
83 \\
2.61\end{array}$ \\
\hline Pert. & j & $\begin{array}{l}\text { Thorp's PAteNt } \\
\text { Allan Plain lot, b'd N. \& } \mathrm{W} \text {. } \\
\text { by the Craig lot, E. by the } \\
\text { Ricketson lot, \& S. by A. } \\
\text { Irwin \& Downs . . . . . . . } \\
\text { Total, } 19,962.60 \text { acres. }\end{array}$ & 154.5 & 40 \\
\hline
\end{tabular}

* Resale. 


\section{DELAIIARE COUNTY}

List of State Lands other than Forest Preserve

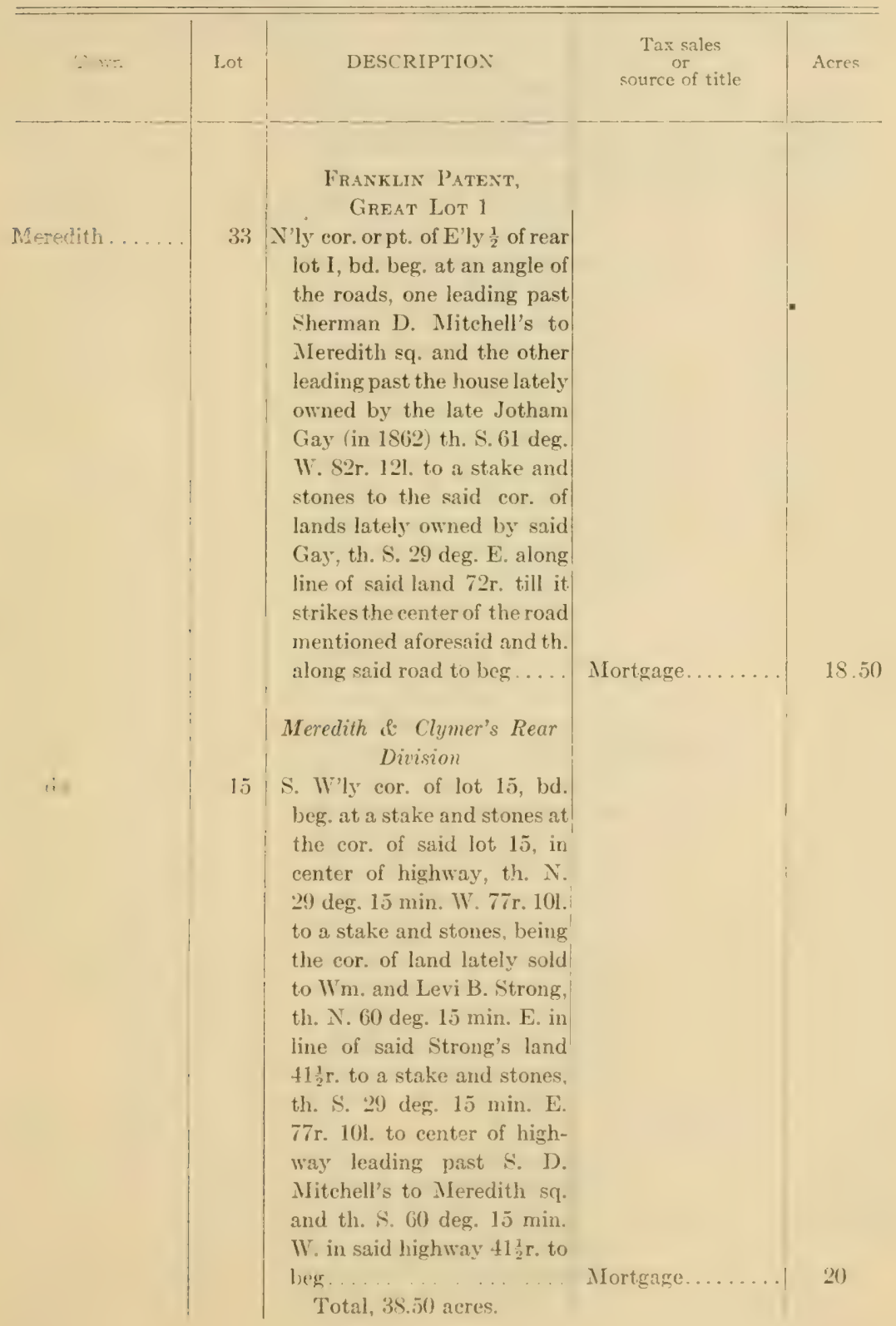




\section{Delaware Countr}

\begin{tabular}{|c|c|c|c|c|}
\hline Town & Lot & DESCRIPTION & $\begin{array}{c}\text { Tax sales } \\
\text { or } \\
\text { source of title }\end{array}$ & Acres \\
\hline Middletown. . & 121 & 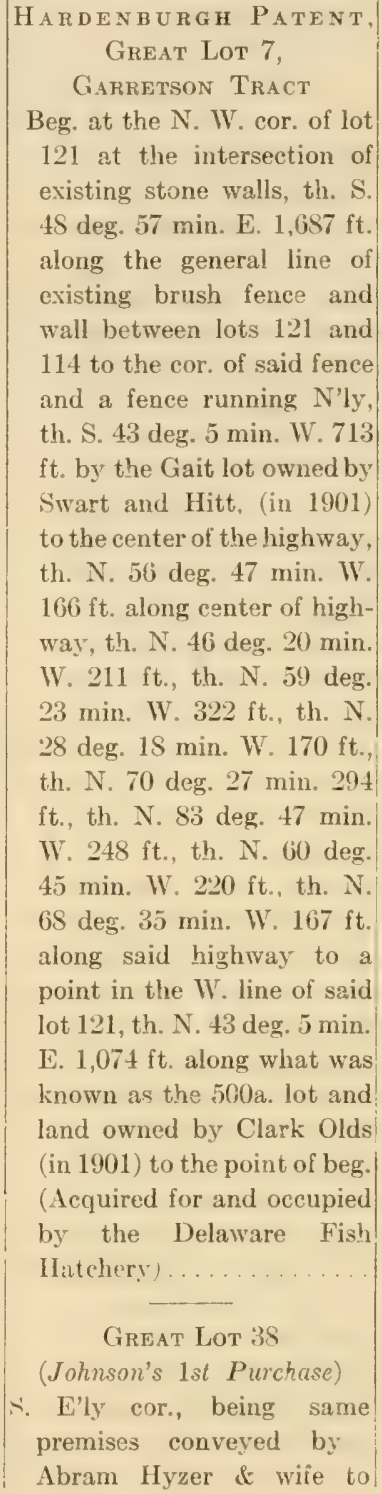 & Purchase. 1901. & 32.06 \\
\hline
\end{tabular}




\section{Delaware County}

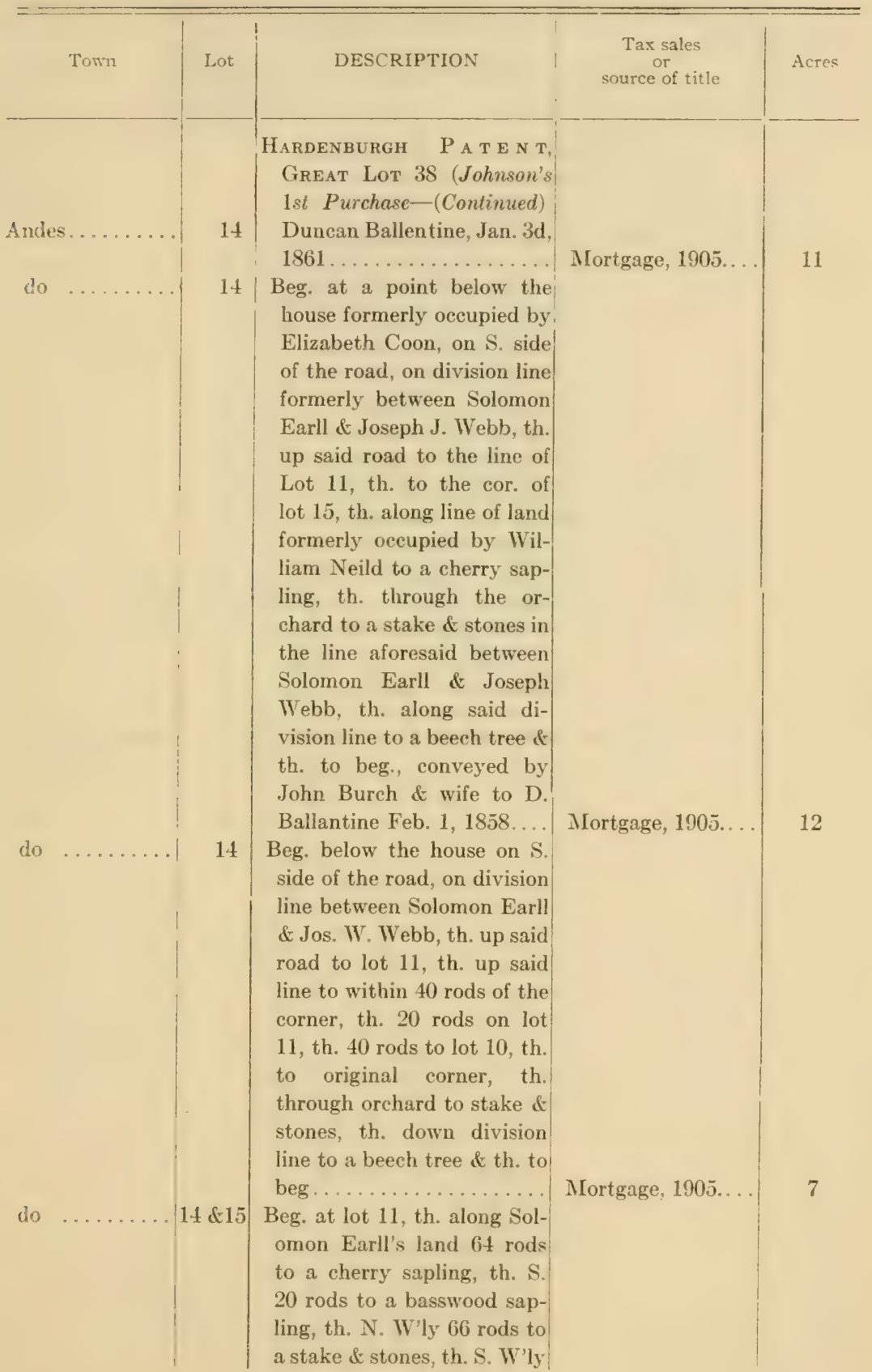


Delaware County

\begin{tabular}{|c|c|c|c|c|}
\hline Town & Lint & DESCRIPTION & $\begin{array}{c}\text { Tax sales } \\
\text { or } \\
\text { source of title }\end{array}$ & Acres: \\
\hline Andex.... & $\mid$ & $\begin{array}{l}\text { HARDENBURGH P A T E N T, } \\
\text { GREAT LoT } 38 \text { (Johnson's, } \\
1 \text { st Purchase - Continued) } \\
\text { to division line of lot } 15 \text {, th. } \\
\text { to beg.; also all that part of } \\
\text { lot } 15 \text { lying N. W'ly at the } \\
\text { highway from Tranperskill } \\
\text { to Wolf Hollow; also beg. } \\
\text { at lot } 15 \text { and N'ly part of } 14 \text {, } \\
\text { th. N'ly to a beech tree, th. } \\
35 \text { rods to a soft maple tree, } \\
\text { th. S. W'ly to a birch tree } \\
27 \text { rods \& th. along the high- } \\
\text { way to beg., all conveyed } \\
\text { by Abram Hyzer \& wife to } \\
\text { Duncan Ballantine } M \text { Iarch } \\
\text { 4, } 1859 . . . . . . . . . . . . . . \\
\text { Total, Great Lot } 38,69 \\
\text { acres. } \\
\text { Total, } 139.50 \text { acres. }\end{array}$ & . & $\therefore t$ \\
\hline
\end{tabular}




\section{ESSEX COUNTY}

List of State Lands other than Forest Preserve

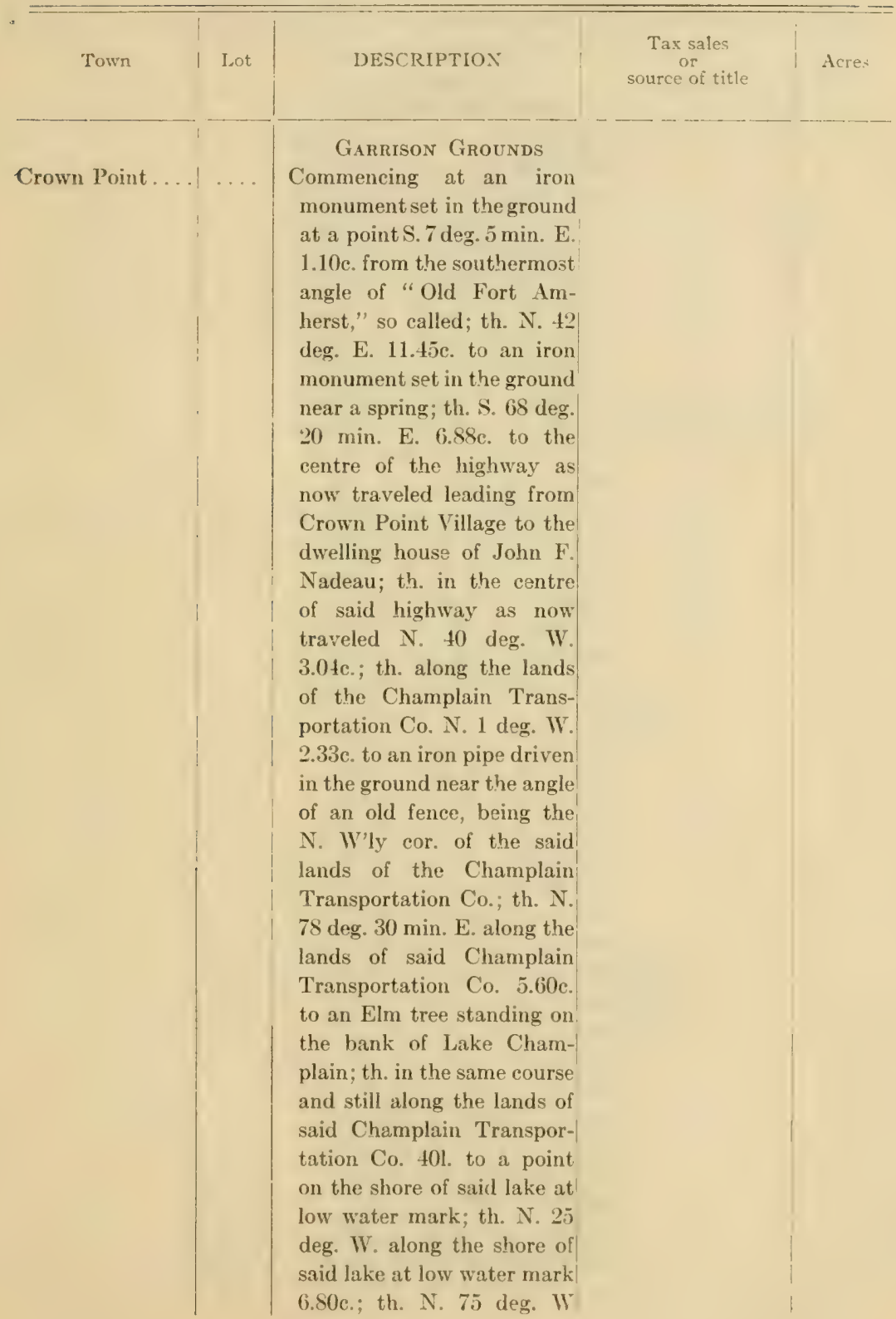




\section{Essex County}

\begin{tabular}{|c|c|c|c|c|}
\hline Town & Lot & DESCRIPTION & $\begin{array}{c}\text { Tax sales } \\
\text { or } \\
\text { source of title }\end{array}$ & Acres \\
\hline Crown Point. & $\ldots$ & 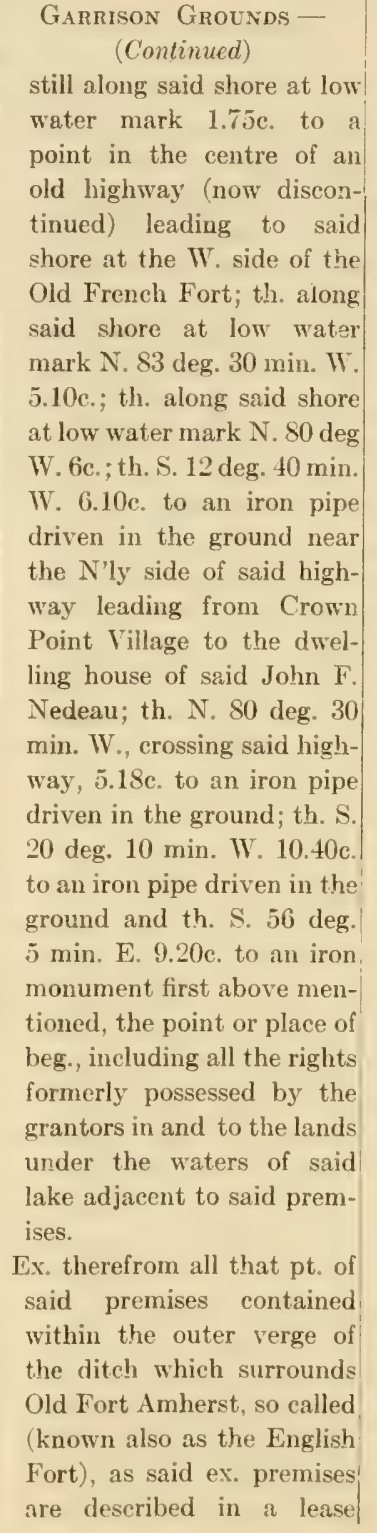 & & \\
\hline
\end{tabular}


Essex Counti

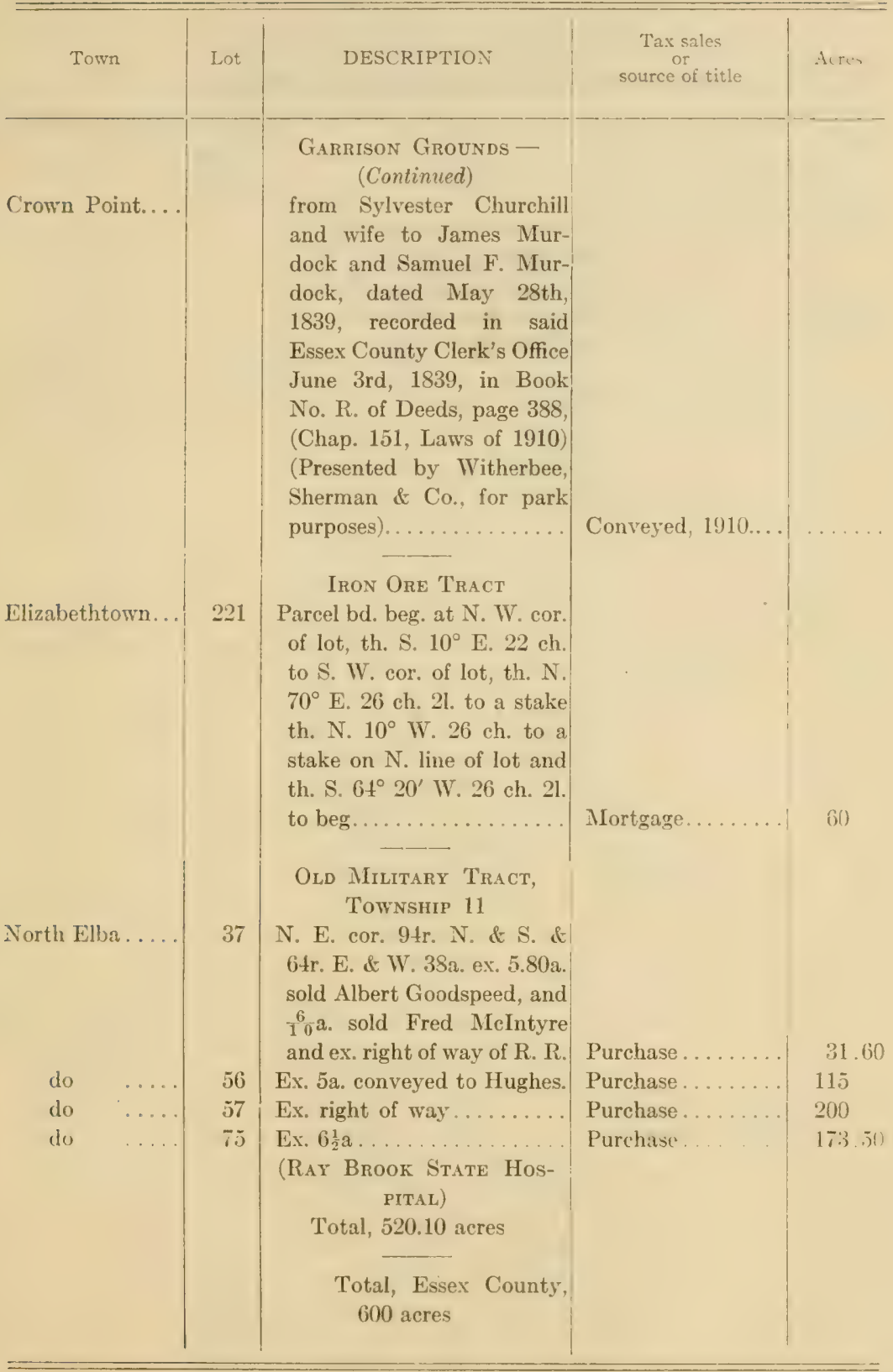




\section{FRANKLIN COUNTY}

List of State Lands other than Forest Preserve

\begin{tabular}{c|c|c|c|c}
\hline Town & Lot & DESCRIPTION & $\begin{array}{c}\text { Tax sales } \\
\text { or } \\
\text { source of title }\end{array}$ & Acres \\
\hline Franklin........ & $243 \mid \begin{array}{c}\text { N. } \frac{1}{2} \text { ex, la. in N. W. cor. on } \\
\text { which is a starch mill ..... }\end{array}$ & Mortgage.......... & 99 \\
\hline \hline
\end{tabular}




\section{FULTON COUNTY}

List of State Lands other than Forest Preserve

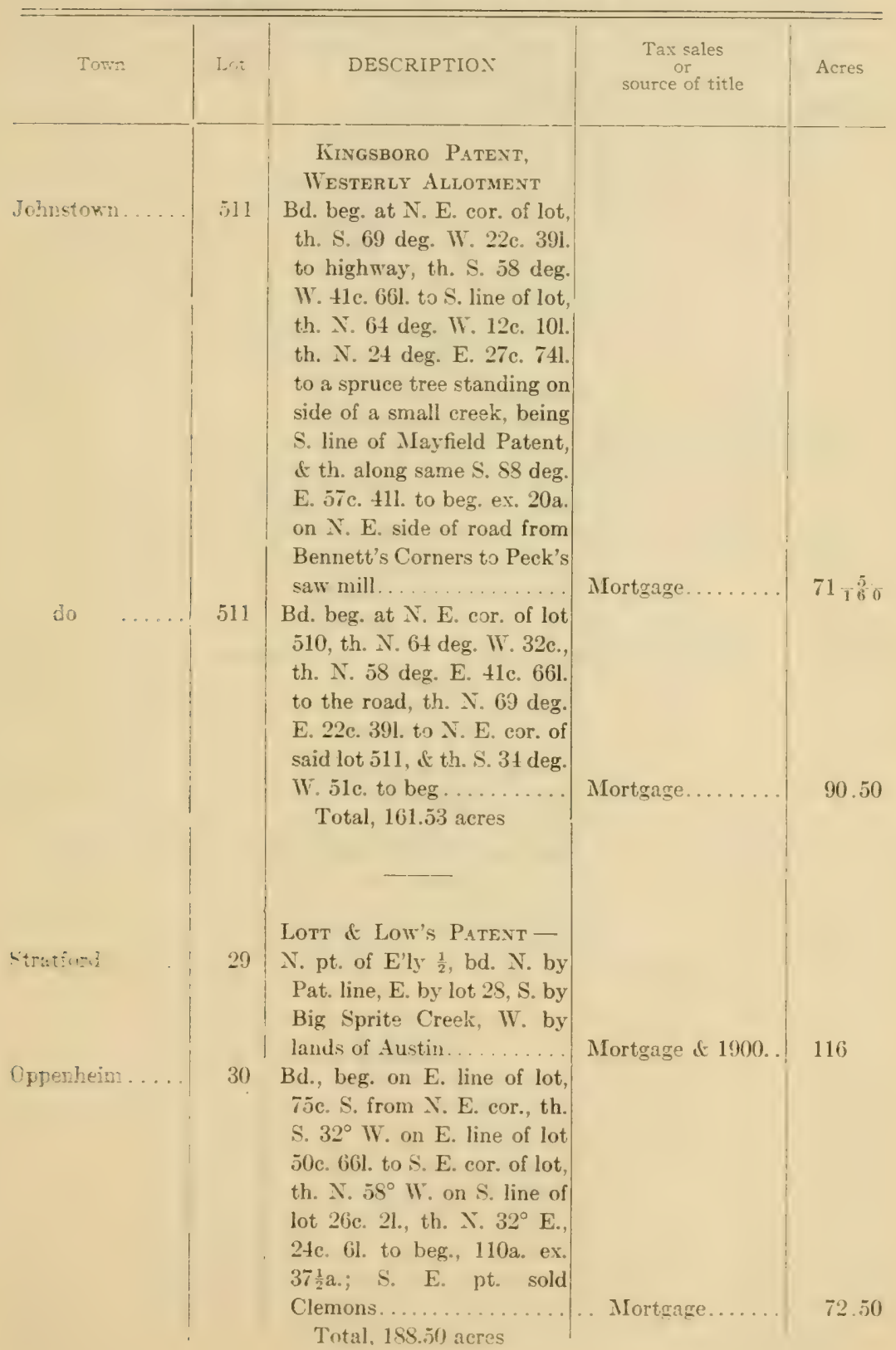




\section{Fulton County}

\begin{tabular}{|c|c|c|c|c|}
\hline Town & Lot & DESCRIPTION & $\begin{array}{c}\text { Tax sales } \\
\text { or } \\
\text { source of title }\end{array}$ & Aires \\
\hline Mayfield.... & $\ldots$ & $\begin{array}{l}\text { MAYFiELD, Town of } \\
\text { All that remains of the und. } \\
\frac{1}{2} \text { of a parcel, bd. N. W'ly } \\
\text { by Kingsboro Patent, S. W. } \\
\text { by lands of Nelson Gardner, } \\
\text { S. by lands of Mr. Rogers } \\
\text { \& E. by lands of Justice } \\
\text { Merchant, 100a. after ex. } \\
5 \text { a. sold by Simon Thurston } \\
\text { to Justice Merchant...... } \\
\text { OppenHeIM, Town of } \\
\text { Bd. N. by land of Hiram } \\
\text { Bixby, E. \& S. by land of } \\
\text { A. Michael and W. by lands } \\
\text { of G. Handy............ } \\
\text { Bd. N. by land of Jas. Demp- } \\
\text { ster, W. by land of Jacob' } \\
\text { Weaver, S. by land of Geo. } \\
\text { Handy and E. by land of } \\
\text { Hiram Bixby ............ } \\
\text { Total, Town of Oppen- } \\
\text { heim, } 51 \text { acres } \\
\text { Total, Fulton County, } \\
\text { 446.03 acres }\end{array}$ & $\begin{array}{l}\text { Mortgage..... } \\
\text { Murtgage .... }\end{array}$ & 4.5 \\
\hline
\end{tabular}




\section{GREENE COUNTY}

List of State Lands other than Forest Preserve

\begin{tabular}{|c|c|c|c|c|}
\hline Town & Lot & DESCRIPTION & $\begin{array}{c}\text { Tax sales } \\
\text { or } \\
\text { source of title }\end{array}$ & Acres \\
\hline Catskill. & 7 & $\begin{array}{l}\text { Catskill PAtent } \\
\text { Bd. N. by land now or re- } \\
\text { cently owed by heirs of } \\
\text { Abraham A. Salisbury, de-' } \\
\text { ceased, \& land of Chas. } \\
\text { Schwalli, E. by land of said } \\
\text { Chas. Schwalli \& land of } \\
\text { Bortle Flegle \& wife, S. by } \\
\text { land of John H. Overbaugh } \\
\text { \& W. by land of said Ovèr- } \\
\text { baugh \& Nathaniel Finch, } \\
\text { known as the farm on which } \\
\text { Geo. Gonnerman resided, } \\
\text { except \& reserving the } \\
\text { burial ground on said pre- } \\
\text { mises, with right of ingress } \\
\text { \& egress for burial purposes } \\
\text { only .................. }\end{array}$ & Mortgage, 1596. & 134.82 \\
\hline Lexington... & 2 & 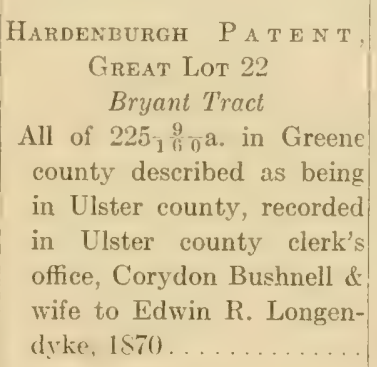 & Mortgage & $1 \times 7.50$ \\
\hline Ashland. & 57 & $\begin{array}{l}\text { VAN BERGEN'S PATENT, } \\
\text { 3d Tract } \\
\text { Bd. beg. at a pile of stones } \\
\text { around a beech bush at S. } \\
\text { E. cor. of land now or } \\
\text { formerly owned or occupied, } \\
\text { by Levi Austin, th. N. } 2^{\circ} \mathrm{W} \text {. } \\
\text { 40c. S0I. to a stake and } \\
\text { stones, on the side of } \\
\text { the Mt., th. S. } 32 \mathrm{c} \text {. } 311 \text {. to } \\
\text { a stake and stones, th. S. }\end{array}$ & & \\
\hline
\end{tabular}




\section{Greene County}

\begin{tabular}{|c|c|c|c|c|}
\hline Town & Lot & DESCRIPTION & $\begin{array}{c}\text { Tax sales } \\
\text { or } \\
\text { source of title }\end{array}$ & Acres \\
\hline Ashland... & . & $\begin{array}{l}\text { VAN BERGEN's PATENT, } \\
3 d \text { Tract-(Continued) } \\
88^{\circ} \text { E. } 30 \mathrm{c} .10 \mathrm{l} \text {. to beg.; bd. } \\
\text { N. by lot } 50, \text { E. by lands } \\
\text { of Wm. Decker, S. by land } \\
\text { of Levi Austin and W. by } \\
\text { lot 56, same lands formerly } \\
\text { of Josiah Tuttle, deeded by } \\
\text { him to John Groat, } 1826 . \\
\text { Total, Greene County, } \\
449.57 \text { acres. }\end{array}$ & Mortgage, 1903. & $127 \cdot 25$ \\
\hline
\end{tabular}




\section{HAMILTON COUNTY}

List of State Lands other than Forest Preserve

\begin{tabular}{|c|c|c|c|c|}
\hline Town & Lot & DESCRIPTION & $\begin{array}{c}\text { Tax sales } \\
\text { or } \\
\text { source of title }\end{array}$ & Acres \\
\hline Benson. & 4 & 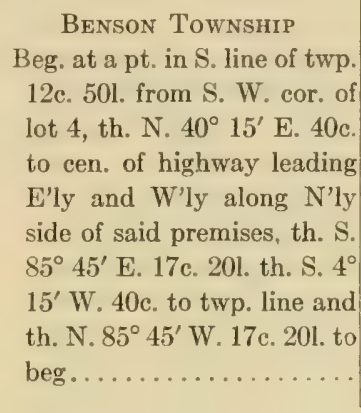 & Mortgage... & 68.81 \\
\hline
\end{tabular}




\section{HERKIMER COUNTY}

List of State Lands other than Forest Preserve

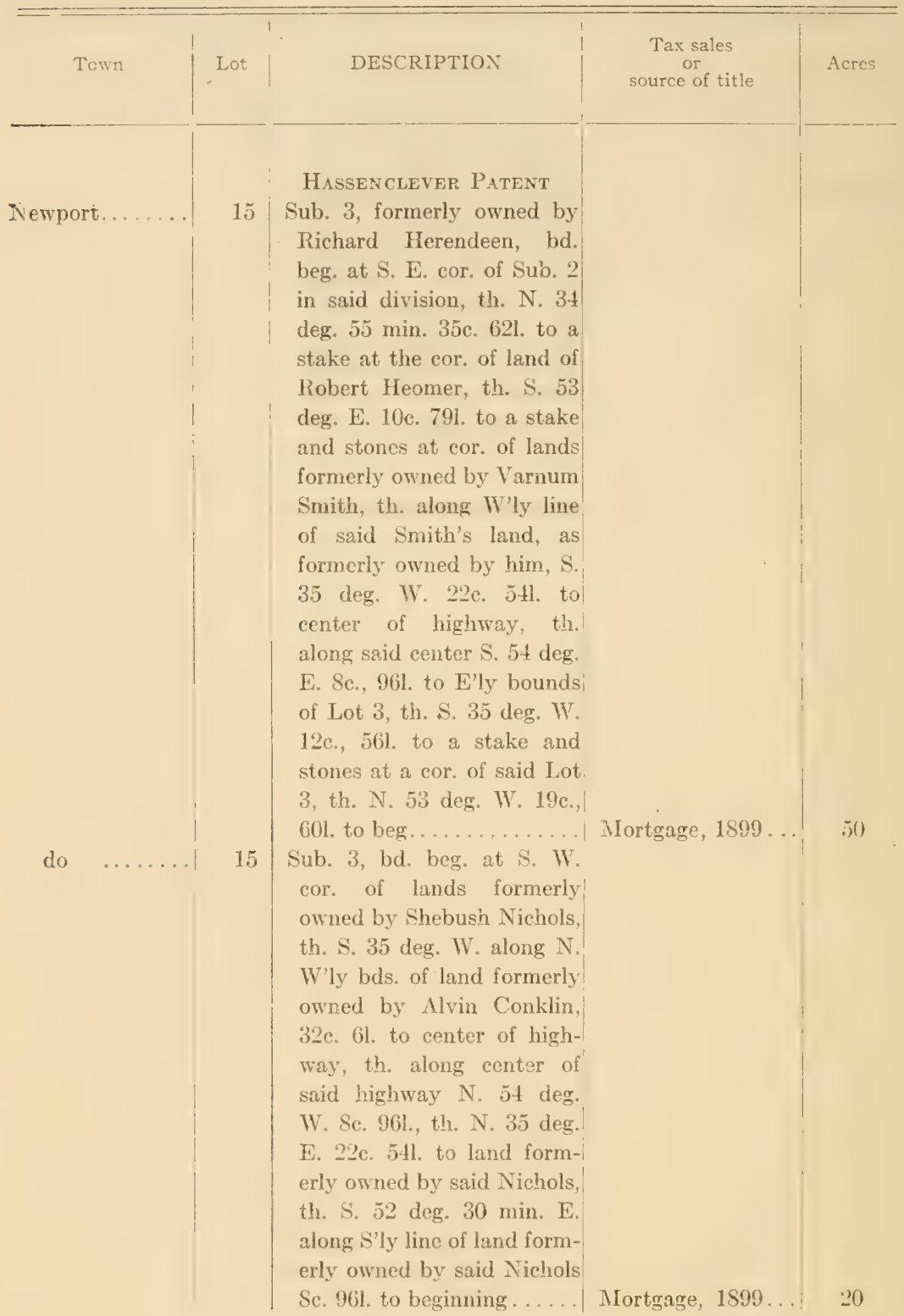


Herkimer County

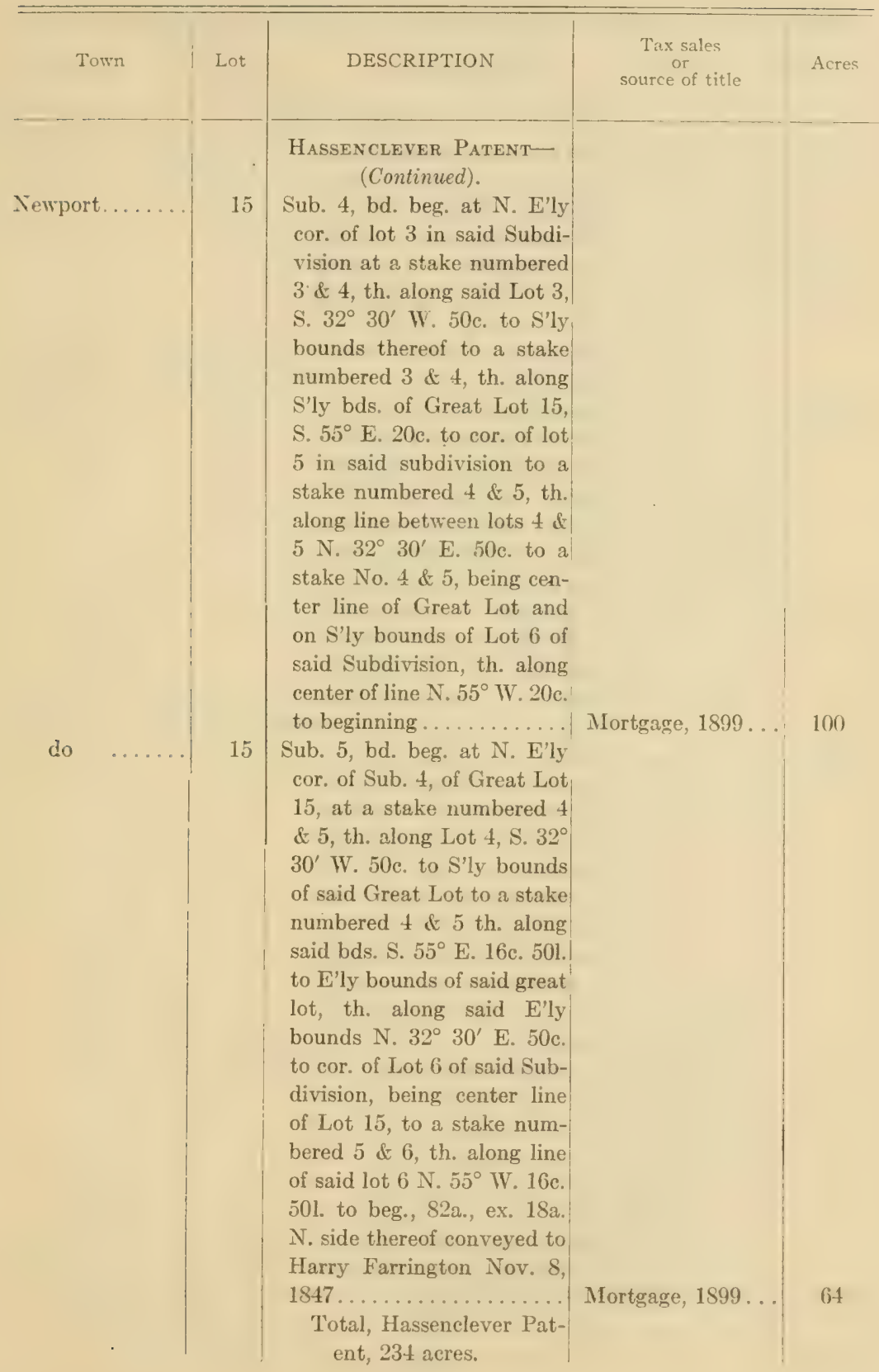


Herkimer County

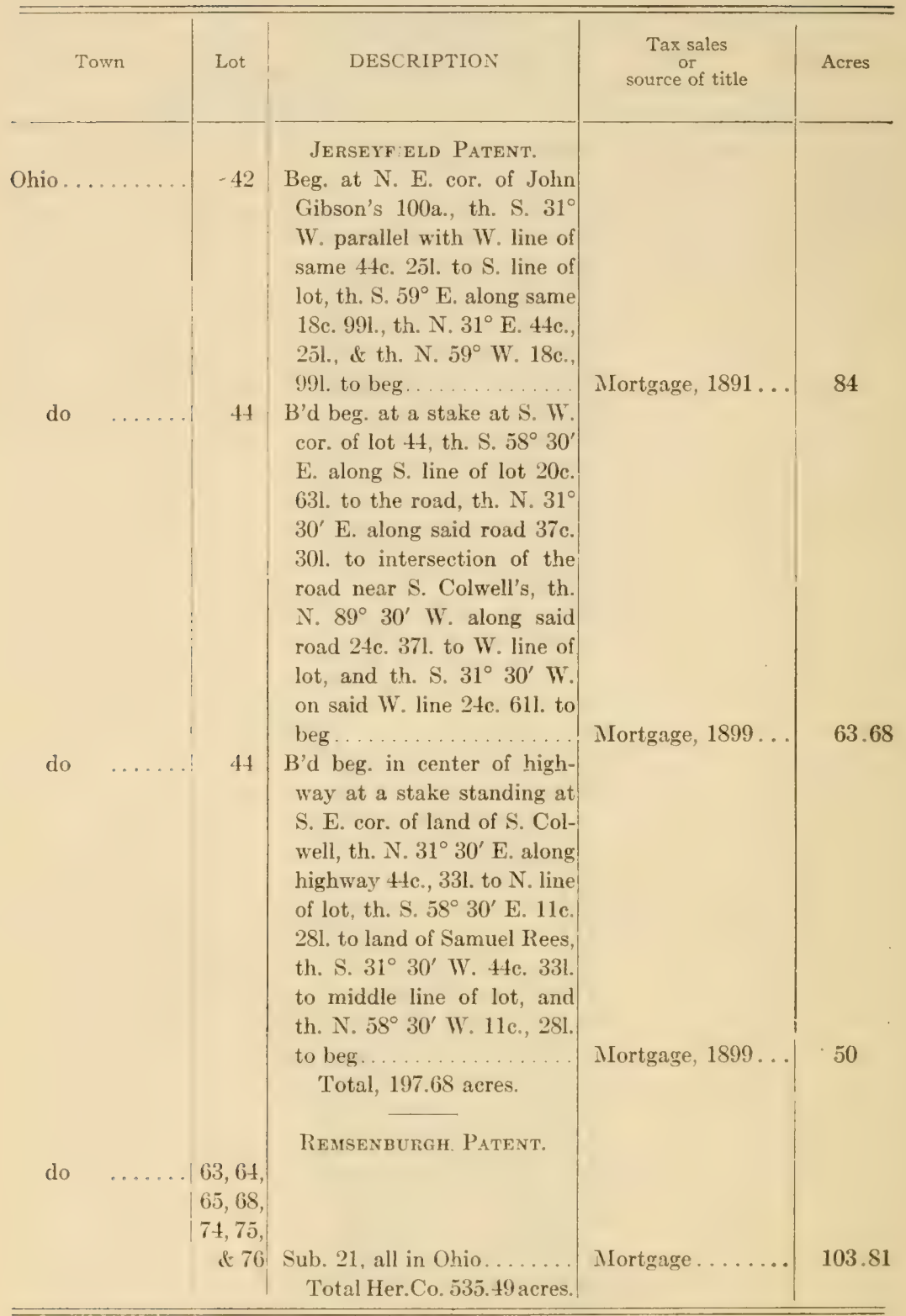




\section{JEFFERSON COUNTY}

List of Lands in the St. Lawrence Reservation or International Park.

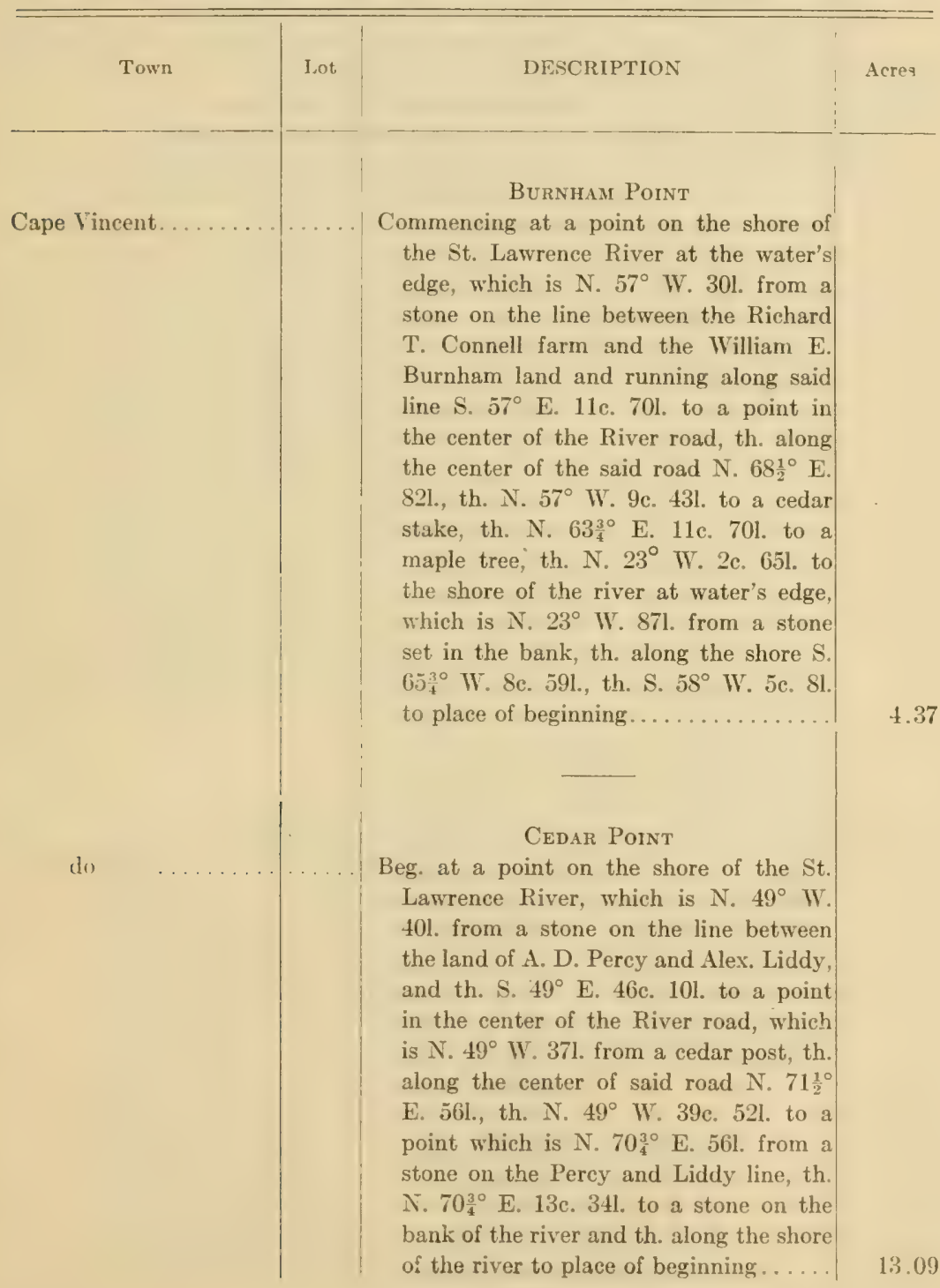




\section{Jefferson County}

ST. LAWRENCE RESERVATION-(Continued)

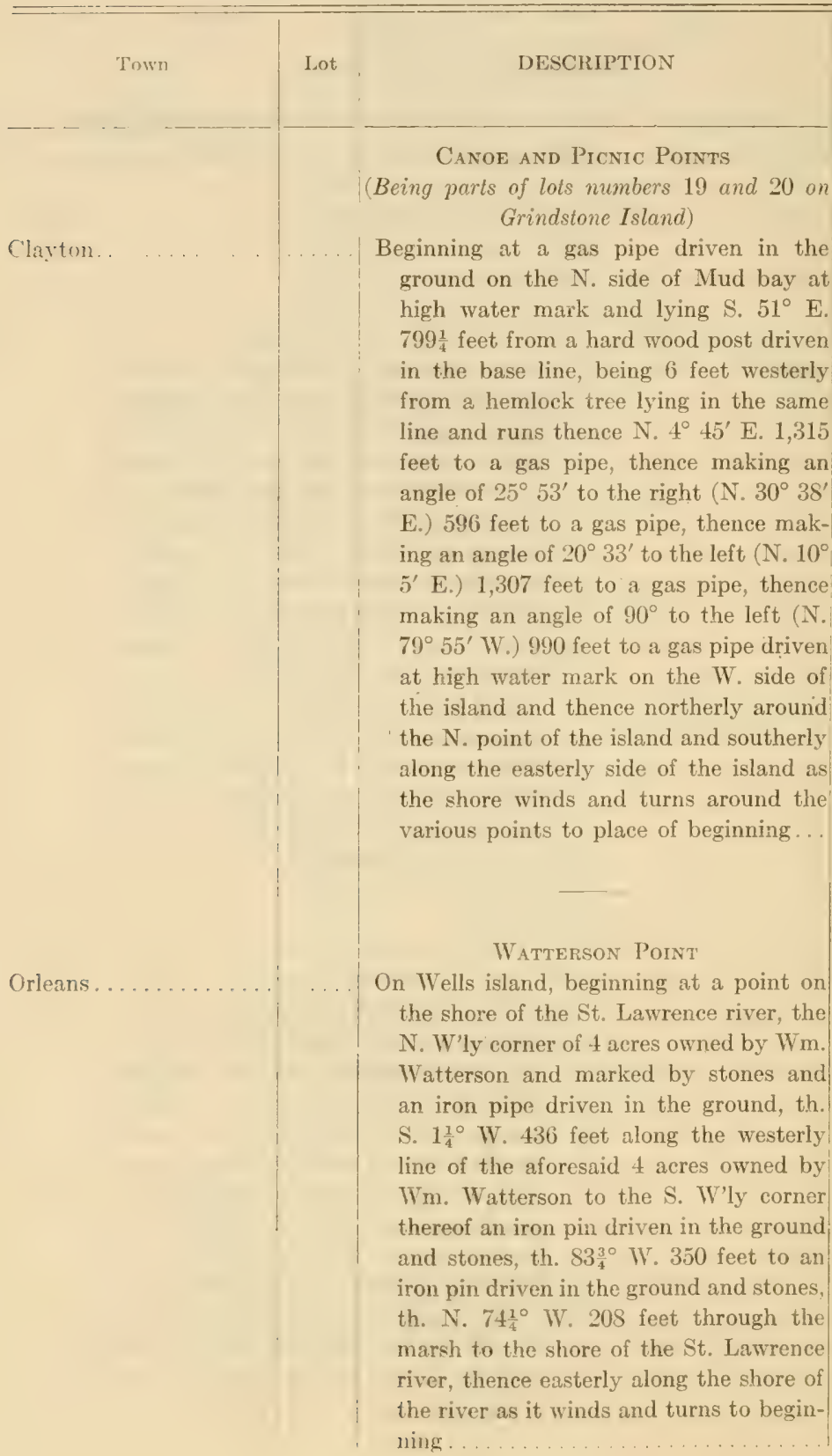

Acres 


\section{Jefferson Countr}

ST. LAWRENCE RESERVATION-(Continued)

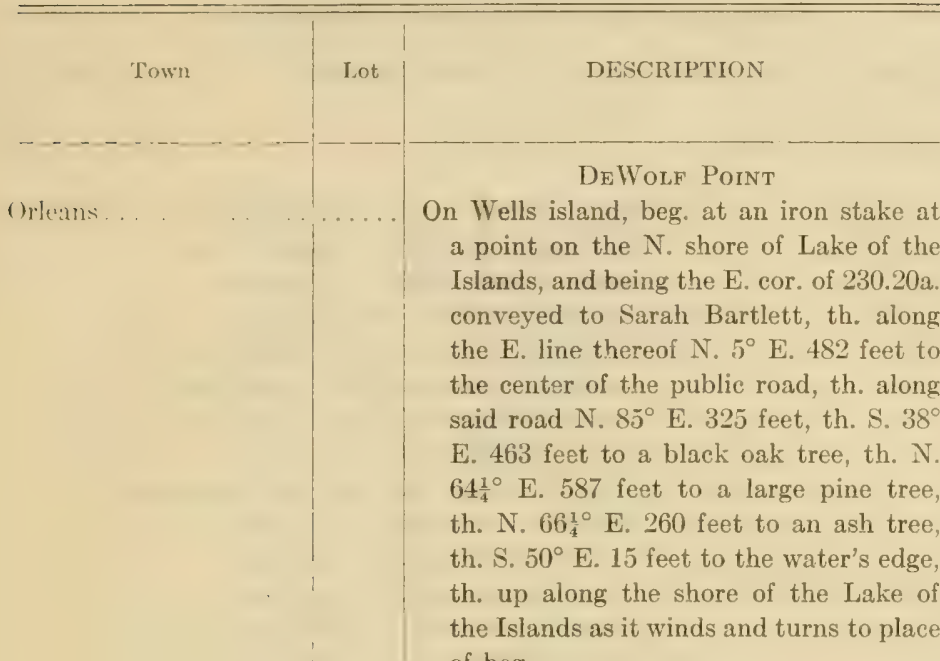
of beg.

\section{Mary IsLand}

Alexandria . . . . . . . . . . Mary Island in St. Lawrence river at the foot of Wells Island and below the canal or hauling place, so called...........

\section{Acres}

\section{Kring Potnt}

Beg. at a stake set in the ground in that part of the Kring farm known as "Kring Point," which stake is 2,800 feet in a straight line from the most $S^{\prime}$ ly part of said point, th. $S \cdot 10^{\circ}$ E. 4 c. $54 \frac{1}{2} \mathrm{l}$. (300 ft.) to a stake set in the shore of Goose bay, th. S'ly along the N. W. shore of Goose bay as it winds and turns to the most S. W'ly part of Kring Point, th. N. and N. E'ly along the shore of Goose bay and the shore of St. Lawrence river as it winds and turns to a stake near the shore of the river, th. N. $87^{\circ}$ E. Sc. $12 \frac{1}{2}$ l.

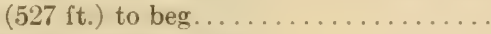

\section{Long Point on Chaumont Bay Macomb's Purchase Great Tract 4 (Ralph French Survey)} E'ly line of land of Samuel Failing in the center of the Shore Road, so called, 


\section{Jefferson County}

ST. LAWRENCE RESERVATION-(Continued)

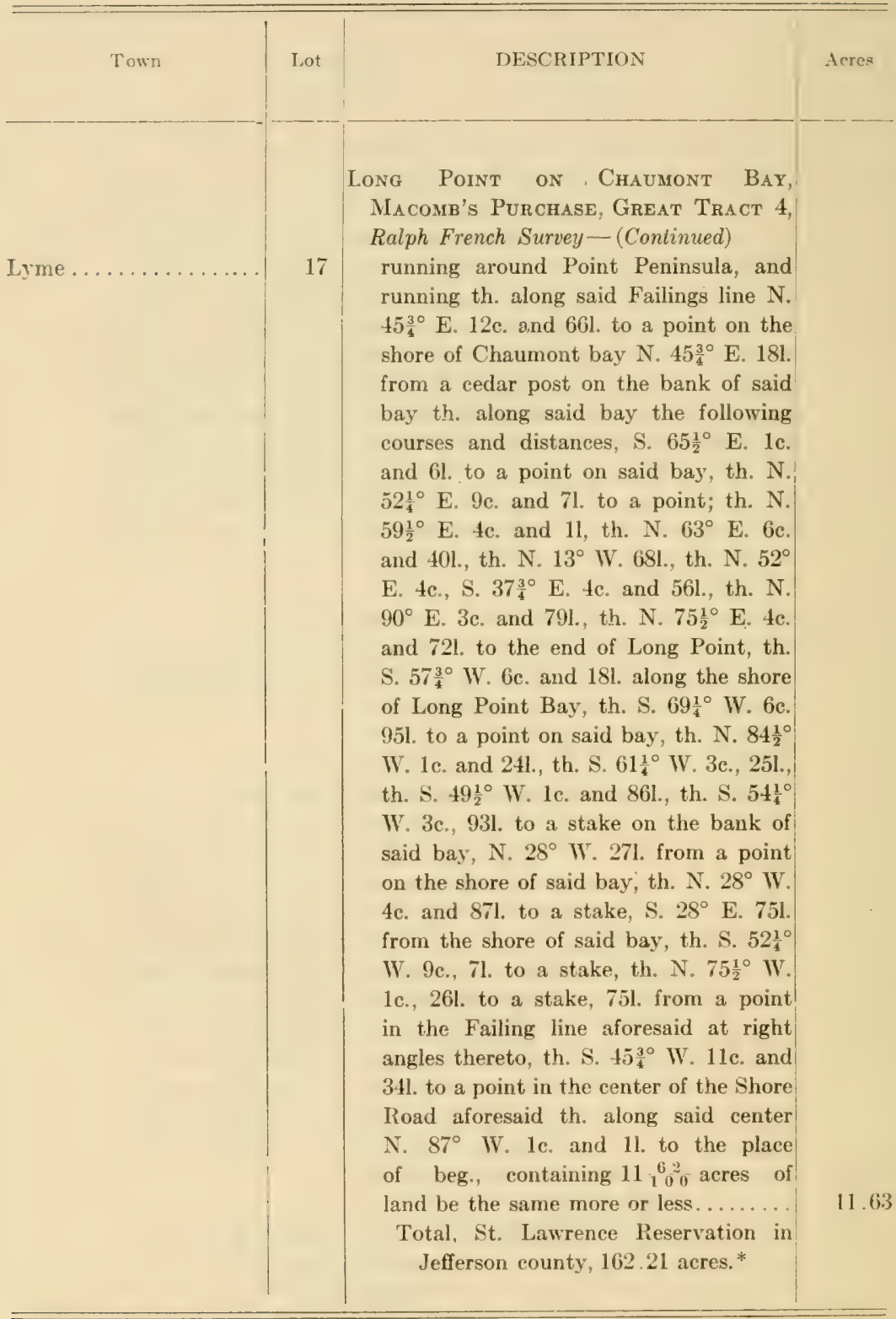

* For other land in the St. Lawrence Reservation, see Lawrence County (Appendix) 


\section{LEWIS COUNTY}

List of State Lands other than Forest Preserve

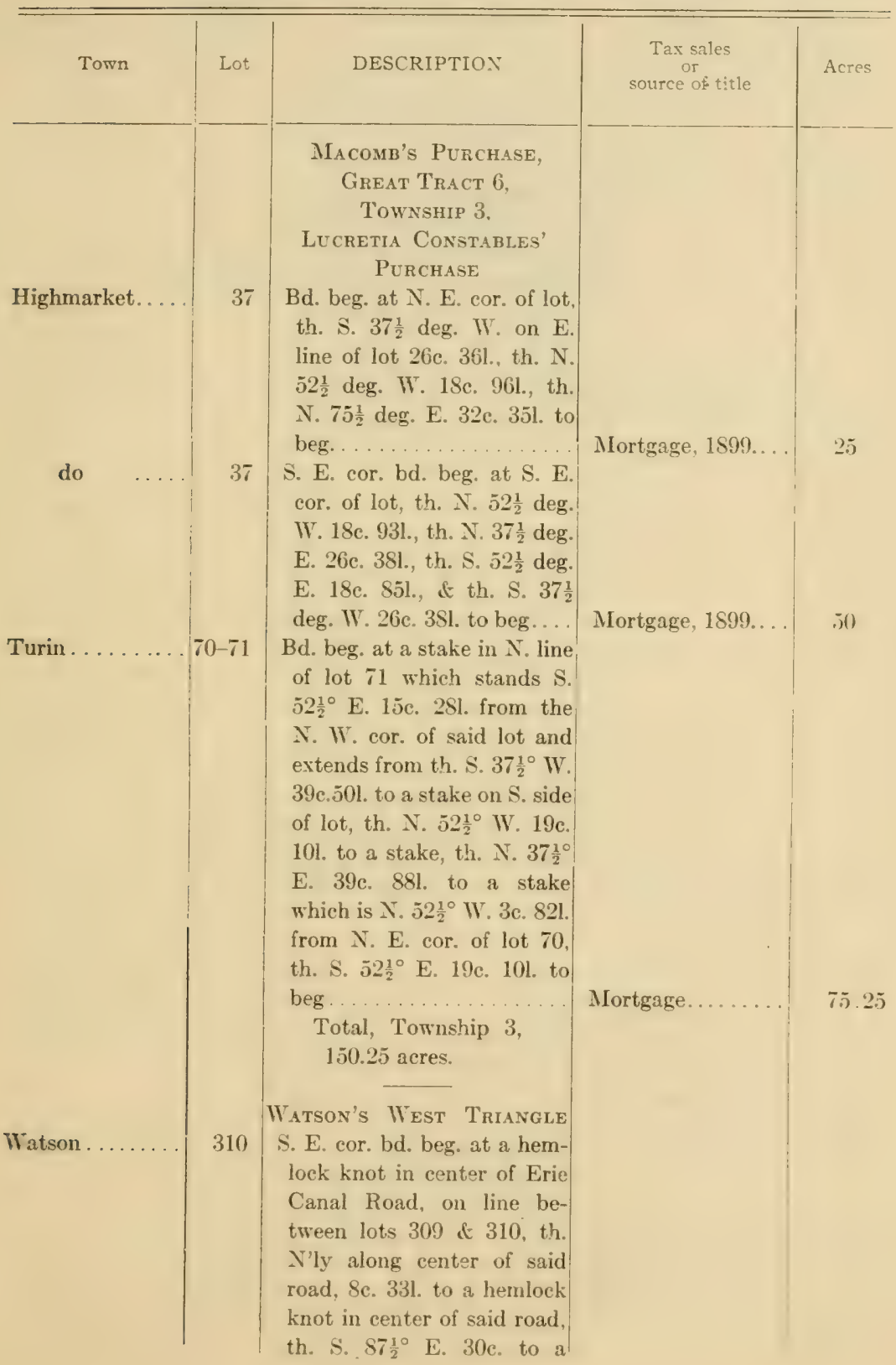


Lewis County

St. Lawrence Reservation - (Continued)

\begin{tabular}{|c|c|c|c|c|}
\hline Town & Lot & DESCRIPTION & $\begin{array}{c}\text { Tax sales } \\
\text { or } \\
\text { source of title }\end{array}$ & Acres \\
\hline Watson. & 310 & $\begin{array}{l}\text { WATSON's WEST TRIANGLE } \\
\text { (Continued) } \\
\text { hemlock post, th. S. on E. } \\
\text { line of lot } 310 \text {, according } \\
\text { to the original survey, th. } \\
\text { W. on line between lots } 309 \\
\text { and } 310 \text { to beg.......... } \\
\text { Total, Lewis Co., } 175.25 \\
\text { acres. }\end{array}$ & Mortgage, $1892 \ldots$ & 25 \\
\hline
\end{tabular}




\section{ONEIDA COUNTY}

List of State Lands other than Forest Preserve

\begin{tabular}{|c|c|c|c|c|}
\hline Town & Lot & DESCRIPTION & $\begin{array}{c}\text { Tax sales } \\
\text { or } \\
\text { source of title }\end{array}$ & Acres \\
\hline Forestport. & 6 & 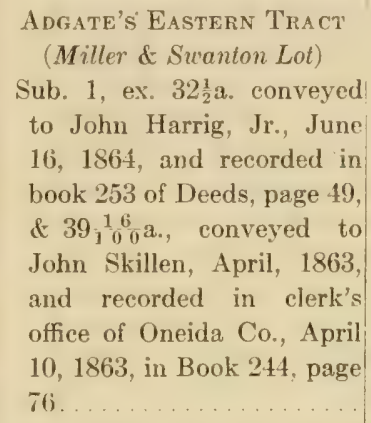 & Mortgage, 1878. & 28.40 \\
\hline Boonville. . & 73 & 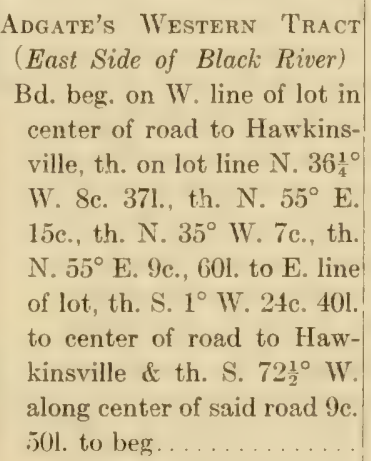 & Mortgage, 1875. & 20 \\
\hline Lee. & 13 & $\begin{array}{l}\text { Banyar's Patent } \\
\text { N. pt. bd. S. by land of Benj. } \\
\text { Rudd................ }\end{array}$ & Mortgage, 1888. & 24.25 \\
\hline Western & 17 & $\begin{array}{l}\text { S. W. cor. bd. E'ly by a deep } \\
\text { gulf and N'ly a line par'l } \\
\text { with S. line................ }\end{array}$ & Mortgage, 188s.. & -1.0 \\
\hline Lees. & 18 & & Mortgage, 1888. & 95 \\
\hline do & 23 & $\begin{array}{l}\frac{1}{2} \text { of } \mathrm{S} . \frac{1}{2} \text { being premises con- } \\
\text { veyed Jan. } 5 \text {, } 1846 \text {, by G. } \\
\text { Leroy Banyar to Alfred } \\
\text { Hartshorn.................. }\end{array}$ & 1888.. & 25.50 \\
\hline (t) & 46 & W. $\frac{1}{2} \ldots \ldots \ldots \ldots \ldots$ & Mortgage, 1907. & 46.25 \\
\hline do & 47 & $\begin{array}{l}\text { E. side adj. the above ...... } \\
\text { Total, Banyar's Patent, } \\
\text { 19Sa. }\end{array}$ & Mortgage, 1907. . & 2 \\
\hline
\end{tabular}


Onema County

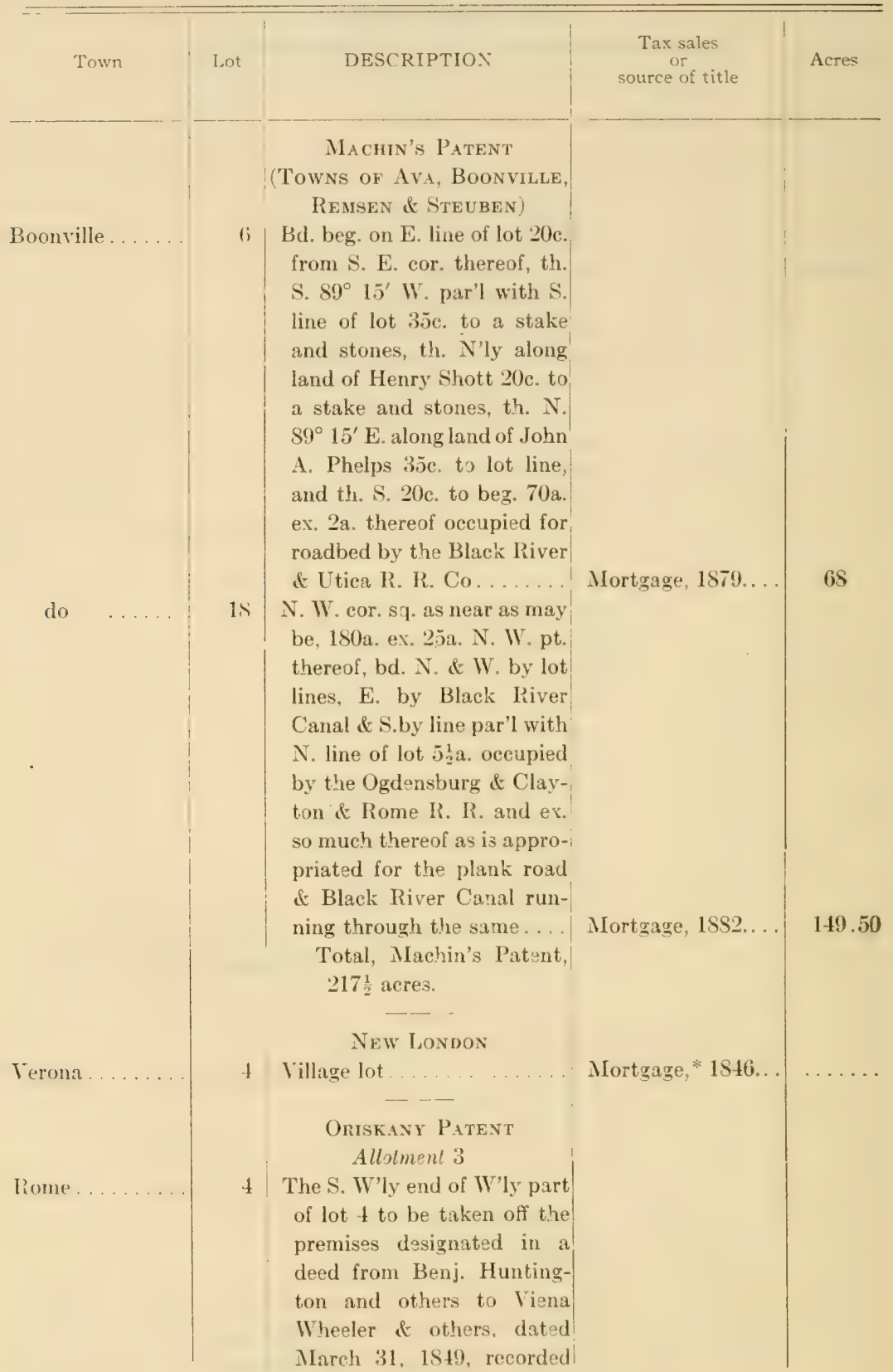


Oneida County

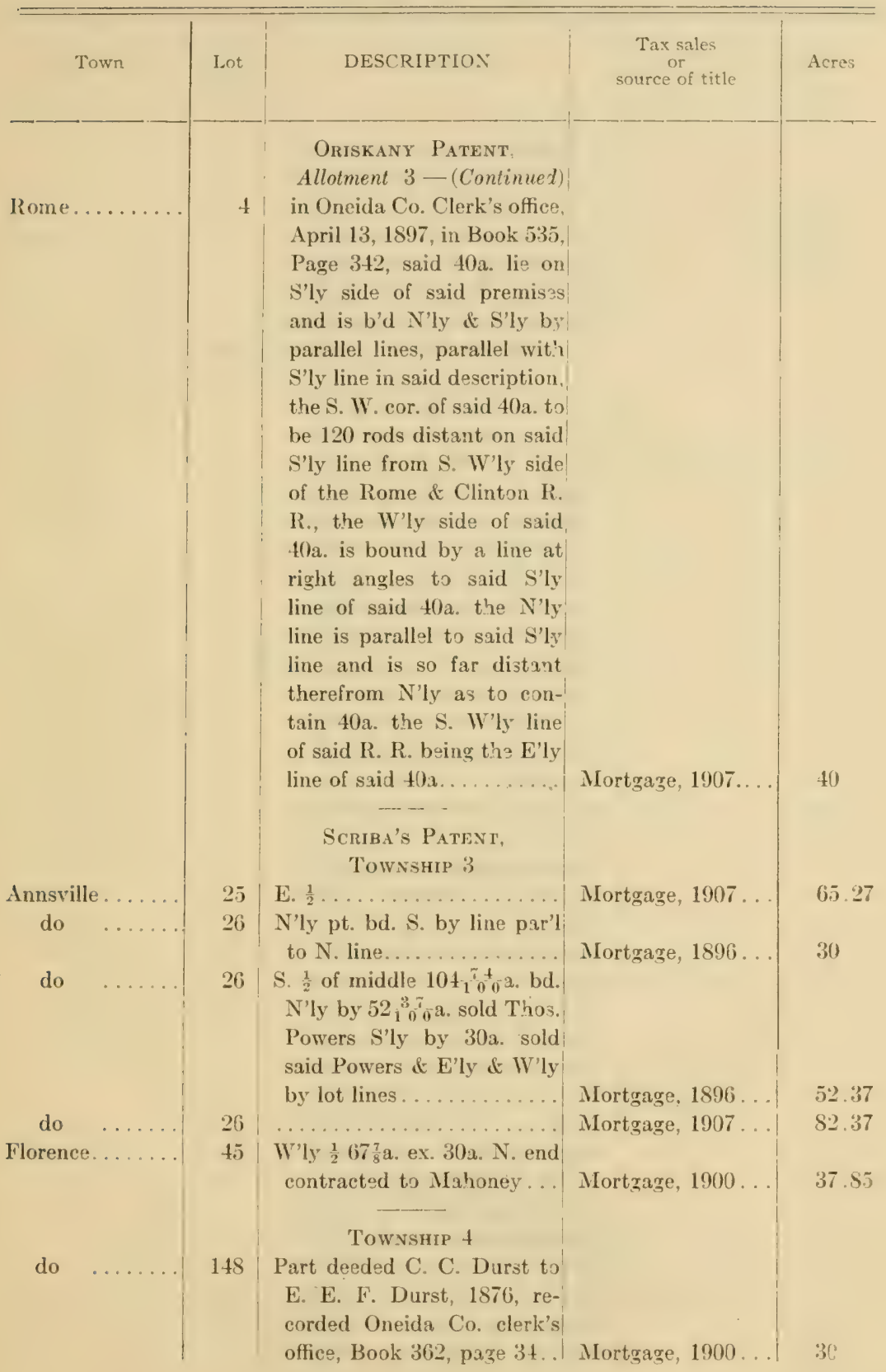




\section{Oneida County}

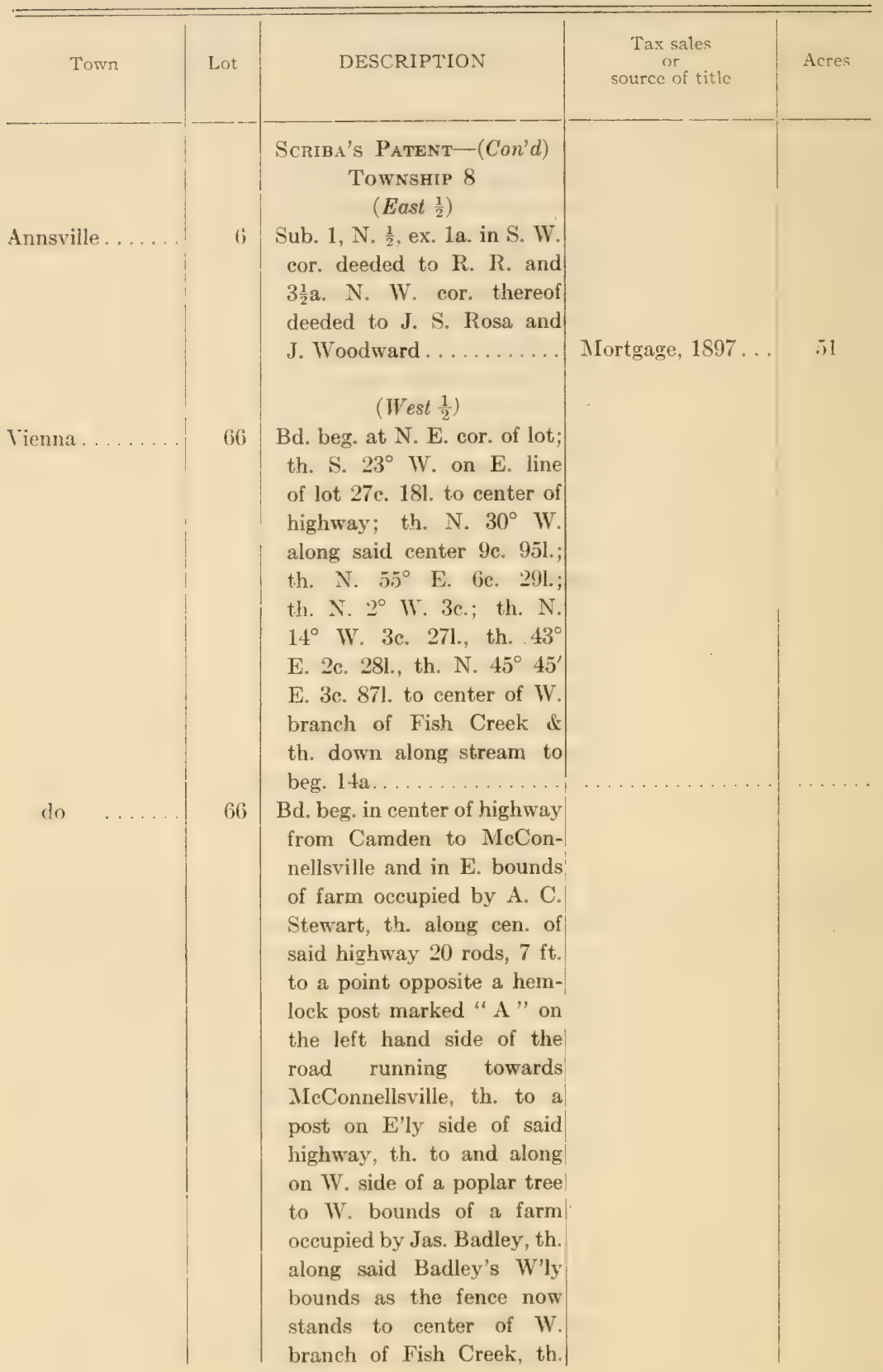


Oneida County

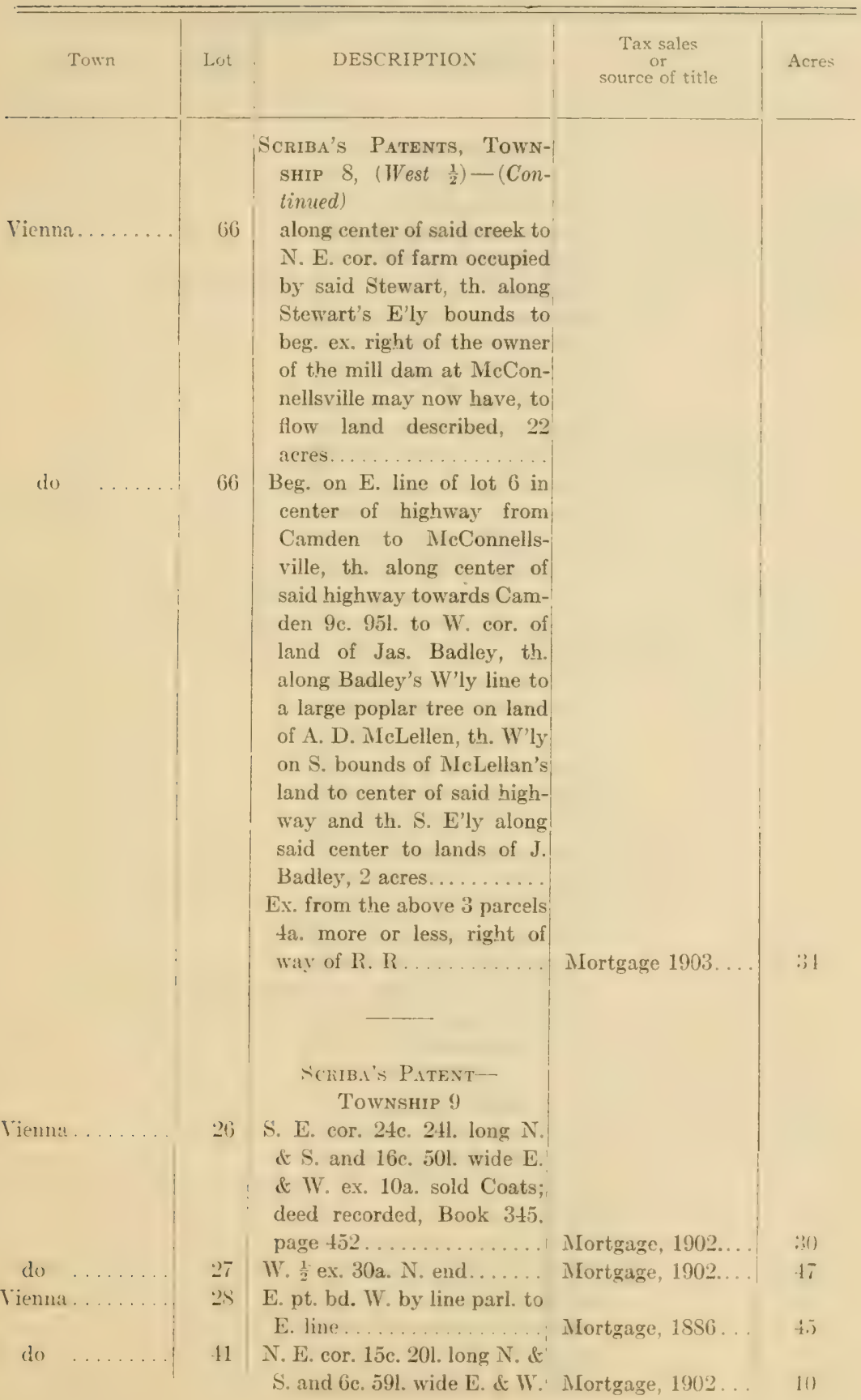




\section{Oneida County}

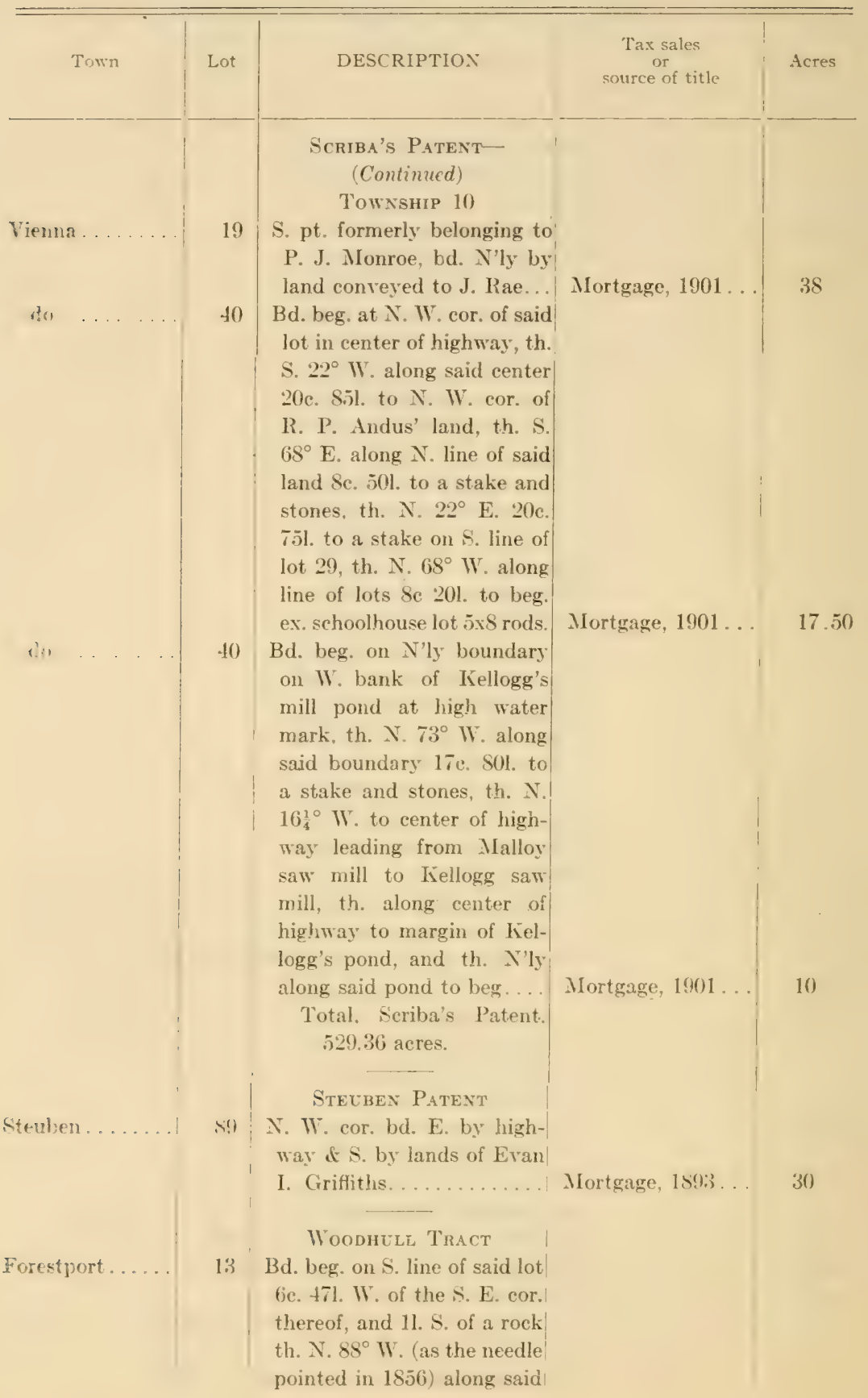


Oneida County

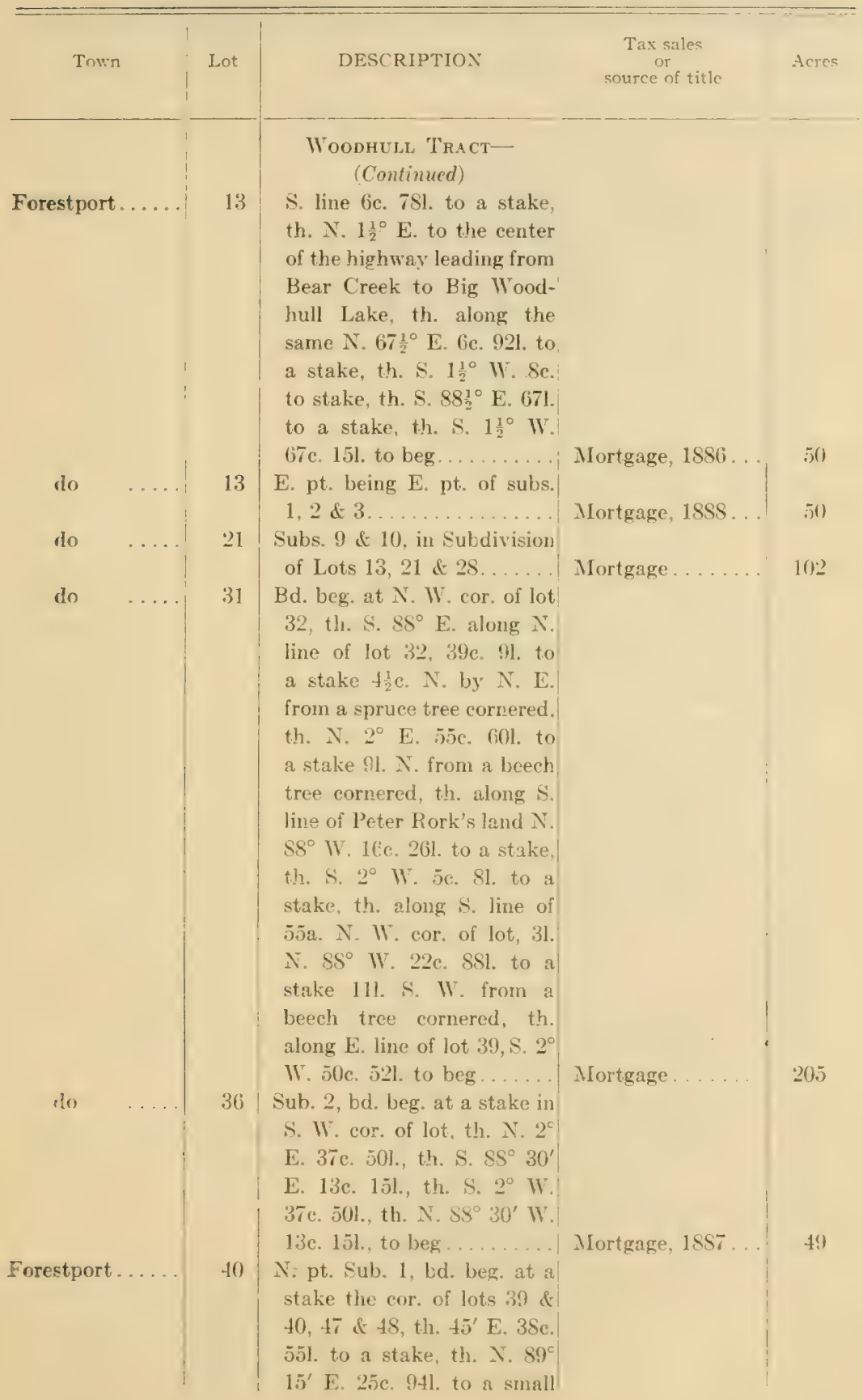




\section{Oneida County}

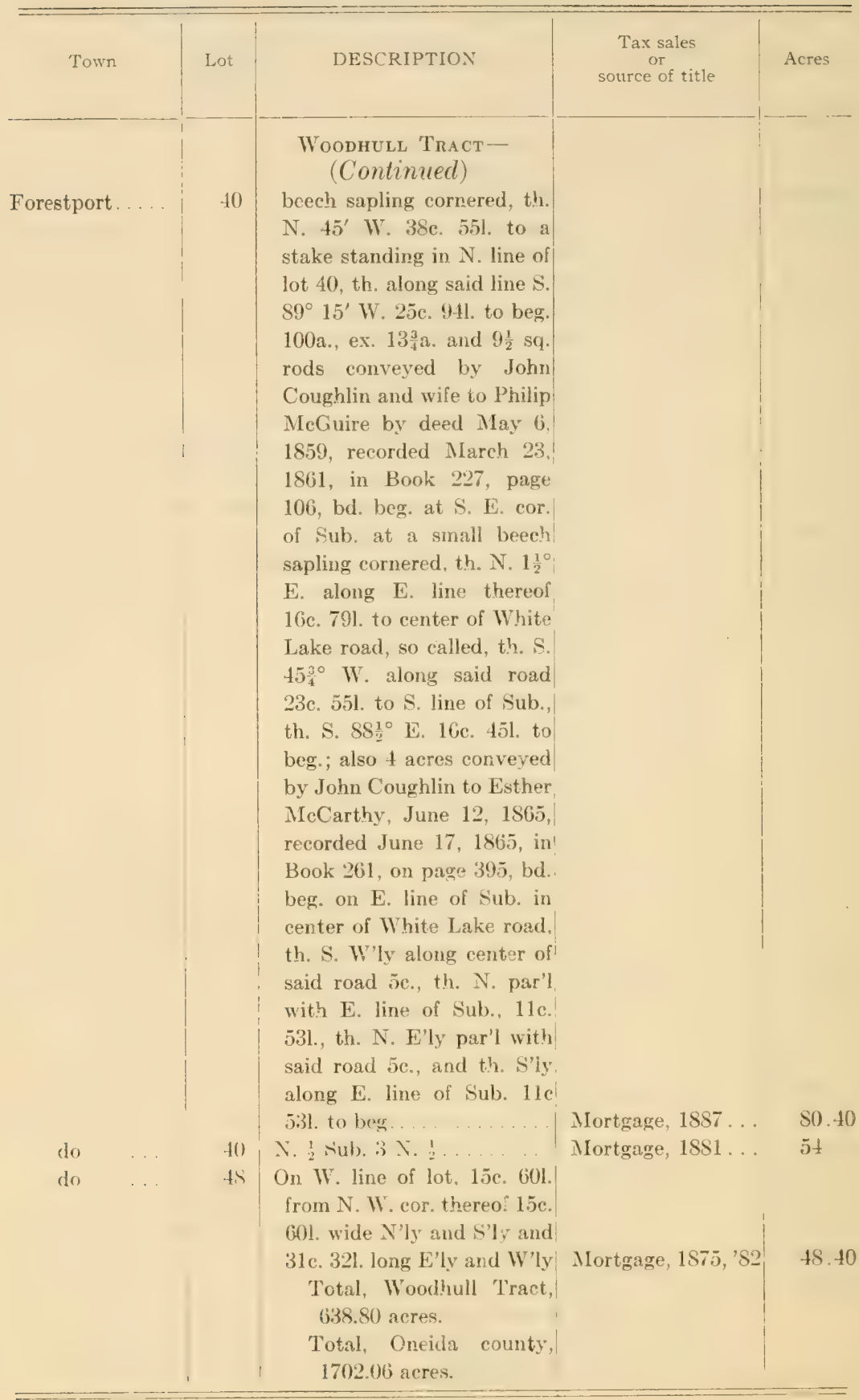


ST. LAWRENCE COUNTY

List of Lands in the St. Lawrence Reservation or International Park*

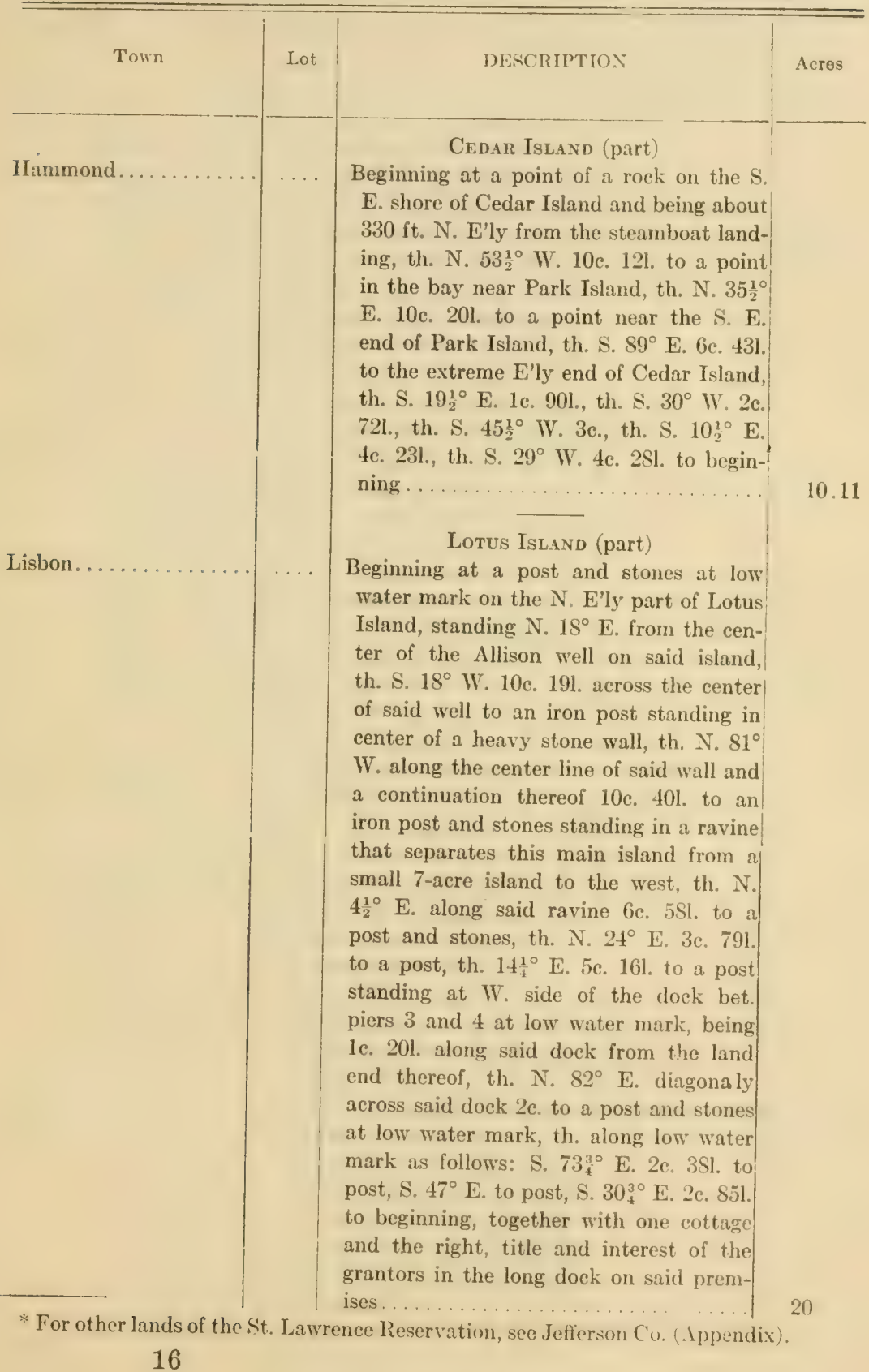


St. Lawrence County

\begin{tabular}{|c|c|c|c|}
\hline 'Town & Lot & DESCRIPTION & $\Lambda$ cres \\
\hline Lisbon. . & & 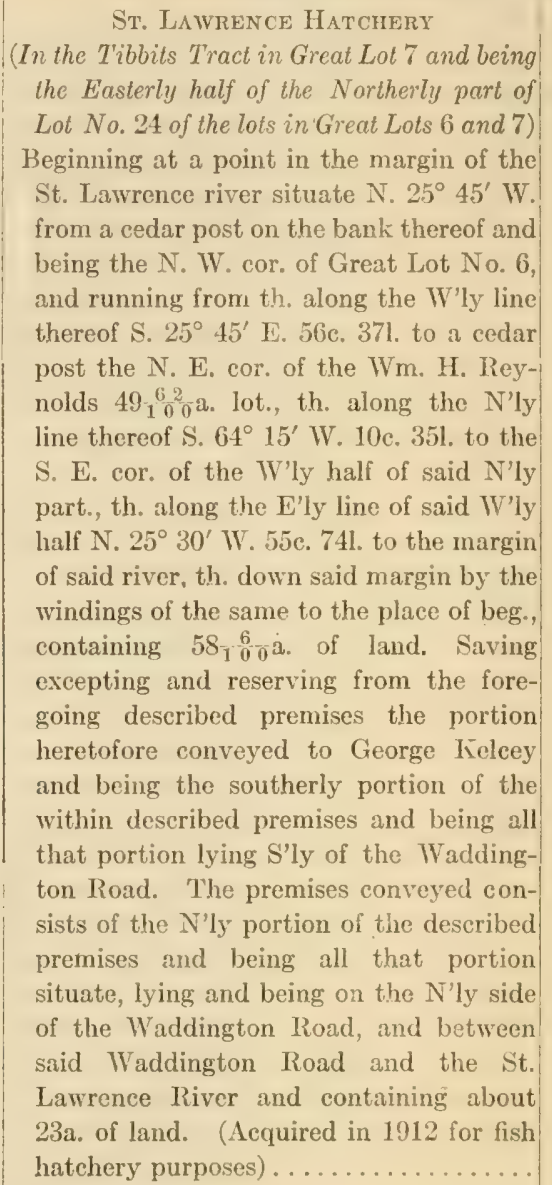 & 23 \\
\hline
\end{tabular}


Str. Lawrence County

\begin{tabular}{|c|c|c|c|c|}
\hline Town & Lot & DESCRIPTION & $\begin{array}{c}\text { Tax sales } \\
\text { or } \\
\text { source of title }\end{array}$ & Acres \\
\hline Hopkinton. . & $\ldots$ & $\begin{array}{l}\text { Hopkinton, Town of } \\
\text { Bd. beg. in center of the } \\
\text { highway leading from } \\
\text { Gaines Sheldon's land to } \\
\text { East Stockholm at a point } \\
\text { in the S. bounds of the town } \\
\text { of Stockholm, th. S'ly 21c. } \\
\text { 95l. to N. W'ly cor. of Asa } \\
\text { Sheldon's land, th. S. 11c. } \\
\text { 36l. to a stake \& stones, th. } \\
\text { W. along N. line of lands } \\
\text { lately under contract of sale } \\
\text { to Sheldon \& Stoddard, 49c. } \\
\text { 831. to Parker Converse } \\
\text { lands, th. N. along E. line } \\
\text { of said Converse lands } 8 \mathrm{c} \text {. } \\
\text { 90l. to a stake in the bounds } \\
\text { of Stockholm, th. E'ly along. } \\
\text { town line } 53 \mathrm{c} \text {. to beg...... }\end{array}$ & Mortgage, 1896... & 104.86 \\
\hline Pierrepont. . & $\ldots$ & $\begin{array}{c}\text { Macomb's Purchase } \\
\text { Great Tract } 3 \\
\text { Township } 3 \\
\text { (De Witt) } \\
\text { Bd. beg. at a point in center } \\
\text { of the highway and on the } \\
\text { town line between Colton } \\
\text { \& Pierrepont and the farms } \\
\text { owned and occupied by } \\
\text { Jesse Oakley \& Ezra Per- } \\
\text { kins, th. S. } 3^{\circ} 45^{\prime} \text { W. along } \\
\text { said town line } 17 \mathrm{c} .761 . \text { to } \\
\text { N. E. cor. of the Shirtliff } \\
\text { lot, th. N. } 86^{\circ} 15^{\prime} \text { W. along } \\
\text { said Shirtliff's N'ly line } 13 \mathrm{c} . \\
\text { 83l. to a stake, th. N. } 15 \mathrm{c} . \\
\text { 691. to center of aforesaid } \\
\text { highway and th. N. } 85^{\circ} \\
\text { east along center of said } \\
\text { highway } 15 \mathrm{c} .41 \mathrm{l} \text {. to beg... }\end{array}$ & Mortgage, 1909.. & 24.16 \\
\hline
\end{tabular}


St. Lawrence County

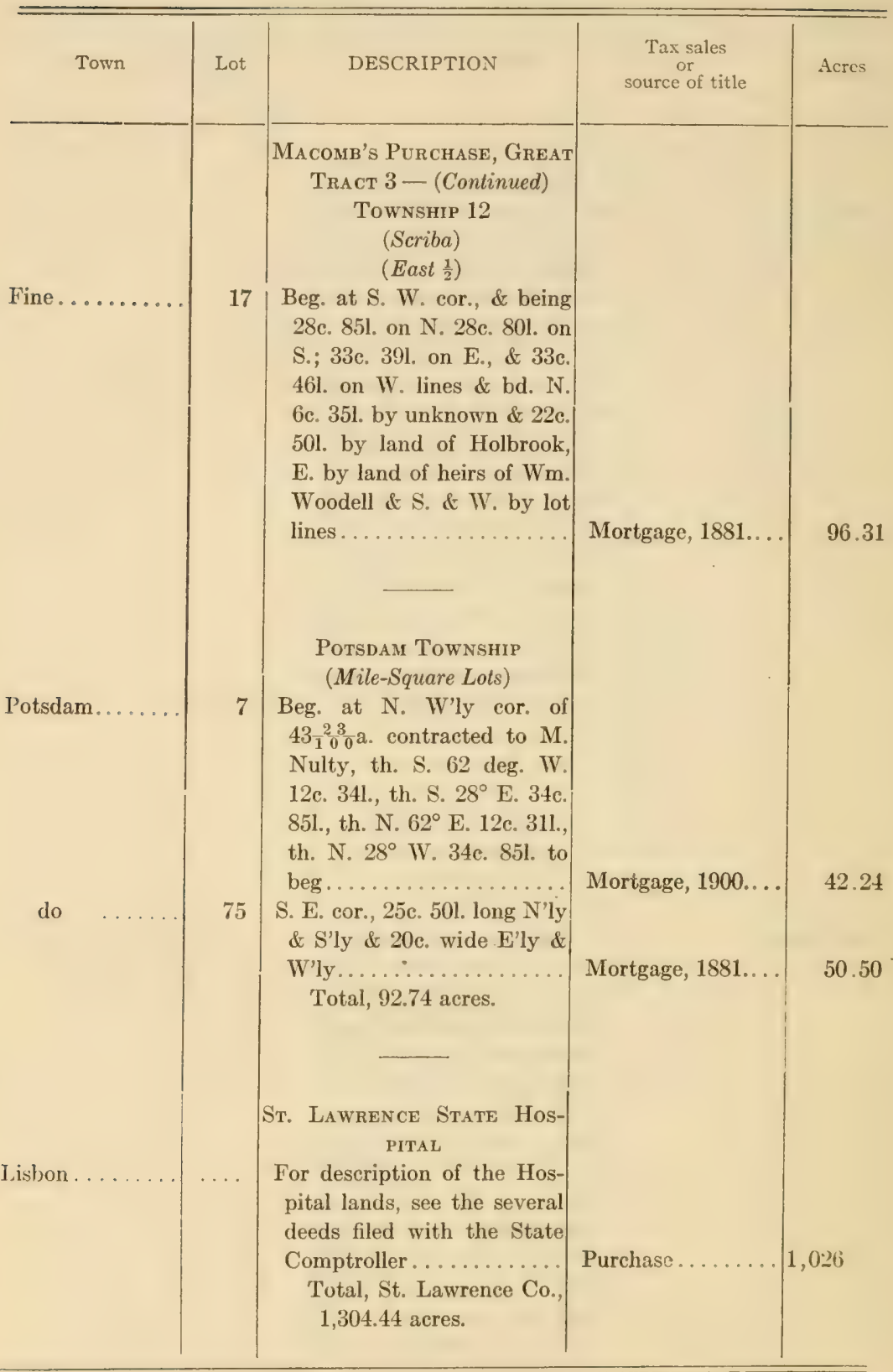




\section{SARATOGA COUNTY}

List of State Lands other than Forest Preserve

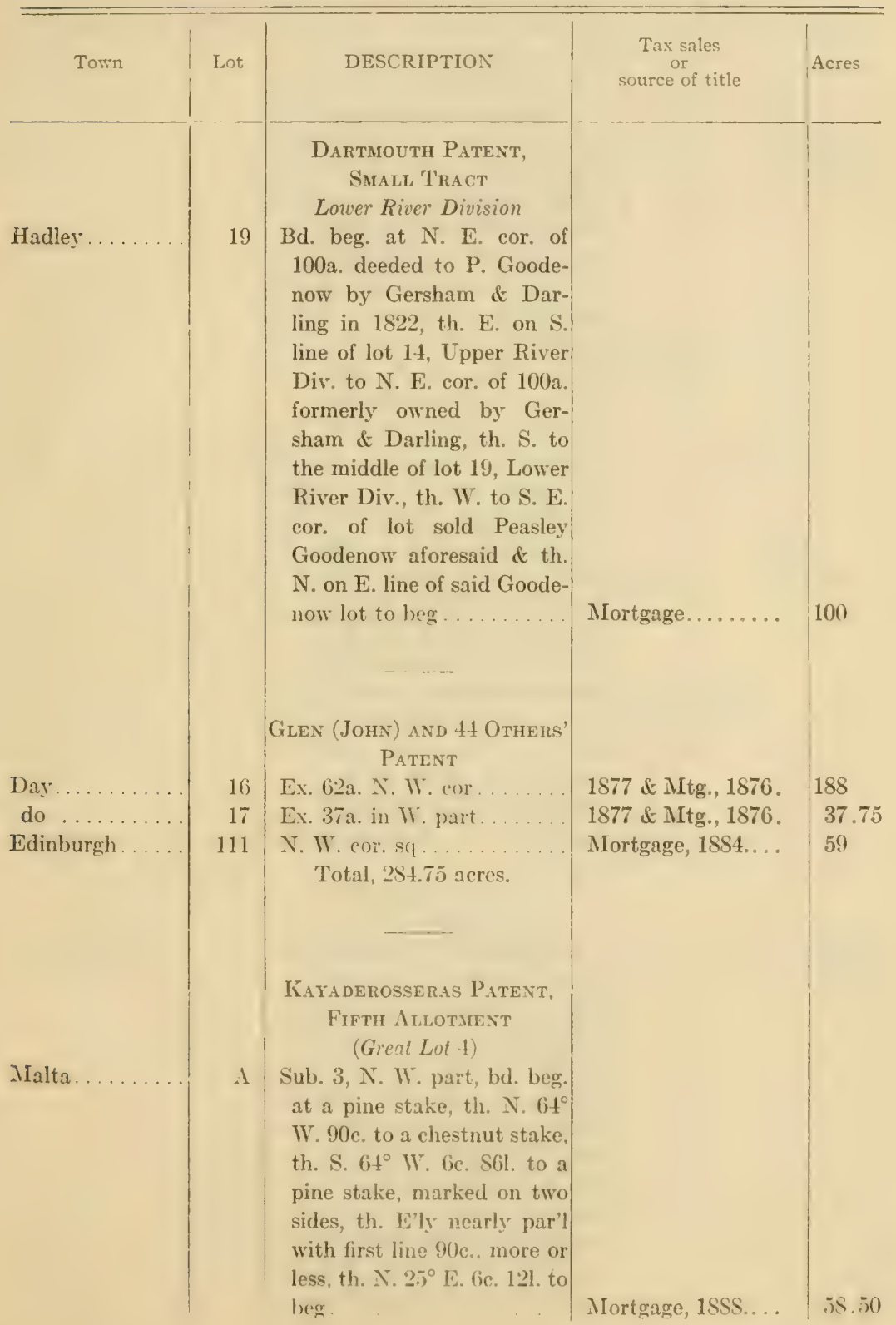


Saratoga County

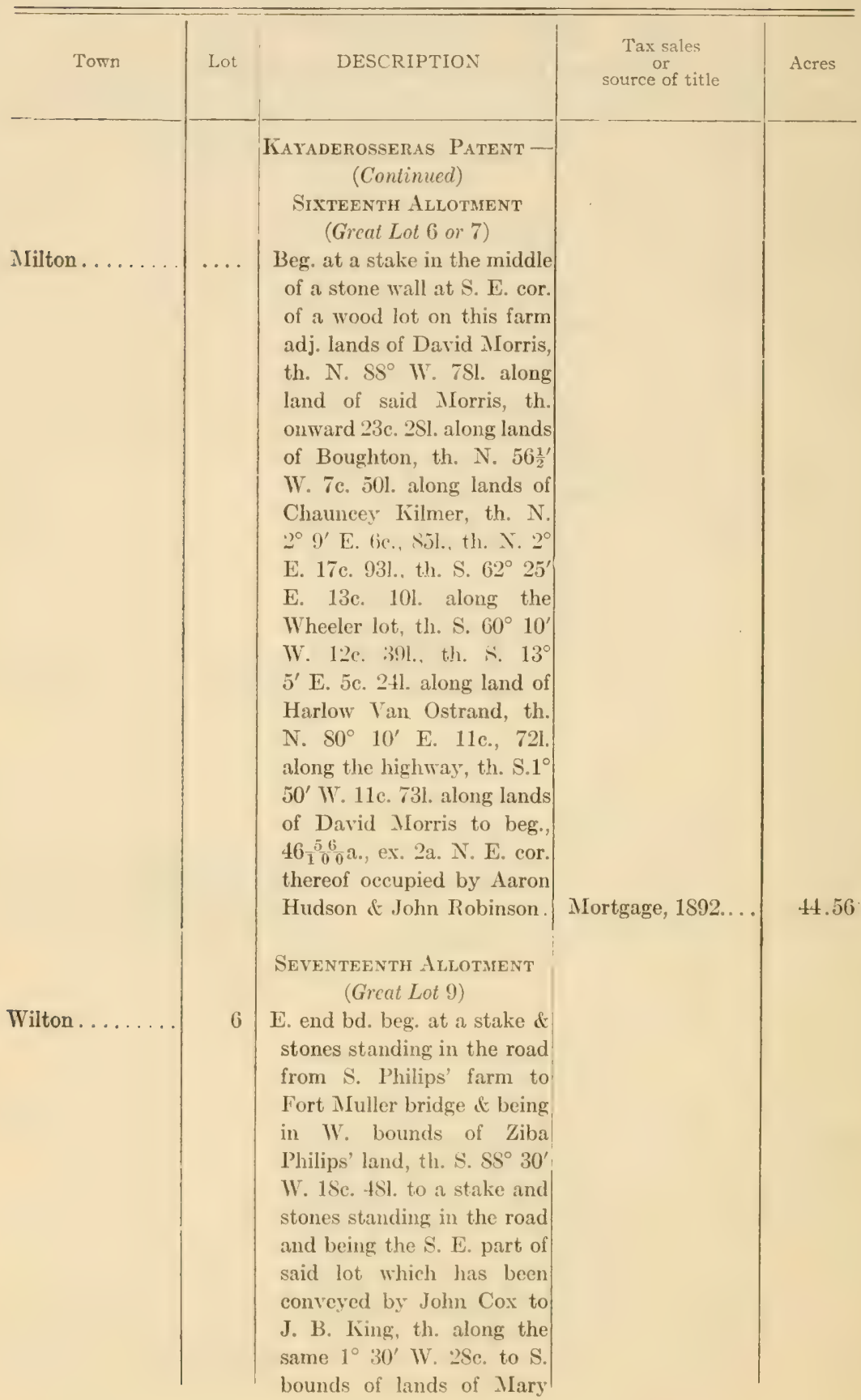




\section{Saratoga County}

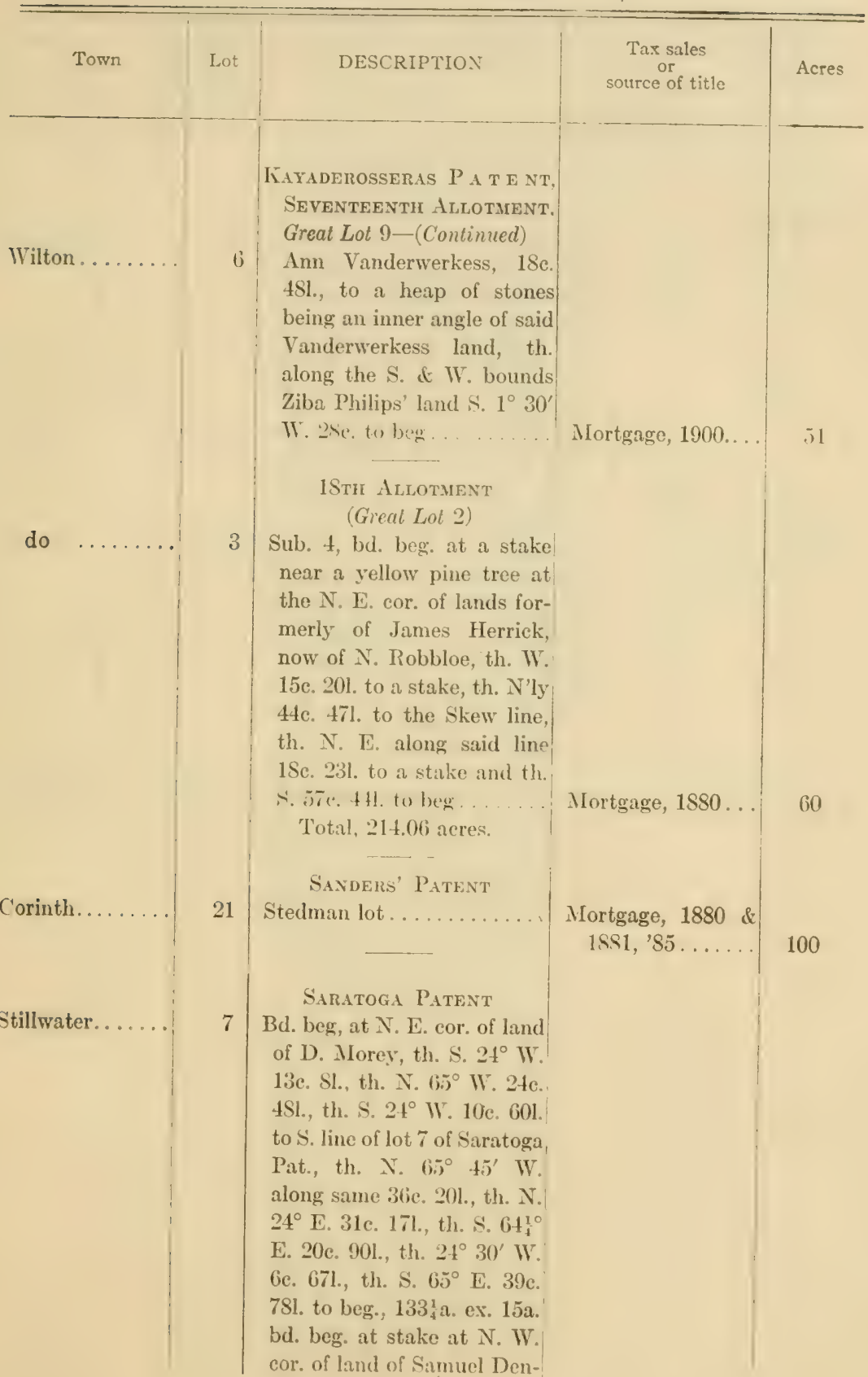


Saratoga County

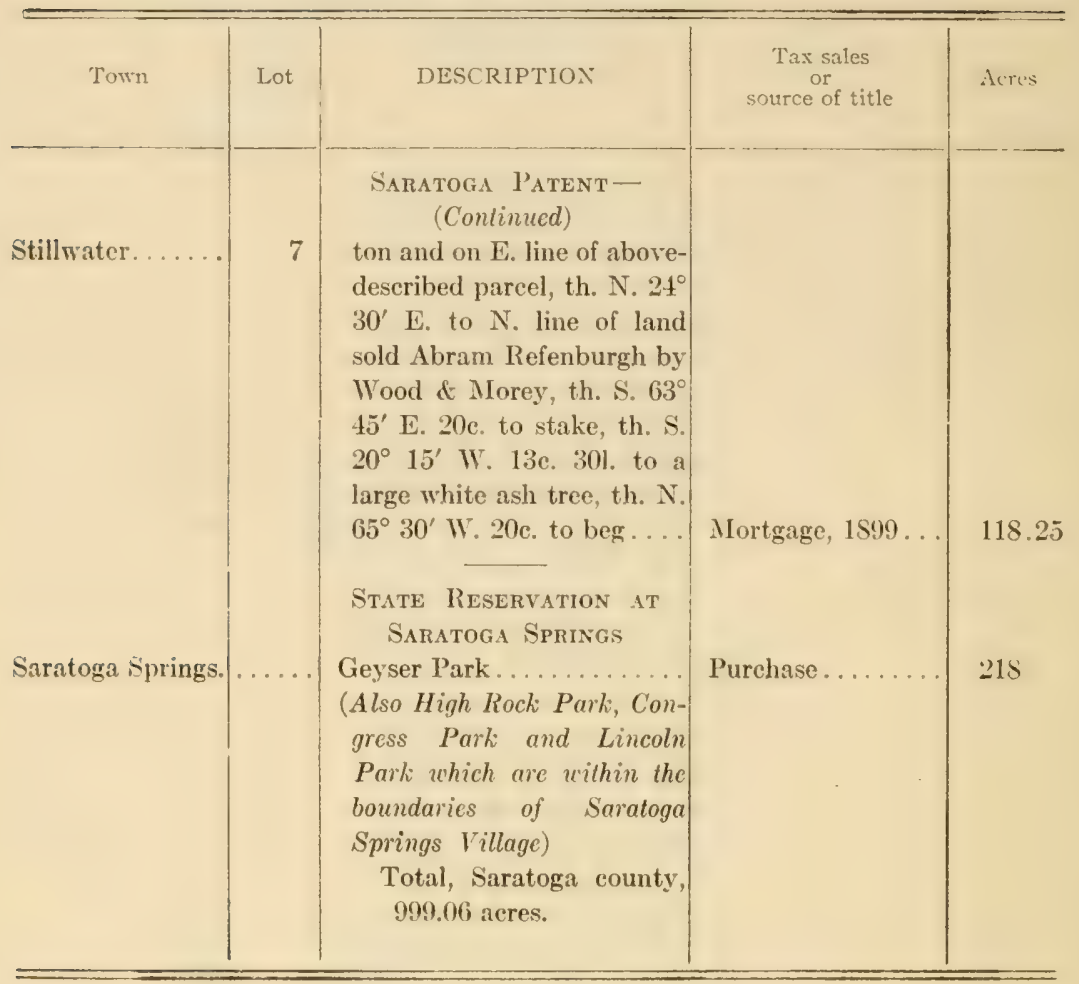


ULSTER COUNTY

List of State Lands other than Forest Preserve

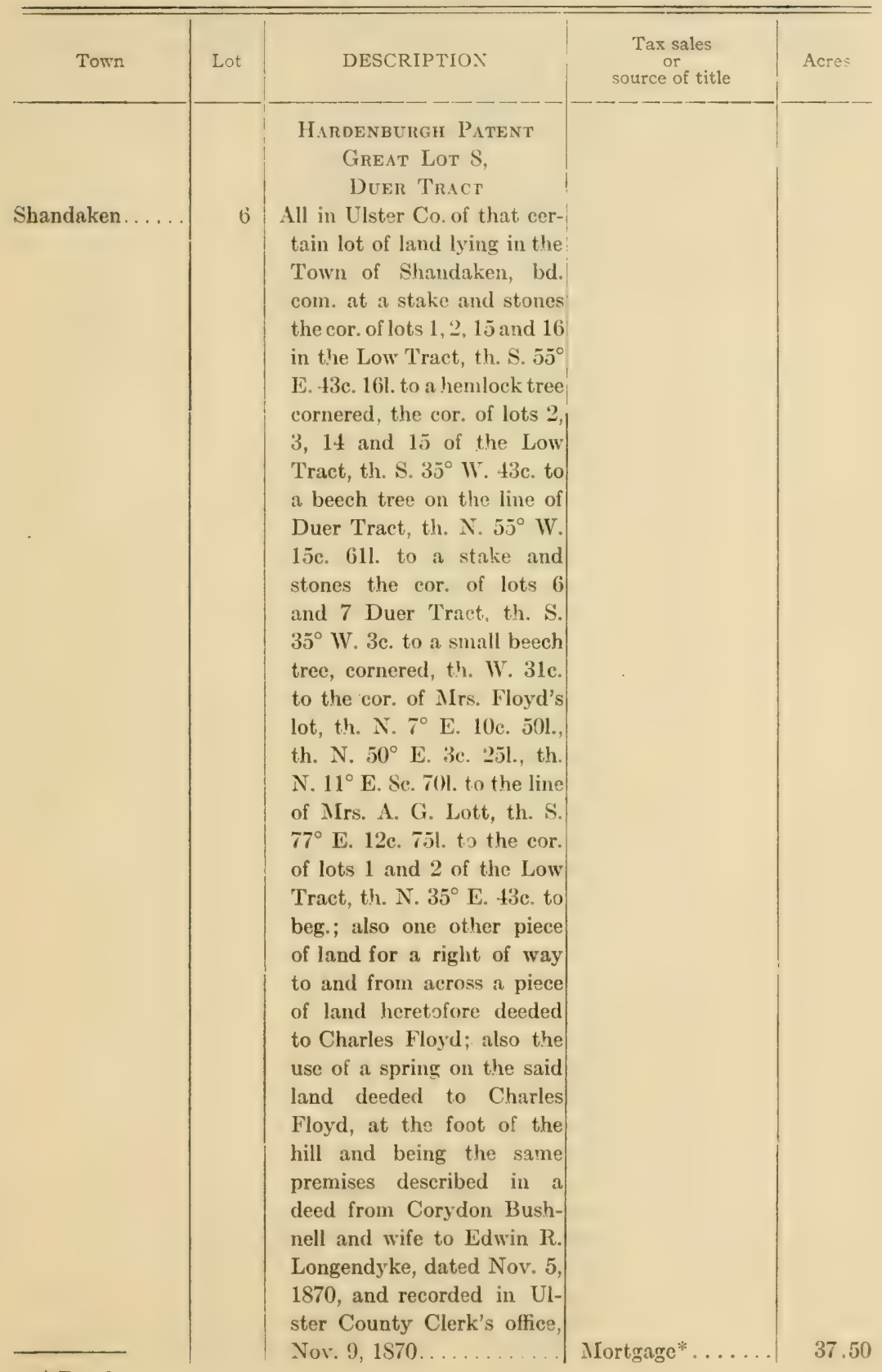


Ulsten County

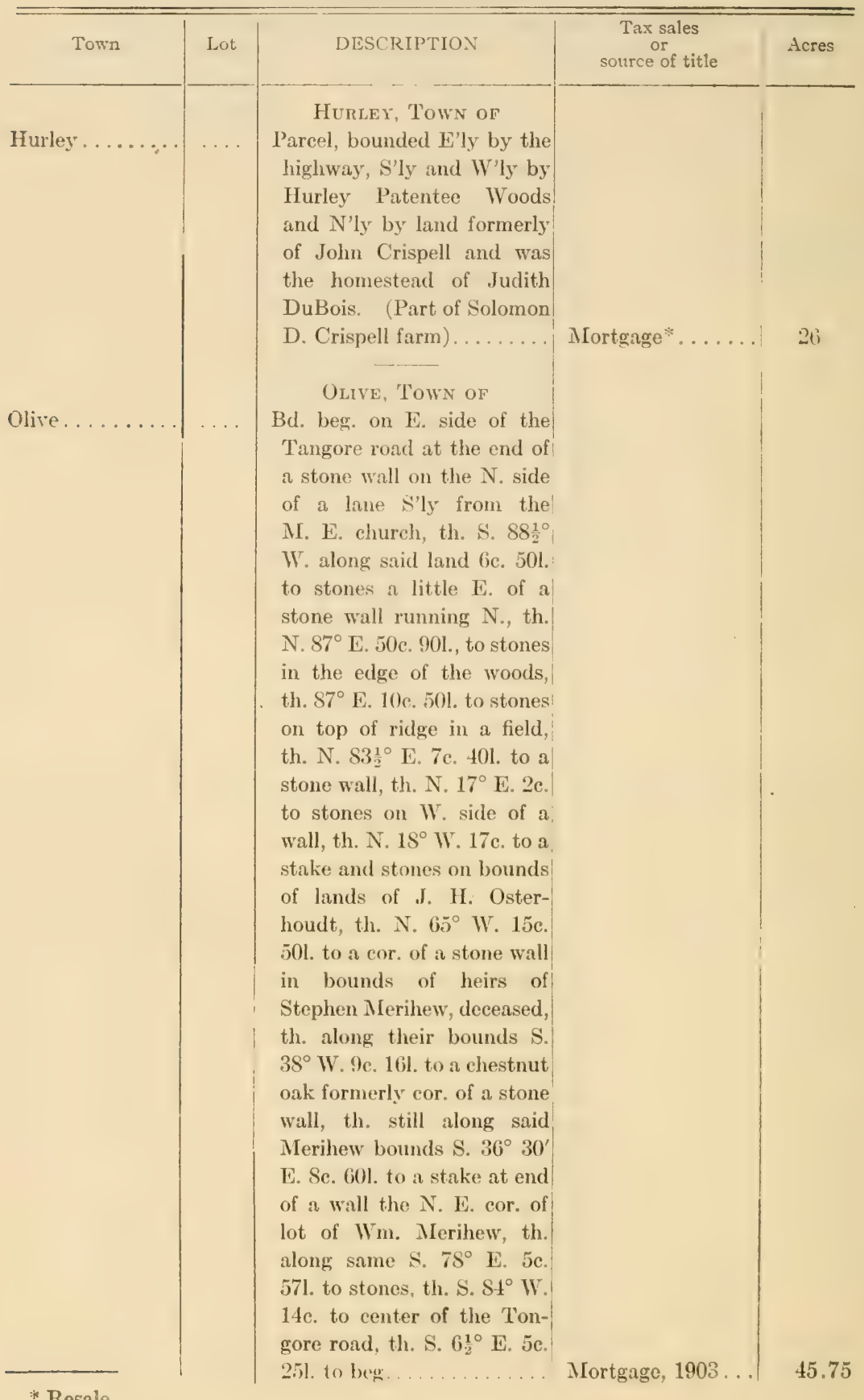




\section{Ulster County}

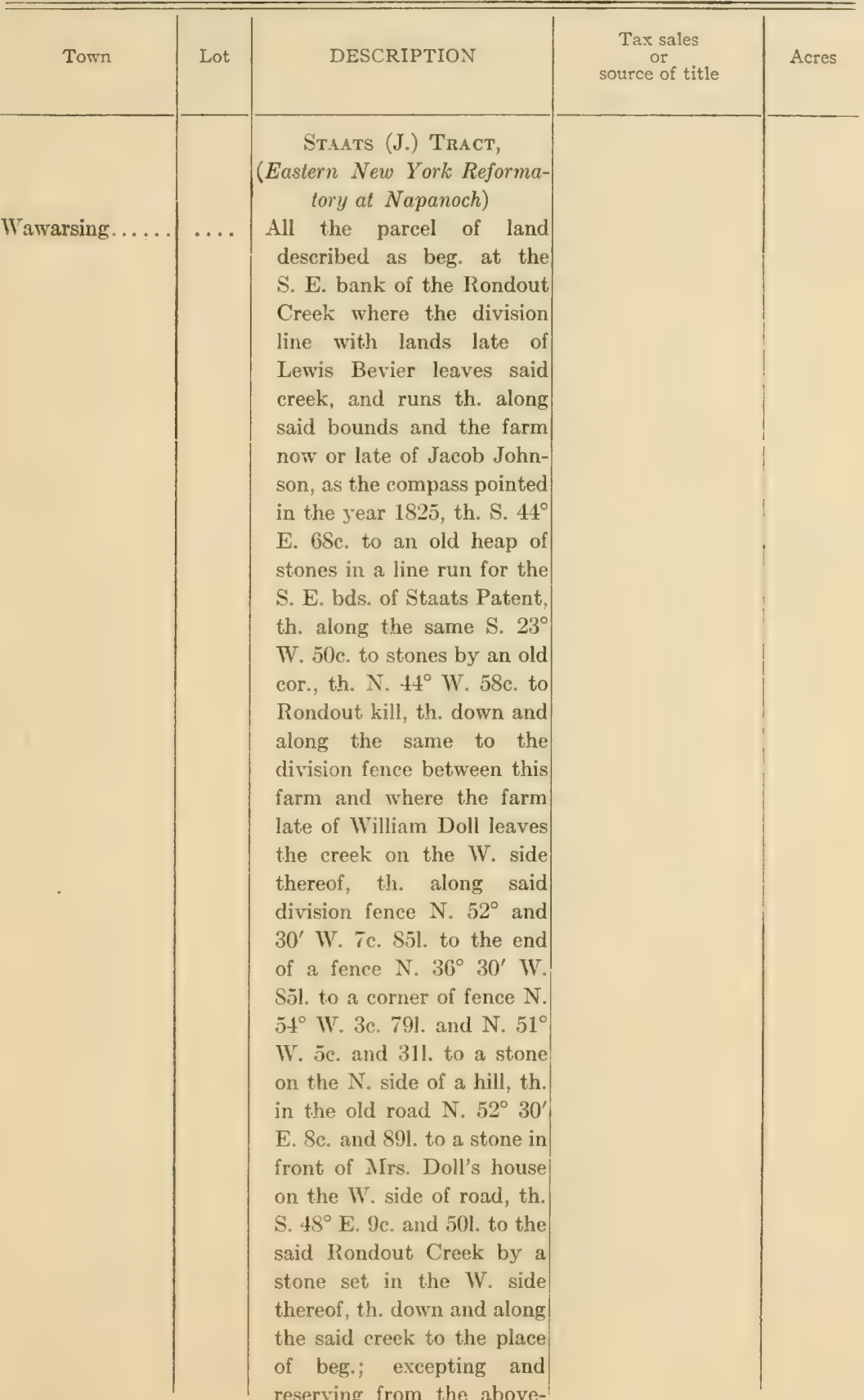




\section{Ulster County}

\begin{tabular}{|c|c|c|c|c|}
\hline Town & Lot & DESCRIPTION & $\begin{array}{c}\text { Tax sales } \\
\text { or } \\
\text { source of title }\end{array}$ & Acres \\
\hline Wawarsing. & $\ldots$ & $\begin{array}{l}\text { STAATS (J.) TRACT, (Eastern } \\
\text { New York Reformatory at } \\
\text { Napanoch) - (Continued) } \\
\text { described premises one lot } \\
\text { heretofore conveyed to } \\
\text { Wm. G. Hill, one lot here- } \\
\text { tofore conveyed to the } \\
\text { Del. \& Hudson Canal } \\
\text { Company and one lot here- } \\
\text { tofore to Adam Zwerval; } \\
\text { also excepting and reserving } \\
\text { all the lands on the W'ly } \\
\text { side of the Delaware \& } \\
\text { Hudson Canal......... } \\
\text { Parcel of land described as } \\
\text { Beg. at a point on the N. } \\
\text { E'ly boundary line of pre- } \\
\text { mises of the Eastern New } \\
\text { York Reformatory at an } \\
\text { iron pipe driven in the } \\
\text { ground on the boundary } \\
\text { line between premises of } \\
\text { Uriah E. Wood and the } \\
\text { New York, Ontario and } \\
\text { Western Railway, and run- } \\
\text { ning th. in a S. E'ly direc- } \\
\text { tion along the N. E'ly } \\
\text { boundary line of the premi- } \\
\text { ses of said Eastern New } \\
\text { York Reformatory and } \\
\text { across the Berme Road a } \\
\text { distance of 1,809.65 feet; } \\
\text { th. in a N. E'ly direction } \\
\text { and at right angles to the } \\
\text { last mentioned course a } \\
\text { distance of } 97 \text { feet to an } \\
\text { iron pipe driven in the } \\
\text { ground; th. in a N. W'ly } \\
\text { direction at right angles } \\
\text { to the last mentioned course } \\
\text { and in a line parallel to the } \\
\text { course first above men- } \\
\text { tioned a distance of } 775.55 \\
\text { feet to an iron pipe driven }\end{array}$ & Purchase. & 281 \\
\hline
\end{tabular}




\section{ULSTER County}

\begin{tabular}{|c|c|c|c|c|}
\hline Town & Lot & DESCRIPTION & $\begin{array}{c}\text { Tax sales } \\
\text { or } \\
\text { source of title }\end{array}$ & Acres \\
\hline Wawarsing. . & & $\begin{array}{l}\text { STAATS (J.) TRACT (Eastern } \\
\text { New York Reformatory at } \\
\text { Napanoch) - (Continued) } \\
\text { in the ground on the N. E'ly } \\
\text { side of the said Berme } \\
\text { Road; th. deflecting to } \\
\text { the right } 111^{\circ} 47^{\prime} \text { along the } \\
\text { said side of the Berme } \\
\text { road a distance of 53.85 } \\
\text { feet to an iron pipe driven } \\
\text { in the ground on the S. E'ly } \\
\text { side of a brook; th. N. W'ly } \\
\text { in a line parallel with the } \\
\text { course first above men- } \\
\text { tioned and a distance of } 147 \\
\text { feet therefrom } 985.05 \text { feet } \\
\text { to an iron pipe driven in } \\
\text { the ground on the line of a } \\
\text { wire fence on the boundary } \\
\text { line between premises of } \\
\text { Uriah E. Wood and the } \\
\text { New York, Ontario and } \\
\text { Western Railway Co.; th. } \\
\text { deflecting to the left } 62^{\circ} \\
2^{\prime} \text { along said wire fence a } \\
\text { distance of } 56.60 \text { feet to an } \\
\text { iron pipe driven in the } \\
\text { ground; th. deflecting to } \\
\text { the left } 4^{\circ} \text { and } 18^{\prime} \text { along } \\
\text { said wire fence a distance } \\
\text { of } 105.90 \text { ft. to an iron pipe } \\
\text { driven in the ground and } \\
\text { constituting the point or } \\
\text { place of beg. Subject to } \\
\text { the easement in the public } \\
\text { in and to the road or high- } \\
\text { way known as the Berme } \\
\text { Road.............. } \\
\text { Total, } 395.25 \text { acres. }\end{array}$ & Purchase. & .j \\
\hline
\end{tabular}


WARREN COUN'TY

List of State Lands other than Forest Preserve

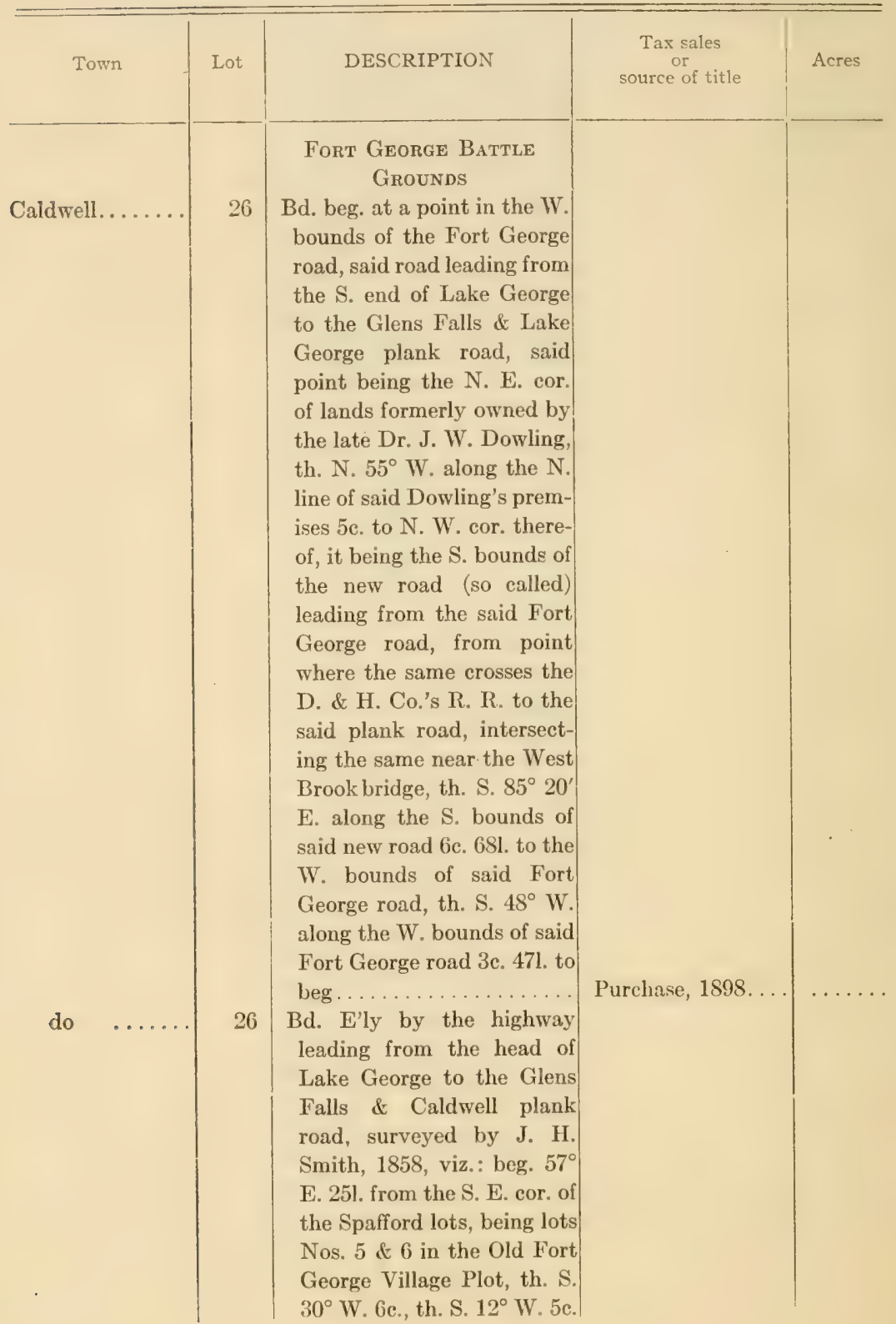




\section{Warren County}

\begin{tabular}{|c|c|c|c|c|}
\hline Town & Lot & DESCRIPTION & $\begin{array}{c}\text { Tax sales } \\
\text { or } \\
\text { source of title }\end{array}$ & Acres \\
\hline Caldwell. . & 26 & 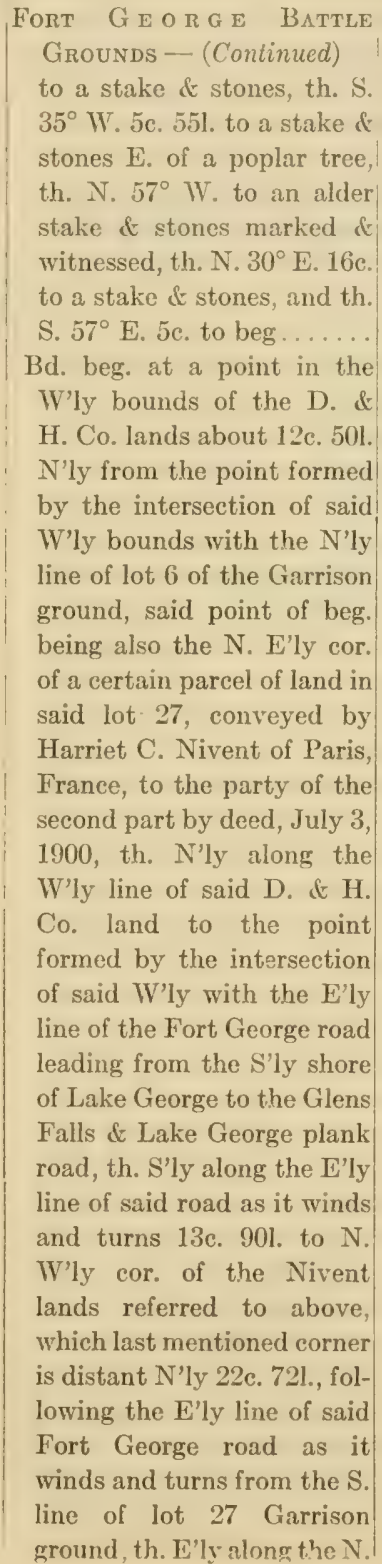 & Purchase, 1898. & 8.7 \\
\hline
\end{tabular}


Warren County

\begin{tabular}{|c|c|c|c|c|}
\hline Town & Lot & DESCRIPTION & $\begin{array}{c}\text { Tax sales } \\
\text { or } \\
\text { source of title }\end{array}$ & Acres \\
\hline $\begin{array}{l}\text { Caldwell... } \\
\text { do } \ldots\end{array}$ & 27 & $\begin{array}{l}\text { FonT G E O G E BATTLE } \\
\text { GrounDS-(Continued) } \\
\text { bounds of said Nivent lands } \\
\text { about } 8 \mathrm{c} \text {. 50l. to beg., in- } \\
\text { cluding the right, title, and } \\
\text { interest in that portion of } \\
\text { the said Fort George road } \\
\text { which bounds the premises } \\
\text { conveyed............. } \\
\text { Fort George lot bd. beg. at a } \\
\text { point in the center of the } \\
\text { highway known as the Fort } \\
\text { George road, leading from } \\
\text { the Glens Falls and Lake } \\
\text { George plank road to the } \\
\text { head waters of Lake George, } \\
\text { th. E'ly along N. line of lots } \\
5 \text { \& } 6 \text { Garrison ground (the } \\
\text { said N. line of lot } 5 \text { being } \\
\text { also the N. line of land } \\
\text { owned by the late W. H. } \\
\text { Bradley, and the N. line of } \\
\text { lot } 6 \text { being also the N. line } \\
\text { of premises now owned or } \\
\text { oceupied by Henry Cham- } \\
\text { bers) about } 17 \mathrm{c} \text {. to a point } \\
\text { in the W. bounds of land } \\
\text { owned by the D. \& H. Co. } \\
\text { R. R., th. N'ly along the } \\
\text { W. bounds of said R. R. } \\
\text { lands about } 12 \frac{1}{2} \text { c. to S. E. } \\
\text { cor. of lands owned by } \\
\text { Catherine T. R. Matthews } \\
\text { and Ann S. Van Cortlandt, } \\
\text { the above described Fort } \\
\text { Th. W'ly along S. bounds of } \\
\text { said Matthews \& Van Cort- } \\
\text { landt lands until the line } \\
\text { intersects the center line of }\end{array}$ & Purchase, 1900 . . & 17.50 \\
\hline
\end{tabular}


WASHINGTON COUNTY

List of State Lands other than Forest Preserve

\begin{tabular}{|c|c|c|c|c|}
\hline Town & Lot & DESCRIPTION & $\begin{array}{c}\text { Tax sales } \\
\text { or } \\
\text { source of title }\end{array}$ & Atwin \\
\hline Fort Ann. & 60 & $\begin{array}{l}\text { ARTILLERY PATENT. } \\
\text { Lying near Parish's lock bd. } \\
\text { N. by lands of I. V. Baker, } \\
\text { Jr., and Widow White, E. } \\
\text { by Wood Creek, S. by land } \\
\text { of Ariel West and Leonard! } \\
\text { Wellen, and W. by lands of } \\
\text { Milo Starks and I. V. Baker, } \\
\text { Jr., lately owned by Wm. } \\
\text { Morton, 70a. ex. about 22a. } \\
\text { on the W. side of the lot } \\
\text { which has been released and } \\
\text { now owned by R. R. Parish. } \\
\text { (Great Meadow Prison } \\
\text { Pands) } \\
\text { point on the E. bank of, } \\
\text { Wood Creek, where the } \\
\text { W'ly line of lot } 74 \text { in Ar- } \\
\text { tillery Patent intersects } \\
\text { said Wood Creek (said } \\
\text { point being distant } 175 \text { ft. } \\
\text { N. from the S. W'ly cor. of } \\
\text { the Albert Johnson farm, } \\
\text { measured on the W. of said } \\
\text { Johnson farm); running th. } \\
\text { S. } 175 \text { ft. to the S. W'ly cor. } \\
\text { of said Johnson farm; th. } \\
\text { E'ly along the S'ly line of } \\
\text { said Johnson farm } 39 \mathrm{c} \text {. } \\
388_{2}^{1} l . ; \text { th. N. on a line at } \\
\text { right angles to the said S'ly } \\
\text { side of lot } 74 \text { and along the } \\
\text { E'ly line of said Johnson } \\
\text { farm } 16 \mathrm{c} \text {. } 77 \frac{1}{2} \text {. more or less } \\
\text { to the N'ly side of said lot } \\
74 \text {; th. running E'ly along } \\
\text { the N'ly side of said lot } 74, \\
39 \mathrm{c} \text {. } 38 \frac{1}{2} l . \text { to the E. side of }\end{array}$ & Mortgage. . & 1.9 \\
\hline
\end{tabular}


Washington County

\begin{tabular}{|c|c|c|c|c|}
\hline Town & Lot & DESCRIPTION & $\begin{array}{c}\text { Tax sales } \\
\text { or } \\
\text { source of title }\end{array}$ & Acres \\
\hline Fort Ann. & $\ldots$ & $\begin{array}{l}\text { ARTILLER PATENT (Greal } \\
\text { Meadow Prison Lands)- } \\
\text { (Continued) } \\
\text { said lot 74; th. running S'ly } \\
\text { along the E'ly line of said } \\
\text { lot 74, 34c. } 621 \text {. more or less } \\
\text { to the S'ly side of said lot } \\
\text { 74; th. running W'ly along } \\
\text { the S'ly side of said lot } 74 \\
\text { to the W. side of land in or } \\
\text { about the year 1869, oc- } \\
\text { cupied by Benjamin Baker; } \\
\text { th. S'ly along the W. side of } \\
\text { said land formerly occupied } \\
\text { by Benjamin Baker to the } \\
\text { S. E'ly cor. of land con- } \\
\text { veyed by Ebenezer Martin } \\
\text { to Peter Comstock by deed } \\
\text { recorded in Liber pp. cp. } \\
\text { 520; th. W'ly along the S'ly } \\
\text { side of said land, S. } 86 \text { deg. } \\
\text { W. until it strikes the land } \\
\text { conveyed by Silas W. Briggs } \\
\text { and Paulina, his wife, to } \\
\text { George W. Baxter, by deed } \\
\text { recorded in Liber 19, cp. } \\
\text { 437; th. S. W'ly along the } \\
\text { S. E'ly side of said land } \\
\text { about 531 ft.; th. N. W'ly } \\
\text { along the S. W'ly side of } \\
\text { said land 165 ft. more or } \\
\text { less to the center of the } \\
\text { highway; th. S. W'ly along } \\
\text { the center of said highway } \\
\text { to a point where it strikes } \\
\text { the dividing line between } \\
\text { lots } 59 \text { and } 60 \text { in the Ar- } \\
\text { tillery Patent at the N. E'ly } \\
\text { cor. of said lot No. } 60 \text {; th. } \\
\text { running S'ly along the E'ly } \\
\text { side of said lot No. } 60 \text { to } \\
\text { the S'ly side of said lot No. } \\
60 \text { and th. W'ly along the } \\
\text { S'ly side of said lot No. } 60\end{array}$ & & \\
\hline
\end{tabular}


Wasinngton CotnTy

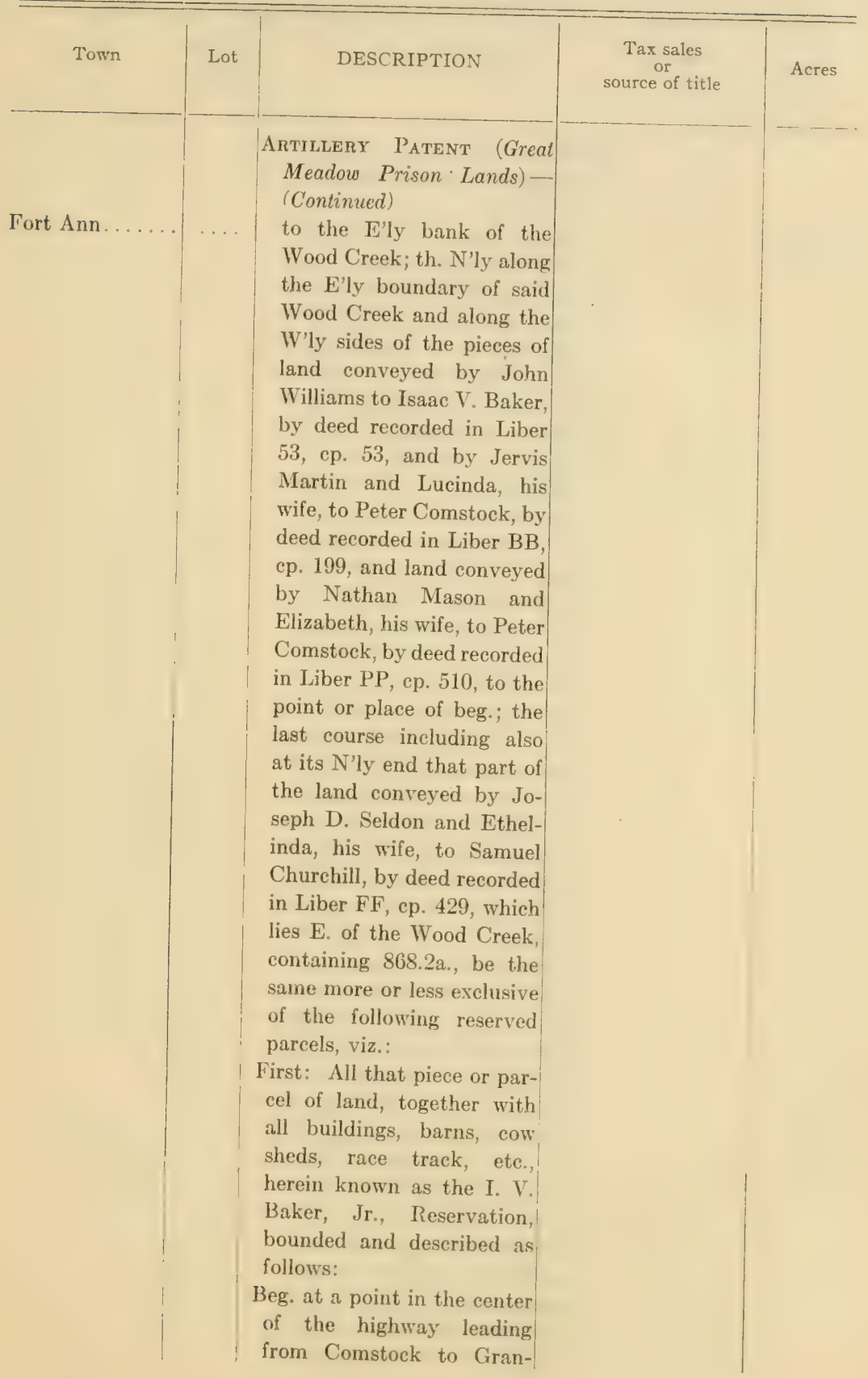


Washington County

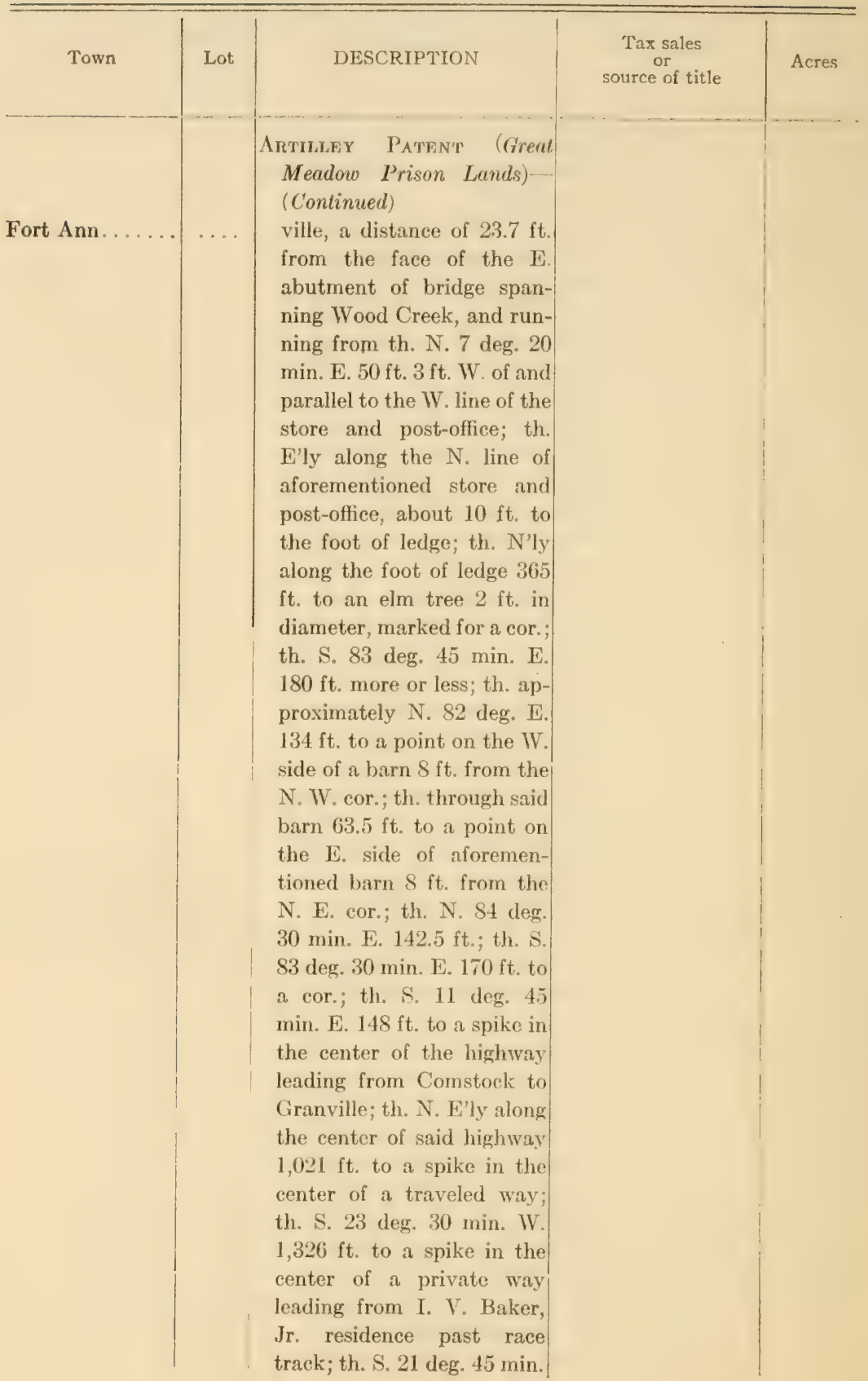


Washington Country

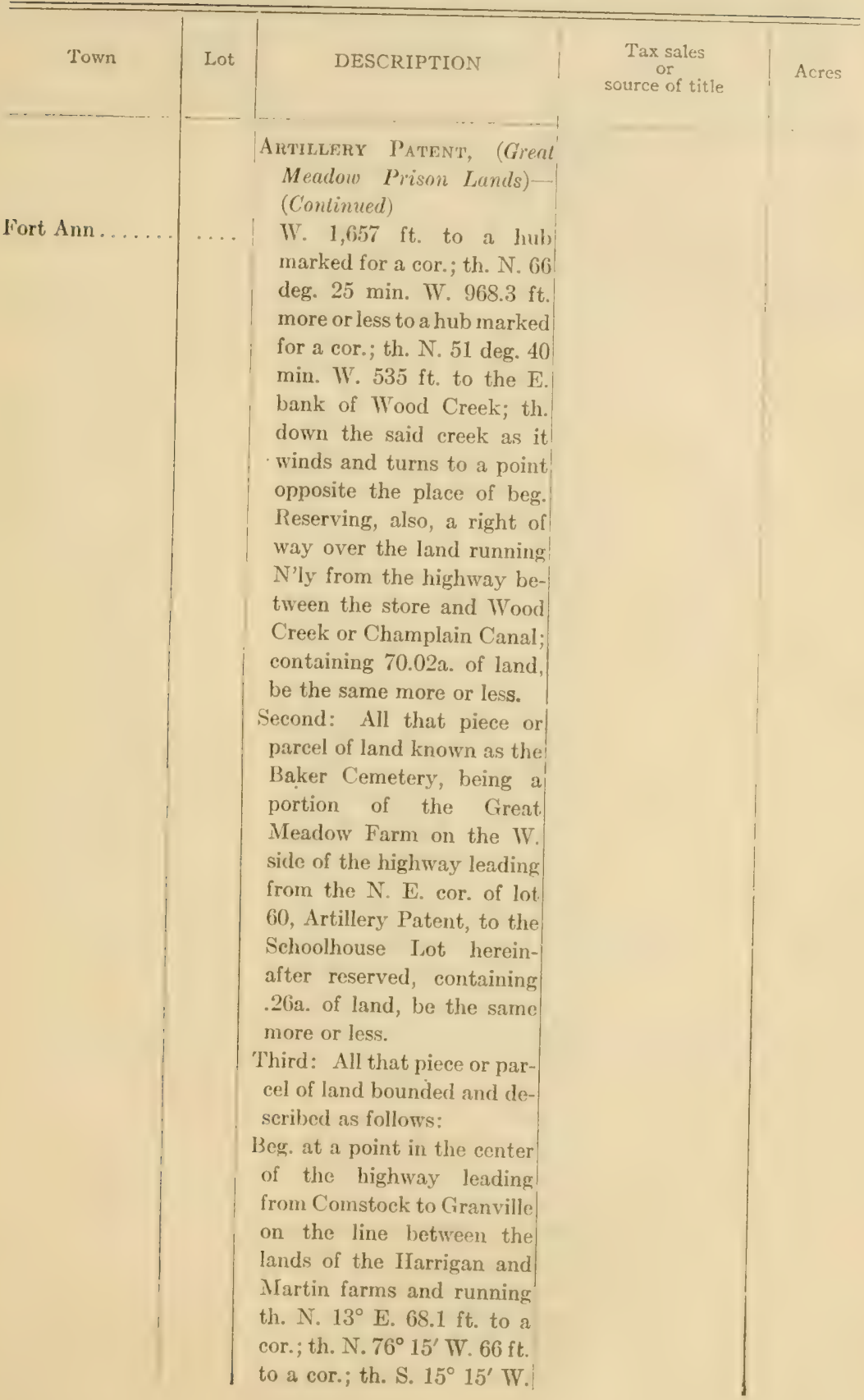


Washington CoUnty

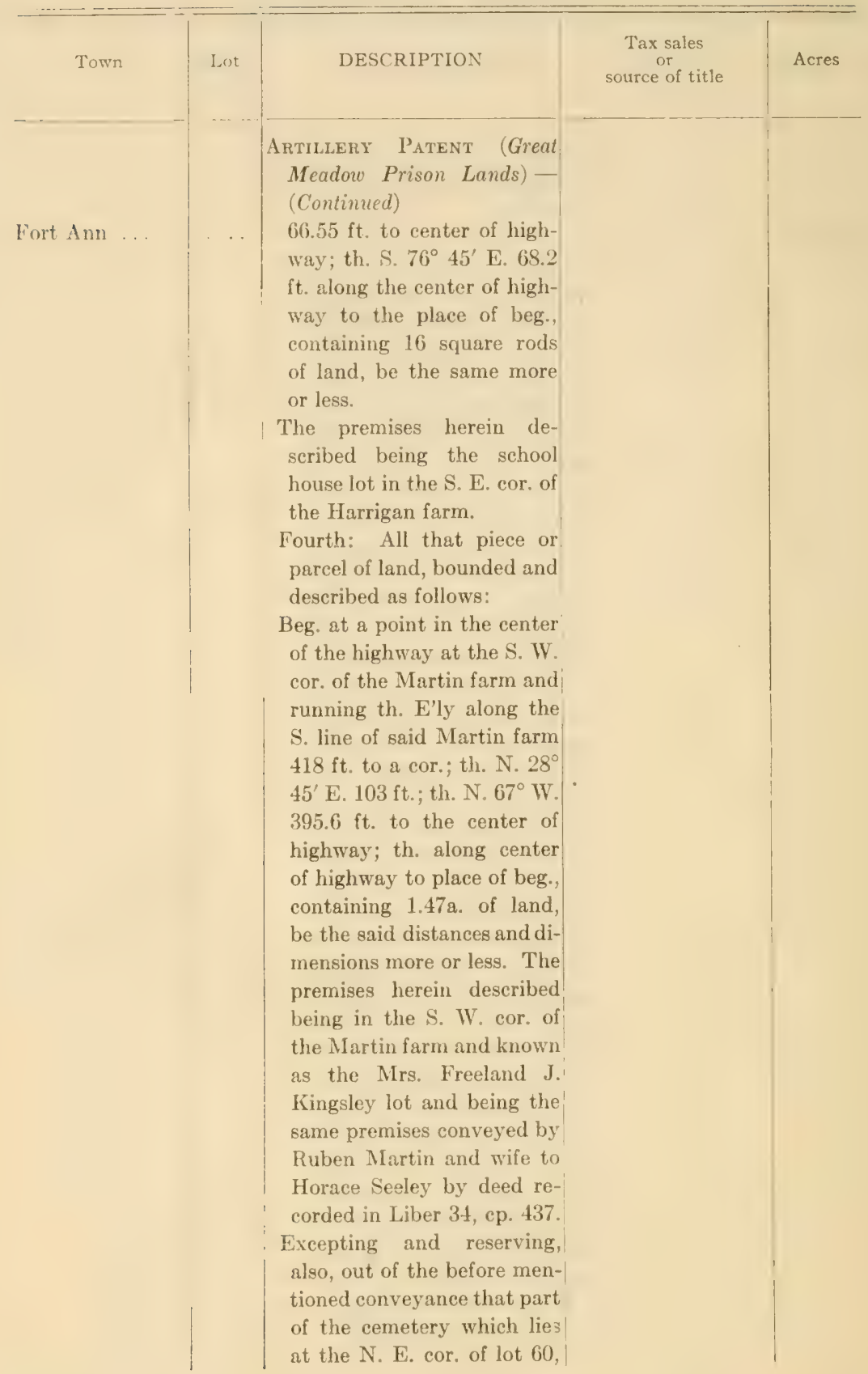


Washington CoUnty

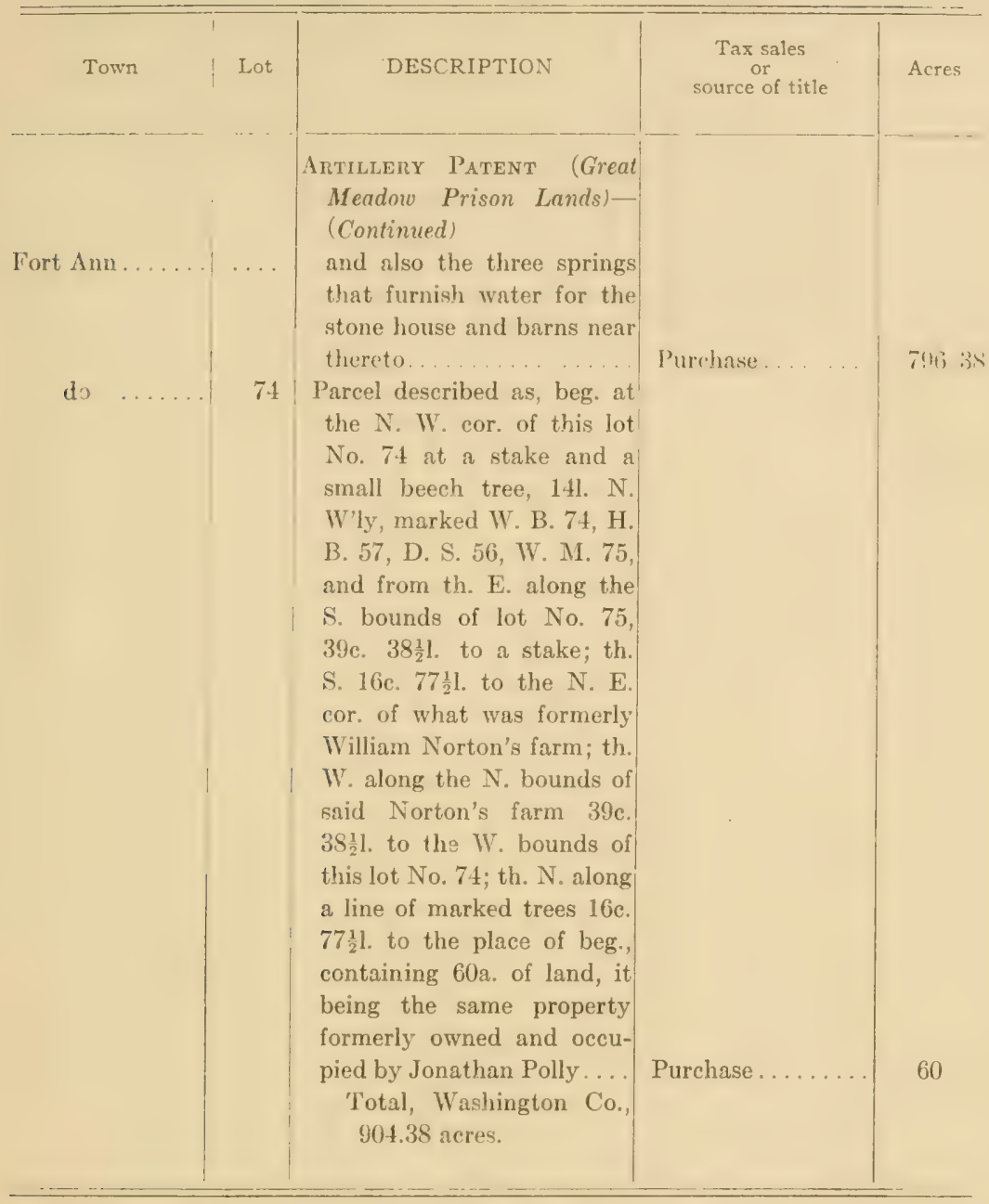








\section{LIBRARY OF CONGRESS

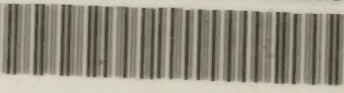 00014558901}

